\title{
Cementitious Waste Option Scoping Study Report
}

\author{
A. E. Lee \\ D. D. Taylor
}

Published February 1998
Idaho National Engineering and Environmental Laboratory Department
High-Level Radioactive Waste (HLW) Programs Department
Lockheed Martin Idaho Technologies Company Idaho Falls, Idaho 83415

Prepared for the U.S. Department of Energy Assistant Secretary for Environment Management Under DOE Idaho Operations Office

Contract DE-AC07-94ID13223

I. HWEHMON OF THIS DOCUMENT IS UNLMMI 


\section{DISCLAIMER}

Portions of this document may be illegible electronic image products. Images are produced from the best available original document. 


\begin{abstract}
A "Settlement Agreement" between the Department of Energy (DOE) and the State of Idaho mandates that all high-level radioactive waste (HLW) now stored at the Idaho Chemical Processing Plant (ICPP) on the Idaho National Engineering and Environmental Laboratory (INEEL) will be treated so that it is ready to be moved out of Idaho for disposal by a target date of 2035. This study investigates the nonseparations Cementitious Waste Option (CWO) as a means to achieve this goal. Under this option all liquid sodium-bearing waste (SBW) and existing HLW calcine would be recalcined with sucrose, grouted, canisterized, and interim stored as a mixed-HLW for eventual preparation and shipment off-Site for disposal. The CWO waste would be transported to a Greater Confinement Disposal Facility (GCDF) located in the southwestern desert of the United States on the Nevada Test Site (NTS). All transport preparation, shipment, and disposal facility activities are beyond the scope of this study. CWO waste processing, packaging, and interim storage would occur over a 5-year period between 2013 and 2017. Waste transport and disposal would occur during the same time period.
\end{abstract}

\title{
DISCLAIMER
}

This report was prepared as an account of work sponsored by an agency of the United States Government. Neither the United States Government nor any agency thereof, nor any of their employees, makes any warranty, express or implied, or assumes any legal liability or responsibility for the accuracy, completeness, or usefulness of any information, apparatus, product, or process disclosed, or represents that its use would not infringe privately owned rights. Reference herein to any specific commercial product, process, or service by trade name, trademark, manufacturer, or otherwise does not necessarily constitute or imply its endorsement, recommendation, or favoring by the United States Government or any agency thereof. The views and opinions of authors expressed herein do not necessarily state or reflect those of the United States Government or any agency thereof. 


\section{SUMMARY}

Treatment of high-level radioactive wastes (HLWs) at the Idaho Chemical Processing Plant (ICPP) at the Idaho National Engineering and Environmental Laboratory (INEEL) is mandated under a "Settlement Agreement" between the Department of Energy (DOE) and the State of Idaho. Among other things, the Settlement Agreement requires that this treated $\mathrm{HLW}$ be ready to be moved out of Idaho for disposal by a target date of 2035 .

As a method of compliance, the nonseparations Cementitious Waste Option (CWO) was proposed by Dr. D. D. Siemer et al., to fulfill the agreement. The proposed CWO process would receive liquid sodium bearing waste (SBW) from the tank farm, HLW calcine from the calcine solids storage facility (CSSF) via the calcine retrieval system, combine the two waste streams plus sucrose into a slurry, then inject the slurry into the modified existing NWCF calciner. The calciner would convert the injected slurry into recalcined solids as a mixed-HIW. The recalcined product would then be transferred to the Direct Cementitious Waste Option grouting facility to be mixed with special cement, sodium hydroxide, and water into a hydroceramic grouted waste matrix. The grouted matrix would be poured into HLW canisters for subsequent steam curing in an autoclave. The canisters would then be dewatered, degassed, sealed, and placed into interim storage to await transfer to a packaging facility and transport to a Greater Confinement Disposal Facility (GCDF) on the Nevada Test Site (NTS). The resulting hydroceramic grouted waste form was designed to be geochemically stable in NTS tuffacious, zeolitic, alluvial soil. The GCDF is a cost saving, proven, alternative to the National HLW Repository (i.e., Yucca Mountain or equal).

The primary thrust of this study is to provide a conceptual design evaluation of the CWO recalcination process and recalcine transport to the grouting facility. The calcine retrieval system, Maximum Achievable Control System (MACT) Compliance Facility, Direct Cementitious Waste Option (DCWO) grouting facility, and Interim Storage Facility (ISF) have been developed separately by others as integral parts of the CWO system. This study also presents the CWO design basis information/data, key assumptions, requirements, process descriptions, system descriptions, costs, uncertainties, Project Data Sheets, project-specific contingencies and variance, recommendations, and conclusions. The timeframe for the completion of all CWO activities from calcine retrieval to GCDF disposal will be 5 years starting in January 2013 and ending in December 2017.

The primary assumptions that may be CWO show stoppers are (a) classification of the GCDF for CWO mixed-HLW disposal, (b) Nuclear Waste Policy Amendment Act (NWPAA) will be revised to include the NTS-GCDF as a disposal site, (c) Nucleart Regulatory Commission (NRC) will license the NTS-GCDF for mixed-HLW, and (d) CWO listed RCRA hazardous waste can be delisted. 
The existing GCD Test conducted on the NTS in the 1980s disposed-of greater than class C waste, tritium, transuranic (TRU) waste, and classified low-level waste (LLW) in large diameter, deep boreholes. It is assumed that the NTS will develop the GCDF Waste Acceptance Criteria (WAC) for CWO mixed-HLW disposal based on a new Performance Assessment to be generated by Sandia National Laboratories (SNL). It is also assumed that the waste will be delisted before it can be transported from the INEEL to the NTS and placed in the GCDF. This assumption is based on the approval of a Resource Conservation and Recovery Act (RCRA) delisting petition for calcined wastes. A delisting petition will require the approval of the Environmental Protection Agency (EPA), the initiating state (Idaho), the receiving state (Nevada), and any state the waste will travel through. If the CWO waste cannot meet the GCDF WAC, then it will require processing for storage in the National HLW Repository, because there would be no other location to dispose of it.

The INEEL CWO canistered waste form, in the grouted, recalcine, condition cannot be placed in the National HLW Repository as-is, because it will not meet the vitrification requirement stated in the repository WAC. Vitrification is the transformation of a waste material into a glass matrix considered, at present, to be the Best Demonstrated Available Technology (DBAT). The waste must also be delisted as previously explained. Therefore, the CWO waste form must be shown to equal or exceed the HLW repository WAC requirements. It is assumed that a Determination of Equivalent Treatment (DET) petition for grouted waste will be approved by the EPA. For this reason, a variance has been developed for the CWO process whereby the CWO recalcined and grouted waste could be Hot Isostatically Pressed (HIP'ed) into a glassceramic waste form. The HIP'ed waste form is expected to meet or exceed the WAC of the National HLW Repository and would be comparable to vitrified glass. This variance is considered too costly for consideration as option. Refer to Section 8.1 for further information and EDF-CWO-001 in Appendix E.

The objective of this study was to scope, bound, estimate cost, and provide a final report for the entire CWO process. The processing facility consists of a calcine retrieval system, modified New Waste Calcining Facility (NWCF), a MACT (Maximum Achievable Control Technology) compliance facility, a grouting facility, and an interim storage facility (ISF). The processing sequence is shown schematically in the Process Flow Diagrams in Appendix B.

This study does not include: Cask design, purchase, or handling; preparation for shipping, transportation to the final disposal site, and the installation of the GCDF. These items are beyond the scope of this study and will be addressed by others at a future date.

The operating period for CWO processing and disposal will be 5 years starting in January 2013 and ending December 2017.

During the performance of this study, available information and empirical data were sought, identified, and evaluated relating to: (a) existing calcine, (b) sodium-bearing waste (SBW), (c) sucrose, (d) recalcined waste (its composition, blending, and total volume), (e) modifications to the existing calciner for the recalcination process, $(f)$ recalcination process, (g) proposed grouting process and facility, (h) Interim Storage Facility (ISF), and (i) requirements and regulations that will apply. Where information and data were not available, engineering judgment was used in generating assumptions. These items are documented in this report. Costs were estimated for required facilities, equipment modification, process equipment, and operations. Failure modes were noted and project risks were identified and evaluated. Project data were estimated and summarized in Project Data Sheets. Additional information and data can be found in the references and attached appendices. 


\section{CONCLUSIONS}

At a scoping level, this study investigated the calcination redesign of the NWCF and indicates that the existing calcine and liquid SBW can be processed into a recalcined product suitable for grouting. The grout matrix poured into canisters can be processed into a durable hydroceramic waste form for storage, transport, and disposal. All waste canisters processed by the CWO can be placed into interim storage, and should be acceptable for GCDF disposal when and if the major assumptions for GCDF classification, RCRA de-listing of waste, waste treatment by grouting, NRC HLW licensing of the NTS-GCDF, and DET approval become fact. It is assumed that the CWO process can be finished within a 5-year timeframe (2013 through 2017) using the production schedules established in this report.

The CWO reasons for recalcining stored $\mathrm{HLW}$ calcine and liquid SBW with sucrose are: (a) to solidify remaining SBW in the tank farm, (b) remove nitrates from the calcine and SBW by about $90 \%$, (c) remove approximately $99 \%$ of mercury from existing calcine and SBW, (d) to blend all wastes and minimize composition variations in the final grouted waste form, (e) redistribute the alkali metals (i.e., sodium and potassium) present in relatively high concentrations in recently-produced calcines and SBW, and (f) reduce the existing calcine and SBW volume by nearly $10 \%$.

Approximately $166,134 \mathrm{ft}^{3}\left(4,705 \mathrm{~m}^{3}\right)$ of recalcined HLW would be generated from existing calcine and liquid SBW. The recalcined waste would be combined with sodium hydroxide, silica-containing cold additives, and water to produce about 404,829 cubic feet (11,625 cubic meters) of grouted-recalcine HLW.

Approximately 15,924 grouted recalcine waste filled canisters would be produced by the Grout Facility for the Nonseparations Cementitious Waste Option. This quantity is based on the use of the Savannah River Site HLW canister (2 foot diameter by 9 foot 10 inches tall) with a nominal waste fill volume of 25.4 cubic feet ( 0.72 cubic meters).

The Total Project Cost (TPC) estimate for the CWO process from calcine retrieval to interim storage, including utilities/infrastructure is $\$ 2,797,920$ with a life-cycle cost (LCC) of $\$ 2,628,901$.

As a variation, in case a GCDF will not take the CWO waste and the waste must be stored in the National HLW Repository, the CWO will add a HIP process. The HIP process would be designed to reduce grouted recalcine final waste volume and provide a waste form similar to vitrified glass. The HLW repository will only accept vitrified waste products in approved containers. The HLW Savannah River Site (SRS) canister used in this study for CWO waste disposal/storage is assumed to be an approved container. The HIIP process would occur over a 20-year timeframe regardless of the 5-year CWO waste production schedule duration. The HIP containers loaded with grouted waste would be placed in the ISF stored until the order to HIP was given. After the HIP process the HIP'ed containers (about 31,000) would be canisterized (about 10,334 containers) and transferred to interim storage awaiting transport to the a final disposal site (e.g., the NTS GCDF or Yucca Mountain). The cost of this variation as a viable waste modification method is too high for the value provided. The TPC for this variance is estimated to be $\$ 3,230,746,000$, with an LCC of approximately $\$ 3,144,080,000$ (1998 dollars) above and beyond the cost of the CWO, over a 20-year timeframe. This variation was developed to show that it was considered; however it is not contemplated to be a viable alternative. 
The CWO process, developed for the treatment, transport preparation, and interim storage of recalcined mixed $\mathrm{HLW}$ within a five year time frame is considered technically and operationally feasible. However, the expense does not appear to justify the advantage gained. For instance, the waste reduction would be less than 10 percent by mass. The most significant advantage of the CWO process is the extraction of most of the mercury and nitrates contained in the existing calcine and liquid sodium bearing waste. The primary reason that mercury and nitrates should be removed from the final waste form is that their presence could be detrimental to the grouting process and final waste form acceptance. Existing calcines and liquid SBW are estimated to contain approximately 61,000 pounds of elemental mercury and once amalgamated using sulfur the mass weight would be about 81,000 pounds.

The amalgamated mercury (cinnabar) from the CWO would technically be an LDR acceptable form for shallow land burial; however, the compound may be radioactive making it a mixed-LLW. It is assumed that the remote waste management complex (RWMC) would accept the waste for disposal.

Construction and operations activities required to conform to the CWO schedules could cause minor environmental impacts. However, no specific impact was determined to be serious.

The HLW processing option that will be implemented will be announced in the Record of Decision (ROD) to be released in late 1999. The final disposal/storage location for INEEL waste must be determined before ROD release because it is a critical issue to the final waste processing option.

\section{RECOMMENDATIONS}

This study has identified a number of technical concerns that could affect the feasibility of the CWO alternative. Based on these concerns, the following recommendations are made:

Additional studies should be initiated to evaluate the method of recalcination and calciner modifications plus any possible improvements for waste transfer, handling, grouting, and storage.

A proof-of-principal grout facility should be funded for research and characteristic studies before full-scale grout facility operations using nonradioactive or simulated calcine material.

The CWO HIP variance should not be used due to high cost. 


\section{ACKNOWLEDGMENTS}

The following team of LMITCO personnel made significant contributions of their time and expertise to this study. Their work is reflected throughout this report and its appendices:

\begin{tabular}{|l|l|}
\hline Allen Lee & $\begin{array}{l}\text { CWO Preliminary Study Technical lead, final report development, DCWO } \\
\text { grout facility review, Risk Assessment, Assumptions development, HIP } \\
\text { variance EDF, and closure. }\end{array}$ \\
\hline Dean Taylor & $\begin{array}{l}\text { Technical leadership, design basis, assumption and requirement definition, } \\
\text { study of engineering issues, schedule, and NWCF recalcination design } \\
\text { concepts. }\end{array}$ \\
\hline $\begin{array}{l}\text { Howard Forsythe } \\
\text { Dave Thompson }\end{array}$ & $\begin{array}{l}\text { NWCF Calciner revision and redesign for CWO Recalcine operations and } \\
\text { transfer, and various support studies. }\end{array}$ \\
\hline $\begin{array}{l}\text { Tracy } \\
\text { Langenwalter }\end{array}$ & $\begin{array}{l}\text { AE Facility design and development of site, plot, and floor plans plus } \\
\text { sections for the CWO. }\end{array}$ \\
\hline Ancil Gibbs & Drafting. \\
\hline $\begin{array}{l}\text { Ron Averill } \\
\text { Bob Evans } \\
\text { John Duggan }\end{array}$ & Instrumentation and control, electrical power. \\
\hline $\begin{array}{l}\text { Jack Prendergast } \\
\text { Bob Jamison }\end{array}$ & Manpower and spatial requirements. \\
\hline Jilla Banaee & Applicable regulations, requirements. \\
\hline $\begin{array}{l}\text { Rick Adams } \\
\text { Bruce Wallace } \\
\text { John Baker }\end{array}$ & Cost estimate. \\
\hline Bob Turk & Life-cycle cost estimate. \\
\hline $\begin{array}{l}\text { Gary Wilson } \\
\text { Rod Kimmit }\end{array}$ & $\begin{array}{l}\text { Information input and development of the Environmental Impact Summary } \\
\text { and Project Data Sheets. }\end{array}$ \\
\hline $\begin{array}{l}\text { Paul Bayless } \\
\text { Jim Cozzoul }\end{array}$ & Risk assessment and Failure Modes \\
\hline Henry Peterson & Radiation and shielding calculations \\
\hline $\begin{array}{l}\text { Ron Dafoe } \\
\text { Syl Losinski }\end{array}$ & Direct Cementitious Grout Facility (DCWO) \\
\hline $\begin{array}{l}\text { John Rawlins } \\
\text { Byron Blakely }\end{array}$ & Interim Storage Facility \\
\hline Sara Gifford & Calcine Retrieval and Transport \\
\hline
\end{tabular}




\section{CONTENTS}

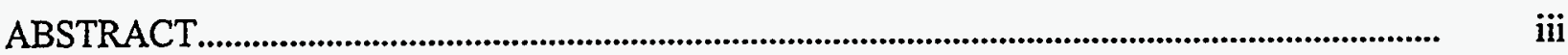

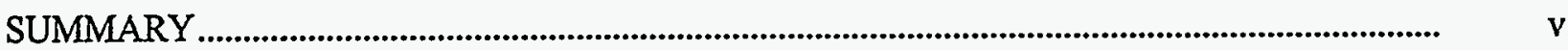

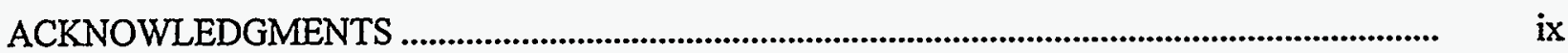

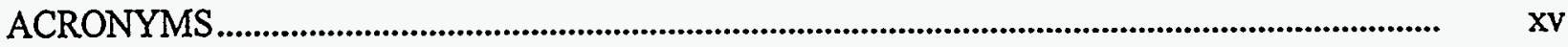

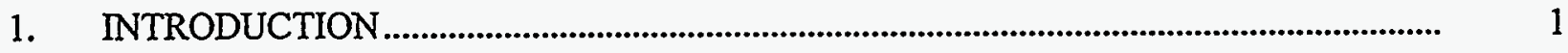

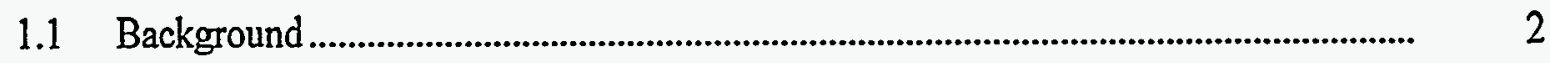

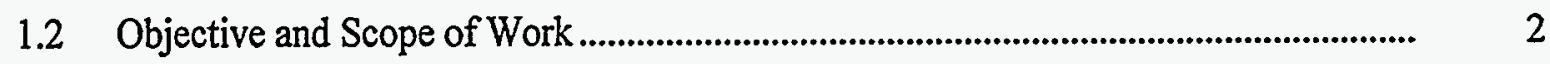

1.3 The Greater Confinement Disposal Facility .......................................................... 3

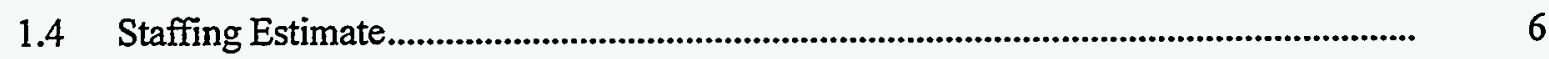

2. DESIGN BASIS/KEY ASSUMPTIONS/REQUIREMENTS ........................................... 7

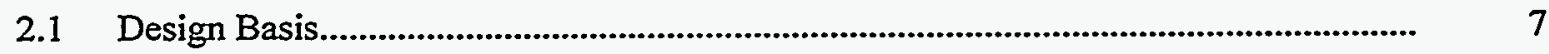

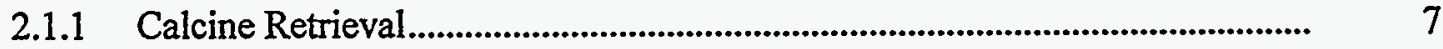

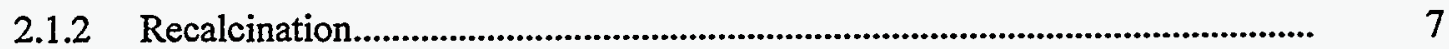

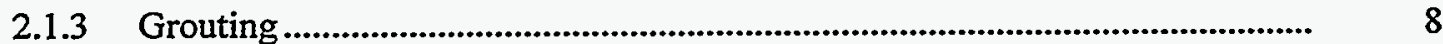

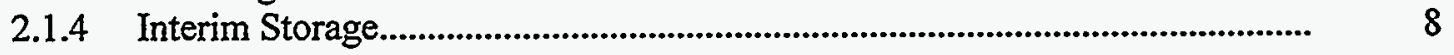

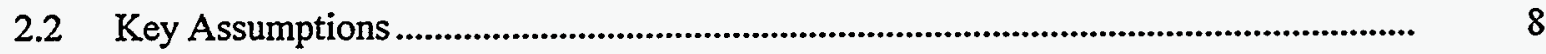

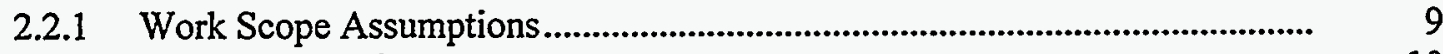

2.2.2 Study Assumptions ........................................................................................ 10

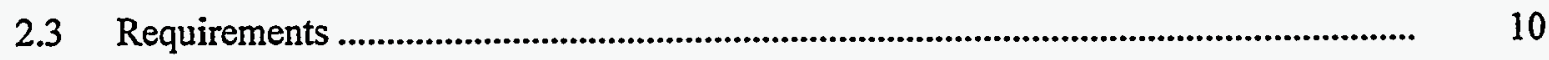

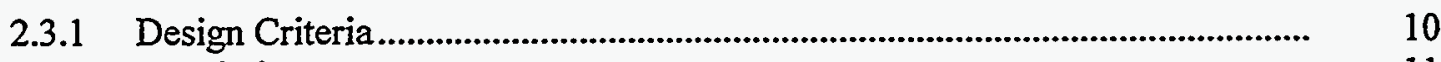

2.3.2 Regulations .................................................................................................... 11

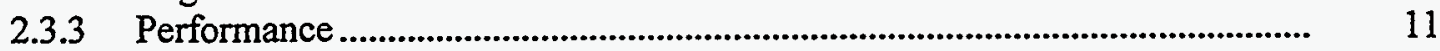

2.3.4 Applicable Codes and Standards....................................................................... 12

2.3.5 HLW Repository WAC........................................................................................ 12

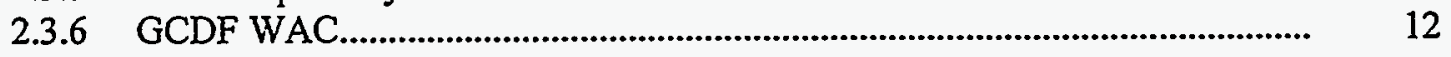

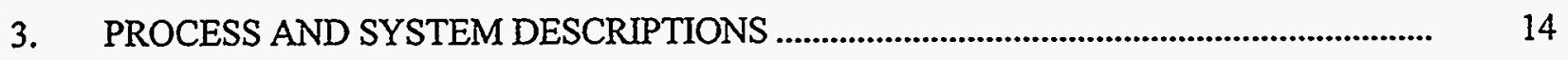

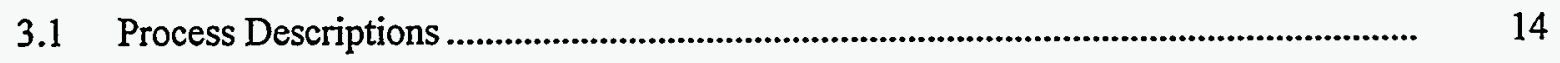

3.1.1 Calcine Retrieval............................................................................................ 14

3.1.2 Sodium Bearing Waste Liquid Retrieval .......................................................... 15

3.1.3 Recalcination.......................................................................................... 15 
3.1.4 Cementitious Waste Grouting Process........................................................... 17

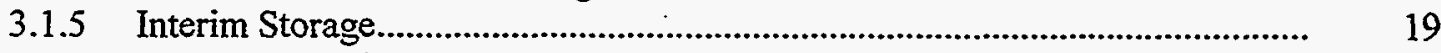

3.1.6 MACT Compliance Facility ................................................................... 19

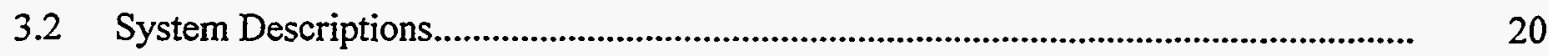

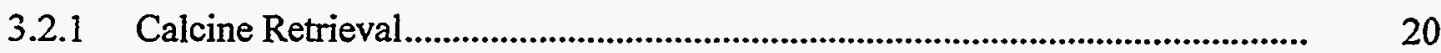

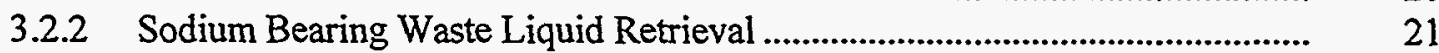

3.2.3 Recalcination System............................................................................... 21

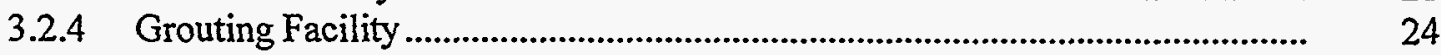

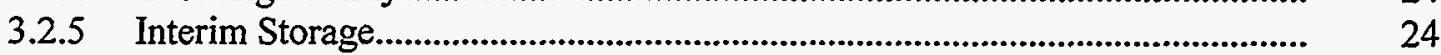

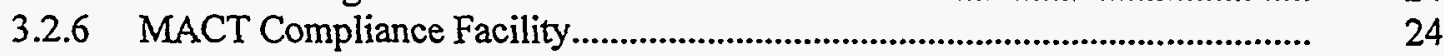

3.3 CWO-NWCF Facility Recalcination Modifications and Additions ........................... 25

3.3.1 NWCF Process Equipment Modifications ...................................................... 25

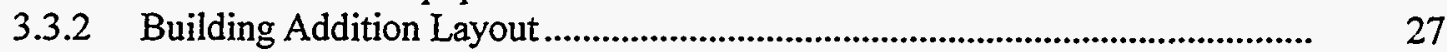

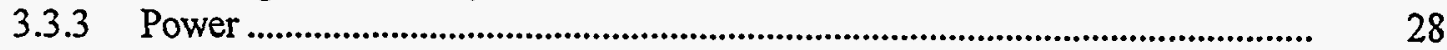

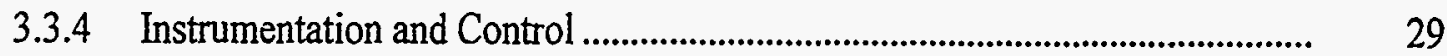

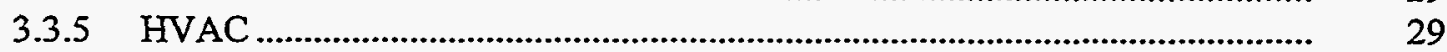

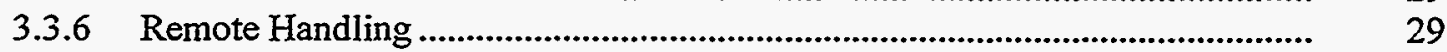

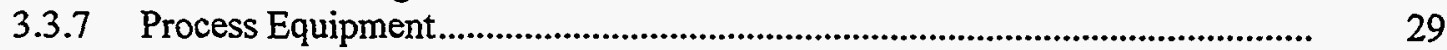

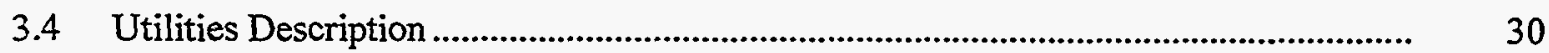

3.4.1 Utilities Summary .................................................................................. 30

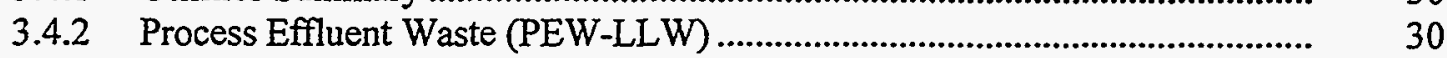

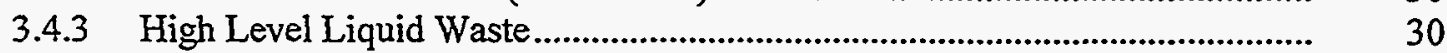

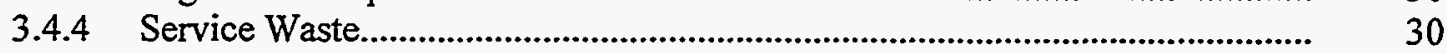

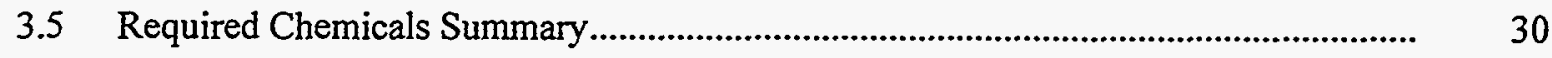

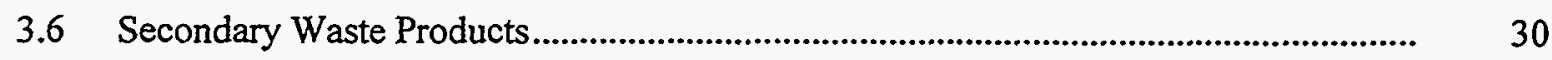

3.6.1 Secondary Wastes Generated by the CWO Process ...................................... $\quad 30$

3.6.2 Solid Radioactive Waste .............................................................................. $\quad 30$

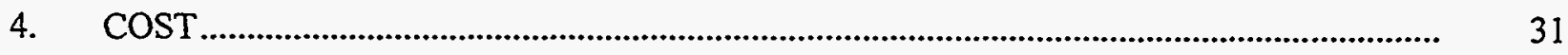

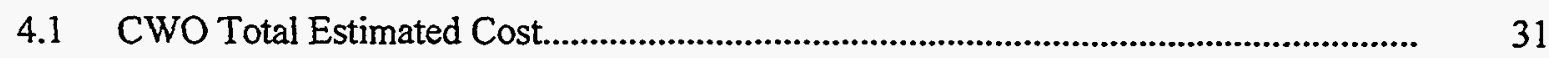

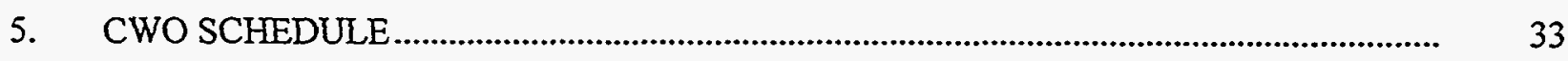

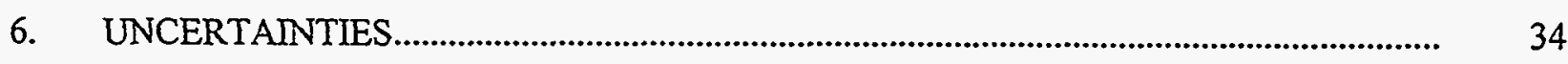

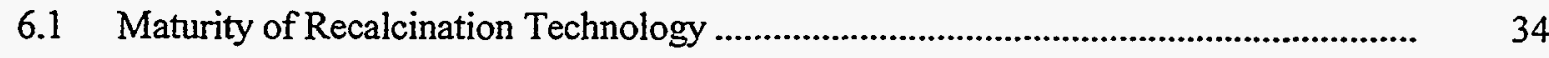

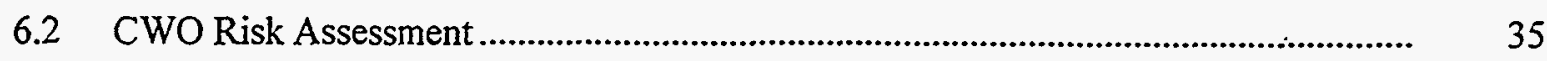

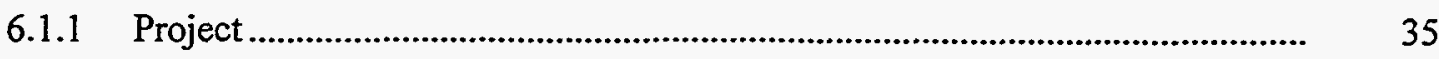


6.1.2 Technical................................................................................................

6.1.3 ES\&H......................................................................................................... 36

6.2 Failure Modes..................................................................................................................... 36

7. PROJECT DATA SHEET ................................................................................................. 38

8. PROJECT-SPECIFIC VARIANCES .................................................................................... 56

8.1 CWO Hot Isostatic Pressed (HIP) Preliminary Plan.................................................. 56

9. POTENTIAL IMPACTS OF NRC LICENSING …….......................................................... 57

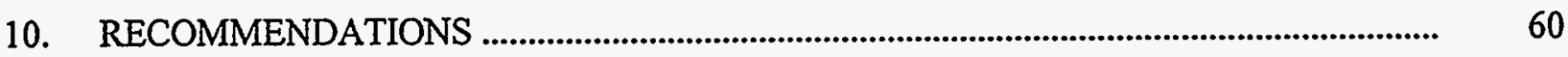

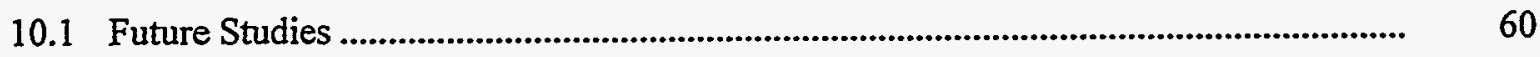

10.2 Process Optimization ................................................................................................ , 61

10.3 Building Optimization ................................................................................................ 61

11. CONCLUSIONS ..............................................................................................................

12. REFERENCES .........................................................................................................

Appendix A-Key Assumptions

Appendix B-Flow Diagram

Appendix C-Cost Estimate

Appendix D-Drawings

Appendix E-Engineering Design File

Appendix F-Risk Assessment Data Sheets

Appendix G-Grouting Facility Project Data Sheet Supporting Data

Appendix $\mathrm{H}-$ Maturity of Technology Guidance

\section{TABLES}

1. Total estimated cost for CWO - design and construction (5yr)................................................ 31

2. CWO project schedule...................................................................................................... 33

3. Project Data Sheet for CWO........................................................................................... 39

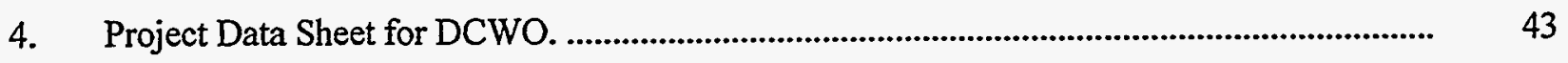




\section{ACRONYMS}

BNFL British Nuclear Fuels Limited

BDAT Best Demonstrated Available Technology

CEMS Continuous Emissions Monitoring System

CMP corrugated metal pipe

CSSF Calcine Solids Storage Facility

CWO Cementitious Waste Option using recalcined materials

DCS Distributed Control System

DET Determination of Equivalent Treatment

DCWO Direct Cementitious Waste Option

DNFSB Defense Nuclear Facilities Safety Board

DOE U.S. Department of Energy

DOE-HQ DOE Headquarters

DOE-NV Nevada

DOT U.S. Department of Transportation

DWG Drawing

DWPF Defense Waste Plant Facility

EDF Engineering Design File

EPA Environmental Protection Agency

ES\&H Environmental, Safety, and Health

FTE full-time equivalent

GC Glass Canister - term used to refer to a $2 \mathrm{ft} \times 10 \mathrm{ft}$ stainless steel canister filled with vitrified waste.

GCD Greater Confinement Disposal

GCDF Greater Confinement Disposal Facility

GCDT Greater Confinement Disposal Test

gph gallons per hour 
GTCC Greater Than Class-C (waste)

HAW High Activity Waste

HEPA high-efficiency particulate air-Filter

HIP Hot Isostatic Press

HLW high-level radioactive waste

HWO Hot Isostatic Press Waste Option

ICPP Idaho Chemical Processing Plant

INEEL Idaho National Engineering and Environmental Laboratory

ISF

Interim Storage Facility

LAW Low Activity Waste

LLW low-level waste

LCC Life-Cycle Cost

LDR Land Disposal Restriction

MACT Maximum Achievable Control Technology

NEPA National Environmental Policy Act

NHLWR National High-Level Waste Repository

NRC Nuclear Regulatory Commission

NTS Nevada Test Site

NWCF New Waste Calcining Facility

NWPAA Nuclear Waste Policy Amendment Act

OCRWM Office of Civilian Radioactive Waste Management (a DOE office)

OSHA Occupational Safety and Health Act

PCDD Polychlorinated-dibenzo-dioxin

PCDF Polychlorinated-dibenzo-furan

PDS Project Data Sheet

PEW process effluent waste

PPA Preliminary Performance Assessment

PS Preliminary Study Assumptions 


\begin{tabular}{|c|c|}
\hline RCRA & Resource Conservation and Recovery Act \\
\hline ROD & Record of Decision \\
\hline RWMC & Radioactive Waste Management Complex \\
\hline $\mathrm{SA}$ & Study Assumptions \\
\hline SBW & sodium bearing waste \\
\hline $\operatorname{scfm}$ & standard cubic feet per minute \\
\hline SLB & shallow land burial \\
\hline SNF & spent nuclear fuel \\
\hline SNL & Sandia National Laboratories \\
\hline SRS & Savannah River Site \\
\hline $\mathrm{TA}$ & transport air \\
\hline TEC & Total Estimated Cost \\
\hline TPC & Total Project Costs \\
\hline TRU & transuranic \\
\hline UPS & uninterruptible power supply \\
\hline VIC & Ventilation, Instrumentation, and Control \\
\hline VOG & vessel offgas \\
\hline VWO & Direct Vitrification Waste Option \\
\hline WAC & Waste Acceptance Criteria \\
\hline WAPS & Waste Acceptance Product Specification \\
\hline WA-SRD & Waste Acceptance System Requirements Document \\
\hline WS & Work Scope \\
\hline WTF & Waste Treatment Facility \\
\hline
\end{tabular}




\section{Cementitious Waste Option Scoping Study Report}

\section{INTRODUCTION}

Treatment of radioactive wastes at the Idaho Chemical Processing Plant (ICPP) at the Idaho National Engineering and Environmental Laboratory (INEEL) is mandated under a "Settlement Agreement" between the Department of Energy (DOE) and the State of Idaho (Reference R7). Among other things, the agreement requires that this treated high-level radioactive waste (HLW) be ready to be moved out of Idaho for disposal by a target date of 2035 .

The objective of this study was to investigate the Cementitious Waste Option (CWO) for mixedHLW treatment. The CWO process would blend existing $H L W$ calcine with liquid sodium-bearing waste (SBW) and sucrose to form a slurry containing high concentration of solids. This slurry would be recalcined in the modified existing New Waste Calcining Facility (NWCF) fluidized bed calciner. The recalcined solid waste would then be transferred to the CWO 5-year grouting facility and mixed with calcined clay, blast furnace slag, sodium hydroxide, and water. This mixture would form a grout mixture that would then be cast into standard HLW canisters. The waste filled canisters would be steam cured in an autoclave and then dewatered. The final waste form would be a zeolitic hydroceramic cementitious waste that would be geochemically and geophysically stable in southwestern Nevada desert alluvial soil. Finally, the canisters would be permanently sealed and then transferred to a separate Interim Storage Facility (ISF) to await preparation and shipment to the Nevada Test Site (NTS) - Greater Confinement Disposal Facility (GCDF) for vertical borehole disposal in alluvium. Each waste canister would be 2 feet ( 0.6 meters) in diameter by 9 feet-10 inches ( 3 meters) high, fabricated of stainless steel, and would comply with the specifications of the Savannah River Site (SRS) HLW canister as identified by the Fluor-Daniel 60\% Design Review Report (Reference R6). The operating period for CWO processing would be 5 years beginning in January 2013 and ending in December 2017.

If the CWO HLW filled canisters cannot meet the Waste Acceptance Criteria (WAC) to be developed by the NTS for the NTS-GCDF, or the GCDF is not allowed to be licensed for HLW, then the waste would require transformation into a material that would meet the WAC of the National HLW Repository (NHLWR) (the only other location to put the CWO waste). To do this, a variation of the CWO process would cast the grouted recalcine material into specialized Hot Isostatic Press (HIP) containers over a 5-year timeframe and place the containers into interim storage ( 31,000 containers). The containers would then be pulled from interim storage and HIP'ed (Hot Isostatically Pressed) over a 20-year period. During the HIP'ing process, three HIP'ed containers would be inserted into a standard HLW canister. Each canister would then be sealed and placed into interim storage awaiting preparation and shipment to the National HLW Repository. Approximately 10,334 waste canisters would be produced by the HIP'ing process. The HIP'ed waste form would be assumed to meet the WAC for the repository and the Best Demonstrated Available Technology (BDAT). Refer to Section 8.1 and EDF-CWO-001 (Appendix E, Item 11) for details. Technically, the CWO HIP'ing variance is not considered a viable variation due to extreme cost and should not be considered a contingency or option.

Many programmatic work assumptions have been made regarding the CWO mixed-HLW, but the most important assumptions are (a) a WAC will be developed by NTS to allow HLW to be disposed of in the GCDF, (b) the Nuclear Waste Policy Act Amendment (NWPAA) will be revised to include the NTS-GCDF as a HLW disposal site, (c) the Nuclear Regulatory Commission (NRC) will license the NTS-GCDF for mixed-HLW disposal, (d) the Resource Conservation and Recovery Act (RCRA listed materials within the mixed-HLW calcine (and recalcine) product will have a delisting petition approved by the Environmental Protection Agency (EPA), DOE, and each state the waste must travel through, 
(e) INEEL waste canisters will be acceptable waste disposal vessels for the GCDF, and (f) all HLW will be ready for movement out of the State of Idaho on or before December 31, 2035.

Cask design, handling, loading, and transportation to the final disposal site plus the design and installation of the GCDF is beyond the scope of this study and will be addressed by others.

\subsection{BACKGROUND}

Calcination is a treatment process performed at the ICPP to transform liquid radioactive waste into a stable granular solid waste form. Past calcining activities produced the large calcine inventories now stored in the Calcine Waste Solid Storage Facility (CSSF) at the ICPP. In addition, liquid SBW is stored in the ICPP tank farm. The calcine is considered a mixed-HLW and the SBW is considered a mixed waste. Various possible HLW separation and nonseparation treatment alternatives are under consideration; however the treatment method considered for this study is the Cementitious Waste Option (CWO).

Under the CWO the wastes would be extracted from storage, treated by blending and recalcining the combined solid and liquid wastes as a slurry, grouting and finish processing the recalcined waste solids in canisters, and then storing the canisters in an interim storage facility awaiting preparation and transport to the NTS-GCDF. A feasibility study similar to the CWO was recently conducted by Fluor-Daniel. This study was called the High Level Liquid Waste Alternative Approach Evaluation (Reference R33) and covered a method proposed to produce a vitrifiable waste form from the INEEL liquid SBW and existing calcines. This alternative approach consists of a process where these wastes would be combined and recalcined, mixed with additives to produce an intermediate vitrifiable concrete, and then vitrified. The intermediate and final concrete would contain all the existing radionuclides. The proposed CWO operations would process all of the wastes in 5 years.

According to Dr. Siemer, the primary developer of the CWO and DCWO (References R27, R27, R34, and R35), the GCDF at the NTS would be the best and least expensive location for INEEL HLW disposal. A proof-of-principle test (GCDT) conducted in the 1980 s on the NTS proved the GCD concept and methodology to be functional, safe, and cost-effective using high concentrations of radioactive and heat producing waste. The test waste possessed higher levels of radiation and heat per borehole than could be produced by the INEEL HLW borehole waste loading. The GCDT (test) borehole contained rad waste with an approximate radionuclide level of 1.11 megacuries and a heat load of about 3.5 kilowatts compared to the CWO-GCDF borehole estimate of 67.7 kilocuries and 676 watts (decayed to the year 2016) per borehole. Presently, no WAC document exists for the only existing GCDF at NTS, because the site and pertinent documentation was closed out in 1989. No waste has been accepted for GCD placement since closure.

The most current information on HLW forms is found in the Waste Acceptance System Requirements Document (WA-SRD)(Reference R10) and the Waste Acceptance Product Specification (WAPS)(Reference R9). One of the acceptable waste form containers described is the SRS DWPF 0.6 meter ( 24 in.) in diameter by 3 meters ( 9 foot- 10 inches) tall stainless steel canister (see Savannah River Site Canister drawing M-1 in Appendix D of Reference R19), which is exactly the same as that described in Reference R6. This canister is used in the CWO Study to contain grouted waste for interim Storage, transport, and disposal. 


\subsection{Objective and Scope of Work}

The primary objective of this preliminary study was to scope the recalcination and recalcine transfer methodology and design for the CWO. The CWO consists of a series of support systems and facilities that were developed by others for use by this option. These consist of: (a) calcine retrieval system, (b) recalcination system (the NWCF with suitable modifications), (3) Maximum Available Control Technology (MACT) compliance facility to treat NWCF offgases, (d) a grouting facility, and (e) an ISF.

In the performance of the study, a number of assumptions were made about the nature of the existing stored calcine, the SBW to be treated, the current calcination process and facility (NWCF), grouted waste (composition and density), and the proposed systems and facilities for calcine retrieval, grouting, and interim storage. Based on these assumptions, the necessary processing steps were identified, together with the needed facilities and equipment. In addition, the requirements and regulations that would apply to the CWO were researched and documented. Costs were estimated for required facilities, process equipment, and operations. Failure modes and project risks were identified and evaluated. Finally, all data required for the Project Data Sheets were estimated and summarized. A plan was developed during the course of this study that addresses a HIP'ing variance for the CWO.

The results of the CWO scoping study are documented in this report. It discusses: (a) CWO design basis, (b) assumptions, (c) requirements, (d) processing steps and required systems, (e) proposed modifications to NWCF, (f) utilities requirements, (g) required chemicals, (h) secondary wastes produced, (i) project cost and schedule, )j) risks, (k) conclusions, and (l) recommendations. Additional detailed information is available in the accompanying appendices and references.

\subsection{Greater Confinement Disposal Facility}

The term Greater Confinement Disposal (GCD) is a disposal strategy that consists of placing radioactive waste in the bottom of a large diameter deep borehole drilled into deep alluvial soil and covering the waste with soil, clay, gravel, sand, or concrete. The GCD was first developed in the early 1980s as a method of disposing LLW not suitable for near-surface disposal in shallow land burial (SLB) sites. Hence the name "greater confinement." Traditionally, LLW has been disposed of in SLB sites that are less than 98.4 feet (30 meters) below the upper surface of the earth (SLB site depths are normally less than 50 feet (15 meters). The minimum GCD disposal depth is required to be equal-to-or-greater-than 98.4 feet (30 meters) relative to current law (10 CFR 60 and 40 CFR 191). To date, greater-than-class-C (GTCC), transuranic (TRU), and classified LLW have been disposed of in GCDs at the NTS. Also, no waste has been accepted for NTS-GCD disposal since 1989.

Although the NWPAA mandates Yucca Mountain to be the only disposal site for HLW, the GCDF is being considered for its potential cost savings based on the assumptions that:

1. GCDF-WAC will accept the INEEL grouted and recalcined HLW

2. INEEL RCRA listed waste will be delisted

3. Canister used for INEEL waste (identical to the Savannah River design) will meet the GCDF-WAC

4. NWPAA will be revised to include the NTS-GCDF as a HLW disposal site

5. NRC will license the NTS-GCDF for mixed-HLW disposal. 
A preliminary design study was developed to investigate a potential GCDF as the disposal site for INEEL HLW ${ }^{(20)}$ for the CWO. The GCDF would be located on the NTS in southeastern Nevada on Frenchman Flats between bomb test craters. This area (also called Area 5) is located on the southeast side of the NTS and has been used since 1961 for the known disposal of on and offsite radioactive LLW, classified LLW, GTCC waste, and TRU waste. The waste was disposed of in SLB sites and large diameter deep boreholes located in deep alluvium deposits. The GCDF would be used for disposal of INEEL canisters containing grouted, recalcined waste in dry deep alluvial desert soil. The GCDF preliminary design was based on the Greater Confinement Disposal Test (GCDT) facility developed, installed, and operated on the NTS at Frenchman Flats during the 1980s. NTS operations will be responsible for final GCDF site engineering, design, and installation.

The NTS is a DOE test site previously dedicated to thermonuclear bomb testing during the $1950 \mathrm{~s}$, and $60 \mathrm{~s}$. Tests were conducted both above and below ground within two primary areas known as Frenchman Flats and Yucca Flats. Both areas are contaminated with radioactive bomb test materials and are unsuitable for anything other than future thermonuclear events or waste disposal.

The CWO would produce a nominal 16,000 NNEEL waste canisters that would require about 210 boreholes drilled 12 -foot in diameter by 150 -foot deep to dispose of all waste canisters. The GCDF would use a 5-year disposal schedule to support the 5-year CWO process. Approximately 42 boreholes would be drilled and loaded per year to support the NEEL schedule.

The CWO-GCDF preliminary design would consist of two individual 43.6-acre GCD sites, each containing three disposal cells, roads, shielding berms, and runoff water control features. The sites would be spaced far enough apart to eliminate radiation interference during remote-controlled loading operations. Two separate sites would be required because of the 42 borehole per year regime needed to support the 5-year disposal schedule. Each site would be capable of providing a maximum of 21 boreholes per year due to the drilling and loading time required. Two sites would allow alternate site operation when one site is closed to all construction activities due to open-air radiation fields during waste loading operations. Each site would be provided with separate boring equipment, remote operated waste handling equipment, a remote operations control center, an instrument-monitoring module, and crew.

Canister design, waste processing, grouting, interim storage, cask development, cask purchase, transport packaging, transportation, and associated costs are beyond the scope of this study and shall be addressed by others. The waste filled canisters would be transported from the INEEL to the NTS either by truck or rail using overpack type transport casks. The waste material contained by the canisters would be mixed-HLW delisted from RCRA for listed hazardous waste and treated for classified RCRA hazardous waste by the grouting method.

The cost of a CWO-GCDF consisting of two identical 43.6 acres sites, containing a nominal 105 boreholes each (or 210 boreholes total), providing 42 drilled, loaded, and closed boreholes per month (21 boreholes per site due to the loading of rad sources), over a 5-year disposal plan (7-year GCDF site development and closure program) is presented as follows (Reference R20):

- Total planning cost estimate

- Total estimated Life-Cycle-Cost

- Final Life-Cycle-Cost for NTS direct Congressional funding

- Final Life-Cycle-Cost for waste generator disposal charges

$$
=\$ 306,400,000
$$$$
=\$ 252,000,000
$$$$
=\$ 44,352,000
$$

$=\$ 207,648,000$. 
Waste Generator Disposal Cost Breakdown:

- $\quad$ Cost per borehole

- Cost per canister

- Cost per cubic meter of grouted waste

- Cost per cubic meter of recalcined waste

- $\quad$ Cost per curie
$=\$ 988,800$ (210 boreholes)

$=\$ 13,040 \quad(15,924$ canisters $)$

$=\$ 18,111 \quad(11,465$ cubic meters $)$

$=\$ 44,134 \quad(4,705$ cubic meters $)$

$=\$ 14.64$ (14,181kilo-Curie inventory).

In conclusion, the GCD and GCDT proven methodology appears to be a functional, effective, and safe means of radioactive waste disposal management that presents a cost effective way to dispose of the HLW generated by the INEEL. The HLW is assumed to be RCRA delisted and would be placed in the Frenchman Flat area of the NTS. This area offers a large GCDF operations zone for an unlimited number of GCD sites and is already contaminated by radioactive bomb test materials both above and belowgrade. The burial area will not, or ever will be, usable for anything other than future bomb tests or waste disposal. The water table existing below bomb craters is, or eventually will be, contaminated by the migration of radioactive bomb test materials, which cannot be mitigated. The canistered waste is not expected to add to the water table contamination for more than 10,000 years, as determined by preliminary performance assessments, thereby meeting EPA regulations. Risk assessments conclude that plant and animal activities are not considered a threat to a GCDF because they normally do not exceed 25.3 feet ( 8 meters) below surface. Excavation by humans is possibly the greatest threat to a GCDF due to drilling activities; however the abovegrade concrete cap, radiation placard, and the corrugated metal pipe (CMP) at the top of each borehole, should theoretically indicate a problem zone. Drilling for drinking or livestock water within the GCDF area is considered a probability once the 100-year government site administrative control has been relinquished. Mining by humans is not practical because metals or minerals of economic value do not exist belowgrade. Also, seismic events are not a problem with alluvium deposits due to soil structure and depth. Interesting to note, the GCDF is located in a seismic zone 2, whereas the National HLW Repository (Yucca Mountain) is located in a seismic zone 3.

The fabrication of a GCDF on the NTS from a technical standpoint is a good choice for the disposal of radioactive LLW and $\mathrm{HLW}$. The waste filled canisters are strong and thick enough to withstand the crushing force of surrounding soil and the grouted waste material contained within is geochemically and geophysically stable in alluvium. The GCDT appears to have proven that gamma radiation and thermal activity associated with radioactive waste products are absorbed by the soil and thermal equilibrium occurs after a number of years. The water contained in the soil (normally $10 \%$ by volume) has dropped to approximately $3 \%$ for soils surrounding the greatest radiation and heat source location near the bottom of the GCDT; this is normal due to the generated heat level, but is not detrimental. Approximately 4 inches of rainfall occurs in this part of the Nevada desert per year, therefore soil water makeup could take many years to reach the normal level at waste canister depths. All INEEL waste canisters would be disposed of at a minimum depth of 98.4 feet ( 30 meters) belowgrade, therefore all existing medium depth EPA waste disposal requirements can be met. The waste canisters can be excavated in the future if necessary; however, this would be a difficult, time consuming, and expensive operation.

It would cost about $\$ 300,000$ per canister (Reference R32) for a waste generator to place 16,000 canisters in the national HLW mined repository ( $\$ 4.8$ billion) and would cost about $\$ 13,040$ per canister for the waste generator to dispose of the waste in a CWO-GCDF ( $\$ 208$ million). Therefore the 
potential exists to save approximately $\$ 4.6$ billion by the use of the CWO-GCDF at NTS compared to the National HLW Repository.

\subsection{Staffing Estimate}

An Engineering Design File (EDF) has been written to address the Staffing Estimate for this study.(Appendix E, Item 6). A total estimated staffing requirement of 132 personnel for the calcination portion of the $\mathrm{CWO}$ has been identified. This staffing estimate is broken down into two general sections; (1) staffing for the current calciner from the "FY-98 Calcined HLW Project Budget Totals Report" dated October 28, 1997, and (2) the Additional Staffing Estimate for Calciner Modification." The estimated total full-time equivalent (FTE) personnel required for the existing and current calciner operations would be 96 . The total estimated additional staffing FTEs required for the recalciner modifications would be 36 personnel. These estimates do not include laboratory support for calciner operations.

This process is based upon a 24 hour per day, 7 day per week operation to be completed in five years commencing on January 1, 2013, and ending December 31, 2017. There would be three, 8-hour shifts per operating day and a floating shift to provide coverage for days off and vacation scheduling. Refer to EDF-CWO-005 (Appendix E, Item 5) for further information and quantity of personnel per work discipline. 


\section{DESIGN BASIS/KEY ASSUMPTIONS/REQUIREMENTS}

\subsection{Design Basis}

The design basis for each of the separate studies that contributed to the CWO Study described herein are listed below. This list is intended as a summary to identify overlaps between this and the other studies, and to provide a clarification of the divisions among the various options.

\subsubsection{Calcine Retrieval}

A calcine retrieval and transportation system was scoped to retrieve calcine from the CSSFs and transport it to the waste treatment facility. The design details are available in EDF-WTS-002. The calcine retrieval and transportation system was designed to supply calcine to the treatment options currently under study [Vitrification Waste Option (VWO), Hot Isostatic Press Waste Option (HWO), Cemetitious Waste Option (CWO), Direct Cementitious Waste Option (DCWO), and TRU Separations). The system was divided into three subsystems: (1) CSSF access method, (2) calcine retrieval system, and (3) calcine transportation system. During CSSF access, the buildings, equipment, and piping are removed from the superstructure of each CSSF and the retrieval risers are installed and accessed. The CSSFs are then prepared for calcine retrieval. The calcine retrieval system presents a viable method to retrieve calcine from the CSSFs and relies on an air jet and a suction nozzle. The calcine transportation system is a closed loop pneumatic system similar to one currently used at the ICPP for transportation of calcine. The scope of this study was limited to the Fluor-Daniel feasibility design (Reference R6). The purpose was to compare this system directly to the Fluor-Daniel system. However, two issues that warranted further review were identified as the removal of corrosion coupons from the bins and the installation of D\&D risers. Separate cost estimates were developed for the removal of corrosion coupons from the bins and installation of $\mathrm{D} \& \mathrm{D}$ risers.

\subsubsection{Recalcination}

The "Cementitious Waste Option, "hereafter referred to as the "CWO Process," is based on work completed earlier at the INEEL (reference R24), and at the Hanford reservation (reference R36). The previous work describes the use of sucrose (sugar) as a reducing agent in the denitration and calcination of radioactive high level liquid wastes, and on work described in references R26, R27, R28, describing processes for solidification of solid calcined wastes using hydroceramic grouting and hot isostatic pressing. The process treats HLW calcine solids and liquids stored at the ICPP for interim storage and eventual transport to a disposal facility. It consists of the following basic steps:

1. Retrieval of calcined solids from existing storage bins at ICPP (CSSF-1 through 7).

2. Slurrying of retrieved calcine solids with remaining liquid SBWs in the ICPP tank farm.

3. The slurrying step extracts leachable, soluble nitrates (primarily $\mathrm{NaNO}_{3}$ and $\mathrm{KNO}_{3}$ ) from the calcine solids into aqueous solution in preparation for reduction of the nitrates to $\mathrm{N}_{2}, \mathrm{O}_{2}$, and lower valence state oxides of nitrogen. The slurrying step also redistributes the alkali metal from the high-alkali sodium waste blends throughout the calcine. This is desirable in achieving the preferred composition for grouting the recalcined solids.

4. Calcination of the slurried calcine solids and liquid SBW together in the existing fluidized bed calciner in the New Waste Calcining Facility (NWCF) at ICPP. 


\section{Transfer of the recalcine material to the DCWO grouting facility.}

\subsubsection{Grouting}

This design is based on hydroceramic stabilization technology developed by Dr. D. D. Siemer and others at the INEEL (reference R19). In this process, the CWO high level recalcined waste would be combined with calcined clay, blast furnace slag, sodium hydroxide, and water to form a hydroceramic grout with analogs of naturally occurring feldspathoids and zeolites.

The resulting stabilized waste form is considered structurally sound, geophysically and geochemically stable in alluvial soil, and is not expected to be RCRA hazardous. The grouted waste form is contemplated to comply with land disposal restrictions for RCRA-treated waste allowing permanent placement in a GCDF. Further discussion on the design basis is documented in EDF-DCWO-011 (Appendix E, Item7).

\subsubsection{Interim Storage}

An interim storage facility (ISF) would be located near the DCWO grouting facility to store HLW canisters from the time they are released from grouting to the time they are packaged for shipment and transported to the NTS-GCDF. The quantity of canisters and their characteristics result from the CWO studies for the processing alternatives. The design life for the facilities is from 40 to 60 years. The SRS canister and the West Valley Demonstration Project HLW canister meet the repository WAC for dimensions, although there are significant differences in these canisters' physical makeup. The Savannah River canister was used as the baseline for this study because it was selected in the ICPP Waste Treatment Facilities Feasibility Study Report. Transport of the waste to the storage facility and from the facility to the repository are not included in this study, however, equipment for handling the canisters for movement into and from the facility are included. A passive cooling system is also desired. The additional safety issues and operating costs of an active system would thus be avoided. Refer to INEEL/EXT-97-01393 for design details.

\subsection{Key Assumptions}

A number of assumptions have been made in developing the CWO process baseline. These assumptions have been categorized as follows:

- Work Scope assumptions - those that provide a framework that directs the formulation of programmatic objectives and constraints. Generally, these assumptions were imposed by the customer, regulatory environment, and general conditions, which are independent of the $\mathrm{HLW}$ program or any of the waste treatment/disposal options being considered.

- Study assumptions - those that were needed to establish the technical basis for the design of a particular option, and which have an appreciable impact on design, cost, or feasibility. Generally these assumptions were imposed by personnel who performed the study, and are based on whatever knowledge, judgment, or data were available to these personnel at the time the study was performed.

- Minor assumptions - those that were needed for calculations, design decisions, or tradeoffs incident to baselining the process, but which have no appreciable impact on design, cost, or feasibility. 
Note that assumptions listed below include only Work Scope and Study assumptions. Minor assumptions were generally noted only in EDFs or other pertinent locations as they were needed. Also, note that CWO involves four major processes:

1. Calcine retrieval

2. Recalcination

3. Grouting

4. Interim Storage.

Only the key assumptions for process (2) recalcination are listed here. Assumptions for the other three processes are given in their respective documents. [(1) Calcine retrieval assumptions in EDF-WTS-002, (3) Grouting assumptions in Appendix A of INEEL/EXT-97-01399, (4) Interim storage assumptions in INEEL/EXT-97-01393.]

\subsubsection{Work Scope Assumptions}

The following Work Scope (WS) Assumptions formed the framework, i.e., the directed charter, for this study, only. Although some of these assumptions are subject to change, it is beyond the scope of this study to evaluate the associated risk.

WS-1 A WAC will be developed by NTS to allow HLW to be disposed of in the GCDF.

WS-2 The NWPAA will be revised to include the NTS-GCDF as a HLW disposal site.

WS-3 The NRC will license the NTS-GCDF for mixed-HLW disposal.

WS-4 The RCRA-listed wastes in the grouted waste form, will be delisted before the grouted waste form being placed in interim storage

WS-5 INEEL waste canisters will be acceptable waste disposal vessels for the GCDF.

WS-6 A performance assessment will be developed by SNL to show that GCDF disposal of HLW is acceptable to 40 CFR 191.

WS-7 A GCDF will be opened by January 1, 2013 to support the CWO schedule.

WS-8 Grouted waste will be cast in cylindrical stainless steel disposal canisters that are 2 feet in diameter by 9 feet- 10 inches high with at least $80 \%$ utilization of the available volume in the canister.

WS-9 The overall treatment facility online factor will be $50 \%$. Calcine retrieval and recalcination of slurried wastes will proceed on a 24-hr/day, 7-day/week schedule (subject to the above assumed online factor). All other processes (e.g., grouting, curing, and storage operations) will proceed on the basis of four 10-hour shifts per week, and 150 actual working days per year (again subject to the assumed online factor). 
WS-10 Operation of the calciner in the anticipated timeframe (see assumption 8, above) will require that the NWCF be modified to comply with MACT requirements.

WS-11 All HLW will be ready for movement out of the State of Idaho on or before December $35,2035$.

WS-12 The grouted waste form will be shown to be "geochemically" and "geophysically"suitable for disposal in the deep alluvial soil structure of the NTS.

\subsubsection{Study Assumptions}

Although some of these assumptions are subject to change, it is beyond the scope of this study to evaluate the associated risk.

SA-1 Tank farm liquid wastes in tanks WM-187, -188 , and -189 will be calcined using the current process from June 1, 1997 to June 1, 1998, and (following a 3-month turnaround) from about September 1, 1998 until December 31, 1999. All liquid tank farm wastes remaining on Jaqnuary 1,2000 , and generated after this date will be slurried with calcine solids in the ICPP CSSF (bin sets) and recalcined.

SA-2 Hydroceramic grout recipes will be developed before commencement of waste processing. A sufficient number of recipes will be developed to accommodate the expected range of blended compositions that will result from blending of alumina, zirconia/fluorinel/blend, and high-sodium calcines with liquid SBW. These recipes are assumed to be sufficiently robust to accommodate the maximum expected variation in calcine composition.

SA-3 A pumping system for radioactive solids slurries will be designed and demonstrated that is not prohibitively expensive to build, install, operate, and maintain.

SA-4 Safety issues of sugar denitration/recalcination can be mitigated to the acceptance of DOE.

SA-5 The pressurized recalcine delivery system from the modified NWCF calciner to the grout facility will be acceptable to DOE.

\subsection{Requirements}

Process requirements are established by statutory laws, DOE orders, and the Batt agreement between DOE and the State of Idaho. These requirements are described in detail in References R5, R7, and R8. Regulatory requirements and criteria can be found in Appendix E, item 1. Labor laws can be found in 10 CFR 1910 and 1926.

\subsubsection{Design Criteria}

EDF-WTS-004 (Appendix E, Item 7) documents the design requirements for the nonseparations and TRU only separations options for the Waste Treatment Facility (WTF) feasibility studies. Although this study is only at a scoping level, the requirements addressed in the EDF are still relevant. The following information has been extrapolated from the EDF. 
The facilities would be designed and constructed under one of three possible regulatory scenarios: (1) performance against DOE orders with maintenance of status quo interfaces with other regulatory and oversight agencies such as the EPA (State of Idaho) and the Defense Nuclear Facilities Safety Board (DNFSB), (2) performance against DOE orders with all current regulatory/oversight relationships maintained and/or NRC oversight to achieve "NRC Equivalency" or, (3) NRC licensing through replacement of DOE orders with NRC regulations and replacement of DNFSB oversight with NRC licensing process. Construction and operations labor will adhere to the Occupational Safety and Health Act (OSHA) regulations found in 29 CFR (Code of Federal Regulations) 1910 and 1926. For the purposes of this study and at the direction of the HLW alternatives feasibility studies project manager, the base case for this study is performance against DOE orders (scenario 1, above). This is consistent with the approach taken by Fluor-Daniel, Inc., at the direction of the HLW Program in the preparation of the planning alternative and will provide an apples-to-apples comparison of the alternatives.

The EDF identifies the applicable DOE orders, regulations and guidance documents that would be used in the design of the facilities. Currently, no NRC regulations are in place for the licensing of waste processing facilities such as those discussed in the EDF. The only WTF activity for which the NRC has been routinely involved is the licensing of waste storage and disposal facilities. Nevertheless, NRC requirements are looming on the horizon. In addition, the waste products to be produced are destined for NRC licensed storage facilities. Therefore, some NRC requirements cannot be ignored. Thus, where appropriate, NRC regulations are explicitly specified in the design requirements. Where specific design criteria are provided under NRC regulations and guidance documents whether directly applicable or for similar facilities, it will be referenced. This will be useful in helping to determine the cost differential between DOE regulatory/oversight and NRC licensing requirements.

The criteria contained in the EDF are based only on the rudimentary descriptions of the processes presented in Section 1.1 of the EDF. Going beyond the scoping level when designs are developed further, some of the criteria may become nonapplicable, and others will be identified. The purpose, as stated in the EDF, is to provide a set of high-level requirements to guide the development of the conceptual designs of the facilities and provide a reasonable basis for cost estimating purposes. In general, the EDF does not attempt to cover criteria outside of the design and construction of the facilities. Process criteria such as the waste form acceptance criteria, treatment standards, etc., will be addressed by others.

\subsubsection{Regulations}

Identification and survey of existing environmental regulations and standard criteria pertaining to the design, construction, operations, and performance of the proposed WTFs at the ICPP, of which the CWO is included, are documented in EDF-WTS-003 (Appendix E, Item 1).

It is expected that DOE-ID will continue to implement the QA Program described in DOE-RW-0333P Quality Assurance Requirements and Description (QARD) (Reference R8) and will apply it to this effort as well. The impact of implementing DOE-RW-0333P is reflected in the capital and operating cost estimates generated by this Scoping Study.

The radiological evaluation EDF-HWO-006 (Appendix E, Item 8) performed for the HIPing Waste variation is also applicable to this study. Included is a regulatory discussion related to air emissions and area-monitoring equipment, hot cell manned entry, and breathing air.

\subsubsection{Performance}

Section C.3 of the Settlement Agreement (Reference R7) between DOE and the State of Idaho states that "DOE shall treat all high-level waste currently at INEEL so that it is ready to be moved out of 
Idaho for disposal by a target date of 2035." The CWO process, as proposed in this Scoping Study, would start producing grout filled canisters in the grout facility by January 1,2013 and finish by December 31,2017 , which is ahead of the target date.

\subsubsection{Applicable Codes and Standards}

Codes and standards, as may be applicable to the proposed facility, are discussed in detail in both EDF-WTS-003 (Appendix E, Item 1) and EDF-WTS-004 (Appendix E, Item 8).

Codes and standards related to radiological issues are listed in EDF-HWO-006 (Appendix E, Item 9).

OSHA labor regulations for personnel shall apply to all aspects of CWO operations relative to 29 CFR 1910 and 1926.

\subsubsection{HLW Repository WAC}

The HLW repository WAC related to vitrified HLW are found in EM-WAPS Rev. 01, Waste Acceptance Product Specifications for Vitrified High-Level Waste Forms, May, 1995 (Reference R9). The WAPS is derived from the requirements listed in DOE/RW-0351P, Revision 02, Waste Acceptance System Requirements Document, December 1996 (Reference R10). A summary of the WAC is documented in the Waste Disposal Options Feasibility Study (Reference R2).

The standard waste form is borosilicate glass, although provisions exist for qualifying other waste forms. RCRA hazardous waste is not allowed in the waste form. In addition to waste form requirements, the WAPS information is useful to this Study because it provides physical requirements and limitations associated with the standard 0.6 meter ( 24 inches) in diameter by 3 meters ( 9 foot- 10 inch) long HLW stainless steel canister described in the WAPS.

As detailed in the Waste Disposal Options Feasibility Study (Reference R2), the grouted waste is not a Land Disposal Restriction (LDR)-specified technology. Therefore, a determination of equivalent treatment (DET) petition must be submitted to the EPA in accordance with 40 CFR 268.42(b) (Reference R14) for the grouting method to be acceptable.

\subsubsection{GCDF WAC}

A discussion of the WAC for the Greater Confinement Disposal Facility (GCDF) at the NTS is found in the Waste Disposal Options Feasibility Study (Reference R2), the Environmental Regulations and Standard Repositories Criteria for the Disposal of Waste Forms from the INEEL Proposed High Level Waste Processing Alternatives (Reference R33), and the Preliminary Design of the Nevada Test Site Greater Confinement Disposal Facility (Reference R20). The term GCD is a disposal strategy that consists of placing the waste in the bottom of large diameter deep boreholes and covering it with soil, clay, gravel, sand, or concrete. The GCD was first developed in the early 1980 s as a method of disposing LLW not suitable for near-surface disposal in SLB sites. Hence the name "greater confinement." Traditionally, LLW has been disposed of in SLB sites that are less than 98.4 feet ( 30 meters) below the upper surface of the earth. The minimum GCD disposal depth for GTCC and HLW is equal to or greater than 98.4 feet ( 30 meters) below grade relative to current law. To date, GTCC, TRU, and classified LLW have been disposed of in GCDs at the NTS. Also, no waste has been accepted for NTS-GCD disposal since 1989. 
Although the NWPAA mandates Yucca Mountain to be the only disposal site for HLW, the GCDF is being considered for its potential cost savings based on the assumptions that: (a) the GCDF-WAC will accept the INEEL grouted and recalcined HLW, (b) the INEEL RCRA listed waste will be delisted, (c) the canister used for INEEL waste (identical to the Savannah River design) will meet the GCDF-WAC.

A Preliminary Performance Assessment (PPA) will be required before the classification of the NTS-GCDF and the related WAC for HLW disposal. Three previous PPAs were developed for the GCD Test performed on the NTS during the 1980s. The first two were developed before and during the GCDT. The third was developed years after the completion of the test (Reference R34) using established information and collected instrumentation data. The behavior of the GCD site was analyzed using a HLW performance assessment methodology developed at SNL for the NRC. This methodology was used to determine if the GCD site could comply with the EPA's requirements for the disposal of spent nuclear fuel (SNF), HLW, and TRU waste, as set forth in 40 CFR 191. The third and last PPA for the GCDT was concerned with EPA compliance only. Political and legal issues were not considered. Results of the PPA analyses were not conclusive because other factors needed to be determined before making a recommendation. The PPA method is only a tool that provides information and data to decision-makers for final judgements.

Currently the NTS-WAC only accepts LLW and mixed LLW for disposal or storage from waste generators outside the State of Nevada. Waste generators must be designated by DOE headquarters (DOE-HQ) and approved by DOE-Nevada (DOE-NV) before waste shipment. Presently, the INEEL is neither a designated or approved waste generator and must obtain this status before any shipment off-Site to the NTS. No GCDF-WAC currently exists on the NTS therefore a GCDF-WAC for HLW must be generated before INEEL waste can be shipped to the NTS for disposal. 


\section{PROCESS AND SYSTEM DESCRIPTIONS}

\subsection{Process Descriptions}

The complete CWO option is based on the following major processes:

- Retrieval of existing calcine from CSSF storage

- Retrieval of liquid SBW from tank farm storage

- Slurrying of calcine and SBW followed by recalcination in the NWCF calciner

- Grouting of recalcined solids into a cementitious waste form

- Transfer of grouted wastes to an interim storage facility (to await final disposal)

- Treatment of offgas from the recalcination process to comply with MACT requirements, before release to the environment.

Each of these major processes is discussed in the following sections.

\subsubsection{Calcine Retrieval}

Since 1963, the ICPP has calcined high-level liquid wastes into solid calcine, and stored the resulting solid wastes in the Calcine Solids Storage Facility (CSSF), which consists of seven bin sets. Each bin set is a separate structure housing one or more stainless steel bins that actually contain the calcine solids. Over the years a number of different calcine types have been generated. For purposes of the CWO waste processing option, these calcines have been categorized into three generic types: type $\mathrm{A}$ (aluminum calcine), type B (zirconium/zirconium blends/fluorinel calcines), and type $C$ (high alkali calcines from processing SBW). The baseline process design requires blending of the three calcine types together to produce a slurry mixture that is recalcined. Refer to EDF-WTS-002 (Appendix E, Item 6).

The calcine retrieval system is designed to retrieve calcine from the CSSF bin sets and transport it to the NWCF slurry-blending cell. This system is described in detail in the Calcine Retrieval and Transportation EDF (Appendix E, Item 9). Retrieval is accomplished by inserting two nozzles into a bin, one injects air to fluidize the calcine while the other provides suction to pneumatically lift the suspended calcine out the top of the bin. After leaving a bin, the calcine travels through a closed loop pneumatic transport system. The baseline calcine retrieval system has two independent suction lines and can extract calcine from two different bin sets at once. The maximum retrieval rate is $5,400 \mathrm{~kg} / \mathrm{hr}(2,700 \mathrm{~kg} / \mathrm{hr}$ in each line). Using this system, calcines of types $\mathrm{A}, \mathrm{B}$, and $\mathrm{C}$ are retrieved and transported separately to dedicated storage bins in the slurry-blending cell. Because the retrieval rate greatly exceeds the processing rate, the two retrieval lines can easily provide the three calcine types at the required average rate (making use of the dedicated storage bins to provide surge capacity). Since type B calcines constitute the majority of the total calcine, one of the two retrieval lines would be dedicated to type $B$ calcine, and the other will be used alternately to retrieve type $A$ and type $C$ calcines.

Precautionary measures and efficiency issues require that one bin within a bin set be completely emptied before retrieving from another bin. However, calcine can be retrieved from two bin sets simultaneously due to the dual transport systems. As recalcination proceeds, retrieval would switch from 
one bin-set to another as the bin sets are emptied or as different calcine types are required for blending purposes.

\subsubsection{Sodium-Bearing Waste Liquid Retrieval}

Sodium-bearing waste (SBW) liquid located in the ICPP tank farm is retrieved for slurrying with existing stored calcines from prior calcination campaigns. SBW retrieval is accomplished using existing steam jetting equipment with transfer lines modified to allow delivery of the tank farm waste directly into the new 4,000 gallon slurrying tanks. The average design retrieval rate is 73 gallons per hour (gph). Two slurrying tanks are used to allow for continuous operation of the calciner. Each tank provides sufficient slurry mixture for about one 24-hour day operation of the calciner. (See EDF-CWO-003 in Appendix E, Item 2).

\subsubsection{Recalcination}

Recalcination is described in detail in EDF-CWO-003 (Appendix E, Item 2). The EDF provides design basis assumptions, process description, design throughputs, process flow diagrams, material balance, process equipment list, utilities requirements, required modifications to the NWCF, required chemicals, generated waste streams, and process concerns.

The recalcination step is included in the CWO process to achieve the following objectives:

- Solidify remaining SBW in the tank farm

- $\quad$ Remove nitrates and mercury from existing calcine and SBW to prevent potential problems during grouting

- Blend all wastes to minimize composition variations in the final grouted waste form

- Redistribute the alkali metals (i.e., sodium and potassium) present in relatively high concentrations in recently-produced calcines and SBW.

The subsections below provide summary descriptions for major processing steps during recalcination.

3.1.3.1 Bulk Ingredients. The principal bulk ingredients of the slurry mixtures that would be recalcined are:

- Sodium bearing waste liquid

- $\quad$ Retrieved calcine from the ICPP Calcine Solid Storage Facilities (CSSFs, or bin sets)

- Demineralized process water

- 65 wt\% sucrose (sugar) solution

- Calcium nitrate.

Use of sucrose as a reducing agent eliminates the need for large quantities of additives such as aluminum nitrate to prevent agglomeration of the calciner bed. In addition, based on pilot plant results to 
date, addition of boric acid to control formation of gamma alumina may not be necessary. However, due to relatively high concentrations of chlorides in SBW $(\sim 1,200 \mathrm{ppm})$, modest quantities of calcium nitrate may be needed to retain the chlorides in the recalcine solids (hereafter referred to as "recalcine"). Refer to EDF-CWO-003 (Appendix E, Item 2).

Stored calcines fall into one of three generic categories:

- Category A consists solely of aluminum calcine

- Category B includes zirconium, zirconium-sodium, and fluorinel calcines

- Category $\mathrm{C}$ includes calcines derived from high-sodium wastes that are generally not first or second order raffinates but result from decontamination activities.

In a given week, calcine from all three categories above are retrieved and stored temporarily in three separate bins. In dispensing calcine into the slurry tanks, the three calcine types are used in the same relative proportions as the total masses of calcine in each category. Blending calcines in this way would reduce the range of variation in composition of the recalcine that is produced, and thus reduce the number of required grouting recipes together with the amount of grouting additives required.

3.1.3.2 Blending/Mixing. No admixing of calcines is performed up to the point of slurrying with SBW liquid. The three calcine types would be fed to the slurrying tanks together with SBW and process water. The water is added to reduce the solids concentration to around 33 weight percent so the mixture can be pumped into the calciner. The slurrying tanks are sparged continuously to keep the solids in suspension, and to ensure that the soluble nitrate species are thoroughly leached from the calcines. Once the dissolved solids concentration in a tank has reached equilibrium, the slurry is sampled and the nitrate concentration is determined in order to set the sugar feed rate. Use of two 4,000-gallon slurry tanks allows continuous operation of the calciner, with each full tank providing feed for about one operating day. While the contents of one tank are being fed to the calciner, the other is charged with slurry ingredients, sparged, and sampled. Refer to EDF-CWO-003 (Appendix E, Item 2).

3.1.3.3 Recalcination Process. Once a slurry mixture is ready for processing, the mixture is pumped through a high velocity recycle loop that traverses the vertical and horizontal distance to the calciner cell (and back). The flow velocity in the loop is adjusted high enough to keep the solids in suspension. Since the resulting mass flow rate of slurry is higher than the required feed rate to the calciner, most of the flow is routed back to the slurry tanks. The recycle loop feeds a manifold around the circumference of the calciner from which three low volume streams are withdrawn to provide feed to the calciner. Refer to EDF-CWO-003 (Appendix E, Item 2).

Recalcination would be done using sugar as a reducing agent in the feed to convert nitrates to the respective metal oxides, plus nitrogen $\left(\mathrm{N}_{2}\right)$, Oxygen $\left(\mathrm{O}_{2}\right)$, and modest quantities of nitrous oxide (Nox). Each of three slurry streams is mixed with a 65 weight percent sucrose solution from storage using in line static mixers. These streams are introduced into the calciner through specially designed nozzles capable of injecting the slurries. The slurry is fed into the calciner bed through existing penetrations in the calciner vessel. An existing unused penetration through the east wall of the calciner cell would be used for the slurry recycle loop. Air, oxygen, and kerosene would be added through existing lines. Sucrose solution would be transported into the ICPP by truck or by rail, and stored in an existing tank in the cold additive mixing area. Offgas is routed to the current calcination offgas treatment facility (with modifications described below), and recalcine is transported via the current product transport system to a secondary (booster) transport system. The latter system carries the recalcine to the grouting facility. 
3.1.3.4 Offgas System. The existing offgas treatment system in NWCF, with some modifications and additions, would be used for the recalcination process. Refer to EDF-CWO-003 (Appendix E, Item 2). Modifications include the following:

- Because of higher solids concentrations and reduced gas flow rate, the existing primary cyclone would be redesigned and replaced

- An electrolytic mercury recovery system would be installed in the valve cubicle to prevent buildup of mercury in tank farm heels (see "Mercury Recovery," below)

- One or more of the existing tanks in the NWCF would be selected for use as.a sugar digestor. This tank will be dedicated to digestion of residual sugar and/or hydrocarbons in scrub solution before deep recycle back to the tank farm.

3.1.3.5 Mercury Recovery. At the 600 to $650^{\circ} \mathrm{C}$ operating temperature of the calciner, virtually all of the mercury in the feed is converted to gaseous form. Most (>90\%) of this mercury accumulates in the offgas scrub solution until it is purged (deep recycled) to the tank farm. In the CWO recalcination process, a slipstream of the scrub solution would be continuously processed through an electrowinning cell designed to extract elemental mercury. This would be done to prevent mercury accumulation in the tank farm as a consequence of periodic deep recycle. Refer to EDF-CWO-003 (Appendix E, Item 2).

3.1.3.6 Recalcine Transfer. The recalcine transport system consists of two independent pneumatic systems coupled together by a booster station. The first leg uses the existing NWCF transport system to move calcine from the calciner to the booster station. The second leg is a closed loop pressurized system that delivers calcine from the booster station to the grouting facility. The booster station is located in a transport cell within the recalcining facility and provides temporary calcine storage plus a method of transferring recalcined solids from the NWCF vacuum system to the pressurized system. Both systems are capable of moving calcine at the expected mass flow rate of $674 \mathrm{lbm} / \mathrm{h}$. Refer to EDF-CWO-003 (Appendix E, Item 2).

3.1.3.7 Mercury Amalgamation. Mercury recovered from NWCF scrub solution would be mixed waste and will require further treatment before disposal. Because BDAT for high-mercury hazardous waste is amalgamation the recovered mercury from the electrowinning system would be mixed with elemental sulfur and blended to form $\mathrm{HgS}$ (i.e., cinnebar--a stable, naturally occurring form of mercury). Quantitative amalgamation readily occurs when excess sulfur is vigorously admixed with elemental mercury. This can be done by placing the $\mathrm{Hg}+\mathrm{S}$ mixture in a suitable container (e.g., a l-gallon paint can), together with a number of small steel balls (to promote mixing), sealing the vessel, and then rotating /vibrating the assembly in a jar mill. This is the proposed process for the CWO process baseline. Refer to EDF-CWO-003 (Appendix E, Item 2).

\subsubsection{Cementitious Waste Grouting Process}

The CWO 5-year grouting process includes the grouting of ICPP recalcine and casting the mixture into 2 foot $(0.6 \mathrm{~m})$ diameter by $9 \mathrm{ft}-10$ in $(3 \mathrm{~m})$ high $\mathrm{HLW}$ stainless steel canisters then placing the canisters into interim storage to await shipment to the Greater Confinement Disposal Facility located at the NTS. The operating period for these activities will be a 5-year time span beginning January 1, 2013.

A variation has been devised for the CWO that involves grouting the recalcine and casting the mixture into special HIP containers over the same 5-year timeframe. The HIP containers would then be 
placed into interim storage to await the HIP process. The container HIP'ing process would occur during the 5-year period and continue after recalcine and grouting process completion for 15 more years to complete a 20-year regime. During the HIP'ing process the HIP'ed containers would be sealed inside HLW canisters (the same canisters used for grouted waste) with three HIP containers per canister. Approximately $31,000 \mathrm{HIP}$ containers will be produced during the five-year recalcine and grouting operation and about $10,334 \mathrm{HLW}$ canisters will be produced throughout the 20-year HIP campaign. The HIP containers and HLW canisters will require interim storage in the same facility. The HLW canisters are the final product of the CWO and will be stored until shipment preparations and transport to the National HLW Repository becomes available. Refer to EDF-CWO-001 (Appendix E, Item 11). The process schematic (HLW Study: Nonseparations Alternative Direct Cementitious Waste Option-Section $B$ ), indicating the pathway and the integrated waste volume, is found in EDF-DCWO-011 (Appendix E, Item 10); also consult Reference R19.

The base DCWO process and facility designs were sized to process the recalcine within a 5-year timeframe to match the 5-year processing time for the CWO. The DCWO process and facility designs for the DCWO Study are scaled down from the base designs. The process description, however, is identical for both the CWO and DCWO grouting facilities. Except as noted in this section, the processes described are detailed further and documented in EDF-DCWO-011 (Appendix E, Item 10). The 5-year to 20-year scaling details are documented in EDF-DCWO-015 (Appendix E, Item 11).

The proposed process for the CWO 5-Year Grouting Facility uses a batch method applied to the 10-hour work-day. The general processing steps proposed under this Scoping Study include:

- $\quad$ Receipt of recalcine from the NWCF on a continuous basis

- Receipt of commercial materials for the grout mix

- $\quad$ Receipt of empty SRS canisters

- Mixing and sampling of recalcine to determine grout "recipe"

- Mixing of recalcine and recipe ingredients to form grout

- Filling the SRS canisters with grout

- Controlling contamination spread through surface surveys and use of high-efficiency particulate air (HEPA) filters

- Autoclaving the filled canisters to "cure" the grout

- Dewatering the "cured" canisters

- Seal welding the canister plug in the top of the canisters

- Verifying fill volume

- Recycling of off-specification product

- Transporting the canisters to interim storage. 
Drawing DCWO-00 (Appendix B) of Reference R19, includes a block diagram showing the general processing steps listed above. Appendix $B$ also includes additional block diagrams showing more detailed operations within each processing step.

EDF-DCWO-010 (Appendix E, Item 12) estimates the reasonable maximum number of canisters produced for the CWO to be 16,000 . The number of canisters produced in a week is based on a grouting work schedule of four, 10-hour days per week. Processes such as the autoclave and dewatering.cycles are on a continuous 24-hour schedule until completed.

All operations involving the recalcine are performed remotely using robotics for activities such as canister movement, canister manipulation, and decontamination surveys and cleaning.

No strategic or critical materials, as listed in the Stockpile Report to the Congress (Reference R4), are being used in the process.

\subsubsection{Interim Storage}

A new storage facility has been scoped to interim store the $16,000 \mathrm{CWO}$ waste canisters. The ISF is discussed in the Interim Storage Study (Reference R11) and the drawings in located in Appendix B. The facility consists of 10 vaults capable of storing 18,900 canisters stacked three canisters high.

The HIP'ing variance plan for the Cementitious Waste Option (EDF-CWO-001, Appendix E, Item 11) would require interim storage of 31,000 special HIP containers until processed. After HIP processing, three HIP containers would be sealed inside a single HLW canister and then interim stored. An estimated $10,334 \mathrm{HLW}$ canisters containing all the HIP containers produced would be the final end product awaiting shipping preparation and transport to the National HLW Repository. A quantity of HIP containers and canisters would be interim stored at the same time.. The HIP container grouting process would occur over a 5 -year period corresponding to the recalcination schedule. The HIP process would take 20-years to complete. The first 5 years of HIP processing and HLW canister loading with HIP containers would overlap the CWO recalcination and grouting process. The remaining 15 years would continue the HIP process and canister loading until complete. It is assumed that the HIP containers can be stored in the same storage sleeves that are planned for the canisters. A special handling grapple would be required to handle the drums. The grapple must be capable of lowering the drums into the storage sleeves and releasing the drum. The grapple must also be capable of the reverse operation to remove the drums for processing. The unprocessed drums would be 56 inches long; thus, six could be stored in each storage sleeve. To store the 32,000 drums, 5,334 storage sleeves would be required. This would necessitate a nine vault storage facility.

\subsubsection{MACT Compliance Facility}

It is assumed that MACT requirements will be imposed on the recalcining process by the State of Idaho and EPA. Thus, offgas from the NWCF will be routed through a new MACT facility, before atmospheric release via the ICPP main stack (Reference R11). Off-gas treatment in the MACT facility will achieve the following objectives: 
- Reduce $\mathrm{NO}_{\mathrm{x}}$ (primarily $\mathrm{NO}_{2}$ ) to Nitrogen $\left(\mathrm{N}_{2}\right.$ ) Oxygen $\left(\mathrm{O}_{2}\right)$ and low levels of nitrous oxides (Though NOx control is not mandated by MACT, it is presumed necessaryl to allow the use of EPA-approved sampling/analysis methods in monitoring emissions of some MACTregulated pollutants.)

- Cut the carbon monoxide (CO) concentration to below the MACT limit

- Cut THC concentrations to below the MACT limit

- Reduce mercury concentrations to below the MACT limit

- Oxidize and/or extract PCDD/PCDF (polychlorinated-dibenzo-dioxin/polychlorinateddibenzo-furan)to below the MACT limit

- Condition the offgas (heat, cool, and demist) to permit filtration through activated carbon and HEPA filters

- Provide sufficient draft to pull the offgas stream through the MACT unit and exhaust it to the main ICPP stack.

\subsection{System Descriptions}

\subsubsection{Calcine Retrieval}

The calcine retrieval/transport system consists of two major subsystems. The first of these subsystems is a series of retrieval units mounted on top of the CSSF bin sets. Each retrieval unit incorporates a suction nozzle and an air jet. The air jet would fluidize the calcine and the suction nozzle would lift the fluidized calcine out of the bin. A Vertical Deployment Apparatus (VDA) will move the retrieval units into the bins during calcine retrieval. Two VDAs are required for each bin set, one for the suction nozzle and one for the air jet. Refer to EDF-WTS-002, Appendix E, Item 6).

The second major subsystem is a set of two closed loop pneumatic transport lines designed to operate under vacuum conditions. The suction nozzles would be connected to the transport subsystem with shielded jumpers. The closed loop lines minimize the amount of released air by recycling transport air, and the vacuum condition reduces the risk of contamination spread. Each transport subsystem consists of a cyclone separator, sintered metal filter, HEPA filters, blower, and aftercooler. Each of the two lines is sized for retrieving and transporting 5,952 pounds/hour (2,700 kilograms/hour) of calcine. When both lines are operating, calcine can be delivered to the NWCF at a rate of 11,905 pounds/hour (5,400 kilograms/hour).

Each transport line consists of a 4-inch (10-centimeter) 304L stainless steel pipe inside a 6-inch (15-cm) stainless steel encasement line. The transport lines are encased in a concrete pipe chase at grade level to minimize excavation. The pipe chase is covered with an earthen berm to provide additional shielding. The pipe chase contains four transport lines: one line for type A and C calcines, one for type B

\footnotetext{
' Based on letter report from M. Fuchs (Radian Corp) to Chad Richert (Lockheed Idaho Technologies Company) dated April 28, 1995: Laboratory Studies Performed to Develop Sampling and Analvtical Methods for Conducting an Emission Test of the New Waste Calcining Facility.
} 
calcines, plus backup lines for each. In addition, the pipe contains two 8-inch (20-centimeter) return air lines. The transport line pipe chase is steam-traced to prevent condensation of water inside the transport lines. Both transport lines can access all seven bin sets.

The calcine retrieval transport lines enter the NWCF slurry-blending cell and are routed through two separate cyclones (CY-101, 102 in Drawing CWO-01, Appendix B). The cyclones extract calcine from the lines and deposit it in three dedicated storage bins for type $A, B$, and $C$ calcines $(B-401,402,403$ in Drawing CWO-04, Appendix B). The type B calcine transport line uses one cyclone, and the type A/C line uses the other. A valve below the latter cyclone directs calcine to either the type $A$ or type $C$ storage bin. Transport air flows through sintered metal prefilters (F-101, 103 in Dwg CWO-01, Appendix B) to remove fines, and then passes through a set of three HEPA filters (F-102, 104 in Drawing CWO-01, Appendix B) to minimize contamination of the transport air blowers (BL-103, 104 in Drawing CWO-01, Appendix B). Each blower delivers 800 cubic feet/minute of transport air to carry 5,952 pounds/hour (2,700 kilograms/hour) of retrieved calcine. The transport air from the blower discharge passes through an aftercooler to remove the heat of compression and then enters the return line to the bin sets.

Each transport line also incorporates an aftercooler (E-101, 102 in Drawing CWO-01, Appendix $B$ )and a reheater to condition the air flow upstream of the HEPA filters and downstream of the blowers.

\subsubsection{Sodium-Bearing Waste Liquid Retrieval}

Sodium-bearing waste (SBW) would be transferred from the ICPP tank farm to the recalcination facility slurry-blending cell using existing equipment. Steam jets are the primary method of transferring waste from the tank farm. Waste would be removed from the 300,000 -gallon storage tanks and placed in existing transfer lines. A new transfer line would be installed from valve box B-11 to the slurry-blending cell. Existing tank farm instrumentation and leak detection equipment would be used to monitor all transfers. Refer to EDF-CWO-003, Appendix E, Item 2).

\subsubsection{Recalcination System}

Recalcination would be done in the modified NWCF. Process throughputs are summarized in EDF-CWO-003 (Appendix E, Item 2).

Mass balances for the recalcination process are given in EDF-CWO-003 (Appendix E). Summary descriptions of the process systems used for recalcination are provided in the following sections.

3.2.3.1 Bulk Ingredients. Calcine retrieved from the bin sets is extracted from the two pneumatic transport lines into two cyclones (CY-101 and CY-102 in Drawing CWO-01, Appendix B) in the new slurying wing of NWCF. The cyclones discharge calcine into temporary storage bins $(B-401, B-$ 402 , and B-403) for calcine types A, B, and C. CY-102 discharges only type B calcine to B-402, and CY101 discharges either type $A$ calcine to $B-401$ or type $C$ calcine to $B-403$, depending on which type is being retrieved.

Sodium-bearing waste (SBW) is steam jetted directly from the ICPP tank farm (tanks WM-180, 181,184 , or 186) to either of the two slurry tanks (V-401A or V-401B). Process water is added directly to the slurry tanks from lines in the slurry cell connected to the ICPP demineralized water supply. Sucrose (sugar) is assumed to be shipped into ICPP as a 65 weight percent solution in water and stored in a new storage tank T-201 located in the existing calcium nitrate mixing room. The sucrose is pumped from $\mathrm{T}-201$ to the calciner cell through new piping installed through an existing penetration (as described in NEL-96/196, page 22). Calcium nitrate is stored in liquid form in the calcium nitrate mixing room 
and is pumped first to the NWCF blend and hold tanks, through existing piping, and from there to the slurry tanks through new interconnecting piping.

\title{
3.2.3.1.1 Processing Rates and Statistics-Refer to EDF-CWO-003 (Appendix E,
} Item 2) for principal processing rates and processing statistics for the CWO process.

\author{
3.2.3.1.2 \\ balance statistics. \\ Mass Balance-Refer to EDF-CWO-003 (Appendix E, Item 2) for mass
}

3.2.3.2 Blending / Mixing. Calcines of types A, B, and C are drawn from the bin sets in relative proportions corresponding to the known total masses in each of the three categories. The calcines are fed gravimetrically from bins (B-401, 402, 403 in Drawing CWO-04, Appendix B) into either of two slurrying tanks (V-401A or B), each sized to hold sufficient SBW/calcine slurry feed for 24 hours of calcination. Use of two tanks in tandem allows continuous operation of the calciner. The slurry tanks also receive process water, liquid SBW, and calcium nitrate, which are then slurried with the solid calcines. Mixing is achieved with air spargers, which also keep the high concentration of undissolved solids ( $\sim 33$ weight percent) in suspension. The tanks also incorporate decon spray systems and heating/cooling coils. Refer to EDF-CWO-003 (Appendix E, Item 2).

Once the slurry mixture is ready for processing, it is pumped through a high velocity recycle loop that traverses the vertical and horizontal distance to the calciner cell (and back). An existing unused penetration through the east wall of the calciner cell would be used for the lines. The flow velocity in the recycle loop is kept high enough to retain the solids in suspension. Since the resulting mass flow rate of slurry is higher than the required feed rate to the calciner, most of the flow is routed back to the slurry tanks. The recycle line feeds a manifold around the circumference of the calciner from which three low volume streams are withdrawn to provide feed to the calciner. Each stream is mixed with a 65 weight percent sucrose solution from storage using inline static mixers (M-501 in Drawing CWO-05, Appendix B). These streams are then introduced into the calciner through three specially designed nozzles (FN-501 in Drawing CWO-05, Appendix B) capable of injecting the slurries. The nozzle design would allow introduction of 0.1-0.5 millimeter calcine particles while maintaining atomization of the liquid phase.

The feed nozzles would interface with the calciner through existing penetrations in the vessel wall. Three flow sensors would be installed just upstream of the nozzles to measure the feed rate of sugar/slurry to each of the calciner feed nozzles. These must measure over the range of $15-45$ gallons/hour and at least 1,268 pounds/hour, and would be used in conjunction with three flow rate controllers interfaced with the existing control system.

Three positive displacement pumps in parallel (P-401 in Drawing CWO-04, Appendix B) would be used to pump the slurry through the recycle loop. These pumps may be of the moving cavity type (Moyno), diaphragm tube type (Toyo), or hydraulic cylinder cement type (Schwing). Although each type has advantages and disadvantages, the Moyno pumps were chosen for the baseline design, and may be best suited for both erosive slurries and high radiation environments since it has fewer seals. The pumps would deliver 250 pounds/square inch at 100 gallons/minute of 33 weight percent solids, using a 20-30 horsepower electric motor.

3.2.3.3 Recalcination. Fluidizing air, oxygen, and kerosene would be added to the calciner through existing lines. Sucrose solution would be transported into ICPP by truck or by rail, and stored in an existing tank in the cold additive mixing area. It would be pumped (P-201 in Drawing CWO-02, Appendix B) from this storage location directly to the injection point in the calciner feed lines. Scrub 
recycle solution would be recycled to the calciner using the existing system. One of the four feed nozzles would not be replaced, but would stay in its current configuration to accept the recycled scrub from the existing blend, hold, and feed tanks. Refer to EDF-CWO-003 (Appendix E, Item 2).

Calciner offgas will be routed to the existing calcination offgas treatment facility (with modifications described below), and recalcine would be transported via the current product transport system to a booster station (CY-701 and B-701 in Drawing CWO-07, Appendix B) in the slurrying/transport cell, as described below.

3.2.3.4 Off-gas System. Treatment of offgas from the calciner is accomplished using the existing offgas system for NWCF with some modifications (see Drawing CWO-06, Appendix B). The current system includes a cyclone, spray quench, venturi scrubber, demisting vessels, ruthenium adsorbers (which act as pre-filters for the HEPA filters), heaters, HEPA filter banks, and a draft system to pull the offgas through the system and propel it into the Atmospheric Protection System and the ICPP main stack.

It is expected that changed offgas flowrates, and higher concentrations of fines in the offgas would necessitate redesign and replacement of the present cyclone (CY-501 in Drawing CWO-05, Appendix B). In addition, a system (described below) to extract elemental mercury from the NWCF scrub solution would be added. This system is needed to prevent accumulation of mercury in tank farm liquids. Such accumulation would result from efficient capture of mercury in the NWCF scrub solution, and subsequent periodic flushing of the high-mercury scrub solution back to the tank farm during deep recycle.

The NWCF offgas system would be upgraded to comply with MACT requirements. The upgrade will include control systems for carbon monoxide, NOx, unburned hydrocarbons, and residual mercury not collected in the scrubbing system. The MACT system is described in a later section.

3.2.3.5 Mercury recovery. The mercury extraction system is an electrowinning cell, consisting of a series of electrolytic recovery tanks (EC-601 in Drawing CWO-06, Appendix B), a backwashable filter (F-603), and a positive displacement pump (P-601), all installed in the valve cubicle. A slipstream of $50 \mathrm{gph}$ is drawn off the scrub hold tank and routed to one of the two tanks. The recovery cells would extract elemental mercury from the scrub solution and store it in one of the tanks. If tank farm wastes and calcine are delisted (as assumed), the recovered elemental mercury would be decanted from the storage tank into 1-gallon paint cans, amalgamated with sulfur in a jar mill, and disposed of as radioactive waste. Otherwise, it will be stored indefinitely, pending availability of a suitable mixed waste disposal facility.

3.2.3.6 Recalcine Transport. The existing NWCF transport air (TA) system is designed to transport solids approximately 230 feet ( 460 feet for a closed loop) at a maximum transfer rate of 300 pound-mass/hour. The recalcination process requires a transfer rate of 674 pound-mass/hour. The increased capacity would be achieved by reducing the transport distance for the existing system and adding a second transport system to carry the recalcined solids from NWCF to the new grouting facility. The new transport system would include a booster station located in the slurrying/transport cell. This cell would be a new addition, adjacent to the NWCF. Since the transport distance from the calciner to the booster station would be shortened from 230 feet to less than 140 feet, the current NWCF TA system should be able to move the increased calcine load, using the existing transport air return jet to provide the required motive force. Transport air from the existing system would be returned to the calciner vessel, as is currently done. The new solids loading ratio would be between 1.7 and 2.0 pound-mass solids/poundmass air.

The existing TA system would remain mostly unchanged within the NWCF. However, upon exiting the building, the transport line would be redirected to the booster station (rather than the CSSF bin 
sets). The booster station would incorporate a cyclone (CY-701 in Drawing CWO-07, Appendix B) that extracts solids from the line and drops them into a 550-cubic foot storage bin (B-701). This bin provides 72 hours of calcine surge capacity at a mass flow rate of 674 pound-mass/hour. A rotary valve at the bin outlet meters calcine into the new TA system line which then delivers the calcine to the grouting facility at the same rate it is received. The new TA system is a closed loop pressurized system capable of transporting calcine 900 feet $(1,800$ feet for a closed loop) at a design rate of 674 pound-mass/hour. A compressor (BL-701 in Drawing CWO-07, Appendix B), located in the recalcining facility, provides 280 standard cubic feet per minute of transport air at 10 pounds/square inch gauge (psig), giving a solids loading of about 0.5 pound-mass solids/pound-mass air. The new transport line enters near the top of the grouting facility where the calcine passes through another cyclone (CY-301 in Drawing CWO-03, Appendix B) and is deposited in the grouting facility storage bins ( $T-201$ in Drawing DCWO-02 of Reference R19). After leaving the cyclone, the TA air passes through a bag filter to remove fines that would otherwise build up in the system, and then is returned to the booster station compressor to be recycled.

The new transport line consists of a 4-inch ( 10 centimeter) $304 \mathrm{~L}$ stainless steel pipe inside a 6 -inch (15-centimeter) stainless steel encasement line. The transport lines are encased in a concrete pipe chase covered with an earthen berm to provide additional shielding. The pipe chase contains two transport lines, a backup line and a 20 centimeter ( 8 -inch) return air line. The transport line pipe chase is steamtraced to prevent water condensation in the transport lines.

3.2.3.7 Mercury Amalgamation. Recovered elemental mercury from the accumulation tank in the mercury recovery unit would be decanted into 1-gallon paint cans containing elemental sulfur in $50 \%$ excess (L-101 in Drawing CWO-10, Appendix B). The Hg-S mixture in each can is amalgamated in a jar mill and removed from the valve cubicle. If either the tank farm wastes or the calcine is successfully delisted, and the activity of the amalgam is sufficiently low, it can be disposed of as lowlevel radioactive waste at the Radioactive Waste Management Complex (RWMC) at the INEEL. Otherwise, the amalgam would be stored as treated mixed waste until such time that a suitable mixed waste disposal facility is available.

\subsubsection{Grouting Facility}

The CWO grouting facility and related equipment are discussed and detailed in the DCWO Study Report (Reference R19). Drawings related to the facility are included in that study.

\subsubsection{Interim Storage}

The ISF and related equipment are discussed and detailed in the Interim Storage Scoping Study (Reference R11). Drawings related to the facility are included in that study.

\subsubsection{MACT Compliance Facility}

The MACT Compliance Facility and related equipment are discussed and detailed in the Feasibility Study Report for NWCF MACT Compliance Facility (Reference R22). Drawings related to the facility are included in that study. 


\subsection{CWO-NWCF Facility Recalcination Modifications and Additions}

\subsubsection{NWCF Process Equipment Modifications}

The NWCF must be modified in order to accommodate the CWO process. Details of the modification are given in Attachments 1 and 2 of EDF-CWO-003 (Appendix E, Item 2). A summary list of the required process equipment modifications is as follows:

1. Additional hot cell space must be added to the east-side of building 659. The building layout and design for this space is discussed in the next section. New process equipment that this space would house is as follows:

\section{- Slurry tanks}

- $\quad$ Pumps, pumping equipment, and a recycle loop for slurry feed from the slurry tanks to the calciner

- Temporary storage bins for calcine types $A, B$, and $C$ above the slurry tanks

- Booster station (consisting of a cyclone and a temporary calcine storage bin) to transfer recalcined solids from the existing NWCF product transport system into a new pressurized transport system from NWCF to the grouting facility

Additional process related requirements for the new NWCF hot cell space include the following:

- Facility would provide separate shielding for each of the above systems to allow separate decontamination and maintenance access without excessive radiation fields

- Labyrinth would be provided for shielding and contamination control

- $\quad$ Piping would be provided for steam, cooling water, and sparging air for the slurry tanks

- $\quad$ Piping would be provided for decon spray systems in all cells

- Transfer lines (with air lifts) would be provided between the slurry tanks and the tank farm, and between the slurry tanks and the blend and hold cell tanks

- Vent lines would be added from the slurry tanks and calcine storage bins to the vessel offgas (VOG) system

- Provision would be made to vent transport air from the calcine retrieval system into the calciner.

2. A slurry recycle loop must be installed between the slurry feed tanks and the calciner cell, which incorporates the following features:

- Moving cavity ("Moyno") pump to move slurried solid calcine and liquid SBW from the slurry tanks to the calciner cell 
- $\quad$-inch piping loop from the pump to the manifold in the calciner cell (described below) and back to the slurry tanks

- Circular pipe manifold around the calciner that receives slurry from the recycle loop, distributes slurry to up to three of the four feed nozzles to the calciner, and then returns the overnlow to the recycle, which routes it back to the slurry tanks.

3. Feed lines to three of the four feed nozzles must be removed and replaced with feed lines connected to the branching tee on the slurry recycle loop manifold described above. The fourth feed nozzle is maintained in the current configuration to allow feeding from the blend and hold tanks to the calciner (for example during startup).

4. The existing cyclone for the calciner offgas must be removed and replaced with a unit designed for the changed offgas and solids flow rates.

5. The existing solids transport system must be modified to transport recalcined solids from NWCF to the booster station, rather than to the bin sets. In addition to the new equipment items described above for the booster station, the following transport system equipment would be installed in the grouting facility:

- Cyclone (CY-702 in Drawing CWO-07, Appendix B) at the grouting facility to transfer calcine from the new transport line and deposit it into the calcine storage tanks (T-201A,B in Drawing DCWO-02 from Reference R19)

- Baghouse (BH-701) to extract fines from recycle transport air, upstream of the blower

- Transport air blower (BL-701) to provide motive force for moving the transport.

Additional detail for the transport system equipment is provided in Attachment 1 of EDF-CWO-003 (Appendix E, Item 2).

6. Additions to the NWCF offgas system, described in Reference R22, would be provided to comply with MACT requirements. These additions are shown schematically in Drawing CWO-09 of Appendix B and include the following equipment:

- $\quad$ NOx and unburned hydrocarbon abatement system (John Zink NOxidizer system)

- Air dilution/spray quench system to control exit temperature of MACT system offgases

- Two granulated activated carbon canister filter units to extract mercury which remains in the offgas after the scrubbing process

- New draft system compressors to handle the increased offgas flows from the MACT facility

- New HEPA filter bank for final filtration before discharge of offgas into the ICPP main stack 
- Continuous Emissions Monitoring System (CEMS) to verify MACT compliance for selected pollutants

- Automatic waste feed cutoff system to stop processing of waste through the calciner when emissions exceed MACT requirements.

7. In addition to the above modifications required for MACT compliance, a system for amalgamation of the mercury collected from the scrub solution would be required. This system is shown schematically in Drawing CWO-10. Mercury collected in the electrowinning cell would be decanted into 1-gallon paint cans, amalgamated with elemental sulfur, and disposed as low-level radioactive waste. (If delisting of NWCF effluents is unsuccessful, or if the mercury contains high radionuclide concentrations and is considered $\mathrm{HLW}$ then it would require storage until a suitable disposal facility is available.)

\subsubsection{Building Addition Layout}

A NWCF process cell large enough to house CWO equipment and close enough to the calciner cell to make construction feasible was not available. Therefore, a separate facility would be built to house the CWO equipment. The new facility would be an external addition to the NWCF located adjacent to the NWCF east wall near the northeast corner.

Shoring would be installed as required to limit the excavation within the construction area. Existing concrete ramps and landings at the NWCF east entrance, east elevator entrance, and ramp/dock shall be demolished and replaced after construction. Existing underground utilities that fall within the construction area include but are not limited to: firewater lines, steam and condensate lines, electrical ductbanks, telephone cable, cathodic protection lines, communication/instrumentation lines, and calcine transport lines. Existing underground utility lines would be rerouted where possible and/or retained and protected during construction.

The existing asphalt access road running along the NWCF east side would be rerouted to the east as required. Existing underground utilities affected by the access road rerouting would be modified as required. Modifications may include rerouting and/or demolition and installation of handholes and manholes.

The addition would be approximately 70 feet long, 38 feet wide, and 90 feet high. Forty feet of the addition would be below the existing grade. The addition is divided into cells and rooms at varying floor levels, each having concrete shielding walls and accesses. The walls, floor, and roof would be constructed out of reinforced structural grade concrete. The wall thickness would be sized to provide adequate shielding. The slurry-blending cell would have concrete walls approximately 3 feet -6 inches thick. The other cell walls would be approximately 2 feet -0 inches thick. The floor of each cell would be covered with stainless steel plate. The mezzanine would be covered by a steel frame structure with metal wall and roof panels. The mezzanine would house a bridge crane for equipment removal.

Equipment (tanks, vessels, pumps, compressors, etc.) would be located such that personnel access is possible for maintenance, replacement, etc. Stainless steel working platforms, stairs, and ladders would provide access to elevated equipment.

The addition would consist of a Slurry-blending Cell, Pump Cell, Sampling Cell, Sample Viewing Room, Transport (transfer) Cell, Recalcine Transport Air Compressor Room and Grout Facility Transport 
Air Compressor Room, Mercury Amalgamation Room with storage, cask transfer, decontamination, and mezzanine.

3.3.2.1 Slurry-Blending Cell. The slurry-blending cell would have two cyclones; two sintered metal filters, three calcine storage bins, and two slurry tanks. The cell design would allow solids to flow by gravity from the calcine retrieval system cyclones to the storage bins and into the slurry tanks. Approximately 64 feet is needed to accommodate an equipment arrangement that would allow solids to flow by gravity. The cell would have a labyrinth entryway for shielding and contamination control.

3.3.2.2 Pump Cell. The pump cell would be located directly south of the slurry-blending cell and adjacent to the NWCF transport air return cubicle. The cell would contain three slurry feed pumps and the associated piping, instrumentation, and valves. The slurry feed line to the calciner cell would exit the pump cell and enter the NWCF building in the return jet cubicle. From the cubicle, the slurry line would pass through an abandoned 18-inch shielded encasement to the calciner cell.

3.3.2.3 Sample Cell. The sample cell would be located directly to the north of the slurryblending cell. The cell would have an adjacent Sample Viewing Room. Remote operational equipment (shielding windows, master slaves, remote valves, etc.) would be used during sampling operations to limit radiation exposure to operators.

3.3.2.4 Transport Cell. The transport cell would be located above the east half of the pump cell and south of the slurry-blending cell. The cell design provides sufficient height for the recalcine transport cyclone to be placed above the recalcine storage bin. The exterior roof of the Slurry-blending Cell and the Transport (transfer) Cell would have removable concrete hatches covered with a removable roofing assembly. The assembly would be constructed of steel framing members and steel roof panels. The roof hatches are for equipment installation and removal.

3.3.2.5 Calcine Retrieval Transport Air Compressor Room. The calcine retrieval transport air compressor room would be located directly above the sample and amalgamation cell. Two HEPA filter banks, two transport air compressors, and two aftercoolers would be located in this room.

3.3.2.6 Grouting Facility Transport Air (TA) Compressor Room. The Grouting Facility Transport Air (TA) Compressor room would be located above the pump cell and west of the transport cell. This room would house two transport air compressors. The concrete ceilings of the Pump Cell and the Grouting Facility TA Compressor room would contain roof hatches for equipment removal.

Mercury Almalgamation Room. The mercury amalgamation room will be adjacent to and west of the sample cell. This room will house a sulfur loading device and a jar mill. These will be used to blend and amalgamate sulfur with elemental mercury collected from NWCF scrub solution as previously discussed. A portion of the cell will be dedicated to temporary storage of the amalgamated mercury.

\subsubsection{Power}

The Recalcining Facility electrical requirements were estimated to be $131 \mathrm{kVA}$. The major load is the process equipment. Power would be carried over existing feeders up to Substation 15. From Substation 15 , new feeders would be routed through new and existing duct banks. The possibility of obtaining power from CPP-659 would be investigated during the conceptual design. Refer to EDF-CWO-004 (Appendix E, Item 4). 
The standby power requirements for the recalcining facility were analyzed and determined to be only lighting and miscellaneous equipment resulting in a standby power requirement of $3 \mathrm{kVA}$. HVAC would be connected to the existing NWCF system, and therefore additional standby power would not be required. Process equipment would not require standby power.

Standby power would be provided from the standby power panels located in the NWCF. Should additional standby power be required, it can be provided to the facility via the normal power distribution system from Substation 60.

A solid state uninterruptible power supply (UPS) with a static transfer switch would be provided. The UPS would be provided with a 20 -minute battery backup. Both the normal feed and the bypass feed to the UPS would be on standby power. The UPS would feed a $208 \mathrm{Y} / 120$ volt panel. The UPS and the panel would be located in the electrical room. The UPS would support the following loads: voice paging/evacuation systems, environmental monitoring system, and other critical loads.

\subsubsection{Instrumentation and Control}

Instrumentation would be provided to monitor process streams and to control process functions. All tanks and bins would have a means of measuring the inventory in the tank or bin, either through level or weight measurements. The pressure and temperature in the tanks and bins would also be measured. The tanks and bins with offgas lines would have a differential pressure measurement between the tank and the offgas line. Control for the remote operated valves and pumps would come from input provided by the various instruments. Where possible, instrumentation would be nonintrusive due to the corrosive nature of the material being measured. The existing NWCF Distributed Control System (DCS) would be used. New instrumentation would connect to the data system through new wiring.

\subsubsection{HVAC}

The HVAC system for the CWO addition would use the existing supply and exhaust air systems at the NWCF. This would entail extending the underground exhaust tunnel to provide exhaust for each cell and room in the addition. The supply air ducts would be extended to the new facility from the existing NWCF supply distribution system. It is assumed the existing NWCF HVAC system would have the capacity to meet the added demands but this would be further analyzed during conceptual design.

\subsubsection{Remote Handling}

The recalcining building addition has been designed as a remote facility. However, the facility was designed with cells and rooms that provide shielding from radiation sources for mechanical equipment. Maintenance activities can take place with a minimum amount of decontamination.

\subsubsection{Process Equipment}

A complete process equipment list for recalcination, referencing the process flow diagrams in Appendix B, is provided in EDF-CWO-003 (Appendix E, Item 2). Sizing information, and required numbers of each equipment item are included.

3.3.6.1 Equipment Labels. To access the equipment labels refer to EDF-CWO-003 (Appendix E, Item 2). 
3.3.6.2 Equipment List. To access the equipment list refer to EDF-CWO-003 (Appendix E, Item 2).

\subsection{Utilities Description}

\subsubsection{Utilizies Summary}

To access the utility summary refer to EDF-CWO-003 (Appendix E, Item 2).

\subsubsection{Process Effluent Waste (PEW-LLW)}

During normal operations at the NWCF, LLW is generated and processed through the PEW evaporator. The volume of LLW is not expected to change substantially as a result of CWO modifications to the NWCF.

\subsubsection{High-Level Liquid Waste}

High-level radioactive waste (HLW) generated at the NWCF is transferred to and stored in the ICPP tank farm. The volume of high level waste generated is not expected to change substantially as a result of CWO modifications to the NWCF. Refer to EDF-CWO-003 (Appendix E, Item 2).

\subsubsection{Service Waste}

The CWO modifications to the NWCF would not require significant increases in cooling water or steam usage. Therefore, the volume of water discharged to the service waste system is not expected to change substantially.

\subsection{Required Chemicals Summary}

Chemicals required for the CWO process are summarized in EDF-CWO-003 (Appendix E, Item 2).

\subsection{Secondary Waste Products}

\subsubsection{Secondary Wastes Generated by the CWO Process}

Secondary waste streams generated by the CWO process (in addition to those generated from grouting of recalcine; see Ref. 10) are summarized in EDF-CWO-003 (Appendix E, Item 2).

\subsubsection{Solid Radioactive Waste}

A large percentage of solid radioactive waste generated at the NWCF is protective clothing. The CWO modifications to the NWCF are not expected to result in an increase in protective clothing usage. Therefore, the volume of solid radioactive waste is not expected to change substantially. 


\section{4. $\operatorname{cosT}$}

The base design for the CWO was developed for a 5 year operating schedule. The CWO grouting facility would also operate on a 5-year schedule to match the CWO.

Total Estimated Cost (TEC) for each part of the complete CWO option is provided below. Details are given in Appendix $\mathrm{C}$ for the CWO 5-year operation.

OPC Other Project Cost

TEC Total Estimated Cost

TPC Total Project Cost

\subsection{CWO Total Estimated Cost}

Table 1. Total estimated cost for CWO - design and construction (5yr)

\begin{tabular}{|c|c|c|c|c|c|c|c|}
\hline Cost Item & $\begin{array}{c}\text { CRTS } \\
(\mathrm{k} \$)\end{array}$ & $\begin{array}{c}\text { Recalcination } \\
(\mathrm{k} \mathrm{S})\end{array}$ & $\begin{array}{c}\text { MACT } \\
(\$) \\
\end{array}$ & $\begin{array}{c}\text { Grout } \\
\text { Facility (k\$) } \\
\end{array}$ & $\begin{array}{c}\text { Interim } \\
\text { Storage }(k \$)\end{array}$ & $\begin{array}{c}\text { Utilities } \\
(\mathrm{k} S)\end{array}$ & $\begin{array}{c}\text { Total } \\
(\$)\end{array}$ \\
\hline OPC (Unescalated) & 21,267 & 56,122 & 15,457 & 86,562 & 16,228 & 1,386 & 197,022 \\
\hline OPC (Escalated) & 9,358 & 12,505 & 2,651 & 12,708 & 4,253 & 264 & 41,739 \\
\hline OPC (Mgmt Reserve) & 0 & 3,011 & 0 & 877 & 0 & 0 & 3,888 \\
\hline OPC (Contingency) & 10,475 & 22,483 & 3,692 & 34,853 & 5,118 & 442 & 77,063 \\
\hline Total OPC & 41,100 & 94,100 & 21,800 & 135,000 & 25,600 & 2,100 & 319,700 \\
\hline TEC (Unescalated) & 123,193 & 120,104 & 34,522 & 682,374 & 417,093 & 10,721 & $1,388,007$ \\
\hline TEC (Eescalated) & 52,330 & 47,750 & 6,286 & 216,257 & 151,004 & 3.873 & 477,500 \\
\hline TEC (Mgmt Reserve) & 13,406 & 10,102 & 2,536 & 64,303 & 44,363 & 1,049 & 135.759 \\
\hline TEC (Contingency) & 49,992 & 57,944 & 9,356 & 287,065 & 69,440 & 3,156 & 476.953 \\
\hline Total TEC & 238,921 & 236,900 & 52,700 & $1,250,000$ & 681,900 & 18,799 & $2,479,220$ \\
\hline TPC (Unescalated) & 144,460 & 176,227 & 49,974 & 768,936 & 433,321 & 12,104 & $1,585,022$ \\
\hline TPC (Escalated) & 61,688 & 60,255 & 8,937 & 228,965 & 155,258 & 4,137 & 519,240 \\
\hline TPC (Mgmt Reserve) & 13.406 & 13,112 & 2,536 & 65,181 & 44,363 & 1,049 & 139,647 \\
\hline TPC (Contingency) & 60,467 & 80,407 & 13.048 & 321,918 & 74,558 & 3,606 & 554.004 \\
\hline Total TPC & 280,021 & 330,000 & $74.500^{\circ}$ & $1,385,000$ & 707.500 & 20.899 & $2,797,920$ \\
\hline Operations (Unescalated) & 19,363 & 400,956 & 10.907 & 404,043 & 48.245 & 58 & 883,572 \\
\hline Operations (Escalated) & 11,085 & 228,337 & 6.479 & 234.917 & 57.554 & 67 & 538.439 \\
\hline Operations (Contingency) & 9,143 & 188,788 & 5,216 & 191.688 & 31,740 & 38 & 426,613 \\
\hline Total Operations & 39.621 & 818080 & 22,601 & 830,648 & 137.538 & 163 & $1,848.651$ \\
\hline Post Operations (Unesc) & 14,713 & 131,822 & 3,671 & 367,385 & 114.497 & 279 & 632,367 \\
\hline Post Operations (Esc)) & 10,712 & 109,432 & 2,682 & 286,339 & 718,639 & 608 & $1,128,412$ \\
\hline
\end{tabular}


Table 1. (continued).

\begin{tabular}{|c|c|c|c|c|c|c|c|}
\hline Cost Item & $\begin{array}{c}\text { CRTS } \\
(\mathrm{k} \$)\end{array}$ & $\begin{array}{c}\text { Recalcination } \\
(\mathrm{k} \$)\end{array}$ & $\begin{array}{c}\text { MACT } \\
(\$)\end{array}$ & $\begin{array}{c}\text { Grout } \\
\text { Facility (k\$) }\end{array}$ & $\begin{array}{c}\text { Interim } \\
\text { Storage }(\mathrm{k} \$)\end{array}$ & $\begin{array}{c}\text { Utilities } \\
(\mathrm{k} \$)\end{array}$ & $\begin{array}{c}\text { Total } \\
(\$)\end{array}$ \\
\hline Post Operations (Contin) & 3,814 & 72.376 & 1,906 & 196,117 & 249,941 & 266 & 524,420 \\
\hline Total Post Operations & 29,239 & 313,631 & 8,259 & & $1,083,077$ & 1,153 & $2,285,200$ \\
\hline $\begin{array}{c}\text { Total Cost } \\
\text { (w/escalation, Mgt } \\
\text { reserve \& Contingency) }\end{array}$ & 348,880 & $1,461,711$ & 105,360 & $3,065,489$ & $1,926,115$ & 22,216 & $6,929,771$ \\
\hline $\begin{array}{c}\text { Dotal Cost (Unescalated) } \\
\text { Discounted Cost } \\
\text { (Escalated) }\end{array}$ & 178,566 & 709005 & 114,531 & $1,540,365$ & 596,063 & 12,445 & $3,150,975$ \\
\hline
\end{tabular}




\section{CWO SCHEDULE}

The CWO Recalcination Facility and Grout Facility would follow a 5-year operation schedule relative to the following table:

Table 2. CWO project schedule.

\begin{tabular}{|c|c|c|c|c|c|c|c|c|c|c|c|c|c|c|c|c|c|c|c|c|c|c|}
\hline ID & Task Name & Duration & 19992000 & 2001 & 2002 & 2003 & 2004 & 2005 & 20062 & 2007 & 2008 & 2009 & 2010 & J2011 & 2012 & 2013 & 2014 & 2015 & 2016 & 2017 & \begin{tabular}{|l|l|}
2018 & 20 \\
\end{tabular} & 1922020 \\
\hline 1 & ROD & & $10 / 1$ & & & & & & & & & & & & & & & & & & & \\
\hline 2 & $R 80$ & 4 Years & & & & & & & & & & & & & & & & & & & & \\
\hline 3 & Feasibility Study & 1Year & & & & يتيеي & & & & & & & & & & & & & & & & \\
\hline 4 & Conceptual Design & 3 Years & & & & & & & & & & & & & & & & & & & & \\
\hline 5 & Tite Design & $1-1 / 2$ Year: & & & & & & & & $=$ & & & & & & & & & & & & \\
\hline 6 & BDAT Equivalent Approv & & & & & & & & & & & & & & & & & & & & & \\
\hline 7 & Construction & 2.12 Years & & & & & & & & & & & & & & & & & & & & \\
\hline 8 & Testing & 1Year & & & & & & & & & & & & & & & & & & & & \\
\hline 9 & ORR \& Startup & 1 Year & & & & & & & & & & & & & & & & & & & & \\
\hline 10 & Processing (recalcining i & 5 Years & & & & & & & & & & & & & & & & & & & & \\
\hline 11 & Processing Ends & & & & & & & & & & & & & & & & & & & & $12 / 31$ & \\
\hline 12 & Intenm Storage & Incefinite & & & & & & & & & & & & & & & & & & & & \\
\hline
\end{tabular}




\section{UNCERTAINTIES}

Uncertainties relate to areas of the CWO that are questionable such as the maturity of recalcination technology, risk assessment, and failure modes. For future CWO activities, the questions raised by these problem areas must be answered.

\subsection{Maturity of Recalcination Technology}

Guidance for assessing the maturity of technologies developed through the Office of Science and Technology (OST) in DOE is found in the Interim Guidance-Office of Science and Technology Technical Decision Process, DOE Standard Operating Procedure (Reference R23). Appendix H provides guidelines whereby a given technology may be classified into one of seven stages of maturity. These stages are titled "Basic Research", "Applied Research", "Exploratory Development", "Advanced Development", "Engineering Development", "Demonstration", and "Implementation". Each stage is characterized with minimum goals, objectives, measures of effectiveness, and actions. In addition, each stage of maturity must include requirements for entry into the next stage, as detailed in the above reference.

The above document is strictly applicable only to technologies developed under the auspices and funding of OST. The CWO process utilizes a number of technologies, most of which are sufficiently mature to have been utilized in commercial applications similar to, but not identical with, the proposed application to mixed-HLW in the CWO. However, because these technologies were not developed under the OST paradigm, direct application of the OST maturity guidelines is not straightforward.

Dr. D. D. Siemer (LMITCO) has successfully grouted surrogates of all three major calcine types (alumina, zirconium, and SBW) using the approach recommended in the CWO, and has objectively demonstrated the leach resistance of the resulting waste forms to be superior to that of borosilicate glass. These demonstrations may satisfy the Stage 5 ("Engineering Development"), Gate 5 requirement (from the OST guidance document) for "Completed and documented preliminary test results and satisfied test plan requirements".

In regard to slurry calcination technology, the Swedish-owned company Studsvik, Inc. is currently constructing a steam-heated commercial-scale fluidized bed processing facility in Erwin, TN for volume and weight reduction of radioactive ion exchange resins, solvents, and sludges. The design of this facility is based on Studsvik's proven THOR (THermal Organic Reduction) technology. Existence of this facility suggests that the slurry calcination portion of the CWO may satisfy the Stage 6 ("Demonstration"), Gate 6 requirement stating "Implementation and commercialization viability have been clearly defined according to accepted business standards".

Extensive experience in pumping of slurry feeds to fluidized bed combustors (and other energy systems) has been accumulated by the Advanced Processes and Technologies group at the Energy \& Environmental Research Center (EERC) at the University of North Dakota, though this group has no separate program focused on slurry pumping, per se. Nonetheless, performance data compiled at EERC would suggest that slurry pumping technology may satisfy the Stage 4 ("Advanced Development"), Gate 4 requirement: "Technology assessed as being the right technology, at the right place, at the right time".

The use of sucrose (sugar) as a reducing agent for inorganic nitrates has been the subject of pilot scale studies at ICPP, and of demonstration testing at Hanford by Vectra, Inc. The latter achieved $>95 \%$ reduction of nitrates in LLW simulant using sugar. Studsvik, Inc. (mentioned above) claim 99\% 
reduction of nitrates ( $<100 \mathrm{ppm}$ total NOx in offgas) from processing of 5.2 Moles of Sodium Hydroxide $\left(\mathrm{NaNO}_{3}\right.$ in water slurry using the THOR process. These developmental activities, again, were not performed according to the OST protocols. However, the objective facts associated with these studies suggest that the use of sugar to reduce nitrates has satisfied the stated goal of Stage 4 ("Advanced Development") in the OST paradigm, which is "Specific DOE-EM application of product, concept, or subsystems that includes studies, advanced analysis, and laboratory-scale models". In addition, the goal of Stage 5 ("Engineering Development") which is to "Scale-up and refine detailed design for prototypes and pilots; clarify DOE deployment strategy and schedules to meet internal/external performance needs" may also have been satisfied.

In summary, the principal technologies applied in the CWO have been developed and applied in a number of applications, some of which are commercial. Unfortunately, not all of these applications have been in the area of nuclear waste handling systems. This indicates the need for some (not necessarily extensive) additional demonstration of these technologies, prior to finalization of design and specifications for the required processing systems for the CWO. The overall technology development process for the CWO is expected to be straightforward, based on the successes described above.

\subsection{CWO Risk Assessment}

Schedule and cost risks identified in this Scoping Study are categorized below according to the source of the risk. Risks were identified for a 5-year schedule. No effort was expended to identify or assess risks related to the HIPing activities since they are covered in the CWO HIP Variance Plan (EDFCWO-001, Appendix E, Item 11). Data sheets for all the identified risks are included in Appendix F, along with explanation of the Risk Rating calculation method.

Most of the risks are derived from the possibility that the Study Assumptions (SA) listed in Section 2.2.2 of this report may be incorrect. The Work Scope (WS) Assumptions provide the framework for this study. If any WS Assumptions are incorrect, they would cause programmatic risks that are not listed or evaluated here, and dealing with the consequences and required contingency plans for a high-rated WS risk is beyond the scope of this study.

In the Project category, the highest risk is rated at " $4 . "$ In the Technical category, the highest risk is rated at "4." In the Environmental Safety and Health (ESH) category, the highest risk is rated at "4." The maximum highest risk rating is "9." The three risk-sets are listed below:

\subsubsection{Project}

P.1 Changing regulatory requirements may change CWO design and delay startup. Risk $=4$

P.2 NWCF building addition for recalcination and the MACT Facility may not be allowed to be installed where specifieded. Risk $=2$

\subsubsection{Technical}

T.1 Higher than expected erosion in the slurry piping system. Risk $=4$

T.2 The NWCF may not accommodate injection of slurried wastes. Risk $=4$

T.3 A high (>90\%) destruction of nitrates in the slurried wastes may not be achieved during recalcination. Risk $=2$. 
T.4 Proper calcine blends may not be achieved due to calcine retrieval difficulties. Risk = TBD

\subsubsection{ES\&H}

ESH.1 Uncontrolled organic and nitrate reaction. Risk $=3$

ESH.2 Recalcine transfer line leaks. Risk $=\mathrm{T} 2$

ESH.3 High incident of equipment failures could lead to excessive radiation exposures. Risk $=4$.

\subsection{Failure Modes}

The following possible failure scenarios were identified; however, their evaluation is beyond the scope of this study.

Failure modes are considered to fall into one of two categories - electrical and mechanical.

The following is a list of possible significant failure modes by major system for the CWO process.

Calcine Retrieval

- Transport line failure, mechanical

- Plugged transfer line, mechanical

- Cyclone failure, mechanical and/or electrical

- Sintered metal filter failure, mechanical

- Transport air blower failure, mechanical and/or electrical

- HEPA filter failure, mechanical

- Transport air aftercooler failure, mechanical and/or electrical

Slurry Blending

- Calcine storage bin rotary valve failure, mechanical

- Calcine diverter valve failure, mechanical

- Slurry tank valve failure, mechanical

Slurry Feed System

- $\quad$ Slurry feed pump failure, mechanical and/or electrical 
- Slurry piping system failure, mechanical

- Slurry feed nozzle failure, mechanical

- $\quad$ Sucrose inline mixer failure, mechanical

- Sucrose pump failure, mechanical and/or electrical

Recalcine Transfer

- Cyclone failure, mechanical and/or electrical

- $\quad$ Return jet failure, mechanical

- Transfer line failure, mechanical

- Plugged transfer line, mechanical

- Grouting Facility Recalcine Transfer System

- Recalcine interim storage rotary valve failure, mechanical

- Transport air blower failure, mechanical and/or electrical

- Transfer line failure, mechanical

- Plugged transfer line, mechanical

- Grouting facility cyclone failure, mechanical and/or electrical

- Grouting facility bag filter failure, mechanical. 


\section{PROJECT DATA SHEET}

Table 3 and 4 contain the Project Data Sheets for CWO, DC, and DCWO. Data are presented for the construction, operation, and decommissioning phases of the project. Estimates are included for the following parameters:

- $\quad$ Cost

- $\quad$ Schedule

- Air Emissions

- $\quad$ Liquid Effluents

- $\quad$ Solid Wastes

- Utilities Used

- Manpower Requirements

- Regulatory Requirements.

Because this project is at a preliminary stage of design, most of the information presented in this PDS is based on representative literature values, existing laboratory data, and engineering judgement. The cost data in this table were obtained from the cost estimates in Appendix F. Appendix $\mathrm{H}$ contains the support information for the remainder of the data in the table.

Project data sheets for the CRTS, MACT Facility, DCWO, ISF, and Utility Support can be located as indicated below:

- $\quad$ CRTS

EDF-WTS-002, Appendix E, Item 6

- MACT Facility Reference R22

- DCWO Reference R19

- ISF Reference R11

- Utility Support Reference R3. 
Table 3. Project Data Sheet for CWO.

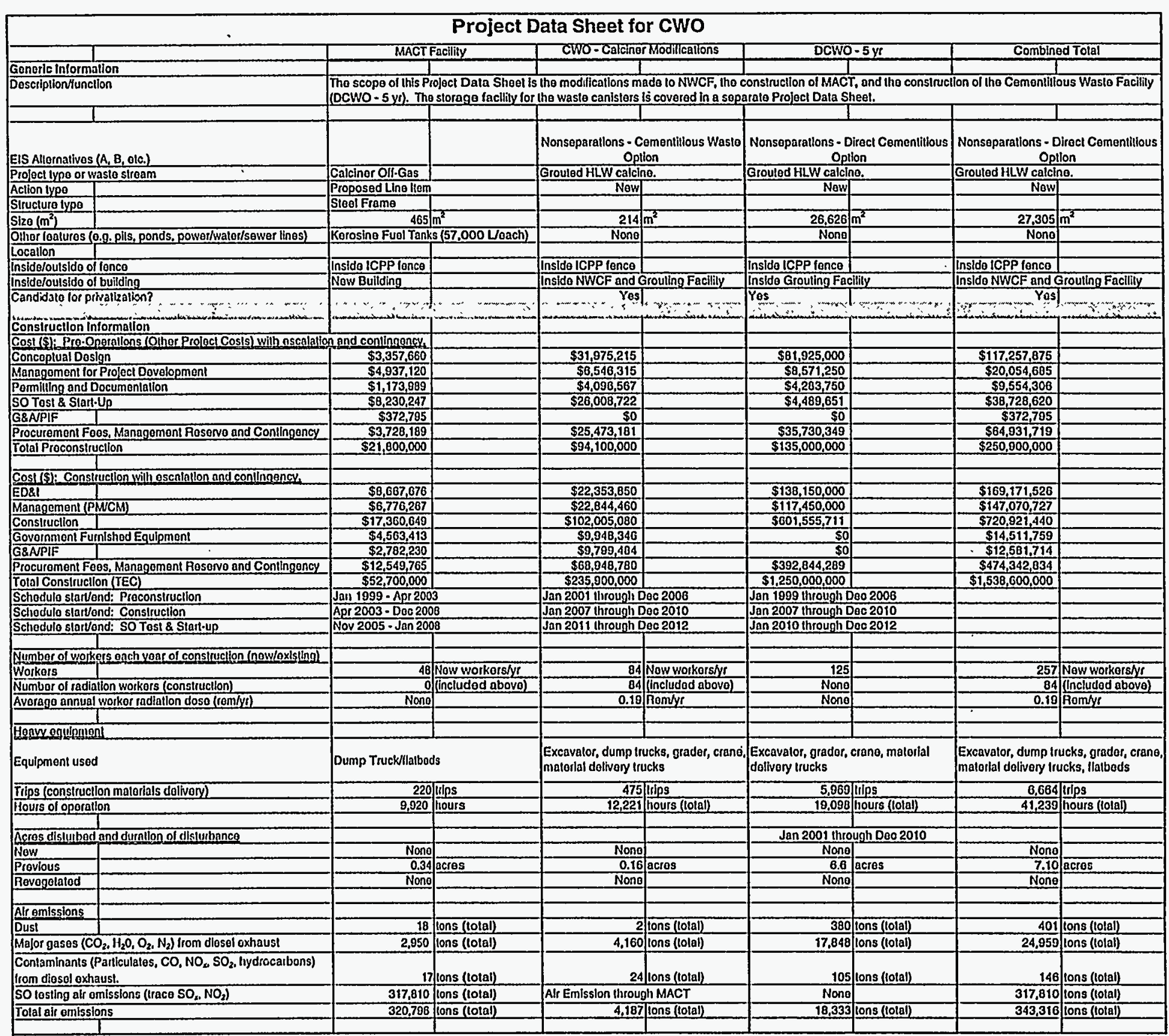


Table 3. (continued).

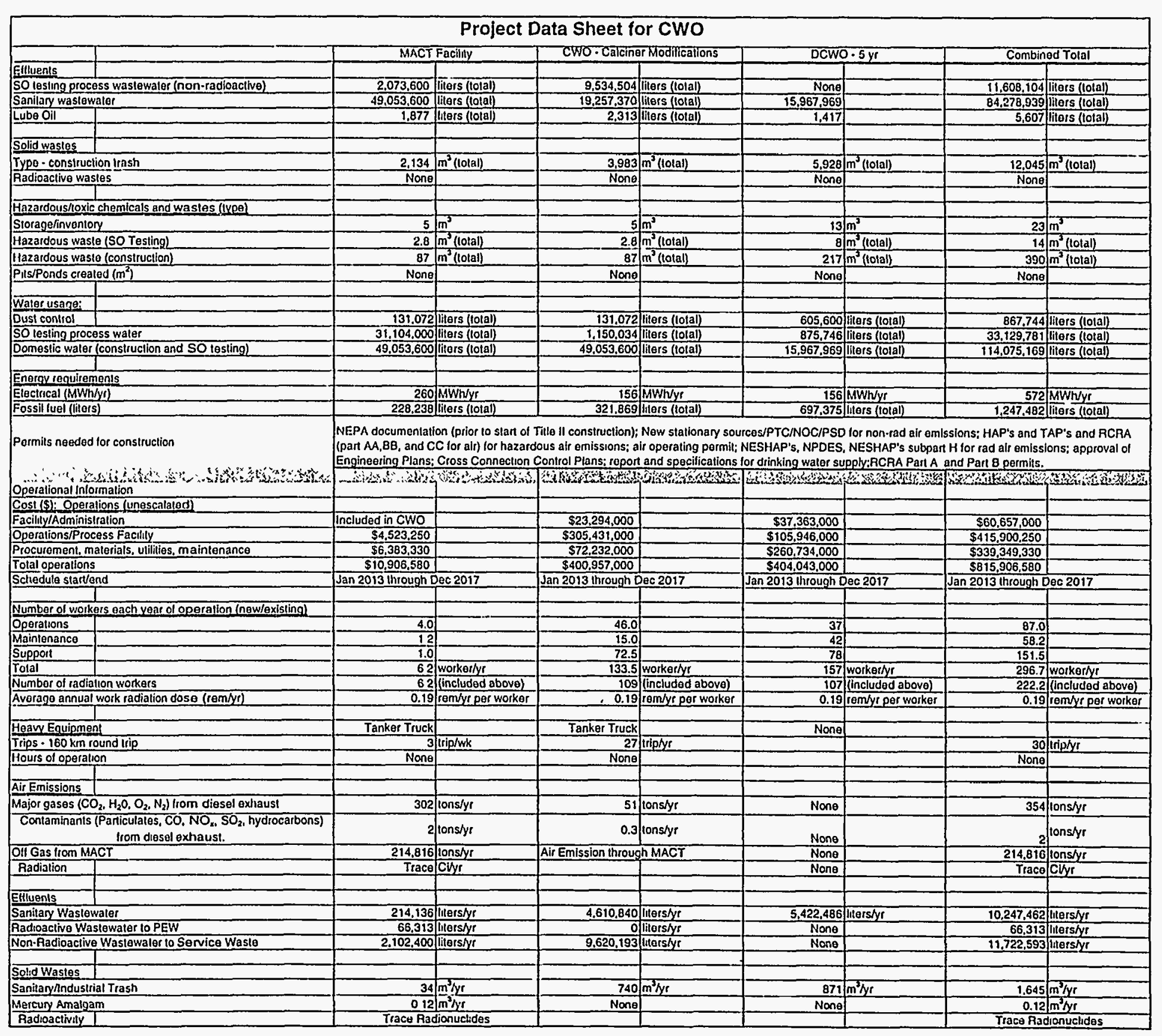


Table 3. (continued).

\begin{tabular}{|c|c|c|c|c|c|c|c|c|c|}
\hline \multicolumn{10}{|c|}{ Project Data Sheet for CWO } \\
\hline \multirow{2}{*}{\multicolumn{2}{|c|}{ Activaled Carbon }} & & \multicolumn{2}{|c|}{ CWO-Calciner Modilications } & \multicolumn{2}{|c|}{ DCWO-5yr } & \multirow{2}{*}{\multicolumn{2}{|c|}{$\frac{\text { Combined Total }}{4 / \mathrm{m}^{2} / y r}$}} \\
\hline & & & & \multicolumn{2}{|l|}{ Nonel } & \multicolumn{2}{|c|}{ None } & & \\
\hline \begin{tabular}{|l|l|} 
Radioactivity \\
\end{tabular} & & \multicolumn{2}{|c|}{$\frac{4.4 / \mathrm{m}^{3} / \mathrm{yr}^{\mathrm{r}}}{\text { Possible/2aco }}$} & & & & & \multicolumn{2}{|c|}{$\frac{4 / \mathrm{m}^{3} / \mathrm{yr}}{\text { Possible Irace l-129 }}$} \\
\hline \multicolumn{2}{|c|}{ Kin Buck Replacement (One Time) } & \multicolumn{2}{|c|}{$10 / \mathrm{n}^{2}$} & None & & None & & \multicolumn{2}{|c|}{$10 / \mathrm{m}^{3}$} \\
\hline Radioactivity & & \multirow{2}{*}{\multicolumn{2}{|c|}{$\frac{0.216: C i}{30} \mathrm{~m}^{3}$}} & & & \multirow{2}{*}{\multicolumn{2}{|c|}{$5 9 \longdiv { m ^ { 3 } / y r }$}} & \\
\hline HEPA Lillers & & & & None & & & & $89 \sqrt{n}$ & \\
\hline Radioaclivity & & 0.071 & & & & Trace & & 0.0716 & \\
\hline Radioaclive wast & es (cánisters) & & & & & 2.304 & $\mathrm{~m}^{3} \mathrm{yr}$ & $2.304 \pi$ & $m^{3} / y r$ \\
\hline & & & & & & $4.730,400$ & $\mathrm{C} / \mathrm{y}_{\mathrm{y}}$ & $4,730,400.0000$ & \\
\hline & & & & & & & & & \\
\hline $\begin{array}{l}\text { Hazardoushoxic } \\
\text { Pits/Ponds usod }\end{array}$ & Chemlcals and wastos & None] & & None & & Nona & & None & \\
\hline Nitric Acid (HNO) & $\frac{(m)}{3) .13 M}$ & None:- & & 65.700 & galyr & & & 65.700 & gably \\
\hline Caustic (material & . not waste) & & & & & 153,113 & kglyn & 153,113 & kgivir \\
\hline Whacrusane & & & & & & & & & \\
\hline$\frac{\text { Nader usdate }}{\text { Process waler }}$ & & $31,536,000$ & liters/yr & $1,150,034$ & ititers/yt & $1,167,662$ & $\overline{\text { liters } / \mathrm{yr}}$ & $33,853,696$ & iiters/yr \\
\hline Domestic waler & & 214,136 & ilers $y$ r & $4,610,840$ & Wiershys & $5,422,486$ & iters $s / y r$ & 10.247 .462 & iiters/yc \\
\hline Fn & & & & & & & & & \\
\hline 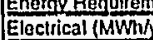 & & 1146 & MWHHYr & 746 & MWWhyr & 5,475 & $\overline{M W h y r}$ & 7,368 & $\overline{\mathrm{MWh} / \mathrm{yr}}$ \\
\hline Fossilluel (Iltorty & & $3,061,809$ & liers/yt & 530,506 & Fierryr & None & & $3,592,315$ & liternyr \\
\hline Sleam (kglyt) & & Nonel & & $110.647,083$ & $\mathrm{~kg} / \mathrm{gr}$ & $32,727,273$ & kgor & $143,374,356$ & $\mathrm{~kg} / \mathrm{gr}$ \\
\hline Permils noeded & (Ior facility oporalions) & $\begin{array}{l}\text { HAP's and TAP's an } \\
\text { air emissions; appro }\end{array}$ & $\begin{array}{l}\text { id RCAA (part AA,E } \\
\text { val of Engineering }\end{array}$ & $\begin{array}{l}38 \text {, and CC lor air) } 10 \\
\text { Pians; Cross Conne }\end{array}$ & $\begin{array}{l}\text { or hazardous air emi } \\
\text { octlon Conirol Plans; }\end{array}$ & $\begin{array}{l}\text { issions; alr operating } \\
\text { report and specilica }\end{array}$ & $\begin{array}{l}\text { g permil; NESHAP's, } \\
\text { alions lor drinking w }\end{array}$ & $\begin{array}{l}\text { NPDES, NESHAP's } \\
\text { aler supply, RCRA P }\end{array}$ & $\begin{array}{l}\text { 's subpart } \mathrm{H} \text { for rad } \\
\text { Part A and Pant B }\end{array}$ \\
\hline Docontaminatlo & 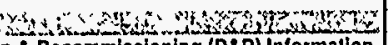 & 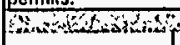 & 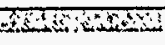 & 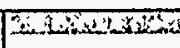 & 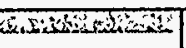 & 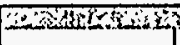 & 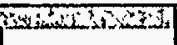 & 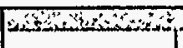 & Fin \\
\hline $\cos (\$): D 8 D(1)$ & $\begin{array}{l}n \text { \& Docommissioning (O\&D) Informalion } \\
\text { nescalaled) }\end{array}$ & & & & & & & & \\
\hline Dacommission & & $\$ 512,500$ & & $\$ 17,275,000$ & & $\$ 67,500,000$ & & $\$ 85,287,500$ & \\
\hline Decontamination & & $\$ 2,317,840$ & & $\$ 44,057,000$ & & $\$ 1115,340,000$ & & $5161,714,840$ & \\
\hline Demolition & & $\$ 864,395$ & & $\$ 70,491,000$ & & \begin{tabular}{|l|}
$184,545,000$ \\
\end{tabular} & & $\$ 255,900,395$ & \\
\hline Tolal D\&D & & $\$ 3,694,735$ & & $\$ 131,823.000$ & & $\$ 367,385,000$ & & $\$ 502,902,735$ & \\
\hline Schedulo starder & nd: D8D & Jan 2018 through D & ec 2018 & $\operatorname{San} 2018$ through D & Dec 2022 & Jan 2018 through D & 2002020 & $\operatorname{Jan} 2018$ through & ec 2022 \\
\hline Number of worke & 10. & $\frac{14}{14}$ & workertyr & 191 & workerlyr & 884 & workersyr & 1.089 & workar/yr \\
\hline Number of radiat & ion workers (D\&D) & 14 & (includad above) & 132 & (included abovo) & 593 & (Included abovo) & 739 & (included above) \\
\hline Average annual & worker radialion doso (rem/yi) & 0.19 & remyry per worker & 0.19 & remproper worker & 0.19 & rem/yr per worker & 0.19 & eern'yr per worker \\
\hline & & & & & & & & & \\
\hline Heave equipmen & & & & & & & & & \\
\hline & & Mobilo Cranos. Roll. & 1.olf Irucks, Dozors, & Mobile Cranos, Roll & il-ofl leucks, Dozors, & Mobilo Cranes, Roll & 11-off lisucks, Dozers, & Moblie Cranes, Roll & 1. oll trucks, Dozers, \\
\hline $\begin{array}{l}\text { Equipmont used } \\
\text { Trips }\end{array}$ & & Load & & & Iders & Loaders & & Loaders & \\
\hline Trips & Roll-oll trucks & $\frac{3}{6750}$ & pours & 43,200 & Hours & $\frac{18}{93.150}$ & Hour day & $\begin{array}{r}24 \\
143.100\end{array}$ & perday \\
\hline & & & & & & & & & \\
\hline Acros dislurbed & and duralion of disfurbance & $\sqrt{\operatorname{san} 2018 \text { ihrough } D}$ & sec2018 & $5 \operatorname{an} 2018$ through D & 0002022 & $\operatorname{San} 2019$ through D & Dec 2020 & Jan 2018 through 0 & 5062022 \\
\hline Now & & Nane & & None & & None & & None & \\
\hline Provious & & 0.34 & acros & 0.16 & acres & 68 & acros & 7.10 & acros \\
\hline Aevegolated & & None & & None & & Nono & & Nong & \\
\hline Áremissions & & & & & & & & & \\
\hline non-radioaclivo & Fuol combustion gases $\left(\mathrm{CO}_{2} \mathrm{H}_{2} \mathrm{O}, \mathrm{O}_{2} \mathrm{~N}_{2}\right)$ & 3,923 & lons (total) & 25,109 & ions (total) & 54,142 & Ions (lotal) & $\overline{83,174}$ & tons (lotal) \\
\hline non-radioaclivo | & $\begin{array}{l}\text { Fuel combustion contaminants (CO, } \\
\text { particulates, } \mathrm{NO}_{2}, \mathrm{SO}_{2} \text {, hydrocartons) }\end{array}$ & & Ions (total) & 147 & Ions (10tal) & & Lons (lotal) & 485 & tons (tolal) \\
\hline radioaclive & HEPA Alllered ofl-gas & 26.173 & Lons (lotal) & 130.864 & Oons (lotal) & 78.518 & Ions (lotali) & 235.554 & Lons (lolal) \\
\hline & & & & & & & & & \\
\hline Ellluenls & & & & & & & & & \\
\hline radioactive & Spent decontaminalion solution & 1.703 .250 & thers (total) & $8,516,250$ & 5 liters (tolal) & $5,109,750$ & liters (1olal) & $15,329,250$ & liters (total) \\
\hline \begin{tabular}{|l|l} 
non-radioaclive \\
\end{tabular} & Sanitary wastewaler & 298,069 & ititers (lotal) & $20,376,827$ & liters (lotal) & 56.445.812 & Riters (total) & $77,120,708$ & itters (lotal) \\
\hline non-radioactive & Lube oil & 1.277 & thers (lotal) & 8.176 & 5 ilers (lotal) & 17,629 & fiters(lotal) & 27,0082 & iters (lotal) \\
\hline & & & & & & & & & \\
\hline Solio wasles: & & & & & & 17001 & & & \\
\hline radioactive & & 454 & $\mathrm{~m}^{3}$ & 388 & 8/ $\mathbf{m}^{\mathbf{2}}$ & 47,943 & $\mathrm{~m}^{3}$ & 48,785 & \\
\hline Non-radioactive & (industrial) & 175 & $\sqrt{\eta^{3}}$ & 292 & $2 \mathrm{~m}^{3}$ & 36,048 & $m^{3}$ & 36.516 & $\mathrm{~m}^{3}$ \\
\hline Hazardous & & 0.29 & & 0.19 & & & $m^{3}$ & & \\
\hline
\end{tabular}




\begin{tabular}{|c|c|c|c|c|c|c|c|c|c|}
\hline \multicolumn{8}{|c|}{ 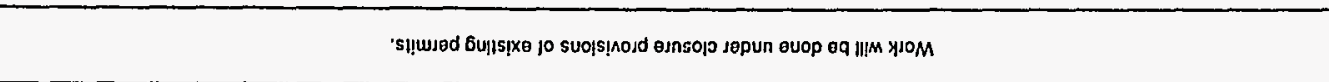 } & \multicolumn{2}{|c|}{ 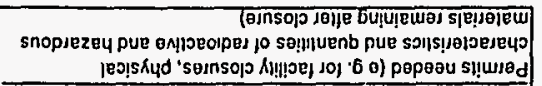 } \\
\hline (7ikion siom & $220^{\prime} 2 z p^{\prime}$ & \multirow{2}{*}{\multicolumn{2}{|c|}{ 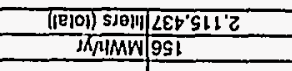 }} & \multirow{2}{*}{\multicolumn{2}{|c|}{ 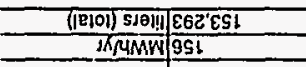 }} & \multirow{2}{*}{\multicolumn{2}{|c|}{ 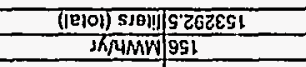 }} & \multirow{2}{*}{\multicolumn{2}{|c|}{ 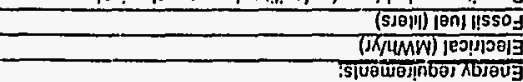 }} \\
\hline & & & & & & & & & \\
\hline & sil| बि बा!s ddग & & 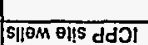 & & 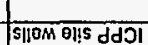 & & 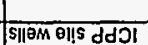 & & 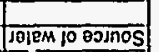 \\
\hline 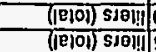 & 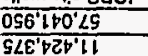 & 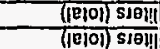 & 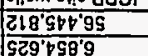 & 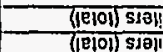 & 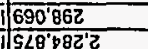 & 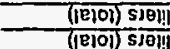 & 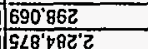 & & 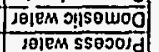 \\
\hline & & & & & & & & & 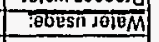 \\
\hline (साi|0|) & $\overline{L L T}$ & (रांख्या) & tor & (घण01111 & जीड़ा' & (10|0) w & & 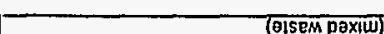 & 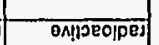 \\
\hline & $\frac{16}{\operatorname{anON}}$ & & BWON & & करण & & $\frac{16}{\partial U O N}$ & $($ wi) & | \\
\hline (11010) & 606 & (180101), w) & $\cos 2$ & 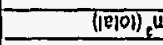 & 4502 & $50 M N 1$ & (10601015 & & Soluenuyobed \\
\hline & & & & & & & & 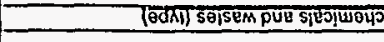 & 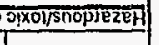 \\
\hline 10101 pos & Ujqüos & $1 K \mathrm{G}-\mathrm{C}$ & $\frac{1}{O M 20}$ & suollevilipow. & 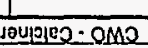 & KIIIPB:= & $\frac{1}{110 W W}$ & & \\
\hline & & & & OMO & $x_{0}$ & & & & \\
\hline
\end{tabular}

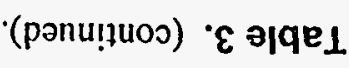


Table 4. Project Data Sheet for DCWO.

\begin{tabular}{|c|c|c|c|c|c|}
\hline \multicolumn{6}{|c|}{ Project Data Sheet for DCWO - 5 Year } \\
\hline \multirow{2}{*}{\multicolumn{6}{|c|}{ Generic Information }} \\
\hline & & & & & \\
\hline \multicolumn{2}{|c|}{ Description/function } & \multicolumn{4}{|c|}{$\begin{array}{l}\text { Directly grout HLW calcine in preparation for road-ready } \\
\text { storage awaiting shipment to a permanent repository }\end{array}$} \\
\hline \multicolumn{2}{|c|}{ EIS Alternatives $(A, B$, etc. $)$} & \multicolumn{3}{|c|}{ Nonseparations - Direct Cementitious Option } & \\
\hline \multicolumn{2}{|c|}{ Project type or waste stream } & \multicolumn{2}{|c|}{ Grouted HLW calcine. } & & \\
\hline Action type & & \begin{tabular}{|l|l|} 
New \\
\end{tabular} & & & \\
\hline \multicolumn{6}{|l|}{ Structure type } \\
\hline Size $\left(m^{2}\right)$ & & 26,626 & $\mathrm{~m}^{2}$ & & \\
\hline \multicolumn{2}{|c|}{ Other features (e.g. pits, ponds, power/water/sewer lines) } & None & & & \\
\hline \multicolumn{6}{|l|}{ Location } \\
\hline \multicolumn{2}{|c|}{ Insideloutside of fence } & Inside ICPP fence | & & & \\
\hline \multicolumn{2}{|c|}{ Inside/outside of building } & Inside Grouting Fac & ility & & \\
\hline \multicolumn{2}{|c|}{ Candidate for privatization? } & Yes & & & \\
\hline \multirow{2}{*}{\multicolumn{6}{|c|}{$\begin{array}{l}\text { Fonstruction Information } \\
\text { Cons }\end{array}$}} \\
\hline & \multicolumn{5}{|c|}{ Construction Information } \\
\hline \multicolumn{6}{|c|}{ Cost (\$): Preconstruction (w/escalation \& contingency) } \\
\hline \multicolumn{2}{|c|}{ Conceptual Design } & $\$ 81,925,000$ & & & \\
\hline \multicolumn{2}{|c|}{ Management Costs } & $\$ 8,571,250$ & & & \\
\hline \multirow{2}{*}{\multicolumn{2}{|c|}{$\begin{array}{l}\text { Permitting and Documentation } \\
\text { Startup Activities }\end{array}$}} & $\$ 4,283,750$ & & & \\
\hline Startup Activities & & $\$ 4,489,651$ & & & \\
\hline \multicolumn{2}{|c|}{ Management Reserve and Contingency } & $\$ 35,730,349$ & & & \\
\hline Total Precons & uction & $\$ 135,000,000$ & & & \\
\hline & & & & & \\
\hline Cost (\$): Con & ruction & & & & \\
\hline ED\&1 & & $\$ 138,150,000$ & & & \\
\hline Management & $\mathrm{M} / \mathrm{CM})$ & $\$ 117,450,000$ & & & \\
\hline Construction & & $\$ 601,555,711$ & & & \\
\hline Procurement & es, Mgt Reserve and Contingency, G\&A & $\$ 392,844,289$ & & & \\
\hline Total Constru & on (TEC) & $\$ 1,250,000,000$ & & & \\
\hline Schedule star & end: Preconstruction & January 1999 throug & gh Decembe & 2006 & \\
\hline Schedule star & end: Construction & January 2007 throus & gh Decembe & 2010 & \\
\hline Schedule star & end: SO Test \& Start-up & January 2010 throu & gh Decembe & 2012 & \\
\hline & & & & & \\
\hline Number of wo & ers each year of construction (new/existing & & & & \\
\hline Nonradiation & & 125 & New workers & & \\
\hline Number of rac & tion workers (construction) & None & & & \\
\hline Average annu & worker radiation dose (rem/yr) & None & & & \\
\hline & & & & & \\
\hline Heavy equipm & & & & & \\
\hline Equipment us & & Excavator, grader, $\mathrm{c}$ & crane, materi & ial delivery truc & \\
\hline Trips (constru & ion materials delivery) & 5,969 & & & \\
\hline Hours of oper & ion (including materials delivery vehicles) & 19,098 & hours (total) & & \\
\hline & & & & & \\
\hline Acres disturbe & and duration of disturbance & January 2001 throu & igh Decembe & 2010 & \\
\hline New & & None & & & \\
\hline Previous & & 6.61 & acres & & \\
\hline Revegetated & & None & & & \\
\hline
\end{tabular}


Table 4. (continuêd).

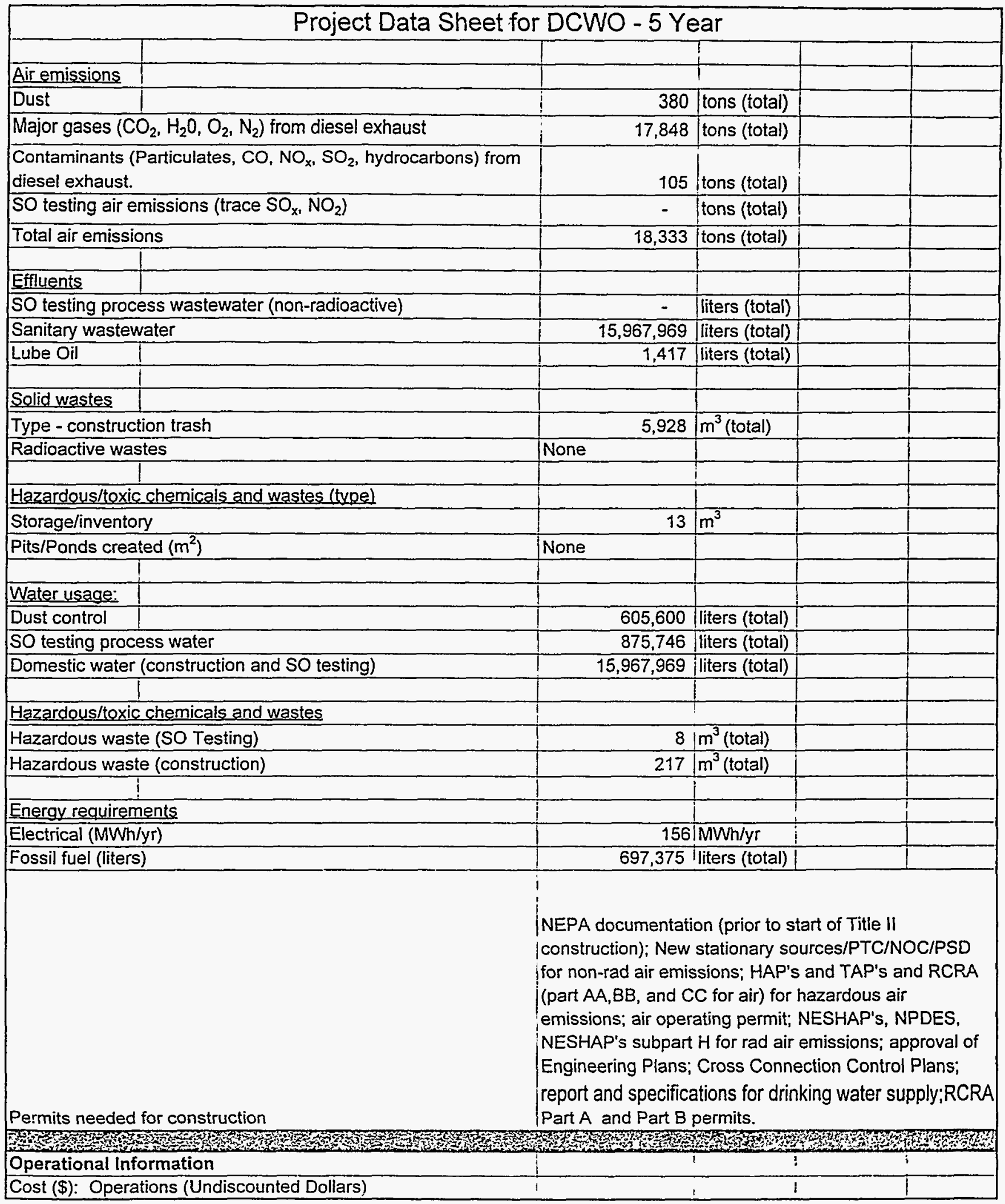


Table 4. (continued).

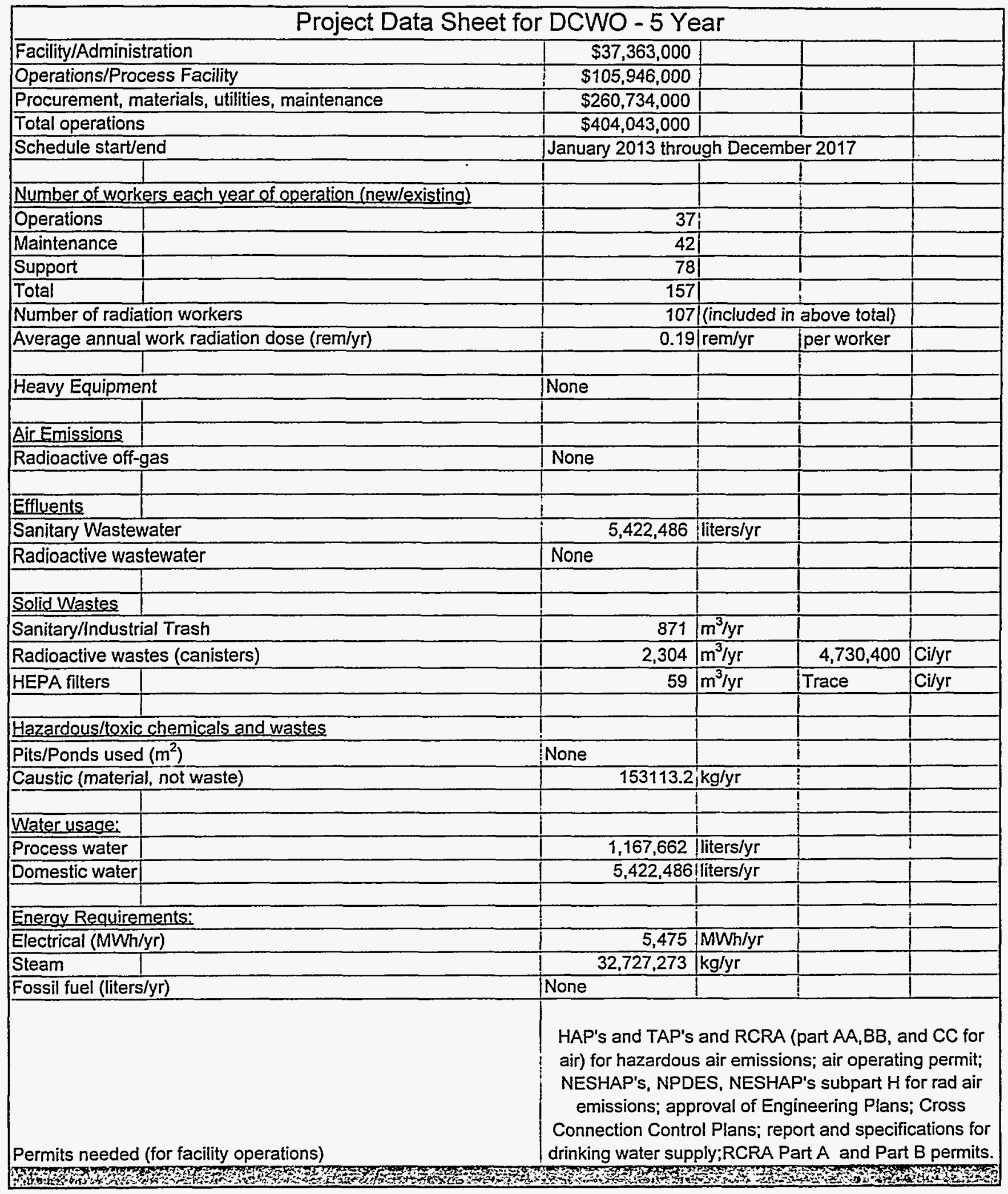


Table 4. (continued).

\begin{tabular}{|c|c|c|c|c|c|}
\hline & Project Data Sheet & $\mathrm{rDCWO}-5 \mathrm{Ye}$ & & & \\
\hline Decontaminati & on \& Decommissioning (D\&D) Information & $T 2+2$ & & & \\
\hline Cost (\$): D\&D & Undiscounted dollars] & & & & \\
\hline Decommission & & $\$ 67,500,000$ & & & \\
\hline Decontaminatio & & $\$ 115,340,000$ & & & \\
\hline Demolition & 1 & $\$ 184,545,000$ & & & \\
\hline Total D\&D & 1 & $\$ 367,385,000$ & & & \\
\hline Schedule start/6 & end: $D \& D$ & January 2018 thro & ugh Decemb & er 2020 & \\
\hline Number of work & ers each year of D\&D (new/existing) & 884 & New worke & $s / y r$ & \\
\hline Number of radie & ation workers (D\&D) & 593 & New worke & $\mathrm{s} / \mathrm{yr}$ & \\
\hline Average annual & worker radiation dose (rem/yr) & 0.19 & rem/yr & per worker & \\
\hline & I & I & & & \\
\hline Heavy equipme & & & & & \\
\hline Equipment usec & & Mobile Cranes, Rol & Il-off trucks, & Dozers, Loade & \\
\hline Trips & Roll-off trucks & 18 & per day & & \\
\hline Hours of operat & ion (all heavy equipment) & 93,150 & Hours & & \\
\hline & & & & & \\
\hline Acres disturbed & and duration of disturbance & January 2019 throu & ugh Decemb & 2020 & \\
\hline New & & None & & & \\
\hline Previous & & 6.6 & acres & & \\
\hline Revegetated & & None & & 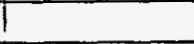 & \\
\hline & & & & & \\
\hline Air emissions & & & & & \\
\hline non-radioactive & Fuel combustion gases $\left(\mathrm{CO}_{2}, \mathrm{H}_{2} \mathrm{O}, \mathrm{O}_{2}, \mathrm{~N}_{2}\right)$ & 54,142 & tons (total) & & \\
\hline non-radioactive & $\begin{array}{l}\text { Fuel combustion contaminants (CO, } \\
\text { particulates, } \mathrm{NO}_{x}, \mathrm{SO}_{2} \text {, hydrocarbons) }\end{array}$ & 316 & tons (total) & & \\
\hline radioactive & HEPA filtered off-gas & 78,518 & tons (total) & & \\
\hline & & & & & \\
\hline Effluents & & & & & \\
\hline radioactive & Spent decontamination solution & $5,109,750$ & liters (total) & 5,110 & $\mid \mathrm{C}$ \\
\hline non-radioactive & Sanitary wastewater & $56,445,812$ & liters (total) & & \\
\hline non-radioactive & Lube oil & 17,629 & liters (total) & & 1 \\
\hline & & & & & \\
\hline Solid wastes: & 1 & & & & \\
\hline radioactive & & 47,943 & $\mathrm{~m}^{3}$ & 479 & $|c|$ \\
\hline Non-radioactive & (industrial) & 36,048 & $\mathrm{~m}^{3}$ & & i \\
\hline Hazardous & $\perp$ & 16 & $\mathrm{~m}^{3}$ & & \\
\hline & 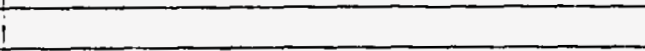 & & & & \\
\hline Hazardous/toxic & chemicals and wastes (type) & & & & 1 \\
\hline Storage/invento & & 205 & $\mathrm{~m}^{3}$ (total) & & 1 \\
\hline Pits/Ponds crea & $\operatorname{ted}\left(m^{2}\right)$ & None & & 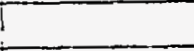 & \\
\hline radioactive & (mixed waste) & 141 & $\mathrm{~m}^{3}$ (total) & 1 & $\mathrm{Ci}$ \\
\hline & & & & & \\
\hline Water usage: & i & 1 & I & & \\
\hline Process water & & $6,854,625$ & |liters (total) & & 1 \\
\hline Domestic water & & $56,445,812$ & |liters (total) & & \\
\hline Source of water & & IICPP site wells & 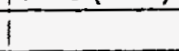 & & \\
\hline & & & & & \\
\hline Energy requiren & nents: & 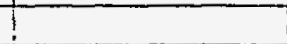 & T & & 1 \\
\hline
\end{tabular}


Table 4. (continued).

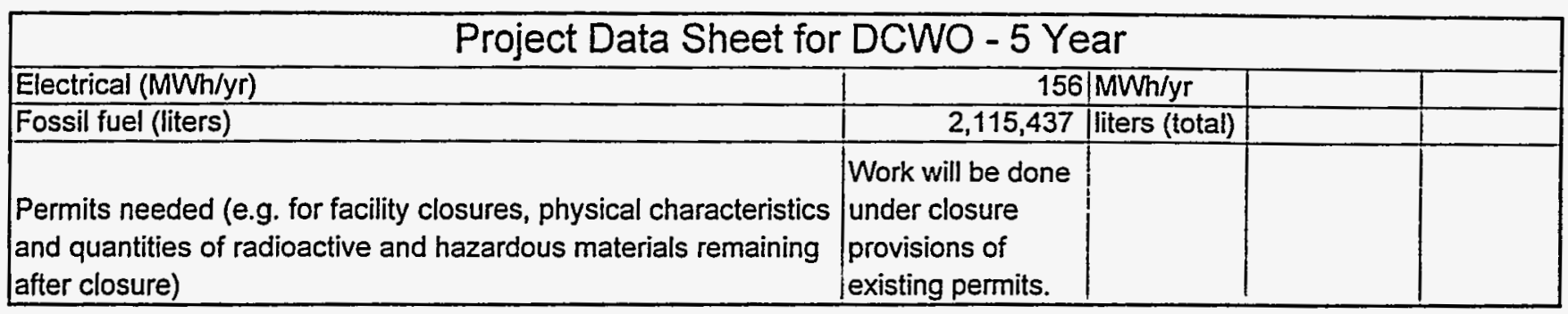


Tabie 4. (continued).

Construction Assumptions

\begin{tabular}{|c|c|c|c|c|c|c|c|}
\hline Construction duration $=$ & & years & & & & & \\
\hline SO testing $=$ & & years & \multicolumn{3}{|c|}{1 year overlaps with construction } & & \\
\hline Total years $=$ & & years & elapsed time & & & & \\
\hline \multirow[t]{2}{*}{ Labor - use a total of } & 125 & new workers/yr & & & & & \\
\hline & & & & & & & \\
\hline Sanitary Wastewater $=$ & 703,125 & gallyr = & $15,967,969$ & liters (total) & & & \\
\hline \multicolumn{4}{|c|}{ (based on 25 gal/person-day and 225 days/year of construction) } & & & & \\
\hline \multicolumn{7}{|c|}{$\begin{array}{l}\text { (Benefield, LD and C.W. Randall, Biological Process Design for Wastewater Treatment, InPrint, Inc., 1987, p. } 104 \text { - } \\
\text { wastewater generation }=15-30 \text { gal/day-person) }\end{array}$} & \\
\hline & & & & & I & ! & \\
\hline Water used for dust control $=$ & 2000 & gal/wk = & 605,600 & liters (total) & & & \\
\hline \multicolumn{8}{|c|}{ (assumes dust contral required 20 weeks/yr ..R. Kimmitt) } \\
\hline & & & & & & & \\
\hline \multicolumn{4}{|c|}{ Electrical usage assumed to be $3,000 \mathrm{kWh}$ (from John Duggan) } & & 1 & & \\
\hline \multirow{2}{*}{\multicolumn{2}{|c|}{$156 \mid \mathrm{MWh} / \mathrm{yr}$}} & & & & & & \\
\hline & & & & & & & \\
\hline \multicolumn{4}{|c|}{$\begin{array}{l}\text { Assume } 3 \text { gallons of lubricating oil and hydraulic fluid generated for every } 60 \\
\text { hours of operation of heavy equipment = }\end{array}$} & 1,417 & \multicolumn{2}{|l|}{ liters (total) } & \\
\hline & & & & & & & \\
\hline Square footage $=$ & 286,600 & sq.ft $=$ & 26,626 & $\mathrm{~m}^{2}$ & & & \\
\hline \multirow{2}{*}{\multicolumn{8}{|c|}{ (Stephanie Austad, 1/12/98) }} \\
\hline & & & & & & & \\
\hline Acres disturbed $=$ & 287,300 & sq.ft. $=$ & 26,691 & $m^{2}=$ & 6.6 & acres (prev & vious) \\
\hline \multicolumn{8}{|l|}{ (Stephanie Austad, 1/12/98) } \\
\hline & & 1 & 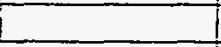 & & & & \\
\hline Heavy equipment $=$ & 3 vehicles @6 & 224 hrs/yr during & construction $=$ & 7,488 & hrs total & & \\
\hline \multicolumn{3}{|c|}{ Equipment fuel usage (see http://wuw/deere.com/ind) = } & & 6 & $g a l / h r$ & & \\
\hline \multicolumn{2}{|c|}{ Total heavy equipment fuel usage $=$} & \multicolumn{2}{|c|}{$44928 \mathrm{gal}=$} & 170,052 & \multicolumn{2}{|l|}{ liters (total) } & \\
\hline & & & & & & & \\
\hline \multicolumn{3}{|c|}{ Dust during construction $=1.2$ tons/month-acre $=$} & 380 & tons (total) & & & \\
\hline \multicolumn{3}{|c|}{ (from USEPA Office of Ar Quality Planning and Standards) } & & & & & \\
\hline & & & & & & & \\
\hline \multicolumn{8}{|c|}{ Construction costs are from life cycle cost estimate (R. Turk) } \\
\hline & & & & & & & \\
\hline \multicolumn{8}{|c|}{ Air emissions from fuel usage are based on the diesel emissions spreadsheet. } \\
\hline & & & & & & & \\
\hline \multicolumn{8}{|c|}{ Alr emissions during SO \& start-up testing are based on non-radioactive operations for 3 years: } \\
\hline 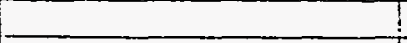 & & & & $T$ & & & \\
\hline 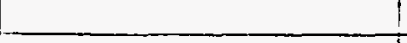 & & & & & & & \\
\hline & & & & & & & \\
\hline Construction trash $=$ & 7,750 & ydi $3($ total $)=$ & & 5.928 & $\mathrm{~m}^{3}$ (total) & & \\
\hline Use 15.5 yd3/yr per capita. Thi & is is twice the $\mathrm{g}$ & eneration rate o & f trash from site o & perations) & & & \\
\hline & & & & & & & \\
\hline Hazardous waste generation & 275 & gal/week = & & 217 & $\mathrm{~m}^{3}$ (total) & & \\
\hline based on an assumed generat & ion rate of 555 & -gallon drum & vaste per week) & & & & \\
\hline & & & & & & & \\
\hline Hazardous waste storage $=$ & 3300 & gal $=$ & 13 & & & & \\
\hline ume & 12 weeks [8 & 84 days] in a & lation & n area & T & & \\
\hline 1 man-year of labor $=$ & 1800 & manhours & & & & & \\
\hline & & & & & & 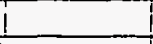 & \\
\hline SO testing liquid effluent = & - & Ibs. total $=$ & & - & liters (total) & & \\
\hline & 1 & & 1 & & & & \\
\hline
\end{tabular}


Table 4. (continued).

Construction Assumptions

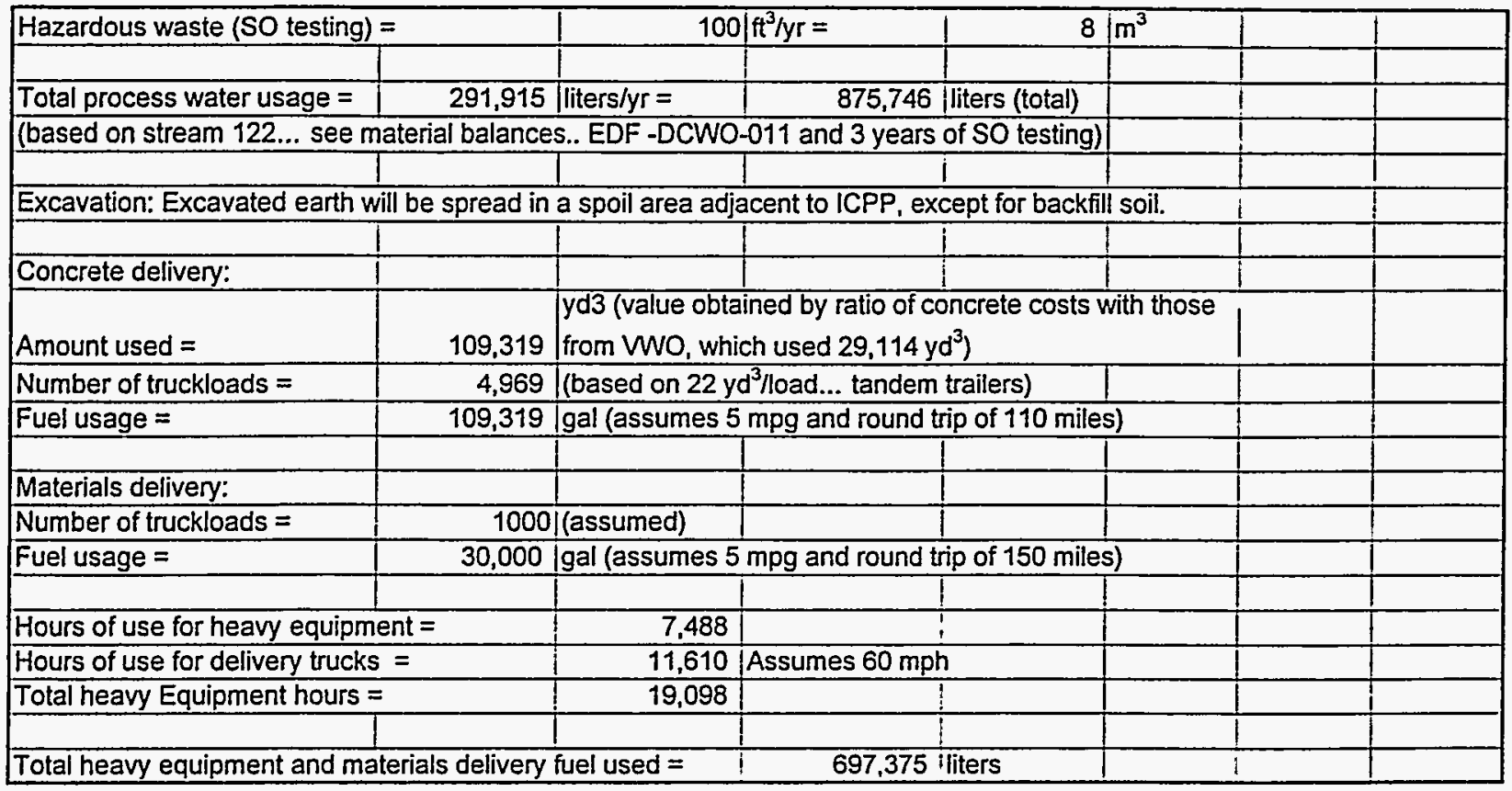


Table 4. (continued).

Operations Assumptions

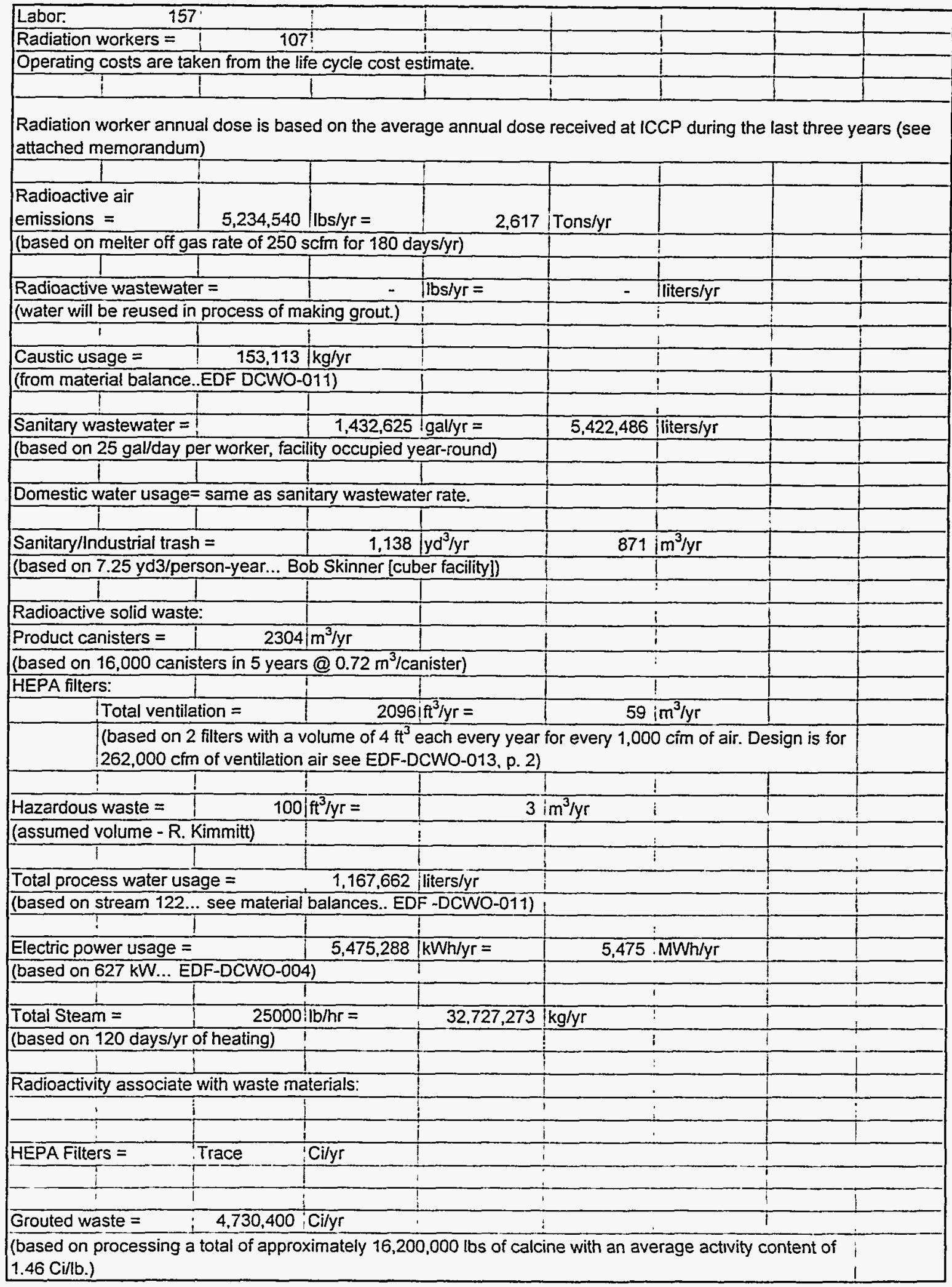


Table 4. (continued).

D\&D Labor

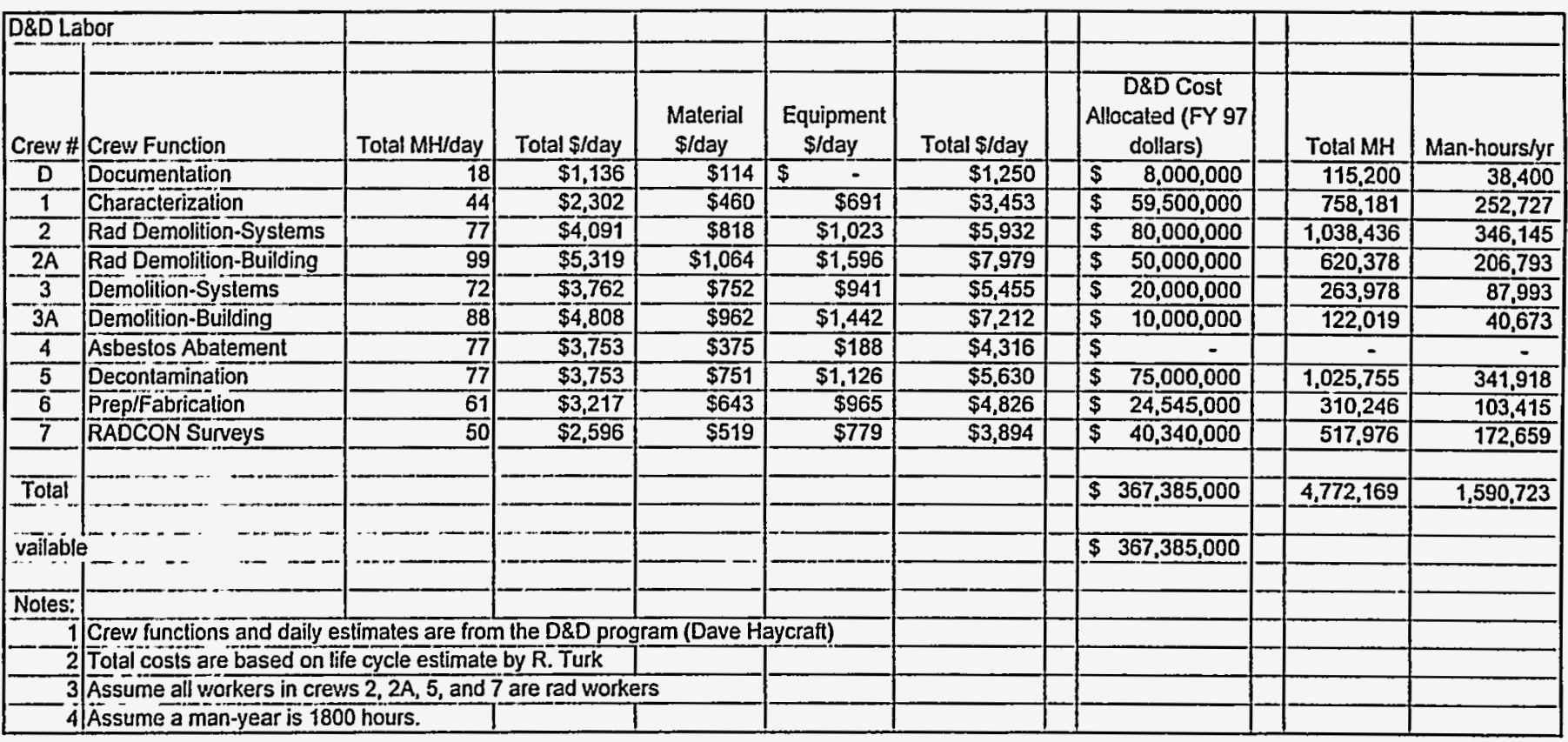


Table 4. (continued).

D D Assumptions

\begin{tabular}{|c|c|c|c|c|c|c|c|}
\hline Duration of $D \& D=$ & 3 & years & & 1 & 1 & I & $!$ \\
\hline & & 1 & & & & & \\
\hline Heavy Equipment & & i\# Used & Hours/day & Days/wk & jWks/yr & Hours/yr & \\
\hline & Mobile Crane & 2 & 3 & 4 & 45 & 1,080 & \\
\hline & Roll-Off Truck & 6 & 8 & 5 & 45 & 10,800 & \\
\hline & Dozer & 2 & 5 & 5 & 45 & 2,250 & \\
\hline & Loader & 61 & 81 & 5 & 45 & 10,800 & \\
\hline & $\begin{array}{l}\text { Scabbler (w/ } \\
\text { Vacuum } \\
\text { System) }\end{array}$ & 2 & 8) & 5 & 45 & 3,600 & \\
\hline & $\begin{array}{l}\text { Pneumatic } \\
\text { Ram }\end{array}$ & 2 & 4 & 4 & 45 & 1,440 & \\
\hline & $\begin{array}{l}\text { Demolition } \\
\text { Machine } \\
\text { (Remote } \\
\text { Control) } \\
\end{array}$ & 2 & 4 & 3 & 45 & 1,080 & \\
\hline & & & & & $T$ & & \\
\hline Total hours/yr & & f & i & & $T$ & 31,050 & \\
\hline & & $!$ & & & & & \\
\hline Total heavy equipment ho & jurs $=$ & & & & & 93,150 & \\
\hline $\begin{array}{l}\text { Assume each piece of eq } \\
\text { Web Site (Construction E }\end{array}$ & $\begin{array}{l}\text { quipment uses } \\
\text { quipment - http }\end{array}$ & 6 gallon of dies & $\begin{array}{l}\text { sel fuel per hour } \\
\text { com/ind/produc }\end{array}$ & $\begin{array}{l}\text { ir. Consumpti } \\
\text { tproduct.htr }\end{array}$ & $\begin{array}{l}\text { tion rate from Joh } \\
\text { ml) }\end{array}$ & hn Deere & \\
\hline No. of gallons of fuel useo & during $D \& D$ & & 558,900 & $\mathrm{gal}=$ & $2.115,437$ & liters (total) & \\
\hline & & & & & & I & \\
\hline Acreage disturbed is the & same as for con & nstruction = & 6.6 & acres & & & \\
\hline & & & & $i$ & $i$ & & \\
\hline D\& D labor requirements & are taken from & D \& D labor ar & ind equipment s & spreadsheet. & & & \\
\hline & & & & & 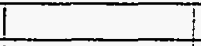 & 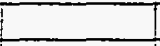 & \\
\hline$D$ \& D costs come from th & ie life cycle cost & it estimate. & & & T & 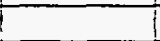 & \\
\hline & & & & & t & 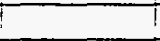 & \\
\hline Assume each roll-off truch & makes 3 trips & per day to $\overline{R W}$ & $\mathrm{VMC}$ & & i & 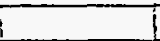 & \\
\hline No. of trips $=$ & 1 & 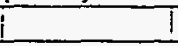 & 181 & & 1 & 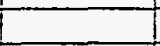 & \\
\hline Miles traveled @ 12 miles & /round trip = & & 216 & miles/day & & & \\
\hline & & & & & I & I & \\
\hline Decontamination solution & stored $=$ & & 2,000 & gallons & 205 & $\mathrm{~m}^{3}$ & \\
\hline & & & & 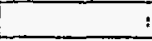 & 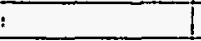 & & \\
\hline Daily process water usag & & & 3000 & gal/day $=$ & $6,854,625$ & liters (tolal) & \\
\hline (assumed for washing, de & con, etc.; basec & d on 225 days & (yr) & & & & \\
\hline & 1 & & & ! & & & \\
\hline Domestic water usage $=$ & & $56,445,812$ & iters (total) & 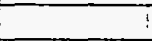 & & & \\
\hline (based on $25 \mathrm{gal} / \mathrm{day}$ for & each worker) & & 1 & 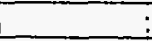 & & & \\
\hline & & & & 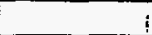 & & & \\
\hline Sanitary wastewater $=$ sar & me as domestic & water usagel & & & & & \\
\hline & & & & & & & \\
\hline Assume portable HEPA s & ystems off-gas & rate $=$ & 2000 & scim $=$ & 78,518 & Tons (total) & \\
\hline (assumes 225 days/yr) & & 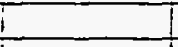 & & & 1 & & \\
\hline & & & & & & & \\
\hline Assume daily spent decor & . solution $=$ & & 2000 & gal/day & $5,109,750$ & liters (total) & \\
\hline (assumes 225 days/yr tot & & & $i$ & & & 1 & \\
\hline & & & & & & & \\
\hline Solid Waste Generation & (factors from D & Jave Kenoyer - & - D\&D Program & & & & \\
\hline Waste Type & $\begin{array}{c}\text { Factor } \\
\text { (cu.ft.sq ft.) }\end{array}$ & $\begin{array}{l}\text { Sq.Ft. in } \\
\text { Facility }\end{array}$ & $\begin{array}{l}\text { Cu.Ft. of } \\
\text { Waste }\end{array}$ & Cu. Meters & & & \\
\hline 1 & 1 & $i$ & & $\div$ & & & \\
\hline $\begin{array}{l}\text { WERF-LLW Combustible } \\
\text { PFES }\end{array}$ & 0.167 & 286,600 & 47,862 & $1,356 !$ & & I & \\
\hline $\begin{array}{l}\text { WERF-LLW Combustible } \\
\text { Building Debris }\end{array}$ & 0.128 & $286,600 !$ & 36.685 & 1.039 & & & \\
\hline
\end{tabular}


Table 4. (continued).

D D Assumptions

\begin{tabular}{|c|c|c|c|c|c|c|}
\hline $\begin{array}{l}\text { WERF-LLW } \\
\text { Compactable Building } \\
\text { Debris }\end{array}$ & 0.195 & 286,600 & 55,887 & 1,583 & & \\
\hline $\begin{array}{l}\text { RWMC-LLW Non- } \\
\text { Compactable Equipment }\end{array}$ & 0.513 & 286,600 & 147,026 & 4,165 & & \\
\hline $\begin{array}{l}\text { RWMC-LLW Non-Compt } \\
\text { Building Debris }\end{array}$ & 0.684 & 286,600 & 196,034 & 5,553 & & \\
\hline $\begin{array}{l}\text { RWMC-LLW Non-Compt } \\
\text { Concrete Rubble }\end{array}$ & 3.44 & 286,600 & 985,904 & 27,929 & $\begin{array}{l}\text { Factor is twice that used by } \\
\text { the D\&D program to } \\
\text { account for that large } \\
\text { amount of concrete used. }\end{array}$ & \\
\hline $\begin{array}{l}\text { RWMC-LLW Non-Compt } \\
\text { Scrap Metal }\end{array}$ & 0.778 & 286,600 & 222,975 & 6,317 & & \\
\hline \begin{tabular}{|l|} 
RWMC-LLW \\
Asbestos/ACM Covered \\
Pipe
\end{tabular} & 여 & 286,600 & - & - & & \\
\hline $\begin{array}{l}\text { CFA Landfill Non-Compt } \\
\text { Building Debris }\end{array}$ & 1.99 & 286,600 & 570.334 & 16,157 & & \\
\hline $\begin{array}{l}\text { CFA Landfill Non-Compt } \\
\text { Concrete Rubble }\end{array}$ & 2.45 & 286,600 & 702,170 & 19,892 & $\begin{array}{l}\text { Factor is twice that used by } \\
\text { the D\&D program to } \\
\text { account for that large } \\
\text { amount of concrete used. }\end{array}$ & \\
\hline CFA Landfill Asbestos & 0 & 286,600 & - & - & & \\
\hline $\begin{array}{l}\text { HWSF Hazardous Mtrls } \\
\text { (Hg/PCBs/etc) }\end{array}$ & 0.002 & 286,600 & 573 & 16 & & 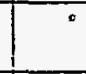 \\
\hline \multirow[t]{2}{*}{ Metal Recycle } & 0.022 & 286,600 & 6,305 & 179 & & \\
\hline & & & & & & \\
\hline$L L W=$ & & & $1,692,373$ & 47,943 & & \\
\hline Non-Rad = & & & $1,272,504$ & 36,048 & & \\
\hline Hazardous $=$ & & & 573 & 16 & & \\
\hline \multirow[t]{2}{*}{ Metal $=$} & & & 6,305 & 179 & & \\
\hline & & & & & & \\
\hline Electric power usage $=$ & 156,000 & $\mathrm{kWh} / \mathrm{yr}$ & 156 & $\mathrm{MWh} / \mathrm{yr}$ & & \\
\hline \multicolumn{7}{|c|}{ (based on $3,000 \mathrm{kWh} / \mathrm{wk}$ - John Duggan) } \\
\hline & & & & I & 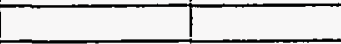 & \\
\hline \multicolumn{5}{|c|}{ Air emissions from fuel are based on the diesel emissions spreadsheet. } & 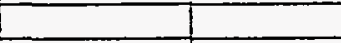 & \\
\hline & & & & 1 & 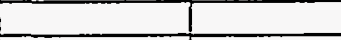 & \\
\hline \multirow[t]{2}{*}{1 manyear of labor $=$} & 1800 & manhours & & i & 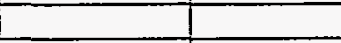 & $i$ \\
\hline & 1 & 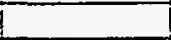 & & 1 & 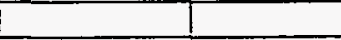 & 1 \\
\hline Lube oil $=$ & 17,629 & liters (total) & & T & 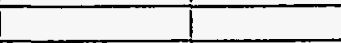 & \\
\hline \multicolumn{3}{|c|}{ (based on 3 gal for every 60 hours of operation) } & & 1 & 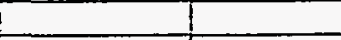 & \\
\hline & & & & 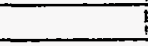 & 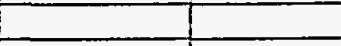 & \\
\hline Mixed waste $=$ & 37,125 & gal (total) $=$ & $141 \mid$ & $\mathrm{m} 3$ (total) & & \\
\hline \multicolumn{7}{|c|}{ (based on an assumed 555 -gallon drums generated per week... work only 45 weeks/yr) } \\
\hline & & & & $-7+2+$ & 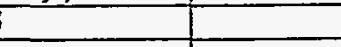 & \\
\hline \multicolumn{3}{|c|}{ Radjoactivity associated with waste materials: } & & & 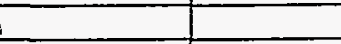 & \\
\hline & & & & & & $\mathrm{i}$ \\
\hline Spent decontamination so & Iution $=$ & 5.110 & $\mathrm{Ci}$ & $i$ & 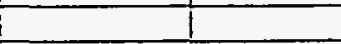 & \\
\hline \multicolumn{4}{|c|}{ (based on an assumed average activity concentration of $1 \mathrm{uCi} / \mathrm{ml}$ ) } & & & \\
\hline & & & & 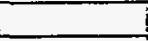 & 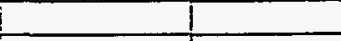 & \\
\hline Radioactive solid waste $=$ & & 479 & $\overline{C i}$ & 1 & 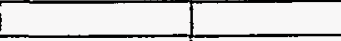 & \\
\hline \multicolumn{7}{|c|}{ (based in an assumed activity concentration of $0.01 \mathrm{uCi} / \mathrm{cc}\left[0.01 \mathrm{Ci} / \mathrm{m}^{3}\right]$ ) } \\
\hline & & & & & & \\
\hline Mixed waste $=$ & & 1 & $\mathrm{Ci}$ & & & \\
\hline \multicolumn{6}{|c|}{ (based on an assumed activity concentration of $0.01 \mathrm{uCi} / \mathrm{cc}\left[0.01 \mathrm{Cl} / \mathrm{m}^{3}\right]$ ) } & $!$ \\
\hline
\end{tabular}


Table 4. (continued).

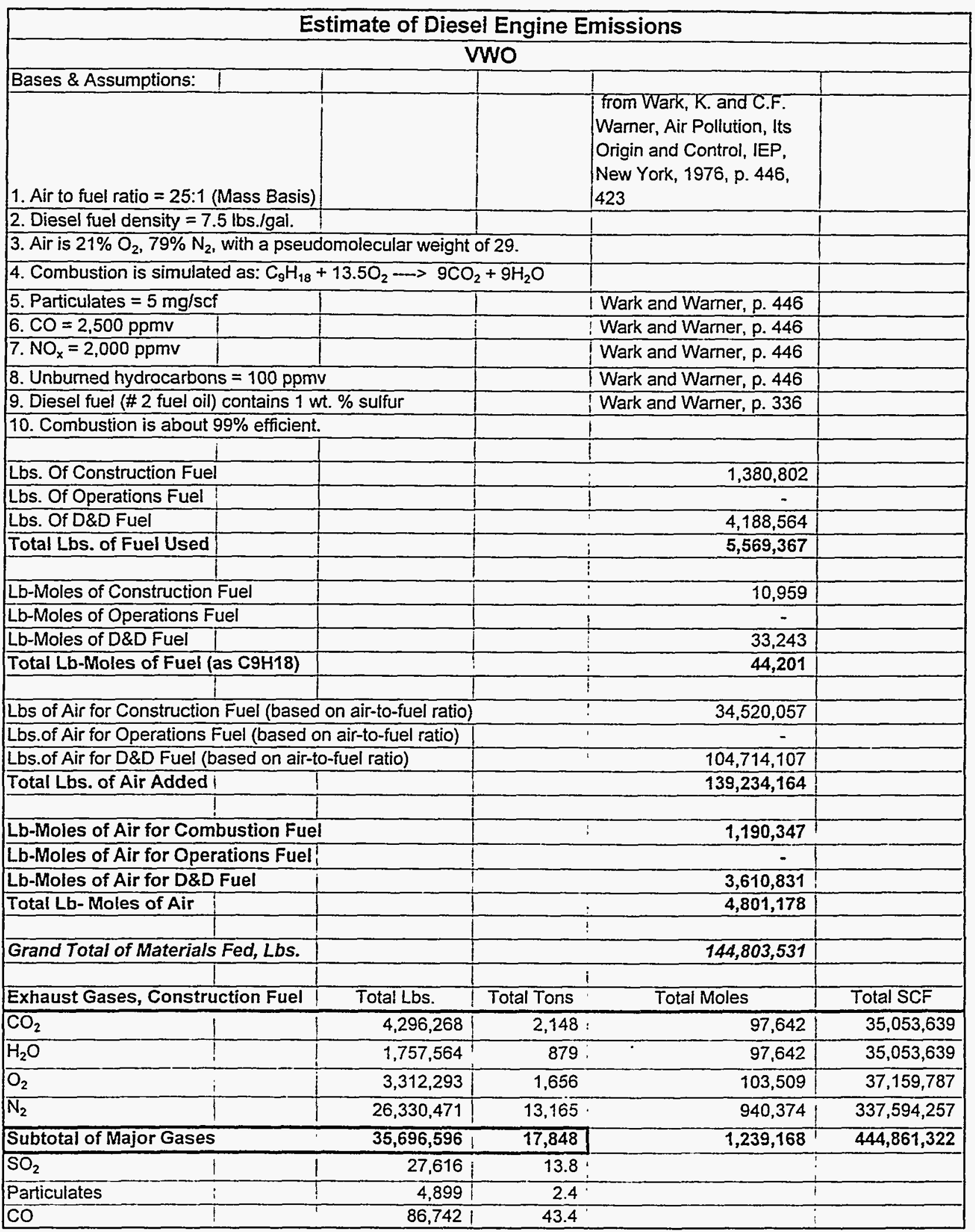


Table 4. (continued).

\begin{tabular}{|c|c|c|c|c|}
\hline $\mathrm{NO}_{\mathrm{x}}$ (assumed $\mathrm{NO}$ ) & 74,350 & 37.2 & & \\
\hline Unburned Hydrocarbons & 15,614 & 7.8 & & \\
\hline Subtotal of Contaminants & 209,221 & 105 & & \\
\hline & & & & \\
\hline Exhaust Gases, Operations Fuel & Total Lbs. & Total Tons & Total Moles & Total SCF \\
\hline $\mathrm{CO}_{2}$ & - & - & - & - \\
\hline $\mathrm{H}_{2} \mathrm{O}$ & - & - & - & - \\
\hline $\mathrm{O}_{2}$ & - & - & - & - \\
\hline$\overline{N_{2}}$ & - & - & - & - \\
\hline Subtotal of Major Gases & - & $=$ & - & - \\
\hline $\mathrm{SO}_{2}$ & - & - & $\cdot$ & \\
\hline Particulates & - & - & & \\
\hline $\mathrm{CO}$ & - & - & & \\
\hline $\mathrm{NO}_{x}$ (assumed $\left.\mathrm{NO}\right)$ & - & - & & \\
\hline Unburned Hydrocarbons ! & - & - & & \\
\hline Subtotal of Contaminants & - & - & & \\
\hline & & & & \\
\hline Exhaust Gases, D\&D Fuel & Total Lbs. & Total Tons & Total Moles & Total SCF \\
\hline \begin{tabular}{|l|l}
$\mathrm{CO}_{2}$ & \\
\end{tabular} & $13,032,419$ & 6,516 & 296,191 & $106,332,688$ \\
\hline$\overline{\mathrm{H}_{2} \mathrm{O}}$ & $5,331,444$ & 2,666 & 296,191 & $106,332,688$ \\
\hline $\mathrm{O}_{2}$ & $10,047,602$ & 5,024 & 313,988 & $112,721,538$ \\
\hline$\overline{N_{2}}$ & $79,871,587.63$ & 39,936 & $2,852,557$ & $1,024,067,856$ \\
\hline Subtotal of Major Gases & $108,283,052$ & 54,142 & $3,758,927$ & $1,349,454,769$ \\
\hline $\mathrm{SO}_{2}$ & 81,153 & 40.6 & & \\
\hline Particulates & 14,862 & 7.4 & & \\
\hline $\mathrm{CO}$ & 263,125 & 131.6 & & \\
\hline $\mathrm{NO}_{x}$ (assumed NO) & 225,536 & 112.8 & & \\
\hline Unburned Hydrocarbons & 47,362 & 23.71 & & \\
\hline Subtotal of Contaminant & 632,038 & $316 !$ & & \\
\hline
\end{tabular}




\section{PROJECT-SPECIFIC VARIANCES}

\subsection{CWO Hot Isostatic Press (HIP) Preliminary Plan}

A variation plan has been devised for the CWO that involves grouting the recalcine and casting the mixture into special HIP containers over the 5-year recalcination time period. The HIP containers would then be placed into Interim Storage to await the HIP process. The container HIP'ing process would occur during the 5-year period and continue after recalcine and grouting process completion for 15 more years to complete a 20-year regime. After the HIP'ing process, the HIP'ed containers would be sealed inside HLW canisters (the same canisters used for CWO grouted waste) with three HIP containers per canister. Approximately 31,000 HIP containers would be produced during the 5-year recalcine and grouting operation and about 10,334 HLW canisters would be produced throughout the 20 -year HIP campaign. The HIP containers and HLW canisters would require interim storage in the same facility. The HLW canisters are the final end product of the CWO and would be interim stored until shipment preparations and transport to the National HLW Repository became available. Refer to EDF-CWO-001 (Appendix E, Item 11). The process schematic (HLW Study: Non-Separations Alternative Direct Cementitious Waste Option-Section B), indicates the pathway and integrated waste volume, can be found in EDF-DCWO-011 (Appendix E, Item 10); also consult Reference R19. 


\section{POTENTIAL IMPACTS OF NRC LICENSING}

The CWO 5-year grout facility used for this study, as presented in a separate report (Reference R19), will not be NRC licensed and therefore, neither the grout facility design nor the cost estimates included were modified to include NRC licensing. The NWCF used for recalcination in this report would not require NRC licensing because it is a DOE waste treatment facility. This section contains a brief discussion, gleaned from data contained in the Regulatory Requirements and Criteria for ICPP Proposed Waste Processing Facilities, EDF-WTS-003 (Appendix E) and from the Waste Treatment Facilities Feasibility Study Report (Reference R6) of the potential impacts of NRC licensing on the Waste Treatment Facilities (WTF).

Existing NRC regulations are compiled in 10 CFRs, "Energy." These regulations follow a similar philosophy advocated by the DOE, EPA, etc. The Commission has also issued regulatory guides (such as NUREGs) that provide acceptable methods to comply with the NRC regulations; they contain criteria for facility design, operations, and health and safety.

The only WTF-type activity that the NRC has routinely licensed is HLW waste storage. Currently, NRC regulations do not exist to license WTF-type HLW separations or treatment facilities. The most applicable licensing process regulations to the WTF are contained in: (a) 10 CFR 2, 10 CFR 30, 10 CFR 51, and 10 CFR 61 for low level waste (LLW) facilities, and (b) 10 CFR 2, 10 CFR 50, 10 CFR 52, 10 CFR 70, and 10 CFR 72 for HLW or HAW facilities. Licensing a nuclear facility requires preparing and submitting an application and supporting documents to the NRC, such as Safety Analysis Reports, Environmental Reports, quality assurance documents, training plans, monitoring plans, and safeguards and security plans. The NRC licensing process is divided into four stages: preapplication, application review, construction and operating license, and decontamination and site closure. The licensing duration from submitting the application to receiving of the license is expected to take 3 to 5 more years, or longer. The benefits of NRC licensing are enhanced operating safety, strengthened relationships with stakeholders, and license-holder participation in future regulation development.

According to data developed in the Fluor Daniel Incorporated "Idaho Chemical Processing Plant Waste Treatment Facilities Feasibility Report (Reference R6), the estimated capital cost percentage increase for NRC licensing of WTS facilities, above current DOE requirements for those facilities are as follows:

$\begin{array}{lc}\text { Waste Separations } & 21 \% \\ \text { Low-Activity Waste Treatment } & 13 \% \\ \text { High-Activity Waste Interim Storage } & 26 \% \\ \text { Low-Activity Waste Collection } & 8 \% \\ \text { Calcine Dissolution } & 15 \% \\ \text { CalcineTransfer } & 14 \% \\ \text { High-Activity Waste Treatment } & 26 \% \\ \text { Infrastructure (Utilities) } & 9 \%\end{array}$


These estimates are hypothetical and highly speculative. The capitol costs listed here are equivalent to the escalated, current dollar TEC in the cost estimate presented in Section 4.

Reference R6, also estimated the operating schedule impact and dollar-value increase in annual operating costs for three groups of systems as follows:

$$
\text { Group I Negligible Schedule Impact } \$ 1.3 \mathrm{M}
$$

Group II Minimal Schedule Impact $\$ 2.7 \mathrm{M}$

Group III Significant Schedule Impact $\$ 4.7 \mathrm{M}$

Group I includes the LAW collection, infrastructure, and tank heel; Group II includes the waste separations, LAW treatment, condensate collection, and environmental analysis laboratory; and Group III includes the HAW interim storage, calcine dissolution, calcine transfer, and HAW treatment. Additional costs resulting from operating schedule impacts experienced due to $\mathrm{NRC}$ involvement are based on a "best guess" reflecting the perceived likelihood of NRC's difficulty in assimilating the group into their new regulatory framework. "Negligible" could range from no costs, to tens of thousands of dollars. "Minimal" could range from a few thousand dollars to several hundred thousand dollars. "Significant" could range from a hundred thousand to several million dollars.

The CWO is best characterized by Group III except it does not have waste separations, low activity waste treatment, or low activity waste collection, therefore, the portion of the increased cost associated with that facility must be subtracted from the $\$ 4,700,000$ Group III cost to determine the CWO increase. Assuming the NRC licensing annual cost increase for the calcine dissolution system is $58 \%$, then the annual cost increase for the CWO (the remainder of the Group III system) is approximately $\$ 2,726,000$ (per year).

The increase of capital cost and 5-year operating cost due to NRC licensing of the CWO is based on the following:

1. $14 \%$ escalated TEC cost increase for the CRTS

2. $26 \%$ escalated TEC cost increase for the ISF

3. $26 \%$ escalated TEC cost increase for the CWO grout facility

4. $9 \%$ escalated TEC cost increase for infrastructure

5. $26 \%$ escalated TEC cost increase for the MACT Facility).

The increase in capital cost is estimated to be $\$ 537,435,000$.

The increase in the 5-year operating cost is estimated to be $\$ 272,370,000$ 
The total increase of capitol cost and five year operating cost is estimated to be $\$ 799,805,000$.

Some of the potential major impacts associated with NRC licensing of WTF, other than cost, are:

- Increased oversight, including more public involvement and input in all decision processes

- More restrictive physical limits on some parameters, including exposure limits, seismic, and tornado

- $\quad$ More strict radiation monitoring

- $\quad$ Restrictions on sharing utilities between facilities

- More stringent evaluations of the impact from off-Site hazards

- Full testing required for emergency utilities

- Physical changes to the plant and equipment

- More elapsed schedule time required

- Methods to comply with some other codes and standards may be complicated and require more time

- $\quad$ Although the NRC may license the WTF, it may not automatically inherit or adopt the same agreements and obligations with the State of Idaho and EPA Region 10 that are in place for DOE and INEEL. 


\section{RECOMMENDATIONS}

This section emphasizes future studies, process optimization, and building optimization

\subsection{Future Studies}

A number of technical concerns have been identified in developing the baseline design for the CWO process. In order to establish the viability of the process these concerns should be addressed in further development work. Specifically, the following items require attention:

1. The baseline slurry feed system for recalcination has not been tested in a high radiation environment, and its reliability, robustness, and resistance to plugging have not been demonstrated. Seals and deformable components in the system (e.g., the flexible diaphragm in the slurry pump) are likely to deteriorate rapidly when exposed to gamma radiation from the calcine. The slurry feed system should be assembled and tested with surrogate wastes to evaluate its viability, and optimize its design. All polymer/rubber components should be irradiated to determine their useful life, and identify components that are likely to require frequent changeout. In addition other commercially available alternatives (see Appendix B) should also be tested and evaluated.

2. Transfer of scrub solutions containing organics (from sugar calcination) back the tank farm would probably be prohibited on the basis of safety considerations unless such solutions were sampled and analyzed prior to transfer, and the organic concentrations shown to be low. Rates of organic digestion in nitric acid appear to be low, and may make digestion infeasible as a means of reducing organic concentrations in the scrub. These rates need to be measured, and the feasibility of digestion of organics re-assessed on the basis of the measurements.

3. Calcines from sugar calcination have been found (in pilot studies) to be hygroscopic. Such calcines readily absorb water and agglomerate, causing packing and leading to difficulties in handling. Measures may be required to ensure that exposure of recalcined solids to humid air be limited from the time it leaves the calciner until the time it is grouted.

4. The process design assumes the following: (a) all nitrates in solid calcine will readily dissolve in liquid SBW and water used for slurrying, (b) the only composition variable of the slurry mixture that will be required to adjust calcination process parameters is the dissolved nitrate composition, (c) the only calcination process parameter that will require adjustment for each slurry tank is the rate of sucrose injection, and (d) nitrate concentrations in the liquid portion of the slurry mixture can readily be determined within less than 24 hrs of slurry blending. turnaround) of slurry mixtures can be done to determine their nitrate content prior to recalcination. These assumptions should be verified by testing and development.

5. An inline mixer (to blend sucrose solution with the liquid in the slurry) in the slurry stream may cause plugging problems due to the high solids content. Whether or not this is the case should be determined, and alternative mixing method developed if necessary.

6. The baseline CWO process assumes that (a) sizing of the calcine and cold additives is not be required, (b) all calcines can be grouted by adjusting additive proportions, (c) a reasonable number of grouting recipes will accommodate variations in calcine composition, and 
(d) autoclaving of grouted waste will be required to make a suitable hydroceramic waste form. These assumptions should be verified with bench scale studies of grouting recipes using non-radioactive pilot plant calcines stored at ICPP. These studies would generate a credible set of reaction conditions required to produce acceptable waste forms from all calcines in the ICPP inventory.

These grouting studies would also provide data to explore suggested steps for process optimization, described in the next section. It is emphasized that in developing the baseline CWO design for this scoping study, in those cases where processing requirements were not accurately known (due to lack of bench scale test data), conservative assumptions were generally made which inflate both facility and lifecycle costs. If valid test data were available, it is likely that the estimated cost for the CWO process could be significantly reduced.

\subsection{Process Optimization}

The baseline CWO process calls for blending and recalcination of stored calcine solids, together with liquid SBW. The benefits likely to be obtained from blending and recalcination prior to grouting have been assessed as described in Thompson/Taylor EDF on blending (EDF-CWO-005, Appendix E, Item 13). The assessment suggests that recalcination and blending would reduce the final waste mass by less than $10 \%$, and would reduce the requirement for added caustic $(\mathrm{NaOH})$ by $90-100 \%$. It also suggests that composition variations in stored calcines are likely to be accommodated by a single grouting "recipe" with no blending at all.

These conclusions indicate that the CWO process costs documented herein could be reduced substantially by eliminating the recalcination step and the requirement that calcines be blended prior to grouting. These changes would reduce the life cycle costs as follows:

- $\quad$ Construction costs for NWCF modifications would be eliminated

- Operational costs for recalcination would be eliminated

- The simplest calcine retrieval system would be sufficient, reducing the cost of retrieval

- Large calcine blending bins (currently part of the baseline grouting process per Reference $\mathrm{R} 19)$ would not be required. This would reduce the capital costs for equipment and the size of the required building.

Based on the estimated cost of modifications to NWCF alone, the potential savings from these optimizing measures would be hundreds of millions of dollars.

\subsection{Building Optimization}

No further building optimization has been determined. The design presented in this study is considered optimum for the scoping level of effort provided. 


\section{CONCLUSIONS}

The baseline CWO process calls for blending and recalcination of stored calcine solids, together with liquid SBW. The benefits likely to be obtained from blending and recalcination prior to grouting have been assessed as described in Thompson/Taylor EDF on blending (EDF-CWO-005, Appendix E, Item 13). The assessment suggests that recalcination and blending would reduce the final waste mass by less than $10 \%$, and would reduce the requirement for added caustic $(\mathrm{NaOH})$ by $90-100 \%$. It also suggests that composition variations in stored calcines are likely to be accommodated by a single grouting "recipe" with no blending at all.

These conclusions indicate that the CWO process costs documented herein could be reduced substantially by eliminating the recalcination step and the requirement that calcines be blended prior to grouting. These changes would reduce the life cycle costs as follows:

- Construction costs for NWCF modifications would be eliminated

- Operational costs for recalcination would be eliminated

- The simplest calcine retrieval system would be sufficient, reducing the cost of retrieval

- Large calcine blending bins (currently part of the baseline grouting process per Reference $\mathrm{R} 19$ ) would not be required. This would reduce the capital costs for equipment and the size of the required building.

Based on the estimated cost of modifications to NWCF alone, the potential savings from these optimizing measures would be hundreds of millions of dollars.

Approximately 166,134 cubic feet $\left(4,705 \mathrm{~m}^{3}\right)$ of mixed-HLW recalcined waste would be generated from existing calcine and liquid SBW. The recalcined waste would be combined with calcined clay, blast furnace slag, sodium hydroxide, and water to produce about 404,829 cubic feet $\left(11,465 \mathrm{~m}^{3}\right)$ of groutedrecalcined mixed-HLW after grout cycle processing.

Approximately 15,924 grouted recalcined waste filled canisters would be produced by the Grout Facility for the Nonseparations Cementitious Waste Option. The quantity is based on the use of the SRS HLW canister with a nominal waste fill volume of 25.4 cubic feet $\left(0.72 \mathrm{~m}^{3}\right)$.

At a scoping level, this study indicates that the existing calcine and liquid SBW can be processed into a recalcined product suitable for grouting, and that the grout matrix poured into canisters can be processed into a durable zeolitic hydroceramic form. All waste canisters processed by the CWO must be placed into interim storage awaiting transport off-Site and are assumed to be acceptable for GCDF disposal. The process should be finished within a 5-year timeframe.

The recalciner operation is based on a 24 hour a day, 7-day week production schedule. The grouting schedule is based on a 4 day 10 -hour weekly shift for the grout mixing and canister filling stages and a 7 day, 24-hour weekly period for the remaining operations. The ISF would operate on a 4 day, 10-hour weekly shift.

The CWO cost estimates for the calcine retrieval, recalcination of waste products, the MACT operation for offgas control, the grout facility operation, and interim storage based on a 5-year schedule are as follows: 


$$
\begin{array}{ll}
\text { Total Estimated Cost (TEC) }= & \$ 2,479220,000 \\
\text { Total Project Cost (TPC) }= & \$ 2,797,920,000
\end{array}
$$

The CWO combined Life Cycle Cost (LCC) for a five-year operation (2013 through 2017) and an assumed 24-year (2001 through 2024) start to closure timeline $=\$ 2,628,856,000$

A variation to the CWO would add a HIP process designed to reduce waste volume and provide a waste form similar and considered superior to the vitrified BDAT. The HIP process would occur over a 20 -year timeframe (2013 through 2033) regardless of the 5-year recalcination duration. The HIP process was assumed to use special HIP drums instead of canisters for processing and would follow the recalcined waste grouting process. After HIPing, three HIP'ed containers would be placed into standard HLW canisters and sealed. The HIP'd container dimensions would be designed to achieve an $80 \%$ or better canister fill volume. After sealing the canisters would be transferred to interim storage to await transport cask insertion and transport to the National HLW Repository. The Total Estimated Cost (TEC) for this variance would be approximately $\$ 2,562,344,000$; the Total Project Cost (TPC) for this variation would be about $\$ 3,230,746,000$, and the LCC for this variation would be approximately $\$ 3,144,080,000$ (in 1999 dollars) over the same 20 -year time period.

Construction and operations activities required to accomplish this schedule could potentially result in various minor environmental impacts. However, no specific impact was determined to be serious.

The Record Of Decision (ROD) to identify the INEEL waste treatment method and waste form will not be finalized until October 1, 1999. There are three individual methodologies that must be considered; (1) no action, (2) separations, and (3) non-separations. The ROD decision is mandated to identify one of the following waste treatment options to comply with the Batt agreement; (1) Tru-Separations, (2) Full Separations, (3) Cementitious Waste Option Non-Sep, (4) Direct Cemetitious Waste Option Non-Sep, (5) HIP Waste Option Non-Sep, or (6) Vitrified Waste Option Non-Sep. The no action decision would not comply with the Batt agreement but may be considered due to cost of waste treatment and/or the lack of a dedicated future national disposal or storage site. The final mixed HLW treatment option decision hinges on the expected method of national disposal or storage perceived as available in the future for the final waste form. If the National HLW Repository is deemed unavailable due to irreconcilable problems or the Greater Confinement Disposal Facility cannot be licensed for HLW disposal, then the waste must be treated by the chosen method and stored indefinitely until further decision making criteria becomes available. 


\section{REFERENCES}

Note: All references can be found in the project reference files.

R1. “Waste Inventories/Characterization Study," INEL/EXT-97-00600, September 1997.

R2. "Waste Disposal Options Feasibility Study," INEEL/EXT-97-01145 Draft, October 1997.

R3. "Utilities and Infrastructure Supporting the Waste Treatment Options," INEEL/EXT-97-01398, January 1998.

R4. Stockpile Report to the Congress - Fiscal Year 1996.

R5. Interim Guidance-Office of Science and Technology Technical Decision Process, DOE Standard Operating Procedure, May 8, 1997.

R6. Fluor Daniel, Inc. Government Services Operating Company, "Idaho Chemical Processing Plant Waste Treatment Facilities Feasibility Study," Delivery Order 94-36, 30\% Design Review Package, June 1997, 60\% Design Review Package, August 1997, and 90\% Design Review, October, 1997.

R7. "Consent Order and Settlement Agreement between DOE and the State of Idaho regarding spent fuel and nuclear waste issues," October 17,1995.

R8. U.S. Department of Energy, Office of Civilian Radioactive Waste Management (OCRWM), "Quality Assurance Requirements and Description (QARD)," DOE/RW-0333P.

R9. EM-WAPS Rev. 01, "Waste Acceptance Product Specifications for Vitrified High-Level Waste Forms," May 1995.

R10. DOE/RW-0351 P Revision 02, "Waste Acceptance System Requirements Document," December 1996.

R11. Rawlins, J.K. et al, “Interim Storage Scoping Study,” INEEL/EXT-97-01393.

R12. Russell, N. E., "Hot Isostatic Pressed (HIP) Waste Option Scoping Study Report," INEEL/EXT-97-01392,

R13. Title 10, Code of Federal Regulations (CFR), Part 72 Licensing Requirements for the Independent Storage of Spent Nuclear Fuel and High-Level Radioactive Waste.

R14. 40 CFR 268.42(b)

R15. "Hot Isostatic Pres (HIP) Vitrification of Radwaste Concretes," INEL-95/00343, 1995.

R16. Raytheon Engineers and Constructors, Inc., “Idaho Chemical Processing Plant Bin Set 1 Calcine Recovery Project: Phase I and II Special Studies Draft Report", Volume I, February 1995.

(EDF-WTS-002 Reference). 
R17. Griffith, D. L., "Status of Calcine Retrieval Development Work -DLG-06096", September 1996. (EDF-WTS-002 Reference).

R18. Bodner, S.S., "Effects of Bend Configuration on Dilute-Phase Pneumatic Transport", ENICO-1084, May 1981. (EDF-WTS-002 Reference).

R19. Dafoe, R. E. et al, "Direct Cementitious Waste Option Scoping Study Report," INEEL/EXT-01399, January 1998.

R20. Lee, A. E., "Preliminary Design of the Nevada Test Site Greater Confinement Disposal Facility." INEEL/INT-97-01316, January 1998.

R21. Interdepartmental Communication to Thomas G. McDonald from Henry K. Peterson, "Update of Radiation Field Definition and Thermal Generation Rates For Calcine Process Packages of Various Geometries," HKP-26-97, Dated December 4, 1997.

R22. Rawlins, J. K., Scoping Study Report for NWCF MACT Compliance Facility," INEEL/INT-00992.

R23. EDF-WTS-003 in the project file.

R24. Petrie, J. C., “Report on Run 12, Twelve-Inch Diameter Calciner", letter Petr-13-65A to E.J. Bailey, December 30, 1965 (INEL report).

R25. Dole, L. R. et al, "Cement-Based Radioactive Waste Hosts formed Under Elevated Temperatures and Pressures (FUETAP Concrete) for Savannah River Plant High-Level Defense Waste, ORNL/TM-8579, March 1983 (ORNL report).

R26. Siemer, D. D., B. E. Scheetz, and M. L. D. Gougar, "Hot Isostatic Press (HIP) Vitrification of Radwaste Concretes", Materials Res. Soc. Symp. Vol 412, 1996, pp. 403-410, (Proceedings of 1995 MRL Symposium on 'Scientific Basis for Nuclear Waste Management XIX', Boston, MA, Nov. 29 - Dec 3, 1995).

R27. Siemer, D. D. "Hot Isostatically Pressed Concrete as a Radwaste Form", Advances in Ceramics, Vol 61, Ceramics Transactions, pp. 657-664, (Proceedings of 1995 American Ceramics Society Symposium on Waste Management, Cincinnati, OH, April 29 - May 4, 1995).

R28. Welland, H., "NWCF Process Modification for Sodium-Bearing Waste Project Conceptual Design," INEL/INT-97-00075, April 1997.

R29. Boardman, R. D., Interdepartment Communication to D. V. Croson, Subject: Transmittal of Status Report on Alternative Calcination Development Accomplishments and Results, April 30, 1997.

R30. Adams, R. D. et al, "The 90\% Draft of the Feasibility Study Report for MACT Compliance Facility," INEL/INT-97-00992, August 1997.

R31. Banaee, J., "Environmental Regulations and Standard Repositories Criteria for the Disposal of Waste Forms from the INEEL Proposed High-Level Waste Processing Alternatives, " INEEL/EXT-97-01 147, October, 1997. 
R32. Stegan, G.E., Numatec, INEEL High-Level Waste Program Impacts Related to Disposal Fees and Ability of Repository to Accept Waste, IN-RPT-001, prepared for LMITCO under Contract C95-175006, Task Order F97.

R33. Fluor-Daniel Inc., "High Level Liquid Waste Alternative Approach Evaluation, for the U.S. Department of Energy," FDI Delivery Order 94-34, DOE Delivery Order DE-AD07-91ID60034, December 1996.

R34. Gougar, M. L., D. D. Siemer, B. E.Sheetz, "Disposal of INEL Spent Nuclear Fuel Reprocessing Waste Using a Glass Forming Cement," ANS Embedded Topical Meeting, DOE Spent Nuclear Fuel and Fissile Material Management, Reno, NV, June 96.

R35. Siemer, D. D., "A Better Way to Prepare the INE(E)L HLW for Disposal," April 14, 1997.

R36. VECTRA GSI Report No. WHC-VIT-03, "Fluid Bed Calciner Test Report - Final", -WHC-SD-WM-VI-031, August 1995 (Hanford report). 
Appendix A

Assumptions 
Appendix A

Key Assumptions

Cementitious Waste Option 


\section{FEASIBILITY S'TUDY(FS) ASSUMPTIONS}

\begin{tabular}{|c|c|c|c|c|c|}
\hline $\begin{array}{l}\text { Number } \\
\text { Latest Rev } \\
\text { Initials }\end{array}$ & Assumption & References & Application to Volumes & $\begin{array}{l}\text { Application to } \\
\text { Alternatives }\end{array}$ & Conlact and Date \\
\hline $\begin{array}{c}\text { WS-1 } \\
2 / 11 / 98 \\
\text { AEL }\end{array}$ & \multicolumn{5}{|c|}{$\Lambda W \Lambda C$ will be developed by NTS to allow $H L W$ to be disposed of in the GCDF. } \\
\hline Basis & (Sce basis for WS-2) & & $\begin{array}{l}\text { This refess to EIS } \\
\text { volume numbers and } \\
\text { will be completed by } \\
\text { others }\end{array}$ & $\begin{array}{l}\text { This refers to the EIS } \\
\text { alternatives and will be } \\
\text { completed by others. }\end{array}$ & \begin{tabular}{|l|} 
A. E. Lee \\
(208)526-9716 \\
xal@inel.gov
\end{tabular} \\
\hline
\end{tabular}

\begin{tabular}{c|c}
\hline WS-2 & The NWPAA will be revised to include the NTS-GCDF as a IILW disposal site. \\
$2 / 11109$ &
\end{tabular}


FEASIBILITY STUDY(FS) ASSUMPTIONS dedicated to thermonuclear bomb testing during the 1950's, and 60 's. Tests were conducted both above and below ground within two primary areas known as Frenchman Flats and Yucca Flats. Both areas are contaminated with radioactive bomb test materials and are unsuitable for anything other than fulure thermonuclear testing or waste disposal (see Reference 1). The water table existing below

bounb craters is, or cventually will be, contaminated by the migration of radioactive bomb test materials, which cannot be mitigated. The waste form to be gencrated by the CWO is assumed to be RCRA delisted (Reference 2) and would be contained in stainless stecl

canisters placed in borcholes in the Frenchman Flat area of the NTS.

The canistered waste is not expected to add to the water table contamination for more than 10,000 years, as determined by preliminary performance assessments (c.g., see Reference 3). Risk assessments have concluded that plant and animal activities are not considered a threat because they normally do not exceed 25.3 feet (8 meters) below surface. Excavation by liumans is possibly the grcatest threat to radioactive waste disposal due to drilling activities; however the above grade concrete cap, radiation placard, and the corrugated metal pipe at the top of each borehole, should alert potential intruders to a problem zone. Drilling for drinking or livestock water within the aren is not considered probable once the 100 year Government sitc administrative control has been relinquished. Mining by humans is not practical because metals or minerals of economic value do not exist below grade. Also, scismic events are not a problem with alluvium deposits due to soil structure and depth. The Frenchman Flat area is located in a scismic zone 2 whereas the national HLW repository (Yucca Mountain) is located in a seimic zone 3.

Placement of 16,000 canisters in the national HLW mined repository is estimated to cost roughly $\$ 4.8$ Billion $(\$ 300,000$ per canister-

Reference 4). The corresponding estimated disposal cost at the N'TS is $\$ 208$ Million $(\$ 13,040$ per canister--Reference 5$)$. Therefore the potential exists to save approximately $\$ 4.6$ Billion by the use of the CWO in conjuction with disposal at N'TS compared to the national IILW repository.

Assumption WS-2 is based on the premise that the information discussed above is credible, and that based on this (and other supporting data) Congress will be convinced to revise the public law, as necessary.
(1) I. J. Winograd [USGS], "Radioactive Waste Disposal in Thick Unsaturated Zoncs", Science, vol 212 no 4502, 26 June 1981, pp. 1457-1464.

\section{This refers to EIS}

volume numbers and

will be completed by

alternatives and will be

completed by others.

(208)526-9716

xal@incl.gov

(2) Idaho Clıemical Processing Plant

High Activity Waste Treatment Project

Regulatory Assessment Report, prepared

by R. G. Morgan and S. E. Leroy, Duke

Engincering Services, Inc. S. E. Leroy

letter to V. L. Jacobson, dated Aptil 25,

1997.

(3) Preliminary Performance Assessment of the Greater Confinement Disposal

Facility at the Nevada Test Site", L.L.

Price, ct al, SAND-91-0047.

(4) Stcgan, G.E., Numatec, INEEL HighLevel Waste Program Impacts Related to Disposal Fecs and Ability of Repository to Accept Waste, IN-RPT-001, prepared for LMITCO under Contract C95-175006, Task Order F97.

(5) Lec, A. E., "Prcliminary Design of the Nevada Test Site Grenter Confinement Disposal Facility." INEEU/INT-9701316, January 1998. others

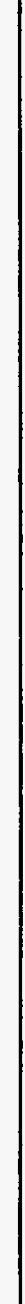




\section{FEASIBILITY STUDY(FS) ASSUMPTIONS}

\begin{tabular}{|c|c|c|c|c|c|}
\hline Basis & $\begin{array}{l}\text { The key component of the INESL waste management plan is the de- } \\
\text { listing of various hazardous wastes in the various waste streams such } \\
\text { that RCR } A \text { requirements will not apply (Reference } 1 \text { ). Ilowever, the } \\
\text { timing for this action is uncertain and RCRA requirements during } \\
\text { treatment of the waste are assumed to apply. }\end{array}$ & $\begin{array}{l}\text { (1) Idalıo Chemical P1ocessing Plant } \\
\text { High Activity Waste Treatment Project } \\
\text { Regulatory Assessment Report, prepared } \\
\text { by R. G. Morgan and S. E. Leroy, Duke } \\
\text { Engineering Services, Inc. S. E. Leroy } \\
\text { letter to V. L. Jacobson, dated April } 25 \text {, } \\
1997 \text {. }\end{array}$ & $\begin{array}{l}\text { This refers to EIS } \\
\text { volume numbers and } \\
\text { will be completed by } \\
\text { others }\end{array}$ & $\begin{array}{l}\text { This refers to the EIS } \\
\text { altematives and will be } \\
\text { completed by others. }\end{array}$ & $\begin{array}{l}\text { A. E. Lec } \\
\text { (208)526-9716 } \\
\text { xal@incl.gov }\end{array}$ \\
\hline
\end{tabular}

\begin{tabular}{|c|c|c|c|c|c|}
\hline & \multicolumn{5}{|c|}{ INEEL Waste canisters will be acceptable waste disposal vessels for the Greater Confinement Disposal Facility (GCDF) at the NTS. } \\
\hline Basis & $\begin{array}{l}\text { A preliminary design study was performed to investigate the Greater } \\
\text { Confinement Disposal Facility (GCDF) at NTS as the disposal site } \\
\text { for Idaho National Engineering and Environmental Laboratory } \\
\text { (INEEL) HLW. The INEEL canisters were used as a basis for that } \\
\text { study, with no finding of infeasibility. }\end{array}$ & $\begin{array}{l}\text { (1) Lee, A. E., "Preliminary Design of the } \\
\text { Nevada Test Site Greater Confinement } \\
\text { Disposal Facility." INEEL/INT-97- } \\
01316 \text {, January } 1998 .\end{array}$ & $\begin{array}{l}\text { This refers to EIS } \\
\text { volume numbers and } \\
\text { will be completed by } \\
\text { others }\end{array}$ & $\begin{array}{l}\text { This refers to the EIS } \\
\text { alternatives and will be } \\
\text { completed by others. }\end{array}$ & $\begin{array}{l}\text { A. E. Lee } \\
\text { (208)526-9716 } \\
\text { xal@inel.gov }\end{array}$ \\
\hline
\end{tabular}

\begin{tabular}{|c|c|c|c|c|c|}
\hline WS-6 & \multicolumn{5}{|c|}{ A performance Assessment will be developed by Sandia Laboratory to show that GCDF disposal of HLW is acceptable to 40 CFR 191.} \\
\hline Basis & $\begin{array}{l}\text { A preliminary performance assessment by Sandia (Reference I) has } \\
\text { already been done which concluded that the above assumption is } \\
\text { reasonable. }\end{array}$ & $\begin{array}{l}\text { (1) Preliminary Performance Asscssment } \\
\text { of the Greater Confinement Disposal } \\
\text { Facility at the Nevada Test Site", L.L. } \\
\text { Price, et al, SAND-91-0047. }\end{array}$ & $\begin{array}{l}\text { This refers to EIS } \\
\text { volume numbers and } \\
\text { will be completed by } \\
\text { ofleers }\end{array}$ & $\begin{array}{l}\text { This refers to the EIS } \\
\text { alternatives and will be } \\
\text { completed by others. }\end{array}$ & $\begin{array}{l}\text { A. E. Lee } \\
\text { (208)526-9716 } \\
\text { xal@incl.gov }\end{array}$ \\
\hline
\end{tabular}

\begin{tabular}{|c|c|c|c|c|}
\hline WS-7 & \multicolumn{4}{|c|}{ A GCDF will be opened by January 1,2013 to support the CWO schedule. } \\
\hline Basis & $\begin{array}{l}\text { There is currently no objective basis for this assumption. It was } \\
\text { made only to indicate that IF the GCDF were opened by this date, } \\
\text { the INEEL waste could be processed and disposed as soon as } \\
\text { December } 31,2017 \text {. }\end{array}$ & $\begin{array}{l}\text { This refers to EIS } \\
\text { volume numbers and } \\
\text { will be completed by } \\
\text { others }\end{array}$ & $\begin{array}{l}\text { This refers to the EIS } \\
\text { alternatives and will be } \\
\text { completed by others. }\end{array}$ & $\begin{array}{l}\text { A. E. L.ec } \\
\text { (208)526-9716 } \\
\text { xal@inel.gov }\end{array}$ \\
\hline
\end{tabular}

\begin{tabular}{|c|c|c|c|c|}
\hline $\begin{array}{c}\text { WS-8 } \\
2 / 11 / 99\end{array}$ & \multicolumn{4}{|c|}{ Grouted waste will be cast in cylindrical stainless steel disposal canisters which are 2 feet in diameter by 9'-10" high with at least $80 \%$ utilization of the available volume in the canister. } \\
\hline Basis & $\begin{array}{l}\text { This assumption was made to provide a consistent basis for } \\
\text { comparison of the CWO process with other alternatives, which also } \\
\text { made this assumption. }\end{array}$ & $\begin{array}{l}\text { This refers to EIS } \\
\text { volume numbers and } \\
\text { will be completed by } \\
\text { others }\end{array}$ & $\begin{array}{l}\text { This refers to the EIS } \\
\text { alternatives and will be } \\
\text { completed by others. }\end{array}$ & $\begin{array}{l}\text { A. E. Lee } \\
\text { (208)526-9716 } \\
\text { xal@incl.gov }\end{array}$ \\
\hline
\end{tabular}

WS-9 $\quad$ The overall treatment facility online factor will be $50 \%$. Calcine retrieval and recalcination of slurried wastes will procced on a $24-1$ r/day, $7-$ day/week schedule (subject to the above-assumed 2/1 1/99 online factor). All other processes (e.g., grouting, curing, storage operations, etc.) will proceed on the basis of four 10-lur shifts per week, and 198 actual working days per year (again subject to the assumed online factor). 


\section{FEASIBILITY STUDY(FS) ASSUMPTIONS}

\begin{tabular}{|c|c|c|c|c|c|}
\hline Basis & $\begin{array}{l}\text { The } 50 \% \text { online factor is reasomable (though conservative), based on } \\
\text { past operating experience at the NWCF (Reference } 1 \text { ). The } 24-\mathrm{ll} \\
\text { operation of the recalcination facility is based on the current } \\
\text { operating schedule at NWCF. The 4-day X } 10 \text {-lir schedule for } \\
\text { grouting operations is based on the current "defaule" work schedule } \\
\text { at the INEEL, and the assumption that grouting operations can } \\
\text { feasibly be performed on this sclicdule. }\end{array}$ & $\begin{array}{l}\text { (1) Welland, H., "NWCF Process } \\
\text { Modification for Sodium-Bearing Waste } \\
\text { Project Conceptual Design," INEL/NT- } \\
\text { 97-00075, April 1997. }\end{array}$ & $\begin{array}{l}\text { This refers to EIS } \\
\text { volume numbers and } \\
\text { will be completed by } \\
\text { others }\end{array}$ & $\begin{array}{l}\text { This refers to the EIS } \\
\text { altematives and will be } \\
\text { completed by others. }\end{array}$ & $\begin{array}{l}\text { A. E. Lec } \\
\text { (208)526-9716 } \\
\text { xal@inel.gov }\end{array}$ \\
\hline
\end{tabular}

\begin{tabular}{|c|c|c|c|c|c|}
\hline $\begin{array}{l}\text { WS-10 } \\
2 / 11 / 99\end{array}$ & \multicolumn{5}{|c|}{$\begin{array}{l}\text { Operation of the calciner in the anticipated time frame (2013-2017) will require that the NWCF be modified to comply with Maximum Achicvable Control Technology (MACT) } \\
\text { requirements. }\end{array}$} \\
\hline Basis & $\begin{array}{l}\text { Recent informal discussions between LMITCO management and } \\
\text { EPA Region X and the Idaho Department of Environmental Quality } \\
\text { suggest that the ICPP calciner will be regulated as a hazardous waste } \\
\text { incinerator in the future. EPA rulemaking is currently underway to } \\
\text { impose MACT requirements on all such facilities within five years } \\
\text { (Reference 1). }\end{array}$ & $\begin{array}{l}\text { (1) Federal Register, } \Lambda \text { pril } 19,1996 \\
\text { Article Number } 96-8 \text {. }\end{array}$ & $\begin{array}{l}\text { This refers to EIS } \\
\text { volume numbers and } \\
\text { will be completed by } \\
\text { others }\end{array}$ & $\begin{array}{l}\text { This refers to the EIS } \\
\text { alternatives and will be } \\
\text { completed by others. }\end{array}$ & $\begin{array}{l}\text { A. E. Lee } \\
\text { (208)526-9716 } \\
\text { xal@inel.gov }\end{array}$ \\
\hline
\end{tabular}

\begin{tabular}{|c|c|c|c|c|}
\hline WS-11 & \multicolumn{4}{|c|}{ All HLW will be ready for movement out of the State of ldaho on or before 12/31/35. } \\
\hline Basis & $\begin{array}{l}\text { This is a requirement of the } 1995 \text { Batt } \Lambda \text { greement between the State } \\
\text { of Idalio and the U.S. Department of Energy. }\end{array}$ & $\begin{array}{l}\text { This refers to EIS } \\
\text { volume numbers and } \\
\text { will be completed by } \\
\text { others }\end{array}$ & $\begin{array}{l}\text { This refers to the EIS } \\
\text { alternatives and wili be } \\
\text { completed by others. }\end{array}$ & $\begin{array}{l}\text { A. E. Lec } \\
\text { (208)526-9716 } \\
\text { xa!@inel.gov }\end{array}$ \\
\hline
\end{tabular}

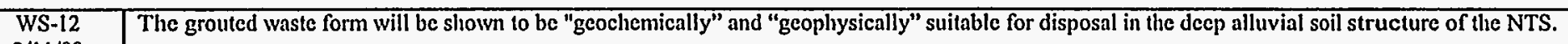

$2 / 11 / 99$

AEL 


\section{FEASIBILITY STUDY(FS) ASSUMPTIONS}

Basis $\quad$ Geochenical stability of the waste form is supported by data that have been published (Reference 1). These data suggest that zeolites are formed during grouting of calcinc simulants, using the proposed CWO grouting process. Zeolites are natural "getters" that are often used in ion exchange processes for extracting radionuclides from aqueous solutions. Moreover, zeolitic minerals formed by the CWO grouting process appear chemically similar to the zcolitized tuff

which is claracteristic of the alluvial soils at the N'TS (Reference 2). The process by which they are formed is similar to the natural zeolite formation process under alkaline soil conditions (Reference 3). The chemical similarity of the waste to the native soils at the NTS implies a paucity of thermodynamic driving forces for geochemical breakdown of the waste after disposal in the soil.

Geophysical stability of the waste is implied by barriers to radionuclide transport to the biosphere which exist in the NTS. Reference 3 discusses a number of these (e.g., highly sorptive soils, low vadose zone water flows, ongoing crustal extension [deposition] large distance to groundwater. etc.). The suitability of the NTS GCDF for disposal of radwaste was also addressed directly in a preliminary assessment performed by Sandia (Reference 4). The latter work concluded that the NTS GCD facility is likely to meet the requirements of 40 CFR 191 as a disposal site for TRU waste, high level waste, and spent fuel.

A seven-year testing program was conducled at the NTS GCDF to study diffusion rates of heat and radionuclides (including tritium) from an actual "hot" disposal hole (Reference 5). The program included tracer measurements of mass diffusion rates, and short- and long-term risk assessments (LTRAs). The only scenario in the LTRA which gave appreciable dose was worst case inundation where the disposal zone becomes saturated and a drinking water well is placed on the sitc boundary.

\section{(1) Journal of the American Ceramic} Suciely, vol 80 no 9, pp. 2449-2453, 1997.

(2) I. J. Winograd [USGS], "Radioactive Waste Disposal in Thick Unsalurated Zones", Science, vol 212 no 4502,26 Junc 1981, pp. 1457-1464.

(3) R. C. Surdam, R. A. Sheppard, "Zcolites in Saline, Alkaline Lake Deposits", pp. 145-174 of book "Natural Zcolites", from Zeolite 76, International Conference on the Occurence, Properties, and Utilization of Natural Zcolites, L. B. Sand \& F. A. Mumpton, editors, Tucson, AZ, June 1976, Pernagon Press.

(4) "Preliminary Performance Assessment of the Greater Confinement Disposal Facility at the Nevada 'lest Site", L.L. Price, et al, SAND-91-0047.

(5) "Greater Confinement Disposal Test at the Nevada Test Site, Final Teclinology Report", P. T. Dickman, SAIC, UNO2608, January 1989.

\section{This refers to EIS} volume numbers and will be completed by others
This refers to the EIS altematives and will be completed by others.
A. E. L.ce (208)526-9716 xal@incl.gov

Hydroceranic grout recipes will be developed before commencement of waste processing. $\lambda$ sufficient number of recipes will be developed to accommodate the expected range of blended compositions which will result from blending of alumina, zirconia/fluorinel/blend, and high-sodium calcines with liquid SBW. These recipes are assumed to be sufficiently robust to accommodate the maximum expected variation in calcine composition.

This refers to EIS
volume numbers and
will be completed by
others

This refers to the EIS alternatives and will be completed by others.

A. E. Lee

(208)526-9716 xal@inel.gov

\begin{tabular}{l|l} 
SA-2 & A pumping system for radioactive solids slurries will be designed and demonstrated which is not prohibitivcly expensive to build, install, operate, and naintain..
\end{tabular} 
FEASIBILITY STUDY(FS) ASSUMPTIONS

\begin{tabular}{|c|c|c|c|c|}
\hline Basis & $\begin{array}{l}\text { This assumption is based on conversations with vendors of sludge \& } \\
\text { cement pumping equipment, and with teclnical personnel at the } \\
\text { Energy \& Environmental Research Center of the University of North } \\
\text { Dakota who have designed and operated slurry systems for granular } \\
\text { fucls. Successful systems are in existence for non-radioactive } \\
\text { slurrics. Though there are potential problems in a radioactive } \\
\text { environment which are not present in "cold" systems, the above } \\
\text { assumption was made on the strength of the mentioned } \\
\text { conversalions, and in the absence of experience to the contrary. }\end{array}$ & $\begin{array}{l}\text { This refers to EIS } \\
\text { volume numbers and } \\
\text { will be completed by } \\
\text { otliers }\end{array}$ & $\begin{array}{l}\text { This refers to the EIS } \\
\text { alternatives and will be } \\
\text { completed by others. }\end{array}$ & $\begin{array}{l}\text { A. E. Lee } \\
\text { (208)526-9716 } \\
\text { xal@inel.gov }\end{array}$ \\
\hline
\end{tabular}

\begin{tabular}{|c|c|c|c|c|}
\hline $\mathrm{S} \Lambda-3$ & \multicolumn{4}{|c|}{ Safely issues of sugar denitration/recalcination can be mitigated to the acceptance of DOE. } \\
\hline Basis & $\begin{array}{l}\text { This assumption was necessary in order for sugar to be considered as } \\
\text { a reducing agent in the calciner. Sensitivity testing of process } \\
\text { residues from simulated sugar calcination during } 1996 \text { at ICPP failed } \\
\text { to show any sign whatsoever of reactivity of these residues, and thus } \\
\text { provide technical support for the above assumption. }\end{array}$ & $\begin{array}{l}\text { This refers to EIS } \\
\text { volume numbers and } \\
\text { will be completed by } \\
\text { others }\end{array}$ & $\begin{array}{l}\text { This refers to the EIS } \\
\text { altematives and will be } \\
\text { completed by others. }\end{array}$ & $\begin{array}{l}\text { A. E. Lec } \\
\text { (208)526-9716 } \\
\text { xal@incl.gov }\end{array}$ \\
\hline
\end{tabular}

\begin{tabular}{|c|c|c|c|c|c|}
\hline $\begin{array}{c}S \Lambda-4 \\
2 / 11 / 99\end{array}$ & \multicolumn{5}{|c|}{ The pressurized recalcine delivery system from the modified NWCF calciner to the grout facility will be acceptable to DOE. } \\
\hline Basis & $\begin{array}{l}\text { The assumption was made because a vacuum delivery system would } \\
\text { require scveral "booster" pumping stations because of the required } \\
\text { transport distance for the calcine. The cost of such a system would } \\
\text { be substantially higher than that of a pressurized system. In addition, } \\
\text { it was reasoned that since most (if not all) past transport system line } \\
\text { failures at ICPP have occurred at cyclones or bends in the transport } \\
\text { lines, design features (e.g., wear plates) at these "pressure points" } \\
\text { could be incorporated to accommodate erosion. Moreover, any } \\
\text { transport line (vacuum or pressurized) will be housed within a } \\
\text { secondary containment line. By maintaining the secondary line } \\
\text { under vacuum, and by monitoring the air in the line for } \\
\text { radioactivity, any breach in the primary line could be controlled and } \\
\text { contained without external contamination. }\end{array}$ & 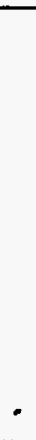 & $\begin{array}{l}\text { This refers to EIS } \\
\text { volume numbers and } \\
\text { will be completed by } \\
\text { others }\end{array}$ & $\begin{array}{l}\text { This refers to the EIS } \\
\text { alternatives and will be } \\
\text { completed by others. }\end{array}$ & $\begin{array}{l}\text { A. E. Lee } \\
\text { (208)526-9716 } \\
\text { xal@inel.gov }\end{array}$ \\
\hline
\end{tabular}


Appendix B

Process Flow Diagrams 


\section{Appendix B}

\section{Process Flow Diagrams}

HLW Study: Non-separations Alternative Cementitious Waste Option

CWO-00

CWO-01

CWO-02

CWO-03

CWO-04

CWO-05

CW0-06

CWO-07

CW0-08

CW0-09

CWO-10 


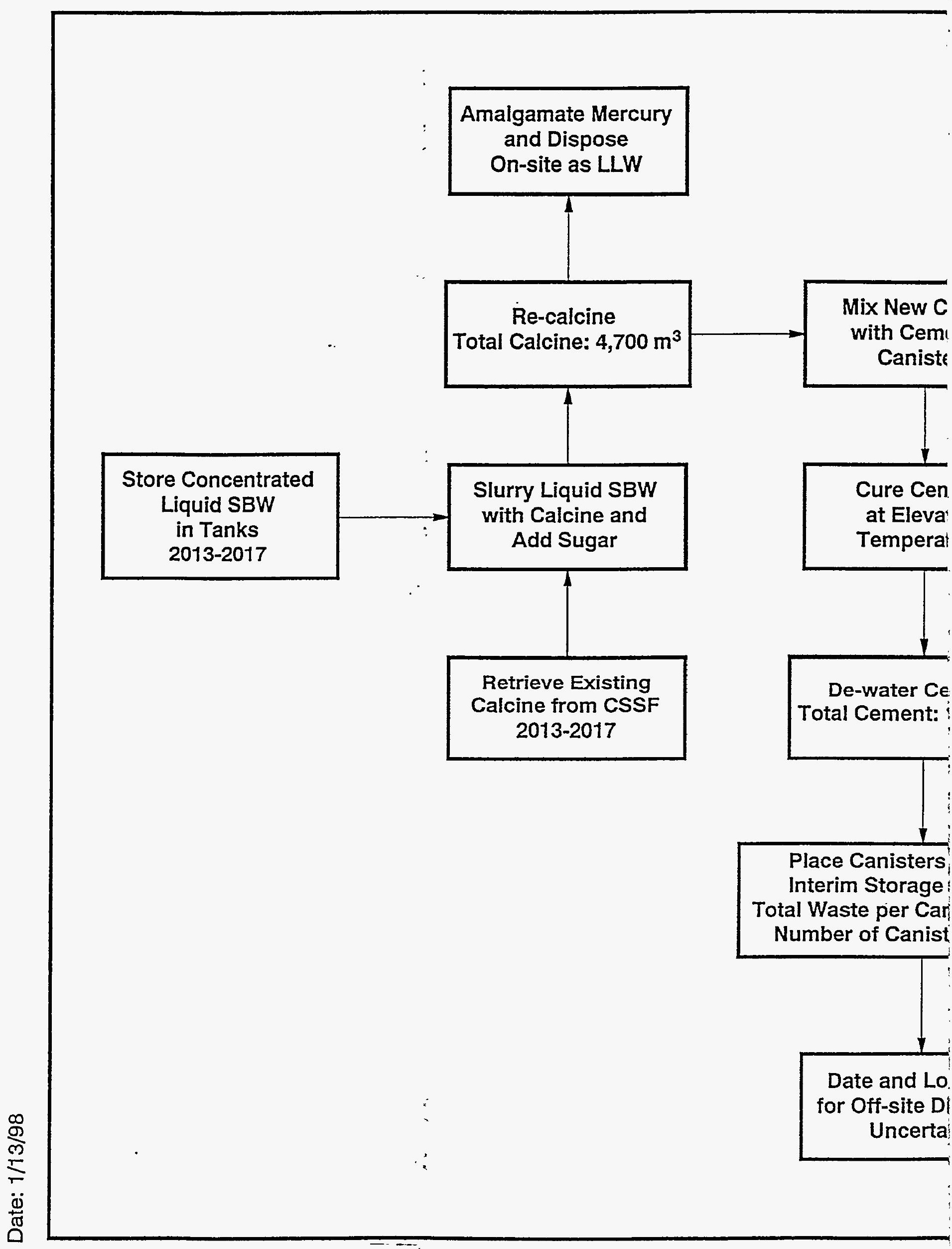




\section{Assumptions}

- The waste forms will meet the LDRs for all RCRAhazardous wastes, and all listed wastes will be de-listed

- Calciner will be modified to accept a slurry-feed

- Cement will be mixed with the new calcine as it is generated

- The cementitious waste form will be ready to be moved out of ldaho for disposal by a target date of 2035

- This waste form will equal or exceed the BDAT to meet waste acceptance criteria

- State of Idaho will allow on-site disposal of LLW

- State of Idano will allow liquid SBW to be treated after 2012 


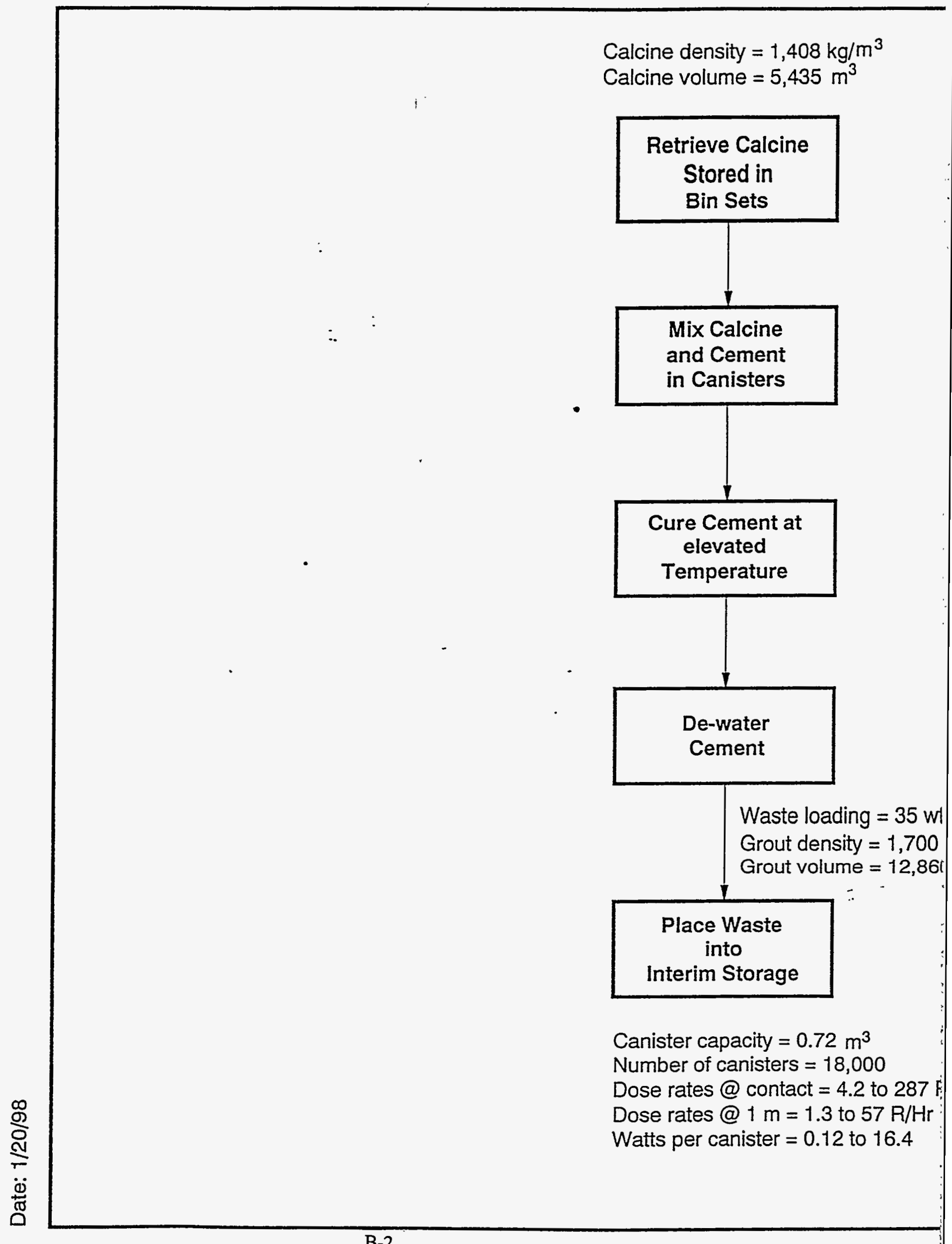




\section{Assumptions}

- All listed hazardous materials will be treated and de-listed

- Non-listed hazardous materials will be treated to meet acceptance criteria

- All waste will be calcined before grouting

- This option will not process new waste generated after 2012

- All direct-grouting processes will occur from 2013 through 2032

- The cement waste form will be ready to be moved out of Idaho for disposal by a target date of 2035

- This waste form will equal or exceed the BDAT 


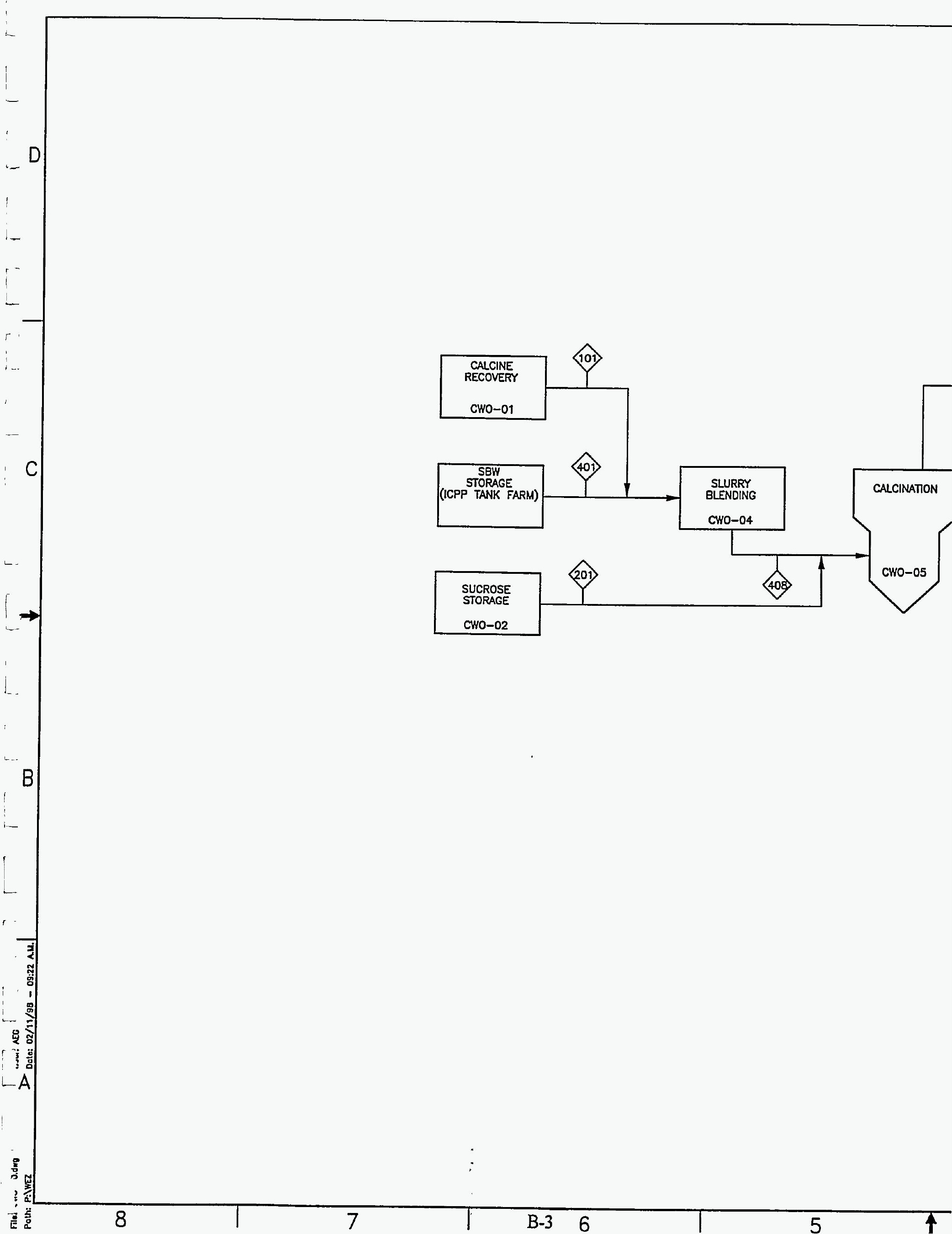



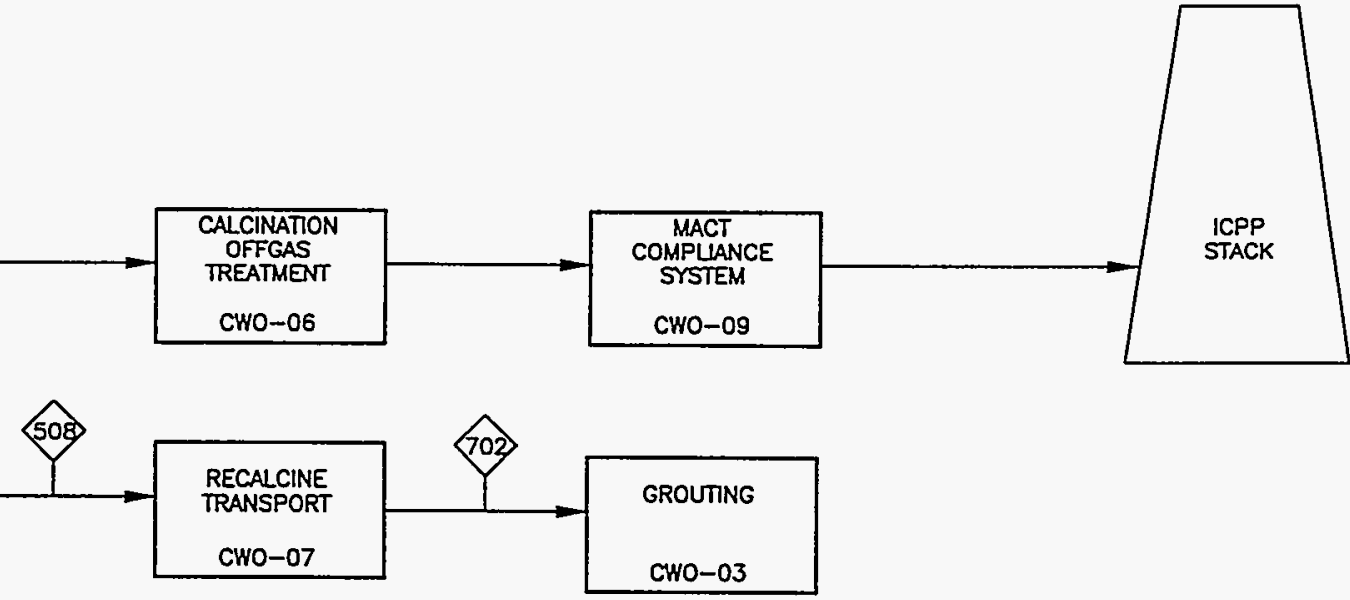

\begin{tabular}{|c|c|c|c|}
\hline Subcormuet Ma. & \multicolumn{3}{|c|}{ LOCKHEEDMATSIN/ } \\
\hline FouESTIF & \multirow{4}{*}{\multicolumn{3}{|c|}{$\begin{array}{c}\text { ICPP } \\
\text { WASTE TREATMENT FACIUTIES } \\
\text { CEMENTITIOUS WASTE OPTION } \\
\text { BLOCK/PROCESS FLOW DIAGRAM }\end{array}$}} \\
\hline bescet: & & & \\
\hline \multirow{3}{*}{\multicolumn{4}{|c|}{$\begin{array}{l}\text { CEMENTTITIOUS WASTE OPTION } \\
\text { BLOCK/PROCESS FLOW DIAGRAM }\end{array}$}} \\
\hline & & & \\
\hline & & & \\
\hline 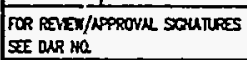 & 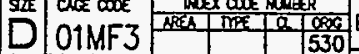 & DHG- & Ry \\
\hline ERECTKE GATE: & SCNE NONE & se्t CW & 00 \\
\hline
\end{tabular}




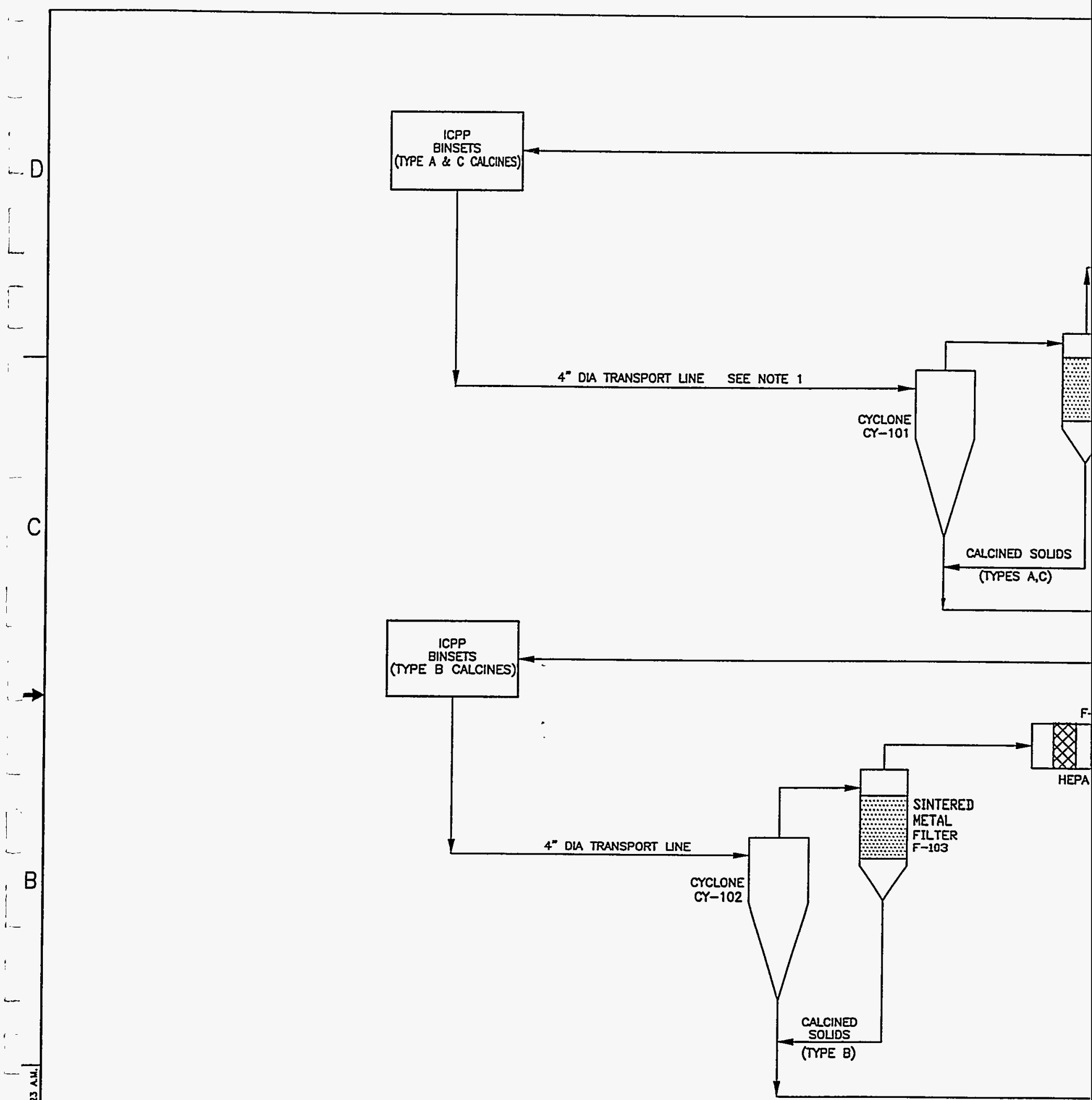




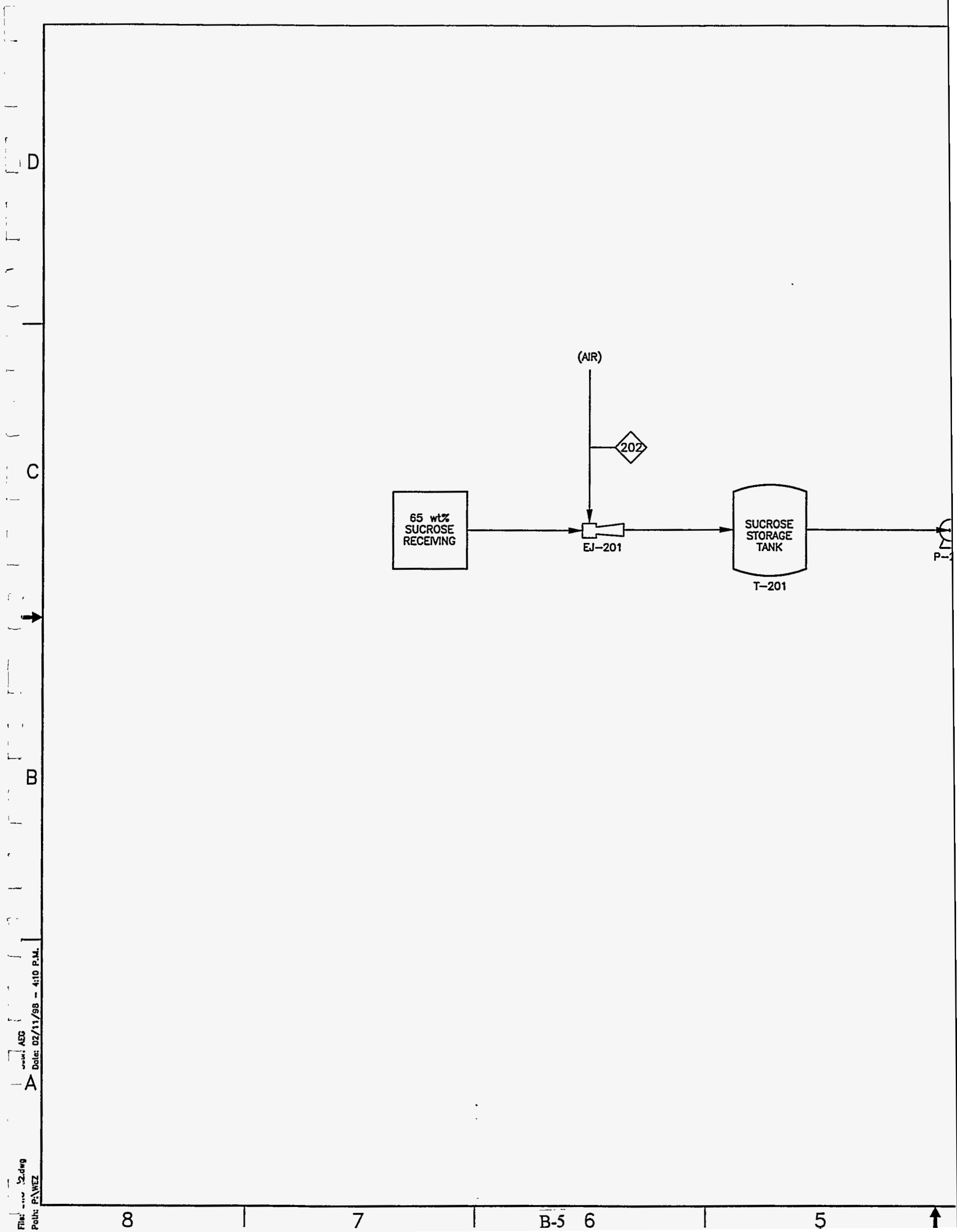


CALCINATON VES-NCC-105

CWO-05

\begin{tabular}{|c|c|c|c|}
\hline Sugcormust ma. & \multicolumn{3}{|c|}{ LOCKHEED MARTIN/ } \\
\hline Fraesta: & \multirow{5}{*}{\multicolumn{2}{|c|}{$\begin{array}{l}\text { ICPP } \\
\text { WASTE TREATMENT FACILTIES } \\
\text { CEMENTIIOUS WASTE OPTION } \\
\text { SUCROSE STORAGE }\end{array}$}} & \\
\hline Descest & & & \\
\hline \multirow{2}{*}{\begin{tabular}{|l|l|} 
DRAwt: \\
Prazct Me \\
\end{tabular}} & & & \\
\hline & & & \\
\hline $\operatorname{sptc} \operatorname{cose}$ & & & \\
\hline 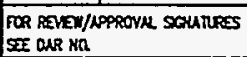 & \begin{tabular}{|l|l|} 
san & Che cook \\
$D$ & $01 \mathrm{MF} 3$ \\
\end{tabular} & 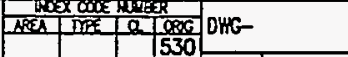 & Ry \\
\hline
\end{tabular}


LSED BAGHOUSE

FILTER

$F-301$

1.GROUTING PROCESS IS IDENTICAL

TO THAT DESCRIBED IN "DIRECT

CEMENTIIOUS OPTION

(NOWO-02

(NOTE 1)

\begin{tabular}{|c|c|c|c|}
\hline suscaninet no. & \multicolumn{3}{|c|}{ LOCKHEED MARTIN/ } \\
\hline Fearster: & \multirow{5}{*}{\multicolumn{3}{|c|}{$\begin{array}{c}\text { ICPP } \\
\text { WASTE TREATMENT FACIUTES } \\
\text { CEMENTITOUS WASTE OPTION } \\
\text { GROUTING }\end{array}$}} \\
\hline Cescat & & & \\
\hline Dramt: & & & \\
\hline Priacet Na & & & \\
\hline PSEC 000 & & & \\
\hline 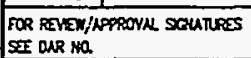 & 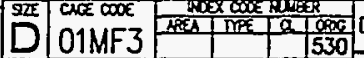 & DHG & $\mathrm{KaV}$ \\
\hline
\end{tabular}




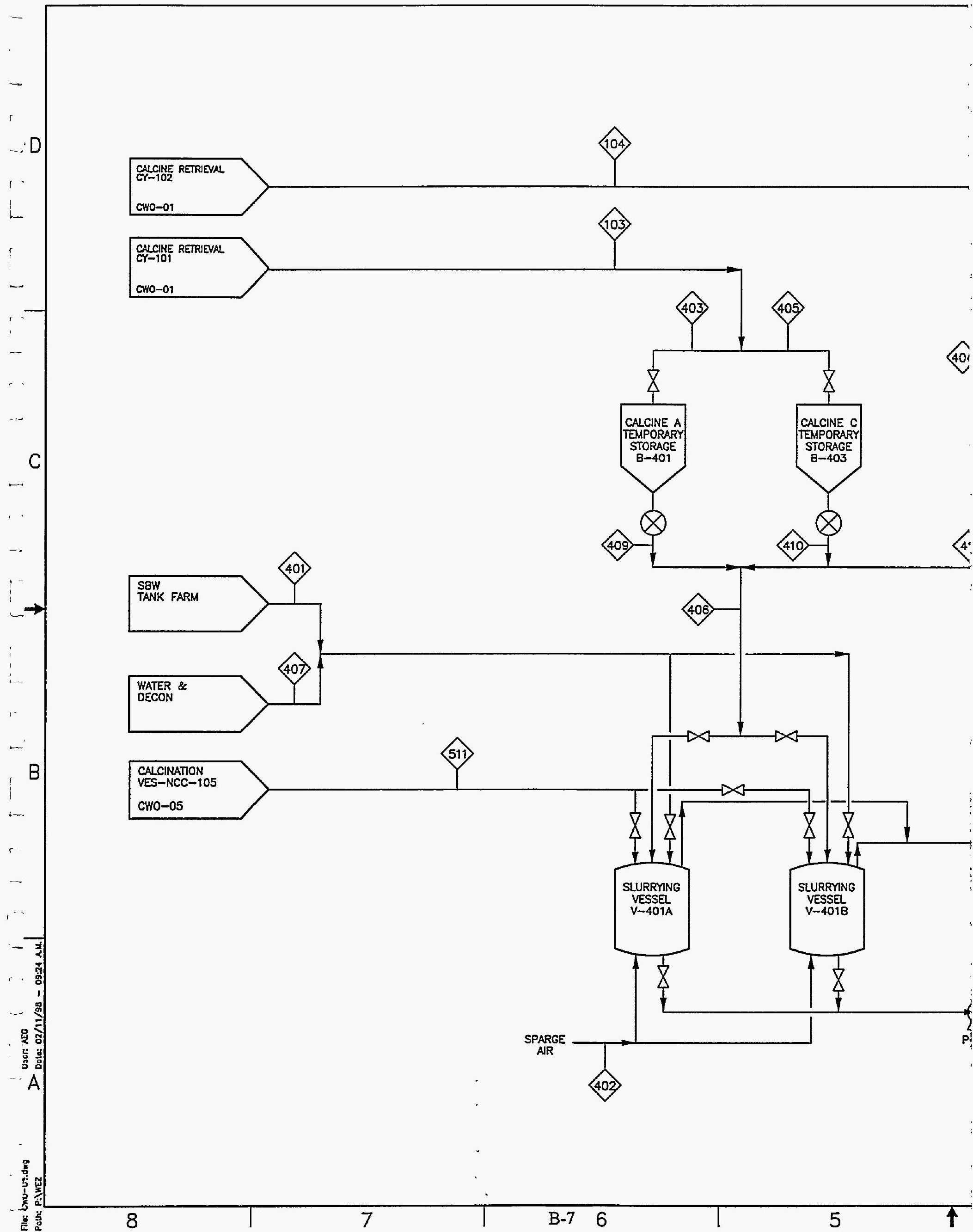




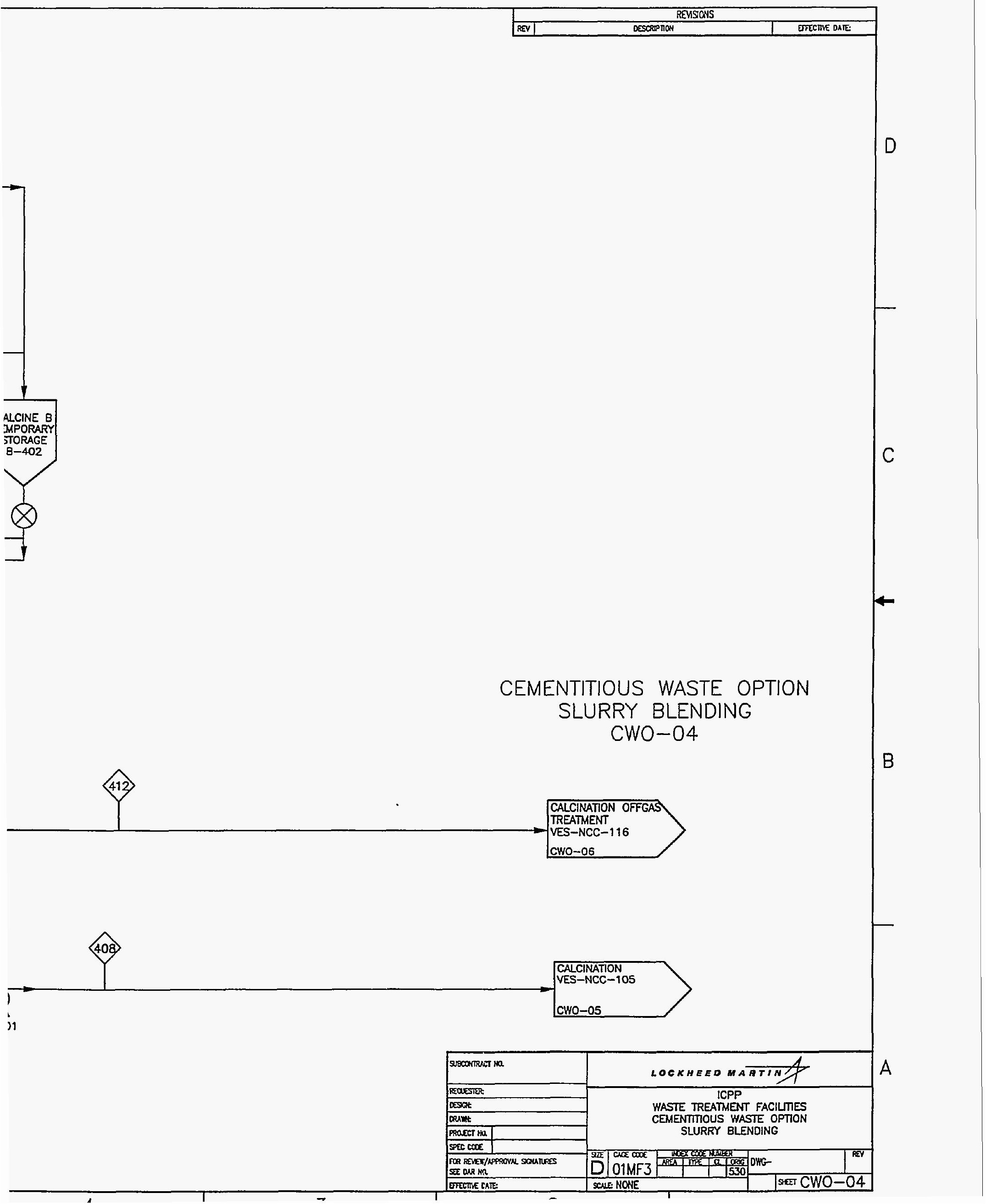




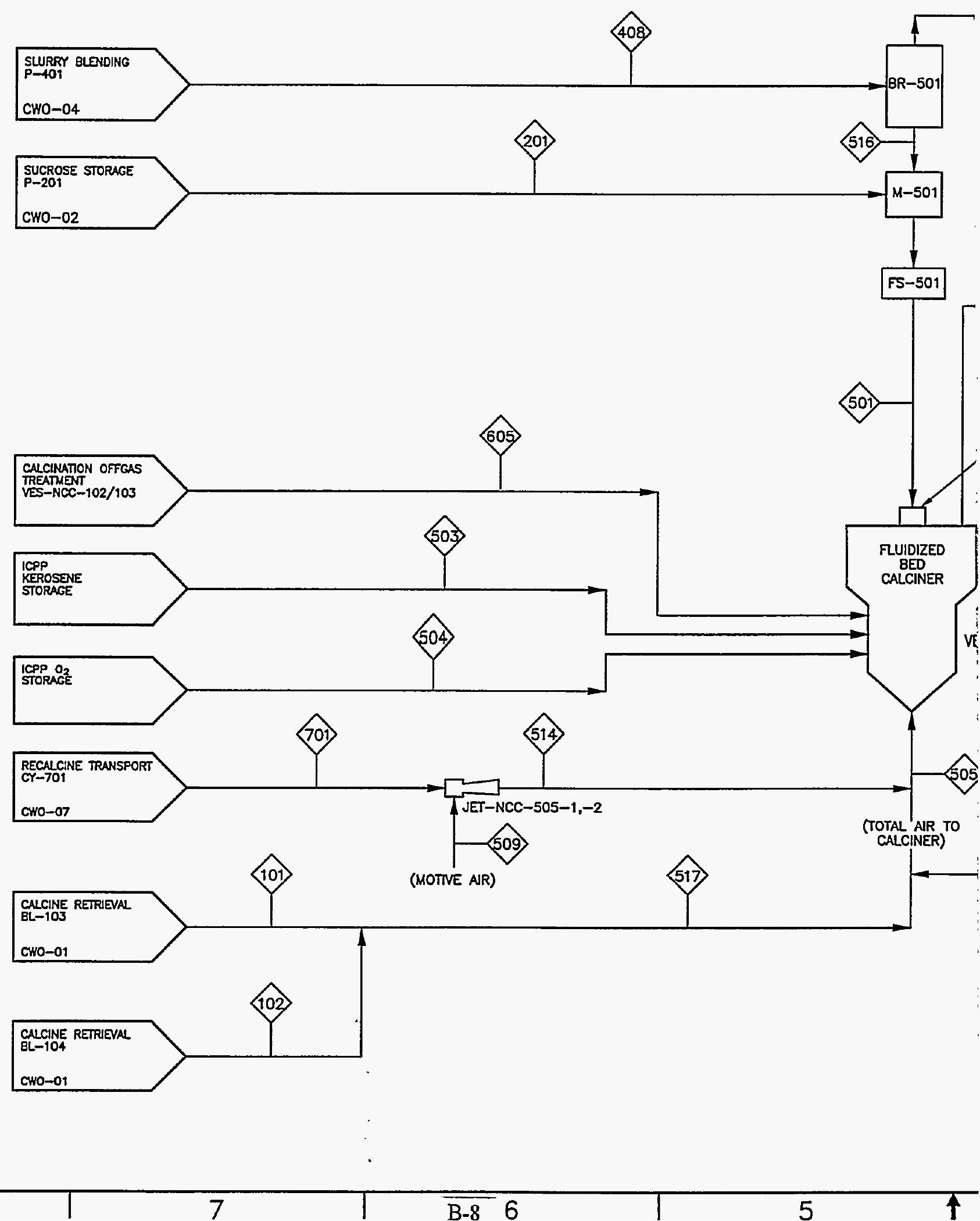



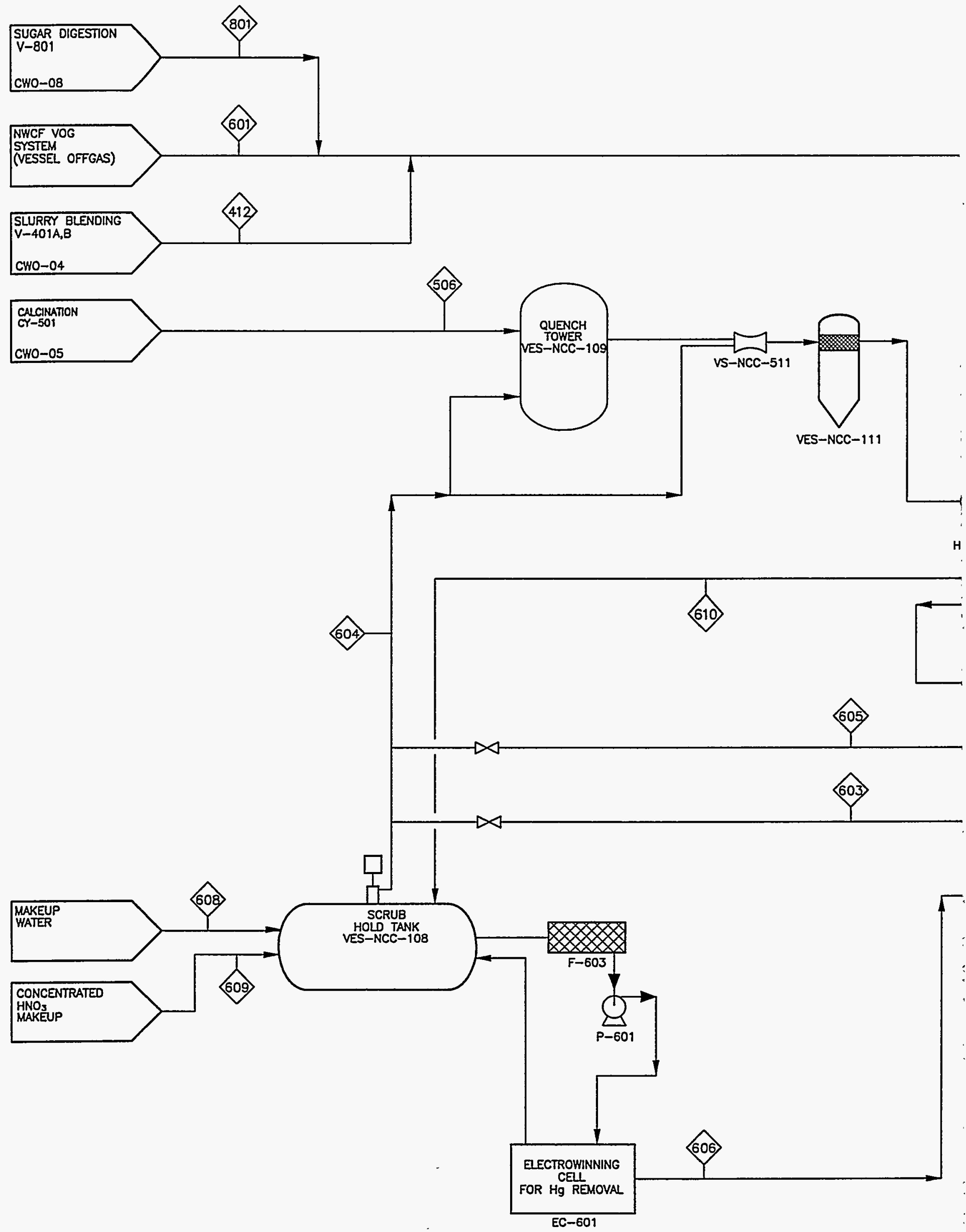


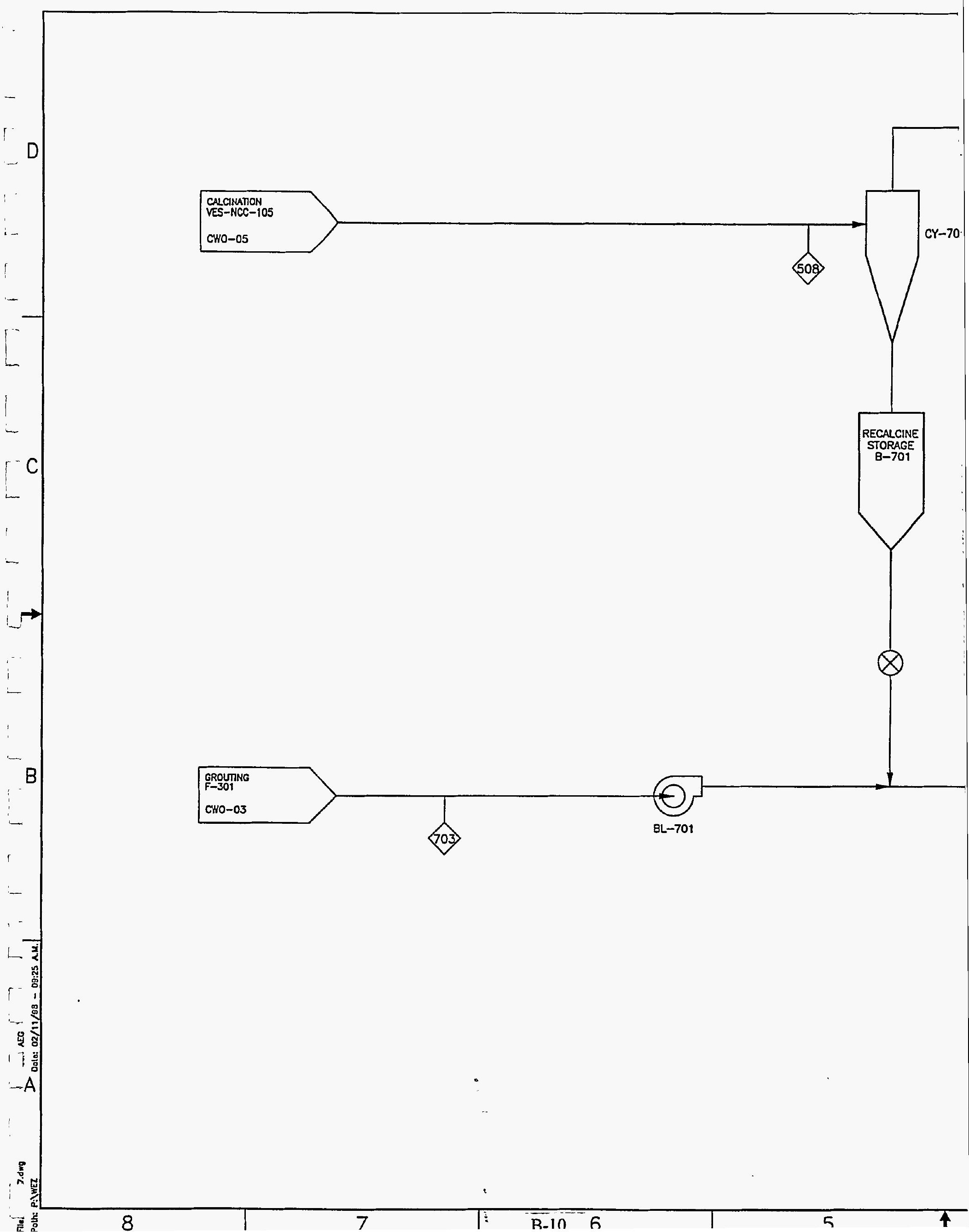




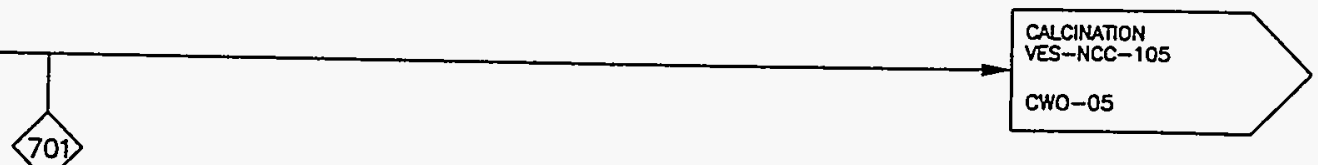

\begin{tabular}{|c|c|c|}
\hline suecormist на & \multicolumn{2}{|l|}{ LOCKHEED MATTIN/A } \\
\hline REQESTR: & \multirow{4}{*}{$\begin{array}{l}\text { ICPP } \\
\text { WASTE TREATMENT FACILIIES } \\
\text { CEMENTIIOUS WASTE OPTION } \\
\text { RECALCINE TRANSPORT }\end{array}$} & \\
\hline DRAW: & & \\
\hline PraECT No. & & \\
\hline $\sec c \cos$ & & \\
\hline 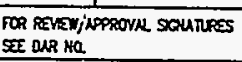 & 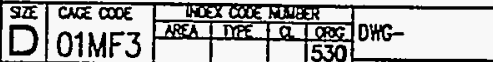 & Fis \\
\hline
\end{tabular}




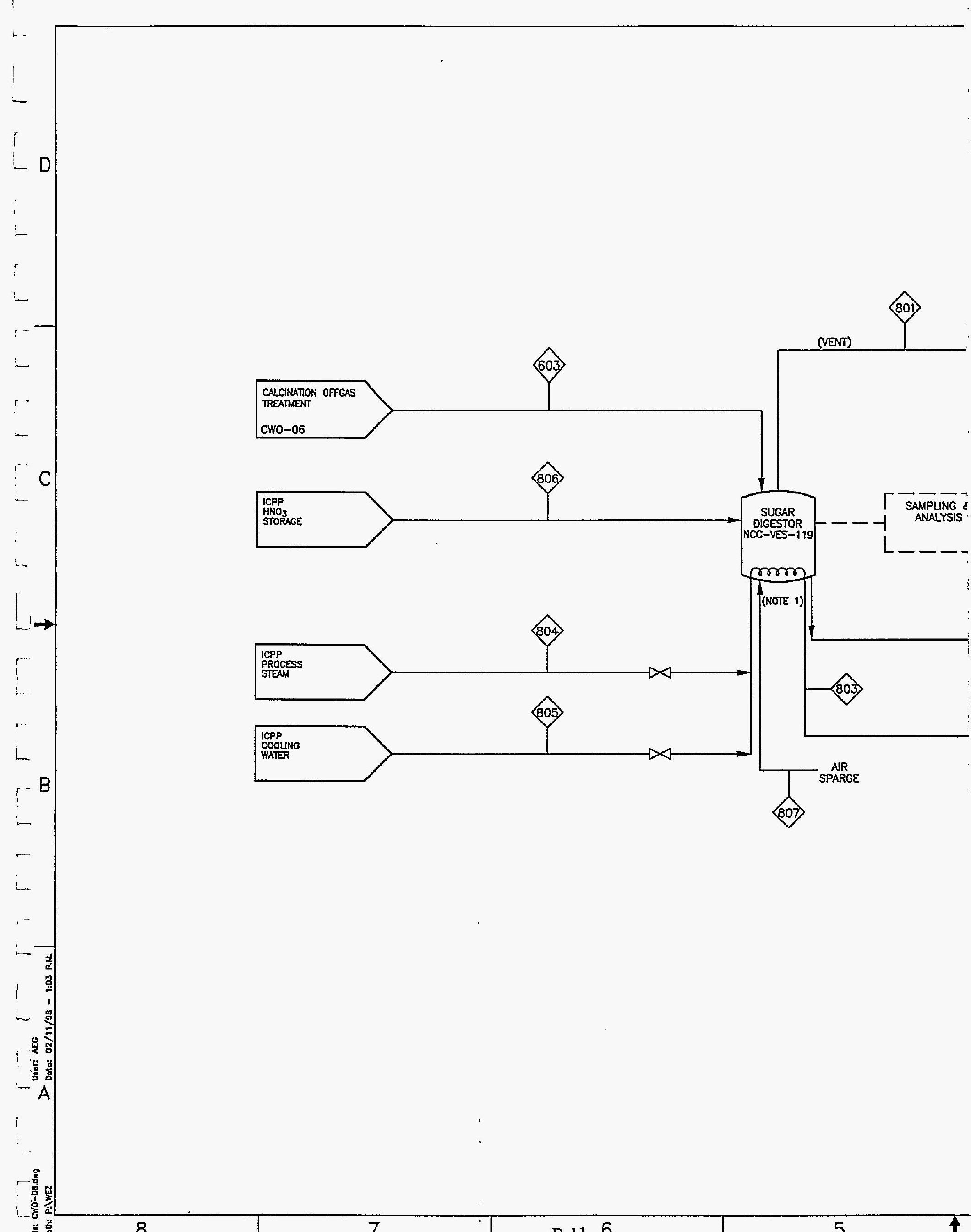




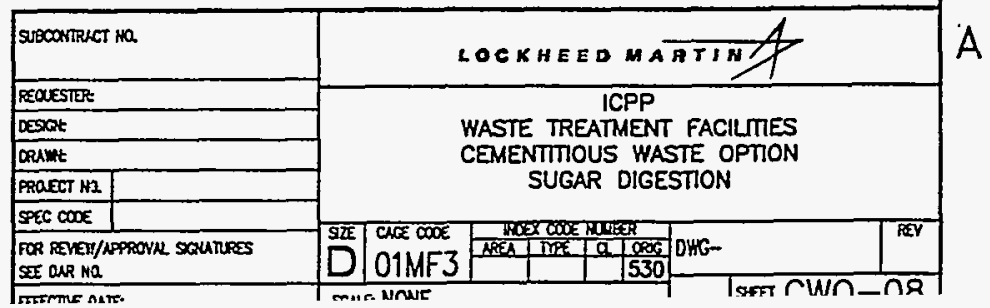




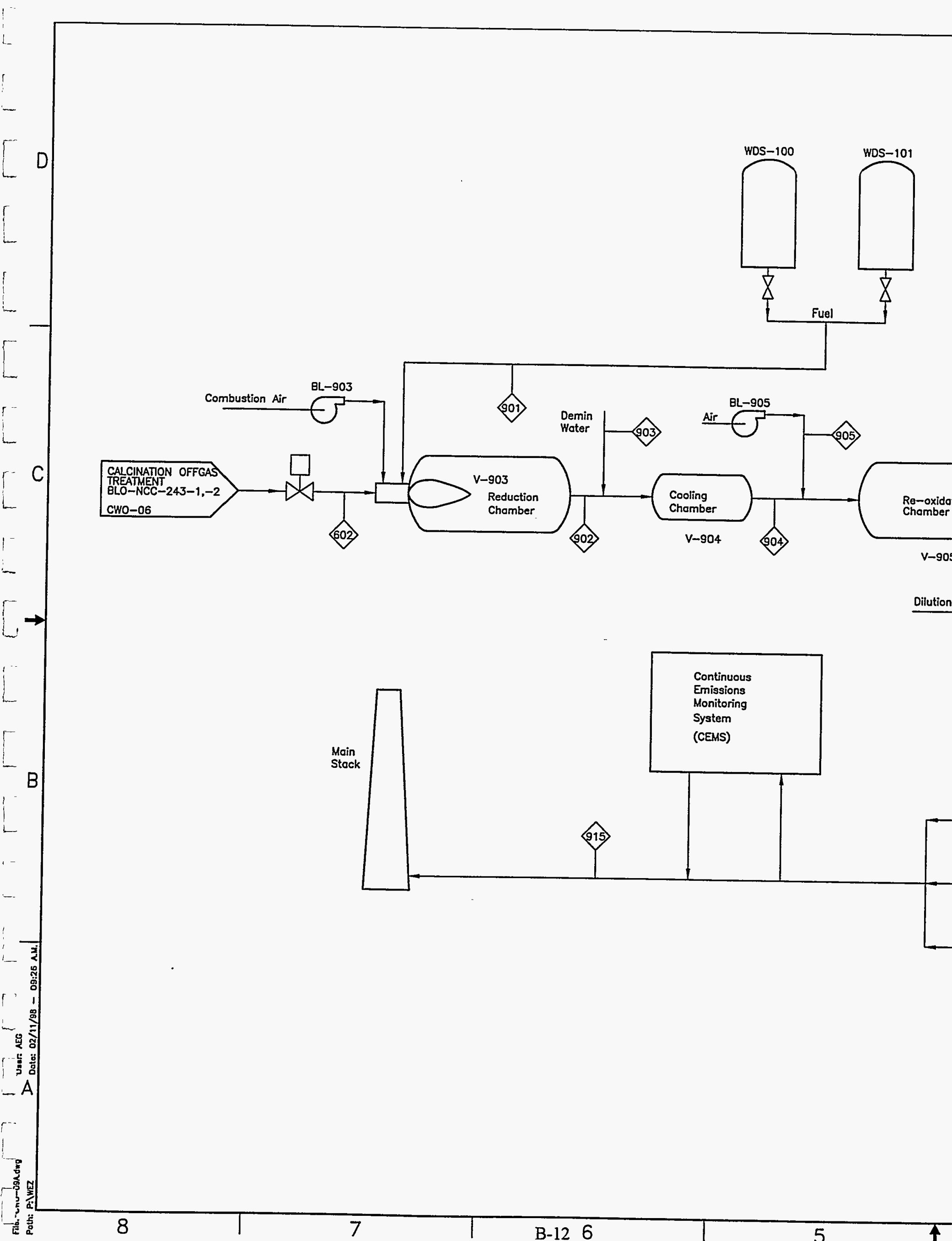




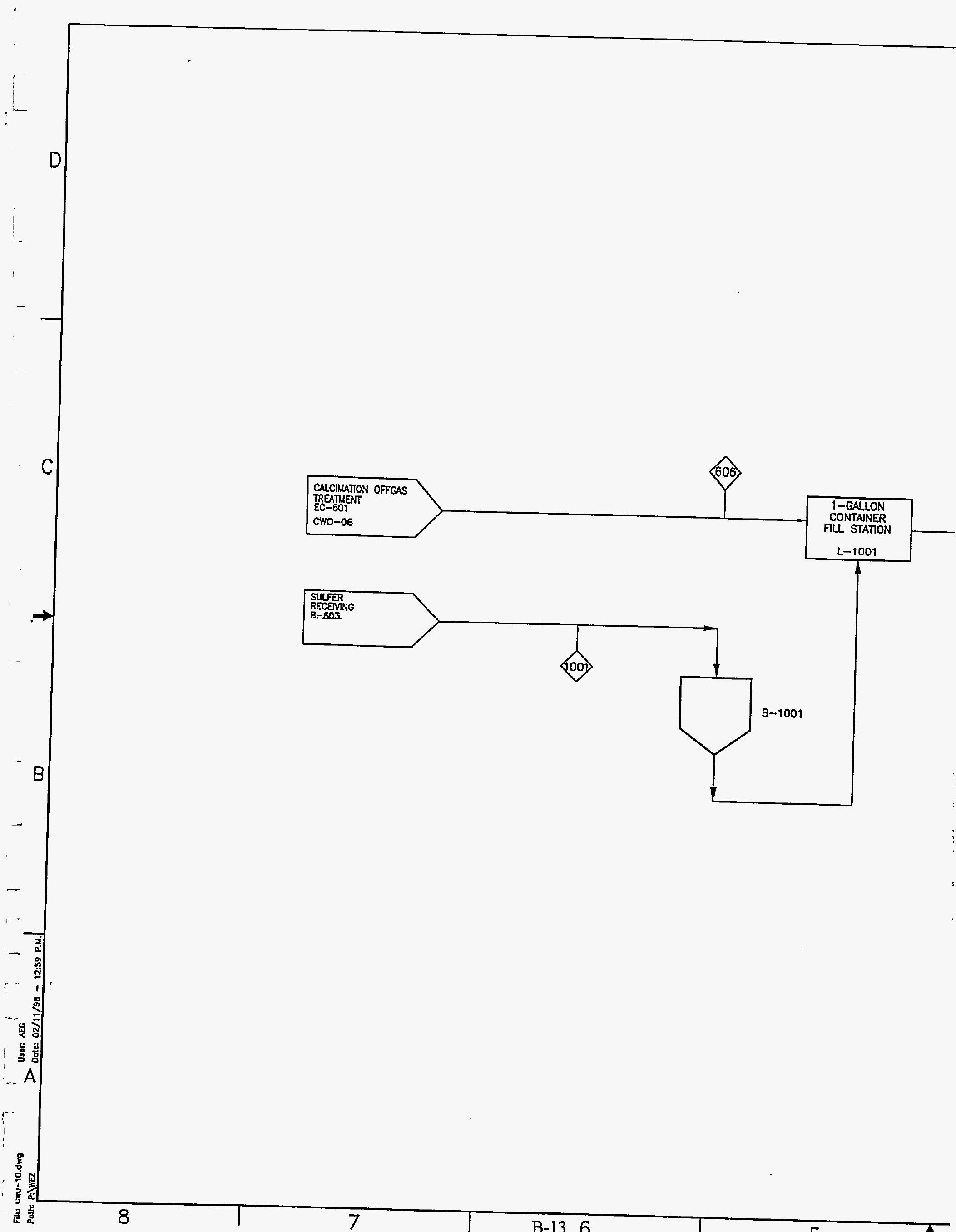




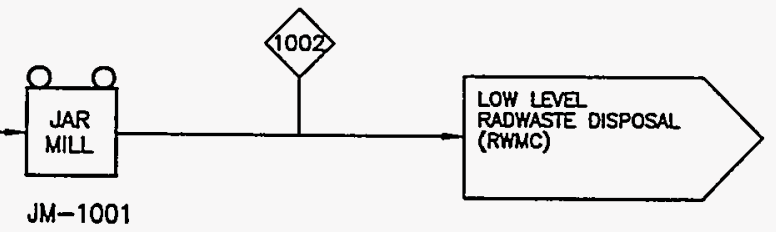

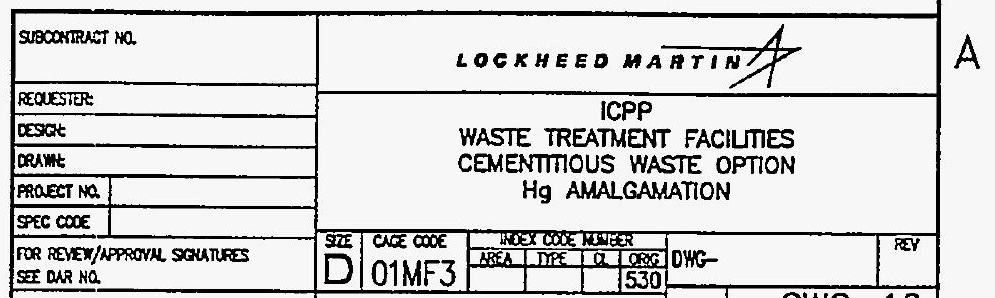




\section{Appendix C}

Cost Estimates 


\section{Appendix C}

\section{Cost Estimates}

1. Grout Facility - 5 year

2. LCC Grout Facility - 5 Year

3. Recalciner -5 year

4. LCC Recalciner - 5 year

5. MACT -5 year

6. LCCMACT - 5 year 


\section{cooxures mantring}

Lockheed Martin Idaho Technologies Company INTERDEPARTMENTAL COMMUNICATION

Date: February 4, 1998

To: $\quad$ A E Eee:

MS 3765

$6-9716$

From:

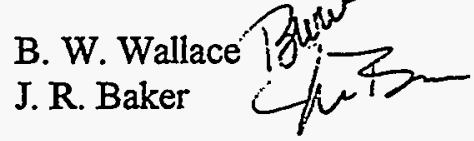

MS 4143

$6-7868$

MS 4143

$6-7140$

Subject: RECALCINATION TREATMENT NON-SEPARATIONS - CEMENTITIOUS WASTE OPTION - BWW-04-98 \& JRB-02-98

Reference: HLW EIS Waste Treatment Study Cost Estimate - Dated 11/25/97 - File Number 2414-1.

Process Sodium Bearing Waste MACT Compliance Cost Estimate - Dated 11/10/97

- File Number 2362-A.

NWCF SBW Process Mods-Sugar Option - Dated 8/23/96 - File Number 2324-K2.

As per your request, Cost Estimating has prepared a Planning Cost Estimate for the abovementioned project located at the Idaho Chemical Processing Plant (ICPP).

The Total Estimated Cost (TEC) in un-escalated dollars is as follows:

Conceptual Design, Management, Permits and Turnover

Title Design, Management and Construction

TOTAL ESTIMATED COST
$\$ 76,000,000$

$\$ 166,885,000$

$\$ 242,885,000$

The Total Estimated Cost (TEC) in escalated dollars is as follows:

Conceptual Design, Management, Permits and Turnover Title Design, Management and Construction TOTAL ESTIMATED COST

$\$ 94,100,000$

$\$ 235,900,000$

$\$ 330,000,000$

Please refer to the attached Cost Estimating Summary, Detail, and the Contingency Analysis work sheets for the cost breakdowns. Also included for your use are the Cost Estimate Recapitulation Sheets describing the assumptions and basis used in the preparation of this estimate and a CWO schedule.

If you have any questions or comments, please do not hesitate to contact us (B. W. Wallace at 6-7868 or Lotus Notes ID BCE, or J. R. Baker at 6-7140 or Lotus Notes ID RBJ).

BWW 
February 4, 1998

BWW-04-98

Page 2 of 2

Attachments

cc: R. D. Adams, MS 3655

B. O. Reyes, MS 3655

Estimate File \#2420

J. R. Baker Files

B. W. Wallace Files

D. T. Peterson Files 


\section{COST ESTIMATE SUPPORT DATA RECAPITULATION}

Project Tite: HLW EIS Scop. Cementitious Waste Option
Aate: 2/04/98
Approved By:
SCOPE OF WORK: Brief description of the proposed project.
The elements included in this option are the receipt of calcined waste from the bin sets, blending of
the waste and the incorporation of sucrose solution to form a slurry through the use of three receipt
bins and two slurry tanks, delivery of the slurry to new nozzles in the existing calciner using a
system of positive displacement pumps and piping routed through existing chases, receipt of the re-
calcined waste at a new booster transfer station and the transport of this waste to a new grouting
facility. Additional elements of this system include a new cyclone installed in the existing calciner,
a new Maximum Achievable Control Transfer (MACT) Facility, the installation of a mercury
scrubber in the existing off-gas system and provisions for receiving and handling of bulk sucrose
solution. Also included is a $40^{\prime} x$ x $70^{\prime} x 24$ ' eave height metal building with a 10 ton bridge crane
erected over the new NWCF wing and the extension of several existing systems at the facility to
provide process stem and cooling, Vent Off Gas handling, and decontamination capabilities.

II. BASIS OF THE ESTIMATE: Drawings, Design Report, Engineers Notes and/or other documentation upon which the estimate is originated.

Basis of the estimate is a CEMENTITIOUS WASTE OPTION (CWO) DESIGN BASIS REPORT dated 11/17/97 furnished by LMITCO Chemical Engineer (D. D. Taylor), Discussions with D. D. Taylor, LMITCO Chemical Engineers, (H. S. Forsythe \& D. N. Thompson) and LMITCO Principal Performer/Technical Coordination, (A. E. Lee), and discussions with all of these same individuals except $\mathrm{D}$. $N$. Thompson during a draft review of the cost estimate. Also used as a basis for the estimate was sketches of the required addition the existing New Waste Calcine Facility and an instrumentation list as provided by LMTCO engineers, (B. P. Evans \& T. A. Langenwalter).

III. ASSUMPTIONS: Conditions statements accepted or supposed true without proof of demonstration. An assumption has a direct impact on total estimated cost.

- The scope of the work provided in this estimate is limited to that identified earlier and does not include other associated elements such as the Grouting Facility, existing waste extraction or transfer back to the slurry bins, interim storage, Hip or vitrification processes.

- The existing infrastructure will support in its current configuration and without the need for laternation the building and processes provided for in this estimate. Extension of and connection to this infrastructure is included.

- Existing chases or transfer lines will be available and adequate to use for conveyance of waste to and from the existing calcine process.

- Existing NWCW process controllers will have adequate capacity and capability to control the new required processes. An allowance for connection to and software re-programming has been included.

- While the original scope identified a sucrose digestion system to be incorporated in this estimate, 


\section{COST ESTIMATE SUPPORT DATA RECAPITULATION}

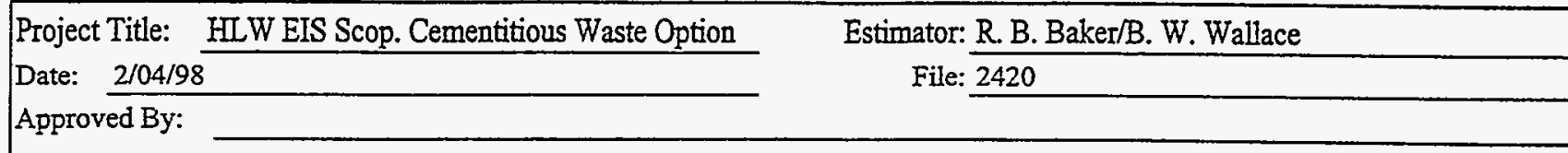

at the direction of D.D. Taylor no such system has been included and the assumption that existing systems in place will be adequate to provide for this process.

- All material which will be in contact with waste is assumed to be 304L Stainless Steel unless an alternate material is specifically identified in the estimate.

- No amalgamation or handling facility/process has been included for the mercury that is expected to be recovered during the re-calcining effort. Allowances for a mercury recovery scrubber have been included.

- The transfer line and transfer booster station included in this estimate is congruent with is expected to be constructed with features similar to other elements in this project.

- The footprint of the building is not expected to exceed 2200 square feet, contain shielding walls greater than $5^{\prime}$ in thickness or be constructed of material other than 4000 PSI standard concrete.

- Supporting infrastructure requirements for equipment not included in this estimate, but which may be contained within the buildings of this estimate have not been provided for. It is expected that these requirements will be provided for in the same estimate they are provided for.

- It is expected that the work will be performed through the use of a Prime Subcontractor and Subtier Subcontractors. Labor and material mark-ups have been included in the amounts of $10 \%$ and $15 \%$ for Profit and Overhead respectively with an additional mark-up of $10 \%$ by the Prime Subcontractor on all Subtier Subcontractors with an overall Bond applied at the rate of $2 \%$.

- Allowances for the rigor of NQA-1 construction practices and requirements have been included and are reflected either in the labor and material for a specific item or as a separate line item in the amount of $30 \%$.

- Development of the estimate has been with the assumption that the stay time allowable within the calciner cell will be for a three hour duration due to the expected ambient temperature.

- That RWMC will accept all demolished materials.

- The cyclone can be removed from the calciner cell in one piece.

- It is expected that the removed cyclone will be sized in the decon area of NWCF and that the remainder of the demolished equipment and materials will be sized in a temporary sizing enclosure.

- Temporary hatch opening "play pen" and mock-up facility costs were based on information received from the HLLW Evaporator Project, these costs were aajusted as needed to represent the requirements of this project.

- The sugar feed lines will route through the valve cubicle. No decon is included in this area. - Reflected in the estimate is an assumed escalation schedule which reflects the midpoint of the design in the year 2009, the midpoint of construction as 2011 and the midpoint of project management as 2012.

- It assumed that the process, as described and used as the basis for this estimate will serve the intended purpose. Contingency has been included to cover the costs of the unknowns as presented, but the contingency included does not provide monies to ensure the design will provide a workable system nor is the contingency included intended to provide an alternate system if the one described proves unsatisfactory.

- It is assumed that the existing systems to be used in conjunction with this process will in fact be available for use in this new process. It is also assumed that these existing systems will have adequate capacity to provide the required service for the new process and that the tie on and 


\section{COST ESTMMATE SUPPORT DATA RECAPITULATION}

\begin{tabular}{|l} 
Project Title: \\
Date: $\frac{\text { HLW EIS Scop. Cementitious Waste Option }}{\text { Approved By: }}$ \\
\hline \\
extension of the systems can be accomplished in a manner not greatly dissimilar to the intentions \\
included in this estimate. \\
- It is assumed that the process steam and cooling piping will be extended from the existing \\
NWCF service and that the lineal footage required for this extension will not be greater than 150 \\
feet. \\
- Additional Vent Off Gas piping required will not be greater than 300 lineal feet; will not require \\
additional HEPA filtering, and will be connected to the existing NWCF Off Gas system.
\end{tabular}

IV. CONTINGENCX GUIDELINE IMPLEMENTATION: The percentage used for contingency as determined by the contingency allowance guidelines can be altered to reflect the type of construction and conditions that may impact the total estimated cost.

The level of contingency included in these estimates is greater than guides would normally indicate for a project at this level. The higher level has been included to address the complexity of the processes and equipment involved, the extreme radiological controls and conditions present and the specialty of the materials required for completion. Also reflected in this contingency level are the uncertainties of the final form and function required for successful execution of the intended procedure.

\section{OTHER COMMENTS/CONCERNS SPECIFIC TO THE ESTIMATE:}

- All FY'98 and later projects are to be assessed a Procurement Fee of 3\%, a G\&A Fee of 23\%, and a Performance Incentive Fee (PIF) of $5.5 \%$. See the attached G\&A/PIF calculation sheet for the method used to calculate these fees. These fees were applied only to material, equipment and subcontract costs. Labor unit rates used in the estimate already contain these fees.

- A procurement fee of one percent of construction was used to cover the operating contractor support to DOE-ID for their contract administration.

- No attempt has been made to determine if the schedule and resource requirements are feasible. There are many phases of this project; many of them constructed concurrently. If this project were attempted with current, known resources, it would definitely overtax available personnel and craft. Whether outside resources could be obtained in sufficient quantities is doubtful. 
1..

$$
\text { C-6 }
$$


Lockheed Martin Idaho Technologies Co.

PROSECT NAME: Recalcination Treatment Non-Separations

Other Project Costs - Escalated

FEOUESTOR: Al Lee 6-9716 MS 3765
COST ESTIMATE SUMMARY

TYPE OF ESTIMATE: Planning PRONECT NO: 2420 PREPARED BY: JRB/BCE

REPORT NAME: Cost Estimate Summary
LOCATION 1: INEEL - ICPP

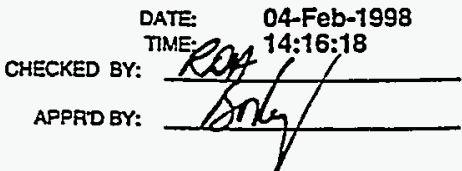

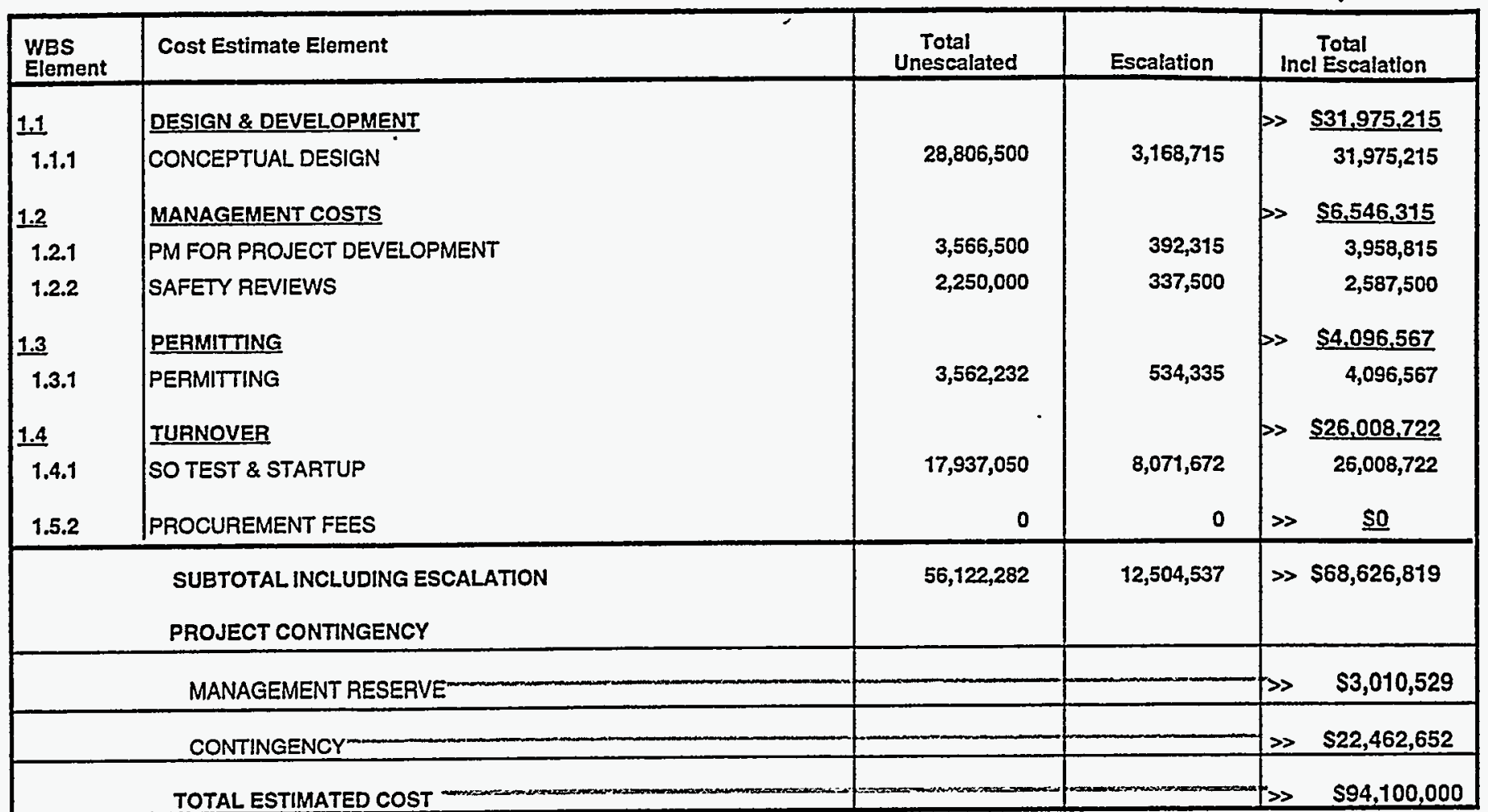

\section{PROJECT COST PARAMETERS}

EDI AS A \% OF CONST. + GFE $=106.00 \%$

CONTINGENCY $=37.12 \%$ 
Lockheed Martin Idaho Technologies Co.

PROAECT NAME: Recalcination Treatment Non-Separations

Other Project Costs - Unescalated

LOCATON 1: INEEL-ICPP

REQUESTOR:

Al Lee 6-9716 MS 3755
COST ESTIMATE SUMMARY

TYPE OF ESTIMATE: $\quad$ Planning
PROJECT NO:
PREPAREO BY:
JRB/BCE
REPOAT NAME: Cost Estimate Summary

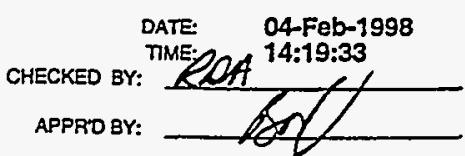

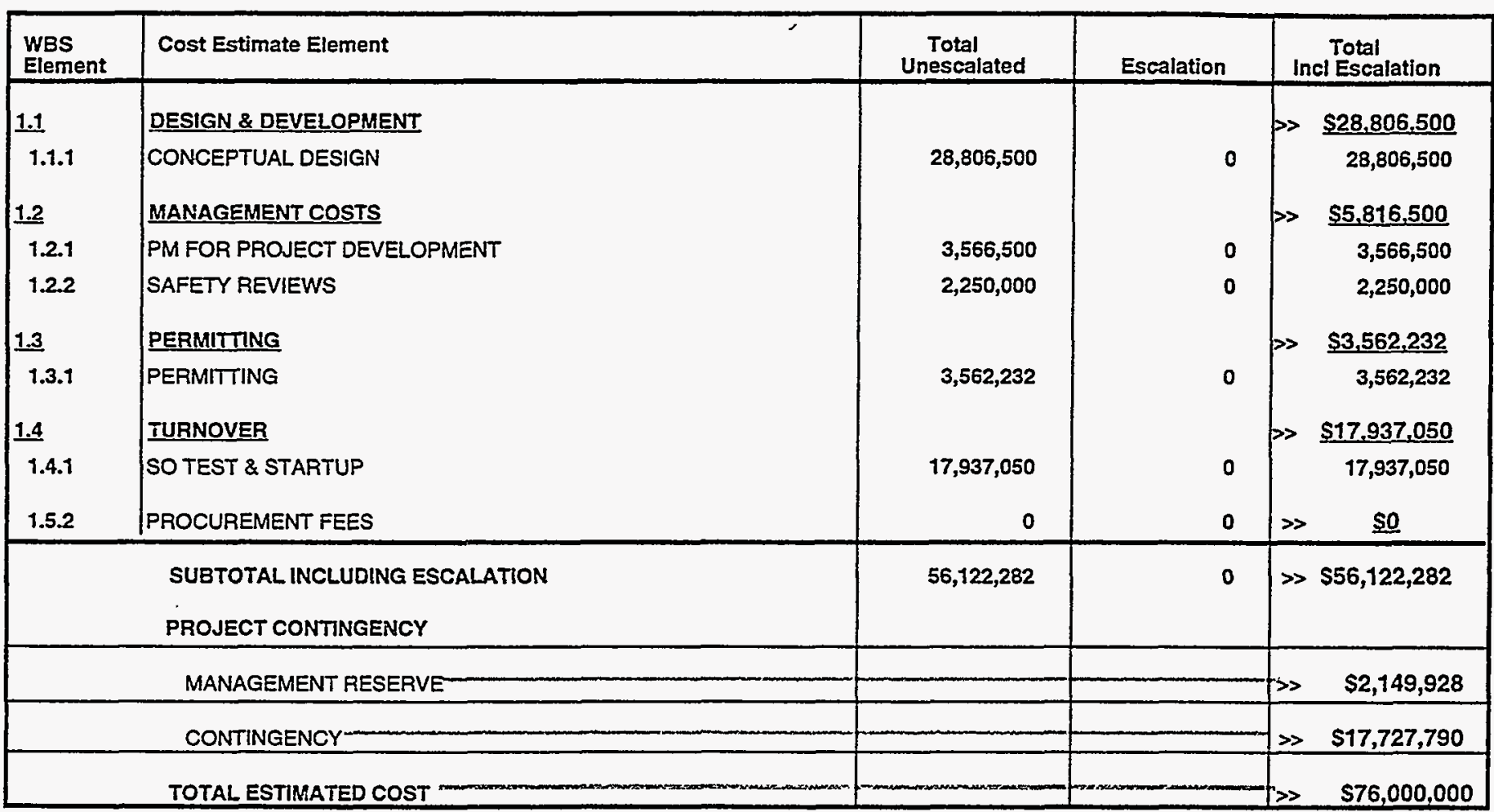

\section{PROJECT COST PARAMETERS}

EDI AS A $\%$ OF CONST. + GFE $=134.00 \%$ 
Lockheed Martin Idaho Technologles Co. Rev. 6/96

PAOJECT NAME: Recalcination Treatment Non-Separations Other Project Costs

INEEL - ICPP

REQUESTOR: Al Lee 6-9716 MS 3765

\section{DETAILED COST ESTIMATE SHEET}

TYPE OF ESTIMATE: Planning

PROJECT NO: 2420

PREPARED BY: JRB/BCE
PAGE 1

DATE 04-Feb-1998

TIME: 14:20:02

REPORT NAME: Detall Cost Estlmate Sheet

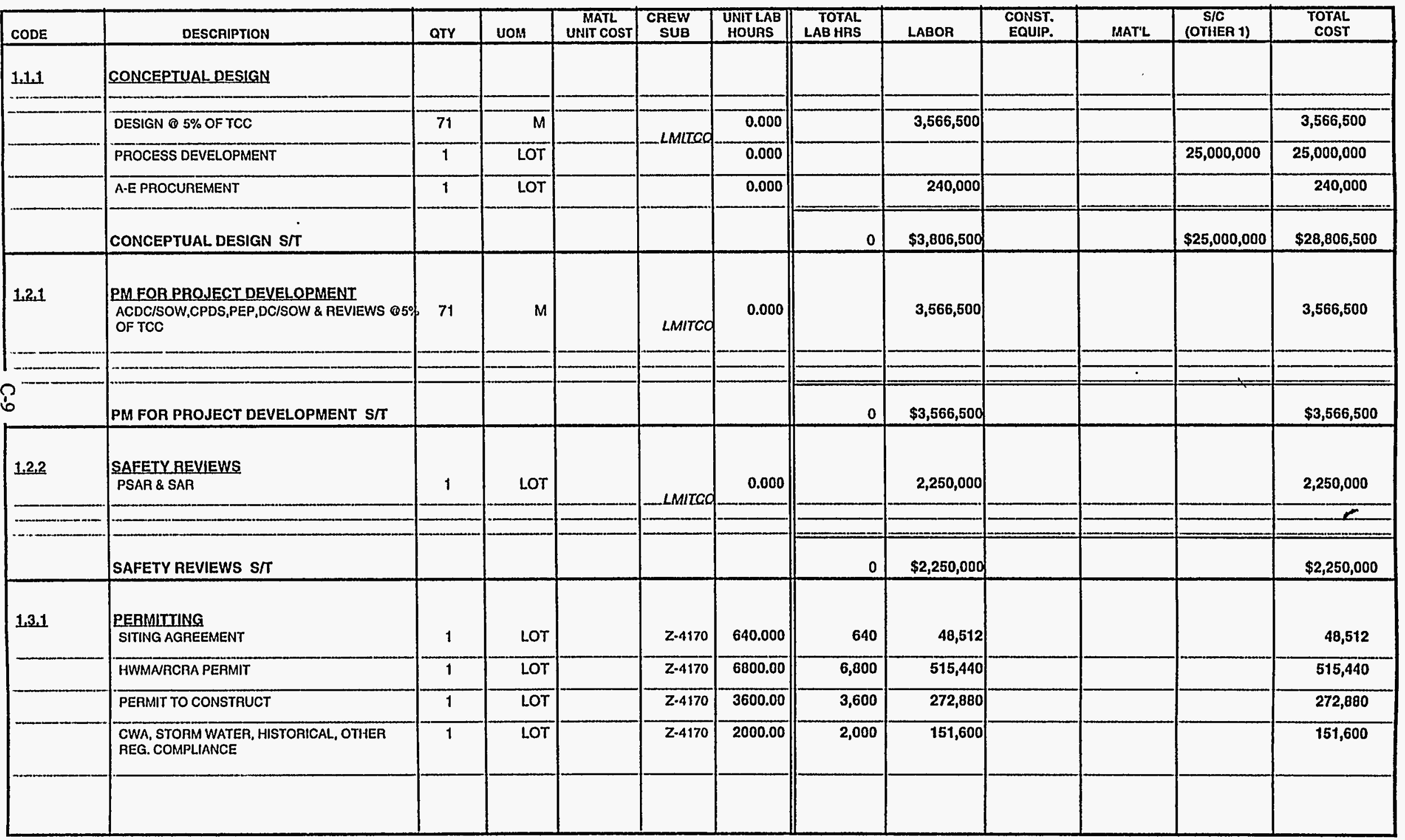


Lockheed Martin Idaho Technologies Co.

PROJECT NAME: Recalcination Treatment Non-Separations Other Project Costs

LOCATION 1: INEEL - ICPP

HEOUESTOR: AI LeO 6-9716 MS 3765
DETAILED COST ESTIMATE SHEET

TYPE OF ESTIMATE: Planning

PROJECT NO: 2420

PREPARED BY: JRB/BCE
PAGE \# 2

DATE O4-Fob-1998

TIME: 14:20:02

REPORT NAME: Detall Cost Estlmate Sheot

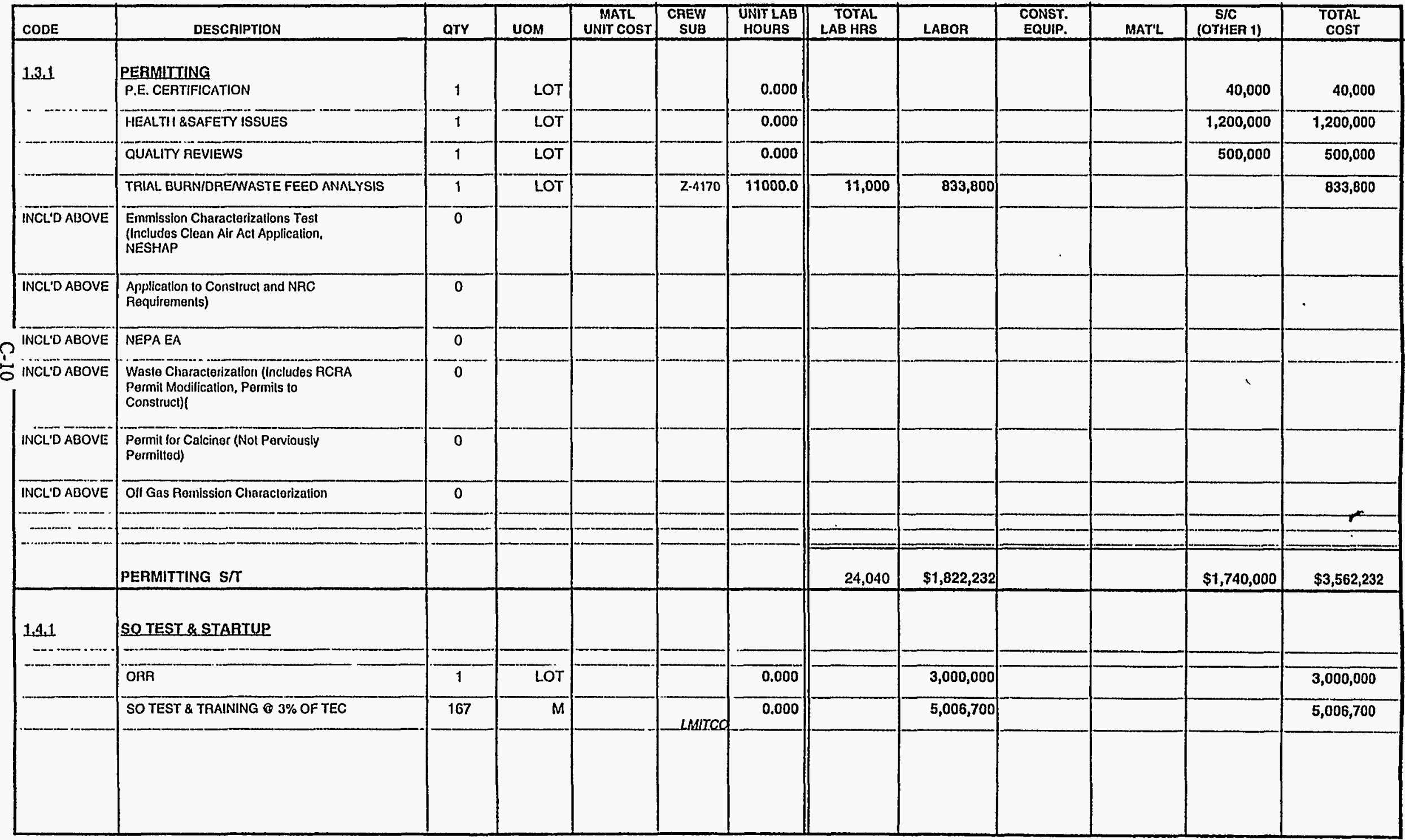


Lockheed Marlin Idaho Technologles Co.

ROJECTNAME: Fecalcination Treatment Non-Separations Other Project Costs

LOCATION 1

REQUESTOR: Al Lee 6-9716 MS 3765
DETAILED COST ESTIMATE SHEET

TYPE OF ESTIMATE: Planning

PROJECT NO: 2420

PREPARED BY: JRE/BCE
PAGE" 3

DATE 04-Feb-1998

TIME: 14:20:02

PEPORT NAME: Detall Cost Estimate Sheot

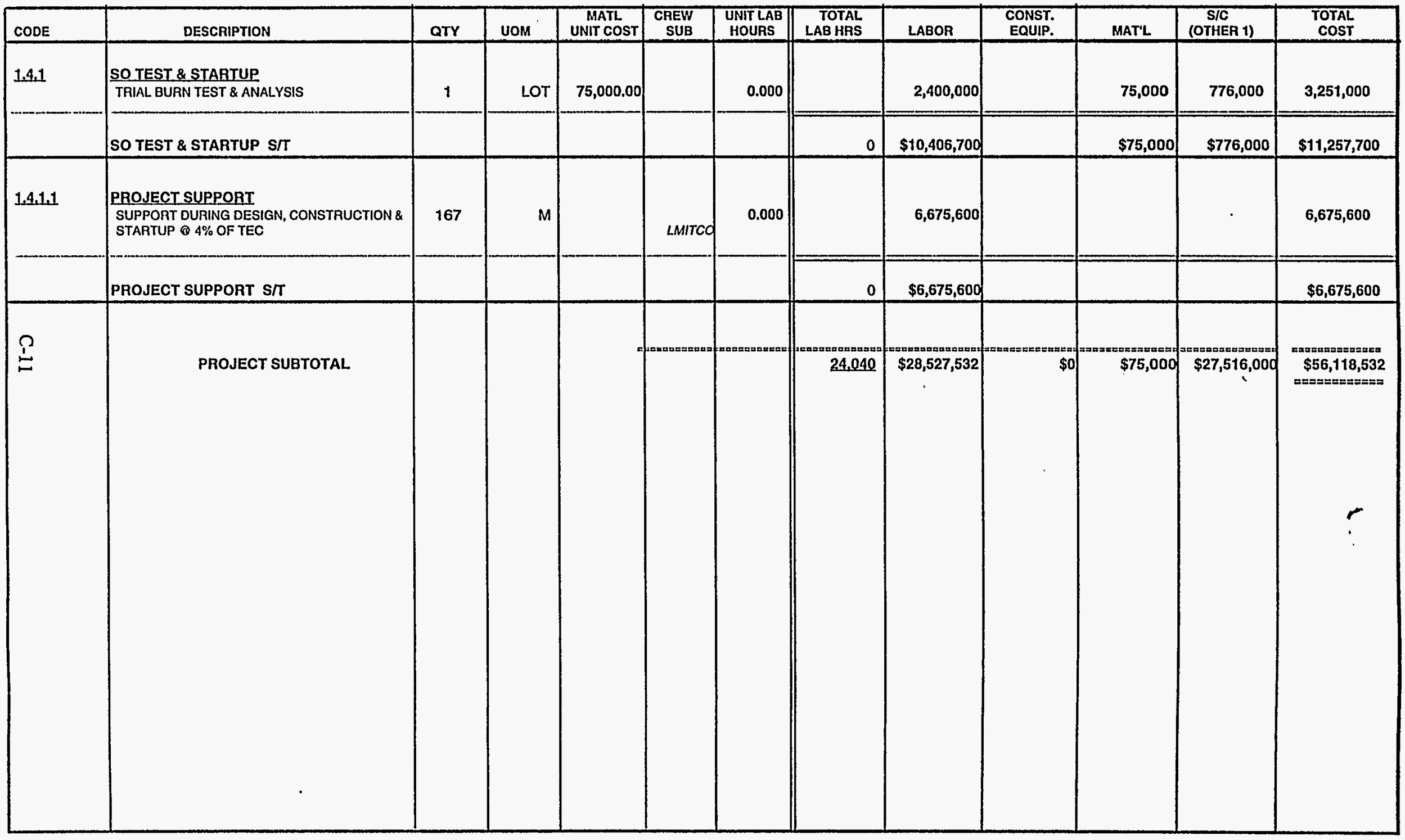


Lockheed Martin Idaho Technologies Co.

PROSECT NANE: Recalcination Treatment Non-Separations Other Project Costs - Unescalated

LOCATION I: INEEL-ICPP

REQUESTOR: Al Lee 6-9716 MS 3765

\section{CONTINGENCY ANALYSIS}

TYPE OF ESTMATE: PROSECTNO: PREPARED BY: Planning
2420
JRB/BCE
1. DATE 04-Feb-1998

TIME: 14:19:38

REPORT NAME: Contingency Analysis

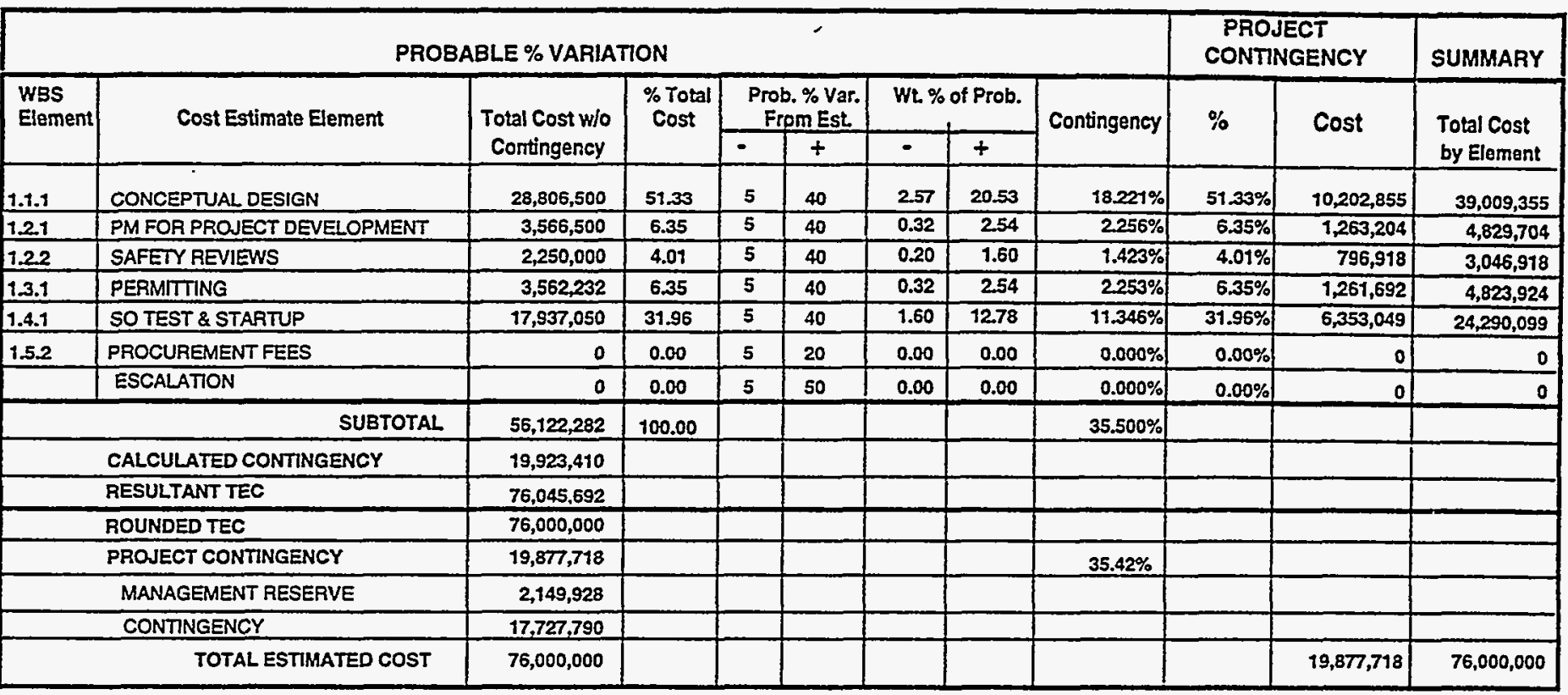

CONFIDENCE LEVEL AND ASSUMED RISKS:

The Lockheed Idaho Technologies Co. Cost Estimate Contingency Analysis

Model is based on the applied contingency and the assumptions upon which

the estimate was predicated. The model is applied with a suggested risk leve

of $18 \%$ and a level of confidence of $90 \%$ the estimate will fall within the bid range.

The Contingency Analysis is based on a weighted average to provide a

$90 \%$ probability of underrun and a $10 \%$ probability of overrun.
CONTINGENCY ANALYSIS GUIDE BY TYPE OF ESTIMATE

Guidelines established by DOEJFM 50, Cost Estimating Guide, Vol. 6 ,

Cost Guide, and as presented in the INEL Cost Estimating Guide.

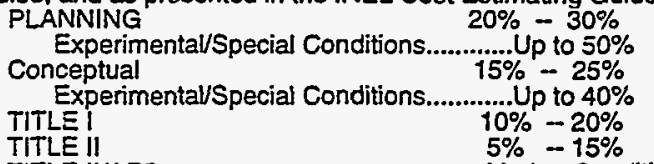

TITLE II/AFC

Market Conditions 


\section{Lockheed Martin Idaho Technologies Co.}

Rev. G56
PROECT NAME: Recalcination Treatment Non-Separations

LOCATION 1: INEEL-ICPP

REQUESTOR: Al LeE 6-9716 MS 3765
CONTINGENCY ANALYSIS

TYPE OFESTIMATE: Planning PROJECT NO: 2420 PREPARED BY: JRBIBCE
1. DATE: 04-Feb-1998
TIME: $14: 16: 23$

REPORT NAME: Contingency Analysis

\begin{tabular}{|c|c|c|c|c|c|c|c|c|c|c|c|}
\hline \multicolumn{9}{|c|}{ PROBABLE \% VARIATION } & \multicolumn{2}{|c|}{$\begin{array}{l}\text { PROJECT } \\
\text { CONTINGENCY }\end{array}$} & \multirow{3}{*}{$\begin{array}{l}\text { SUMMARY } \\
\text { Total cost } \\
\text { by Element }\end{array}$} \\
\hline \multirow[t]{2}{*}{$\begin{array}{l}\text { WBS } \\
\text { Element }\end{array}$} & \multirow[t]{2}{*}{ Cost Estimate Element } & \multirow{2}{*}{$\begin{array}{c}\text { Total Cost w/o } \\
\text { Contingency }\end{array}$} & \multirow[t]{2}{*}{$\begin{array}{l}\text { \% Total } \\
\text { Cost }\end{array}$} & \multicolumn{2}{|c|}{$\begin{array}{c}\text { Prob. \% Var. } \\
\text { From Est } \\
\end{array}$} & \multicolumn{2}{|c|}{ Wt. \% of Prob. } & \multirow[t]{2}{*}{ Contingency } & \multirow[t]{2}{*}{$\%$} & \multirow[t]{2}{*}{ Cost } & \\
\hline & & & & - & \pm & - & + & & & & \\
\hline 1.1 .1 & CONCEPTUAL DESIGN & $28,806,500$ & 41.98 & 5 & 40 & 2.10 & 16.79 & $14.901 \%$ & $40.12 \%$ & $10,220,362$ & $39,026,862$ \\
\hline 1.2 .1 & PM FOR PROJECT DEVELOPMENT & $3,566,500$ & 5.20 & 5 & 40 & 0.26 & 2.08 & $1.845 \%$ & $4.97 \%$ & $1,265,371$ & $4,831,871$ \\
\hline 122 & SAFETY REVIEWS & $2,250,000$ & 3.28 & 5 & 40 & 0.16 & 1.31 & $1.164 \%$ & $3.13 \%$ & 798,286 & $3,048,286$ \\
\hline 1.3.1 & PERMITTING & $3,562,232$ & 5.19 & 5 & 40 & 0.26 & 208 & $1.843 \%$ & $4.96 \%$ & $1,263,857$ & $4,826,089$ \\
\hline 1.4 .1 & SO TEST \& STARTUP & $17,937,050$ & 26.14 & 5 & 40 & 1.31 & 10.45 & $9.279 \%$ & $24.98 \%$ & $6,363,950$ & $24,301,000$ \\
\hline \multirow[t]{2}{*}{1.52} & PROCUREMENT FEES & 0 & 0.00 & 5 & 20 & 0.00 & 0.00 & $0.000 \%$ & $0.00 \%$ & 0 & 0 \\
\hline & ESCALATION & $12,504,537$ & 18.22 & 5 & 50 & 0.91 & 9.11 & $8.108 \%$ & $21.83 \%$ & $5,561,355$ & $18,055,892$ \\
\hline & SUBTOTAL & $68,626,819$ & 100.00 & & & & & $37.140 \%$ & & & \\
\hline & CALCULATED CONTINGENCY & $25,487,929$ & & & & & & & & & \\
\hline & RESULTANT TEC & $94,114,748$ & & & & & & & & & \\
\hline & ROUNDED TEC & $94,100,000$ & & & & & & & & & \\
\hline & PROJECT CONTINGENCY & $25,473,181$ & & & & & & $37.12 \%$ & & & \\
\hline & MANAGEMENT RESERVE & $3,010,529$ & & & & & & & & & \\
\hline & CONTINGENCY & $22,462,652$ & & & & & & & & & \\
\hline & TOTAL ESTIMATED COST & $94,100,000$ & & & & & & & & $25,473,181$ & $94,100,000$ \\
\hline
\end{tabular}

CONFIDENCE LEVEL AND ASSUMED RISKS:

The Lockheed Idaho Technologies Co. Cost Estimate Contingency Analysis Model is based on the applied contingency and the assumptions upon which

the estimate was predicated. The model is applied with a suggested risk level

of $18 \%$ and a level of confidence of $90 \%$ the estimate will fall within the bid range.

The Contingency Analysis is based on a weighted average to provide a

$90 \%$ probability of underrun and a $10 \%$ probability of overnn.
CONTINGENCY ANALYSIS GUIDE BY TYPE OF ESTIMATE

Guidelines established by DOEJFM 50, Cost Estimating Guide, Vol. 6.

Cost Guide, and as presented in the INEL Cost Estimating Guide.

PLANNING $20 \%-30 \%$

Experimental/Special Conditions.............Up to $50 \%$

Conceptual

$15 \%-25 \%$

Experimental/Special Conditions............. $10 \%$ to $40 \%$

TITLE II

TITLE II/AFC 
1.

$$
\text { ; }
$$

C-14 
Lockheed Martin Idaho Technologies Co.

PROUECT NAME: Recalcination Treatment Non-Separations

Cementitious Waste Option - Escalated
COST ESTIMATE SUMMARY

TYPE OFESTIMATE: Planning 1 : PROSECT NO: 2420

REPORT NAME: Cost Estimate Summary
LOCATION 1: INEEL-ICPP

REQUESTOR: AI Lee 6-9716 MS 3765

PREPARED BY: JRB/BCE

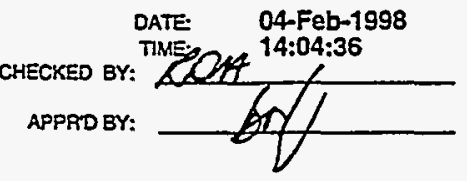

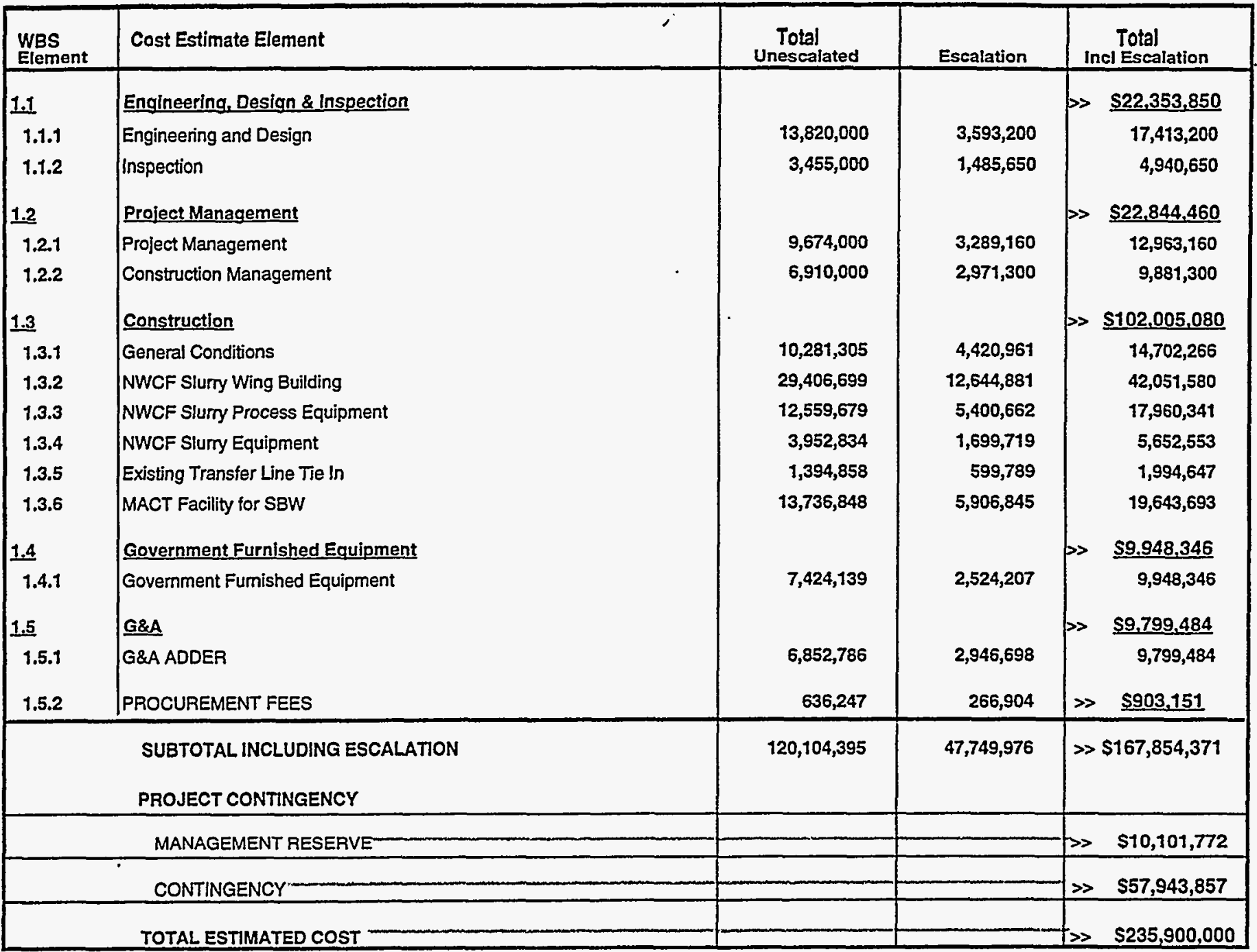

PROJECT COST PARAMETERS

EDI AS A \% OF CONST. + GFE= $25.00 \%$ CONTINGENCY $=40.54 \%$ 
Lockheed Martin Idaho Technologies Co.

PROSECT NAME: Recalcination Treatment Non-Separations Cementitious Waste Option - Unescalated

LOCATION : REOUESTOR:

\section{COST ESTIMATE SUMMARY}

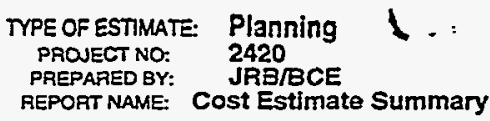

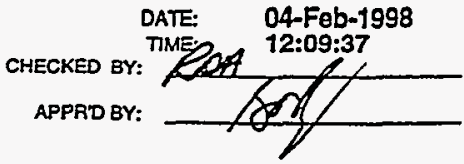

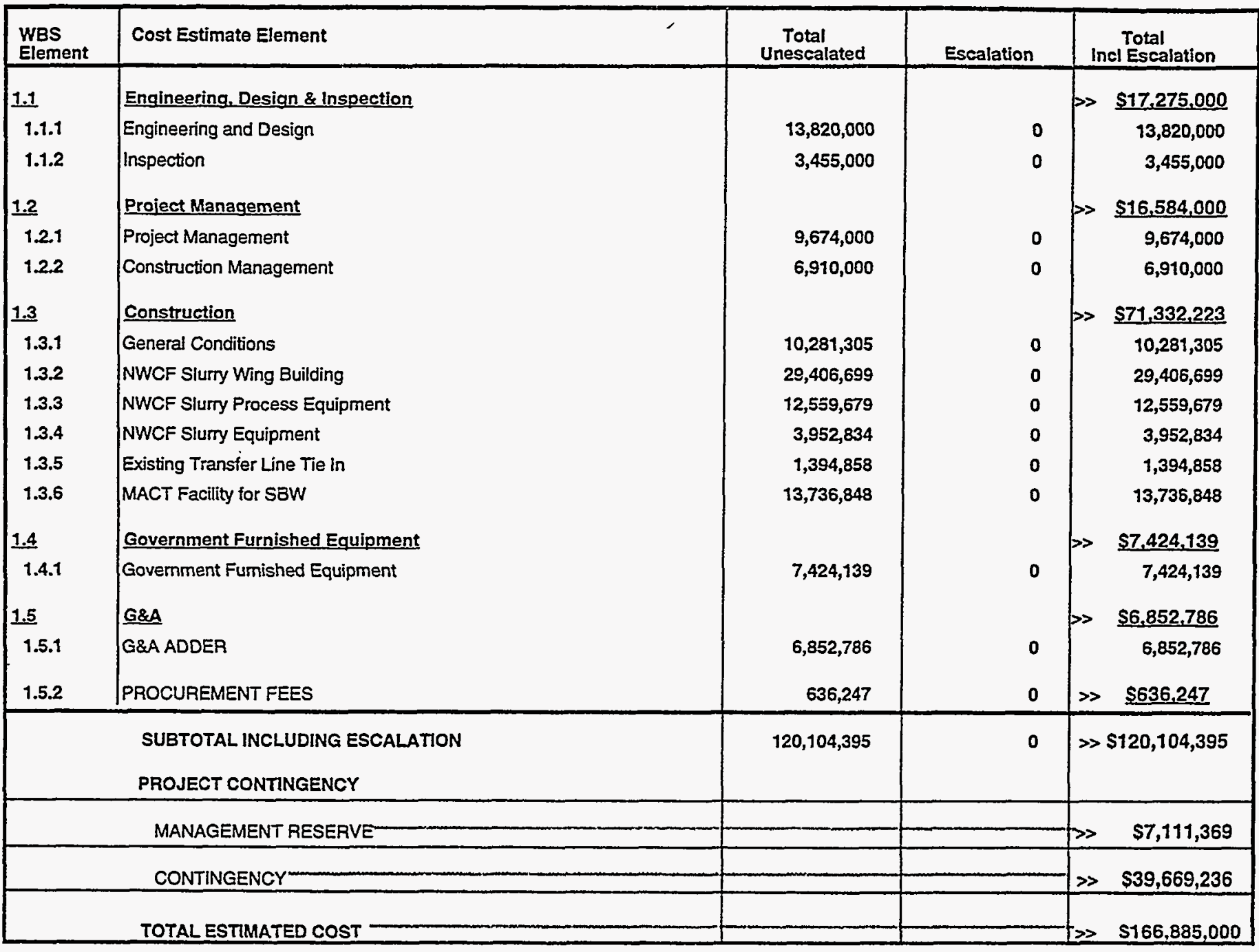

\section{PROJECT COST PARAMETERS}

EDI AS A $\%$ OF CONST. + GFE $=27.00 \%$

CONTINGENCY $=38.95 \%$ 
Lockheed Martin Idaho Technologies Co. Rov. 6/96 PROJECT NAME: Recalcination Treatment Non-Separations Cementitious Waste Option

LOCATION 1: INEEL - ICPP

PEQUESTOR: ALLe日 6-9716 MS 3765

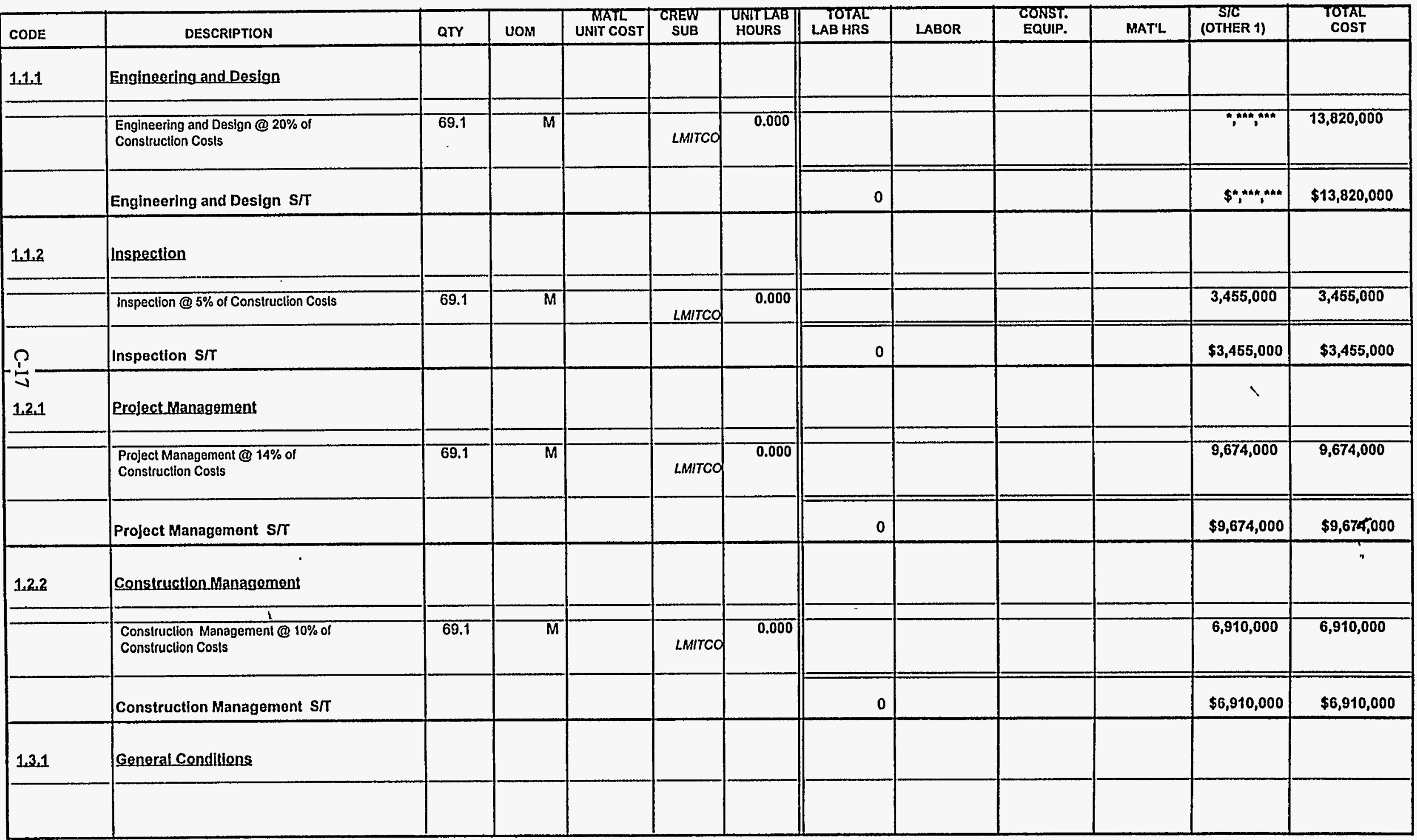

\section{DETAILED COST ESTIMATE SHEET}

TYPE OF estimate: Planning

PREPARED BY: JRB/BCE
PROJECT NO.: 2420
PAGE \# 1

DATE 03-Feb-1998

TIME: 16:40:26

REPORT NAME: Detail Cost Estimate Sheot 
Lockheed Martin Idaho Technologies Co.

Rov $6 / 06$

PROJECT NAME: Recalcination Treatment Non-Separatlons Cementitlous Waste Option

LOCATION I: INEEL - ICPP

REQUESTOR: Al LeQ 6-9716 MS 3765

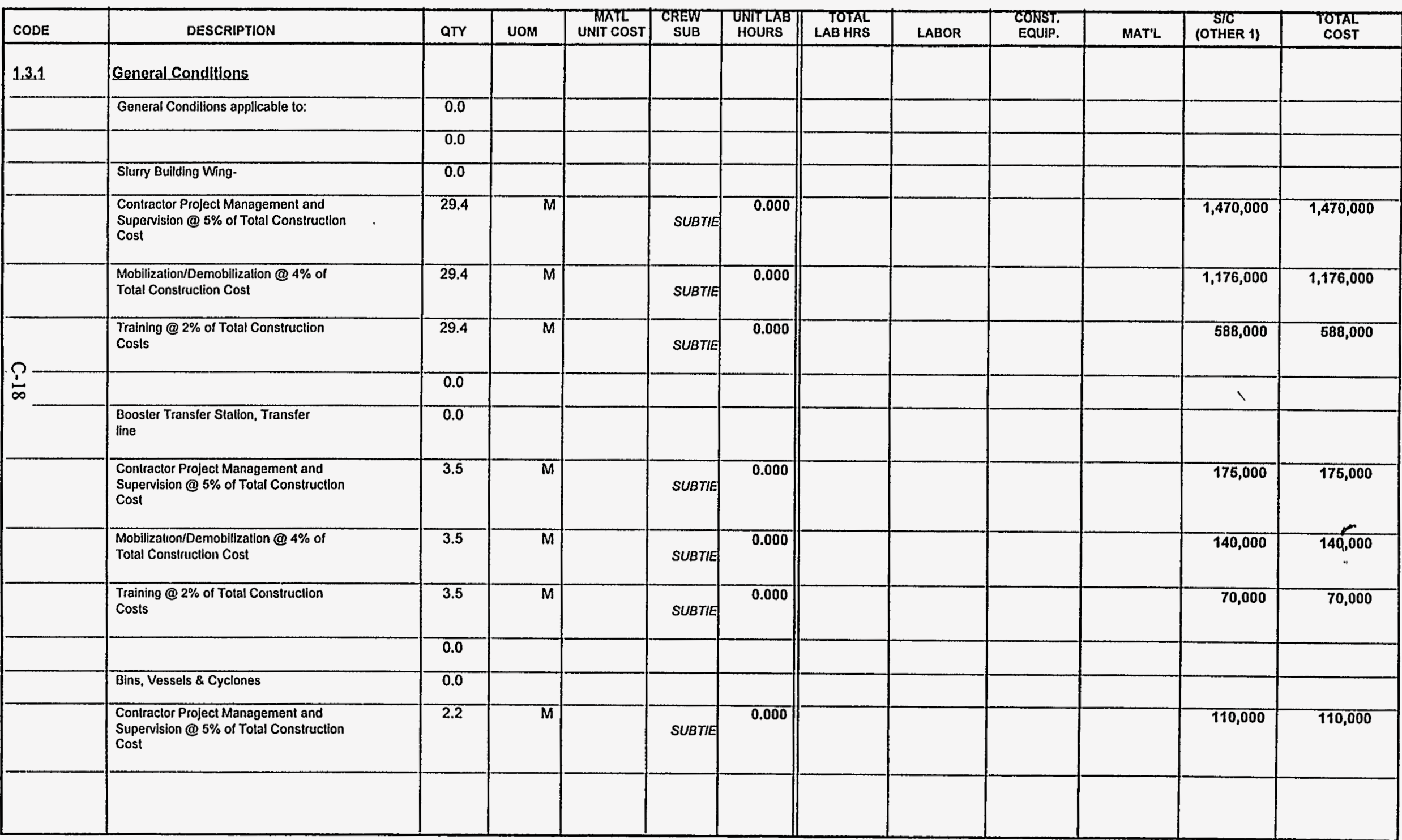

DETAILED COST ESTIMATE SHEET

TYPE OF ESTIMATE: Planning

PROJECT NO:: $\quad 2420$

PREPARED BY. JRB/BCE
PAGE $\# 2$

DATE 03-Feh-1998

TIME: $16: 40: 26$

REPORT NAME: Detail Cost Estlmate Sheet 
Lockheed Martin Idaho Technologies Co.

Rov. 6/96
PROJECT NAME: Recalcination Treatment Non-Separations

Cementitious Waste Option

LOCATION 1: INEEL - ICPP

REQUESTOR: AI Le 6-9716 MS 3765

\section{DETAILED COST ESTIMATE SHEET}

TYPE OF ESTIMATE: Planning

PROJECT NO: 2420

PREPARED BY: JRB/BCE
PAGE \# 3

DATE 03-Feb-1998

TIME: 16:40:26

REPORT NAME: Detall Cost Estimate Sheet

\begin{tabular}{|c|c|c|c|c|c|c|c|c|c|c|c|c|}
\hline CODE & DESCRIPTION & aTr & บoM & $\begin{array}{l}\text { MATL } \\
\text { UNIT COST }\end{array}$ & $\begin{array}{l}\text { CREW } \\
\text { SUB }\end{array}$ & $\begin{array}{l}\text { ONITLAE } \\
\text { HOURS }\end{array}$ & $\begin{array}{l}\text { TOTAL } \\
\text { LAB HRS }\end{array}$ & LABOR & $\begin{array}{l}\text { CONST. } \\
\text { EQUIP. }\end{array}$ & MATL & $\begin{array}{l}\text { STC } \\
\text { (OTHER 1) }\end{array}$ & $\begin{array}{l}\text { TOTAL } \\
\text { Cost }\end{array}$ \\
\hline \multirow[t]{5}{*}{1.3 .1} & $\begin{array}{l}\text { General Conditions } \\
\text { Mobilizalion/Demobilization @ 4\% of } \\
\text { Tolal Construction Cost }\end{array}$ & 2.2 & $M$ & & SUBTIE & 0.000 & & & & & 88,000 & 88,000 \\
\hline & $\begin{array}{l}\text { Tralning @ 2\% of Total Conslruclion } \\
\text { Cosis }\end{array}$ & 2.2 & $\bar{M}$ & & SUBTIE & 0.000 & & & & & 44,000 & 44,000 \\
\hline & & 0.0 & & & & & & & & & & \\
\hline & Pumps \& Mixers & 0.0 & & & & & & & & & & \\
\hline & $\begin{array}{l}\text { Contraclor Projecl Management and } \\
\text { Supervision @ 5\% ol Total Conslruction } \\
\text { Cost }\end{array}$ & 0.4 & $\bar{M}$ & & SUBTIE & $\overline{0.000}$ & & & & & 20,000 & 20,000 \\
\hline \multirow{9}{*}{$-\frac{2}{\sigma}$} & $\begin{array}{l}\text { Mobilizalion/Demobilization @ A\% of } \\
\text { Total Construction Cost }\end{array}$ & 0.4 & $\bar{M}$ & & SUBTIE & $\overline{0.000}$ & & & & & 16,000 & 16,000 \\
\hline & $\begin{array}{l}\text { Training @ 2\% of Total Construction } \\
\text { Costs }\end{array}$ & 0.4 & $\bar{M}$ & & SUBTIE & 0.000 & & & & & 8,000 & 8,000 \\
\hline & & 0.0 & & & & & & & & & & \\
\hline & Slury Equipment Instrumentation & 0.0 & & & & & & & & & & \\
\hline & $\begin{array}{l}\text { Contraclor Project Management and } \\
\text { Supervision @ 5\% of Tolal Construction } \\
\text { Cost }\end{array}$ & 2.8 & $\bar{M}$ & & SUBTIE & $\overline{0.000}$ & & & & & 140,000 & $\stackrel{140,000}{r}$ \\
\hline & $\begin{array}{l}\text { Mobilizallon/Demobilizalion @ } 4 \% \text { of } \\
\text { Tolal Construction Cost }\end{array}$ & 2.8 & $\bar{M}$ & & SUBTIA & 0.000 & & & & & 112,000 & 112,000 \\
\hline & $\begin{array}{l}\text { Training @ } 2 \% \text { of Total Construction } \\
\text { Cosis }\end{array}$ & 2.8 & $\bar{M}$ & & SUBTIE & 0.000 & & & & & 56,000 & 56,000 \\
\hline & & 0.0 & & & & & & & & & & \\
\hline & Existing Transfer Line Tie.In & 0.0 & & & & & & & & & & \\
\hline & & & & & & & & & & & & \\
\hline & & & & & & & & & & & & \\
\hline
\end{tabular}


Lockheed Martin Idaho Technologies Co. Rov 6/06

PROJECT NAME' Recalcination Treatment Non-Separatlons Cementitious Waste Optlon

LOCATION 1: INEEL - ICPP

REQUESTOR AI Lee 6-9716 MS 3765

\section{DETAILED COST ESTIMATE SHEET}

TYPE OF ESTIMATE. Planning

PROJECT NO:: 2420

PREPARED BY: JRB/BCE
PAGE \# 4

DATE 03-Fob-1998

TIME: 16:40:26

REPORT NAME: Detall Cost Estimato Sheot

\begin{tabular}{|c|c|c|c|c|c|c|c|c|c|c|c|c|}
\hline CODE & DESCRIPTION & QTY & uom & $\begin{array}{l}\text { MATL } \\
\text { UNIT COST }\end{array}$ & $\begin{array}{l}\text { CREW } \\
\text { SUB }\end{array}$ & $\begin{array}{l}\text { DNITLAB } \\
\text { HOURS }\end{array}$ & $\begin{array}{l}\text { TOTAL } \\
\text { LAB HRS }\end{array}$ & LABOR & $\begin{array}{l}\text { CONST: } \\
\text { EQUIP. }\end{array}$ & MATLL & STC & $\begin{array}{l}\text { TOTAL } \\
\text { COST }\end{array}$ \\
\hline \multirow[t]{4}{*}{$1,3,1$} & $\begin{array}{l}\text { General Conditions } \\
\text { Contractor Project Management and } \\
\text { Supervision @ } 5 \% \text { of Tolal Construction } \\
\text { Cost }\end{array}$ & 1.0 & M & & SUBTIE & 0.000 & & & & & 60,000 & 50,000 \\
\hline & $\begin{array}{l}\text { Mobilization/Demobilization @ 4\% of } \\
\text { Total Construction Cost }\end{array}$ & 1.0 & $\bar{M}$ & & SUBTIE & $\overline{0.000}$ & & & . & & 40,000 & 40,000 \\
\hline & $\begin{array}{l}\text { Training @ 2\% of Total Consiruction } \\
\text { Costs }\end{array}$ & 1.0 & $\vec{M}$ & & SUBTIE & 0.000 & & & & & 20,000 & 20,000 \\
\hline & General Conditions $S / T$ & & & & & & 0 & & & & $\$ 4,323,000$ & $\$ 4,323,000$ \\
\hline \multirow{5}{*}{$\overbrace{0}^{1.3 .1}$} & $\begin{array}{l}\text { Bulk Sucrose General Conditions } \\
\text { RAD BOXES }\end{array}$ & 4.0 & EA & & $\begin{array}{l}\text { LABR } \\
\text { SUBTIE }\end{array}$ & 8.000 & 32 & 929 & & & & 929 \\
\hline & GENERAL MATERIAL HANDLIING \& CLEANUP & 1.0 & LOT & & $\begin{array}{l}\text { LABR } \\
\text { SUBTIA }\end{array}$ & 1200.00 & 1,200 & 34,848 & & & T & 34,848 \\
\hline & OVERTIME ALLOWANCE & 1.0 & LOT & & SUBTIE & 0.000 & & 241,339 & & & & 241,339 \\
\hline & SMALL TOOLS \& CONSUMABLES & 1.0 & LOT & $77,228.00$ & SUBTIE & $\overline{0.000}$ & & & & 77,228 & & 77,228 \\
\hline & Bulk Sucrose General Conditions $\mathbf{S} / \mathbf{T}$ & & & & & & 1,232 & $\$ 277,116$ & & $\$ 77,228$ & & $\$ 354,344$ \\
\hline \multirow[t]{5}{*}{$\frac{1.3 .1 .1}{100}$} & $\begin{array}{l}\text { Bulk Sucrose Training } \\
\text { PIPEFITTER SITE TRAINING }\end{array}$ & 0.0 & & & & & & & & & & : \\
\hline & 1ST YEAR & 17.0 & $\overline{E A}$ & & $\begin{array}{l}\text { PIPE } \\
\text { SUBTIE }\end{array}$ & 120.000 & 2,040 & 76,052 & & & 12,750 & 87,802 \\
\hline & $\begin{array}{l}\text { 2ND YEAR UPDATE TRAINING FOR MOST OF } \\
\text { ABOVE }\end{array}$ & 15.0 & $E A$ & & $\begin{array}{c}\text { PIPE } \\
\text { SUBTIE }\end{array}$ & 40.000 & 600 & 22,074 & & & 3,750 & 25,824 \\
\hline & $\begin{array}{l}\text { 2ND YEAR TRAINING FOR REMAINDER OF } \\
\text { WORK FORCE }\end{array}$ & 41.0 & $\overline{E A}$ & & $\begin{array}{l}\text { PIPE } \\
\text { SUBTIE }\end{array}$ & 120.000 & $\overline{4,920}$ & 181,007 & & & 30,750 & 211,757 \\
\hline & 3RD YEAR & 57.0 & EA & & $\begin{array}{l}\text { PIPE } \\
\text { SUBTIA } \\
\end{array}$ & 120.000 & 6,840 & 251,644 & & & 42,750 & 294,394 \\
\hline & & & & & & & & & & & & \\
\hline
\end{tabular}


Lockheed Martin Idaho Technologies Co. Rov. 6196

ROJECT NAME: Recalcination Treatment Non-Separations Cementitlous Waste Optlon

LOCATION 1: INEEL - ICPP

REQUESTOR: Al Lee 6-9716 MS 3765
DETAILED COST ESTIMATE SHEET

TYPE OF ESTIMATE: Planning

PROJECT NO: 2420

PREPARED BY: JRB/BCE
PAGE \# 5

DATE 03-Feb-1998

TIME: 16:40:26

REPORT NAME: Detall Cost Estlmate Sheet

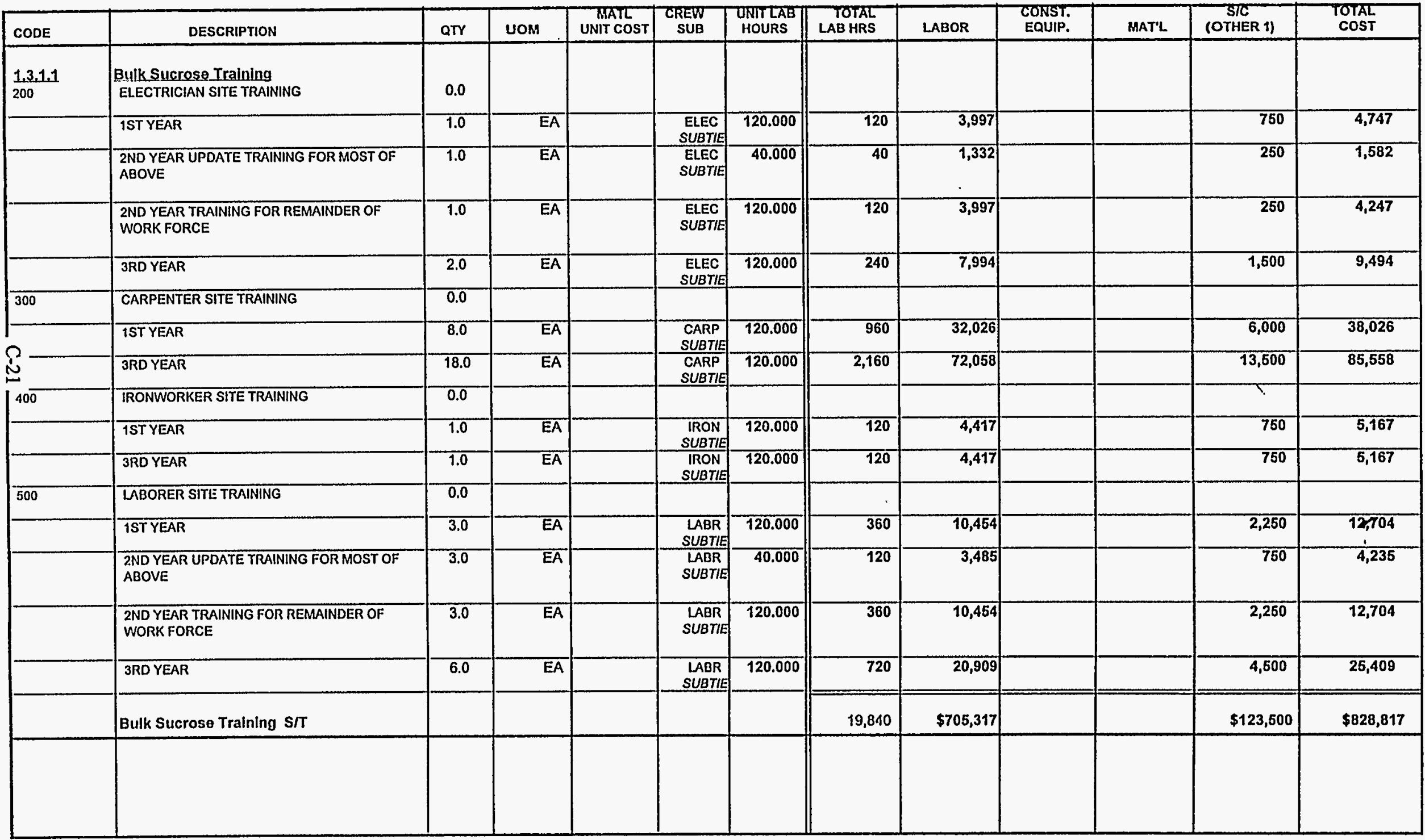


Lockheed Martin Idaho Tochnologies Co. Rav $6 / 96$

PROJECT NAME: Recalcination Treatment Non-Separations Cementitlous Wasto Option

LOCATION 1: INEEL - ICPP

REQUESTOR: Al Lee 6-9716 MS 3765

\section{DETAILED COST ESTIMATE SHEET}

TPE OF ESTIMATE: Planning

PROJECTNO: 2420

PREPARED BY: JRB/BCE
PAGE\# 6

DATE 03-Fob-1998

TIME: 16:40:26

REPORT NAME: Detail Cost Estimate Sheot

\begin{tabular}{|c|c|c|c|c|c|c|c|c|c|c|c|c|}
\hline CODE & DESCRIPTION & ary & vom & MARTL & $\begin{array}{l}\text { CREW } \\
\text { SUB }\end{array}$ & $\begin{array}{l}\text { UNITLAB } \\
\text { HOURS }\end{array}$ & $\begin{array}{l}\text { TOTAL } \\
\text { LAB HRS }\end{array}$ & LABOR & $\begin{array}{l}\text { CONST. } \\
\text { EQUIP. }\end{array}$ & MAT'L & SIC & $\begin{array}{l}\text { TOTAL } \\
\text { COST }\end{array}$ \\
\hline \multirow[t]{4}{*}{$1,3.1 .2$} & $\begin{array}{l}\text { Bulk Sucrose Burnout \& Mockup } \\
\text { Training } \\
\text { PIPEFITTER BURNOUT/MOCKUP TRAINING } \\
\text { ALLOWANCE }\end{array}$ & 118.0 & EA & & $\begin{array}{c}\text { PIPE } \\
\text { SUBTIE }\end{array}$ & 40.000 & 4,720 & 173,649 & & & & 173,649 \\
\hline & $\begin{array}{l}\text { ELECTRICIAN BURNOUTMMOCKUP TRAINING } \\
\text { ALLOWANCE }\end{array}$ & 5.0 & $\overline{E A}$ & & $\begin{array}{l}\text { ELEC } \\
\text { SUBTIA }\end{array}$ & 40.000 & 200 & $\overline{6,662}$ & & & & 6,662 \\
\hline & $\begin{array}{l}\text { CARPENTER BURNOUT/MOCKUP TRAINING } \\
\text { ALLOWANCE }\end{array}$ & 23.0 & $\overline{E A}$ & & $\begin{array}{l}\text { CARP } \\
\text { SUBTIA }\end{array}$ & 40.000 & 920 & $\overline{30,691}$ & & & & 30,691 \\
\hline & $\begin{array}{l}\text { IRONWORKER BURNOUTIMOCKUP TRAINING } \\
\text { ALLOWANCE }\end{array}$ & 1.0 & $\overline{E A}$ & & $\begin{array}{c}\text { IRON } \\
\text { SUBTIA }\end{array}$ & 40.000 & 40 & $\overline{1,472}$ & & & & 1,472 \\
\hline \multirow{2}{*}{ 足 } & $\begin{array}{l}\text { LABORER BURNOUT/MOCKUP TRAINING } \\
\text { ALLOWANCE }\end{array}$ & $\overline{15.0}$ & $\overline{E A}$ & & $\begin{array}{l}\text { LABR } \\
\text { SUBTIE }\end{array}$ & $\overline{40.000}$ & 600 & 17,424 & & & & 17,424 \\
\hline & Bulk Sucrose Burnout \& Mockup Training \$ & $\mathbf{T}$ & & & & & 6,480 & $\$ 229,898$ & & & 1 & $\$ 229,898$ \\
\hline \multirow[t]{4}{*}{1.3 .1} & $\begin{array}{l}\text { Sample Room General Conditions } \\
\text { SUPERVISION }\end{array}$ & 1.0 & LOT & & $\begin{array}{c}\text { PIPE } \\
\text { SUBTIE }\end{array}$ & 40.000 & 40 & 1,472 & & & & 1,472 \\
\hline & TRAINING - 3 MEN & 1.0 & LOT & & $\begin{array}{c}\text { PIPE } \\
\text { SUBTIE }\end{array}$ & 240.000 & 240 & 8,830 & & & & 8,830 \\
\hline & $\begin{array}{l}\text { ADD 10\% TO MATERIAL FOR DOE/RW/0333P } \\
\text { QUALITY STANDARDS }\end{array}$ & 1.0 & LOT & $75,370.00$ & SUBTIE & $\overline{0.000}$ & & & & 76,370 & & 75,370 \\
\hline & Sample Room General Conditions S/T & & & & & & 280 & $\$ 10,301$ & & $\$ 75,370$ & & $\$ 85,671$ \\
\hline \multirow[t]{2}{*}{1.3 .1} & $\begin{array}{l}\text { MACT Facillty General Condltions } \\
\text { SUPERVISION @ 5\% OF CONSTRUCTION HOURS }\end{array}$ & 1.0 & LOT & & $\begin{array}{c}\text { CARF } \\
\text { SUBTIE }\end{array}$ & 4713.00 & 4,713 & 162,551 & & & & 162,551 \\
\hline & TRAINING @ 3\% OF CONSTRUCTION HOURS & 1.0 & LOT & & $\begin{array}{l}\text { SSWK } \\
\text { SUBTIE }\end{array}$ & 2828.00 & 2,828 & 104,099 & & & & 104,099 \\
\hline & & & & & & & & & & & & \\
\hline & & & & & & & & & & & & \\
\hline
\end{tabular}


Lockheed Martin Idaho Technologies Co.

Rov. $6 / 96$
PROJECT NAME: Recalcination Treatment Non-Separations Cementitious Waste Option

LOCATION 1: INEEL - ICPP

REQUESTOR: Al LeE 6-9716 MS 3765
DETAILED COST ESTIMATE SHEET

TYPE OF ESTIMATE: Planning

PROJECTNO:: 2420

PREPARED BY: JRBIBCE
PAGE \# 7

DATE 03-Feb-1998

TIME: 16:40:26

REPORT NAME: Detall Cost Estimate Sheet

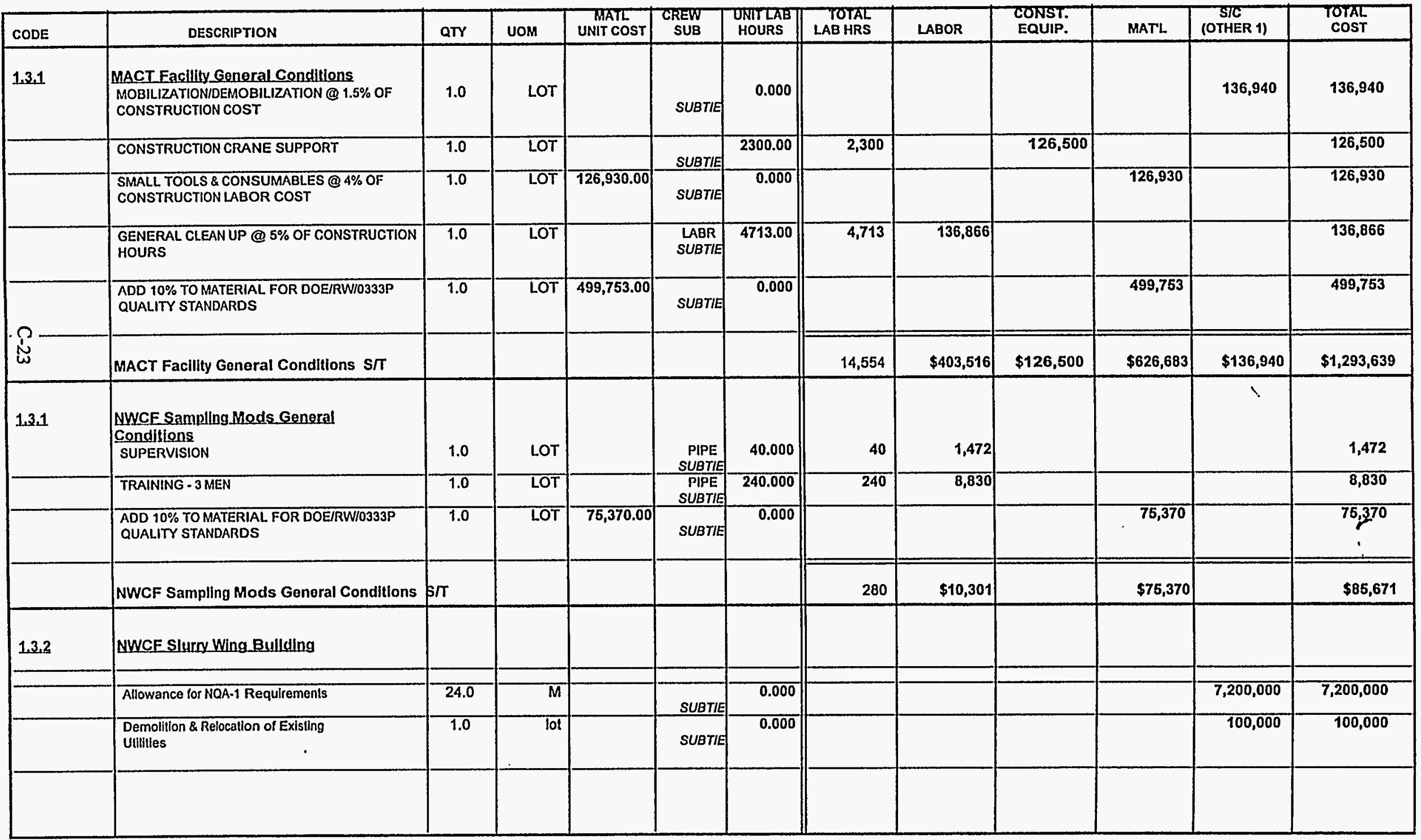


Lockheed Martin Idaho Technologies Co.

Rov. 6/96

PROJECr NAME Recalcination Treatment Non-Separations

Cementitious Waste Option

LOCATION 1: INEEL - ICPP

DETAILED COST ESTIMATE SHEET

TYPE OF ESTIMATE: Planning

PROJECTNO.: 2420

PREPAREO QY: JRB/BCE
PAGE\# 8

DATE 03-Fob-1998

TME: 16:40:26

REPORT NAME. Detali Cost Estimate Sheet

REQUESTOR. AI LeE 6-9716 MS 3765

\begin{tabular}{|c|c|c|c|c|c|c|c|c|c|c|c|c|}
\hline CODE & DESCRIPTION & QTY & บOM & $\begin{array}{l}\text { MATL } \\
\text { UNIT COST }\end{array}$ & $\begin{array}{l}\text { CREW } \\
\text { SUB }\end{array}$ & $\begin{array}{l}\text { UNITLAB } \\
\text { HOURS }\end{array}$ & $\begin{array}{l}\text { TOTAL } \\
\text { LAB HRS }\end{array}$ & LABOR & $\begin{array}{l}\text { CONST. } \\
\text { EQUIP. }\end{array}$ & MAT'L & $\begin{array}{l}\text { STC } \\
\text { (OTHER 1) }\end{array}$ & $\begin{array}{l}\text { TOTAL } \\
\text { COST }\end{array}$ \\
\hline \multirow[t]{6}{*}{1.3 .2} & $\begin{array}{l}\text { NWCF Slurry Wing Bullding } \\
\text { Wire Saw Openings for Demolition }\end{array}$ & 56.0 & sqit & & SUBTIE & 0.000 & & & & & 8,400 & 8,400 \\
\hline & $\begin{array}{l}\text { Demolition For Openings from Exisiting } \\
\text { Bullding to New Building }\end{array}$ & 168.0 & cuft & & SUBTIE & 0.000 & & & & & 5,040 & 5,040 \\
\hline & $\begin{array}{l}\text { Install/Remove Shoring and Piles for } \\
\text { Excavation }\end{array}$ & $11,500.0$ & sqft & & SUBTIE & 0.000 & & & & & 345,000 & 345,000 \\
\hline & $\begin{array}{l}\text { Excavation for Buildling incl's export } \\
\text { \&backfill }\end{array}$ & $13,535,0$ & cuyd & & SUBTIE & 0.000 & & & & & 135,350 & 135,350 \\
\hline & $\begin{array}{l}\text { Palch Back Silework After Building is } \\
\text { Completed }\end{array}$ & 1.0 & $10 t$ & & SUBTIE & 0.000 & & & & & 50,000 & 50,000 \\
\hline & $\begin{array}{l}\text { Concrete Walls, Foolings, Slabs, } \\
\text { etc.... }\end{array}$ & $4,237.0$ & cuyd & & SUBTIA & 0.000 & & & & & $3,601,450$ & $3,601,450$ \\
\hline \multirow{11}{*}{ 点 } & Struclural Steel & $2,500.0$ & sqfi & & cur rid & 0.000 & & & & & 187,600 & 187,500 \\
\hline & Plafforms/Decking & $1,600.0$ & sqfit & & דa & 0.000 & & & & & 240,000 & 240,000 \\
\hline & Metal Railings/Ladders & 600.0 & Inft & & and & $\overline{0.000}$ & & & & & 75,000 & 75,000 \\
\hline & Waterproofing & $6,368.0$ & sqft & & ad & 0.000 & & & & & 4,776 & 4,776 \\
\hline & Roofing & $2,300.0$ & sqft & & 1070 & 0.000 & & & & & 8,050 & 89050 \\
\hline & Flashings & 1.0 & Tot & & anumd & 0.000 & & & & & 5,000 & 5,000 \\
\hline & $\begin{array}{l}\text { Exterior Finish on Bullding (metal, } \\
\text { dryvil, elc... }\end{array}$ & $3,000.0$ & sqfit & & SUBTIE & 0.000 & & & & & 27,000 & 27,000 \\
\hline & Personnel Shlelding Doors & 8.0 & each & & CuOTd & 0.000 & & & & & 160,000 & 160,000 \\
\hline & Shielding Windows & 4.0 & each & & המדח & 0.000 & & & & & 744,000 & 744,000 \\
\hline & Decon Painting & $11,500.0$ & saft & & and & $\overline{0.000}$ & & & & & 57,500 & 57,600 \\
\hline & Stainless Steel liner & $13,091.0$ & sqfi & & & 0.000 & & & & & 130,910 & 130,910 \\
\hline & & & & & & & & & & & & \\
\hline & & & & & & & & & & & & \\
\hline
\end{tabular}


Lockheed Martin Idaho Technologies Co. Rev. 6196

PROJECT NAME: Recalcination Treatment Non-Separations Cementitious Waste Option

LOCATION 1: INEEL - ICPP

REQUESTOR. AILOO 6-9716 MS 3765

\section{DETAILED COST ESTIMATE SHEET}

TYPE OF ESTIMATE: Planning

PROJECTNO: 2420

PREPARED BY: JRB/BCE
PAGE \# 9

DATE 03-Feb-1998

TIME: 16:40:26

REPORT NAME: Detall Cost Estimate Sheot

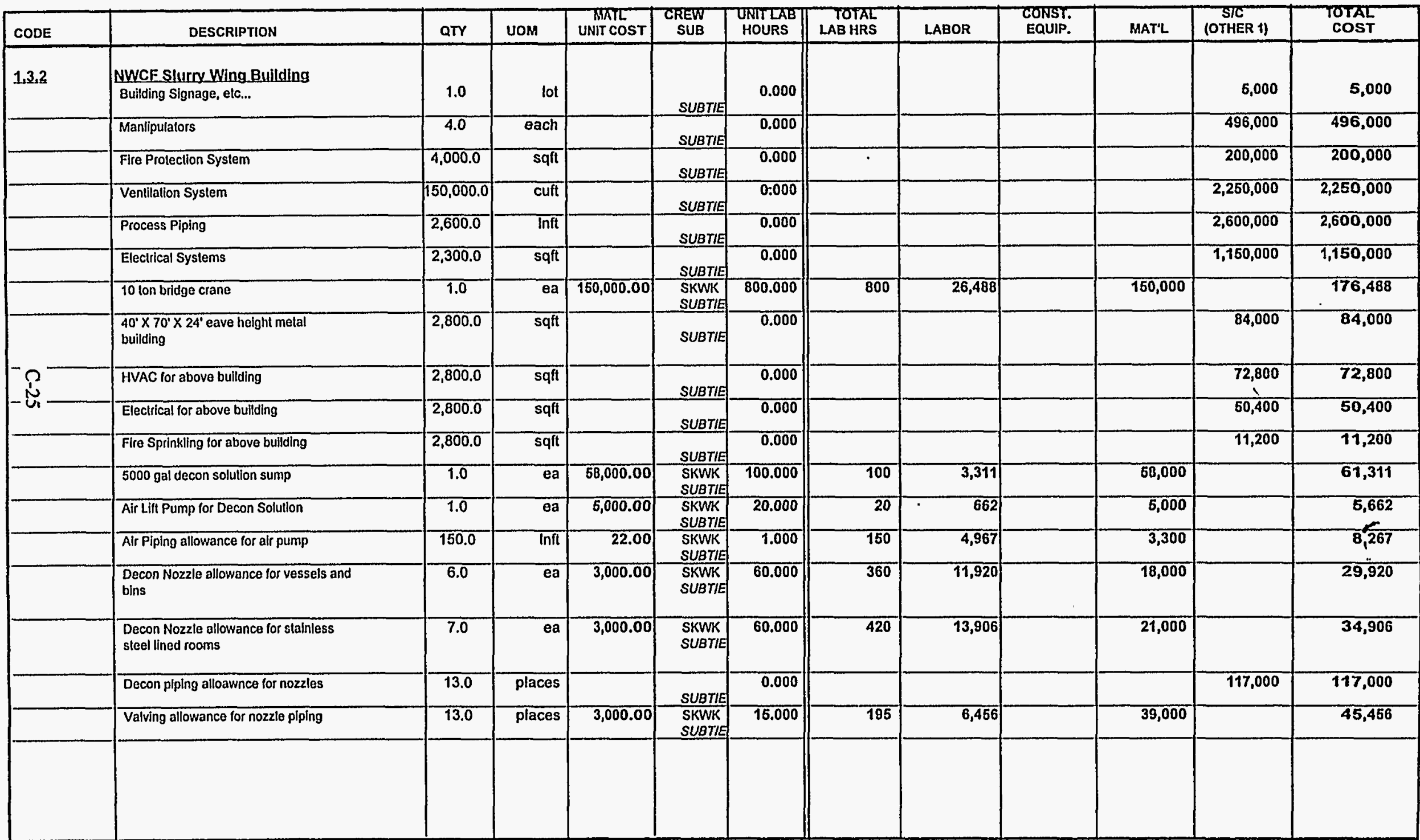


Lockheed Martin Idaho Technologies Co.

Rov $6 / 96$

PROJECT NAME Recalcination Treatment Non-Separations

Cementitious Waste Option

LOCATION 1: INEEL - ICPP

REQUESTOR: Al Lee 6-9716 MS 3766
DETAILED COST ESTIMATE SHEET

TYPE OF ESTIMATE: Planning

PROJECT NO:: 2420

PREPARED QY: JRB/BCE
PAGE\# 10

DATE 03-Fob-199

TIME: 16:40:26

RePort name: Detail Cost Estimate Sheet

\begin{tabular}{|c|c|c|c|c|c|c|c|c|c|c|c|c|}
\hline CODE & DESCRIPTION & ary & UOM & $\begin{array}{l}\text { WATL } \\
\text { UNIT COST }\end{array}$ & $\begin{array}{l}\text { CREW } \\
\text { SUB }\end{array}$ & $\begin{array}{l}\text { UNITLAB } \\
\text { HOURS }\end{array}$ & $\begin{array}{l}\text { TOTAL } \\
\text { LAB HRS }\end{array}$ & LABOR & $\begin{array}{l}\text { COWST: } \\
\text { EQUIP. }\end{array}$ & MAT'L & $\begin{array}{l}\text { STC } \\
\text { (OTHER 1) }\end{array}$ & $\begin{array}{l}\text { TOTAL } \\
\text { COST }\end{array}$ \\
\hline $1,3,2$ & $\begin{array}{l}\text { NWCE Slurry_Wing_Building } \\
\text { Electrical allowance for valving } \\
\text { conirol }\end{array}$ & 13.0 & places & $3,000.00$ & $\begin{array}{l}\text { SKWK } \\
\text { SUBTIE }\end{array}$ & 16.000 & 196 & 6,466 & & 39,000 & & 45,456 \\
\hline & $\begin{array}{l}\text { Tie in to existing decon solution } \\
\text { system }\end{array}$ & 1.0 & 10t & & SUBTIE & 0.000 & & & & & 1,000 & 1,000 \\
\hline & Supply line for Decon Solution & 150.0 & Inft & 22.00 & $\begin{array}{l}\text { SKWK } \\
\text { SUBTIE }\end{array}$ & 1.000 & 150 & 4,967 & & 3,300 & & $\overline{8,267}$ \\
\hline & Return L.ine for Decon Solulion & 150.0 & Inft & 22.00 & $\begin{array}{l}\text { SKWK } \\
\text { SUBTIE }\end{array}$ & 1.000 & 160 & 4,967 & & 3,300 & & 8,267 \\
\hline & $\begin{array}{l}\text { Valving allowance for Decon Solution } \\
\text { Supply }\end{array}$ & 1.0 & place & $3,000.00$ & $\begin{array}{l}\text { SKWK } \\
\text { SUBTIE }\end{array}$ & 30.000 & 30 & 993 & & 3,000 & & 3,993 \\
\hline & $\begin{array}{l}\text { Valving allowance for Decon Solution } \\
\text { Return }\end{array}$ & 1.0 & place & $3,000.00$ & $\begin{array}{l}\text { SKWK } \\
\text { SUBTIA }\end{array}$ & $\overline{30.000}$ & 30 & 993 & & 3,000 & & 3,993 \\
\hline 0 & $\begin{array}{l}\text { Process Steam and Cooling piping } \\
\text { extensions }\end{array}$ & 150.0 & Inft & & SUBTIE & 0.000 & & & & & 18,000 & 18,000 \\
\hline & $\begin{array}{l}\text { Addilional relurn capability to tank } \\
\text { farm }\end{array}$ & 500.0 & Inft & & SUBTIE & 0.000 & & & & & 60,000 & 60,000 \\
\hline & Tle In for return line at tank farm & 1.0 & place & $4,000.00$ & $\begin{array}{l}\text { SKWK } \\
\text { SUBTIE }\end{array}$ & 200.000 & 200 & 6,622 & & 4,000 & & 10,622 \\
\hline & $\begin{array}{l}\text { NWCF penetrations from cold area to } \\
\text { hot area }\end{array}$ & 1.0 & place & $1,500.00$ & $\begin{array}{l}\text { SKWK } \\
\text { SUBTIE }\end{array}$ & 100.000 & 100 & 3,311 & & 1,500 & & 4,811 \\
\hline & $\begin{array}{l}\text { NWCF penetrations from hot area to hot } \\
\text { area }\end{array}$ & 2.0 & places & $1,500.00$ & $\begin{array}{l}\text { SKWK } \\
\text { SUBTIE }\end{array}$ & 200.000 & $\overline{400}$ & 13,244 & & 3,000 & & 16,244 \\
\hline & $\begin{array}{l}\text { NWCF penelratlons from cold area to } \\
\text { cold area }\end{array}$ & 1.0 & place & 500.00 & $\begin{array}{l}\text { SKWK } \\
\text { SUBTIE }\end{array}$ & 20.000 & 20 & 662 & & 500 & & $t, 162$ \\
\hline & $\begin{array}{l}\text { Piping allowance from filler room to } \\
\text { NWCF diverter valve }\end{array}$ & 100.0 & Inft & & SUBTIE & 0.000 & & & & & 12,000 & 12,000 \\
\hline & & & & & & & & & & & & \\
\hline & & & & & & & & & & & & \\
\hline
\end{tabular}


Lockheed Martin Idaho Technologies Co.

Rav. $6 / 96$

PROJECT NAME: Recalcination Treatment Non-Separations

Cementitious Waste Option

LOCATION 1: INEEL - ICPP

REQUESTOR: Al Le日 6.9716 MS 3765

\section{DETAILED COST ESTIMATE SHEET}

TYPE OF ESTMATE: Planning

PROJECT NO: 2420

PREPARED BY: JRBIBCE
PAGE \# 11

DATE 03-Fob-1998

TIME: 16:40:26

RePORT NAME: Detail Cost Estimate Sheet

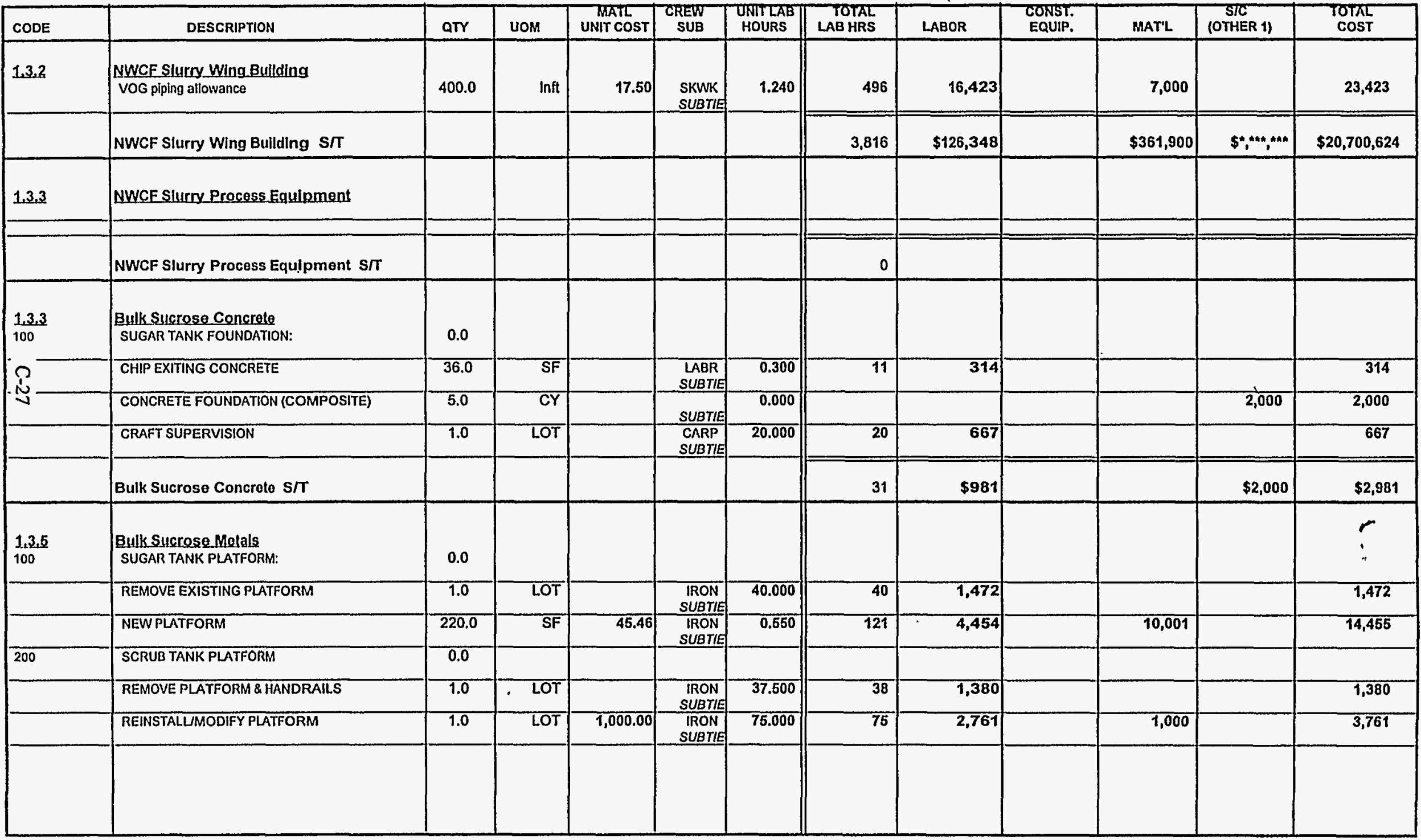


Lockheed Martin Idaho Technologies Co.

nov 6196

PROJECr NAME Recalcination Treatment Non-Separations Cementitious Waste Option

INEEL - ICPP

AI Lee 6-9716 MS 3766

\section{DETAILED COST ESTIMATE SHEET}

TYPE OF ESTIMATE Planning

PROJECT NO: 2420

PREPARED BY: JRB/BCE
PAgE \# 12

DATE 03-Fob-1998

TIME: 16:40:26

REPORT NAME: Dotall Cost Estimato Sheot

\begin{tabular}{|c|c|c|c|c|c|c|c|c|c|c|c|c|}
\hline CODE & - DESCRIPTION & ary & UOM & UNITTE & $\begin{array}{l}\text { CREW } \\
\text { SUB }\end{array}$ & $\begin{array}{l}\text { DINITLAB } \\
\text { HOURS }\end{array}$ & $\begin{array}{l}\text { TOTKL } \\
\text { LAB HRS }\end{array}$ & LABOR & $\begin{array}{l}\text { CONST. } \\
\text { EQUIP. }\end{array}$ & MAT'L & $\begin{array}{l}\text { STC } \\
\text { (OTHER 1) }\end{array}$ & $\begin{array}{l}\text { TOTRL } \\
\text { COST }\end{array}$ \\
\hline \multirow[t]{2}{*}{$1,3,5$} & $\begin{array}{l}\text { Bulk Sucrose Metals } \\
\text { CRAFT SUPERVISION }\end{array}$ & 1.0 & LOT & & $\begin{array}{c}\text { IRON } \\
\text { SUBTIE }\end{array}$ & 91.000 & 91 & 3,350 & & & & 3,350 \\
\hline & Bulk Sucrose Metals S/T & & & & & & 365 & $\$ 13,417$ & & $\$ 11,001$ & & $\$ 24,418$ \\
\hline \multirow[t]{3}{*}{1.3 .2} & $\begin{array}{l}\text { Bulk Sucrose Finishes } \\
\text { PAINT EYEWASH/SAFETY SHOWER \& FL.OOR }\end{array}$ & 1.0 & LOT & 50.00 & $\begin{array}{c}\text { PAIN } \\
\text { SUBTIE }\end{array}$ & 15.000 & 15 & 419 & & 50 & & 469 \\
\hline & PAINT CARBON STEEL. PIPE \& SUPPORTS & 1.0 & LOT & & SUBTIE & 0.000 & & & & & 2,000 & 2,000 \\
\hline & PAINT TANK PLATFORM & 1.0 & LOT & & SUBTIE & 0.000 & & & & & 3,000 & 3,000 \\
\hline \multirow{2}{*}{ 莕 } & CRAFT SUPERVISION & 1.0 & LOT & & $\begin{array}{l}\text { PAIN } \\
\text { SUBTIE }\end{array}$ & 20.000 & 20 & 559 & & & & 559 \\
\hline & Bulk Sucrose Finishes $\mathbf{S} / \mathrm{T}$ & & & & & & 35 & $\$ 978$ & & $\$ 50$ & $\$ 5,000$ & $\$ 6,028$ \\
\hline \multirow[t]{3}{*}{$\begin{array}{l}1.3 .11 .1 \\
100\end{array}$} & $\begin{array}{l}\text { Calciner Nozzles } \\
\text { FUEL NOZZLES (REMOTE OPERATION, NO IN- } \\
\text { CELL): }\end{array}$ & 0.0 & & & & & & & & & ? & \\
\hline & REMOVE FUEL NOZZLES & 4.0 & $\overline{E A}$ & & $\begin{array}{c}\text { PIPE } \\
\text { SUBTIE }\end{array}$ & 2.000 & 8 & 294 & & & & 294 \\
\hline & INSTALL NEW NOZZLES & 4.0 & $\overline{E A}$ & $8,000.00$ & $\begin{array}{c}\text { PIPE } \\
\text { SUBTIE }\end{array}$ & 2.000 & $\overline{8}$ & 294 & & 32,000 & & 32,294 \\
\hline \multirow[t]{2}{*}{200} & CALCINER NOZZLE PLUGS & 0.0 & & & & & & & & & & s \\
\hline & PLUGS FOR DECON VESSEL. FILLING & 8.0 & $\overline{E A}$ & $2,000.00$ & $\begin{array}{c}\text { PIPE } \\
\text { SUBTIE }\end{array}$ & 1.900 & 15 & 559 & & 16,000 & & 16,559 \\
\hline \multirow[t]{5}{*}{300} & $\begin{array}{l}\text { FEED NOZZLES (REMOTE OPERATION, NO IN- } \\
\text { CELL): }\end{array}$ & 0.0 & & & & & & & & & & \\
\hline & REMOVE FEED NOZZLES & 4.0 & $\overline{E A}$ & & $\begin{array}{c}\text { PIPE } \\
\text { SUBTIE } \\
\end{array}$ & 2.000 & 8 & 294 & & & & 294 \\
\hline & INSTALL NEW NOZZLES & 4.0 & EA & $8,000.00$ & $\begin{array}{c}\text { PIPE } \\
\text { SUBTIE }\end{array}$ & 2.000 & $\overline{8}$ & 294 & & 32,000 & & 32,294 \\
\hline & . & & & & & & & & & & & \\
\hline & & & & & & & & & & & & \\
\hline
\end{tabular}


Lockheed Martin Idaho Technologies Co.

Rov. 6196

PROJECT NAME: Recalcination Treatment Non-Separations

Cementillous Waste Option

OCATION 1: INEEL - ICPP

REOUESTOR: Al Lee 6-9716 MS 3765

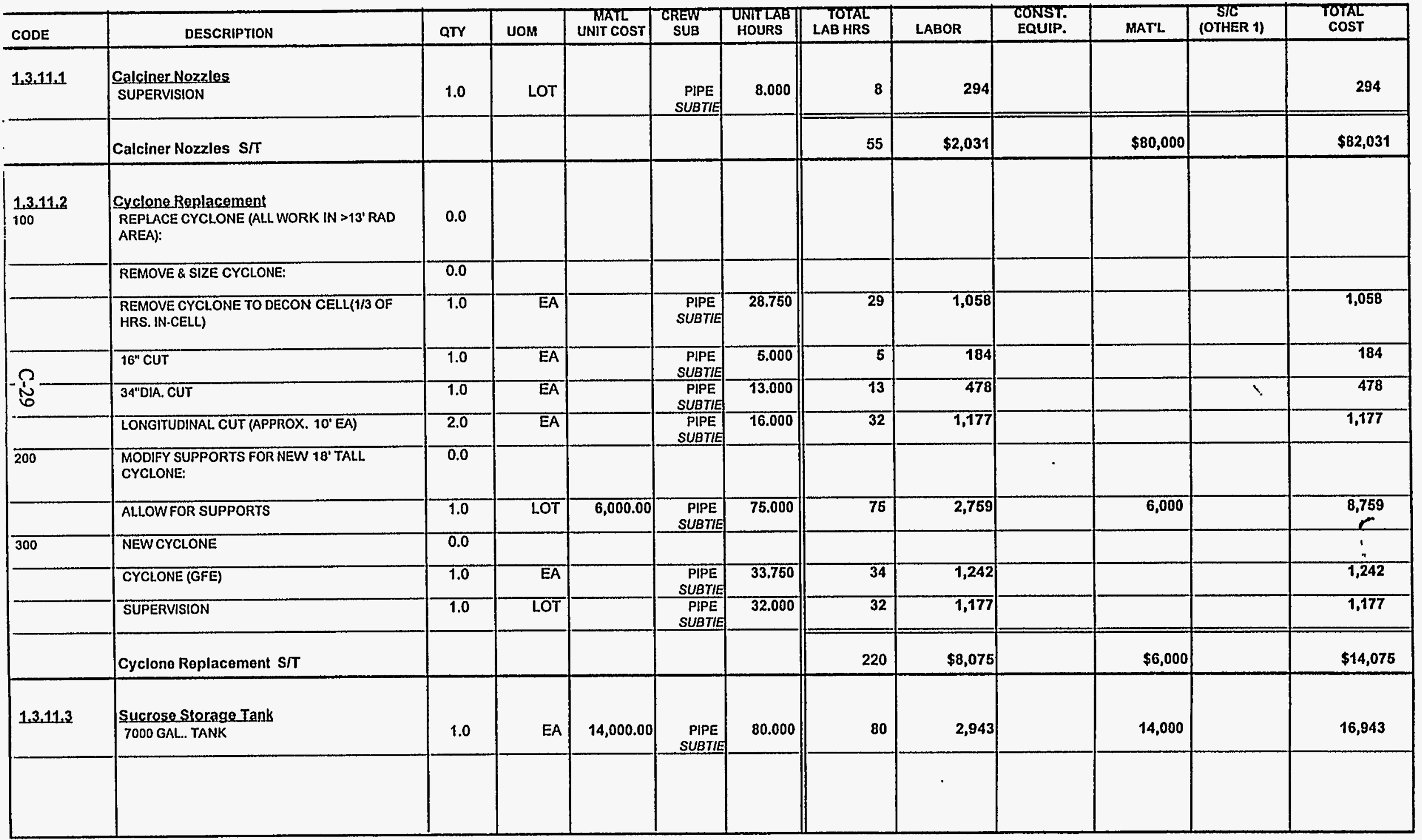

\section{DETAILED COST ESTIMATE SHEET}

TYPE OF ESTIMATE: Planning

PROJECT NO: 2420

PREPARED BY: JRB/BCE
PAGE* 13

DATE 03-Feb-1998

TIME: 16:40:26

REPORT NAME: Detail Cost Estimate Sheet 
Lockheed Martin Idaho Technologies Co. Rov 6190

PROJECT NAME: Recalcination Treatment Non-Separations Cementitious Waste Option

LOCATION 1: INEEL - ICPP

REQUESTOR. AI Lee 6-9716 MS 3765

\section{DETAILED COST ESTIMATE SHEET}

TPE OF ESTIMATE- Planning

PROJECTNO.: 2420

PREPARED BY: JRB/BCE
PAGE \# 14

DATE 03-Feb-1998

TIME: 16:40:26

REPORT NAME: Detail Cost Estimate Sheet

\begin{tabular}{|c|c|c|c|c|c|c|c|c|c|c|c|c|}
\hline CODE & DESCRIPTION & arv & UOM & $\begin{array}{l}\text { MATL } \\
\text { UNIT COST }\end{array}$ & $\begin{array}{r}\text { CREW } \\
\text { SUB } \\
\end{array}$ & $\begin{array}{l}\text { UNITLAB } \\
\text { HOURS }\end{array}$ & $\begin{array}{l}\text { TOTAL } \\
\text { LAB HIRS }\end{array}$ & LABOR & $\begin{array}{l}\text { CONST. } \\
\text { EQUIP. }\end{array}$ & MAT'L & $\begin{array}{c}\text { STC } \\
\text { (OTHER 1) }\end{array}$ & $\begin{array}{r}\text { TOTAL } \\
\text { COST }\end{array}$ \\
\hline \multirow[t]{6}{*}{1.3 .11 .3} & $\begin{array}{l}\text { SUcrose Storage Tank } \\
\text { TANK STEAM JACKET (ALLOW) }\end{array}$ & 1.0 & LOT & $3,000.00$ & SURTIE & 0.000 & & & & 3,000 & & 3,000 \\
\hline & TANK INSULATION & 570.0 & SF & $\overline{4.00}$ & $\begin{array}{c}\text { ASBE } \\
\text { SUBTIE }\end{array}$ & 0.300 & 171 & 6,392 & & 2,280 & & $\overline{8,672}$ \\
\hline & TANK AGITATOR & 1.0 & $E A$ & $17,000,00$ & $\begin{array}{l}\text { PIPE } \\
\text { SUBTIE }\end{array}$ & 40.000 & 40 & 1,472 & & 17,000 & & 18,472 \\
\hline & SUGAR PUMP & 1.0 & $E A$ & $2,500.00$ & $\begin{array}{c}\text { PIPE } \\
\text { SUBTIE }\end{array}$ & 24.000 & 24 & 883 & & 2,500 & & 3,383 \\
\hline & CRAFT SUPERVISION & 1.0 & LOT & & $\begin{array}{c}\text { PIPE } \\
\text { SUBTIE } \\
\end{array}$ & 100.000 & 100 & 3,679 & & & & 3,679 \\
\hline & Sucrose Storage Tank $\mathbf{S} / \mathrm{T}$ & & & & & & 415 & $\$ 15,369$ & & $\$ 38,780$ & & $\$ 54,149$ \\
\hline $1,3.11 .4$ & $\begin{array}{l}\text { Mercury Scrubber } \\
\text { ALLOWANCE FOR NITRONIC } 50 \text { SCRUBBER }\end{array}$ & 1.0 & EA & $500,000.00$ & $\begin{array}{c}\text { PIPE } \\
\text { SUBTIE }\end{array}$ & 900.000 & 900 & 33,111 & & 600,000 & & 533,111 \\
\hline \multirow{3}{*}{$-\stackrel{8}{0}$} & ALLOWANCE FOR PLATUM ELECTRODE & 1.0 & EA & $50,000.00$ & SUBTIE & 0.000 & & & & 50,000 & 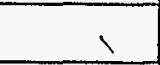 & 50,000 \\
\hline & Allowance for NQA-1 Requirements & 1.0 & $\bar{M}$ & & SUBTIE & 0.000 & & & & & 300,000 & 300,000 \\
\hline & Mercury Scrubber $\mathbf{s} / \mathrm{T}$ & & & & & & 900 & $\$ 33,111$ & & $\$ 550,000$ & $\$ 300,000$ & $\$ 883,111$ \\
\hline \multirow[t]{3}{*}{1.3 .11 .5} & $\begin{array}{l}\text { Jet Grinder } \\
\text { JET GRINDER NOZZLE \& FLANGE }\end{array}$ & 2.0 & EA & $3,000.00$ & $\begin{array}{c}\text { PIPE } \\
\text { SUBTIA }\end{array}$ & 15.000 & 30 & 1,104 & & 6,000 & & 7,504 \\
\hline & SUPERVISION 1 & 0.0 & EA & & $\begin{array}{c}\text { PIPE } \\
\text { SUBTIE } \\
\end{array}$ & & & & & & & \\
\hline & Jet Grinder $\mathbf{S} / \mathrm{T}$ & & & & & & 30 & $\$ 1,104$ & & $\$ 6,000$ & & $\$ 7,104$ \\
\hline \multirow[t]{3}{*}{1.3 .11 .6} & $\begin{array}{l}\text { Scrub Holding Tank } \\
\text { REMOVE PUMPS \& JUMPERS REMOTELYY }\end{array}$ & 1.0 & LOT & & $\begin{array}{c}\text { PIPE } \\
\text { SUBTIE }\end{array}$ & 40.000 & 40 & 1,472 & & & & 1,472 \\
\hline & REMOVE TANK & 1.0 & EA & & $\begin{array}{c}\text { PIPE } \\
\text { SUBTIE }\end{array}$ & 71.250 & 71 & 2,621 & & & & 2,621 \\
\hline & CUT UP TANK IN ENCLOSURE & 70.0 & IF & & $\begin{array}{l}\text { PIPE } \\
\text { SUBTIE }\end{array}$ & 1.000 & 70 & 2,575 & & & & 2,575 \\
\hline & & & & & & & & & & & & \\
\hline
\end{tabular}


Lockheed Martin Idaho Technologies Co. Rav. 6196 PROJECT NAME: Recalcination Treatment Non-Separations Cementitious Waste Option

LOCATION 1: INEEL - ICPP

DETAILED COST ESTIMATE SHEET

TYPE OF ESTMATE: Planning

PROJECTNO: 2420

PREPARED BY: JRB/BCE
PAGE\# 15

DATE 03-Fob-1998

TIME: 16:40:26

REPORT NAME: Detall Cost Estimate Sheet

REQUESTOR: AI Lee 6-9716 MS 3765

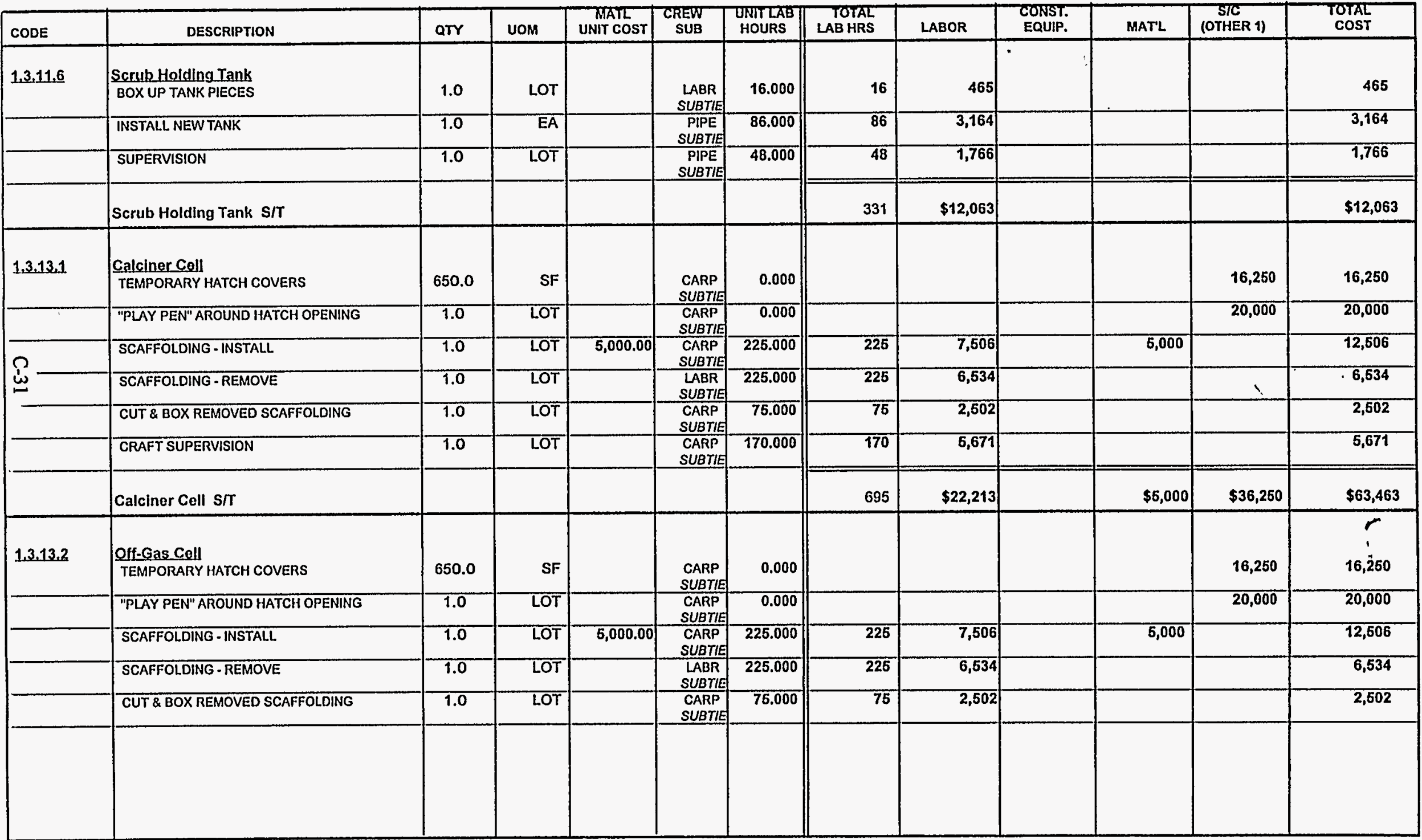


Lockhoed Martin Idaho Technologies Co.

Rov $6 / 00$

PROJECT NAME: Recalcination Treatment Non-Separations Cementitious Waste Option

LOCATION 1: INEEL - ICPP

REQUESTOR AILe日 6-9716 MS 3765

\section{DETAILED COST ESTIMATE SHEET}

TYPE OF ESTIMATE. Planning

PROJECTNO:: 2420

PREPARED BY: JRB/BCE
PAGEH 16

DATE 03-Feb-1998

TIME: 16:40:26

REPORT NAME: Detall Cost Estimate Sheet

\begin{tabular}{|c|c|c|c|c|c|c|c|c|c|c|c|c|}
\hline CODE & DESCRIPTION & arv & UOM & UNATL COST & $\begin{array}{l}\text { CREW } \\
\text { SUB }\end{array}$ & $\begin{array}{l}\text { UNITLAB } \\
\text { HOURS }\end{array}$ & $\begin{array}{l}\text { TOTAL } \\
\text { LAB HRS }\end{array}$ & LABOR & $\begin{array}{l}\text { CONST: } \\
\text { EQUIP. }\end{array}$ & MAT'L & (OTHER 1) & $\begin{array}{l}\text { TOTAL } \\
\text { COST }\end{array}$ \\
\hline \multirow[t]{2}{*}{1.3 .13 .2} & $\begin{array}{l}\text { Off-Gas Cell } \\
\text { CRAFT SUPERVISION }\end{array}$ & 1.0 & LOT & & $\begin{array}{l}\text { CARP } \\
\text { SUBTIES } \\
\end{array}$ & 170.000 & 170 & 5,671 & & & & 5,671 \\
\hline & Off-Gas Cell $\mathrm{S} / \mathrm{T}$ & & & & & & 695 & $\$ 22,213$ & & $\$ 6,000$ & $\$ 36,250$ & $\$ 63,463$ \\
\hline \multirow[t]{3}{*}{$1,3,13.3$} & $\begin{array}{l}\text { Bulk Sucrose, Nezzle \& Cyclone } \\
\text { Mock-UpS } \\
\text { MOCK-UP FACILITY - CACINER CELL. }\end{array}$ & 1.0 & LOT & & SUBTIE & 0.000 & & & & & 150,000 & 150,000 \\
\hline & MOCK-UP FACILITY - OFF-GAS CELL & 1.0 & LOT & & SUBTIE & 0.000 & & & & & 125,000 & 125,000 \\
\hline & Bulk Sucrose, Nozzle \& Cyclone Mock-Ups & $s \pi$ & & & & & 0 & & & & $\$ 275,000$ & $\$ 275,000$ \\
\hline \multirow[t]{2}{*}{1.3 .13 .4} & $\begin{array}{l}\text { pemolished Equipment SIzing } \\
\text { Enclosure } \\
\text { ENCLOSURE, CHANGE AREA, ENTRY \& } \\
\text { VENTILATION }\end{array}$ & 800.0 & $S F$ & & SUBTIE & 0.000 & & & & & 20,000 & 20,000 \\
\hline & Demolished Equipment Sizing Enclosure s & $\mathbf{T}$ & & & & & 0 & & & & $\$ 20,000$ & $\$ 20,000$ \\
\hline \multirow[t]{4}{*}{1.3 .15 .1 .1} & $\begin{array}{l}\text { Bulk Sucrose. Nozzle \& Cyclone Fan } \\
\text { Pulleys } \\
\text { CHANGE SUPPLY FAN PULLEYS }\end{array}$ & 3.0 & SETS & 600.00 & $\begin{array}{c}\text { MILL. } \\
\text { SUBTIE } \\
\end{array}$ & 20.000 & 60 & 1,877 & & 1,800 & & $\stackrel{r}{3,677}$ \\
\hline & CHANGE EXHAUST FAN PULLEYS & 3.0 & SETS & 600.00 & $\begin{array}{c}\text { MILL } \\
\text { SUBTIE }\end{array}$ & 75.000 & 225 & 7,038 & & 1,800 & & 8,838 \\
\hline & CRAFT SUPERVISION & 1.0 & LOT & & $\begin{array}{c}\text { MILL } \\
\text { SUBTIE } \\
\end{array}$ & 143.000 & $\overline{143}$ & 4,473 & . & & & 4,473 \\
\hline & Bulk Sucrose, Nozzle \& Cyclone Fan Pulley & $\mathbf{s} \pi$ & & & & & 428 & $\$ 13,388$ & & $\$ 3,600$ & & $\$ 16,988$ \\
\hline 1.3 .15 .1 .2 & $\begin{array}{l}\text { Bulk Sucrose. Nozzle \& Cyclone } \\
\text { System Balancing }\end{array}$ & & & & & & & & & & & \\
\hline
\end{tabular}


Lockheed Martin Idaho Technologies Co. Rov. 6196

PROJECT NAME. Recalcination Treatment Non-Separations Cementitious Waste Option

LOCATION 1: INEEL - ICPP

REQUESTOR: Al Lee 6.9716 MS 3765

\section{DETAILED COST ESTIMATE SHEET}

TYPE OF ESTIMATE: Planning

PROJECT NO.: 2420

PREPARED BY: JRB/BCE
PAGE 17

DATE 03-Feb-1998

TIME: 16:40:26

REPORT NAME: Detall Cost Estimate Sheet

\begin{tabular}{|c|c|c|c|c|c|c|c|c|c|c|c|c|}
\hline CODE & DESCRIPTION & aTr & Uom & $\begin{array}{c}\text { MATL } \\
\text { UNIT COST }\end{array}$ & $\begin{array}{l}\text { CREW } \\
\text { SUB }\end{array}$ & $\begin{array}{l}\text { UNITLAB } \\
\text { HOURS }\end{array}$ & $\begin{array}{l}\text { TOTAL } \\
\text { LAB HRS }\end{array}$ & LABOR & $\begin{array}{l}\text { CONST. } \\
\text { EQUIP. }\end{array}$ & MATLL & $\begin{array}{c}\text { STC } \\
\text { (OTHER 1) }\end{array}$ & $\begin{array}{l}\text { TOTAL } \\
\text { COST }\end{array}$ \\
\hline & BALANCE SYSTEM AFTER PULLLEY CHANGES & 1.0 & LOT & & SUBTIE & 0.000 & & & & & 40,000 & 40,000 \\
\hline & $\begin{array}{l}\text { Bulk Sucrose, Nozzle \& Cyclone System Ba } \\
\text { S/T }\end{array}$ & ancing & & & & & 0 & & & & $\$ 40,000$ & $\$ 40,000$ \\
\hline \multirow[t]{4}{*}{$\frac{1.3 .15,2.1}{100}$} & $\begin{array}{l}\text { Cyclone Replacement } \\
\text { DISCONNECT PIPING FROM CYCLONE: }\end{array}$ & 0.0 & & & & & & & & & & \\
\hline & CUT \& REMOVE PIPE: $1 / 2$ " TO $11 / 2 "$ & 24.0 & $\overline{E A}$ & & $\begin{array}{c}\text { PIPE } \\
\text { SUBTIE }\end{array}$ & 3.750 & 90 & 3,311 & & & & 3,311 \\
\hline & CUT \& REMOVE PIPE: $6^{\prime \prime}$ & 2.0 & $\overline{\mathrm{EA}}$ & & $\begin{array}{c}\text { PIPE } \\
\text { SUBTIE }\end{array}$ & 7.500 & 15 & 562 & & & & 552 \\
\hline & CUT \& REMOVE PIPE: $16^{\prime \prime}$ & 4.0 & $\overline{E A}$ & & $\begin{array}{c}\text { PIPE } \\
\text { SUBTIE }\end{array}$ & 18.750 & 75 & 2,759 & & & & 2,769 \\
\hline \multirow{4}{*}{$\begin{array}{l}200 \\
\stackrel{\leftrightarrow}{w}\end{array}$} & $\begin{array}{l}\text { REMOVE \& RELOCATE PIPING TO INSTALL } \\
18^{\prime} \text { CYCLONE (1/2" TO } 1 " \text { PIPES AT } 8^{\prime} \text { TO } \\
\left.13^{\prime}\right)\end{array}$ & 0.0 & & & & & & & & & & \\
\hline & PIPE CUTS & 20.0 & $\overline{E A}$ & & $\begin{array}{c}\text { PIPE } \\
\text { SUBTIE }\end{array}$ & 3.750 & 76 & 2,759 & & & & 2,769 \\
\hline & NEWPIPE & 50.0 & $\overline{L F}$ & 6.00 & $\begin{array}{c}\text { PIPE } \\
\text { SUBTIA }\end{array}$ & 0.560 & 28 & 1,030 & & 300 & & 1,330 \\
\hline & ELBOWS & 40.0 & EA & 20.00 & $\begin{array}{c}\text { PIPE } \\
\text { SUBTIE }\end{array}$ & 15.000 & 600 & 22,074 & & 800 & & $\overline{22,874}$ \\
\hline \multirow[t]{9}{*}{$\overline{300}$} & NEW CYCLONE & 0.0 & & & & & & & & & & \\
\hline & 16" TEE TAPER BORED TWO PLACES & 1.0 & $\mathrm{EA}$ & $2,600.00$ & $\begin{array}{c}\text { PIPE } \\
\text { SUBTIE }\end{array}$ & 3.760 & $\overline{4}$ & $\overline{138}$ & & 2,600 & & 2,738 \\
\hline & $16^{\prime \prime}$ ELBOW & 2,0 & $E A$ & $1,300.00$ & $\begin{array}{c}\text { PIPE } \\
\text { SUBTIE }\end{array}$ & 3.750 & 8 & 276 & & 2,600 & & 2,876 \\
\hline & $16^{\prime \prime}$ FLANGE & 1.0 & $\mathrm{EA}$ & $1,500.00$ & \begin{tabular}{|} 
PIPE \\
SUBTIE \\
\end{tabular} & 1.900 & 2 & 70 & & 1,600 & & 1,570 \\
\hline & 16" BLIND FLANGE & 1.0 & $E A$ & $1,400.00$ & $\begin{array}{c}\text { PIPE } \\
\text { SUBTIE }\end{array}$ & 1.900 & 2 & 70 & & 1,400 & & 1,470 \\
\hline & $16^{\prime \prime}$ BOLT \& GASKET SET & 1.0 & EA & 200.00 & $\begin{array}{c}\text { PIPE } \\
\text { SUBTIE } \\
\end{array}$ & 26.250 & 26 & 966 & & 200 & & 1,166 \\
\hline & $16^{\prime \prime}$ PIPE & 10.0 & LF & 300.00 & $\begin{array}{l}\text { PIPE } \\
\text { SUBTIE }\end{array}$ & 3.750 & 38 & 1,380 & & 3,000 & & 4,380 \\
\hline & $10^{\prime \prime}$ SHOP WELD & 2.0 & $\overline{E A}$ & & $\begin{array}{c}\text { PIPE } \\
\text { SUBTIE }\end{array}$ & 14.000 & 28 & 1,030 & & & & 1,030 \\
\hline & $16 "$ FIELD WELD & 8.0 & $E A$ & & $\begin{array}{c}\text { PIPE } \\
\text { SUBTIE }\end{array}$ & 78.750 & 630 & 23,178 & & & & 23,178 \\
\hline & & & & & & & & & & & & \\
\hline
\end{tabular}


1.

C-34 
Lockheed Martin Idaho Technologies Co.

Rov. 6/96 Cementilious Waste Option

OCATION 1: INEEL - ICPP

REOUESTOR: AI LeE 6-9716 MS 3765
DETAILED COST ESTIMATE SHEET

TYPE OF ESTIMATE: Planning

PROJECTNO: 2420

PREPARED BY: JRB/BCE
PAGE \# 18

DATE 03-Feb-1998

TIME: 16:40:26

REPORT NAME: Detail Cost Estimate Sheet

\begin{tabular}{|c|c|c|c|c|c|c|c|c|c|c|c|c|}
\hline CODE & DESCRIPTION & QTY & UOM & $\begin{array}{l}\text { MATL } \\
\text { UNIT COST } \\
\end{array}$ & $\begin{array}{l}\text { CREW } \\
\text { SUB }\end{array}$ & $\begin{array}{l}\text { UNITLABT } \\
\text { HOURS }\end{array}$ & $\begin{array}{l}\text { TOTAL } \\
\text { LAB HRS }\end{array}$ & LABOR & $\begin{array}{l}\text { CONST. } \\
\text { EQUIP. }\end{array}$ & MATLL & $\begin{array}{l}\text { STC } \\
\text { (OTHER 1) }\end{array}$ & $\begin{array}{l}\text { TOTRE: } \\
\text { COST }\end{array}$ \\
\hline \multirow[t]{9}{*}{1.3 .15 .2 .1} & $\begin{array}{l}\text { Cyclone Replacement } \\
\text { 6" PIPE }\end{array}$ & 6.0 & LF & 60.00 & $\begin{array}{c}\text { PIPE } \\
\text { SUBTIE }\end{array}$ & 1.500 & 9 & 331 & & 360 & & 691 \\
\hline & 6" PIPE BENDS & 2.0 & $\overline{E A}$ & 300.00 & $\begin{array}{l}\text { PIPE } \\
\text { SUBTIE }\end{array}$ & $\overline{0.000}$ & & & & 600 & & 600 \\
\hline & 6" WN FLANGE & 1.0 & $E \bar{A}$ & 150.00 & $\begin{array}{c}\text { PIPE } \\
\text { SUBTIE }\end{array}$ & 0.750 & 1 & 28) & & 150 & & 178 \\
\hline & 6" BOLT \& GASKET SET & 1.0 & EA & 80.00 & $\begin{array}{c}\text { PIPE } \\
\text { SUBTIE }\end{array}$ & 11.250 & 11 & 414 & & 80 & & 494 \\
\hline & $11 / 2 "$ PIPE & 5.0 & $\mathrm{LF}$ & 9.00 & $\begin{array}{r}\text { PIPE } \\
\text { SUBTIA } \\
\end{array}$ & 0.560 & 3 & $10 \overline{3}$ & & 45 & & 148 \\
\hline & $11 / 2 "$ FIELD BW & 2.0 & $E A$ & & $\begin{array}{c}\text { PIPE } \\
\text { SUBTIE } \\
\end{array}$ & 9.400 & 19 & 692 & & & & 692 \\
\hline & 1"PIPE & 5.0 & LF & 6.00 & $\begin{array}{r}\text { PIPE } \\
\text { SUBTIE }\end{array}$ & 0.560 & 3 & 103 & & 30 & & 133 \\
\hline & 1"WOL & 1.0 & $\overline{E A}$ & 60.00 & $\begin{array}{c}\text { PIPE } \\
\text { SUBTIE }\end{array}$ & 0.000 & & & & 60 & & 60 \\
\hline & T"FIELD BW & 2.0 & $E A$ & & $\begin{array}{c}\text { PIPE } \\
\text { SUBTIE }\end{array}$ & 7.500 & 15 & 552 & & & & 552 \\
\hline \multirow{4}{*}{$\underset{w}{W}$} & $1 / 2^{\prime \prime}$ PIPE & 60.0 & $\mathrm{LF}$ & 6.00 & $\begin{array}{c}\text { PIPE } \\
\text { SUBTIE }\end{array}$ & 0.560 & 34 & 1,236 & & 300 & ! & $\overline{1,636}$ \\
\hline & 1/2" BENDS & 40.0 & EA & 100.00 & $\begin{array}{c}\text { PIPE } \\
\text { SUBTIL } \\
\end{array}$ & 0.000 & & & & 4,000 & & 4,000 \\
\hline & $1 / 2 " \mathrm{WOL}$ & 1.0 & $E A$ & 60.00 & $\begin{array}{r}\text { PIPE } \\
\text { SUBTIE }\end{array}$ & 0.000 & & & & 50 & & 50 \\
\hline & 1/2" FIELD BW & 24.0 & $\mathrm{EA}$ & & $\begin{array}{c}\text { PIPE } \\
\text { SUBTIE } \\
\end{array}$ & 7.500 & 180 & 6,622 & & & & 6,622 \\
\hline \multirow[t]{8}{*}{400} & PIPE TESTING: & 0.0 & & & & & & & & & & $r$ \\
\hline & VISUAL \& LP YEST-IN SHOP $=16^{\prime \prime}$ & 2.0 & EA & & $\begin{array}{c}\text { PIPE } \\
\text { SUBTIE }\end{array}$ & 2.000 & 4 & 147 & & & & 147 \\
\hline & VISUAL \& LP TEST-IN SHOP <16" & 40.0 & $\overline{E A}$ & & $\begin{array}{c}\text { PIPE } \\
\text { SUBTIE } \\
\end{array}$ & 0.500 & 20 & 736 & & & & 736 \\
\hline & VISUAL \& LP TEST- $<16^{\prime \prime}\left(8^{\prime}\right.$ TO 13') & 40.0 & $E A$ & & $\begin{array}{c}\text { PIPE } \\
\text { SUBTIE }\end{array}$ & 1.900 & 76 & 2,796 & & & & 2,796 \\
\hline & VISUAL. \& LP TEST- $<16^{\prime \prime}\left(>13^{\prime}\right)$ & 29.0 & $E A$ & & $\begin{array}{c}\text { PIPE } \\
\text { SUBTIE } \\
\end{array}$ & 1.900 & 55 & 2,027 & & & & 2,027 \\
\hline & VISUAL \& LP TEST $=16^{\prime \prime}\left(>13^{\prime}\right)$ & 8.0 & EA & & $\begin{array}{c}\text { PIPE } \\
\text { SUBTIEE }\end{array}$ & 7.500 & 60 & 2,207 & & & & 2,207 \\
\hline & X-RAY SHOP & 4.0 & $E A$ & & SUBTIE & 0.000 & & & & & 200 & 200 \\
\hline & X-RAY - $\angle 16^{\prime \prime}\left(8^{\prime}\right.$ TO $\left.13^{\prime}\right)$ & 4.0 & $E A$ & & SUBTIE & 0.000 & & & & & 2,200 & 2,200 \\
\hline & & & & & & & & & & & & \\
\hline & & & & & & & & & & & & \\
\hline
\end{tabular}


Lockheed Martin Idaho Technologies Co.

Rov 6/93

Cementltious Waste Option

LOCATION 1. INEEL - ICPP

REQUESTOR. Al Lee 6-9716 MS 3765

\section{DETAILED COST ESTIMATE SHEET}

TYPE OF Estimate: Planning

PROJECT NO.: 2420

PREPARED BY: JRB/BCE
PAGE\# 19

DATE 03-Fob-1998

TIME: 16:40:26

REPORT NAME: Detail Cost Estimate Sheet

\begin{tabular}{|c|c|c|c|c|c|c|c|c|c|c|c|c|}
\hline CODE & DESCRIPTION & QTY & UOM & $\begin{array}{l}\text { MATL } \\
\text { UNIT COST }\end{array}$ & $\begin{array}{l}\text { CREW } \\
\text { SUB }\end{array}$ & $\begin{array}{l}\text { UNITLAB } \\
\text { HOURS }\end{array}$ & $\begin{array}{l}\text { TOTAL } \\
\text { LAB HRS }\end{array}$ & LABOR & $\begin{array}{l}\text { CONST. } \\
\text { EQUIP. }\end{array}$ & MAT'L & $\begin{array}{l}\text { STC } \\
\text { (OTHER 1) }\end{array}$ & $\begin{array}{l}\text { TOTAL } \\
\text { COST }\end{array}$ \\
\hline \multirow[t]{2}{*}{$1.3 .15,2.1$} & $\begin{array}{l}\text { Cyclone Replacement } \\
X-R A Y-<16^{\prime \prime}\left(>13^{\prime}\right)\end{array}$ & 3.0 & EA & & SURTIE & 0.000 & & & & & 1,650 & 1,650 \\
\hline & $X-R A Y==16^{\prime \prime}\left(>13^{\prime \prime}\right)$ & 3.0 & $\overline{E A}$ & & & 0.000 & & & & & 4,500 & 4,500 \\
\hline \multirow[t]{2}{*}{500} & CRAFT SUPERVISION & 1.0 & LOT & & $\begin{array}{c}\text { PIPE } \\
\text { SUBTIE }\end{array}$ & 352.000 & 352 & 12,950 & & & & 12,950 \\
\hline & Cyclone Replacement S/T & & & & & & 2,461 & $\$ 90,537$ & & $\$ 18,075$ & $\$ 8,650$ & $\$ 117,162$ \\
\hline \multirow[t]{2}{*}{$\begin{array}{l}1,3,15,2,2 \\
100\end{array}$} & $\begin{array}{l}\text { Sucrose Addition Pining } \\
\text { EYEWASHISAFETY SHOWER: }\end{array}$ & 0.0 & & & & & & & & & & \\
\hline & RELOCATE EYEWASH/SAFETY SHOWER & 1.0 & LOT & 150.00 & $\begin{array}{l}\text { PIPE } \\
\text { SUBTIE }\end{array}$ & 15.000 & 15 & 552 & & 150 & & 702 \\
\hline \multirow{13}{*}{$\begin{array}{l}200 \\
-\stackrel{\Omega}{\alpha} \\
-\end{array}$} & SUGAR PIPING IN RM.427: & 0.0 & & & & & & & & & & \\
\hline & $11 / 2 "$ FILL LINE A106 PIPE & 100.0 & LF & 2.00 & $\begin{array}{c}\text { PIPE } \\
\text { SUBTIE }\end{array}$ & 0.150 & 15 & 562 & & 200 & $\backslash$ & 752 \\
\hline & ELBOWS & 5.0 & $E A$ & 7.00 & $\begin{array}{l}\text { PIPE } \\
\text { SUBTIE }\end{array}$ & 5.000 & 25 & 920 & & 35 & & 855 \\
\hline & VALVE & 1.0 & $\overline{E A}$ & 200.00 & $\begin{array}{l}\text { PIPE } \\
\text { SUBTIE }\end{array}$ & 6.000 & 6 & 221 & & 200 & & 421 \\
\hline & 1 1/2" RAWWATER LINEA106 PIPE & 20.0 & LF & 2.00 & $\begin{array}{l}\text { PIPE } \\
\text { SUBTIE }\end{array}$ & 0.150 & 3 & 110 & & 40 & & 150 \\
\hline & ELBOW & 3.0 & EA & 7.00 & $\begin{array}{l}\text { PIPE } \\
\text { SUBTIE }\end{array}$ & 5.000 & 16 & 552 & & 21 & & 573 \\
\hline & TEE & 1.0 & $E A$ & 20.00 & $\begin{array}{l}\text { PIPE } \\
\text { SUBTIE }\end{array}$ & 8.000 & 8 & 294 & & 20 & & .314 \\
\hline & VALVE & 2.0 & $E A$ & 200.00 & $\begin{array}{l}\text { PIPE } \\
\text { SUBTIE }\end{array}$ & 6.000 & 12 & 441 & & 400 & & 841 \\
\hline & 1" STEAM \& CONDENSATE LINES A106 PIPE & 100.0 & $\mathrm{LF}$ & 1.50 & $\begin{array}{c}\text { PIPE } \\
\text { SUBTIE }\end{array}$ & 0.160 & 15 & 552 & & 150 & & 702 \\
\hline & ELBOW & 10.0 & $E A$ & 7.00 & $\begin{array}{l}\text { PIPE } \\
\text { SUBTIE }\end{array}$ & 5.000 & 60 & 1,840 & & 70 & & 1,910 \\
\hline & TEE & 2.0 & $\overline{E A}$ & 20.00 & $\begin{array}{c}\text { PIPE } \\
\text { SUBTIE }\end{array}$ & $\overline{8.000}$ & 16 & 689 & & 40 & & 629 \\
\hline & VALVE & 4.0 & EA & 120.00 & $\begin{array}{l}\text { PIPE } \\
\text { SUBTIE }\end{array}$ & 6.000 & 24 & $\overline{883}$ & & 480 & & 1,363 \\
\hline & INSULATION & 150.0 & ELF & 3.00 & $\begin{array}{l}\text { ASBE } \\
\text { SUBTIA }\end{array}$ & 0.250 & 38 & 1,402 & & 450 & & 1,852 \\
\hline & & & & & & & & & & & & \\
\hline & & & & & & & & & & & & \\
\hline
\end{tabular}


Lockheed Martin Idaho Technologies Co. Rov $0 / 96$ PROJECT NAME: Recalcination Treatment Non-Separations Cementitious Waste Option

LOCATION 1: INEEL - ICPP

REQUESTOR: Al Le日 6.9716 MS 3765

\section{DETAILED COST ESTIMATE SHEET}

TYPE OF ESTIMATE: Planning

PROJECTNO:: 2420

PREPARED BY: JRB/BCE
PAGE* 20

DATE 03-Feb-1998

TIME: 16:40:26

REPORT NAME: Detall Cost Estimate Sheet

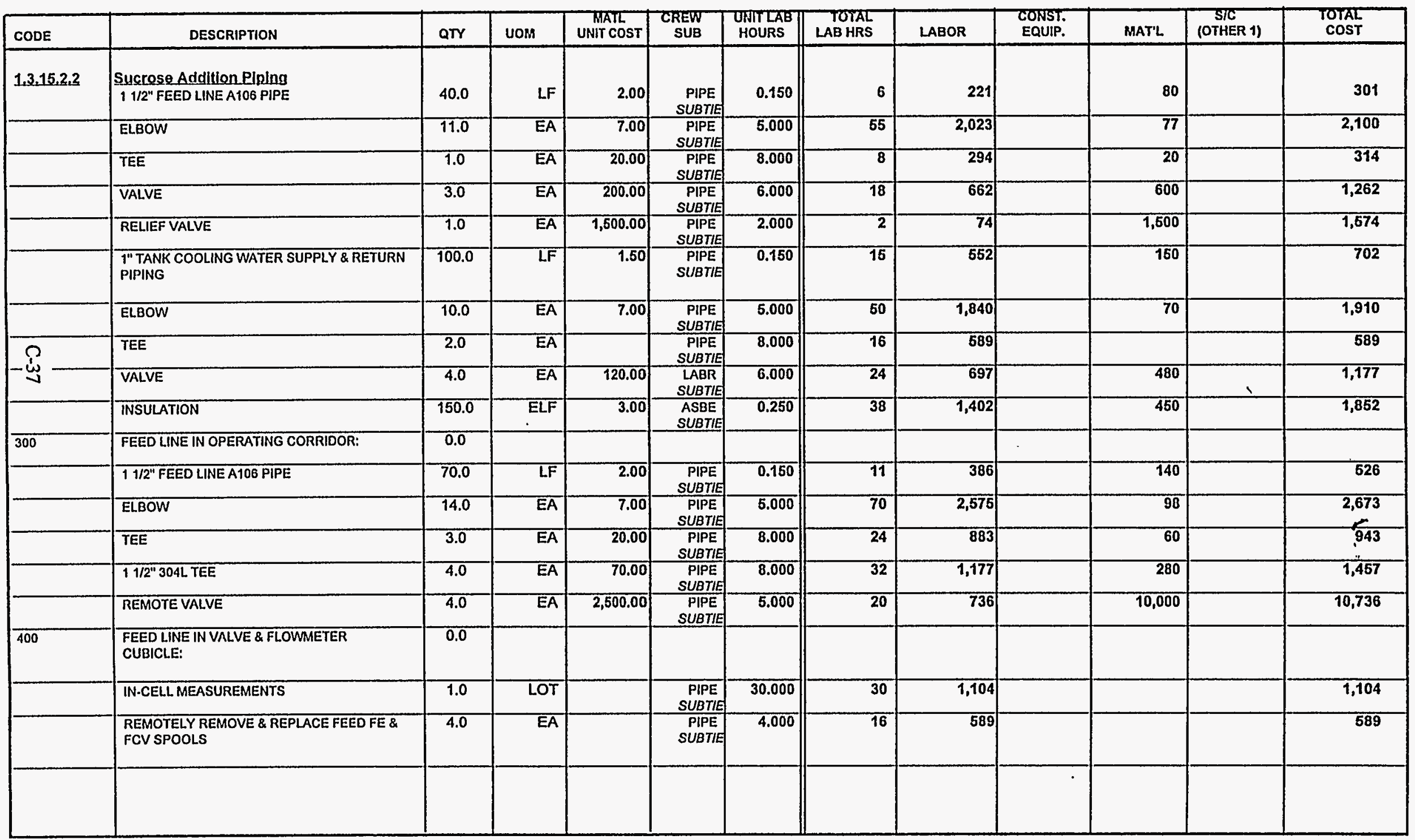


Lockheed Martin Idaho Technologles Co.

$\operatorname{Rav} 6 / 90$

PROJECT NAME: Recalcination Treatment Non-Separations

Cementitious Wasto Option

LOCATION 1: INEEL - ICPP

REQUESTOR' AI Lee 6-9716 MS 3765

\section{DETAILED COST ESTIMATE SHEET}

TYPE OF ESTMATE. Planning

PROJECT NO: 2120

PREPARED BY: JRB/BCE
PAGE \# 21

DATE 03-Fob-1998

TIME: 16:40:26

REPORT NAME: Detail Cost Estimate Sheet

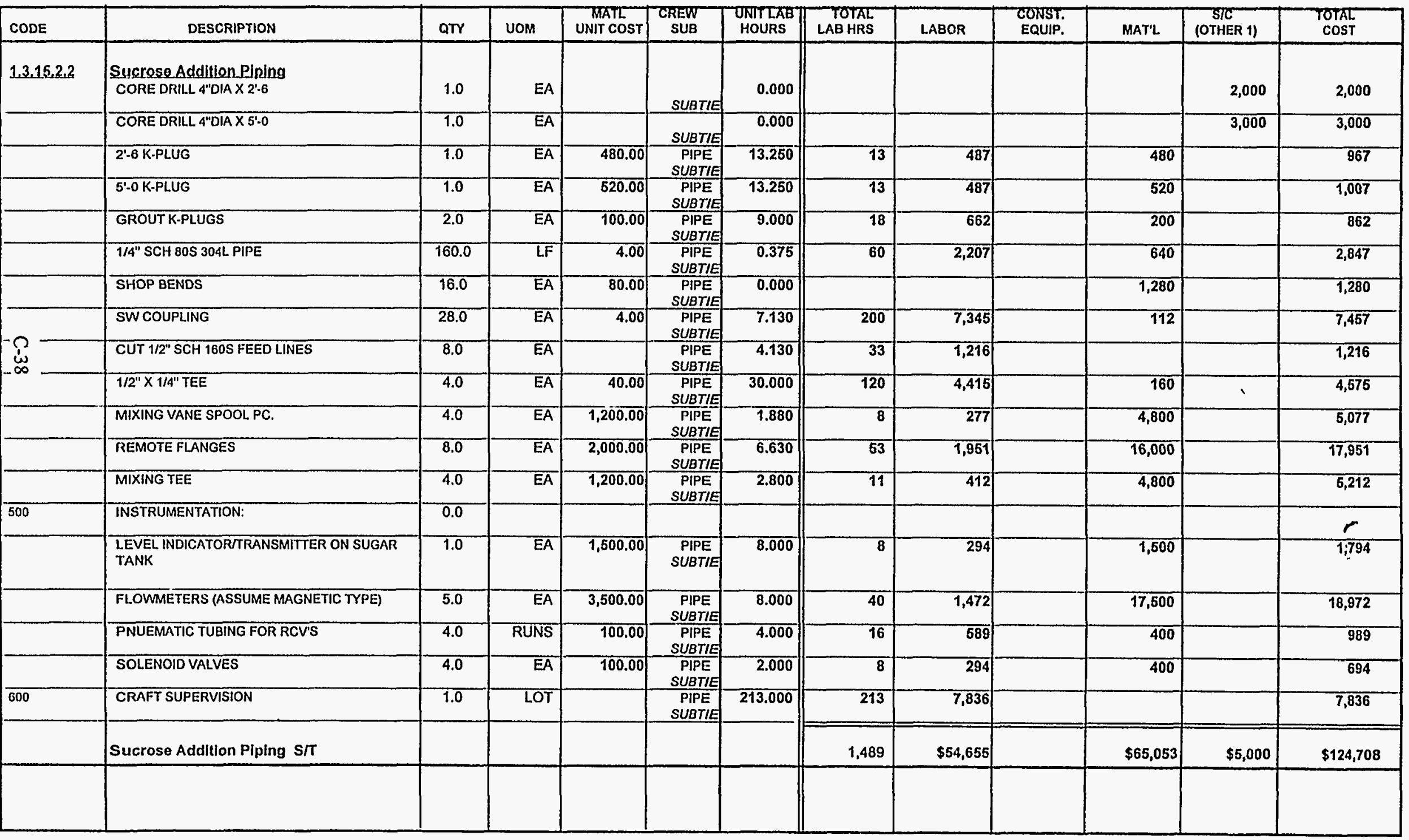


Lockheed Martin Idaho Technologies Co. Rev. G/96 PROJECT NAME: Recalcination Treatment Non-Separations Cementitious Waste Option

LOCATION 1: INEEL - ICPP

REQUESTOR: Al LeO 6-9716 MS 3765

\section{DETAILED COST ESTIMATE SHEET}

TYPE OF Estimate: Planning

PROJECT NO:: 2420

PREPARED BY: JRBIBCE
PAGE \# 22

DATE 03-Feb-1998

TIME: 16:40:26

REPORT NAME: Detail Cost Estimate Sheet

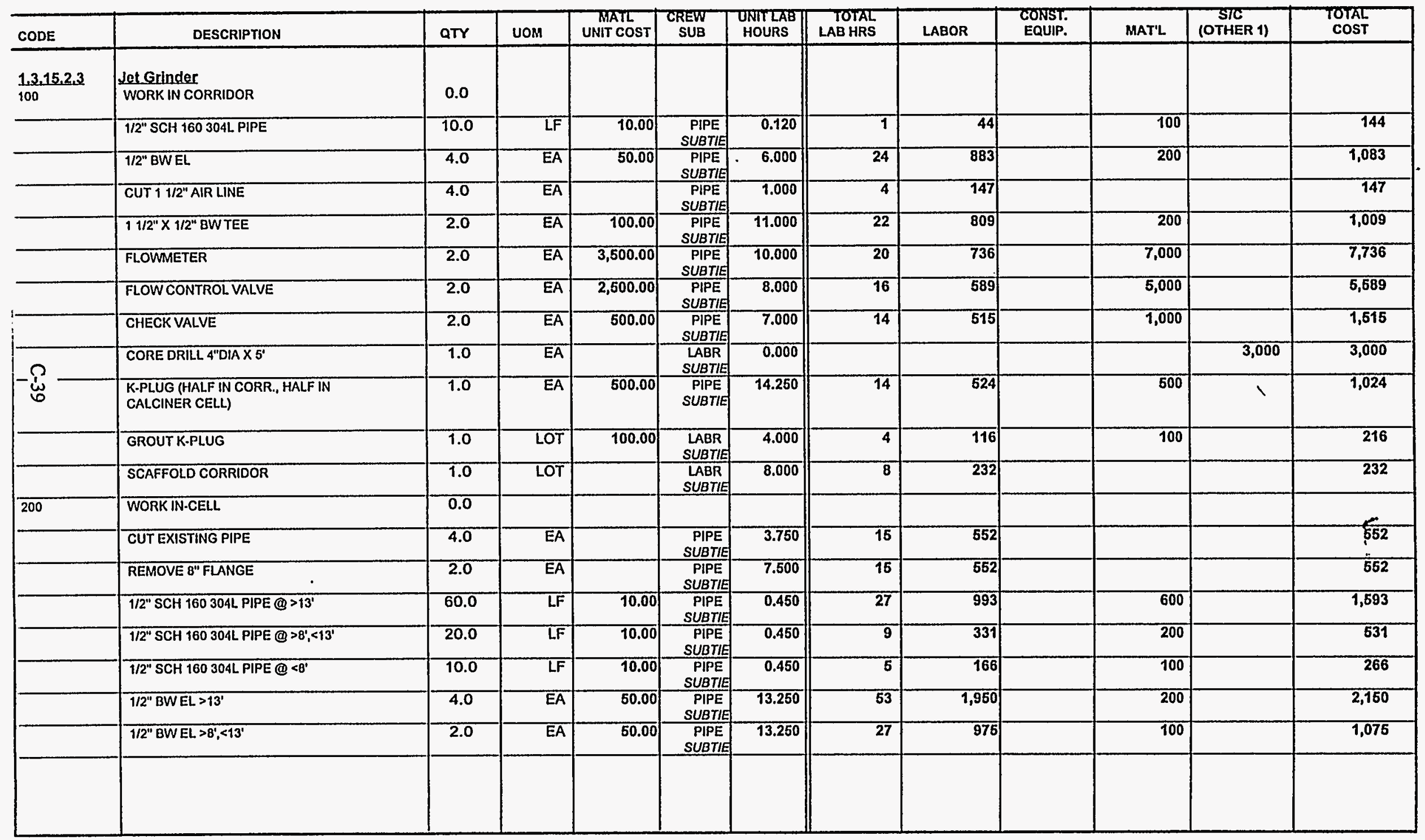


Lockheed Martin Idaho Technologies Co.

Rov 6/96
PROJECT NAME: Recalcination Treatmont Non-Separations Cementitious Wasto Option

LOCATION 1: INEEL - ICPP

REQUESTOR. AI Lee 6-9716 MS 3765

\section{DETAILED COST ESTIMATE SHEET}

TYPE Of Estimate: Planning

PROJECT NO.: 2420

PREPARED BY: JRB/BCE
PAGE \# 23

DATE 03-Feb-1998

TIME: $16: 40: 26$

REPORT NAME: Detail Cost Estimate Sheot

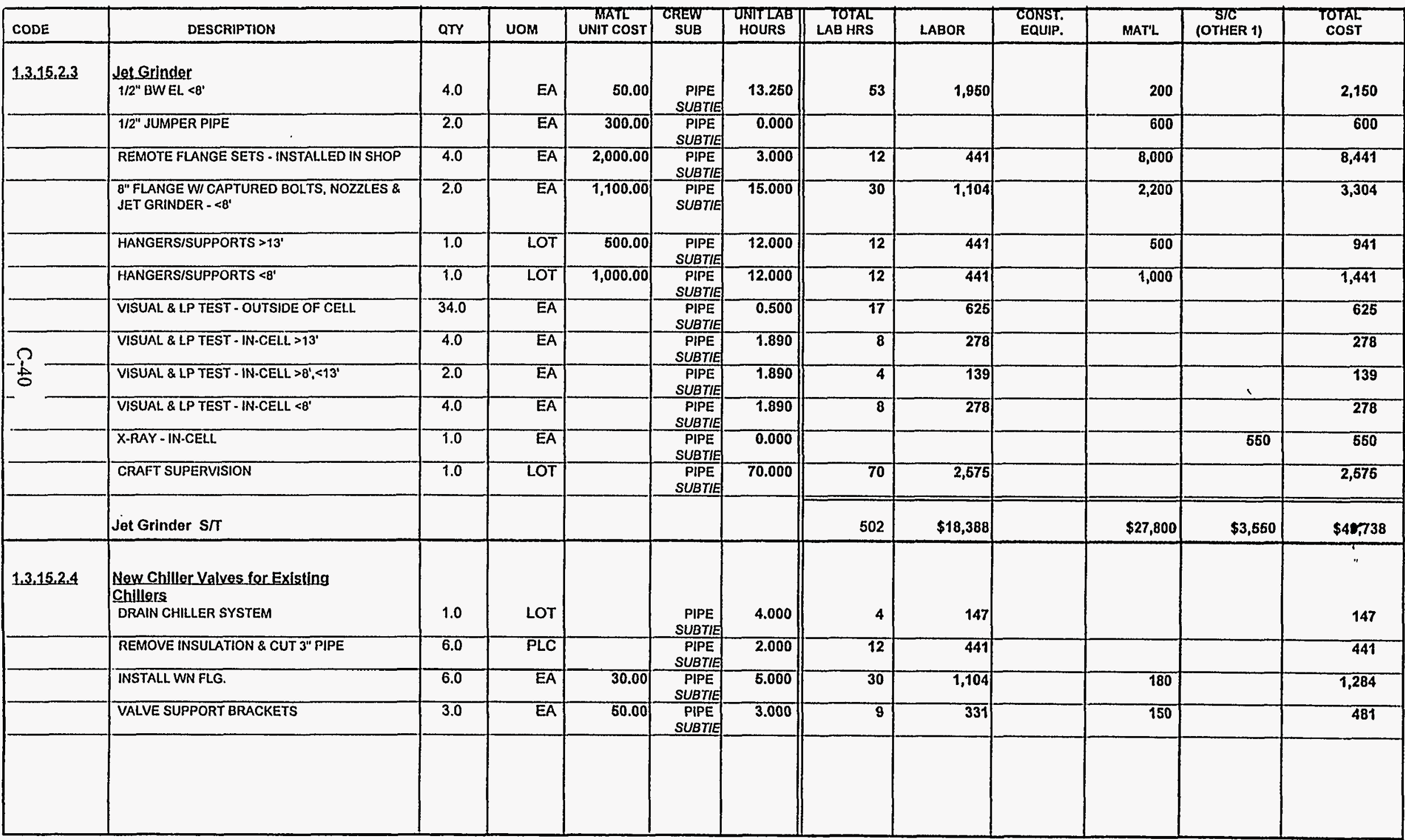


Lockheed Martin Idaho Technologies Co.

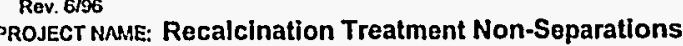

Comentitious Waste Optlon

LOCATION 1: INEEL - ICPP

REQUESTOR: Al Lee 6-9716 MS 3765
DETAILED COST ESTIMATE SHEET

TYPE OF ESTIMATE: Planning

PROJECT NO:: 2420

PREPARED 8Y: JRB/BCE
PAGE \# 24

DATE 03-Feb-1998

TIME: 16:40:26

REPORT NAME: Detall Cost Estimate Sheot

\begin{tabular}{|c|c|c|c|c|c|c|c|c|c|c|c|c|}
\hline CODE & DESCRIPTION & aTY & UOM & $\begin{array}{l}\text { MATL } \\
\text { UNIT COST }\end{array}$ & $\begin{array}{l}\text { CREW } \\
\text { SUB }\end{array}$ & $\begin{array}{l}\text { UNITLAB } \\
\text { HOURS }\end{array}$ & $\begin{array}{l}\text { TOTAL } \\
\text { LAB HRS }\end{array}$ & LABOR & $\begin{array}{l}\text { CONST. } \\
\text { EQUIP. }\end{array}$ & MAT'L & $\begin{array}{l}\text { STC } \\
\text { (OTHER 1) }\end{array}$ & $\begin{array}{l}\text { TOTAL } \\
\text { COST }\end{array}$ \\
\hline $1.3,15,2.4$ & $\begin{array}{l}\text { New Chiller Valves for Existing } \\
\text { Chillers } \\
\text { FLGD.MOTOR-OPERATED VALVES WILIMIT } \\
\text { SWITCHES }\end{array}$ & 3.0 & EA & $3,400.00$ & $\begin{array}{c}\text { PIPE } \\
\text { SUBTIE }\end{array}$ & 4.000 & 12 & 441 & & 10,200 & & 10,641 \\
\hline & 3" BOLT \& GASKET SETS & 6.0 & $\overline{E A}$ & 10.00 & $\begin{array}{l}\text { PIPE } \\
\text { SUBTIE }\end{array}$ & 1.000 & 6 & 221 & & 60 & & 281 \\
\hline & INSULATE VALVES & 3.0 & $\overline{E A}$ & 20.00 & $\begin{array}{c}\text { PIPE } \\
\text { SUBTIE }\end{array}$ & 2.670 & 8 & 295 & & 60 & & 355 \\
\hline & TEST \& CLEAN SYSTEM & 1.0 & LOT & 20.00 & $\begin{aligned} \text { PIPE } \\
\text { SUBTIR } \\
\end{aligned}$ & 16.000 & 16 & 589 & & 20 & & 609 \\
\hline & S.O. TEST & 1.0 & LOT & & $\begin{array}{l}\text { PIPE } \\
\text { SUBTIE }\end{array}$ & 40.000 & 40 & 1,472 & & & & 1,472 \\
\hline & SUPERVISION & 1.0 & LOT & & $\begin{aligned} \text { PIPE } \\
\text { SUBTIE }\end{aligned}$ & 23.000 & 23 & $\overrightarrow{846}$ & & & & 846 \\
\hline$\stackrel{p}{1}$ & Now Chiller Valves for Existing Chillers $\mathrm{S} / \mathrm{T}$ & & & & & $T$ & 160 & $\$ 6,887$ & & $\$ 10,670$ & & $\$ 16,657$ \\
\hline$\frac{1.3,15,2.6}{100}$ & $\begin{array}{l}\text { Scrub Holding Tank Piplng } \\
\text { DISCONNECT \& RECONNECT TANK PIPING }\end{array}$ & 0.0 & & & & & & & & & $\checkmark$ & \\
\hline & $\overline{\text { CUT }<1 " \text { PIPE }}$ & 22.0 & $\overline{E A}$ & & $\begin{array}{l}\text { PIPE } \\
\text { SUBTIE }\end{array}$ & $\overline{3.750}$ & 83 & 3,035 & & & & 3,035 \\
\hline & CUT 11/2" PIPE & 6.0 & $\overline{E A}$ & & $\begin{array}{c}\text { PIPE } \\
\text { SUBTIE }\end{array}$ & $\overline{4.900}$ & $\overline{20}$ & 1,082 & & & & 1,002 \\
\hline & CUT 2" PIPE & 4.0 & $\overline{E A}$ & & $\begin{array}{l}\text { PIPE } \\
\text { SUBTIE } \\
\end{array}$ & 5.600 & $\overline{22}$ & 824 & & & & 824 \\
\hline & CUT 3" PIPE & 8.0 & $\overline{E A}$ & & $\begin{array}{c}\text { PIPE } \\
\text { SUBTIE }\end{array}$ & 7.500 & 60 & 2,207 & & & & $2 ; 207$ \\
\hline & 1/4" PIPE & 20.0 & LF & 4.00 & $\begin{array}{l}\text { PIPE } \\
\text { SUBTIE }\end{array}$ & 0.380 & 8 & 280 & & 80 & & 360 \\
\hline & $\overline{B W}$ & 10.0 & $\overline{E A}$ & & $\begin{array}{l}\text { PIPE } \\
\text { SUBTIE }\end{array}$ & 9.400 & 9 & 3,468 & & & & 3,458 \\
\hline & 1/2" PIPE & 16.0 & LF & 10.00 & $\begin{array}{l}\text { PIPE } \\
\text { SUBTIE }\end{array}$ & 0.450 & 7 & 265 & & 160 & & 425 \\
\hline & $\overline{B W}$ & 8.0 & $\overline{E A}$ & & $\begin{array}{c}\text { PIPE } \\
\text { SUBTIE }\end{array}$ & 11.260 & 90 & 3,311 & & & & 3,311 \\
\hline & 1"PIPE & 8.0 & $\overline{L F}$ & 14.00 & $\begin{array}{c}\text { PIPE } \\
\text { SUBTIE }\end{array}$ & 0.490 & 4 & 144 & & 112 & & 256 \\
\hline & $\overline{B W}$ & 4.0 & $\overline{E A}$ & & $\begin{array}{l}\text { PIPE } \\
\text { SUBTIE }\end{array}$ & 15.000 & 60 & 2,207 & & & & 2,207 \\
\hline & & & & & & & & & & & & \\
\hline
\end{tabular}


Lockheed Martin Idaho Technologles Co. Rav. $6 / 90$

ROJECT NAME: Recalcination Treatment Non-Separations Cementitious Waste Option

LOCATION 1: INEEL. - ICPP

DETAILED COST ESTIMATE SHEET

TYPE OF ESTIMATE: Planning

PROJECT NO:: 2420

PREPARED BY: JRBIBCE
PAgE \# 25

DNTE 03-Fob-1998

TIME: 16:40:26

REPORT NAME: Detall Cost Estimato Sheot

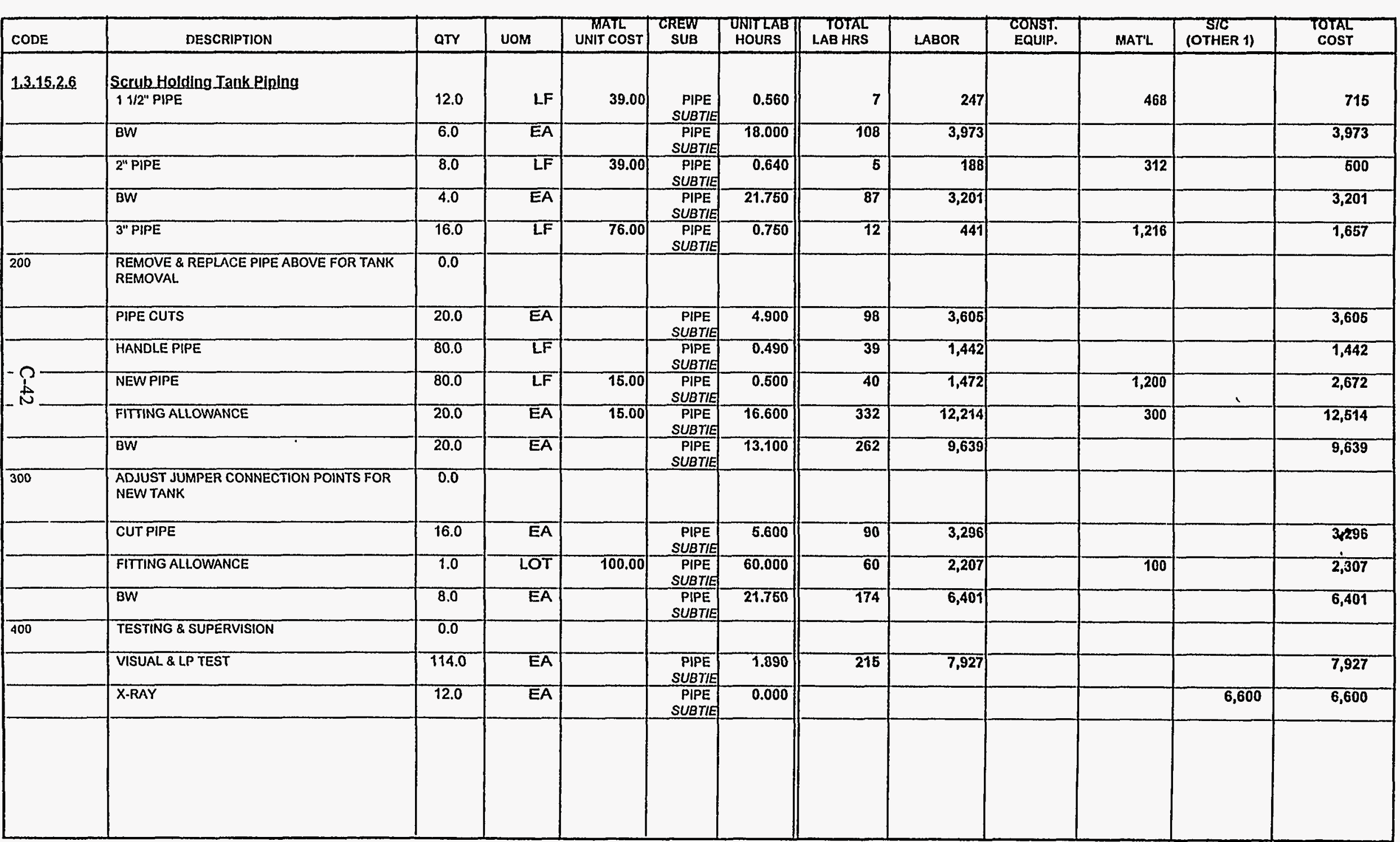


Lockheed Martin Idaho Technologies Co. Rov. 6/96

PROJECT NAME: Recalcination Treatment Non-Separations Comentitious Waste Option

LOCATION 1: INEEL - ICPP

REQUESTOR: AILO 6-9716 MS 3765

\section{DETAILED COST ESTIMATE SHEET}

TYPE OF ESTIMATE: Planning

PROJECTNO:: 2420

PREPARED BY: JRB/BCE
PAGE \# 26

DATE 03-Feb-1998

TIME: 16:40:26

REPORT NAME: Detall Cost Estimate Sheet

\begin{tabular}{|c|c|c|c|c|c|c|c|c|c|c|c|c|}
\hline CODE & DESCRIPTION & QTY & UOM & $\begin{array}{c}\text { MATL } \\
\text { UNIT COST }\end{array}$ & $\begin{array}{l}\text { CREW } \\
\text { SUB }\end{array}$ & $\begin{array}{l}\text { UNITLAB } \\
\text { HOURS }\end{array}$ & $\begin{array}{l}\text { TOTAL } \\
\text { LAB HRS }\end{array}$ & LABOR & $\begin{array}{l}\text { CONST. } \\
\text { EQUIP. }\end{array}$ & MATLL & (OTHER 1) & $\begin{array}{l}\text { TOTAL } \\
\text { COST }\end{array}$ \\
\hline \multirow[t]{2}{*}{1.3 .15 .2 .6} & $\begin{array}{l}\text { Sarub Holding Tank Piping } \\
\text { CRAFT SUPERVISION }\end{array}$ & 1.0 & Lor & & $\begin{array}{c}\text { PIPE } \\
\text { SUBTIE }\end{array}$ & 331.000 & 331 & 12,177 & & & & 12,177 \\
\hline & Scrub Holding Tank Piping S/T & & & & & & 2,317 & $\$ 85,247$ & & $\$ 3,948$ & $\$ 6,600$ & $\$ 95,795$ \\
\hline \multirow[t]{2}{*}{1.3 .16 .1} & $\begin{array}{l}\text { Corresion Monitor } \\
\text { CORROSION MONITORS, MOUNTING \& SIGNAL }\end{array}$ & 1.0 & LOT & & SUBTIE & 0.000 & & & & & 150,000 & 150,000 \\
\hline & Corrosion Monitor S/T & & & & & & 0 & & & & $\$ 150,000$ & $\$ 150,000$ \\
\hline \multirow[t]{4}{*}{$\begin{array}{l}1.3 .16 .2 \\
100\end{array}$} & $\begin{array}{l}\text { Sucrose Addilion } \\
\text { RCV \& INSTRUMENT SIGNALS: }\end{array}$ & 0.0 & & & SUBTIE & & & & & & & \\
\hline & RCV LIMIT SWITCHES & 4.0 & SETS & 600.00 & $\begin{array}{l}\text { ELECC } \\
\text { SUBTIE } \\
\end{array}$ & 4.000 & 16 & 533 & & 2,400 & & 2,933 \\
\hline & RCV CONDUIT \& WIRE & 4.0 & SETS & 500.00 & $\begin{array}{l}\text { ELEC } \\
\text { SUBTIE } \\
\end{array}$ & 20.000 & 80 & 2,665 & & 2,000 & & 4,666 \\
\hline & INSTRUMENT CONDUIT \& WIRE & 6.0 & SETS & 400.00 & \begin{tabular}{|c|} 
ELEEC \\
SUBTIS \\
\end{tabular} & 18.000 & 96 & 3,198 & & 2,400 & & 6,598 \\
\hline \multirow[t]{3}{*}{200} & SUGAR PUMP POWER \& CONTROL: & 0.0 & & & & & & & & & & \\
\hline & $\begin{array}{l}\text { MOTOR STARTER, DISCONNECTS, CONDUIT \& } \\
\text { WIRE }\end{array}$ & 1.0 & LOT & 600.00 & $\begin{array}{l}\text { ELEC } \\
\text { SUBTIE }\end{array}$ & 24.000 & 24 & 799 & & 500 & & $\begin{array}{l}1,299 \\
r\end{array}$ \\
\hline & TESTING & 1.0 & LOT & & $\begin{array}{l}\text { ELEC } \\
\text { SUBTIE }\end{array}$ & 10.000 & 10 & 333 & & & & .333 \\
\hline \multirow[t]{2}{*}{$\overline{300}$} & CRAFT SUPERVISION & 1.0 & LOT & & $\begin{array}{l}\text { ELEC } \\
\text { SUBTIE }\end{array}$ & 100.000 & 100 & 3,331 & & & & 3,331 \\
\hline & Sucrose Addition $\mathrm{S} / \mathrm{T}$ & & & & & & 326 & $\$ 10,859$ & & $\$ 7,300$ & & $\$ 18,169$ \\
\hline \multirow[t]{2}{*}{1.3 .16 .3} & $\begin{array}{l}\text { Jet Grinder } \\
\text { CONDUIT \& WIRE TO DCS FOR FCV }\end{array}$ & 100.0 & LF & 3.00 & $\begin{array}{c}\text { ELEC } \\
\text { SUBTIE } \\
\end{array}$ & 0.300 & 30 & 999 & & 300 & & 1,299 \\
\hline & CONDUIT \& WIRE TO DCS FOR FE & 100.0 & $\overline{\mathrm{LF}}$ & 3.00 & $\begin{array}{l}\text { ELEC } \\
\text { SUBTIE } \\
\end{array}$ & 0.300 & 30 & 999 & & 300 & & 1,299 \\
\hline & & & & & & & & & & & & \\
\hline
\end{tabular}


Lockheed Martin Idaho Technologies Co.

Rov. $6 / 96$ REJECT NAME: Recalcination Treatment Non-Separations Cementitious Waste Option

LOCATION Y INEEL - ICPP

REQUESTOR. Al Lee 6-9716 MS 3765

\section{DETAILED COST ESTIMATE SHEET}

TYPE OF estimate: Planning

PROJECT NO:: 2420

PREPARED BY: JRB/BCE
PAGE 27

DATE 03-Fob-1998

TIME: $16: 40: 26$

REPORT NAME: Detall Cost Estlmate Sheol

\begin{tabular}{|c|c|c|c|c|c|c|c|c|c|c|c|c|}
\hline CODE & DESCRIPTION & QTY & UOM & $\begin{array}{l}\text { MATL } \\
\text { UNIT COST }\end{array}$ & $\begin{array}{l}\text { CREW } \\
\text { SUB }\end{array}$ & $\begin{array}{l}\text { UNITLAB } \\
\text { HOURS }\end{array}$ & $\begin{array}{l}\text { TOTAL } \\
\text { LAB HRS }\end{array}$ & LABOR & $\begin{array}{l}\text { CONST. } \\
\text { EQUIP. }\end{array}$ & MAT'L & $\begin{array}{l}\text { STC } \\
\text { (OTHER 1) }\end{array}$ & $\begin{array}{l}\text { TOTAL } \\
\text { COST }\end{array}$ \\
\hline \multirow[t]{3}{*}{1.3 .16 .3} & $\begin{array}{l}\text { Jet_Grinder } \\
\text { TESTING }\end{array}$ & 1.0 & LOT & & $\begin{array}{c}\text { ELEC } \\
\text { SUBTIE }\end{array}$ & 5.000 & 5 & 167 & & & & 167 \\
\hline & SUPERVISION & 1.0 & $\overline{\text { LOT }}$ & & $\begin{array}{l}\text { ELEC } \\
\text { SUBTIIE }\end{array}$ & 33.000 & $\overline{33}$ & 1,099 & & & & 1,099 \\
\hline & Jet Grinder S $/ T$ & & & & & & 98 & $\$ 3,264$ & & $\$ 600$ & & $\$ 3,864$ \\
\hline \multirow[t]{3}{*}{$1.3,16,4$} & $\begin{array}{l}\text { Cyclone Replacement } \\
\text { CUT \& REMOVE TE CONDUITS }\end{array}$ & 4.0 & EA & & $\begin{array}{c}\text { ELEC } \\
\text { SUBTIE }\end{array}$ & 3.000 & 12 & 400 & & & & 400 \\
\hline & RECONNECT TE TO NEW CYCLONE & 4.0 & $\overline{E A}$ & 130.00 & $\begin{array}{c}\text { ELEC } \\
\text { SUBTIE }\end{array}$ & 15.750 & $\overline{63}$ & 2,099 & & 620 & & 2,619 \\
\hline & CRAFT SUPERVISION & 1.0 & LOT & & $\begin{array}{c}\text { ELEC } \\
\text { SUBTIE }\end{array}$ & 25.000 & 25 & 833 & & & & 833 \\
\hline$\stackrel{b}{t}$ & Cyclone Replacement S/T & & & & & & 100 & $\$ 3,331$ & & $\$ 520$ & & $\$ 3,851$ \\
\hline \multirow[t]{3}{*}{1.3 .16 .5} & $\begin{array}{l}\text { New Chiller Valves } \\
\text { POWER \& CONTROLS FOR VALVES }\end{array}$ & 3.0 & EA & $1,000.00$ & $\begin{array}{c}\text { ELEC } \\
\text { SUBTIE } \\
\end{array}$ & 20.000 & 60 & 1,999 & 60 & 3,000 & & 5,059 \\
\hline & CRAFT SUPERVISION & 1.0 & LOT & & $\begin{array}{c}\text { ELEC } \\
\text { SUBTIE }\end{array}$ & 20.000 & 20 & 666 & & & & 666 \\
\hline & New Chiller Valves $\mathrm{S} / \mathrm{T}$ & & & & & & 80 & $\$ 2,665$ & $\$ 60$ & $\$ 3,000$ & & $\$ 5 ; 725$ \\
\hline \multirow[t]{7}{*}{$\frac{1.3 .16 .5}{100}$} & $\begin{array}{l}\text { Quench Tower Modifications } \\
\text { TETT }\end{array}$ & 1.0 & $E A$ & $1,100.00$ & $\begin{array}{c}\text { ELEC } \\
\text { SUBTIE }\end{array}$ & 30.000 & 30 & 999 & & 1,100 & & 2,089 \\
\hline & TEITT TO TANK & 1.0 & $\overline{E A}$ & $1,100.00$ & $\begin{array}{l}\text { ELEC } \\
\text { SUBTII }\end{array}$ & 30.000 & 30 & 999 & & 1,100 & & 2,099 \\
\hline & TE/TT TO QUENCH TANK DRAIN LINE & 1.0 & $\overline{E A}$ & $1,100.00$ & $\begin{array}{l}\text { ELEC } \\
\text { SUBTIE }\end{array}$ & 30.000 & 30 & 999 & & 1,100 & & 2,099 \\
\hline & SST FLEX WHIP TO TANK TE & 1.0 & $\overline{E A}$ & 100.00 & $\begin{array}{c}\text { ELEC } \\
\text { SUBTIE }\end{array}$ & 11.250 & 11 & 375 & & 100 & & 475 \\
\hline & GULTON PLUG FOR TANK TE & 1.0 & $E A$ & $1,000.00$ & $\begin{array}{l}\text { ELEC } \\
\text { SUBTIE }\end{array}$ & 11.900 & 12 & 396 & & 1,000 & & 1,396 \\
\hline & CONDUIT, IN.CELLSST & 80.0 & LF & 30.00 & $\begin{array}{l}\text { ELEC } \\
\text { SUBTIII }\end{array}$ & 1.500 & 120 & $\begin{array}{l}, 997 \\
\end{array}$ & & 2,400 & & 6,397 \\
\hline & & & & & & & & & & & & \\
\hline
\end{tabular}


Lockheed Martin Idaho Technologies Co.

ROJECT NAME: Recalcination Treatment Non-Separations Cementitious Waste Option

LOCATION 1: INEEL - ICPP

REQUESTOR: Al LeE 6-9716 MS 3765
DETAILED COST ESTIMATE SHEET

TYPE OF ESTIMATE: Planning

PROJECTNO: 2420

PREPARED OY: JRBIBCE
PAGE \# 28

DATE 03-Feb-1998

TIME: 16:40:26

REPORT NAME: Detall Cost Estimate Sheet

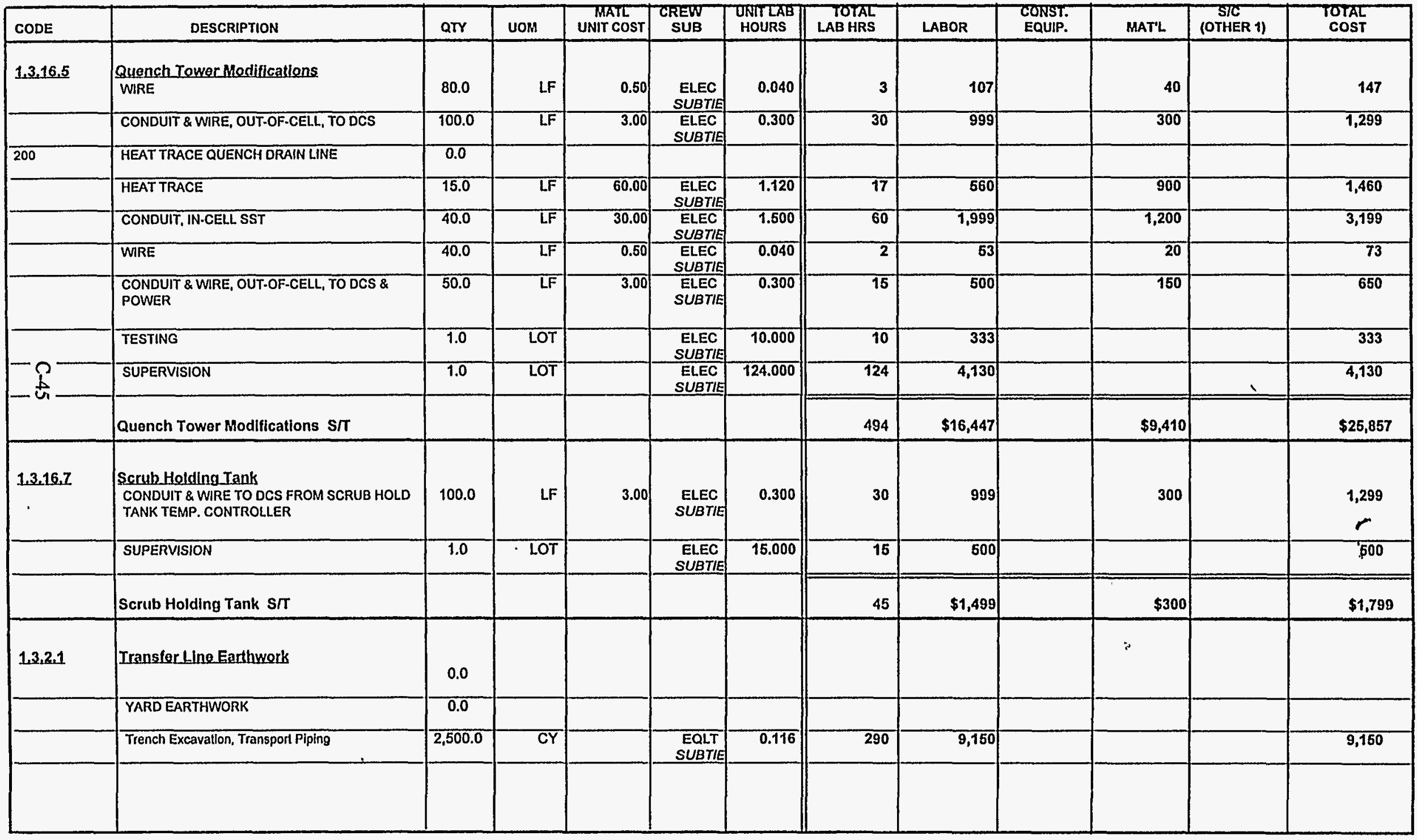


Lockheed Martin Idaho Tochnologies Co. Rov. 6/96

PROJECT NAME- Recalcination Treatmont Non-Separations Cemontitious Waste Option

LOCATION 1: INEEL - ICPP

DETAILED COST ESTIMATE SHEET

TYPE OF ESTIMATE: Planning

PROJECT NO: 2420

PREPARED BY. JRB/BCE
PAGE \# 29

DATE 03-Fob-1998

TIME: 16:40:26

REPORT NAME: Dotall Cost Estimate Sheet

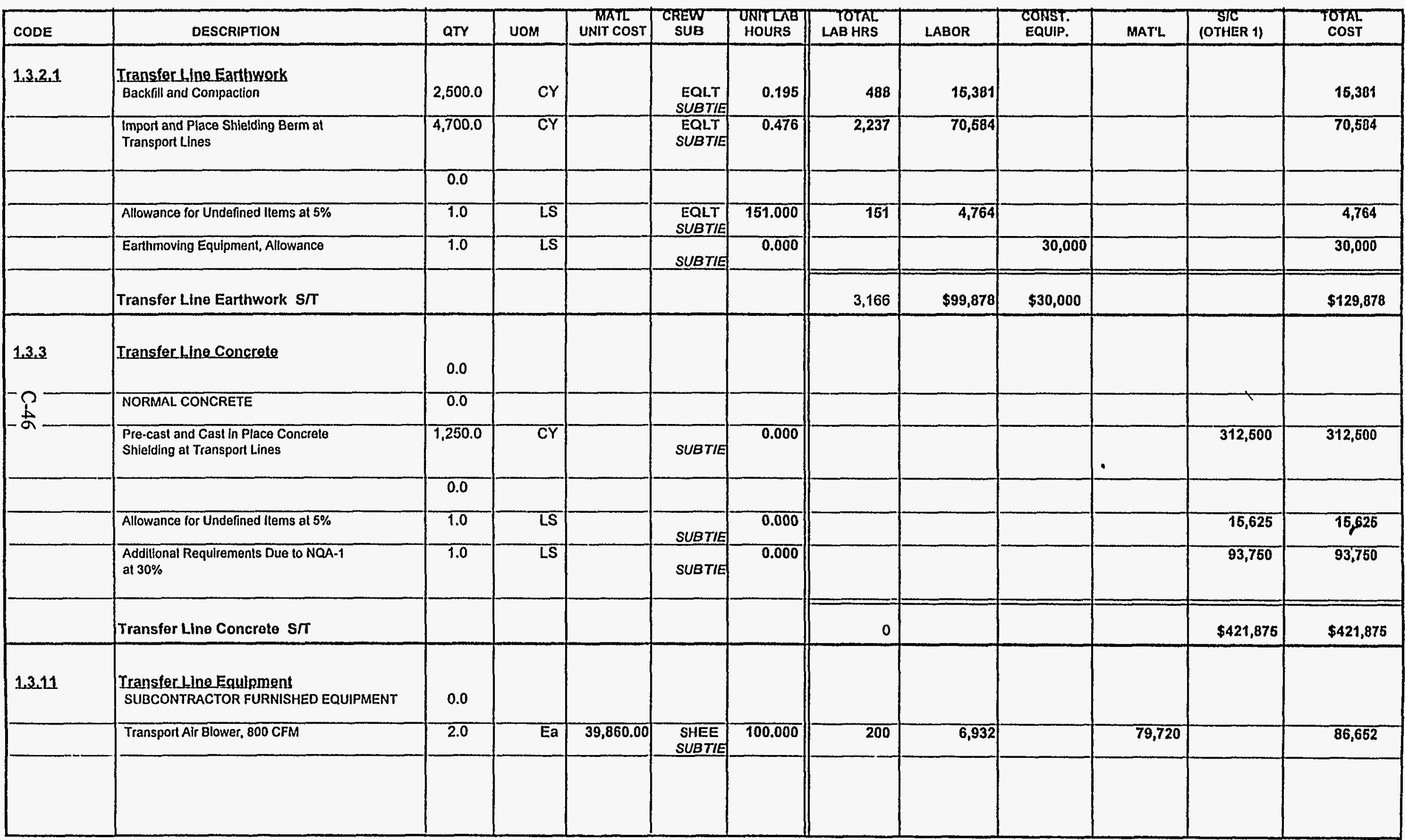


Lockheed Martin Idaho Technologies Co.

Rev 6/96
PROJECT NAME: Recalclnation Treatment Non-Separations Cementitious Waste Option

LOCATION 1: INEEL - ICPP

DETAILED COST ESTIMATE SHEET

TYPE OF EstIMATE: Planning

PROJECT NO:: 2420

PREPARED BY: JRBIBCE
PAGE \# 30

DATE 03-Fob-1998

TIME: 16:40:26

REPORT NAME: Detall Cost Estimate Sheot

REQUESTOR: AI LeO 6-9716 MS 3765

\begin{tabular}{|c|c|c|c|c|c|c|c|c|c|c|c|c|}
\hline CODE & DESCRIPTION & are & UOM & $\begin{array}{l}\text { MATL } \\
\text { UNIT COST }\end{array}$ & $\begin{array}{l}\text { CREW } \\
\text { SUB }\end{array}$ & $\begin{array}{l}\text { UNITLAB } \\
\text { HOURS }\end{array}$ & $\begin{array}{l}\text { TOTAL } \\
\text { LAB HRS }\end{array}$ & LABOR & $\begin{array}{l}\text { CONST. } \\
\text { EQUIP. }\end{array}$ & MAT'L & $\begin{array}{l}\text { SIC } \\
\text { (OTHER 1) }\end{array}$ & $\begin{array}{l}\text { TOTAL } \\
\text { COST }\end{array}$ \\
\hline \multirow[t]{12}{*}{1.3 .11} & $\begin{array}{l}\text { Transfer Line Egulnment } \\
\text { Balancing Air Blower, } 80 \text { CFM }\end{array}$ & 2.0 & Ea & $1,643.00$ & SHEE & 40.000 & 80 & 2,773 & & 3,286 & & 6,059 \\
\hline & Heal Exchanger & 2.0 & $\mathbf{E a}$ & $11,026.00$ & $\begin{array}{c}\text { SHEE } \\
\text { SUBTII }\end{array}$ & 40.000 & 80 & 2,773 & & 22,052 & & 24,825 \\
\hline & Exhaust Fan, 1,600 CFM (EF-4) & 2.0 & $\mathrm{Ea}$ & $1,778.00$ & $\begin{array}{l}\text { SHEE } \\
\text { SUBTIE }\end{array}$ & 12.000 & 24 & 832 & & 3,556 & & 4,388 \\
\hline & & 0.0 & & & & & & & & & & \\
\hline & INSTALL GOVT FURNISHED EQUIPMENT & 0.0 & & & & & & & & & & \\
\hline & Sintered Metal Filler & 2.0 & Ea & & $\begin{array}{l}\text { SHEE } \\
\text { SUBTIE }\end{array}$ & 400.000 & 800 & 27,728 & & & & 27,728 \\
\hline & Rolary Valve w/Removable Works & 2.0 & $\mathbf{E a}$ & & $\begin{array}{l}\text { PIPE } \\
\text { SUBTIE }\end{array}$ & 12.000 & 24 & 883 & & & & 883 \\
\hline & Glove Poils & 2.0 & $\mathrm{Ea}$ & & $\begin{array}{l}\text { SHEE } \\
\text { SUBTIE }\end{array}$ & 50.000 & 100 & 3,466 & & & & 3,466 \\
\hline & & 0.0 & & & & & & & & & & \\
\hline & Allowance for Undefined llems al $5 \%$ & 1.0 & LS & $6,675.00$ & $\begin{array}{l}\text { SHEE } \\
\text { SUBTIE }\end{array}$ & 69.000 & 69 & 2,392 & & 5,675 & $\checkmark$ & 8,067 \\
\hline & $\begin{array}{l}\text { Addilional Requirements Due to NQA-1 } \\
\text { at } 30 \%\end{array}$ & 1.0 & LS & $35,754.00$ & $\begin{array}{l}\text { SHEE } \\
\text { SUBTIE }\end{array}$ & 437.000 & 437 & 15,146 & & 35,754 & & 50,900 \\
\hline & Transfer Line Equipment $S / T$ & & & & & & 1,814 & $\$ 62,924$ & & $\$ 150,043$ & & $\$ 212,967$ \\
\hline \multirow[t]{6}{*}{1.3 .15 .1} & $\begin{array}{l}\text { Transfer Line PInlug Systems } \\
\text { Transport Air Piplng, 8" Sch } 40 \text { SST }\end{array}$ & $1,800.0$ & LF & 288.00 & $\begin{array}{c}\text { PIPE } \\
\text { SUBTIE }\end{array}$ & 2.760 & 4,968 & 182,773 & & 518,400 & & $\frac{r}{701,173}$ \\
\hline & $\begin{array}{l}\text { Calcine Transport Piplng, \&" Sch } 40 \\
\text { SST }\end{array}$ & $\overline{3,500.0}$ & LF & 96.00 & $\begin{array}{l}\text { PIPE } \\
\text { SUBTIE }\end{array}$ & 1.660 & 5,810 & 213,750 & & 336,000 & & 649,760 \\
\hline & Steam Piping. 1 1/2" Sch 40 C Sul & $3,500.0$ & $\overline{L F}$ & 6.25 & $\begin{array}{c}\text { PIPE } \\
\text { SUBTIE }\end{array}$ & 1.080 & 3,780 & 139,066 & & 21,875 & & 160,941 \\
\hline & Encasement Piping, 6" Sch 40 C SII & $3,500.0$ & LF & 25.00 & $\begin{array}{c}\text { PIPE } \\
\text { SUBTIE } \\
\end{array}$ & 1.960 & 6,860 & 252,379 & & 87,500 & & 339,879 \\
\hline & Rod Out Stations & 5.0 & PICs & $4,000.00$ & $\begin{array}{c}\text { PIPE } \\
\text { SUBTIE }\end{array}$ & 170.000 & 850 & 31,272 & & 20,000 & & 61,272 \\
\hline & Flat Side Diverter Valve, Allowance & 21.0 & Ea & $9,500.00$ & $\begin{array}{c}\text { PIPE } \\
\text { SUBTIE } \\
\end{array}$ & 10.000 & 210 & 7,726 & & 199,500 & & 207,226 \\
\hline & & & & & & & & & & & & \\
\hline
\end{tabular}


Lockheed Martin Idaho Technologies Co. Rov. 6/96

PROJECT NAME: Rocalcination Treatmont Non-Separations Cementilious Waste Option

LOCATION 1: INEEL - ICPP

REQUESTOR AI Lee 6-9716 MS 3765

\section{DETAILED COST ESTIMATE SHEET}

TYPE OF ESTIMATE: Planning

PROJECTNO:: 2420

PREPARED BY: JRB/BCE
PAGE \# 31

DATE 03-Fob-1998

TIME: 16:40:26

REPORT NAME: Detail Cost Estimate Sheet

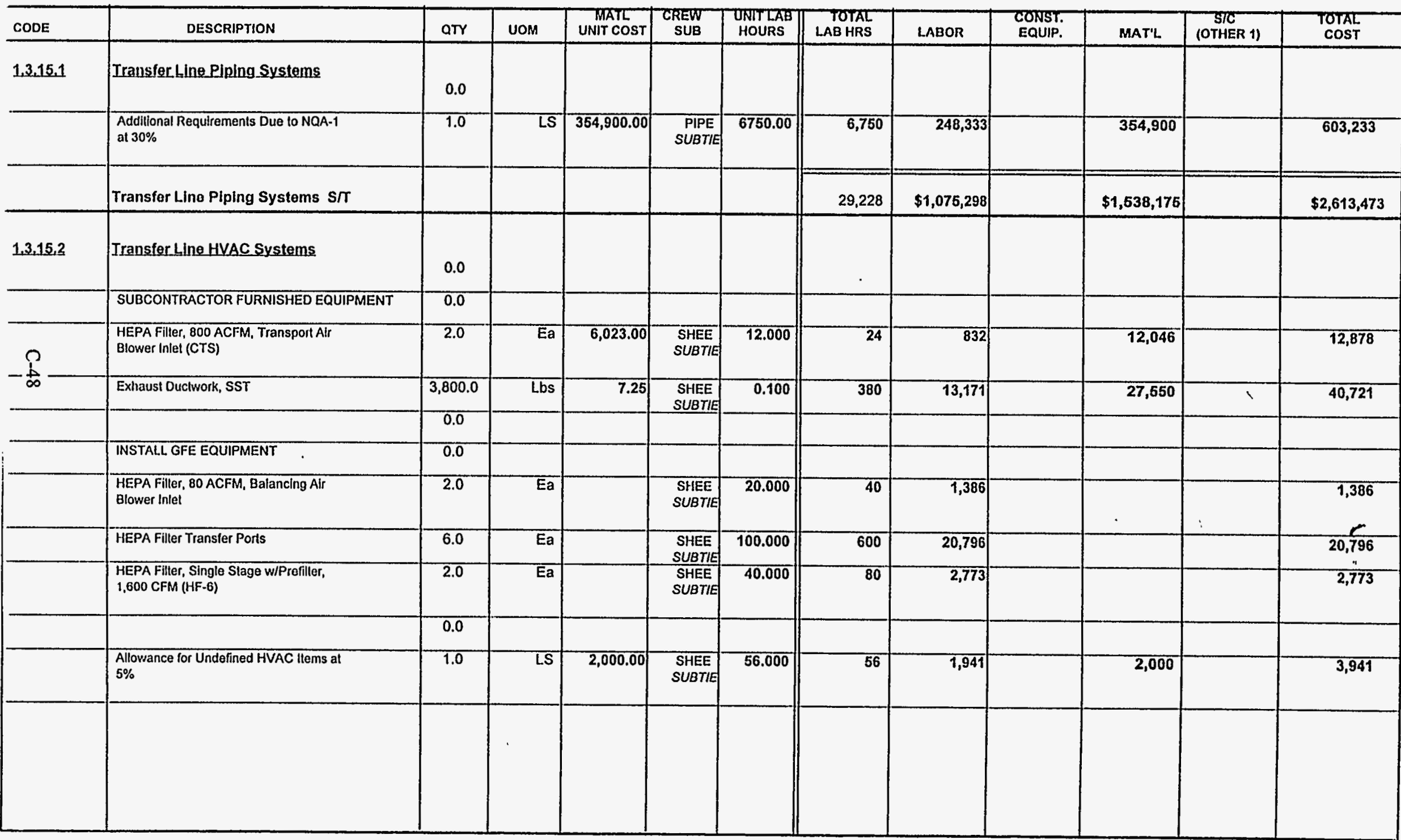


Lockheed Martin Idaho Technologies Co. Rov. $6 / 96$

PROJECT NAME: Recalcination Treatment Non-Separations Cementitlous Waste Option

LOCATION 1: INEEL - ICPP

REQUESTOR: Al Lee 6-9716 MS 3765

\section{DETAILED COST ESTIMATE SHEET}

TYPE OF ESTIMATE: Planning

PROJECTNO: 2420

PREPARED BY: JRB/BCE
PAGE 32

DATE 03-Feb-1998

TIME: 16:40:26

REPORT NAME: Detall Cost Estimate Sheot

\begin{tabular}{|c|c|c|c|c|c|c|c|c|c|c|c|c|}
\hline CODE & DESCRIPTION & QTY & Uom & $\begin{array}{l}\text { MAIL } \\
\text { UNIT COST }\end{array}$ & $\begin{array}{l}\text { CREW } \\
\text { SUB }\end{array}$ & $\begin{array}{l}\text { UNITLAB] } \\
\text { HOURS }\end{array}$ & $\begin{array}{l}\text { TOTAL } \\
\text { LAB HRS }\end{array}$ & LABOR & $\begin{array}{l}\text { CONST. } \\
\text { EQUIP. }\end{array}$ & MAT'L & (OTHER 1) & $\begin{array}{l}\text { TOTAL } \\
\text { COST }\end{array}$ \\
\hline \multirow[t]{2}{*}{$1,3,15,2$} & $\begin{array}{l}\text { Iransfer LineHVAC Systems } \\
\text { Addillonal Requirements Due to NQA-1 } \\
\text { at } 30 \%\end{array}$ & 1.0 & LS & $12,000.00$ & $\begin{array}{l}\text { SHEE } \\
\text { SUBTIE }\end{array}$ & 340.000 & 340 & 11,784 & & 12,000 & & 23,784 \\
\hline & Transfer Line HVAC Systems S/T & & & & & & 1,520 & $\$ 52,683$ & & $\$ 53,596$ & & $\$ 106,279$ \\
\hline \multirow[t]{5}{*}{1.3 .5} & $\begin{array}{l}\text { Bins, Yessels and_Cyclones } \\
\text { Slorage Bins }(15.57 \mathrm{~cm})\end{array}$ & 3.0 & each & $116,000.00$ & $\begin{array}{c}\text { MILL } \\
\text { SUBTIA }\end{array}$ & 200.000 & 600 & 18,768 & & 348,000 & & 366,768 \\
\hline & Cyclones & 2.0 & each & $300,000.00$ & $\begin{array}{c}\text { MILL } \\
\text { SUBTIE }\end{array}$ & 100.000 & 200 & 6,256 & & 600,000 & & 606,256 \\
\hline & Slurry Vessels $(113.26 \mathrm{~cm})$ & 2.0 & $\overline{\text { each }}$ & $273,000.00$ & $\begin{array}{c}\text { MILL } \\
\text { SUBTIL }\end{array}$ & 250.000 & 500 & 15,640 & & 546,000 & & 561,640 \\
\hline & Allowance for NQA-1 Requirements & 2.0 & $\bar{M}$ & & SUBTIE & 0.000 & & & & & 600,000 & 600,000 \\
\hline & Bins, Vessels and Cyclones $S / T$ & & & & & & 1,300 & $\$ 40,664$ & & $\$ 1,494,000$ & $\$ 600,000$ & $\$ 2,134,664$ \\
\hline \multirow[t]{6}{*}{$1,3.7$} & $\begin{array}{l}\text { Pumps and Mlxers } \\
15 \mathrm{gph} \text { Sucrose Pump }\end{array}$ & 1.0 & each & $2,278.00$ & $\begin{array}{c}\text { PIPE } \\
\text { SUBTIE }\end{array}$ & 40.000 & 40 & 1,472 & & 2,278 & & 3,750 \\
\hline & Slurry Pumps & 3.0 & each & $55,000.00$ & $\begin{array}{c}\text { PIPE } \\
\text { SUBTIE }\end{array}$ & 80.000 & 240 & 8,830 & & 165,000 & & 173,830 \\
\hline & Air Sparge Systems & 2.0 & $\overline{\text { each }}$ & $13,650.00$ & $\begin{array}{c}\text { PIPE } \\
\text { SUBTIE }\end{array}$ & 25.000 & 50 & $\overline{1,840}$ & & 27,300 & & $29 / 40$ \\
\hline & $\begin{array}{l}\text { Stalic in Line Mixers (Helical } \\
\text { Nitronic 50) }\end{array}$ & 3.0 & each & $8,000.00$ & $\begin{array}{l}\text { PIPE } \\
\text { SUBTIE }\end{array}$ & 80.000 & 240 & 8,830 & & 24,000 & & 32,830 \\
\hline & Allowance for NQA-1 Requirements & 0.5 & $\bar{M}$ & & SUBTIE & 0.000 & & & & & 150,000 & 150,000 \\
\hline & Pumps and Mixers $S / T$ & & & & & & 570 & $\$ 20,970$ & & $\$ 218,678$ & $\$ 160,000$ & $\$ 389,648$ \\
\hline 1.3 .5 & $\begin{array}{l}\text { Samplo Room Metals } \\
\text { SAMPLER TABLE - ALLOW }\end{array}$ & 1.0 & EA & 600.00 & $\begin{array}{c}\text { PIPE } \\
\text { SUBTIE }\end{array}$ & 15.000 & 16 & 652 & & 600 & & 1,152 \\
\hline & & & & & & & & & & & & \\
\hline
\end{tabular}


Lockhoed Martin Idaho Technologies Co.

nov 6196

PROJECT NAME: Rocalcination Treatment Non-Separations

Comentitious Wasto Option

INEEL - ICPP

REQUESTOR: AI Lee 6-9716 MS 3765

\section{DETAILED COST ESTIMATE SHEET}

TYPE OF ESTIMATE: PJanning

PROJECT NO.: 2420

PREPARED BY. JRB/BCE
PAGE \# 33

DATE 03-Fob-1998

TIME: 16:40:26

REPORT NMME: Detail Cost Estimate Sheet

\begin{tabular}{|c|c|c|c|c|c|c|c|c|c|c|c|c|}
\hline CODE & DESCRIPTION & QTY & UOM & $\begin{array}{l}\text { MATL } \\
\text { UNIT COST }\end{array}$ & $\begin{array}{l}\text { CIREW } \\
\text { SUB }\end{array}$ & $\begin{array}{l}\text { DNITLAB } \\
\text { HOURS }\end{array}$ & $\begin{array}{l}\text { TOTAL } \\
\text { LAB HRS }\end{array}$ & LABOR & $\begin{array}{l}\text { CONST. } \\
\text { EQUIP. }\end{array}$ & MAT'L & $\begin{array}{l}\text { STC } \\
\text { (OTHER 1) }\end{array}$ & $\begin{array}{l}\text { TOTAL } \\
\text { COST }\end{array}$ \\
\hline \multirow[t]{2}{*}{1.3 .5} & Sample Room Metals & & & & & & & & & & & \\
\hline & Sample Room Metals $\mathbf{S} / \mathrm{T}$ & & & & & & 15 & $\$ 552$ & & $\$ 600$ & & $\$ 1,152$ \\
\hline \multirow[t]{2}{*}{1.3 .11} & $\begin{array}{l}\text { Sample Room Equipment } \\
\text { SAMPLERS }\end{array}$ & 2.0 & EA & $10,000.00$ & $\begin{array}{r}\text { PIPE } \\
\text { SUBTIE } \\
\end{array}$ & 45.000 & 90 & 3,311 & & 20,000 & & 23,311 \\
\hline & $\begin{array}{l}\text { TRANSPORT CONTAINERS WI SHIELDED } \\
\text { INSERTS }\end{array}$ & 2.0 & $E A$ & $200,000.00$ & SUBTIE & 0.000 & & & & 400,000 & & 400,000 \\
\hline$\tilde{u}_{-}$ & Sample Room Equipment $\mathbf{S} / \mathrm{T}$ & & & & & & 90 & $\$ 3,311$ & & $\$ 420,000$ & & $\$ 423,311$ \\
\hline \multirow[t]{2}{*}{1.3 .15} & $\begin{array}{l}\text { Sample Room Mechanical } \\
\text { QUICK DISCONNECT HOSES }\end{array}$ & 4.0 & EA & 200.00 & $\begin{array}{l}\text { PIPE } \\
\text { SUBTIA } \\
\end{array}$ & 4.000 & 16 & 689 & & 800 & & 1,389 \\
\hline & Sample Room Mechanical S/T & & & & & & 16 & $\$ 589$ & & $\$ 800$ & & $\$ 1,389$ \\
\hline \multirow[t]{10}{*}{$1,3.4$} & $\begin{array}{l}\text { NWCE Slurny Equlpment } \\
\text { Instrumentalion }\end{array}$ & & & & & & & & & & & \\
\hline & & & & & & & & & & & & 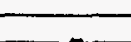 \\
\hline & Differential Pressure & 8.0 & each & & SUBTIE & 0.000 & & & & & 120,000 & 120,000 \\
\hline & Pressure & 15.0 & each & & SUBTIE & 0.000 & & & & & 75,000 & 75,000 \\
\hline & Level & 10.0 & $\overline{\text { each }}$ & & SUBTIE & 0.000 & & & & & 150,000 & 160,000 \\
\hline & Flow & 13.0 & each & & SUBTIE & 0.000 & & & & & 325,000 & 325,000 \\
\hline & Current & 4.0 & each & & SUBTIE & 0.000 & & & & & 40,000 & 40,000 \\
\hline & Temperature & 7.0 & each & & SUBTIE & $\overline{0.000}$ & & & & & 70,000 & 70,000 \\
\hline & Variable Speed Drive & 3.0 & each & & SUBTIE & 0.000 & & & & & 90,000 & 90,000 \\
\hline & Valve Control & 27.0 & each & & SUBTIE & 0.000 & & & & & 405,000 & 405,000 \\
\hline & & & & & & & & & & & & \\
\hline
\end{tabular}


Lockheed Martin Idaho Technologies Co.

Rov 6196

PROJECT NAME: Recalcination Treatment Non-Separations Cementitious Waste Option

LOCATION 1: INEEL - ICPP

REQUESTOR: AIL Le 6-9716 MS 3765

\section{DETAILED COST ESTIMATE SHEET}

TYPE OF ESTIMATE: Planning

PROJECT NO:: 2420

PREPARED BY: JRB/BCE
PAGE * 34

DATE 03-Fob-1998

TIME: 16:40:26

REPORT NAME: Detail Cost Estimate Sheet

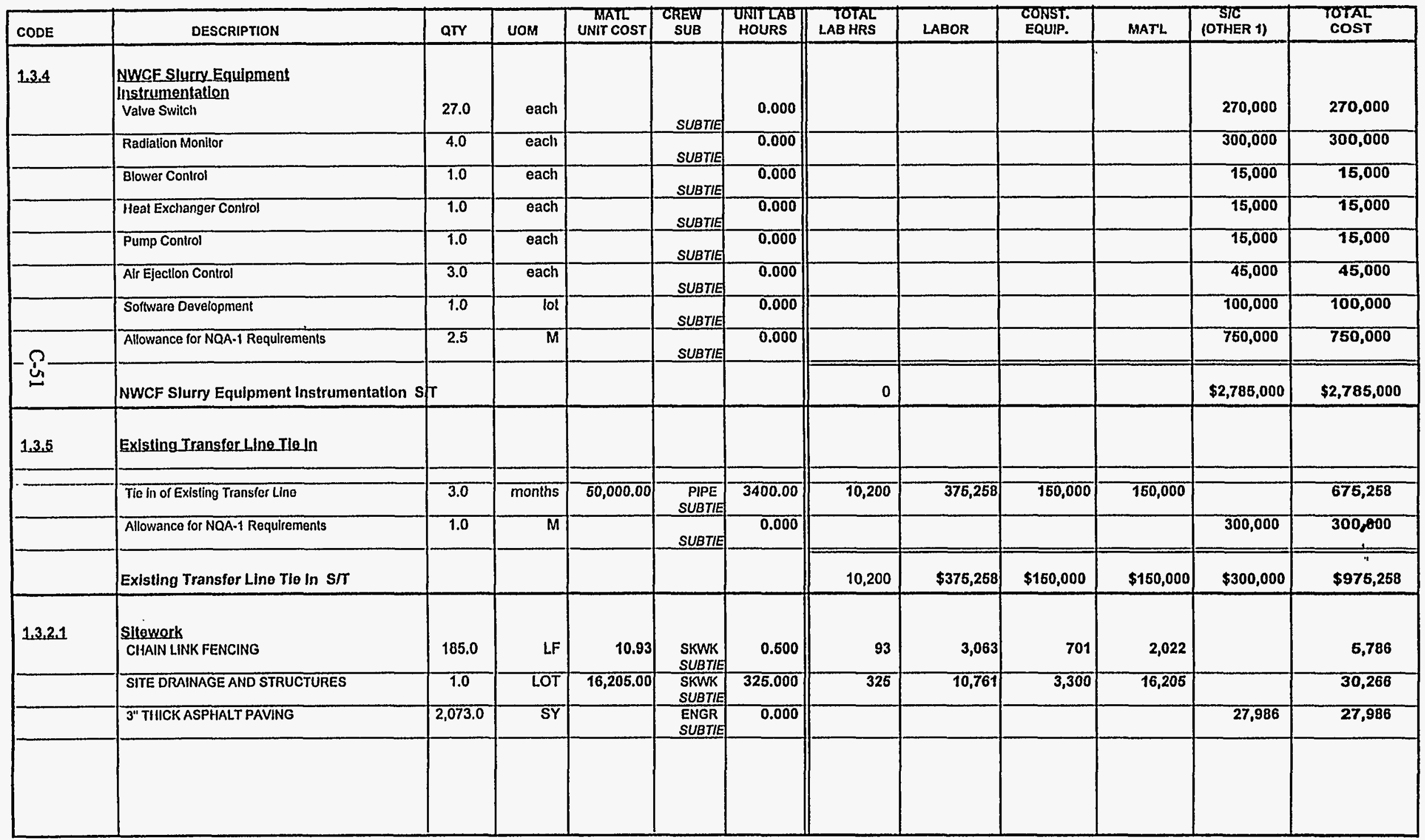


Lockheed Martin Idaho Technologies Co.

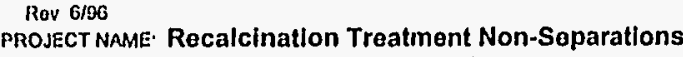

Cementitious Waste Option

LOCATION 1: INEEL - ICPP

REQUESTOR AILO0 G-9716 MS 376

\section{DETAILED COST ESTIMATE SHEET}

TYPE OF estimate: Planning

PROJECTNO.: 2420

PREPARED BY: JRB/BCE
PAGE \# 35

OATE 03-Feb-1998

TIME: 16:40:26

REPort NAME: Detail Cost Estimate Shoet

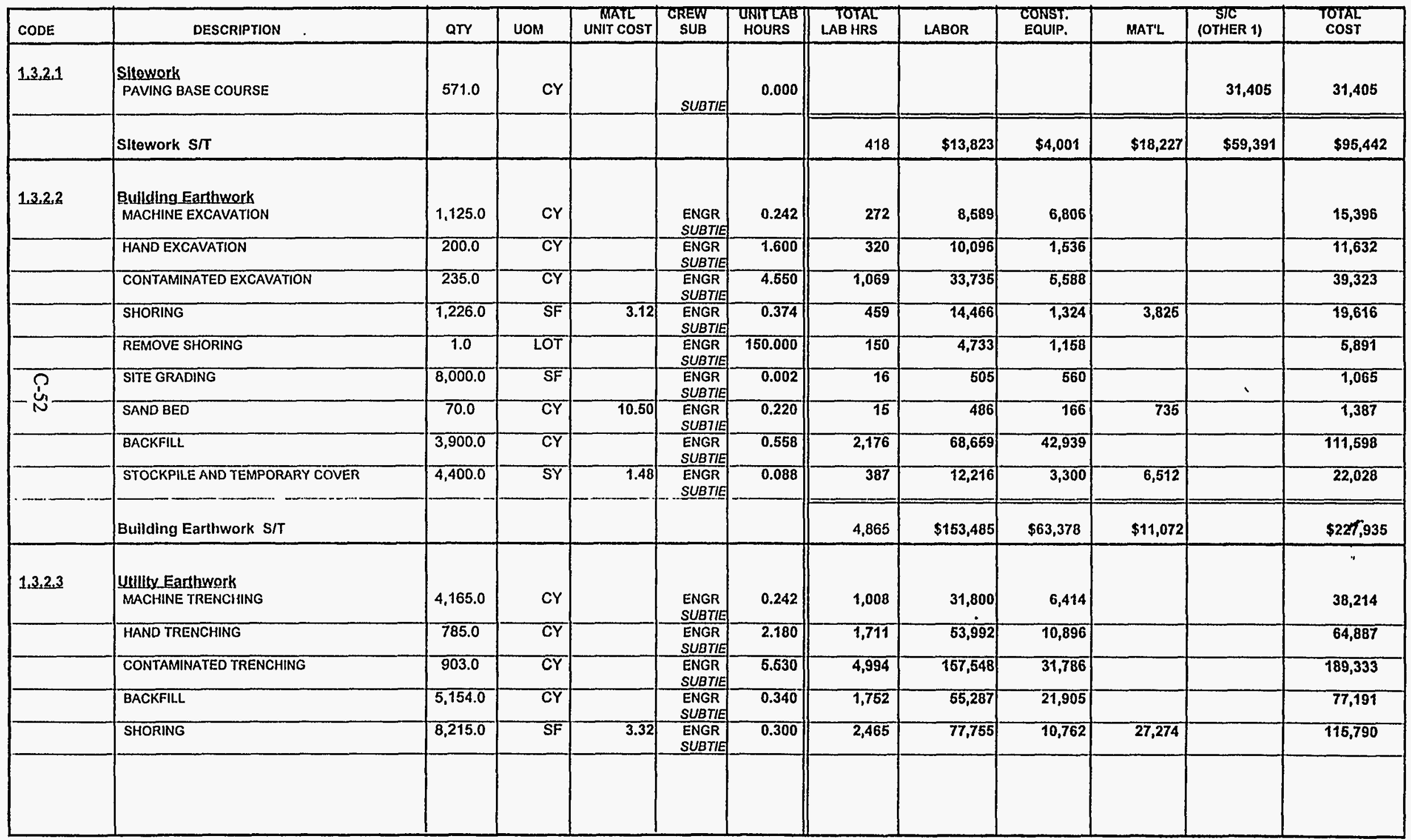


Lockheed Martin Idaho Technologies Co. Rev. $6 / 96$

PROJECT NAME: Recalcination Treatment Non-Separations Cementitious Waste Option

LOCATION 1: INEEL - ICPP

REQUESTOR: AI Le8 6-9716 MS 3765

\section{DETAILẸD COST ESTIMATE SHEET}

TYPE OF ESTIMATE: Planning

PROJECT NO.: 2420

PREPARED BY: JRBIBCE
PAGE* 36

03-Feb-1998

TIME: 16:40:26

REPORT NAME: Detail Cost Estimate Sheet

\begin{tabular}{|c|c|c|c|c|c|c|c|c|c|c|c|c|}
\hline CODE & DESCRIPTION & arr & UOM & $\begin{array}{l}\text { MRTL } \\
\text { UNIT COST }\end{array}$ & $\begin{array}{l}\text { CREW } \\
\text { SUB }\end{array}$ & $\begin{array}{l}\text { UNIILAB } \\
\text { HOURS }\end{array}$ & $\begin{array}{l}\text { TOIAL } \\
\text { LAB HRS }\end{array}$ & LABOR & $\begin{array}{l}\text { CONST. } \\
\text { EQUIP. }\end{array}$ & MATL & $\begin{array}{l}\text { STC } \\
\text { (OTHER 1) }\end{array}$ & $\begin{array}{l}\text { TOTAL } \\
\text { COST }\end{array}$ \\
\hline \multirow[t]{3}{*}{$1,3.2 .3$} & $\begin{array}{l}\text { Utility Earthwork } \\
\text { SAND BEDDING }\end{array}$ & 70.0 & CY & 10.45 & $\begin{array}{l}\text { ENGR } \\
\text { SUBTIE }\end{array}$ & 0.180 & 13 & 398 & 206 & 732 & & 1,335 \\
\hline & STOCKPILE COVER & 650.0 & SY & 1.33 & $\begin{array}{l}\text { ENGR } \\
\text { SUBTIE }\end{array}$ & 0.034 & 22 & 697 & 66 & 865 & & 1,627 \\
\hline & Utility Earthwork $\mathbf{S} \pi$ & & & & & & 11,964 & $\$ 377,476$ & $\$ 82,032$ & $\$ 28,870$ & & $\$ 488,378$ \\
\hline \multirow[t]{3}{*}{$1,3,2,4$} & $\begin{array}{l}\text { Demolition } \\
\text { CUT \& REMOVE ASPHALT }\end{array}$ & $2,100.0$ & sY & & $\begin{array}{l}\text { ENGR } \\
\text { SUBTIE }\end{array}$ & 0.000 & & & & & 18,900 & 18,900 \\
\hline & REMOVE SEPTIC SYSTEM & 1.0 & LOT & & $\begin{array}{l}\text { ENGR } \\
\text { SUBTIE }\end{array}$ & 120.000 & $\overline{120}$ & 3,786 & 1,300 & & 5,000 & 10,086 \\
\hline & REMOVE SIDEWALK & 45.0 & LF & & $\begin{array}{l}\text { ENGR } \\
\text { SUBTIE }\end{array}$ & 0.000 & & & & & 225 & 225 \\
\hline \multirow{4}{*}{$-\stackrel{?}{\dot{w}}$} & REMOVE CONTAMINATED PIPING & 980.0 & $\overline{\mathrm{LF}}$ & & $\begin{array}{l}\text { PIPE } \\
\text { SUBTIE }\end{array}$ & 0.440 & $\overline{431}$ & 15,864 & 1,960 & & & 17,824 \\
\hline & REMOVE GUARD POSTS & 1.0 & LOT & & $\begin{array}{l}\text { ENGR } \\
\text { SUBTIE }\end{array}$ & 15.000 & 15 & 473 & 40 & & ' & 513 \\
\hline & REMOVE FENCE & 107.0 & LF & & $\begin{array}{l}\text { LABR } \\
\text { SUBTIA } \\
\end{array}$ & 0.040 & 4 & 124 & 33 & & & 157 \\
\hline & Demolltion $\mathrm{s} / \mathrm{T}$ & & & & & & 570 & $\$ 20,247$ & $\$ 3,333$ & & $\$ 24,125$ & $\$ 47,706$ \\
\hline \multirow[t]{7}{*}{$1,3,3,1$} & $\begin{array}{l}\text { Utility Concrete } \\
\text { ELECT. DUCTBANK CONCRETE }\end{array}$ & 135.0 & cY & 75.00 & $\begin{array}{l}\text { SSWK } \\
\text { SUBTIE }\end{array}$ & 0.340 & 46 & 1,690 & 406 & 10,125 & & 12,221 \\
\hline & ELECT. DUCTBANK FORMWORK & $\overline{2,515.0}$ & SF & 0.83 & $\begin{array}{l}\text { CARP } \\
\text { SUBTIE }\end{array}$ & 0.110 & 277 & 9,229 & 780 & 2,087 & & 12,096 \\
\hline & ELECT. DUCTBANK REBAR & 5.0 & TON & 700.00 & $\begin{array}{l}\text { RODM } \\
\text { SUBTIE } \\
\text { SU }\end{array}$ & 13.700 & 69 & 2,247 & 870 & 3,600 & & 6,617 \\
\hline & ELECTRICAL MANHOLES & 5.0 & $\overline{E A}$ & $5,170,00$ & $\begin{array}{l}\text { ENGR } \\
\text { SUBTII }\end{array}$ & 35.600 & $\overline{178}$ & $\overline{6,616}$ & 1,630 & 26,850 & & 33,096 \\
\hline & ELECTRICAL HIANDHOLES & 2.0 & $\overline{\mathrm{EA}}$ & 553.00 & $\begin{array}{l}\text { ENGR } \\
\text { SUBTIE }\end{array}$ & 13.100 & 26 & 827 & 254 & 1,106 & & 2,187 \\
\hline & ELECTRICAL EQUIPMENT PADS & 59.0 & $\overline{C Y}$ & 113.00 & $\begin{array}{l}\text { SSWK } \\
\text { SUBTIE }\end{array}$ & 2.000 & 118 & $\overline{4,344}$ & $\overline{912}$ & 6,667 & & 11,922 \\
\hline & Utility Concrete $S / T$ & & & & & & 713 & $\$ 23,952$ & $\$ 4,852$ & $\$ 49,335$ & & $\$ 78,139$ \\
\hline & & & & & & & & & & & & \\
\hline
\end{tabular}


Lockheed Martin Idaho Technologies Co.

Rov $6 / 96$

ROJECT NAME: Recalcination Treatment Non-Separations Cementitious Waste Option

LOCATION 1: INEEL - ICPP

REQUESTOR: Al Lee 6-9716 MS 3765

\section{DETAILED COST ESTIMATE SHEET}

TYPE OF ESTIMATE: Planning

PROJECT NO:: 2420

PREPARED BY: JRB/BCE
PAGE\# 37

DATE 03-Feb-1998

TIME: $16: 40: 26$

REPORT NAME: Detail Cost Estimate Sheet

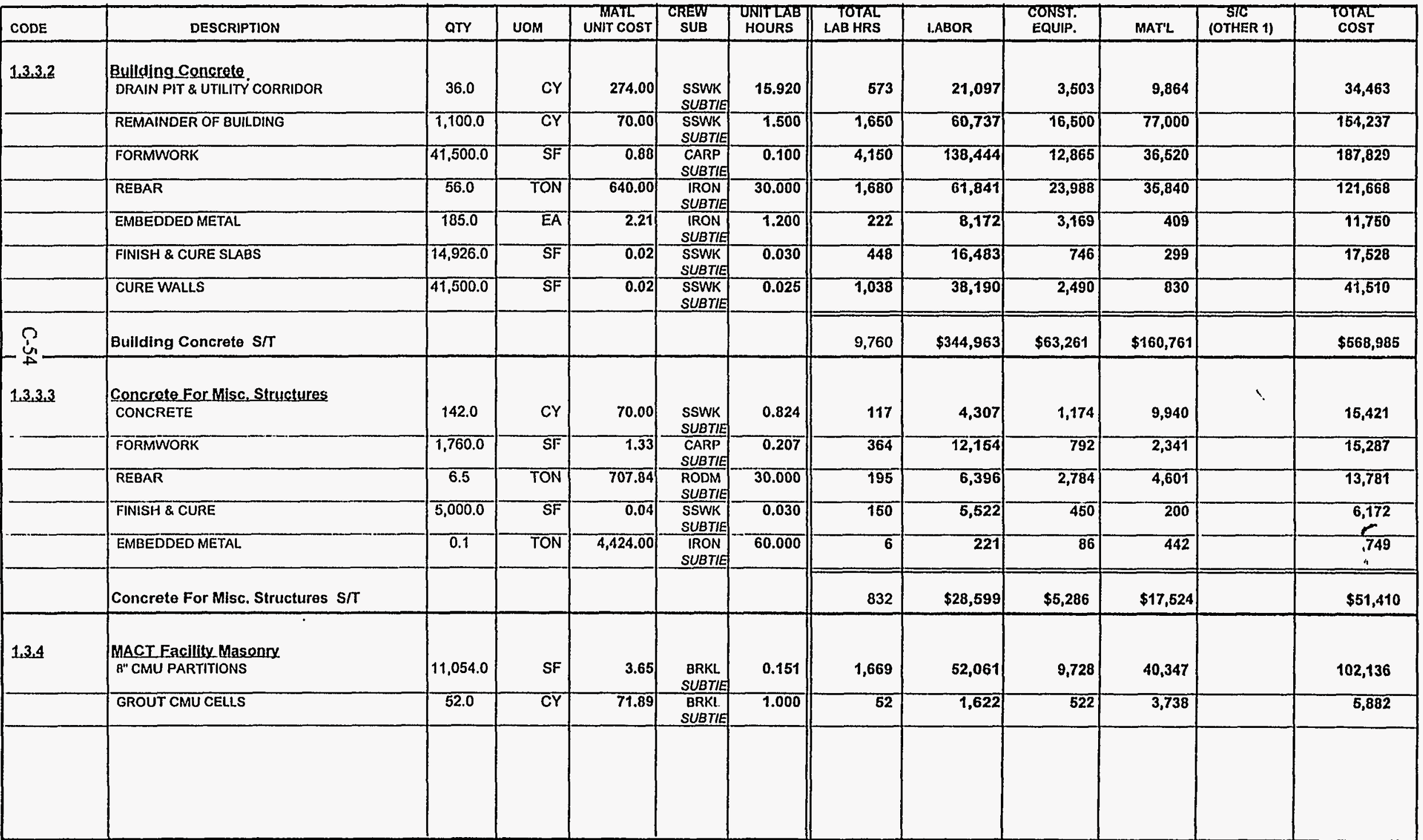


Lockheed Martin Idaho Technologies Co. Rov $6 / 96$

PROJECT NAME: Recalcination Treatment Non-Separations Cementitlous Waste Option

LOCATION 1: INEEL - ICPP

REQUESTOR: AI Le日 6-9716 MS 3765

\section{DETAILED COST ESTIMATE SHEET}

TYPE OF ESTIMATE: Planning

PROJECT NO: 2420

PREPARED BY: JRB/BCE
PAGE 38

DATE 03-Fob-1998

TIME: 16:40:26

REPORT NAME: Detall Cost Estimato Sheet

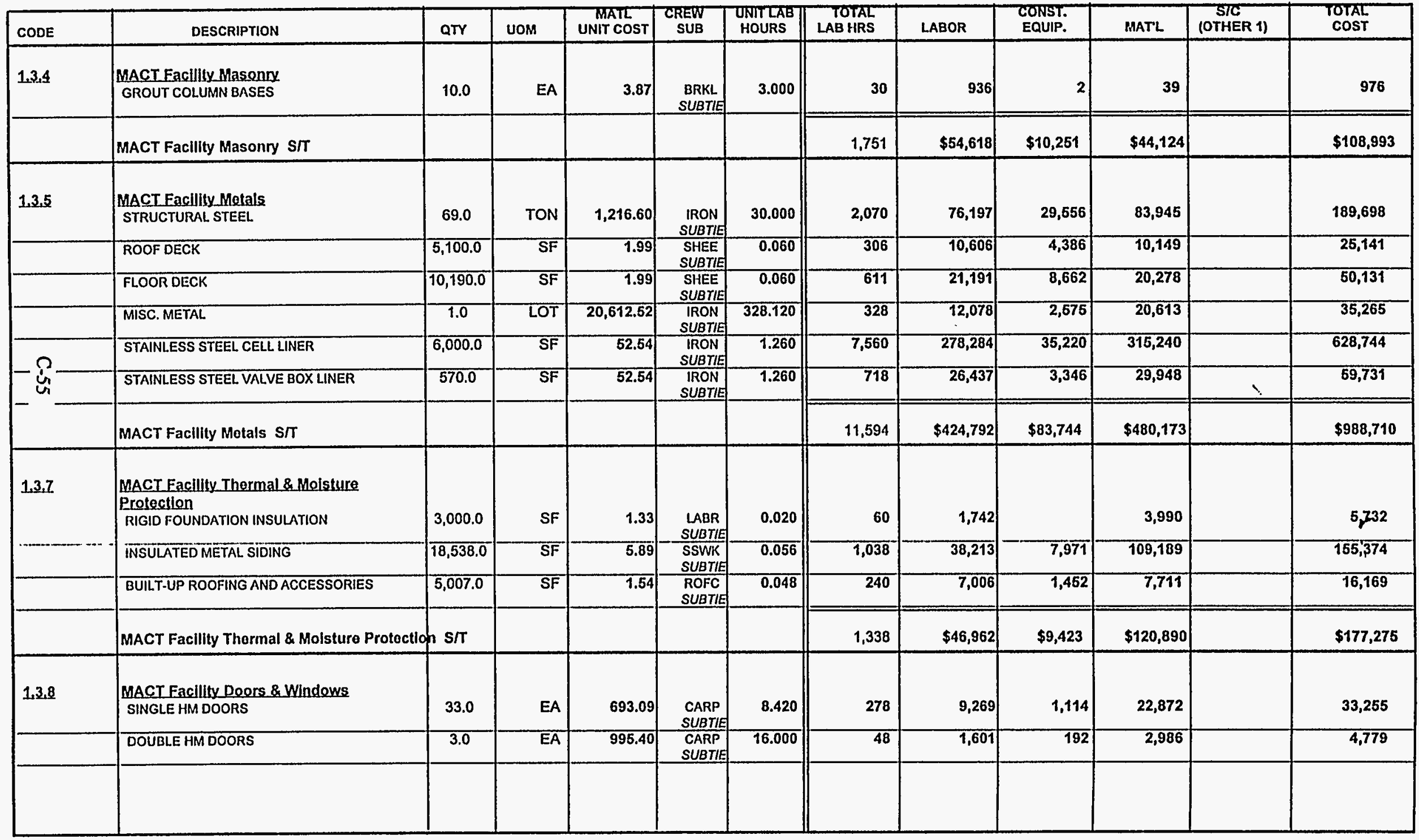


Lockheed Martin Idaho Technologies Co.

Rov $6 / 96$

PROJECT NAME Recalcination Treatment Non-Separations

Cementitious Waste Option

LOCATION: INEEL - ICPP

REQUESTOR: AI LeO 6-9716 MS 3765

\section{DETAILED COST ESTIMATE SHEET}

TYPE OF ESTIMATE: Planning

PROJECT NO: 2420

PREPARED BY: JRB/BCE
PAGE \# 39

DATE 03-Fob-1998

TIME: 16:40:26

REPORT NAME: Detall Cost Estimate Sheet

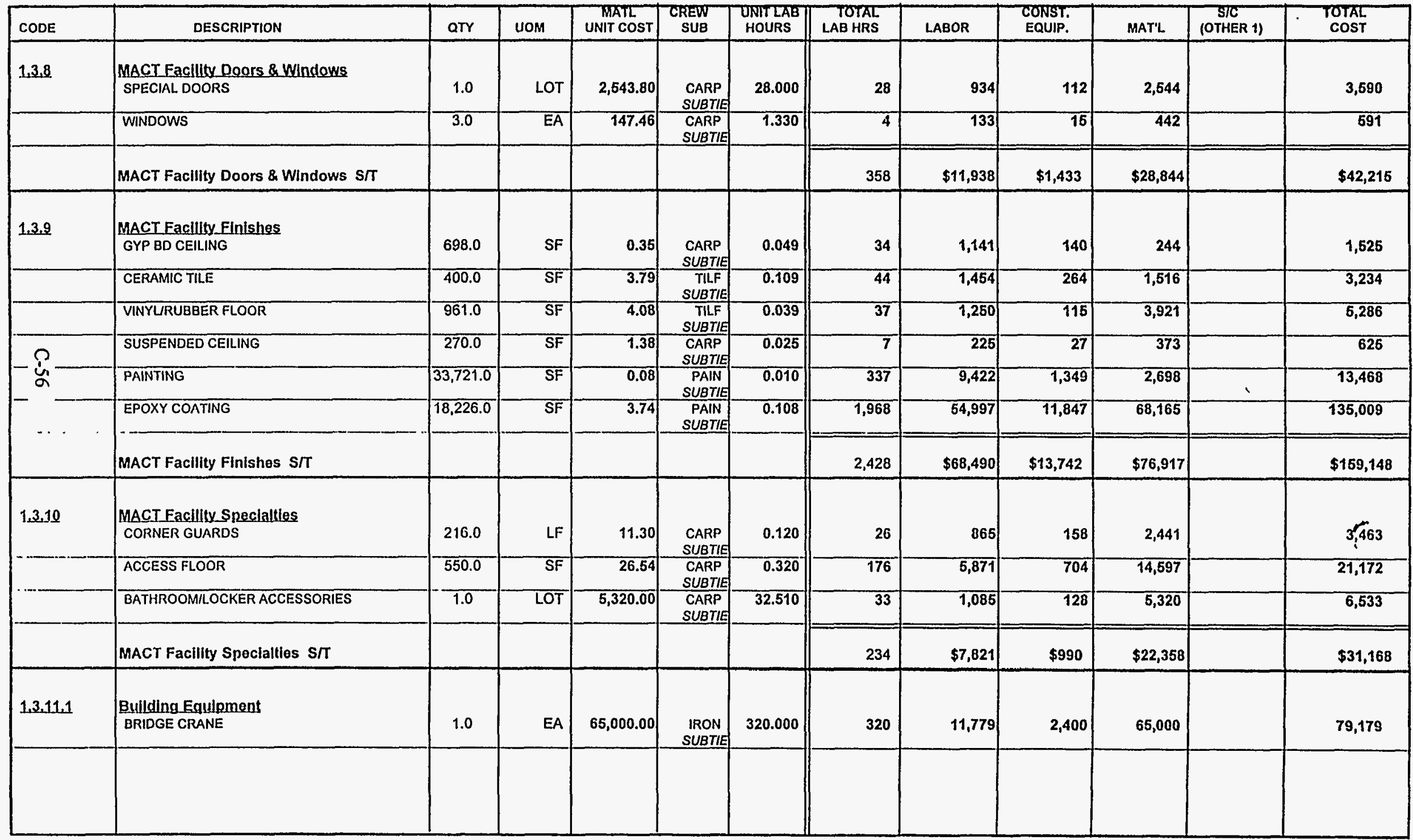


Lockheed Martin Idaho Technologies Co.

Rov. 6/96

PROJECT NAME: Recalcination Treatment Non-Separations

Cementitious Waste Option

INEEL - ICPP

REQUESTOR: AI LOQ 6-9716 MS 3765

\section{DETAILED COST ESTIMATE SHEET}

TYPE OF Estimate: Planning

PROJECT NO:: 2420

PREPARED BY: JRB/BCE
PAGE \# 40

DATE 03-Fob-1998

TIME: 16:40:26

REPORT NAME: Detall Cost Estlmate Sheot

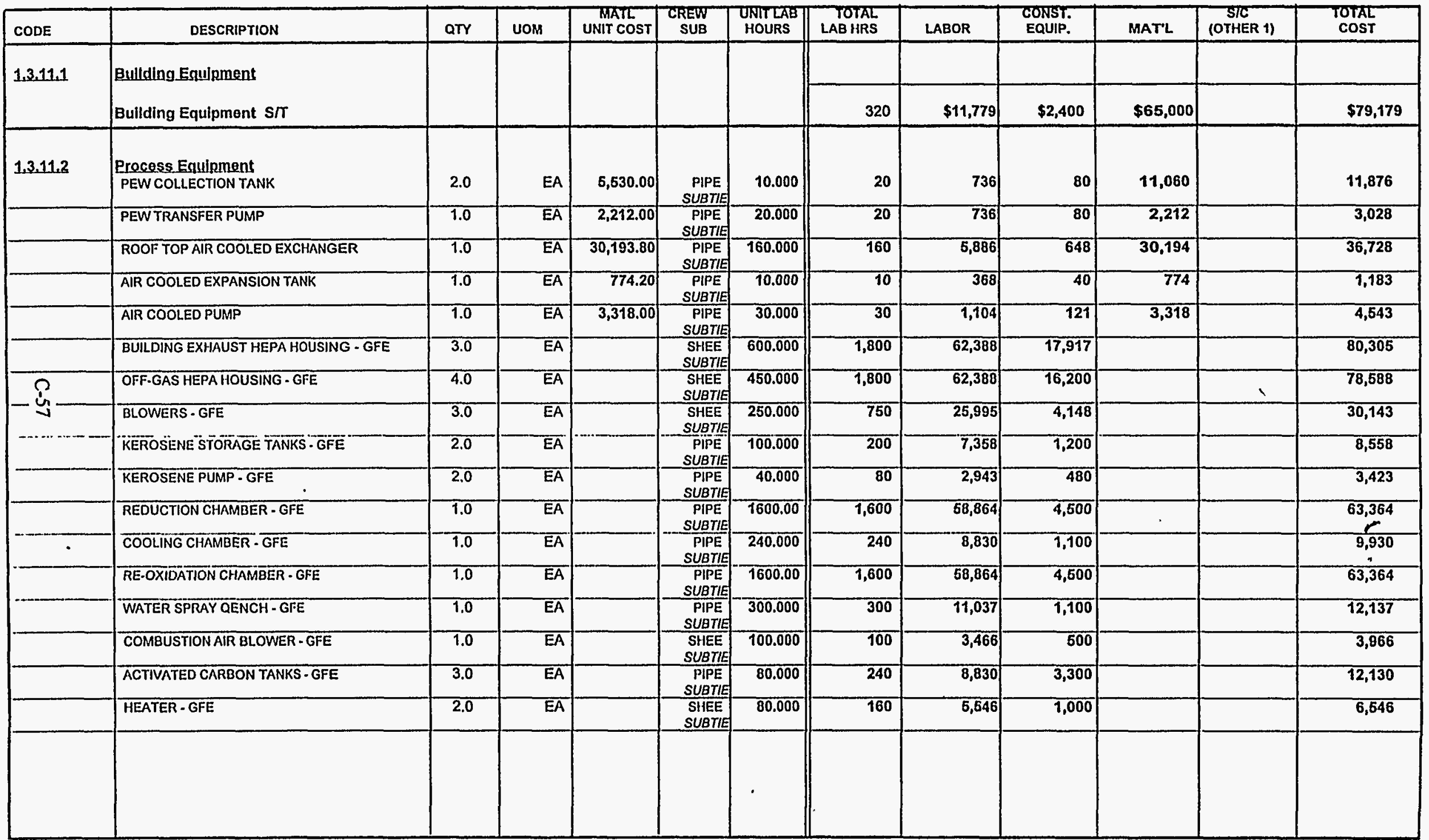


Lockheed Martin Idaho Technologles Co.

Rov. $6 / 96$

PROJECT NAME. Rocalcinatlon Treatment Non-Separations Comentitlous Waste Option

LOCATION 1: INEEL - ICPP

REQUESTOR: AILOQ 6-9716 MS 3765

\section{DETAILED COST ESTIMATE SHEET}

TYPE OF estimate: Planning

PROJECT NO.: 2420

PREPARED BY: JRB/BCE
PAGE \# 41

DATE 03-Fob-1998

TIME: 16:40:26

REPORT NAME: Detall Cost Estimate Sheet

\begin{tabular}{|c|c|c|c|c|c|c|c|c|c|c|c|c|}
\hline CODE & OESCRIPTION & QTY & Uom & MNIT $\cos r$ & $\begin{array}{l}\text { CREW } \\
\text { SUB }\end{array}$ & $\begin{array}{l}\text { UNTILAB } \\
\text { HOURS }\end{array}$ & $\begin{array}{l}\text { TOTAL } \\
\text { LAB HRS }\end{array}$ & LABOR & $\begin{array}{l}\text { CONST. } \\
\text { ERUIP. }\end{array}$ & MAT'L. & $\begin{array}{l}\text { STC } \\
\text { (OTHER 1) }\end{array}$ & $\begin{array}{l}\text { TOTAL } \\
\text { COST }\end{array}$ \\
\hline \multirow[t]{2}{*}{$1,3.11,2$} & $\begin{array}{l}\text { Procoss_Equinment } \\
\text { DEMISTER - GFE }\end{array}$ & 1.0 & EA & & $\begin{array}{c}\text { PIPE } \\
\text { SUBTIA } \\
\end{array}$ & 60.000 & 60 & 2,207 & 375 & & & 2,582 \\
\hline & Process Equipment $\mathrm{S} / \mathrm{T}$ & & & & & & 9,170 & $\$ 327,645$ & $\$ 57,289$ & $\$ 47,558$ & & $\$ 432,392$ \\
\hline \multirow[t]{2}{*}{1.3 .13} & $\begin{array}{l}\text { MACT Facillty Special Consfruction } \\
\text { KEROSENE PUMP PRE-ENGINEERED BUILDING }\end{array}$ & 200.0 & SF & & SUBTIE & 0.000 & & & & & 9,216 & 9,216 \\
\hline & MACT Facility Special Construction $\mathrm{s} / \mathrm{r}$ & & & & & & 0 & & & & $\$ 9,216$ & $\$ 9,216$ \\
\hline 1.3 .15 .1 .1 & $\begin{array}{l}\text { Utility Piping } \\
\text { 4" SEWER PIPE }\end{array}$ & 160.0 & $L F$ & 10.45 & PIPE & 0.633 & 101 & 3,726 & 701 & 1,672 & & 6,099 \\
\hline \multirow{3}{*}{$-\Omega_{\infty}$} & 2" POTABLE WATER PIPE & 325.0 & LF & $\overline{5.46}$ & $\begin{array}{c}\text { PIPE } \\
\text { SUBTIE }\end{array}$ & $\overline{0.329}$ & 107 & $\overline{3,934}$ & 1,063 & 1,775 & & 6,771 \\
\hline & 8" FW PIPE & 400.0 & LF & 61.99 & $\begin{array}{c}\text { PIPE } \\
\text { SUBTIL } \\
\end{array}$ & 4.180 & $\overline{1,672}$ & $\overline{61,513}$ & $\overline{11,568}$ & 24,796 & & 97,877 \\
\hline & G" FW PIPE & 50.0 & {$[F$} & $\overline{49.06}$ & $\begin{array}{c}\text { PIPE } \\
\text { SUBTIE }\end{array}$ & $\overline{1.460}$ & 73 & 2,686 & 505 & 2,453 & & 5,644 \\
\hline \multirow{5}{*}{$\cdots-\cdots$} & 3"FW FOAM PIPE & 130.0 & LF & 17.56 & $\begin{array}{r}\text { PIPE } \\
\text { SUBTIE } \\
\end{array}$ & 0.650 & 85 & $\overline{3,109}$ & 584 & 2,283 & & 5,975 \\
\hline & RELOCATE FW PIPE, HYD., ETC. & & LOT & & $\begin{array}{l}\text { PIPE } \\
\text { SUBTIE }\end{array}$ & 20.000 & 20 & 736 & 134 & & & 870 \\
\hline & i/2" INSTR. AIR PIPE & 120.0 & {$[F$} & 7.78 & $\begin{array}{c}\text { PIPE } \\
\text { SUBTIE }\end{array}$ & 0.830 & 100 & 3,664 & $\overline{691}$ & 934 & & 5,289 \\
\hline & COAT \& WRAP PIPE & $1,225.0$ & $\overline{L F}$ & $\overline{3.32}$ & $\begin{array}{r}\text { PIPE } \\
\text { SUBTIL } \\
\end{array}$ & $0 . \overline{200}$ & $\overline{245}$ & 9,014 & 735 & 4,067 & & 13,816 \\
\hline & Utility Piping S/T & & & & & & 2,402 & $\$ 88,381$ & $\$ 15,980$ & $\$ 37,979$ & & $\$ 142,340$ \\
\hline \multirow[t]{2}{*}{1.3 .15 .1 .2} & $\begin{array}{l}\text { Building.PIping } \\
\text { FIRE PROTECTION PIPE \& EQUIPMENT(C.S. } \\
\text { \& SST }\end{array}$ & $10,235.0$ & SF & 7.51 & $\begin{array}{c}\text { SPRI } \\
\text { SUBTIE }\end{array}$ & 0.164 & 1,679 & 60,662 & 12,896 & 76,865 & & 160,423 \\
\hline & FIRE PROTECTION DETECTION \& CONTROL & 1.0 & LOT & $26,939.00$ & $\begin{array}{c}\text { ELEC } \\
\text { SUBTII }\end{array}$ & 337.960 & 338 & 11,257 & 1,350 & 26,939 & & 39,546 \\
\hline & & & & & & & & & & & & \\
\hline
\end{tabular}


Lockheed Martin Idaho Technologies Co.

Rev. 6/96
ROJECT NAME: Recalcination Treatment Non-Separations Cementitious Waste Option

LOCATION 1: INEEL - ICPP

REQUESTOR. Al LEQ 6-9716 MS 3765

\section{DETAILED COST ESTIMATE SHEET}

TYPE OF EsTIMATE: Planning

PROJECT NO.: 2420

PREPARED BY: JRB/BCE
PAGE * 42

DATE 03-Feb-1998

TIME: 16:40:26

REPORT NAME: Detail Cost Estimato Sheet

\begin{tabular}{|c|c|c|c|c|c|c|c|c|c|c|c|c|}
\hline CODE & DESCRIPTION & atr & vom & $\begin{array}{l}\text { MATL } \\
\text { UNIT COST }\end{array}$ & $\begin{array}{l}\text { CREW } \\
\text { SUB }\end{array}$ & $\begin{array}{l}\text { UNITLAB } \\
\text { HOURS }\end{array}$ & $\begin{array}{l}\text { TOTAL } \\
\text { LAB HRS }\end{array}$ & LABOR & $\begin{array}{l}\text { CONST: } \\
\text { EQUIP. }\end{array}$ & MAT'L & $\begin{array}{c}\text { STC } \\
\text { (OTHER 1) }\end{array}$ & $\begin{array}{l}\text { TOTALL } \\
\text { COST }\end{array}$ \\
\hline \multirow[t]{7}{*}{1.3 .15 .1 .2} & $\begin{array}{l}\text { Building Piping } \\
\text { SANITARY WASTE FIXTURES \& PIPING }\end{array}$ & 1.0 & LOT & $3,565.74$ & $\begin{array}{c}\text { PIPE } \\
\text { SUBTE }\end{array}$ & 93.000 & 93 & 3,421 & 372 & 3,566 & & 7,360 \\
\hline & $\begin{array}{l}\text { POTABLE WATER TO KEROSENE AREA SAFETY } \\
\text { SHOWERS }\end{array}$ & 117.0 & LF & 12.99 & $\begin{array}{c}\text { PIPE } \\
\text { SUBTIE }\end{array}$ & 0.200 & 23 & $\overline{861}$ & 94 & 1,520 & & 2,474 \\
\hline & FREEZLESS SAFETY SHOWERS & 2.0 & $E A$ & 498.00 & $\begin{array}{c}\text { PIPE } \\
\text { SUBTIE }\end{array}$ & 4.000 & 8 & 294 & 32 & 996 & & 1,322 \\
\hline & POTABLE WATER IN BUILDING & 260.0 & $\overline{L F}$ & 12.85 & $\begin{array}{l}\text { PIPE } \\
\text { SUBTIE }\end{array}$ & 0.400 & 104 & 3,826 & 419 & 3,341 & & 7,586 \\
\hline & SAFETY SHOWERS & 4.0 & EA & 221.20 & $\begin{array}{c}\text { PIPE } \\
\text { SUBTIE }\end{array}$ & 8.000 & 32 & 1,177 & 128 & 885 & & 2,190 \\
\hline & EYEWASH & 4.0 & EA & 154.84 & $\begin{array}{c}\text { PIPE } \\
\text { SUBTIE }\end{array}$ & 8.000 & $\overline{32}$ & 1,177 & 128 & 619 & & 1,925 \\
\hline & WATER HEATER & 1.0 & $\overline{E A}$ & 331.80 & $\begin{array}{c}\text { PIPE } \\
\text { SUBTIE } \\
\end{array}$ & 8.000 & 8 & 294 & $\overline{32}$ & 332 & & 658 \\
\hline$-\dot{u}_{0}^{\prime}$ & Bullding Plping $\mathrm{s} \pi$ & & & & & & 2,317 & $\$ 82,972$ & $\$ 15,451$ & $\$ 115,062$ & & $\$ 213,485$ \\
\hline \multirow[t]{11}{*}{$1,3,15,1,3$} & $\begin{array}{l}\text { Process. PIpIng } \\
\text { DBL CONTAINED KEROSENE LINE }\end{array}$ & 400.0 & LF & 57.74 & $\begin{array}{c}\text { PIPE } \\
\text { SUBTIE }\end{array}$ & 2.426 & 970 & 35,701 & 8,480 & 23,096 & 8,848 & 76,125 \\
\hline & OFF.GAS TIE-IN & 1.0 & LOT & $18,627.00$ & $\begin{array}{r}\text { PIPE } \\
\text { SUBTIE } \\
\end{array}$ & 1743.00 & 1,743 & 64,125 & 16,100 & 18,627 & 8,295 & 107,147 \\
\hline & DBL CONTAINED LLW LINE & 75.0 & LF & 83.13 & $\begin{array}{c}\text { PIPE } \\
\text { SUBTIE }\end{array}$ & $\overline{26.330}$ & 1,975 & 72,651 & 650 & 6,235 & 10,507 & 90,042 \\
\hline & $\overline{\text { COAT \& WRAP }}$ & 475.0 & LF & 3.32 & $\begin{array}{c}\text { PIPE } \\
\text { SUBTIE }\end{array}$ & 0.200 & 95 & $\overline{3,495}$ & 285 & 1,577 & & 5,657 \\
\hline & PEW DBL.CONTAINED PIPING & 905.0 & $\mathrm{LF}$ & 138.25 & $\begin{array}{l}\text { PIPE } \\
\text { SUBTIEE }\end{array}$ & 1.357 & 1,228 & 45,181 & 4,914 & $\overline{125,116}$ & & 175,212 \\
\hline & OFF-GAS PIPING & 725.0 & $\overline{L F}$ & $1,300.00$ & $\begin{array}{c}\text { PIPE } \\
\text { SUBTIE }\end{array}$ & 4.500 & $\overline{3,263}$ & 120,027 & 13,050 & 942,600 & & $1,075,577$ \\
\hline & ADD FOR THIRD CARBON VESSEL PIPING & 1.0 & LOT & $30,000.00$ & $\begin{array}{c}\text { PIPE } \\
\text { SUBTIL }\end{array}$ & $\overline{250.000}$ & 250 & 9,198 & 625 & 30,000 & & 39,823 \\
\hline & OFF-GAS COOLING LOOP PIPING & 175.0 & $\overline{\mathrm{LF}}$ & 282.44 & $\begin{array}{l}\text { PIPE } \\
\text { SUBTIE }\end{array}$ & 2.550 & 446 & 16,418 & 1,806 & 49,427 & & 67,651 \\
\hline & COOLING AIR PIPING & 162.0 & LF & 305.27 & $\begin{array}{c}\text { PIPE } \\
\text { SUBTIE }\end{array}$ & 2.150 & 348 & 12,814 & 1,413 & 49,454 & & 63,680 \\
\hline & COMBUSTION AIR PIPING & 190.0 & LF & 197.43 & $\begin{array}{c}\text { PIPE } \\
\text { SUBTIL }\end{array}$ & 1.953 & 371 & 13,662 & 1,601 & $\overline{37,612}$ & & 62,664 \\
\hline & DECON PIPING & 680.0 & $\mathrm{LF}$ & 32.75 & $\begin{array}{c}\text { PIPE } \\
\text { SUBTIE }\end{array}$ & 0.487 & 331 & 12,183 & 1,340 & 22,270 & & 36,793 \\
\hline & & & & & & & & & & & & \\
\hline
\end{tabular}


Lockheed Martin Idaho Technologies Co. Rov, 6196

PROJECT NAME: Recalcination Treatment Non-Separations Cementitious Waste Option

LOCATION 1: INEEL - ICPP

REOUESTOR: AILe日 6-9716 MS 3765

\section{DETAILED COST ESTIMATE SHEET}

TYPE OF ESTIMATE: Planning

PROJECT NO.: 2420

PREPARED BY: JRB/BCE
PAGE \# 43

DATE 03-Fob-1998

TIME: 16:40:26

REPORT NAME: Detail Cost Estimate Sheet

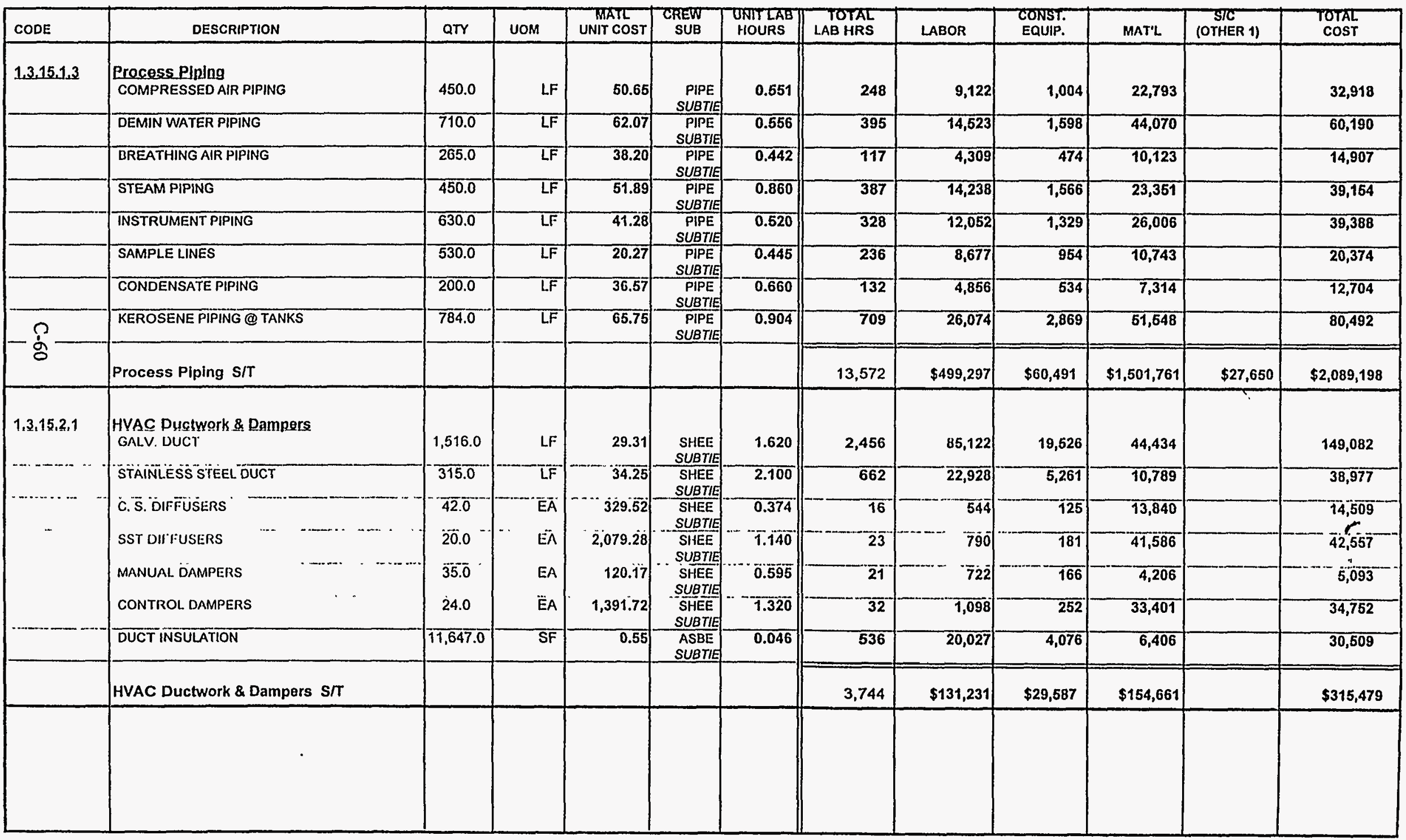


Lockheed Martin Idaho Technologies Co.

Rov $6 / 96$

PROJECT NAME: Recalcination Treatment Non-Separations Cementitious Wasto Option

LOCATION 1: INEEL - ICPP

DETAILED COST ESTIMATE SHEET.

TYPE OF ESTIMATE: Planning

PROJECT NO: 2420

PREPAREO BY: JRB/BCE
PAGE* 44

DATE 03-Feb-199

TIME: 16:40:26

REPORT NAME: Detail Cost Estimate Sheet

REQUESTOR: AILe日 6-9716 MS 3765

\section{UWAT}

\begin{tabular}{|c|c|c|c|c|c|c|c|c|c|c|c|c|}
\hline CODE & DESCRIPTION • & QTY & UOM & $\begin{array}{l}\text { MATL } \\
\text { UNIT COST }\end{array}$ & $\begin{array}{l}\text { CREW } \\
\text { SUB }\end{array}$ & $\begin{array}{l}\text { UNITLAB } \\
\text { HOURS }\end{array}$ & $\begin{array}{l}\text { TOTAL } \\
\text { LAB HRS }\end{array}$ & LABOR & $\begin{array}{l}\text { CONST. } \\
\text { EQUIP. }\end{array}$ & MATL & $\begin{array}{l}\text { STC } \\
\text { (OTHER 1) }\end{array}$ & $\begin{array}{l}\text { TOTAL } \\
\text { COST }\end{array}$ \\
\hline \multirow[t]{6}{*}{1.3 .15 .2 .2} & $\begin{array}{l}\text { HVAC Equipment } \\
\text { SUPPLY AIR HANDLER } 22,270 \text { CFM }\end{array}$ & 1.0 & EA & $33,180.00$ & $\begin{array}{l}\text { SHEE } \\
\text { SUBTIE }\end{array}$ & 80.000 & 80 & 2,773 & 636 & 33,180 & & 36,589 \\
\hline & AIR CONDITIONER 1500CFM & 2.0 & EA & $2,212.00$ & $\begin{array}{l}\text { SHEE } \\
\text { SUBTIL }\end{array}$ & 8.000 & 16 & 555 & 127 & 4,424 & & $\overline{5,106}$ \\
\hline & EXHAUST FAN 15,000 CFM & 3.0 & $\overline{E A}$ & $24,332.00$ & $\begin{array}{l}\text { SHEE } \\
\text { SUBTIE }\end{array}$ & 80.000 & 240 & 8,318 & 1,908 & 72,996 & & 83,222 \\
\hline & BUILDING HEPA FILTERS & 8.0 & $\overline{E A}$ & $4,838.75$ & $\begin{array}{l}\text { SHEE } \\
\text { SUBTIE }\end{array}$ & 8.500 & 68 & 2,357 & $\overline{541}$ & 38,710 & & 41,608 \\
\hline & BALANCE \& TEST SYSTEM & 1.0 & LOT & & $\begin{array}{l}\text { SHEE } \\
\text { SUBTIE }\end{array}$ & 0.000 & & & & & & \\
\hline & HVAC Equipment $\mathrm{S} / \mathrm{T}$ & & & & & & 404 & $\$ 14,003$ & $\$ 3,212$ & $\$ 149,310$ & & $\$ 166,524$ \\
\hline \multirow[t]{2}{*}{$1,3.15 .2 .3$} & $\begin{array}{l}\text { HYAC Conirols \& Instrumentation } \\
\text { CONTROLS \& INSTRUMENTS }\end{array}$ & 1.0 & LOT & $47,436.34$ & $\begin{array}{c}\text { ELEC } \\
\text { SUBTII } \\
\end{array}$ & 686.340 & 686 & 22,862 & 2,740 & 47,436 & & 73,038 \\
\hline & HVAC Controls \& Instrumentation SIT & & & & & & 686 & $\$ 22,862$ & $\$ 2,740$ & $\$ 47,436$ & $\because$ & $\$ 73,038$ \\
\hline \multirow[t]{6}{*}{$1,3,16,1$} & $\begin{array}{l}\text { Electrical Utilities } \\
\text { 3" PVC CONDUIT \& FITTINGS }\end{array}$ & $6,510.0$ & $L F$ & 4.92 & $\begin{array}{l}\text { ELEC } \\
\text { SUBTIE }\end{array}$ & 0.170 & 1,107 & 36,864 & 8,333 & 32,029 & & 77,226 \\
\hline & 2" PVC CONDUIT \& FITTINGS & $\overline{375.0}$ & LF & 2.69 & $\begin{array}{l}\text { ELIEC } \\
\text { SUBTIE }\end{array}$ & $0.170^{-1}$ & 64 & 2,124 & $\overline{480}$ & 1,009 & & 3,612 \\
\hline & RELOCATE ELECTRICAL EQUIPMENT & 1.0 & LOT & & EIEC & 375.000 & 375 & 12,491 & 2,132 & & & 14,623 \\
\hline & CÁTHOD̈IC PROTECTION & 1.0 & LOT & & TELC & 0.000 & & & & & 6,600 & 6,600 \\
\hline & POWLK REIED CNBLES & $5,075.0$ & LF & 7.85 & $\begin{array}{l}\text { ELEC } \\
\text { SUBTIE }\end{array}$ & $\overline{0.100}$ & 508 & 16,905 & $\overline{2,030}$ & 39,839 & & 58,774 \\
\hline & Electrical Utilltles $S / T$ & & & & & & 2,053 & $\$ 68,384$ & $\$ 12,975$ & $\$ 72,877$ & $\$ 6,600$ & $\$ 160,836$ \\
\hline \multirow[t]{2}{*}{1.3 .16 .2} & $\begin{array}{l}\text { Building Eloctrlical } \\
\text { PANELS, SWITCHES \& TRANSFORMERS }\end{array}$ & 5.0 & EA & 822.86 & $\begin{array}{l}\text { ELEC } \\
\text { SUBTIE }\end{array}$ & 16.800 & 84 & 2,798 & 336 & 4,114 & & 7,248 \\
\hline & GROUNDING SYSTEM & 1.0 & LOT & $6,029.00$ & $\begin{array}{l}\text { ELEC } \\
\text { SUBTIE }\end{array}$ & 445.800 & 446 & 14,860 & 1,805 & 5,029 & & 21,683 \\
\hline & & & & & & & & & & & & \\
\hline
\end{tabular}

EXHAUST FAN 15,000 CFM

BUILDING HEPA FILTERS

BALANCE \& TEST SYSTEM

\begin{tabular}{|c|c|c|c|c|c|c|c|c|c|c|c|c|}
\hline CODE & DESCRIPTION • & QTY & UOM & $\begin{array}{l}\text { MAIL } \\
\text { UNIT COST }\end{array}$ & $\begin{array}{l}\text { CREW } \\
\text { SUB }\end{array}$ & $\begin{array}{l}\text { UNITLAB } \\
\text { HOURS }\end{array}$ & $\begin{array}{l}\text { TOTAL } \\
\text { LAB HRS }\end{array}$ & LABOR & $\begin{array}{l}\text { CONST. } \\
\text { EQUIP. }\end{array}$ & MATL & $\begin{array}{l}\text { STC } \\
\text { (OTHER 1) }\end{array}$ & $\begin{array}{r}\text { TOTAL } \\
\text { COST }\end{array}$ \\
\hline \multirow[t]{6}{*}{1.3 .15 .2 .2} & $\begin{array}{l}\text { HVAC Equipment } \\
\text { SUPPLY AIR HANDLER 22,270 CFM }\end{array}$ & 1.0 & EA & $33,180.00$ & SHEE & 80.000 & 80 & 2,773 & 636 & 33,180 & & 36,589 \\
\hline & AIR CONDITIONER 1500CFM & 2.0 & $E A$ & $2,212.00$ & $\begin{array}{l}\text { SHEE } \\
\text { SUBTIA }\end{array}$ & 8.000 & 16 & 555 & 127 & 4,424 & & 5,106 \\
\hline & EXHAUST FAN 15,000 CFM & 3.0 & $\overline{E A}$ & $24,332.00$ & $\begin{array}{l}\text { SHEE } \\
\text { SUBTII }\end{array}$ & 80.000 & 240 & $\overline{8,318}$ & 1,908 & 72,996 & & 83,222 \\
\hline & BUILDING HEPA FILTERS & 8.0 & $\overline{E A}$ & $4,838.75$ & $\begin{array}{l}\text { SHEE } \\
\text { SUBTII }\end{array}$ & 8.500 & 68 & 2,357 & 541 & $\mathbf{3 8 , 7 1 0}$ & & 41,608 \\
\hline & BALANCE \& TEST SYSTEM & 1.0 & LOT & & $\begin{array}{l}\text { SHEE } \\
\text { SUBTIE }\end{array}$ & 0.000 & & & & & & \\
\hline & HVAC Equipment $S / T$ & & & & & & 404 & $\$ 14,003$ & $\$ 3,212$ & $\$ 149,310$ & & $\$ 166,524$ \\
\hline \multirow[t]{2}{*}{1.3 .15 .2 .3} & $\begin{array}{l}\text { HVAC Controls \& Instrumentation } \\
\text { CONTROLS \& INSTRUMENTS }\end{array}$ & 1.0 & LOT & $47,436.34$ & $\begin{array}{l}\text { ELEC } \\
\text { SUBTIE } \\
\end{array}$ & 686.340 & 686 & 22,862 & 2,740 & 47,436 & & 73,038 \\
\hline & HVAC Controls \& Instrumentation SIT & & & & & & 686 & $\$ 22,862$ & $\$ 2,740$ & $\$ 47,436$ & $\because$ & $\$ 73,038$ \\
\hline \multirow[t]{6}{*}{$1,3.16,1$} & $\begin{array}{l}\text { Electrical Utilities } \\
\text { 3" PVC CONDUIT \& FITTINGS }\end{array}$ & $6,510.0$ & LF & 4.92 & $\begin{array}{l}\text { ELEC } \\
\text { SUBTIE }\end{array}$ & 0.170 & 1,107 & 36,864 & 8,333 & 32,029 & & 77,226 \\
\hline & 2" PVC CONDUIT \& FITTINGS & $\overline{375.0}$ & LF & $\overline{2.69}$ & $\begin{array}{l}\text { EL.EC } \\
\text { SUBTIE }\end{array}$ & $0.170^{-1}$ & 64 & $\overline{2,124}$ & $\overline{480}$ & 1,009 & & 3,612 \\
\hline & RELOCATE ELECTRICAL EQUIPMENT & 1.0 & LOT'- & & $\begin{array}{l}\text { EIEC } \\
\text { SUBTIE }\end{array}$ & 376.000 & $\overline{375}$ & $\overline{12,491}$ & 2,132 & & & 14,623 \\
\hline & CATTHOD̈IC̈ PROTTECTION & 1.0 & LOT & & $\begin{array}{l}\text { E:LEC } \\
\text { SUDTIS }\end{array}$ & 0.000 & & & & & 6,600 & 6,600 \\
\hline & POWLKIVEEDCABLES & $5,075.0$ & LF & 7.85 & $\begin{array}{l}\text { ELLC } \\
\text { SUBTIE }\end{array}$ & 0.100 & 508 & 16,905 & $\overline{2,030}$ & 39,839 & & 58,774 \\
\hline & Electrical Utilltios s/T & & & & & & 2,053 & $\$ 68,384$ & $\$ 12,975$ & $\$ 72,877$ & $\$ 6,600$ & $\$ 160,836$ \\
\hline \multirow[t]{2}{*}{1.3 .16 .2} & $\begin{array}{l}\text { Building Eloctrical } \\
\text { PANELS, SWITCHES \& TRANSFORMERS }\end{array}$ & 5.0 & EA & 822.86 & $\begin{array}{c}\text { ELEC } \\
\text { SUBTIE }\end{array}$ & 16.800 & 84 & 2,798 & 336 & 4,114 & & 7,248 \\
\hline & GROUNDING SYSTEM & 1.0 & LOT & $6,029.00$ & $\begin{array}{l}\text { ELEC } \\
\text { SUBTIE }\end{array}$ & 445.800 & $\overline{446}$ & 14,860 & 1,805 & 5,029 & & 21,683 \\
\hline & & & & & & & & & & & & \\
\hline
\end{tabular}

UNIT COST CREW 
Lockheed Martin Idaho Technologies Co. Rov 6/96

PROJECT NAME Rocalcination Troatment Non-Separations Cementitious Waste Option

LOCATION 1: INEEL - ICPP

REQUESTOR: AI Le日 6-9716 MS 3765

\section{DETAILED COST ESTIMATE SHEET}

TYPE OF ESTIMATE: Planning

PROJECT NO.: 2420

PREPAREO BY: JRB/BCE
PAGE \# 45

DATE 03-Fob-1098

TIME: 16:40:26

REPORT NAME: Detall Cost Estimate She日t

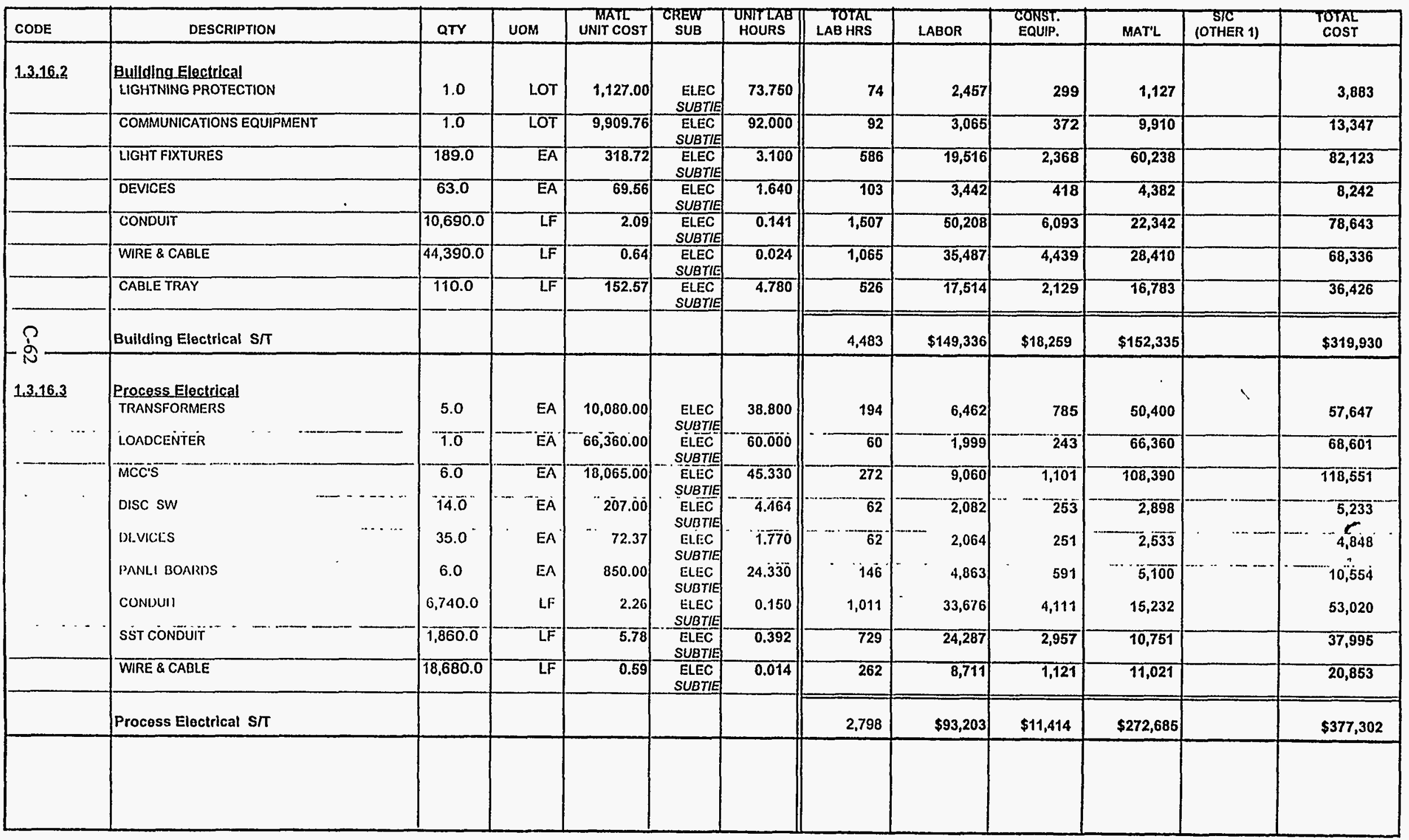


Lockheed Martin Idaho Technologies Co.

Rov. 6/96 PROJECT NAME: Rocalchitious Waste Option

LOCATION 1: INEEL - ICPP

REQUESTOR: AI Lee 6-9716 MS 3766

\section{DETAILED COST ESTIMATE SHEET}

TPPE OF EstiMaATE: Planning

PROJECT NO: 2420

PREPARED QY: JRB/BCE
PAGE * 46

DATE 03-Feb-1998

TIME: 16:40:26

REPORT NAME: Detall Cost Estlmate Sheet

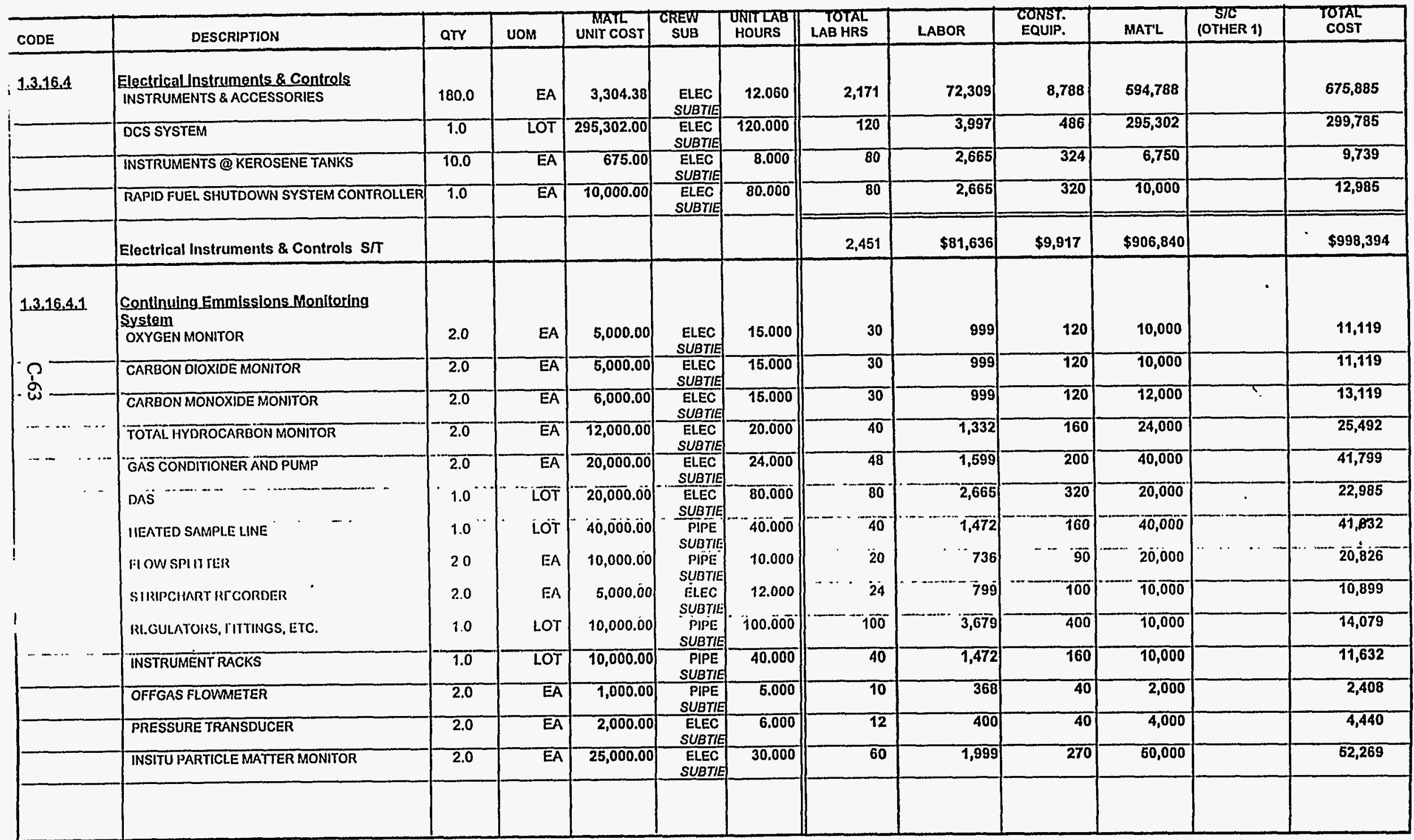


Lockheed Martin Idaho Technologies Co. Rev $6 / 96$

PROJECT NAME Recalcination Treatment Non-Separations Comentifious Waste Option

LOCATION 1 . INEEL - ICPP

RERUESTOR Al Lee 6-9716 MS 3765
DETAILED COST ESTIMATE SHEET

TYPE OF ESTIMATE: Planning

PROJECT NO.: 2420

PREPAREO BY: JRB/BCE
PAgE: 47

DATE 03-Fab-1988

TIME: 16:40:26

REPORT MAME: Detall Cost Estimate Sheet

\begin{tabular}{|c|c|c|c|c|c|c|c|c|c|c|c|c|}
\hline CODE & DESCRIPTION & QTY & UOM & $\begin{array}{l}\text { WATL } \\
\text { UNIT COST }\end{array}$ & $\begin{array}{l}\text { CREW } \\
\text { SUB }\end{array}$ & $\begin{array}{l}\text { UNITLAB } \\
\text { HOURS }\end{array}$ & $\begin{array}{l}\text { TOTAL } \\
\text { LAB HRS }\end{array}$ & LABOR & $\begin{array}{l}\text { CONST. } \\
\text { EQUIP. }\end{array}$ & MAT'L & SIC & $\begin{array}{l}\text { TOTAL } \\
\text { COST }\end{array}$ \\
\hline $1,3,16.4 .1$ & $\begin{array}{l}\text { Continuing Emmissiens. Monitering } \\
\text { System } \\
\text { MISC. FITTINGS, VALVES, TUBING,GAUGES }\end{array}$ & 1.0 & LOT & $26,000.00$ & $\begin{array}{c}\text { PIPE } \\
\text { SUBTIE } \\
\end{array}$ & 160.000 & 160 & 6,886 & 640 & 26,000 & & 32,626 \\
\hline & Continuing Emmissions Monitoring System & $s \pi$ & & & & & 724 & $\$ 25,404$ & $\$ 2,940$ & $\$ 288,000$ & & $\$ 316,344$ \\
\hline 1.0 & NWCE Sampling Modifications & & & & & & & & & & & \\
\hline & & & & & & & & & & & & \\
\hline & NWCF Sampling Modifications $S / T$ & & & & & & 0 & & & & & \\
\hline 1.3 .2 & $\begin{array}{l}\text { NWCF Sampling Mods Sitéwork } \\
\text { DECON SAMPLING CELL }\end{array}$ & 1.0 & LOT & & $\begin{array}{l}\text { LABR } \\
\text { SUBTIE } \\
\end{array}$ & 750.000 & 760 & 21,780 & & & & 21,780 \\
\hline$\stackrel{1}{\AA}$ & NWCF Sampling Mods Silework S/T & & & & & & 750 & $\$ 21,780$ & & & $T$ & $\$ 21,780$ \\
\hline $1,3.5$ & $\begin{array}{l}\text { NWCF Sampling Mods Metals } \\
\text { SAMPLER TABLE - NLLOW }\end{array}$ & 1.0 & EA & 600.00 & $\begin{array}{l}\text { PIPE } \\
\text { SUBTIE }\end{array}$ & 15.000 & 15 & 552 & & 600 & & 1,152 \\
\hline & NWCF Sampling Mods Molals S/T & & & & & & 15 & $\$ 552$ & & $\$ 600$ & & $\$ 1,152$ \\
\hline $1,3,11$ & $\begin{array}{l}\text { NWCF:Sannuliug Mods_Eguipment } \\
\text { SAMPLERS }\end{array}$ & 2.0 & $\mathrm{EA}$ & $10,000.00$ & $\begin{array}{c}\text { PIPE } \\
\text { SUBTIE }\end{array}$ & 45.000 & 90 & 3,311 & & 20,000 & & $\begin{array}{c}" \\
23,311\end{array}$ \\
\hline & $\begin{array}{l}\text { TRANSPORT CONTAINERS W/ SHIELDED } \\
\text { INSERTS }\end{array}$ & 2.0 & $\overline{E A}$ & $200,000.00$ & SUBTIE & 0.000 & & & & 400,000 & ..... & $-400,000^{-}$ \\
\hline & GAS CHROMATOGRAPHIMASS SPECTROMETER & 1.0 & $\overline{E A}$ & $65,000.00$ & SUBTIE & 0.000 & & & & 65,000 & & 65,000 \\
\hline & $\begin{array}{l}\text { INDUCTIVELY COUPLED PLASMA } \\
\text { SPECTROPHOTOMETERS }\end{array}$ & 1.0 & $E A$ & $92,000.00$ & SUBTIE & 0.000 & & & & 92,000 & & 92,000 \\
\hline & & & & & & & & & & & & \\
\hline
\end{tabular}


Lockheed Martin Idaho Technologies Co.

Rav 6196

PROJECT NAME: Recalcination Treatmont Non-Separations Cementitious Wasto Option

LOCATION 1: INEEL - ICPP

REQUESTOR: Al Lee 6-9716 MS 3765
DETAILED COST ESTIMATE SHEET

TPPE OF ESTIMATE: Planning

PROJECT NO: 2420

PREPARED BY: JRBIBCE
PAGE \# 48

DATE 03-Fob-1998

TIME: 16:40:26

REPORT NaMe: Detail Cost Estimate Sheet

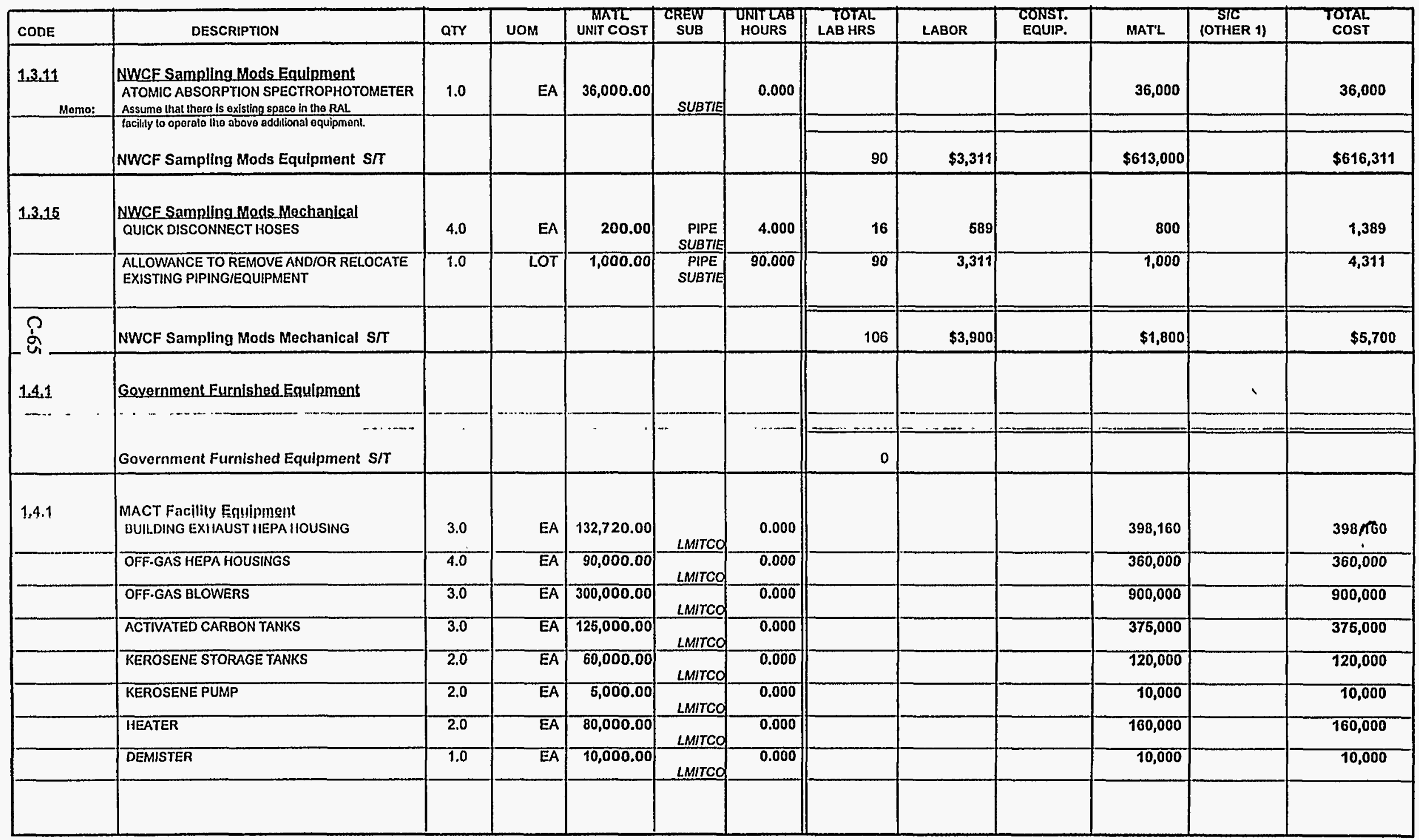


Lockheed Martin Idaho Technologies Co.

Rov. 6/86

PROJECr NAME: Recalcination Treatment Non-Separations

Cementitious Waste Option

LOCATION : INEEL - ICPP

REQUESTOR: Al Lee 6-9716 MS 3765
DETAILED COST ESTIMATE SHEET

TYPE OF ESTIMATE: Planning

PROJECTNO.: 2420

PREPARED BY: JRB/BCE
PAGE \# 49

DATE 03-Fob-1998

TIME: 16:40:26

REPORT NAME: Detall Cost Estimate Sheet

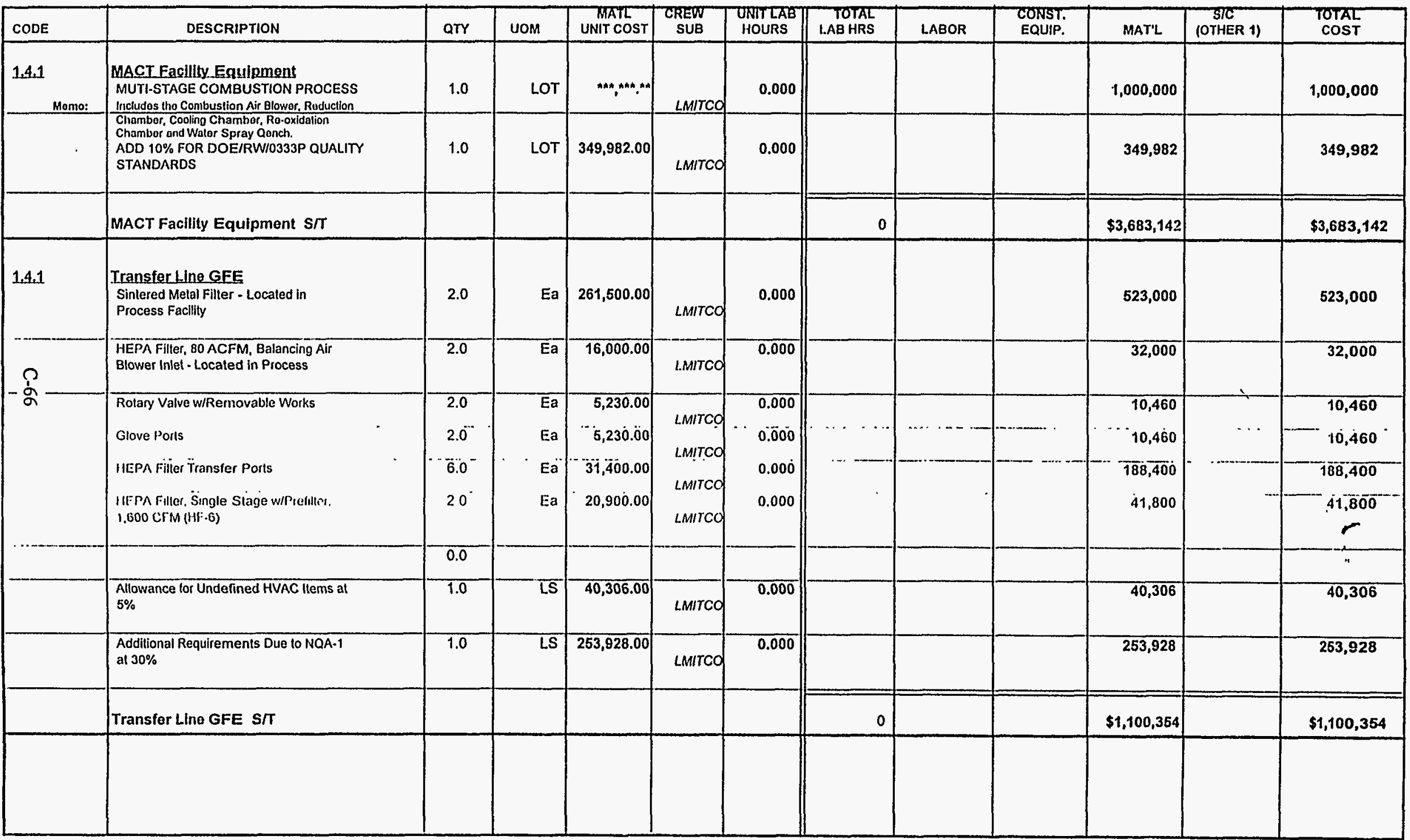


Lockheed Martin Idaho Technologies Co.

ReV. $6 / 96$
PROJECT NAME: Recalcination Treatment Non-Separations

Cementitlous Waste Option

LOCATION 1: INEEL - ICPP

REQUESTOR: Al Lee 6-9716 MS 3765

\section{DETAILED COST ESTIMATE SHEET}

TYPE OF ESTIMATE: Planning

PROJECT NO:: 2420

PREPARED BY: JRB/BCE
PAGE \# 50

DATE 03-Fob-1998

TIME: 16:40:26

REPORT NAME: Detail Cost Estimate Sheet

\begin{tabular}{|c|c|c|c|c|c|c|c|c|c|c|c|c|}
\hline CODE & DESCRIPTION & aTY & UOM & $\begin{array}{l}\text { MATL } \\
\text { UNIT COST }\end{array}$ & $\begin{array}{c}\text { CREW } \\
\text { SUB }\end{array}$ & $\begin{array}{l}\text { TNITLAB } \\
\text { HOURS }\end{array}$ & $\begin{array}{l}\text { TOTAL } \\
\text { LAB HRS }\end{array}$ & LABOR & $\begin{array}{l}\text { CONST. } \\
\text { EQUIP. }\end{array}$ & MAT'L & $\begin{array}{l}\text { STC } \\
\text { (OTHER 1) }\end{array}$ & $\begin{array}{l}\text { TOTAL } \\
\text { COST }\end{array}$ \\
\hline \multirow[t]{9}{*}{1.4 .1} & $\begin{array}{l}\text { Sucrose Equipment GFE } \\
\text { NEW CYCLONE }\end{array}$ & 1.0 & EA & $300,000.00$ & LMITCO & 0.000 & & & & 300,000 & & 300,000 \\
\hline & VENTURI SCRUBBER & 1.0 & $\overline{E A}$ & $51,000.00$ & LMITCO & 0.000 & & & & 51,000 & & 51,000 \\
\hline & NEW SCRUB RECYCLE PUMPS & 2.0 & $\overline{E A}$ & $215,000.00$ & LMITCO & 0.000 & & & & 430,000 & & 430,000 \\
\hline & SCRUB HOLD TANK & 1.0 & $\overline{E A}$ & $385,000.00$ & LMITCO & 0.000 & & - & & 385,000 & & 385,000 \\
\hline & SPIN-JET - HIGH PRESSURE PUMP & 1.0 & $\overline{E A}$ & $55,000.00$ & LMITCO & $\overline{0.000}$ & & & & 55,000 & & 55,000 \\
\hline & $\begin{array}{l}\text { SPIN-JET - REMOTELYY OPERATED SPIN } \\
\text { HEAD }\end{array}$ & 1.0 & EA & $80,000.00$ & LMITCO & 0.000 & & & & 80,000 & & 80,000 \\
\hline & SPIN-JET - MISCELLLANEOUS & 1.0 & LOT & $10,000,00$ & LMITCO & 0.000 & & & & 10,000 & & 10,000 \\
\hline & SPIN-JET - LINE JET & 1.0 & $\overline{E A}$ & $10,000.00$ & LMITCO & 0.000 & & & & 10,000 & & 10,000 \\
\hline & RAD BOXES & $14: 0$ & EA & 500.00 & LMITCO & $\overline{0.000}$ & & & & 7,000 & 、 & 7,000 \\
\hline \multirow[t]{2}{*}{$\begin{array}{c}-2 \\
9 \\
9\end{array}$} & RAD CLOTIIING \& SUPPLIES & $\begin{array}{l}936.0 \\
\cdots\end{array}$ & ENTRY & 100.00 & LMITCO & 0.000 & & & & 93,600 & & 93,600 \\
\hline & Sucrose Equipment GFE S/T & & & & & & 0 & & & $\$ 1,421,600$ & & $\$ 1,421,600$ \\
\hline \multirow[t]{8}{*}{$1,3,14,4$} & $\begin{array}{l}\text { Călcinger Cell Pecollapulinatiol } \\
\text { BASELINE DECONTAMINATION OPERATIONS }\end{array}$ & 1.0 & LOT & & $\begin{array}{l}\text { L.5210 } \\
\text { LMITCO }\end{array}$ & 1600.00 & 1,600 & 93,296 & & & & 93,296 \\
\hline & SPIN-JET - MODIFICATION ENGINEERING & 1.0 & LOT & & $\begin{array}{l}\text { L.4100 } \\
\text { LMITCO }\end{array}$ & 320.000 & 320 & 33,085 & & & & 33,085 \\
\hline & $\overline{D E C O N}$ CYCLONE & 1.0 & LOT & & $\begin{array}{l}\text { L-5210 } \\
\text { LMITCO }\end{array}$ & 1000.00 & 1,000 & 58,310 & & & & 58,310 \\
\hline & DECON CALCINER & 1.0 & LOT & & $\begin{array}{l}\text { L-5210 } \\
\text { LMITCO }\end{array}$ & 1600.00 & 1,600 & 93,296 & & & & 93,296 \\
\hline & DECON PRODUCT TAKE-OFF LINES & 1.0 & LOT & & $\begin{array}{l}\text { L.5210 } \\
\text { LMITCO }\end{array}$ & 250.000 & $26 \overline{0}$ & 14,678 & & & & 14,578 \\
\hline & DECON FLUIDIZING AIR LINE & 1.0 & LOT & & $\begin{array}{l}\text { L.5210 } \\
\text { L.MITCO }\end{array}$ & 300.000 & 300 & 17,493 & & & & 17,493 \\
\hline & DECON CALCINER INSULATION \& EXTERIOR & 1.0 & LOT & & $\begin{array}{l}\text { L-5210 } \\
\text { LMITCO }\end{array}$ & 150.000 & 160 & 8,747 & & & & 8,747 \\
\hline & FINAL CALCINER CELL FLOOR DECON & 1.0 & LOT & & $\begin{array}{l}\text { L-5210 } \\
\text { LMITCO } \\
\end{array}$ & 100.000 & 100 & 5,831 & & & & 5,831 \\
\hline & & & & & & & & & & & & \\
\hline
\end{tabular}


Lockheed Martin Idaho Technologies Co. Rav 6190

PROJECT NAME: RecalcInatlon Trealment Non-Separatlons

Cementitlous Waste Optlon

INEEL - ICPP

INEEL - ICPP

REQUESTOR. Al LeQ 6.9716 MS 3765
DETAILED COST ESTIMATE SHEET

TYPE OF ESTIMATE: Planning

PROJECTNO:: 2420

PREPARED QY: JRB/BCE
PAGE H 51

DATE 03-Feb-1998

TIME: 16:40:26

RePort name: Detall Cost Estlmate Sheet

\begin{tabular}{|c|c|c|c|c|c|c|c|c|c|c|c|c|}
\hline CODE & DESCRIPTION & aTr & uom & $\begin{array}{l}\text { PAATL } \\
\text { UNIT COST }\end{array}$ & $\begin{array}{l}\text { CREW } \\
\text { SUB }\end{array}$ & $\begin{array}{l}\text { UNITLAB } \\
\text { HOURS }\end{array}$ & $\begin{array}{l}\text { TOTAL } \\
\text { LAB HRS }\end{array}$ & LABOR & $\begin{array}{l}\text { CONST. } \\
\text { EQUIP. }\end{array}$ & MAT'L & $\begin{array}{l}\text { STC } \\
\text { (OTHER t) }\end{array}$ & $\begin{array}{l}\text { TOThL } \\
\text { COST }\end{array}$ \\
\hline \multirow[t]{2}{*}{1.3 .14 .4} & $\begin{array}{l}\text { Calciner Cell Decontaminatien } \\
\text { RADCON TECH SUPPORT }\end{array}$ & 1.0 & LOT & & $\begin{array}{l}\text { L-1342 } \\
\text { LMITCO }\end{array}$ & 660.000 & 660 & 32,155 & & & & 32,165 \\
\hline & Calciner Cell Decontamination $\mathrm{S} / \mathrm{T}$ & & & & & & 5,980 & $\$ 356,790$ & & & & $\$ 356,790$ \\
\hline 1.4 .3 & $\begin{array}{l}\text { Off-Gas Coll Decontamination } \\
\text { BASELINE DECONTAMINATION REMOTE } \\
\text { OPERATIONS }\end{array}$ & 4.0 & FTE & & $\begin{array}{l}\text { L.5210 } \\
\text { LMITCO }\end{array}$ & 160.000 & 640 & 37,318 & & & & 37,318 \\
\hline \multirow[t]{2}{*}{$\cdots-$} & RADCON TECH SUPPORT FOR REMOTE DECON & 2.0 & $\overline{\text { FTE }}$ & & $\begin{array}{l}\text { L.1342 } \\
\text { LMITCO }\end{array}$ & 160.000 & 320 & $\overline{15,590}$ & & & & $15, \overline{590}$ \\
\hline & IN-CELL. DECON & 2.0 & FTE & & $\begin{array}{l}\text { L.5210 } \\
\text { LMITCO }\end{array}$ & 45.000 & 90 & 6,248 & & & & 5,248 \\
\hline \multirow{3}{*}{$\hat{\Omega}^{\beta}$} & "TOOL PASSER" OUT-OF.CELL & 1.0 & FTE & & $\begin{array}{l}\text { L-5210 } \\
\text { LMITCO }\end{array}$ & 45.000 & 45 & 2,624 & & & & 2,624 \\
\hline & RADCON TECH SUPPORT & 1.0 & FTE & & $\begin{array}{l}\text { L-1342 } \\
\text { LMITCO }\end{array}$ & 45.000 & 46 & $\overline{2,192}$ & & & & 2,192 \\
\hline & Off-Gas Cell Decontamination $\mathrm{s} / \mathrm{T}$ & & . & & & & 1.140 & $\$ 62,973$ & & & & $\$ 62,973$ \\
\hline \multirow{3}{*}{$\begin{array}{l}1.4 .4 \\
00401500 \\
. .\end{array}$} & $\begin{array}{l}\text { Misc. OC SUPPOH } \\
\text { AlIOWANCF FOR OC OPERATION OF OII CRANI } \\
\text { C. BAN }\end{array}$ & 05 & FTE & & $\begin{array}{l}\text { L-5210 } \\
\text { IMIICOC }\end{array}$ & 4140.00 & 2,070 & 120,702 & & & & $\begin{array}{c}120,702 \\
\end{array}$ \\
\hline & Rad Control Techs & 1.8 & FTE & & $\begin{array}{l}\text { L-1342 } \\
\text { LMITCO } \\
\end{array}$ & 4200.00 & 7,560 & 368,323 & & & & $368 ; 323$ \\
\hline & Misc. OC Support S/T & & & & & & 9,630 & $\$ 489,025$ & & & & $\$ 489,026$ \\
\hline $\begin{array}{l}1.5 .1 \\
00701000\end{array}$ & $\begin{array}{l}\text { G\&A ADDER } \\
\text { Tolal G\&A }\end{array}$ & 1.0 & LS & & & 0.000 & & & & & $2,348,692$ & $2,348,692$ \\
\hline 00702000 & Total PIF & 1.0 & LS & & & 0.000 & & & & & $4,604,094$ & $4,504,094$ \\
\hline & & & & & & & & & & & & \\
\hline & & & & & & & & & & & & \\
\hline
\end{tabular}


Lockheed Martin Idaho Technologies Co.

Rev. 6196

Recalcination Treatment Non-Separations Cementitlous Waste Option

LOCATION 1: INEEL - ICPP

REQUESTOR: AI Le日 6-9716 MS 3765
DETAILED COST ESTIMATE SHEET

TYPE OF ESTMMATE: Planning

PROJECT NO: 2420

PREPARED BY: JRB/BCE
PAGE* 52

DATE 03-Fob-1998

TME: $16: 40: 26$

REporT

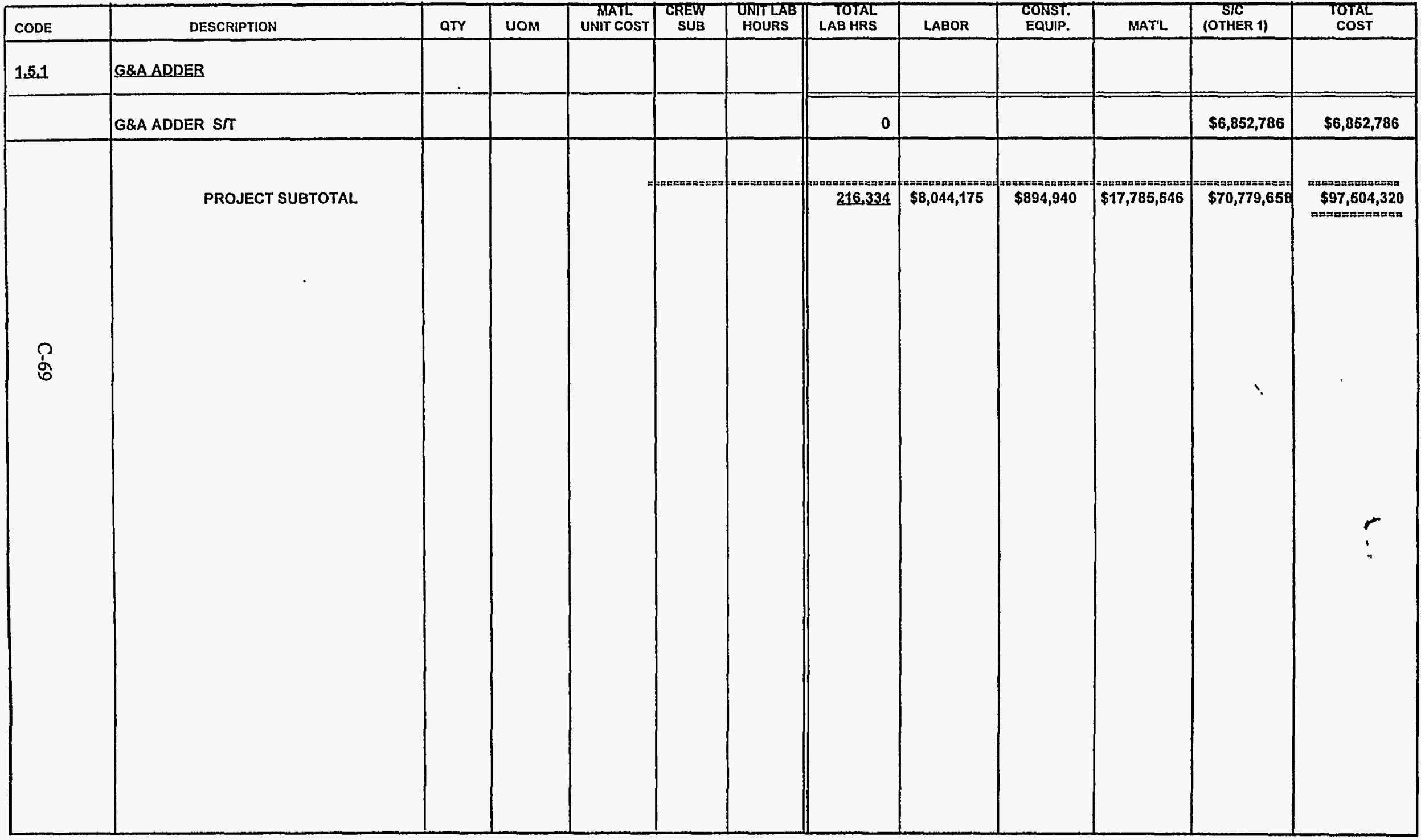


Lockheed Martin Idaho Technologies Co.

PROJECT NAME: Recalcination Treatment Non-Separations Cementitious Waste Option

LOCATION 9: INEEL - ICPP

REQUESTOR: Al LeE 6-9716 MS 3765
CONTINGENCY ANALYSIS

TYPE OF ESTIMATE: Planning PROJECT NO: 2420 PREPARED BY:
1.

DATE: $03-F e b-1998$

TIME: $16: 35: 23$

REPORT NAME: Contingency Analysis

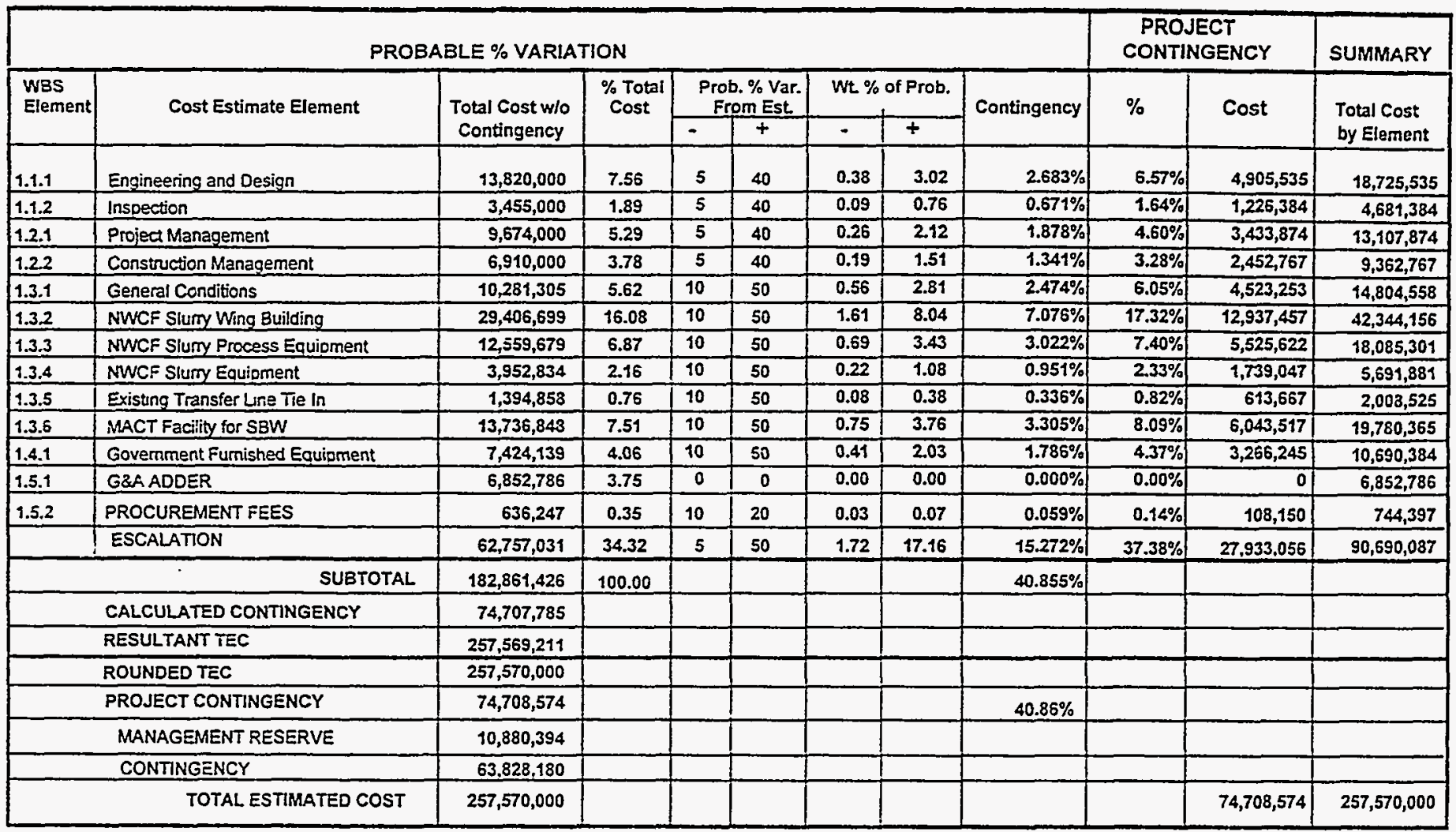

CONFIDENCE LEVEL AND ASSUMED RISKS:

The Lockheed Idaho Technologies Co. Cost Estimate Contingency Analysis Model is based on the applied contingency and the assumptions upon which the estimate was predicated. The model is applied with a suggested risk level of $18 \%$ and a level of confidence of $90 \%$ the estimate will fall within the bid range. The Contingency Analysis is based on a weighted average to provide a $90 \%$ probability of underrun and a $10 \%$ probability of overrun.
CONTINGENCY ANALYSIS GUIDE BY TYPE OF ESTIMATE

Guidelines established by DOE/FM 50 , Cost Estimating Guide, Vol. 6 , Cost Guide, and as presented in the INEL Cost Estimating Guide. PLANNING $20 \%-30 \%$ . Conceptual $\quad 15 \%-25 \%$ . Experimental/Special Conditions.............Up to $40 \%$ TITLE I

TITLE II $10 \%-20 \%$ Market Conditions 
Lockheed Martin Idaho Technologies Co. Rev. $6 / 96$

PROSECT NAME: Recalcination Treatment Non-Separations Cementitious Waste Option - Unescalated

LOCATION $1:$

INEEL - ICPP

REQUESTOR: AI Lee 6-9716 MS 3765
CONTINGENCY ANALYSIS

TYPE OFESTIMATE: Planning

PROSECT NO:

PREPARED BY:
2420

JRB/BCE
DATE: 04-Feb-1998

TME: 12:09:58

REPORT NAME: Contingency Analysis

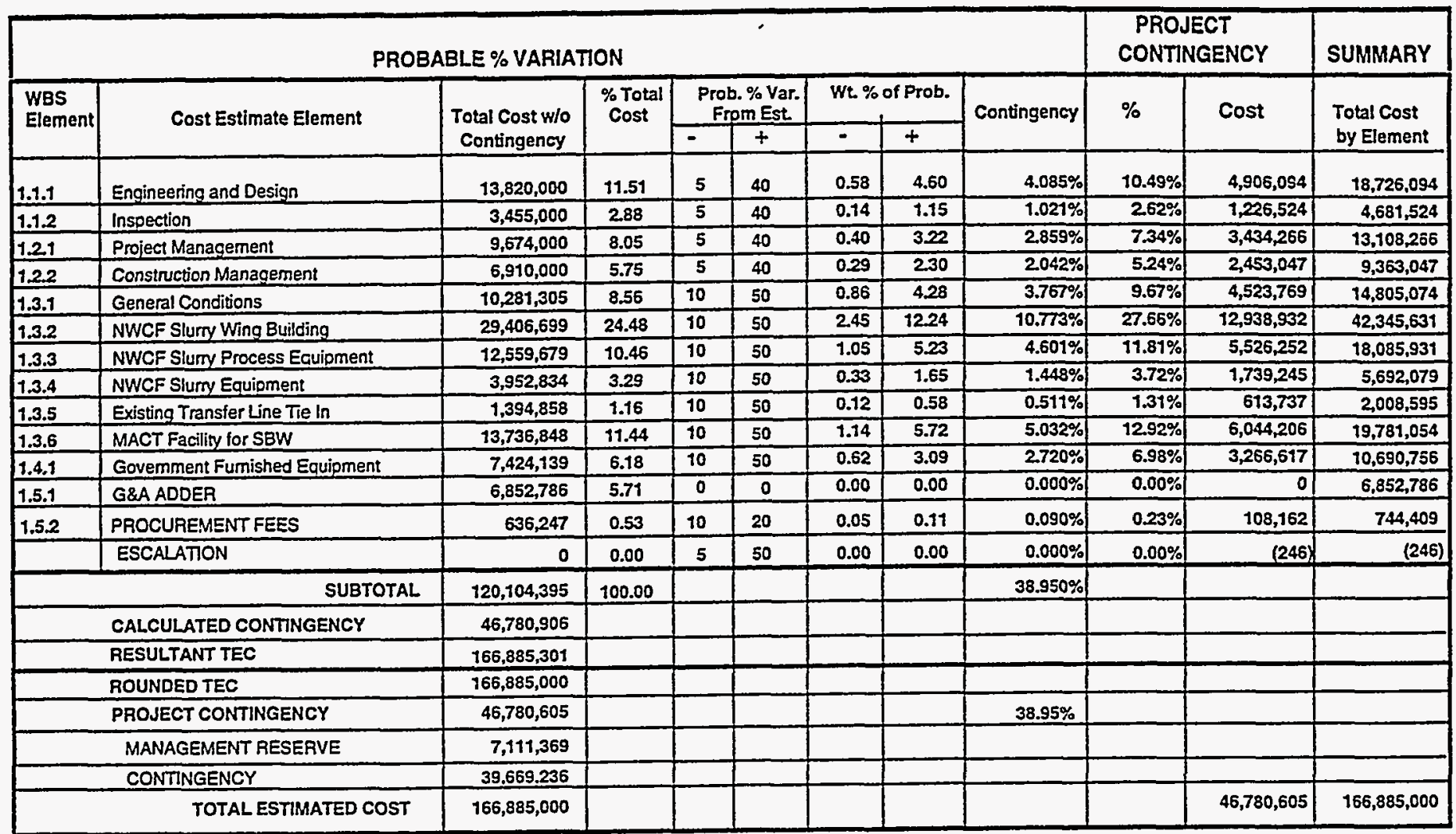

CONFIDENCE LEVEL AND ASSUMED RISKS:

The Lockheed Idaho Technologies Co. Cost Estimate Contingency Analysis

Model is based on the applied contingency and the assumptions upon which

the estimate was predicated. The model is applied with a suggested risk level

of $18 \%$ and a level of confidence of $90 \%$ the estimate will fall within the bid range.

The Contingency Analysis is based on a weighted average to provide a

$90 \%$ probability of underrun and a $10 \%$ probability of overrun.
CONTINGENCY ANALYSIS GUIDE BY TYPE OF ESTIMATE

Guidelines established by DOE/FM 50, Cost Estimating Guide, Vol. 6.

Cost Guide, and as presented in the INEL Cost Estimating Guide.

PLANNING

Conceptual $15 \%-25 \%$

Experimental/Special Conditions............. Up to $40 \%$

TITLEI

TITLE U

TITLE II/AFC

$5 \%$ - $15 \%$

Market Conditions 
Lockheed Martin Idaho Technologies Co.

PROJECT NAME: Recalcination Treatment Non-Separations Cementitious Waste Option - Escalated

LOCATICN 1

REQUESTOR: Al Lee 6-9716 MS 3765
CONTINGENCY ANALYSIS

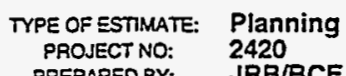

$\begin{array}{ll}\text { PROJECT NO: } & 2420 \\ \text { PREPARED BY: } & \text { JRB/BCE }\end{array}$
DATE: $04-F e b-1998$
TIME: $14: 04: 46$

REPORT NAME: Contingency Analysis

\begin{tabular}{|c|c|c|c|c|c|c|c|c|c|c|c|}
\hline & PROE & BLE \% VARIA & ON & & & & & & $\begin{array}{l}\text { PROJ } \\
\text { CONTI }\end{array}$ & $\begin{array}{l}\text { CT } \\
\text { IENCY }\end{array}$ & SUMMARY \\
\hline \begin{tabular}{|l|} 
WBS \\
Element
\end{tabular} & Cost Estimate Element & Total Cost w/o & $\begin{array}{c}\% \text { Total } \\
\text { Cost }\end{array}$ & & $\begin{array}{l}\% \text { Var. } \\
\text { m Est. }\end{array}$ & Wt. \% & Prob. & Contingency & $\%$ & Cost & Total Cost \\
\hline & & Contingency & & $=$ & + & - & + & & & & by Elament \\
\hline 1.1 .1 & Engineering and Design & $13,820,000$ & 8.23 & 5 & 40 & 0.41 & 3.29 & $2.923 \%$ & $7.21 \%$ & $4,907,249$ & $18,727,249$ \\
\hline 1.1 .2 & Inspection & $3,455,000$ & 2.06 & 5 & 40 & 0.10 & 0.82 & $0.731 \%$ & $1.80 \%$ & $1,226,812$ & $4,681,812$ \\
\hline 1.2 .1 & Project Management & $9,674,000$ & 5.76 & 5 & 40 & 0.29 & 2.31 & $2.046 \%$ & $5.05 \%$ & $3,435,074$ & $13,109,074$ \\
\hline 1.2 .2 & Construction Management & $6,910,000$ & 4.12 & 5 & 40 & 0.21 & 1.65 & $1.461 \%$ & $3.61 \%$ & $2,453,624$ & $9,363,624$ \\
\hline 1.3 .1 & General Conditions & $10,281,305$ & 6.13 & 10 & 50 & 0.61 & 3.06 & $2.695 \%$ & $6.65 \%$ & $4,524,834$ & $14,806,139$ \\
\hline 1.3 .2 & NWCF Slurry Wing Building & $29,405,699$ & 17.52 & 10 & 50 & 1.75 & 8.76 & $7.708 \%$ & $19.02 \%$ & $12,941,978$ & $42,348,677$ \\
\hline 1.3 .3 & NWCF Slumy Process Equipment & $12,539,679$ & 7.48 & 10 & 50 & 0.75 & 3.74 & $3.292 \%$ & $8.12 \%$ & $5,527,553$ & $18,087,232$ \\
\hline 1.3 .4 & NWCF Slumy Equipment & $3,952,834$ & 2.35 & 10 & 50 & 0.24 & 1.18 & $1.036 \%$ & $256 \%$ & $1,739,654$ & $5,692,488$ \\
\hline 1.3 .5 & Existing Transfer Line Tie in & $1,394,858$ & 0.83 & 10 & 50 & 0.08 & 0.42 & $0.366 \%$ & $0.50 \%$ & 613,881 & $2,008,739$ \\
\hline 1.3.6 & MACT Facility for SBW & $13,736,848$ & 8.18 & 10 & 50 & 0.82 & 4.09 & $3.601 \%$ & $8.88 \%$ & $6,045,629$ & $19,782,477$ \\
\hline 1.4 .1 & Government Fumished Equioment & $7,424,139$ & 4.42 & 10 & 50 & 0.44 & 221 & $1.946 \%$ & $4.80 \%$ & $3,267,386$ & $10,691,525$ \\
\hline 1.5 .1 & G\&A ADDER & $6,852,786$ & 4.08 & 0 & 0 & 0.00 & 0.00 & $0.000 \%$ & $0.00 \%$ & 0 & $6,852,786$ \\
\hline 1.5 .2 & PROCUREMENT FEES & 636,247 & 0.38 & 10 & 20 & 0.04 & 0.08 & $0.054 \%$ & $0.16 \%$ & 108,187 & 744,434 \\
\hline & ESCALATION & $47,749,976$ & 28.45 & 5 & 50 & 1.42 & 14.22 & $12.659 \%$ & $31.23 \%$ & $21,253,768$ & $69,003,744$ \\
\hline & SUBTOTAL & $167,854,371$ & 100.00 & & & & & $40.529 \%$ & & & \\
\hline & CALCULATED CONTINGENCY & $68,029,646$ & & & & & & & & & \\
\hline & RESULTANT TEC & $235,834,017$ & & & & & & & & & \\
\hline & ROUNDED TEC & $235,900,000$ & & & & & & & & & \\
\hline & PROJECT CONTINGENCY & $68,045,629$ & & & & & & $40.54 \%$ & & & \\
\hline & MANAGEMENT RESERVE & $10,101,772$ & & & & & & & & & \\
\hline & CONTINGENCY & $57,943,857$ & & & & & & & & & \\
\hline & TOTAL ESTIMATED COST & $235,900,000$ & & & & & & & & $68,045,629$ & $235,900,000$ \\
\hline
\end{tabular}

\section{CONFIDENCE LEVEL AND ASSUMED RISKS:}

The Lockheed Idaho Technologies Co. Cost Estimate Contingency Analysis Model is based on the applied contingency and the assumptions upon which

the estimate was predicated. The model is applied with a suggested risk level of $18 \%$ and a level of confidence of $90 \%$ the estimate will fall within the bid range. The Contingency Analysis is based on a weighted average to provide a

$90 \%$ probability of underrun and a $10 \%$ probability of overrun.
CONTINGENCY ANALYSIS GUIDE BY TYPE OF ESTIMATE

Guidelines established by DOEIFM 50, Cost Estimating Guide, Vol. 6, Cost Guide, and as presented in the INEL Cost Estimating Guide. PLANNING $20 \%-30 \%$ Experimental/Special Conditions............. Up to $50 \%$ Conceptual

Experimental/Special Conditions $15 \%-25 \%$ TITLE I TITLE UI $\quad 5 \%-15 \%$ TITLE II/AFC Market Conditions 
1.

\section{G\&A/PIF ADDER CALCULATION SHEET}

File $\$ 2420$

PROCUREMENT FEE:

CONSTRUCTION $=$
GFE $=$
$\quad$ Subtotal
$\begin{array}{r}\$ 71,332,221 \\ \$ 7,424,139 \\ \$ 78,756,360\end{array}$

FEE @ $1 \%=$

$\$ 78,756,360 \cdot 0.01=$

$\$ 787,564$

G\&A @ 23\% (with a ceiling of $\$ 500,000$ imposed per year

CONSTRUCTION OR

CEILING *4 YEARS =

GFE $=$

$\$ 2,000,000$

$\$ 7,424,139$

PROCUREMENT FEE $=$

$\$ 787,564$

Subtotal $\$ \frac{\$ 10,211,703}{}$

FEE @ 23\%=

$\$ 10,211,703 * 0.23=\quad \$ 2,348,692$

PIF @ 5.5\%

\begin{tabular}{|c|c|}
\hline CONSTRUCTION $=$ & $\$ 71,332,221$ \\
\hline GFE $=$ & $\$ 7,424,139$ \\
\hline PROCUREMENT FEE = & $\$ 787,564$ \\
\hline$G \& A=$ & $\$ 2,348,692$ \\
\hline
\end{tabular}

FEE @ $5.5 \%=$

$\$ 81,892,615 * 0.055=\quad \$ 4,504,094$

TOTAL PROCUREMENT FEE:

$\$ 787,564$

TOTAL G\&A FEE:

$\$ 2,348,692$

TOTAL PIF:

$\$ 4,504,094$ 
$1 \ldots$

,

\begin{abstract}
.
\end{abstract}




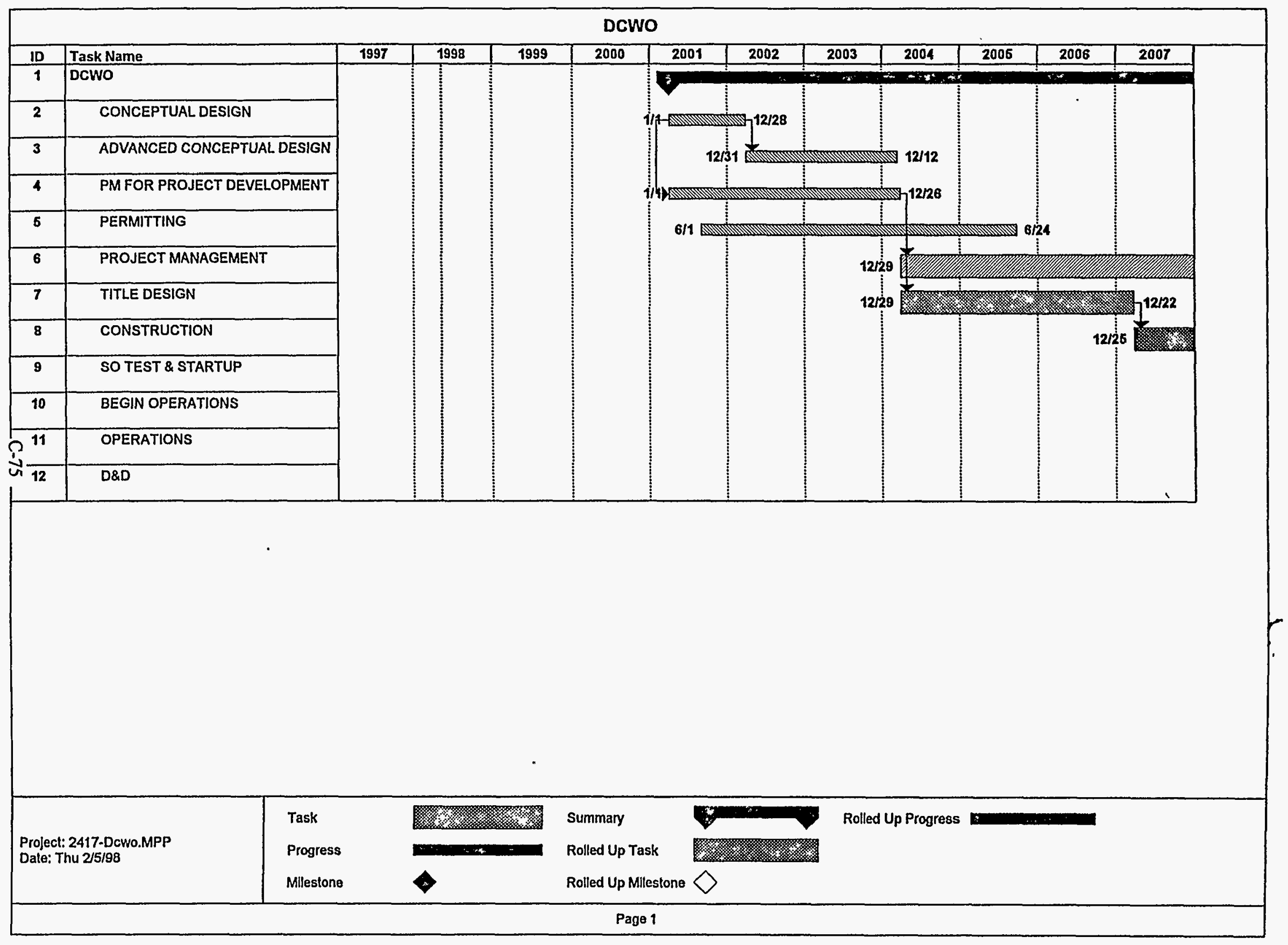




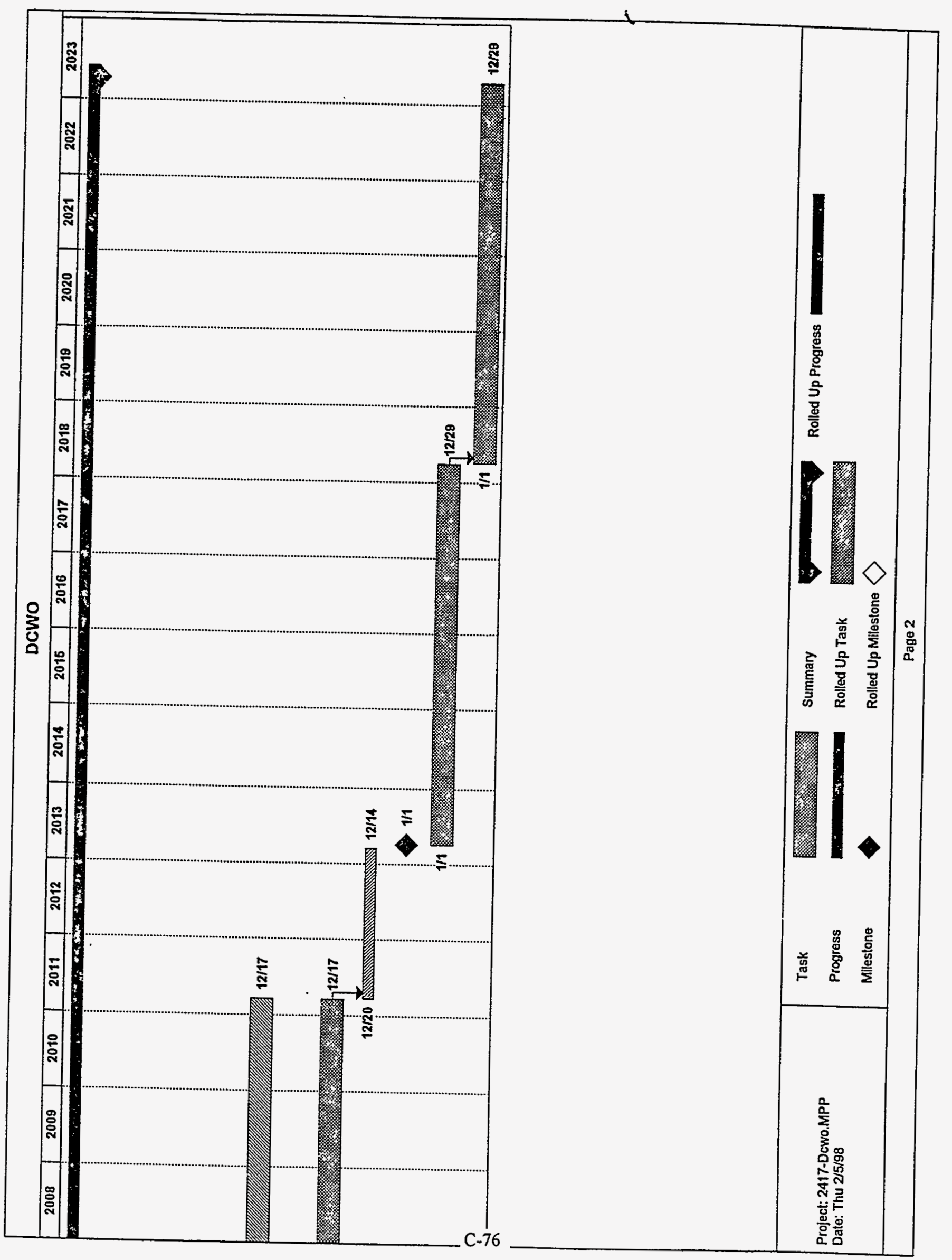


Date: $\quad$ February 7, 1998

To:

A. E. Lee

MS 3765

$6-9716$

From:

R. . Turk Ly fach

MS 3875

6-3611

Subject: ECONOMIC AND LIFE CYCLE ANALYSIS CONDUCTED FOR WASTE TREATMENT FACILITIES CEMENTITIOUS WASTE OPTION (CWO)-RJT$29 / 98$

\section{Purpose:}

As requested an Economic and Life-Cycle Cost (LCC) has been conducted to evaluate the NONSEPARATIONS CEMENTITIOUS WASTE OPTION (CWO). This process is proposed to treat all existing calcine with liquid Sodium Bearing Waste (SBW), by recalcining the resulting slurry using sucrose. The recalcined waste will be mixed with a grout, transferred into a canister, cured, sealed and then transported to an interim storage awaiting final disposition in a repository. The CWO facility will include a calcine receiving system, grouting material receiving and handling system, a blending system, a grouting process, product lag storage, an off-gas treatment system with supporting utilities system and a new Maximum Achievable Control Transfer (MACT) facility.

This economic analysis is based on information provided Sara Gifford, D. D. Taylor, H. S. Forsythe, D. N. Thompson, and A. E. LEE, Byron Blakely, John Duggan and other team members. R. B. Baker and B. W. Wallace provided cost estimates. Jack Prendergast provided process personnel modeling.

\section{Methodology:}

The Economic Evaluation assumed a 24-year period, (2001-2024) since this is the estimated time required to complete all of the anticipated remediation activities. The LCC identifies evaluated the initial development, construction, operating and post operating costs over the life-cycle. A discounted LCC analysis assumes a current 1998 -dollar basis, discounted at $6.30 \%$ per the Office of Management and Budget (OMB) Circular A-94. All costs are conservatively discounted assuming the end-of-year convention. 


\section{Assumptions:}

The scope of work and requirements of all related activities are vague at this time. Facility and processing costs were developed from historical experience associated with DD\&D work at the INEEL. The LCC analysis was generated to match cost estimating cost structure. These costs include Permitting, Direct and Indirect Construction, G\&A, Procurement Fee, Engineering, Inspection, Project Management, Construction Management, Escalation and Contingency costs. The design period is assumed to be accomplished in six years with construction completed in four years, followed by two years of start-up and testing. Labor rates were assumed as follows: Managers, $\$ 125 / \mathrm{hr}$; Engineers, $\$ 108 \$ / \mathrm{hr}$; Other Technicians $\$ 85 / \mathrm{hr}$; Administration/Support staff $\$ 65 / \mathrm{hr}$; Operators and Maintenance personnel $\$ 65 / \mathrm{hr}$. The operational period for this facility was assumed to be five years, followed by five years of post-operations activities. Utilities were assumed to cost $\$ .0824 / \mathrm{kWh}$ for the facility. Grouting material is assumed to cost $\$ 150 /$ ton, Sucrose \$1.50/gal, Kerosene \$1.50/gal, Nitric Acid \$1.07/gal, Sodium Hydroxide $\$ 660 /$ ton, Granular Carbon $\$ 8,000 /$ ton. Due to this projects lack of complexity and relative cleanliness this analysis assumed a decommissioning cost equal to $20 \%$ of the unescalated engineering design cost, decontamination costs equal to $5 \%$ of total unescalated TPC and demolition costs equal to $8 \%$ of total unescalated TPC cost.

\section{Results:}

The Five-year Cementitious Option has a Discounted LCC of $\$ 574$ million.

Attachments:

cc: R. J. Turk File 


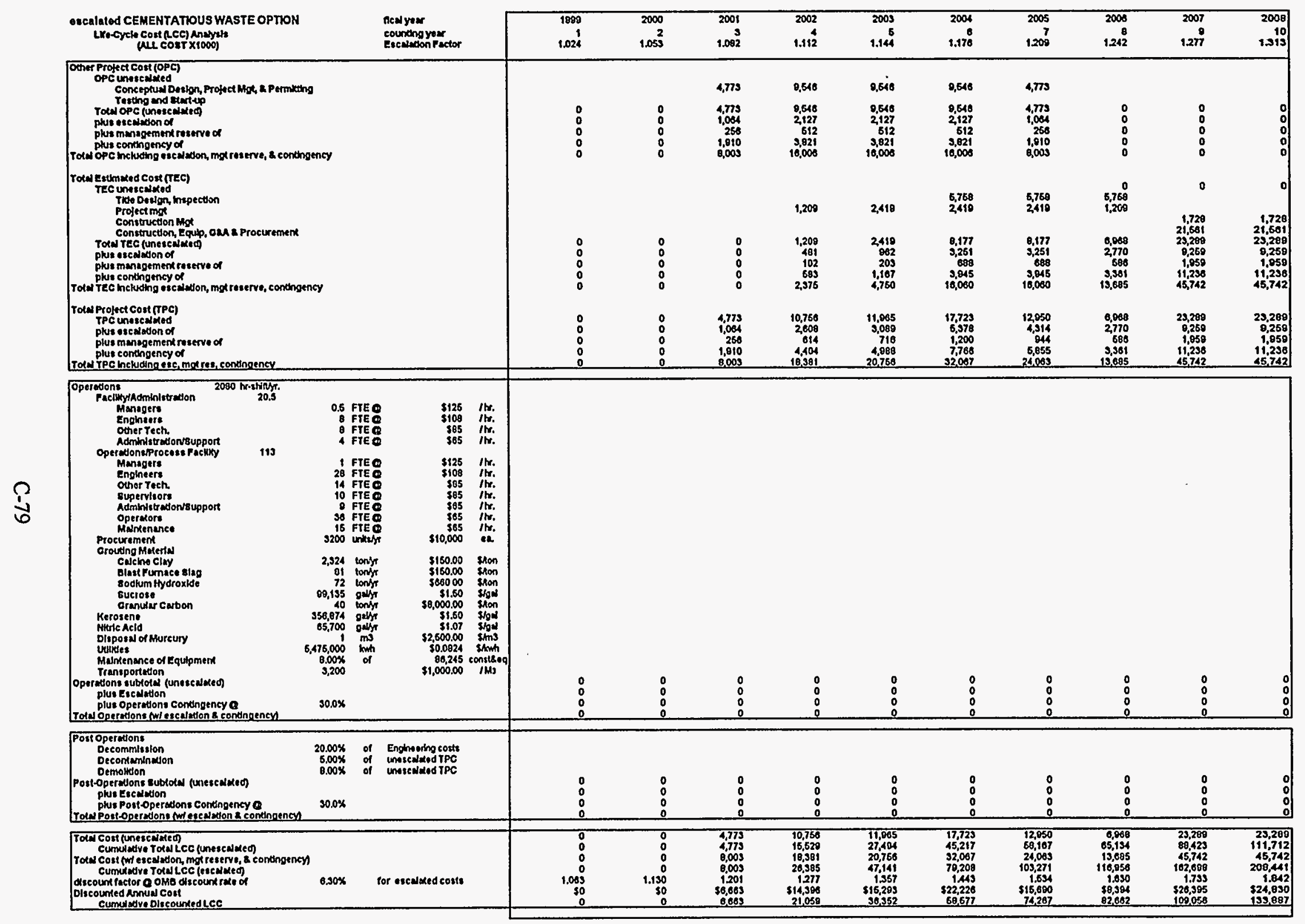




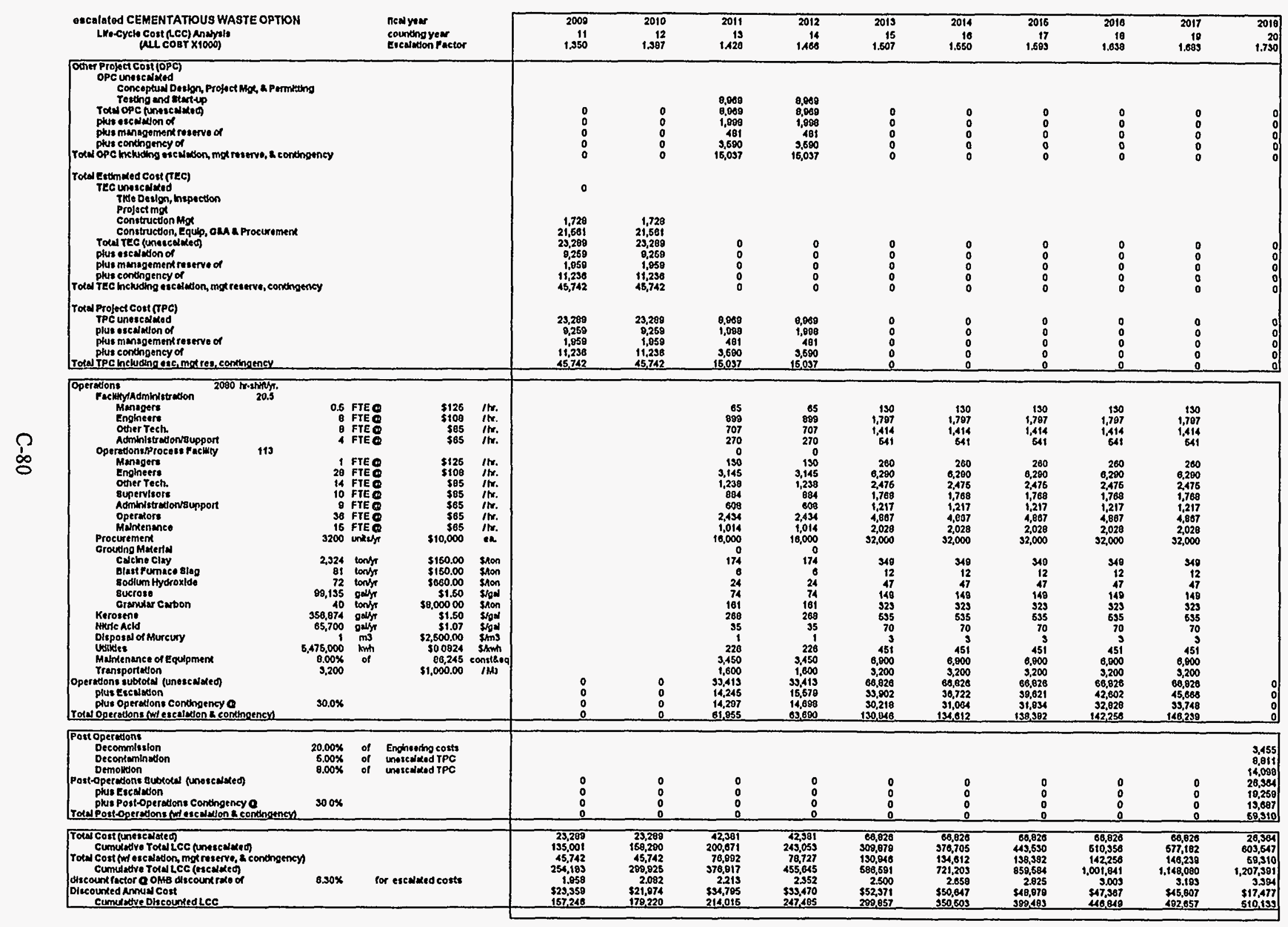




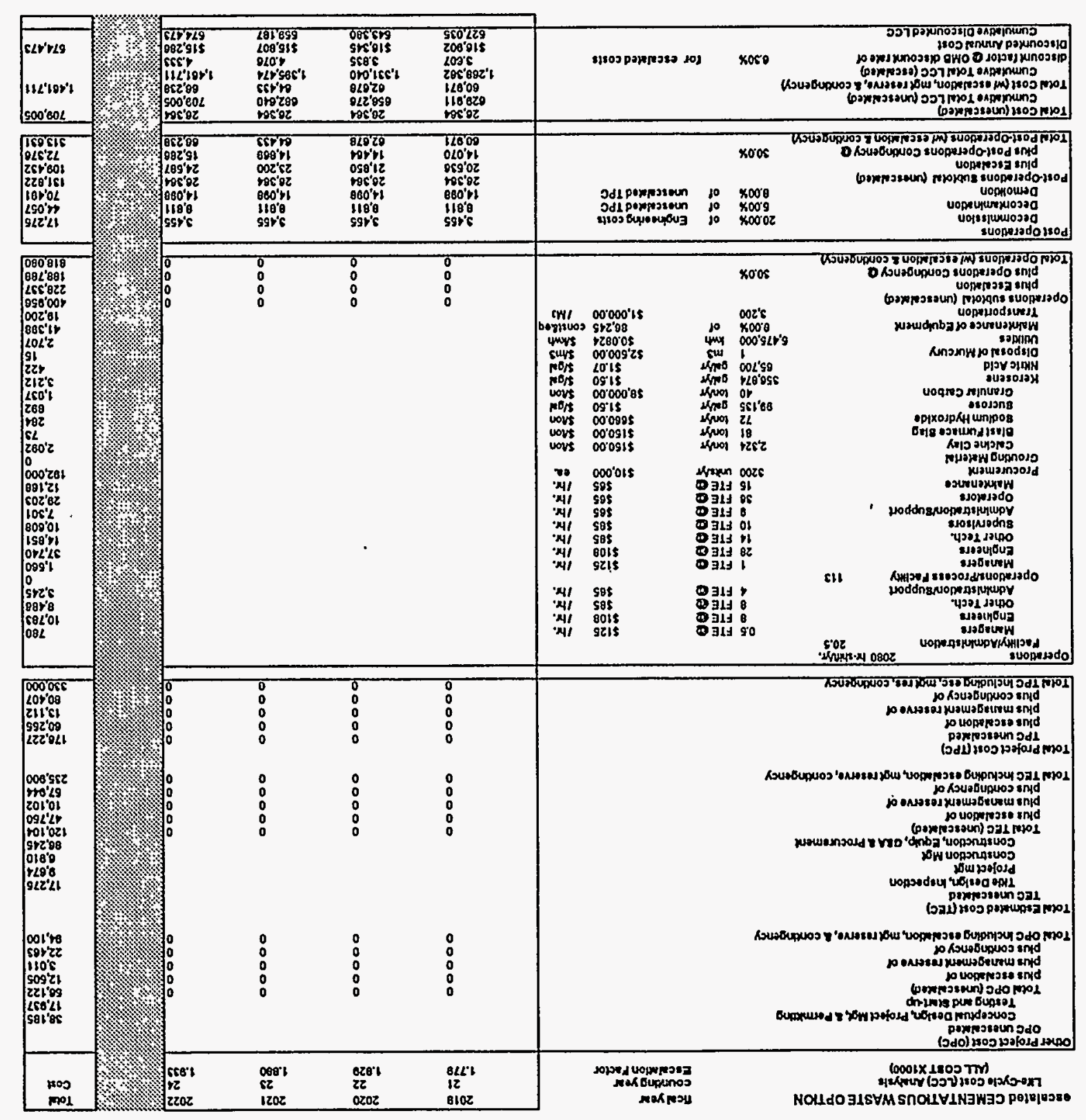




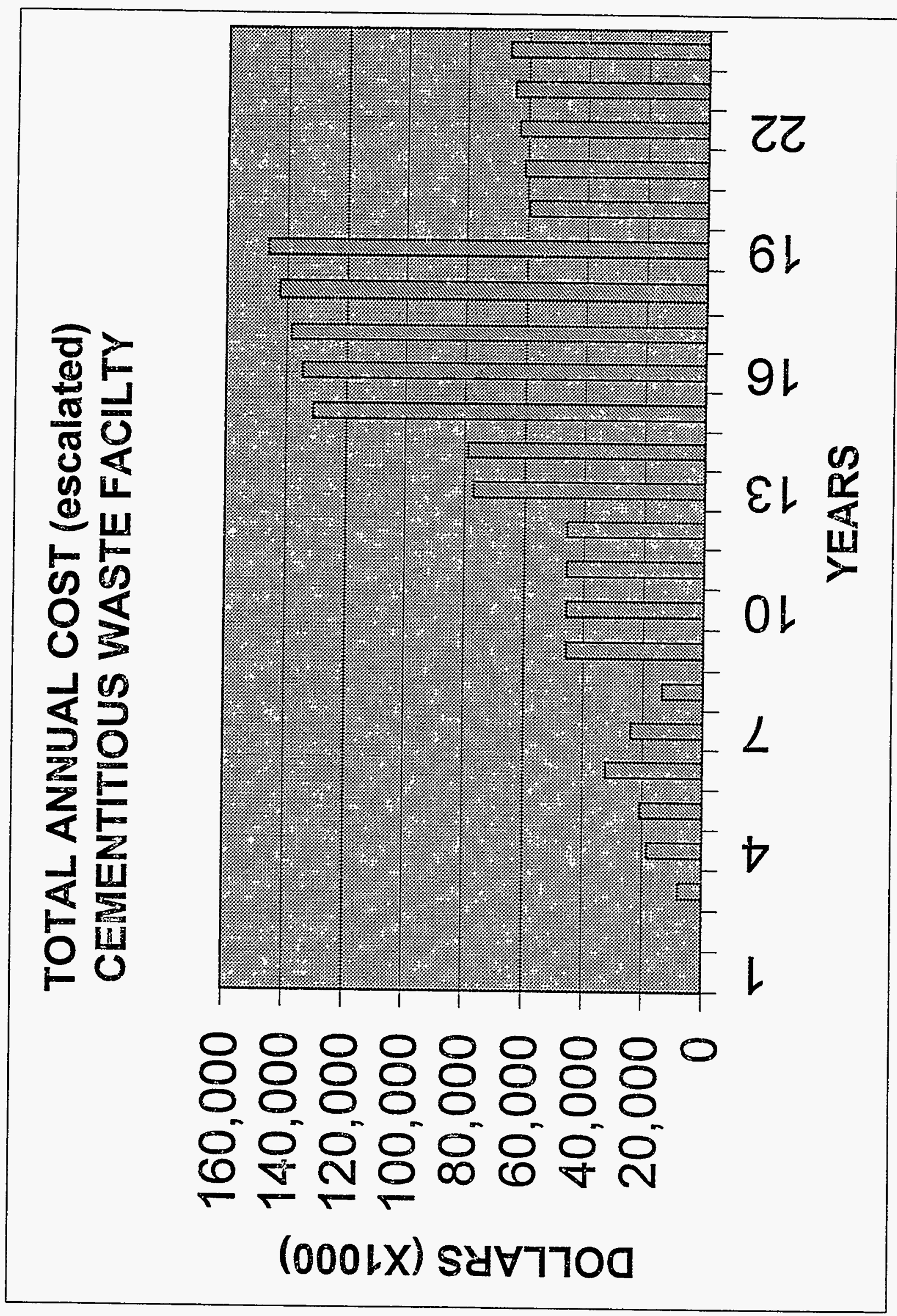




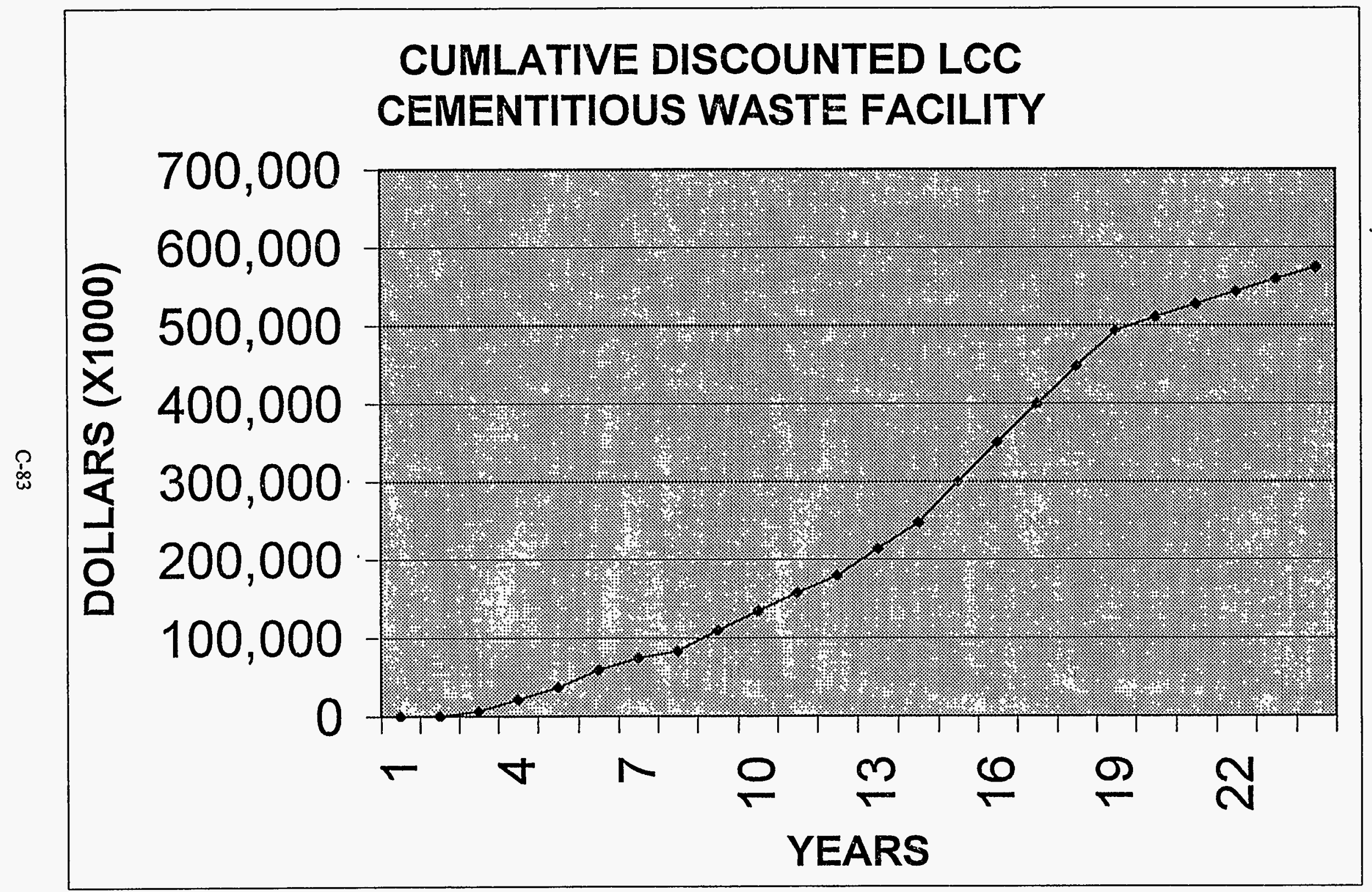




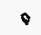

C-84 
MACT Operations Cost Estimate

$2013-2017$

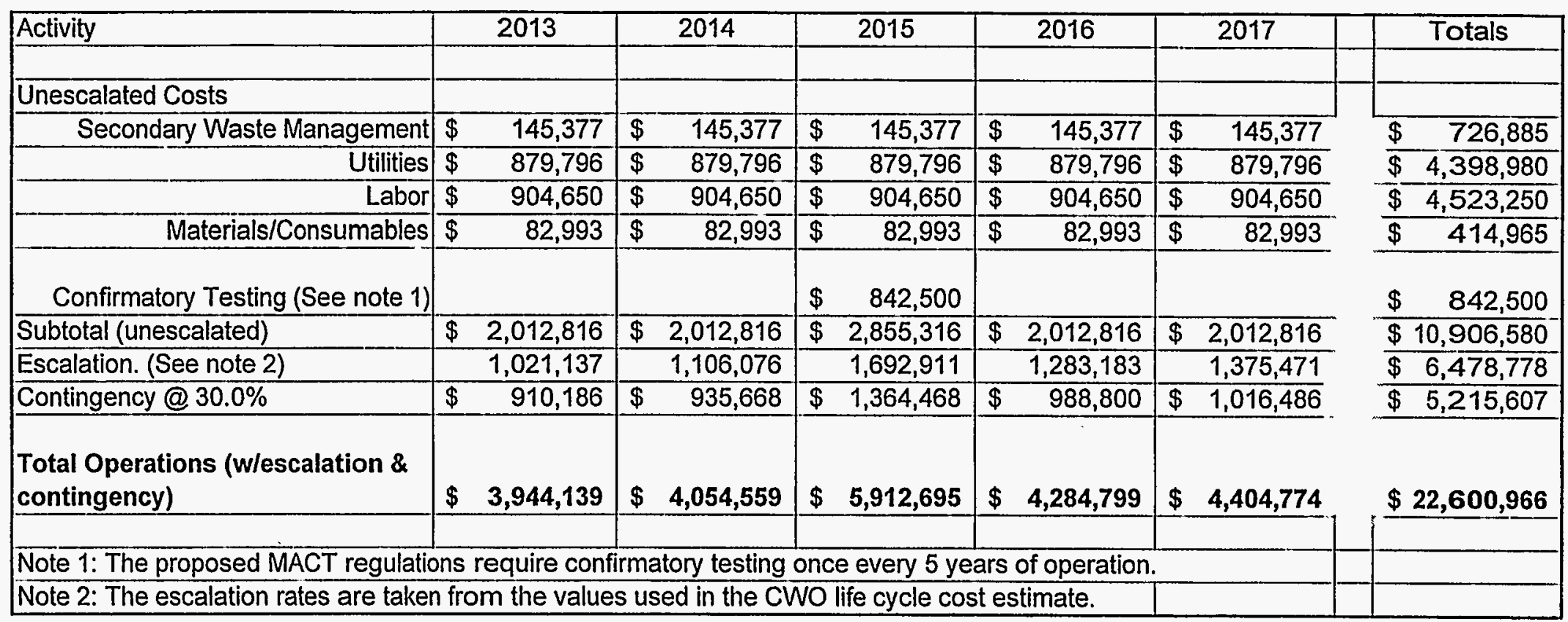




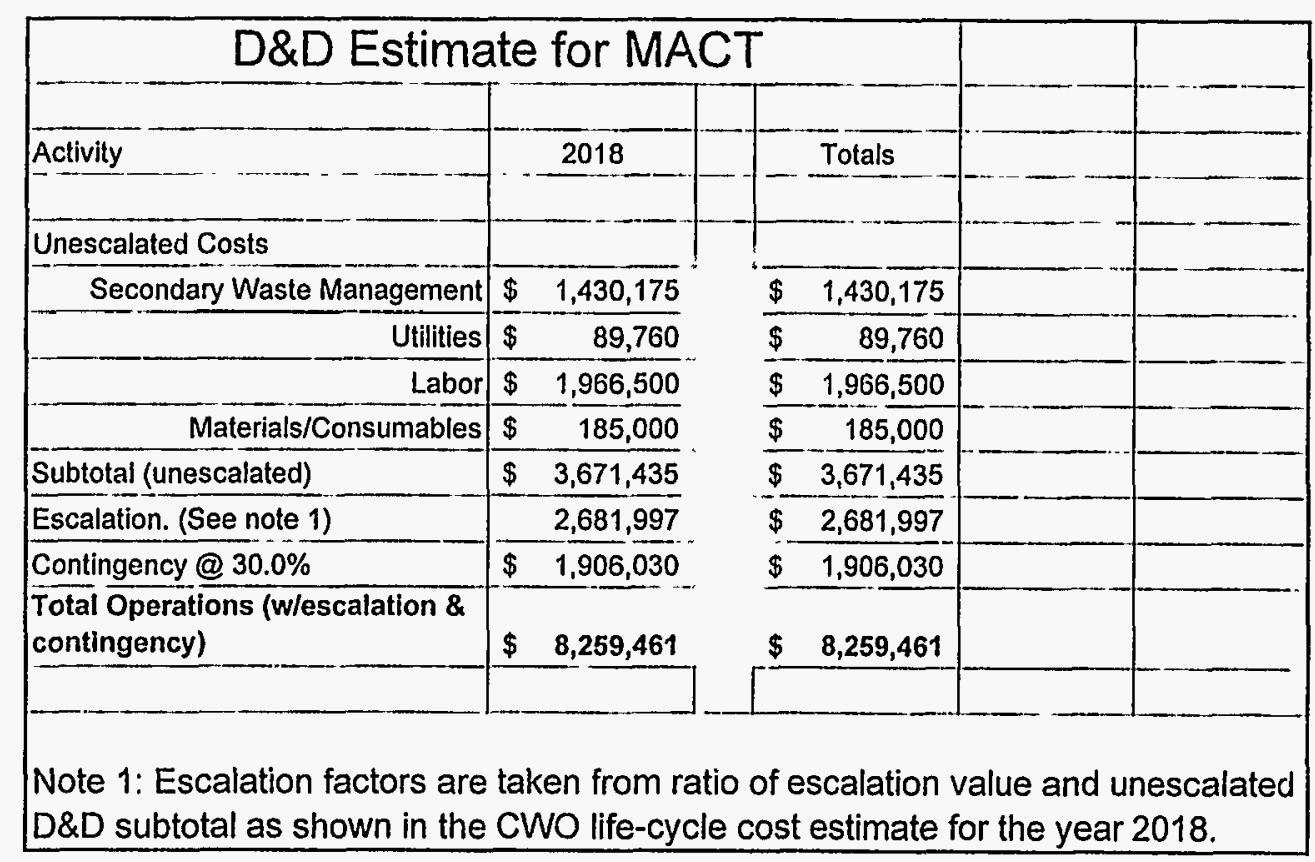




\section{Appendix D}

\section{Drawings and Sketches}




\section{Appendix D}

\section{Drawings and Sketches}

CWO-CI

CWO-C2

CWO-A1

CWO-A2

CWO-A3

CWO-A4

CWO-E1 


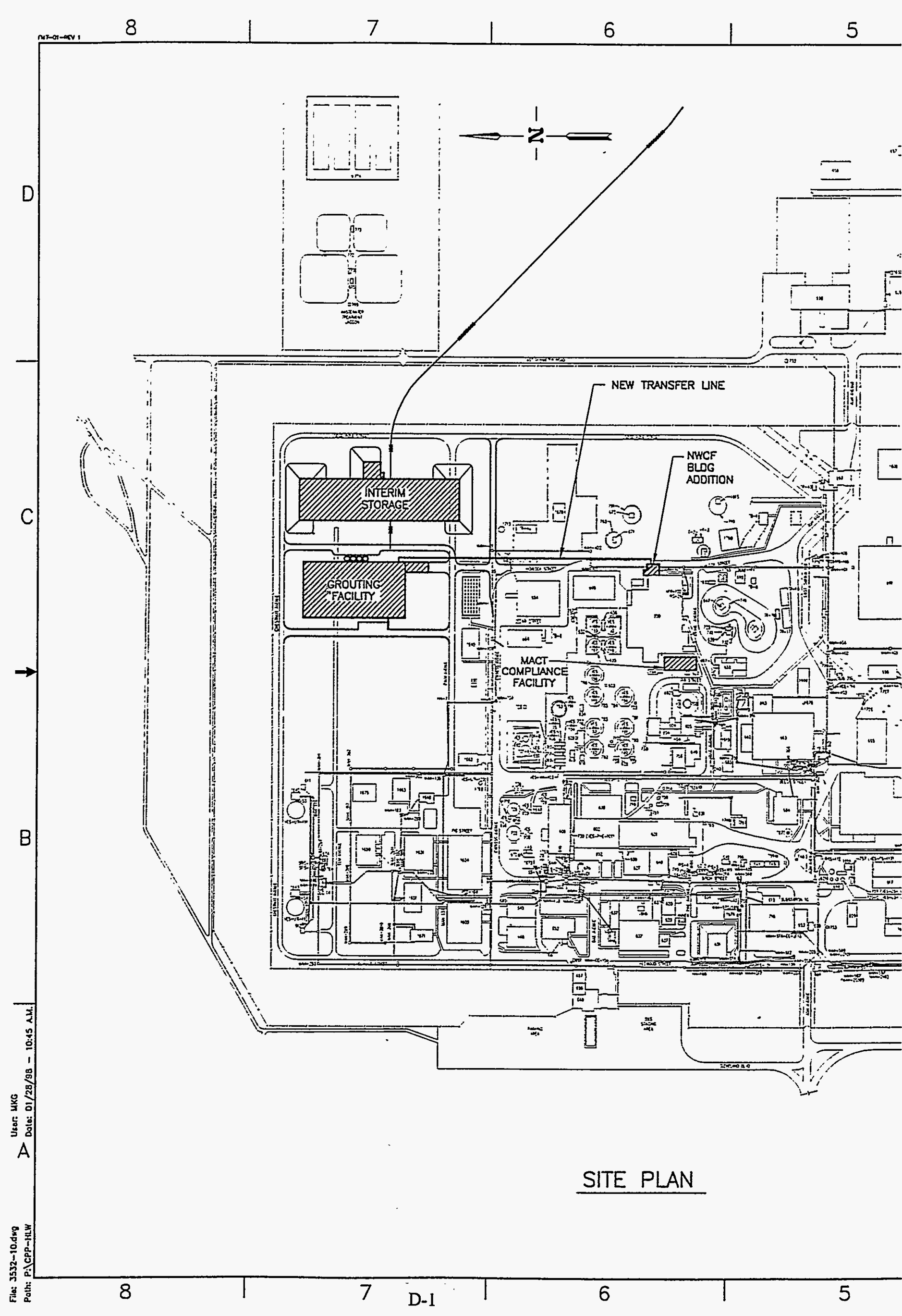




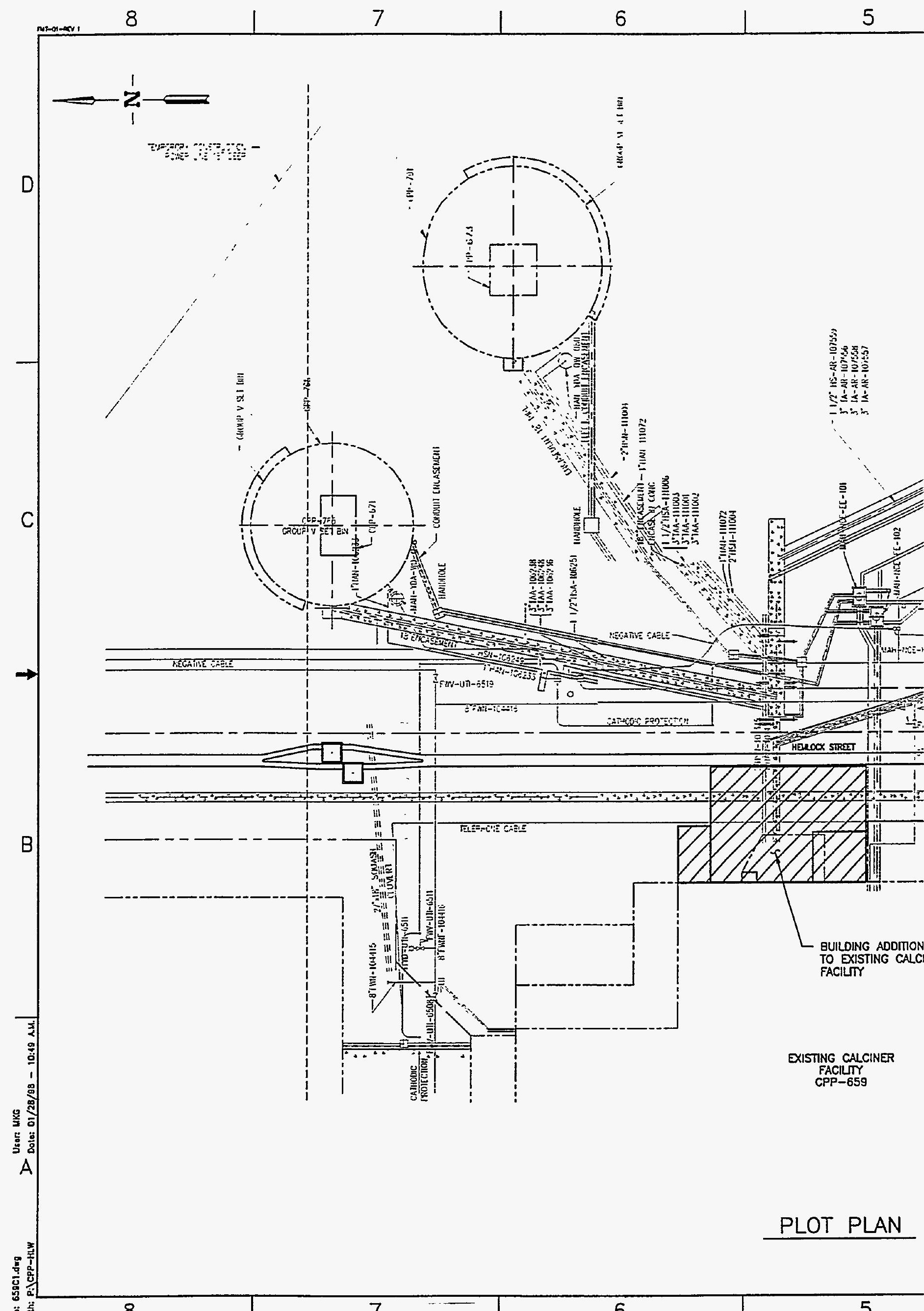




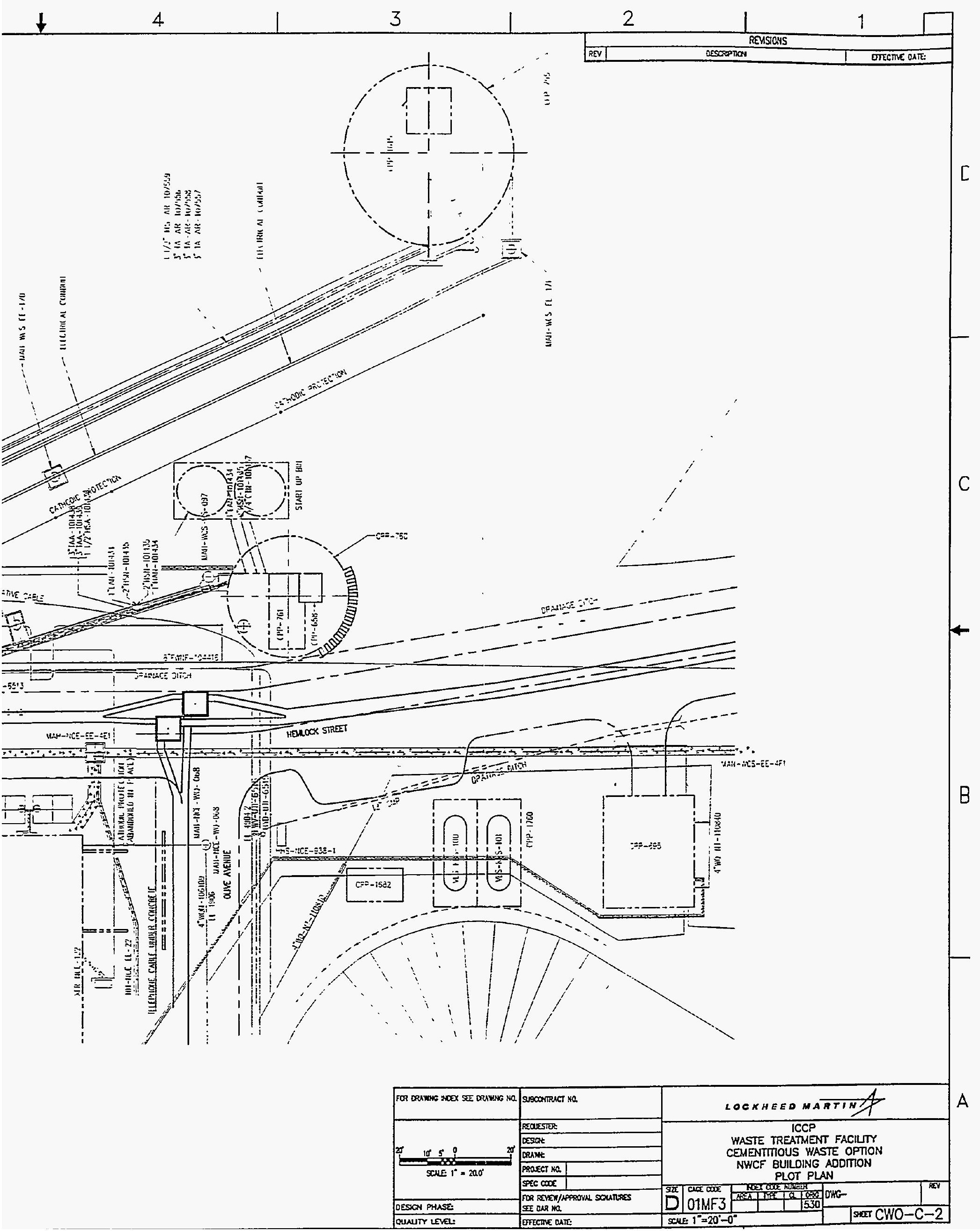




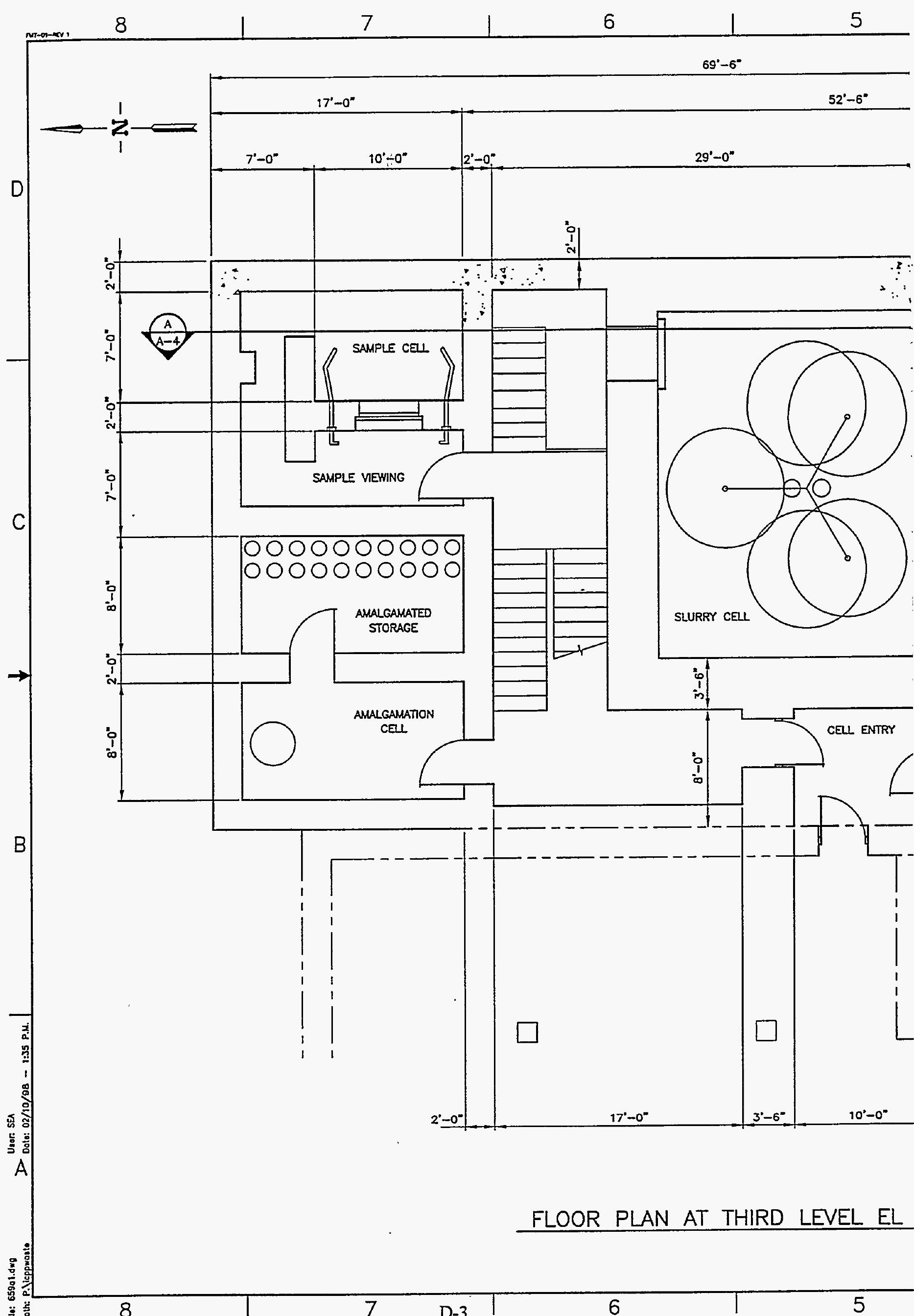




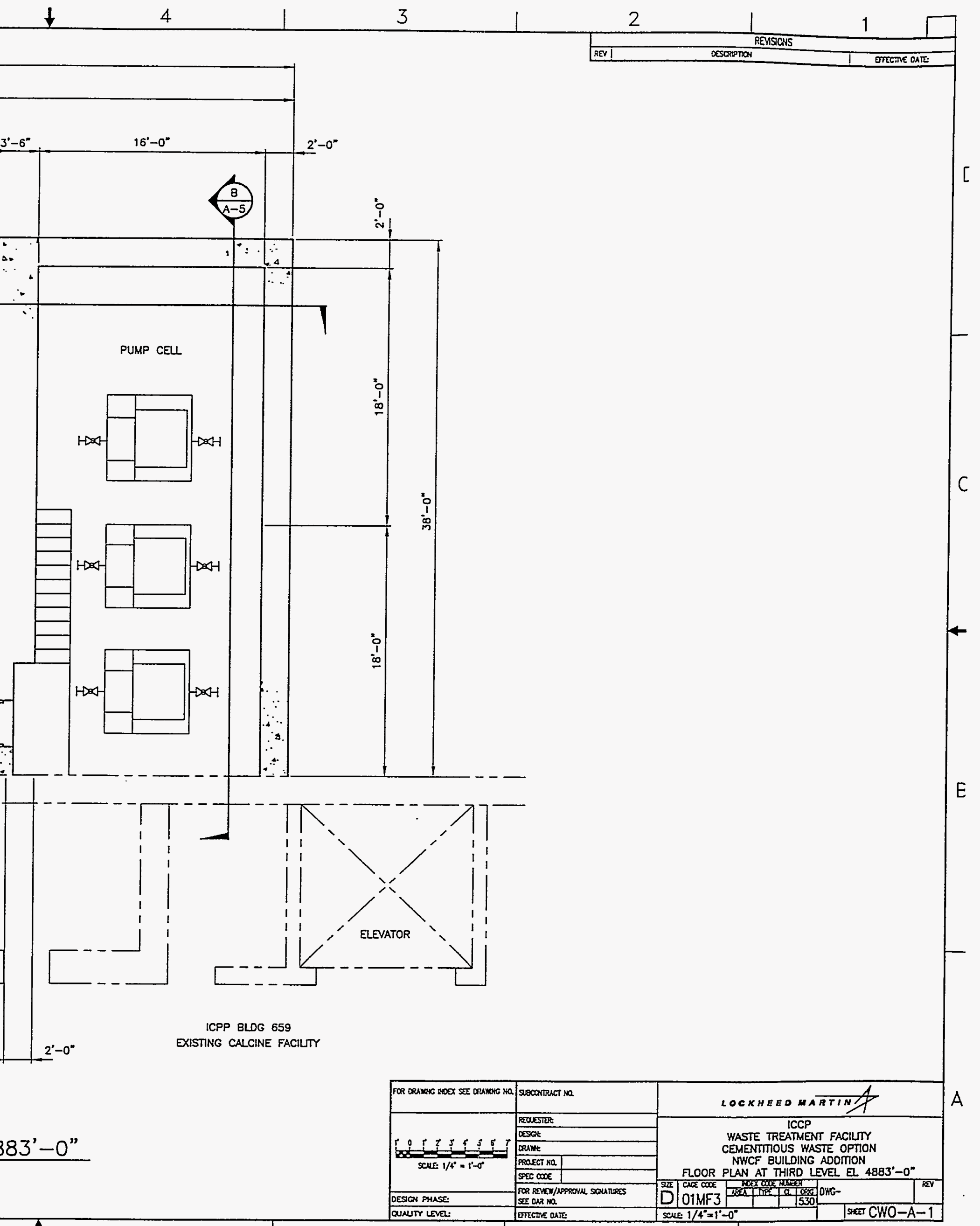




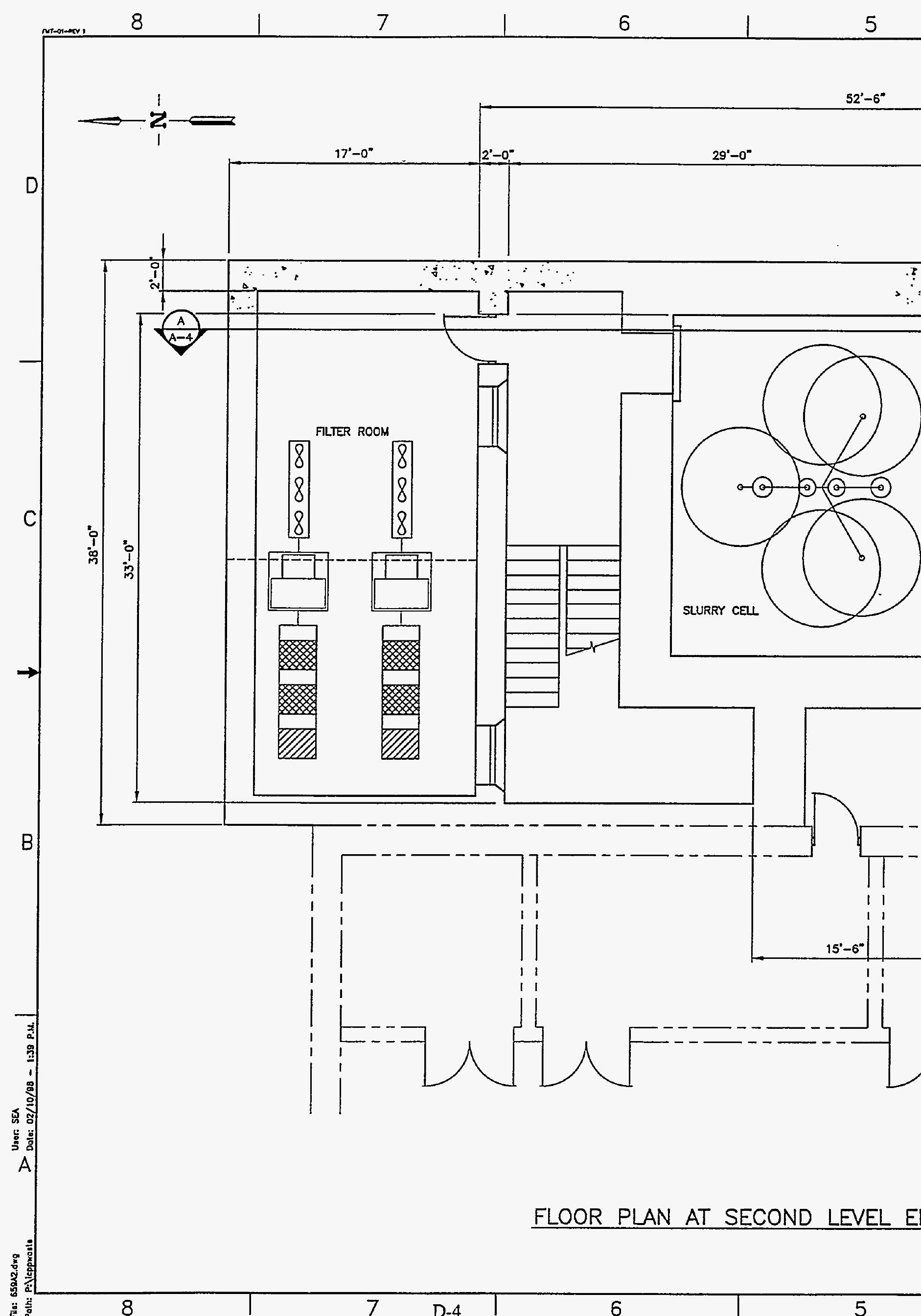




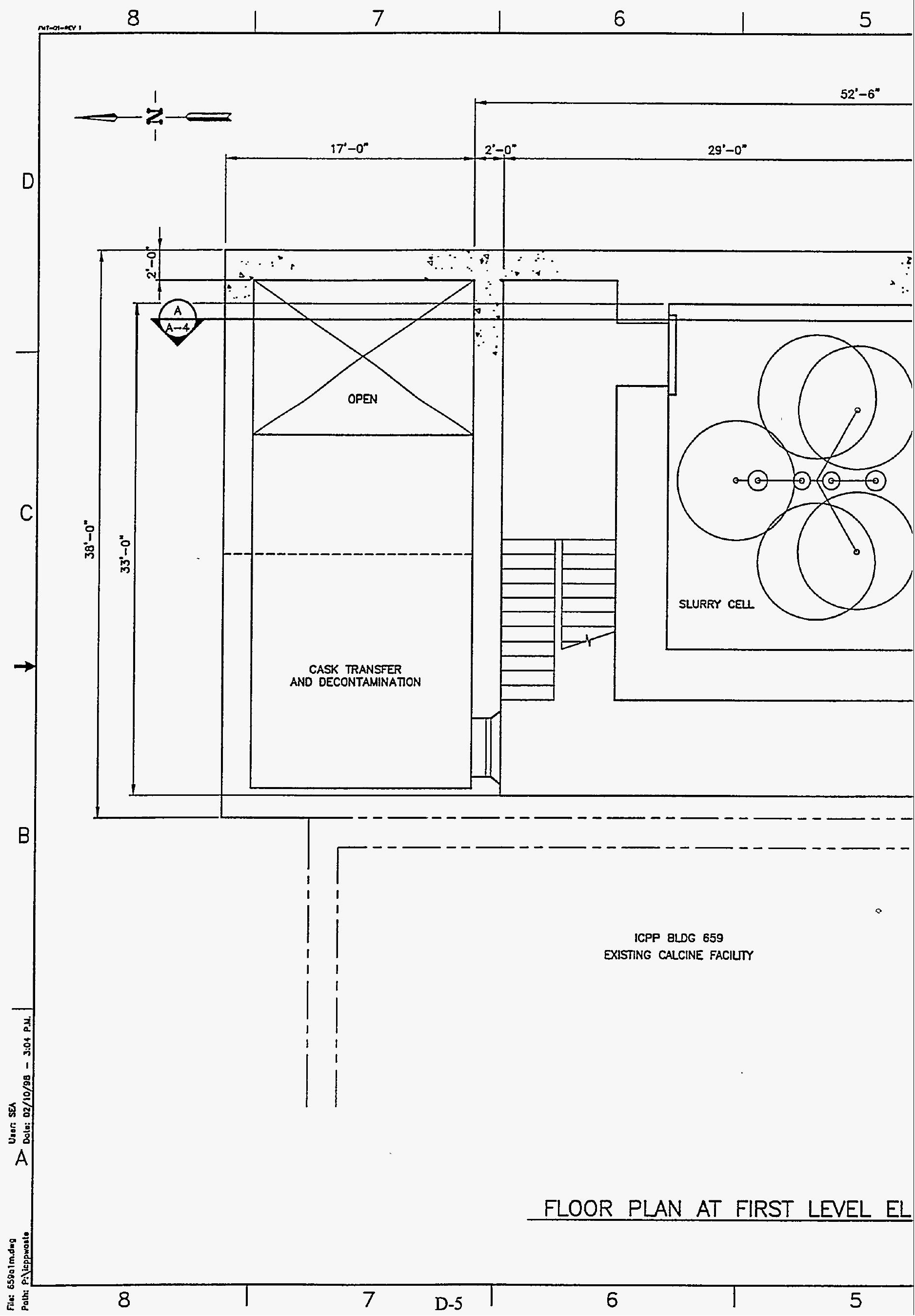




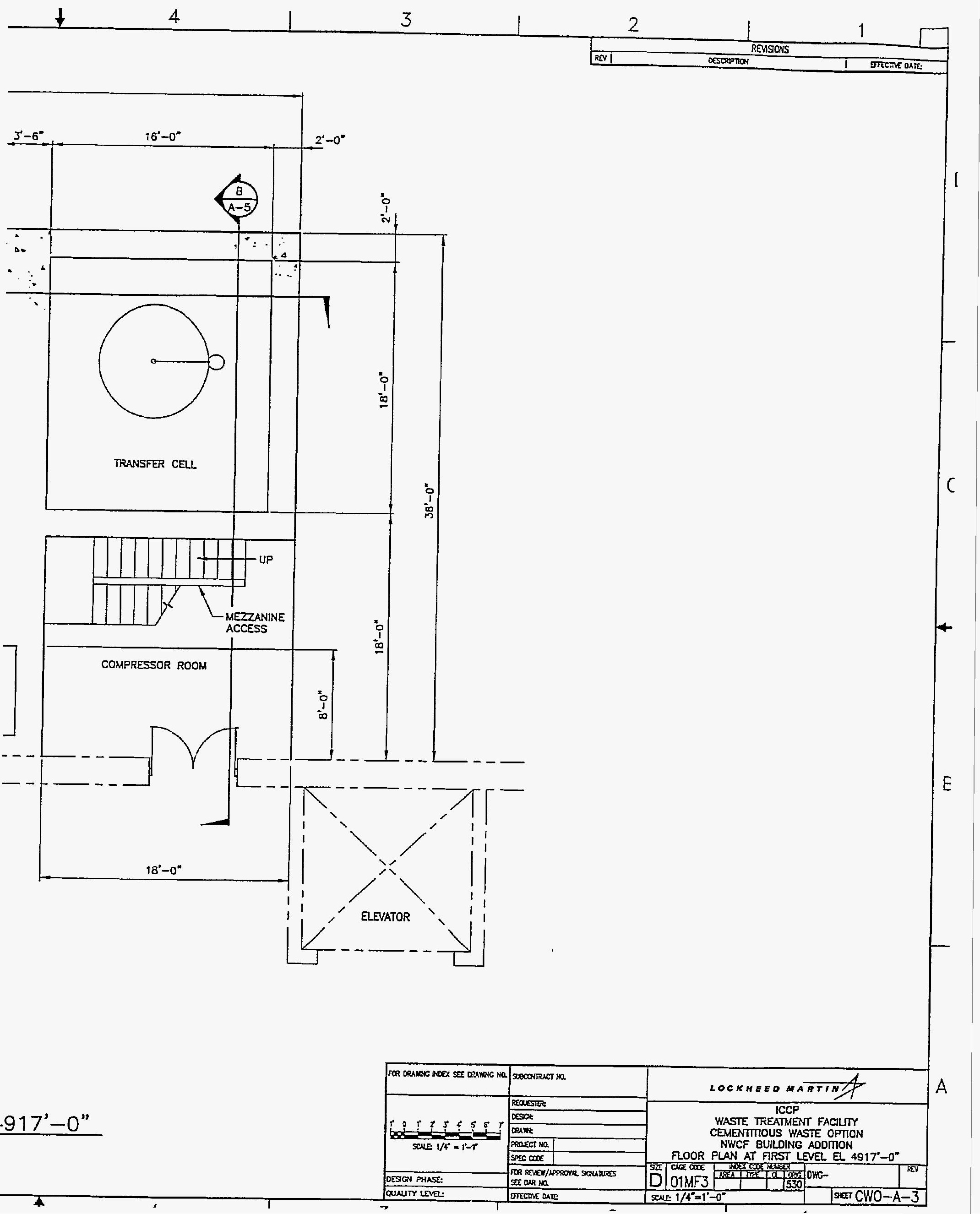




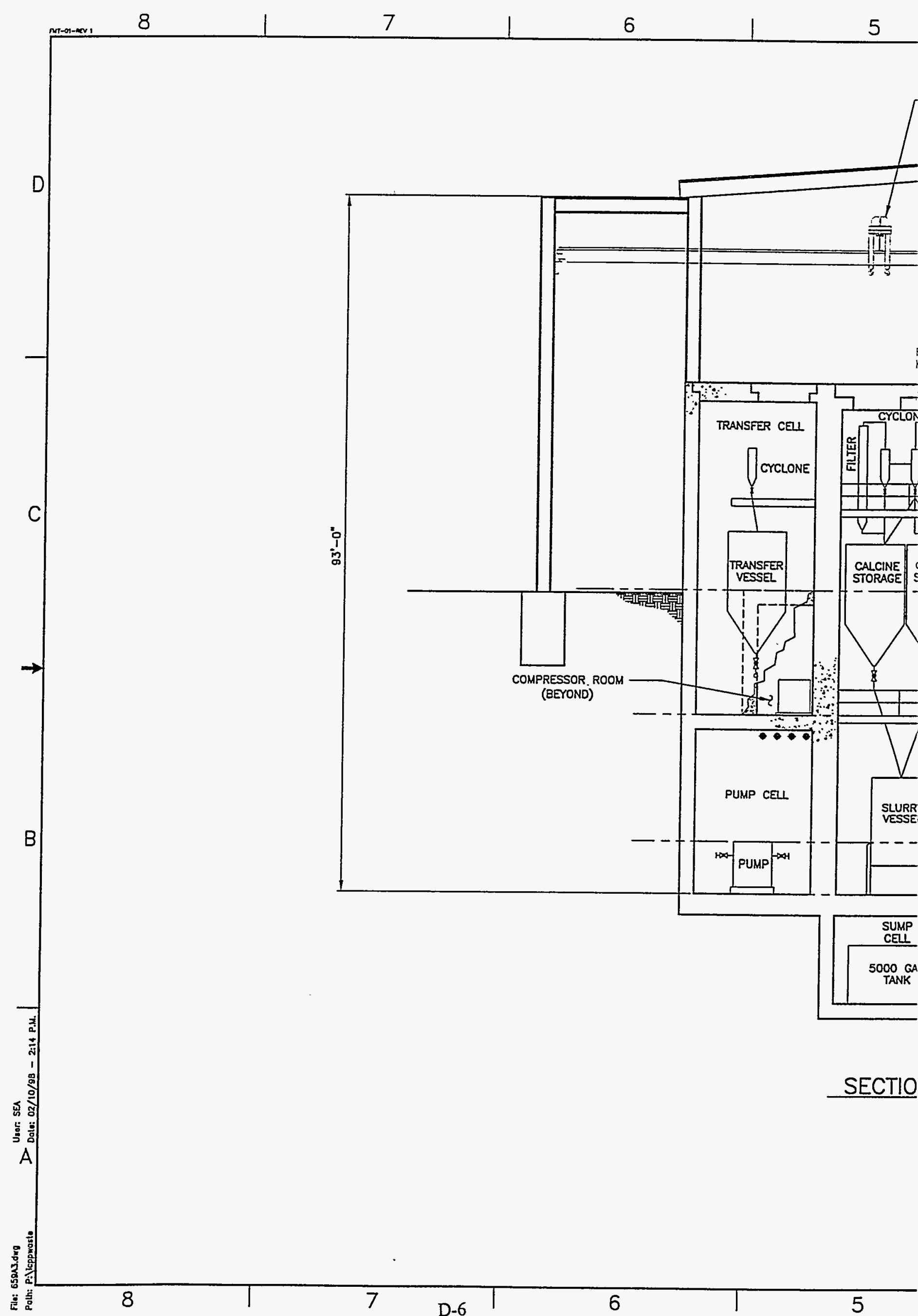




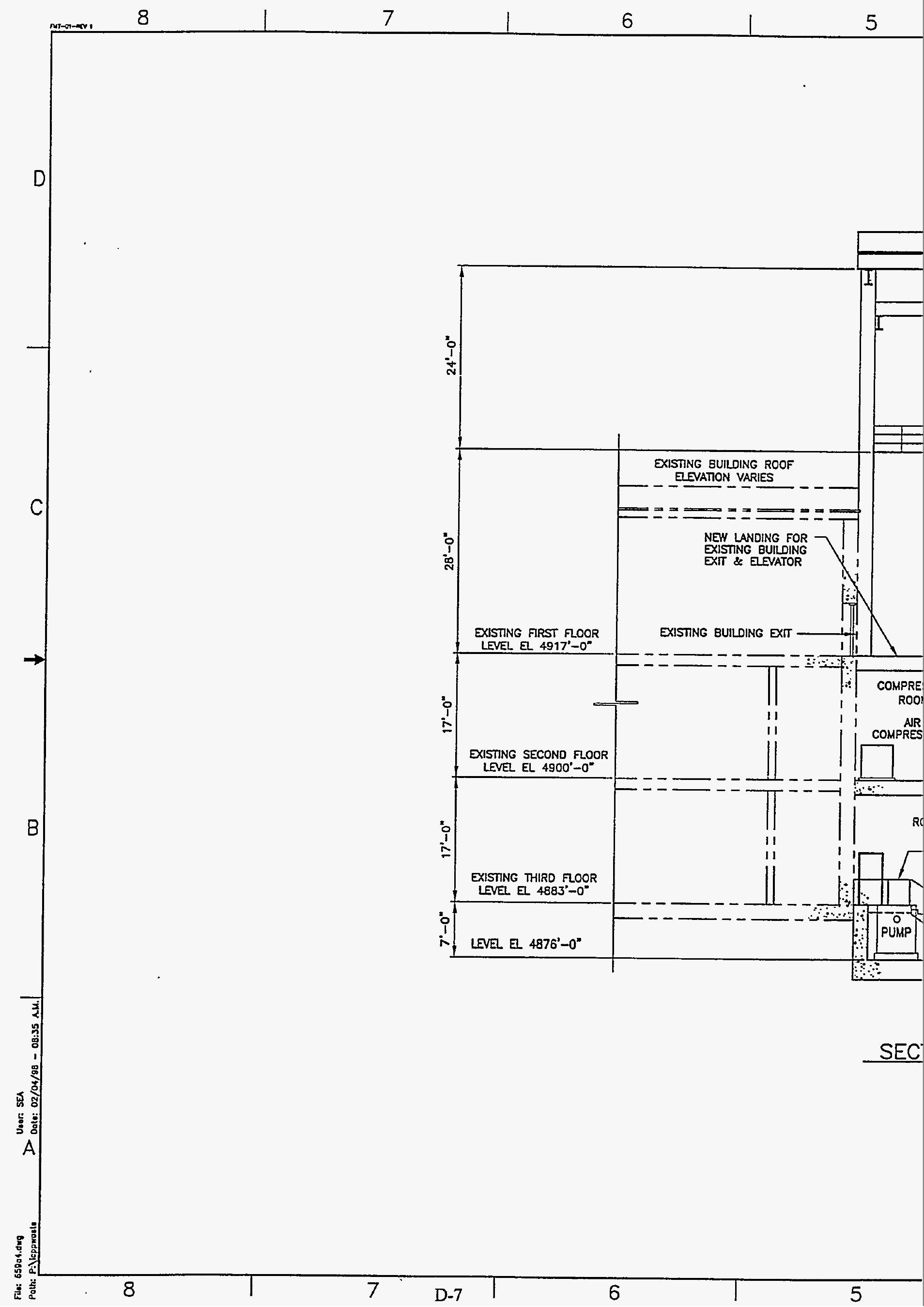




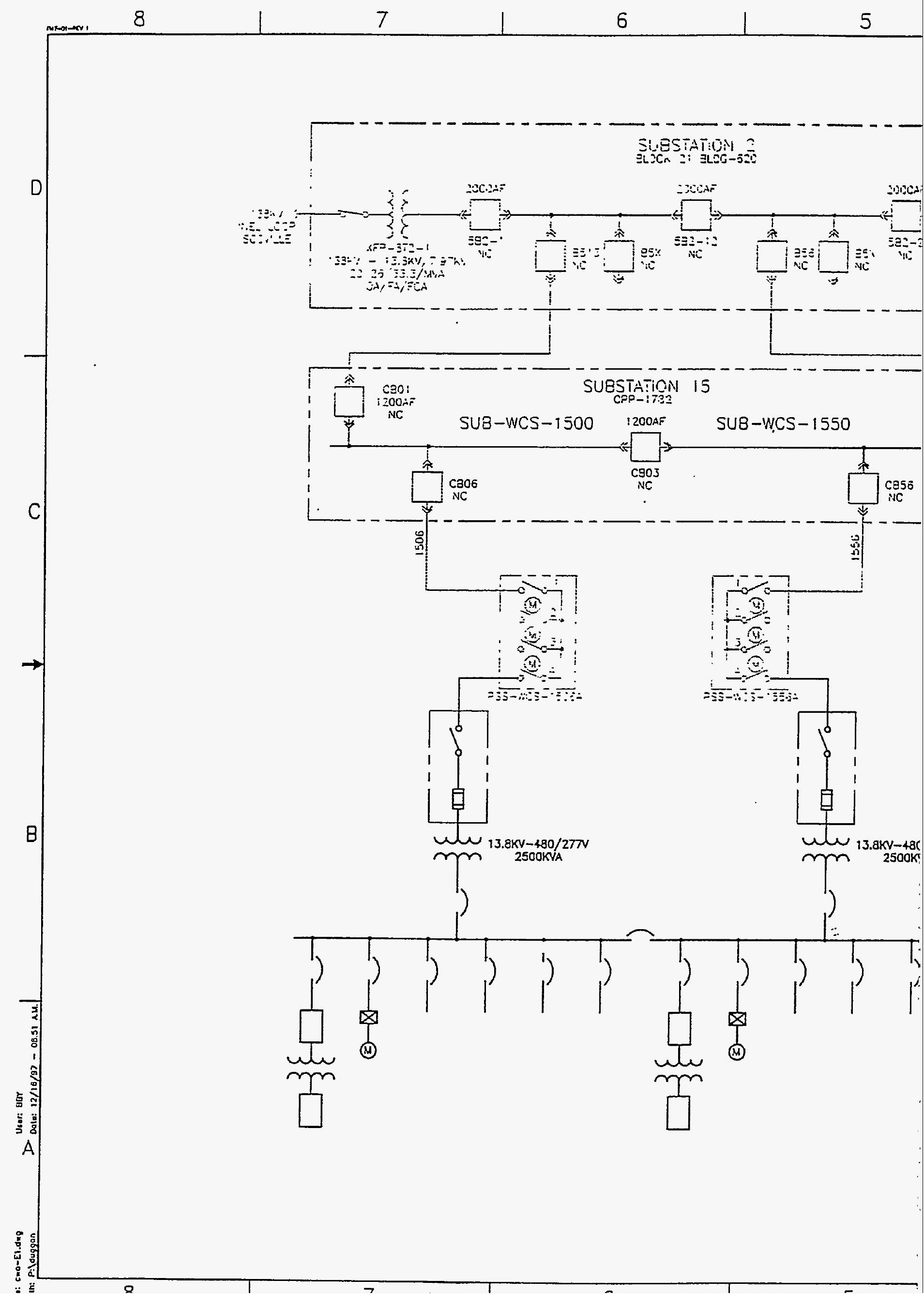




\section{Appendix E}

\section{Engineering Design Files}




\section{Appendix E}

\section{Engineering Design Files}

1. Regulatory Requirements and Criteria for the ICPP Proposed Waste Processing Facilities, EDF-WTS-003

2. CWO Scoping Study - Design Basis, EDF-CWO-003-(DDT)

3. CWO Instrumentation for the Cementitious Waste Treatment Scoping Study, EDF-CWO002-(RPE)

4. CWO Electrical Requirements - EDF-CWO-004-ED-(JED)

5. CWO Scoping Study Staffing Estimate - EDF-CWO 006-SRP-(J P)

6. Waste Treatment Facility Study, Calcine Retrieval and Transportation - EDF-WTS-002 (SEG)

7. Regulatory and Design Requirements for Waste Treatment Facilities, EDF-WTS-004

8. Radiological Evaluation of the Calcine HIP Study, EDF-HWO-006

9. DCWO Process Design, EDF-DCWO-011 (RED)

10. DCWO 20 Year Scaling, EDF-DCWO-015

11. Cementitious Waste Option Hot Isostatic Pressed Variance Plan, EDF-CWO-001 (AEL)

12. Canister Loading, EDF-DCWO-010

13. Impact of Blending/Recalcination of Calcines Before Cementation, EDF-CWO-005 (DDT) 
Project File Number O2BDO

Project/Task

Non-Separations Alternatives

Subtask CEMENTITIOUS WASTE OPTION (CWO)

Title: Cementitious Waste Option Hot Isostatic Pressed Variation

Summary: This EDF investigates the Hot Isostatic Pressed (HIP) variation of the CWO grouted recalcine mixed-high-level waste. It identifies the process, grout materials, waste volumes, modifications to the base case, scaleup factor, estimated cost, and discounted Life Cycle Cost estimate. The entire HWO system would be doubled in size including estimated cost and life cycle cost (LCC) for the CWO HIP Variance Plan. The CWO HIP facility estimated Total TPC (escalated) $=k \$ 3,320,746$, and the discounted LCC (escalated) $=k \$ 3,114,080$.

The CWO HIP Variation Plan is based upon the "Hot Isostatic Pressed Waste Option (HWO) Study," INEEL/EXT-98-01392 and EDF-HWO-012 found in the Project Files. The CWO HIP facility will have a 20 year operation phase and would use the same type building, equipment, time frame and flow sequence as depicted in the HWO study. The significant differences between the CWO HIP facility and the HWO facility are waste volume, quantity of HIP containers produced, and the quantity of HLW canisters filled and placed into interim storage. The initial volume of waste to be HIP'ed by the CWO HIP facility would be approximately 11,500 cubic meters where the waste volume to be HIP'ed by the HWO facility would be about 6,000 cubic meters. The quantity of HIP containers filled with waste material for the CWO HIP facility (and interim storage) would be approximately 31,000, where the quantity for the HWO facility would be about 17,000 . The quantity of HLW canisters filled with three HIP'ed containers each for the CWO HIP facility (and interim storage) would be approximately 10,300 where the quantity for the HWO facility would be about 5,700.

In each instance of differential between the CWO-HIP facility and the HWO facility volume or container/canister quantities, the HWO appears to be slightly more than one-half of the CWCO-HIP facility values. Therefore, a direct factor of 2 to 1 will be applied in this EDF to upscale the size of the CWO HIP facility relative to the size of the HWO facility, including costs. This EDF assumes a rough-order-of-magnitude (ROM) for all upscaled features and costs for the CWO HIP facility compared to the HWO. The upscale factor is considered conservative due to the elimination of some HWO facility equipment items and consequent building size reduction for the CWO HIP facility; however, there would be increased cost associated with the HIP container modifications needed to update the CWO grout facility. Therefore the 2:1 upscale factor appears to be a reasonable assumption. A ROM is allowable for scoping studies.

Therefore, the CWO HIP facility will be considered twice the size of the HWO facility for the building footprint, rooms and cells within the building, equipment quantity, operating personnel, maintenance staff, utilities and all other features not identified. The HWO calcine waste storage and mixing equipment would not be required for the CWO HIP facility due to the use of the CWO grouting facility for HIP container grouting functions and would reduce the building size. Mercury extraction equipment would also be unnecessary. The cost estimate and life cycle cost (LCC); however, will be directly doubled.

There are various differences between the CWO HIP facility and the HWO facility; however, with the exception of HIP materials storage and mixing systems and the elimination of the mercury removal system, the HIP process is the same. The major differences between the facilities are: 
1. the waste material would be CWO recalcine transferred from modified New Waste Calcining Facility (NWCF) to the CWO grouting facility for HIP container grout casting

2. the CWO grout matrix uses calcined clay, blast furnace slag, sodium hydroxide, and water and would require no other additives

3. the grout filled HIP containers would be transferred to interim storage awaiting HIP'ing

4. the grout filled HIP containers would be transferred from interim storage to the CWO HIP facility for HIP operations and canisterization lit may be necessary for the HIP'ed containers to be returned to interim storage to await return to the CWO HIP facility for canisterization).

Another difference between the CWO HIP facility and HWO facility relates to mercury removal, amalgamation, and storage. Most of the mercury would be removed at the NWCF by the CWO recalcination process. The elemental mercury would be, extracted, amalgamated, and stored by the NWCF and the MACT facility. This means the mercury removal system provided by the HWO design would be considered dormant and not be installed

The same HIP containers, HLW canisters, and processing equipment (with noted exceptions) would be used by both HIP operations. Refer to the cost estimate and LCC estimate contained in the "Hot Isostatic Press Waste Option Study Report," INEEL/EXT-98-01392 for support data.

The estimated cost and LCC (doubled) for the CWO HIP Facility is as follows:

Total OPC $\left({ }^{*}\right.$ escalated $)=$ $k \$ 334,201 \times 2=k \$ 662,402$

Total TEC $(*$ escalated $)=$ $k \$ 1,281,172 \times 2=k \$ 2,562,344$

Total TPC $(*$ escalated $)=$ $k \$ 1,615,373 \times 2=k \$ 3,230,746$

Total Operations $\left({ }^{*}\right.$ esc $)=$ $k \$ 2,574,115 \times 2=k \$ 5,148,230$

Total Post Operations $(*$ esc) $=$

$$
k \$ 1,574,514=k \$ 3,149,028
$$

Total Cost (unescalated) $=$ $k \$ 2,263,435 \times 2=k \$ 4,526,870$

Total Cost ${ }^{*}$ escalated $)=$ $k \$ 5,763,998 \times 2=k \$ 11,527,996$

Discounted Cost (escalated) $=k \$ 1,572,040 \times 2=k \$ 3,144,080$

* with management reserve and contingency

OPC - Other Project Cost, TEC - Total Estimated Cost, TPC - Total Project Cost Distribution (complete package): WTP EIS Studies Library, D. D. Taylor MS-3625, R. E. Dafoe MS-3765, B. R. Helm MS-3765, K. T. Williams MS_3765, A. E. Lee Files MS-3765

Distribution (summary package only):

\begin{tabular}{|c|c|c|c|c|c|}
\hline $\begin{array}{l}\text { Author } \\
\text { A. E. Lee }\end{array}$ & $\begin{array}{l}\text { Dept. } \\
4130\end{array}$ & $\begin{array}{l}\text { Reviewed } \\
\text { D. D. Taylor } \\
\text { P. To }\end{array}$ & $\begin{array}{l}\text { Date } \\
z / 12 / 98\end{array}$ & Approved & $\begin{array}{l}\text { Date } \\
2 / 12\end{array}$ \\
\hline & 211396 & $\begin{array}{l}\text { LMITCO } \\
\text { Review }\end{array}$ & Date & $\begin{array}{l}\text { LMITCO } \\
\text { Approval }\end{array}$ & Date \\
\hline
\end{tabular}


Project File Number O2BDO

Project/Task Waste Treatment Project Feasibility Studies

\section{Subtask Cementitious Waste Option}

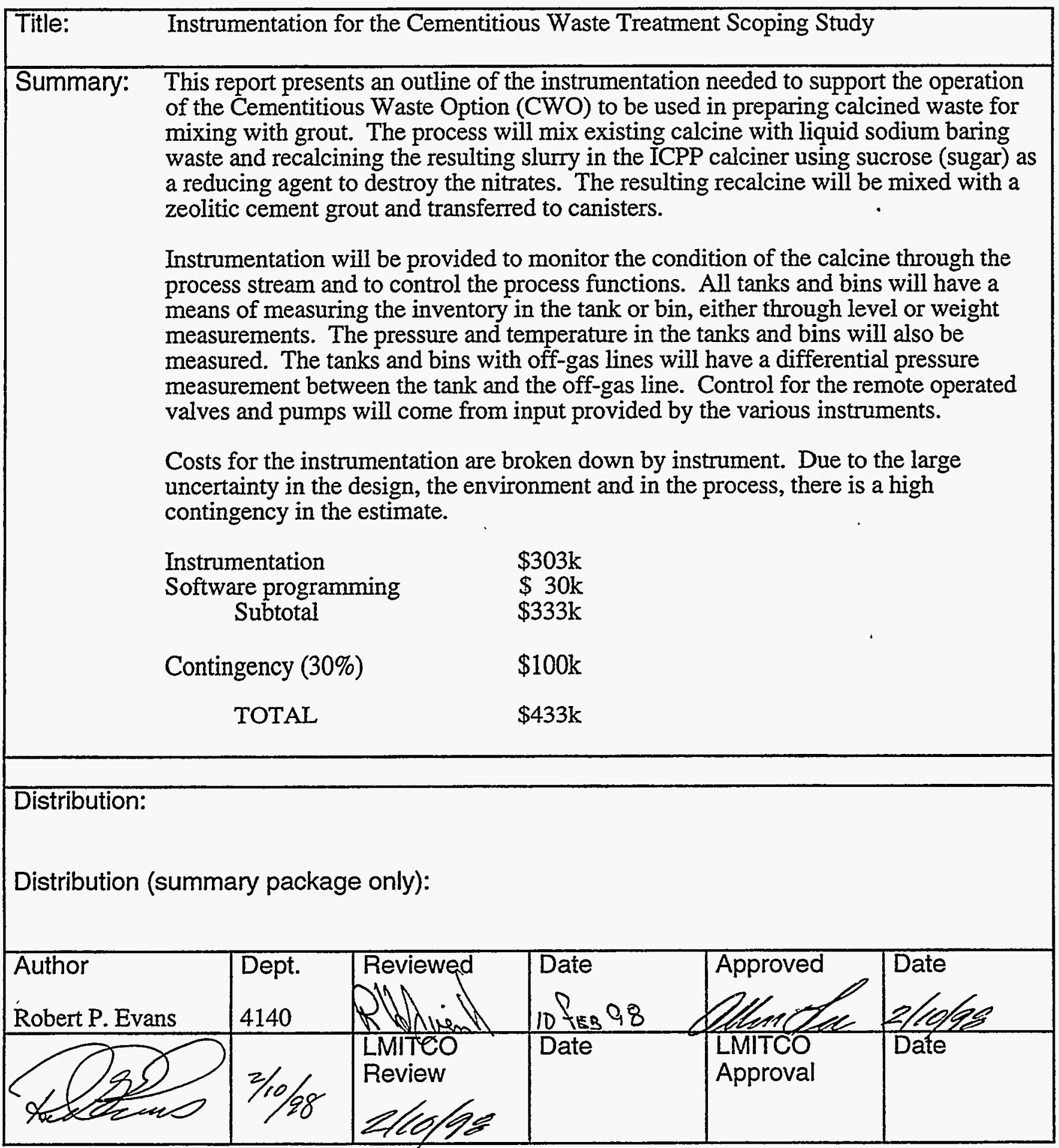




\section{Instrumentation for the Cementitious Waste Treatment Scoping Study}

\section{Introduction}

This report presents an outline of the instrumentation needed to support the operation of the Cementitious Waste Option (CWO) to be used in preparing calcined waste for mixing with grout. The process will mix existing calcine with liquid sodium baring waste and recalcining the resulting slurry in the ICPP calciner using sucrose (sugar) as a reducing agent to destroy the nitrates. The resulting recalcine will be mixed with a zeolitic cement grout and transferred to canisters.

\section{Process}

Existing calcine will be metered into two slurring vessels (see attached figures for equipment and flow path) from three bins holding different calcines. The slurring vessels will be filled with liquid sodium barring waste prior to loading the calcine. Water may also be added to obtain the proper mixture. Sparge air will be introduced into the vessels to keep the calcine in suspension while filling. (see Sketch CWO-01) When the proper mixture is achieved, the slurry will be circulated through the loop. Three pumps will be used to circulate the slurry. A portion of the slurry will be taken from the loop and mixed with the sucrose solution and introduced into the calciner. (see Sketch CWO-02). The recalcined product will pass through a cyclone separator and into a storage bin. The calcine will then be pneumatically fed from the bins to at grouting facility about 700 feet away.

\section{Instrumentation}

Instrumentation will be provided to monitor the condition of the calcine through the process stream and to control the process functions. All tanks and bins will have a means of measuring the inventory in the tank or bin, either through level or weight measurements. The pressure and temperature in the tanks and bins will also be measured. The tanks and bins with off-gas lines will have a differential pressure measurement between the tank and the off-gas line. Control for the remote operated valves and pumps will come from input provided by the various instruments.

Where possible, instrumentation will be non-intrusive due to the corrosive nature of the material being measured.

\section{Requirements}

The instrumentation provided will be for system control; therefore standard system accuracies will be sufficient.

The existing data system will be used. New instrumentation will connect to the data system through new wiring.

None of the instrumentation is considered safety related and hence no redundancy is required. The environment is not considered hazardous and hence explosion proof equipment is not required. 


\section{Assumptions}

System design life is 5 years. The hardware will be required to operate for the design life in a radiation field of about $70 \mathrm{R} / \mathrm{hr}$ and in a corrosive environment.

The instrumentation provided will be for system control; therefore standard system accuracies will be sufficient.

The existing data system will be used. New instrumentation will connect to the data system through new wiring.

None of the instrumentation is considered safety related and hence no redundancy is required. The environment is not considered hazardous and hence explosion proof equipment is not required.

Wiring from the instruments to the data system will use twisted-shielded pair run in conduit. The majority of the signals will be $4-20 \mathrm{~mA}$.

All remote operated valves will have positive feedback of valve position.

Costs are for instrumentation hardware (i.e., transmitter, transducer, valve and pump controllers, wire, conduit, and a data channel); it does not include the valves or pumps.

Costs for programming the control system are based on a best-experience-based guess.

\section{Instrument List}

The attached list is for the proposed instrumentation, as it has been identified to date. This list is extremely tentative and there is a very high uncertainty factor involved. The costs shown for an instrument include the instrument itself, a data channel (or part thereof), wire and conduit. No installation or demolition costs are included. Instruments are listed by measurement type and not by instrument type; i.e., a level measurement could refer to a differential pressure based instrument, a weight based instrument, an ultrasonic based level instrument, a float based level instrument, etc. No effort has been made at this point to identify specific types of instruments to meet the intended requirements.

Associated hardware, such as pumps, valves, and bins, is also included on the list, for the sake of completeness. No costs are associated here with this hardware.

\section{Costs}

Costs for the instrumentation are broken down by instrument on the attached instrument list. Costs shown do not include installation. Due to the large uncertainty in the design, the environment and in the process, there is a high contingency in the estimate.

$\begin{array}{cc}\begin{array}{c}\text { Instrumentation } \\ \text { Software programming } \\ \text { Subtotal }\end{array} & \begin{array}{l}\$ 303 \mathrm{k} \\ \$ 30 \mathrm{k}\end{array} \\ \$ 333 \mathrm{k}\end{array}$




\section{Sketches}

Four sketches are included for reference. The first, identified as CWO-00 is an overview of the system and the main hardware. No instrumentation is shown on this sketch. This sketch does show the areas covered by the other sketches.

Sketch CWO-01 shows the calcine feed and slurring systems in greater detail. Instrumentation numbers are given to cross-reference with the instrument list.

Sketch CWO-02 shows the sucrose supply and mixing systems.

Sketch CWO-03 shows the transport system.

All sketches are tentative and based on the latest available knowledge. 


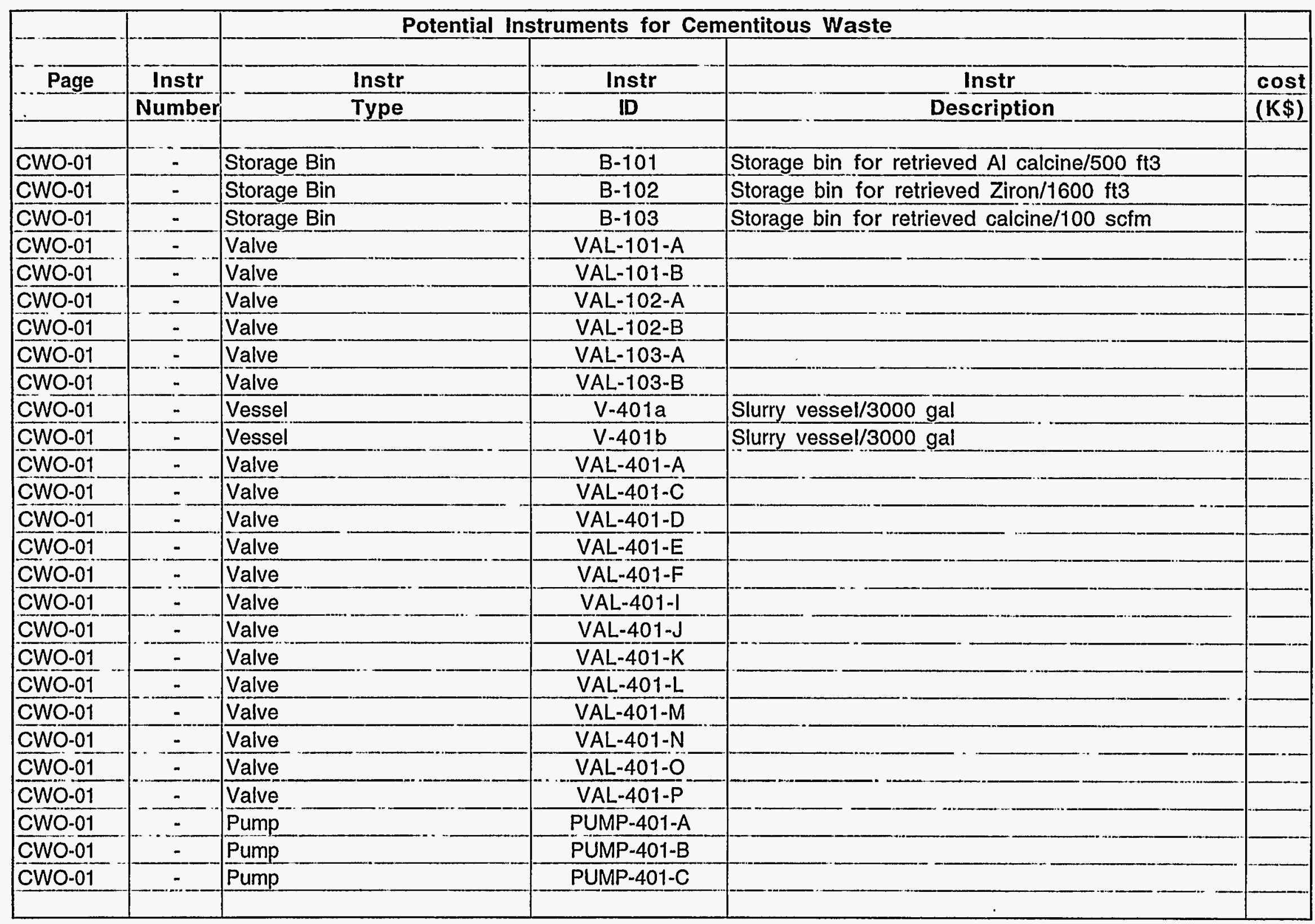




\begin{tabular}{|c|c|c|c|c|c|}
\hline \multicolumn{6}{|c|}{ Potential Instruments for Cementitous Waste } \\
\hline Page & $\begin{array}{l}\text { Instr } \\
\text { Number }\end{array}$ & $\begin{array}{l}\text { Instr } \\
\text { Type }\end{array}$ & $\begin{array}{l}\text { Instr } \\
\text { ID }\end{array}$ & $\begin{array}{c}\text { Instr } \\
\text { Description }\end{array}$ & $\begin{array}{l}\text { cost } \\
(\mathrm{K} \$)\end{array}$ \\
\hline CWO-01 & 1 & Differential pressure & PDT-101-A & Off gas flow/mixer/B-101 & 3 \\
\hline CWO-01 & 2 & Differential pressure & PDT-102-A & Off gas flow/mixer/B-102 & 3 \\
\hline CWO-01 & 3 & Differential pressure & PDT-103-A & Off gas flow/mixer/B-103 & 3 \\
\hline CWO-01 & 4 & Differential pressure & PDT-401-A & Off gas flow/Slurry vessel/V-401a & 3 \\
\hline CWO-01 & 5 & Differential pressure & PDT-401-B & Off gas flow/Slurry vessel/V-401b & 3 \\
\hline CWO-01 & 6 & Pressure & PT-101-A & & 2 \\
\hline CWO-01 & 7 & Pressure & PT-102-A & & 2 \\
\hline CWO-01 & 8 & Pressure & PT-103-A & - & 2 \\
\hline CWO-01 & 9 & Pressure & PT-401-A & & 2 \\
\hline CWO-01 & 10 & Pressure & PT-401-B & & 2 \\
\hline CWO-01 & 11 & Pressure & PT-401-C & & 2 \\
\hline CWO-01 & 12 & Pressure & PT-401-D & & 2 \\
\hline CWO-01 & 13 & Level & LT-101-A & . & 3 \\
\hline CWO-01 & 14 & Level & LT-102-A & & 3 \\
\hline CWO-01 & 15 & Level & $L T-103-A$ & & 3 \\
\hline CWO-01 & 16 & Level & $L T-401-A$ & . & 3 \\
\hline CWO-01 & 17 & Level & LT-401-B & & 3 \\
\hline CWO-01 & 18 & Flow & FT-401-A & & 5 \\
\hline CWO-01 & 19 & Flow & FT-401-B & & 5 \\
\hline CWO-01 & 20 & Current & IT-401-A & & 2 \\
\hline CWO-01 & 21 & Current & IT-401-B & & 2 \\
\hline CWO-01 & 22 & Current & IT-401-C & & 2 \\
\hline CWO-01 & 23 & Temperature & TT-401-A & & 2 \\
\hline CWO-01 & 24 & Variable Speed Drive & VSD-401-A & & 6 \\
\hline CWO-01 & 25 & Variable Speed Drive & VSD-401-B & & 6 \\
\hline CWO-01 & 26 & Variable Speed Drive & VSD-401-C & & 6 \\
\hline CWO-01 & 27 & Valve control & VAL-101-A & & 3 \\
\hline
\end{tabular}




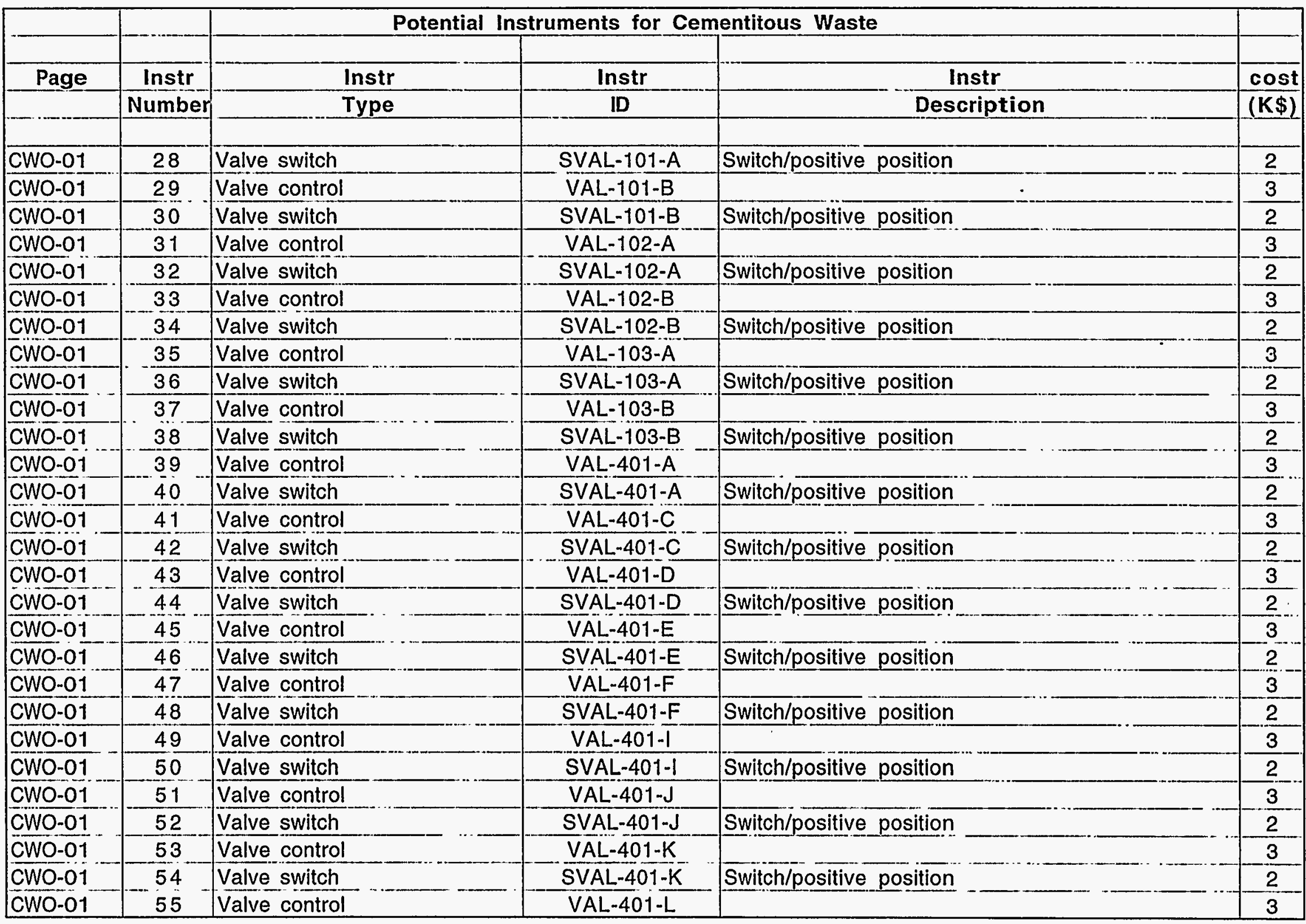




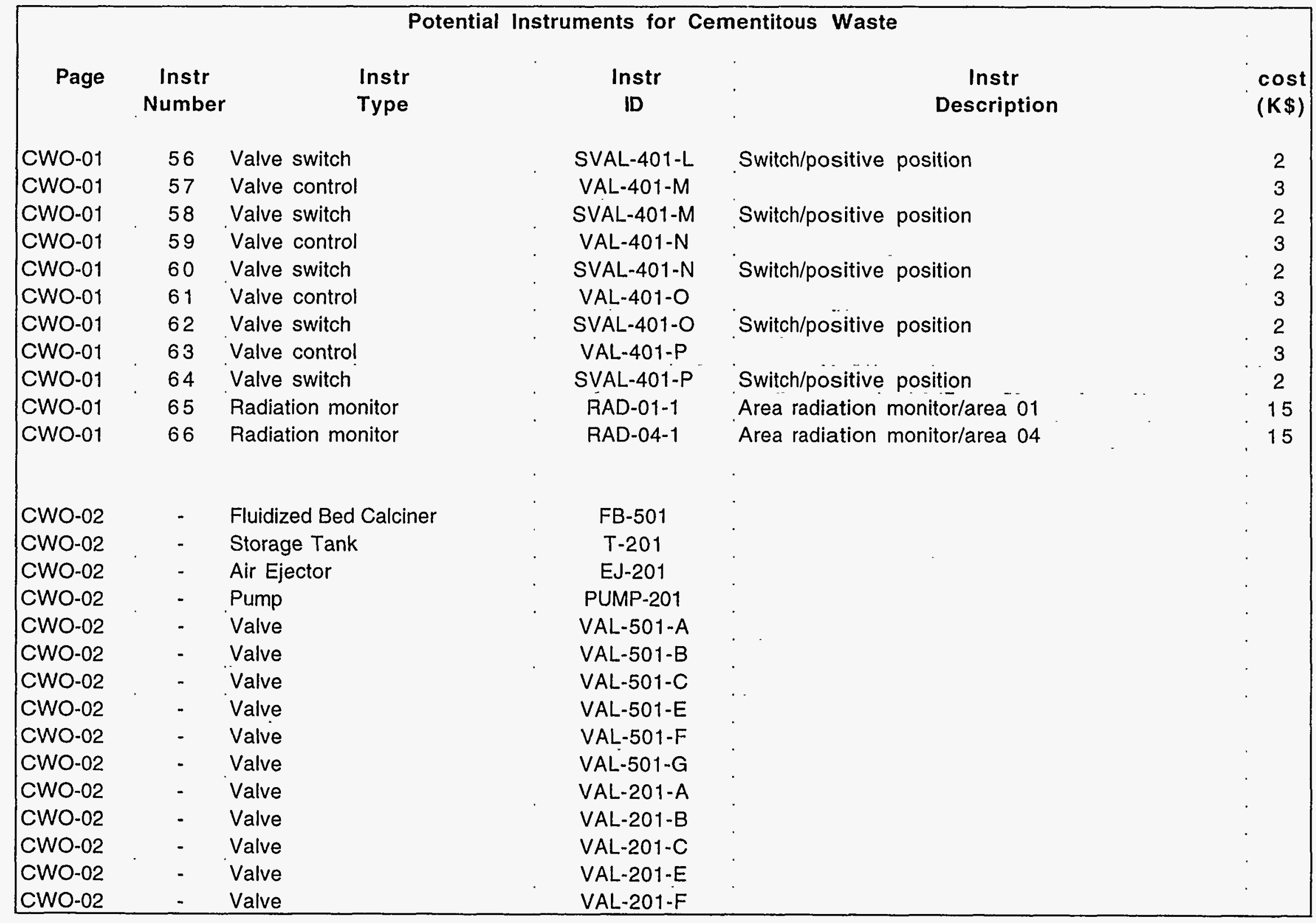




\begin{tabular}{|c|c|c|c|c|c|}
\hline & & \multicolumn{3}{|c|}{ Potential Instruments for Cementitous Waste } & \\
\hline & & & & & \\
\hline Page & Instr. & Instr & Instr & Instr & cost \\
\hline & Number & Type & ID & Description & $(\mathrm{K} \$)$ \\
\hline & & & & & \\
\hline CWO-02 & $=$ & Valve & VAL-201-G & & \\
\hline CWO-02 & - & Mixer & MIX-501-A & & \\
\hline cWo-02 & $=$ & Mixer & MIX-501-B & & \\
\hline CWO-02 & - & Mixer & MIX-501-C & & \\
\hline & & & & & \\
\hline CWO-02 & 67 & Flow & FT-501-A & & 5 \\
\hline CWO-02 & 68 & Flow & FT-501-B & & 5 \\
\hline CWO-02 & 69 & Flow & FT-501-C & & 5 \\
\hline CWO-02 & 70 & Flow. & FT $-501-E$ & & 5 \\
\hline CWO-02 & 71. & Flow & FT-501-F & & 5 \\
\hline CWO-02 & 72 & Flow. & FT-501-G & & 5 \\
\hline CWO-02 & 73 & Flow & FT-501-H & & 5 \\
\hline CWO-02 & 74 & Flow & FT-201-A & & 5 \\
\hline CWO-02 & 75 & Flow & FT-201-B & & 5 \\
\hline CWO-02 & 76 & Flow & FT-201-C & & 5 \\
\hline CWO-02 & 77 & Flow & FT-201-E & & 5 \\
\hline CWO-02 & 78 & Pressure & PT-501-A & & 2 \\
\hline CWO-02 & 79 & Pressure & PT-501-B & & 2 \\
\hline CWO-02 & 80 & Pressure & PT-501-C & & 2 \\
\hline CWO-02 & 81 & Pressure & PT-201-A & & 2 \\
\hline $\bar{c} \overline{0-02}$ & 82 & Level & LT-201-A & & 3 \\
\hline CWO-02 & 83 & Current & IT-201-A & & 2 \\
\hline CWO-02 & 84 & Valve Control & VAL-501-E & & 3 \\
\hline CWO-02 & 85 & Valve switch & SVAL-501-E & Switch/positive position & 2 \\
\hline CWO-02 & 86 & Valve Control & VAL-501-F & & 3 \\
\hline CWO-02 & 87 & Valve switch & SVAL-501-F & Switch/positive position & 2 \\
\hline CWO-02 & 88 & Valve Control & VAL-501-G & & 3 \\
\hline CWO-02 & 89 & Valve switch & SVAL-501-G & Switch/positive position & 2 \\
\hline
\end{tabular}




\begin{tabular}{|c|c|c|c|c|c|}
\hline \multicolumn{6}{|c|}{ Potential Instruments for Cementitous Waste } \\
\hline Page & $\begin{array}{l}\text { Instr } \\
\text { Number }\end{array}$ & $\begin{array}{l}\text { Instr } \\
\text { Type }\end{array}$ & $\begin{array}{l}\text { Instr } \\
\text { ID }\end{array}$ & $\begin{array}{c}\text { Instr } \\
\text { Description }\end{array}$ & $\begin{array}{l}\text { cost } \\
(K \$)\end{array}$ \\
\hline CWO-02 & 90 & Pump Control & VSD-201-A & & 3 \\
\hline CWO-02 & 91 & Air Ejector Control & EJ-201-A & & 3 \\
\hline CWO-02 & 92 & Radiation monitor & RAD-05-1 & Area radiation monitor/area 05 & 15 \\
\hline CWO-03 & - & Cyclone & CY-701 & Extract solids (line to mixing) $/ 100 \mathrm{scfg} / 10 \mathrm{lb} / \mathrm{min}$ & \\
\hline CWO-03 & - & Cyclone & or & & \\
\hline CWO-03 & - & Storage bin & MB-701a & Storage bin for blend recalcine to grout $/ 400 \mathrm{ft} 3$ & \\
\hline CWO-03 & - & Storage bin & $M B-701 b$ & Storage bin for blend recalcine to grout $/ 400 \mathrm{ft} 3$ & \\
\hline CWO-03 & . & Storage bin & $M B-701 c^{\circ}$ & Storage bin for blend recalcine to grout $/ 400 \mathrm{ft} 3$ & \\
\hline CWO-03 & - & Storage bin & & & \\
\hline CWO-03 & - & Blower & BLO-701-A & & \\
\hline CWO-03 & . & Heat Exchanger & E-701-A & & \\
\hline CWO-03 & . & Valve & VAL-701-A & & \\
\hline CWO-03 & - & Valve & VAL-701-C & 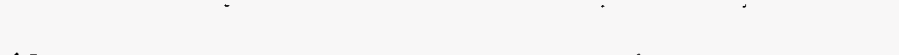 & \\
\hline CWO-03 & - & Valve & VAL-701-F & & \\
\hline CWO-03 & - & Valve & VAL-701-G & & \\
\hline CWO-03 & 93 & Temperature & TT-701-A & & 2 \\
\hline CWO-03 & 94 & Temperature & TT-701-B & & 2 \\
\hline CWO-03 & 95 & Temperature & TT-701-D & & 2 \\
\hline CWO-03 & 96 & Temperature & TT-701-F & & 2 \\
\hline CWO-03 & 97 & Level & LT-701-A & & 3 \\
\hline CWO-03 & 98 & Level & LT-701-B & & 3 \\
\hline CWO-03 & 99 & Level & LT-701-C & & 3 \\
\hline CWO-03 & 100 & Level & LT-701-D & & 3 \\
\hline CWO-03 & 101 & Pressure & PT-701-B & & 2 \\
\hline CWO-03 & 102 & Pressure & PT-701-D & & 2 \\
\hline
\end{tabular}




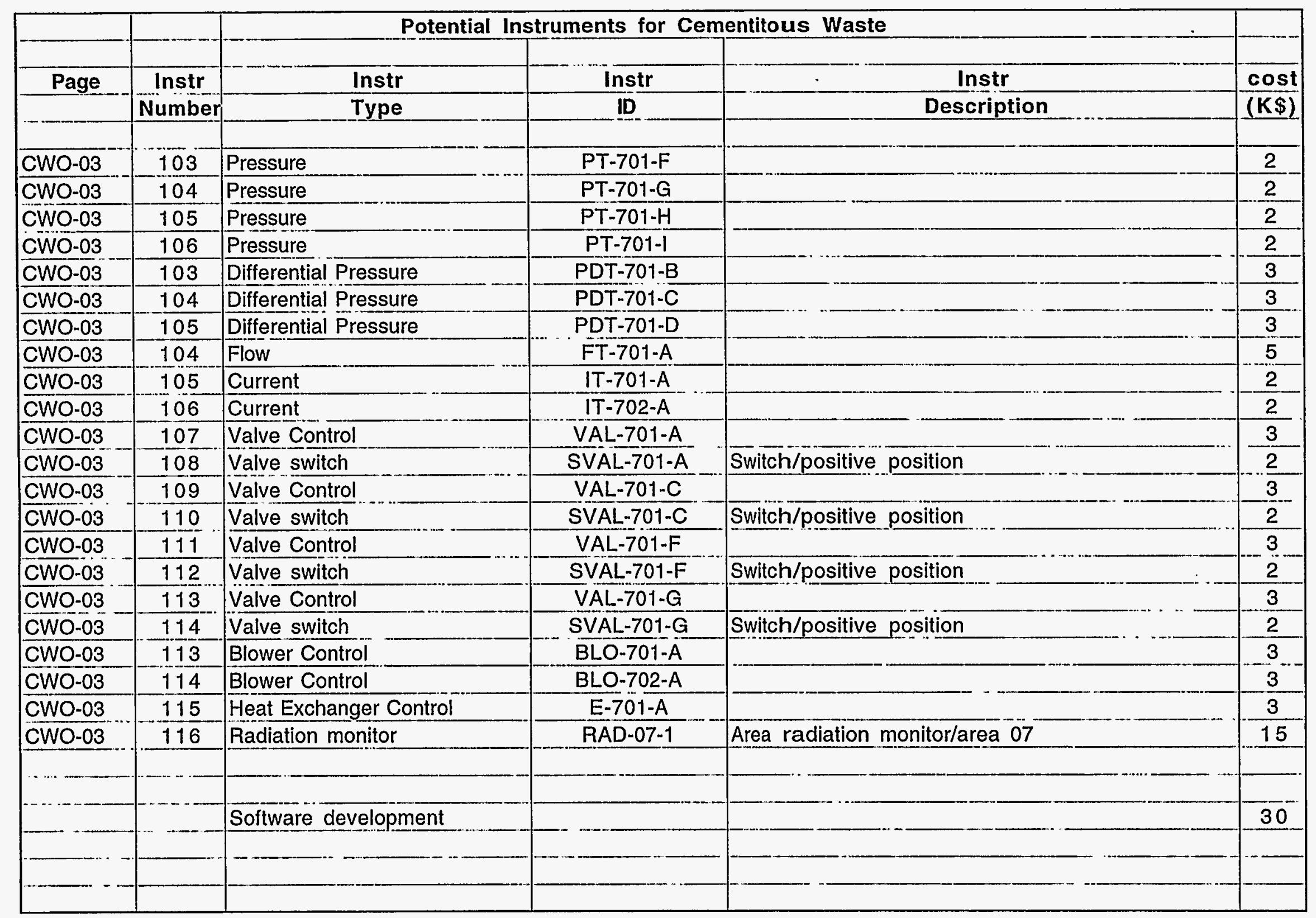




\begin{tabular}{|c|c|c|c|c|c|}
\hline \multicolumn{6}{|c|}{ Potential Instruments for Cementitous Waste } \\
\hline Page & $\begin{array}{c}\text { Instr } \\
\text { Number }\end{array}$ & $\begin{array}{l}\text { Instr } \\
\text { Type }\end{array}$ & $\begin{array}{l}\text { Instr } \\
\text { ID }\end{array}$ & $\begin{array}{c}\text { Instr } \\
\text { Description }\end{array}$ & $\begin{array}{l}\text { cost } \\
(K \$)\end{array}$ \\
\hline & & & & & 433 \\
\hline
\end{tabular}




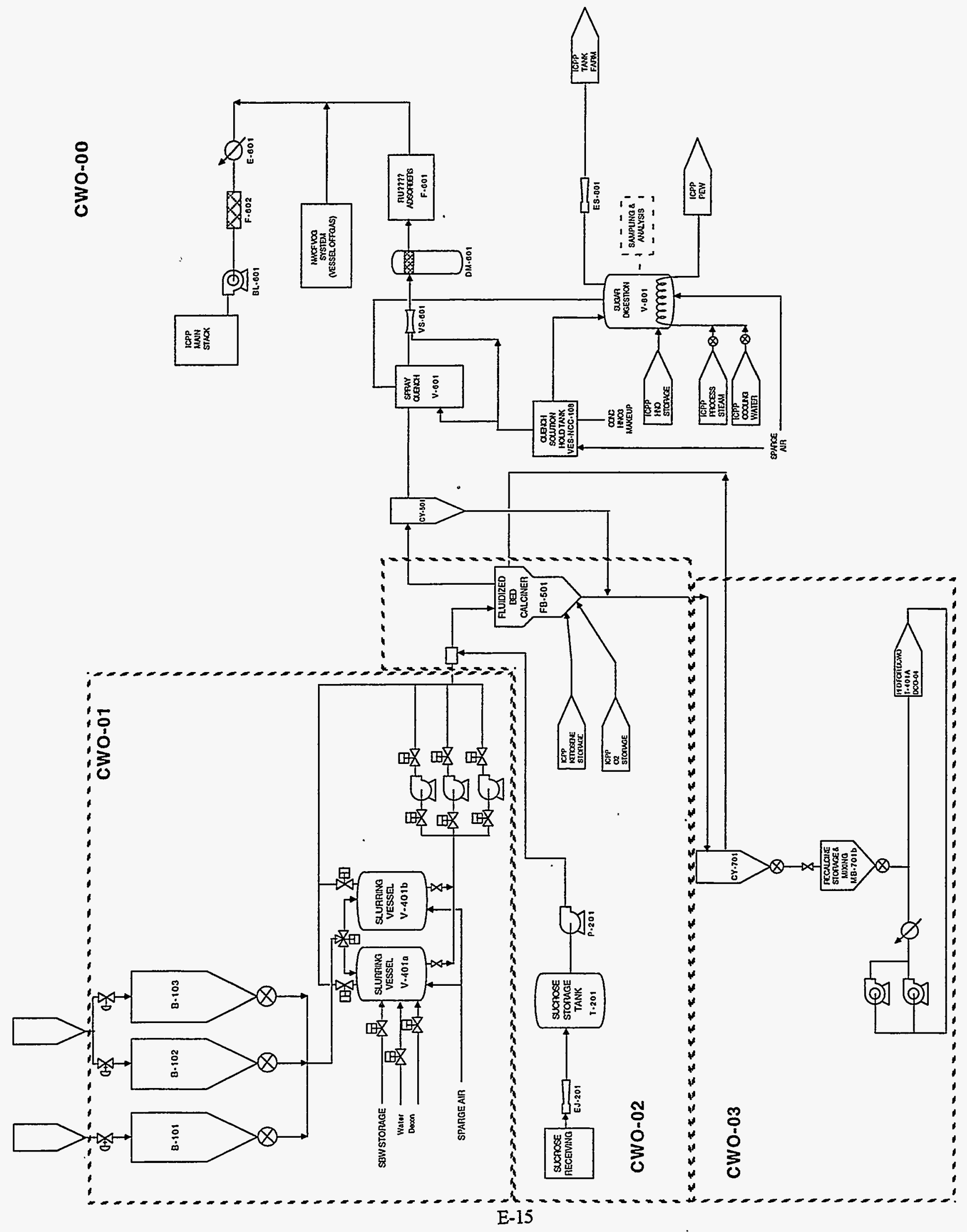




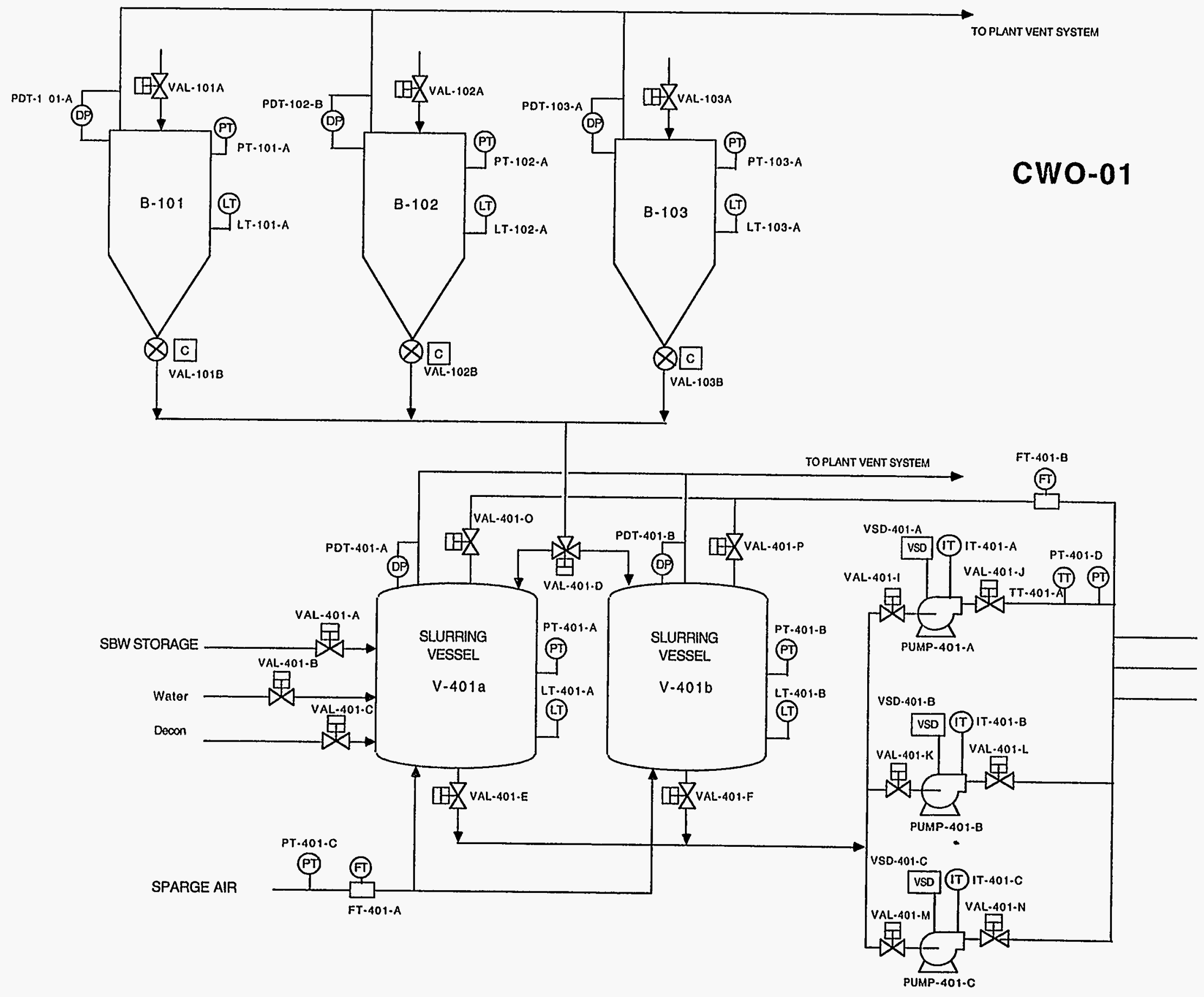




\section{CWO-02}

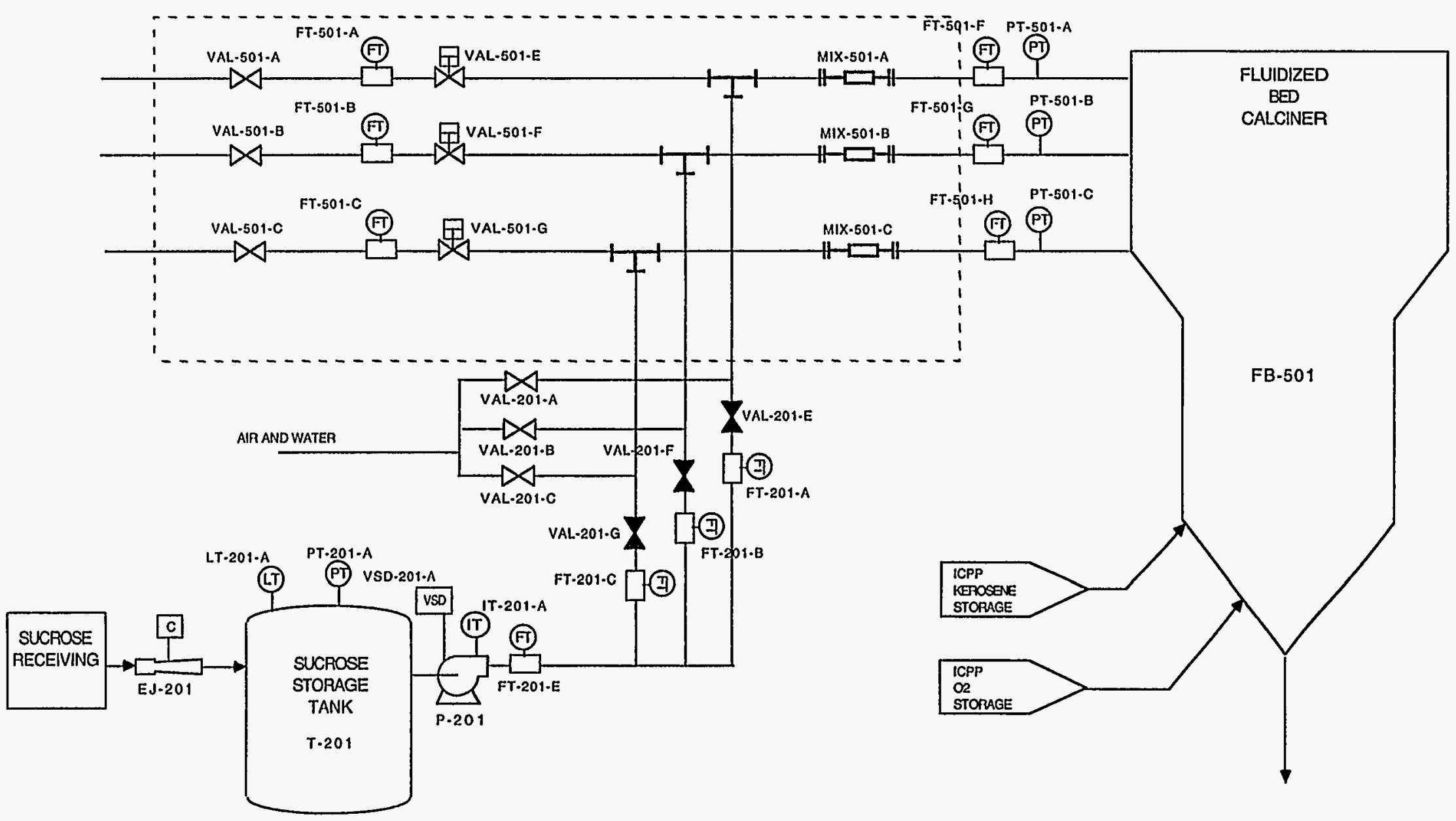




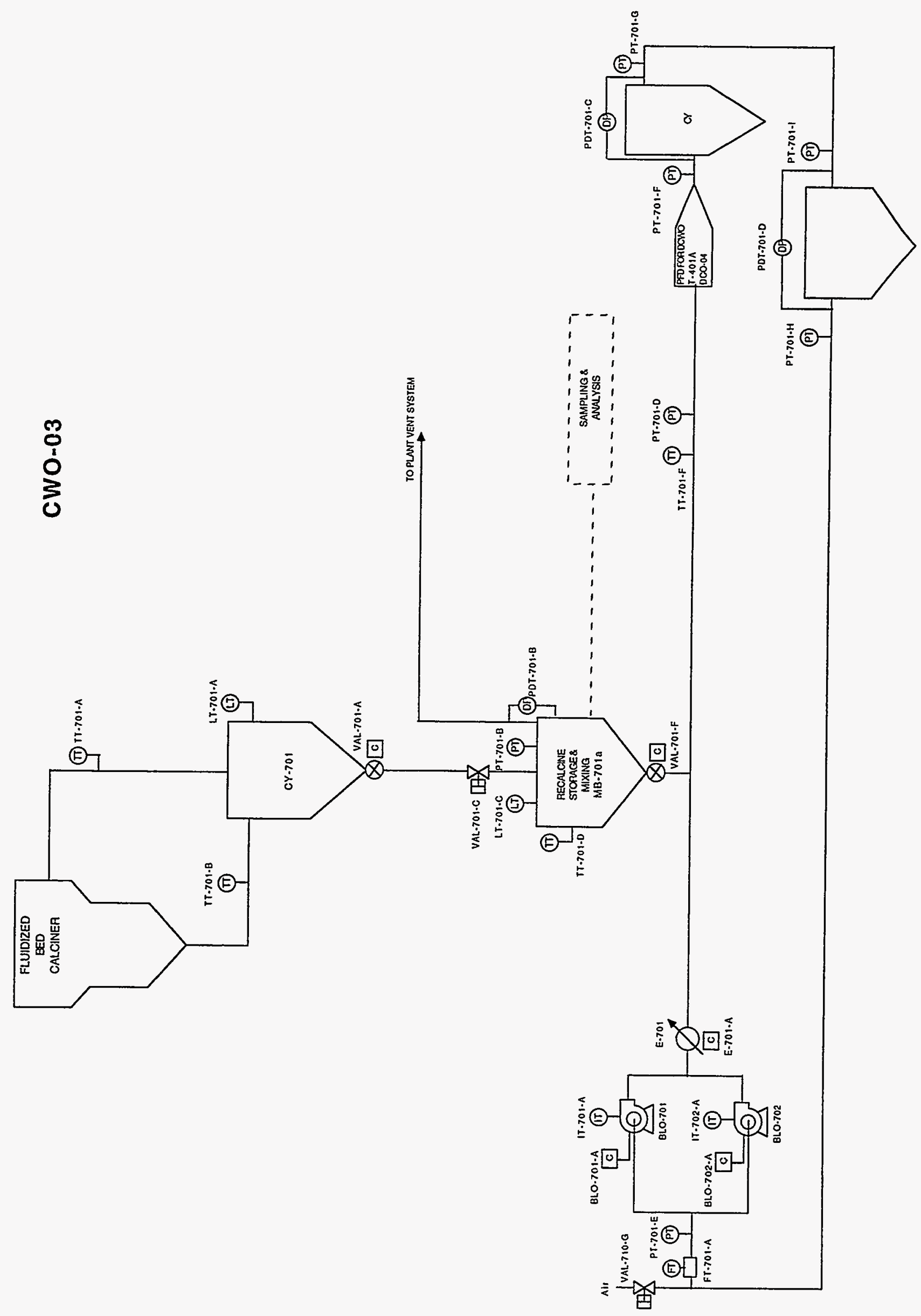

E-18 
Project File Number $\quad$ 02BD0

Project/Task Waste Treatment Project Feasibility Studies

Subtask CWO Scoping Study

Title: $\quad$ Cementitious Waste Option (CWO) Design Basis

Summary: This EDF describes the proposed process for the Cementitious Waste Option (CWO). The description includes design basis and assumptions, process description, process flow diagrams, material balances, new equipment list, utilities summary, chemical requirements summary, generated waste streams, and process concerns.

Distribution (complete package): WTP EIS Studies Library, A. E. Lee M.S. 3765, R. E. Dafoe M.S. 3765, K. L. Williams M.S. 3765

Distribution (summary package only):

\begin{tabular}{|c|c|c|c|c|c|}
\hline $\begin{array}{l}\text { Author } \\
\text { D. D. Taylor }\end{array}$ & $\begin{array}{l}\text { Dept. } \\
4170\end{array}$ & $\begin{array}{l}\text { Reviewed } \\
\text { S. J. Losinski }\end{array}$ & \begin{tabular}{|l|} 
Date \\
2/10/98
\end{tabular} & Approved & $\begin{array}{l}\text { Date } \\
2 / 1 / 195\end{array}$ \\
\hline & & LMIICO Review & Date & LMITCO Approval & Date \\
\hline
\end{tabular}




\section{REQUIREMENTS}

Process requirements are established by statutory laws, DOE orders, and the Batt agreement between DOE and the State of Idaho. These requirements are described in detail in Ref. 1.

\section{DESIGN BASIS}

\subsection{Background}

The process described below (the "Cementitious Waste Option", hereafter referred to as the CWO process) is based on work done earlier at the $\mathrm{INEL}^{2}$ and at the Hanford reservation ${ }^{3}$ describing the use of sugar as a reducing agent in the denitration and calcination of radioactive high level liquid wastes, and on work described in references 4,5 , and 6 describing processes for solidification of solid calcined wastes using hydroceramic grouting and hot isostatic pressing. The process treats high level radioactive waste calcine solids and liquids stored at the Idaho Waste Processing Plant (ICPP) for transport to and disposal in a suitable repository. It consists of the following basic steps:

1) Retrieval of calcined solids from existing storage bins at ICPP (CSSF-1 through 7);

2) Slurrying of retrieved calcine solids with remaining liquid sodium bearing wastes (SBW) in the ICPP tank farm.

The slurrying step extracts leachable, soluble nitrates (primarily $\mathrm{NaNO}_{3}$ and $\mathrm{KNO}_{3}$ ) from the calcine solids into aqueous solution in preparation for reduction of the nitrates to $\mathrm{N}_{2}, \mathrm{O}_{2}$, and lower valence state oxides of nitrogen. The slurrying step also redistributes the alkali metal from the high-alkali sodium waste blends throughout the calcine. This is desirable in achieving the desired composition for grouting the recalcined solids;

3) Recalcination of the slurried calcine solids and liquid SBW using sucrose (in $65 \mathrm{wt} \%$ solution) as a reducing agent in the existing fluidized bed calciner in the New Waste Calcining Facility (NWCF) at ICPP;

4) Blending of the recalcined solids with water and $\mathrm{NaOH}$ to form a hydroceramic grout which is cast into 2'x10' stainless steel disposal canisters;

5) Curing of the grout to a centerline temperature of $200^{\circ} \mathrm{C}$ in saturated steam;

6) Dewatering of the cured grout by heating to $250^{\circ} \mathrm{C}$;

7) Transfer to an interim storage facility, pending transport to and disposal at a national high level radioactive waste repository.

The steps involving the slurrying of calcine solids with SBW and recalcination with sugar are included in the CWO process to achieve the following objectives: 
- Remove nitrates from the wastes. This is desirable because (a) it reduces the total mass (and volume) of the final waste form by roughly 10 percent, and (b) it eliminates most of the NOX emissions from the calciner that would otherwise result from calcination of SBW

- Remove mercury from the wastes prior to grouting. This is desirable because it eliminates the need for developing special additives for immobilizing mercury in the final waste form. Such immobilization may be required in order to remove the RCRA toxicity characteristic for mercury in preparation for final disposal.

The intended disposal site for the final grouted waste is the deep alluvial deposits at the Nevada Test Site. The grouting process is tailored to produce a grout which will be geochemically stable in these deposits, and which complies with Land Disposal Restrictions for RCRA-treated wastes.

\subsection{Design Basis Assumptions}

The following assumptions have been made in developing the CWO process:

1) Tank farm liquid wastes in tanks WM-187, -188 , and -189. will be calcined using the current process from $6 / 1 / 97$ to $6 / 1 / 98$, and (following a three month downturn) from about $9 / 1 / 98$ until $12 / 31 / 99$.

2) All liquid tank farm wastes remaining on $1 / 1 / 2000$, and generated after this date will be slurried with solid calcine from the binsets in a constant ratio of liquid volume:solid mass. The total volume of liquids to be slurried is assumed to be $1,588,021$ gal.

3) Processing of wastes will begin on $1 / 1 / 13$ and be completed in 5 years.

4) Slurried liquid/calcine can be recalcined in NWCF with sucrose (sugar) as the only additive, in the proportion $38 \mathrm{gm}$ sucrose per mole nitrate in the slurry.

5) Solids generated from recalcination of slurried wastes are grouted as they are produced. The only storage requirement is to accommodate surge capacity and batch mixing of grout.

6) Grouted waste will be cast in cylindrical stainless steel disposal canisters which are $2 \mathrm{ft}$ in diameter by $10 \mathrm{ft}$ high. Total volume of grout in each canister will be $0.72 \mathrm{~m}^{3}$.

7) The retrieval system for the calcine will allow retrieval from any binset on any given day. The retrieval rate will be $2,700 \mathrm{~kg} / \mathrm{hr}$ in each of two retrieval from two different binsets.

8) Alumina calcine and calcine from campaigns $\mathrm{H}-4$ and $\mathrm{H}-5$ can be retrieved separately and segregated from all remaining calcines. The latter category is assumed to consist primarily of zirconium, zirconium/sodium blends, and fluorinel type calcines.

9) Hydroceramic grout recipes will be developed before processing of wastes commences. A sufficient number of recipes will be developed to accommodate the expected range of blended compositions, which will result from blending of calcines from the three categories described in item 8 with SBW. These recipes are assumed to be sufficiently robust to accommodate the maximum expected variation in calcine composition. 
10) The fluidized bed calcination process (with suitable feed nozzle modifications) can accommodate injection of slurried wastes (i.e., liquid together with high concentrations of undissolved solids) via existing waste feed ports in the calciner vessel.

11) Sugar calcination will reduce at least $90 \%$ of the nitrates in the waste. The resulting nitrate concentration in the recalcined waste will be acceptable for grouting.

12) Radionuclide concentrations in alumina and zirconia calcine are as described by Doug Wenzel on $7 / 22 / 97$ and 8/11/97, respectively. Radionuclide concentrations in SBW calcine are as described in EDF-FDO-001 (C. M. Barnes, "Estimates of Feed and Waste Volumes, Compositions and Properties"). Radionuclide concentrations in liquid wastes are as described in KJR-02-04/JAN-03-94.

13) The overall treatment facility online factor will be $50 \%$. Calcine retrieval and recalcination of slurried wastes will proceed on a 24-hr/day, 7-day/week schedule (subject to the above assumed online factor). All other processes (e.g., grouting, curing, storage operations, etc.) will proceed on the basis of four 10-hr shifts per week, and 199 normal working days per year (again subject to the assumed online factor).

14) Radionuclide contamination during all waste processing operations is controlled by maintaining negative pressure inside all waste handling areas. Building air is double HEPA filtered to remove airborne contamination.

15) Mercury contained in slurried wastes will be collected by the NWCF scrub system with $99 \%$ efficiency. Mercury recovered in the scrub solution will be extracted as elemental mercury in an electrowinning cell, by continuous treatment of a slipstream of the scrubbing solution. Elemental mercury collected by this method will be amalgamated with sulfur to produce a disposable radioactive waste. Mercury remaining in the offgas will be removed to comply with MACT requirements.

16) Consistent with the INEL Site Treatment Plan, a mercury retorting facility will be constructed at $W_{E R F^{l}}$ in time for waste processing by the CWO option. Such a facility would be available (if necessary) to treat mercury-laden activated carbon used to extract mercury from the offgas generated by recalcination.

17) Operation of the calciner in the anticipated time frame (see assumption 3 , above) will require that the NWCF be modified to comply with Maximum Achievable Control Technology (MACT) requirements.

18) A delisting petition for all ICPP calcines will be approved by EPA.

${ }^{1}$ Waste Experimental Reduction Facility 
ENGINEERING DESIGN FILE

\section{PROCESS DESCRIPTION}

\subsection{Process Summary (Dwg CWO-00)}

The major processing steps for the Cementitious Waste Option (CWO) are shown schematically on the process flow diagrams for the CWO process (Section 4) and in the process flow diagrams for the Direct Cementitious Waste Option (DCWO) in Ref. 10. The essential steps in the process are as follows:

1) Pneumatic recovery of calcine from the Calcine Solids Storage Facility (CSSF) binset and transport to temporary mixing/storage bins;

2) SBW retrieval using existing steam jetting system, with transfer lines modified to allow delivery of the tank farm waste directly into slurrying tanks.

3) Blending of calcines with liquid sodium-bearing wastes (SBW), demineralized process water, and calcium nitrate in slurrying tanks to dissolve leachable nitrates into the aqueous phase;

4) Calcination of the slurried mixture (SBW liquid and undissolved calcine solids) in the NWCF fluidized bed calciner, with addition of sucrose (sugar) as a reducing agent to destroy nitrates, with no other additives (solids produced from this step are termed "recalcine" in what follows);

5) Pneumatic transport of recalcine via the current product transfer system from the calciner to a booster station, and from there to a grouting facility;

6) Grouting recalcine into a hydroceramic waste form using the FUETAP ${ }^{2}$ process. The final waste form from this process resembles naturally occurring solids in alluvial deposits;

7) Casting the grout directly into 2'x10' SRS waste disposal canisters;

8) Curing the grout with saturated steam at $250^{\circ} \mathrm{C}$ for a sufficient time to reach a centerline grout temperature of at least $200^{\circ} \mathrm{C}$ (the curing period is estimated to require approximately 48 hours);

9) Dewatering the cured grout by heating to $250^{\circ} \mathrm{C}$ in a mild vacuum (the dewatering period is estimated to require approximately 168 hours [7 days]);

10) Sealing the 2 ' $x 10^{\prime}$ disposal canisters and transporting them to interim storage.

The above processing steps are depicted schematically in drawings CWO-00 through CWO-10. Additional detail for the steps in the CWO process is provided in Section 3.3. Overall processing rates and statistics for the process are given in the following section.

\footnotetext{
${ }^{2}$ Formed Under Elevated Temperature and Pressure (see Ref. 4).
} 
$431.02 \#$

$2: 11 / 98$

Rev. \#01
ENGINEERING DESIGN FILE

Function File Number - SPR-02

EDF Serial Number - EDF-CWO-003

Page 6 of 34

\subsection{Processing Rates and Statistics} follows:

The principal processing rates and overall processing statistics for the CWO process are as

TABLE 1: PROCESSING RATES AND STATISTICS FOR CWO PROCESS

\begin{tabular}{|c|c|c|}
\hline DESCRIPTION & RATE & UNITS \\
\hline Processing rate for " $A$ " type calcine (alumina) & 101 & $\mathrm{lbm} / \mathrm{hr}$ \\
\hline Processing rate for " $\mathrm{B}$ " type calcine (zirocnia/blends) & 465 & $\mathrm{lbm} / \mathrm{hr}$ \\
\hline Processing rate for " $\mathrm{C}$ " type calcine $(\mathrm{H}-4, \mathrm{H}-5)$ & 47 & $\mathrm{lbm} / \mathrm{hr}$ \\
\hline Slurrying rate of SBW liquid & 73 & $\mathrm{gph}$ \\
\hline Slurrying rate of dilution water & 54 & $\mathrm{gph}$ \\
\hline Total liquid slurry rate & 127 & gph \\
\hline Slurrying rate of calcine solids & 613 & $\mathrm{lbm} / \mathrm{hr}$ \\
\hline Solids concentration in slurry & $33 \%$ & weight\% \\
\hline Sucrose feed to calciner & 23 & gph \\
\hline Net slurry liquid feed to calciner & 127 & gph \\
\hline Recycle liquid feed to calciner & 30 & gph \\
\hline Total liquid feed to nozzles & 180 & $\mathrm{gph}$ \\
\hline Undissolved solids feed to calciner & 613 & $\mathrm{lbm} / \mathrm{hr}$ \\
\hline Kerosene feed to calciner & 16 & gph \\
\hline Recalcined solids production rate & 673 & $\mathrm{lbm} / \mathrm{hr}$ \\
\hline Recalcined solids grouting rate & 29,709 & $\mathrm{lbm} / \mathrm{shift}$ \\
\hline Canister filling rate & 32.1 & canisters/day \\
\hline Overall mass of recalcined solids produced & 6,696 & metric tons \\
\hline Overall volume of recalcined solids produced & 4,705 & $\mathrm{~m}^{3}$ \\
\hline Overall mass of grout produced & 19,131 & metric tons \\
\hline
\end{tabular}




\begin{tabular}{l|r|l} 
DESCRIPTION & RATE & UNITS \\
\hline \hline Overall volume of grout produced & 11,465 & $\mathrm{~m}^{3}$ \\
\hline Overall number of canisters produced & 15,924 & canisters \\
\hline & & \\
\hline
\end{tabular}

\subsection{Process Steps}

\subsubsection{Calcine Retrieval (Dwg CWO-01)}

The system for recovering existing calcine from the CSSF is described elsewhere ${ }^{9}$. This system allows for retrieving calcine from any of the seven binsets on any given day. (Note, however, that simultaneous recovery from multiple binsets is not assumed.) As described in Section 2.2 (item 8) existing calcine is categorized into three classes of calcine:

- Calcine A--aluminum calcine,

- Calcine B--zirconium calcine (including zirconium/sodium blends and fluorinel calcine), and

- Calcine C--SBW calcine (from WM-188 and WM-189 during campaigns $\mathrm{H}-4$ and $\mathrm{H}-5$ ).

It is assumed that calcine $A$ is physically segregated into binsets CSSF-1 (all bins) and CSSF-2 (bins 3, 4, and the top of bin 5). It is also assumed that calcine $C$ is physically segregated into CSSF-6 (top of all bins). Since the total quantities of calcine in the three categories are known (from past flowsheet data), the known segregation of calcines $A$ and $C$ allows the retrieval and segregation of the three categories of calcine into three distinct temporary storage bins (B-401, 402, 403 in Dwg CWO-04). Calcine is retrieved from the binsets in a manner that maintains a minimum inventory in each bin corresponding to one batch of slurry feed (see Section 3.3.2, below). Calcine types A, B, and $C$ (from B-101, 102, and 103, respectively) are drawn from the bins in relative proportions corresponding to the known total masses in each of the three categories.

\subsubsection{Sucrose Storage (Dwg CWO-02)}

Sucrose is assumed to be shipped into ICPP as a $65 \mathrm{wt} \%$ solution in water, and stored in tank $\mathrm{T}$-201. The sugar solution is pumped via P-201 from storage directly to the liquid feed lines into the calciner.

\subsubsection{Grouting (Dwg CWO-03)}

Recalcine will be grouted in a new facility. The grouting process for CWO is the same as for the DCWO from the point at which recalcine enters tank DCWO T-201A or B (see Ref. 10).

\subsubsection{Slurry Blending (Dwg CWO-04)}

Calcine solids of type A, B, and C flow gravimetrically from B-401, 402, 403 into one of two slurrying vessels $(\mathrm{V}-401 \mathrm{a}, \mathrm{b})$. The bins and slurrying vessels are sized to hold sufficient calcine and slurry for four days and 24 hours of calcination, respectively. Two slurrying vessels are used to allow continuous operation of the calciner. The masses of the three calcine types transferred are in the same proportion as the total inventories of each calcine type. Once either vessel is filled and blended, it is 
$431.02 \#$

$2 / 11 / 98$

Rev.
Function File Number - SPR-02

EDF Serial Number - EDF-CWO-003

Page 8 of 34

sampled and characterized sufficiently to set the rate of sucrose injection for recalcination. The setpoint is chosen by reference to pre-determined flowsheets developed for the range of expected calcine and SBW compositions. This range is also pre-determined based on the known range of variation of composition within the $\mathrm{A}, \mathrm{B}$, and $\mathrm{C}$ calcine categories, the manner in which these calcines are blended prior to slurrying, and the composition of the liquid SBW. After characterization is complete, the vessel contents are fed to the calciner. While one vessel is fed, the other is being filled, blended, and characterized for the succeeding batch.

The slurry tanks are aggressively mixed with air spargers to keep the undissolved solids suspended and uniformly dispersed through the slurry mixture, and to effect the dissolution of the soluble nitrates from the calcine solids. The homogeneous slurry mixture is pumped from the slurry tanks with a progressive cavity pump and into a recycle loop extending from the slurry tanks to the calciner and back to the tanks. The flow rate in the recycle loop is far in excess of the feed rate to the calciner, and is determined so as to generate sufficient turbulence to keep the solids in suspension in the horizontal piping run to the calciner. At the calciner the recycle loop feeds a manifold to which the calciner feed nozzles are connected. The rate of delivery of slurry mixture to the calciner nozzles is controlled by servo-controlled valves at the entrances to the nozzles. The overflow in the recycle loop from the manifold is routed back to the slurry tanks, and provides mixing to maintain the tank solids in suspension. To achieve the required pressure in the manifold to force the required slurry flow rate through the calciner feed nozzles, a valve on the return line to the slurry tanks is adjusted.

One progressive cavity pump is needed for each slurry tank. The pump attached to the tank being mixed circulates slurry from the tank, through a short loop within the slurrying cell, and back to the tank to promote mixing and maintain the solids in suspension. A third pump is plumbed in to provide a backup.

The process flow diagram for slurry blending indicates addition of process water to the slurry vessel. This addition is assumed to be required to reduce the solids concentration in the slurry to facilitate pumping through the recycle loop and into the feed nozzles. To achieve a solids weight loading of $33 \%$ in the slurry requires water addition in the ratio 0.75 volumes water per volume of SBW liquid. This addition is assumed for the process flows and mass balances provided herein.

Finally, calcium nitrate solution from the cold mixing area is transferred to the blend \& hold cell, and from there to the slurrying tanks in quantities sufficient to complex corrosive anions (e.g., fluorides).

\subsubsection{Calcination (Dwg CWO-05)}

Calcination of the slurried wastes is done in the existing NWCF, with modifications to incorporate the following unit operations:

- Feeding of slurried waste to the bed, and

- Addition of sucrose solution to the liquid feed upstream of the feed nozzles.

The slurry is fed through existing feed nozzle penetrations in the calciner for the current allliquid feed system. Three of the four feed nozzles and the corresponding feed lines from the recycle loop manifold (see Section 3.3.2) will be designed to accommodate the changed flowrates, the abrasiveness of the slurry mixture, and the required physical breakup of the slurry as it emerges from the feed nozzles into the calciner. Breakup of the slurry will be accomplished with high-pressure air jets around the slurry feed port, similar to existing feed nozzles. 
The fourth feed nozzle and feed line will not be replaced and will not be tied to the slurry recycle loop, but will continue to be fed by the existing feed system. Scrub solution will be recycled to the calciner via the fourth nozzle.

Sucrose will be added to the slurry just downstream of the takeoff point from the slurry recycle loop (BR-501 in Dwg CWO-05). The sucrose will be added in a $65 \mathrm{wt} \%$ aqueous solution, and a static inline mixer will be installed in the line between the recycle takeoff point and the feed nozzles to achieve the required mixing of the sucrose solution and the slurry. Since the slurry will be fed to the nozzles under pressure (from a progressive cavity pump at the slurry tanks), the pressure drop associated with the static mixer may easily be accommodated by suitable adjustment of the pump speed.

\subsubsection{Calcination Offgas Treatment (Dwg CWO-06)}

Treatment of offgas from the calciner will be accomplished using the existing offgas system for NWCF with some modifications. The current system includes a cyclone, spray quench, venturi scrubber, demisting vessels, ruthenium adsorbers (which act as pre-filters for the HEPA filters), heaters, HEPA filter banks, and a draft system to pull the offgas through the system and propel it into the Atmospheric Protection System and the ICPP main stack.

It is expected that changed offgas flowrates, and higher concentrations of fines in the offgas will necessitate redesign and replacement of the present cyclone. In addition, a system to extract elemental mercury from the NWCF scrub solution will be added in the valve cubicle. This system is needed to prevent accumulation of mercury in tank farm liquids. Such accumulation would result from efficient capture of mercury in the NWCF scrub solution, and subsequent periodic flushing of the highmercury scrub solution back to the tank farm during deep recycle. The mercury extraction system will be a series of electrolytic reduction cells, designed to reduce oxidized mercury to the elemental state, and deposit it in mercury pool electrodes at the bottom of the cells. Mercury recovered in the cells will llow by gravity into a separate accumulation tank, and dispensed from there into an amalgamation system (see Section 3.3.10, below).

\subsubsection{Recalcine Transport (Dwg CWO-07)}

The existing pneumatic transport system for calcine solids in NWCF is designed to transport calcine roughly 230 feet ( 460 feet for the closed loop) at a maximum rate of $300 \mathrm{lbs} / \mathrm{hr}$. However, the transport rate can be increased to the required a rate of $674 \mathrm{lbm} / \mathrm{hr}$, provided the length of the transport line is shortened. This will be accomplished by installation of a second pneumatic transport system with a booster (or transfer) station within 140 feet of the caliner ( 280 feet for the closed loop). Recalcine solids will be transported by the existing transport system from the calciner to this booster station. There, the solids will be extracted by a cyclone and re-entrained into the motive air ( $280 \mathrm{scfm}$ ) of the new system. The new transport line will extend from NWCF to the new grouting facility.

Recalcine will be extracted from the secondary system into a cyclone atop storage/static mixing vessels T-201A,B in the grouting facility (see Dwg CWO-07 from this report and Dwg DCWO-02 from Ref. . $10)$.

The existing transport system remains virtually unchanged within the NWCF, except that the lines will be shortened and routed to the new booster station. The solids loading ratio in the system will be between 1.7 and $2.0 \mathrm{lbm}$ solids/lbm air. The corresponding ratio in the new tranpsort system will be about $0.5 \mathrm{lbm}$ solids/lbm air 


\subsubsection{Sugar Digestion (Dwg CWO-08)}

Scrub solution must be periodically flushed to the tank farm when chlorides, undissolved solids, or total dissolved solids exceed threshold levels. It is expected that incomplete oxidation of the sucrose will result in some buildup of carbon and/or hydrocarbons in the scrub. Such substances would constitute an explosion hazard if sent to the tank farm (due to potential nitration of organic substances). Thus, all scrub solution sent to the tank farm will first be digested in a suitable accumulation/reaction vessel prior to being sent to the tank farm. This digestion process involves the oxidation of hydrocarbons by extended exposure to concentrated $\mathrm{HNO}_{3}$ at elevated temperature $\left(50-90^{\circ} \mathrm{C}\right)$. Tank digestion of the hydrocarbons from the scrub solution takes placed slowly ${ }^{7}$, and could require 3-6 weeks.

It is assumed that existing tanks in NWCF could be used for this purpose. Among those that might be used are the blend \& hold tanks (NCC-VES-101, 102, 103) and the hot and cold sump tanks (NCC-VES-119 and 122, respectively. Each of these tanks has a capacity of 3,000 gal (or more), can be heated, cooled, and/or air sparged, and can be filled from the scrub hold tank and drained to the tank farm.

\subsubsection{MACT Compliance Facility (Dwg CWO-09)}

Per assumption 17 in Section 2.2, the NWCF offgas system will be upgraded to comply with MACT requirements, as outlined in Ref. 11 . The upgrade will include control systems for carbon monoxide, NOx, unburned hydrocarbons, and residual mercury not collected in the scrubbing system. Details of this system are provided in Section 8 (item 7).

\subsubsection{Mercury Amalgamation (Dwg CWO-10)}

Recovered elemental mercury from the scrub solution will be mixed waste and will require treatment prior to disposal. This will be done by amalgamation with sulfur, as described in Section 8 (item 8).

\section{PROCESS FLOW DIAGRAMS}

Process flow diagrams are provided as Attachment 3 (CWO-00 through CWO-10). See also drawings for the Direct Cementitious Waste Option in Ref. 10.

\section{MATERIAL FLOW RATES}

Material flow rates for process streams shown in the drawings in Attachment 3 are provided in Table 2, below. 
TABLE 2: MATERIAL FLOW RATES

MATERIAL FLOWS FOR CEM ENTITIOUS WASTE OPTION (CWO):

\begin{tabular}{|c|c|c|c|c|c|c|c|c|c|}
\hline & & 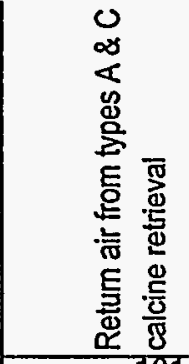 & 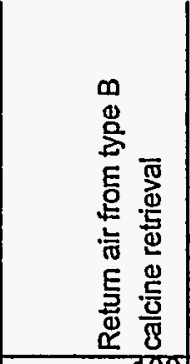 & 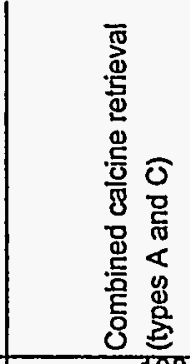 & 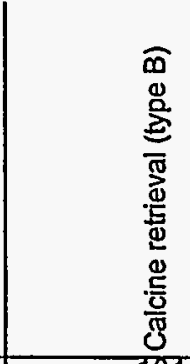 & 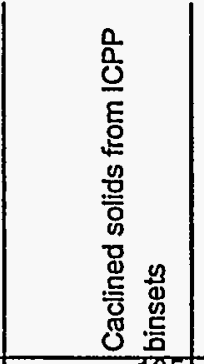 & 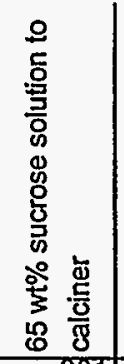 & 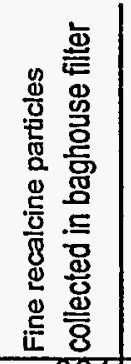 & 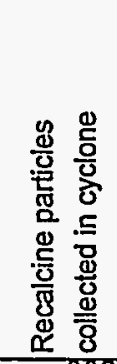 \\
\hline MateriallStream ID & Units* & 101 & 102 & 103 & 704 & 105 & 207 & 301 & 302 \\
\hline Total sollds & $\mathrm{lbm} / \mathrm{hr}$ & & & $\begin{array}{l}2860 \text { (non- } \\
\text { continuous) }\end{array}$ & $\begin{array}{l}9020 \text { (non- } \\
\text { continuous) }\end{array}$ & $\begin{array}{l}11880 \text { (non- } \\
\text { continuous) }\end{array}$ & & 67 & 606 \\
\hline Totantquids & gallhr & & & & & & 23 & & \\
\hline Total volumetric flow & gallhr & & & & & & 23 & & \\
\hline Sucrose & $76 \mathrm{~m} / \mathrm{hr}$ & & & & & & 150 & & \\
\hline Air & scfm & $\begin{array}{l}800 \text { (non- } \\
\text { continuous) }\end{array}$ & $\begin{array}{l}800 \text { (non- } \\
\text { continuous) }\end{array}$ & & & $\begin{array}{l}7600 \text { (non- } \\
\text { continuous) }\end{array}$ & & & \\
\hline Dissolved solids (excl. sucrose) & $76 \mathrm{~m} / \mathrm{hr}$ & & & & & & of & & \\
\hline Undissolved sollds & $76 m / h r$ & & & & & & & & \\
\hline Dissolved solids (incl. sucrose) & $76 \mathrm{~m} / \mathrm{hr}$ & & & & & & 159 & & \\
\hline Total gas flow & scfm & $\begin{array}{l}800 \text { (non- } \\
\text { continuous) }\end{array}$ & $\begin{array}{l}800 \text { (non- } \\
\text { continuous) }\end{array}$ & & & & & & \\
\hline Kerosene & $\mathrm{gph}$ & & & & & & & & \\
\hline 02 & scrm & & & & & & & & \\
\hline H2O & $7 \mathrm{bm} / \mathrm{hr}$ & & & & & & 86 & & \\
\hline RNO3 (13M) & $\mathrm{gpm}$ & & & & & & & & \\
\hline $\mathrm{Hg}$ & $76 m / h r$ & & & & & & & & \\
\hline
\end{tabular}

- Units are as indicated in this column, except where explicilly stated 


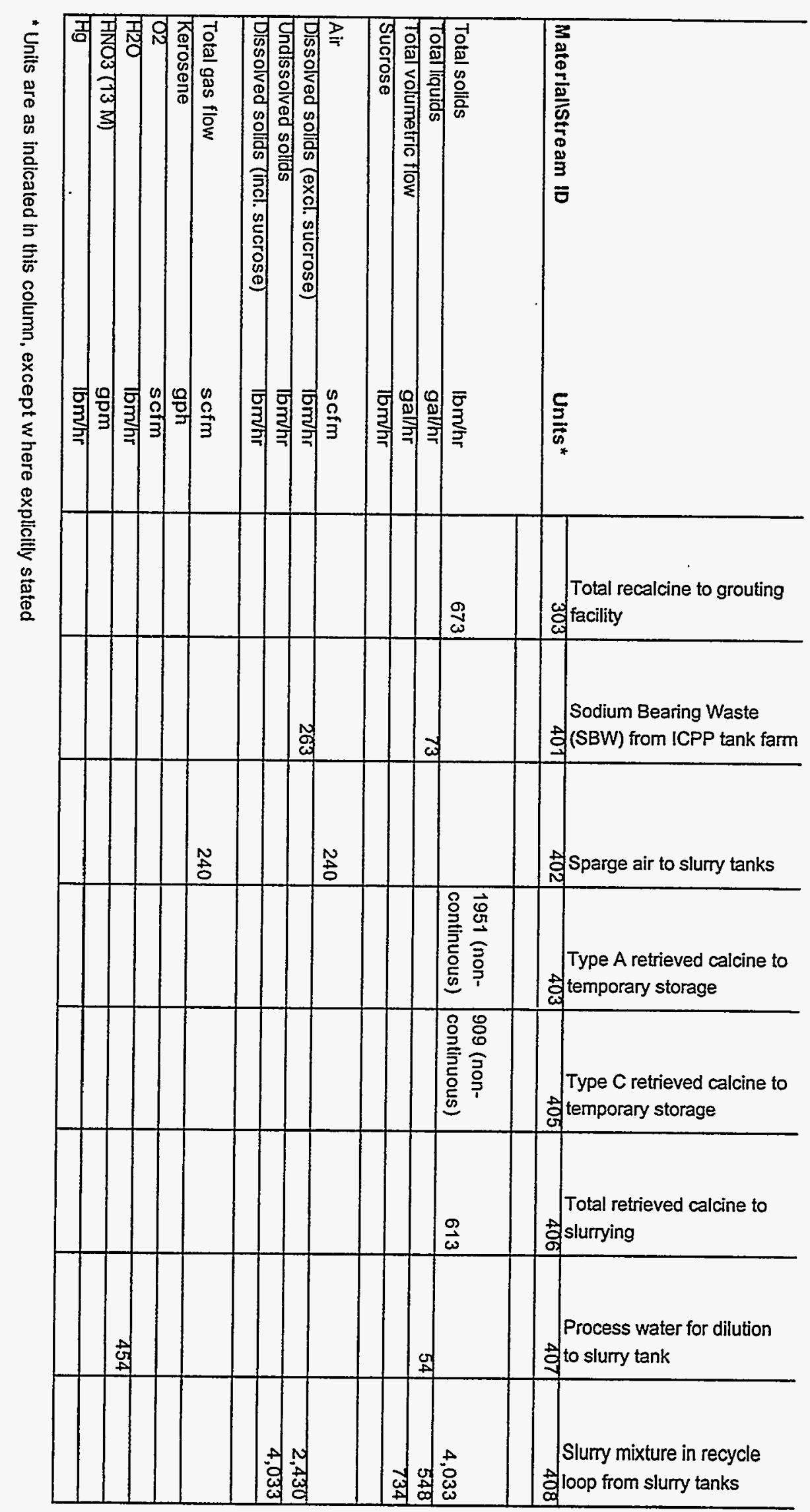


$\overline{\mathrm{I} \varepsilon}-\exists$

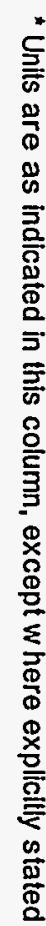

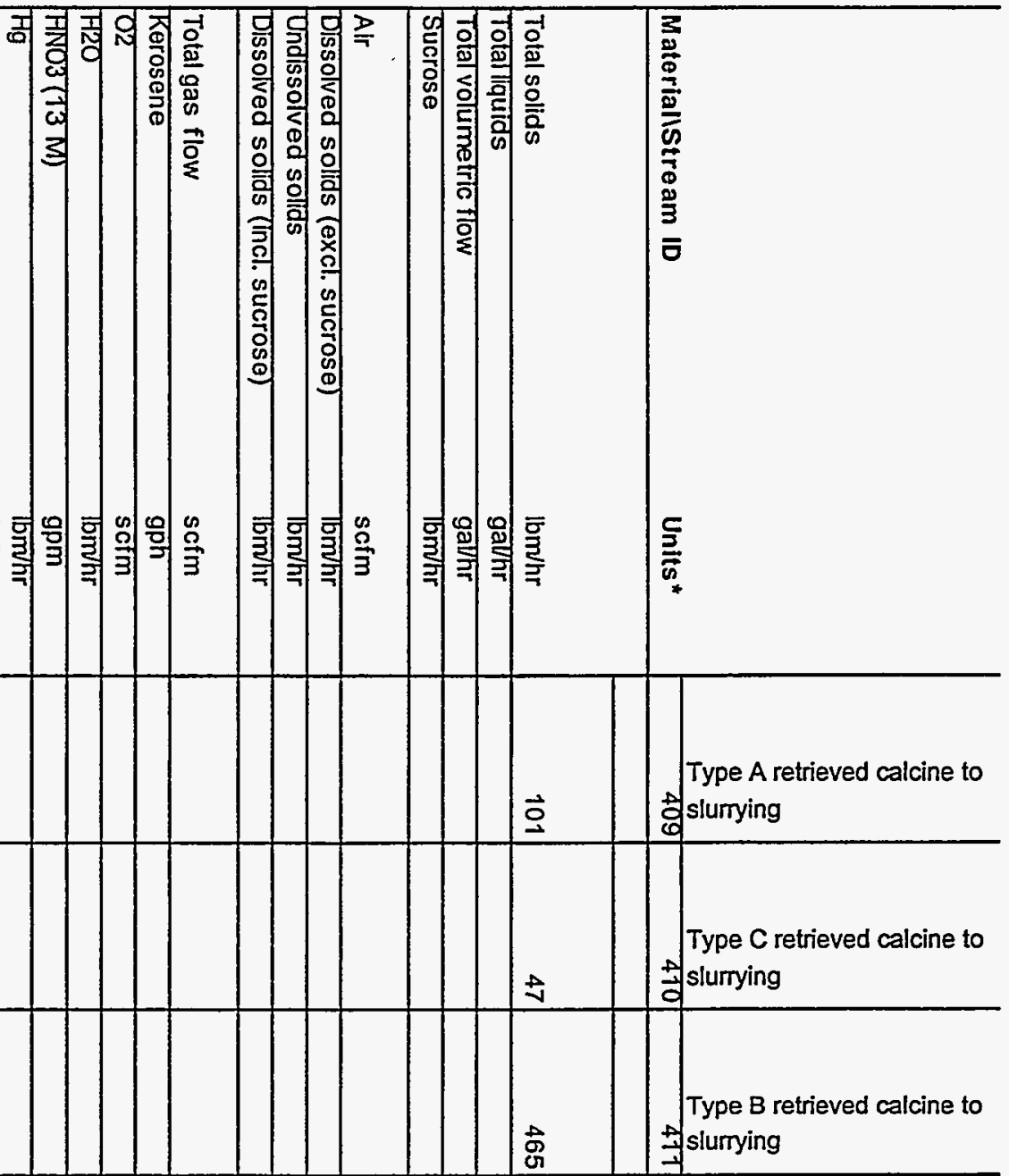

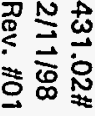

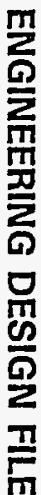

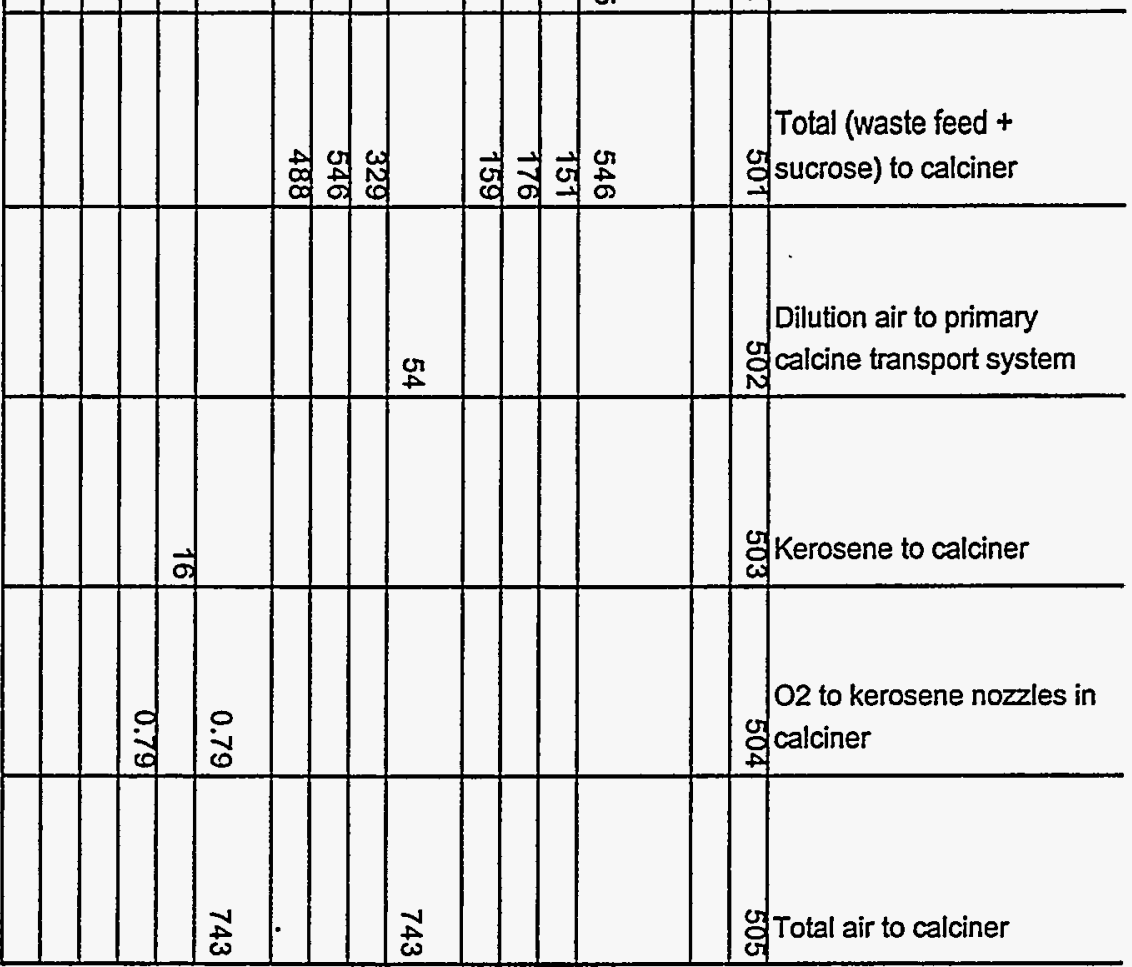

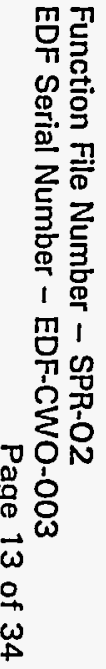




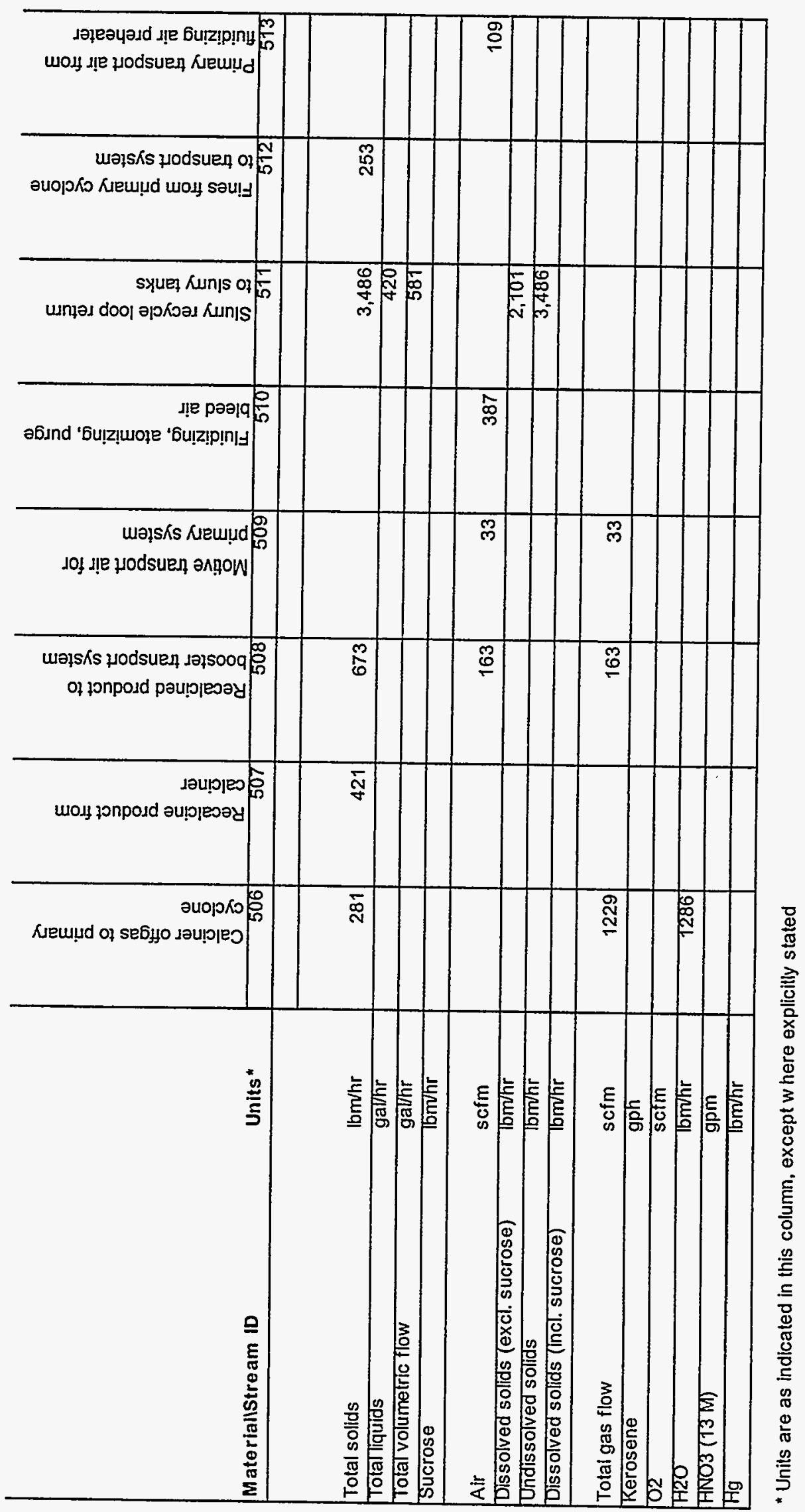




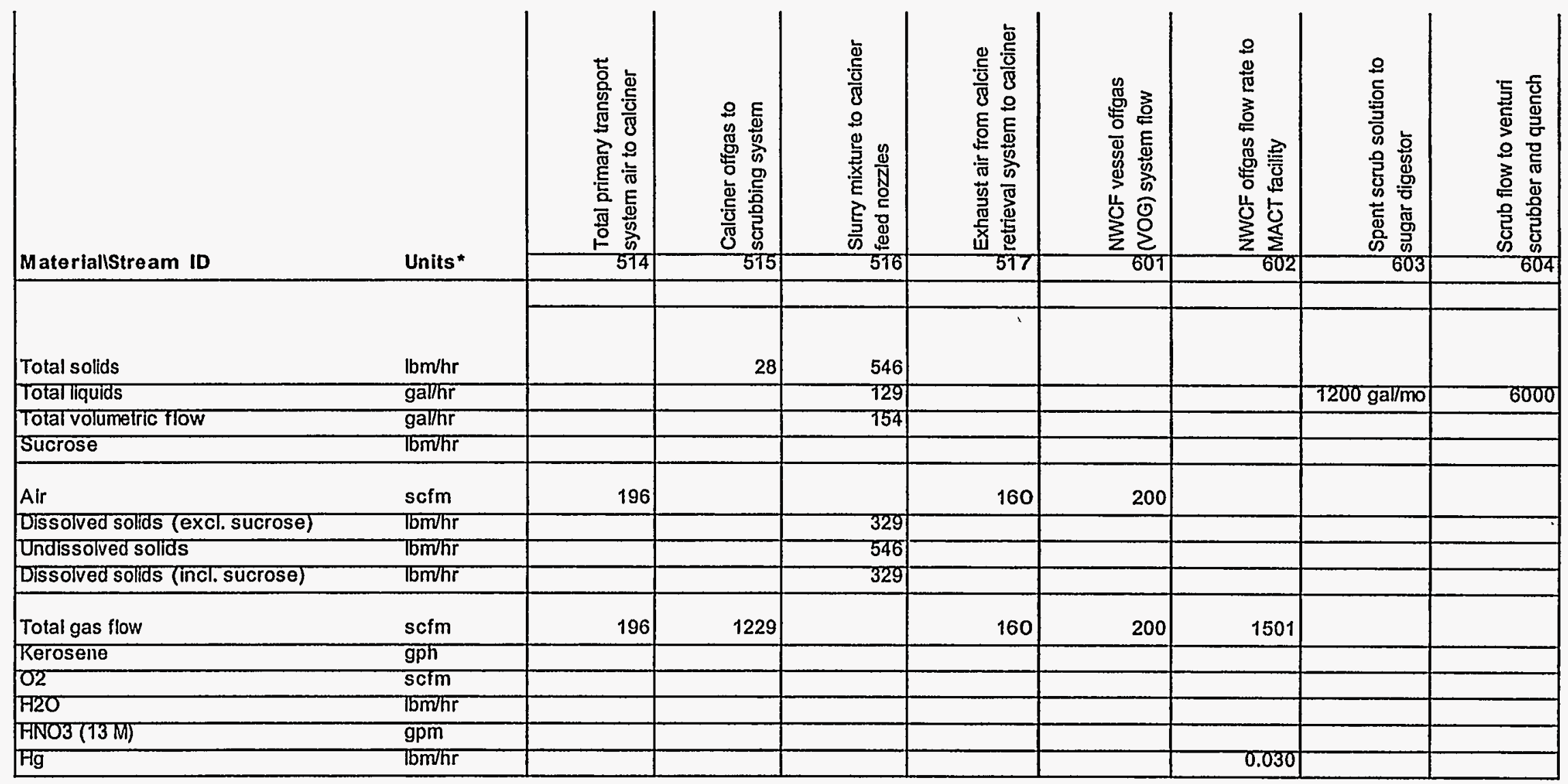

- Unils are as indicated in this column, except $w$ here expllitly stated 

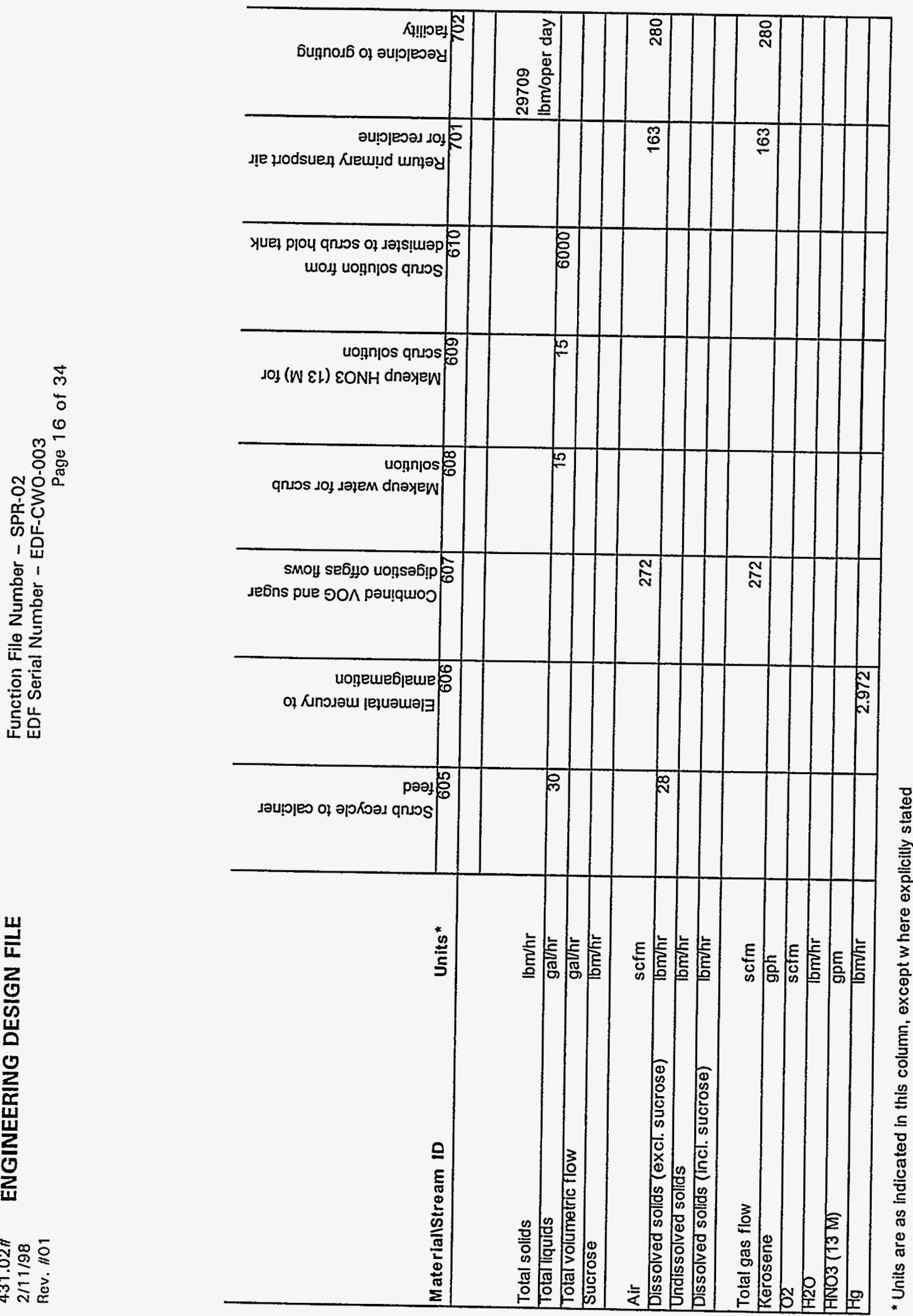

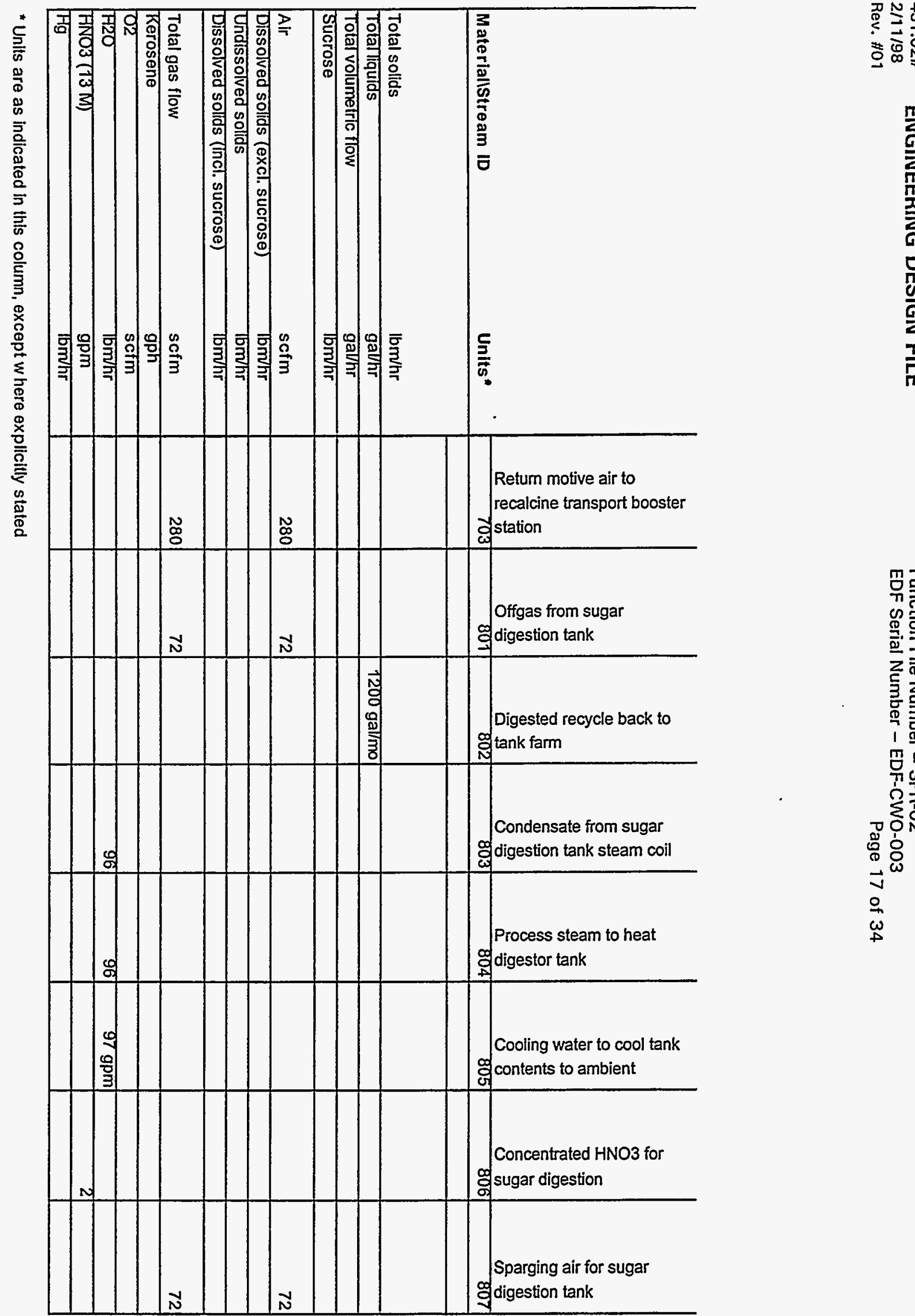


\begin{tabular}{|c|c|c|c|c|c|c|c|c|c|}
\hline & & 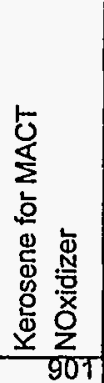 & 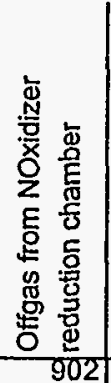 & 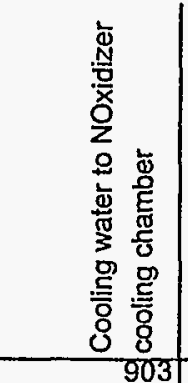 & 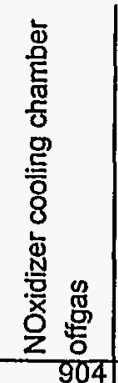 & 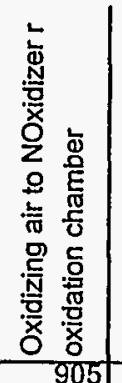 & 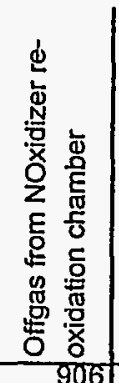 & 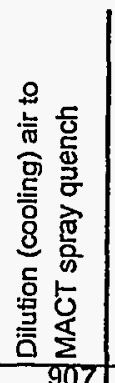 & 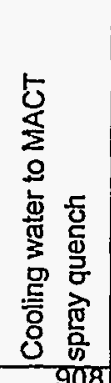 \\
\hline Materianstream ID & Units" & & & & & & & 907 & \\
\hline Total solids & $\mathrm{lbm} / \mathrm{hr}$ & & & & & & & & \\
\hline Totaliquids & gallhr & & & & & & & & \\
\hline Total volumetric flow & gal/hr & & & 149 & & & & & 952 \\
\hline Sucrose & $76 m / h r$ & & & & & & & & \\
\hline Air & scfm & & & & & 579 & & 3742 & \\
\hline Dissolved solids (excl. sucrose) & $75 \mathrm{~m} / \mathrm{hr}$ & & & & & & & & \\
\hline Undissolved solids & $76 m / h r$ & & & & & & & & \\
\hline Dissolved sollds (incl. sucrose) & $76 m / h r$ & & & & & & & & \\
\hline Total gas flow & scfm & & 2656 & & 3069 & 579 & 3742 & 3742 & \\
\hline Kerosene & $\mathrm{gph}$ & 72 & & & & & & & \\
\hline 02 & sctm & & & & & & & & \\
\hline $\mathrm{H} 2 \mathrm{O}$ & Tom $/ h r$ & & & TBD & & & & & 7940 \\
\hline $\mathrm{FNO3}$ (13M) & $\mathrm{gpm}$ & & & & & & & & \\
\hline $\mathrm{Hg}$ & Tbmhr & & 0.030 & & 0.030 & & 0.030 & & \\
\hline
\end{tabular}

* Units are as indicated in this column, except where explicitly stated 


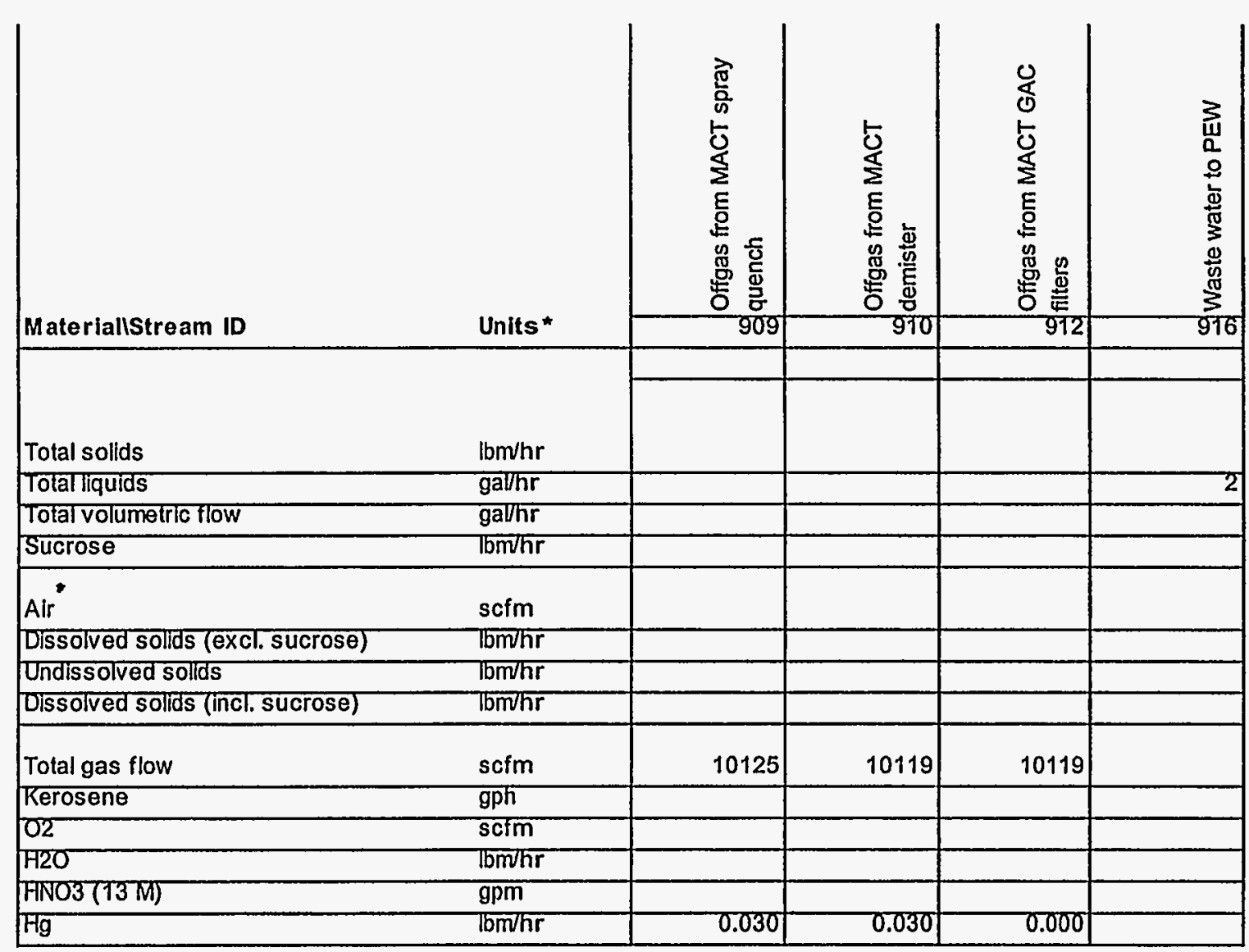

- Units are as indicated in this column, except $w$ here explicitly stated 


\section{NEW EQUIPMENT LIST}

\subsection{Equipment Labels}

The following labels are used to specify equipment needed in the process flow diagrams:

B

$\mathrm{BH}$

$\mathrm{BL}$

BR

CY

DM

E

EC

EJ

F

FB

FN

FS

$\mathrm{P}$

$\mathrm{T}$

V

WS
Storage bin/Hopper

Baghouse filter system

Blower/Fan

Branch (tee) in piping system

Cyclone separator

Demister vessel

Heat exchanger

Electrowinning cell (electrolytic Hg extraction device)

Ejector

Filter

Fluidized bed calciner

Liquid feed nozzle for calciner

Flow sensor

Pump

Tank

Vessel

Welding station

\subsection{Equipment Needed}

New equipment items required for the CWO process (in addition to those described in the new equipment list for the DCWO process; see Ref. 10) are described in Table 3, below:

TABLE 3: NEW EQUIPMENT LIST FOR CWO PROCESS

\begin{tabular}{l|l|l|l} 
ID & $\begin{array}{l}\text { DWG } \\
\text { NO. }\end{array}$ & DESCRIPTION & SIZING INFORMATION \\
\hline \hline CY-101 & CWO-01 & $\begin{array}{l}\text { Cyclone to extract retrieved calcine } \\
\text { (Types A and C) from the pneumatic } \\
\text { retrieval system line \#1 }\end{array}$ & $\begin{array}{l}\text { (This item is called out in Ref. 9, } \\
\text { and in included here for information } \\
\text { purposes only.) }\end{array}$ \\
\hline
\end{tabular}




\begin{tabular}{|c|c|c|c|}
\hline ID & $\begin{array}{l}\text { DWG } \\
\text { NO. }\end{array}$ & DESCRIPTION & SIZING INFORMATION \\
\hline F-101 & CWO-01 & $\begin{array}{l}\text { Sintered metal prefilter for recycled } \\
\text { transport air in line \#1 of the } \\
\text { pneumatic retrieval system }\end{array}$ & $\begin{array}{l}\text { (This item is called out in Ref. 9, } \\
\text { and is included here for information } \\
\text { purposes only.) }\end{array}$ \\
\hline F-102 & CWO-01 & $\begin{array}{l}\text { HEPA filter bank for recycled } \\
\text { transport air in line \#1 of the } \\
\text { pneumatic retrieval system }\end{array}$ & $\begin{array}{l}\text { (This item is called out in Ref. } 9 \text {, } \\
\text { and is included here for information } \\
\text { purposes only.) }\end{array}$ \\
\hline BL-103 & CWO-01 & $\begin{array}{l}\text { Blower for transport of calcine } \\
\text { (Types A and C) in line \#1 of the } \\
\text { pneumatic retrieval system }\end{array}$ & $\begin{array}{l}\text { (This item is called out in Ref. 9, } \\
\text { and is included here for information } \\
\text { purposes only.) }\end{array}$ \\
\hline E-101 & $\begin{array}{l}\text { CWO-01 } \\
\end{array}$ & $\begin{array}{l}\text { Heat exchanger to cool air in line \#1 } \\
\text { of the pneumatic retrieval system } \\
\text { after blower compression }\end{array}$ & $\begin{array}{l}\text { (This item is called out in Ref. } 9 \text {, } \\
\text { and is included here for information } \\
\text { purposes only.) }\end{array}$ \\
\hline CY-102 & CWO-01 & $\begin{array}{l}\text { Cyclone to extract retrieved calcine } \\
\text { (Type B) from the pneumatic } \\
\text { retrieval system line } \# 2\end{array}$ & $\begin{array}{l}\text { (This item is called out in Ref. 9, } \\
\text { and is included here for information } \\
\text { purposes only.) }\end{array}$ \\
\hline F-103 & CWO-01 & $\begin{array}{l}\text { Sintered metal prefilter for recycled } \\
\text { transport air in line } \# 2 \text { of the } \\
\text { pneumatic retrieval system }\end{array}$ & $\begin{array}{l}\text { (This item is called out in Ref. 9, } \\
\text { and is included here for information } \\
\text { purposes only.) }\end{array}$ \\
\hline F-104 & CWO-01 & $\begin{array}{l}\text { HEPA filter bank for recycled } \\
\text { transport air in line } \# 2 \text { of the } \\
\text { pneumatic retrieval system }\end{array}$ & $\begin{array}{l}\text { (This item is called out in Ref. 9, } \\
\text { and is included here for information } \\
\text { purposes only.) }\end{array}$ \\
\hline BL-104 & CWO-01 & $\begin{array}{l}\text { Blower for transport of calcine } \\
\text { (Type B) in line \#2 of the pneumatic } \\
\text { retrieval system }\end{array}$ & $\begin{array}{l}\text { (This item is called out in Ref. 9, } \\
\text { and is included here for information } \\
\text { purposes only.) }\end{array}$ \\
\hline E-102 & CWO-01 & $\begin{array}{l}\text { Heat exchanger to cool air in line } \# 2 \\
\text { of the pneumatic retrieval system } \\
\text { after blower compression }\end{array}$ & $\begin{array}{l}\text { (This item is called out in Ref. 9, } \\
\text { and is included here for information } \\
\text { purposes only.) }\end{array}$ \\
\hline P-201 & CWO-02 & $\begin{array}{l}\text { Positive displacement pump to } \\
\text { supply controlled feed rate of } \\
\text { sucrose solution from storage tank to } \\
\text { static mixer in calciner waste feed } \\
\text { line }\end{array}$ & $23 \mathrm{gpm}$ \\
\hline T-201 & CWO-02 & $\begin{array}{l}\text { Tank to store } 65 \mathrm{wt} \% \text { sucrose } \\
\text { solution for use in calcination }\end{array}$ & $\begin{array}{l}8,150 \text { gal capacity (sufficient for } \\
15 \text { days' calcination) } \\
\text { Material: } 304 \mathrm{~L} \text { SS }\end{array}$ \\
\hline EJ-201 & CWO-02 & $\begin{array}{l}\text { Air ejector for transport of } 65 \mathrm{wt} \% \\
\text { sucrose solution from bulk receiving }\end{array}$ & Transport 23 gpm sucrose solution \\
\hline
\end{tabular}




\begin{tabular}{|c|c|c|c|}
\hline ID & $\begin{array}{l}\text { DWG } \\
\text { NO. }\end{array}$ & DESCRIPTION & SIZING INFORMATION \\
\hline & & terminal to storage & $152 \mathrm{scfm}$ estimated air requirement \\
\hline CY-301 & CWO-03 & $\begin{array}{l}\text { Cyclone in grouting facility to } \\
\text { extract recalcine from the secondary } \\
\text { (booster) pneumatic transport system }\end{array}$ & $\begin{array}{l}280 \mathrm{scfm} \text { air flow with } 673 \mathrm{lbm} / \mathrm{min} \\
\text { solids flow }\end{array}$ \\
\hline F-301 & CWO-03 & $\begin{array}{l}\text { Pulsed baghouse filter to extract fine } \\
\text { particles from the secondary } \\
\text { (booster) pneumatic transport system }\end{array}$ & $\begin{array}{l}280 \text { scfm gas flow with } 67 \mathrm{lbm} / \mathrm{min} \\
\text { solids flow }\end{array}$ \\
\hline B-401 & CWO-04 & $\begin{array}{l}\text { Storage bin for retrieved Type A } \\
\text { (aluminum) calcine }\end{array}$ & $550 \mathrm{ft}^{3}$ storage capacity \\
\hline $\mathrm{B}-402$ & CWO-04 & $\begin{array}{l}\text { Storage bin for retrieved Type B } \\
\text { (zironcia/zironia blends/fluorinel) } \\
\text { calcines }\end{array}$ & $550 \mathrm{ft}^{3}$ storage capacity \\
\hline B-403 & CWO-04 & $\begin{array}{l}\text { Storage bin for retrieved Type } \mathrm{C} \\
\text { (from } \mathrm{H}-4, \mathrm{H}-5 \text { campaigns ) calcine }\end{array}$ & $550 \mathrm{ft}^{3}$ storage capacity \\
\hline $\mathrm{V}-401 \mathrm{~A}, \mathrm{~B}$ & CWO-04 & $\begin{array}{l}\text { Two (2) slurrying vessels to blend } \\
\text { solid calcine with liquid SBW }\end{array}$ & $\begin{array}{l}\text { 4,000 gal } \\
\text { Slurrying vessels also require air } \\
\text { spargers to achieve required mixing, } \\
\text { decon spray systems, and heating } \\
\text { and cooling coils } \\
480 \text { scfm estimated requirement for } \\
\text { sparging air for two.vessels } \\
\text { combined } \\
\text { Material: Nitronic } 50 \text {-or equivalent }\end{array}$ \\
\hline P-401 & CWO-04 & $\begin{array}{l}\text { Three (3) progressive cavity pumps } \\
\text { to move slurry from vessels } \\
\text { V-401a,b to the calciner feed } \\
\text { nozzles }\end{array}$ & $\begin{array}{l}100 \text { gpm @ } 33 \% \text { solids content (by } \\
\text { weight) } \\
\text { Discharge head } 200 \mathrm{ft} \\
\text { Pump power } 20 \mathrm{hp} \\
\text { Material: Nitronic } 50 \text { or equivalent }\end{array}$ \\
\hline FN-501 & CWO-05 & $\begin{array}{l}\text { Three (3) redesigned feed nozzles } \\
\text { for slurry feed to calciner }\end{array}$ & $\begin{array}{l}\text { Nozzles incorporate liquid feed and } \\
\text { atomizing air } \\
\text { Material: Inconnel }\end{array}$ \\
\hline BR-501 & CWO-05 & $\begin{array}{l}\text { Three (3) specially-designed } \\
\text { branching tees to take off a portion } \\
\text { of the slurry recycle loop flow, and } \\
\text { route it to the calciner feed nozzles }\end{array}$ & $\begin{array}{l}\text { Tees must be developed to minimize } \\
\text { fluid/solid separation at the junction } \\
\text { Material: Inconnel }\end{array}$ \\
\hline
\end{tabular}




\begin{tabular}{|c|c|c|c|}
\hline ID & $\begin{array}{l}\text { DWG } \\
\text { NO. }\end{array}$ & DESCRIPTION & SIZING INFORMATION \\
\hline M-501 & CWO-05 & $\begin{array}{l}\text { Three (3) static inline mixers to } \\
\text { blend } 65 \text { wt\% sucrose solution with } \\
\text { slurry feed from slurry recycle loop }\end{array}$ & $\begin{array}{l}\text { Slurry flow rate } 73 \mathrm{gph} \text { liquid, } \\
546 \mathrm{lbm} / \mathrm{hr} \text { solids } \\
\text { Sucrose solution flow rate } 23 \mathrm{gph} \\
\text { (No restriction on pressure drop } \\
\text { through mixer) }\end{array}$ \\
\hline FS-501 & CWO-05 & $\begin{array}{l}\text { Three ( } 3 \text { ) flow sensors to measure } \\
\text { the flow rate of slurry to each of the } \\
\text { three slurry feed nozzles }\end{array}$ & $\begin{array}{l}\text { Measure volumetric flow rate of } \\
\text { slurry from } 15-45 \mathrm{gph} \\
\text { (mass flow rate of } 1268 \mathrm{lbm} / \mathrm{hr} \text { ) }\end{array}$ \\
\hline CY-501 & CWO-05 & $\begin{array}{l}\text { Redesigned replacement cyclone for } \\
\text { calciner offgas fines (replacing } \\
\text { VES-NCC-107) }\end{array}$ & $\begin{array}{l}\text { Gas flow rate } 1229 \mathrm{scfm} \\
\text { Solids (calcine fines) flow rate } \\
281 \mathrm{lbm} / \mathrm{hr}\end{array}$ \\
\hline EC-601 & CWO-06 & $\begin{array}{l}\text { Electrowinning (electrolytic) cell for } \\
\text { recovery of mercury from scrub } \\
\text { solution }\end{array}$ & $\begin{array}{l}\text { Cell requires: } \\
\text { - } \quad \text { six Nitronic } 50 \text { tanks, }(2 \mathrm{ft} \\
\text { diameter X } 2 \mathrm{ft} \text { high) } \\
\text { - } 2 \text { gpm positive displacement } \\
\text { pump } \\
\text { - } \text { Back-washable metal filter } \\
\text { - } \quad \text { four } 3.1 \mathrm{ft}^{2} \text { platinum electrodes } \\
\text { - } 1 \text { " Nitronic } 50 \text { lines from } \\
\text { electrowinning tanks in valve } \\
\text { cubicle to/from the scrub hold } \\
\text { tank (NCC-VES-108) }\end{array}$ \\
\hline$\overline{F-603}$ & CWO-06 & $\begin{array}{l}\text { Backwashable sintered metal filter } \\
\text { for filtering undissolved solids from } \\
\text { scrub solution }\end{array}$ & $\begin{array}{l}480 \mathrm{gpm} \text { liquid rate } \\
20 \mathrm{lbm} / \mathrm{hr} \text { solids removal rate }\end{array}$ \\
\hline P-601 & CWO-06 & $\begin{array}{l}\text { Positive displacement pump to } \\
\text { transer scrub solution to the mercury } \\
\text { removal cell }\end{array}$ & 480 gph capacity \\
\hline CY-701 & CWO-07 & $\begin{array}{l}\text { Cyclone to extract recalcine solids } \\
\text { from primary pneumatic transport } \\
\text { system into accumulation bin } \\
\text { (B-701) }\end{array}$ & $\begin{array}{l}163 \mathrm{scfm} \text { gas flow } \\
673 \mathrm{lbm} / \mathrm{hr} \text { solids flow }\end{array}$ \\
\hline B-701 & CWO-07 & $\begin{array}{l}\text { Recalcine accumulation bin to accept } \\
\text { recalcine from the primary } \\
\text { pneumatic transport system, prior to } \\
\text { introduction into secondary }\end{array}$ & $\begin{array}{l}550 \mathrm{ft}^{3} \text { capacity, cone angle } 65^{\circ} \text {, } \\
\text { equipped with rotary valve } \\
\text { Material: } 304 \mathrm{~L} \text { SS }\end{array}$ \\
\hline
\end{tabular}




\begin{tabular}{|c|c|c|c|}
\hline ID & $\begin{array}{l}\text { DWG } \\
\text { NO. }\end{array}$ & DESCRIPTION & SIZING INFORMATION \\
\hline & & $\begin{array}{l}\text { pneumatic transport system to } \\
\text { grouting facility }\end{array}$ & 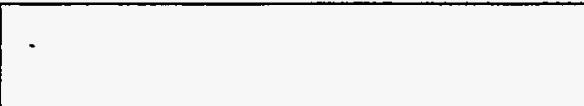 \\
\hline BL-701 & CWO-07 & $\begin{array}{l}\text { Blower to provide motive force for } \\
\text { secondary pneumatic transport } \\
\text { system }\end{array}$ & 25 psig pressure rise @ $280 \mathrm{scfm}$ \\
\hline BL-903 & CWO-09 & $\begin{array}{l}\text { Blower to provide combustion air to } \\
\text { NOxidizer reduction chamber }\end{array}$ & 0 scfm@0.5 psia pressure rise \\
\hline V-903 & CWO-09 & $\begin{array}{l}\text { Reduction chamber for MACT } \\
\text { NOxidizer system }\end{array}$ & $2,000 \mathrm{scfm}$ capacity \\
\hline V-904 & CWO-09 & $\begin{array}{l}\text { Cooling chamber for MACT } \\
\text { NOxidizer system }\end{array}$ & 2,000 scfm capacity \\
\hline BL-905 & CWO-09 & $\begin{array}{l}\text { Blower to provide combustion air to } \\
\text { NOxidizer re-oxidation chamber }\end{array}$ & 579 scfm@0.5 psia pressure rise \\
\hline V-905 & CWO-09 & $\begin{array}{l}\text { Oxidation chamber for MACT } \\
\text { NOxidizer system }\end{array}$ & $2,000 \mathrm{scfm}$ capacity \\
\hline BL-906 & CWO-09 & $\begin{array}{l}\text { Blower to provide dilution (cooling) } \\
\text { air to MACT system spray quench }\end{array}$ & 3,742 scfm @ 0.5 psia pressure rise \\
\hline V-906 & CWO-09 & $\begin{array}{l}\text { Water spray quench tower for } \\
\text { MACT system }\end{array}$ & $\begin{array}{l}10,125 \text { scfm gas flow } \\
16 \text { gpm liquid flow }\end{array}$ \\
\hline DM-907 & CWO-09 & $\begin{array}{l}\text { Demister vessel (with meshes) for } \\
\text { post-quench MACT offgas }\end{array}$ & $\begin{array}{l}10,125 \text { scfm gas flow, } 2 \text { gpm liquid } \\
\text { flow }\end{array}$ \\
\hline E-908 & CWO-09 & $\begin{array}{l}\text { Heat exchanger to reheat MACT } \\
\text { offgas prior to entering GAC filters }\end{array}$ & $798,449 \mathrm{Btu} / \mathrm{hr}$ capacity \\
\hline F-909A,B,C & CWO-09 & $\begin{array}{l}\text { Three (3) sulfur-impregnated } \\
\text { granular activated carbon filter } \\
\text { canisters }\end{array}$ & $\begin{array}{l}\text { 10,119 scfm gas flow } \\
\text { Size: } 10 \mathrm{ft} \text { dia } x 5 \mathrm{ft} \text { high }\end{array}$ \\
\hline E-910 & CWO-09 & $\begin{array}{l}\text { Heat exchanger to reheat MACT } \\
\text { offgas prior to entering HEPA filters }\end{array}$ & $798,449 \mathrm{Btu} / \mathrm{hr}$ capacity \\
\hline F-911A,B,C,D & CWO-09 & $\begin{array}{l}\text { HEPA filters for final polishing of } \\
\text { MACT offgas prior to stack release }\end{array}$ & 10,119 scfm gas flow \\
\hline \multirow[t]{2}{*}{$\mathrm{BL}-912 \mathrm{~A}, \mathrm{~B}, \mathrm{C}$} & CWO-09 & $\begin{array}{l}\text { Three (3) blowers to provide draft } \\
\text { for MACT offgas system }\end{array}$ & $\begin{array}{l}\text { 10,119 scfm@1.0 psia pressure } \\
\text { rise }\end{array}$ \\
\hline & CWO-09 & $\begin{array}{l}\text { Continuous Emissions Monitoring } \\
\text { System (CEMS) for MACT system }\end{array}$ & $\begin{array}{l}\text { Instrumentation needed: } \\
\text { - Offgas flow rate }\end{array}$ \\
\hline
\end{tabular}




\begin{tabular}{|c|c|c|c|}
\hline ID & $\begin{array}{l}\text { DWG } \\
\text { NO. }\end{array}$ & DESCRIPTION & SIZING INFORMATION \\
\hline & & & $\begin{array}{l}\text { Paramagnetic } \mathrm{O}_{2} \text { measurement } \\
\text { - } \\
\text { - } \text { Hon-dispersive infrared } \mathrm{CO} \\
\text { total hydrocarbons } \\
\text { - } \\
\text { - } \\
\text { - Non-dispersiculate concentration } \\
\mathrm{CO}_{2}\end{array}$ \\
\hline$\cdot$ & CWO-09 & $\begin{array}{l}\text { Automatic Waste Feed Cutoff } \\
\text { System (AWFCS) for MACT system }\end{array}$ & $\begin{array}{l}\text { This is a control system tied to } \\
\text { process monitoring instrumentation } \\
\text { (including the CEMS), and provides } \\
\text { an automatic shutoff of the waste } \\
\text { feed in the event that the } \\
\text { instrumentation detects a significant } \\
\text { excursion outside the permitted } \\
\text { operating envelope. }\end{array}$ \\
\hline B-1001 & CWO-10 & $\begin{array}{l}\text { Storage/metering bin for elemental } \\
\text { sulfur during amalgamation of } \mathrm{Hg}\end{array}$ & $\begin{array}{l}4.1 \mathrm{ft}^{3} \text { capacity, with metering } \\
\text { capability }\end{array}$ \\
\hline JM-1001 & CWO-10 & $\begin{array}{l}\text { Jar mill to amalgamate } \mathrm{Hg}-\mathrm{S} \text { mixture } \\
\text { in 1-gal paint cans }\end{array}$ & $1.57 \mathrm{gpd} \mathrm{HgS}$ \\
\hline L-1001 & CWO-10 & $\begin{array}{l}\text { Loading station to decant elemental } \\
\text { mercury from the collection tank in } \\
\text { the electrowinning system into 1-gal } \\
\text { paint cans for amalgamation with } \\
\text { sulfur }\end{array}$ & $\begin{array}{l}0.63 \mathrm{gpd} \text { elemental } \mathrm{Hg} \text {, } \\
17.1 \mathrm{lbm} / \text { day elemental sulfur }\end{array}$ \\
\hline
\end{tabular}

The process equipment listed in Table 3 includes only those items which are specific to the CWO process. In addition to these, all equipment items listed in Ref. 10 for grouting of calcine will be required in order to complete waste treatment under the CWO process. Equipment required for MACT compliance (see Section 8 ) is also specific to the CWO process, and will be required.

\section{UTILITESS SUMMARY}

Table 4 summarizes the new utilities requirements for the NWCF after modifications to accommodate the CWO process: 
TABLE 4: RECALCINATION UTILITIES SUMMARY

\begin{tabular}{l|l|l|l} 
EQUIPMENT ID & $\begin{array}{l}\text { DWG } \\
\text { NO. }\end{array}$ & DESCRIPTION & REQUIREMENT \\
\hline \hline
\end{tabular}

\section{PROCESS STEAM:}

\begin{tabular}{l|l|l|l}
\hline V-108 & CWO-08 & Steam for sugar digestor & $96 \mathrm{lbm} / \mathrm{hr}$ \\
\hline V-401 & CWO-04 & $\begin{array}{l}\text { Steam for heating coils in } \\
\text { slurrying tanks }\end{array}$ & $119 \mathrm{lbm} / \mathrm{hr}$ \\
\hline E-908 & CWO-09 & $\begin{array}{l}\text { Steam to reheat offgas upstream } \\
\text { of GAC filters in MACT system }\end{array}$ & $726 \mathrm{lbm} / \mathrm{hr}$ \\
\hline E-910 & CWO-09 & $\begin{array}{l}\text { Steam to reheat offgas upstream } \\
\text { of HEPA filters in MACT } \\
\text { system }\end{array}$ & $726 \mathrm{lbm} / \mathrm{hr}$ \\
\hline TOTAL & $\begin{array}{l}\text { Steam for normal NWCF } \\
\text { operations }\end{array}$ & $5000 \mathrm{lbm} / \mathrm{hr}$ \\
\hline
\end{tabular}

\begin{tabular}{l|l|l|l}
\hline AIR: & CBO-04 & $\begin{array}{l}\text { Motive air for rotary valves on } \\
\text { calcine storage bins }\end{array}$ & $30 \mathrm{scfm}$ \\
\hline B-101,-102,-103 & CWo-04 & Sparge air for slurrying tanks & $176 \mathrm{scfm}$ \\
\hline V-401 & CWO-02 & $\begin{array}{l}\text { Pneumatic transport air for } \\
\text { transfer of sucrose from } \\
\text { receiving to storage tank }\end{array}$ & $152 \mathrm{scfm}$ \\
\hline EJ-201 & CWO-08 & $\begin{array}{l}\text { Sparge air for sugar digestion } \\
\text { tank }\end{array}$ & $90 \mathrm{scfm}$ \\
\hline V-801 & CWO-04 & Process water for slurrying & $1 \mathrm{gpm}$ \\
\hline WATER: & & $\begin{array}{l}\text { Cooling water for normal } \\
\text { NWCF processes }\end{array}$ & $185 \mathrm{gpm}$ \\
\hline V-401 & CWO-09 & $\begin{array}{l}\text { Cooling water for MACT } \\
\text { reduction chamber effluent }\end{array}$ & $2 \mathrm{gpm}$ \\
\hline V-904 & CWO-09 & $\begin{array}{l}\text { Cooling water for MACT spray } \\
\text { quench }\end{array}$ & $16 \mathrm{gpm}$ \\
\hline V-906 & CWO-01 & $\begin{array}{l}\text { Cooling water for calcine } \\
\text { retrieval system aftercoolers }\end{array}$ & $15 \mathrm{gpm}$ \\
\hline E-101,102 & &
\end{tabular}




\begin{tabular}{l|l|l|l} 
EQUIPMENT ID & $\begin{array}{l}\text { DWG } \\
\text { NO. }\end{array}$ & DESCRIPTION & REQUIREMENT \\
\hline \hline TOTAL & & & $219 \mathrm{gpm}$ \\
\hline
\end{tabular}

\section{ELECTRICAL:}

\begin{tabular}{l|l|l|l}
\hline P-401 & CWO-04 & Slurry pumps & $25 \mathrm{~kW}$ \\
\hline BL-103,104 & CWO-01 & $\begin{array}{l}\text { Blowers for pneumatic transport } \\
\text { of retrieved calcine from binsets }\end{array}$ & $213 \mathrm{~kW}$ \\
\hline BL-701 & CWO-07 & $\begin{array}{l}\text { Blowers for pneumatic transport } \\
\text { of recalcine }\end{array}$ & $27 \mathrm{~kW}$ \\
\hline & & $\begin{array}{l}\text { Normal (existing) NWCF } \\
\text { operations/processes }\end{array}$ & $1000 \mathrm{~kW}$ \\
\hline P-201 & CWO-02 & Sucrose pump & $0.10 \mathrm{~kW}$ \\
\hline BL-905 & CWO-09 & $\begin{array}{l}\text { MACT system oxidizing } \\
\text { chamber air blower }\end{array}$ & $2 \mathrm{~kW}$ \\
\hline BL-906 & CWO-09 & $\begin{array}{l}\text { MACT system dilution air } \\
\text { blower }\end{array}$ & $12 \mathrm{~kW}$ \\
\hline BL-912A,B,C & CWO-09 & MACT draft system blowers & $0 \mathrm{~kW}$ \\
\hline TOTAL & & & $1252 \mathrm{~kW}$ \\
\hline
\end{tabular}

\begin{tabular}{l|l|l|l}
\hline FOSSIL FUELS: & CWO-05 & Kerosene for recalcination & $16.2 \mathrm{gal} / \mathrm{hr}$ \\
\hline VES-NCC-105 & CWO-09 & $\begin{array}{l}\text { Kerosene for NOxidizer } \\
\text { reduction chamber in MACT } \\
\text { system }\end{array}$ & $71.6 \mathrm{gal} / \mathrm{hr}$ \\
\hline V-903 & & & $87.8 \mathrm{gal} / \mathrm{hr}$
\end{tabular}

\section{NWCF MODIFICATIONS}

The NWCF must be modified in order to accommodate the CWO process. Details of the modification are given in Appendices A and B. A summary list of the required modifications is as follows:

1) Additional hot cell space must be added to the east side of building 659. This space is to house: 
- Slurry tanks

- Pumps, valves, piping, and a recycle loop for slurry feed to calciner

- Temporary storage bins for calcine types $A, B$, and $C$ above the slurry tanks

- A booster station to pneumatically transport recalcine from NWCF to the grouting facility located roughly $650 \mathrm{ft}$ distant from NWCF in the northeast corner of the ICPP facility

- Blowers for the calcine transport booster station

- Filters and blowers for the pneumatic calcine retrieval system

- A slurry sampling system

- Equipment for amalgamation of elemental mercury with sulfur

Requirements for the new hot cell space include the following:

- Approximate dimensions should be 52' X 38' X 70';

- The facility should provide separate shielding for each of the above systems to allow separate decontamination and maintenance access without excessive radiation fields;

- A labyrinth should be provided for shielding and contamination control;

- Piping should be provided for steam, cooling water, and sparging air for the slurry tanks;

- Piping should be provided for decon spray systems in all cells;

- Transfer lines (with air lifts) must be provided between the slurry tanks and the tank farm, and between the slurry tanks and the blend and hold cell tanks;

- Vent lines must be added from the slurry tanks and calcine storage bins to the vessel offgas (VOG) system;

- The existing NWCF HVAC system should be modified to provide for heating/cooling in the new cells, and to provide 0.5 "W.C. negative building pressure;

- Provision must be made to vent transport air from the calcine retrieval system into the calciner. This will be accomplished by core drilling a penetration between the new cell housing the temporary calcine storage bins and the existing return jet cubicle, and connecting a vent line between the retrieval system offgas duct and one of the lines from the diverter valve (TA-8) to the calciner. The diverter valve will be removed, and one of the two lines from the valve to the calciner will be permanently plumbed to carry return air from the primary calcine pneumatic transport system to the calciner. The second line from the diverter valve will be permanently plumbed to carry vented air from the calcine retrieval system to the calciner.

- A sump must be provided at the lowest level of the hot cell addition to collect decontamination liquids. This sump must be interconnected with transfer lines between the slurry cell and the tank farm.

Additional detail for the new hot cell space is given in Attachment 1, together with the rationale for building additional space rather than placing the above equipment in existing NWCF hot cells. 
2) A slurry recycle loop must be installed between the slurry feed tanks and the calciner cell, which incorporates the following features:

- A pump to move slurried solid calcine and liquid SBW from the slurry tanks to the calciner cell. The pump must be have the capabilities described for item P-401 in Table 3. Several different pump types are discussed in Attachment 2. A moving cavity ("Moyno") type pump was selected for the baseline design.

- A 1-inch piping loop from the pump to the manifold in the calciner cell (described below) and back to the slurry tanks.

- A circular pipe manifold around the calciner which receives slurry from the recycle loop, distributes slurry to up to three of the four feed nozzles to the calciner, and then returns the overflow to the recycle, which routes it back to the slurry tanks.

Additional detail for the slurry delivery system equipment is provided in Attachment 2.

3) Feed lines to three of the four feed nozzles must be removed and replaced with feed lines connected to the a branching tee on the slurry recycle loop manifold described above. The fourth feed nozzle is maintained in the current configuration to allow feeding from the blend and hold tanks to the calciner (for example during startup).

4) The existing cyclone for the calciner offgas must be removed and replaced with a unit designed for a different operating condition, as described for item CY-501 in Table 3.

5) The existing solids transport system must be rerouted to transport recalcine from the calciner to a booster station within $140 \mathrm{ft}$ of the calciner, instead of to the binsets. At the booster station the calcine will be de-entrained from the transport air of the primary system, and introduced into the transport air of a new, secondary system. The latter system will carry the recalcine from the NWCF to the grouting facility. Equipment needed for the new system includes the following items, which are to be installed at the booster station:

- A cyclone (CY-701) to extract recalcined solids from the primary (existing) pneumatic transport line out of the calciner,

- A calcine storage bin (B-701) to transfer recalcine from the cyclone into the new transport line.

In addition to the above items, the following transport system equipment will be installed in the grouting facility:

- A cyclone (CY-702) at the grouting facility to extract the recalcine and deposit it into the calcine storage tank (T-201 in Dwg DCWO-02 from Ref. 10) in the grouting facility;

- A baghouse (BH-701) to extract fines from recycle transport air, upstream of the blower,

- A transport air blower (BL-701) to provide motive force for moving the transport.

Additional detail for the transport system equipment is provided in Attachment 1.

6) An electrowinning system to extract mercury collected in the scrub solution will be installed, as discussed in Section 3.3.6. The mercury extraction system consists of a set of four to five identical 
electrowinning cells (EC-601), each consisting of a 2-5 gal tank incorporating a platinum electrode of roughly 2-3 $\mathrm{ft}^{2}$ of active surface area. The system also includes a backwashable filter (F-603) and a positive displacement pump (P-601) capable of pumping a slipstream of about $480 \mathrm{gph}$ from the scrub hold tank to the electrowinning cells and back to the scrub tank. The system will be installed in the valve cubicle as desribed in Appendix $\mathrm{K}$ of Ref. 7 (space permitting) or in the new hot cell (item 1, above).

The system will extract elemental mercury from NWCF scrub solution into the mercury pool electrode of each electrowinning cell, and extracted mercury will flow by gravity into a separate temporary storage tank. If tank farm wastes and calcine are delisted, the recovered elemental mercury will be amalgamated with sulfur and disposed as radioactive waste (see item 8 , below). Otherwise, it will be stored indefinitely, pending availability of a suitable mixed waste disposal facility.

7) Additions to the NWCF offgas system, described in Ref. 11, will be provided to comply with MACT requirements. These additions are shown schematically in Dwg CWO-09. While it is anticipated that the use of sugar as a reducing agent in the calciner will reduce $\mathrm{NO}$ and $\mathrm{NO}_{2}$ levels substantially below current levels, and may thus obviate the need for NOx abatement, control of unburned hydrocarbon emissions will still be required. Since the NOx control system which was scoped in Ref. 11 (a John Zink NOxidizer system) is designed to control both NOx and unburned hydrocarbons, and since the cost of this system is believed comparable (for purposes of the current scoping study) to that of a system designed to control hydrocarbons only, the entire MACT system design from Ref. 11 assumed as a basis for the CWO process.

Equipment and modifications required for MACT compliance include the following:

- A NOx and unburned hydrocarbon abatement process, consisting of a John Zink NOxidizer system;

- An air dilution/spray quench system to lower the exit temperature of NWCF offgas leaving the NOxidizer;

- A series of two granulated activated carbon canister filter units to scavenge most of the mercury which remains in the offgas after the scrubbing process;

- A new draft system compressor to handle the increased offgas flows resulting from operation of the NOxidizer;

- A new HEPA filter bank for final filtration prior to discharge of offgas into the ICPP main stack (filtration is currently provided by the APS system; however, increased offgas flows will exceed this system's flow capacities, necessitating the new filter bank);

- A Continuous Emissions Monitoring System (CEMS) to verify MACT compliance for selected pollutants;

- An automatic waste feed cutoff system to stop processing of waste through the calciner when emissions exceed MACT requirements.

8) A system for amalgamation of the mercury collected from the scrub solution will be required. This system is shown schematically in Dwg CWO-10. Subject to assumption 18 in Section 2.2, mercury 
collected in the electrowinning cell will be decanted into 1-gal paint cans (L-1001). These cans will be capped, decontaminated in the NWCF decontamination cell, shielded (if necessary), and transferred to an amalgamation cell in the NWCF hot cell addition, where sufficient elemental sulfur will be added for amalgamation of the mercury. The mixture will be blended in a jar mill (JM-1001) until amalgamation is complete. The paint cans containing amalgamated mercury will be temporarily stored in a storage vault in the amalgamation cell, until they can be disposed as radioactive waste (presumably low-level).

If delisting of NWCF effluents is unsuccessful, or if the mercury collected in the electrowinning cell contains sufficient concentrations of radionuclides to be considered high-level waste (HLW) then it will require indefinite storage (after treatment) until a suitable disposal facility is available.

9) The NWCF hot cell addition will include a slurrying cell to house the temporary calcine storage bins, the cyclones to de-entrain calcine from the pneumatic calcine retrieval system, and the slurrying tanks. In addition, this cell will house sintered metal filters for the retrieval system. A separate cell in the NWCF addition will house HEPA filters and blowers for the retrieval system. This cell will incorporate leaded viewing windows, a PAR manipulator for remote changeout of the filters, and a loading station where spent filters can be loaded into transport casks. The upper portion of the cell will house a decontamination area to prepare loaded casks for transport out of the hot cell to the ICPP filter leach facility.

\section{REQUIRED CHEMICALS SUMMARY}

Chemicals required for the CWO process are summarized below in Table 5:

TABLE 5: REQUIRED CHEMICALS SUMMARY

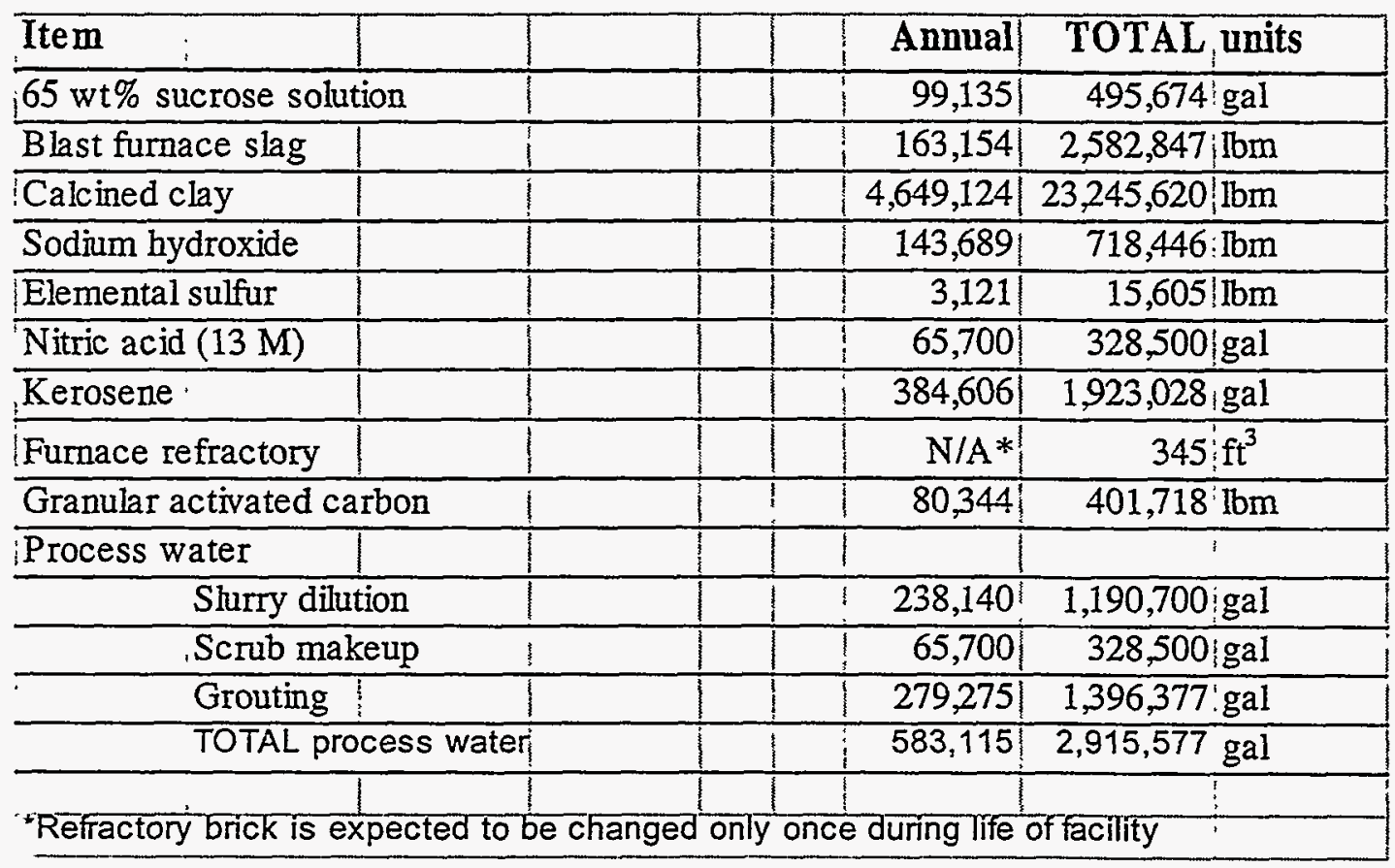




\section{GENERATED WASTE STREAMS}

Secondary waste streams generated by the CWO process (in addition to those generated from grouting of recalcine; see Ref. 10) are summarized below in Table 6:

TABLE 6: SECONDARY WASTES GENERATED BY CWO PROCESS

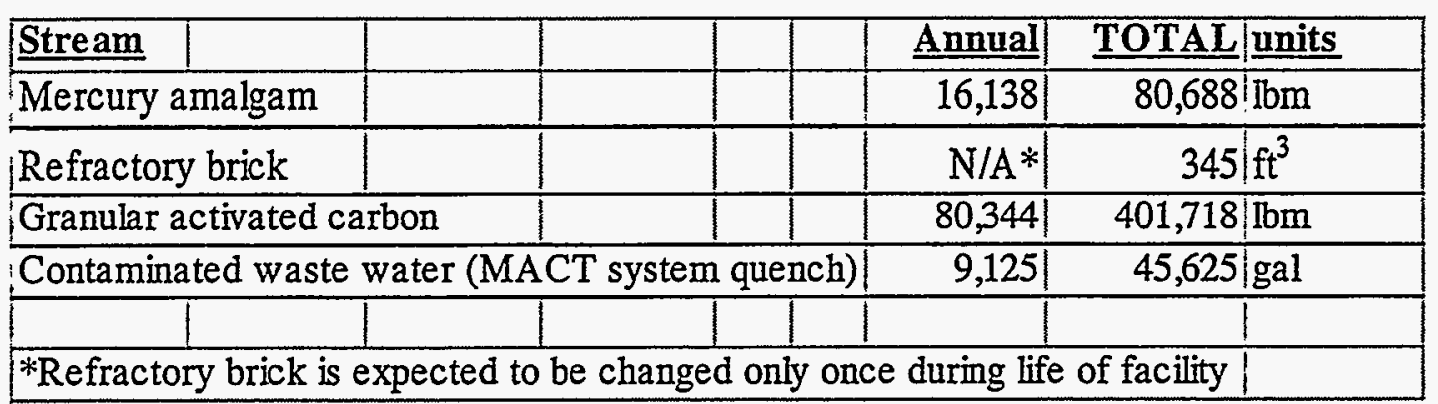

\section{CONCERNS}

The following concerns have been identified relative to the CWO process:

1) The slurry feed system described in item 2 of Section 8 has not been tested in a high radiation environment, and its reliability, robustness, and resistance to plugging have not been demonstrated. It is highly likely that the seals and deformable components of the baselined system (e.g., the elastomeric stator in the slurry pump) may rapidly deteriorate when exposed to the gamma radiation from the calcine. The baselined system should be assembled and tested with surrogate wastes to evaluate its viability, and optimize the design. All polymer/rubber components should be irradiated to determine their useful life, and identify components that are likely to require frequent changeout. In addition other commercially available alternatives (see Attachment 2) should also be tested and evaluated.

2) Transfer of scrub solutions containing organics (i.e., unburned carbon and/or hydrocarbons from sugar calcination) back the tank farm would probably be prohibited by safety oversight personnel (e.g., the Defense Nuclear Facilities Safety Board) unless such solutions were sampled and analyzed prior to transfer, and the organic concentrations shown to be low. Rates of organic digestion in nitric acid appear to be low (half life approximately 1 week, based on information in Ref. 7), and may make digestion infeasible as a means of reducing organic concentrations in the scrub.

3) Calcines from sugar calcination have been generated in pilot scale studies at ICPP, and have been found $^{8}$ to be extremely hygroscopic. Such calcines readily absorb water and aggomerate, causing packing and leading to difficulties in handling. Measures may be required to ensure that exposure of recalcined solids to humid air be limited from the time it leaves the calciner until the time it is grouted. 
$431.02 \#$

$2 / 11 / 98$

Rev. \#01
ENGINEERING DESIGN FILE
Function File Number - SPR-02

EDF Serial Number - EDF-CWO-003

4) The process design described herein assumes the following: (a) all nitrates in solid calcine will readily dissolve in liquid SBW and water used for slurrying, (b) the only composition variable of the slurry mixture that will be required to adjust calcination process parameters is the dissolved nitrate composition, (c) the only calcination process parameter that will require adjustment for each slurry tank is the rate of sucrose injection, and (d) nitrate concentrations in the liquid portion of the slurry mixture can readily be determined within less than $24 \mathrm{hrs}$ of slurry blending. turnaround) of slurry mixtures can be done to determine their nitrate content prior to recalcination. These assumptions should be verified by testing and development.

5) An inline mixer (to blend sucrose solution with the liquid in the slurry) in the slurry stream may cause plugging problems due to the high solids content. Whether or not this is the case should be determined, and alternative mixing method developed if necessary.

6) The baseline grouting process for the CWO assumes that sizing of the calcine and the required additives will not be required. In addition, it assumes that all calcines can be accommodated by suitable adjustments of the additive proportions, and that a reasonable number of grouting recipes will accommodate the entire range of variation of calcine composition. Finally, the baseline process makes very conservative assumptions about the curing conditions (e.g., elevated temperature in autoclaves) that will be required to make the desired hydroceramic waste form. These assumptions should be verified with bench scale studies of grouting recipes using nonradioactive pilot plant calcines stored at ICPP. These studies would generate a credible set of reaction conditions required to produce acceptable waste forms from all calcines in the ICPP inventory.

\section{REFERENCES}

1. EDF-WTS-003 in the project file ("Requirements for the Design, Construction, and Operations of the ICPP Proposed Waste Processing Facilities").

2. J. C. Petrie, "Report on Run 12, Twelve-Inch Diameter Calciner", letter Petr-13-65A to E.J. Bailey, December 30, 1965 (INEL report).

3. VECTRA GSI Report No. WHC-VIT-03, "Fluid Bed Calciner Test Report - Final", -WHC-SD-WM-VI-031, August 1995 (Hanford report).

4. L. R. Dole, et al, "Cement-Based Radioactive Waste Hosts formed Under Elevated Temperatures and Pressures (FUETAP Concretes) for Savannah River Plant High-Level Defense Waste, ORNL/TM-8579, March 1983 (ORNL report).

5. D. D. Siemer, B. E. Scheetz, and M. L. D. Gougar, "Hot Isostatic Press (HIP) Vitrification of Radwaste Concretes", Materials Res. Soc. Symp. Vol 412, 1996, pp. 403-410, (Proceedings of 1995 MRL Symposium on 'Scientific Basis for Nuclear Waste Management XIX', Boston, MA, Nov. 29 - Dec 3, 1995).

6. D. D. Siemer, "Hot Isostatically Pressed Concrete as a Radwaste Form", Advances in Ceramics, Vol 61, Ceramics Transactions, pp. 657-664, (Proceedings of 1995 American Ceramics Society Symposium on Waste Management, Cincinnati, OH, April 29 - May 4,1995). 
7. Henry Welland, "NWCF Process Modification for Sodium-Bearing Waste Project Conceptual Design", INEL/INT-97-00075, April 1997.

8. R. D. Boardman, Interdepartment Communication to D. V. Croson, Subject: Transmittal of Status Report on Alternative Calcination Development Accomplishments and Results, April 30, 1997.

9. EDF-WTS-002 in the project file ("Calcine Retrieval and Transportation").

10. "Direct Cementitious Waste Option Scoping Study", INEEL/EXT-97-01399, December 1997.

11. R. D. Adams, et al, "The $90 \%$ Draft of the Feasibility Study Report for MACT Compliance Facility", INEL/INT-97-00992, August 1997. 


\section{Attachment 1}




\section{Attachment 1 \\ Waste Treatment Facilities - Non-Separations Options: Cementitious Waste Option (CWO) Scoping Study}

Modifications/Additions to NWCF for Calcination of Slurry Feeds

H. S. Forsythe

\section{Purpose}

This scoping study covered the following design tasks.

- Determine the calcine transport needs.

- Determine the best location for the calcine storage bins and slurry tanks.

- Investigate the possibility of using an existing NWCF vessel as a sugar digester.

\section{Calcine Transport System}

The existing NWCF transport system is limited to transporting solids $\sim 160$ feet ( 320 feet for a closed loop) at a maximum transfer rate of $300 \mathrm{lbs} / \mathrm{hr}$. The solids loading ratio is maintained between 0.8 and $1.0 \mathrm{lbm}$ solids $/ \mathrm{lbm}$ air. Erosion is kept to a minimum by restricting transport air velocity to $<80 \mathrm{ft} / \mathrm{sec}$.

The new grouting facility will be located in the Northeast corner of CPP. This location is 650 feet from the New Waste Calcining Facility (NWCF). In addition, the grouting facility will be 200 feet tall and the transport system must be capable of delivering solids to the top of the facility. If the transport system is a closed loop, there will be in excess of 1,700 feet of transport piping.

The current NWCF transport system cannot deliver solids to the grouting facility for the following reasons.

- The estimated CWO calcine flowrate is $674 \mathrm{lb} / \mathrm{hr}$. The current system is limited to $300 \mathrm{lb} / \mathrm{hr}$.

- The estimated transport distance to the grouting facility is $>1,700$ feet. The current system is limited to 320 feet.

- The current system is a vacuum system and is, therefore, pressure drop limited.

The optimum transport system will have two independent pneumatic systems coupled together by a booster station (See Figure-2). The first leg will be a vacuum system moving calcine from the calciner to the booster station. The second leg will be a pressure system delivering calcine to the grouting facility. 


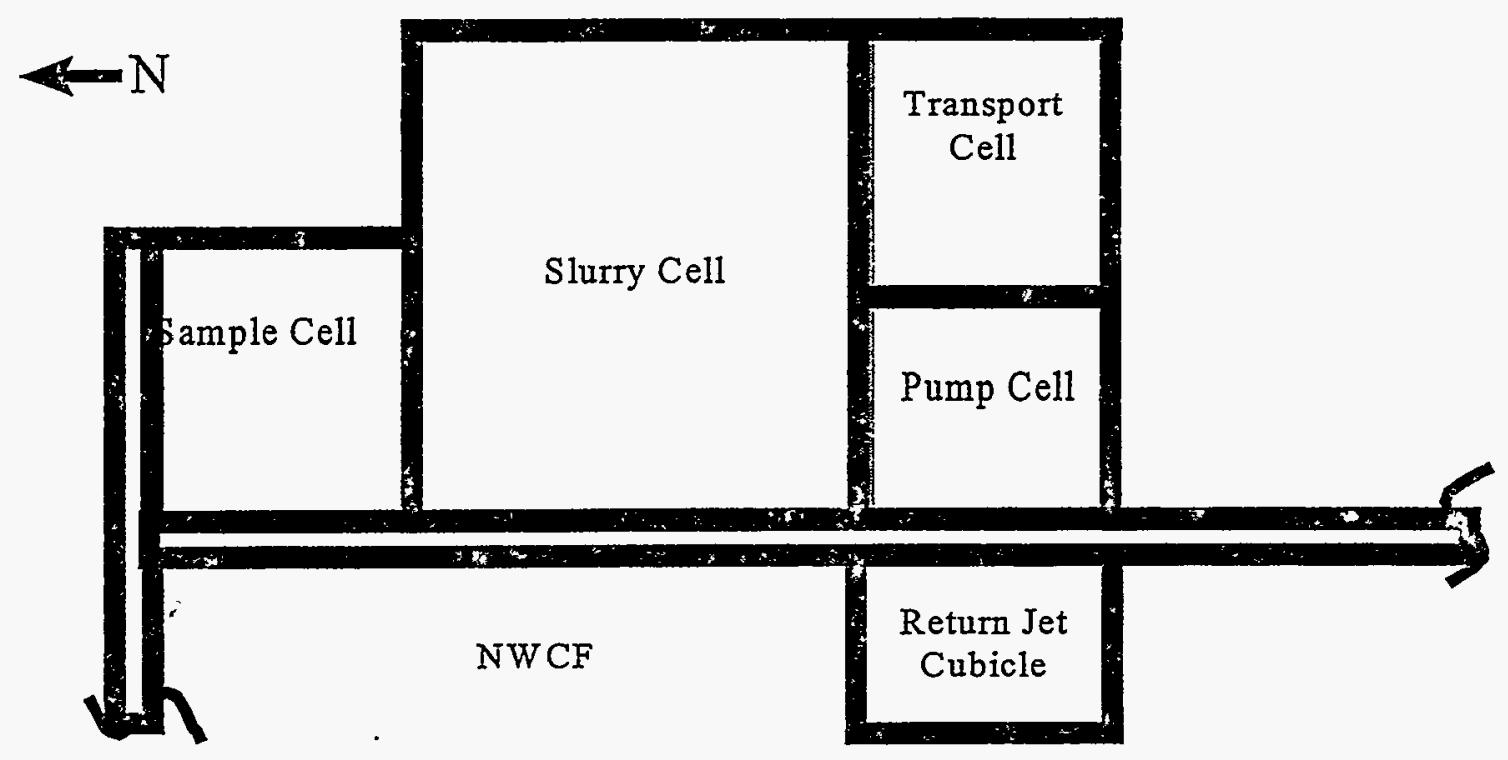

Figue-1

New Hot Cell Layout

If the booster station can be located adjacent to the NWCF, the transport distance from the calciner to the booster station will be $<50$ feet. At $<50$ feet, the current TA system pressure drop would be cut up to $50 \%$. Reducing the TA system pressure drop would allow the system capacity to nearly double. As a result, the current NWCF TA system could be used to move calcine to the booster station. Therefore, the assumption is made that the current NWCF TA system can adequately transport solids at the design flowrate of $674 \mathrm{lbm} / \mathrm{hr}$.

The second leg of the transport system will have equipment located in both the booster cell as well as the grouting facility. The following equipment is needed.

- One cyclone

One surge hopper, $\sim 550 \mathrm{ft}^{3}$; incoming flow $\sim 7.2 \mathrm{ft}^{3} / \mathrm{hr}$ ( $\sim 3$ days operation)

- Two transport air compressors

- Mechanism for feeding calcine into the TA system

- One cyclone at the grouting facility

- One bag filter at the grouting facility

$\therefore \sim 1,750$ feet of 4 " pipe

The bag filter is needed to remove fines that would otherwise buildup in the system. Because the second leg is a pressure system, it can move solids to the grouting facility without the aid of additional booster stations. Additional characteristics of the grouting facility TA system are provided below.

- Closed loop system

$\therefore$ Total pipe length $>1,750$ feet

- Minimum transport velocity of $40 \mathrm{ft} / \mathrm{sec}$ 
- 4" I.D. transport pipe

- Solids loading ratio of $\sim 0.5 \mathrm{lb}$ solids $/ \mathrm{lb}$ air.

- Average transport capacity of $674 \mathrm{lb} / \mathrm{hr}$.

- Compressor discharge pressure of $\sim 25$ psig.

- Air flow of $280 \mathrm{scfm}$.

Average system DP of 15 psi.

The table below contains pressure drop estimates for the grouting facility transport system.

\begin{tabular}{rccc}
\hline Piping Section $\left({ }^{*}\right)$ & $\begin{array}{c}\text { Piping } \\
\text { Section }\end{array}$ & $\begin{array}{c}\text { Pressure } \\
\text { (psi) }\end{array}$ & $\begin{array}{c}\text { Velocity } \\
\text { (ft/sec) }\end{array}$ \\
\hline Compressor Discharge & P1 & 25.0 & 35.4 \\
Exiting Booster Cell & P2 & 24.6 & 36.1 \\
Bottom of Grouting Facility & P3 & 19.9 & 44.5 \\
Inlet to Grouting Facility Cyclone & P4 & 19.3 & 45.8 \\
Return, bottom of Grouting Facility & P5 & 14.9 & 59.3 \\
Compressor Suction & P6 & 10.0 & 88.2 \\
& & & \\
Solids/Air Raito & 0.5 & & \\
Solids (lb/hr) & 674.0 & & \\
Air (lb/hr) & $1,348.0$ & & \\
Pipe I.D. (inches) & 4 & & \\
Compressor DP & 15.0 & & \\
SCFM & 277.9 & & \\
$\mathrm{hp}$ & 18.5 & \\
$\mathrm{~kW}$ & 24.8 & \\
\hline
\end{tabular}

* See Figure-2

\section{Location of Calcine Storage Bins and Slurry Tanks}

The only option for placing the slurry tanks inside the existing NWCF building is the blend and hold cell. This cell currently contains one 5,000 gallon hold tank, two 4,000 gallon mix tanks, one 100 gallon feed head tank, and one 2,000 gallon High Level Liquid Waste Evaporator (HLLWE). This cell is not a viable option for the following reasons.

1. The cell does not provide sufficient height in order to place the calcine storage bins in the cell in a configuration that would allow gravity flow of solids to the slurry tanks.

2. The current tanks would have to be removed. The high cost and excessive radiation exposure to complete this task were deemed unacceptable.

3. The existing feed system will be needed during startup, shutdown and upset conditions. Therefore, a third of the tanks and equipment must remain in the cell. 
If the slurry tanks cannot be placed inside the NWCF, then the best option is to build a new hot cell adjacent to the NWCF. The ideal location for this new hot cell is along the East Side of the.NWCF. This location is close to the calcine storage facilities and close to the calciner cell. Therefore, this new hot cell should be placed against, and have in common, the east wall of CPP-659. The new hot cell will have four separate cells; slurry cell, pump cell, sampling cell and a transport booster cell (See Figure-1).

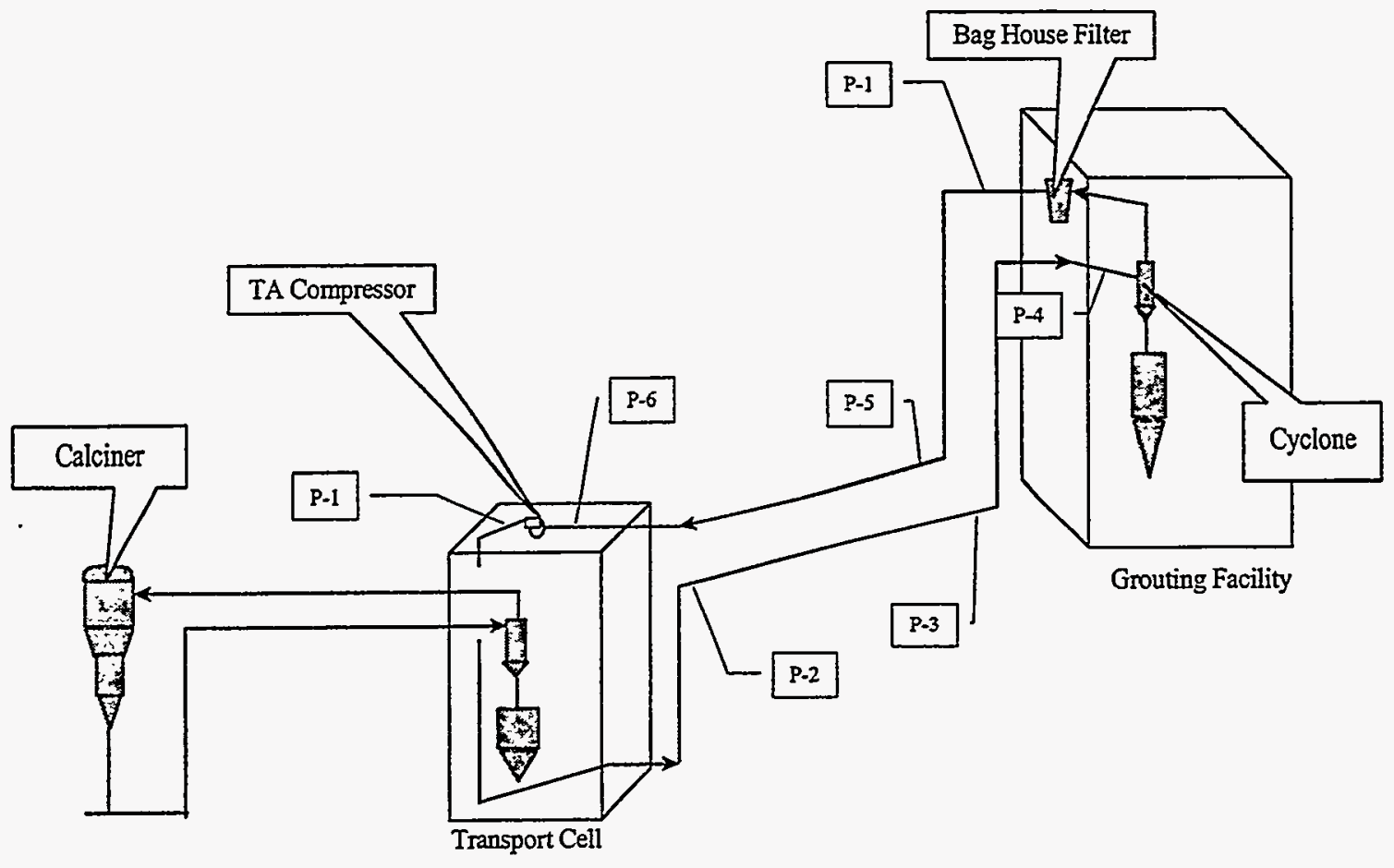

Figure-2

CWO Calcine Transport System

\section{Slurry Cell}

The slurry cell will have two cyclones, three calcine storage bins, and two slurry tanks. The cell design should allow for gravity flow of solids from the retrieval system TA cyclones through the storage bins and into the slurry tanks. The cell dimensions are estimated to be $30^{\prime} \times 30^{\prime} \times 64^{\prime}$. 64 feet is needed to accommodate equipment arrangement to allow for the desired gravity flow of solids. The cell should have a labyrinth for shielding and contamination control.

The slurry cell will require the following utilities and capabilities.

- Utilities
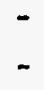

Steam for heating the slurry tanks

Water for diluting the slurry

Air for sparging the slurry tanks 
- $\quad$ Instrument Air

- $\quad$ Decon solution for deconing the slurry tanks

- Drains: the cell needs a drain system connected to the building sump tanks, NCC-119/122.

- Transfer lines: transfer lines from the tankfarm and to the blend and hold cell must be provided.

- HVAC: the cell needs a HVAC system capable heating/cooling and providing 0.5 " $\mathrm{H} 2 \mathrm{O}$ vacuum.

- Equipment vent: the slurry tanks and calcine storage bins need to be connected to the NWCF vessel offgas (VOG) system.

- Electrical: the cell needs power lighting.

- Access: personnel access from the NWCF $3^{\text {rd }}$ level is needed to the pump cell, sample cell, and slurry cell. In addition, crane access is needed to the equipment and pump cells through the roof.

- Platforms and ladder access is needed to all equipment in the cell.

- All piping and equipment in the pump and slurry cell must be stainless steel.

- A stainless steel floor liner is required. This liner should come up the wall four feet.

The slurry cell will contain the following equipment.

- $\quad$ CY-401 and CY-402 (Cvclones)

$800 \mathrm{scfm}$ gas flow (assume $1.5 \mathrm{lbm}$ solids/1 lbm air)

$230 \mathrm{ft} / \mathrm{sec}$ (assume a 4" TA pipe)

$100 \mathrm{lbm} / \mathrm{min}$ solids flow (assume calcine retrieval will provide $2,700 \mathrm{~kg} / \mathrm{hr}$ )

Material: $\quad 316 L$ Stainless Steel

The type $\mathrm{B}$ calcine bin ( $\mathrm{Zr} / \mathrm{Zr}-\mathrm{Na} /$ fluorinel) will have a dedicated calcine retrieval transfer line and cyclone. The type A calcine bin (aluminum) and the type $\mathrm{C}$ calcine bin $(\mathrm{H}-4 / \mathrm{H}-5)$ will share a transfer line and cyclone. In addition, the shared cyclone will need a diverter valve to direct the calcine to the appropriate calcine bin.

Each cyclone will need the following instrumentation.
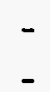

$-$

$-$

$-$

$-$
Transport Air (TA) pressure in

TA pressure out

DP across the cyclone

Cyclone temperature

Flow and velocity of $T A$ return

- B-101/102/103 (Calcine Storage Bins)

$\begin{array}{ll}\text { Height } & 16.5^{\prime} \\ \text { Diameter } & 8.0^{\circ} \\ \text { Capacity } & 550 \mathrm{ft}^{3}\end{array}$


The calcine bins are assumed to have a cone angle of $65^{\circ}$. Each bin will have a rotary valve and a load cell to allow for metering out a specific mass of calcine. Following the rotary valve, each bin transfer line will have a diverter valve. The diverter valve will direct the calcine to the selected slurry vessel. The calcine transfer lines are assumed to require an angle of $65^{\circ}$ to prevent plugging during transfers.

Each bin will need the following instrumentation.

$\begin{array}{ll}- & \text { Bin weight (determined by a load cell) } \\ - & \text { Variable speed rotary valve } \\ - & \text { Bin pressure } \\ - & \text { Solids level }\end{array}$

\begin{tabular}{ll} 
- V-401A.B (Slurry Vessels) \\
\hline Height & $16.0 \mathrm{ft}$ \\
Diameter & $8.0 \mathrm{ft}$ \\
Capacity & 4,000 gallons \\
Material & $316 \mathrm{~L}$ Stainless Steel
\end{tabular}

Slurry vessels need the capability of having water or decon solution added. The vessels must have a method of being mixed (ex: air sparger, recirculation line). Each vessel needs a sampler capable of handling slurry with up to $50 \mathrm{wt} \%$ UDS. The vessels also must have internal heating/cooling coils. The capability must exist to transfer the slurry tanks to each other, NCC-102/103 and NCC-119 (high activity sump tank). If a slurry batch must be disposed of, it can be transferred to NCC-102/103 and fully digested with nitric acid prior to being sent to the tank farm.

Each vessel will need the following instrumentation.

$\begin{array}{ll}- & \text { Level } \\ - & \text { Volume } \\ - & \text { Density } \\ - & \text { Slurry temperature } \\ - & \text { Pressure }\end{array}$

\section{Pump Cell}

The pump cell will be located directly south of the slurry cell and adjacent to the NWCF transport air return cubicle. The cell dimensions are estimated to be 12 ' X 14' $\mathrm{X} 64^{\prime}$. The cell will contain three slurry feed pumps, slurry piping, slurry instrumentation, and valves. 
The slurry line to the calciner cell will exit the pump cell and enter the NWCF building in the return jet cubicle. The return jet cubicle is a radiologically controlled area and will provide shielded access to the NWCF. From the cubicle, the slurry line will pass through an abandoned 18" shielded encasement to the calciner cell. This layout provides the shortest distance to the calciner with the least expense.

The pump cell will need the following utilities and capabilities.

- Utilities

$$
\text { - Instrument Air }
$$

- Drains: the cell needs a drain system connected to the building sump tanks, NCC-119/122.

- HVAC: the cell needs a HVAC system capable heating/cooling and providing $0.5^{\prime \prime} \mathrm{H} 2 \mathrm{O}$ vacuum.

- Electrical: the cell needs power for the pumps and for lighting.

- Access: personnel access is needed from the slurry cell labyrinth In addition, crane access is needed through the roof.

- Platforms and ladder access is needed to all equipment in the cell.

- All piping and equipment in the cell must be stainless steel.

- A stainless steel floor liner is required. This liner should come up the wall four feet.

The pump cell will contain the following equipment.

- P-401A.B.C (Slurry Feed Pumps)

$\begin{array}{ll}\text { Type } & \text { progressive cavity } \\ \text { Head } & 200.0 \mathrm{ft} \\ \text { Motor } & 20 \mathrm{hp} \\ \text { Capacity } & 100 \mathrm{gpm} \\ \text { Material } & 3 X X \text { Stainless Steel } \\ \text { Power } & \sim 30 \mathrm{hp}\end{array}$

Each pump will need the following instrumentation.

$\begin{array}{ll}- & \text { Amps } \\ - & \text { Mass flow }(\mathrm{lb} / \mathrm{hr}) \\ - & \text { Density } \\ - & \text { Temperature } \\ - & \text { Pressure }\end{array}$

\section{$\underline{\text { Sample Cell }}$}

The sample cell will be located directly to the north of the slurry cell. The estimated cell dimensions are14' X 14'. 
The sample system should be a remote operation, similar to the existing NWCF sample cell, in order to limit radiation exposure to operators. (shielding windows, master slaves, remote valves, etc.). In addition, the sampling system should have access to the Remote Analytical Laboratory (RAL) via the pneumatic sample transfer system.

The sample cell will require the following utilities and capabilities.

- Utilities

$\begin{array}{ll}- & \text { Steam } \\ - & \text { Water } \\ - & \text { Air } \\ - & \text { Instrument Air } \\ - & \text { Decon solution }\end{array}$

- Drains: the cell needs a drain system connected to the building sump tanks, NCC-119/122.

- Transfer lines: transfer lines to and from the slurry tanks.

- HVAC: the cell needs a HVAC system capable heating/cooling and providing 0.5 " $\mathrm{H} 2 \mathrm{O}$ vacuum.

- Electrical: the cell needs power for lighting and for masterslaves.

- Access: personnel access from the NWCF $3^{\text {rd }}$ level is needed.

- All piping and equipment in contact with the samples must be stainless steel.

The sample cell will have the following equipment.

- Slurry Vessel Sampling System

The sampling system must be capable of sampling $50 \mathrm{wt} \%$ UDS slurry from either slurry vessel.

\section{Transport Air Booster Cell}

The transport air booster cell will be located directly east of the pump cell and south of the slurry cell. The cell will have two rooms, one stacked on top of the other. The estimated dimensions for the bottom cell are $12^{\prime} \mathrm{X} 14^{\prime} \mathrm{X} 36^{\prime}$. The estimated dimensions for the upper room are $12^{\prime} \times 14^{\prime} \times 17^{\prime}$.

The NWCF transport line exits the building through the return jet cubicle. Upon leaving the building, the TA line will pass through the pump cell and into the bottom room of the booster cell. The bottom room will contain the TA cyclone and calcine storage bin. The cyclone will be positioned directly above the calcine storage bin, which will allow for gravity flow of the solids. The upper room will be isolated and shielded from the lower room and will contain the TA compressors for the grouting facility TA system. 
The transport air booster cell will require the following utilities and capabilities.

- Utilities

$\begin{array}{ll}- & \text { Steam } \\ - & \text { Water } \\ - & \text { Air } \\ - & \text { Instrument Air } \\ \text { - } & \text { Decon solution }\end{array}$

- Drains: the cell needs a drain system connected to the building sump tanks, NCC-119/122.

- HVAC: the cell needs a HVAC system capable heating/cooling and providing 0.5 " $\mathrm{H} 2 \mathrm{O}$ vacuum.

- Equipment vent: the calcine storage bin should be connected to the NWCF vent system.

- Electrical: the cell needs power for lighting and for the transport air compressors.

- Access: personnel access from the pump cell is needed. In addition, crane access is needed to the storage bin and the transport air compressors through the roof.

- Platforms and ladder access is needed to all equipment in the cell.

- All piping and equipment in the cell must be stainless steel.

- A stainless steel floor liner is required. This liner should come up the wall four feet.

The transport air booster cell will have the following equipment.

- CY-701(Cyclone)

$170 \mathrm{scfm}$ gas flow (assume $0.8 \mathrm{lbm}$ solids/1 $\mathrm{lbm}$ air)

$50 \mathrm{ft} / \mathrm{sec}$ (assume a 3" TA pipe)

$11.2 \mathrm{lbm} / \mathrm{min}$ solids flow (assume calcine production at $674 \mathrm{lbm} / \mathrm{hr}$ )

Material 3XX Stainless Steel

The cyclone will need the following instrumentation.

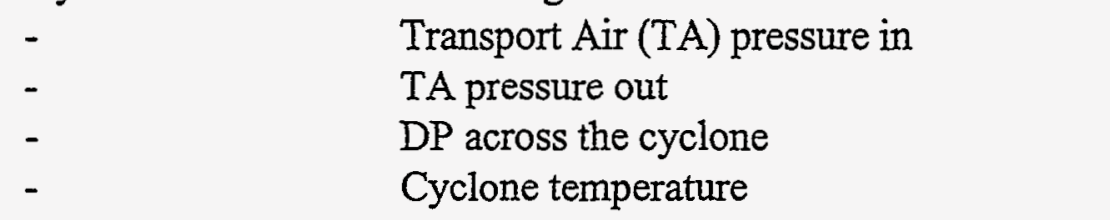

- B-701 (Calcine Storage Bin)

$\begin{array}{ll}\text { Height } & 16.5^{\prime} \\ \text { Diameter } & 8.0^{\prime} \\ \text { Capacity } & 550 \mathrm{ft}^{3}\end{array}$




\section{Material}

3XX Stainless Steel

The bin is assumed to have a cone angle of $65^{\circ}$. The bin will have a rotary valve designed for metering out a specific volume of calcine.

The bin will need the following instrumentation.

$\begin{array}{ll}- & \text { Bin weight (determined by a load cell) } \\ - & \text { Variable speed rotary valve } \\ - & \text { Bin pressure } \\ - & \text { Solids level }\end{array}$

- BL-701A/B (Transport Air Blower)

$\begin{array}{ll}\text { Type } & \text { positive displacement } \\ \text { Head } & \text { XXX ft (25 psi) } \\ \text { Capacity } & 280 \mathrm{scfm} \\ \text { Motor } & 20 \mathrm{hp} \\ \mathrm{kW} & 25 \\ \text { Material } & \text { Stainless steel }\end{array}$

Each blower will need the following instrumentation.

$\begin{array}{ll}- & \text { Amps } \\ - & \text { Pressure } \\ - & \text { Temperature } \\ - & \text { Flow (scfm) }\end{array}$

\section{Sugar Digester Vessel}

The off-gas quench solution tank, NCC-108, has an operating range of 750 to 3,750 gallons. A typical deep recycle can range from 500 to 1,500 gallons and contain between 2500 and $5000 \mathrm{ppm}$ chloride. Therefore, the digester must be capable of processing 1,500 gallons of waste with up to $5,000 \mathrm{ppm}$ chloride. Five existing vessels could be used as a sugar digester, NCC-102, NCC-103 NCC-119, NCC-122 and NCC-150.

NCC-102 and 103 have operating volumes of 3,200 gallons each. The tanks are constructed of $3 \mathrm{XX}$ stainless steel. Each tank has internal heating and cooling coils. These tanks will no longer be the primary feed blend tanks; therefore, one could be used as a sugar digester.

NCC-119 has an operating capacity of 4,500 gallons and is constructed of $3 X X$ stainless steel. This vessel has internal heating and cooling coils. This vessel is designated for fluoride bearing high activity waste destined for the tank farm. Presently, deep recycled solution is sent to NCC-119 before being sent to the tank 
farm. This vessel could be used as a sugar digester with the understanding that corrosion may be a problem.

NCC-122 has an operating capacity of 3,800 gallons and is constructed of $3 X X$ stainless steel. This vessel also has internal heating and cooling coils. However, NCC-122 cannot be used as a sugar digester. This tank is designated for non-fluoride low activity waste destined for the PEW evaporator and should be kept as such.

NCC-150, the HLW Evaporator, has an operating capacity of 2,000 gallons and is constructed of Hastalloy G-30. The evaporator is a thermo-siphon design. As a result, the liquid must be brought to boiling in the reboiler before the siphon system will work. The overheads are condensed and collected in NCC-122. The concentrate is allowed to cool before being transferred to the tank farm. The HLWE is probably the best option providing operations can adequately control the temperature in the evaporator. 


\section{Attachment 2}




\title{
Attachment 2 \\ Waste Treatment Facilities - Non-Separations Options: Cementitious Waste Option (CWO) Scoping Study
}

\author{
Selection of Delivery System for Slurry to Calciner - Design Summary
}

David N. Thompson

\section{Purpose}

The purpose of this task was to investigate ways to deliver calcines slurried in sodium bearing waste (SBW) to the calciner at the new waste calcining facility (NWCF). This task was a part of the overall Cementitious Waste Design Option (CWO) design task, which was concerned with extracting the nitrates from the calcines presently stored in the bin sets at ICPP, and recalcining the wastes with the addition of SBW.

\section{Objectives}

The objectives of this task were:

1. Identify candidate pumping system for delivering slurried calcines to the calciner

2. Roughly specify piping, pressure drops, valves, tees, bends, etc.

3. Provide method for mixing sucrose with the slurried solids before introduction to the calciner

4. Suggest control method for pump operation and slurry addition to the calciner

\section{Results}

Task 1: Choice of pump -- Pump choice for this application depends on a number of important factors. The high radiation environment necessitates a reliable pump which will operate uninterrupted for a long period of time. The density $\left(2.7 \mathrm{~g} / \mathrm{cm}^{3}\right)$ and size $(0.3 \mathrm{~mm}$ diameter $)$ of the particles make potential settling of the particles in the feed lines or in the nozzles a real possibility. The concentration of solids in the slurry is 41 to $43 \mathrm{wt} \%$, which limits pump choices mainly to positive displacement pumps rather than centrifugal systems. The calcines are generally very hard particles, which makes it necessary to have an abrasion-resistant pump. Finally, ease of maintenance (and less need for maintenance) is important.

After speaking with several vendors and consultants in the areas of fluidized bed coal combustion and sludge disposal, three possible pumps were selected: (1) Horizontal diaphragm pump (HDP pump, Toyo Pumps North America, Inc., Bumaby, British Columbia, Canada); (2) Moving cavity displacement pump (MCD pump, Monoflo, Houston, TX); and (3)

Sludge/Cement pump (SCP pump, Schwing America, Inc., Danbury, CT). There are pros and cons for each pump. Scaleup is easiest for the MCD pump, since it can be scaled up simply by adding stages. All three are relatively abrasion resistant. The HDP pump is probably the most resistant because the slurry contacts a polymer tube rather than hard steel alloys, although the stability of the covalent bonds in a high $\gamma$-radiation environment could potentially limit use of the HDP pump for this application. The HDP pump's footprint is generally smaller than the other 
pumps, although it is generally wider than the other two options (for our application, a rectangular area about $6 \mathrm{ft}$ by $6 \mathrm{ft}$ by $7 \mathrm{ft}$ (high)). The MCD pump is a little more narrow, but it occupies a length of about $15 \mathrm{ft}$. The SCP pump is generally larger than the other pumps because of its hydraulic cylinders. All three pumps can easily cover the pressure range required (a consultant from the coal processing industry indicated that $100 \mathrm{psig}$ should be enough pressure to force the slurry through an 0.5 in opening). None of the choices could be expected to keep the solids in slurry at the low flow rates needed into the calciner (total of $97 \mathrm{gal} / \mathrm{hr}$ or $546 \mathrm{lb} / \mathrm{hr}$ of solids plus $73 \mathrm{gal} / \mathrm{hr}$ of SBW). Thus, a high velocity slurry recirculation loop was added to keep the solids in slurry. The velocity in this line was chosen to be $10 \mathrm{ft} / \mathrm{s}$, which is a conservative order of magnitude larger than the minimum fluidization velocity for silica sand. The $97 \mathrm{gal} / \mathrm{hr}$ of slurry fed to the calciner would then be drawn from this line, mixed with the sucrose solution, and injected into the calciner. The manner in which the slurry is drawn into the nozzle lines would need to ensure that the slurry concentration remains constant, necessitating a novel tee in the line. The preferred tee would look essentially like a Pitot tube, with a cross section of the flow through the recirculation loop sufficient to represent the calciner feed (divided by three) exiting into the nozzle line. A diagram of the slurry supply system with recirculation loop and injection lines is shown in Figure 1, with a description of the system. Specifications are given in the figure caption for convenience.

Task 2: Piping. valves, and fittings -- Because of space limitations, the pumps would need to be located at a distance from the calciner, and $22 \mathrm{ft}$ below the existing shielded conduit for piping into the calciner cell. This vertical section could present problems with the SCP system, since the flow would be pulsed and thus potentially allow solids to settle in this section. A coal processing group at the University of North Dakota who have been using a recirculation system similar to our design to feed a coal slurry to a fluidized coal combustion bed communicated to us that the HDP pumps were generally more reliable and required less maintenance than the MCD pumps. Thus, the HDP pump was initially chosen. However, previous operator experience in pumping high rad liquids at ICPP indicated that polymer pump seals do not generally last more than a week or so. Because of this, the HDP pump was excluded and the MCD pump became more preferred because it uses fewer seals. However, the operating lifetime of the pump seals will potentially be a limiting issue in this design option, regardless of the type of pump chosen. The pipe section lengths, fittings, valves, flowmeters, etc. are listed in Table 1 . The $\mathrm{P}_{i}$ locations listed refer to the labels in Figure 1. No nozzle data are included, since it was assumed that a nozzle could be designed which would give the proper pressure drop. Thus, valves (2), mass flowmeters (3), pressure taps (6), and about 20-30 ft of pipe are not included in Table 1.

Task 3: Addition of sucrose to the calciner feed lines - It is important that the sucrose be well mixcd with the calcine/SBW slurry before introduction to the calciner; otherwise, complete reaction of the extracted nitrates will not occur. Originally a mixing tank was planned, and this still is a good option. However, this may require an additional pump. Another potentially viable option is to introduce the sucrose into the nozzle lines using a static mixer.

Task 4: Control options for the pumping system - The control system envisioned for this system is a feedback loop controlling the pump motor based on downstream pressures and flowrates. The pump cannot be allowed to run dry, so a cutoff switch should be installed in case of a drop 
of mass flow rate in the recirculation loop to near zero. Likewise, a large increase in pressure at any of the pressure taps between $P_{2}$ and $P_{3}$ or between $P_{4}$ and $P_{5}$ could indicate a blockage, which would signal the pump to shut down. The valves in the nozzle tube entrances would be used, with the mass flowmeters just upstream, to control the mass flowrate of slurry in each nozzle line. The pressure sensors at the flowmeters and upstream of the sucrose mixing point would signal blockages of the nozzle line and nozzles and close the feed valve for that line, as well as provide data for controller estimation of the sucrose addition rate. The valve at the sucrose pump would be tied to the recirculation loop flowmeter and to the nozzle tube flowmeters such that no sucrose was added if some or all of those flows were zero. The remaining valves would be remotely operated. The valves on either side of the pump would be used to isolate the pump for changing of its diaphragm tube and check valves. 


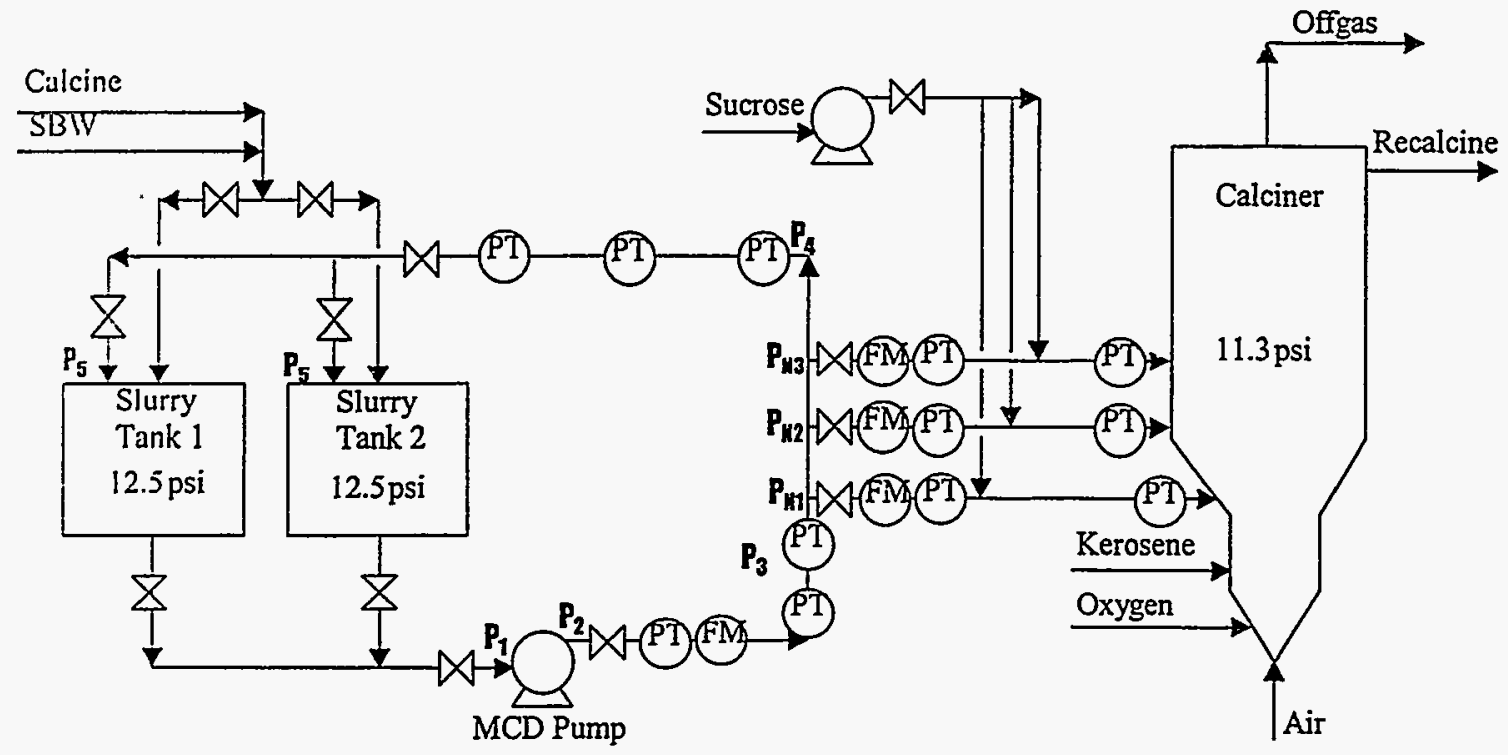

Figure 1: Recirculation loop and nozzle system for slurry addition to the calciner, indicating locations of valves, magnetic mass flowmeters (FM), and pressure taps (PT). The pressure taps between $\mathrm{P}_{2}$ and $\mathrm{P}_{3}$ and between $\mathrm{P}_{4}$ and $\mathrm{P}_{5}$ are used to indicate blockages in the lines and are placed at each point where the line passes through a wall or ceiling. Pressures estimated in Table 1 below are indicated in bolded letters $\left(\mathrm{P}_{i}\right)$ at their locations. The slurry is withdrawn from the tanks at the bottom, and the recycle line is designed to enter the tanks at the top, although introduction at the bottom could assist in mixing the slurry. Mixing in the slurry tanks will be by air sparging. A sampling line is to be teed into the recycle line before the tanks. The MCD pump will need to deliver a $\Delta \mathrm{P}$ of at least $200 \mathrm{psi}$, and a volumetric flowrate of $1.63 \mathrm{gal} / \mathrm{s}$. The pump motor should be at least $30 \mathrm{hp}$.

Table 1: Pressure drop calculations for the recirculation loop with a pipe diameter of 2 in I.D. These calculations do not include any piping or flows into the tees to the nozzle lines. The slurry vclocity was assumed to be $10 \mathrm{ft} / \mathrm{s}$. A conservative total length of pipe needed for this section of the process (including parallel pump lines and nozzle lines) is $250 \mathrm{ft}$.

\begin{tabular}{|c|c|c|c|c|c|c|c|}
\hline $\begin{array}{l}\text { Piping } \\
\text { Section } \\
\text { (2 in ID) }\end{array}$ & $\begin{array}{l}\text { Pressure at } \\
\text { terminus } \\
\text { (psi) }\end{array}$ & $\begin{array}{c}\text { Length of } \\
\text { Pipe } \\
\text { (ft) }\end{array}$ & $\begin{array}{c}30^{\circ}-90^{\circ} \\
\text { Elbows }\end{array}$ & Tees & $\begin{array}{c}\text { Ball } \\
\text { Valves }\end{array}$ & $\begin{array}{l}\text { Flow } \\
\text { Meters }\end{array}$ & $\begin{array}{c}\text { Pressure } \\
\text { Taps }\end{array}$ \\
\hline Tank i to $P 1^{2}$ & $15^{b}$ & 27.5 & 1.5 & 0.5 & 2 & 0 & 0 \\
\hline$P 1$ to $P 2$ & 166 & $0^{c}$ & 0 & 0 & 0 & 0 & 0 \\
\hline ?2 to P3 & 120 & 62.0 & 6 & 1 & 1 & 1 & 3 \\
\hline$P_{3}$ to $P_{4}$ & 117 & 12.5 & 0 & 1 & 0 & 0 & 0 \\
\hline$P_{Y_{1}}$ to $P_{x_{2}}$ & 111 & 23.0 & 1 & 1 & 0 & 0 & 0 \\
\hline$P_{1}$ to $P_{x_{3}}$ & 105 & 23.0 & 1 & 1 & 0 & 0 & 0 \\
\hline$P=$ to $P 4$ & 104 & 12.5 & 1 & 0 & 0 & 0 & 0 \\
\hline$P_{4}^{4}$ to $P_{5}$ & 70 & 94.5 & 6.50 & 1 & 1 & 0 & 3 \\
\hline P亏 וo Tank i & 12.5 & $0^{d}$ & 0 & 0 & 0 & 0 & 0 \\
\hline
\end{tabular}

a Average for the two slurry tanks.

b Il 1 as, assumed that a slurry delivery system can be designed to maximize use of the static pressure head present 
from the slurry in the tanks.

c Tilis section is the MCD pump.

d T"is section is the slurry tank; the pressure is decreased to atmospheric by passing the slurry through an orifice. 
$431.02 \#$

$2 / 11 / 98$

Rev. \#01
ENGINEERING DESIGN FILE
Function File Number - SPR-02

EDF Serial Number - EDF-CWO-003

\section{Attachment 3}


$431.02 \#$

$2 / 11 / 88$

Rev. $\# 01$
ENGINEERING DESIGN FILE
Function File Number - SPR-02

EDF Serial Number - EDF-CWO-003

(Process flow diagrams in this attachment are provided in Appendix B of the main (summary) document for the CWO process, and are not duplicated here.) 
Project File Number $\underline{\text { O2BDO }}$

Project/Task Non-Separation Alternatives

Subtask Cementitious Waste Option

\begin{tabular}{|c|c|c|c|c|c|}
\hline \multicolumn{6}{|c|}{ Title: $\quad$ Electrical Requirements } \\
\hline \multicolumn{6}{|c|}{$\begin{array}{l}\text { Summary: This EDF evaluated the electrical requirements for the Cementitious } \\
\text { Waste Option. It is assumed that a separate source of normal would be } \\
\text { provided for the facility, however, the possibility of obtaining power } \\
\text { from CPP-659 will be investigated during the conceptual design. The } \\
\text { process is such that standby power would not be required. If the } \\
\text { power is interrupted, it is safe to stop the process and resume when } \\
\text { normal power is restored. Based on this assumption, standby power } \\
\text { will be available from CPP-659. Additional standby power, if required, } \\
\text { would be obtained from Substation } 60 \text { via the normal power } \\
\text { distribution system. }\end{array}$} \\
\hline \multicolumn{6}{|c|}{ Distribution (complete package): } \\
\hline $\begin{array}{l}\text { Author } \\
\text { John E. } \\
\text { Duggan }\end{array}$ & Dept. & Reviewed & Date & 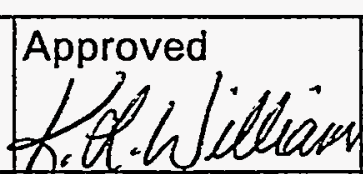 & $\begin{array}{l}\text { Date } \\
2 / 10 / 98\end{array}$ \\
\hline & & $\begin{array}{l}\text { LMITCO } \\
\text { Review lolag } \\
\text { Clllos doe }\end{array}$ & Date & $\begin{array}{l}\text { LMITCO } \\
\text { Approval }\end{array}$ & Date \\
\hline
\end{tabular}

See Management Control Procedure (MCP) 6 for instructions on use of this form. 


\section{CEMENTITIOUS WASTE OPTION}

\subsection{ELECTRICAL REQUIREMENTS}

\subsection{EXISTING AND PLANNED ELECTRICAL UTILTIES AT ICPP}

The ICPP ties into the $138 \mathrm{kV}$ INEL loop at Substation 2 which is located outside the area fence to the south. Substation 2 transforms the $138 \mathrm{kV}$ to $13.8 \mathrm{kV}$ and provides power to Substation 10 which is located within the ICPP complex. Power at $13.8 \mathrm{kV}$ is than distributed from Substation 10 through the complex. The Electrical and Utility System Upgrade (EUSU) project is currently under construction. The EUSU project will install a new $13.8 \mathrm{kV}$ electrical distribution system throughout the complex. This new system will provide greater safety, additional capacity and greater reliability.

Currently, standby power is provided by each facility. Standby generators are located at various facilities and operate as an island of power during a normal power outage. The EUSU project will construct a standby power plant, install new standby generators and tie existing generators into the standby power system. Standby power will than be distributed through the complex by the new $13.8 \mathrm{kV}$ distribution system, The Utility Control System (UCS) will control the usage of standby power.

\subsection{POWER REQUIREMENTS}

\subsubsection{NORMAL POWER}

The electrical requirements of the Cementitious waste option were analyzed and determined to be $131 \mathrm{kVA}$. The results of the analysis is shown in Table 1. The major load of Cementitious waste option the is the process equipment.

Normal power will be supplied to the Cementitious waste option by $13.8 \mathrm{kV}$ feeders from substation 15. A combination of new and existing duct banks will be used to route the feeders.

This study will assume that a separate source of power will be required for the Cementitious waste option. During the conceptual design, the possibility of obtaining power from CPP-659 will be investigated. 


\subsubsection{STANDBY POWER}

The requirements for standby power for the Cementitious waste option were analyzed and determined to be only lighting and miscellaneous equipment resulting in a Standby power requirement of $3 \mathrm{kVA}$. HVAC will be connected to the existing facility and therefore, additional standby power will not be required. Process equipment will not require standby power.

Standby power to the Cementitious waste option will be provided from the standby power panels located in the existing building. Should additional standby power be required, it will be provided to the facility via the normal power distribution system from Substation 60.

\subsubsection{DISTRIBUTION SYSTEM}

Both normal and standby power will be provided by one set of redundant feeders. The EUSU project will install a redundant pair of sectionalizing switches in the north east quadrant of the complex. These switches, PSS-NCE-1507A and PSSNCE-1557A are supplied by one set of 500KCMIL cables each. These are switches are fed directly from Substation 15 and are very lightly loaded. New duct banks will be run from these switches to a new load center. The load center will be double ended and will provide a redundant source of power to the Cementitious waste option

\subsubsection{UNINTERRUBTABLE POWER SUPPLY (UPS)}

A solid state UPS with a static transfer switch will be provided. The UPS will be provided with a 20 minute battery backup. Both the normal feed and the bypass feed to the UPS will be on standby power. The UPS will feed a 208Y/120 Volt panel. The UPS and the panel will be located in the electrical room. The UPS will support the following loads: Voice paging/evacuation systems, environmental monitoring system and other critical loads.

\subsection{LIGHTING}

\subsubsection{INTERIOR LIGHTING}

Lighting in office areas and other low ceiling areas will be supplied by recessed of pendant mounted fluorescent fixtures. These fixtures will be operated at 277 Volts and will be locally switched. Motion detectors will be utilized areas of low occupancy. Lighting in high bay areas will be will be supplied by metal halide fixtures operating at 277 Volts. The metal halide fixtures will be switched at the lighting panel. 


\subsubsection{CELL LIGHTING}

Lighting in the cells will be designed to allow for remote operation of the equipment via an in cell CCTV system or by operation through cell windows. Lighting of the cells will be indirect. Translucent panels will be provided on the top of the cells and on the side walls as required. Fixtures will be mounted so that maximum light is directed into the cell and so that the fixtures are easily accessible for maintenance. The light source will be metal halide. The lighting design will take into account light loss through the translucent panels as well as the light lost in viewing operations through the cell windows.

\subsubsection{EXTERTOR LIGHTING}

The exterior of the building will be illuminated with high pressure sodium wall pack fixtures mounted over each personnel door. Loading areas will be provided with high pressure sodium pall pack fixtures on each side of the doors. The need for area lighting in maneuvering areas will be evaluated during the conceptual and final designs.

\subsubsection{EMERGENCY AND EXIT LIGHTING}

Emergency egress lighting will be in accordance with NFPA 101, Life Safety Code. In areas where illumination is provided by fluorescent fixtures, selected fixtures will be provided with integral battery back up. In areas where illumination is provided by metal halide fixtures, selected fixtures will be provided with a quartz lamp which will be used for emergency lighting. The quartz lamp will be connected to the UPS system or will be provided with an integral battery.

\subsection{DESIGN DESCRIPTION}

\subsubsection{SITE CONDITIONS}

Electrical equipment will be rated for continuous operation at an elevation of 5,000 feet above sea level.

\subsubsection{HAZARDOUS LOCATIONS}

Several areas within the facility will be used for storing or handling of hazardous materials. An evaluation will be performed during the conceptual design and the final design to determine the effects of these materials on the installation of electrical equipment. Flammable and Combustible Liquids Codes NFPA 30: 
National Electrical Code, NFPA 70; and Recommended Practice for Classification of Class I Hazardous Locations for Electrical Installations in Chemical Process Areas, NFPA 497A will be used in making the determination.

\subsubsection{SERVICES}

\subsubsection{PRIMARY SERVICE}

Two $13.8 \mathrm{kV}$ feeders will provided the primary service to the site. The feeders will originate from Substation 2 located outside the fence. Power for the Cementitious waste option will be carried over existing feeders up to Substation 15. From Substation 15, new feeders will be routed through new and existing duct banks.

\subsubsection{STANDBY POWER}

Standby power will be supplied form building CPP-659

\subsubsection{EQUIPMENT}

\subsubsection{LOAD CENTERS}

One load center will be provided. The load center will be located outside the Cementitious waste option and will be a NEMA 3R Walk-in type similar the load centers currently in use at the ICPP. The load center will provide 480Y/277 Volts and will be double ended. Each transformer will be sized to provide service to all loads on the load center. The load centers will be provided with all equipment and wiring to insure that it is fully compatible with the existing UCS System.

\subsubsection{TRANSFORMERS}

Transformers 5,000 kVA and below will be cast coil, dry type transformers. Transformers over $5,000 \mathrm{kVA}$ will be oil filled. 


\subsubsection{LIGHTING}

Lighting levels will conform the Illuminating Engineer's Society (IES) handbook and standard practices at the ICPP. In general, the design will provide the following illumination levels:

- Work Stations 70 foot candles

- Work Areas 30 to 50 foot candles depending on activity

- Non-Work Areas 10 foot candles, 50 where data is obtained

\subsubsection{GROUNDING}

Grounding at the ICPP is accomplished with bare copper conductors installed in all duct banks and ground rods installed in every manhole this in turn is solidly connected to the casing of the deep wells. Facilities and structures throughout the ICPP are connected to this ground system. As well, the Cementitious waste option will be connected to the ground system. Grounding within the facility and at the outdoor load centers and other structures will be accomplished in accordance with the National Electrical Code and IEEE Standard 142-1991, IEEE Recommended Practice for Grounding of Industrial and Commercial Power Systems.

\subsubsection{LIGHTNING PROTECTION}

Lightning protection will be provided in accordance with NFPA 78, Lightning Protection Code. 


\section{TABLE 1}

WASTE TREATMENT PROJECT

FEASIBILITY STUDIES

CONNECTED LOAD

CEMENTITIOUS WASTE OPTION

\begin{tabular}{|l|r|}
\hline \multicolumn{1}{|c|}{ LOAD } & \multicolumn{1}{|c|}{ KVA } \\
\hline General Building Lighting & \\
4,300 sq. Ft @ 2.0 Watts per sq. Ft $=8,600$ Watts & 9 \\
\hline Miscellaneous Loads & 4 \\
4,300 sq. Ft. @ 1.0 Watts per sq. Ft $=4,300$ Watts & 60 \\
\hline Transport System 60 HP & 58 \\
\hline Process Equipment: $58.2 \mathrm{~kW}$ & \\
\hline & 131 \\
\hline Total connected kVA & \\
\hline
\end{tabular}




\section{TABLE 2}

\section{WASTE TREATMENT PROJECT \\ FEASIBIITY STUDIES}

STANDBY LOAD

CEMENTITIOUS WASTE OPTION

\begin{tabular}{|l|l|r|}
\hline \multicolumn{1}{|c|}{ LOAD } & STANDBY REQUREMENTS & kVA \\
\hline General Building Lighting & & \\
4,300 sq. Ft @ 2.0 Watts per sq. Ft $=8,600$ Watts & $25 \%$ of $8,600=2,150$ Watts & 2 \\
\hline $\begin{array}{l}\text { Miscellaneous Loads } \\
\text { 4,300 sq. Ft. @ 1.0 Watts per sq. Ft }=4,300 \text { Watts }\end{array}$ & $25 \%$ of 4,300 $=1,075$ & 1 \\
\hline Transport System 60 HP & 0 & \\
\hline Process Equipment: 58.2 kW & 0 & \\
\hline & & 3 \\
\hline Total connected Standby kVA & & \\
\hline
\end{tabular}


Project File Number $\quad \underline{02 B D 0}$

Project/Task Waste Treatment Project Feasibility Studies

\section{Subtask CWO Scoping Study}

Title: Impact of Blending/Recalcination of Calcines Prior to Cementation

Summary: This EDF describes a brief study that was conducted to examine the impacts of blending and recalcination on grouting of ICPP high level radioactive waste calcines. Specific impacts considered were: (a) key element ratios in grouted waste forms, (b) required additives, and (c) final waste mass. The study was done in support of scoping design efforts for the DCWO and CWO high level waste processing options, currently under consideration by DOE for treating radioactive wastes at ICPP. The study concludes blending and recalcination may not be necessary.

Distribution (complete package):

WTP EIS Studies Library, A. E. Lee M.S. 3765, R. E. Dafoe M.S. 3765, K. L. Williams M.S. 3765

Distribution (summary package only):

\begin{tabular}{|c|c|c|c|c|c|}
\hline $\begin{array}{l}\text { Authors } \\
\text { D. N. } \\
\text { Thompson } \\
\text { D. D. Taylor } \\
\end{array}$ & $\begin{array}{l}\text { Dept. } \\
4170\end{array}$ & $\begin{array}{l}\text { Reviewed } \\
\text { S. J. Losinski } \\
0.1 \text { Juensh }^{2}\end{array}$ & $\begin{array}{l}\text { Date } \\
02 / 05 / 98\end{array}$ & Approved & Date \\
\hline O.D rayke & & $\begin{array}{c}\text { LMITCO Review } \\
2 / 1 / 190\end{array}$ & Date & LMITCO Approval & Date \\
\hline
\end{tabular}




\section{INTRODUCTION}

The Cementitious Waste Option (CWO) for treating high level radioactive wastes at the Idaho Waste Processing Plant (ICPP) calls for recalcination of all existing waste calcines by slurrying them with liquid sodium bearing waste (SBW), water, and a $65 \mathrm{wt} \%$ aqueous solution of sucrose, and injecting the slurry mixture into the fluidized bed calciner at the New Waste Calcining Facility (NWCF). Recalcining of solid calcines in this way is intended to achieve the following objectives:

1) Solidify remaining SBW in the tank farm,

2) Remove nitrates and mercury from existing calcine and SBW to prevent potential problems during grouting,

3) Blend all wastes to minimize composition variations that must be accommodated in the grouting recipe(s), and

4) Redistribute sodium and potassium (alkali is a required ingredient of the proposed grouting process), present in relatively high concentrations in recently-produced calcines and SBW, throughout the rest of the calcine.

In order to achieve items 3 and 4 the CWO process design must include some type of blending scheme. The baseline process calls for blending of three generic calcine types (alumina, zirconia/flourinel/blend, and high-sodium calcines) as they are retrieved from the ICPP binsets. The present study was undertaken to investigate composition variations in existing calcines and their potential effect on grouting recipes. This information was needed to determine the design requirements for the CWO process blending system.

\section{APPROACH}

\subsection{Assumptions}

\subsubsection{Required Ratios in Grout}

Both the Direct Cementitious Waste Option (DCWO) and the CWO processes are based on published studies ${ }^{1}$ on formation of hydroceramic waste forms by D. D. Siemer and his coworkers. Their work, in turn, is based on extensive studies of the FUETAP ${ }^{2}$ grouting process at Oak Ridge National Laboratory. Siemer recommends a "properly formulated concrete" as one that (a) does not contain excessive amounts of nitrate, chloride, or sulfate, and (b) has a gross composition that approximates natural assemblages of aluminosilicate minerals (rocks). Specifically, the following conditions should be satisfied (molar basis):

${ }^{1}$ D. D. Siemer. B. E. Scheetz, and M. L. D. Gougar, "Hot Isostatic Press (HIP) Vitrification of Radwaste Concretes", Materials Res. Soc. Symp. Vol 412, 1996, pp. 403-410. Proceedings of the 1995 MRL Symposium on "Scientific Basis for Nuclear Waste Management, XIX", Boston, MA, Nov 29-Dec 3, 1995).

2 L. R. Dole, et al, "Cement-Based Radioactive Waste Hosts formed Under Elevated Temperatures and Pressures (FUETAP Concretes) for Savannah River Plant High-Level Defense Waste, ORNL/TM-8579, March 1983 (ORNL report). 
Function File Number - SPR-03

EDF Serial Number - EDF-CWO-005

Page 3 of 11

(a) $(\mathrm{Al}+\mathrm{Fe}) /(\mathrm{K}+\mathrm{Na}) \geq 1.0$

(b) $(\mathrm{Si}) /(\mathrm{K}+\mathrm{Na}) \geq 2.0$

In addition to the above, it is noted ${ }^{3}$ that in a number of aluminosilicate minerals (e.g., herschelite, pollucite, gobbinsite, chabazite, and a number of zeolites) the ratio of aluminum to silicon is approximately 0.5 . However, Siemer has noted ${ }^{4}$ that successful grouting of calcine-like materials has been accomplished with $\mathrm{Al}$ :Si ratios as high as 1.87 . Thus, it was also assumed that the following is a desirable condition (but probably not strictly necessary according to Siemer) in the formulation of a hydroceramic grout for ICPP calcines:

(c) $0.5 \leq(\mathrm{Al}+\mathrm{Fe}) /(\mathrm{Si}) \leq 2.0$

Finally, alkali metal (e.g., $\mathrm{Na}, \mathrm{K}$ ) is a necessary ingredient of the grouting mixture. Siemer distinguishes two functions which these species serve. First, alkali metal immobilizes soluble anions (e.g., nitrates) in the final waste form. About four equivalents of alkali per equivalent of anion are required for this function. Second, so-called "free alkali" is needed to activate silica in the mixture and thus promote the formation of the desired aluminosilicate minerals. For this study it was assumed that all alkali in excess of that needed to bind the anions is free alkali. Siemer recommends a minimum of about 1 wt\% of free alkali in the final grouted waste (excluding water).

Based on the above considerations, depending on the quantity of alkali present in the calcine and the dry additives (i.e., calcined clay and blast furnace slag; see Section 2.1.2 below), additional alkali may be required in each grouted batch. It was assumed that additional alkali would be provided by adding pure caustic $(\mathrm{NaOH})$. The quantity required for each batch was calculated based on the following assumptions:

(d) total moles $(\mathrm{K}+\mathrm{Na}) \geq 4$ (total moles $\mathrm{NO}_{3}^{-}$)

(e) total mass of free alkali $=0.01$ (mass calcine + clay + BFS + caustic)

\subsubsection{Bulk Grouting Mix}

Again, per suggestion by $\mathrm{D}$. D. Siemer, the following assumptions were made regarding the bulk ingredients of grouted calcine:

(a) The grout mix will consist of calcine, calcined (Troy) clay, blast furnace slag (BFS) containing $\mathrm{CaS}$, caustic $(\mathrm{NaOH})$, and water;

(b) The waste loading of dewatered grout (2\% water) is $35 \%$ (percentages are wt\%)

(c) The weight ratio of calcined clay to BFS is $9: 1$

\footnotetext{
${ }^{3}$ M. W. Grutzeck, D. D. Siemer, "Zeolites Synthesized from Class F Fly Ash and Sodium Aluminate Slurry", J. Am. Ceram. Soc., 80 [9] 2449-53 (1997).

${ }^{4}$ Telecon on $12 / 11 / 97$ between D. D. Siemer, D. D. Taylor, and D. N. Thompson.
} 
ENGINEERING DESIGN FILE

Using the above assumptions, the following weight proportions of calcined clay and BFS were used in the current study:

(d) $1.576 \mathrm{lbm}$ calcined clay per lbm calcine

(e) $0.175 \mathrm{lbm}$ calcined clay per lbm calcine
Function File Number - SPR-03

EDF Serial Number - EDF-CWO-005

Page 4 of 11

\subsubsection{Composition of Additives}

The compositions of calcined clay and BFS were assumed to be as follows:

\begin{tabular}{|c|c|c|c|c|c|c|c|c|c|c|}
\hline & $\mathrm{Al}_{2} \mathrm{O}_{3}$ & $\mathrm{~B}_{2} \mathrm{O}_{3}$ & $\mathrm{CaO}$ & $\mathrm{Fe}_{2} \mathrm{O}_{3}$ & $\mathrm{~K}_{2} \mathrm{O}$ & $\mathrm{MgO}$ & $\mathrm{Na}_{2} \mathrm{O}$ & $\mathrm{SiO}_{2}$ & $\mathrm{ZrO}$ & $\mathrm{CaS}$ \\
\hline זרa & $27.71 \%$ & $0.00 \%$ & $0.13 \%$ & $1.21 \%$ & $0.93 \%$ & $0.93 \%$ & $0.13 \%$ & $68.37 \%$ & $0.58 \%$ & $0.00 \%$ \\
\hline $\mathrm{ES}$ & $7.30 \%$ & $0.02 \%$ & $41.00 \%$ & $0.34 \%$ & $0.47 \%$ & $10.30 \%$ & $0.36 \%$ & $34.00 \%$ & $0.10 \%$ & $6.11 \%$ \\
\hline
\end{tabular}

\subsection{Calcine Data}

Since 1963, the ICPP has calcined high level liquid wastes into solid calcine, and stored the resulting solid wastes in the Calcine Solids Storage Facility (CSSF) consisting of seven (7) binsets. Each binset is a separate structure housing one or more stainless steel bins which actually contain the calcine solids. Over the years a number of different types of liquid waste have been calcined. Thus, the binsets contain a variety of different calcine types, stratified in layers throughout the bins.

M. D. Staiger has assembled a database of nominal calcine compositions, based on available data for the liquid waste batches that have been processed up to 1993 in both NWCF and its predecessor the Waste Calcining Facility (WCF). This database provides volumes and calculated elemental compositions of each batch of calcine in tabular form, and was provided for this study. This data was used with two modifications. First, Staiger's composition data were computed on an oxygenfree basis. Thus, for proper mass accounting, the data were corrected by assuming oxygen to be stoichiometrically present to establish electrical neutrality. That is, for each calcine batch, likely valence states were assigned to all cations and anions present in the calcine. The mass of oxygen was then estimated on the basis of these valence states, the listed weight percentages of each specie, an assumed calcine density, and the listed volume of calcine from each batch. With this estimate the weight percentages of all species were then recalculated.

The second modification to Staiger's database was to add a single projection of the average composition ${ }^{5}$ and mass $^{6}$ for calcines that will be produced in the current campaigns (H4 and H5), scheduled to be completed by the time the Record of Decision (ROD) is announced for the treatment of ICPP high level wastes. The batch-by-batch composition data in the modified database provides a worst case estimate for composition variations that will be experienced during retrieval of calcine for grouting.

Assumed densities for calcine in these calculations were as follows:

\footnotetext{
${ }^{5}$ Based on information provided by B. H. O'Brien on 12/08/97.

${ }^{6}$ From Charles Barnes' projections documented in EDF-FDO-001 ("Estimates of Feed and Waste Volumes, Compositions, and Properties").
} 

(a) Alumina caline:
$1.1 \mathrm{gm} / \mathrm{cm}^{3}$
(b) All other calcines:
$1.6 \mathrm{gm} / \mathrm{cm}^{3}$

\subsection{Analysis Approach}

The first step in the analysis was to determine the range of variation of key calcine constituents. This was done by tabulating all batches in the order they were processed, and then calculating mean and standard deviation statistics for key species. The results of this exercise are discussed below in Section 3.1.

The second step in the analysis was to look at three idealized waste grouting possibilities:

Case (1): Direct grouting of the existing calcines, without recalcination and without blending Case (2): Grouting after recalcination of existing calcines, without blending to remove all nitrates Case (3): Grouting after recalcination of existing calcines, with perfect blending to remove all nitrates.

For each of these cases the mass of calcine was used to determine the required masses of calcined clay and BFS [using the Siemer grouting recipe (see Section 2.1)]. For Case (1) the batchwise masses of calcine were used for this calculation. For Case (2) the batchwise calcine mass after denitration was calculated, assuming that every mole of nitrate is replaced with $1 / 2$ mole of oxygen $\left(\mathrm{O}\right.$ not $\left.\mathrm{O}_{2}\right)$. For Case (3), the denitrated calcine mass was also used, but instead of using batchwise data, a single batch of calcine was assumed, with a composition corresponding to the mass weighted average of all batches in the modified Staiger database.

In all cases, once the masses of calcine, clay, and BFS were determined, the quantity of caustic $(\mathrm{NaOH})$ to be added was calculated which ensures (a) binding of all nitrate, and (b) satisfaction of the 1 wt\% alkali requirement (see Section 2.1.1). Once these calculations were complete, the resulting grout formulation was examined from the perspective of the target ratios mentioned in the assumptions. In addition the total mass of grout and required additives (clay $+\mathrm{BFS}$ and $\mathrm{NaOH}$ ) were examined. Based on these data, inferences were drawn about the value of calcine blending prior to grouting.

\section{RESULTS}

\subsection{Variabiliy of Calcine Compositions in Binsets}

Figure 1 shows the raw data from Staiger's database showing the weight fractions of key elements ( $\mathrm{Al}, \mathrm{K}+\mathrm{Na}, \mathrm{Zr}, \mathrm{Fe}, \mathrm{F}$, and $\mathrm{NO}_{3}$ ) in the grout formulation. The standard statistical measures of the composition variability are given in the following table:

$\circ$

Mean (wt\%)

\section{Table 1: Composition Variations in Calcines}

\begin{tabular}{cccccc}
$\mathrm{Al}$ & $\mathrm{K}+\mathrm{Na}$ & $\mathrm{Zr}$ & $\mathrm{Fe}$ & $\mathrm{F}$ & $\mathrm{NO}_{3}$ \\
\hline 24.6 & 2.1 & 8.0 & 0.3 & 12.1 & 3.4
\end{tabular}




\begin{tabular}{l|cccccc} 
Std Dev (\% of mean) & $71 \%$ & $83 \%$ & $84 \%$ & $116 \%$ & $82 \%$ & $83 \%$ \\
Maximum (wt\%) & 51.2 & 8.1 & 21.6 & 2.1 & 29.1 & 13.1 \\
Minimum (wt\%) & 2.1 & 0.0 & 0.0 & 0.0 & 0.0 & 0.0
\end{tabular}

Taken at face value the above results indicate significant variation in the calcine composition, and suggest that blending of calcines would be desirable, if not necessary, from the standpoint of optimizing the number of different grouting recipes to be used. The next step in the analysis was performed to further investigate this implication.

\subsection{Key Grouting Ratios}

After calculating the required quantities of grout additives as described above, the following molar ratios were calculated for each calcine batch in Staiger's database:

- $(\mathrm{Si}) /(\mathrm{K}+\mathrm{Na})$

- $(\mathrm{Al}+\mathrm{Fe}) /(\mathrm{K}+\mathrm{Na})$

- $(\mathrm{Al}+\mathrm{Fe}) /(\mathrm{Si})$.

The results are shown in Figures 2 and 3. The above ratios are shown for Case(1) (DCWO process with no blending) in Figure 2(a), (b), (c), and for Case (2) (CWO process with no blending) in Figure 3(a), (b), (c). The corresponding ratios for case (3) (ideal CWO process, with recalcination and perfect blending of all calcines into a single homogeneous mixture) are shown as the dotted lines labeled 'BLENDED CWO' in all figures.

\section{DISCUSSION OF RESULTS}

\subsection{Range of Key Grouting Ratios}

The data in the above figures are summarized in the following table which gives the range of variation for each of the parameters in the figures:

Table 2: Ranges of Variation of Key Parameters

\begin{tabular}{lcccc} 
& $\begin{array}{c}\text { Case (1) } \\
\text { (DCWO) }\end{array}$ & $\begin{array}{c}\text { Case (2) } \\
\text { (CWO w/o blending) }\end{array}$ & $\begin{array}{c}\text { Case (3) } \\
\text { (Ideal CWO) }\end{array}$ & Target \\
\cline { 2 - 5 } $\mathrm{Si} /(\mathrm{K}+\mathrm{Na})$ & $1.9-19.4$ & $15.0-22.0$ & 12.9 & $>2.0$ \\
$(\mathrm{Al}+\mathrm{Fe}) /(\mathrm{K}+\mathrm{Na})$ & $1.9-28.7$ & $4.9-29.3$ & 10.7 & $>1.0$ \\
$(\mathrm{Al}+\mathrm{Fe}) /(\mathrm{Si})$ & $0.5-1.5$ & $0.5-1.5$ & 0.8 & $0.5-1.87$
\end{tabular}

For both Case (1) and (2), while the range of variation of the $\mathrm{Si} /(\mathrm{K}+\mathrm{Na})$ ratio is fairly large, all batches have a value above 2.0 , which satisfies the nominal recipe requirement for this ratio. By comparison, for the case of perfect blending (Case (3)) the value of this ratio is 12.9 , also satisfying the 
nominal requirement, but also significantly different from the "target" value of 2.0. Similarly, for all three cases, the variation of the $(\mathrm{Al}+\mathrm{Fe}) /(\mathrm{K}+\mathrm{Na})$ ratio is large, but all values satisfy the nominal requirement. The value for the case of perfect blending is 10.7 , which exceeds the target value of 1.0 significantly, but again not by as great a margin as the extreme values for Cases (1) and (2).

Finally, the $(\mathrm{Al}+\mathrm{Fe}) / \mathrm{Si}$ ratio for Cases (1), (2), and (3) is above the target value of 0.5 but below the limit of 1.87 . The latter value is the highest value of this ratio for which has been successfully tested ${ }^{7}$.

\subsection{Final Waste Produced/Required Additives}

The calculated masses of grout produced from each of the three cases considered were compared to evaluate the impacts of blending and recalcination on this measure of process performance. The results are summarized in Table 3, below, which gives the normalized masses (normalizing factors were the Case (1) values).

Table 3: Stabilized Grout Mass and Additives Required

\begin{tabular}{llll}
\multicolumn{1}{c}{} & Case (1) & Case (2) & Case (3) \\
\cline { 2 - 4 } Clay + Slag added & 1 & 0.965 & 0.965 \\
$\mathrm{NaOH}$ added & 1 & 0.072 & 0.000 \\
Final waste mass & 1 & 0.935 & 0.933
\end{tabular}

The table indicates that recalcination without blending [Case (2)] reduces the mass of required dry additives by about $3.5 \%$, the amount of $\mathrm{NaOH}$ required by about $92.8 \%$, and the final waste mass by about $6.5 \%$. The values for Case (3) indicate that recalcination with perfect blending only alters these numbers slightly.

\section{CONCLUSIONS \& DISCUSSION}

From this study the following four conclusions were drawn:

1) Concentrations of $\mathrm{Al}, \mathrm{K}+\mathrm{Na}, \mathrm{Zr}, \mathrm{Fe}, \mathrm{F}$, and $\mathrm{NO}_{3}$ in the existing calcines vary over relatively wide ranges. At first sight, this variation suggests that blending/homogenization of the calcines may be needed prior to calcining. However, based on available data for grouting recipes using calcined materials, the range of variation of three key ratios examined in the study $[(\mathrm{Al}+\mathrm{Fe}) /(\mathrm{Na}+\mathrm{K})$, $(\mathrm{Si}) /(\mathrm{Na}+\mathrm{K}),(\mathrm{Al}) /(\mathrm{Si}))]$ may very likely be tolerable and still produce a viable grouted waste form.

2) If complete homogenization of existing calcines were possible, then it is likely that no additional alkali would be need in producing the final grouted waste form. In addition, the quantity of required dry additives (calcined clay and blast furnace slag) would be reduced by about $3.5 \%$, and the total final waste mass produced would be reduced by about $6.7 \%$.

\footnotetext{
${ }^{7}$ Per D. D. Siemer in a telecon on $12 / 11 / 97$.
} 
3) Recalcination of existing calcines without any blending whatsoever is almost as effective in reducing the quantity of required additives (alkali, calcined clay, and blast furnace slag) and the final waste mass as recalcination plus homogenization.

4) The benefits of either recalcination or blending are likely to be quite modest if composition variations in the calcine coming from the bins can be accommodated by the grouting recipe as these results suggest they might. However, testing of cold calcines should be undertaken to confirm this tentative conclusion.

Recalcination of existing calcines may still be desirable from two standpoints. First, it would remove mercury from the final waste form. However, grout testing should be undertaken to determine whether this is actually necessary. It is possible that high mercury concentrations could be accommodated in the grouted waste (e.g., FeS may prove successful as an additive to bind mercury as $\mathrm{HgS}$ in the final grouted waste form). The second argument for recalcination (with sugar as a reducing agent) is that it would reduce NOx concentrations in the NWCF plume during processing of SBW, and minimize the amount of nitrate in the waste to be grouted. However, grout testing may again demonstrate that nitrate in grouted waste can be tolerated. This underscores the need for testing/development of grouting formulas.

\section{FIGURES}


ENGINEERING DESIGN FILE

(a) Al

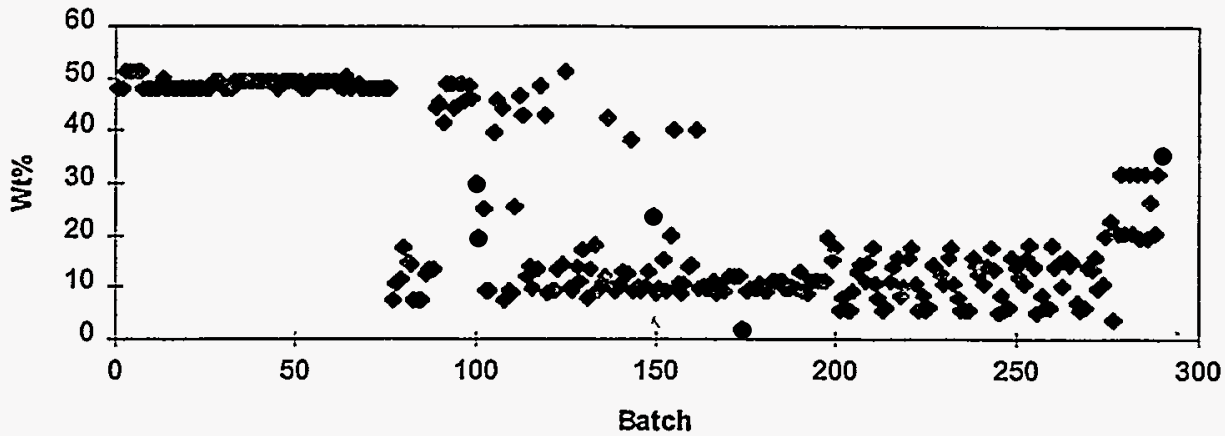

(b) $\mathrm{K}+\mathrm{Na}$

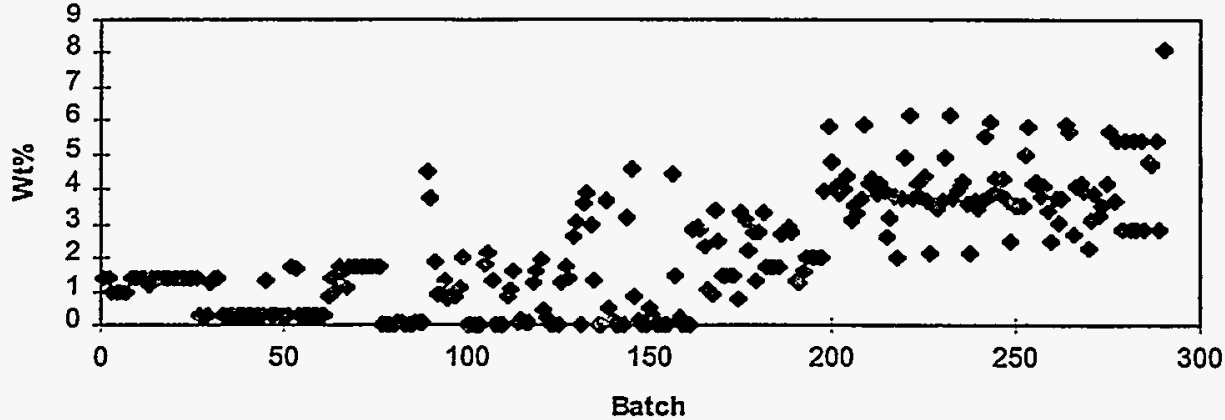

(c) Zr

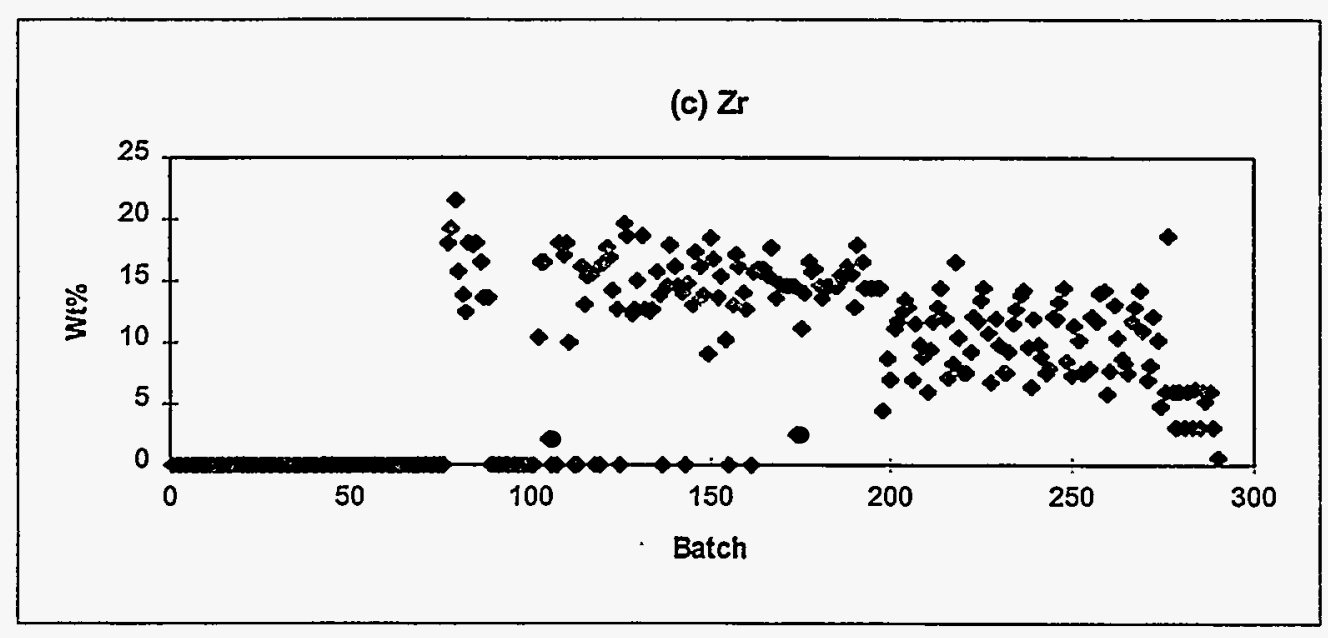

Figure 1

Function File Number - SPR-03 EDF Serial Number - EDF-CWO-005

Page 9 of 11 
ENGINEERING DESIGN FILE

Function File Number - SPR-O3

EDF Serial Number - EDF-CWO-005

Page 10 of 11
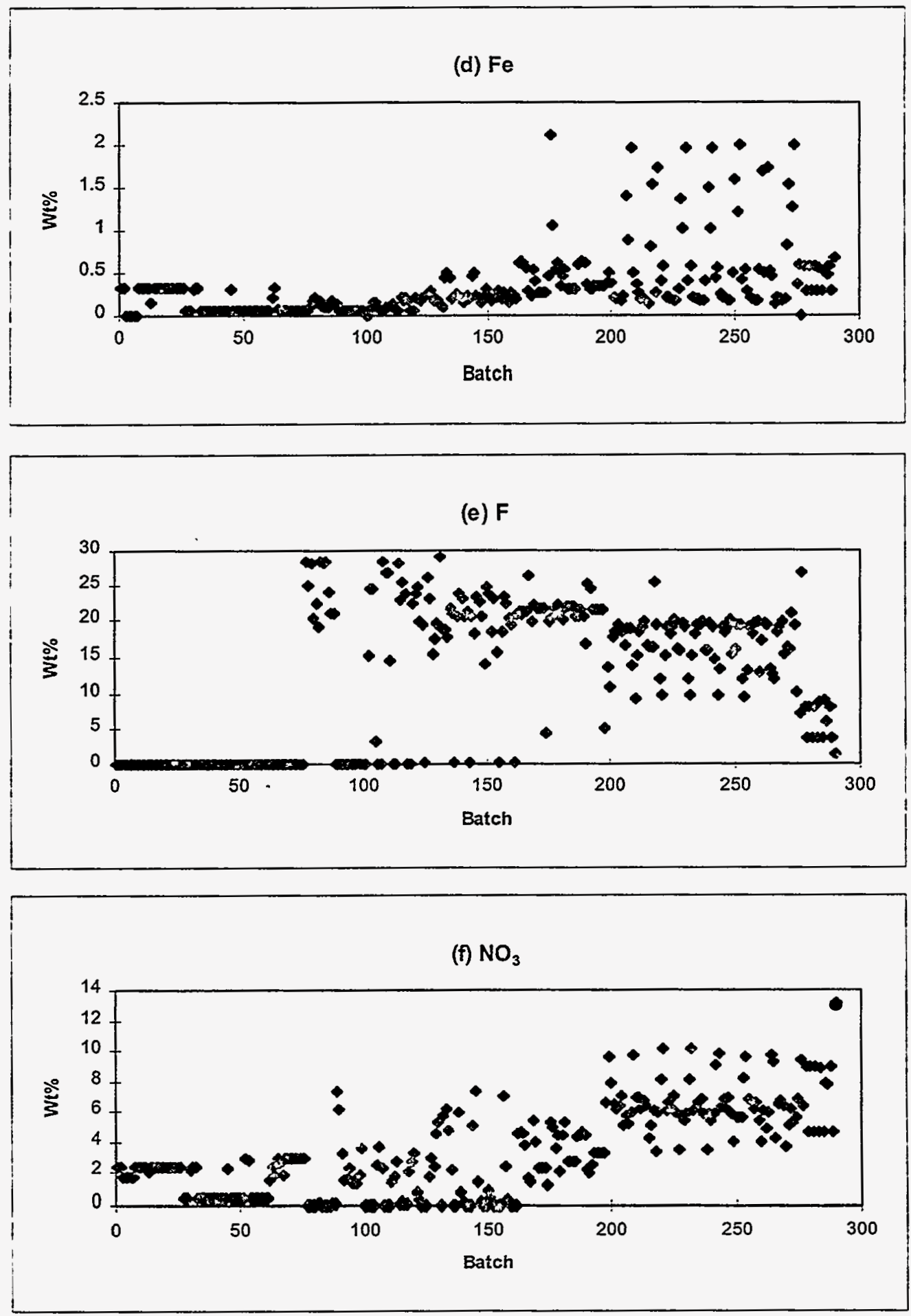

Figure 1 (cont'd) 
431.02\#

2/11/98

Rev. $\$ 01$
Function File Number - SPR-03

EDF Serial Number - EDF-CWO-005

Page 11 of 11 (a) Batchwise DCWO Option No Blending)

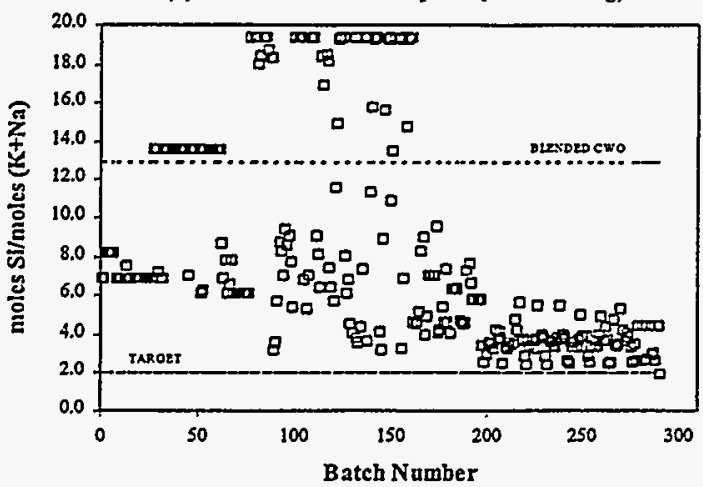

(b) Batchwise DCWo Option (No Blending)

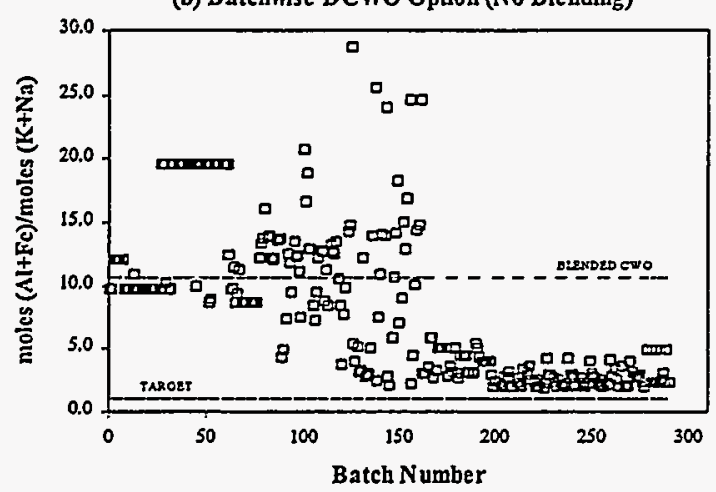

(c) Batchwise DCWO Option (No Blending)

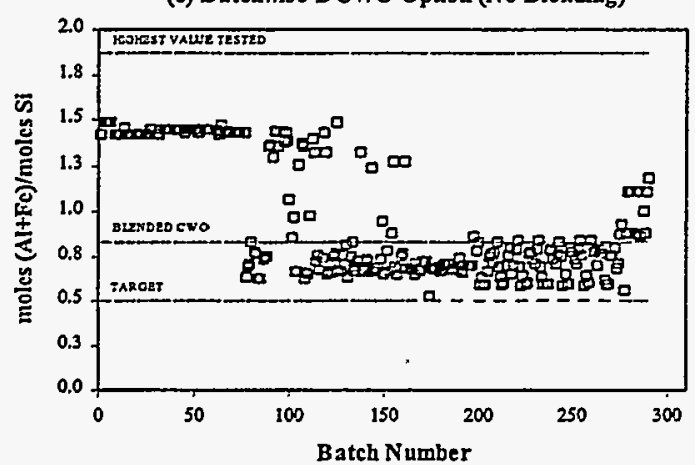

Figure 2 (a) Batchwise Cwo Option (No Blending)

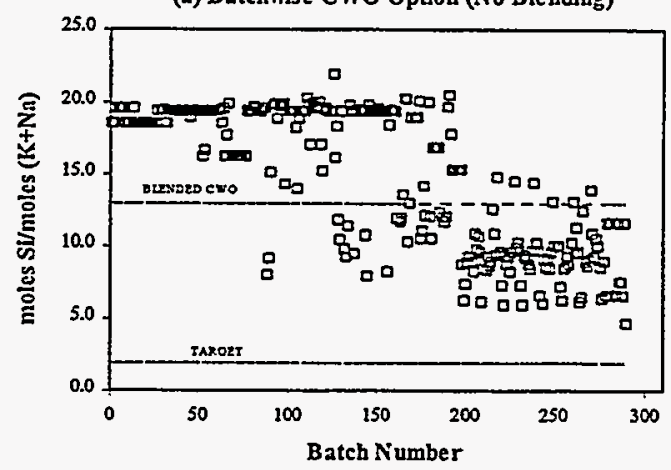

(b) Batchrise CWo Option (No Blending)

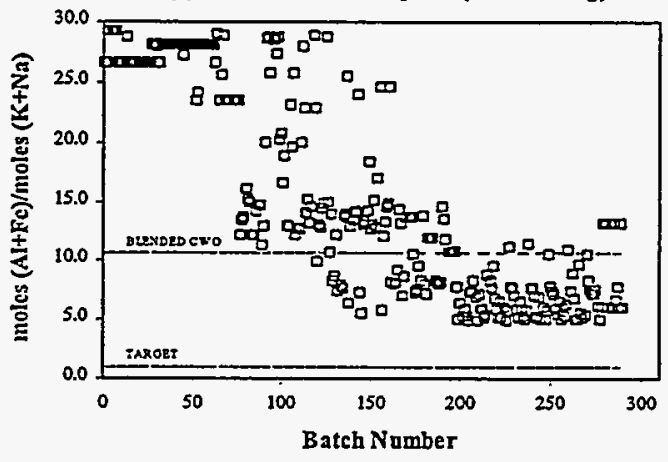

(c) Batchwise Cwo Option (No Blending)

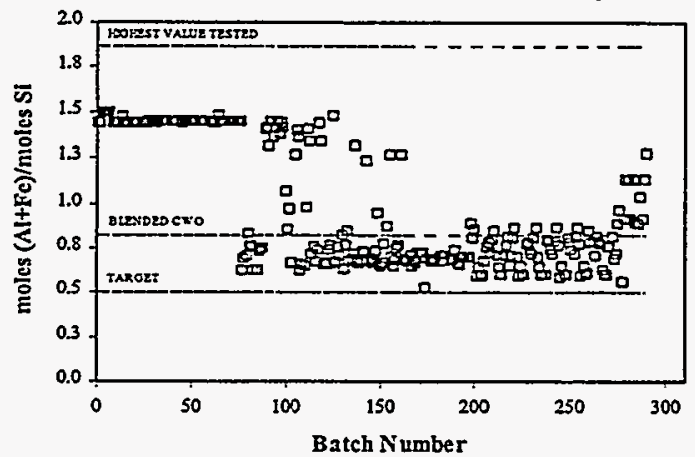

Figure 3 
E-94 
Project/Task Non-Separation Alternatives

Subtask Cementitious Waste Option (CWO)

\section{Title: CWO Scoping Study Staffing Estimate}

\section{Summary:}

This EDF addresses the estimate of the total staffing requirement for the calcination process for the Cementitious Waste Option (CWO). In the CWO scoping study, there are two major processing requirements that have been addressed separately for the required staffing; the calcination process and the grouting process. This EDF focuses on the staffing requirement for the calcination process. The calcination process is an existing process but in the CWO, the calcination process has been modified from the current calcination process to a recalcination process to feed previously calcined waste mixed with sodium bearing waste (SBW) and a 65 weight \% sugar solution. This process is based upon a 24 $\mathrm{hr} /$ day, 7 day/wk operation to be completed in 5 years commencing on $1 / 1 / 13$ and finishing on $12 / 31 / 17$. There are 3-8 hour shifts per operating day and a floating shift to provide coverage for days-off and vacation scheduling. The method used to estimate the staffing requirements for the CWO calcining process consists of two steps; first, the existing staffing at the ICPP calciner was used for the FY-98 projected baseline determination and second, additions to the existing calciner facility staffing were estimated to include the slurrying (mixing) step of solid calcine and liquid SBW and the addition of sugar to the calcine-SBW/slurry mixture. The baseline calciner staffing is 96 personnel and the additional calciner staffing to address the CWO modifications is 36 personnel to yield a total estimate for this requirement of 132 personnel or FTEs. The breakout of the labor needs for this estimate are identified by the following resource groupings in the FY-98 NWCF projected budget totals; Shift Operators, Engineering Support, Craft Support, Shift Supervision, Administration, ICPP Rad Con and all other support.

Distribution (complete package): WTP EIS Studies Library, D.J.Harrell MS 3211, B.R.Helm MS 3765, V.L.Jacobson MS 3211, R.T.Jamison MS 4146, K.L.Williams MS 3765, W.J.Prendergast MS 3710

Distribution (summary package only):

\begin{tabular}{|c|c|c|c|c|c|}
\hline $\begin{array}{l}\text { Author } \\
\text { W.J. } \\
\text { Prendergast }\end{array}$ & $\begin{array}{l}\text { Dept. } \\
4170\end{array}$ & $\begin{array}{l}\text { Reviewed } \\
\text { B Tamison } \\
\text { halmosun }\end{array}$ & 2110190 & $\begin{array}{l}\text { Approved } \\
\text { Allen Lee }\end{array}$ & $\begin{array}{l}\text { Date } \\
\text { Zllo kes }\end{array}$ \\
\hline & - & $\begin{array}{l}\text { LMIfCo } \\
\text { Review } \\
\text { zflofys }\end{array}$ & Date & $\begin{array}{l}\text { LMITCO } \\
\text { Approval }\end{array}$ & Daté \\
\hline
\end{tabular}




\section{Summary:}

This EDF identifies a total estimated staffing requirement of 132 personnel for the calcination portion of the CWO. This staffing estimate is broken down into two general sections below; staffing for current calciner from the "FY-98 Calcined HLW Project Budget Totals" report dated October 28, 1997, and another section for "Additional Staffing Estimate for Recalcining Modification". The total FTEs for the existing and current Calciner operations is 96 personnel. The total FTEs for the additional staffing for the recalcining modifications is 36 personnel. The grand total staffing requirement estimate for the calcining portion of the CWO is 132 personnel or FTEs. NOTE: These estimates do not include laboratory support for calciner operations.

\section{FY-98 CALCINED HLW PROJECT BUDGET TOTALS}

1. Shift Operators: $\frac{\text { FTES }}{28}$

2. Shift Supervision: 6

3. ICPP Rad Con: $\quad 7$

4. Engineering Support: 28

5. Craft Support: 15

6. Administration: 2

7. All Other Support: 10

Total FTEs $\quad \overline{96}$ 


\section{ADDITIONAL STAFFING FOR RECALCINING MODIFICATIONS}

1. Shift Operators: 2

Per Shift $\quad$ FTEs for 4 Shifts

8

2. Shift Supervision:

1

4

3. ICPP Rad Con:

1

4

4. Engineering Support:

2

8

5. Craft Support:

2

8

6. All Other Support:

1

4

Total FTEs:

$\overline{36}$

Grand Total for CWO Calcining Process: 132 
E-98 
EDF Serial Number CPP-97083 EDF-HWO-006

Functional File Number 4000-14

SPR-01

\title{
ENGINEERING DESIGN FILE
}

\author{
Radiological Evaluation \\ for the Calcine \\ Hot Isostatic Press (HIP) \\ Feasibility Study
}

\author{
By \\ B. J. Schrader \\ November 26, 1997
}


EDF Serial Number CPP-97083

Functional File Number 4000-14

\title{
ENGINEERING DESIGN FILE
}

\author{
Radiological Evaluation \\ For the Calcine \\ Hot Isostatic Press (HIP) \\ Feasibility Study
}

By

B.J. Schrader

November 26, 1997 
Project File Number N/A

\section{Project/Task N/A}

Subtask N/A

Title: $\quad$ Radiological Evaluation for the Calcine Hot Isostatic Press (HIP) Feasiblity Study

Summary: This summary briefly defines the problem or activity to be addressed in the EDF, gives a summary of the activities performed in addressing the problem and states the conclusions, recommendations, or results arrived at from this task.

This EDF evaluates and documents the radiological requirements for the feasibility of the Hot Isostatic Press (HIP) option for immobilization of the calcine. Areas of discussion in this evaluation are as follows:

1) Applicable codes and Standards

2) Radiological Monitoring Equipment

3) Hot Cell Manned Entry Requirements

4) Breathing Air Requirements

5) Radiological Risk Evaluation of Process

The discussion of each of the listed topics includes references to mandatory and nonmandatory requirements. The basis for this evaluation is the Department of Energy (DOE) would operate the facility under all currently applicable constraints and requirements. No attempt was made to evaluate the probability of changing requirements.

Distribution (complete package): N.E. Russell MS-3765 G.W. Clarke MS-4145 B.J. Schrader MS-5209 File

Distribution (summary package only):

\begin{tabular}{|c|c|c|c|c|c|}
\hline $\begin{array}{l}\text { Author } \\
\text { B.J. Schrader } \\
\text { BO D O }\end{array}$ & $\begin{array}{l}\text { Dept. } \\
\text { Radiological } \\
\text { Support }\end{array}$ & $\begin{array}{l}\text { Reviewed } \\
\text { Exfusel }\end{array}$ & $\begin{array}{l}\text { Date } \\
\text { infoi/git }\end{array}$ & Approved & Date 19.9 \\
\hline$\pi$ & & $\begin{array}{l}\text { LMITCO } \\
\text { Review }\end{array}$ & Date & $\begin{array}{l}\text { LMITC } \varnothing \\
\text { Approval }\end{array}$ & Date \\
\hline
\end{tabular}

See Management Control Procedure (MCP) 6 for instructions on use of this form. 


\section{SUMMARY}

The Hot Isostatic Press (HIP) option of the Calcine Immobilization Program evaluates the feasibility of using the process to convert calcine into an acceptable glassceramic waste form suitable for terminal storage in a federal geologic repository. The . fact that the calcine is highly radioactive requires that a radiological evaluation of the process and flow paths is performed to ensure that the process can be performed safely and maintain personnel exposure As Low As Reasonably Achievable (ALARA).

This EDF evaluates and documents the radiological requirements for the feasibility of the Hot Isostatic Press (HIP) option for immobilization of the calcine. Areas of discussion in this evaluation are as follows:

1) Applicable codes and Standards

2) Radiological Monitoring Equipment

3) Hot Cell Manned Entry Requirements

4) Breathing Air Requirements

5) Radiological Risk Evaluation of Process

The discussion of each of the listed topics includes references to mandatory and nonmandatory requirements. The basis for this evaluation is the Department of Energy (DOE) would operate the facility under all currently applicable constraints and requirements. No attempt was made to evaluate the probability of changing requirements.

The evaluation and recommendations of this EDF are based on existing technology and proven techniques and methods for handling highly radioactive material. The lessons learned from the NWCF Calciner, GSF canning station and the FSA fuel handling areas were evaluated for inclusion in this document.

This EDF does not include an evaluation of how the calcine will be removed from the Bin sets and moved into the facility. The process evaluation starts with the addition of the mixing agent with the calcine. 


\section{Key Assumptions}

The assumptions made during the development of this EDF are included below. These assumptions will require verification during follow-on design efiorts. In some cases, the assumptions may have a strong bearing on the direction of the design; in other cases, the assumptions simply identify important issues that need to be addressed as the design progresses.

- The calcine will be processed in a facility that will be operated under the requirements and oversight of the Department of Energy. All applicable regulations and standards for the Department of Energy will be enforced.

- The mixing agent used in the process will be non-radiological or of such low level activity that shielding will not be required.

- Exposures to personnel both on and off-site will be maintained within the codified requirements of 10CFR20 and 10CFR835.

- The exhaust system will be monitored in accordance with ANSI-N42.17B-1989 to ensure and validate regulatory compliance with emission requirements.

- ANSI/ANS 57.8-1992 is the primary design document for configuring the HVAC systems and identifying contamination control zones. The DOE-ID ArchitectEngineer (A-E) Standard was used for guidance when specific direction was not provided in ANSI/ANS 57.8.

- Siting of the facility will ensure that adequate utilities and process systems are available. Siting will also include an evaluation on methods of movement of the calcine from the bin sets to the facility that maintains exposures ALARA. 


\section{CODES and STANDARDS}

\begin{tabular}{|c|c|}
\hline Code/Standard & TITLE \\
\hline 10 CFR 20 & Standards for Protection Against Radiation \\
\hline 10 CFR 71 & Packaging and Transportation of Radioactive Material \\
\hline 10 CFR 830 & Nuclear Safety Management \\
\hline 10 CFR 835 & Occupational Radiation Protection \\
\hline 29 CFR 1910 & $\begin{array}{l}\text { Occupational Safety and Health Standards for } \\
\text { General Industry }\end{array}$ \\
\hline 29 CFR 1926 & $\begin{array}{l}\text { Occupational Safety and Health Standards for } \\
\text { Construction }\end{array}$ \\
\hline 40 CFR 53 & $\begin{array}{l}\text { Ambient Air Monitoring Reference and Equivalent } \\
\text { Methods }\end{array}$ \\
\hline 40 CFR 58 & Ambient Air Quality Surveillance \\
\hline $\begin{array}{l}49 \text { CFR } 173 \\
\text { Sub Part } 1\end{array}$ & $\begin{array}{l}\text { Shippers - General Requirements for Shipments and } \\
\text { Packages of Radioactive Materials }\end{array}$ \\
\hline $\begin{array}{l}\text { ANSI/ASME N510- } \\
1989\end{array}$ & Testing of Nuclear Air-Cleaning Systems \\
\hline $\begin{array}{l}\text { ANSI/ASME NQA- } \\
1-1989\end{array}$ & Quality Assurance Program \\
\hline $\begin{array}{l}\text { ANSI/NFPA } 801 \text { - } \\
1995\end{array}$ & Facilities Handling Radioactive Materials \\
\hline ANSI N13.1-1969 & $\begin{array}{l}\text { Guide to Sampling Airborne Radioactive Materials in } \\
\text { Nuclear Facilities }\end{array}$ \\
\hline $\begin{array}{l}\text { ANSI N42.17B- } \\
1989\end{array}$ & $\begin{array}{l}\text { Performance Specifications for Health Physics } \\
\text { Instrumentation Occupational Airborne Radioactivity } \\
\text { Monitoring Instrumentation }\end{array}$ \\
\hline $\begin{array}{l}\text { ANSI/ANS 57.8- } \\
1992\end{array}$ & HEPA Systems \\
\hline $\begin{array}{l}\text { DOE-ID A-E } \\
\text { Manual }\end{array}$ & Architectural - Engineering Manual \\
\hline $\begin{array}{l}\text { INEL RadCon } \\
\text { Manual }\end{array}$ & Radiological Control Manual for the INEEL \\
\hline
\end{tabular}

The table listed above is a brief summary of the requirements and documents that are the basis for the design. It is also assumed that the applicable DOE orders will be implemented. Currently DOE is phasing out the order system in favor of codifying the requirements. Therefore no effort was made to determine the applicable orders and estimate if they will be changed over to CFR status. Let it be sufficient that at the time of title design a document search of the remaining DOE orders should be performed. 


\section{Radiological Monitoring Equipment}

The following is a list of the radiation monitoring equipment that would be required under the configuration as it exists on 11/20/97. Any deviation from this configuration would require evaluation/modification of this list. Most of the instruments listed must be capable of transmitting data to a microprocessor-based system for display, recording, alarm and trending. The instruments will also be connected to the Radiation, Environmental and Safety (RE\&S) computer system for remote monitoring. The RE\&S interface is available at several locations in the plant and is available in the utility tunnel.

\begin{tabular}{|c|c|c|c|c|c|}
\hline $\begin{array}{c}\text { Instrument } \\
\text { Type }\end{array}$ & $\begin{array}{c}\text { Radiation } \\
\text { Area } \\
\text { Monitor }\end{array}$ & $\begin{array}{l}\text { Constant } \\
\text { Air } \\
\text { Monitor }\end{array}$ & $\begin{array}{l}\text { Stack } \\
\text { Monitor }\end{array}$ & $\begin{array}{l}\text { Liquid } \\
\text { Effluent } \\
\text { Monitor }\end{array}$ & $\begin{array}{c}\text { Personnel } \\
\text { Contamination } \\
\text { Monitor }\end{array}$ \\
\hline \multicolumn{6}{|l|}{ Location } \\
\hline $\begin{array}{c}\text { Can } \\
\text { Loading } \\
\text { Bay }\end{array}$ & & $x$ & & & $X$ \\
\hline $\begin{array}{l}\text { Control } \\
\text { Room }\end{array}$ & $\bar{x}$ & $x$ & & & $\bar{x}$ \\
\hline $\begin{array}{c}\text { Calcine } \\
\text { Storage } \\
\text { Room }\end{array}$ & $\bar{x}$ & & & & \\
\hline HVAC & $\begin{array}{c}X \\
\text { On Filter } \\
\text { Banks }\end{array}$ & $x$ & $x$ & & $\bar{x}$ \\
\hline $\begin{array}{c}\text { Main } \\
\text { Floor } \\
\text { Control } \\
\text { Area } \\
\end{array}$ & $\begin{array}{c}x \\
4 \text { for each } \\
\text { Train }\end{array}$ & $\begin{array}{c}x \\
4 \text { for each } \\
\text { Level }\end{array}$ & & $\begin{array}{c}x \\
\text { All drains }\end{array}$ & $\begin{array}{c}x \\
\text { At all exit points } \\
\text { from the facility. }\end{array}$ \\
\hline $\begin{array}{l}\text { Transfer } \\
\text { Tunnel }\end{array}$ & $\bar{x}$ & $\bar{x}$ & & $\bar{x}$ & \\
\hline $\begin{array}{c}\text { Electrical } \\
\text { Room }\end{array}$ & & $\bar{x}$ & & & $\bar{x}$ \\
\hline $\begin{array}{l}\text { Change } \\
\text { Rooms }\end{array}$ & & $\bar{x}$ & & & $\bar{x}$ \\
\hline $\begin{array}{c}\text { Outer } \\
\text { Offices }\end{array}$ & & & & & $\begin{array}{c}X \\
\text { Hand Held }\end{array}$ \\
\hline Elevator & & & & & $\begin{array}{c}X \\
\text { At exit Points }\end{array}$ \\
\hline
\end{tabular}




\section{Hot Cell Manned Entry Requirements}

The Hot cell manned entry requirements will change if the methods of processing or the configuration changes.

Although the facility will be designed for the maximum remote operation and maintenance, the cells will require manned entry to perform non-periodic maintenance. The facility as reierred to here is the process train area. The entry requirements for the facility are as follows:

- The facility shall be equipped with a breathing air system compliant with the applicable sections of 29CFR1910.

- The facility entrance will need to have a change out area that can isolate the operating area from the hot cells. This change out area should be air locked from the hot cells as a minimum with Zoned ventilation as defined in ANSI/ANS 57.81992.

- The cells shall be isolated from the access corridor by means of a door as a minimum. It is not necessary to airlock the cell entryways.

- The access corridor shall be wide enough to allow unencumbered access and egress. In addition, it is required that an outer pair of anti-contamination clothing be removed at the exit from the cell. The access corridor must be wide enough to allow an anti-c doffing station.

- An area large enough to store contaminated tools and equipment should be available inside of the contamination area.

- The access corridor will be maintained as a Contamination Area (CA) and Radiation Area (RA) in accordance with the requirements of 10CFR835. The cells will be controlled as High Contamination Areas (HCAs) and High Radiation Areas (HRAs) also in accordance with the requirements of 10CFR835.

- The cells must be capable of remote decon operations in addition to the requirements for manned entry decon. The cells shall have RCRA compliant drains and supporting systems.

- Fire suppression systems and alarms compliant with applicable codes and standards shall be available.

- The access corridor will need to be shielded from the cells. The shielding does not need to be as thick as the exterior walls. 


\section{Breathing Air Requirements}

Compressed air used for respiration shall comply with the quality requirements contained in 29CFR1910 Part 134(d). Breathing air shall meet at least the requirements of the specification for Grade D breathing air as described in the Compressed Gas Association Commodity Specification G-7.1-1966.

The Breathing Air supply that will provide air for airline respirators is contained in 29CFR1910 Part 134. The system should supply air to a minimum of two OSHA compliant manifolds capable of supporting 6 airlines. One system per train would be required. The system will need to be provided with backup compressed gas cylinder air. The standby air shall comply with 29CFR1910(d)(2)(ii). Alarms to indicate compressor failure and overineating would be required.

The breathing air system will need to be capable of supporting personnel in an atmosphere as defined by OSHA - Immediately Dangerous to Life or Health (ILDH).

Although not all cells in the facility will need the requirement for ILDH supply air, some of them will.

\section{Radiological Risk Evaluation of Process}

The process has several parts that could increase the risk of radiological exposure and should be further evaluated in the design process.

- The ventilation system must be adequate to contain the radiological contaminants. The Hot cell area must be controlled as a process contamination area. Supply air ducting and exhaust ducting will be routed to and from the cells. These cell penetrations are a potential source of radiation streaming. The design must include a shielding evaluation.

- The utility support penetrations into the cells are a potential source of radiation exposure outside the cells and into the operating corridors.

- The Transfer tunnel could potentially be a source of very high exposure if maintenance is required while calcine is stuck in the tunnel. Consideration should be given to a method of remotely removing cans stuck in transit.

- The insertion of calcine into the process cell will be a weak point in the process. Calcine in notorious for solidifying in lines. Lessons learned in the process development of transferring the calcine to the bin sets from the calciner should be considered in further development of this option. 
- Containment of the contaminants within the cell will be difficult. Spread of radiological contamination must be minimized through the use of ventilation and air locks.

\section{Summary}

The evaluation and recommendations of this EDF are based on existing technology and proven techniques and methods for handling highly radioactive material. The lessons learned from the NWCF Calciner, GSF canning station and the FSA fuel handling areas were evaluated for inclusion in this document. The process and facilities as currently defined provide a strong measure of radiological control and will maintain personnel exposures ALARA. Further studies should consider the recommendations and concerns as defined in this EDF. 
Project File Number $\quad$ O2BEO

Project/Task Waste Treatment Project Feasibility Studies

Subtask Direct Cementitious Waste Option Scoping Study

\begin{tabular}{|c|c|c|c|c|c|}
\hline \multicolumn{6}{|c|}{ Title: $\quad$ Canister Loading } \\
\hline \multicolumn{6}{|l|}{ Summary: } \\
\hline \multirow{2}{*}{\multicolumn{6}{|c|}{$\begin{array}{l}\text { This EDF documents the total number of canisters to be filled, canister } \\
\text { throughput rate in the grout facility, and canister weight loading. } \\
\text { References: } \\
\text { 1. EDF-FDO-001, Estimates of Feed and Waste Volumes, Compositions and Properties, } \\
\text { C.M. Barnes, October, } 1997 \\
\text { 2. Savannah River Site HLW Canister drawing, ORNL DWG 90-418 }\end{array}$}} \\
\hline & & & & & \\
\hline \multirow{2}{*}{\multicolumn{6}{|c|}{$\begin{array}{l}\text { Conclusions: } \\
\text { Number of canisters to be filled }=18,000 \\
\text { Loaded canister weight }=1700 \mathrm{~kg}(3748 \mathrm{lb}) \\
5 \text { year schedule throughput }=72 \text { canisters per week } \\
20 \text { year schedule throughput }=18 \text { canisters per week } \\
\text { Distribution (complete package): WTP EIS Studies Library, R. E. Dafoe M.S. 3765, D. J. } \\
\text { Harrell M. S. } 3211, \text { B. R. Helm M. S. } 3765 \text {, S. J. Losinski M.S. 3625, K. L. Williams M. S. } \\
3765\end{array}$}} \\
\hline & & & & & \\
\hline \multicolumn{6}{|c|}{ Distribution (summary package only): } \\
\hline Ayther & $\begin{array}{l}\text { Dept. } \\
\text { i//30. }\end{array}$ & Reviewyed & $\begin{array}{l}\text { Date } \\
12 / 9197\end{array}$ & $\begin{array}{l}\text { Approved } \\
\text { Ayl Losinsti }\end{array}$ & \\
\hline 12t & & $\begin{array}{l}\text { LMITCO } \\
\text { Review }\end{array}$ & Date & $\begin{array}{l}\text { LMITCO } \\
\text { Approval }\end{array}$ & Date \\
\hline
\end{tabular}


The following statistics relating to calcine and the waste loading percentage, grout density, and resulting grout volume for the Direct Cemetitious Waste Option are discussed and documented in EDF-FDO-001, Rev 1. The calcine volume and density are provided in ranges, but for this and the other non-separations options, program direction requires the use of the quantities listed below.

Calcine volume $=5435 \mathrm{~m}^{3}$

Calcine density $=1408 \mathrm{~kg} / \mathrm{m}^{3}$

Waste Loading $=35 \mathrm{wt} \%$

Grout density $=1700 \mathrm{~kg} / \mathrm{m}^{3}$

Grout volume $=12,860 \mathrm{~m}^{3}$

The Savannah River Site canister fill volume capacity is listed as $25.3 \mathrm{ft}^{3}\left(0.72 \mathrm{~m}^{3}\right)$ as shown on the drawing. Empty canister weight is listed at $1000 \mathrm{lb}(454 \mathrm{~kg})$.

Based on the above information the following results are obtained:

Number of canisters produced $=12,860 \mathrm{~m}^{3} / 0.72^{3} /$ canister $=17,861$

Rounded to 2 significant digits $=18,000$ canisters

Loaded canister weight $=1700 \mathrm{~kg} / \mathrm{m}^{3} \times 0.72 \mathrm{~m}^{3} /$ canister $+454 \mathrm{~kg}$

$$
=1224 \mathrm{~kg}+454 \mathrm{~kg}=1678 \mathrm{~kg}
$$

Rounded to 2 significant digits $=1700 \mathrm{~kg}(3748 \mathrm{lb})$ per canister

Throughput for a 5 year schedule $=18,000$ canisters $/ 5 y r / 52$ weeks $=69.2$ canisters per week.

To accommodate using even numbers, say that for a 4 day work week, 18 canisters a day are required, or 72 canisters per week.

Throughput for a 20 year schedule is $1 / 4$ of the 5 year and is 18 canisters per week. 
Project File Number $\quad 02 B D 7$

Project/Task Waste Treatment Facility Study

Subtask Retrieve calcine from CSSFs and deliver it to the Waste Treatment Facility

Title: Calcine Retrieval and Transportation

Summary:

A calcine retrieval and transportation system is presented to retrieve calcine from the CSSFs and transport it to the Waste Treatment Facility. The calcine retrieval and transportation system is designed to supply calcine to the treatment options currently understudy (Cementitious Waste Option (CWO), Direct Cementitious Waste Option (DCWO), Hot Isostatic Press (HIP) Waste Option (HWO), Vitrification Waste Option (VWO), and TRU Separations options). The system is divided into three subsystems: CSSF access method, calcine retrieval system, and calcine transportation system. During CSSF access, the buildings, equipment, and piping are removed from the superstructure of each CSSF. Retrieval risers are installed and accessed. The CSSFs are prepared for calcine retrieval. The calcine retrieval system presents a viable method to retrieve calcine from the CSSFs. The system relies on an air jet and a suction nozzle. The calcine transportation system is a pneumatic system similar to one currently used at the ICPP for transportation of calcine. A process data sheet and cost estimate were developed for the calcine retrieval and transportation system.

Three cost estimates are presented to meet the needs of each waste treatment option. Each waste treatment option requires the same access activities for the seven CSSFs. The retrieval method is the same for each waste treatment option. The locations of the waste treatment facilities developed by each option necessitates variations in the transport system. The length of the transport system is the basic difference between the transport systems. The cost estimate includes costs associated with removing corrosion coupons prior to retrieving the calcine and installing D\&D risers after retrieval activities are complete.

The first cost estimate was developed to meet the 5-year operating schedule of the Cementitious Waste Option (CWO). This system delivers calcine from the CSSFs to an NWCF addition. A second estimate was developed to deliver calcine to the TRU Separations options' calcine dissolution facility. A third cost estimate for the calcine retrieval and transportation system is presented for the DCWO, HWO, and VWO options which require an intermediate transport station (ITS) to deliver the calcine to the Waste Treatment Facility.

The CWO option has a five year operating period that begins $1 / 1 / 2013$. The total unescalated cost for the calcine retrieval and transportation system is $\$ 176,566,000$. The total cost including escalation, management reserve, and contingency is $\$ 348,880,000$. The discounted annual cost is $\$ 166,409,000$.

The TRU-Separations Options have twenty year operating periods that begin $1 / 1 / 2013$. The total unescalated cost for the calcine retrieval and transportation system is $\$ 237,389,000$. The total 
Project File Number $\quad 02 B D 7$

cost including escalation, management reserve, and contingency is $\$ 531,023,000$. The discounted annual cost is $\$ 192,309,000$.

The DCWO, HWO, and VWO options have twenty year operating periods that begin 1/1/2013. The total unescalated cost for the calcine retrieval and transportation system is $\$ 243,039,000$. The total cost including escalation, management reserve, and contingency is $\$ 543,371,000$. The discounted annual cost is $\$ 196,878,000$.

The scope of this study was limited to the Fluor-Daniels feasibility design. The purpose was to compare this system directly to the Fluor-Daniels system. However, two issues that warranted further review and inclusion in the cost estimate were identified. Separate cost estimates were developed for the removal of corrosion coupons from the bins and installation of D\&D risers.

Distribution (complete package): A complete copy of this EDF will be included in the following reports:

R. E. Dafoe, Direct Cementitious Waste Option Study Report, INEEL/EXT-97-01399, February 1998.

W. H. Landman, TRU Separations Options Study Report, INEEL/EXT-97-01428, February 1998.

A. E. Lee, Cementitious Waste Option Preliminary Study Report, INEEL/EXT-9701400, February 1998.

D. A. Lopez, Vitrified Waste Option Study Report, INEEL/EXT-97-01389, February 1998.

N. E. Russell, Hot Isostatic Press (HIP) Waste Option Study Report,INEEL/EXT-9701392 , February 1998.

Distribution (summary package only):

\begin{tabular}{|l|l|l|l|l|l|}
\hline $\begin{array}{l}\text { Author } \\
\text { S.E. Gifford } \\
\text { Sarac.giffyid } \\
2 / 3 / 98\end{array}$ & $\begin{array}{l}\text { Dept. } \\
\text { M C\&I E }\end{array}$ & $\begin{array}{l}\text { Reviewed } \\
\text { Heforsythe }\end{array}$ & $\begin{array}{l}\text { Date } \\
\text { K. L. Williams }\end{array}$ & Date \\
&
\end{tabular}

See Management Control Procedure (MCP) 6 for instructions on use of this form. 


\section{Outline}

'1.0 Introduction

1.1 Background

1.2 Purpose and Scope

2.0 Design Basis

2.1 Design Criteria

2.2 Key Assumptions

2.3 Requirements

3.0 Process Design

3.1 CSSF Access

3.1.1 Process Basis

3.1.2 Process Description

3.1.3 Process Equipment Description

3.1.4 Process Issues

3.2 Calcine Retrieval

3.2.1 Process Basis

3.2.2 Process Description

3.2.3 Process Equipment Description

3.2.4 Process Issues

3.3 Calcine Transport

3.3.1 Process Basis

3.3.2 Process Description

3.3.3 Process Equipment Description

3.3.4 Process Issues

3.4 Implementation

4.0 Input to Project Data Sheet

5.0 Program Schedule

6.0 Costs

7.0 Recommendations for Further Study

8.0 Uncertainties

8.1 Maturity of Technology

8.2 Risk Assessment

8.2.1 Project Risk

8.2.2 Technical Risk

8.2.3 ES\&H Risk

8.3 Failure Modes

8.4 Cost Estimate Uncertainties

9.0 Potential Impacts of NRC Licensing

10.0 Summary and Conclusions

Appendices
A. Drawings
B. References
C. Equipment List 
$431.02 \#$

$01 / 29 / 98$

Rev. $\# 00$

\section{ENGINEERING DESIGN FILE}

D. Background Information for Project Data Sheet

E. Risk Assessment Data Sheets

F. Cost Estimate
Function File Number - SPR-WTS-01

EDF Serial Number - EDF-WTS-002

Page 4 of 62 


\section{Acronyms}

CSSF Calcined Solids Storage Facility

CWO Cementitious Waste Option

DCWO Direct Cementitious Waste Option

HAW High Activity Waste

HLW High Level Waste

HWO Hot Isostatic Pressing (HIP) Waste Option

ICPP Idaho Chemical Processing Plant

INEEL Idaho National Engineering and Environmental Laboratory

ITS Intermediate Transport Station

LAW Low Activity Waste

NRC Nuclear Regulatory Commission

NWCF New Waste Calcining Facility

SBW Sodium Bearing Waste

VDA Vertical Deployment Apparatus

VIC

VWO

WCF

WTF

WTS

Ventilation Instrumentation and Control Building

Vitrified Waste Option

Waste Calcining Facility

Waste Treatment Facility (generic facility name for the facility developed in each waste treatment option)

Waste Treatment Study 


\subsection{Introduction}

At the ICPP, a fluidized bed calcination process changes the chemical composition of high-level radioactive mixed liquid waste generated from the reprocessing of spent nuclear fuel and sodium bearing waste (SBW) generated from decontamination activities. The calcination process converts the liquid waste to a solid waste and reduces the volume of the waste by a factor of 7 . After the calcination process, the resulting solid waste, called calcine, is pneumatically transported from the calciner to one of seven storage facilities, named calcined solids storage facilities (CSSF).

The settlement agreement between the Department of Energy and the State of Idaho mandates that high level waste be ready for removal from Idaho by a target date of 2035 for disposal. The calcine in the CSSFs must be retrieved from the CSSFs and treated. This EDF details a method to access and prepare the CSSFs for calcine retrieval, a calcine retrieval system, and a transport system to deliver the calcine to the Waste Treatment Facility. The information presented is applicable to the non-separations waste treatment options (DCWO, HWO, and VWO) and the TRU Separations waste treatment options.

\subsection{Background Information}

The calcine is stored in cylindrical steel bins within a CSSF. The number of separate, self contained bins in a CSSF varies from 3 to 7 . The bins are either cylindrical or annular. The outside diameter of the bins is approximately $12 \mathrm{ft}$. The length of the bins range from $24 \mathrm{ft}$ to 61 $\mathrm{ft}$. A passive convection cooling system is used to cool the bins inside a large concrete vault. Above the bin vault are structures that house the necessary equipment to receive the calcine. These structures form the superstructure of each CSSF. Figure 1 shows a sketch of each CSSF.

Calcine production began in November $1963^{1}$. The first six CSSFs store several forms of calcine. Currently, CSSF 6 is being filled while CSSF 7 remains empty. As of 1996, the CSSFs housed approximately $134,500 \mathrm{ft}^{3}$ of calcine. There are three main calcine types: alumina calcine, zirconia calcine, and calcine blends. The amount of calcine stored in each CSSF is shown in Table 1. The information for this Table was taken from reference 1. 

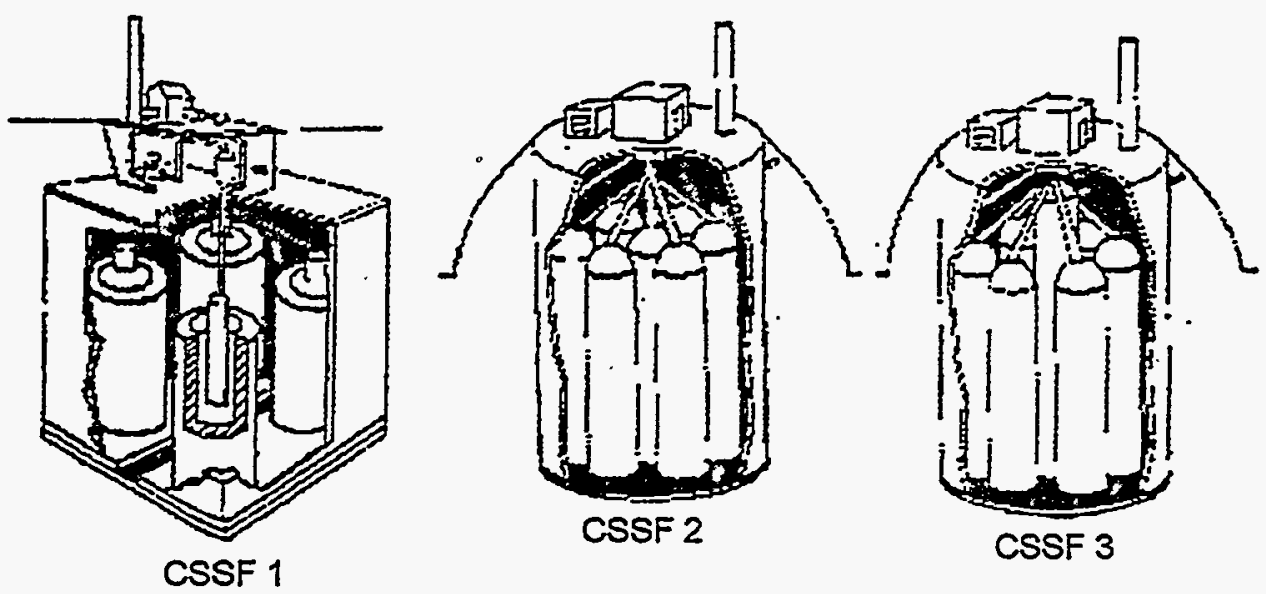

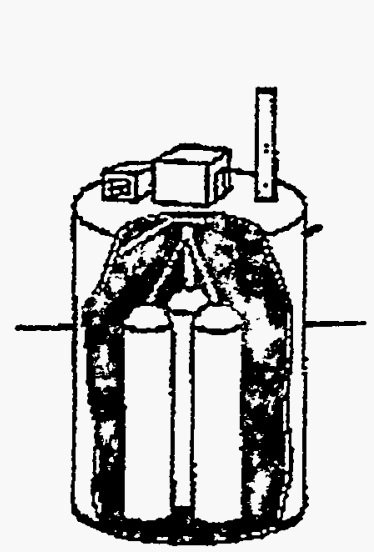

CSSF 4

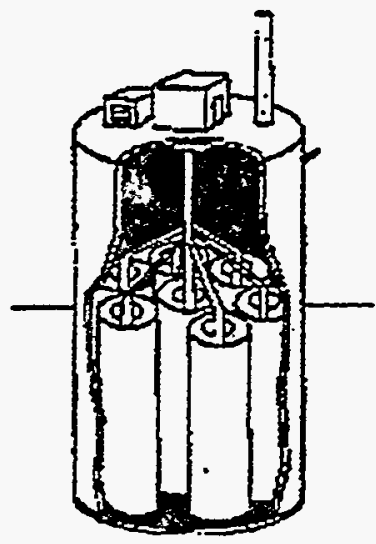

CSSF 5

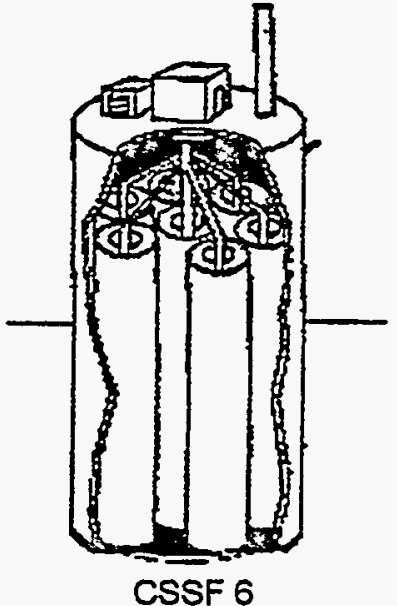

CSSF 6

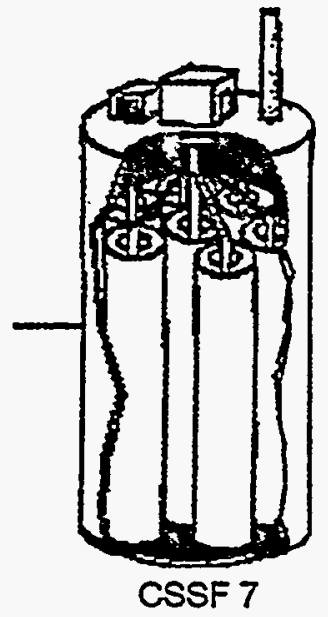

Figure 1. Calcined Solids Storage Facilities (CSSFs).

Table 1. Volume of Calcine Type in Each CSSF As of 1997.

\begin{tabular}{|c|c|c|c|c|c|c|}
\hline CSSF & $\begin{array}{c}\text { Alumina } \\
\text { Calcine } \\
\left(\mathrm{ft}^{3}\right)\end{array}$ & $\begin{array}{c}\text { Zirconia } \\
\text { Calcine } \\
\left(\mathrm{ft}^{3}\right)\end{array}$ & $\begin{array}{c}\text { Other } \\
\left(\mathrm{ft}^{3}\right)\end{array}$ & $\begin{array}{c}\text { Calcine } \\
\text { Blends } \\
\left(\mathrm{ft}^{\hat{3}}\right)\end{array}$ & $\begin{array}{c}\text { Cold/ } \\
\text { Dolomite } \\
\left(\mathrm{ft}^{3}\right)\end{array}$ & $\begin{array}{l}\text { Total } \\
\left(\mathrm{ft}^{3}\right)\end{array}$ \\
\hline & & & & Description & & \\
\hline 1 & 7292 & & & & 373 & 7665 \\
\hline 2 & 10,754 & 18,582 & & & 900 & 30,236 \\
\hline 3 & 2,250 & 24,844 & 50 & $\begin{array}{c}5,580 \\
\mathrm{Zr}-\mathrm{SBW}\end{array}$ & 5,810 & 38,534 \\
\hline$\overline{4}$ & & 5,210 & & $\begin{array}{c}11,130 \\
1 \% \mathrm{Al}-\mathrm{Zr} \& \\
99 \% \mathrm{Zr}-\mathrm{SBW}\end{array}$ & 910 & 17,250 \\
\hline 5 & & & 50 & $\begin{array}{c}31,303 \\
\mathrm{~A} \mathrm{l}-\mathrm{Zr}-\mathrm{SBW}\end{array}$ & 3,670 & 35,023 \\
\hline 6 & & & & $\frac{5,010}{\mathrm{~A} \mathrm{l}-\mathrm{Z} \mathrm{r}-\mathrm{SBW}}$ & 730 & 5,740 \\
\hline 7 & & & & & & 0 \\
\hline
\end{tabular}




\subsection{Purpose and Scope}

The purpose of this study is to define a calcine retrieval and transportation plan that is compatible with the calcine processing options currently under study by the WTF program (namely the CWO, DCWO, HWO, VWO, and the TRU Separations Options). The plan will consist of accessing the calcine in each CSSF, removing the calcine from the bins, and transporting the calcine from each CSSF to the Waste Treatment Facility (the processing facility developed in each option study). Three versions of this plan were necessary to meet the needs of each waste treatment option. The equipment necessary for CSSF access, calcine retrieval and transportation will be approximately sized. A cost estimate for each option will be developed. The cost estimate will include capital equipment costs as well as operating and maintenance costs over the life of the project. As necessary, sketches will be included to clarify the systems and processes developed.

The scope of EDF is limited to the scope of the Fluor-Daniels ${ }^{2}$ design for the calcine retrieval and transportation system as presented in reference 2 . The design presented in this EDF should not seek to define unique systems to accomplish the overall tasks. Modifications to the systems presented in the Fluor-Daniels ${ }^{2}$ design should only be made to accommodate the unique needs of the five processing options currently under study. The issues and recommendations for future study identified during this study will be documented. Additional activities, that are necessary to fully implement the calcine retrieval and transportation system but are beyond the scope of the Fluor-Daniels design, will be included in the cost estimate.

\subsection{Design Basis}

Although the overall design is based on the Fluor-Daniels ${ }^{2}$ design, design criteria and key assumptions were made. The requirements that must be met are explained in section 2.3 . The criteria, assumptions, and requirements were independently developed and applied to the calcine retrieval and transportation system.

\subsection{Design Criteria}

The design criteria are listed below. All portions of the design must satisfy criteria in the general category. These criteria are essential to satisfy ES\&H goals.

\section{General:}

1. Minimize worker radiation exposure and spread of contamination.

2. The systems should be designed to withstand any credible fire or other applicable accidents and still serve as a confinement barrier.

3. The systems should be designed to withstand appropriate natural phenomena hazards.

4. Provide primary and secondary confinement at all times while minimizing the confinement volumes.

5. Adequately heat and cool occupied areas of enclosures.

6. Provide instrumentation and control for operation and data acquisition. 
7. Facility equipment and calcine retrieval and transport systems must be capable of being decontaminated and/or replaced safely and easily.

8. The systems should minimize the generation of hazardous wastes.

\section{$\underline{\text { Retrieval: }}$}

1. Minimize the amount of remote mechanical equipment placed in the bins. In effect, minimize the potential for equipment breakdown.

2. The bin pressure must remain slightly more negative than the surrounding vault space during retrieval. Controls are required to maintain the negative-pressure confinement.

3. Calcine will be retrieved from one bin in a CSSF a time. Although, calcine from more than one CSSF can be retrieved from at a given time.

4. Retrieval will be performed only if the transportation systems are operational.

5. Retrieve and deliver calcine to the Waste Treatment Facility according to the demand of the process.

\section{Transportation:}

1. Design the transportation system for a 30 year service life.

2. The transportation system should be readily maintainable.

3. Provide the appropriate amount of rod-out stations for the pneumatic transport system.

\subsection{Key Assumptions}

The scope of this study mandates the first and foremost assumption is that Fluor-Daniels ${ }^{2}$ has developed a viable and competitive option for calcine retrieval and transportation. The remaining assumptions are outlined below. They are divided into three categories: CSSF access, calcine retrieval, and transportation. A basis for each assumption is provided.

\begin{tabular}{|c|c|}
\hline Assumption & Basis \\
\hline \multicolumn{2}{|l|}{ CSSF Access: } \\
\hline $\begin{array}{l}\text { Installation of risers can be accomplished } \\
\text { with little modification to existing } \\
\text { technology. }\end{array}$ & $\begin{array}{l}\text { Similar risers were installed on hazardous } \\
\text { waste bins. The technology was developed } \\
\text { by West Valley Nuclear, Inc. and applied } \\
\text { to the CSSFs by Raytheon Engineers and } \\
\text { Constructors. } \\
\text { References: } 3 \text { and } 4\end{array}$ \\
\hline $\begin{array}{l}10 \% \text { of the ground fill removed for } \\
\text { construction and CSSF } 1 \text { access is } \\
\text { contaminated. }\end{array}$ & References: 2 and 3 \\
\hline $\begin{array}{l}\text { Transport piping from WCF and NWCF to } \\
\text { the CSSFs will be decontaminated as }\end{array}$ & $\begin{array}{l}\text { Closure plans of WCF and NWCF } \\
\text { Reference: } 5 \text { and } 6\end{array}$ \\
\hline
\end{tabular}




\begin{tabular}{|c|c|}
\hline $\begin{array}{l}\text { outlined in the WCF and NWCF closure } \\
\text { plans. This piping will remain accessible } \\
\text { for D\&D without endangering personnel. }\end{array}$ & \\
\hline $\begin{array}{l}\text { Access activities do not reduce CSSF } \\
\text { integrity. Additionally, superstructure } \\
\text { demolition reduces static stress on the } \\
\text { CSSF. }\end{array}$ & $\begin{array}{l}\text { Shoring activities will occur during fill } \\
\text { removal. The Raytheon study analyzed the } \\
\text { roof of CSSF } 1 \text { and found that these } \\
\text { activities would help to stabilize CSSF } 1 \text {. } \\
\text { Reference: } 3\end{array}$ \\
\hline $\begin{array}{l}\text { An adequate place for retrieval riser } \\
\text { attachment can be located on each bin. } \\
\text { Piping inside the bin vault will not interfere } \\
\text { with riser location. }\end{array}$ & $\begin{array}{l}\text { The exact location of retrieval risers was } \\
\text { not determined because it is highly } \\
\text { dependent on the requirements of the riser } \\
\text { welding method. This method is currently } \\
\text { being studied at the ICPP. However, } \\
\text { locations for } 40 \text { retrieval risers were found } \\
\text { for CSSF } 1 \text {. This number far exceeds the } \\
24 \text { retrieval risers required for CSSF1. The } \\
\text { remaining CSSFs at most require an } \\
\text { additional } 8 \text { retrieval risers to be installed. } \\
\text { Reference: } 3\end{array}$ \\
\hline $\begin{array}{l}\text { Bins will not be structurally weakened by } \\
\text { the attachment of retrieval risers. Measures } \\
\text { to avoid this (support retrieval riser weight } \\
\text { above bin and installation of a self- } \\
\text { supporting floor) can be taken but are not } \\
\text { documented. }\end{array}$ & Reference: 3 and 4 \\
\hline $\begin{array}{l}\text { Remote equipment can be sized } \\
\text { appropriately from equipment developed } \\
\text { for previous projects. }\end{array}$ & Reference: 4 \\
\hline \multicolumn{2}{|l|}{ Retrieval: } \\
\hline $\begin{array}{l}\text { All types of calcine in all the bins are } \\
\text { retrievable as a dilute phase using the } \\
\text { retrieval method provided. }\end{array}$ & Reference: 2 \\
\hline $\begin{array}{l}\text { The retrieval method is a viable option for } \\
\text { all bin shapes. This is pertinent to the } \\
\text { cylindrical bins. }\end{array}$ & $\begin{array}{l}\text { Reference: } 8 \text { and S. E. Gifford, telephone } \\
\text { conversation with Dan Griffith, INEEL. }\end{array}$ \\
\hline $\begin{array}{l}\text { Relocation of retrieval equipment (VDA } \\
\text { and jumper) from one CSSF to another will } \\
\text { take approximately } 1 \text { week. }\end{array}$ & $\begin{array}{l}\text { In the Fluor-Daniels }{ }^{2} \text { cost estimate } \\
\text { relocation of the confinement enclosure } \\
\text { and all associated equipment is } \\
\text { accomplished in two weeks. } \\
\text { Reference: } 2\end{array}$ \\
\hline $\begin{array}{l}\text { Calcine is assumed to have remained } \\
\text { unchanged. This mainly means that it has } \\
\text { not agglomerated in any of the CSSFs. }\end{array}$ & $\begin{array}{l}\text { This assumption allows the same retrieval } \\
\text { equipment design to be used in all the } \\
\text { CSSFs. Samples from CSSF } 2 \text { indicate } \\
\text { that the calcine can remain unchanged }\end{array}$ \\
\hline
\end{tabular}




\begin{tabular}{|l|l|}
\hline & $\begin{array}{l}\text { during storage. Samples from all CSSFs } \\
\text { are necessary to validate this assumption } \\
\text { for future studies. } \\
\text { Reference: } 7\end{array}$ \\
\hline $\begin{array}{l}\text { During calcine retrieval no less than 95\% } \\
\text { of the calcine in a bin will be removed. } \\
\text { Beyond this level, as much of the calcine as } \\
\text { is reasonable will be retrieved. }\end{array}$ & $\begin{array}{l}\text { The 95\% of the calcine in a bin has been } \\
\text { demonstrated to be retrievable at a high } \\
\text { rate. The next 4.7\% of the calcine is } \\
\text { retrievable at a significantly lower rate. } \\
\text { Reference: } 8\end{array}$ \\
\hline $\begin{array}{l}\text { No internal obstructions (stiffening rods, } \\
\text { thermowells, etc.) will interfere with } \\
\text { extending the retrieval lines into the bins. }\end{array}$ & $\begin{array}{l}\text { The internal obstructions are well } \\
\text { documented. It is anticipated that the } \\
\text { retrieval risers can be located to avoid these } \\
\text { obstructions. The scope of this study does } \\
\text { not permit an in depth examination of this } \\
\text { issue. } \\
\text { Reference: INEEL drawings }\end{array}$ \\
\hline $\begin{array}{l}\text { No miscellaneous materials will enter the } \\
\text { suction nozzle. Where possible, such } \\
\text { material will be removed from the bins } \\
\text { prior to retrieval activities. }\end{array}$ & $\begin{array}{l}\text { The scope of this study does not permit an } \\
\text { in depth examination of this issue. } \\
\text { However, this is necessary that } \\
\text { miscellaneous materials present in the bins } \\
\text { do not enter the transportation system. } \\
\text { References: } 3 \text { and S. E. Gifford, separate } \\
\text { conversations with Dan Griffith and Dan } \\
\text { Staiger, INEEL. }\end{array}$ \\
\hline $\begin{array}{l}\text { Transportation: } \\
\text { including the cyclone, should be reinforced } \\
\text { with nitronic wear plates to prevent erosion } \\
\text { failures. }\end{array}$ & $\begin{array}{l}\text { Reference: } 3 \\
\text { the concrete chase and earthen berm are } \\
\text { adequate to meet the radiological shielding } \\
\text { needs of dilute phase calcine. }\end{array}$ \\
$\begin{array}{l}\text { All directional changes will utilize blinded } \\
\text { Reference: } 11\end{array}$ \\
\hline
\end{tabular}

\subsection{Requirements}

Statutory law, DOE orders, and the Batt Agreement establish the requirements for the calcine retrieval and transportation system. These requirements are examined in reference 9. 


\subsection{Process Design}

The process design has been split into three sections to provide clarity to the description. CSSF access (section 3.1) will prepare each bin for calcine retrieval by decontaminating the CSSF superstructure and installing or accessing the retrieval risers. Calcine retrieval (section 3.2) will remove the calcine from each bin and place it directly in the transportation system. The transportation system (section 3.3) will deliver the calcine to the Waste Treatment Facility and provide the motive for $\mathrm{r}$. A basis, process description, equipment description, and process issues are detailed for each section of the design.

\subsection{CSSF Access}

\subsubsection{Process Basis}

CSSF access prepares each bin for calcine retrieval by reducing radiation exposure, adding necessary retrieval risers, and accessing existing retrieval risers. The CSSFs will be prepared for calcine retrieval by erecting permanent confinement enclosures. The access method presented by Fluor-Daniels ${ }^{2}$ was originally developed in the Raytheon ${ }^{3}$ design for CSSF 1 . The specific details for each CSSF may vary but the overall the process is the same for all CSSFs.

\subsubsection{Process Description}

The primary goal of this phase of the retrieval and transport system is to prepare the CSSFs for retrieval. Bin vault ventilation systems will be replaced, confinement enclosures will be constructed, and retrieval lines will be installed and accessed. All modifications to the CSSFs and construction of new buildings will comply with the general design criteria outlined in reference 10. The CSSF access process is outlined in 9 steps. In order to protect the integrity of each CSSF some of these steps may overiap in the schedule.

1. Earthwork. Extensive excavation of CSSF 1 will expose the superstructure down to the bin vault roof. The excavation necessary to reach CSSF 1 will affect the earthen berms of CSSFs 2 and 3. Retaining walls must be installed to preserve these shielding berms. The uncontaminated portion of excavated fill, which must be covered, is estimated at $90 \%$. The contaminated fill must be disposed of appropriately. This work begins in an uncontaminated work area. Shoring of the CSSFs, construction of the retaining wall for CSSF 1, and removal of $10 \%$ contaminated fill require work in a radiation zone. Therefore, shielded or remote equipment should be used during this step.

An equipment ramp must be built to CSSF 1. This ramp will be used during the installation of the bridge crane and the construction of the confinement buildings. For CSSF 2-7, a mobile crane will place the equipment on the CSSF roof. This work will primarily occur under a temporary decontamination tent. The cooling air stacks will interfere with locating the confinement enclosure on the bin vault roof. Relocation of the cooling air stacks will begin in this step. 
2. Construct ventilation, instrumentation, and control (VIC) building for each CSSF. The primary purpose of the VIC building is to house the ventilation, instrumentation, and control equipment. It will be a pre-manufactured steel building placed adjacent to each CSSF. During this step the HVAC, instrumentation, and control equipment will be installed. The HVAC equipment will regulate and decontaminate air from the bin vault, confinement enclosure, and this building. The interface between the VIC building and confinement enclosure for each CSSF is shown in sketch CRT-01. Temperature control will be maintained in the control room and operating areas with this system. Air flow will be monitored to minimize the risk of contamination spread by circulating air from low risk areas to high risk areas. Sampling ports will be provided to ensure the HVAC equipment is compliant with the applicable standards.

Installation of instrumentation will consist of relocating panels and rewiring because the instrumentation is functional in each CSSF. The control room will house the remote operations control and instrumentation. It will allow for internal viewing of the bins through the CCTV system as well as aide in installation of retrieval risers. Construction of this building includes floor, lights, windows, doors, $\mathrm{OH}$ doors, insulation, and fire protection. For all the CSSFs, this building will have the same layout and size. The VIC building for all CSSFs will require electricity, water, heating and/or cooling, breathing air, and plant air.

3. Construct confinement enclosure for each CSSF. The confinement enclosure is a nonreactor nuclear facility. It is pictured in figure 2 as it would appear on CSSF 5, 6, or 7 . For CSSFs 1,2,3, and 4 the confinement enclosure will be constructed on the roof of the bin vault. It acts as a confinement barrier during retrieval activities. This enclosure is a premanufactured steel building. This enclosure also includes ladder, guardrail, steel plate, and structural steel. It will be complete with fire protection equipment, lighting, equipment wiring and CCTV capabilities. The interior of the confinement enclosure will be coated with strippable coatings for decontamination purposes.

A negative pressure will be maintained inside the enclosure in order to reduce the risk of contamination spread. The confinement enclosure will house a bridge crane, core drilling platform, welding equipment, vertical deployment equipment, and shielded jumpers. The bridge crane will be installed for use during decontamination of the superstructure equipment and piping, core drilling procedures, and calcine retrieval.

This building will be decontaminated several times throughout the calcine retrieval process. The confinement enclosures for all the CSSFs will require electricity, water, breathing air, plant air, high pressure steam, service water, and instrument air. After construction of the confinement enclosure, the temporary confinement tent over the construction site may be removed.

4. Access vaults that require decontamination and decommissioning. In this step, equipment inside the vaults will not be disturbed. CSSFs $1,2,3$, and 4 require installation of access ways to the vaults. This will be accomplished by core drilling into the inaccessible rooms. Existing access ways to the vaults (CSSFs 5, 6, and 7) will be cleared. Necessary portable shielding should be placed at the vault entry locations. For all CSSFs, external 


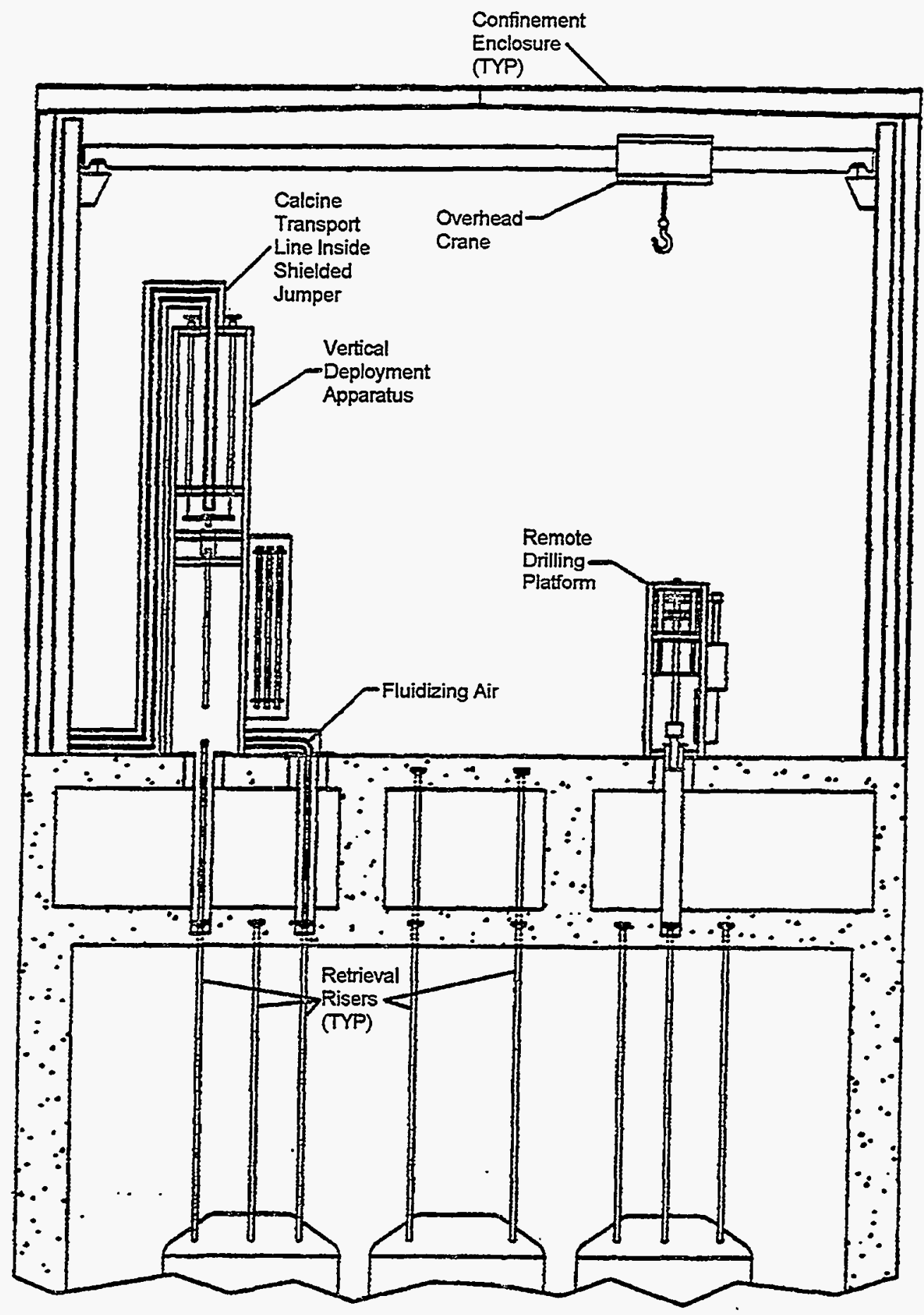

Figure 2. Confinement Enclosure and equipment pictured for CSSF 5,6 , or 7 . 
structures and equipment such as corrugated metal buildings, cooling stack ductwork, cooling air blowers, and their associated ductwork will be removed. As necessary, installation of plugs in ductwork penetrations will reduce worker exposure. Lead brick and conduit will be removed during this step. This work will be performed in a low radiation zone.

5. Decontaminate superstructure vaults and rooms. The vaults and rooms above the large concrete vault housing the bins comprise the superstructure of the CSSFs. The cyclone vault, equipment vault, instrument room, fan room, inlet plenum room, and exhaust plenum room will be decontaminated during this step. The vault that contains the calcine storage bins will not be decontaminated during CSSF access. Portable shielding should be placed in the vaults to reduce the exposure to acceptable limits. Remotely vacuum vault floors before entering with HEPA filtered vacuums. These vacuums have the ability to remove small debris and dust but not large objects. Strippable coating should be applied to the walls and floors of vaults that will be entered. Cut existing transport, probe, off-gas, rod out, monitoring and other nonessential piping that penetrate vault and room floors. The cut lines should then be plugged to prevent contamination spread during calcine retrieval. In CSSF 1,2,3, and 4 the pipes must be accessible after the new concrete floor is poured for closure activities. Access to the pipes should be provided in a similar manner to the accesses of existing retrieval lines in CSSFs 5, 6, and 7.

Once access to the vaults has been gained, decontamination activities begin. These activities require extensive shielding if it is to be accomplished manually. A better alternative is to employ remote equipment. This remote equipment must be designed and tested. The relative level of radiation in the superstructure vault is shown in Table 2 . These relative levels of radiation are based on the function of the vault and if calcine was ever present in the vault. In the high radiation areas, shielding concrete floors should be poured before temporary shielding is installed. Furthermore, Table 2 notes vaults that may have solids accumulations ${ }^{2}$. These accumulations are a result of damage to transport piping during filling of several CSSFs. Efforts may have been made to clean and repair these vaults but a conservative approach is to assume these vaults are extremely contaminated.

During this step, piping, vessels, and conduit will be disassembled and packaged in appropriate containers. The bridge crane will be used to remove the packages from the CSSF. Wall penetrations should be plugged when the piping is removed. Contaminated lines that lead away from the CSSFs (such as transport lines from the WCF or the NWCF) should be decontaminated and flushed. Flushing activities are discussed in section 3.1.4. 
Table 2. Assumed Bin Vault Relative Radiation Levels

\begin{tabular}{|c|c|c|c|}
\hline $\mathrm{CSSF}$ & $\begin{array}{c}\text { Low } \\
\text { Radiation } \\
\text { Zone } \\
\end{array}$ & $\begin{array}{c}\text { High } \\
\text { Radiation } \\
\text { Zone } \\
\end{array}$ & $\begin{array}{c}\text { Known } \\
\text { Solids } \\
\text { Accumulations } \\
\end{array}$ \\
\hline 1 & Ventilation Exhaust Room & Cyclone Vault & \\
\hline 2 & Instrument Building & Cyclone Vault & Cyclone Vault \\
\hline$\overline{3}$ & $\begin{array}{l}\text { Instrument Building } \\
\text { Equipment Vault }\end{array}$ & Cyclone Vault & Cyclone Vault \\
\hline 4 & Instrument Building & $\begin{array}{l}\text { Cyclone Vault } \\
\text { Ventilation } \\
\text { Equipment Room }\end{array}$ & \\
\hline 5 & $\begin{array}{l}\text { Instrument Room } \\
\text { Access Cell }\end{array}$ & Cyclone Vault & \\
\hline 6 & $\begin{array}{l}\text { Instrument Room } \\
\text { Fan Room } \\
\text { Inlet Plenum Room } \\
\text { Exhaust Plenum Room }\end{array}$ & $\begin{array}{l}\text { Cyclone Vault } \\
\text { Off-Gas Filter Room }\end{array}$ & \\
\hline 7 & $\begin{array}{l}\text { Instrument Room } \\
\text { Fan Room } \\
\text { Inlet Plenum Room } \\
\text { Exhaust Plenum Room }\end{array}$ & $\begin{array}{l}\text { Cyclone Vault } \\
\text { Off-Gas Filter Room }\end{array}$ & \\
\hline
\end{tabular}

6. Demolish CSSF superstructure. This step will only be conducted for CSSF 1 . The superstructures of CSSF 2,3, and 4 will have been removed during step 1 , as the superstructure enclosures are metal buildings and not concrete. During step 5 , the superstructure vaults of CSSF 5, 6, and 7 will be decontaminated. The superstructures of the last 3 CSSFs are much more robust than superstructures of previous CSSFs. The walls and roof of these will be left in place.

For CSSF 1, the temporary shielding and structural support steel will be removed from the vaults with the bridge crane. Demolish vault roof and walls. It is not necessary to demolish the CSSF superstructure for CSSFs 5,6 , and 7 because of the lower radiation levels in these CSSFs. The vault superstructure is a more permanent and integral part of the CSSF structure. At this point, the superstructure roof of CSSFs 5,6, and 7 will be core drilled to allow access to the existing retrieval risers located in the superstructure floor.

7. Prepare confinement enclosure for bin access. This is accomplished by removing and replacing strippable coatings. Portable $\mathrm{CO}_{2}$ decontamination equipment will be used to remove any residue contamination on the walls and floors. After the confinement enclosure is decontaminated, the shielding concrete pad will be poured on the bin vault roof. Attention must be given to maintaining access to all piping exiting the bin vault through the floor and existing retrieval risers. The concrete pad will be 21 in. thick for CSSF 1 and 18 in. thick for 
CSSFs 2,3 , and 4 . CSSFs 5,6 , and 7 do not require shielding floors because the superstructure will provide shielding.

8. Access bins. The distinction between CSSFs is significant in this step. CSSF 1 requires all new access lines. Retrieval lines must be uncapped for CSSFs 4, 5, 6, and 7. In CSSFs 2 and 3 , the existing retrieval lines must be uncapped and new retrieval lines must be added. Table 3 details the location of existing risers by CSSFs and superstructure location. Retrieval lines in CSSFs 5, 6, and 7 have multiple lengths. The shorter lines rise from the bins to the floor - of the superstructure. The longer lines pass through the cyclone vault.

Table 3. Location of Existing Retrieval Risers

\begin{tabular}{|c|c|c|c|c|c|c|c|}
\hline CSSF & 1 & 2 & 3 & 4 & 5 & 6 & 7 \\
\hline Vault Roof & & 7 & 7 & 6 & & & \\
\hline $\begin{array}{c}\text { Instrument } \\
\text { Room }\end{array}$ & & & & & 13 & 7 & 7 \\
\hline $\begin{array}{c}\text { Cyclone } \\
\text { Room Floor }\end{array}$ & & & & & & 4 & 2 \\
\hline $\begin{array}{c}\text { Cyclone } \\
\text { Room Roof }\end{array}$ & & & & & 6 & 4 & 4 \\
\hline Fan Room & & & & & & 6 & $\overline{8}$ \\
\hline Access Cell & & & & & 9 & & \\
\hline $\begin{array}{l}\text { Exhaust } \\
\text { Plenum }\end{array}$ & & & & & & 4 & 4 \\
\hline $\begin{array}{c}\text { Off-Gas } \\
\text { Filter Room }\end{array}$ & & & & & & 1 & 1 \\
\hline Inlet Plenum & & & & & & 2 & 2 \\
\hline $\begin{array}{c}\text { Total } \\
\text { Existing } \\
\text { Retrieval } \\
\text { Risers } \\
\end{array}$ & $\overline{0}$ & $\begin{array}{c}7 \\
(1 / \mathrm{bin})\end{array}$ & $\begin{array}{c}7 \\
(1 / \text { bin })\end{array}$ & $\begin{array}{c}6 \\
(2 / \mathrm{bin})\end{array}$ & $\begin{array}{c}28 \\
(4 / \mathrm{bin})\end{array}$ & $\begin{array}{c}28 \\
(4 / \text { bin })\end{array}$ & $\begin{array}{c}28 \\
(4 / \mathrm{bin})\end{array}$ \\
\hline $\begin{array}{l}\text { Additional } \\
\text { Lines } \\
\text { Required } \\
\text { (Length) }\end{array}$ & $\begin{array}{c}24 \\
(8 \operatorname{are~} 16 \mathrm{ft} \\
\& \\
16 \operatorname{are~} 23 \mathrm{ft})\end{array}$ & $8(26 \mathrm{ft})$ & $7(20 \mathrm{ft})$ & 0 & 0 & 0 & 0 \\
\hline $\begin{array}{c}\text { Retrieval } \\
\text { Line } \\
\text { Required per } \\
\text { Bin } \\
\end{array}$ & $\overline{2}$ & $\begin{array}{c}2 \text { in WC- } \\
136-1 \text { and } \\
1 \text { in the } \\
\text { remaining } \\
\text { bins }\end{array}$ & 1 & 0 & 0 & 0 & 0 \\
\hline $\begin{array}{l}\text { Current Rise } \\
\text { in Retrieval } \\
\text { Line from } \\
\text { Bin to End }\end{array}$ & 0 & $28 \mathrm{ft}$ & $18 \mathrm{ft}$ & $18 \mathrm{ft}$ & $\begin{array}{l}4 \operatorname{are~} 38 \mathrm{ft} \\
2 \operatorname{are~} 37 \mathrm{ft} \\
22 \operatorname{are~} 24 \mathrm{ft}\end{array}$ & $\begin{array}{l}4 \operatorname{are} 39 \mathrm{ft} \\
24 \operatorname{are~} 26 \mathrm{ft}\end{array}$ & $\begin{array}{l}4 \text { are } 39 \mathrm{ft} \\
24 \text { are } 26 \mathrm{ft}\end{array}$ \\
\hline $\begin{array}{c}\text { Total Length } \\
\text { of Retrieval }\end{array}$ & $1016 \mathrm{ft}$ & $959 \mathrm{ft}$ & $1038 \mathrm{ft}$ & $438 \mathrm{ft}$ & $1232 \mathrm{ft}$ & $1309 \mathrm{ft}$ & $1309 \mathrm{ft}$ \\
\hline
\end{tabular}




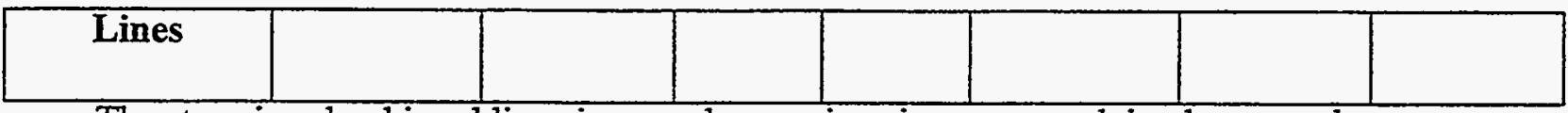

The steps involved in adding risers and accessing risers are explained separately.

Additional Retrieval Line Access: The core drilling equipment will be placed with the overhead crane. A 1 in. pilot hole will be drilled through the concrete floor to the bin vault. A 9 in. diameter hole will then be drilled through the concrete floor. Core capture equipment will prevent the core from falling into the bin vault and damaging the bins. The 8 in. retrieval lines will be extended and welded to the top of each bin. Inspection of the welds is necessary to ensure that calcine will not be released. Each bin requires two retrieval risers for calcine retrieval. The system used to install the risers will require further development and testing. It was used to access bins at the West Valley Demonstration Project ${ }^{4}$. In that project, the pipe diameter was half the size and the concrete floor was nearly twice as thick. A throughway between the riser and the bin will be made with remote equipment. A remotely operated hole saw could be used to cut a hole in the bin. Then a plug will be placed on the top end of the retrieval riser to minimize contamination of the confinement enclosure. CSSFs 1-3 and the bins will be inspected for safety hazards prior to welding and cutting operations. If hazards are identified (such as the presence of explosive gases) these hazards will be mitigated or a different welding or cutting method will be used.

Existing Retrieval Line Access: The flanged and welded terminations of the existing retrieval lines should have been located in the vault demolition step. These lines will be opened and capped until needed for calcine retrieval. It will be necessary to core drill through the confinement enclosure floor to access some of the retrieval lines in CSSF 5,6 , and 7. The existing retrieval risers will be plugged with steel lined, concrete plugs. At the conclusion of each of these activities the radiation levels of the confinement enclosure should be inspected. If they are found to be excessive the enclosure should be decontaminated.

At the conclusion of step 9, the CSSF is prepared for calcine retrieval activities. The retrieval lines will remain in the bins. The retrieval risers will be plugged with the steel lined, concrete, stepped plugs. Step 10 occurs during CSSF closure.

9. CSSF D\&D preparation. The bins will be prepared for D\&D by adding 18 in. diameter risers. The method used to install retrieval risers will be used to install the D\&D risers. It is anticipated the same core drilling platform can be used to install the D\&D risers and the new retrieval risers. However, a larger drill bit will be required. The riser will be welded to the bin and extended to the floor of the confinement enclosure. A steel lined plug will be placed on top of the rișer. As a safety precaution, the bin will not be opened into the D\&D risers until D\&D begins. Annular bins require two D\&D risers (CSSF 1, 5, 6, and 7). Cylindrical bins require one D\&D riser (CSSF 1 center bins, 2, 3, and 4). The riser will be used to insert a robot to aid in the retrieval of the final $5 \%$ of the calcine in the bins. The installation of the D\&D risers is essential to CSSF closure plan presented in reference 12 . The cost of installing the D\&D risers is included in the cost estimate (see section 6.0). 
The retrieval system will be available for $\mathrm{D} \& \mathrm{D}$. The positive displacement blower, vertical deployment apparatus (VDA), and shielded jumper will be available for D\&D. The retrieval lines will remain in each bin. The retrieval lines are basically too hot to do anything else. Some minor adjustment to the retrieval system may be necessary (such as relocating the shielded jumper and VDA within a CSSF). The installation of the D\&D risers are costed separately from the capital equipment. The operation of the retrieval system, once the CSSF is declared empty, is the responsibility of the CSSF closure team. These costs are documented in section 6.0 .

\subsubsection{Process Equipment Description}

The equipment necessary for CSSF access mainly consists of ventilation and riser installation equipment. The ventilation equipment will satisfy the ventilation requirements of the bin vault and the confinement enclosure. This equipment is described in the equipment list in appendix $A$. The riser installation equipment consists of an overhead crane (used to remove packaged, contaminated equipment and place the remote drilling platform), remote drilling platform, and remote welding equipment.

The remote drilling platform will drill penetrations through the bin vault roof ( $2 \mathrm{ft}$ to $3 \mathrm{ft}$ of concrete) of each CSSF. Retrieval risers and D\&D risers will be inserted through these penetrations. The basic operation is as follows: drill a pilot hole ( 1 in. to 2 in.), insert a toggle type capture mechanism (to prevent the core from falling into the CSSF and damaging the bins), finally, drill a larger diameter hole (allowing installation of the risers). Secondary confinement will be provided during drilling operations by a tent confinement around the drilling platform. The tent confinement will reduce exposure to workers and contamination spread within the confinement enclosure. The remote drilling platform will be relocated from bin to bin within a CSSF. This will require coordinating the construction schedules. The platform will be secured to the confinement enclosure floor with anchor bolts. Safety barriers and warning signs will prevent exposure to shine radiation directly above the open penetration. A riser plug will be installed to provide a shielding barrier once the penetrations have been drilled.

The remote welding device will weld the new risers to the bins. Commercially, resistance welding is often used to join tubs to surfaces in a hands on manner. A test program is currently underway at the INEEL to better understand the parameters involved in converting this technique to remote operation. In general terms, flanged risers, approximately $4 \mathrm{ft}$ to $5 \mathrm{ft}$ long, will be welded to the top of each bin. The remaining length of the riser will be bolted to the flanged riser. It is anticipated that the welds will need to be inspected prior to opening the bins through the risers. At this stage, a remote weld inspection technique has not been identified. However, it should be a simple matter to convert a test method for remoter inspection of the welds. The best time to develop a remote weld inspection method is during the weld test program.

The remote hole drill will be used to provide a throughway into the bin from the new retrieval riser. It will cut a circular hole, inside a new retrieval riser, on the top of a bin. The retrieval lines will be inserted into the bins through these holes. Bins with existing retrieval risers will not require the use of this equipment. This equipment should be purchased off-the-shelf and then converted for remote operation. The core should be captured to prevent it from falling into the 
$431.02 \#$

$01 / 29 / 98$

Rev. $\$ 00$

bins.

\subsubsection{Process Issues}

The CSSF access plan described above is not perfect, complete, or final. It is based on methods developed by Raytheon and Fluor-Daniels ${ }^{2}$ in previous and current studies. The details of the CSSF access plan are broad. They do not address the requirements of each CSSF individually. The specific points remain to be identified in a feasibility report. This section attempts to point out potential errors and concerns in the CSSF access method. They are not expected to impact the cost or schedule of the project but they warrant further examination and study.

\section{Radiation Levels in CSSF Superstructure:}

The radiation levels vary throughout the vault superstructure of each CSSF. The cost of demolishing the vault superstructure increases as the radiation level increases. At this stage in the design process, relative radiation levels were used to develop the cost estimate. The function of the room and evidence of operational anomalies determined the relative radiation level. Not all of the CSSFs contain all of these rooms. For future studies and cost estimates, a survey of each superstructure vault should be completed to quantify the level of radiation in the vaults.

The cyclone vault and the off-gas filter room were considered to be the highest radiation areas. These areas were exposed to the greatest levels of calcine solids. The cyclone vault houses the cyclone and the distribution piping. In CSSF 2 and 3, erosion failures are known to have occurred in this vault $\mathrm{a}^{\mathrm{a}}$. The accumulated calcine was cleaned up but higher levels should be expected in the cyclone vault for CSSF 2 and 3 . All equipment housed in this vault should be considered highly contaminated. During filling, the bins were vented through filters in the offgas filter room. The HEPA filters in this room are highly contaminated. After filling, this room was isolated to minimize contamination spread. Shielding is necessary for work in this room. The cyclone vaults and the off-gas filter rooms should be considered high radiation areas.

The instrument room, equipment room, and access cell are assumed to be low radiation zones. These rooms house the instrumentation and equipment used to monitor the CSSF. Calcine did not enter these rooms. The inlet and exhaust plenum rooms are used to passively cool the bin vault. It is unlikely these rooms have levels of contamination beyond that of a radiation area. The fan room contains HEPA filters used only if contamination is detected in the exhaust plenum room. This system has never permanently activated in any of the CSSFs. It is unlikely the HEPA filters in this room are contaminated but they should be treated as such for disposal purposes. The equipment and ductwork downstream from the HEPA filters should be considered uncontaminated. The remaining rooms in the superstructure are assumed to be radiation areas. Unless noted above, the piping or bins in the vault are assumed to be intact.

Existing Lines from the Calcining Facilities (WCF \& NWCF) to the CSSFs: The CSSF access plan calls for the original calcine transport lines to be cut and capped at the CSSFs. The cost of cutting and capping these lines is reflected in the cost estimate (section 6.0). However, the cost of decontaminating these lines is not included. The cost of decontaminating, cutting, and

a Staiger, D., "Review of High-Level Wastes Stored at the ICPP", draft, September 1997. 
capping the lines from the NWCF to the CSSFs is part of the NWCF closure cost. The WCF closure plan calls for the line from CSSF 1 to be used to flush the calcine transport line back to the WCF. The line will be flushed with a grout slurry. Eventually, the line will back up. The grout slurry will solidify just past the first wye. This makes it difficult to flush the lines that run from CSSFs 2 and 3 to the WCF. It would be advantageous to develop detailed plans to flush and properly close these lines. Closure plans for other contaminated lines should be developed as they are identified.

Weight Loading of CSSFs: The weight loading on the CSSFs during access activities and retrieval operations is not expected to be a significant problem. The amount of equipment used during these activities has been minimized to reduce the weight loading on the CSSFs. The final design of the calcine retrieval and transportation system will incorporate the weight loading restrictions of the CSSFs. This issue will be resolved during the design stages of the calcine retrieval and transportation system.

\subsection{Calcine Retrieval}

\subsubsection{Process Basis}

The calcine retrieval system will remove the calcine from a bin and place it directly in the pneumatic transport system. The system minimizes the number of moving parts used to retrieve the calcine to reduce the risk of failure and downtime. The remote operation design of the calcine retrieval system reduces worker exposure.

The calcine retrieval system consists of the equipment necessary to remove calcine from a bin. Each CSSF is equipped with a complete set of retrieval equipment. The retrieval system relies on the transport system to provide the means for the calcine retrieval. There are two transport systems. The calcine retrieval system and the calcine transportation system are separate and distinct. Neither system can operate without the other. The interface between the calcine retrieval system and the calcine transportation system is shown in sketches CRT-02 and CRT-03.

Several modifications have been made to Fluor-Daniels ${ }^{2}$ design to satisfy the needs of the processing options. The basic design, equipment, and process are based on the Fluor-Daniels ${ }^{2}$ design for calcine retrieval. The Fluor-Daniels ${ }^{2}$ design specifies that alumina calcine and zirconia calcine are retrieved and transported using separate systems. The overall system will be more efficient if there are no restrictions placed on the type of calcine that can be retrieved and transported by a system. The alumina calcine system would be idle a large part of the time because the CSSFs contain more zirconia calcine. The duplicated systems allow two bins, in separate CSSFs, to be retrieved from at one time. Safety and efficiency issues require that one bin be completely emptied before another bin is retrieved from within a CSSF.

\subsubsection{Process Description}


Calcine retrieval will be accomplished pneumatically with a suction nozzle and an air jet. The air jet will fluidize the calcine inside the bin. The suction nozzle will lift the fluidized calcine out of the bin. Calcine will enter the transport system through a shielded jumper from the retrieval line. A retrieval system has been mocked up at the INEEL as a pilot plant facility. It is the only system to have successfully demonstrated solids retrieval from a full-scale bin model.

Two retrieval lines will be placed $180^{\circ}$ apart inside the bin. The retrieval lines will be composed of rigid, concentric pipes. The outer diameter of the retrieval lines will be 5 in. The inner pipe will function as the suction line. It will be fitted with a nozzle designed to prevent clogging and uptake of miscellaneous items in the bins. The annulus will be an air jet. It will be fitted with an appropriate fluidizing nozzle. At a given time, a retrieval line will function as a suction line or an air jet. The suction nozzle and air jet will not be housed in the same retrieval line. The function of the retrieval lines will alternate, one line will act as the suction line while the other acts as the air jet. Calcine retrieval will be more efficient. Each line will have the capability to sweep a full circle but it will not be continuously rotating. This will aid in fluidizing agglomerated calcine.

The pressure inside a bin during retrieval should be slightly negative. The negative pressure reduces the risk of contamination spread while a vault is open for retrieval. The negative pressure will provide an additional safety if the confinement were to fail. The negative pressure must not maximum exceed the design pressure of the bins. The design pressure rating varies by CSSF.

A vertical deployment apparatus (VDA) will be used to keep the retrieval lines near the surface of the bulk calcine. This equipment is similar to well-drilling equipment. The retrieval lines will be sectioned. The length of each retrieval line can be extended as the calcine is retrieved. The retrieval system functions most effectively when the suction nozzle and air jet are near the calcine surface. However, it is necessary to adjust the level of each line independently. After the bin is emptied, the lines will be disconnected from the VDA and remain in the bin. The retrieval risers will be plugged, with steel-lined stepped plugs, before the VDA is decontaminated and moved to the next bin.

The calcine will be retrieved at a rate of $2700 \mathrm{~kg} / \mathrm{hr}$ from each bin. When both transport systems are operating, calcine will be delivered to the Waste Treatment Facility at a rate of $5400 \mathrm{~kg} / \mathrm{hr}$. The retrieval rate is set by Fluor-Daniels ${ }^{2}$. The mock-up tests ${ }^{8}$ reveal that retrieval rates above $2300 \mathrm{~kg} / \mathrm{hr}$ can be achieved with 3 in. retrieval lines and a maximum air flow rate of $300 \mathrm{scfm}$ at $12-15$ psig. The higher retrieval rate could be reached by increasing the air flow rate and the blower capacity. More testing is necessary to show that this is a sustainable retrieval rate. The retrieval rate is dependent upon how agglomerated the calcine is. This issue is discussed further in section 3.2.3.

The retrieval system will retrieve at least $95 \%$ of the calcine each bin. This estimate is based on results from a pilot plant program (reference 8 ). The $1 / 4$ and full-scale mockup tests provide the best information about the amount of calcine that can be retrieved using this retrieval method. The details of the tests are not explained in this EDF. The target retrieval rate was not maintained after $95 \%$ of the solids were retrieved. In the $1 / 4$ scale test, less than $1 \%$ of the initial retrieval 
rate could be maintained during retrieval of the final $3 \%$ of solids. A significant amount of time is necessary to retrieve the final $3-4 \%$ of calcine at the bottom of the bin. For the full-scale tests, the retrieval rate dropped as the amount of solids in the bin decreased. The retrieval rate dropped below the target retrieval rate when $95.8 \%$ of the solids had been retrieved.

A conservative retrieval estimate is $95 \%$. A conservative approach is taken for several reasons. The calcine in the "nooks and crannies" created by thermowells, internal stiffening rods, and the bottom of the bins will be difficult to remove with this retrieval method. This system has not been tested on a caked material. It is highly possible that calcine in some of the bins may be caked. Such a material would have a greater tendency to stick to the walls, floors, and internal structure of the bins. It should be noted that in the actual operation of the retrieval system, retrieval should continue as long as a reasonable retrieval rate can be maintained. The schedule and other processing requirements must define that rate.

Throughout calcine processing, retrieval will switch from CSSF to CSSF as CSSFs are emptied or different calcine types are reached. For example, the CWO option requires frequent switching between CSSFs to achieve the correct calcine blend. The other processes need to switch CSSFs once a CSSF is emptied. Safety issues prevent switching from bin to bin within a CSSF before the first bin is emptied. Additionally, such a feature is not necessary to meet the process requirements. The process of switching the retrieval to another CSSF is outlined below: Let bin $A$ be the bin that is currently being retrieved and let bin $B$ be the bin that retrieval is being switched to.

1. Turn off retrieval system for bin A: This is accomplished by shutting off the transport air blower connected to the suction nozzle and air jet. Flush the shielded jumper and upper line with dolomite to remove any calcine deposited in the lines. Close the valve at the CSSF transport line connection. Disconnect the vertical deployment apparatus from the retrieval lines. The lines will rest on the bottom surface of the bin. Place the steel-lined, concrete plugs on the retrieval risers. Disconnect the shielded jumper from the VDA. Decontaminate and relocate the VDA to bin $B$.

2. Adjust Transport System: Diverter valves will be used to connect the CSSF leads to the main transport system. The diverter valves will be pneumatically controlled from the control room adjacent to each CSSF. The steam traced lines in the transportation system will condensation in the return air lines.

3. Turn on retrieval system for bin B: If necessary, the VDA must be transported to and correctly positioned at bin B. The correct set of retrieval line extensions must be placed in the extension tube carousel. The confinement tenting will be replaced around the VDA. The shielded jumper will connect the retrieval lines to the transport system. The plugs on the retrieval risers will be pulled. Retrieval lines will be extended to the top of the calcine in bin B. The power to the retrieval blower and suction jet will be turned on. Retrieval from bin $B$ begins.

\subsubsection{Process Equipment Description}

The Vertical Deployment Apparatus (VDA) will extend the retrieval lines into the bins during calcine retrieval. The VDA will extend the retrieval lines independently. The equipment is 
similar to well drilling equipment. Temporary ventilation and confinement shielding is required. This will be accomplished with a confinement tent and a portable blower. A catch pan will collect radioactive dust and runoff water introduced in the VDA confinement area. The VDA is relocated with the bridge crane. It will be stabilized with anchor bolts attached to the confinement enclosure floor. The apparatus is $5 \mathrm{ft}$ to $6 \mathrm{ft}$ square and $25 \mathrm{ft}$ tall. External shielding panels, $2 \frac{1}{2}$ in. thick, reduce radiation fields inside the confinement enclosure during retrieval. An external ladier and gate provide maintenance access to the VDAs. When the plug is removed from the retrieval riser, the confinement area within the VDA has the same ventilation requirements as the calcine bin.

The VDA is equipped with a plug removal hoist, extension tube carousel, and extension tubes and couplings. A detailed description of their function appears in reference 3 . The plug removal hoist lifts the plug from the retrieval riser and stores it inside the VDA. The motor, brake, controls, and cable require periodic inspection. The load carrying capacity should also be regular checked. The extension tube carousel stores the extension tubes for the risers and retrieval lines.

The extension tubes with couplings form the retrieval line sections. As the retrieval lines are lowered into the bins, a section of tube is added. Each CSSF requires a particular set of extension tubes. These must be loaded into the VDA each time it is relocated.

The shielded jumper connects the discharge of the calcine retrieval line (top of the VDA) to the transport line. The jumper configuration will allow this connection to be made regardless of location of VDA. The shielded jumper is made of double walled, heavy pipe. The jumper is composed of $4 \mathrm{ft}$ to $5 \mathrm{ft}$ sections that are equipped with lifting lugs for easy handling. The jumper must be flushed with high velocity dolomite to reduce the amount of contamination in the line before it is moved.

The CCTV system contains a camera, light, boom, and control system. This system is installed to allow viewing inside the bins. It is not an essential component for the retrieval operation but it will be useful if unforeseen problems develop.

The retrieval equipment described in this section is located inside the confinement enclosure. This equipment must interface with the transport system in order for calcine to be retrieved.

\subsubsection{Process Issues}

The calcine retrieval system described has been tested at the INEEL. Even so, some unresolved issues exist regarding the system. Raytheon ${ }^{3}$ developed the equipment used in this system in a previous study. The purpose of this section is to ensure the feasibility of the calcine retrieval system by expressing concerns and issues that warrant further examination and study.

\section{Corrosion Coupons and Miscellaneous Items in CSSFs:}

Calcine retrieval will be hampered by internal obstructions inside the bins. At some point during the retrieval process, these obstructions should be removed from the bins. For example, the corrosion coupons should be retrieved from each CSSF prior to decontamination of the confinement enclosure during CSSF access. The corrosion coupons in each bin are hung from $1 / 4$ 
in. J hooks. In 1978, some of the corrosion coupon sets were retrieved from CSSF 2. A crane was used to pull the coupons from the bins. However, at least one of the coupon sets was dropped during this operation. In order to minimize the risk of dropping additional corrosion coupon sets the CCTV system should oversee the corrosion coupon removal. The bridge crane can be used to retrieve the corrosion coupons sets. Upon removal of the corrosion coupons, they should be placed in a shielded cask. The coupons should be examined for evidence of corrosion. This information is valuable for retrieval and closure of the CSSFs. The analysis should take place at ICPP in a shielded cell. After the coupons have been analyzed they should be disposed of as radioactive waste. Table 4 describes the corrosion coupons in each CSSF.

\section{Table 4. Corrosion Coupon Description}

\begin{tabular}{|c|c|l|l|}
\hline CSSF & $\begin{array}{c}\text { Number Remaining } \\
\text { in Bin }\end{array}$ & \multicolumn{1}{|c|}{ Bin Location } & \multicolumn{1}{|c|}{ Description } \\
\hline 1 & 0 & & \\
\hline 2 & $3 /$ bin & $\begin{array}{l}\text { VES-WCS-136-1 and } \\
\text { VES-WCS-136-4 }\end{array}$ & Wall mounted \\
\hline 3 & 5 & VES-WC-140-1 & Inside riser \\
\hline 4 & $5 /$ bin & All bins & Wall mounted \\
\hline 5 & $5 /$ bin & $\begin{array}{l}\text { VES-WS5-149 and } \\
\text { VES-WS5-151 }\end{array}$ & $\begin{array}{l}\text { Corrosion coupon } \\
\text { retrieval through nozzle } \\
\text { J (6 in. Sch 40) }\end{array}$ \\
\hline 6 & $5 /$ bin & $\begin{array}{l}\text { VES-WS5-156 and } \\
\text { VES-WS6-159 }\end{array}$ & $\begin{array}{l}\text { Corrosion coupon } \\
\text { retrieval through nozzle } \\
\text { ( 8 in.) }\end{array}$ \\
\hline 7 & & VES-WS7-164 & $\begin{array}{l}\text { Corrosion coupons in } \\
\text { retrieval nozzle F }\end{array}$ \\
\hline
\end{tabular}

There are other miscellaneous items (such as rodout lines, weighted lines, lost samplers and penetrometer points) in the bins ${ }^{b}$. These items will not be as easy to retrieve as the corrosion coupons because their location is not known. Some of these items may be below the calcine surface while others may be on the surface of the calcine. Something must be done to reduce the risk of these items damaging the bins, the retrieval lines, and the transportation system. The air jet could pick up these items. The bin walls may not survive an impact. The bin could be breached. If these items enter the transportation system they could clog or puncture the system. The ideal solution is to detect and remove these items from the bins.

It is necessary to remove the corrosion coupons from the bins before retrieval begins. It is assumed that this activity will occur during the construction phase of the project for each CSSF. For purposes of developing a bounding cost estimate, the corrosion coupon removal activity was included in the cost estimate. A cost estimate was developed to remove the corrosion coupons. The escalated cost of planning and equipment to remove the corrosion coupons from all the

\footnotetext{
'Staiger, D. "Review of High-Level Wastes Stored at the ICPP", draft, September 1997.
} 
CSSFs is $\$ 1,620,000$. This activity was also included in the operating and maintenance cost estimate (See section 6.0 for details).

\section{Control of Retrieval Rate:}

The retrieval rate cannot be rigidly controlled due to the nature of the system. The retrieval rate depends upon several factors. As the calcine supply in the bin diminishes the retrieval rate decreases. The retrieval rate also depends upon how free flowing the calcine is. This retrieval method has been extensively tested with free flowing solids. It is anticipated that agglomerated calcine can be broken up and placed in a dilute phase by the air jet. However, retrieval of agglomerated solids in this manner has not been tested. This issue has not been examined because of the difficulty of simulating agglomerated solids on a large scale. The bins should be sampled to determine if the calcine has agglomerated. If agglomerated calcine is found, a pilot plant study should be conducted to determine the ability of agglomerated calcine to be retrieved with this retrieval method.

\section{Location and Number of Retrieval Lines:}

Limited testing has been conducted to identify the optimum location and number of retrieval lines. The number, size and location of the air jet and suction nozzles will effect the efficiency of the calcine retrieval system. It is necessary to determine if the most efficient configuration has been selected. Reference 8 suggests that two suction nozzles located $\pm 90^{\circ}$ from the air jet may be more efficient. The costs associated with this activity are included in the cost estimate for the design of the retrieval system.

Additionally, the performance of the retrieval system in a cylindrical bin is not known. Tests ${ }^{3}$ have been conducted on an annular bin mock-up. Preliminary indications from the annular bin mock up tests suggest that the retrieval system will be even more effective for these bins. This is a source of concern because the optimal suction nozzle and air jet configuration may vary significantly from that of the annular bin.

\section{CSSF Retrieval Order:}

The order that the CSSFs will be retrieved is not identified in this EDF. Each CSSF will be ready for retrieval by $1 / 1 / 2013$. The order of retrieval is heavily dependent upon the operation of the Waste Treatment Facility. The operation of the Waste Treatment Facility determines when the calcine will be retrieved. The majority of the treatment options do not require a specific blend of calcine. Therefore, they do not have a preference for the CSSF retrieval order. However, it would be beneficial to the Waste Treatment Facility operating crews to retrieve and process the more homogeneous calcine in CSSF 1 and 2 first. The closure study ${ }^{12}$ for the CSSF facilities would prefer the placement of class $C$ grout in the better constructed CSSF 5, 6 , and 7 . The order the CSSFs should be retrieved to accommodate the closure schedule is CSSF1, CSSF 5, CSSF 6, CSSF 7, CSSF 2, CSSF 3, and finally CSSF 4. This is an issue that must be coordinated between the waste treatment options and CSSF closure. Political input should be considered a major factor in determining the retrieval order. 


\subsection{Calcine Transport}

\subsubsection{Process Basis}

The calcine transportation system will deliver the retrieved calcine from the CSSFs to the calcine Waste Treatment Facility with a pneumatic transport system. It also provides the motivation for the suction nozzle and air jet necessary for calcine retrieval (see sketches CRT-02 and CRT-03). Pneumatic transport systems have been used to transport calcine from the calcining facility (NWCF or WCF) to the CSSFs for more than 30 years. Additionally, Raytheon ${ }^{3}$ studied the advantages and disadvantages of open-loop, closed-loop, and combined loop (combining a retrieval system with a closed-loop transport system) pneumatic transportation systems. The combined loop pneumatic transport system provides the best alternative for calcine transportation because recycling of the transport air minimizes the amount of air released.

The Fluor-Daniels ${ }^{2}$ design consists of two independent transportation systems. The independent transportation systems were specified to transport zirconia and alumina calcine separately. There appears to be no advantage, to the non-separations options and TRU Separations options, to keep the zirconia calcine separate from the alumina calcine. The two independent transportation systems, in this study, will allow retrieval and transportation of calcine from 2 CSSFs at one time.

\subsubsection{Process Design}

The calcine transport system will deliver calcine from the CSSFs to the Waste Treatment Facility. Each CSSF will be connected to one of the two pneumatic transport systems. The double transport systems will provide greater flexibility during calcine retrieval (allow more desirable calcine blends to be achieved) and CSSF closure (allow retrieval of the remaining 5\% of calcine in the bins during off-peak hours).

The pneumatic transport system reduces the risk of calcine release by conveying the calcine under negative pressure. If a break develops in the transport line, air will flow in rather than out. This minimizes the risk of calcine release by reducing the potential for calcine to leave the transport line. The momentum of large particles may overcome the negative pressure and escape at an erosion failure (particularly those in bends or other direction changes). The risk of calcine release is lower for a vacuum system than for a pressure system. In a pressure system the calcine particles would be pushed out of the transport system at all erosion failures. Additionally, erosion failures in the transport piping can be minimized through proper layout of the transport system $^{11}$. The costs associated with properly designing the transport system are reflected in the cost estimate (section 6.0).

Each of the two transport systems will convey calcine in a $10 \mathrm{~mm}(4 \mathrm{in}) 304 \mathrm{~L}$ stainless steel line encased in $15 \mathrm{~mm}$ ( 6 in) 304L stainless steel line. Each transport system will have a backup calcine transport line, to be used if the original line becomes permanently clogged or otherwise unusable. Rod out stations will be placed along straight runs to clear clogged transport lines. The transport lines will be accompanied by two return air lines, one for each transport system. 
The return air lines will be $20 \mathrm{~mm}$ ( 8 in) 304L stainless steel. These lines will be steam-traced to prevent water from condensing.

The transport lines and the return air lines will be surrounded by a concrete pipe chase. The pipe chase will be designed to allow access to the diverter valves and rod out stations. The pipe chase will minimize "shine" radiation at accessible locations. An earthen berm will shield the pipe chase. Raytheon ${ }^{3}$ examined many routings and found a large number of obstructions from CSSF 1 to CSSF 6 that prevent burying the transport lines. For purposes of this study, it is assumed that it is more convenient and cost efficient to lay the transport lines above grade. Although, above ground placement does not preclude necessary relocation of some utility lines.

Diverter valves will be used to prevent back flow of calcine into CSSF connections. These valves will be manufactured out of stainless steel. Diverter valves are a typical component of pneumatic systems. Three diverter valves will be necessary to ensure that calcine can be transported from the CSSFs to the Waste Treatment Facility in either line of the transport system. These valves are ideal for use in the calcine transport system because they require little maintenance over their service life.

It is generally accepted in industry, that a vacuum transport system can transport fluidized solids up to $300 \mathrm{ft}$ efficiently. The CWO and TRU Separations options require calcine to be delivered to the NWCF and the Calcine Dissolution Facility, respectively. The site plan for the CWO option is shown in sketch CRT-05. The site plan for the TRU Separations options can be found in the TRU Separations options study report (sketches TRU-C-6 and TRU-C-12). Calcine can be transported from all the CSSFs to these facilities without exceeding the transport distance limitation. The Waste Treatment Facility in the DCWO, HWO, and VWO options are located in the north east corner of ICPP. The required transport distance from CSSF 3 to the Waste Treatment Facility is approximately $550 \mathrm{ft}$. An Intermediate Transport Station (ITS) and two separate transport system legs must be included in the transport system design to deliver calcine from the CSSFs to the Waste Treatment Facility for the DCWO, HWO, and VWO options. The relationship between the ITS and the transport system is shown in sketches CRT-03 and CRT-04.

For the DCWO, HWO, and VWO options, the calcine transport from the CSSFs to the Waste Treatment Facility is accomplished with two transport system legs (each leg contains piping and equipment for each transport system). The first leg transports calcine from the CSSFs to the ITS. At the ITS, the transport air is separated from the fluidized calcine with a cyclone and sintered metal filter. The calcine enters a storage hopper. As the calcine is metered out of the hopper it is fluidized by the second leg of the pneumatic transport system. The calcine is then transported from the ITS to the Waste Treatment Facility, as shown in CRT-04.

\subsubsection{Process Equipment Description}

For the CWO and TRU-Separations Options, there are two independent transportation systems. For the DCWO, HWO, and VWO options, there are four transportation systems (two from the CSSFs to the ITS and two from the ITS to the Waste Treatment Facility). Each transportation system has a backup calcine transport line installed. Two sets of transport equipment (transport air blower, cyclone, sintered metal filter, heat exchanger, and balancing air blower) are located 
inside the Waste Treatment Facility for all the waste treatment options. The DCWO, HWO, and VWO options have two additional sets of transport equipment located in the ITS. The shielding concrete chase is located between the CSSFs and the Waste Treatment Facility.

The transport air blower is a positive displacement blower. It functions as the transport air blower. For the CWO and TRU Separations options, it also serves as the retrieval blower. It has a capacity of $800 \mathrm{cfm}$ and $150 \mathrm{kPa}$ (10psi). The blower sized in the Fluor-Daniels ${ }^{2}$ design was examined in reference 11. It was found that it is slightly oversized for the longest transport line length (CSSF 3 to the Calcine Dissolution Facility). The pressure drop and solids velocity are higher than optimum for a vacuum system. However, the blower is also used to retrieve the calcine from the bins. The extra capacity may be necessary to retrieve the calcine from the bottom of the bins.

The cyclone separates approximately $99 \%$ of the calcine solids from the transport air. It is sized at $2 \mathrm{ft}$ ID X $6 \mathrm{ft}$. This size is slightly higher than the one specified by Fluor-Daniels ${ }^{2}$. A larger cyclone is suggested by the Raytheon studies. It will be reinforced with nitronic plating to reduce the risk of erosion failures.

The sintered metal filter separates the entrained calcine particulates after the transport air leaves the cyclone. About $99.9 \%$ of these particles will be removed as the transport air passes through the sintered metal filter. Air will be back blown through the sintered metal filter to deliver calcine to the process batch bin.

The heat exchanger cools the air after it leaves the transport air blower. It is anticipated this will be water cooled heat exchanger. After the air leaves the heat exchanger it will be separated. Most of the air will be recycled but $10 \%$ of it will be exhausted through the process facility exhaust system.

The balancing air blower removes excess air from transport system. It is used to maintain a slightly negative pressure in the bin being retrieved. This is necessary to provide an additional contamination confinement.

The concrete shielding chase provides shielding to the transport lines that run from CSSFs to Waste Treatment Facility. The radiation fields are reduced to acceptable levels outside the chase. The design of the chase is similar to Raytheon design. The wall thickness is increased to account for the higher transport rate. It will protect the transport lines from weather damage.

\subsubsection{Process Issues}

Even though pneumatic transport systems have been used to transport calcine for over 30 years, some process issues remain for future study. Concerns and areas requiring further study that were identified during this study are detailed in this section.

\section{Centrally Locate the Waste Treatment Facility:}

Central location of the Waste Treatment Facility will serve to minimize the length of pipe runs. This will raise the efficiency of the transport equipment. Currently, the Waste Treatment Facility 
for the DCWO, HWO, and VWO options is located far from the CSSFs (as shown in sketch CRT-04). The location chosen by the TRU Separations Options (as shown in sketches TRU-C-6 and TRU-C-12.) is a better location considering the limitations of a vacuum transport system. However, even this location has its own unresolved issues. The requirements to move the ICPP fence must be identified. The Waste Treatment Facility will extend off the current ICPP site boundary marked by the fence. It is not known if the fence can simply be moved. The Waste Treatment Facility should be located as close to the CSSFs as possible.

\subsection{Implementation}

The calcine retrieval and transportation system could be implemented in several ways. Initially, two options were examined for the capital cost estimate. Alternative $A$ was designed to meet the minimum need of the majority of the processing options (DCWO, HWO, VWO, and TRU Separations). Alternative $B$ was designed to satisfy the requirements of CWO and CSSF closure activities. Both of these options differ from the implementation plan presented by FluorDaniels ${ }^{2}$. Alternative $B$ was selected as the best implementation plan. A cost estimate is included in section 6.0 based on alternative $B$.

Alternative $\mathrm{A}$ was based on the requirements of the majority of the waste treatment options. The DCWO, HWO, VWO, and TRU Separations options require a well-blended and accurately categorized batch of calcine. These treatment options do not require a specific blend of calcine. In alternative $A$, the CSSFs were accessed (a confinement enclosure and VIC building were constructed for each CSSF) during the construction phase of the project. Two sets of retrieval equipment (consisting of a VDA and a shielded jumper) will be purchased. The retrieval equipment will be moved from CSSF to CSSF as the CSSFs are emptied. The retrieval equipment must be decontaminated before relocation. Moving the retrieval equipment between CSSFs is assumed to take less than one week.

The drawback to this implementation alternative is the impact it will have on D\&D work. CSSF closure requires that as much calcine as possible be removed from each bin. After, calcine retrieval as much as $5 \%$ of the calcine may remain in each bin. The CSSF closure study ${ }^{12}$ expects to use the retrieval equipment to remove as much as possible of the remaining calcine. The capital cost estimate for alternative $A$ was not significantly lower that the estimate for alternative $B$ (a copy of the preliminary capital cost estimate for alternative $A$ is located in the project file). For these reasons, alternative $A$ was not selected for further study.

Alternative B satisfies the requirements of the remaining waste treatment option, $\mathrm{CWO}$, and CSSF closure. CWO requires a specific blend of calcine for each processing batch. The calcine blend is achieved with calcine from up to four CSSFs (see reference 13 for details). Switching between CSSFs is required to maintain the blend as large layers of calcine are encountered in the bins. The large number of required moves between CSSFs make purchasing seven sets of retrieval equipment the most cost effective selection. It is not necessary, in this alternative to move the VDAs and shielded jumpers from CSSF to CSSF. The retrieval equipment will be moved between bins within a CSSF. It is expected that moving the retrieval equipment within a CSSF can be accomplished in less than a day. 
Alternative B does not adversely impact CSSF closures. Closure can begin on each CSSF when it is emptied. The calcine remaining in the bins will be retrieved when the high calcine retrieval rate is not necessary. Alternative B also satisfies the requirements of the DCWO, HWO, VWO, and TRU Separations options. Table 5 outlines the similarities and differences between the implementation alternatives.

Table 5. Characteristics of Calcine Retrieval System Options

\begin{tabular}{|l|c|c|}
\hline \multicolumn{1}{|c|}{ Characteristic } & Option A & Option B \\
\hline $\begin{array}{l}\text { Retrieval Rate / Transport } \\
\text { System }\end{array}$ & $2700 \mathrm{~kg} / \mathrm{hr}$ & $2700 \mathrm{~kg} / \mathrm{hr}$ \\
\hline $\begin{array}{l}\text { Number of Independent } \\
\text { Transport Systems }\end{array}$ & 2 & 2 \\
\hline $\begin{array}{l}\text { Number of Confinement } \\
\text { Enclosure and VIC } \\
\text { Building Sets }\end{array}$ & 7 & 7 \\
\hline Number of VDAs Required & 2 & 7 \\
\hline $\begin{array}{l}\text { Relocation of VDAs } \\
\text { between CSSFs (Relocation } \\
\text { Time) }\end{array}$ & $\begin{array}{c}\text { Y I week due to } \\
\text { decontamination activities) }\end{array}$ & Not required \\
\hline $\begin{array}{l}\text { CSSFs Costed with New } \\
\text { VDAs }\end{array}$ & 1 and 2 & $1,2,3,4,5,6$, and 7 \\
\hline VDAs Relocated to CSSFs & $3,4,5,6$, and 7 & - \\
\hline $\begin{array}{l}\text { Tasks to Utilize Alternative } \\
\text { DCWO, HWO, VWO, } \\
\text { TRU Separations }\end{array}$ & $\begin{array}{c}\text { CWO (also DCWO, HWO, } \\
\text { VWO, TRU Separations) }\end{array}$ \\
\hline $\begin{array}{l}\text { Transport System Available } \\
\text { for Closure of CSSFs } \\
\text { during operations }\end{array}$ & No & Yes \\
\hline
\end{tabular}

Alternative B was selected as the best implementation option. It was used as the basis for the cost estimate.

\subsection{Input to Project Data Sheet}

A project data sheet was not completed for the calcine retrieval and transportation system. Instead the data was appended to the project data sheets for each waste treatment option. Table 6 shows the data that should be incorporated into the project data sheets of the each waste treatment option. It summarizes construction, operations, and some D\&D project data. Appendix $\mathrm{D}$ contains the calculations and justification for the data presented in Table 6.

The closure of the CSSFs is currently being studied, therefore, the D\&D project data for the CSSFs will be reported in reference 12. Reference 12 will cover closing of the CSSF structures, added confinement enclosures, VIC buildings, and the retrieval equipment. D\&D project data is included in Table 6 for closure of the transport lines, ITS (building and transport equipment), and 
transport equipment located in the WTF. The D\&D project data included in Table 6 is not comprehensive because a closure method for the transport lines, ITS, and transport equipment has not been developed. It was assumed that the D\&D portion of the project would have a 1year duration. The work may be more effectively accomplished over a longer period of time. A comprehensive examination of the D\&D requirements of the transportation system, based on a closure method, should be completed.

\section{Table 6. Input to Project Data Sheets}

\section{Generic Information}

Structure Size $\left(\mathrm{m}^{2}\right)$

Location

\section{Construction Information}

Cost (\$): Preconstruction (escalation included)

Conceptual Design

Project Management

Permitting and Documentation

Start Up Activities

Contingency

Total Preconstruction

Cost (\$): Construction (escalation included)

Engineering, Design, and Inspection

Management Reserve (PM/CM)

Construction

Government Furnished Eqiupment

G\&A/PIF
Seven Confinement Enclosures which are each 40 $\mathrm{ft} X 40 \mathrm{ft}\left(978 \mathrm{~m}^{2}\right.$ total)

Seven VIC Buildings which are each $40 \mathrm{ft} X 60 \mathrm{ft}$ $\left(1560 \mathrm{~m}^{2}\right.$ total)

One ITS Building that is $600 \mathrm{ft}^{2}\left(55.7 \mathrm{~m}^{2}\right)$

A Confinement Enclosure will be built on the roof of each CSSF.

A VIC Building will be built adjacent to each CSSF.

An ITS Building will be built mid way between the CSSFs and the Waste Treatment Facility.

CWO, DCWO, HWO, VWO \& TRU Separations Options

\$ $18,000,000$

$\$ 2,700,000$

\$ $4,800,000$

$\$ 5,100,000$

$\$ 10,500,000$

$\$ 41,100,000$

CWO \& TRU DCWO, HWO, \& VWO Separations Options
$\$ 18,500,000$
\$ $19,400,000$
$\$ 22,900,000$
$\$ 23,500,000$
$\$ 104,200,000$
$\$ 106,500,000$
$\$ 18,200,000$
$\$ 19,300,000$
$\$ 10,400,000$
$\$ 10,700,000$ 
Procurement Fees, Management Reserve, and Contingency

Total Construction

Schedule start/end: Preconstruction

Schedule start/end: Construction

Number of workers each year of construction

Number of radiation workers (construction)

Average annual worker radiation dose (rem/yr)

Heavy equipment

Equipment used

Trips

Hours of operation

Acres disturbed and duration of disturbance

Air emissions

Major gases $(\mathrm{CO} 2, \mathrm{H} 2 \mathrm{O}, \mathrm{O} 2, \mathrm{~N} 2)$

Contaminants (Particulates, $\mathrm{CO}, \mathrm{NOx}$, SO2, hydrocarbons)

Radioactive wastes

Type

Energy requirements

Electrical (MWh/yr)

Fossil fuel (liters)

Permits needed for construction

\section{Operational Information}

Cost (\$): Operations (not escalated)
S $64,600,000$

\$ $66,600,000$

$\$ 238,800,000$

$\$ 246,100,000$

$1 / 1 / 2004-12 / 31 / 2007$

$1 / 1 / 2008-12 / 32 / 2012$

100 workers per year

90 workers per year

$252 \mathrm{mrem} / \mathrm{yr}$ per worker

Mobile crane, roll off truck, loader, bulldozer, and cement truck

Cycle time/operation not applicable

$12,480 \mathrm{hr}$ (total)

0.5 acres for VIC Buildings, Transport Line Chase, ITS building and ramps

7,255 tons

42.5 tons

Contaminated fill (1500 $\left.\mathrm{yd}^{3}\right)$

Steel/asbestos $(279,000 \mathrm{lb})$

Lead bricks/mixed wastes $(4,900 \mathrm{lb})$

$156 \mathrm{MWh} / \mathrm{yr}$

283,452 liters (total)

NEPA documentation (prior to start of Title II construction); New stationary sources/PTC/NOC/PSD for non-rad air emissions; HAP's and TAP's and RCRA (part AA, BB, and $C C$ for air) for hazardous air emissions; air operating permit; NESHAP's, NPDES, NESHAP's subpart $\mathrm{H}$ for rad air emissions; approval of Engineering Plans; Cross Connection Control Plans; reports and specifications for drinking water supply; RCRA Part A and Part $B$ permits. 
Facility Operations

Utilities

Maintenance of Equipment

Building Maintenance

Total Operations

Schedule start/end

Number of workers each year of operation

Managers

Engineers and other technicians

Supervisors and Administation/Support

Operators

Maintenance

Number of radiation workers

Average annual work radiation dose (rem/year)

Air Emissions

Type (radioactive/chemical)

Quantity (Ci/year, ton/year)

Energy Requirements

Electrical $(\mathrm{MWh} / \mathrm{yr})$

Permits needed (for facility operations)
$\$ 1,218,000$

S $4,661,000$

$\$ 4,761,000$

S $1,054,000$

$\$ 4,158,000$

\$ $4,158,000$

S $7,866,000$

$\$ 28,840,000$

$\$ 28,840,000$

\$ $9,255,000$

$\$ 33,933,00$

$\$ 35,120,000$

$\$ 19,393,000$

$\$ 71,594,000$

$\$ 72,781,000$

5-Year Operation: 1/1/2013-12/31/2017

20-Year Operation: 1/1/2013 - 12/31/2032

5-Year Operation 20-Year Operation

$\begin{array}{ll}0.5 & 0.25 \\ 0.75 & 0.5 \\ 3 & 2 \\ 6 & 3 \\ 1 & 1\end{array}$

5-Year Operation: 10 workers

20-Year Operation: 6 workers

$192 \mathrm{mrem} /$ year per worker

Calcine

CWO Option: $5.6 \times 10^{-6}$ ton/year

TRU Separations Options: $1.4 \times 10^{-6}$ ton/year

DCWO, HWO, VWO Options: $2.8 \times 10^{-6}$ ton/year

Release of $\mathrm{Ci} /$ year dependent on the type and storage length of the calcine released.

CWO Option: $93.2 \mathrm{MWh} / \mathrm{yr}$

TRU Separations Options: $74.1 \mathrm{MWh} / \mathrm{yr}$

DCWO, HWO, VWO Options: $88.7 \mathrm{MWh} / \mathrm{yr}$

HAP's and TAP's and RCRA (part AA, BB, and $C C$ for air) for hazardous air emissions; air operating permit; NESHAP's, NPDES, NESHAP's subpart $\mathrm{H}$ for rad air emissions; approval of Engineering Plans; Cross Connection Control Plans; report specifications for drinking water supply; RCRA Part A and Part $B$ permits.
Decontamination \& Decommissioning (D\&D) Information 
Cost (\$): DD\&D (Unescalated)

Decommission

Decontamination

Demolition

Total DD\&D

Schedule start/end: D\&D

Number of workers each year of D\&D (new/existing)

Number of radiation workers (D\&D)
CWO $\begin{array}{cc}\text { TRU- } & \text { DCWO, HWO, } \\ \text { Separations } & \& \text { VWO } \\ \text { Options } & \end{array}$

S $2,555,000$ \& $2,555,000$ \& $2,681,000$

\& $7,223,000$ \& $7,223,000$ \& 7,415,000

\& $4,935,000 \quad \$ 11,557,000 \quad \$ 11,864,000$

$\$ 14,713,000 \quad \$ 21,335,000 \quad \$ 21,960,000$

CWO: $1 / 1 / 2018-12 / 31 / 2018$

TRU-Separations Options: 1/1/2034-12/31/2034

DCWO, HWO, \& VWO: 1/1/2034-12/31/2034

CWO: 108

TRU-Separations Options: 155

DCWO, HWO, \& VWO: 160

CWO: 75

TRU-Separations Options: 104

DCWO, HWO, VWO: 102

$252 \mathrm{mrem} / \mathrm{yr}$ for each worker

Heavy equipment

Equipment used

Hours of operation

Acres disturbed and duration of disturbance

Mobile Cranes, Roll-off Trucks, Dozers, Loaders, cement trucks, and other specialty equipment used during D\&D

CWO: $4,992 \mathrm{hr}$

TRU-Separations Options: $6,240 \mathrm{hr}$

DCWO, HWO, \& VWO: $7,488 \mathrm{hr}$

CWO \& TRU-Separations Options (transport lines only): 0.09 acre

DCWO, HWO, \& VWO (ITS \& transport lines):

0.11 acre

Air emissions

Major gases (CO2, $\mathrm{H} 2 \mathrm{O}, \mathrm{O} 2, \mathrm{~N} 2)$

CWO: 2,902 tons (total)

TRU-Separations: 3,627 tons (total)

DCWO, HWO \& VWO: 4,353 tons (total)

Contaminants (Particulates, $\mathrm{CO}, \mathrm{NOx}, \mathrm{SO}, \mathrm{CWO}: 17$ tons (total)

hydrocarbons)

TRU-Separations: 21 tons (total)

DCWO, HWO, \& VWO: 25 tons (total)

Solid wastes 


\author{
Type \\ Metal building and uncontaminated transport \\ equipment \\ Quantity $\left(\mathrm{m}^{3}\right)$ \\ CWO \& TRU-Separations Options: $45 \mathrm{~m}^{3}$ \\ DCWO, HWO, \& VWO options: $90 \mathrm{~m}^{3}$ \\ Radioactive wastes \\ Cyclones, metal filters, and hoppers from transport \\ system \\ Type and Quantity \\ CWO \& TRU-Separations Options: $1.2 \mathrm{~m}^{3}$ \\ DCWO, HWO, \& VWO options: $2.6 \mathrm{~m}^{3}$ \\ Energy requirements \\ Electrical (MWh/yr) \\ $156 \mathrm{MWh} / \mathrm{yr}$ (based on John Duggan's estimate of \\ $3000 \mathrm{kWh} / \mathrm{wk}, 52 \mathrm{wk} / \mathrm{yr}$ ) \\ Fossil fuel (liters) \\ CWO: 113,380 liters \\ TRU-Separations Options: 141,725 liters \\ DCWO, HWO, \& VWO: 170,070 liters \\ Permits needed (e.g. for facility closures, \\ physical characteristics and quantities of \\ Work will be done under closure provisions $f$ \\ existing permits \\ radioactive and hazardous materials remaining \\ after closure) \\ Some differences are present between the input to project data sheets and the more formal \\ project data sheet. The primary difference involves the waste streams generated by the system. \\ Quantities for effluents, solid wastes, hazardous/toxic chemicals and wastes, and pits/ponds were \\ not reported. These values are negligible, therefore, the categories have been eliminated from \\ Table 6 . The most significant waste stream is the radioactive waste removed from the CSSFs \\ during construction activities.
}

\title{
5.0 Program Schedule
}

The calcine retrieval and transportation system will observe the same construction schedule for each waste treatment option (CWO, DCWO, HWO, VWO, and TRU Separations Options). The DCWO, HWO, VWO and TRU Separations options operate under a 20-year schedule. The CWO option has a 5-year operational schedule. The calcine retrieval system has the capacity to meet both of these schedules.
Conceptual Design
$1 / 1 / 2004-12 / 31 / 2005$
Title Design
$1 / 1 / 2006-12 / 31 / 2007$
Construction
$1 / 1 / 2008-12 / 31 / 2012$
Operations
$1 / 1 / 2013-12 / 31 / 2032$ or $1 / 1 / 2013-12 / 31 / 2017$

At the end of construction all of the CSSFs should be ready for retrieval by $1 / 1 / 2013$. SO testing and operational readiness reviews will be conducted during the construction period. The waste treatment options require that calcine retrieval begin on $1 / 1 / 2013$ to allow time for $D \& D$ of the 
Waste Treatment Facility and the CSSFs by the target date of 2035 for storage of road ready HLW.

The calcine retrieval and transportation system described above has the capacity to retrieve the estimated total volume of calcine during the operations time period. The total estimated volume of calcine present in the CSSFs by 2013 is $5435 \mathrm{~m}^{3}$ (determined in reference 14). The average bulk density of calcine is $1400 \mathrm{~kg} / \mathrm{m}^{3}$ (according to reference 1 ). The calcine retrieval and transportation system has the capacity to retrieve calcine at a combined retrieval rate of 5400 $\mathrm{kg} / \mathrm{hr}$ (two independent systems with a retrieval rate of $2700 \mathrm{~kg} / \mathrm{hr}$ ). The calcine retrieval and transportation system will need to operate for $1409 \mathrm{hr}$ to retrieve the total estimated volume of calcine. The waste treatment facilities generally assume a downtime factor of $50 \%$ to allow for equipment maintenance. Accounting for the $50 \%$ downtime factor, the calcine retrieval and transportation system will need to operate for $10.8 \mathrm{hr} / \mathrm{wk}$ and $2.7 \mathrm{hr} / \mathrm{wk}$ for the 5 -year and 20year operation schedules, respectively. The short operating time suggests that the optimum retrieval rate could be reduced without adversely impacting the schedule. The calcine retrieval and transportation system has the capacity to retrieve the total estimated volume of calcine during the required operational periods.

A retrieval schedule for the CSSFs not presented in this EDF. There are too many unknown parameters to develop a complete and optimum calcine retrieval schedule. The retrieval schedule must be coordinated between the Waste Treatment Facility and the CSSF closure study $^{12}$. This section is merely intended to show that the proposed retrieval system has the capacity to retrieve the estimated volume of calcine in the time period outlined by the Consent Order. The Consent Order requires that all high level waste be placed in a road ready form by a target date of 2035. The above calculation shows the total estimated calcine volume can be retrieved and delivered to the Waste Treatment Facility in the necessary time frame.

\subsection{Costs}

The cost estimate for the calcine retrieval and transportation system was completed in modular sections to accommodate the differences in the waste treatment options. Each CSSF was independently evaluated. CSSFs 5,6 , and 7 are similar therefore one cost estimate, which may be applied to CSSF 5,6, and 7, was developed. Two transport system costs were developed. Transport system A reflects the cost of transporting calcine from the CSSF to the necessary facilities for the CWO and TRU Separations options. Transport system B reflects the cost of transporting calcine from the CSSFs to the Waste Treatment Facility for the DCWO, HWO, and VWO options. Estimates to purchase and install D\&D risers and remove corrosion coupons are also included. The cost estimate from implementation alternative B (see section 4.0) is presented below. A preliminary cost estimate for implementation of alternative $A$ can be found in the project file.

Appendix F contains the Cost Estimate Support Data Recapitulation, summary sheets, and contingency analysis for each module of the capital cost estimate and the complete life cycle cost estimate. The detail sheets used to develop the capital cost estimate are not included in this EDF. They are located in the project file and are available upon request. 
Although the Fluor-Daniels study was the basis for this study, the total estimated cost (TEC) of the calcine retrieval and transportation system developed in this EDF is nearly twice the TEC for the Fluor-Daniels ${ }^{2}$ design. The higher cost estimate can be attributed to 4 factors. First, more demolition work during the CSSF access phase was estimated for this design than for the FluorDaniels design. The higher cost of demolition work is a result of higher estimated radiation levels in the superstructure and removal of more equipment (including the cyclone and its associated piping). Second, Fluor-Daniels specified that 2 sets of retrieval equipment (confinement enclosures, bridge cranes, VDAs, and shielded jumpers) were necessary. The sets of retrieval equipment would be moved from CSSF to CSSF. Seven sets of retrieval equipment were necessary for the design presented in this EDF because of the interfaces with the Waste Treatment Facility and the CSSF closure study. Third, the location of the Waste Treatment Facility for the DCWO, HWO, and VWO options requires an intermediate transport station to deliver the calcine to the Waste Treatment Facility. This resulted in 2 more sets of transport equipment (transport air blowers, balancing air blowers, HEPA filters, heat exchangers, cyclones, and sintered metal filters) as well as additional transport piping. This additional equipment resulted in a higher cost for the transport system. Fourth, the Fluor-Daniels study did not examine removal of corrosion coupons or installation of D\&D risers. During this study it was found to be necessary to remove the corrosion coupons prior to retrieval activities. The corrosion coupons present a significant risk to the safe and efficient operation of the calcine retrieval and transportation system. D\&D risers, installed after retrieval operations are complete, are necessary to interface with the CSSF closure project. The TEC of the calcine retrieval and transportation system developed in this EDF is nearly twice the TEC for Fluor-Daniels ${ }^{2}$ calcine retrieval and transportation system. 
Table 7. Cost Summary of the Calcine Retrieval and Transportation System (Costs Shown are $\mathrm{x} 1000$ )

\begin{tabular}{|c|c|c|c|c|c|}
\hline Costitem & TCWO & JUCWO & pHWO & jWWO & Thu separations \\
\hline $\begin{array}{l}\text { OPC (unescalated) } \\
\text { OPC Escalation } \\
\text { OPC Mgmt Reserve } \\
\text { OPC Contingency } \\
\text { Total OPC }\end{array}$ & $\begin{array}{r}27,267 \\
9,358 \\
0 \\
10,475 \\
41,100\end{array}$ & $\begin{array}{r}27,257 \\
9,358 \\
0 \\
10,475 \\
41,100\end{array}$ & $\begin{array}{r}21,267 \\
9,358 \\
0 \\
10,475 \\
41,100\end{array}$ & $\begin{array}{r}27,267 \\
9,358 \\
0 \\
10,475 \\
41,100\end{array}$ & $\begin{array}{r}21,267 \\
9,358 \\
0 \\
10,475 \\
41,100\end{array}$ \\
\hline $\begin{array}{l}\text { TEC (Unescalated) } \\
\text { TEC Escalation } \\
\text { TEC Mgmt Reserve } \\
\text { TEC Contingency } \\
\text { Total TEC }\end{array}$ & $\begin{array}{r}123,193 \\
52,330 \\
13,406 \\
49,992 \\
238,921\end{array}$ & $\begin{array}{r}127,031 \\
53,841 \\
13,788 \\
51,611 \\
246,271\end{array}$ & $\begin{array}{r}127,031 \\
53,841 \\
13,788 \\
51,611 \\
246,271\end{array}$ & $\begin{array}{r}127,031 \\
53,841 \\
13,788 \\
51,611 \\
246,271\end{array}$ & $\begin{array}{r}123,193 \\
52,330 \\
13,406 \\
49,992 \\
238,921\end{array}$ \\
\hline TPC (unescalated) & 144,460 & 148,298 & 148,298 & 148,298 & 144,460 \\
\hline $\begin{array}{l}\text { TPC Escalation } \\
\text { TPC Mgmt Reserve } \\
\text { TPC Contingency } \\
\text { Total TPC }\end{array}$ & $\begin{array}{r}61,688 \\
13,406 \\
60,467 \\
280,021\end{array}$ & $\begin{array}{r}63,199 \\
13,788 \\
62,086 \\
287,371\end{array}$ & $\begin{array}{r}63,199 \\
13,788 \\
62,086 \\
287,371\end{array}$ & $\begin{array}{r}63,199 \\
13,788 \\
62,086 \\
287,371\end{array}$ & $\begin{array}{r}61,688 \\
13,406 \\
60,467 \\
280,021\end{array}$ \\
\hline $\begin{array}{l}\text { Operations (unescalated) } \\
\text { Operations Escalation } \\
\text { Operations Contingency } \\
\text { Total Operations }\end{array}$ & $\begin{array}{r}19,393 \\
11,085 \\
9,143 \\
39,621\end{array}$ & $\begin{array}{r}72,781 \\
71,923 \\
43,411 \\
188,115\end{array}$ & $\begin{array}{r}72,781 \\
71,923 \\
43,411 \\
188,115\end{array}$ & $\begin{array}{r}72,781 \\
71,923 \\
43,411 \\
188,115\end{array}$ & $\begin{array}{r}71,594 \\
70,751 \\
42,704 \\
185,049\end{array}$ \\
\hline $\begin{array}{l}\text { Post Operations (unescalated) } \\
\text { Post Operations Escalation } \\
\text { Post Operations Contingency } \\
\text { Total Post Operations }\end{array}$ & $\begin{array}{r}14,713 \\
10,712 \\
3,814 \\
29,239\end{array}$ & $\begin{array}{r}21,960 \\
37,071 \\
8,855 \\
67,886\end{array}$ & $\begin{array}{r}21,960 \\
37,071 \\
8,855 \\
67,886\end{array}$ & $\begin{array}{r}21,960 \\
37,071 \\
8,855 \\
67,886\end{array}$ & $\begin{array}{r}21,335 \\
36,016 \\
8,603 \\
65,954\end{array}$ \\
\hline $\begin{array}{l}\text { Total Cost (unescalated) } \\
\text { Total Cost (w/escalation, mgt reserve, \& contingency) } \\
\text { Discounted Cost (escalated) }\end{array}$ & $\begin{array}{l}778,566 \\
348,880 \\
166,409\end{array}$ & $\begin{array}{l}243,039 \\
543,371 \\
196,876\end{array}$ & $\begin{array}{l}243,039 \\
543,371 \\
196,876\end{array}$ & $\begin{array}{l}243,039 \\
543,371 \\
196,876\end{array}$ & $\begin{array}{l}237,389 \\
531,023 \\
192,309\end{array}$ \\
\hline
\end{tabular}

\subsection{Recommendations for Further Study}

Issues that should be further studied are identified in sections 3.1.4, 3.2.4, and 3.3.4. The majority of these issues will be resolved as feasibility studies and title design are completed. Several have significantly more impact on whether or not the design is viable. Efforts should be made to characterize the state of calcine in the bins as well as the radiation levels in the CSSF superstructures. The limits on the transport length of a vacuum system should be identified. The feasibility of an intermediate transport station and its configuration should be examined. A closure method should be developed for the transportation system. There are several issues that require coordination with the Waste Treatment Facility and the CSSF closure study ${ }^{12}$. These issues must be resolved before title design can begin.

\subsection{Uncertainties}

Many issues and their associated uncertainties were discussed in sections 3.1.4, 3.2.4, and 3.3.4. These issues will not be repeated in this section. The major uncertainties, which apply to the overall project, will be discussed in this section. 


\subsection{Maturity of Technology}

Guidance for determining the maturity of technology is found in the U.S. Department of Energy Standard Operating Procedure, Interim Guidance, Office of Science and Technology,Technology Decision Process, May 8, 1997. The technological maturity is classified into stages 1 through 7. They are titled Basic Research, Applied Research, Exploratory Development, Advanced Development, Engineering Development, Demonstration, and Implementation. This reference details the minimum goals, objectives, measures of effectiveness, actions, and responsibilities to include requirements for entry into the next stage.

The basic technologies (brief descriptions of these basic technologies can be found in sections 3.1.2, 3.2.2, and 3.3.2) necessary to implement the calcine retrieval and transportation system are generally well developed. The classifications of the various technologies range from the "Exploratory Development" stage to the "Implementation" stage. Some of these technologies (particularly the remote welding device and VDA) have been proven in industry in hands on applications. They must be converted for remote use prior to implementation in the calcine retrieval and transportation system. The majority of the remote technologies necessary for decontamination have been demonstrated at the INEEL and other facilities. Applied research on INEEL surrogate calcine has shown that free flowing calcine is retrievable using this technology. These systems require development and testing to ensure their reliability and performance in this application. Some aspects of engineering development require considerably more work.

\subsection{Risk Assessment}

Schedule and cost risks are identified in this EDF. They are categorized below according to the source of the risk. The risks are the same for both implementation options and project schedules. Risk Assessment Forms for all identified risks are included in Appendix E along with an explanation of the Risk Rating calculation method.

The risk ratings can vary from "1" to "9". The highest risk in each category is rated at " 4 ". The risks with the highest ratings will be discussed in this section.

\subsubsection{Project Risk}

P.1 Integrity of CSSF maintained

P.2 Location of retrieval risers

P.3 Estimated retrieval percentage too high

P.4 Internal obstructions prevent retrieval

P.5 Waste Treatment Facility too slow

P.6 Miscellaneous materials prevent retrieval

$$
\begin{aligned}
\text { risk } & =3 \\
\text { risk } & =3 \\
\text { risk } & =2 \\
\text { risk } & =2 \\
\text { risk } & =2 \\
& \text { risk }=4
\end{aligned}
$$

This risk is discussed in some detail in section 3.2.4. However, it bears repeating here. During filling of the CSSFs, miscellaneous materials entered the bins. No attempt has been made to remove these materials from the bins. There is a high potential that the material is scattered throughout the bins. These materials may damage the bins or the calcine retrieval and transportation system if they were to be picked by the suction nozzle or air jet. Therefore, this 
risk was assigned a probability of " 2 ." There is some potential that the schedule will be disrupted, costs will increase, and the performance of the system may be degraded. The impact of this risk is rated at " 2 " because the calcine retrieval and transportation system minimizes the impact. The potential for schedule disruption and increased costs are reduced because each transport system has a back up transport line and retrieval could be switched to another bin or CSSF.

\subsubsection{Technical Risk}

T.1 Objective retrieval rate too high

T.2 All calcines are not retrievable risk $=3$

risk $=4$

This risk is based on an assumption. It was necessary to assume at the onset that all types of calcine could be retrieved with one system. It is possible that some of the calcine in the CSSFs is agglomerated. If it is agglomerated the potential exists that it is not retrievable. Schedule disruptions and increased costs are possible if a significant amount of calcine is not retrievable using the calcine retrieval and transportation system. This risk was assigned a probability and impact of " 2 " because it is possible that it will occur and adversely affect the schedule and costs.

\subsubsection{ES\&H Risk}

\section{ESH.1 Construction radiation dose rates incorrect risk $=4$}

The radiation levels in the CSSF superstructures are not known. A comprehensive survey of these areas has not been conducted. The relative radiation levels used to develop the cost estimates were assumed. It is likely that these levels were incorrectly estimated. The radiation levels may be significantly higher in the CSSF superstructure. If the actual radiation levels are different from the estimated levels there is a potential to impact the schedule and costs. Therefore, the probability and impact were each assigned a value of " 2 ." The risk is given a value of " $4 . "$

\subsection{Failure Modes}

Possible failure scenarios are identified in this section. It is outside the work scope of this study to evaluate these scenarios. However, efforts have been made to ensure that double confinement of the calcine is maintained at all times. This reduces the risk of a source-term release due to a failure of the calcine retrieval and transportation system. The failure modes include:

- Loss of negative pressure in the confinement enclosure

- Leakage in the confinement enclosure

- Bin breach caused during installation of additional retrieval risers leading to a calcine release into the bin vault

- Loss of calcine confinement around the retrieval equipment during operation of retrieval equipment

- Erosion failure in the transport lines 
431.02\#

$01 / 29 / 98$

Rev. $\# 00$

\section{ENGINEERING DESIGN FILE}

Function File Number - SPR-WTS-01

EDF Serial Number - EDF-WTS-002

Page 42 of 62

- Erosion failure in the cyclone

- Power failure during calcine retrieval

A failure would most significantly affect the schedule. The level of significance is dependent upon the failure. Any failure is expected to halt all activities involving calcine retrieval and transportation until the cause of the failure is resolved and necessary modifications are made. The current schedule for calcine retrieval allows $50 \%$ downtime for maintenance. This allows some slack time for unanticipated failures.

\subsection{Cost Estimate Uncertainties}

Competent cost estimators who are familiar with work conducted at the ICPP prepared the cost estimate. Efforts have been made to ensure the cost estimate for the calcine retrieval and transportation system is consistent with the cost estimates for the non-separations options and the TRU Separation option. Radiation zone work has been conservatively estimated based on known incidents during filling of the CSSFs (Dan Staiger's draft report "Review of High Level Wastes Stored at the ICPP"). The assumptions used to develop the cost estimate are outlined in the introductory letter. The cost estimate is well detailed.

\subsection{Potential Impacts of NRC Licensing}

Licensing a nuclear facility requires preparing and submitting an application and supporting documents to the NRC, such as Safety Analysis Reports, an Environmental Reports, quality assurance documents, training plans, monitoring plans, and safeguards and security plans. The NRC licensing process is divided into four stages: pre-application stage, application review stage, construction and operating license stage, and decontamination and site closure stage. The licensing duration from submitting the application to receiving the license is expected to require a minimum of an additional three to five years. The benefits of NRC licensing are enhanced operating safety, strengthened relationships with stakeholders, and license-holder participation in future regulation development.

According to data developed by Fluor-Daniels ${ }^{2}$, the estimated cost for NRC licensing is $14 \%$ of the escalated Total Estimated Cost to comply with the NRC requirements that exceed the current DOE requirements. A NRC licensed facility will experience greater costs during the operating period. These additional costs are discussed in the reports for the separate processing options (in the section titled "Potential Impacts of NRC Licensing").

Some of the potential major impacts associated with NRC licensing of Waste Treatment Facilities, other than cost, are:

- Increased oversight, including more public involvement and input in all decision processes

- More restrictive physical limits on some parameters, including exposure limits, seismic, and tornado 
- More strict radiation monitoring

- Restrictions on sharing utilities between facilities

- More stringent evaluations of the impact from off-site hazards.

- Full testing required for emergency utilities

- Physical changes to the plant and equipment

- More elapsed schedule time required

- The methods to comply with some other codes and standards may be complicated and require more time

- Although the NRC may license the WTS facilities, it may not automatically inherit or adopt the same agreements and obligations with the State of Idaho and EPA Region 10 that are in place for DOE and INEEL

\subsection{Summary and Conclusions}

The calcine retrieval and transportation system presented above combines the best elements of previous studies (references 2,3 , and 8 ), pilot plant tests, and experience. The system meets the requirements set forth in the Consent Order, statutory law, and DOE orders. The assumptions used to develop this system have strong bases.

The system was discussed in three sections: CSSF access, calcine retrieval system, and calcine transportation system. During CSSF access, the CSSFs will be prepared for calcine retrieval. Superstructure buildings, equipment, and piping will be decontaminated and removed from CSSFs 1-4. The concrete vaults of CSSFs 5-7 will be decontaminated but not removed. Existing retrieval lines will be accessed. New retrieval lines will be added to CSSFs 1,2, and 3 . The calcine retrieval and transportation systems will function simultaneously. Calcine will be retrieved from the CSSFs using a fluidizing air jet and a suction nozzle. Then the calcine will be directly placed in the pneumatic transport system for transport to the Waste Treatment Facility.

There are several issues that must be resolved before the calcine retrieval and transportation system can be termed feasible. A plan to deal with the corrosion coupons and other miscellaneous items in the bins should be developed. These items should be removed from the bins or prevented from entering the transport system. The transport system currently calls for longer transport distances than are accepted by industry. It was proposed that testing be done to verify that a vacuum system (with intermediate transport stations, if necessary) can be efficiently used over long distances. Resolving these issues will increase the feasibility of the calcine retrieval and transportation system. The remaining issues (sections 3.1.4, 3.2.4, and 3.3.4) should be resolved over the course of feasibility studies and title design. 
The cost estimate was developed in modules to allow an "apples to apples" comparison with the Fluor-Daniels retrieval and transportation system. Initially, two alternatives for implementation were examined. The first alternative was removed from consideration. It did not satisfy all the processing options and adversely impacted CSSF closure study ${ }^{12}$ because only 2 sets of retrieval equipment were specified. The second alternative represents a retrieval and transportation system that interfaces with the Waste Treatment Facility and the CSSF closure study ${ }^{12}$. A cost estimate based on the second alternative bounds the cost of the project. Separate capital cost estimates were developed for installation of $D \& D$ risers and removal of corrosion coupons.

Life cycle costs for construction, operation, and closure of the calcine retrieval and transportation system were developed. Removal of corrosion coupons and installation of D\&D risers are included in the life cycle cost estimate for each waste treatment option.

The CWO option requires a 5-year operating period. This option uses transport system $A$ to deliver the calcine from the CSSFs to the NWCF. The total unescalated cost for the CWO option is $\$ 178,566,000$. The total cost with escalation, management reserve, and contingency is $\$ 348,880,000$. The discounted annual cost for the CWO option is $\$ 166,409,000$.

The TRU-Separations Options requires the calcine retrieval and transportation system to operate for 20 years. This option employs transport system A to deliver the calcine from the CSSFs to the Calcine Dissolution Facility. The total unescalated cost for the CWO option is $\$ 237,389,000$. The total cost with escalation, management reserve, and contingency is $\$ 531,023,000$. The discounted annual cost for the CWO option is $\$ 192,309,000$.

The DCWO, HWO, and VWO options have a 20 year operating period. These options require transport system $B$ to deliver the calcine from the CSSFs to the Waste Treatment Facilities. The total unescalated cost for the CWO option is $\$ 243,039,000$. The total cost with escalation, management reserve, and contingency is $\$ 543,371,000$. The discounted annual cost for the CWO option is $\$ 196,876,000$. 


\section{Appendix A}

\section{Drawing}

CRT-01 HVAC Flow Diagram

CRT-02 Pneumatic Transport System

CRT-03 Intermediate Transport Station (ITS)

CRT-04 Site Plan for DCWO, HWO, and VWO

CRT-05 Site Plan for CWO 


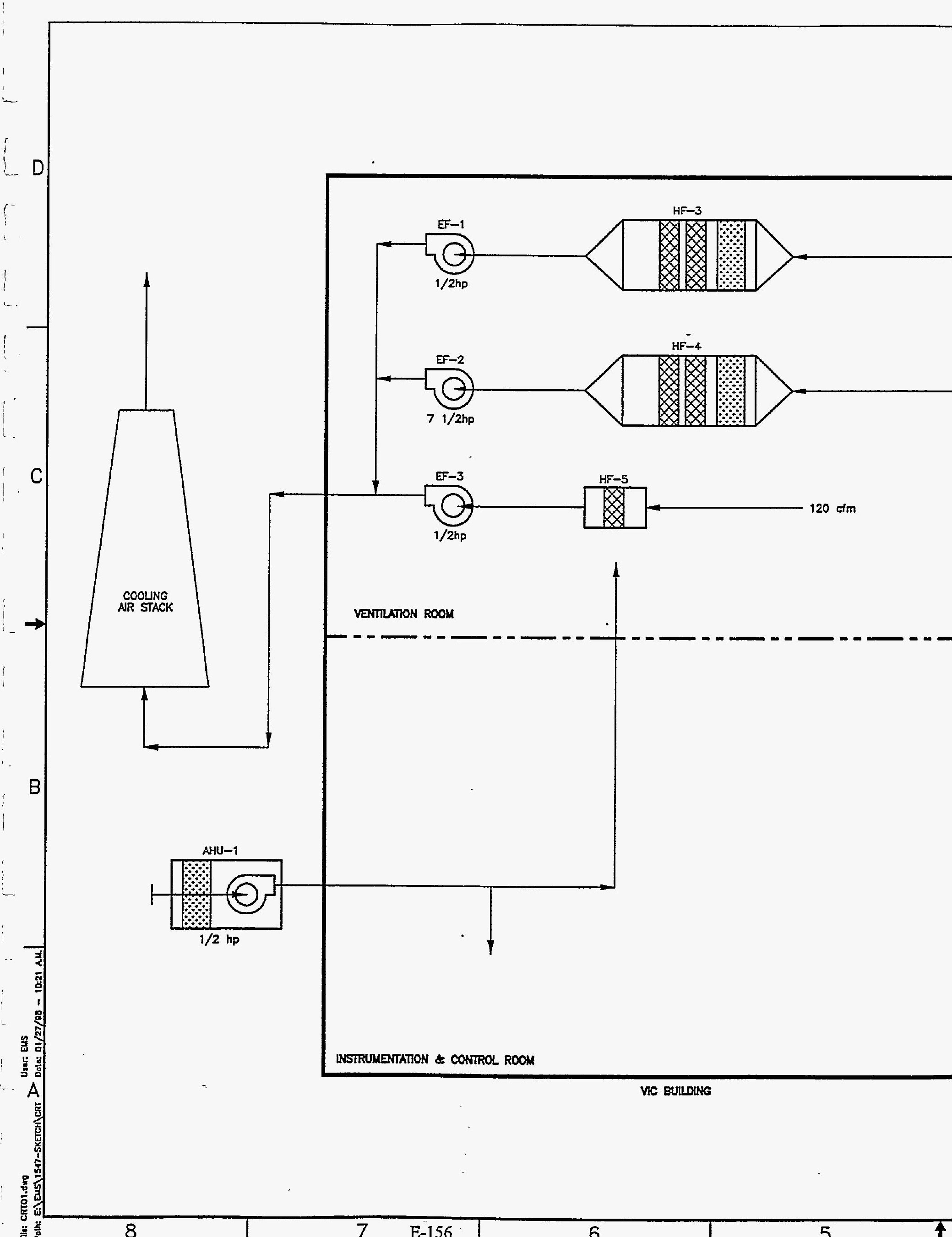




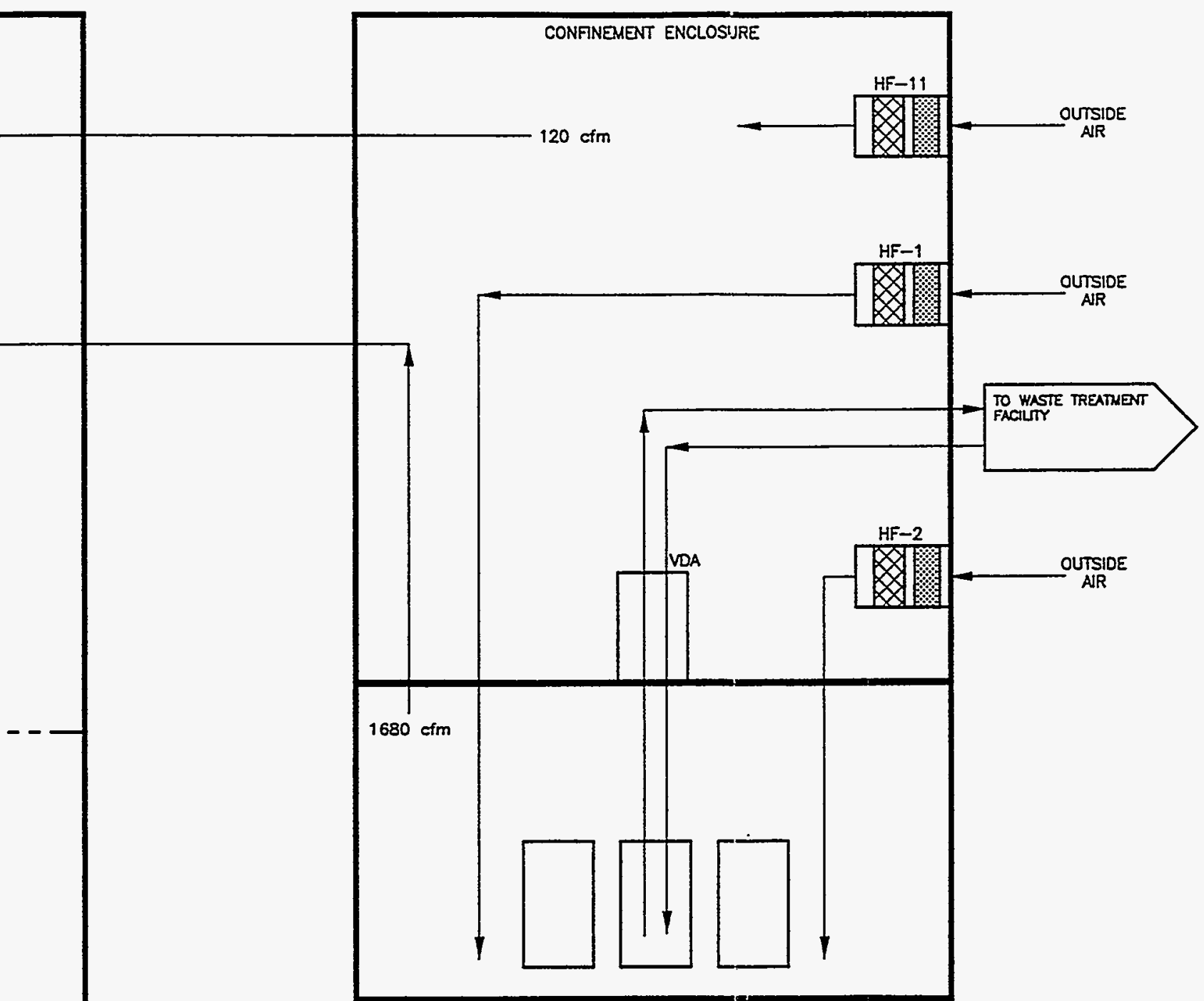

TPPYCAL CSSF

\section{LEGEND:}

- $-\ldots$ - INDICATES ROOMS

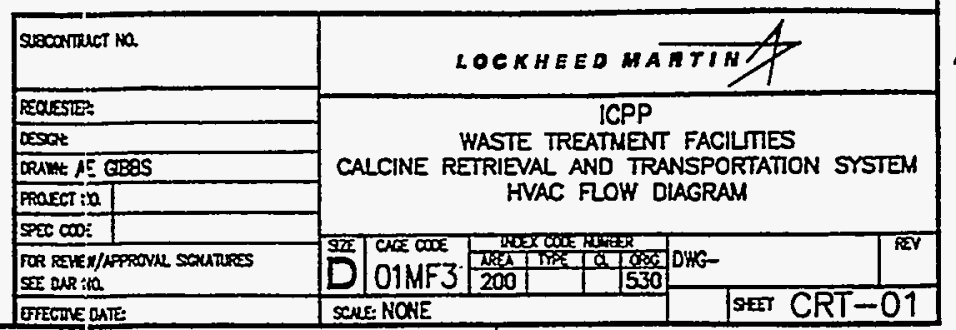




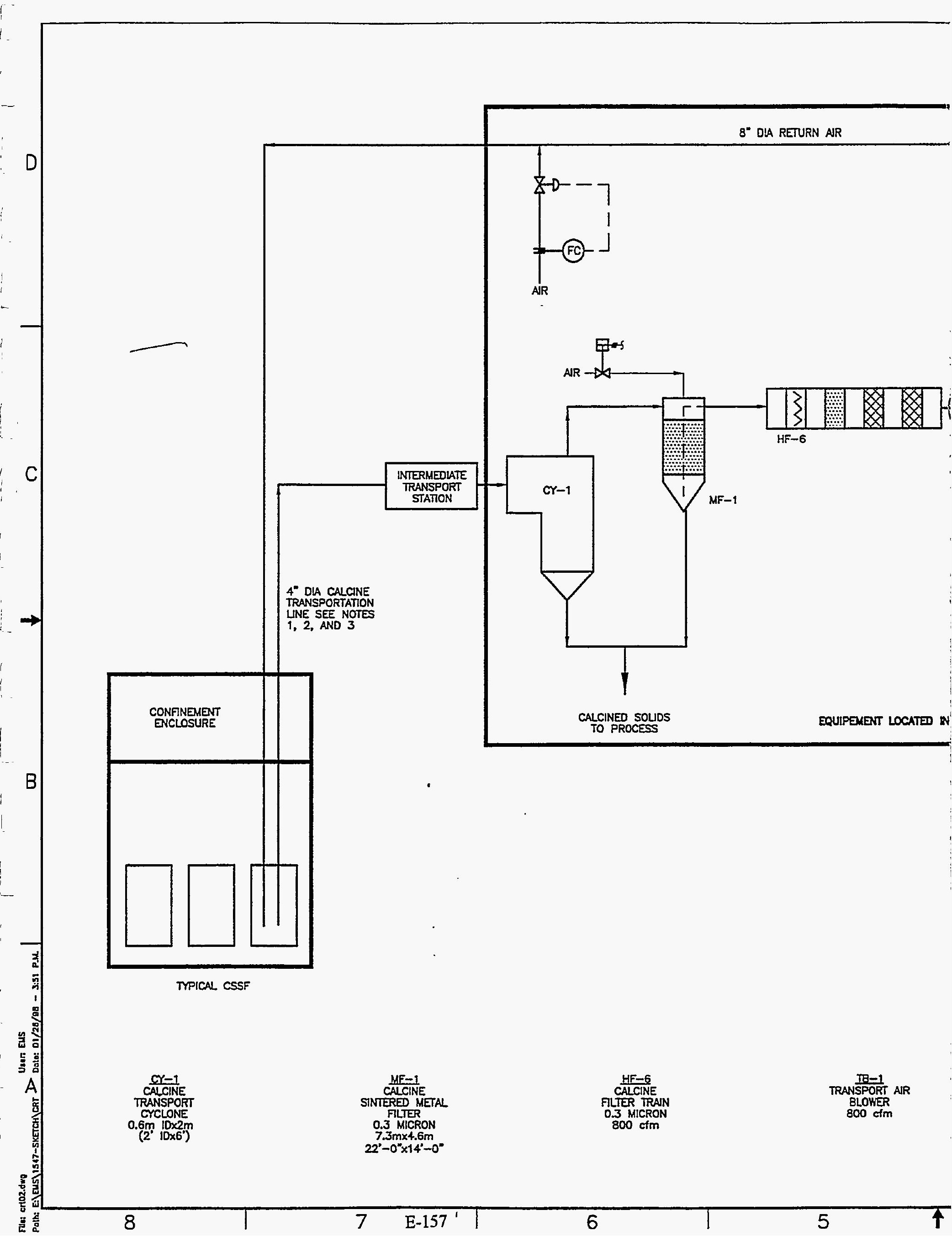




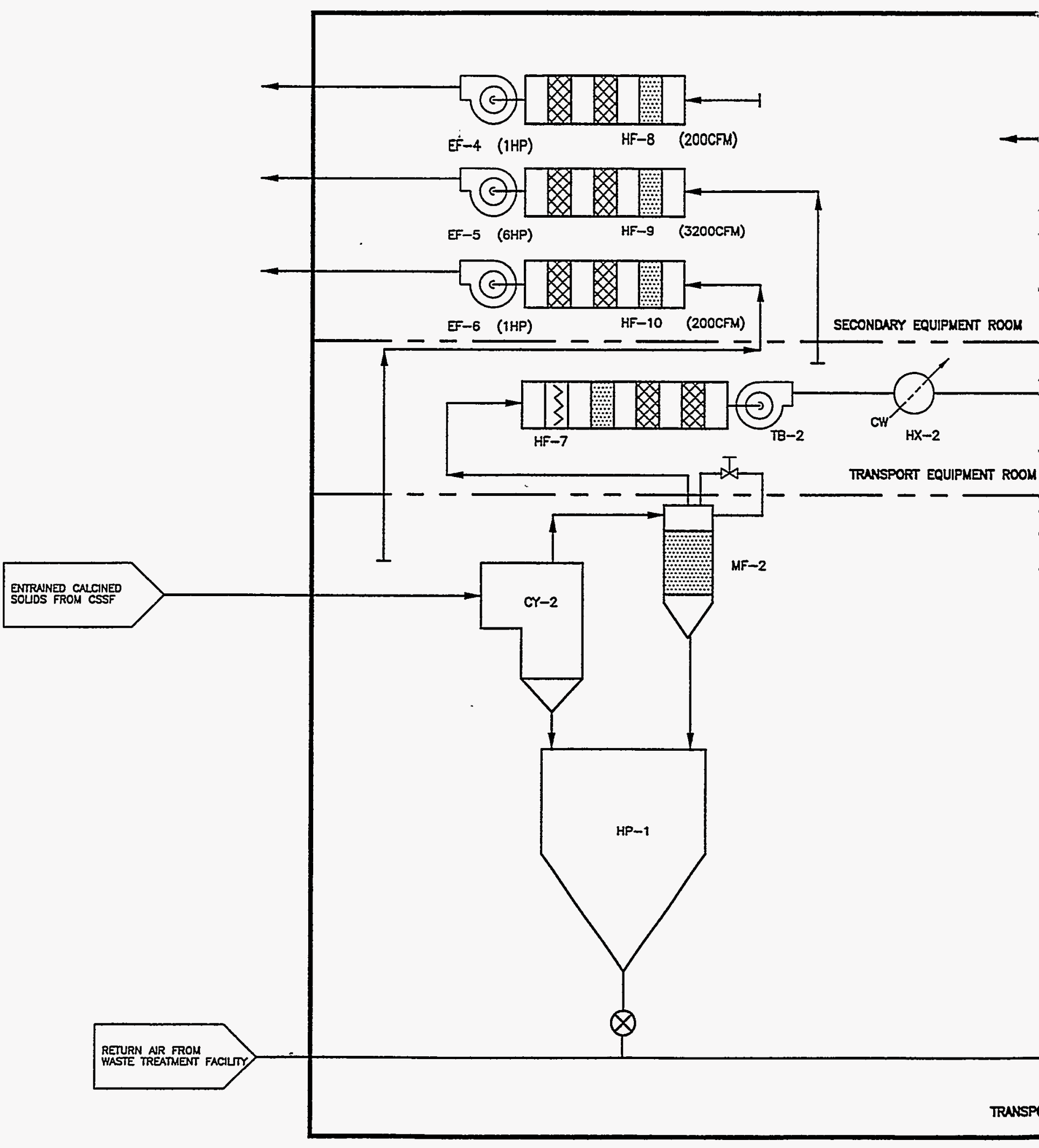

TRANSPORT

CYCLONE

$0.6 \mathrm{~m} 10 \times 2 \mathrm{~m}$

$\left(2^{\prime} 10 \times 6^{\prime}\right)$

INTERMEDLATE SINTERED

METAL FILTER

0.3 MICRON

$7.3 \mathrm{~m} \times 4.6 \mathrm{~m}$

$22^{\circ}-0^{\circ} \times 14^{\circ}-0^{\prime \prime}$
IB-2
INTERMEDIATE TRANSPORT
AIR BLOWER $800 \mathrm{cfm}$
INTERMEDLATE BALANCING

AIR BLOWER 


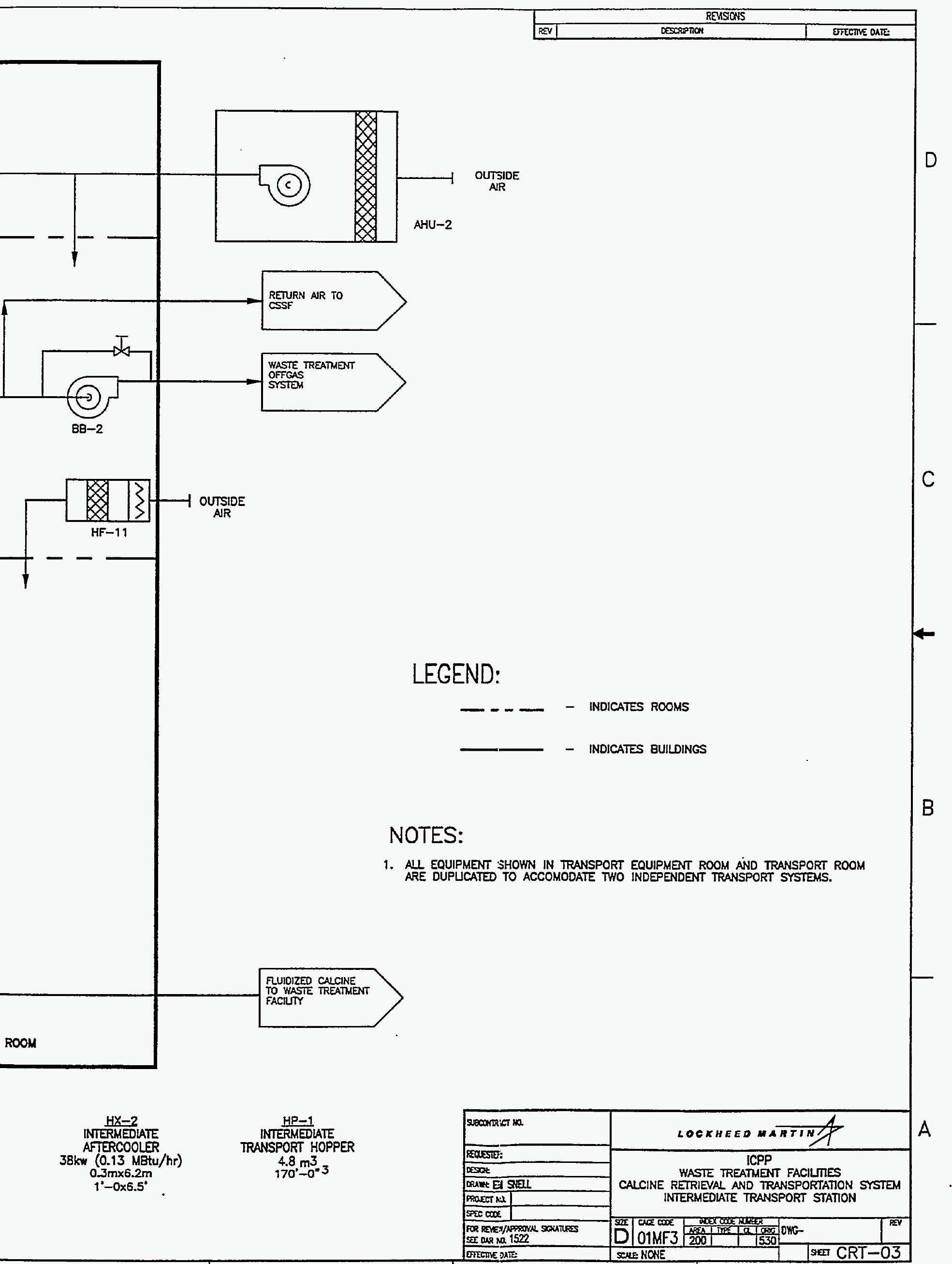




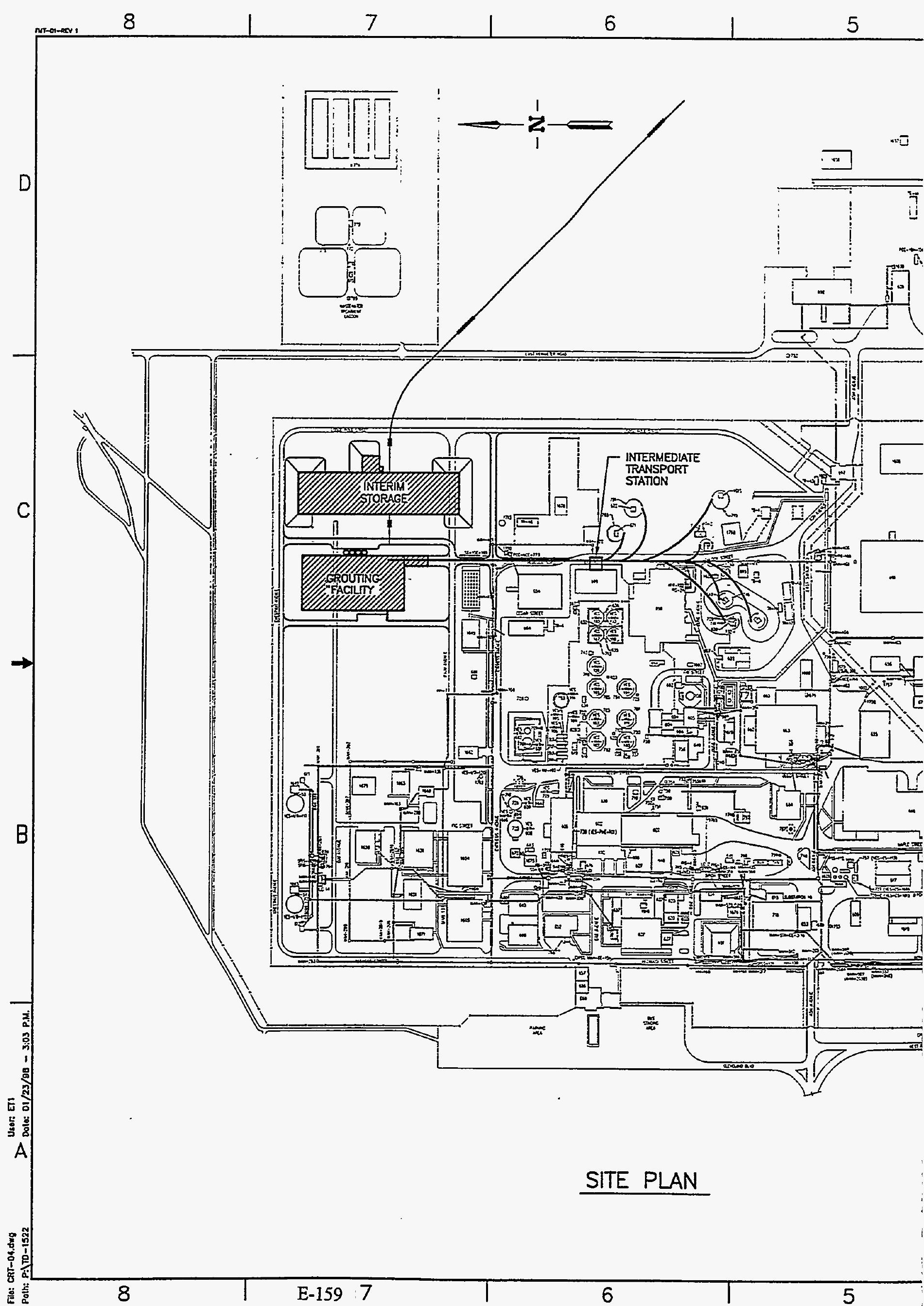


N甘7d $\exists$ IIS

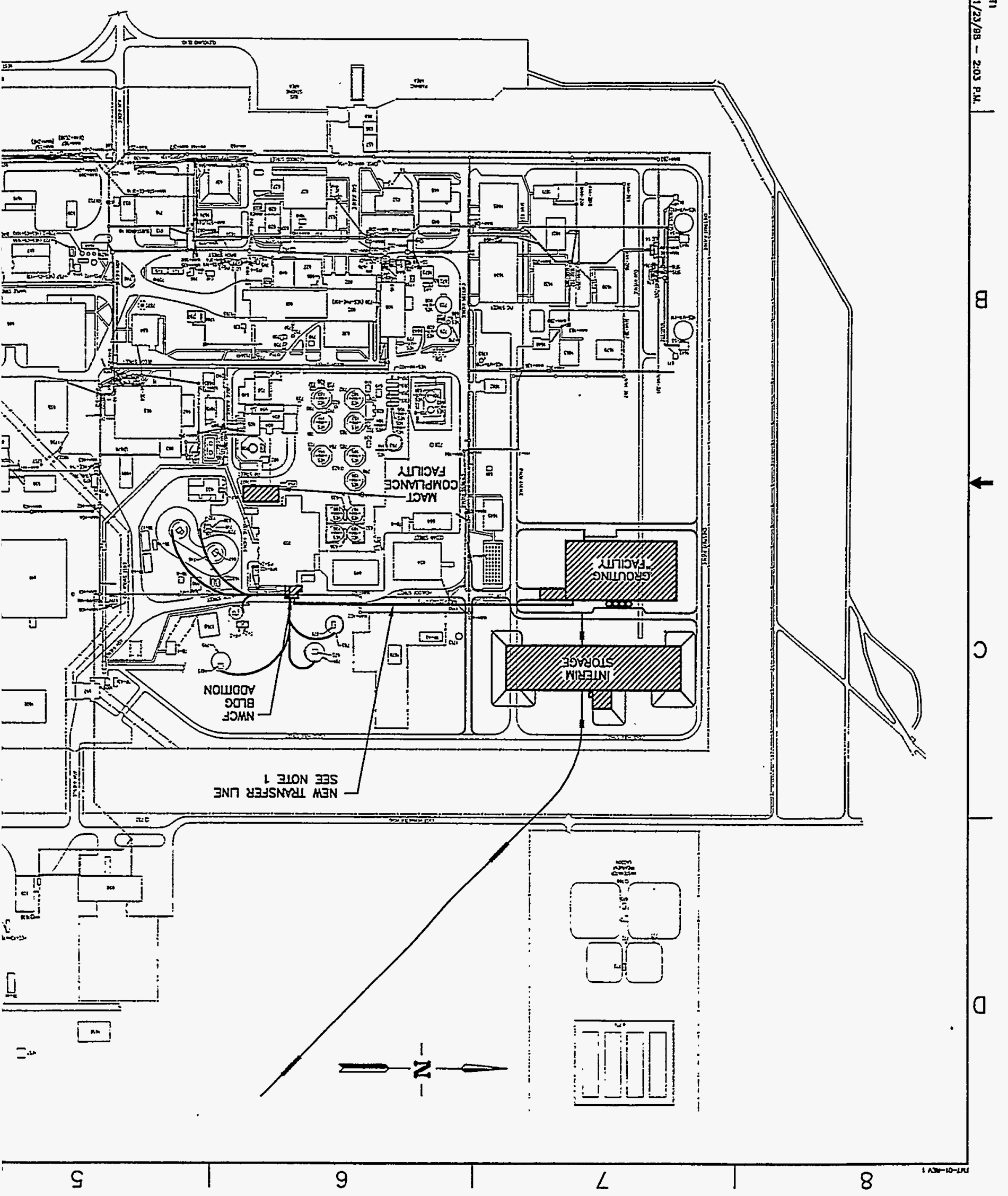




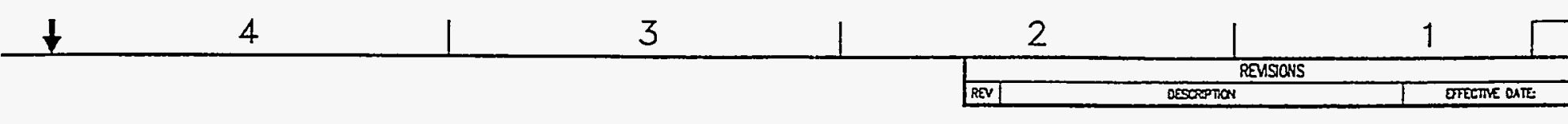

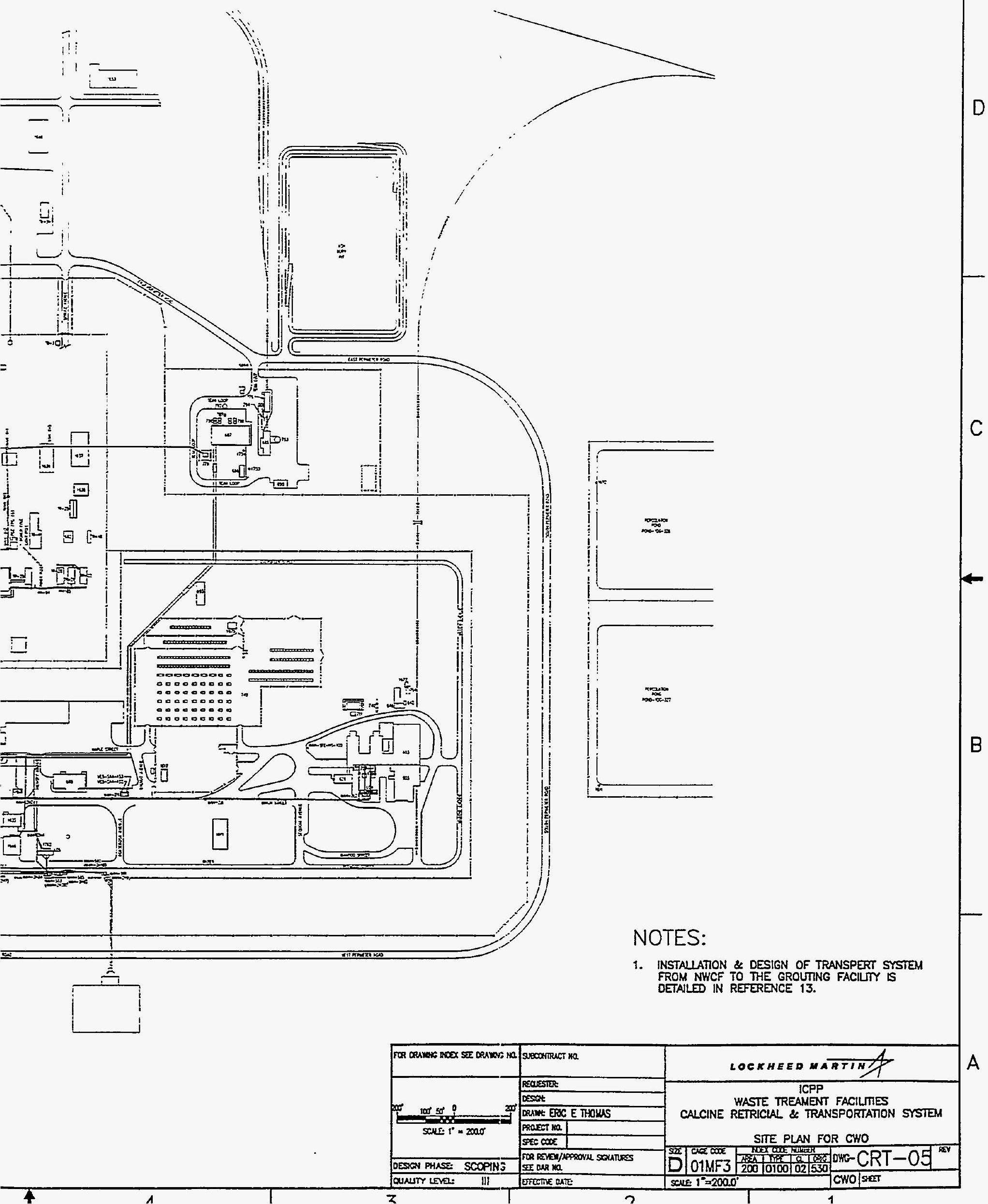


Appendix B

\section{References}

1. INEEL, Waste Inventories / Characterization Study, INEEL/EXT-97-00600, September 1997.

2. Fluor-Daniels, Inc. (Government Services Operating Company), Idaho Chemical Processing Plant Waste Treatment Facilities Feasibility Study, Delivery Order 94-36, 30\% Design Review Package, June 1997, 60\% Design Review Package, August 1997, and 90\% Design Review, October 1997.

3. Raytheon Engineers and Constructors, Inc., Idaho Chemical Processing Plant Bin Set I Calcine Recovery Project: Phase I and II Special Studies Report Volume I, DE-AC07-89ID12679, February 1995.

4. West Valley Nuclear Services Inc., West Valley Nuclear Services, Inc. Remote Installation of Risers on Underground Nuclear Waste Storage Tanks, DOE/NE/44139-45 (DE88010531), 1988.

5. INEEL, HWMA Closure Plan for the Waste Calcining Facility at the Idaho National Engineering and Environmental Laboratory rev 2, INEEL-96/0189, June 1997.

6. INEEL, New Waste Calcining Facility Deactivation Option for Low-level Waste Grout Disposal, INEEL/EXT-97/01076, December 1997.

7. Westra, A.G., J.S. Schofield, S.J. Horn, J.A. Hendricks, G.S. Pomiak, Sampling of Stored High-Level Radioactive Calcined Waste at ICPP, ICP-1186, May 1979

8. D. L. Griffith, Status of Calcine Retrieval Development Work-DLG-06-96, September 1996.

9. Regulatory Requirements and Standard Criteria for the ICPP Proposed Waste Processing Facilities, EDF-WTF-003.

10. Regulatory Design Requirements for Waste Treatment Facilities, EDF-WTS-004.

11. Calculations by Dan Griffith, December 1997 (included in this Appendix).

12. INEEL, Calcined Solids Storage Facility Closure Study, INEEL/EXT-97-01296, 1998.

13. INEEL, Cementitious Waste Option Study Report, INEEL/EXT-97-01400, 1998.

14.Estimates of Feed and Waste Volumes, Compositions, and Properties, EDF-FDO-011 rev. 1, 1998. 


\section{Memo of Conversation}

Name

Dan Griffith

Sara Gifford
Organization

LMITCO Chief Engineer

MC\&I Engineering

$6-5162$

Date: $\quad 12$ November 1997

Subject: Nozzle design and retrieval system efficiency

Discussion:

In the mock up facility, the retrieval method appears to be just as efficient (if not more so) for cylindrical bins as it is for annular bins. This is evidenced by returning the solids to the outer bin.

Previous designs indicate that it is not too difficult to design a nozzle that will prevent uptake of extraneous materials. The nozzles used in the Rover project shown that it not be a significant design effort to develop a nozzle for the retrieval lines. 
Date: Novenibar 19, 1997

To: $\quad$ Sara Gifford

From: Dan Griffith

Subject: Pressure Drop in Proposed Pneumatic Transport System

As requested I have calculated pressure drop for four cases as shown below. All cases are vacuum systems with a length of 315 feet. Inlet pressure to the transport line is 12.3 psia. I assumed the transfer line was steel pipe.

\begin{tabular}{|c|c|c|c|c|c|c|}
\hline $\begin{array}{l}\text { Diameter } \\
\text { Inches }\end{array}$ & $\begin{array}{l}\text { Flow Rate } \\
\text { at Blower } \\
\text { fin. }\end{array}$ & $\begin{array}{l}\text { Solids } \\
\text { to Air Ratio } \\
\text { tb/lb }\end{array}$ & $\begin{array}{l}\text { Solids } \\
\text { Flow } \\
\text { kglhr }\end{array}$ & $\begin{array}{l}\text { Inlet } \\
\text { Velocity } \\
\text { fom }\end{array}$ & $\begin{array}{l}\text { Out. } \\
\text { Yel. } \\
\end{array}$ & $\begin{array}{l}\text { Pres. } \\
\text { Drop } \\
\text { psi }\end{array}$ \\
\hline 4.026 & 800 & 3.9 & 2700 & 4635 & 9049 & 6.0 \\
\hline 26 & 350 & 6.0 & 2700 & 2993 & 3959 & 3.0 \\
\hline 4.026 & 350 & 2.7 & 1360 & 3380 & 3959 & 1.8 \\
\hline 3.068 & 175 & 2.7 & 680 & 2896 & 3409 & 1.9 \\
\hline
\end{tabular}

These results do not include a return air line.

Note that for the first two cases the pressure at the blower will fall below $10 \mathrm{psia}$ which is the pressure you specified. The first case may not be feasible for a vacunn system because of the high pressure drop. The second case may not be feasible for a vacunim system because the high solids to air ratio might result in transport line plugging. The other two cases also have a fairly high solids to air ratios, but I believe they ars feasible. If it is important to achieve such high transfer rates, we should mock this up. I could test anything but the first case in my existing pilot plant. All we would need is a feeder to meter solids in at the desired rate and 300 feet of pipe. We could consider using plastic pipe to save money. We probably have components in the old pilot plant which we could use for the feeder. 
Appedix C

Equipment List 
Appendix C

Equipment List

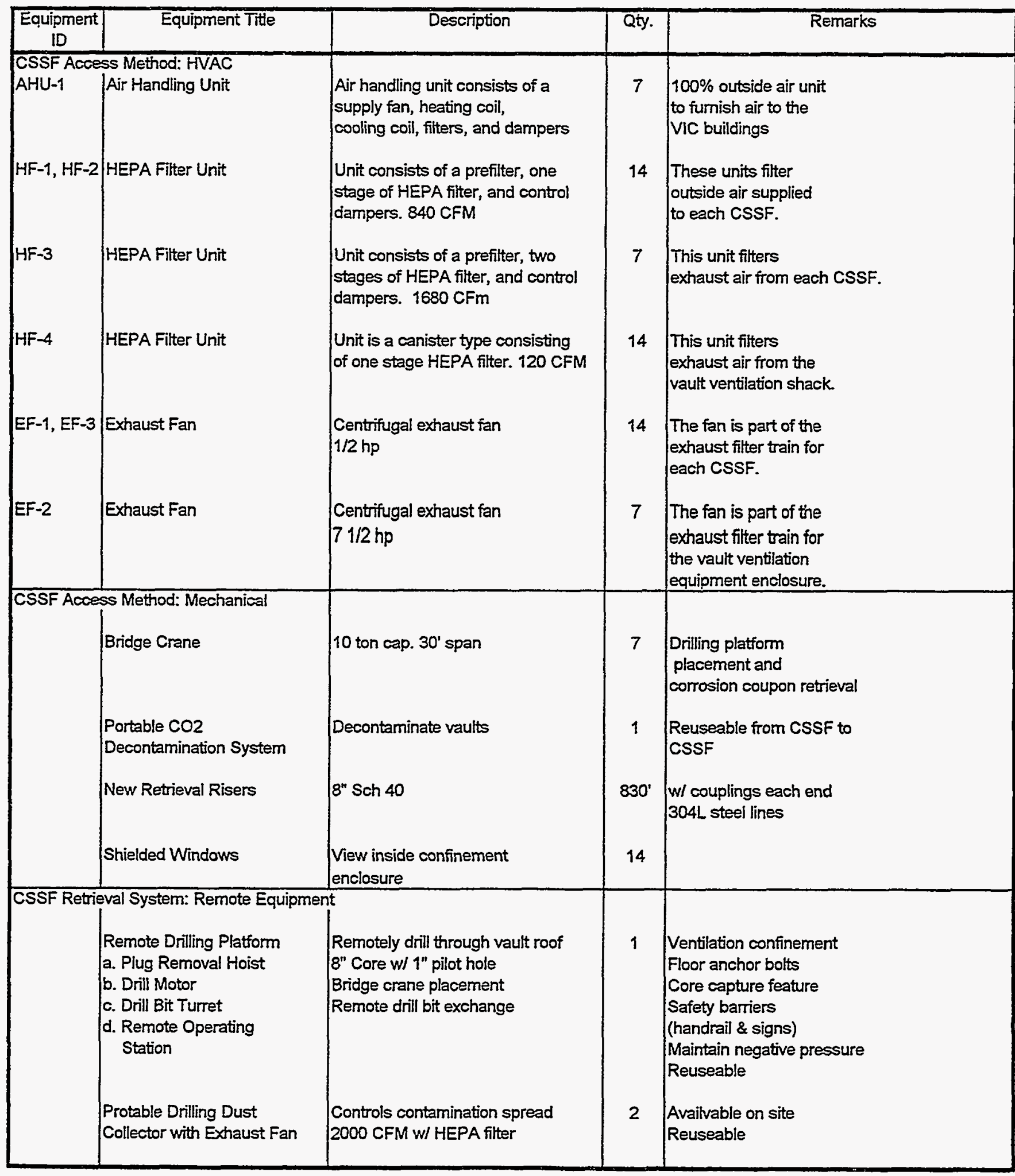




\begin{tabular}{|c|c|c|c|c|}
\hline $\begin{array}{c}\text { Equipment } \\
\text { ID }\end{array}$ & Equipment Title & Description & Qty. & Remarks \\
\hline & $\begin{array}{l}\text { Remote Welding } \\
\text { Equipment including } \\
\text { Weld Inspection Unit } \\
\text { Remote Hole Saw } \\
\\
\text { CCTV Equipment } \\
\text { a. Camera Lens \& Lighting } \\
\text { b. Extension Tubes } \\
\text { c. Video Workstation w/ } \\
\text { Camera } \\
\text { d. Switching Panel, } 2 \\
\text { Monitors, Light Control } \\
\text { e. TPZ Head Control, } \\
\text { Drive Interface Patch } \\
\text { Panel }\end{array}$ & $\begin{array}{l}\text { Remotely vreids the risers to the } \\
\text { bins. Then inspect the integrity } \\
\text { of the welds. } \\
\text { Open bins into added retrieval } \\
\text { risers } \\
\text { Direct during retrieval riser } \\
\text { installation, View inside of bins to } \\
\text { ensure retrieval is complete. }\end{array}$ & $\begin{array}{l}1 \\
7\end{array}$ & $\begin{array}{l}\text { Similar technology used in } \\
\text { Reference } 4 \\
\text { Reuseable } \\
\text { Capture core to prevent } \\
\text { problems during retrieval } \\
\text { Reuseable } \\
\text { Inspect riser welds }\end{array}$ \\
\hline Calcine Retr & $\begin{array}{l}\text { ieval System: Mechanical } \\
\text { Retrieval lines }\end{array}$ & $\begin{array}{l}\text { rigid lines that form } \\
\text { the suction and air } \\
\text { jet lines; stainless steel }\end{array}$ & $2310^{\prime}$ & $\begin{array}{l}\text { coupling at each end } \\
\text { size:5" diameter }\end{array}$ \\
\hline Calcine Retr & $\begin{array}{l}\text { Veval System: Remote Equipn } \\
\text { Vertical Deployment } \\
\text { Apparatus } \\
\text { a. Plug Removal Hoist } \\
\text { b. Rotation Drive } \\
\text { c. Extension Tube } \\
\text { Carousel/Turret } \\
\text { d. Air Supply House Reel } \\
\text { e. Confinement Casing } \\
\text { f. Ladder \& Platforms } \\
\text { g. External Drives } \\
\text { h. Telescoping Line with } \\
\text { Lower Seal } \\
\text { i. Vertical Position Indicator } \\
\text { Shielded Jumper }\end{array}$ & $\begin{array}{l}\text { Deploys extension pipe for } \\
\text { retrieval risers } \\
25^{\prime} \text { tall, } 6 \text { 'X6' base maximum } \\
\text { Vestibule houses carousel } \\
\text { Ventilation connection } \\
\text { Connects discharge } \\
\text { of retrieval system to } \\
\text { the transport system }\end{array}$ & 7 & $\begin{array}{l}\text { Ventilation confinement } \\
\text { SS casing for shielding } \\
\text { Anchor to fioor bolts } \\
\text { Safety barrier } \\
\text { (handrail \& signs) } \\
\text { Should be fitted so that it } \\
\text { can be used to install } \\
\text { retrieval risers as well as } \\
\text { extend retrieval lines. } \\
\\
\qquad \\
\text { double wall, heavy wall } \\
\text { pipe, shielded and } \\
\text { independently mounted } \\
\text { (steel flanged, gasketed) }\end{array}$ \\
\hline $\begin{array}{l}\text { Calcine Tr } \\
\text { HF-6 }\end{array}$ & $\begin{array}{l}\text { Isportation System: HVAC (ec } \\
\text { Filter Train }\end{array}$ & $\begin{array}{l}\text { Prefilter and } 2 \text { stage } \\
\text { HEPA filters } \\
380 \text { L/s ( } 800 \mathrm{cfm}) \\
304 \mathrm{~L} \text { stainless steel }\end{array}$ & $\begin{array}{l}\text { ility) } \\
2\end{array}$ & $\begin{array}{l}\text { Filter air before entering } \\
\text { transport air blower } \\
380 \mathrm{~L} / \mathrm{s}(800 \mathrm{cfm})\end{array}$ \\
\hline $\begin{array}{l}\text { Calcine Tra } \\
\text { BB-1 } \\
\text { TB-1 }\end{array}$ & $\begin{array}{l}\text { Sportation System: Mechanic } \\
\text { Balancing Air Blower } \\
\text { Transport Air Blower }\end{array}$ & $\begin{array}{l}\text { (equipment located in Waste Treat } \\
80 \mathrm{cfm} \\
380 \mathrm{Ls}(800 \mathrm{cfm}) \\
\text { at } 69 \mathrm{kPa}(10 \mathrm{psi}) \\
304 \mathrm{~L} \text { stainless steel }\end{array}$ & $\begin{array}{c}2 \\
2 \\
2\end{array}$ & $\begin{array}{l}\text { Provide transport } \\
\text { air for system as well as } \\
\text { suction and air jets }\end{array}$ \\
\hline
\end{tabular}




\begin{tabular}{|c|c|c|c|c|}
\hline $\begin{array}{c}\text { Equipment } \\
\text { ID }\end{array}$ & Equipment Title & Description & Qty. & Remarks \\
\hline MF-1 & Sintered Metal Filter & $\begin{array}{l}0.3 \text { micron } \\
380 \text { Us }(800 \mathrm{~cm}) \\
22^{\prime \prime} \times 14^{\prime} \\
304 L \text { stainless steel }\end{array}$ & 2 & $\begin{array}{l}\text { Removes calcine fines } \\
\text { entrained in air exiting the } \\
\text { cyclone and delivers to hopper }\end{array}$ \\
\hline CY-1 & Cyclone & $\begin{array}{l}0.3 \mathrm{~m} \text { ID } \times 0.6 \mathrm{~m} \\
\left(1^{\prime} \text { ID } \times 2^{\prime}\right) \\
\text { Raytheon recommends } \\
\text { a longer length } 3^{\prime}-6^{\prime}\end{array}$ & 2 & $\begin{array}{l}\text { Separates calcine from } \\
\text { transport air and delivers it } \\
\text { to facility hopper }\end{array}$ \\
\hline \multirow[t]{5}{*}{$H X-1$} & Heat Exchanger & $\begin{array}{l}38 \mathrm{~kW}(0.13 \mathrm{Mbtu} / \mathrm{hr}) \\
0.3 \mathrm{~m} \times 2.4 \mathrm{~m} \\
380 \mathrm{~L} / \mathrm{s}(800 \mathrm{cim}) \\
304 \mathrm{~L} \text { stainless steel }\end{array}$ & 2 & $\begin{array}{l}\text { Cools air from transport } \\
\text { blower to acceptable } \\
\text { operating temperature } \\
\text { of the balancing air blower }\end{array}$ \\
\hline & $\begin{array}{l}\text { Flat Side } \\
\text { Diverter Valve }\end{array}$ & $\begin{array}{l}\text { Two-way stainless } \\
\text { steel valve, electric } \\
\text { motor controlled }\end{array}$ & 21 & $\begin{array}{l}\text { Air tight valves } \\
\text { void of leakage } \\
\text { motor located outside of } \\
\text { shielding to ease repair and } \\
\text { maintenance }\end{array}$ \\
\hline & Transport Air Lines & $\begin{array}{l}20 \mathrm{~mm} \text { ( } 8 \mathrm{in} \text { ) } 304 \mathrm{~L} \\
\text { stainless steel } \\
\text { (length depends on location of } \\
\text { Waste Treatment Facility) }\end{array}$ & 2 & $\begin{array}{l}\text { Provides air for the } \\
\text { pneumatic transport } \\
\text { system; recycled to reduce } \\
\text { releases to the environment }\end{array}$ \\
\hline & Calcine Transport Lines & $\begin{array}{l}10 \mathrm{~mm} \text { ( } 4 \mathrm{in} \text { ) } 304 \mathrm{~L} \\
\text { stainless steel } \\
\text { (length depends on location of } \\
\text { Waste Treatment Facility) }\end{array}$ & 4 & $\begin{array}{l}\text { Transport calcine from the } \\
\text { CSSF to processing } \\
\text { facility }\end{array}$ \\
\hline & Encasement Lines & $\begin{array}{l}15 \mathrm{~mm} \text { ( } 6 \mathrm{in} \text { ) } 304 \mathrm{~L} \text { stainless } \\
\text { steel } \\
\text { (length depends on location of } \\
\text { Waste Treatment Facility) }\end{array}$ & 4 & Encase calcine transport lines \\
\hline \multicolumn{5}{|c|}{ Calcine Transportation System: HVAC (Additional for DCWO, HWO, WWO: equipment located in Intermediate Transport Station) } \\
\hline AHU-2 & Air Handling Unit & $\begin{array}{l}\text { Air handling unit consists of a } \\
\text { supply fan, heating coil, cooling } \\
\text { coil, fitters, and dampers }\end{array}$ & 1 & $\begin{array}{l}100 \% \text { outside air unit } \\
\text { to fumish air to the TSS }\end{array}$ \\
\hline $\mathrm{HF}-7$ & Filter Train & $\begin{array}{l}\text { Prefilter and } 2 \text { stage } \\
\text { HEPA filters } \\
380 \mathrm{~L} / \mathrm{s}(800 \mathrm{cfm}) \\
304 \mathrm{~L} \text { stainless steel }\end{array}$ & 2 & $\begin{array}{l}\text { Filter air before entering } \\
\text { transport air blower } \\
380 \mathrm{~L} / \mathrm{s}(800 \mathrm{cfm})\end{array}$ \\
\hline HF-8 & Filter Train & $\begin{array}{l}\text { Prefilter and } 2 \text { stage } \\
\text { HEPA filters } \\
200 \mathrm{cfm}\end{array}$ & 1 & $\begin{array}{l}\text { Filter air exiting Secondary } \\
\text { Equipment Room in ITS }\end{array}$ \\
\hline HF-9 & Filter Train & $\begin{array}{l}\text { Prefilter and } 2 \text { stage } \\
\text { HEPA filters } \\
3200 \mathrm{cfm}\end{array}$ & 1 & $\begin{array}{l}\text { Filter air exiting Transport } \\
\text { Equipment Room in ITS }\end{array}$ \\
\hline$H F-10$ & Filter Train & $\begin{array}{l}\text { Prefilter and } 2 \text { stage } \\
\text { HEPA filters } \\
200 \mathrm{cfm}\end{array}$ & 1 & $\begin{array}{l}\text { Filter air exiting Transport } \\
\text { Room in ITS }\end{array}$ \\
\hline
\end{tabular}




\begin{tabular}{|c|c|c|c|c|}
\hline $\begin{array}{c}\text { Equipment } \\
\text { ID }\end{array}$ & Equipment Title & Description & Qty. & Remarks \\
\hline HF-11 & Prefilter & $\begin{array}{l}\text { Prefilter and single } \\
\text { stage HEPA filter }\end{array}$ & 1 & $\begin{array}{l}\text { Filter air entering Transport } \\
\text { Room in ITS }\end{array}$ \\
\hline EF-4 & Exhaust Fan & $\begin{array}{l}\text { Centrifugal exhaust fan } \\
1 \mathrm{hp} \text {. }\end{array}$ & 1 & $\begin{array}{l}\text { Exhaust fan for Secondary } \\
\text { Equipment Room in ITS }\end{array}$ \\
\hline EF-5 & Exhaust Fan & $\begin{array}{l}\text { Centrifugal exhaust fan } \\
6 \mathrm{hp}\end{array}$ & 1 & $\begin{array}{l}\text { Exhaust fan for Transport } \\
\text { Equipment Room in ITS }\end{array}$ \\
\hline$E F-6$ & Exhaust Fan & $\begin{array}{l}\text { Centrifugal exhaust fan } \\
1 \mathrm{hp}\end{array}$ & 1 & $\begin{array}{l}\text { Exhaust fan for Transport } \\
\text { Room in ITS }\end{array}$ \\
\hline \multicolumn{5}{|c|}{ Calcine Transportation System: Mechanical (For DCWO, WWO, HWO: equipment located in Intermediate Transport Station) } \\
\hline BB-2 & Balancing Air Blower & $80 \mathrm{~cm}$ & 2 & \\
\hline TB-2 & Transport Air Blower & $\begin{array}{l}380 \mathrm{Ls}(800 \mathrm{cfm}) \\
\text { at } 69 \mathrm{kPa}(10 \mathrm{psi}) \\
304 \mathrm{~L} \text { stainless steel }\end{array}$ & 2 & $\begin{array}{l}\text { Provide transport } \\
\text { air for system as well as } \\
\text { suction and air jets }\end{array}$ \\
\hline MF-2 & Sintered Metal Filter & $\begin{array}{l}0.3 \text { micron } \\
380 \text { Us }(800 \mathrm{cfm}) \\
22^{n} \times 14^{\prime} \\
304 \mathrm{~L} \text { stainless steel }\end{array}$ & 2 & $\begin{array}{l}\text { Removes calcine fines } \\
\text { entrained in air exiting the } \\
\text { cyclone and delivers to hopper }\end{array}$ \\
\hline CY-2 & Cyclone & $\begin{array}{l}0.3 \mathrm{~m} \text { ID } \times 0.6 \mathrm{~m} \\
\left(1^{\prime} \mathrm{ID} \times 2^{\prime}\right) \\
\text { Raytheon recommends } \\
\text { a longer length } 3^{\prime}-6^{\prime}\end{array}$ & 2 & $\begin{array}{l}\text { Separates calcine from } \\
\text { transport air and delivers it } \\
\text { to facility hopper }\end{array}$ \\
\hline$H X-2$ & Heat Exchanger & $\begin{array}{l}38 \mathrm{~kW}(0.13 \mathrm{Mbtu} / \mathrm{hr}) \\
0.3 \mathrm{~m} \times 2.4 \mathrm{~m} \\
380 \mathrm{U} / \mathrm{s}(800 \mathrm{cfm}) \\
304 \mathrm{~L} \text { stainless steel }\end{array}$ & 2 & $\begin{array}{l}\text { Cools air from transport } \\
\text { blower to acceptable } \\
\text { operating temperature } \\
\text { of the balancing air blower }\end{array}$ \\
\hline $\mathrm{HP}-1$ & Transport Hopper & $4.8 \mathrm{~m} 3$ & 2 & $\begin{array}{l}\text { Collects calcine from first leg of } \\
\text { transport system and delivers it } \\
\text { to the second leg of the transport } \\
\text { system }\end{array}$ \\
\hline
\end{tabular}




\section{Appendix D}

\section{Background for Project Data Sheets}

Table 6 is repeated here for convenience. The justifications for the data entered in this table are found below along with brief explanations. Reference letters coordinate the data.

\section{Table D-1. Input to Project Data Sheet}

\section{Generic Information}

Structure Size $\left(\mathrm{m}^{2}\right)$

Location

\section{Construction Information}

Cost (\$): Preconstruction (escalated included)

Conceptual Design

Project Management

Permitting and Documentation

Start Up Activities

Contingency

Total Preconstruction

Cost (\$): Construction (escalation included)

Engineering, Design, and Inspection

Management Reserve (PM/CM)

Construction

Government Furnished Eqiupment

G\&A/PIF
Seven Confinement Enclosures which are each $40 \mathrm{ft}$ X 40

ft

(978 $\mathrm{m}^{2}$ total)

Seven VIC Buildings which are each $40 \mathrm{ft} \times 60 \mathrm{ft}$ (1560 $\mathrm{m}^{2}$ total)

One ITS Building that is $600 \mathrm{ft}^{2}\left(55.7 \mathrm{~m}^{2}\right)$

A

A Confinement Enclosure will be built on the roof of each CSSF.

A VIC Building will be built adjacent to each CSSF.

An ITS Building will be built mid way between the CSSFs and the Waste Treatment Facility.

CWO, DCWO, HWO, VWO \& TRU Separations Options

B

$$
\begin{array}{lr}
\$ 18,000,000 \\
\$ 2,700,000 \\
\$ \quad 4,800,000 \\
\$ \quad 5,100,000 \\
\$ 10,500,000 \\
\text { \$ } 41,100,000
\end{array}
$$

CWO \& TRU

DCWO, HWO, \& VWO Separations Options
\$ $18,500,000$
S $19,400,000 \mathrm{C}$
\$ $22,900,000$
$\$ 23,500,000$
$\$ 104,200,000$
$\$ 106,500,000$
$\$ 18,200,000$
\& $19,300,000$
$\$ 10,400,000$
\$ $10,700,000$ 
Procurement Fees, Management Reserve, and Contingency

Total Construction

Schedule start/end: Preconstruction

Schedule start/end: Construction

Number of workers each year of construction

Number of radiation workers (construction)

Average annual worker radiation dose (rem/yr)

Heavy equipment

Equipment used

Trips

Hours of operation

Acres disturbed and duration of disturbance

Air emissions

Major gases (CO2, $\mathrm{H} 2 \mathrm{O}, \mathrm{O} 2, \mathrm{~N} 2)$

Contaminants (Particulates, $\mathrm{CO}, \mathrm{NO}, \mathrm{SO}$, hydrocarbons)

Radioactive wastes

Type (Quantity)

Energy requirements

Electrical (MWh/yr)

Fossil fuel (liters)

Permits needed for construction
$\$ 64,600,000$

$\$ 238,800,000$

$\$ 246,100,000$

$1 / 1 / 2004-12 / 31 / 2007$

$1 / 1 / 2008-12 / 31 / 2013$

100 workers per year

$\mathrm{D}$

90 workers per year

$252 \mathrm{mrem} / \mathrm{yr}$ per worker

E

F

Mobile crane, roll off truck, loader, bulldozer, and cement truck

Cycle time/operation not applicable

$12,480 \mathrm{hr}$ (total)

0.5 acres for VIC Buildings, Transport Line Chase, ITS

building and ramps

G

$\mathrm{H}$

7,255 tons (total)

42.5 tons (total)

I

Contaminated fill $\left(1500 \mathrm{yd}^{3}\right)$

Steel/asbestos $(279,000 \mathrm{lb})$

Lead bricks/mixed wastes $(4,900 \mathrm{lb})$

$156 \mathrm{MWh} / \mathrm{yr}$

283,452 liters (total)

NEPA documentation (prior to start of Title II construction); New stationary sources/PTC/NOC/PSD for non-rad air emissions; HAP's and TAP's and RCRA (part $\mathrm{AA}, \mathrm{BB}$, and $\mathrm{CC}$ for air) for hazardous air emissions; air operating permit; NESHAP's, NPDES, NESHAP's subpart $\mathrm{H}$ for rad air emissions; approval of Engineering Plans; Cross Connection Control Plans; reports and specifications for drinking water supply; RCRA Part A and Part B permits.

\section{Operational Information}


Cost (\$): Operations (includes contingency but not escalation)

$$
\text { Facility Operations }
$$

Utilities

Maintenance of Equipment

Building Maintenance

Total Operations

Schedule start/end

Number of workers each year of operation (new/existing)

\section{Managers}

Engineers and other technicians

Supervisors and Administration/Support

Operators

Maintenance

Number of radiation workers

Average annual work radiation dose (rem/yr)

Air Emissions

Type (radioactive/chemical)

Quantity (Ci/year, tons/year)

Energy Requirements

Electrical $(\mathrm{MWh} / \mathrm{yr})$

Permits needed (for facility operations)
CWO TRU Separations DCWO, HWO, VWO Options

$\mathrm{K}$
\$ $1,218,000$
$S 4,661,000$
$\$ 4,761,000$
\& $1,054,000$
$\$ 4,158,000$
S $4,158,000$
S $7,866,000$
S $28,840,000$
$\$ 28,840,000$
\$ $9,255,000$
$\$ 33,933,00$
$\$ 35,120,000$
\& $19,393,000$
$\$ 71,594,000$
$\$ 72,781,000$

5-Year Operation: 1/1/13-1/1/18

20-Year Operation: 1/1/13 - 1/1/33

L

5-Year Operation

20-Year Operation

0.5

0.25

0.75

0.5

3

2

6

3

1

1

5-Year Operation: 10 workers

20-Year Operation: 6 workers

$192 \mathrm{mrem} / \mathrm{year}$ per worker

M

$N$

Calcine

CWO Option: $5.6 \times 10^{-6}$ ton/year

TRU Separations Options: $1.4 \times 10^{-6}$ ton/year

DCWO, HWO, VWO Options: $2.8 \times 10^{-6}$ ton/year

Release of $\mathrm{Ci}$ /year dependent on the type and storage length of the calcine released.

CWO Option: $93.2 \mathrm{MWh} / \mathrm{yr}$

TRU Separations Options: $74.1 \mathrm{MWh} / \mathrm{yr}$

DCWO, HWO, VWO Options: $88.7 \mathrm{MWh} / \mathrm{yr}$

HAP's and TAP's and RCRA (part AA, BB, and CC for air) for hazardous air emissions; air operating permit; NESHAP's, NPDES, NESHAP's subpart $H$ for rad air emissions; approval of Engineering Plans; Cross Connection Control Plans; report specifications for drinking water supply; RCRA Part A and Part B permits. 
Decontamination \& Decommissioning (D\&D) Information

Cost (\$): DD\&D (Unescalated)

Decommission

Decontamination

Demolition

Total DD\&D

Schedule start/end: D\&D

Number of workers each year of D\&D (new/existing)

Number of radiation workers (D\&D)
CWO

\$ $2,555,000$

S $7,223,000$

S $4,935,000$

$\$ 14,713,000$
TRU-Separations Options

DCWO, HWO, \& VWO $\mathrm{P}$

S $2,555,000$ \& $2,681,000$

\& $7,223,000$ \& $7,415,000$

$\$ 11,557,000 \quad \$ 11,864,000$

$\$ 21,335,000$

$\$ 21,960,000$

CWO: $1 / 1 / 2018-12 / 31 / 2018$

TRU-Separations Options: 1/1/2034-12/31/2034

DCWO, HWO, \& VWO: 1/1/2034-12/31/2034

CWO: 108

TRU-Separations Options: 155

DCWO, HWO, \& VWO: 160

CWO: 75

TRU-Separations Options: 104

DCWO, HWO, VWO: 102

Average annual worker radiation dose (rem/yr)

$252 \mathrm{mrem} / \mathrm{yr}$ for each worker

$\mathrm{R}$

Heavy equipment

$\mathrm{S}$

Equipment used

Hours of operation

Mobile Cranes, Roll-off Trucks, Dozers, Loaders, cement trucks, and other specialty equipment used during D\&D

CWO: $4,992 \mathrm{hr}$

TRU-Separations Options: $6,240 \mathrm{hr}$

DCWO, HWO, \& VWO: $7,488 \mathrm{hr}$

Acres disturbed and duration of disturbance

CWO \& TRU-Separations Options (transport lines only):

0.09 acre

$\mathrm{T}$

DCWO, HWO, \& VWO (ITS \& transport lines): 0.11 acre

Air emissions

Major gases ( $\mathrm{CO} 2, \mathrm{H} 2 \mathrm{O}, \mathrm{O} 2, \mathrm{~N} 2)$

CWO: 2,902 tons (total)

TRU-Separations: 3,627 tons (total)

DCWO, HWO \& VWO: 4,353 tons (total)

Contaminants (Particulates, $\mathrm{CO}, \mathrm{NO}$, $\mathrm{SO} 2$, hydrocarbons)

CWO: 17 tons (total)

TRU-Separations: 21 tons (total)

DCWO, HWO, \& VWO: 25 tons (total) 
Solid wastes

Type

Quantity $\left(\mathrm{m}^{3}\right)$

Radioactive wastes

Type and Quantity

Energy requirements

Electrical (MWh/yr)

Fossil fuel (liters)

Permits needed (e.g. for facility closures, physical characteristics and quantities of radioactive and hazardous materials remaining after closure)

\section{V}

Metal building and uncontaminated transport equipment

CWO \& TRU-Separations Options: $45 \mathrm{~m}^{3}$

DCWO, HWO, \& VWO options: $90 \mathrm{~m}^{3}$

Cyclones, metal filters, and hoppers from transport system

W

CWO \& TRU-Separations Options: $1.2 \mathrm{~m}^{3}$

DCWO, HWO, \& VWO options: $2.6 \mathrm{~m}^{3}$

X

$156 \mathrm{MWh} / \mathrm{yr}$ (based on John Duggan's estimate of $3000 \mathrm{kWh} / \mathrm{wk}, 52 \mathrm{wk} / \mathrm{yr}$ )

CWO: 113,380 liters

TRU-Separations Options: 141,725 liters

DCWO, HWO, \& VWO: 170,070 liters

Work will be done under closure provisions f existing permits

A. Confinement Enclosures: ( $30 \mathrm{ft} 6$ in. $X 30 \mathrm{ft} 6 \mathrm{in}.)+6(40 \mathrm{ft} X 40 \mathrm{ft})=10530 \mathrm{ft}^{2}=978 \mathrm{~m}^{2}$ VIC Buildings: $7(40 \mathrm{ft} X 60 \mathrm{ft})=16,800 \mathrm{ft}^{2}=1560 \mathrm{~m}^{2}$ ITS building: $600 \mathrm{ft}^{2}=55.7 \mathrm{~m}^{2}$

B. Preconstruction costs taken from the Cost Estimate prepared by Frosty Hanson (see escalated summary sheets in Appendix F). The reported costs reflect the cost associated with CSSFs 1-7, installation of D\&D risers, removal of corrosion coupons, and the appropriate transportation system. The values reported include escalation, contingency, and management and management reserve.

C. Construction costs taken from the Cost Estimate prepared by Frosty Hanson (see escalated summary sheets in Appendix F). The reported costs reflect the cost associated with CSSFs $1-7$, installation of D\&D risers, removal of corrosion coupons, and the appropriate transportation system. The values reported include escalaton.

D. The number of construction workers per year was developed from information provided by F. P. Hanson in the capital cost estimate. A spreadsheet details how the number of construction workers was developed. The number of rad workers was taken as a high percentage (90\%) of the total number of workers due to the nature of the construction work. Typically, the number of rad workers is closer to $80 \%$ of the total work force. In this case, the electricians, welders, and other finishing personnel should receive rad worker training. 
E. Entry into and demolition work will occur in highly contaminated areas throughout the construction phase of the project. The historical dose rates at ICPP were examined to develop a reasonable estimate of the worker dose. The average annual worker dose rates at ICPP were examined for the period 1994-1996. The dose rates were highest in 1995 when a significant amount of work was completed on the tank farm. It was assumed that the 1995 dose would be representative of the average annual worker dose rate during construction. The average annual worker dose rates for 1994-1996 at ICPP are reported in a memo "Average Worker Dose Rate at ICPP" dated 11/24/97.

F. The most prominent pieces of heavy equipment used during the construction phase of this project include a mobile crane, roll off truck, loader, bulldozer, and cement truck. It is estimated that at any given time an average of 4 vehicles will be in operation during the 5year construction period. Each vehicle was assumed to operate for $624 \mathrm{hr} / \mathrm{yr}$ (which is approximately $1 / 3$ of a man-year). The hours of operation are ( 4 vehicles)(624 $\mathrm{hr} /$ vehicle* $\mathrm{yr})(5 \mathrm{yr})=12,480 \mathrm{hr}$.

G. For CWO and TRU-Separations Options:

VIC Buildings: $1560 \mathrm{~m}^{2}=0.39$ acre (approximately the VIC building footprint) Transport Lines: $(800 \mathrm{ft})(5 \mathrm{ft})=4000 \mathrm{ft}^{2}=0.09$ acre (approximately the footprint of the shielding chase)

Total $=0.48$ acre which is approximately 0.5 acre

For DCWO, HWO, and VWO options:

VIC Buildings: $1560 \mathrm{~m}^{2}=0.39$ acre (approximately the VIC building footprint) Transport Lines: $(925 \mathrm{ft})(5 \mathrm{ft})=4625 \mathrm{ft}^{2}=0.1$ acre (approximately the footprint of the shielding chase)

ITS building: $600 \mathrm{ft}^{2}=0.01$ acre

Total $=0.5$ acre

H. The construction air emissions are primarily derived from emissions of heavy equipment. A spreadsheet was developed by Rod Kimmitt to analyze the air emissions produced by heavy equipment. A copy of the spreadsheet is attached for the calcine retrieval and transportation system.

I. An estimate of the radioactive wastes removed from CSSF 1 is made in reference 3 . This estimate includes removal of equipment and concrete vaults. The removed contaminated fill is estimated to be $1,500 \mathrm{yd}^{3}$. The removed steel and suspected asbestos material is estimated to weigh $5,300 \mathrm{lb}$. The lead bricks are estimated to weigh $300 \mathrm{lb}$. These estimates were used as the basis for the radiactive wastes removed from CSSF 2-7.

CSSF 2 and 3 are estimated to be similar to CSSF 1 because similar construction will occur on these CSSFs. However, the amount of contaminated fill is assumed to be negligible. There is no need to (except no contaminated fill is removed).

CSSF 4, 5, 6, and 7 are expected to have less concrete and steel removed because the superstructure vaults will not be demolished. There is no fill removal for these CSSFs. 
Equipment (steel) that is removed is estimated to weigh about $3,000 \mathrm{lb}$. The lead bricks are assumed to weigh $1,000 \mathrm{lb}$.

Contaminated fill:

Steel and suspected asbestos material:

Lead bricks and mixed waste:

$$
1,500 \mathrm{yd}^{3}
$$$$
3(5,300 \mathrm{lb})+4(3,000 \mathrm{lb})=279,000 \mathrm{lb}
$$$$
3(300 \mathrm{lb})+4(1,000 \mathrm{lb})=4,900 \mathrm{lb}
$$

J. The electrical energy requirement is derived from John Duggan's suggestion that construction requires $3000 \mathrm{kWh} / \mathrm{wk}$. $(3000 \mathrm{kWh} / \mathrm{wk})(52 \mathrm{wk} / \mathrm{yr})=156 \mathrm{MWh} / \mathrm{yr}$. The estimated fossil fuel consumed during the construction phase of this project was determined from an average value for fuel consumption of heavy equipment. The John Deere Construction equipment web page (www.deere.com) indicates an average fuel consumption of heavy equipment to be $6 \mathrm{gal} / \mathrm{hr}$. $(6 \mathrm{gal} / \mathrm{hr})(12,480 \mathrm{hr})=74,880 \mathrm{gal}=283,452$ liters.

$\mathrm{K}$. Operational costs are derived from the Cost Estimate prepared by Bob Turk. The operational costs associated with retrieving calcine from CSSF 1-7 and transport system, installation of $D \& D$ risers, and removal of corrosion coupons are included. These values are not escalated.

L. Operational crew requirements were developed with the assistance of Jack Prendergast for the 5-year and 20-year operations options. The number of radiation workers was derived from the number of operators, maintenance workers, and other technicians.

M. The average value of the average annual dose rates at ICPP for 1994-1996 was used to determine the average annual work radiation does rate during operations. The average over the 3 year period is more representative of work that would occur during the operations phase of the calcine retrieval and transportation system. $(180 \mathrm{mrem} / \mathrm{yr}+252 \mathrm{mrem} / \mathrm{yr}+143$ $\mathrm{mrem} / \mathrm{yr}$ ) $/ 3=192 \mathrm{mrem} / \mathrm{yr}$. See attached memo (also referenced in E).

N. During normal operations and idle time for each CSSF, air emissions are assumed to be negligible due to the multiple layers of confinement. These layers include several stages of HEPA filters. Air emissions were estimated for the exhausted transport air. This estimate is developed for the transport air after it has been through a two stage HEPA filter. The air emissions are primarily composed of calcine particles. The radioactivity of the calcine emitted to the environment varies depending upon the type of calcine and its length of storage.

Assuming the cyclone will have $99 \%$ efficiency, the sintered metal filter will have $99.9 \%$ efficiency, and the HEPA filter will have $99.97 \%$ efficiency. Rod Kimmitt provided these efficiencies.

Calcine remaining in the transport air after HEPA filters: $(0.01)(0.001)(0.0003)(2700 \mathrm{~kg} / \mathrm{hr})=0.009 \mathrm{~g} / \mathrm{hr}$

Total hours each transport system must operate:

$\left(5435 \mathrm{~m}^{3}\right)\left(1400 \mathrm{~kg} / \mathrm{m}^{3}\right)(1 \mathrm{hr} / 5400 \mathrm{~kg})=1409 \mathrm{hr}$ 
For CWO (5-Year Operations):

Assuming all the transport air is exhausted through the Waste Treatment Facility off-gas system.

The transportation system will release:

$$
2(0.009 \mathrm{~g} / \mathrm{hr})(1409 \mathrm{hr})=25 \mathrm{~g}=2.8 \times 10^{-5} \text { ton }
$$

The total air emissions from both transportation systems are $2.8 \times 10^{-5}$ ton for 5-years (5.6 x $10^{-6}$ ton/yr).

For TRU Separations Options (20-Year Operation):

Assuming all the transport air is exhausted through the Waste Treatment Facility off-gas system.

The transportation system will release:

$$
2(0.009 \mathrm{~g} / \mathrm{hr})(1409 \mathrm{hr})=25 \mathrm{~g}=2.8 \times 10^{-5} \text { ton }
$$

The total air emissions from both transportation systems are $2.8 \times 10^{-5}$ ton for 20-years $\left(1.4 \times 10^{-6}\right.$ ton/yr).

For DCWO, HWO, and VWO (20-Year Operation):

Assuming all the transport air is exhausted through the Waste Treatment Facility off-gas system.

The transportation system will release:

$$
4(0.009 \mathrm{~g} / \mathrm{hr})(1409 \mathrm{hr})=50 \mathrm{~g}=5.6 \times 10^{-5} \text { ton }
$$

The total air emissions from the 4 transportation system legs are $5.6 \times 10^{-5}$ ton for 20-years $\left(2.8 \times 10^{-6}\right.$ ton $\left./ y r\right)$.

For actual operations the air emissions would be much lower because $90 \%$ of the air is recycled in the transportation system. These calculations assume that all of the transport air is released through the facility off-gas system.

0 . The power requirement is determined by the power needs of the transportation equipment, ventilation equipment, and VDA. The transport air blower and balancing air blower require $37.8 \mathrm{~kW}$ and $4 \mathrm{~kW}$ of power, respectively. The exhaust fans (EF-1 and EF-3) require $50 \mathrm{~W}$ of power. The remaining exhaust fan (EF-2) requires $1 \mathrm{~kW}$. The VDA is estimated to require $15 \mathrm{~kW}$. These power consumption values used to compute the power requirement are estimates based on manufacturer specifications.

For CWO (5-Year Operation):

The transportation system operates $10.8 \mathrm{hr} / \mathrm{wk}$ for $26 \mathrm{wk} / \mathrm{yr}$. The ventilation blowers in each VIC building operated $24 \mathrm{hr} /$ day everyday. The VDA is estimated to be in operation approximately $3 \mathrm{hr} / \mathrm{wk}$ during calcine retrieval. 


$$
\begin{aligned}
& \text { Power }=2\left(10.8 \frac{h r}{w k}\right)\left(26 \frac{w k}{y r}\right)(37.8 k W+4 k W) \\
& +7\left(24 \frac{h r}{d a y}\right)\left(365 \frac{d a y}{y r}\right)(2(0.05 k W)+1 k W) \\
& +2\left(3 \frac{h r}{w k}\right)\left(26 \frac{w k}{y r}\right)(15 k W)=93.2 \frac{M W \cdot h r}{y r}
\end{aligned}
$$

For TRU Separations Options (20-Year Operation):

The transportation system operates $2.7 \mathrm{hr} / \mathrm{wk}$ for $26 \mathrm{wk} / \mathrm{yr}$ in this alternative. The ventilation blowers in each VIC̀ building operate $24 \mathrm{hr} /$ day every day. The VDA is estimated to be in operation approximately $1 \mathrm{hr} / \mathrm{wk}$ during calcine retrieval.

$$
\begin{aligned}
& \text { Power }=2\left(2.7 \frac{h r}{w k}\right)\left(26 \frac{w k}{y r}\right)(37.8 k W+4 k W) \\
& +7\left(24 \frac{h r}{d a y}\right)\left(365 \frac{d a y}{y r}\right)(2(0.05 k W)+1 k W) \\
& +2\left(1 \frac{h r}{w k}\right)\left(26 \frac{w k}{y r}\right)(15 k W)=74.1 \frac{M W \cdot h r}{y r}
\end{aligned}
$$

For DCWO, HWO, VWO (20-Year Operation):

There are 4 transportation system legs operating $2.7 \mathrm{hr} / \mathrm{wk}$ for $26 \mathrm{wk} / \mathrm{yr}$ in these alternatives. Therefore, there are 4 transport air blowers and 4 balancing air blowers. The ventilation blowers in each VIC building operate $24 \mathrm{hr} / \mathrm{day}$ every day. The VDA is estimated to be in operation approximately $1 \mathrm{hr} / \mathrm{wk}$ during calcine retrieval. Additionally, the ventilation blowers in the ITS run $24 \mathrm{hr} /$ day every day. The power requirement for EF-4 and EF- 6 is estimated to be $100 \mathrm{~W}$ each. The power requirement for EF- 5 is estimated to $800 \mathrm{~W}$ based on the estimate for EF-2.

$$
\begin{aligned}
& \text { Power }=4\left(2.7 \frac{h r}{w k}\right)\left(26 \frac{w k}{y r}\right)(37.8 k W+4 k W) \\
& +7\left(24 \frac{h r}{d a y}\right)\left(365 \frac{d a y}{y r}\right)(2(0.05 k W)+1 k W) \\
& +2\left(1 \frac{h r}{w k}\right)\left(26 \frac{w k}{y r}\right)(15 k W)+\left(24 \frac{h r}{d a y}\right)\left(365 \frac{d a y}{y r}\right)(2(0.1 k W)+0.8 k W) \\
& =88.7 \frac{M W \cdot h r}{y r}
\end{aligned}
$$


P. D\&D costs taken from life cycle cost estimate developed by Bob Turk. See Appendix F. The numbers presented are not escalated.

Q. The number of workers needed during $D \& D$ was developed from the unescalated $D \& D$ costs (See Appendix F). The unescalated costs for each option was inputed into a spreadsheet developed by Rod Kimmitt. The spreadsheets are attached.

R. D\&D work will occur in highly contaminated areas of the transportation system. The historical dose rates at ICPP were examined to develop a reasonable estimate of the worker dose. The average annual worker dose rates at ICPP were examined for the period 19941996. The dose rates were highest in 1995 when a significant amount of work was completed on the tank farm. It was assumed that the 1995 dose would be representative of the average annual worker dose rate during construction. The average annual worker dose rates for 1994-1996 at ICPP are reported in a memo "Average Worker Dose Rate at ICPP" dated 11/24/97. The dose during the D\&D phase was assumed to be the same as during the construction phase of the project.

S. The most prominent pieces of heavy equipment used during the $D \& D$ phase of this project include a mobile crane, roll off truck, loader, bulldozer, cement trucks, and other specialized demolition equipment.

For the CWO options, it is estimated that at any given time an average of 8 vehicles will be in operation during the 1-year construction period. Each vehicle was assumed to operate for $624 \mathrm{hr} / \mathrm{yr}$ (which is approximately $1 / 3$ of a man-year). The hours of operation are ( 8 vehicles $)\left(624 \mathrm{hr} /\right.$ vehicle $\left.^{*} \mathrm{yr}\right)(1 \mathrm{yr})=4,992 \mathrm{hr}$.

For the TRU-Separations Options, it is estimated that at any given time an average of 10 vehicles will be in operation during the 1-year construction period. Each vehicle was assumed to operate for $624 \mathrm{hr} / \mathrm{yr}$ (which is approximately $1 / 3$ of a man-year). The hours of operation are $(10$ vehicles $)\left(624 \mathrm{hr} /\right.$ vehicle $\left.^{*} \mathrm{yr}\right)(1 \mathrm{yr})=6,240 \mathrm{hr}$.

For the DCWO, HWO, and VWO options, it is estimated that at any given time an average of 12 vehicles will be in operation during the 1-year construction period. Each vehicle was assumed to operate for $624 \mathrm{hr} / \mathrm{yr}$ (which is approximately $1 / 3$ of a man-year). The hours of operation are (12 vehicles) $\left(624 \mathrm{hr} /\right.$ vehicle $\left.^{*} \mathrm{yr}\right)(1 \mathrm{yr})=7,488 \mathrm{hr}$.

T. For the CWO and TRU-Separations Options, only the transport lines are D\&D. Transport Lines: $(800 \mathrm{ft})(5 \mathrm{ft})=4000 \mathrm{ft}^{2}=0.09$ acre (approximately the footprint of the shielding chase)

Total $=0.09$ acre which is approximately 0.5 acre

For DCWO, HWO, and VWO options:

Transport Lines: $(925 \mathrm{ft})(5 \mathrm{ft})=4625 \mathrm{ft}^{2}=0.1$ acre (approximately the footprint of the shielding chase)

ITS building: $600 \mathrm{ft}^{2}=0.01$ acre 
Total $=0.11$ acre

T. The D\&D air emissions are primarily derived from emissions of heavy equipment. $A$ spreadsheet was developed by Rod Kimmitt to analyze the air emissions produced by heavy equipment. A copy of the spreadsheet is attached for D\&D work on the calcine transportation system.

U. For the CWO and TRU-Separations Options:

The solid wastes disposed of during the D\&D of the transport system include the 2 transport system blowers, 2 heat exchangers, and the associated piping and duct work. The transport equipment for a single transport system is located in a $10 \mathrm{ft} \mathrm{X} 20 \mathrm{ft}$ area. The equipment is assumed to be no greater than $5 \mathrm{ft}$ tall. The transport equipment is assumed to occupy $60 \%$ of the area. The associated piping is assumed to occupy $20 \%$ of the transport equipment area.

$$
\begin{aligned}
& \text { Volume }=2(0.6)(10 f t \times 20 f t \times 5 f t)+2(0.2)(10 f t \times 20 f t \times 5 f t) \\
& =1200 \mathrm{ft}^{3}+400 \mathrm{ft}^{3}=1600 \mathrm{ft}^{3}=45 \mathrm{~m}^{3}
\end{aligned}
$$

For the DCWO, HWO, and VWO options:

The solid wastes include metal from the metal building in the ITS, 4 transport system blowers, 4 heat exchangers, and the associated piping and duct work. The upper level of the ITS is $20 \mathrm{ft} X 30 \mathrm{ft} \mathrm{X} 14 \mathrm{ft}$. It is assumed that the metal can be compacted into no more than $5 \mathrm{~m}^{3}$.

$$
\begin{aligned}
& \text { Volume }=4(0.6)(10 f t \times 20 f t \times 5 f t)+4(0.2)(10 f t \times 20 f t \times 5 f t)+5 m^{3} \\
& =2400 f^{3}+800 f^{3}+5 m^{3}=95 m^{3}
\end{aligned}
$$

W. For the CWO and TRU-Separations Options:

The radioactive wastes include the 2 cyclones and 2 sintered metal filters. The cyclone has a $2 \mathrm{ft}$ diameter and is $6 \mathrm{ft}$ long. The sintered metal filter has a volume of $3.1 \mathrm{ft}^{3}$. This equipment is located in the Waste Treatment Facility.

$$
\text { Volume }=(2)\left[\pi\left(\frac{2 f t}{2}\right)^{2}(6 f t)\right]+(2)\left(3.1 f t^{3}\right)=44 f^{3}=1.2 m^{3}
$$

For the DCWO, HWO, and VWO options:

The radioactive wastes include 4 cyclones, 2 sintered metal filters, and hoppers. The additional equipment is located in the ITS. The hoppers hold $4.8 \mathrm{~m}^{3}$ it is assumed that it may be compacted $50 \%$.

$$
\text { Volume }=(4)\left[\pi\left(\frac{2 f t}{2}\right)^{2}(6 f t)\right]+(4)\left(3.1 f^{3}\right)+(0.5)(2)\left(4.8 m^{3}\right)=92.8 f^{3}=2.6 m^{3}
$$


$\mathrm{X}$. The electrical energy requirement is derived from John Duggan's suggestion that construction requires $3000 \mathrm{kWh} / \mathrm{wk}$. $(3000 \mathrm{kWh} / \mathrm{wk})(52 \mathrm{wk} / \mathrm{yr})(1 \mathrm{yr})=156 \mathrm{MWh}$

The estimated fossil fuel consumed during the construction phase of this project was determined from an average value for fuel consumption of heavy equipment. The John Deere Construction equipment web page (www.deere.com) indicates an average fuel consumption of heavy equipment to be $6 \mathrm{gal} / \mathrm{hr}$.

For CWO: $(6 \mathrm{gal} / \mathrm{hr})(4,992 \mathrm{hr})=29,952 \mathrm{gal}=113,380$ liters

For TRU-Separations Options: $(6 \mathrm{gal} / \mathrm{hr})(6,240 \mathrm{hr})=37,440 \mathrm{gal}=141,725$ liters

For DCWO, HWO, and VWO options: $(6 \mathrm{gal} / \mathrm{hr})(7,488 \mathrm{hr})=44,928 \mathrm{gal}=170,070$ liters 
Backup to

$E_{1} M_{1} \Psi R$

Memo of Telephone Conversation

Name . $\cdot$ Organization

Steve Aitken

Bill Landman

Date:
LMITCO S\&H

ICPP ALARA Coordinator
Phone

$6-3174$

Chemical and Environmental Eng'g - 6-5279

Subject: . Average Worker Dose Rate at ICPP

Discussion:

Steve was contacted for information to support development of the Project Data Sheet for the Separations Options. He provided the following information for the ICPP site:

Average dose (per worker) for $1996 \quad 180 \mathrm{mR} / \mathrm{hr}$

Average dose (per worker) for 1995 . ' $252 \mathrm{mR} / \mathrm{hr}$

Average dose (per worker) for $1994.143 \mathrm{mR} / \mathrm{hr}$

The 1995 year average was, higher due to extensive work on the tank farm.

E-182 
Back up to $H$

\section{Construction}

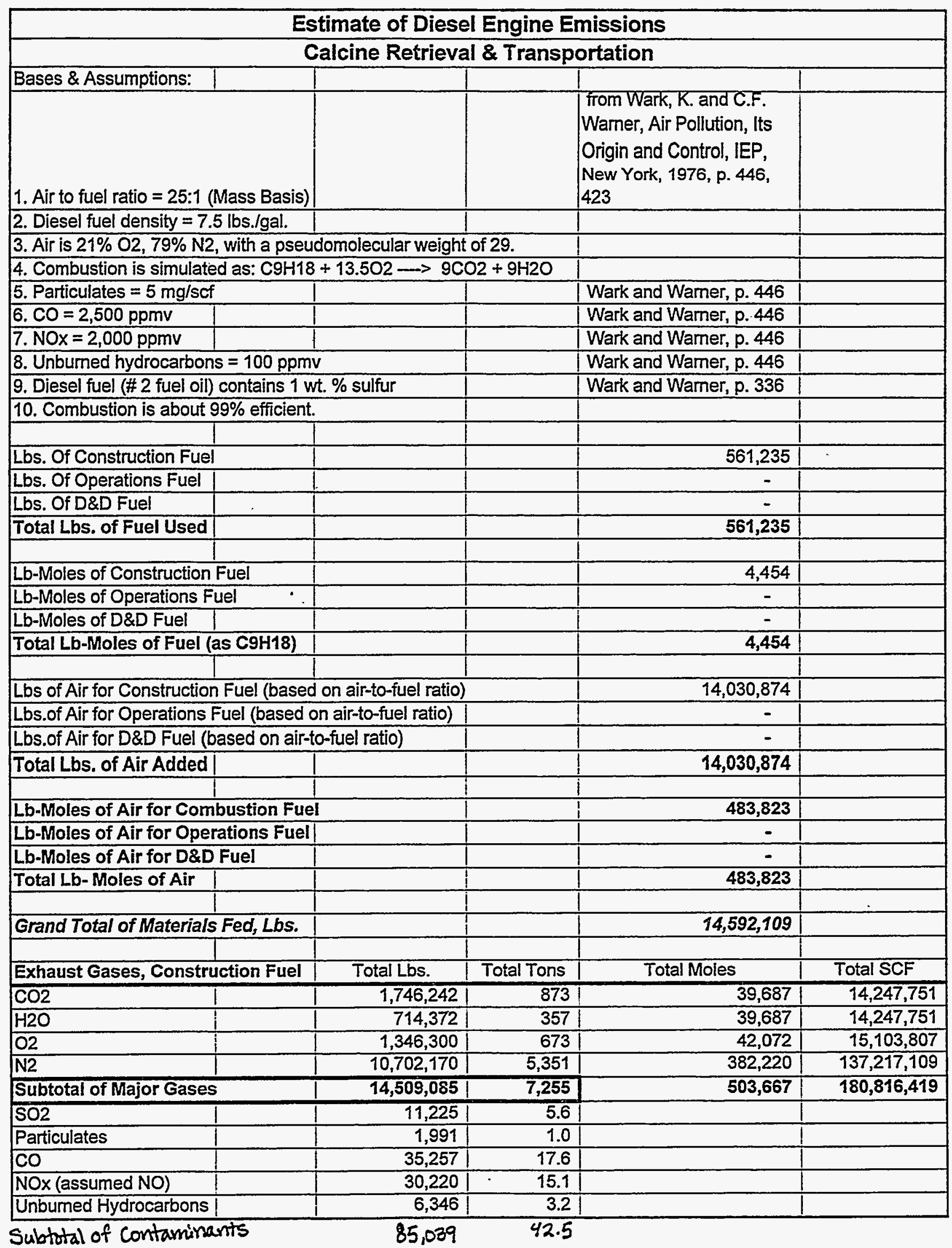




\begin{tabular}{|c|c|c|c|c|c|}
\hline & Project Data Sheet for C & cine Retrieval & - CWO & & \\
\hline Decontaminatic & on \& Decommissioning (D\&D) Information & & & & \\
\hline Cost (\$):D\&D II & Undiscounted dollars) & & & & \\
\hline Decommission & & $\$ 2,555,000$ & & & \\
\hline Decontaminatior & & $\$ 7,223,000$ & & & \\
\hline Demolition & & $\$ 4,935,000$ & & & \\
\hline Total D\&D & & $\$ 14,713,000$ & & & \\
\hline Schedule startle & end: D\&D & January 2033 throl & ugh Decembe & er 2037 & \\
\hline Number of work & ers each year of D\&D (new/existing) & 108 & New worker: & $\mathrm{rs} / \mathrm{yr}$ & \\
\hline Number of radia & tion workers (D\&D) & 75 & New worker: & $\mathrm{rs} / \mathrm{yr}$ & \\
\hline Average annual & worker radiation dose (rem/yr) & 0.19 & rem/yr & Tper worker & \\
\hline & & & & & \\
\hline Heavy equipmer & & & & & \\
\hline Equipment used & & Mobile Cranes, Ro & II-off trucks, $\mathrm{C}$ & Dozers, Loader & \\
\hline Trips & Roll-off trucks & 15 & per day & & \\
\hline Hours of operati & on (all heavy equipment) & 27,990 & Hours & & \\
\hline & & & & & \\
\hline Acres disturbed & and duration of disturbance & January 2033 thro & ugh Decembe & er 2037 & \\
\hline New & & None & & & \\
\hline Previous & & 2.7 & acres & & \\
\hline Revegetated & & None & & & \\
\hline Air emissions & & & & & \\
\hline non-radioactive & Fuel combustion gases $\left(\mathrm{CO}_{2}, \mathrm{H}_{2} \mathrm{O}, \mathrm{O}_{2}, \mathrm{~N}_{2}\right)$ & 16,269 & tons (total) & & \\
\hline non-radioactive & $\begin{array}{l}\text { Fuel combustion contaminants ( } \mathrm{CO}, \\
\text { particulates, } \mathrm{NO}_{\mathrm{x}} \mathrm{SO}_{2}, \text { hydrocarbons) }\end{array}$ & 95 & tons (total) & & \\
\hline radioactive & HEPA filtered off-gas & 26,173 & Itons (total) & & \\
\hline & & & & & \\
\hline Effluents & & & & & \\
\hline radioactive & Spent decontamination solution & $1,703,250$ & |iters (total) & 1,703 & $\overline{C i}$ \\
\hline non-radioactive & Sanitary wastewater & $2,295,160$ & |iters (total) & & \\
\hline non-radioactive & Lube oil & 5,297 & liters (total) & & \\
\hline Solid wastes: & & & & & \\
\hline radioactive & & 29,421 & $\mathrm{~m}^{3}$ & 294 & $\mathrm{Ci}$ \\
\hline Non-radioactive & (industrial) & 22,122 & $\mathrm{~m}^{3}$ & & \\
\hline Hazardous & & 10 & $1 \mathrm{~m}^{3}$ & & \\
\hline Hazardous/foxic & chemicals and wastes (fvpe) & & & & \\
\hline Storage/inventol & & 205 & $\mathrm{~m}^{3}$ (total) & & \\
\hline Pits/Ponds creal & $\operatorname{ted}\left(m^{2}\right)$ & None & & & \\
\hline radioactive & (mixed waste) & 47 & Im $^{3}$ (total) & 01 & $\mathrm{Ci}$ \\
\hline & & & & & \\
\hline Water usage: & & & & & \\
\hline Process water & & $2,284,875$ & liters (total) & & \\
\hline Domestic water & & $2,295,160$ & liters (total) & & \\
\hline Source of water & & |ICPP site wells & & & \\
\hline & I & & & & \\
\hline Eneray requirem & nents: & & & & \\
\hline
\end{tabular}


D\&D Labor

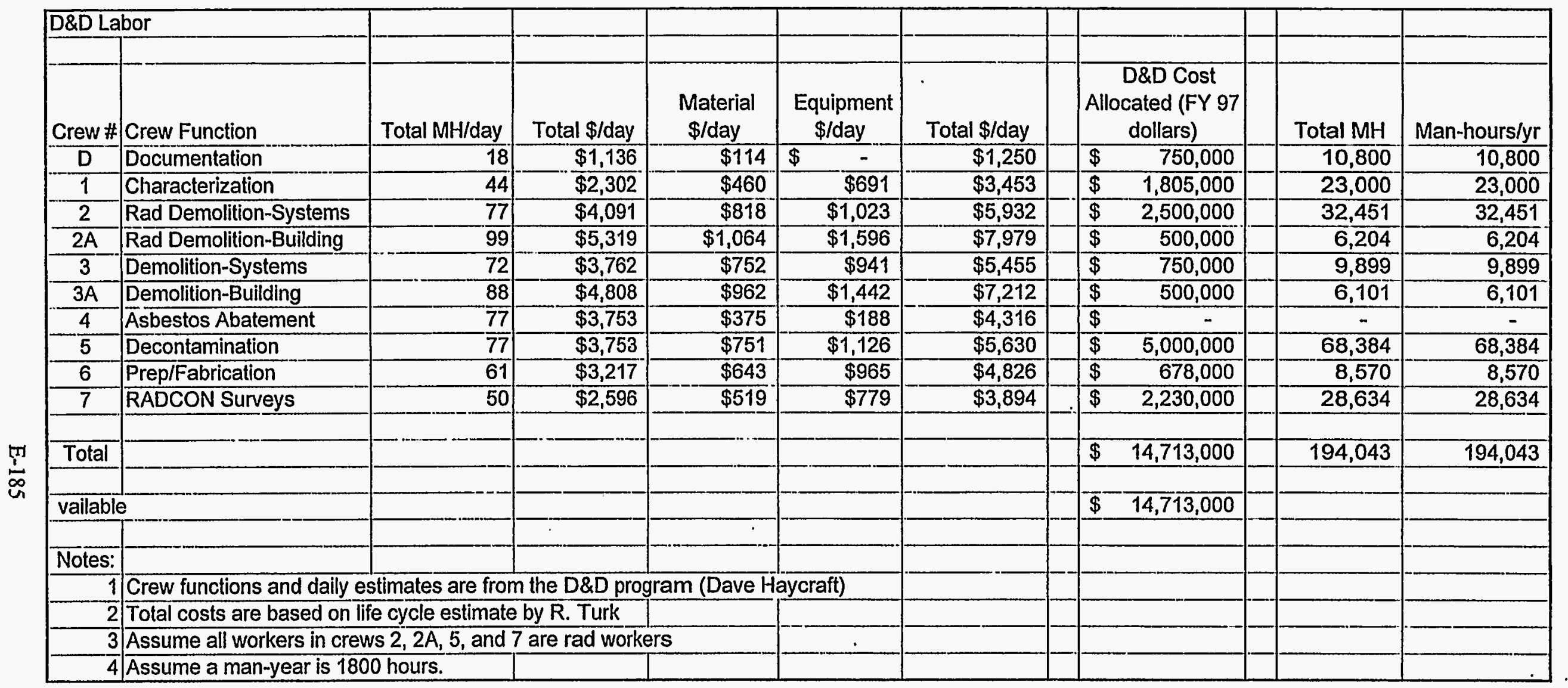




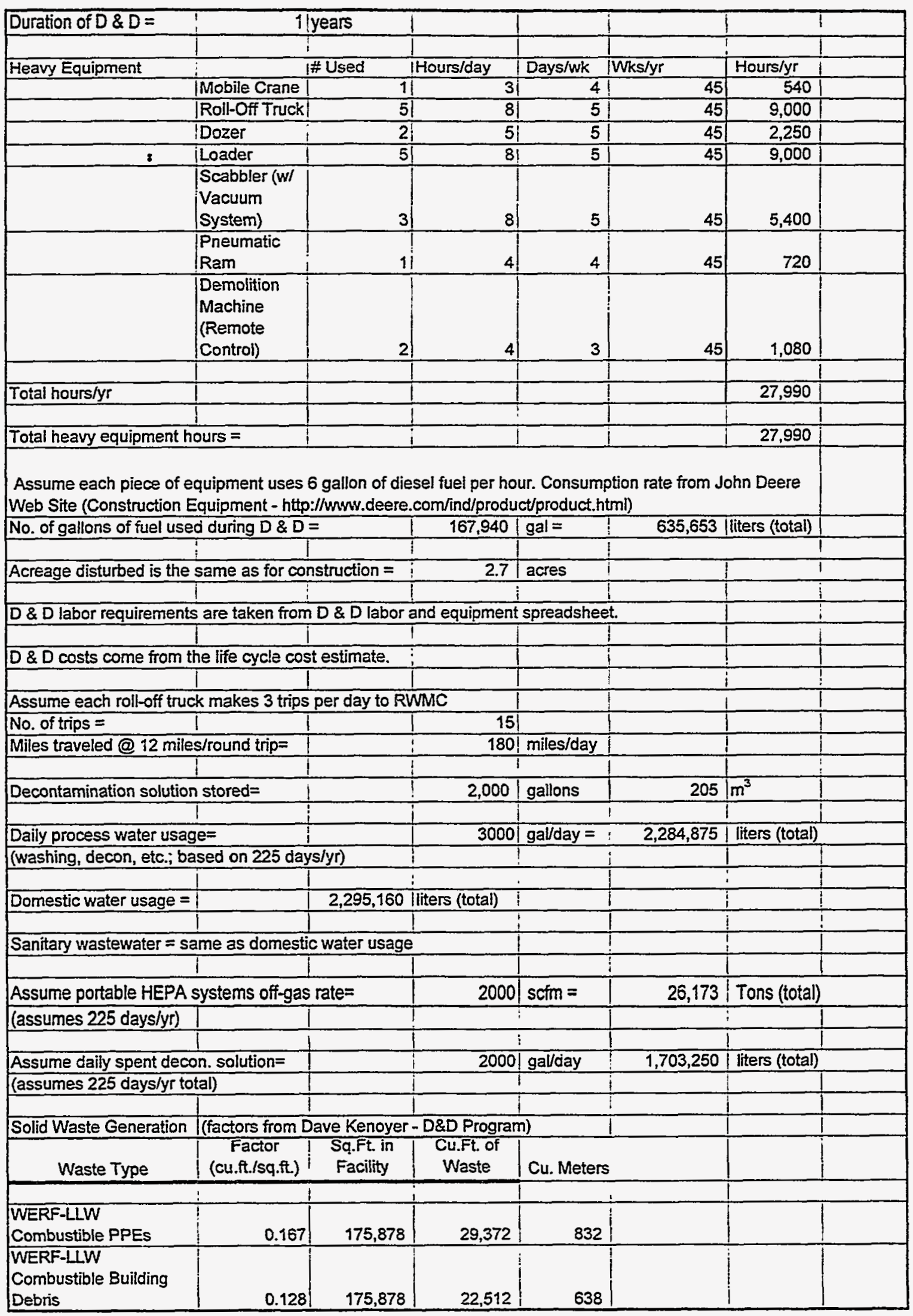




\begin{tabular}{|c|c|c|c|c|c|c|}
\hline $\begin{array}{l}\text { WERF-LLW } \\
\text { Compactable Building } \\
\text { Debris }\end{array}$ & 0.195 & 175,878 & 34,296 & 972 & & \\
\hline $\begin{array}{l}\text { RWMC-LLW Non- } \\
\text { Compactable Equipment }\end{array}$ & 0.513 & 175,878 & 90,225 & 2,556 & & \\
\hline $\begin{array}{l}\text { RWMC-LLW Non-Compt } \\
\text { Building Debris }\end{array}$ & 0.684 & 175,878 & 120,301 & 3,408 & & \\
\hline $\begin{array}{l}\text { RWMC-LLW Non-Compt } \\
\text { Concrete Rubble }\end{array}$ & 3.44 & 175,878 & 605,020 & 17,139 & $\begin{array}{l}\text { This factor is twice as large } \\
\text { as that recommended by } \\
\text { the D\&D program to } \\
\text { account for that large } \\
\text { amounts of concrete used. }\end{array}$ & \\
\hline $\begin{array}{l}\text { RWMC-LLW Non-Compt } \\
\text { Scrap Metal }\end{array}$ & $0.778 \mid$ & 175,878 & 136,833 & 3,876 & & \\
\hline $\begin{array}{l}\text { RWMC-LLW } \\
\text { Asbestos/ACM Covered } \\
\text { Pipe }\end{array}$ & o) & 175,878 & $\begin{array}{ll}- \\
-\end{array}$ & 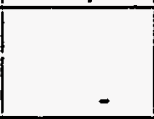 & . & \\
\hline \begin{tabular}{|l|} 
CFA Landfill Non-Compt \\
Building Debris
\end{tabular} & 1.99 & 175,878 & 349,997 & 9,915 & & \\
\hline $\mid \begin{array}{l}\text { CFA Landfill Non-Compt } \\
\text { Concrete Rubble }\end{array}$ & 2.45 & 175,878 & 430,901 & 12,207 & $\begin{array}{l}\text { This factor is twice as large } \\
\text { as that recommended by } \\
\text { the D\&D program to } \\
\text { account for that large } \\
\text { amounts of concrete used. }\end{array}$ & \\
\hline CFA Landfill Asbestos & 0 & 175,878 & - & - & & \\
\hline $\begin{array}{l}\text { HWSF Hazardous Mitris } \\
\text { (Hg/PCBs/etc) }\end{array}$ & 0.002 & 175,878 & 352 & 10 & & \\
\hline Metal Recycle & 0.022 & 175,878 & 3,869 & 110 & & \\
\hline & & & & & & \\
\hline LLW $=$ & & & $1,038,560$ & 29,421 & & \\
\hline Non-Rad $=$ & & & 780,898 & 22,122 & & \\
\hline Hazardous $=$ & & & 352 & 10 & & \\
\hline Metal $=$ & & & 3,869 & 110 & & \\
\hline & & & & & & \\
\hline Electric power usage = & $156,000 \mid$ & |kWh/yr & 156 & $M W h / y r$ & & \\
\hline (based on 3,000 kWh/wk & - John Duggan) & & & & & \\
\hline & & & & & & \\
\hline Air emissions from fuel ar & re based on the & e diesel emiss & ions spreadshe & & & \\
\hline & & & & & & \\
\hline 1 manyear of labor $=$ & 1800 & Imanhours & & & & \\
\hline & & & & & & \\
\hline Lube oil $=$ & $5,297 \mid$ & liters (total) & & & & \\
\hline (based on 3 gal for every & 60 hours of ope & eration) & & & & \\
\hline & & & & $i$ & & \\
\hline Mixed waste $=$ & 12,375 & |gal (total) $=$ & & $\mathrm{m} 3$ (total) & & \\
\hline (based on an assumed 5 & 55-gallon drum & ns generated & ger week... wo & k only 45 we & eeks/yr) & \\
\hline & & & & & & \\
\hline Radioactivity associated v & with waste mate & terials: & & & & \\
\hline & & & & & & \\
\hline Spent decontamination so & olution = & 1,703 & $\mathrm{Ci}$ & & & \\
\hline (based on an assumed av & verage activity & concentration & of $1 \mathrm{uCi} / \mathrm{ml})$ & & & \\
\hline & & & & & & \\
\hline Radioactive solid waste $=$ & & 294 & & & & \\
\hline (based in an assumed act & tivity concentra & ation of $0.01 \mathrm{u}$ & $\mathrm{Ci} / \mathrm{cc}[0.01 \mathrm{Ci} / \mathrm{m}$ & & & \\
\hline & & & & & & \\
\hline Mixed waste $=$ & & & $\mathrm{Ci}$ & & & \\
\hline (based on an assumed ac & ctivity concentre & ration of 0.01 & $\mathrm{Ci} / \mathrm{cc}[0.01 \mathrm{Cl}$ & $\left.\left(m^{3}\right]\right)$ & & \\
\hline
\end{tabular}




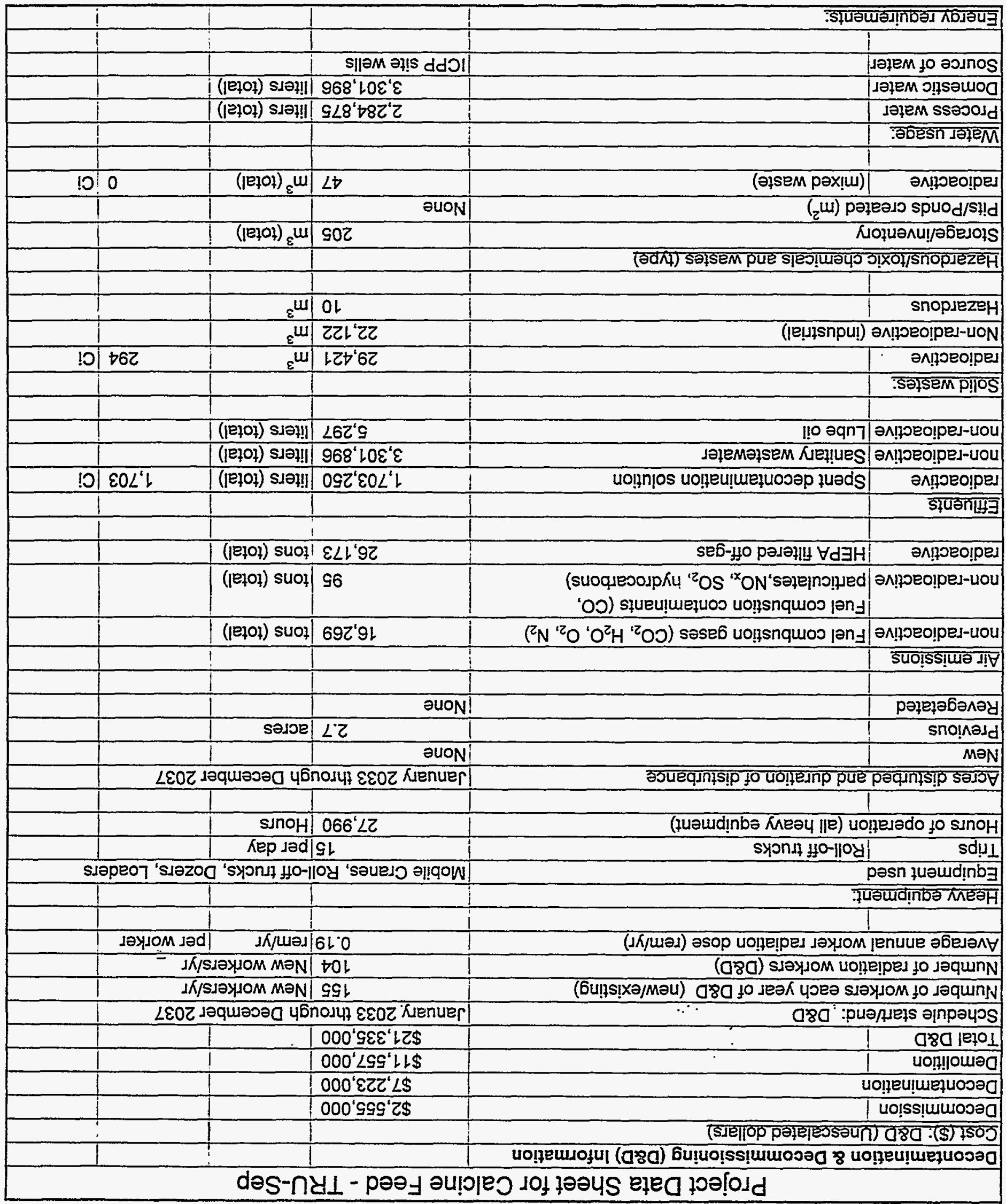

"musto susyprodas-ma1 \& ynigosnddo saporpu! 


\begin{tabular}{|c|c|c|c|c|c|c|c|c|c|c|}
\hline & & & & & & & & \multicolumn{2}{|c|}{ s.nou $008 \mathrm{l}$ s! jeaK-uem e aunss } & 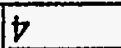 \\
\hline & & & & & & \multicolumn{4}{|c|}{ 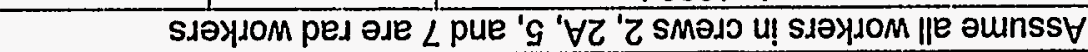 } & $\varepsilon$ \\
\hline & & & & & & \multicolumn{4}{|c|}{ 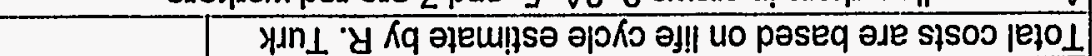 } & 2 \\
\hline & & & & & \multicolumn{5}{|c|}{ 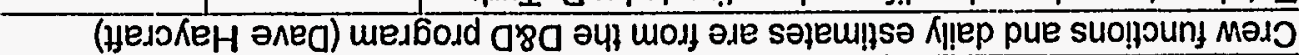 } & $\underline{l}$ \\
\hline & & & & & & & & & & :SOION \\
\hline & & & & & & & & & & \\
\hline & & $000^{\prime} G \varepsilon \varepsilon^{\prime} L Z \quad \$$ & & & & & & & & әjqe!!en \\
\hline & & & & & & & & & . & \\
\hline $99 L^{\prime} 6 L Z$ & $9 G L^{\prime} 6 L Z$ & $000^{\prime} S \varepsilon \varepsilon^{\prime} L 乙 \quad \$$ & & & & & & & & $1 \mathrm{ejO1}$ \\
\hline & & & & & & & & & & \\
\hline$\nabla \varepsilon 9^{\prime} 82$ & $\nabla \varepsilon 9^{\prime} 8 Z$ & $000^{\prime} 0 \varepsilon Z^{\prime} Z$ & & $\nabla 68^{\prime} \varepsilon \$$ & $6 \angle L \$$ & $619 \$$ & $969^{\prime} Z \$$ & 09 & SKaninS NOOQ $\forall$ Y & $L$ \\
\hline$\varepsilon 9 \varepsilon^{\prime} \varepsilon l$ & $\varepsilon 9 \varepsilon^{\prime} \varepsilon l$ & $0+Z^{\prime} \angle 9 O^{\prime} L^{\circ}$ & & $9 z 8^{\prime} \triangleright \$$ & $996 \$$ & $\varepsilon \mapsto 9 \$$ & $\angle L Z^{\prime} E \$$ & 19 & uoḷeo!lue & 9 \\
\hline $78 \varepsilon^{\prime} 89$ & $78 \varepsilon^{\prime} 89$ & $000^{\prime} 000^{\prime} \mathrm{s}$ & 5 & $0 \varepsilon 9^{\prime} \$ \$$ & $9 Z L^{\prime} L \$$ & $19 \angle \$$ & $E G L^{\prime} E \$$ & $\angle L$ & ио!̣eu!uejuosa & $\mathrm{g}$ \\
\hline- & - & - & 5 & $91 \varepsilon^{\prime} \downarrow \$$ & $881 \$$ & GLE\$ & $\varepsilon G L^{\prime} E \$$ & $L L$ & juamejeq $\forall$ solsaqs $\forall$ & $t$ \\
\hline $609^{\prime} \angle L$ & $\forall 09^{\prime} \angle L$ & $09 L^{\prime} Z \nabla \nabla^{\prime} l$ & & $Z 1 Z^{\prime} \angle \$$ & $2 t t^{\prime} l \$$ & $296 \$$ & $808^{1} \triangleright \$$ & 88 & 6u!p|!ng-uo!!!|ouөg & $\forall \varepsilon$ \\
\hline $81 L^{\prime} L Z$ & $8 L L^{\prime} L Z$ & $000^{\prime} 00 L^{\prime} Z$ & 5 & sqt's\$ & $166 \$$ & $29 \angle \$$ & $Z 9 L^{\prime} \varepsilon \$$ & $Z L$ & smə]sKS-Uon!nomag & $\varepsilon$ \\
\hline $80 b^{\prime} Z L$ & $80 t^{\prime} 21$ & $000^{\prime} 000^{\prime}$ & $\frac{b}{b}$ & $6 \angle 6^{\prime} \angle \$$ & $969^{\prime} L \$$ & $690^{\prime} 1 \$$ & 6LE'S\$ & 66 & 6u!p!!ng-uo!!!|ower pey & $\forall Z$ \\
\hline$\varepsilon 88^{\prime} \angle L$ & $\varepsilon 88^{\prime} \mathrm{LL}$ & $000^{\prime} 000^{\prime} 9$ & $b$ & $286^{\circ} 9 \$$ & $\varepsilon 20^{\circ} L \$$ & $818 \$$ & $160^{\prime}+\$$ & $L$ & 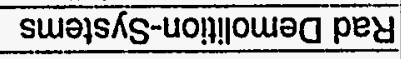 & $\underline{z}$ \\
\hline$\varepsilon 9 \varepsilon^{\prime} z 2$ & $\varepsilon 9 \varepsilon^{\prime} z 2$ & $000^{\prime} G 9 L^{\prime} !$ & & $\varepsilon 9 b^{\prime} \varepsilon \$$ & $169 \$$ & $090 \$$ & $20 \varepsilon^{\prime} Z \$$ & 06 & 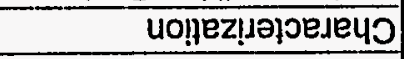 & 1 \\
\hline $008^{\prime} 01$ & $008^{\prime} 01$ & $000^{\prime} 09 L$ & & $09 Z^{\prime} L \$$ & - & จLL\$ & $9 \varepsilon L^{\prime} L \$$ & 81 & uo!nęuəunoog & a \\
\hline$د K /$ sanoy-ueW & HW IEIOL & 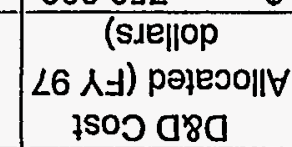 & & Kep/\$ $\mid$ elOL & $\begin{array}{c}\text { Kep/\$ } \\
\text { fuəud!̣nb }\end{array}$ & $\begin{array}{c}\text { Kep/\$ } \\
\text { ןe!ləjew }\end{array}$ & Kep/\$ 1 elOL & Kep/HW IEIOI & uo!!ounঘ Ma」O & \# MәIO \\
\hline & & & & & & & & & & \\
\hline & & & & & & & & & 109 & 107980 \\
\hline
\end{tabular}




\section{D Assumptions}

\begin{tabular}{|c|c|c|c|c|c|c|}
\hline $\begin{array}{l}\text { WERF-LLW } \\
\text { Compactable Building } \\
\text { Debris }\end{array}$ & 0.195 & 175,878 & 34,296 & 972 & & \\
\hline $\begin{array}{l}\text { RWMC-LLW Non- } \\
\text { Compactable Equipment }\end{array}$ & 0.513 & 175,878 & 90,225 & 2,556 & & \\
\hline $\begin{array}{l}\text { RWMC-LLW Non-Compt } \\
\text { Builoing Debris }\end{array}$ & 0.684 & 175,878 & 120,301 & 3,408 & & \\
\hline $\begin{array}{l}\text { RWMC-LLW Non-Compt } \\
\text { Concrete Rubble }\end{array}$ & 3.44 & 175,878 & 605,020 & 17,139 & $\begin{array}{l}\text { This factor is twice as large } \\
\text { as that recommended by } \\
\text { the D\&D program to } \\
\text { account for that large } \\
\text { amounts of concrete used. }\end{array}$ & \\
\hline $\begin{array}{l}\text { RWMC-LLW Non-Compt } \\
\text { Scrap Metal }\end{array}$ & 0.778 & 175,878 & 136,833 & 3,876 & & \\
\hline $\begin{array}{l}\text { RWMC-LLW } \\
\text { Asbestos/ACM Covered } \\
\text { Pipe }\end{array}$ & $0 \mid$ & 175,878 & - & - & & \\
\hline \begin{tabular}{l|} 
CFA Landfill Non-Compt \\
Building Debris
\end{tabular} & 1.99 & 175,878 & 349,997 & 9,915 & & \\
\hline $\begin{array}{l}\text { CFA Landfill Non-Compt } \\
\text { Concrete Rubble }\end{array}$ & 2.45 & 175,878 & 430,901 & 12,207 & $\begin{array}{l}\text { This factor is twice as large } \\
\text { as that recommended by } \\
\text { the D\&D program to } \\
\text { account for that large } \\
\text { amounts of concrete used. }\end{array}$ & \\
\hline CFA Landfill Asbestos & $0 !$ & 175,878 & - & - & & \\
\hline $\begin{array}{l}\text { HWSF Hazardous Mtrls } \\
\text { (Hg/PCBs/etc) }\end{array}$ & 0.002 & 175,878 & 352 & 10 & & \\
\hline Metal Recycle & 0.022 & 175,878 & 3,869 & 110 & & \\
\hline & & & & & & 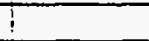 \\
\hline LLW $=$ & & & $1,038,560$ & 29,421 & & \\
\hline Non-Rad = & & & 780,898 & 22,122 & & \\
\hline Hazardous $=$ & & 4 & 352 & 10 & & $\frac{1}{!}$ \\
\hline Metal $=$ & & 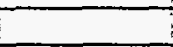 & 3,869 & 110 & & \\
\hline & & & & & $T$ & \\
\hline Electric power usage $=1$ & 156,000 & $\mathrm{kWh} / \mathrm{yr}$ & 156 & MWh/yr & & \\
\hline (based on $3,000 \mathrm{kWh} / \mathrm{wk}$ & - John Duggan) & & & & & \\
\hline & 1 & & & & & \\
\hline Air emissions from fuel are & e based on the & diesel emissi & ions spreadshe & & & ! \\
\hline & & & & & 1 & \\
\hline 1 manyear of labor $=$ & 1800 & manhours & & & i & \\
\hline & & & & & & \\
\hline Lube oil = & 5,297 & iiters (total) & & & $T$ & \\
\hline (based on 3 gal for every & 60 hours of ope & eration) & & & $i$ & \\
\hline & & & & & & \\
\hline Mixed waste $=$ & 12,375 & gal (total) $=$ & 47 & $\mathrm{~m} 3$ (total) & & T \\
\hline (based on an assumed 5 & 55-gallon drum & is generated $p$ & er week... wo & $k$ only 45 we & eeks/yr) & $i$ \\
\hline$i$ & & & & i & & \\
\hline Radioactivity associated $\mathrm{v}$ & with waste mate & erials: & & & & \\
\hline & & & & & & \\
\hline Spent decontamination so & olution $=$ & 1,703 & $\mathrm{Ci}$ & & & \\
\hline (based on an assumed av & verage activity & concentration & of $1 \mathrm{uCi} / \mathrm{ml})$ & & & \\
\hline & & & & & & \\
\hline Radioactive solid waste $=$ & & 294 & & & & \\
\hline (based in an assumed act & tivity concentra & tion of 0.01 uc & $\mathrm{Ci} / \mathrm{cc}[0.01 \mathrm{Ci} / \mathrm{m}$ & & & \\
\hline$i$ & i & & & & 1 & \\
\hline Mixed waste $=$ & & 0 & $\overline{\mathrm{Ci}}$ & & 1 & i \\
\hline
\end{tabular}




\begin{tabular}{|c|c|c|c|c|c|}
\hline & Project Data Sheet for Calcine & trieval - WWO, & DCWO, & HWO & \\
\hline Decontaminatic & on \& Decommissioning (D\&D) Information & & & & \\
\hline Cost (\$):D\&D ( & Indiscounted dollars) & & & & \\
\hline Decommission & & $\$ 2,681,000$ & & & \\
\hline Decontaminatior & & $\$ 7,415,000$ & & & \\
\hline Demolition & & $\$ 11,864,000$ & & & \\
\hline Total D\&D & & $\$ 21,960,000$ & & & \\
\hline Schedule start/e & nd: D\&D & January 2033 throl & ugh Decemb & er 2037 & \\
\hline Number of work & ers each year of D\&D (new/existing) & 160 & New worke & rs/yr & \\
\hline Number of radia & tion workers (D\&D) & 102 & New worke & rs/yr & \\
\hline Average annual & worker radiation dose (rem/yr) & 0.19 & rem/yr & |per worker & \\
\hline & & & & & \\
\hline Heavy equipmer & & & & & \\
\hline Equipment used & & Mobile Cranes, Rol & ll-off trucks, & Dozers, Loadel & \\
\hline Trips & Roll-off trucks & 15 & per day & & \\
\hline Hours of operati & on (all heavy equipment) & 27,990 & Hours & & \\
\hline & & & & & \\
\hline Acres disturbed & and duration of disturbance & January 2033 throu & ugh Decemb & er 2037 & \\
\hline New & & None & & & \\
\hline Previous & & 2.7 & lacres & & \\
\hline Revegetated & & None & & & \\
\hline Air emissions & & & & & \\
\hline non-radioactive & Fuel combustion gases $\left(\mathrm{CO}_{2}, \mathrm{H}_{2} \mathrm{O}, \mathrm{O}_{2}, \mathrm{~N}_{2}\right)$ & 16,269 & |tons (total) & & \\
\hline non-radioactive & $\begin{array}{l}\text { Fuel combustion contaminants (CO, } \\
\text { particulates, } \mathrm{NO}_{x}, \mathrm{SO}_{2} \text {, hydrocarbons) }\end{array}$ & 95 & tons (total) & & \\
\hline radioactive & HEPA filtered off-gas & 26,173 & |tons (total) & & \\
\hline Effluents & & & & & \\
\hline radioactive & Spent decontamination solution & $1,703,250$ & |liters (total) & 1,703 & $\mathrm{Ci}$ \\
\hline non-radioactive & Sanitary wastewater & $3,412,304$ & |liters (total) & & \\
\hline non-radioactive & Lube oil & 5,297 & liters (totai) & & \\
\hline Solid wastos. & & & & & \\
\hline radioactive & & 29,421 & $\mathrm{Im}^{3}$ & 294 & Ci \\
\hline Non-radioactive & (industrial) & 22,122 & $\mathrm{~m}^{3}$ & & \\
\hline Hazardous & & 10 & $1 \mathrm{~m}^{3}$ & & \\
\hline Hazardous/toxic & chemicals and wastes (type) & & & & \\
\hline Storage/inventor & & 205 & $\mathrm{~m}^{3}$ (total) & & \\
\hline Pits/Ponds creat & ed $\left(m^{2}\right)$ & None & & & \\
\hline radioactive & (mixed waste) & 47 & $\mathrm{~m}^{3}$ (total) & 0 & $\mathrm{Ci}$ \\
\hline & & & & & \\
\hline Water usage: & & & & & \\
\hline Process water & & $2,284,875$ & |iters (total) & & \\
\hline Domestic water| & & $3,412,304$ & |liters (total) & & \\
\hline Source of water & & ICPP site wells & & & \\
\hline & & & & & \\
\hline Energy requirem & eents: & & & 1 & \\
\hline
\end{tabular}




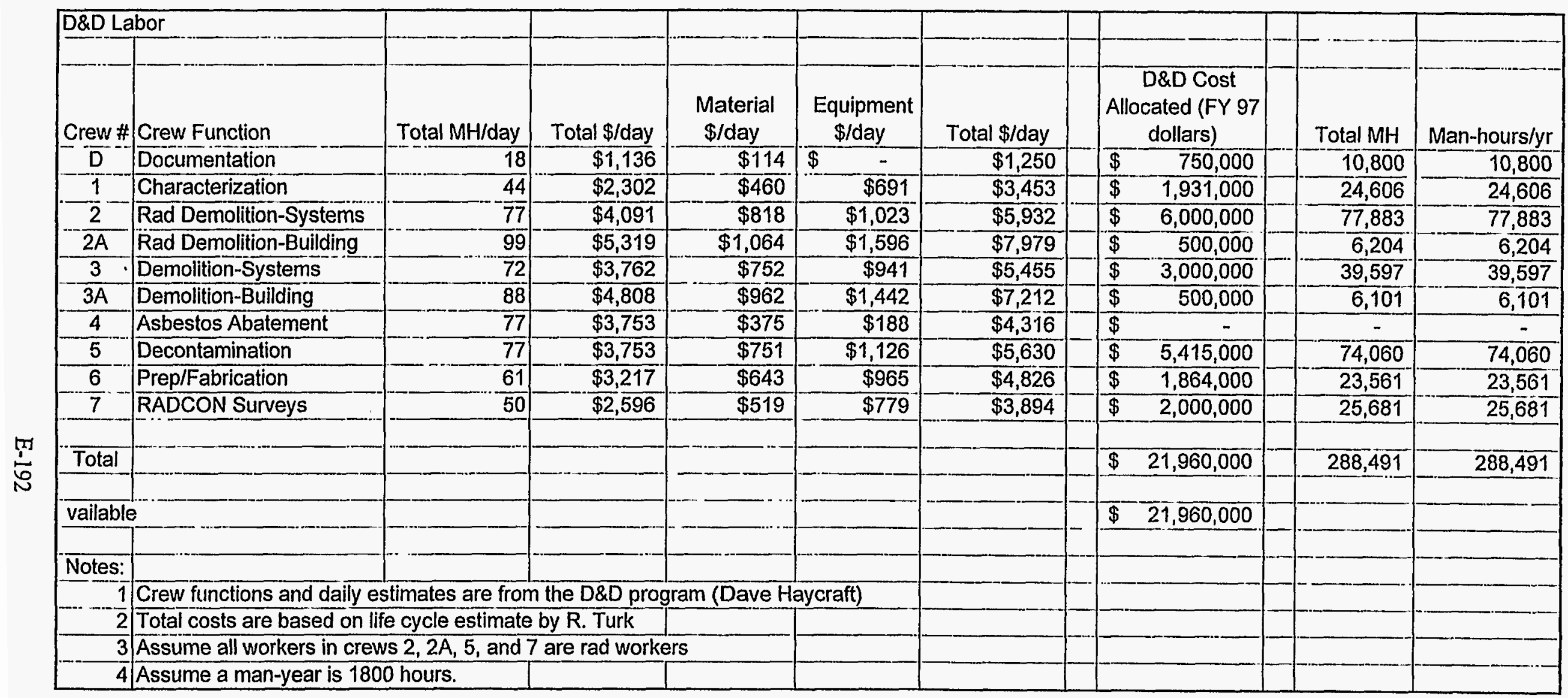




\section{D Assumptions}

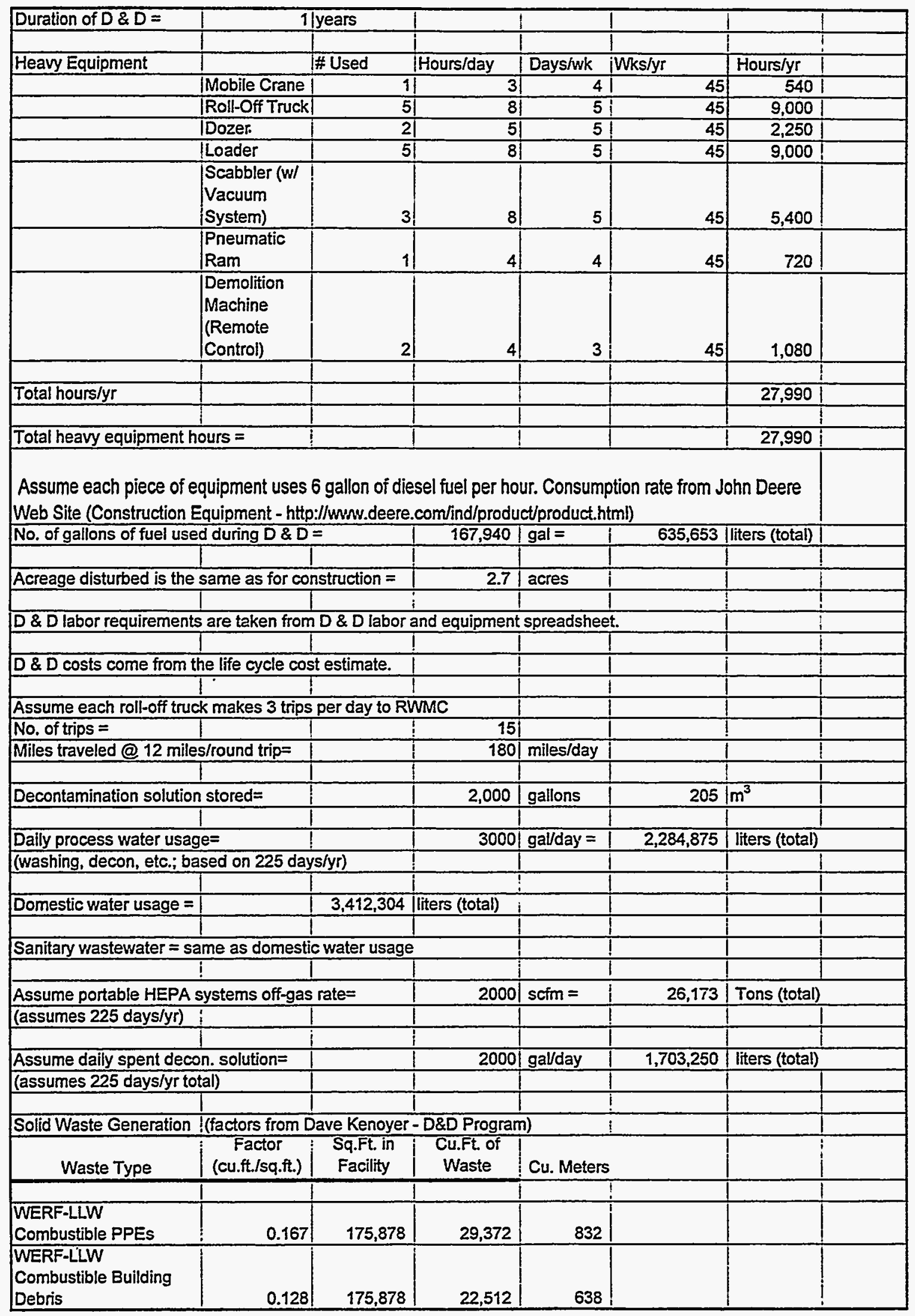




\begin{tabular}{|c|c|c|c|c|c|c|}
\hline $\begin{array}{l}\text { WERF-LLW } \\
\text { Compactable Suilding } \\
\text { Debris }\end{array}$ & 0.195 & $\{75,878$ & 34,296 & 972 & & \\
\hline $\begin{array}{l}\text { RWMC-LLW Non- } \\
\text { Compactable Equipment }\end{array}$ & $0.513 !$ & 175,878 & 90,225 & 2,556 & & \\
\hline $\begin{array}{l}\text { RWMC-LLW Non-Compt } \\
\text { Building Debris }\end{array}$ & 0.684 & 175,878 & $120,301 !$ & 3,408 & & \\
\hline $\begin{array}{l}\text { RWMC-LLW Non-Compt } \\
\text { Concrete Rubble }\end{array}$ & 3.44 & 175,878 & 605,020 & 17,139 & $\begin{array}{l}\text { This factor is twice as large } \\
\text { as that recommended by } \\
\text { the D\&D program to } \\
\text { account for that large } \\
\text { lamounts of concrete used. }\end{array}$ & \\
\hline $\begin{array}{l}\text { RWMC-LLW Non-Compt } \\
\text { Scrap Metal }\end{array}$ & $0.778 !$ & 175,878 & 136,833 & 3,876 & & \\
\hline $\begin{array}{l}\text { RWMC-LLW } \\
\text { Asbestos/ACM Covered } \\
\text { Pipe }\end{array}$ & of & 175,878 & - & - & & \\
\hline $\begin{array}{l}\text { CFA Landfill Non-Compt } \\
\text { Building Debris }\end{array}$ & 1.99 & 175,878 & 349,997 & 9,915 & & \\
\hline $\begin{array}{l}\text { CFA Landfill Non-Compt } \\
\text { Concrete Rubble }\end{array}$ & 2.45 & 175,878 & 430,901 & 12,207 & $\begin{array}{l}\text { This factor is twice as large } \\
\text { as that recommended by } \\
\text { the D\&D program to } \\
\text { account for that large } \\
\text { amounts of concrete used. }\end{array}$ & \\
\hline CFA Landfill Asbestos & $0 !$ & 175,878 & - & - & & \\
\hline $\begin{array}{l}\text { HWSF Hazardous Mitrls } \\
\text { (Hg/PCBs/etc) }\end{array}$ & 0.002 & 175,878 & 352 & 10 & & \\
\hline Metal Recycle & 0.022 & 175,878 & 3,869 & 110 & & \\
\hline & & & & & & \\
\hline LLW $=$ & & & $1,038,560$ & 29,421 & & \\
\hline Non-Rad = & & & 780,898 & 22,122 & & \\
\hline Hazardous $=$ & & & 352 & 10 & & \\
\hline Metal = & $\mathrm{T}$ & & 3,869 & 110 & & \\
\hline & & & & & & \\
\hline Electric power usage $=$ ! & 156,000 & $\mathrm{kWh} / \mathrm{yr}$ & $156 \mid$ & MWh/yr & & \\
\hline (based on $3,000 \mathrm{kWh} / \mathrm{wk}$ - & - John Duggan) & & & & & \\
\hline T & & & & I & & \\
\hline Air emissions from fuel are & e based on the & diesel emissi & ons spreadshe & eet. & & \\
\hline & & & & 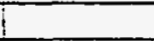 & & \\
\hline 1 manyear of labor $=$ & 1800 & manhours & & i & & \\
\hline 1 & & & 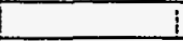 & 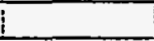 & & \\
\hline Lube oil = & 5,297 & liters (total) & & & & \\
\hline (based on 3 gal for every & 60 hours of ope & eration) & & $!$ & & \\
\hline $\bar{\vdots}$ & & & & & & \\
\hline Mixed waste $=$ & 12,375 & gal (totai) $=$ & 47 & $\mathrm{~m} 3$ (total) & & \\
\hline (based on an assumed 5 & 55-gallon drum & is generated p & er week... wor & rik only 45 we & eeks/yr) & \\
\hline & & & & & 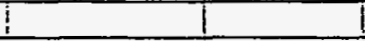 & \\
\hline Radioactivity associated $v$ & with waste mate & erials: & & & $!$ & \\
\hline & & & & & 1 & \\
\hline Spent decontamination so & olution = & 1,703 & $\overline{C i}$ & & $i$ & \\
\hline (based on an assumed av & verage activity & concentration & of $1 \mathrm{uCi} / \mathrm{ml})$ & & & \\
\hline 1 & 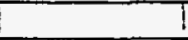 & & & & 1 & \\
\hline Radioactive solid waste $=$ & 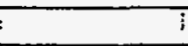 & 294 & $\overline{C i}$ & & 1 & \\
\hline (based in an assumed act & tivity concentra & tion of $0.01 \mathrm{u}$ & $\mathrm{Ci} / \mathrm{cc}[0.01 \mathrm{Ci} / \mathrm{m}$ & & 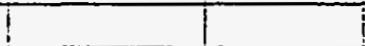 & \\
\hline 1 & & 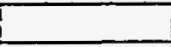 & i & & 1 & \\
\hline Mixed waste $=$ & 1 & 0 & $\mathrm{Ci}$ & \pm & & \\
\hline (based on an assumed ac & ctivity concentra & ation of $0.01 \mathrm{u}$ & $\mathrm{Ci} / \operatorname{cc}[0.01 \mathrm{Cl} / \mathrm{I}$ & $\left.\left(m^{3}\right]\right)$ & & \\
\hline
\end{tabular}




\section{Back up to 4}

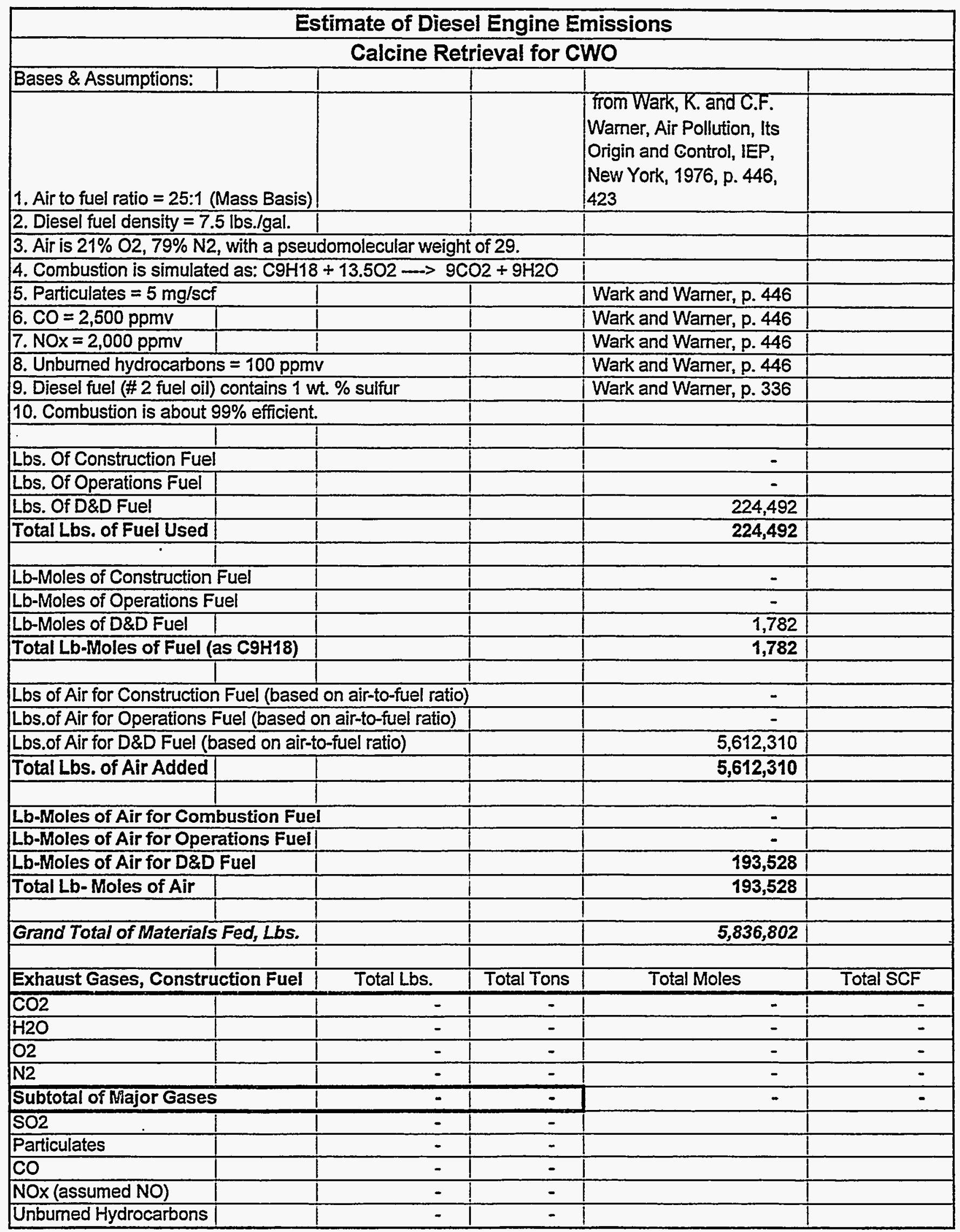




\begin{tabular}{|c|c|c|c|c|}
\hline Subtotal of Contaminants & - & - & & \\
\hline & & & & \\
\hline & & & & \\
\hline Exhaust Gases, Operations Fuel & Total Lbs. & Total Tons & Total Moles & Total SCF \\
\hline $\mathrm{CO} 2$ & - & - & - & - \\
\hline $\mathrm{H} 2 \mathrm{O}$ & - & - & - & - \\
\hline 02 & - & - & - & - \\
\hline N2 & - & - & - & - \\
\hline Subtotal of Major Gases & $=$ & - & - & - \\
\hline$\widehat{\mathrm{SO} 2}$ & - & - & & \\
\hline Particulates & - & - & & \\
\hline $\mathrm{CO}$ & - & - & & \\
\hline NOx (assumed NO) & - & - & & \\
\hline Unburned Hydrocarbons & - & - & & \\
\hline Subtotal of Contaminants & $=$ & - & & \\
\hline & & & & \\
\hline & & & & \\
\hline Exhaust Gases, D\&D Fuel & Total Lbs. & Total Tons & Total Moles & Total SCF \\
\hline $\mathrm{CO} 2$ & 698,492 & 349 & 15,875 & $5,699,060$ \\
\hline $\mathrm{H} 2 \mathrm{O}$ & 285,747 & 143 & 15,875 & $5,699,060$ \\
\hline 02 & 538,516 & 269 & 16,829 & $6,041,480$ \\
\hline $\mathrm{N} 2$ & $4,280,837.83$ & 2,140 & 152,887 & $54,886,457$ \\
\hline Subtotal of Major Gases & $5,803,593$ & 2,902 & 201,465 & $72,326,057$ \\
\hline $\mathrm{SO} 2$ & 4,350 & 2.2 & & \\
\hline Particulates & 797 & 0.4 & & \\
\hline $\mathrm{CO}$ & 14,103 & 7.1 & & \\
\hline NOx (assumed NO) & 12,088 & 6.0 & & \\
\hline Unburned Hydrocarbons! & 2,538 & 1.3 & & \\
\hline Subtotal of Contaminants & 33,875 & 17 & & \\
\hline
\end{tabular}


Back up tou

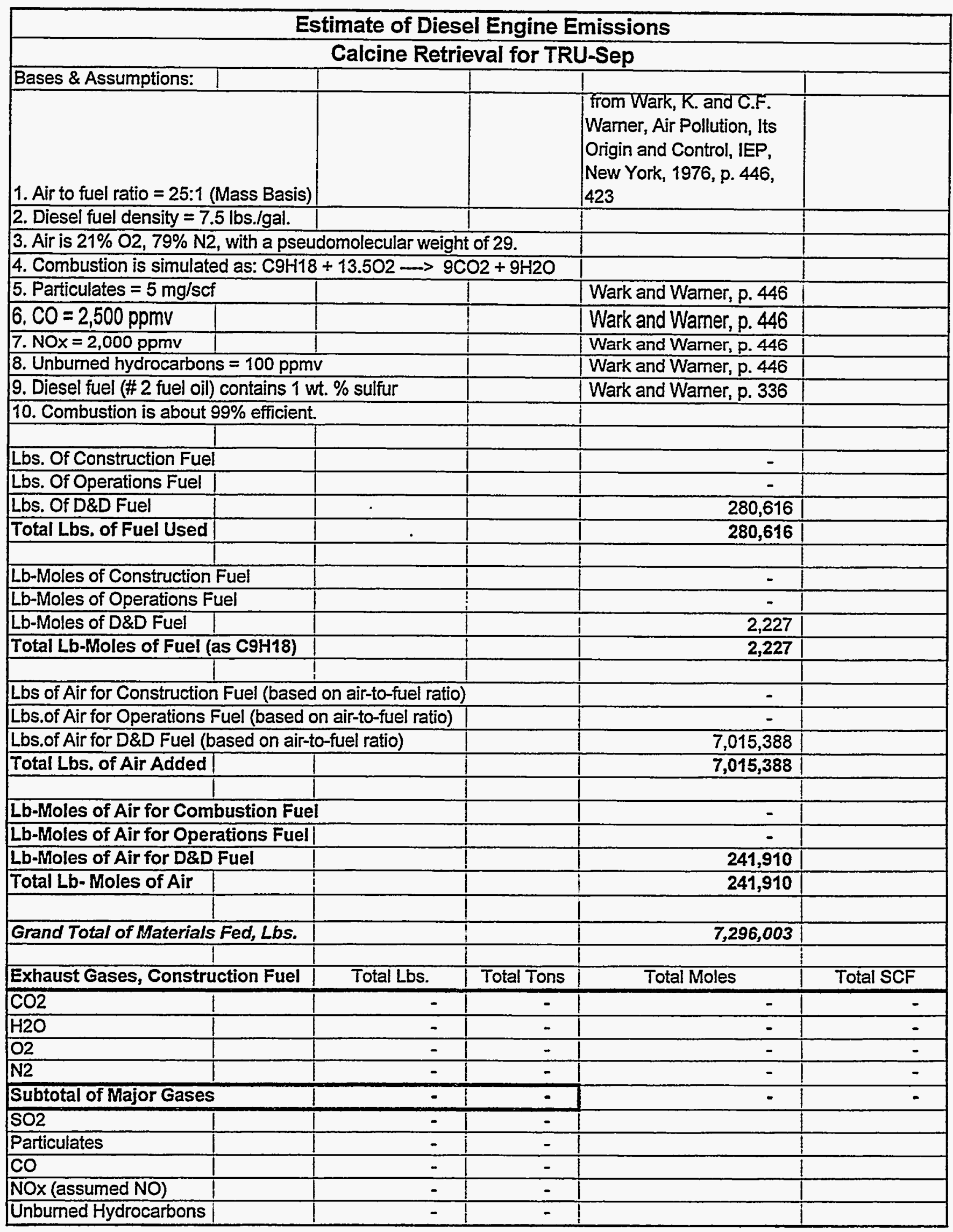




\begin{tabular}{|c|c|c|c|c|}
\hline Subtotal of Contaminants & - & $=$ & & \\
\hline $1+2-1$ & & & & \\
\hline$T$ & & & & \\
\hline Exhaust Gases, Operations Fuel & Total Lbs. & Total Tons & Total Moles & Total SCF \\
\hline $\mathrm{CO} 2$ & - & - & - & $=$ \\
\hline $\mathrm{H} 2 \mathrm{O}$ & - & - & - & - \\
\hline 02 & - & - & - & - \\
\hline N2 & - & - & - & - \\
\hline Subtotal of Major Gases & - & - & - & - \\
\hline $\mathrm{SO} 2$ & - & - & & \\
\hline Particulates & - & - & T. & \\
\hline CO & - & - & & \\
\hline NOx (assumed NO) & - & - & & \\
\hline Unburned Hydrocarbons & - & - & & \\
\hline Subtotal of Contaminants & - & - & & \\
\hline 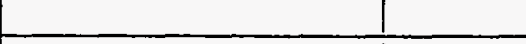 & & & & \\
\hline & & & & \\
\hline Exhaust Gases, D\&D Fuel & Total Lbs. & Total Tons & Total Moles & Total SCF \\
\hline $\mathrm{CO} 2$ & 873,115 & 437 & 19,844 & $7,123,825$ \\
\hline $\mathrm{H} 2 \mathrm{O}$ & 357,183 & 179 & 19,844 & $7,123,825$ \\
\hline $\mathrm{O} 2$ & 673,145 & 337 & 21,036 & $7,551,850$ \\
\hline N2 & $5,351,047.29$ & 2,676 & 191,109 & $68,608,071$ \\
\hline Subtotal of Major Gases & $7,254,491$ & 3,627 & 251,832 & $90,407,572$ \\
\hline SO2 & 5,437 & $\overline{2.7}$ & & \\
\hline Particulates & 996 & 0.5 & & \\
\hline $\mathrm{CO}$ & 17,628 & 8.8 & & \\
\hline NOx (assumed NO) & 15,110 & 7.6 & & \\
\hline Unburned Hydrocarbons & 3,173 & 1.6 & & \\
\hline Subtotal of Contaminants & 42,344 & 21 & & \\
\hline
\end{tabular}


Backup to $u$

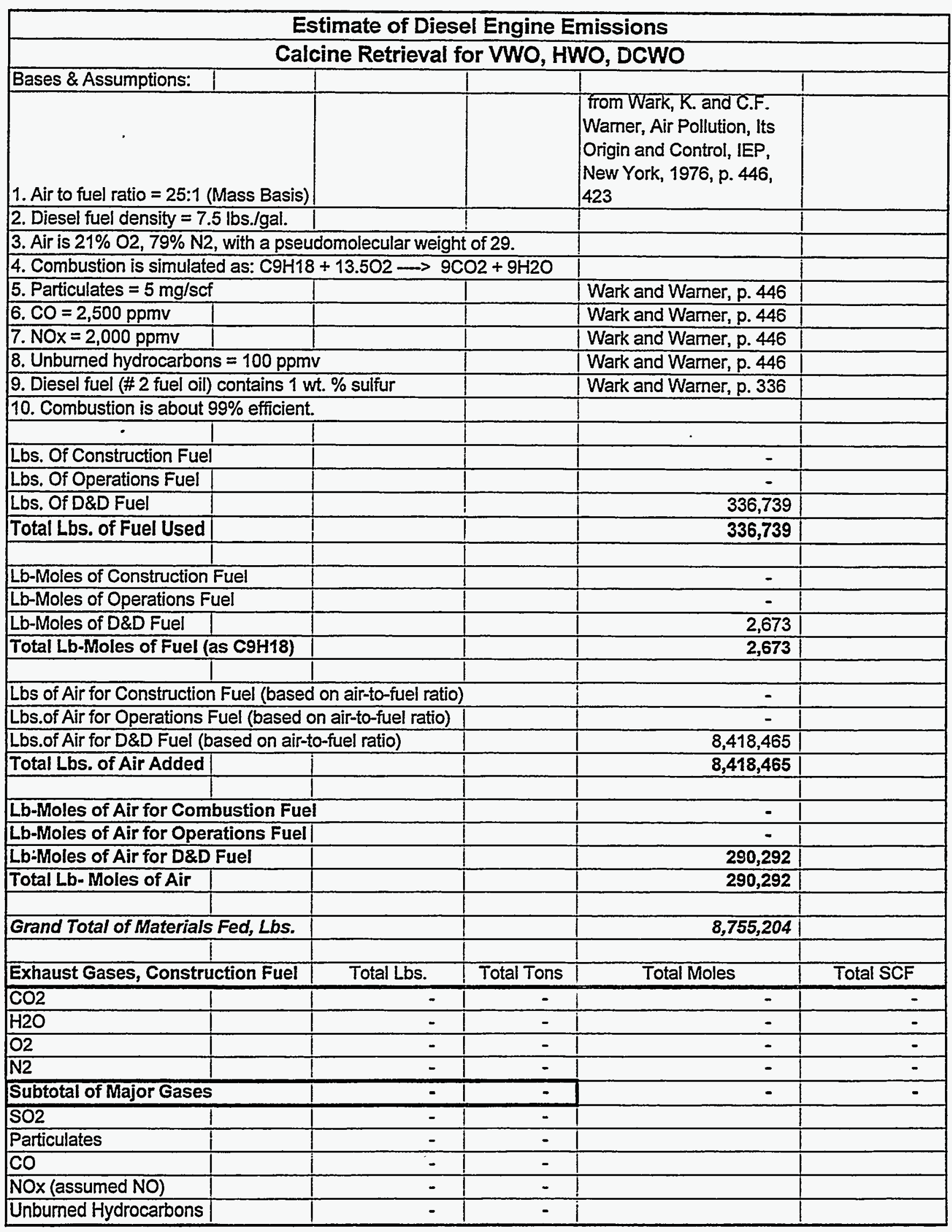




\begin{tabular}{|c|c|c|c|c|}
\hline Subtotal of Contaminants & - & - & & \\
\hline & & & 1 & \\
\hline$!$ & & & & \\
\hline Exhaust Gases, Operations Fuel & Total Lbs. & Total Tons & Total Moles & Total SCF \\
\hline $\mathrm{CO} 2$ & - & - & $\overline{-}$ & $\overline{-}$ \\
\hline $\mathrm{H} 2 \mathrm{O}$ & - & - & - & - \\
\hline $\mathrm{O} 2$ & - & - & - & - \\
\hline N2 & - & - & - & - \\
\hline Subtotal of Major Gases & $=$ & $=$ & - & - \\
\hline $\mathrm{SO} 2$ & - & - & & \\
\hline Particulates & - & - & & \\
\hline $\mathrm{CO}$ & - & - & & \\
\hline NOX (assumed NO) & - & - & & \\
\hline Unburned Hydrocarbons & - & - & & \\
\hline Subtotal of Contaminants & $=$ & - & & \\
\hline & & & 1 & \\
\hline & & & & \\
\hline Exhaust Gases, D\&D Fuel & Total Lbs. & Total Tons & Total Moles & Total SCF \\
\hline $\mathrm{CO} 2$ & $1,047,738$ & 524 & 23,812 & $8,548,590$ \\
\hline $\mathrm{H} 2 \mathrm{O}$ & 428,620 & 214 & 23,812 & $8,548,590$ \\
\hline 02 & 807,775 & 404 & 25,243 & $9,062,220$ \\
\hline N2 & $6,421,256.75$ & 3,211 & 229,331 & $82,329,685$ \\
\hline Subtotal of Major Gases & $8,705,390$ & 4,353 & 302,198 & $108,489,086$ \\
\hline SO2 & 6,524 & 3.3 & & \\
\hline Particulates & 1,195 & 0.6 & & \\
\hline $\mathrm{CO}$ & 21,154 & 10.6 & & \\
\hline NOx (assumed NO) & 18,132 & 9.1 & & \\
\hline Unbumed Hydrocarbons & 3,808 & 1.9 & & \\
\hline Subtotal of Contaminants & 50,813 & 25 & & \\
\hline
\end{tabular}


Appendix E

\section{Risk Assessment Data Sheets}

This appendix contains a Risk Assessment Form for each significant risk identified in the calcine retrieval and transportation system. The risks have been assigned to one of three categories:

Project, Technical, and ES\&H (Environmental, Health, and Safety).

Project Risk (cost or schedule):

P.1 Integrity of CSSF maintained

P. 2 Location of retrieval risers

P.3 Estimated retrieval percentage too high

P.4 Internal obstructions prevent retrieval

P.5 Waste Treatment Facility too slow

P.6 Miscellaneous materials prevent retrieval

$$
\begin{aligned}
& \text { Risk }=3 \\
& \text { Risk }=3 \\
& \text { Risk }=2 \\
& \text { Risk }=2 \\
& \text { Risk }=2 \\
& \text { Risk }=4
\end{aligned}
$$

Technical Risk:

T.1 Objective retrieval rate too high

T.2 All calcines are not retrievable

$$
\begin{aligned}
& \text { Risk }=3 \\
& \text { Risk }=4
\end{aligned}
$$

ES\&H Risk (environment, safety, or health):

ESH.1 Construction radiation dose rates incorrect Risk $=4$

As risks were evaluated, the probability of occurrence and the impact if the risk is realized were rated. The probability and impact were assigned numerical values of " 3 " for high, " 2 " for medium, and " 1 " for low. The equation for risk calculation is Risk = Probability X Impact. The maximum risk rating is "9." 
The risk matrix, Table E-1, shows what action to take as a function of risk rating. These actions are defined in table E-2.

\author{
Table E-1. Risk Matrix

$$
\text { Risk }=\text { Probability X Impact }
$$

\begin{tabular}{l|c|c|c|}
\multicolumn{3}{c}{ Probability } & \multicolumn{3}{c}{ Impact } \\
\cline { 1 - 3 } & Low (1) & Medium (2) & High (3) \\
\cline { 2 - 4 } High (3) & Evaluate (3) & Contingency Plan (6) & Eliminate Risk (9) \\
\cline { 2 - 4 } Medium (2) & Monitor (2) & Contingency Plan (6) & Contingency Plan (6) \\
\cline { 2 - 4 } Low (1) & Forget (1) & Monitor (2) & Evaluate (3) \\
\cline { 2 - 4 } &
\end{tabular}

\title{
Table E-2. Risk Action Definition
}

\begin{tabular}{|c|l|}
\hline Risk Rating & \multicolumn{1}{|c|}{ Action } \\
\hline 9 & $\begin{array}{l}\text { Any risk determined to be in this category will be mitigated through } \\
\text { additional design or analysis until the risk is not longer in this category. }\end{array}$ \\
\hline 6 and 4 & $\begin{array}{l}\text { Risks in these categories will be mitigated to the extent feasible within } \\
\text { the cost and schedule guidelines. For those risks that cannot be } \\
\text { mitigated, contingency plans have been made to deal with the risk if it } \\
\text { occurs. }\end{array}$ \\
\hline 3 & $\begin{array}{l}\text { Risks in this category will be evaluated during the project to identify if } \\
\text { they are becoming problems. }\end{array}$ \\
\hline 2 & These risks will be monitored with no specific action identified. \\
\hline 1 & These risks are noted for interest only. \\
\hline
\end{tabular}




\section{Risk Assessment - Data Sheet}

Risk Type OProject OTechnical OESH

RISK: Integrity of CSSFs maintained

Significant construction activities will occur on the roof of each CSSF. For nearly all CSSFs, 8" holes must be drilled through the concrete roof. There is a possibility that the structural integrity of the CSSFs will be reduced.

\section{PREVENTIVE PLANS}

As part of locating the retrieval risers, a structural analysis of the CSSF roofs will be conducted.

\section{CONSEQUENCES IF RISK NOT RESOLVED}

The schedule and cost would be effected by this risk. The CSS roof would need to be shored up. This would slow down the construction schedule and increase the cost.

Structural integrity
access activities.

CONTINGENCY PLAN(S) IF RISK IS REALIZED:

The CSSF storage vault roof should be shored up.

\section{Risk $=3$}

Risk $=$ Probability $\times$ Impact

\section{Probability Definition}

High - Likely to occur during the project.

Medium - Has the potential to occur during the project

Low - Has little potential to occur during the project.

\section{Impact Definition}

High - Likely to cause significant disruption of schedule, increase in cost, or degradation of performance.

Medium - Has the potential to cause some disrutpion to schedule, increase in cost, or degradation of performance.

Low - Has little potential to cause disruption to schedule, increase in cost, or degradation of performance.

\section{TRIGGER POINT(S) FOR EARLY RISK} IDENTIFICATION:

During feasibility studies, a structural analysis of the CSSF roofs should be conducted. 


\title{
Risk Assessment - Data Sheet
}

\author{
Risk Type $\odot$ Project OTechnical OESH
}

\section{RISK: Location of retrieval risers}

Locations for installation of retrieval risers must be identified. Enough locations may not exist on each bin to install the necessary retrieval risers.

\section{PREVENTIVE PLANS}

This risk has been significantly minimized by decreasing the number of retrieval lines need for calcine retrieval.

\section{PROBABLE CAUSE(S):}

The requirements for installing retrieval risers are not currently known. However, pipes in the bin vault may prevent the installation of the retrieval risers. There may not be adequate space on the bins to install the retrieval risers.

\section{CONSEQUENCES IF RISK NOT RESOLVED}

Calcine retrieval using the proposed calcine retrieval and transportation system would be prevented if the retrieval risers could not be installed. This would significantly impact the schedule and costs.

\section{PROBABILITY}
High (3)
Medium (2)
(-) Low (1)

\section{IMPACT}

\begin{tabular}{ll|}
$\bigcirc$ & High (3) \\
0 & Medium (2) \\
$\bigcirc$ & Low (1) \\
\hline
\end{tabular}

\section{Risk $=3$}

Risk $=$ Probability $\times$ Impact

Probability Definition

High - Likely to occur during the project.

Medium - Has the potential to occur during the project.

Low - Has little potential to occur during the project.

Impact Definition

High - Likely to cause significant disruption of schedule, increase in cost, or degradation of perfomance.

Medium - Has the potential to cause some disrutpion to schedule, increase in cost, or degradation of performance.

Low - Has little potential to cause disuption to schedule, increase in cost, or degradation of performance.

\section{CONTINGENCY PLAN(S) IF RISK IS REALIZED:}

Develop a less restrictive method for installing the retrieval lines.

\section{TRIGGER POINT(S) FOR EARLY RISK}

\section{IDENTIFICATION:}

The requirements of the riser installation process must be know prior to the early identification of this risk. 


\section{RISK: Estimated retrieval percentage too high}

It is estimated that $95 \%$ of the calcine in each bin can be retrieved. This estimate is based on the best available data fro pilot plant tests (ref. 9)

\section{PREVENTIVE PLANS}

The optimum configuration of suction nozzles and air jets should be identified through pilot plant study. The optimum configuratio should minimize the number of retrieval lines while maximizing the retrieval rate.

\section{PROBABLE CAUSE(S):}

Calcine may stick to the walls of the bin. It may get wedged in the "nooks and crannies" created by themowells and internal stiffening rings and rods. The more agglomerated the calcine is the harder it is to retrieve.

\section{CONSEQUENCES IF RISK NOT RESOLVED}

This risk will effect the closure of the CSSFs the most. The mor calcine left in each bin will increase closure costs and lengthen the closure schedule. Pilot plant tests show that $97 \%$ of the calcine (ref. 9) is retrievable at a high retrieval rate.

\section{PROBABILITY}

High (3)

Medium (2)

(-) Low (1)

\section{IMPACT}

High (3)

(-) Medium (2)

$\bigcirc \operatorname{Low}(1)$

\section{Risk $=2$}

Risk $=$ Probability $\times$ Impact

\section{Probability Definition}

High - Likely to occur during the project.

Medium - Has the potential to occur during the project.

Low - Has little potential to occur during the project.

\section{Impact Definition}

High - Likely to cause significant disnuption of schedule, increase in cost, or degradation of performance.

Medium - Has the potential to cause some disrutpion to schedule, increase in cost, or degradation of performance.

Low - Has little potential to cause disruption to schedule, increase in cost, or degradation of performance.

\section{CONTINGENCY PLAN(S) IF RISK IS REALIZED:}

The best that can be done is to provide ample time in the schedule for CSSF closure.

\section{TRIGGER POINT(S) FOR EARLY RISK IDENTIFICATION:}

During operation of the calcine retrieval and transportation system. 


\title{
Risk Assessment - Data Sheet
}

\author{
Risk Type OProject OTechnical OESH
}

\section{RISK: Intemal obstructions prevent retrieval}

Intemal obstructions may interfere with extension of the rigid retrieval lines into the bins.

\section{PREVENTIVE PLANS}

Examine the available CSSF drawings to determine retrieval ris locations that will not interfere with existing, intemal obstructions

\section{PROBABLE CAUSE(S):}

Each bin has its own set of stiffening rods and thermowelis. The retrieval lines may run into these internal obstructions.

\section{CONSEQUENCES IF RISK NOT RESOLVED}

Calcine retrieval may be completely stopped from a bin. This would adversely impact the schedule and drive costs up.
PROBABILITY

High (3)

Medium (2)

(-) Low (1)

\section{IMPACT}

High (3)

(-) Medium (2)

Low (1)

\section{CONTINGENCY PLAN(S) IF RISK IS REALIZED:}

None.

Risk $=2$

Risk $=$ Probability $\times$ Impact

Probability Definition

High - Likely to occur during the project.

Medium - Has the potential to ocur during the project.

Low - Has ittle potential to ocaur during the project.

\section{Inpact Definition}

High - Likely to cause significant disruption of schedule, increase in cost, or degradation of performance.

Medium - Has the potential to cause some disutpion to schedule. increase in cost, or degradation of perfomance.

Low - Has little potential to cause disruption to schedule, increase in cost, or degradation of performance.

\section{TRIGGER POINT(S) FOR EARLY RISK IDENTIFICATION:}

During operation of the calcine retrieval and transportation system. 


\section{Risk Assessment - Data Sheet}

Risk Type $\odot$ Project OTechnical OESH

\section{RISK: Waste Treatment Facility too slow}

The waste treatment facility cannot process calcine fast enough. It falls behind the retrieval rate of calcine.

\section{PREVENTIVE PLANS}

The processing capacity of the Waste Treatment Facility should be defined during the conceptual design phase. The operation o the Waste Treatment Facility and the calcine retrieval and transportation system must be coordinated.

\section{PROBABLE CAUSE(S):}

The waste treatment facility may be slowed down by an equipment failure. The processing rate may be over estimated $i$ the adjoining scoping studies.

\section{CONSEQUENCES IF RISK NOT RESOLVED}

The calcine retrieval and transportation system should not operate unless the WTF requires additional calcine. The $50 \%$ u time estimate provides for some operational lapses. The risk is that the calcine batch bins will overflow with calcine. This will further contaminate the shielded cell when calcine is delivered $t$ the Waste Treatment Facility.
PROBABILITY
High (3)
Medium (2)
- $\operatorname{Low}(1)$

\section{Risk $=2$}

Risk $=$ Probability $\times$ Impact

\section{Probability Definition}

High - Likely to occur during the project.

Medium - Has the potential to occur during the project.

Low - Has little potential to oceur during the project.

\section{Impact Definition}

High - Likely to cause significant disnuption of schedule, increase in cost, or degradation of perfomance.

Medium - Has the potential to cause some disutpion to schedule, increase in cost, or degradation of performance.

Low - Has little potential to cause disnuption to schedule, increase in cost, or degradation of performance.

\section{CONTINGENCY PLAN(S) IF RISK IS REALIZED:}

The amount of calcine retrieved should be adjusted to match the amount of calcine processed in each batch.

\section{TRIGGER POINT(S) FOR EARLY RISK} IDENTIFICATION:

Changes in operations of the Waste Treatment Facility would identify the need to adjust the calcine retrieval rate. 


\title{
Risk Assessment - Data Sheet
}

\author{
Risk Type $\odot$ Project OTechnical OESH
}

RISK: Miscellaneous material prevents retrieval

The miscellaneous material may damage or plug the calcine retrieval and transportation system. Damage tot he calcine retrieval and transportation system or the bins may occur when this material is picked up by the air jet. Clogging can occur if an item enters the transportation system

\section{PREVENTIVE PLANS}

As much of this miscellaneous materias should be removed as possible.

\section{PROBABLE CAUSE(S):}

Over the years, non-calcine materials have entered the CSSFs 2 , and 3. Rod out lines have been lost. Weighted lines have purposefully fallen into the bins. And other foreign materials hav entered the bins. This material is scattered throughout the calcine. It is at different levels. It is not known exactly what material entered the CSSFs 1, 2, and 3 .

\section{CONSEQUENCES IF RISK NOT RESOLVED}

The schedule can tolerate a fair amount of down time (currently operated $50 \%$ of the time). The backup transportation lines should minimize delays in the schedule. This will impact the cos if the damage is extensive.
PROBABILITY

\begin{tabular}{|ll|}
\hline$\bigcirc$ & High (3) \\
$\bigcirc$ & Medium (2) \\
$\bigcirc$ & Low (1) \\
\hline
\end{tabular}

\section{Risk $=4$}

Risk $=$ Probability $\times$ Impact

\section{CONTINGENCY PLAN(S) IF RISK IS REALIZED:}

implement a method to remove these items before they enter th transport system.

\section{Probability Definition}

High - Likely to occur during the project.

Medium - Has the potential to occur during the project.

Low - Has litte potential to ocaur during the project.

\section{Impact Definition}

High - Likely to cause significant disruption of schedule, increase in cost, or degradation of performance.

Medium - Has the potential to cause some disrutpion to schedule, increase in cost, or degradation of performance.

Low - Has little potential to cause disnuption to schedule, increase in cost, or degradation of performance.

\section{TRIGGER POINT(S) FOR EARLY RISK} IDENTIFICATION:

During operation of the calcine retrieval and transport system. 


\section{RISK: Objective retrieval rate too high}

The objective retrieval rate is $2700 \mathrm{~kg} / \mathrm{hr}$. It is expected that calcine can be retrieved near this rate. This rate is based on the Fluor-Daniels design retrieval rate. Retrieval tests (ref. 9) show that it is difficult to maintain any given retrieval rate.

\section{PREVENTIVE PLANS}

A minimum and maximum retrieval rate should be defined. The operator should be able to control, to some extent, the retrieval rate by changing the heights of the suction and air jet nozzles.

\section{PROBABLE CAUSE(S):}

The retrieval rate appears to be directly related to the amount of calcine in the bin. The less calcine there is to retrieve the less calcine can be retrieved. Aggiomerated calcine is more difficult to retrieve than free flowing calcine. The transport air blower, which provides the air jet and suction nozzles, may be undersized to retrieve such a large volume of calcine near the bottom of the bins.

\section{CONSEQUENCES IF RISK NOT RESOLVED}

The schedule should not be adversely impacted as long as the calcine can be retrieved at a rate greater than approximately 50 $\mathrm{kg} / \mathrm{hr}$. However, the cost will increase for operations if the calcine retrieval and transportation system must be operated for more than two $10 \mathrm{hr}$ days/week.

\section{PROBABILITY}

O High (3)

O Medium (2)

○ Low (1)
IMPACT

\begin{tabular}{|ll|}
\hline$\bigcirc$ & High (3) \\
$\bigcirc$ & Medium (2) \\
$\bigcirc$ & Low (1) \\
\hline
\end{tabular}

\section{CONTINGENCY PLAN(S) IF RISK IS REALIZED:}

Calcine retrieval will be conducted using extra shifts if the calcin can be retrieved at a reasonable rate. The reasonable rate will be determined by the need's of the waste treatment process. A good general number would be on the order of $500 \mathrm{lb} / \mathrm{hr}$.

\section{Risk $=3$}

Risk $=$ Probability $\times$ Impact

\section{Probability Definition}

High - Likely to occur during the project.

Medium - Has the potential to occur during the project.

Low - Has little potential to occur during the project.

\section{Impact Definition}

High - Likely to cause significant disruption of schedule, increase in cost, or degradation of performance.

Medium - Has the potential to cause some disrutpion to schedule, increase in cost, or degradation of performance.

Low - Has little polential to cause disruption to schedule, increase in cost, or degradation of performance.
TRIGGER POINT(S) FOR EARLY RISK IDENTIFICATION:

Operation of the calcine retrieval and transportation system 


\title{
Risk Assessment - Data Sheet
}

\author{
Risk Type OProject OTechnical OESH
}

\section{RISK: All calcines are not retrievable}

All types of calcine are retrievable as a dilute phase using this calcine retrieval and transportation system.

\section{PREVENTIVE PLANS}

Pilot plant tests should be conducted on agglomerated calcine. Also samples of the calcine in the CSSFs should be taken and analyzed to determine if the calcine has agglomerated.

\section{PROBABLE CAUSE(S):}

The calcine may be agglomerated. It is anticipated that agglomerated calcine will not fluidize as readily as free flowing calcine

\section{CONSEQUENCES IF RISK NOT RESOLVED}

The retrieval rate could be significantly lower than expected. Th operating costs will increase. The schedule may fall behind.

\section{PROBABILITY}
High (3)
(C) Medium (2)
$\bigcirc$ Low (1)

\section{IMPACT}

\begin{tabular}{ll}
$\bigcirc$ & High (3) \\
$\odot$ & Medium (2) \\
$\bigcirc$ & Low (1) \\
\hline
\end{tabular}

\section{CONTINGENCY PLAN(S) IF RISK IS REALIZED:}

The agglomerated calcine must be broken up before it retrieved. If the potential for agglomerated calcine is high for a particular bin, a vibrator should be added to the end of the retrieval line before it is inserted into the bin. If agglomerated calcine is not suspected, then the air jet should be increased.

Risk $=4$

Risk $=$ Probability $\times$ Impact

Probability Definition

High - Likely to occur during the project.

Medium - Has the potential to occur during the project.

Low - Has little potential to occur during the project.

Inpact Definition

High - Likely to cause significant disruption of schedule, increase in cost, or degradation of perfomance.

Medium - Has the potential to cause some disutpion to schedule, increase in cost, or degradation of performance.

Low - Has Iitte polential to cause disruption to schedule, increase in cost, or degradation of performance.

\section{TRIGGER POINT(S) FOR EARLY RISK} IDENTIFICATION:

Operation of the calcine retrieval and transportation system on actual calcine. 


\section{Risk Type OProject OTechnical OESH}

RISK: Construction radiation dose rates incorrect

The radiation dose rates are estimated from the best available data. The big unknown is the radiation levels in the CSSF superstructures.

\section{PREVENTIVE PLANS}

Proposed radiological surveys of each CSSF superstructure.

\section{PROBABLE CAUSE(S):}

A comprehensive radiological survey of the CSSF superstructures has not been conducted. The radiation levels in the superstructures are not known. They may be significantly higher than estimated.

\section{CONSEQUENCES IF RISK NOT RESOLVED}

More workers will be needed during the construction phase of th project. This will negatively impact the schedule and costs during construction. There is a high confidence that the radiatio levels can be maintained at lower levels during the operations phase of the project.

\section{PROBABILITY}

High (3)

- Medium (2)

$\bigcirc \operatorname{Low}(1)$

\section{Risk $=4$}

Risk $=$ Probability $\times$ Impact

\section{Probability Definition}

High - Likely to occur during the projed.

Medium - Has the potential to occur during the project.

Low - Has fittle potential to occur during the project.

\section{Impact Definition}

High - Likely to cause significant disruption of schedule, increase in cost, or degradation of performance.

Medium - Has the potential to cause some disutpion to schedule, increase in cost, or degradation of performance.

Low - Has little potential to cause disnuption to schedule, increase in cost, or degradation of performance.

\section{CONTINGENCY PLAN(S) IF RISK IS REALIZED:}

More workers and time will b e necessary during the constructio phase of the project.

\section{TRIGGER POINT(S) FOR EARLY RISK}

\section{IDENTIFICATION:}

This risk should be addressed during the conceptual design phase. If not, it well become evident during construction. 
E-212 
431.02\#

$02 / 37 / 98$

Rev. $\# 00$
ENGINEERING DESIGN FILE

Appendix F

Cost Estimates
Function File Number - SPR-WTS-01

EDF Serial Number - EDF-WTS-002

Page 62 of 62 


\begin{tabular}{|c|c|c|c|c|c|c|c|c|c|c|c|c|c|c|c|}
\hline ID & Task Name & 2003 & 2004 & 2005 & 2006 & 2007 & 2008 & 2009 & 2010 & 2011 & 2012 & 2013 & 2014 & 2015 & 2016 \\
\hline 1 & CALCINE TRANSPORT SYSTEM & & & & & & & & & & & & & & \\
\hline 2 & CONCEPTUAL DESIGN & & & & & & & & & & & & & & \\
\hline 3 & ADVANCED CONCEPTUAL DESIGN & & & & & & & & & & & & & & \\
\hline 4 & PM FOR PROJECT DEVELOPMENT & & & & & & & & & & & & & & \\
\hline 5 & PERMITTING & $6 / 2$ & & & & & & & & & & & & & \\
\hline 6 & PROJECT MANAGEMENT & & & & & & & & & & & & & & \\
\hline 7 & TITLE DESIGN & & & $12 / 2$ & 8 & & $712 / 2$ & & & & & & & & \\
\hline 8 & CONSTRUCTION & & & & & & & & & & & & & & \\
\hline 9 & CORROSION COUPON REMOVAL & & & & & & & & & & & & 12 & & \\
\hline 10 & D\&D RISER INSTALLATION & & & & & & & & & & & 12 & & & \\
\hline 11 & CALCINE REMOVAL OPERATIONS & & & & & & & & & & & & 4 & 34 & 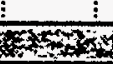 \\
\hline
\end{tabular}




\begin{tabular}{|c|c|c|c|c|c|c|c|c|c|c|c|c|c|c|}
\hline ID & Task Name & 2003 & 2004 & 2005 & 2006 & 2007 & 2008 & 2009 & 2010 & 2011 & 2012 & 2013 & 2014 & 2015 \\
\hline 1 & CALCINE TRANSPORT SYSTEM & & & & & & & & & & & & & \\
\hline 2 & CONCEPTUAL DESIGN & & $2 / 3$ & & & & & & & & & & & \\
\hline 3 & ADVANCED CONCEPTUAL DESIGN & $12 / 3$ & & & & & & & & & & & & \\
\hline$\overline{4}$ & PM FOR PROJECT DEVELOPMENT & & & & & & & & & & & & & \\
\hline 5 & PERMITTING & $6 / 2$ & Deses & 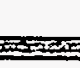 & 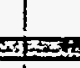 & تحيس & & & & & & & & \\
\hline 6 & PROJECT MANAGEMENT & & & 12 & & & & & & & & & & \\
\hline 7 & TITLE DESIGN & & & $12 / 2$ & & & $12 / 25$ & & & & & & & \\
\hline 8 & CONSTRUCTION & & & & & & & & & & & & & \\
\hline 9 & CORROSION COUPON REMOVAL & & & & & 12 & & & & & & & & \\
\hline 10 & D\&D RISER.INSTALLATION & & & & & & & & & & & & & \\
\hline 11 & CALCINE REMOVAL OPERATIONS & & & & & & & & & & & & & \\
\hline 12 & BIN SET 1 & & & & & & & & & & & & & \\
\hline 13 & BIN SET 5 & & & & & & & & & & & & & \\
\hline 14 & BIN SET 6 & & & & & & & & & & & & & \\
\hline 15 & BIN SET 7 & & & & & & & & & & & & & \\
\hline 16 & BIN SET 2 & & & & & & & & & & & & & \\
\hline 17 & BIN SET 3 & & & & & & & & & & & & & \\
\hline 18 & BIN SET 4 & & & & & & & & & & & & & \\
\hline
\end{tabular}

This schedule represents calcine retrieval and transportation activities for the DCWO, HWO, VWO and TRU-Separations Options. Activities 12-18 represent retrieval of calcine from a specific CSSF. The CSSFs retrieval order has not been determined. The order must be coordinated between CSSF closure and the Waste Treatment Facility needs. All CSSFs will be prepared for retrieval by 1/1/2013. 


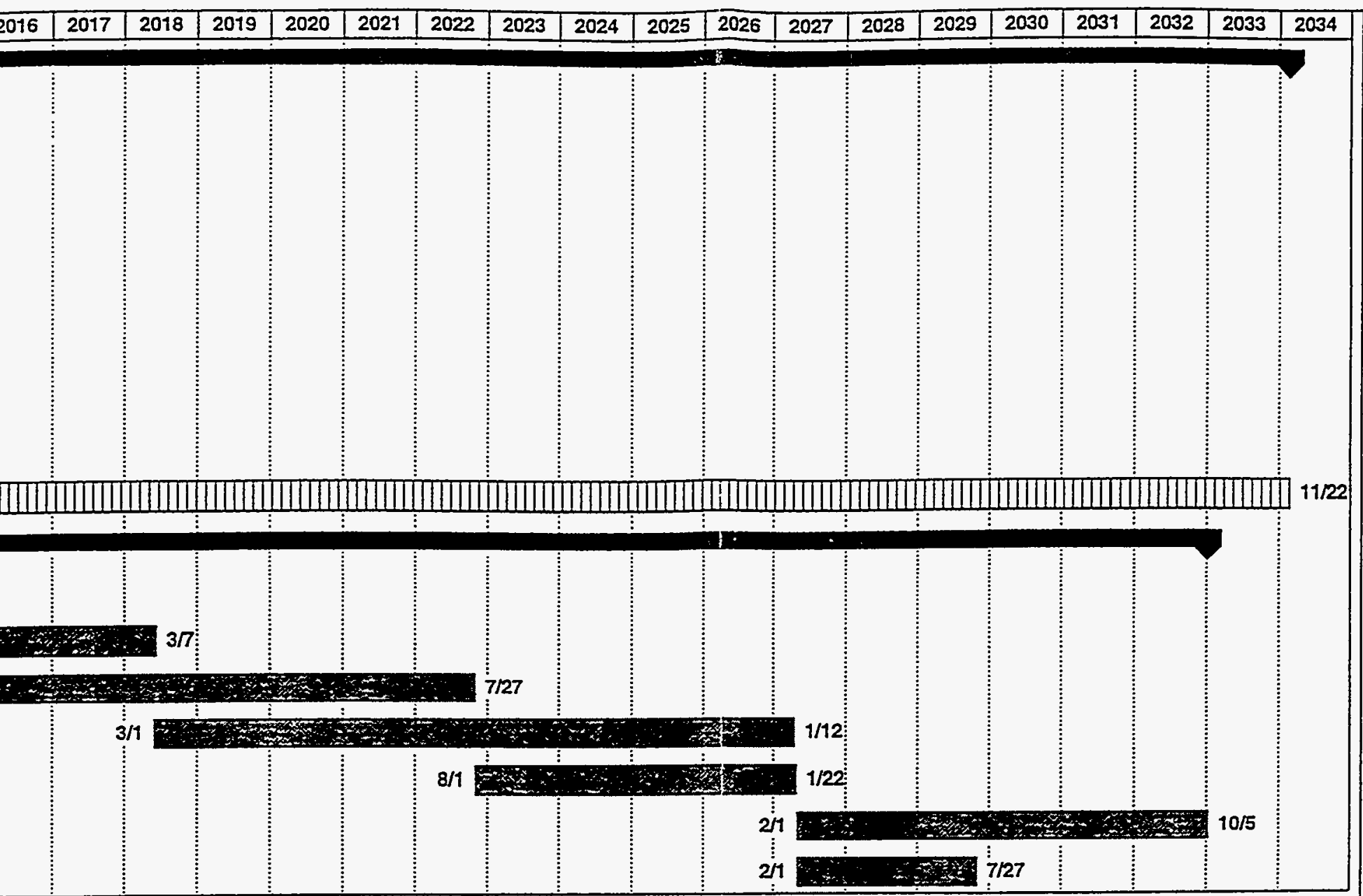


Date: January 24,1998

To:

S. E. Gifford

MS 3765

$6-5162$

From:

F. P. Hanson

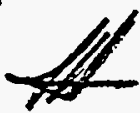

MS 3655

$6-0548$

Subject: HLH EIS WASTE TREATMENT SCOPING STUDIES - FPH-01-98

Reference: F. P. Hanson letter to S. E. Gifford, FPH-33-97, Same Subject, December 9, 1997

Cost Estimating has reviewed the referenced draft cost estimates and prepared the attached final Planning Cost Estimates for the subject project. Nine estimates have been prepared for the activities shown below; the work scope for each activity is described in the respective estimate.

1. Other Project Costs (OPC) including conceptual design, proof of process, permitting and documentation, start-up activities, and related project management costs.

2. One estimate each for Option B for Calcined Solids Storage Facilities (CSSFs) 1 through 4, and one estimate for CSSFs 5 through 7. Since the scope and nature of work for CSSFs 5 through 7 are nearly identical, one estimate has been prepared showing costs which may be applied to each of those CSSFs.

3. Installation of D\&D risers

4. Removal of corrosion coupons

5. One estimate each for Option A and Option B for the Calcine Transport System. Option B incorporates an Intermediate Transport Station with associated equipment.

The general scope of work covered by the estimates includes necessary modification of the CSSF vaults and CSSFs; construction of a new Containment Enclosure and a new Ventilation, Instrumentation, and Control (VIC) Building at each CSSF; construction of a new calcine transport system; and removal of corrosion coupons from the CSSFs.

The estimates incorporate all comments received to date, including your review of the draft estimates dated December 9, 1997 and intermediate draft estimates for the Calcine Transport System, as well as appropriate internal reviews. In addition, G\&A and Performance Incentive Factor (PIF) fees, not previously applied, have been added to the estimates as appropriate. 
S. E. Gifford

January 24,1998

FPH-01-98

Page 2

Option A for modification of the CSSFs incorporated relocation and reuse of certain operational equipment. An examination of this option showed that it did not fully meet the needs of the system and was not sufficiently cost effective to warrant further consideration; therefore, in accordance with your directive, estimates are provided for Option B only.

Assumptions which form the bases of the estimates and any concerns that may affect the costs are shown in the attached Support Data Recapitulation Sheets. Cost Estimate Summary Sheets, Detail Sheets, and Contingency Analysis Sheets for each estimate are also attached for your information. These describe in detai1 the scope of work and unit costs upon which the estimates are based. In addition, a Summary of Cost Estimates has been prepared showing the Total Project cost for each estimate. If you have further comments or questions regarding the estimates, please feel free to call.

fph

Áttachment

CC: Estimate File \#2414-1 F. P. Hanson File

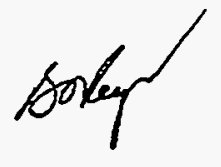


Lockheed Martin Idaho Technologies Co.

(Rev. 06/96)

\section{COST ESTIMATE SUPPORT DATA RECAPITULATIOH}

Project TitTe: HLW EIS Haste Treatment Scoping Studies

Calcine Retrieval and Transport

Type of Estimate: Planning

File No: $2414-1$
Estimator: F. P. Hanson Date: $1-24-98$

Approved By:

I. SCOPE OF WORK: Brief description of the proposed project.

This project will prepare Calcined Solids Storage Facilities (CSSFs) through $\# 7$ for retrieval and transport of calcined waste to a processing facility. Each CSSF vauit and CSSF will be modified as required, a new Containment Enclosure, and a new Ventilation, Instrumentation, and Control (VIC) Building will be constructed at each CSSF, and a new calcine transport system will be constructed. Construction of treatment and process facilities are not in the scope of the attached estimates.

\section{BASIS OF THE ESTIMATE: Drawings, Design Report, Engineers notes, and/or other documentation upon which the estimate is originated.}

a. Cost Estimate for Alternative 3, Phase I \& II Special Studies, Bin Set 1. Bin Set 1 Calcine Retrieval System, Raytheon Engineers and Constructors, Inc:, 11-8-94.

b. Feasibility Study Cost Estimate, Haste Treatment Facilities, Fluor Danie] Northwest, 10-22-97.

c. Draft Bin Set Access Plan with accompanying tables, provided by S. E. Gifford, cognizant technical lead.

d. Drawings, sketches, and miscellaneous supporting information provided by $S$. E. Gifford.

e. Title II Cost Estimate, Waste Characterization Facility, Lockheed Idaho Technologies Co., 11-22-94. This estimate provides the basis for costs for. the Calcine Transport System Intermediate Transport Station. Costs are updated to current costs. 
Lockheed Martin Idaho Technologies Co.

\section{COST ESTIMATE SUPPORT DATA RECAPITULATION \\ (CONTINUATION)}

File No: $2414-1$

Page 2 of 6

III. ASSUMPTIONS: Condition statements accepted or supposed true without proof or demonstration. An assumption has a direct impact on total estimated cost.

1. The Raytheon estimate provides a comprehensive breakdown of work tasks and associated costs for work required to prepare CSSF " I. The Fluor Daniel estimate addresses CSSFs $\# 2$ and $\# 3$, and closely reflects the Raytheon estimate, with no further scope or significant cost development. Because the Raytheon estimate represents the primary scope and cost development, it has been used as the basis of costs and scope of work addressed by the attached estimates. Work scopes and associated labor and material costs have been adjusted as deemed appropriate for subsequent CSSFs.

2. It has been assumed that the Raytheon estimate was developed from a reasonably well developed description of work scope, therefore, quantities, unit costs, and labor effort have been incorporated as presented in the Raytheon estimate, except as otherwise determined by factors specific to this estimate effort. The costs provided in the attached estimates are dependent upon the underlying assumptions, inclusions, exclusions, and basis of quantity development and pricing for the Raytheon estimate.

3. Design, Title III Inspection, and Management costs were applied in the Raytheon estimate as a percentage of construction costs. Those rates have been modified for the attached estimates as follow: Title Design at $20 \%$ of construction and GFE; Title III at 5\% of construction and GFE; and PM and $C M$ each at $10 \%$ of construction and GFE. Title design modifications for CSSF $\# 2$ through $\# 7$ have been assumed at $20 \%$ of Tit7e design costs for CSSF \#1, assuming only relatively minor design modifications would be required.

4. Conceptual design and process development costs have been assumed at $30 \%$ of Title design costs for CSSF $\# 1$. It is assumed that conceptual design costs would not be impacted by subsequent Title design modifications for the remaining CSSFs. See Section IV for further comments.

6. It has been assumed that removal of corrosion coupons will be accomplished by LMITCO operating personnel.

(Continued) 
Lockheed Martin Idaho Technologies Co.

\section{COST ESTIMATE SUPPORT DATA RECAPITULATION.}

(CONTINUATION)

File No: $2414-1$

Page 3 of 6

\section{ASSUMPTIONS: (Continued)}

5. It is assumed that all demolition and new construction work will be competitively bid and performed by a general contractor as the prime subcontractor, with specialty lower tier subcontractors as appropriate. One tier of subcontractor markups has been applied, at $40 \%$ for overhead and profit, plus $1 \%$ for bonding, in accordance with the Raytheon estimate.

7. The Raytheon estimate does not specifically identify costs for subcontractor supervision. It has been assumed that the labor figures incorporated include allowances for subcontractor project coordination and supervision. Costs for personnel $O S \& H$ and site specific training have been included at $2 \%$ of project labor, per the Raytheon estimate, for CSSF $\# 1$, and at $1 \%$ of labor for the remaining CSSFs, assuming primarily refresher and update training for subsequent CSSFs. . Construction of the Calcine Transport System and installation of the D\&D risers are assumed to require additional personnel, therefor, the $2 \%$ allowance for training has been applied to those estimates.

8. Unit costs are assumed to include all costs necessary to accomplish the work including, but not necessarily limited to, site preparation; installation and removal of waste-products, decontamination and cleanup, mobilization and demobilization, and cost of supporting organizations.

9. The labor hours shown in the Raytheon estimate have been incorporated; however, current INEEL Site Stabilization. Agreement rates have been used, which may differ from the rates shown in the reference.

10. Allowances for undefined costs and for NQA-1 have been applied as shown in the Raytheon estimate, and are assumed to be appropriate for the project.

11. Material costs have been applied as shown in the Raytheon estimate, as appropriate, and further escalated at approximately $1.5 \%$ per year from the date of the estimate to the current date.

12. The Raytheon estimate does not include costs for the Containment Enclosure or the VIC Building. The attached estimates assume both the structures to be pre-erigineered metal buildings, complete with appropriate services nd equipment. The Containment Enclosure is assumed to be $30^{\prime} \times 30^{\prime}$ or $40^{\prime} \times$ $40^{\prime}$, depending upon the vault dimension, with a $60^{\prime}$ eave height. The VIC buitding is assumed to be $40^{\prime} \times 60^{\prime} \times 14^{\prime}$ eave height in all cases. Costs -are based on historical data and estimating judgement.

(Continued) 
Lockheed Martin ldaho Technologies Co.

\section{COST ESTIMATE SUPPORT DATA RECAPITULATION (CONTINUATION)}

File No: $2414-1$

Page 4 of 6

III. ASSUMPTIONS: (Continued)

13. It is assumed that minimal site preparation will be required. Costs have not been included for additional service and access roads.

14. The following equipment is associated with the Calcine Transport System and is to be located in the processing facility; costs are assumed to be addressed in the appropriate facility cost estimate and have not been included herein. Equipment numbers are identified in appropriate drawings and equipment 1 ists.

- Air Handiing Unit, 2,130 CFM

- Exhaust Fan, 830 CFM

- Exhaust. Fan, 230 CFM

- HEPA Filter w/Prefilter, 530 CFM

- HEPA Canisters, w/Prefilters, 230 CFM

15. The cost estimate for Other Project costs (OPC) is intended to show all permitting costs associated with the entire CSSF modification and. construction of the transport system. Costs for permitting and documentation are assumed to encompass all activities required.

16. The estimates identify certain activities related to demolition, earthwork, and construction which are to be accomplished during the overall time frame shown, rather than showing labor hours for the specific activities. It is assumed that the time frames and labor loading shown are adequate to accomplish the activities.

17. The proposed schedule shown in the Raytheon estimate calls for engineering from 10/1997 to 10/1999, with a mid-pint of 10/1998, and all other activities from $12 / 2000$ to $5 / 2005$, with a mid-point of $11 / 2002$. Using the schedule of operations provided, the following activity mid-points have been established for purposes of calculating escalation:

- Conceptual design: 2003 - 2005, mid-point 2004.

- Tit7e design: 2005 - 2007, mid-point 2006.

- Construction: 2008 - 2013, mid-point 2011.

(Continued) 
Lockheed Martin Idaho Technologies Co.

\section{COST ESTIMATE SUPPORT DATA RECAPITULATION (CONTINUATION)}

File No: $2414-1$

Page 5 of 6

III. ASSUMPTIONS: (Continued)

- Installation of D\&D risers: Mid-point of operations (2014 - 2033), 2023

- Removal of corrosion coupons: Mid-point of construction, 2011

19. It is assumed that the Calcine Transport System wi11 consist of two, separate systems. The quantities shown in the estimate reflect total quantities for the two systems.

21. It is assumed that the Intermediate Transport Station for Option B will be of reinforced concrete construction housing process equipment, with a preengineered metal building portion housing utilities and support equipment, and that the facility will require normal features for equipment shielding and accessibility, personnel protection, and normal utilities, HVAC, and electrical services.

20. Information available to the cognizant technical lead indicates that, due to corrosion failure of piping in CSSFs 2 and 3 , radiological conditions

arising from contamination are similar-to CSSF $\frac{1}{\pi} 1$. The estimates for CSSFs 2 and 3 have been prepared with that factor in mind, and Tabor allowances incorporated accordingty.

22. LMITCO G\&A has been applied at $23 \%$ of construction and GFE, with a $\$ 500,000$ ceiling applied, and the Performance Incentive Fee (PIF) has been applied at $5.5 \%$. Procurement fees have been assumed at $1 \%$, as procurement support to DOE, rather than the normal $3 \%$ fee. Adders are applied to each estimate based on the assumption the work addressed therein will be accomplished in one year. 
Lockheed Martin Idaho Technologies Co.

\section{COST ESTIMATE SUPPORT DATA RECAPITULATION \\ (CONTINUATION)}

File No: $2414-1$

Page 6 of 6

IV. CONTINGENCY GUIDELINE IMPLEMENTATION: The percentage used for contingency as determined by the contingency allowance guidelines can be altered to reflect the type of construction and conditions that may impact the total estimated cost.

Time constraints and lack of definitive design and project requirements have been taken into consideration in generating the costs and attendant rates of contingency shown in the estimate. Very little is known at this time regarding specific facility and process requirements, and there is a very real potential for encountering radiological conditions beyond those anticipated, resulting in increased labor and equipment costs. This, together with the unknowns associated with subsurface work and radiological. conditions, with the inherent possibility of encountering differing conditions as existing work is opened up, create a high potential for increased costs. Some equipment may not be standard and may require development, and the scheduTe extends over many years. For these reasons, the rate of contingency typically exceeds the guidelines for a planning estimate and extends into the range for special conditions, as defined by DOE/FM 50, Cost Estimating Guide, VoT. 6, and the INEEL Cost Estimating Guide.

Overall contingency rates for escalated estimates are slightly higher than unescalated estimates, due to the assumed higher degree of associated risk as activities and costs are extended into the out years.

\section{OTHER COMMENTS/CONCERNS SPECIFIC TO THE ESTIMATE:}

Individual estimates have been prepared for other Project Costs (OPC); two options for the Calcine Transport System; and each of CSSFs 1 through 4. Since the scope and nature of work for CSSFs 5 through 7 are nearly identical, one estimate has been prepared showing costs which may be applied to each of those CSSFs. After analyzing draft cost estimates for Option A and Option B, for CSSF modifications, it was determined by the cognizant technical lead that Option A did not sufficientiy meet system requirements and would not be further pursued; therefore, complete estimates are provided only for option B for each case, and Summary Sheets are provided for both escalated and unescalated costs for each estimate. Separate estimates have also been prepared for installing D\&D risers and for removing corrosion coupons.

The allowance of $30 \%$ of construction for Title Design included in the Raytheon estimate appears excessive for this magnitude of work. It was determined by the cognizant technical lead that this allowance was based on the total lack of a.proven retrieval method at the time the Raytheon estimate was prepared. Because of subsequent design development, the allowance is reduced to $20 \%$ in the attached estimates. 
Lockheed Martin Idaho Technologies Co.

\section{SUMMARY OF COST ESTIMATES}

\begin{tabular}{|c|c|c|}
\hline $\begin{array}{l}\text { HLW EIS WASTE TREATMENT } \\
\text { SCOPING STUDIES } \\
\text { CALCINE RETRIEVAL AND TRANSPORT } \\
\text { LOCATION: INEEL-ICPP } \\
\text { REQUESTOR: KL WILLLAMS }\end{array}$ & $\begin{array}{l}\text { PLANNING ESTIMATE } \\
\text { ESTIMATE NO. } 24141 \\
\text { PREPARED BY: F.P. HANSON }\end{array}$ & $\begin{array}{l}\text { DATE: 24 JAN } 1998 \\
\text { CHECKED } \\
\text { APPROVED } \\
\end{array}$ \\
\hline \multirow{2}{*}{ ACTIVITY } & \multicolumn{2}{|c|}{ OPTION B } \\
\hline & $\begin{array}{l}\text { UNESCALATED } \\
\text { COSTS }\end{array}$ & $\begin{array}{l}\text { ESCALATED } \\
\text { COSTS }\end{array}$ \\
\hline OTHE்R PROJECT COSTS (OPC) & $\$ 28,300,000$ & $\$ 41,100,000$ \\
\hline CSSF \#1 & $\$ 37,100,000$ & $\$ 52,750,000$ \\
\hline CSSF \#2 & $\$ 24,000,000$ & $\$ 34,000,000$ \\
\hline CSSF \#3 & $\$ 23,400,000$ & $\$ 33,100,000$ \\
\hline CSSF \#4 & $\$ 16,000,000$ & $\$ 22,500,000$ \\
\hline CSSF \#5 & $\$ 14,700,000$ & $\$ 21,000,000$ \\
\hline CSSF \#6 & $\$ 14,700,000$ & $\$ 21,000,000$ \\
\hline CSSF \#7 & $\$ 14,700,000$ & $\$ 21,000,000$ \\
\hline INSTALL D\&D RISẸRS & $\$ 5,100,000$ & $\$ 10,300,000$ \\
\hline REMOVE CORROSION COUPONS & $\$ 1,125,000$ & $\$ 1,620,000$ \\
\hline $\begin{array}{l}\text { TOTAL PROJECT COST } \\
\text { (LESS TRANSPORT SYSTEM) }\end{array}$ & $\$ 179,125,000$ & $\$ 258,370,000$ \\
\hline CALCINE TRANSPORT SYSTEM OPTIONS & & \\
\hline CWO \& TRU SEPARATION OPTIONS (A) & $\$ 15,300,000$ & $\$ 21,650,000$ \\
\hline HWO, WWO, DCWO OPTIONS (B) & $\$ 20,400,000$ & $\$ 29,000,000$ \\
\hline
\end{tabular}


Lockheed Martin Idaho Technologies Co.

COST ESTIMATE SUMMARY

PROIECT NAME: HLW EIS -WASTE TREATMENT SCOPING STUDIES TYPEOFESTIMATE: PLANNING

LOCATION 1: CALCINE RETRIEVAL AND TRANSPORT

INEEL - ICPP

PROSECT NO:

PAEPAAED BY:

2414-1 (OPC)

REQUESTOR:

K. L WILLIAMS

REPOFT NAME

F. P. HANSON

Cost Estimate Summary

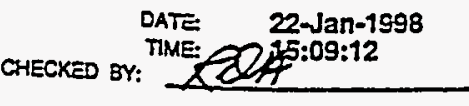

APPRO BY:

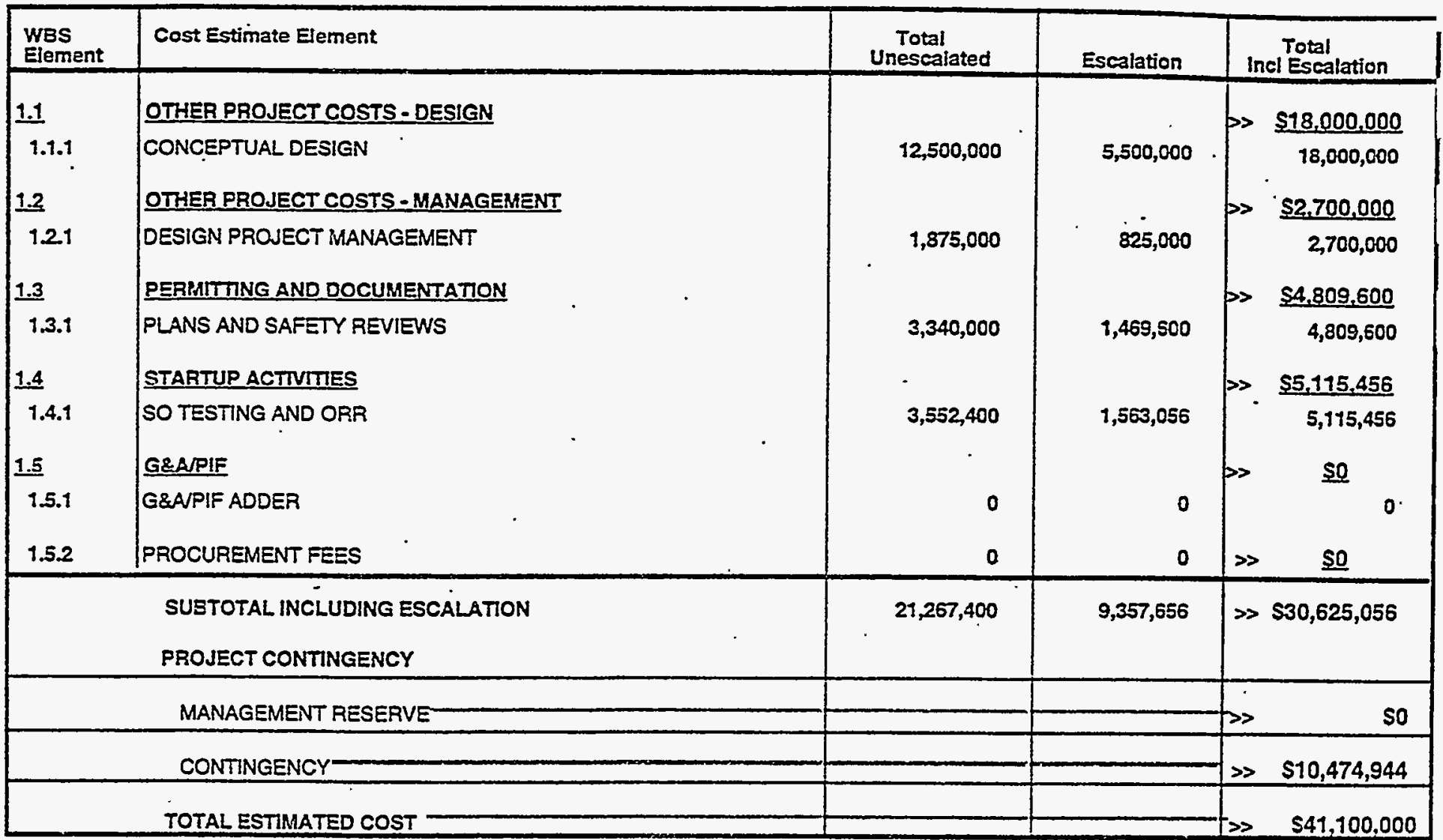

\section{PROJECT COST PARAMETERS}

EDI AS A \% OF CONST, + GFE= $\quad \cdots \%$ 
Lockheed Martin Idaho Technologies Co.

PROJECT NAME: HLW EIS -WASTE TREATMENT SCOPING STUDIES TYPEOFESTIMATE:

LOCATION 1: ' CALCINE REIRIEVAL AND TRANSPORT

REQUESTOR: K.L. WILLAMS
COST ESTIMATE SUMMARY

PROFETIMATE

PREPARED BY: REPORT NAME:
PLANNING

2414-1 (OPC)

F. P. HANSON

Cost Estimate Summary

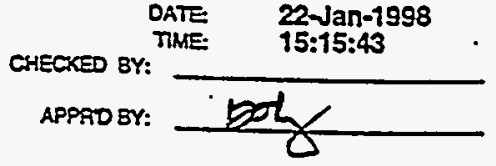

\begin{tabular}{|c|c|c|c|c|}
\hline $\begin{array}{l}\text { WBS } \\
\text { Element }\end{array}$ & Cost Estimate Element & $\begin{array}{c}\text { Total } \\
\text { Unescalated }\end{array}$ & Escalation & $\begin{array}{l}\text { Total } \\
\text { Inel Escalation }\end{array}$ \\
\hline $\begin{array}{l}1.1 \\
1.1 .1 \\
\frac{1.2}{1.2 .1} \\
\frac{1.3}{1.3 .1} \\
1.4 \\
1.4 .1 \\
1.5 \\
1.5 .1 \\
1.5 .2\end{array}$ & $\begin{array}{l}\text { OTHER PROJECT COSTS-DESIGN } \\
\text { CONCEPTUAL DESIGN } \\
\text { OTHER PROJECT COSTS-MANAGEMENT } \\
\text { DESIGN PROJECT MANAGEMENT } \\
\text { PERMITING AND DOCUMENTATION } \\
\text { PLANS AND SAFETY REVIEWS } \\
\text { STARTUP ACTIVITIES } \\
\text { SO TESTING AND ORR } \\
\text { G\&AMPIF . } \\
\text { G\&APIF ADDER } \\
\text { PROCUREMENT FEES }\end{array}$ & $\begin{array}{c}12,500,000 \\
1,875,000 \\
3,340,000 \\
3,552,400\end{array}$ & $\begin{array}{l}0 \\
0 \\
0 \\
0 \\
0\end{array}$ & 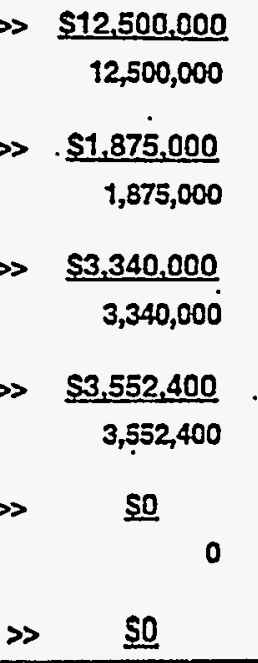 \\
\hline & $\begin{array}{l}\text { SUBTOTALINCLUDING ESTCALATION } \\
\text { PROJECT CONTINGENCY }\end{array}$ & $21,267,400$ & 0 & $\gg \$ 21,267,400$ \\
\hline & MANAGEMENT RESERVE & & & so \\
\hline & CONTINGENCY & & & $\$ 7,032,600$ \\
\hline & TOTAL ESTIMATED COST & & & $\gg \quad \$ 28,300,000$ \\
\hline
\end{tabular}

PROJECT COST PARAMETERS

EDI AS A \% OF CONST. + GFE= $\cdots$ 
Lockheed Martin Idaho Technologies Co. E: HLW EIS -WASTE TREATMENT SCOPING STUDIES TYPE OF ESTMATE: CALCINE REIRIEVAL AND TRANSPORT - OPTION 3 PROSECT NO: INEEL - ICPP

LOCATON I:

K.L. WILLIAMS

PREPARED BY:

REPORT NAME:

2414-1-1 (CSSF 1)

F. P. HANSON

Cost Estimate Summary

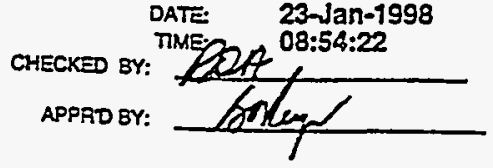

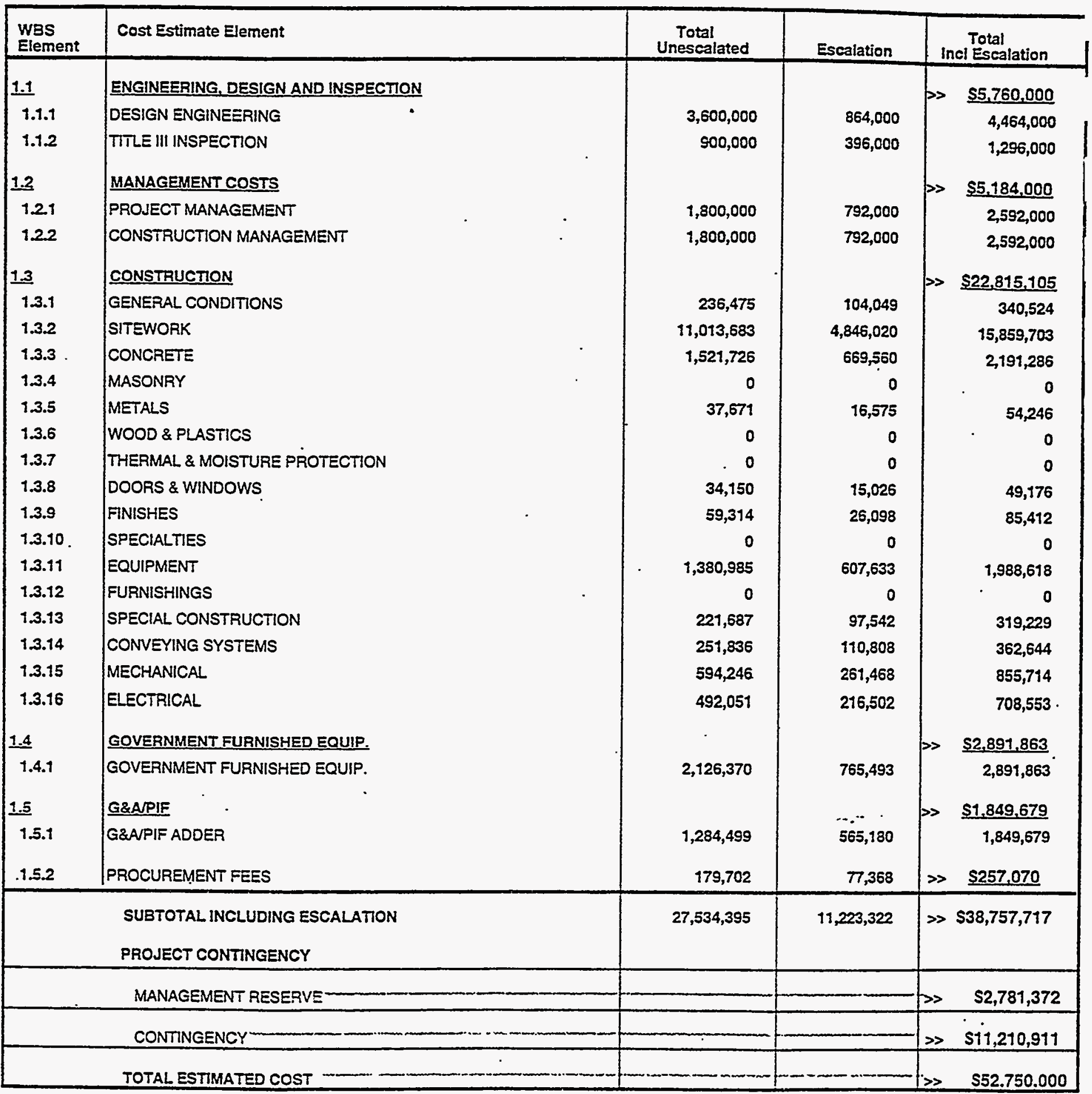

\section{PROJECT COST PARAMETERS}

EDI AS A $\%$ OF CONST. + GFE $=22.00 \%$ 

Lockheed Martin Idaho Technologies Co. PROLECT NAME:

LOCATION 1: REOUESTOR: HLW EIS -WASTE TREATMENT SCO INEEL - ICPP

K.L. WILLAAMS
COST ESTIMATE SUMMARY

PLANNING

2414-1-1 (CSSF 1)

F. P. HANSON

PRCUECT NO:

REPCRT NAME:

Cost Estimate Summary

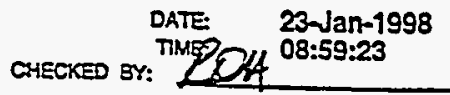

APPRD BY:

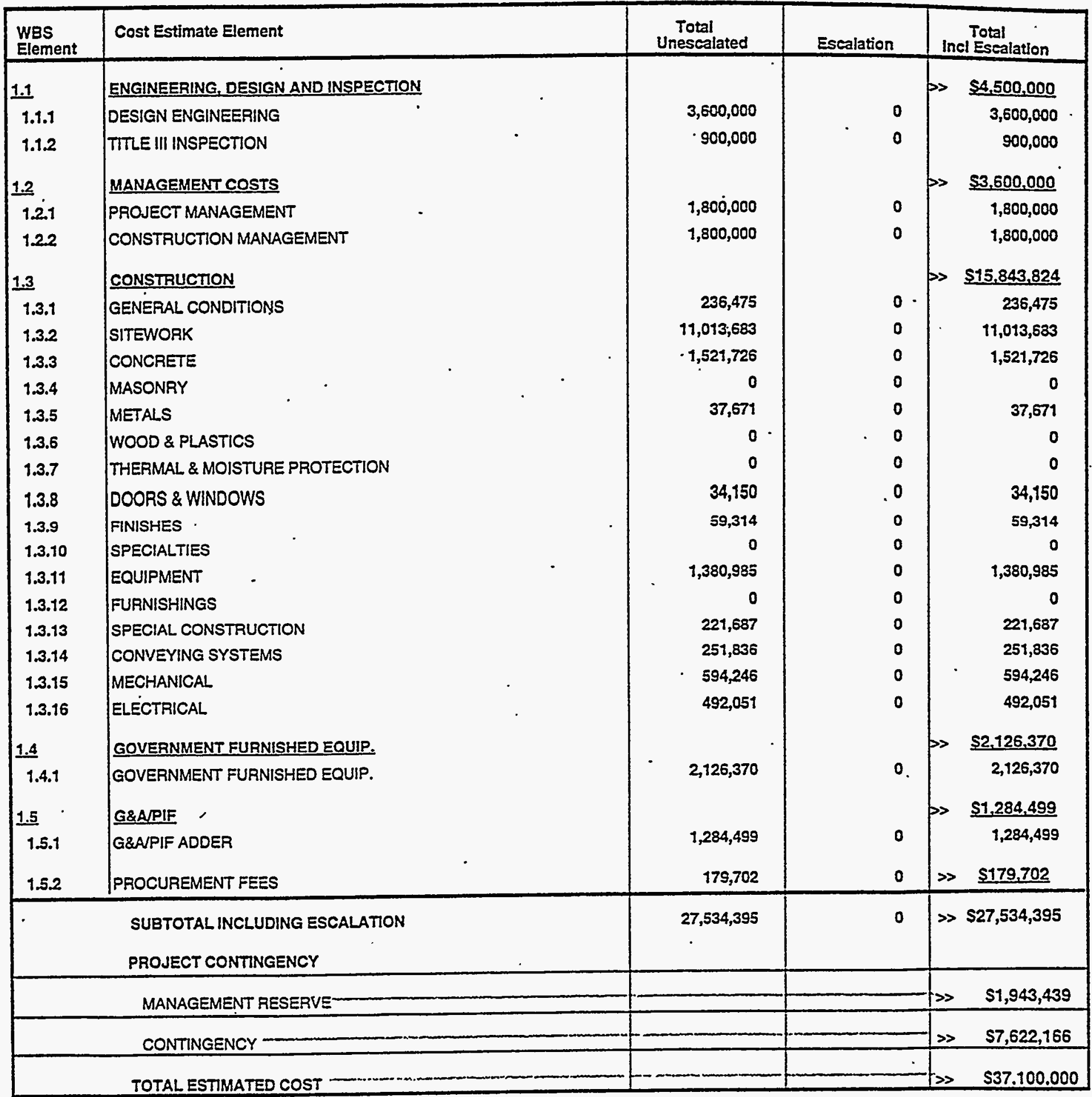

PROJECT COST PARAMETERS

EDI AS A $\%$ OF CONST. + GFE $=25.00 \%$

CONTINGENCY $=34.74 \%$ 
Lockheed Martin Idaho Technologies Co. PROUECT NAME

LCCATION : REQUESTOR:
COST ESTIMATE SUMMARY

PLANNING

2414-1-2 (CSSF 2)

PREPARED BY: F. P. HANSON
REPORT NAME: Cost Estimate Summary
HLW EIS -WASTE TREATMENT SCOPING STUDIES
CALCINE REITIEVAL AND TRANSPORT-OPTION
INEEL-ICPO
K.L WILLAMS

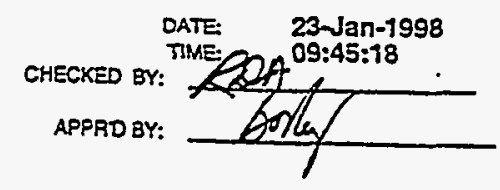

\begin{tabular}{|c|c|c|c|c|}
\hline $\begin{array}{l}\text { WBS } \\
\text { Element }\end{array}$ & Cost Estimate Element & $\begin{array}{c}\text { Total } \\
\text { Unescalated }\end{array}$ & Escalation & $\begin{array}{c}\text { Total } \\
\text { Incl Essalation }\end{array}$ \\
\hline 1.1 & ENGINEERING, DESIGN AND INSPECTION & & & $\$ 1,972,800$ \\
\hline 1.1 .1 & DESIGN ENGINEERING & 720,000 & 316,800 & $1,036,800$ \\
\hline 1.1 .2 & TITLE III INSPECTION & 650,000 & 286,000 & 936,000 \\
\hline 12 & MANAGEMENT COSTS & & & $\$ 3.111,000$ \\
\hline 1.2 .1 & PROJECT MANAGEMENT & $1,275,000$ & 0 & $1,275,000$ \\
\hline 1.2 .2 & CONSTRUCTION MANAGEMENT & $1,275,000$ & 561,000 & $1,836,000$ \\
\hline 1.3 & CONSTRUCTION & & & $\$ 15,903,115$ \\
\hline 1.3.1 & GENERAL CONDITIONS & 79,168 & 34,834 & 114,002 \\
\hline 1.3 .2 & SITEWORK & $6,912,013$ & $3,041,286$ & $9,953,299$ \\
\hline 1.3 .3 & CONCRETE & $1,020,501$ & 449,020 & $1,469,521$ \\
\hline 1.3 .4 & MASONRY & 0 & 0 & 0 \\
\hline 1.3 .5 & METALS & 37,671 & 16,575 & 54,246 \\
\hline 1.3 .6 & WOOD \& PLASTICS & 0 & $\therefore 0$ & 0 \\
\hline 1.3 .7 & THERMAL \& MOISTURE PROTECTION & 0 & 0 & 0 \\
\hline $\mathbf{1 . 3 . 8}$ & DOORS \& WINDOWS & 34,150 & 15,026 & 49,176 \\
\hline 1.3 .9 & FINISHES & 64,411 & 28,341 & 92,752 \\
\hline 1.3 .10 & SPECIALTIES & 0 & 0 & 0 \\
\hline 1.3 .11 & EQUIPMENT & $1,199,716$ & 527,875 & $1,727,591$ \\
\hline 1.3.12 & FURNISHINGS & 0 & 0 & 0 \\
\hline 1.3.13 & SPECIAL CONSTRUCTION & 291,171 & 128,115 & 419,286 \\
\hline 1.3 .14 & CONVEYING SYSTEMS & 278,560 & 122,557 & 401,127 \\
\hline 1.3.15 & MECHANICAL & 596,103 & 262,285 & 858,388 \\
\hline 1.3 .16 & ELECTRICAL & 530,366 & 233,361 & 763,727 \\
\hline 1.4 & GOVERNMENT FURNISHED EQUIP. & & & $\gg \quad \$ 2,290,530$ \\
\hline 1.4 .1 & GOVERNMENT FURNISHED EQUIP. & $1,684,213$ & 606,317 & $2,290,530$ \\
\hline 1.5 & GEAPIF & & & $\gg \quad \$ 1,412,031$ \\
\hline 1.5 .1 & G\&AVIF ADDER & 980,577 & 431,454 & $1,412,031$ \\
\hline \multirow[t]{4}{*}{1.5 .2} & PROCUREMENT FEES & & 54,656 & $\gg \quad \$ 181,936$ \\
\hline & $\begin{array}{l}\text { SUBTOTAL INCLUDING ESCALATION } \\
\text { PROJECT CONTINGENCY }\end{array}$ & $17,755,900$ & $7,115,512$ & >> $\$ 24,871,412$ \\
\hline & MANAGEMENT RESERVE- & & & $\$ 1,978,761$ \\
\hline & CONTINGENCY - & & & $57,149,827$ \\
\hline & TOTAL ESTIMATED COST - & & & $\gg \quad 534.000 .000$ \\
\hline
\end{tabular}

\section{PROJECT COST PARAMETERS}

EDI AS A $\%$ OF CONST. + GFE $=11.00 \%$

CONTINGENCY $=36.70 \%$ 
Lockheed Martin Idaho Technologies Co. Rev. Es6 PROSECT

LOCATION 1:

REQUESTOR:
HIW EIS -WASTE TREATMENT SCOPING STUDIES TYPE CF ESTMATE CALCINE RETRITVALAND TRANSPORT - OPTION 8 PROEETNO

INEEL - ICPP

K. L. WILLIAMS
COST ESTIMATE SUMMARY

PRONECTNOE: PLANNING

PREPARED BY: F.P. 1-2 (CSSF

REPORT NAME: Cost Estimate Summary

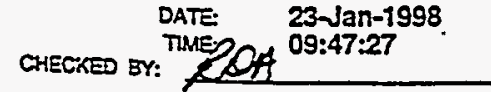

APPRD BY.

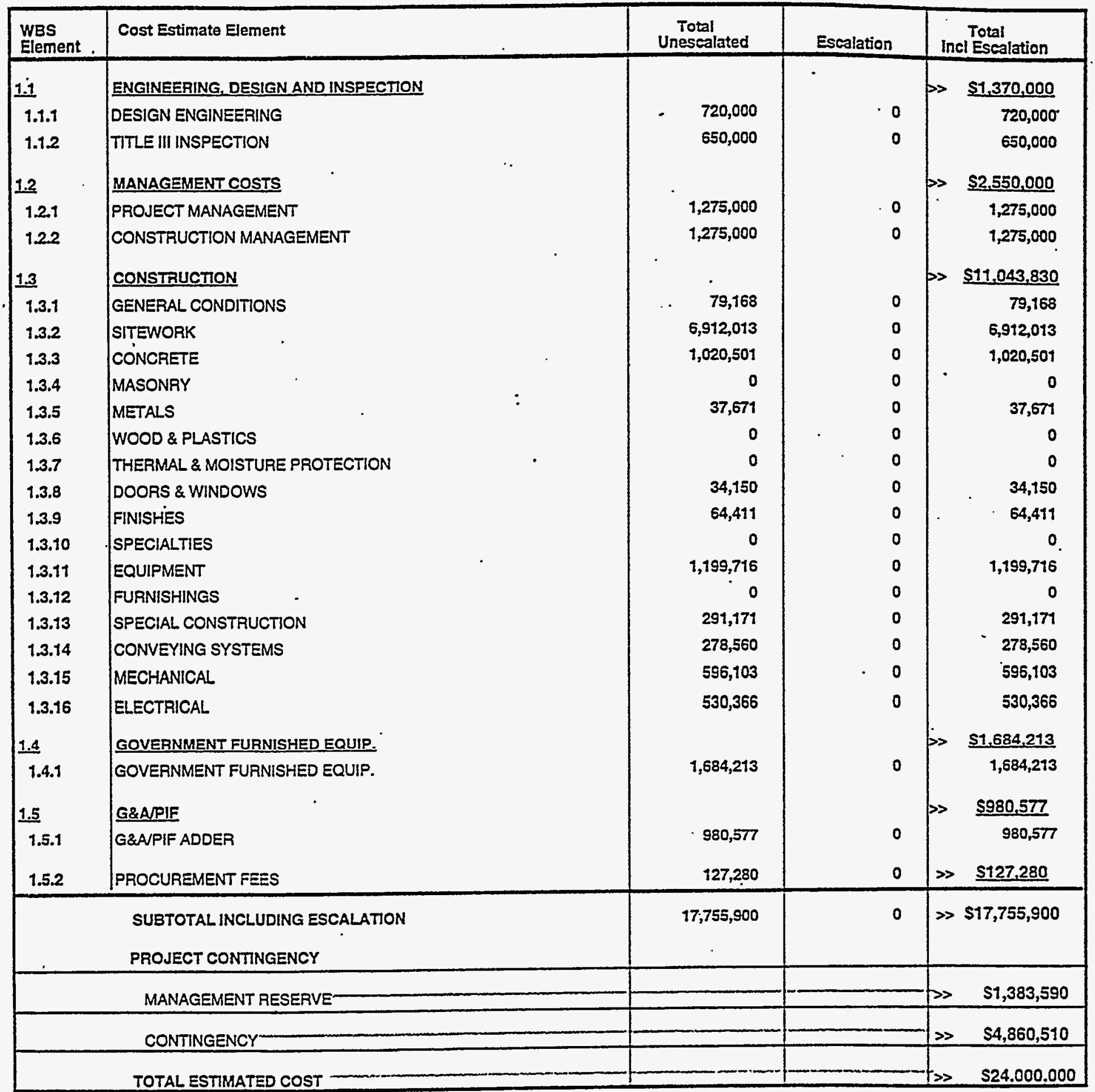

PROJECT COST PARAMETERS

EDI AS A \% OF CONST. + GFE $=11.00 \%$

CONTINGENCY $=35.17 \%$ 
Lockheed Martin Idaho Technologies Co.

PROEET NAME: HLW EIS -WASTE TREATMENT SCOPING STUDIES TYPEOF ESTIMATE:

LCOATON 1: CALCINE RETRIEYAL AND TRANSPORT - OPTIONB INEEL - ICPP

REQUESTOR:
PLANNING

PROLECT NO: 2414-13 (CSSF 3)

PREPARED EY:

REPORT NAME: Cost Estimate Summary

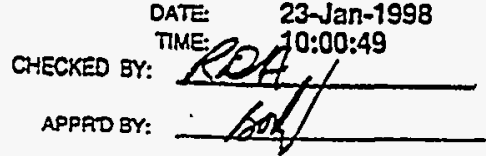

\begin{tabular}{|c|c|c|c|c|}
\hline $\begin{array}{l}\text { WBS } \\
\text { Element }\end{array}$ & Cost Estimate Element & $\begin{array}{c}\text { Total } \\
\text { Unescalated }\end{array}$ & Escalation & $\begin{array}{c}\text { Total } \\
\text { Incl Escalation }\end{array}$ \\
\hline 1.1 & ENGINEERING, DESIGN AND INSPECTION & & & $\$ 1,929,600$ \\
\hline 1.1.1 & DESIGN ENGINEERING & 720,000 & 316,800 & $1,036,800$ \\
\hline 1.1 .2 & TITLE III INSPECTION & 620,000 & 272,800 & - $\quad 892,800^{\circ}$ \\
\hline 1.2 & MANAGEMENT COSTS & & & $\$ 3,050,000$ \\
\hline 1.2 .1 & PROJECT MANAGEMENT & $1,250,000$ & 0 & $1,250,000$ \\
\hline 1.2 .2 & CONSTRUCTION MANAGEMENT & $1,250,000$ & 550,000 & $1,800,000$ \\
\hline 1.3 & CONSTRUCTION & & & $\Rightarrow \quad \$ 15,447,410$ \\
\hline 1.3 .1 & GENERAL CONDITIONS & 79,215 & 34,855 & 114,070 \\
\hline 1.3 .2 & SITEWORK & $6,564,926$ & $2,888,567$ & $9,453,493$ \\
\hline 1.3 .3 & CONCRETE & $1,020,501$ & 449,020 & $1,469,521$ \\
\hline 1.3 .4 & MASONRY & 0 & 0 & 0 \\
\hline 1.3 .5 & METALS & 37,671 & 16,575 & 54,246 \\
\hline 1.3 .6 & WOOD \& PLASTICS & 0 & .0 & 0 \\
\hline 1.3.7 & THERMAL \& MOISTURE PROTECTION & 0 & 0 & 0 \\
\hline 1.3 .8 & DOORS \& WINDOWS & 34,150 & 15,026 & 49,176 \\
\hline 1.3 .9 & FINISHES & 64,411 & 28,341 & 92,752 \\
\hline 1.3 .10 & SPECIALTIES & 0 & 0 & 0 \\
\hline 1.3 .11 & EQUIPMENT & $1,207,349$ & 531,234 & $1,738,583$ \\
\hline 1.3 .12 & FURNISHINGS & $\cdot$ & 0 & 0 \\
\hline 1.3 .13 & SPECIAL CONSTRUCTION & 291,171 & 128,115 & 419,286 \\
\hline 1.3.14 & CONVEYING SYSTEMS & 278,560 & 122,567 & 401,127 \\
\hline 1.3 .15 & MECHANICAL & 619,048 & 272,381 & 891,429 \\
\hline 1.3.16 & ELECTRICAL & 530,366 & 233,361 & 763,727 \\
\hline 1.4 & GOVERNMENT FURNISHED EQUIP. & & & $\gg>\quad \$ 2.290 .530$ \\
\hline 1.4 .1 & GOVERNMENT FURNISHED EQUIP. & $1, \dot{684,213}$ & 606,317 & $2,290,530$ \\
\hline 1.5 & G\&APIF & & & $\Rightarrow \quad \$ 1,385,611$ \\
\hline 1.5 .1 & G\&APIF ADDER & 962,230 & 423,381 & $1,385,611$ \\
\hline \multirow[t]{3}{*}{1.5 .2} & PROCUREMENT FEES & 124,116 & 53,264 & $\gg \quad \$ 177,380$ \\
\hline & SUBTOTALINCLUDING ESCALATION & $17,337,927$ & $6,942,604$ & \multirow[t]{2}{*}{$>\$ 24,280,531$} \\
\hline & PROJECT CONTINGENCY & & & \\
\hline \multicolumn{4}{|c|}{ MANAGEMENT RESERVE } & $\gg \quad \$ 1,930,093$ \\
\hline \multicolumn{2}{|r|}{ CONTINGENCY } & & & $\gg \quad S 6,889,376$ \\
\hline & TOTAL ESTIMATED COST - & & & $\$ 33,100,000$ \\
\hline
\end{tabular}

PROJECT COST PARAMETERS

EDI AS A \% OF CONST. + GFE= $11.00 \%$

CONTINGENCY $=36.32 \%$ 
Lockheed Martin Idaho Technologies Co. COST ESTTMATE SUMMARY

PROSECT NAME: HLW EIS -WASTE TREATMENT SCOPING STUDIES TYPE OF ESTMMTE: PLANNING CALCINE REIRIEVAL AND TRANSPORT-OPTION B PROEECTNO 2414-13 (CSSF 3 )
LOCATON 1: INEEL-ICPP REQUESTOR: K. L. WILLIAMS

REPORT NAME: Cost Estimate Summary

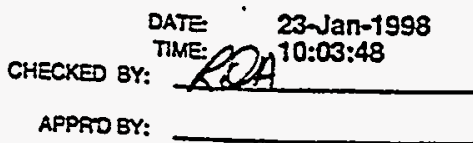

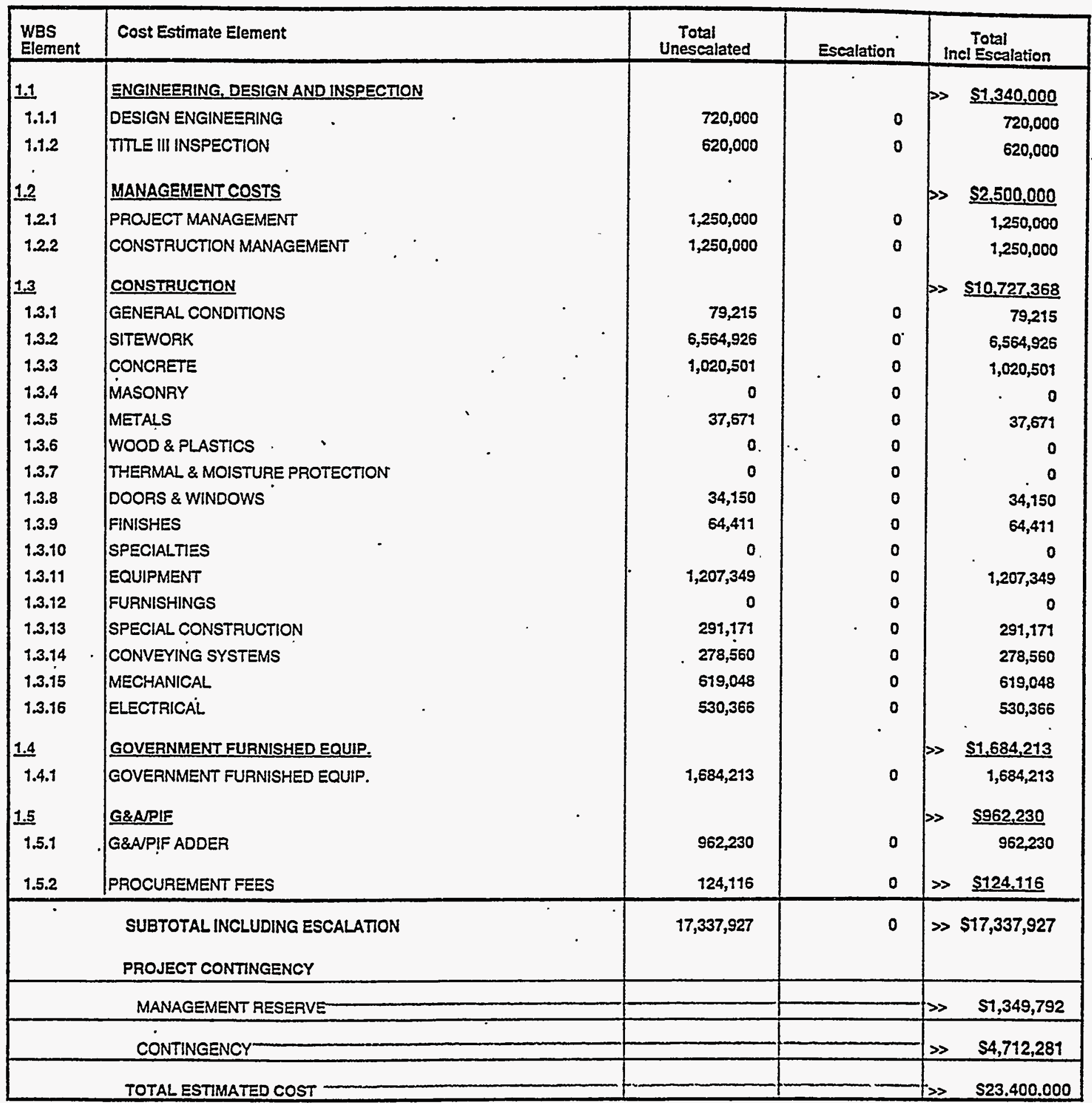

\section{PROJECT COST PARAMETERS}

EDI AS A \% OF CONST. + GFE= $11.00 \%$ 
Lockheed Martin ldaho Technologies Co. COST ESTIMATE SUMMARY

PROUECT NAME: HLW ES -WASTE TREATMENT SCOPING STUDIES TMPE OF ESTIMATE: PLANNING

LCCATION 1 :

CALCINE RETRIEVAL AND TRANSPORT - OPTION B PROLCTNO

2414-1-4 (CSSF 4)

PAEPARED BY: F.P. HANSON

RECUESTOR: KL WILLIAMS

REPORT NAME: Cost Estimate Summary

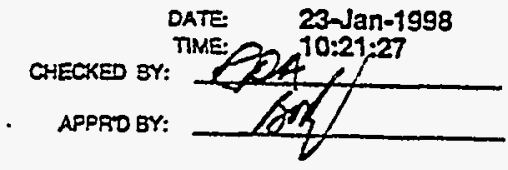

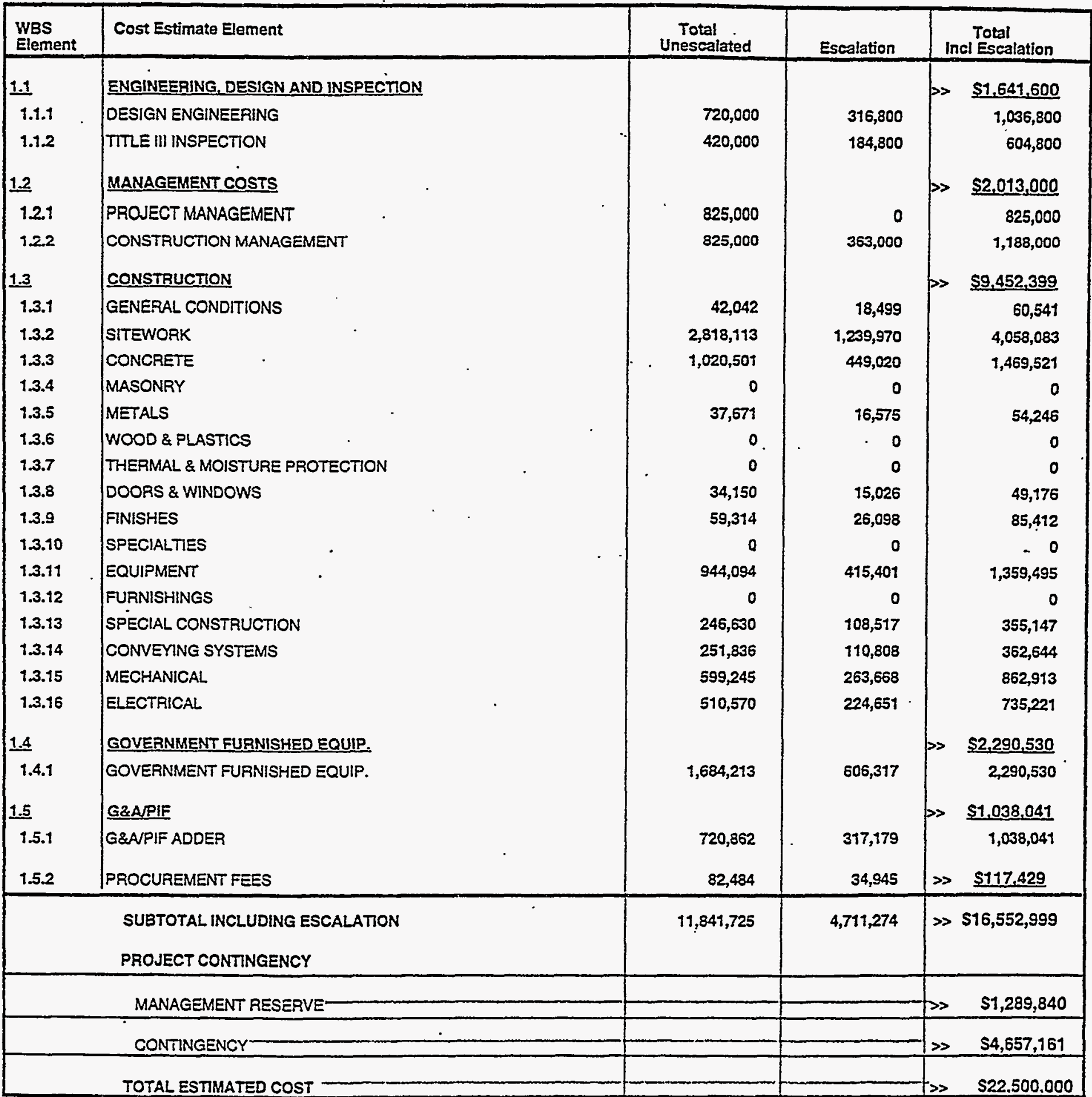

\section{PROJECT COST PARAMETERS}

EDI AS A \% OF CONST. + GFE- $14.00 \%$ 
Lockheed Martin Idaho Technologies Co. PRONECT NAME

LOCATION 1: REOUESTOR:
HLW ES -WASTE TREATMENT SCOPING STUDIES TYPEOF ESTMATE: CALCINE RETRIEVAL AND TRANSPORT - OPTION B PROJECTNO INEEL - ICPP

K. L. WILLIAMS
PREPARED BY: REPORT NAME:
PLANNING

2414-1-4 (CSSF 4)

F. P. HANSON Cost Estimate Summary

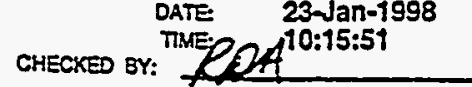

APPRO BY:

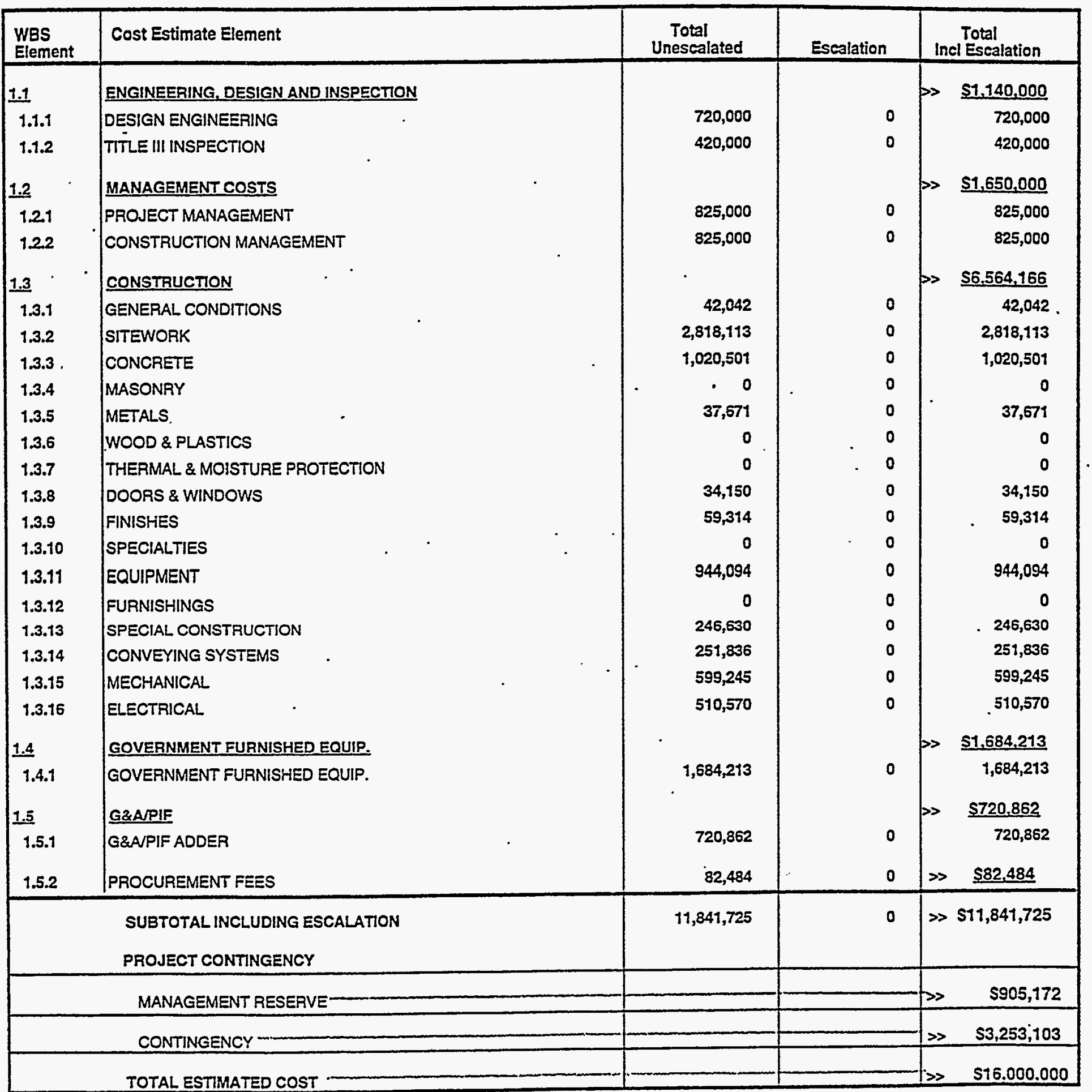

PROJECT COST PARAMETERS

EDI AS A \% OF CONST. + GFE $=14.00 \%$

CONTINGENCY $=35.12 \%$ 
Lockheed Martin Idaho Technologies Co.

COST ESTIMATE SUMMARY

PROIECT NAME: HLW EIS -WASTE TREATMENT SCOPING STUDIES TYPE OF ESTMMATE: PLANNING

CALCINE RETRIEVAL AND TRANSPORT - OPTION B PROEECTNO: 24141-5(CSSFS 5 - 7)
PREPAEDSY: F.P.HANSON

REQUESTOF: K.LWILLLAMS . REPOAT NAME: COSt Estimate Summary

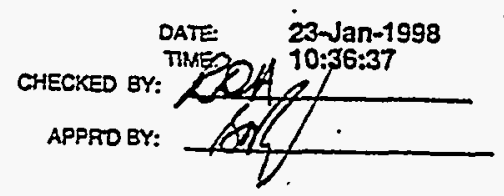

\begin{tabular}{|c|c|c|c|c|}
\hline $\begin{array}{l}\text { WBS } \\
\text { Element } \\
\end{array}$ & Cost Estimate Element & $\begin{array}{c}\text { Total } \\
\text { Unescalated }\end{array}$ & Escalation & $\begin{array}{c}\text { Total } \\
\text { Inci Escalation }\end{array}$ \\
\hline 1.1 & ENGINEERING, DESIGN AND INSPECTION & & & $\$ 1,332,000$ \\
\hline 1.1 .1 & DESIGN ENGINEERING & 720,000 & 172,800 & 892,800 \\
\hline 1.1 .2 & TITLE III INSPECTION & 305,000 & 134,200 & 439,200 \\
\hline 12 & MANAGEMENT COSTS & & & $\$ 2,188,800$ \\
\hline 1.2 .1 & PROJECT MANAGEMENT & 760,000 & 334,400 & $1,094,400$ \\
\hline 1.22 & CONSTRUCTION MANAGEMENT & 760,000 & 334,400 & $1,094,400$ \\
\hline 1.3 & CONSTRUCTION & & & $\$ 8,533,071$ \\
\hline 1.3.1 & GENERAL CONDITIONS & 33,568 & 14,770 & 48,338 \\
\hline 1.3 .2 & SITEWORK & $2,818,113$ & $1,239,970$ & $4,058,083$ \\
\hline 1.3 .3 & CONCRETE & 113,649 & 50,006 & 163,655 \\
\hline 1.3.4 & MASONRY & 0 & 0 & 0 \\
\hline 1.3 .5 & METALS & 37,571 & 16,575 & 54,246 \\
\hline 1.3.6 $\left.{ }^{\circ}-10\right)$ & WOOD \& PLASTICS & 0 & 0 & 0 \\
\hline 1.3.7 & THERMAL \& MOISTURE PROTECTION & 0 & 0 & 0 \\
\hline 1.3.8 & DOORS \& WINDOWS & 34,150 & 15,026 & 49,176 \\
\hline 1.3 .9 & FINISHES & 64,411 & 28,341 & 92,752 \\
\hline 1.3.10 & SPECIALTIES & 0 & 0 & 0 \\
\hline 1.3.11 & EQUIPMENT & $1,116,330$ & 491,185 & $1,607,515$ \\
\hline 1.3.12 & FURNISHINGS & 0 & 0 & . 0 \\
\hline 1.3.13 & SPECIAL CONSTRUCTION & 291,171 & 128,115 & 419,286 \\
\hline 1.3.14 & CONVEYING SYSTEMS & 278,560 & 122,567 & 401,127 \\
\hline 1.3 .15 & MECHANICAL & 619,048 & 272,381 & 891,429 \\
\hline 1.3.16 & ELECTRICAL & 519,072 & 228,392 & 747,464 \\
\hline 1.4 & GOVERNMENT FURNISHED EOUIP. & & & $\$ 2,290,530$ \\
\hline 7.4.1 & GOVERNMENT FURNISHED EQUIP. & §,684,213 & 606,317 & $2,290,530$ \\
\hline$\underline{1.5}$ & G\&APIF & & & $\$ 984,743$ \\
\hline 3.5.1 & G\&APIF ADDER & 683,849 & 300,894 & 984,743 \\
\hline \multirow[t]{2}{*}{1.5 .2} & PROCUREMENT FEES & 76,100 & 32,136 & $\gg \quad \$ 108,236$ \\
\hline & $\begin{array}{l}\text { SUBTOTALINCLUDING ESCALATION } \\
\text { PROJECT CONTINGENCY }\end{array}$ & $10,914,905$ & $4,522,475$ & $\gg \$ 15,437,380$ \\
\hline \multicolumn{2}{|r|}{ MANAGEMENT RESERVE- } & & & $\gg \quad s 1,191,658$ \\
\hline \multicolumn{2}{|r|}{ CONTINGENCY } & & & $\$ 4,370,962$ \\
\hline & OTAL ESTIMATED COST & & & $\$ 21.000 .000$ \\
\hline
\end{tabular}

\section{PROJECT COST PARAMETERS}

EDI AS A \% OF CONST. + GFE $=12.00 \%$

CONTINGENCY $=36.03 \%$ 
Lockheed Martin Idaho Technologies Co. PROLET NAME

LOCATION I: AEQUESTOR: HLW EIS -WASTE TREATMENT SCOPING STUDIES TYPE OF ESTIMATE CALCINE RETRIEVAL AND TRANSPORT - OPTION B PROJECTNO: INEEL - ICPP

K. L. WILLIAMS
COST ESTIMATE SUMMARY

2414 1-5 (CSSFS 5 - 7 ) REPORT NAME: Cost Estimate Summary

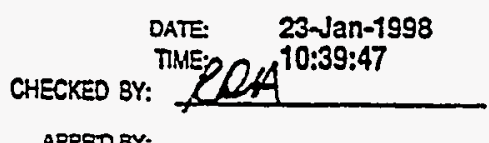

APPRD BY:

\begin{tabular}{|c|c|c|c|c|}
\hline $\begin{array}{l}\text { WBS } \\
\text { Element }\end{array}$ & Cost Estimate Element & $\begin{array}{l}\text { Total } \\
\text { Unescalated }\end{array}$ & Escalation & $\begin{array}{l}\text { Total } \\
\text { Incl Escalation }\end{array}$ \\
\hline $\begin{array}{l}1.1 \\
1.1 .1 \\
1.1 .2 \\
1.2 \\
1.2 .1 \\
1.2 .2 \\
1.3 \\
1.3 .1 \\
1.3 .2 \\
1.3 .3 \\
1.3 .4 \\
1.3 .5 \\
1.3 .6 \\
1.3 .7 \\
1.3 .8 \\
1.3 .9 \\
1.3 .10 \\
1.3 .11 \\
1.3 .12 \\
1.3 .13 \\
1.3 .14 \\
1.3 .15 \\
1.3 .16 \\
1.4 \\
1.4 .1 \\
1.5 \\
1.5 .1 \\
1.5 .2\end{array}$ & $\begin{array}{l}\text { ENGINEERING, DESIGN AND INSPECTION } \\
\text { DESIGN ENGINEERING } \\
\text { TITLE III INSPECTION } \\
\text { MANAGEMENT COSTS } \\
\text { PROJECT MANAGEMENT } \\
\text { CONSTRUCTION MANAGEMENT } \\
\text { CONSTRUCTION } \\
\text { GENERAL CONDITIONS } \\
\text { SITEWORK } \\
\text { CONCRETE } \\
\text { MASONRY } \\
\text { METALS } \\
\text { WOOD \& PLASTICS } \\
\text { THERMAL \& MOISTURE PROTECTION } \\
\text { DOORS \& WINDOWS } \\
\text { FINISHES } \\
\text { SPECIALTIES } \\
\text { EQUIPMENT } \\
\text { FURNISHINGS } \\
\text { SPECIAL CONSTRUCTION } \\
\text { CONVEYING SYSTEMS } \\
\text { MECHANICAL } \\
\text { ELECTRICAL } \\
\text { GOVERNMENT FURNISHED EQUIP. } \\
\text { GOVERNMENT FURNISHED EQUIP. } \\
\text { G\&AIPIF } \\
\text { G\&APIF ADDER } \\
\text { PROCUREMENT FEES } \\
. \\
.\end{array}$ & $\begin{array}{r}720,000 \\
305,000 \\
\\
760,000 \\
760,000 \\
\\
\\
33,568 \\
2,318,113 \\
113,649 \\
0 \\
37,671 \\
0 \\
0 \\
34,150 \\
64,411 \\
0 \\
1,116,330 \\
0 \\
291,171 \\
278,560 \\
619,048 \\
519,072 \\
\end{array}$ & $\begin{array}{l}0 \\
0 \\
0 \\
0 \\
0 \\
0 \\
0 \\
0 \\
0 \\
0 \\
0 \\
0 \\
0 \\
0 \\
0 \\
0 \\
0 \\
0 \\
0 \\
0 \\
0\end{array}$ & 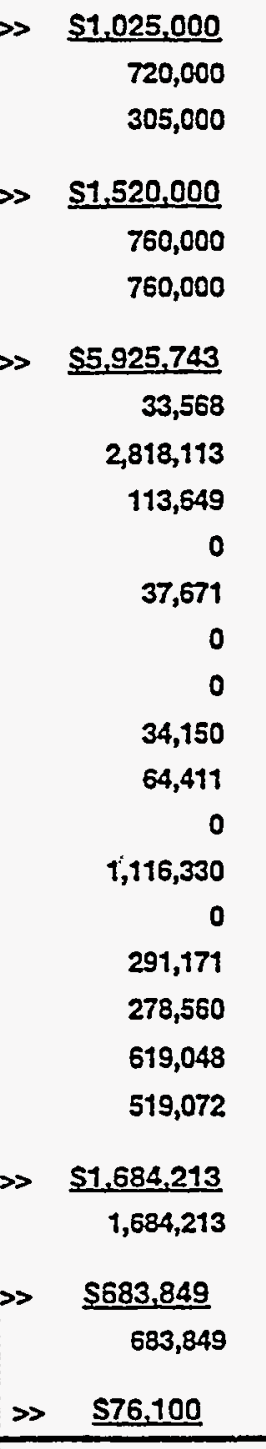 \\
\hline & $\begin{array}{l}\text { SUBTOTAL INCLUDING ESCALATION } \\
\text { PROJECT CONTINGENCY }\end{array}$ & $10,914,905$ & 0 & >> $\$ 10,914,905$ \\
\hline & MANAGEMENT RESERVE & & & $\$ 836,990$ \\
\hline & CONTINGENCY - & & & $\$ 2,948,105$ \\
\hline & TOTAL ESTMMATED COST & & & $\gg \quad \$ 14.700 .000$ \\
\hline
\end{tabular}

PROJECT COST PARAMETERS

EDI AS A \% OF CONST. + GFE $=13.00 \%$

CONTINGENCY $=34.68 \%$ 
Lockheed Martin ldaho Technologies Co.

COST ESTIMATE SUMMARY

PROLET NAME: HLW EIS -WASTE TREATMENT SCOPING STUDIES TYPEOFESTMATE PLANNING

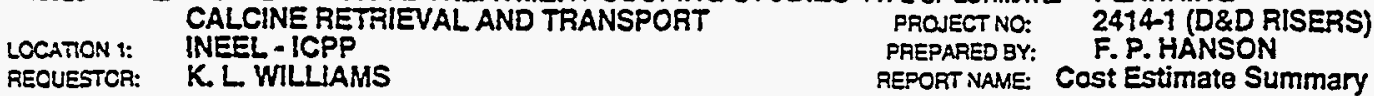


Lockheed Martin Idaho Technologies Co. PROUECT vase

LOCATION 1: REQUESTOR: INEEL - ICPP

K.LWLLIAMS
COST ESTIMATE SUMMARY TO CALCINE RETRIEVAL AND TRANSPORT
PROEETNO: 2414-1 (D\&D RISERS) PAEPARED BY: F.P. HANSON REPORTNAME: Cost Estimate Summary

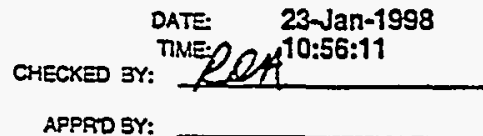

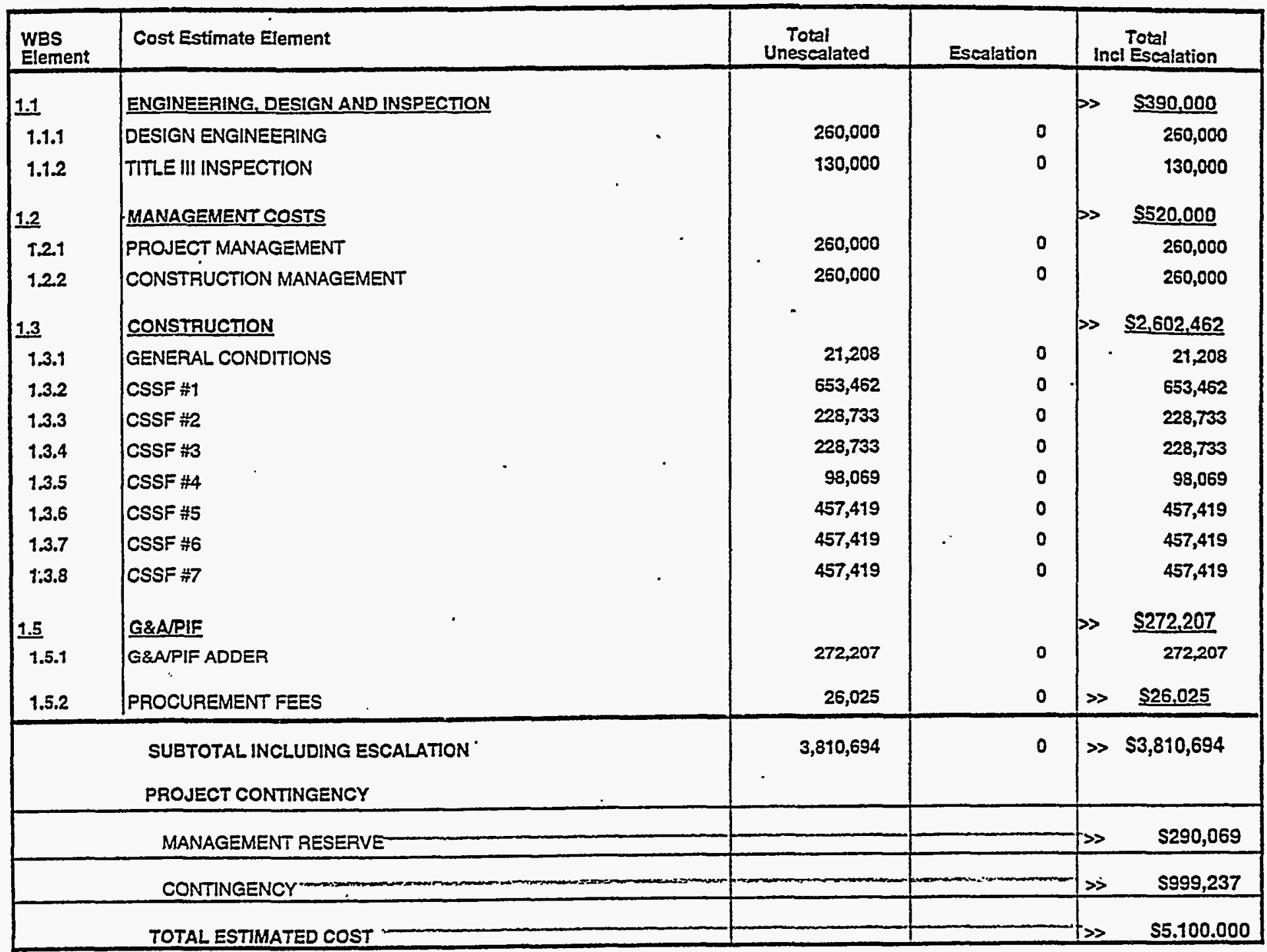

\section{PROJECT COST PARAMETERS}

EDI AS A \% OF CONST + GFE= $15.00 \%$ 
Lockheed Martin Idaho Technologies Co.

LOCATION 1:

RECUESTOR:

HLW EIS -WASTE TREATMENT SCOPINC

CALCINE RETRIEVAL AND TRANSPORT

INEEL - ICPP

K. L. WILLIAMS
COST ESTIMATE SUMMARY

MPE OF ESTIMATE: PLANNING

PROJECT NO: ' 2414-1 (COPR CPNS)

PREPAFED BY:

F. P. HANSON

REPORT NAME: Cost Estimate Summary

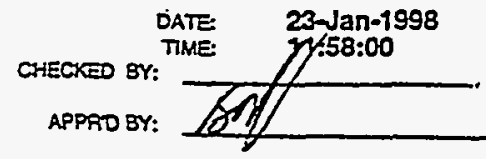

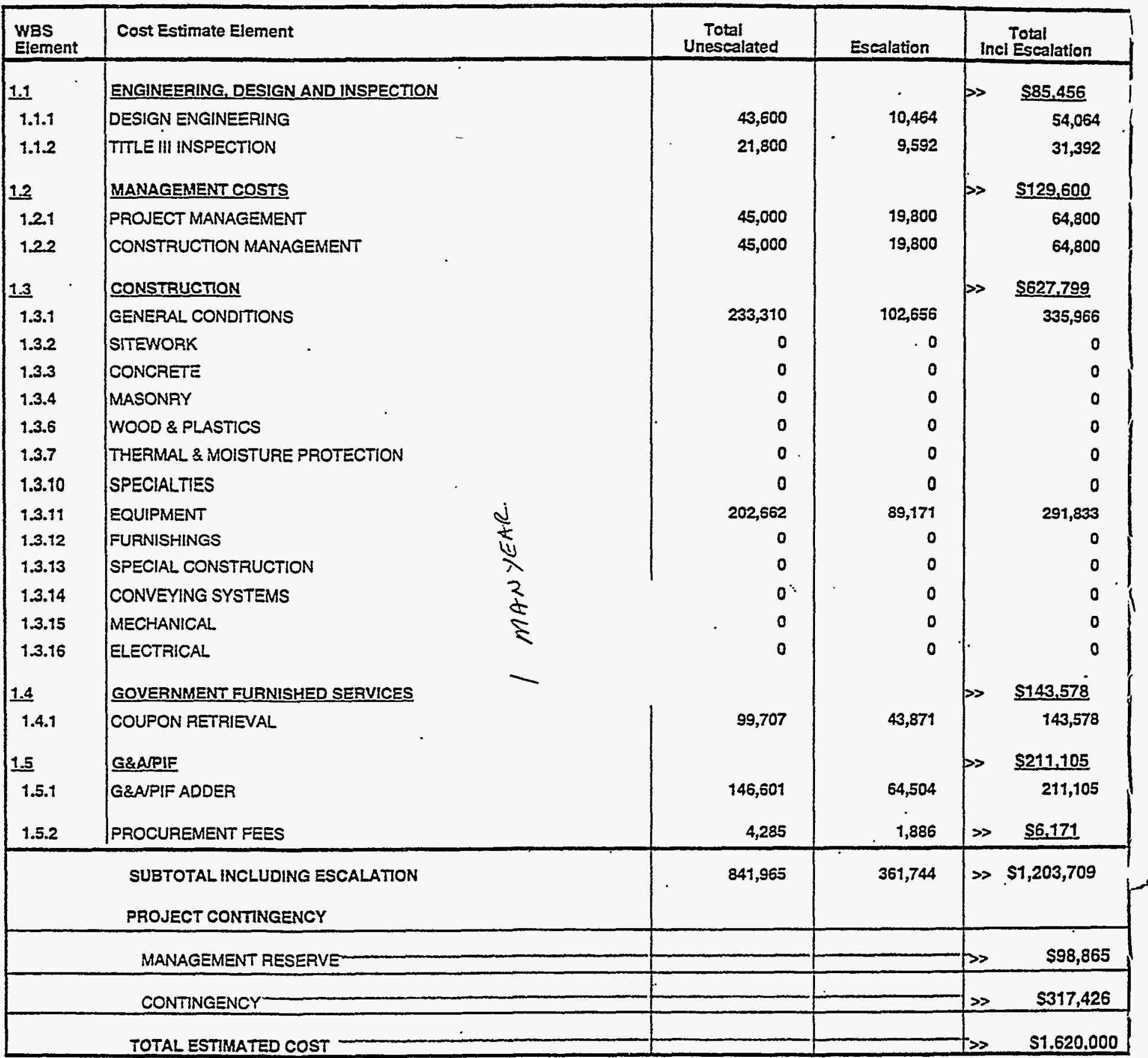

PROJECT COST PARAMETERS

EDI AS A $\%$ OF CONST. + GFE $=11.00 \%$ 
Lockheed Martin Idaho Technologies Co.

COST ESTIMATE SUMMARY

PROFCT NAME: HLW EIS -WASTE TREATMENT SCOPING STUDIES TYPE OF ESTMATE: PLANNING CALCINE RETRIEVAL AND TRANSPORT INEEL - ICPP

PROAECT NO:

FREPARED BY:

2414-1 (CORR CPNS)

LOCATION 1:

K. L. WILLJAMS

REPORT NANE: Cost Estimate Summary

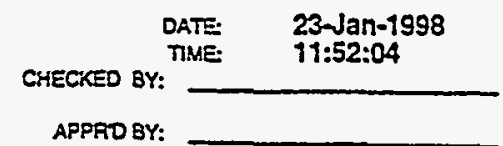

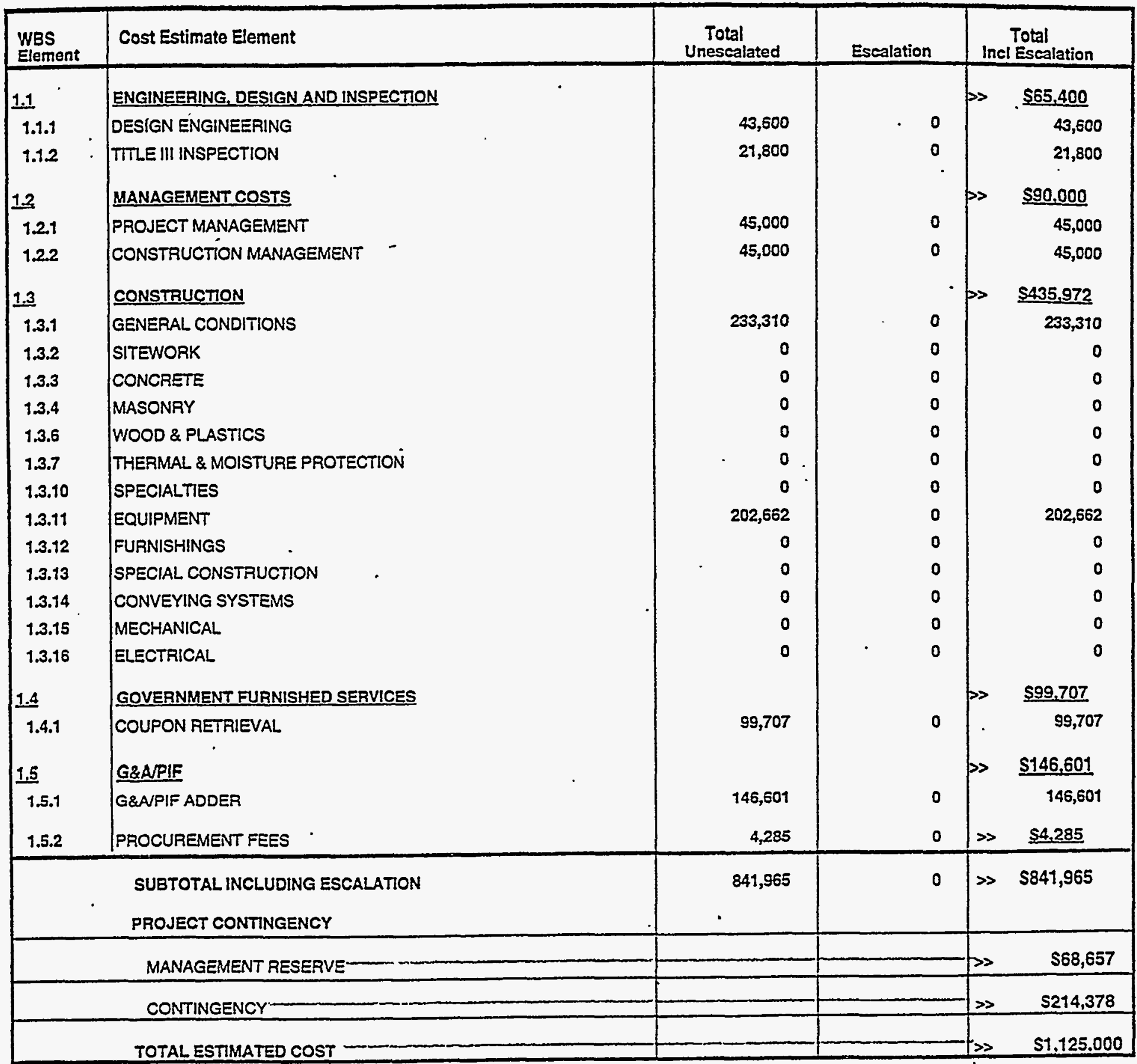

\section{PROJECT COST PARAMETERS}

EDI AS A \% OF CONST. + GFE $=12.00 \%$

CONTINGENCY $=33.62 \%$ 
Lockheed Martin Idaho Technologies Co.

COST ESTIMATE SUMMARY

PRONECT NAME: HLW EIS -WASTE TREATMENT SCOPING STUDIES TYPEOFESTMATE: PLANNING CALCINE RETRIEVAL AND TRANSPORT - OPTION B PROJECT NO: 2414-1 (TRANS SYS A)
INEEL -ICPP
PAEPARED BY: F.P.HANSON

LOCATION :: REPORT NAME: Cost Estimate Summary

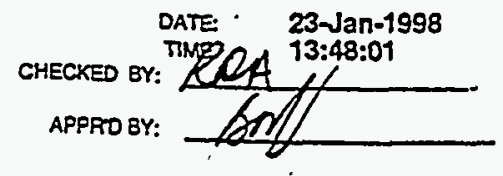

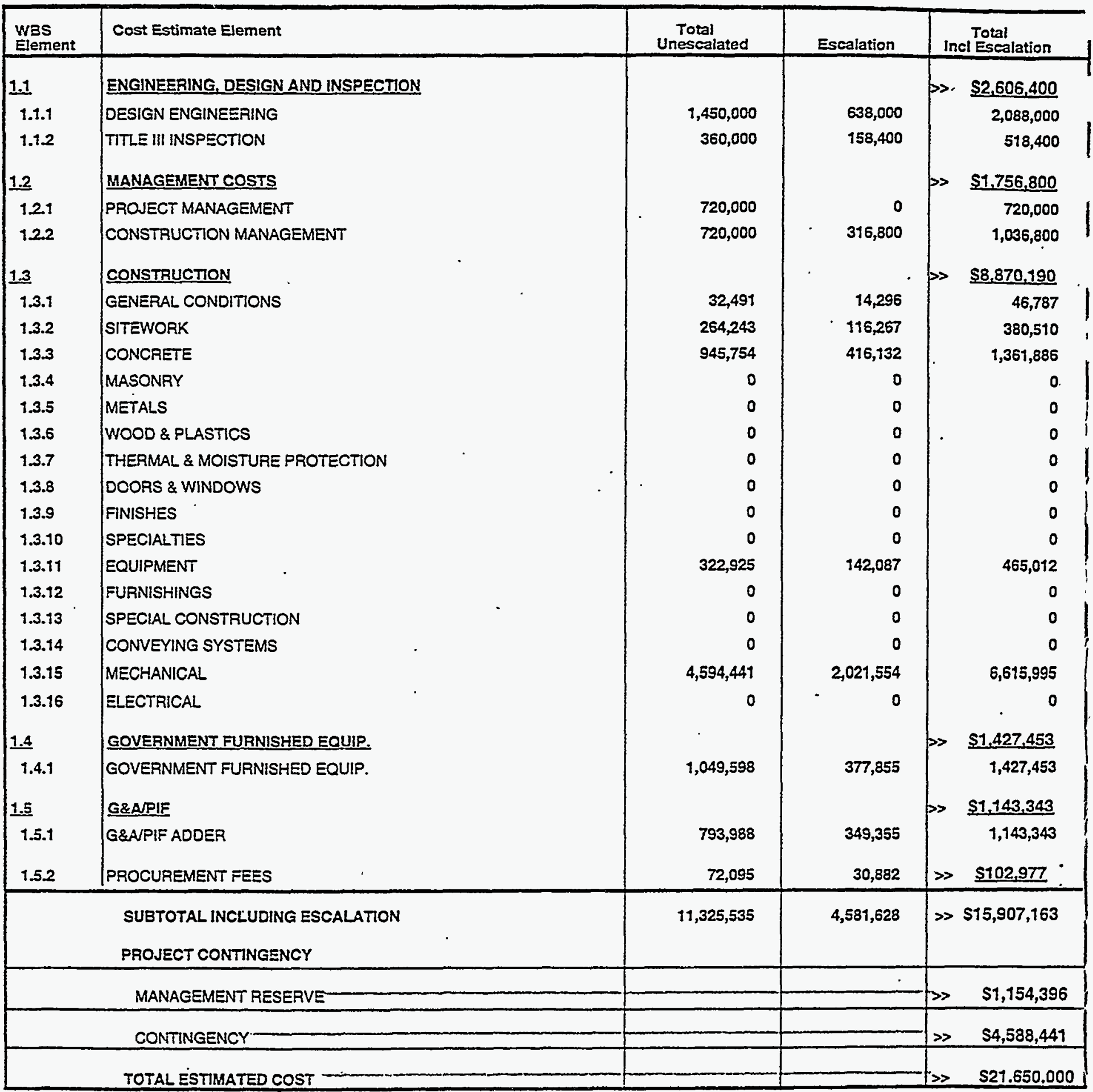

\section{PROJECT COST PARAMETERS}

EDI AS A $\%$ OF CONST. $\bullet$ GFE $=25.00 \%$

CONTINGENCY $=36.10 \%$ 
Lockheed Martin Idaho Technologies Co.

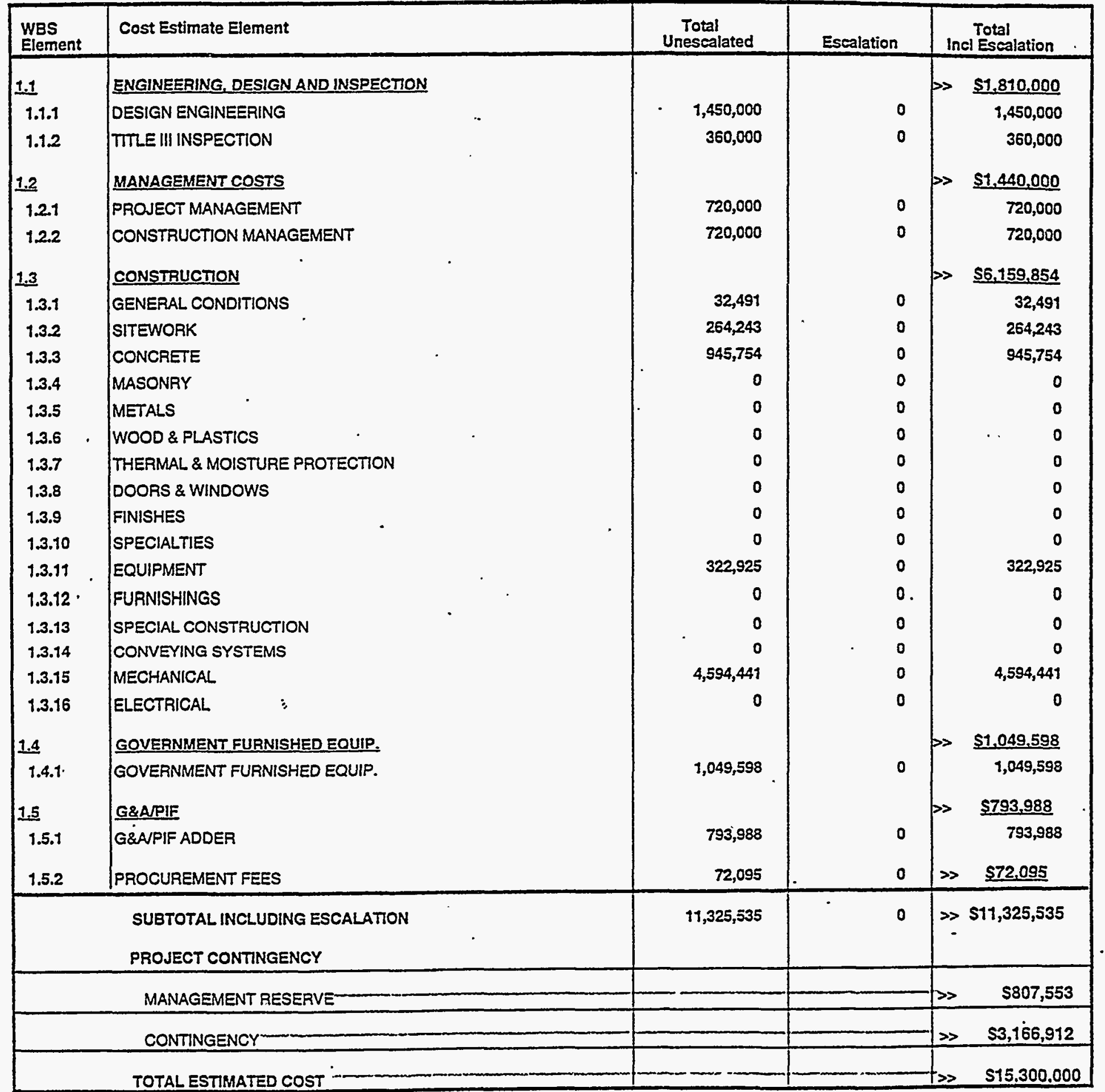

\section{PROJECT COST PARAMETERS}

EDI AS A $\%$ OF CONST. + GFE $=25.00 \%$ 
Lockheed Martin Idaho Technologies Co.

COST ESTIMATE SUMMARY

PROSECT NAME: HLW EIS -WASTE TREATMENT SCOPING STUDIES TYPEOF ESTIMATE PLANNING

CALCINE RETRIEVAL AND TRANSPORT - OPTION B PRONECTNO: 2414-1 (TRANS

INEEL - ICPP

REQUESTCR: K.L WILLIAMS

REPORT NAME: COSt Estimate Summary

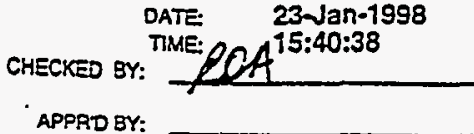

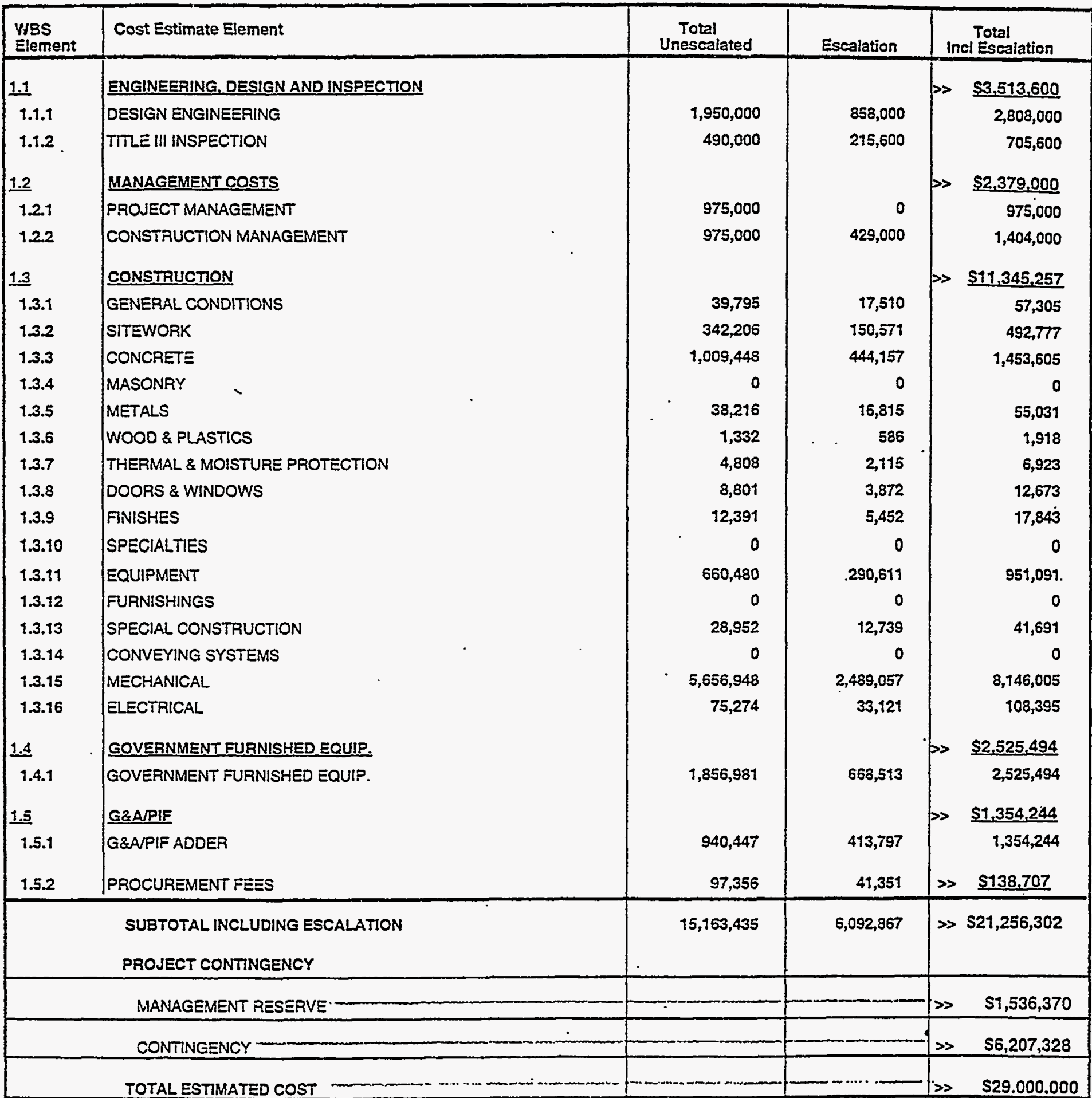

PROJECT COST PARAMETERS

EDI AS A $\%$ OF CONST. + GFE $=25.00 \%$

CONTINGENCY $=36.43 \%$ 
Lockheed Martin Idaho Technologies Co.

COST ESTIMATE SUMMARY

PROIECT NAME: HLW EIS -WASTE TREATMENT SCOPING STUDIES TYPE OFESTMATE: PLANNING

PROJECTNO:

LOCATION 1: INEEL-ICPP

AEQUESTOR:. K. L. WILLIAMS

PAEPARED BY: F. P. HANSON
REPORT NAME: Cost Estimate Summary

2414-1 (TRANS SYS B)

F. P. HANSON

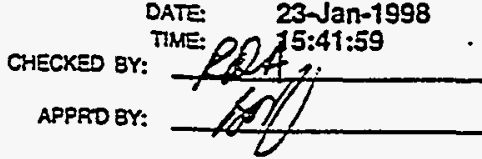

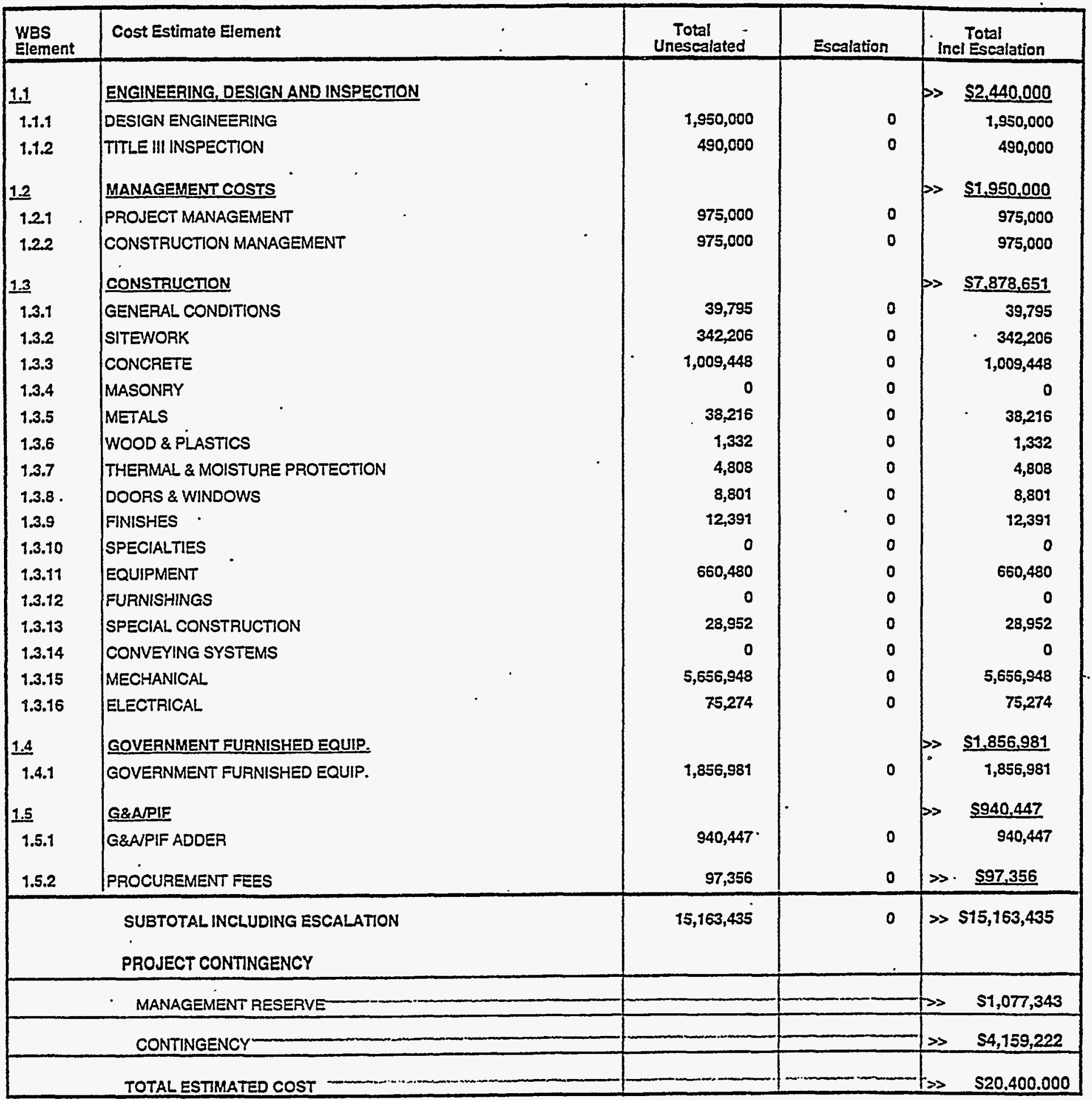

PROJECT COST PARAMETERS

EDI AS A \% OF CONST. + GFE $=25.00 \%$

CONTINGENCY $=34.53 \%$ 
Date: $\quad$ January 28, 1998

To:

S. E. Gifford

MS 3765

$6-5162$

From:

R. J. Turk Pflach

MS 3875

6-3611

Subject: $\quad$ ECONOMIC AND LIFE CYCLE ANALYSIS CONDUCTED FOR CALCINE RETRIEVAL SYSTEM -RJT-08-98

\section{Purpose:}

As requested an Economic and Life-Cycle Cost (LCC) has been conducted to evaluate the CALCINE RETRIEVAL SYSTEM. This process is proposed to retrieve calcine from the Calcine Solids Storage Facilities (CSSF) and transport it to the waste treatment facility. The calcine retrieval and transportation system is designed to supply calcine to the treatment options of Vitrification, Direct Cementitious, Cementitious, Hot Isostatic Pressing and TRU currently understudy. A five and a twenty-year option utilizing a transport system "A or B" per your direction was conducted to coincide with various waste treatment activities.

This economic analysis is based on information provided by Karen Williams, Sara Gifford, A. E. Lee, Ron DaFoe, Dan Griffith, D. Lopez, N. Russell, B. Landman, R. Kimmett and other team members.

F. P. Hanson provided cost estimates. Jack Prendergast provided process personnel modeling.

\section{Methodology:}

The Economic Evaluation assumed a five-year and a 20-year operations period since this is the estimated time required to supply the calcine to the waste treatment options currently being evaluated. The LCC identifies and evaluates the initial development, construction, operation and post-operating costs over the life-cycle. A discounted LCC assumes a current 1998-dollar basis, discounted at $6.30 \%$ annually per the Office of Management and Budget (OMB) Circular A-94. All costs are conservatively discounted assuming the end-of-year convention. 
Page 2

\section{Assumptions:}

The scope of work and requirements of all related activities are vague at this time. Facility and processing costs were developed from historical experience associated with DD\&D work at the INEEL. The LCC analysis was generated to match cost estimating cost structure. These costs include Permitting, Direct and Indirect Construction, G\&A, Procurement Fee, Engineering, Inspection, Project Management, Construction Management, Escalation and Contingency costs. The design period was assumed to be accomplished in five years with construction completed in six years, complete with eighteen months of start-up and testing. Labor rates were assumed as follows: Managers, \$125/hr; Engineers, $\$ 108$ \$/hr; Other Technicians $\$ 85 / \mathrm{hr}$; Administration/Support staff $\$ 65 / \mathrm{hr}$; Operators and Maintenance personnel $\$ 65 / \mathrm{hr}$. The operational period for these facilities was modeled for five and twenty years, followed by one year of post-operations activities. Utilities were assumed to cost $\$ 3.00 /$ sf for the facility. Due to this projects lack of complexity and relative cleanliness this analysis assumed a decommissioning cost equal to $20 \%$ of the unescalated engineering design cost, decontamination costs equal to $5 \%$ of total unescalated pre-operation cost, and demolition costs equal to $8 \%$ of total unescalated pre-operation cost.

\section{Results:}

The Five-year operation of the CALCINE RETRIEVAL SYSTEM "A"Option has a Discounted LCC of $\$ 167$ million.

The Twenty-year operation of CALCINE RETRIEVAL SYSTEM " $A$ " Option has a Discounted LCC of $\$ 192$ million.

The Twenty-year operation of CALCINE RETRIEVAL SYSTEM "B" Option has a Discounted LCC of \$ 197 million.

Attachments:

cc: R. J. Turk File 


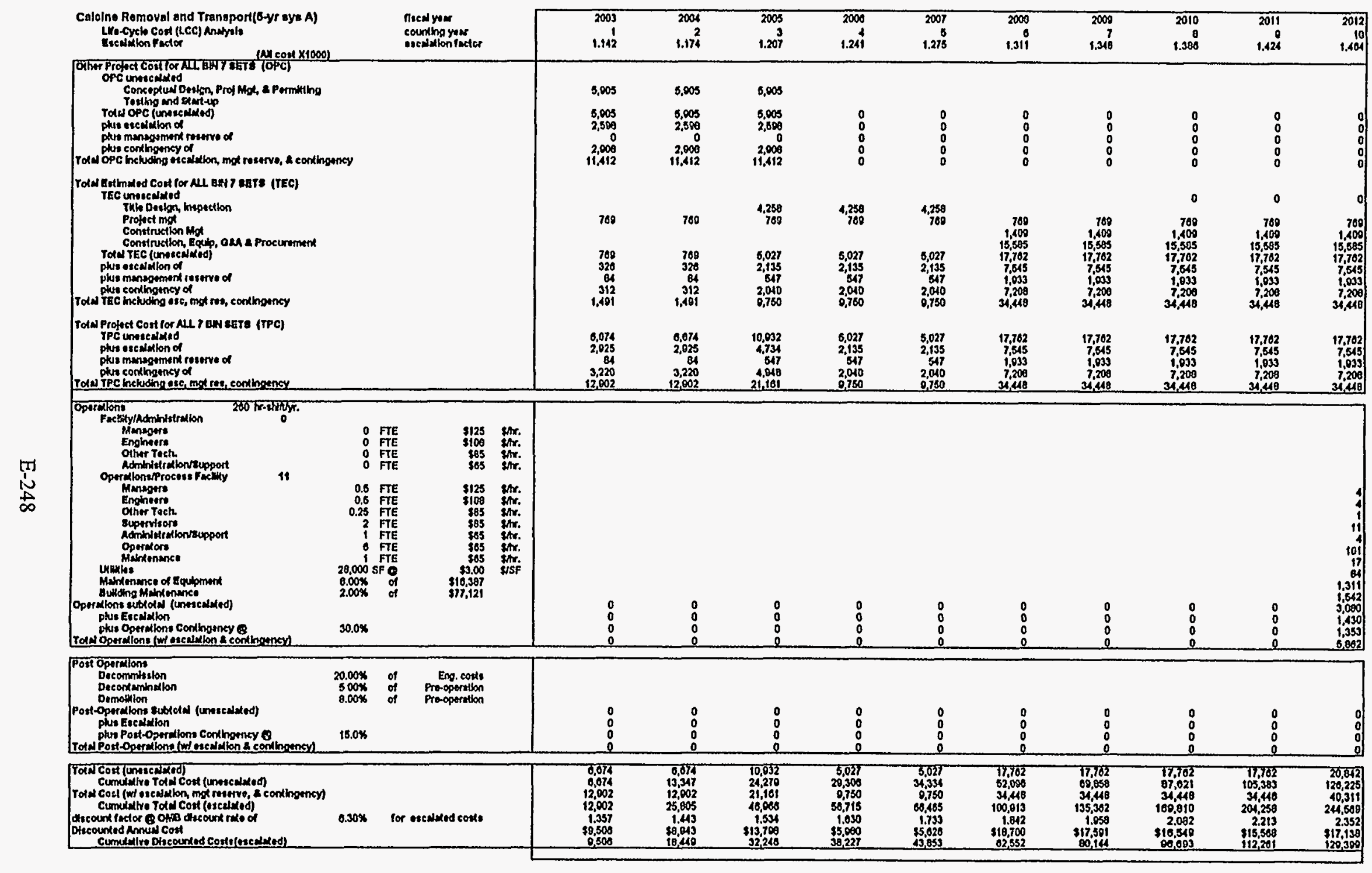




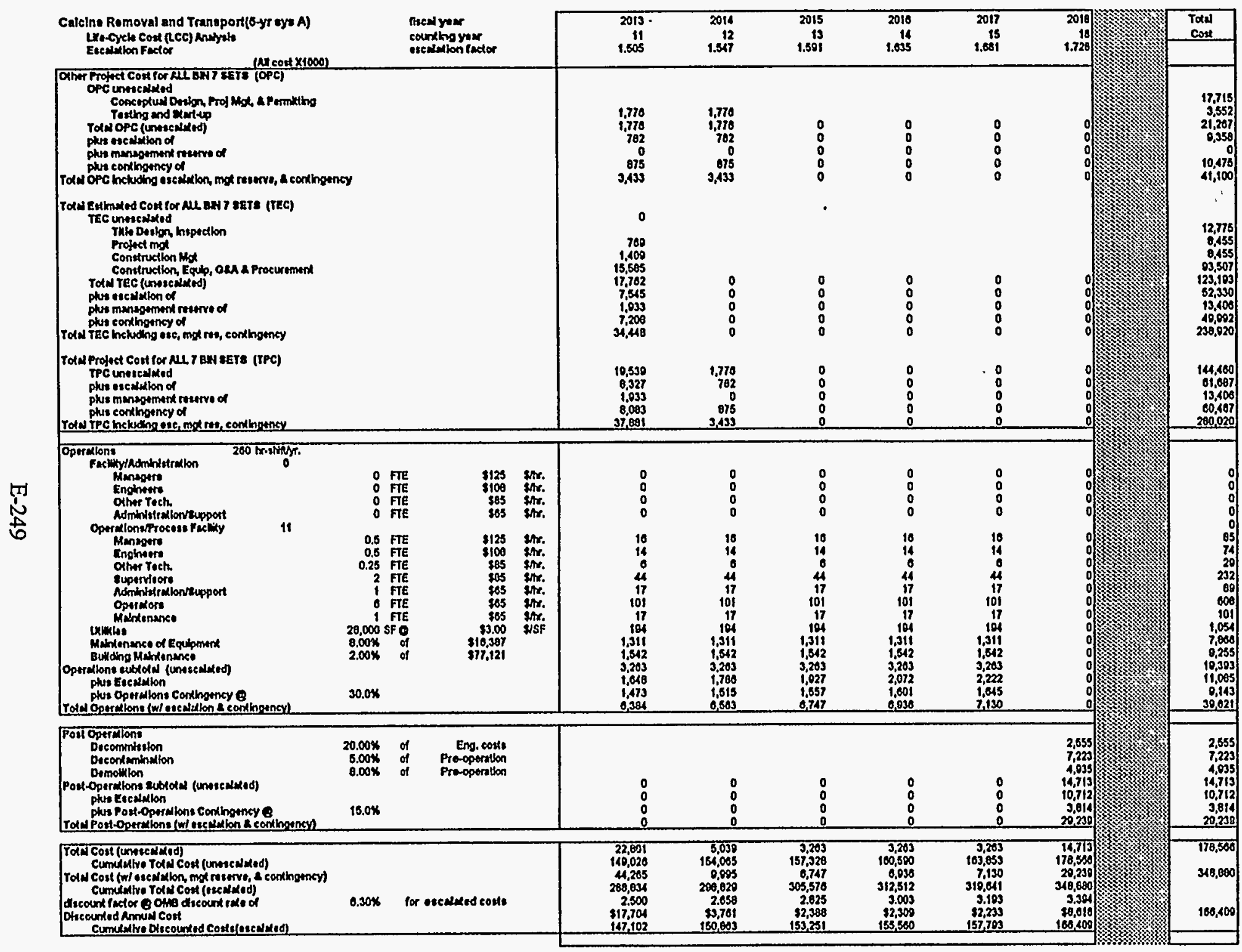




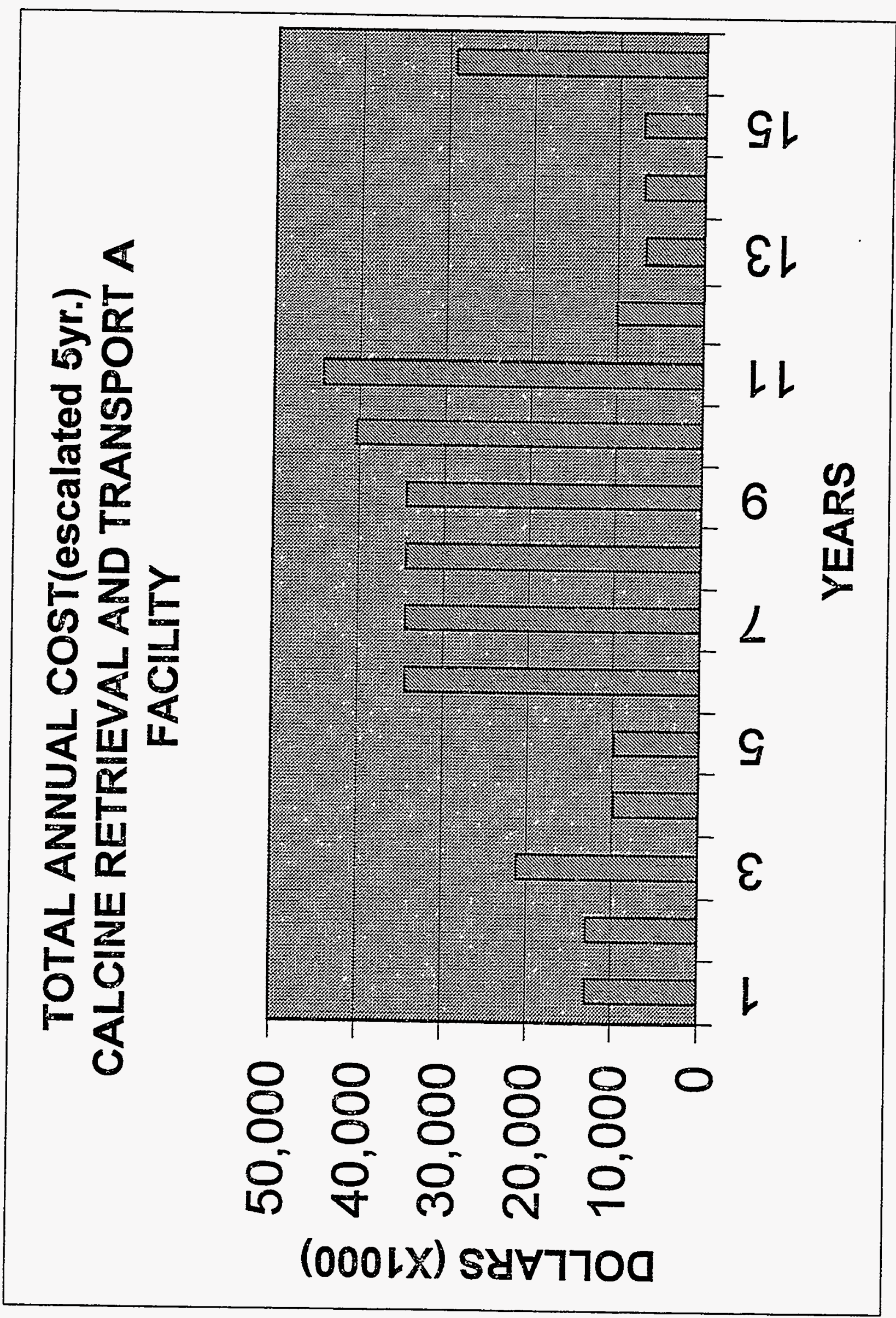




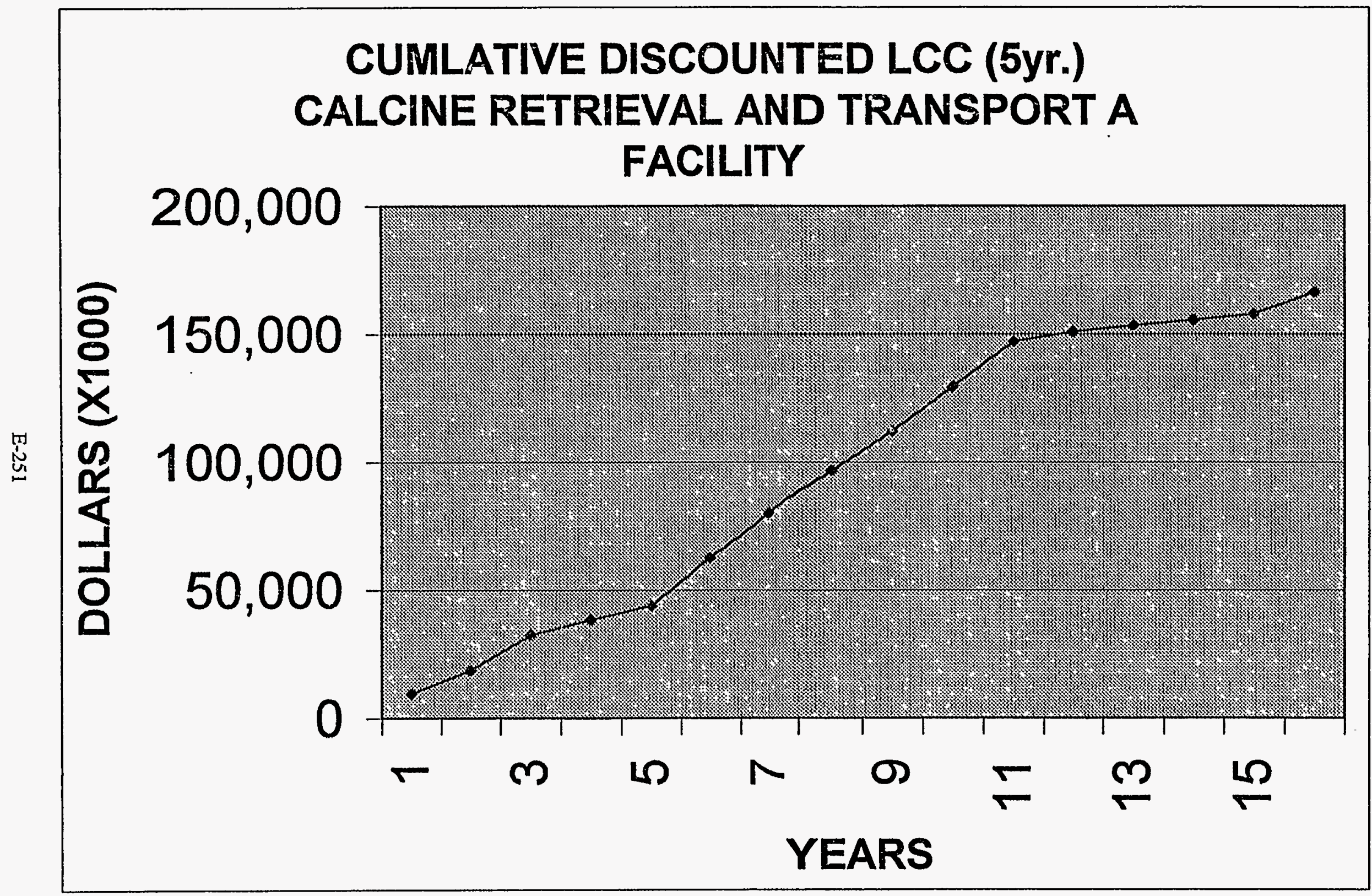




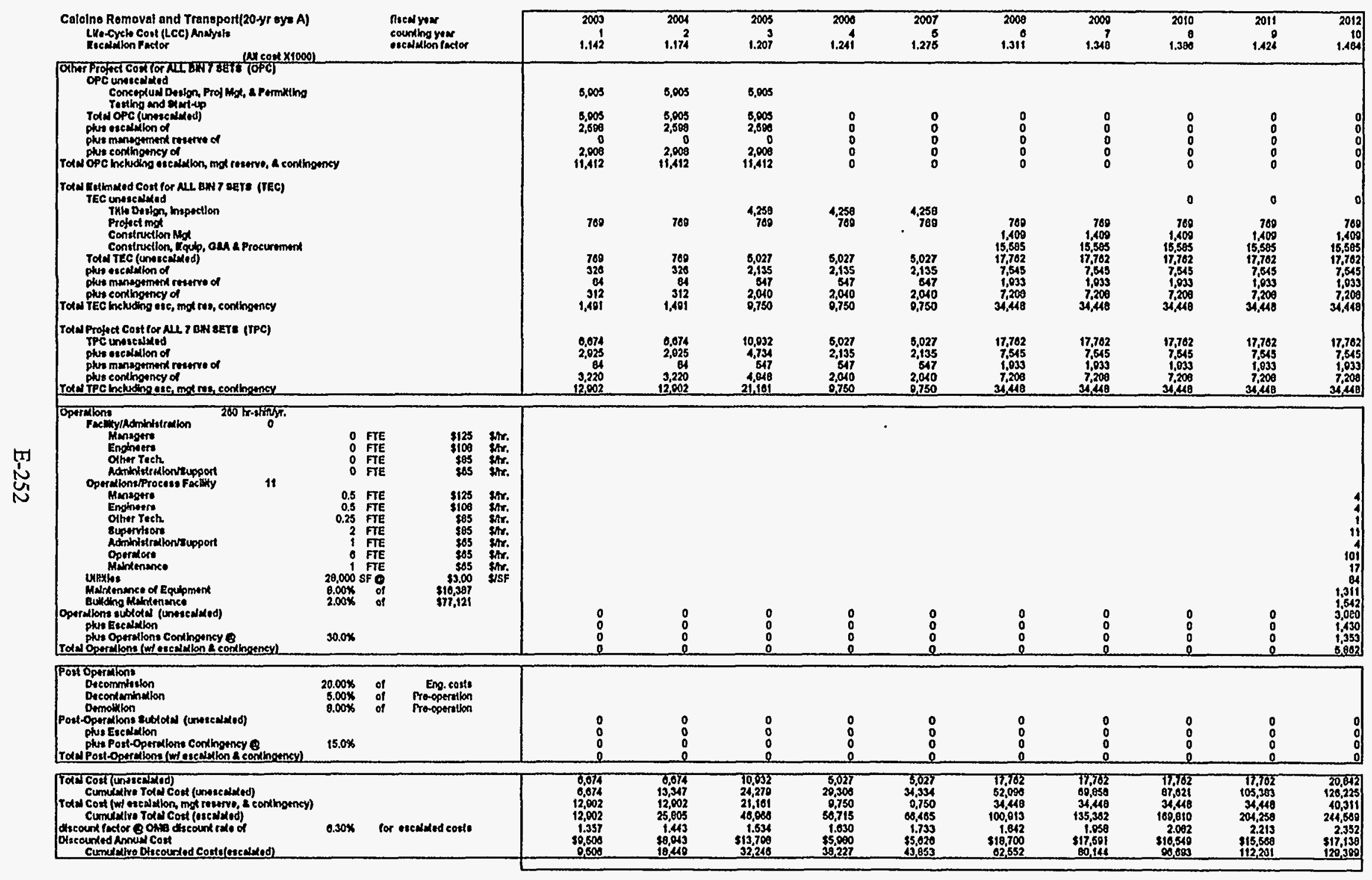




\begin{tabular}{|c|c|c|c|c|c|c|c|c|c|c|c|c|c|}
\hline \multirow{2}{*}{ 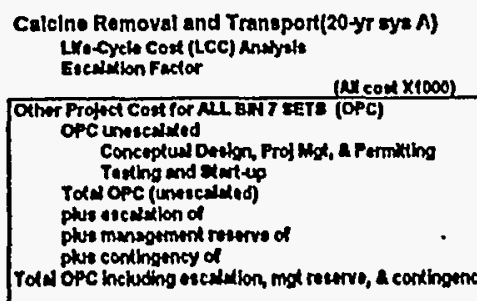 } & \multicolumn{3}{|c|}{ 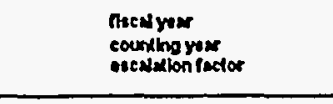 } & $\begin{array}{r}2019 \\
11 \\
1.505\end{array}$ & $\begin{array}{r}2014 \\
12 \\
1.547\end{array}$ & $\begin{array}{r}2015 \\
13 \\
1.591\end{array}$ & $\begin{array}{r}2018 \\
114 \\
1.035\end{array}$ & $\begin{array}{r}2017 \\
15 \\
1.081\end{array}$ & $\begin{array}{r}2010 \\
10 \\
1.728\end{array}$ & $\begin{array}{r}2010 \\
177 \\
1.776\end{array}$ & $\begin{array}{r}2020 \\
10 \\
1.026\end{array}$ & $\begin{array}{r}2021 \\
19 \\
1.871\end{array}$ & $\begin{array}{r}2022 \\
20 \\
1.030\end{array}$ \\
\hline & may & & & $\begin{array}{r}1,778 \\
1,770 \\
702 \\
80 \\
875 \\
3,433\end{array}$ & $\begin{array}{r}1,778 \\
1,776 \\
782 \\
0 \\
8,75 \\
3,439\end{array}$ & $\begin{array}{l}0 \\
0 \\
0 \\
0 \\
0\end{array}$ & $\begin{array}{l}0 \\
0 \\
0 \\
0 \\
0\end{array}$ & $\begin{array}{l}0 \\
0 \\
0 \\
0 \\
0\end{array}$ & $\begin{array}{l}0 \\
0 \\
0 \\
0 \\
0\end{array}$ & $\begin{array}{l}0 \\
0 \\
0 \\
0 \\
0\end{array}$ & $\begin{array}{l}0 \\
0 \\
0 \\
0 \\
0\end{array}$ & $\begin{array}{l}0 \\
0 \\
0 \\
0 \\
0\end{array}$ & $\begin{array}{l}0 \\
: \\
0 \\
0\end{array}$ \\
\hline 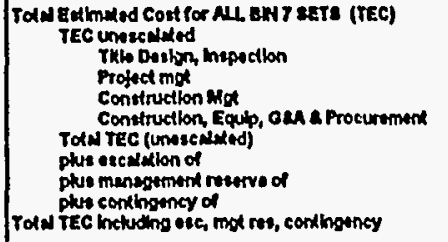 & & & & 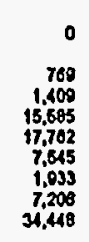 & $\begin{array}{l}0 \\
0 \\
0 \\
0\end{array}$ & $\begin{array}{l}0 \\
0 \\
0 \\
0\end{array}$ & $\begin{array}{l}0 \\
0 \\
0 \\
0 \\
0\end{array}$ & $\begin{array}{l}0 \\
0 \\
0 \\
0 \\
0\end{array}$ & $\begin{array}{l}0 \\
0 \\
0 \\
0 \\
0\end{array}$ & $\begin{array}{l}0 \\
0 \\
0 \\
0 \\
0\end{array}$ & $\begin{array}{l}0 \\
: \\
0 \\
0\end{array}$ & $\begin{array}{l}0 \\
0 \\
0 \\
0 \\
0\end{array}$ & $\begin{array}{l}: \\
0 \\
0 \\
0\end{array}$ \\
\hline 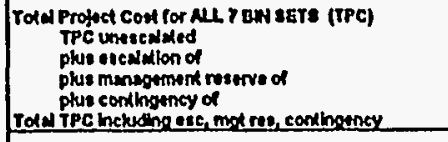 & & & & $\begin{array}{r}10,839 \\
9,327 \\
1,033 \\
8,003 \\
37,881 \\
\end{array}$ & $\begin{array}{r}1,770 \\
782 \\
0 \\
075 \\
0,133 \\
\end{array}$ & $\begin{array}{l}0 \\
0 \\
0 \\
0 \\
0 \\
\end{array}$ & $\begin{array}{l}0 \\
0 \\
0 \\
0 \\
0\end{array}$ & $\begin{array}{l}0 \\
0 \\
0 \\
0 \\
0 \\
\end{array}$ & $\begin{array}{l}0 \\
0 \\
0 \\
0 \\
0 \\
\end{array}$ & $\begin{array}{l}0 \\
0 \\
0 \\
0 \\
0 \\
\end{array}$ & $\begin{array}{l}0 \\
0 \\
0 \\
0 \\
0 \\
\end{array}$ & $\begin{array}{l}0 \\
0 \\
0 \\
0 \\
0 \\
\end{array}$ & $\begin{array}{l}0 \\
0 \\
0 \\
0\end{array}$ \\
\hline 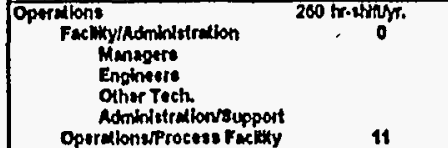 & 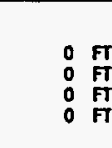 & $\begin{array}{l}\$ 125 \\
\$ 108 \\
\$ 85 \\
\$ 85\end{array}$ & $\begin{array}{l}\text { Shr. } \\
\text { thr. } \\
\text { whr. } \\
\text { inr. }\end{array}$ & $\begin{array}{l}0 \\
0 \\
0 \\
0\end{array}$ & $\begin{array}{l}0 \\
0 \\
0 \\
0\end{array}$ & $\begin{array}{l}0 \\
0 \\
0 \\
0\end{array}$ & $\begin{array}{l}0 \\
0 \\
0 \\
0\end{array}$ & $\begin{array}{l}0 \\
0 \\
0 \\
0\end{array}$ & $\begin{array}{l}0 \\
0 \\
0 \\
0\end{array}$ & $\begin{array}{l}0 \\
0 \\
0 \\
0\end{array}$ & $\begin{array}{l}0 \\
0 \\
0 \\
0\end{array}$ & $\begin{array}{l}0 \\
0 \\
0 \\
0\end{array}$ & $\begin{array}{l}0 \\
0 \\
0 \\
0\end{array}$ \\
\hline 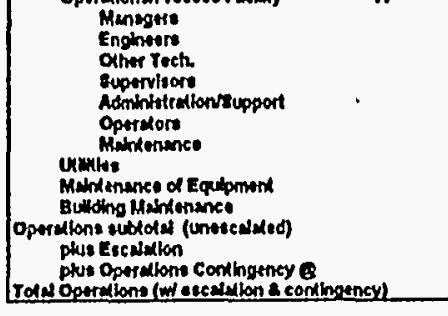 & 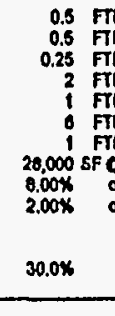 & 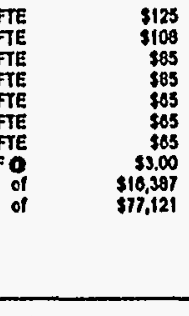 & 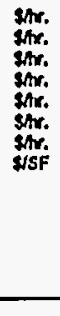 & $\begin{array}{r}10 \\
14 \\
0 \\
14 \\
17 \\
101 \\
117 \\
104 \\
1,311 \\
1,312 \\
0,263 \\
1,018 \\
1,413 \\
0,384 \\
\end{array}$ & 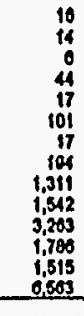 & $\begin{array}{r}10 \\
14 \\
0 \\
14 \\
17 \\
101 \\
17 \\
14 \\
1,311 \\
1,512 \\
3,203 \\
1,027 \\
1,557 \\
0,747 \\
\end{array}$ & $\begin{array}{r}10 \\
14 \\
6 \\
11 \\
17 \\
101 \\
17 \\
107 \\
1,311 \\
1,312 \\
3,203 \\
2,072 \\
1,001 \\
0,030 \\
\end{array}$ & 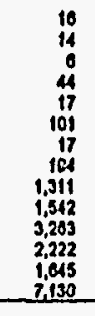 & $\begin{array}{r}16 \\
14 \\
0 \\
4 \\
17 \\
101 \\
17 \\
104 \\
1,311 \\
1,542 \\
3,263 \\
2,373 \\
1,091 \\
7,320 \\
\end{array}$ & $\begin{array}{r}10 \\
14 \\
0 \\
44 \\
17 \\
101 \\
17 \\
194 \\
1,311 \\
1,542 \\
1,203 \\
2,533 \\
1,739 \\
7,535 \\
\end{array}$ & $\begin{array}{r}10 \\
14 \\
0 \\
41 \\
17 \\
101 \\
17 \\
104 \\
1,311 \\
1,412 \\
3,203 \\
2,008 \\
1,787 \\
7,740 \\
\end{array}$ & $\begin{array}{r}10 \\
14 \\
0 \\
41 \\
17 \\
101 \\
17 \\
194 \\
1,311 \\
1,442 \\
3,243 \\
2,002 \\
1,037 \\
7,002 \\
\end{array}$ & 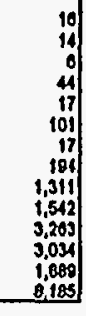 \\
\hline 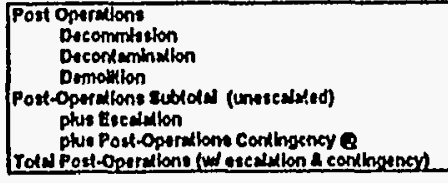 & $\begin{array}{l}20.00 \% \\
5.00 \% \\
0.00 \% \\
18.0 \%\end{array}$ & 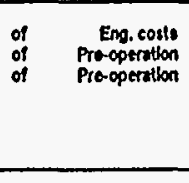 & & $\begin{array}{l}0 \\
0 \\
0 \\
0 \\
\end{array}$ & $\begin{array}{l}0 \\
0 \\
0 \\
0 \\
\end{array}$ & $\begin{array}{l}0 \\
0 \\
0 \\
0 \\
\end{array}$ & $\begin{array}{l}0 \\
0 \\
0 \\
0\end{array}$ & $\begin{array}{l}0 \\
0 \\
0 \\
0 \\
\end{array}$ & $\begin{array}{l}0 \\
0 \\
0 \\
0 \\
\end{array}$ & $\begin{array}{l}0 \\
0 \\
0 \\
0\end{array}$ & $\begin{array}{l}0 \\
0 \\
0 \\
0\end{array}$ & $\begin{array}{r}0 \\
0 \\
0 \\
0\end{array}$ & $\begin{array}{l}0 \\
0 \\
0 \\
0\end{array}$ \\
\hline 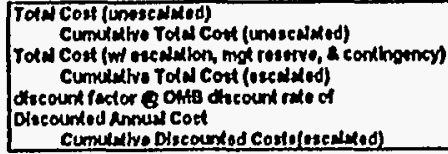 & $0.30 x$ & lor meduled conte & & $\begin{array}{r}22,601 \\
110,020 \\
4,285 \\
269,94 \\
22,600 \\
317,704 \\
147,102 \\
\end{array}$ & 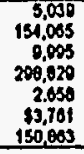 & $\begin{array}{r}15,203 \\
0,328 \\
0,747 \\
305,376 \\
2.023 \\
32,398 \\
153,251 \\
\end{array}$ & $\begin{array}{r}10,203 \\
6,600 \\
312036 \\
31,512 \\
32.003 \\
32,309 \\
135,500 \\
\end{array}$ & 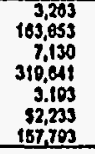 & 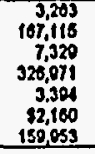 & $\begin{array}{r}17,203 \\
170,398 \\
7,635 \\
34,035 \\
3,007 \\
32,099 \\
102,042 \\
\end{array}$ & 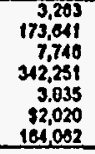 & $\begin{array}{r}3,203 \\
170,003 \\
7,092 \\
350,213 \\
4.070 \\
31,053 \\
160,018 \\
\end{array}$ & 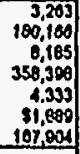 \\
\hline
\end{tabular}




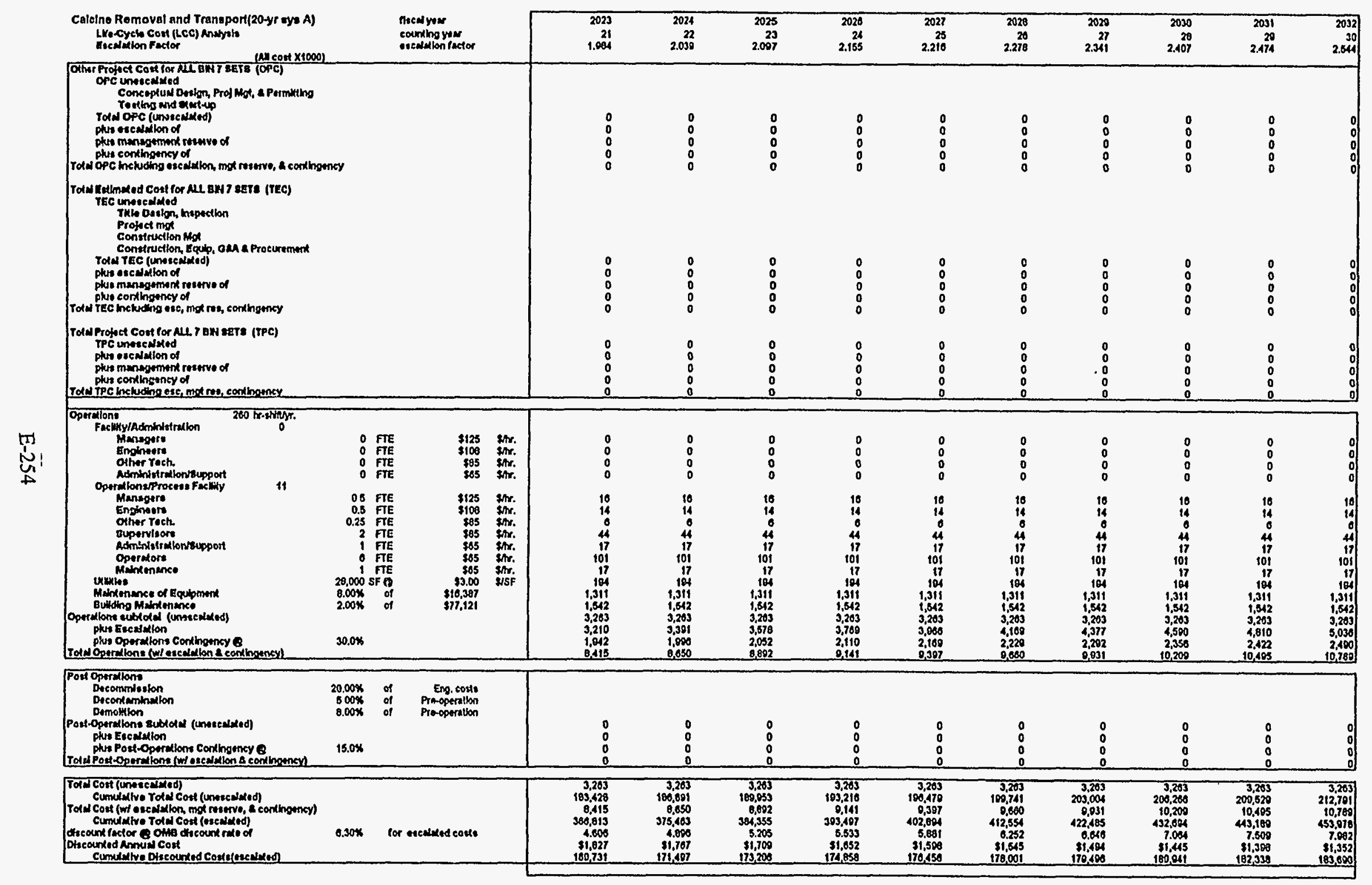




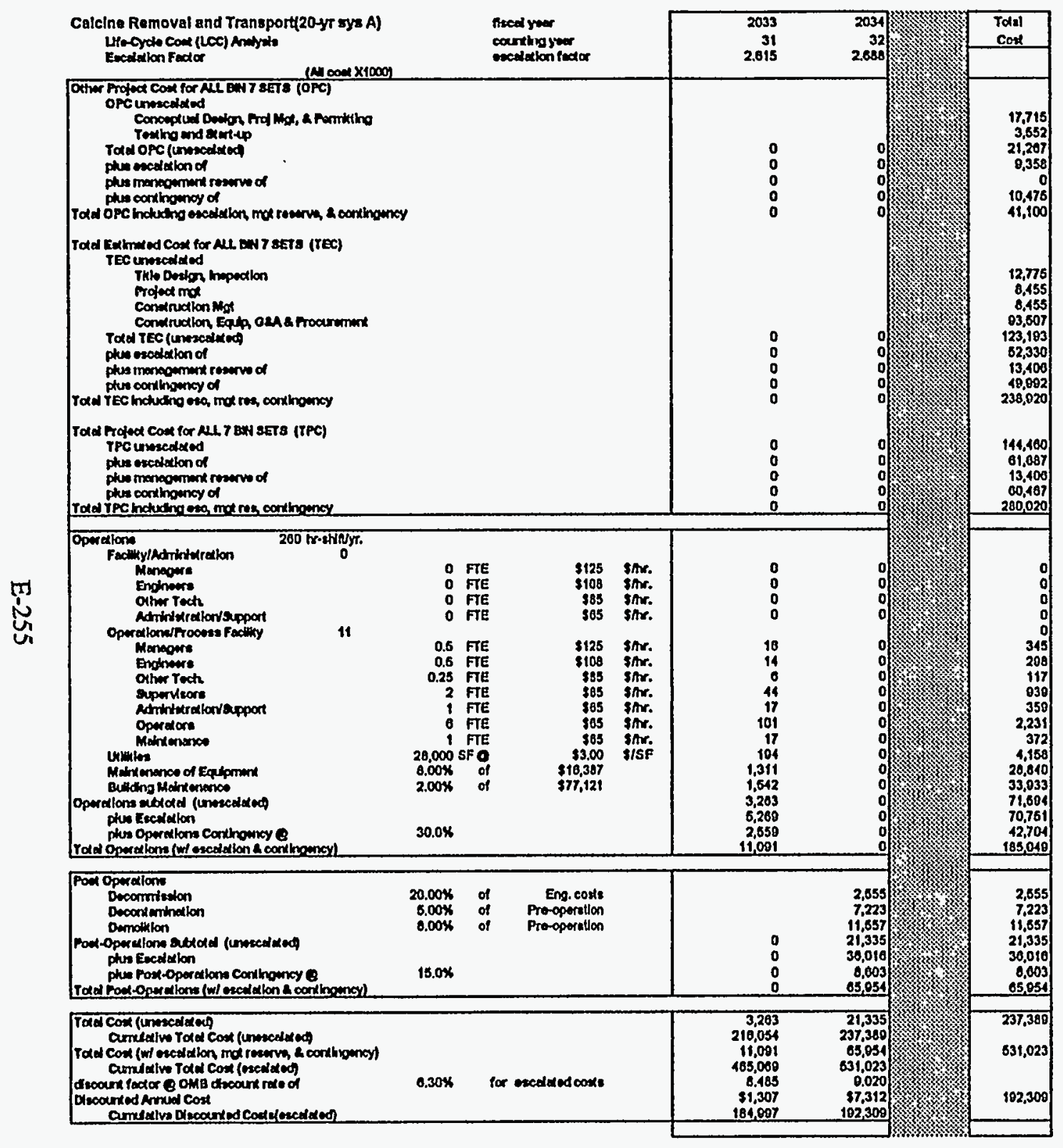




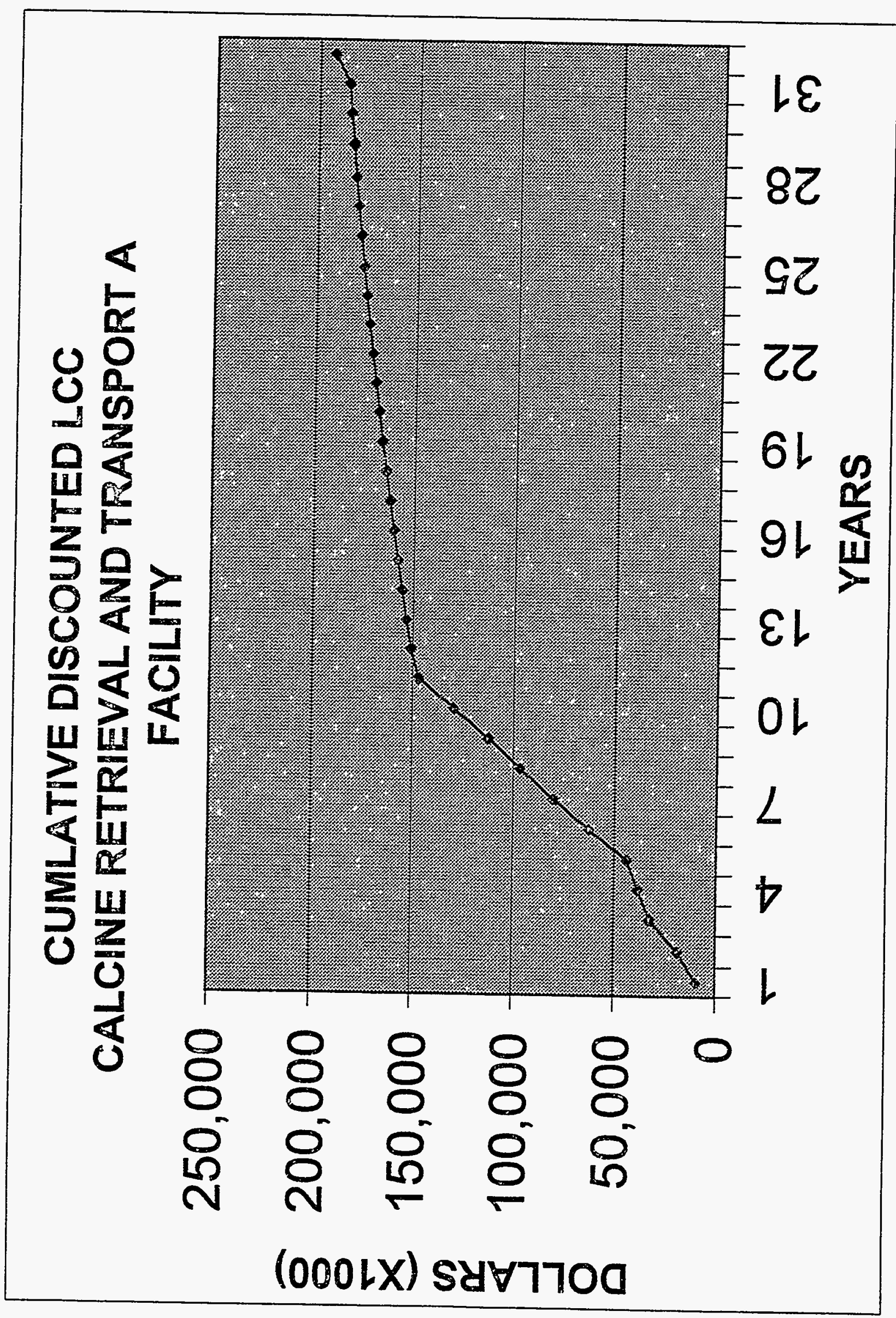




\section{TOTAL ANNUAL COST(escalated) CALCINE RETRIEVAL AND TRANSPORT A FACILITY}

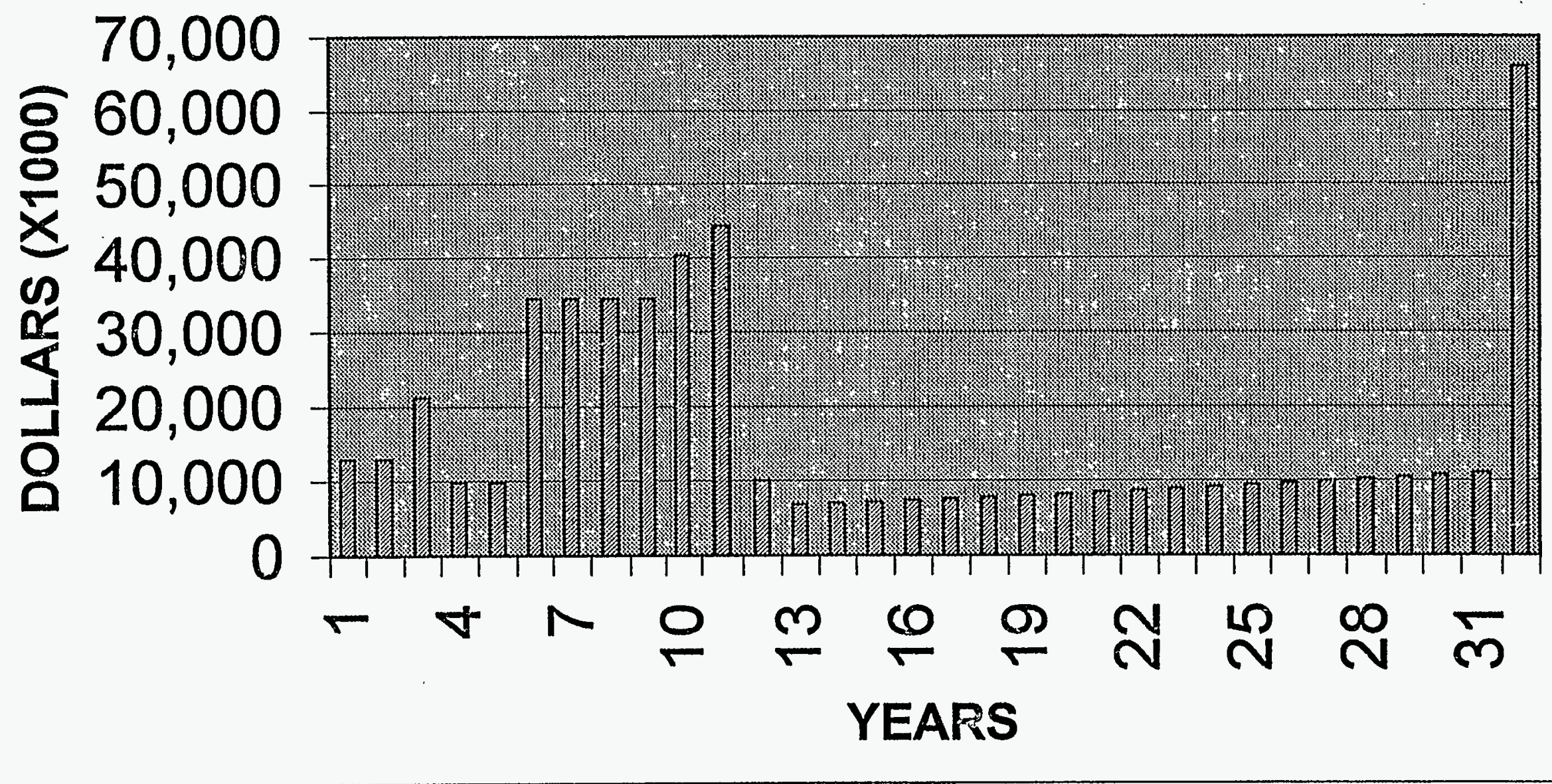




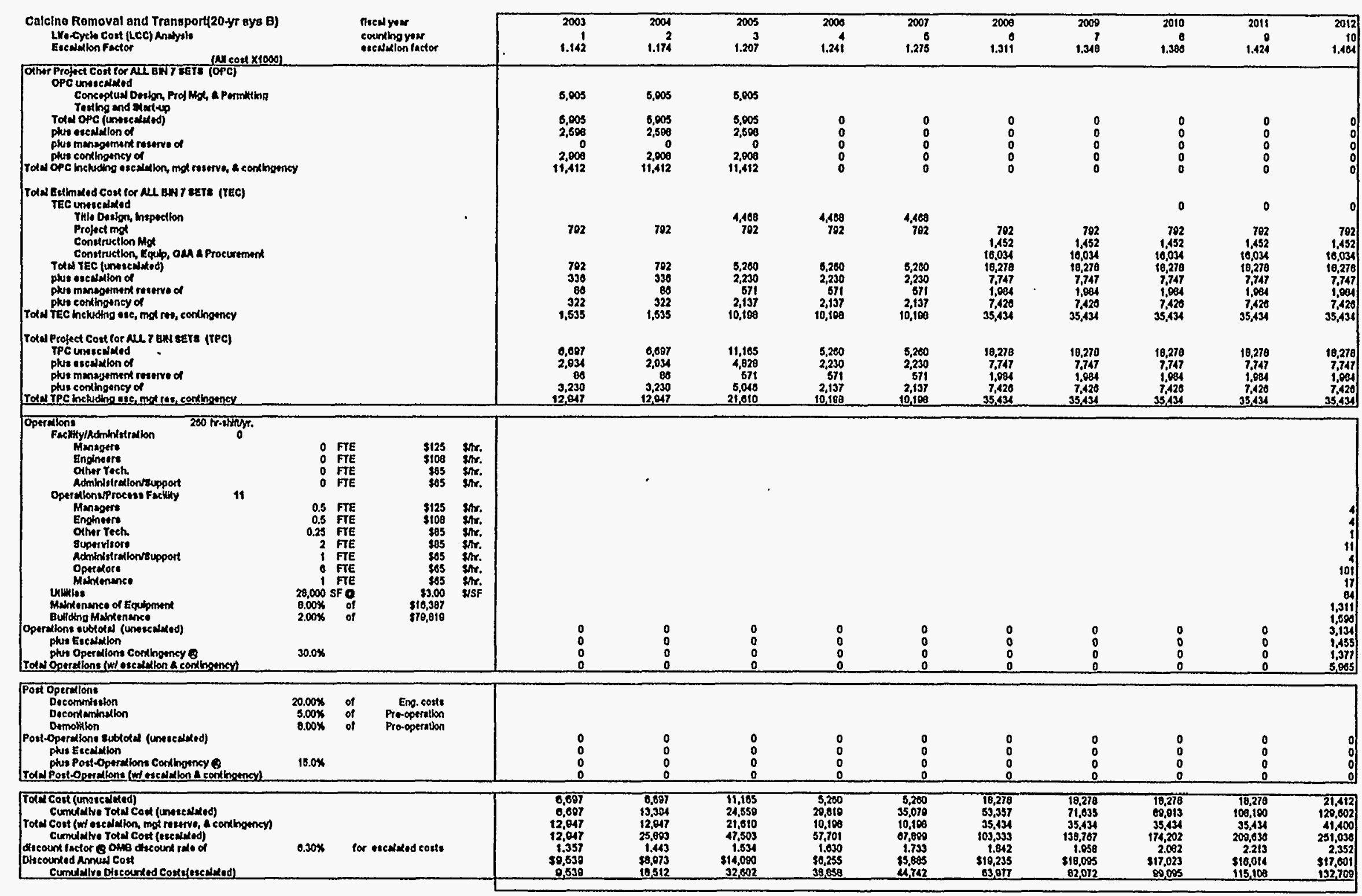




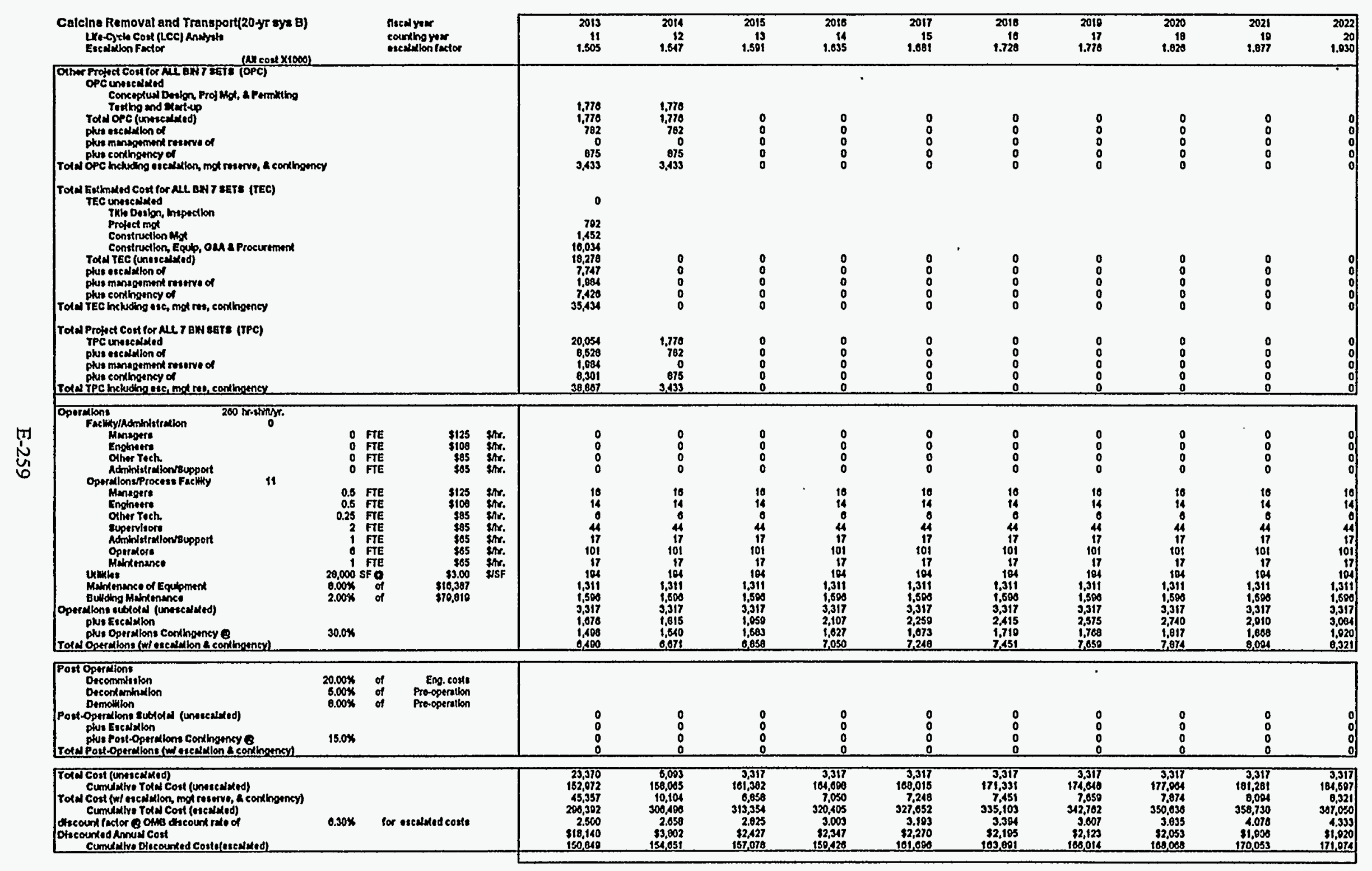




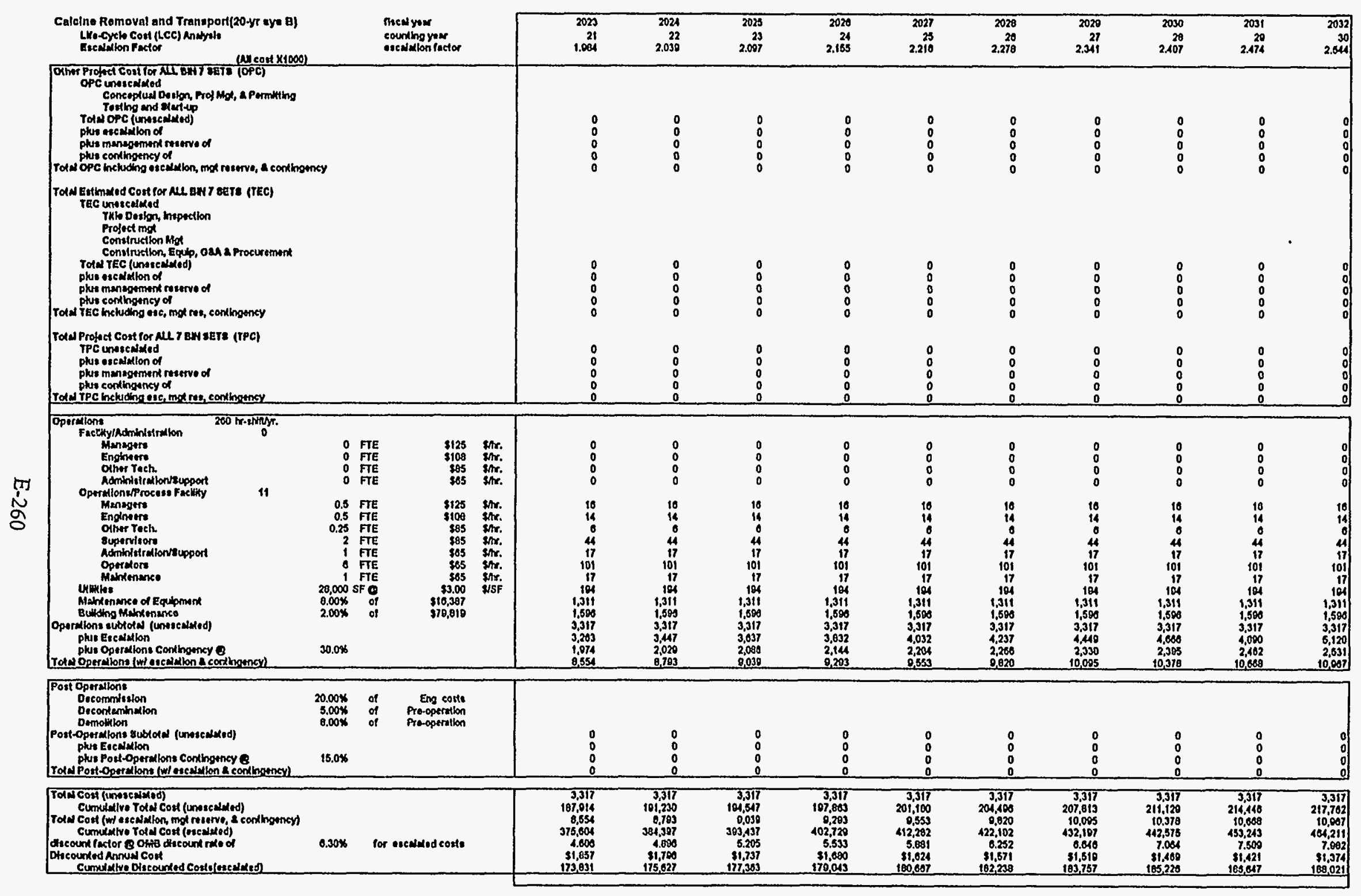




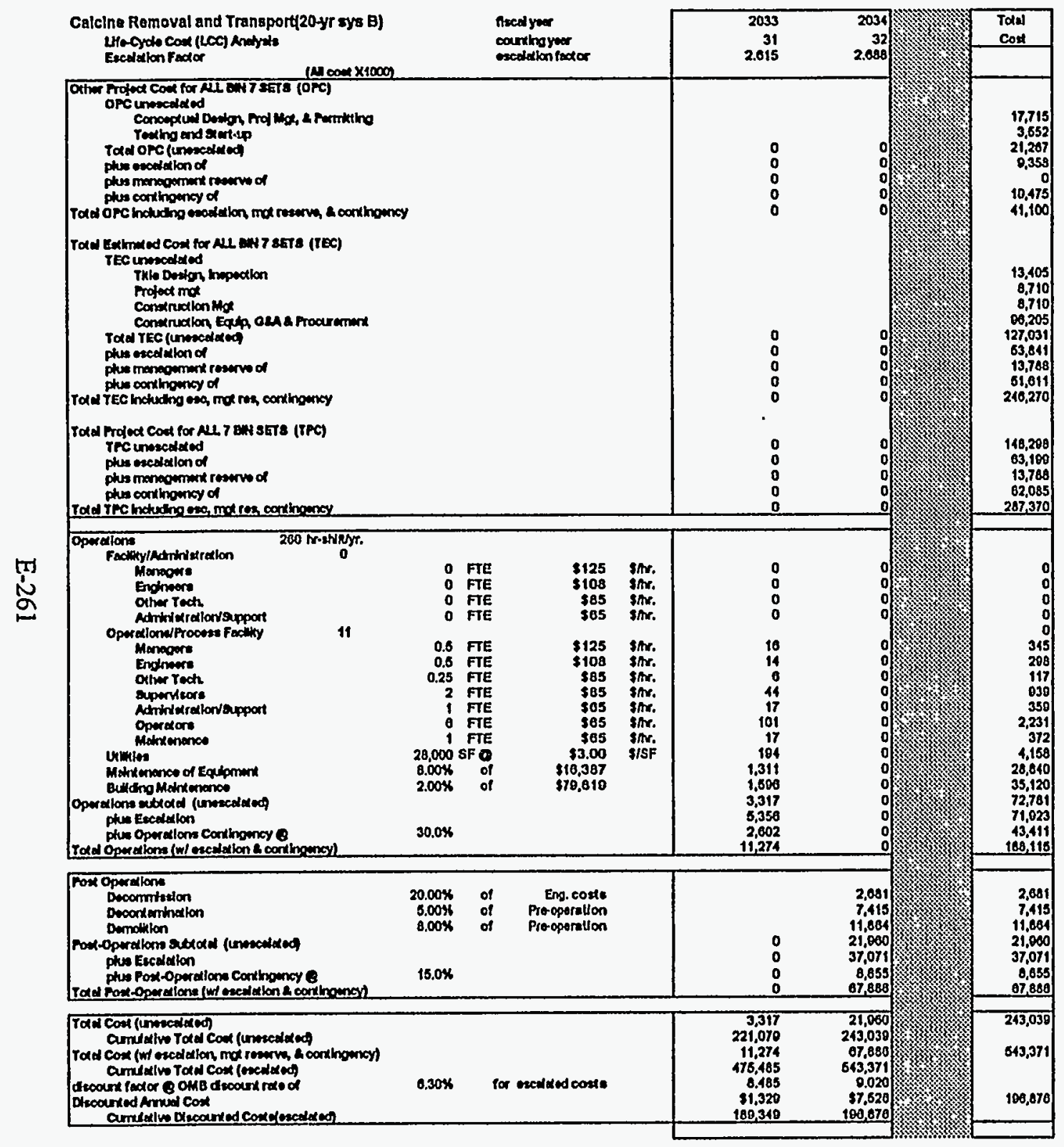




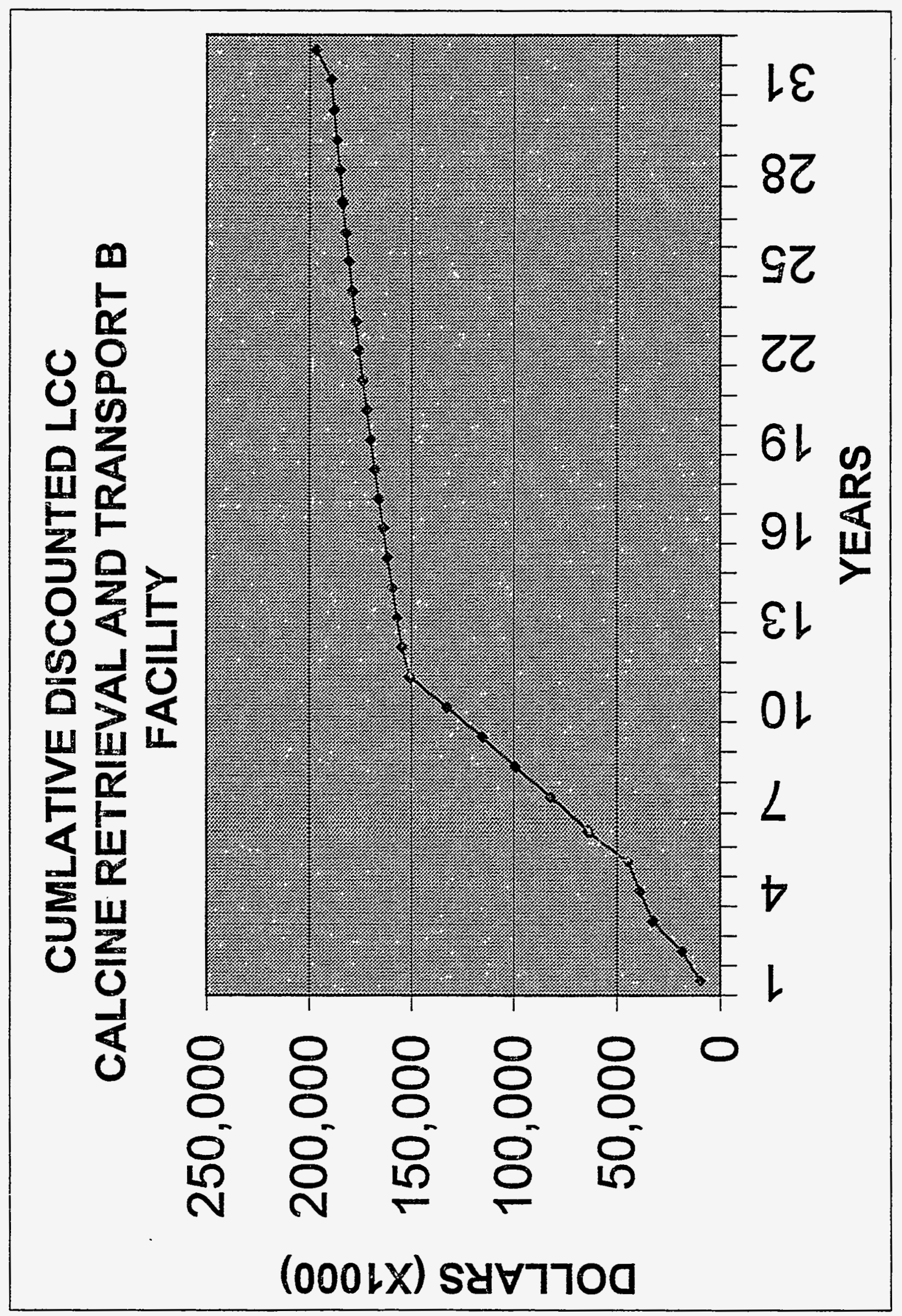




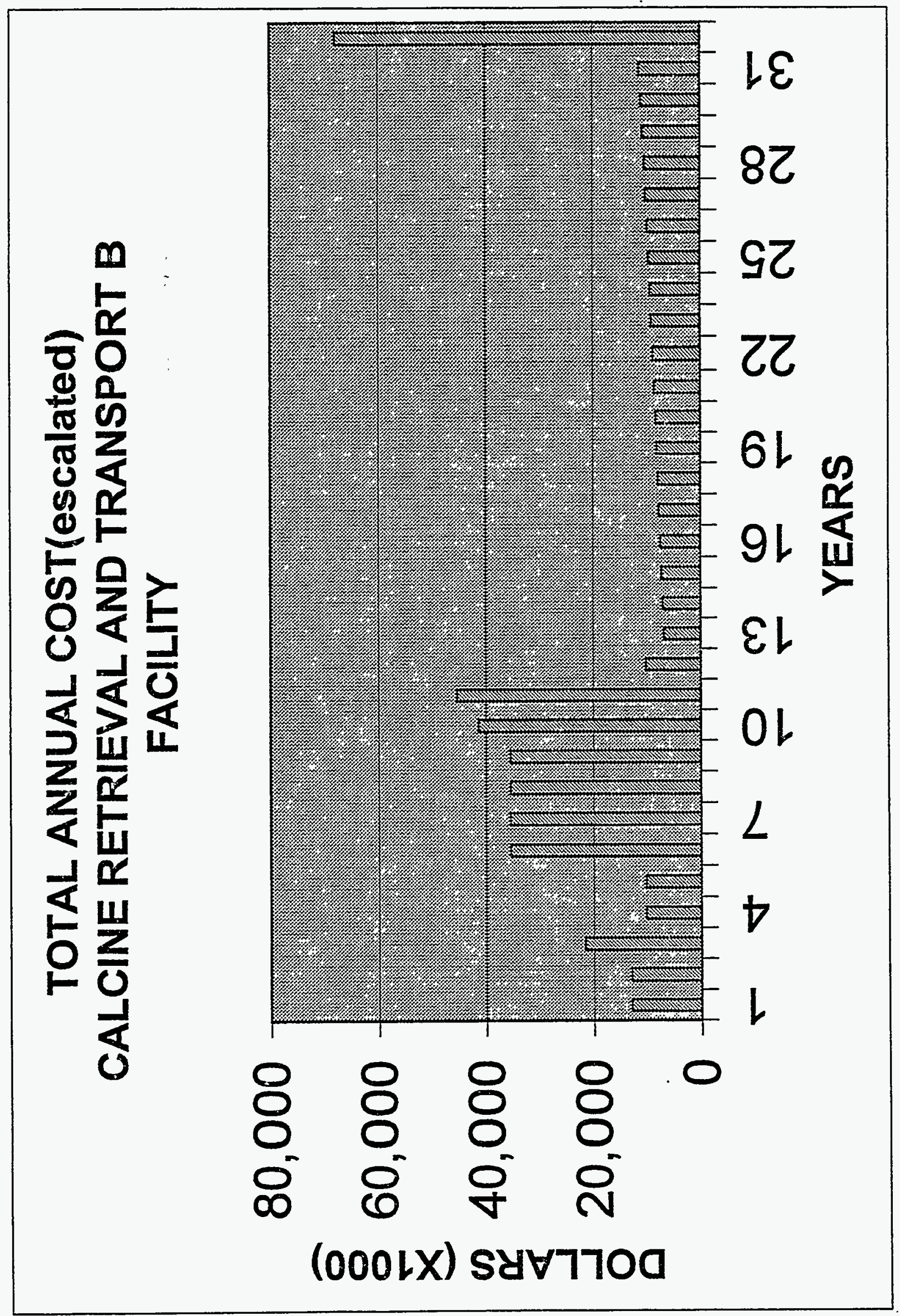


Subtask

Title: Regulatory Requirements for the Design, Construction, and Operations of the ICPP Proposed Waste Processing Facilities

Summary: This EDF identifies and provides the existing environmental regulations and codes pertaining to the design, construction, operations, and performance of the proposed waste treatment and storage facilities at the Idaho Chemical Processing Plant (ICPP). This study also presents an assessment of the current NRC regulations and their potential applicability to the proposed facilities if the facilities were to be licensed by the NRC in the future. The NRC requirements for regulating DOE facilities or activities have not been defined yet. The NRC requirements to be applied will need to be determined by the appropriate NRC and DOE Task Forces.

The principal sources of requirements for the design, construction, and operations presented here are the Department of Energy (DOE), the DOE Idaho Operations Office (DOE-ID), the Environmental Protection Agency (EPA), the Idaho laws and regulations, the National Environmental Policy Act (NEPA), and other local codes and standards. The proposed facilities under consideration in this stidy will provide waste retrieval, treatment, and interim storage capabilities. They will process various wastes that are considered mixed wastes. These are wastes that contain both radioactive and RCRA hazardous constituents. The RCRA constituents include characteristic heavy metals and "listed" hazardous constituents, as defined in 40 CFR 261, subparts $C$ and $D$. The management of the wastes, as well as the facilities, is subject to the - requirements of both the EPA and the Atomic Energy Act (AEA). The specific requirements for radioactive waste management developed under the AEA are administered through the DOE. The proposed treatment facilities are expected to process several types of waste and to convert them to distinct waste forms that are suitable for disposal. The regulatory requirements for the disposal of the various waste forms resulting from the proposed treatment options and the criteria of the potential target repositories are described in detail in INEEL/EXT-97-01147. It is assumed that the wastes resulting from the treatment options will be delisted and will no longer be considered RCRA hazardous waste prior to being sent to interim storage facilities.

Existing NRC requirements apply to commercial, non-DOE, facilities. The degree of applicability of these requirements to the proposed facilities should be determined by the NRC and the DOE, with input from the DOE contractor. Of the existing NRC regulations, it has been determined in this study that 10 CFR 61 will apply to the proposed near-surface disposal facility for the grouted Low-Activity Waste (LAW) or grouted LLW, and 10 CFR 72 will be applicable to the proposed interim storage facilities for the vitrified, Hot Isostatic Pressed (HIPed), or grouted High-Level Waste (HLW), and for the liquid High-Activity Waste (HAW) and vitrified HAW storage facilities. Independently, it was determined by Leroy and Morgan in "Nuclear Regulatory Commission (NRC) Licensing Assessment for the Idaho National Engineering and Environmental Laboratory (INEEL) High-Level Waste program," April 23, 1997, that 10 CFR 30 and 10 CFR 70 
Project File Number $\quad$ 02BD7

will apply to the following facilities:

- 10.CFR 30 for the LAW collection and grouting facilities and for the collection and treatment of the LLW from the INEEL ongoing operations.

- 10 CFR 70 for the separations facility, for the interim storage of liquid HAW resuiting from the separation processes; and for the HAW vitrification facility.

- 10 CFR 70 for the $H I W$ vitrification, HIPing, or grouting facilities.

- 10 CFR 70 for the calcine retrieval and dissolution facilities.

Additional NRC regulations applicable to the proposed facilities are in 10 CFR 2, 10 CFR 19, 10 CFR 20, 10 CFR 21, 10 CFR 50, $10^{\prime}$ CFR 51, 10 CFR 52, and 10 CFR 73. 10 CFR 71 and 49. CFR 173 (Department of Transportation) contain requirements for the packaging and transportation of waste. These requirements could have impact on the design and operations of the storage facilities. The existing facilities that will be modified to be used for storage of treated HLW or HAW are anticipated to be exempted by DOE from any further jurisdiction of NRC. Such a jurisdiction would be excessively, difficuit, costly, and complex to apply. All the work requirements for the modification of the existing facilities are expected to be performed in accordance with the DOE/RW/0333P, "Quality Assurance Requirements and Description.".

If DOE facilities become regulated by NRC, the jurisdiction of other currently government applicable authorities will not automatically or necessarily cease. In particular, it is expected that. local, State, Federal EPA, and some DOE regulatory requirements would still apply.

In addition to all the regulatory requirements established by the various government authorities discussed above, the schedule of the construction and operations of the proposed facilities must meet the terms and the dates of the commitments as stated in the Settlement Agreement between the State of Idaho and the DOE.

Distribution (complete package): HIL EIS Library, also contained in INEEL-EXT-01389 (VWO), INEELEXT-97-01392 (HWO), INEEI-EXT-97-01400 (CWO), INEEL-EXT-97-01399 (DCWO), and INEEL-EXT97-01428 (TRU Separations), S. L. Austad MS 3650, J. B. Bosley MS 3428, R. E. Dafoe MS 3765, W. H. Landman MS 3625, A. E. Lee MS 3765, D. A. Lopez MS 3765, B. R. Helm MS 3765, J. J. McCarthy MS 3625, T. A. Solle MS 3428, N. E. Russell MS 3765, D. S. Vandel MS 3625.

Distribution (summary package only): D. J. Harrell MS 3211, K. L. Williams MS 3765.

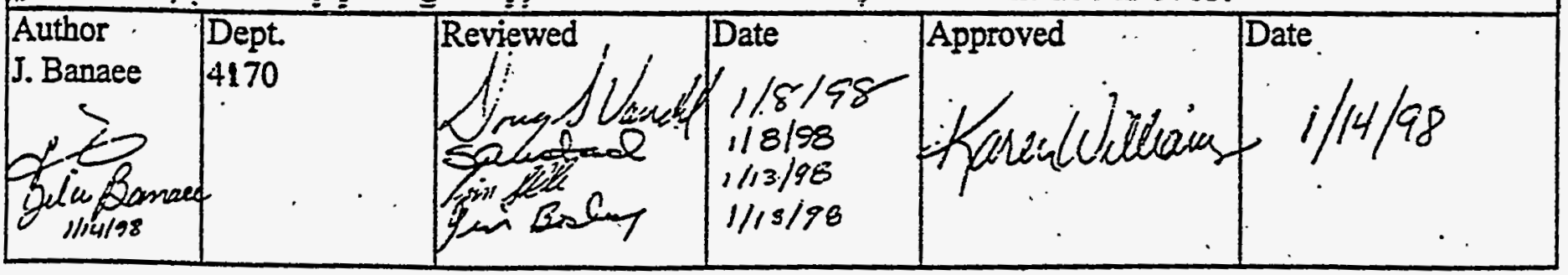




\section{INTRODUCTION}

This study identifies and presents the existing environmental regulations and standard codes for the design, construction, operations, and performance of the proposed waste treatment and storage facilities at the Idaho Chemical Processing Plant (ICPP). Also, it provides the applicable existing Nuclear Regulatory Commission (NRC) regulations and guidance documents to the proposed facilities if they were to be licensed by the NRC in the future. The existing licensing process and related licensing issues applicable to the proposed facilities are also discussed.

The environmental regulations are primarily based on safety and health considerations. The standards define the requirements that protect human health and the environment. The principal sources for the standard regulations and codes presented here are:

- Environmental Protection Agency

- Idaho Laws and regulations

- National Environmental Policy Act (NEPA)

- Department of Energy

- DOE Idaho Operations Office (DOE-ID)

- Other applicable standards and codes

In addition to the current regulations, the governing standards for the facility design and performance considerations of the future (i.e., DOE Orders 435.1) drafted at the time of preparation of this report are also presented.

The facilities under consideration in this study will provide waste retrieval, treatment, and interim storage capabilities. They will process various wastes that are considered mixed wastes. These are wastes that contain both radioactive and Resource Conservation and Recovery Act (RCRA) hazardous constituents. The RCRA constituents include characteristic heavy metals and "listed" hazardous constituents, as defined in 40 CFR 261, subparts C and D. The management of the wastes, as well as the facilities, is subject to the requirements of both the EPA and the Atomic Energy Act (AEA). The specific standards for radioactive waste management developed under $\mathrm{AEA}$ are administered through the $\mathrm{DOE}$.

The proposed treatment facilities are expected to process several types of waste to convert them to distinct waste forms that are suitable for disposal. The wastes resulting from the proposed treatment options are assumed to be delisted prior to being transported to the proposed interim. storage facilities. The waste forms are produced starting from a variety of mixed wastes including high-level liquid waste (HLLW), Sodium-bearing liquid waste (SBW), and other radioactive wastes form ongoing operations (1996-2012), known as the newly generated wastes. (NGW). These wastes have been stored in the Tank Farm at the ICPP. Most of the HLLW have been calcined and stored at the Calcined Solids Storage Facility (CSSF).

The EPA has established treatment standards under the RCRA Land Disposal Restrictions (LDRs), in 40 CFR 268, for hazardous waste constituents prior to land disposal. The regulatory 
requirements for the disposal of the various waste forms resulting from the proposed options and the criteria of the potential target repositories are described in detail in INEEL/EXT-97-01147.

\section{PROPOSED WASTE TREATMENT OPTIONS}

Several treatment options are being proposed including four non-separations and two separation processes. In addition, a no-action alternative will likely be considered, defined as the continuation of the current practice of calcination and storage in stainless steel bins at ICPP. The non-separation options include: (a) vitrified waste option (VWO), (b) Hot Isostatic Pressed (HII) waste option (HWO), (c) direct cementitious waste option (DCWO), and (d) cementitious waste option (CWO). The separation processes are expected to generate up to three different waste streams; designated as high activity waste (HAW), low activity waste (LAW), and . transuranic (TRU) waste. These options are summarized below:

\section{Vitrified Waste Option}

This option involves the following steps: 1) calcination of HLLW, SBW, and the NGW, 2) vitrification of all the calcine wastes (existing and future) and placing in canisters $\left(2^{\prime} \times 10^{\prime}\right.$ or other canisters approved by the repository/NRC), and 3) interim storage prior to shipment to a HLW repository.

Process duration: 20 year Schedule

\section{HIP. Waste Option (HWO)}

As in the VWO, the wastes will be calcined but instead of being vitrified, they will be directly HIP processed and placed in canisters (2' $\times 10^{\prime}$ or other canisters approved by the repository (NRC), and 3 ) will be sent to an interim storage facility prior to shipment to and disposal at a HLW repository.

Process duration: 20 year schedule

\section{Cementitious Waste Option (CWO)}

This option includes calcining the HLLW, retrieving the calcine wastes, and recalcining with the SBW in the modified New Waste Calcining Facility (NWCF), grouting in canisters ( $2^{\prime} \times 10^{\prime}$ canisters), and sending to an interim storage facility for transport to and ultimate disposal at an off-site HIW disposal facility. It is proposed that the cementitious waste would be suitable for disposal at the Nevada Test Site (NTS) using Greater. Confinement Disposal (GCD) facility. 
Currently, the GCD facility has not been approved for disposal of HLW and waste acceptance requirements for the GCD facility have not been defined. Pursuant to the 1987 Nuclear Waste Policy Amendments Act (NWPAA), the Yucca Mountain in Nevada is designated for characterization as the only candidate site for a HLW geologic repository. However, projections of future wastes suggests a need for a second repository at some time in the future; or expansion of the first potential repository. Criteria for acceptance and disposal of waste at the potential HLW repository at the Yucca Mountain have not been finalized. The current waste acceptance criteria are preliminary at the present time. These criteria are covered in detail in INEEL/EXT97-01147.

Process duration: 5 year schedule

\section{Direct Cementitious Waste Option}

The DCWO consists of step 1 of the VWO, then direct grouting of all the calcined wastes and packaging in canisters (2' $\times 10^{\prime}$ or other canisters approved by the repository/NRC), and interim storage prior to shipment to an off-site HLW repository such as the NTS-GCD, if approved for the HLW disposal, or possibly to a potential HLW geologic repository at the Yucca Mountain. This option is planned to have the same processing time as the CWO with the difference in starting date.

\section{Separations Options}

Two waste separation options have been proposed: HAW/LAW, known as full separations, and TRU separations. The full separations option involves calcining the HLW and SBW, retrieving and dissolving the calcine, and feeding the dissolved calcine and the remaining liquid SBW and the NGW to a waste separations facility to separate them into the HAW and LAW streams. The NGW, if classified as LLW, would bypass the separations facility and would ultimately be combined with the LAW:

In the TRU separations, as in the HAW/LAW separation option, the dissolved calcine and the remaining liquid SBW, and the NGW will be fed to a waste separations system. Two separation alternatives are being considered under this option, designated as (1) TRU/LAW Class $C$ and (2) TRU/LAW Class A/HAW. In the first alternative, the wastes would be separated into TRU waste and LAW. The TRU waste fraction is expected to contain alpha-emitting TRU radionuclides with half-lives greater than twenty years. The remaining waste stream, designated as LAW Class C, would contain Cesium (Cs) and strontium (St) isotopes, and low activity waste portion. It is anticipated that the LAW in this alternative would meet the definition of NRC LLW Class C. In the second alternative, $\mathrm{Cs}$ and $\mathrm{Sr}$ will be separated as HAW, and the remaining waste from separations will be designated as TRU and LAW. The LAW is expected to meet the definition of NRC LLW Class A. 
The HAW is planned to be vitrified using the same process described in the VWO and be shipped to a HLW geological repository for permanent disposal. The HAW containing isolated $\mathrm{Cs}$ and $\mathrm{Sr}$ would be stored at the INEEL awaiting disposal in a HLW geologic repository or an alternate approved disposal facility. The TRU waste stream would be converted to a solid form to be send to the Waste Isolation Pilot Plant (WIPP) for disposal. The LAW would be grouted and shipped to a LLW disposal facility. The disposal options being considered for the grouted LAW are the CSSF, the tanks at ICPP, or a LIW near-surface disposal facility. The -requirements for the design and operations of a near-surface LLW disposal facility are covered in detail in EDF-FDO-008. This reference is contained in INEEL/EXT-98-00051.

Based on the NRC source term definition of HLW (10 CFR 60.2), the HAW, LAW and the TRU waste streams are actually considered HIW. These wastes do not conform to the existing classification for radioactive waste. Although, the separations altematives and the resultant waste streams may be technically and economically feasible and attractive, they will need to receive evaluation, redefinition of types of waste, and the concurrence of applicable government authorities. It is assumed that a determination will be made by the appropriate authorities (e.g., the DOE and the NRC) that the TRU waste and the LAW streams meet the TRU waste and the NRC LLW-Class A or Class C definition, respectively. The LAW also meets the definition of incidental waste in the NRC evaluation of HLW separation processes at Hanford Site (58 FR, "State of Washington and Oregon, Denial for Petition for Rulemaking," U.S. Nuclear. Regulatory Commission, "March 4, 1993, p. 12342.). The HAW would be considered HLW and can be classified as HIL.

\section{ASSUMPTIONS}

1) All of the wastes produced from the treatment and storage facilities will meet the requirements enforced by the EPA, the DOE, the DOT, the NRC, and other potential target repositories for the disposal.

2) Under EPA 40 CFR 268.42(b), an equivalency petition for using alternative waste treatment technologies including CWO and DCWO to borosilicate glass ${ }^{a}$ will be granted by the EPA.

3) All of the wastes resulting from the various treatment options will be delisted prior to being transported to the interim storage facilities. The EPA Upfront Exclusions for the petitioned wastes will be granted to delist the RCRA listed hazardous waste codes. The EPA delisting

\footnotetext{
"Vitrification using borosilicate glass technology is considered by the EPA a best demonstrated available technology (BDAT) for treatment of mixed HLW (55 Federal Register (FR), June 1, 1990, p.22627). DOE's studies of glass-ceramic process and comparison of glass-ceramic process and waste form with borosilicate glass waste have shown that glass-ceramic waste form meets the definition of EPA vitrification and borosilicate glass. EPA has concurred with the DOE conclusion and has determined that the glass-ceramic process is an acceptable technology to meet BDAT (see 57 FR, May 26, 1992, p. 22024).
} 
criteria and processes for preparing a delisting petition are contained in "Petitions to Delist Hazardous Wastes: A Guidance Manual," Second Edition, PB93-169365, March 1993. The Upfront Exclusions may be granted for wastes and/or waste residues that have not been generated, but will be generated in the future. The EPA will evaluate the petitioned wastes based on available information such as the characteristics of the untreated wastes, process description, and bench-scale or pilot scale treatment data.

4) An off-site facility would need to be approved for the disposal of $H L W$ resulting from the CWO, DCWO, and HWO.

\section{ENVIRONMENTAL REGULATIONS, DOE CRITERIA, AND OTHER STANDARD CODES}

\subsection{FEDERAL AND STATE LAWS AND REGULATIONS}

\section{Resource Conservation and Recovery Act}

The RCRA has established minimum national standard requirements which apply to owners or operators of all facilities that treat, store, or dispose of hazardous waste. The State of Idaho has the authority to implement the RCRA requirements through the Idaho Department of Health and Welfare (IDHW): The State of Idaho adopted the Federal RCRA regulations, pursuant to the Idaho Hazardous Waste Management Act of 1983. The regulations are incorporated by reference as provided in the Federal requirements under 40 CFR into the "Idaho Rules and Standards for Hazardous Waste", under administrative code.known as Idaho Administrative Procedures Act (IDAPA) 16.01.05.

The RCRA requirements applicable to the hazardous waste facilities are defined in 40 CFR 264 (IDAPA 16.01.05.008), "Standards for Owners and Operators of Hazardous Waste Treatment, Storage, and Disposal Facilities" and in 40 CFR 270 (IDAPA 16.01.05.012), "EPA Administered Permit Programs: the Hazardous Waste Permit Program."

40 CFR 264 sets regulatory requirements for the design, construction, and operation of the facility, quality assurance program, testing and maintenance of the equipment, air emission standards, groundwater protection standards, security, inspection, personnel training, preparedness and prevention, contingency plan and emergency procedures, manifest system and record keeping, closure and post-closure, financial requirements, and use and management of containers.

The existing hazardous waste facilities used for any future hazardous waste management activities can continue operations while meeting the requirements in 40 CFR 265 (16.01.05.009), 
"Interim Status Standards for Owners and Operators of Hazardous Waste Treatment; Storage, and.Disposal Facilities". This will allow the existing facilities to continue operations while meeting minimum operational requirements defined in 40 CFR 265. To be qualified for interim status, the existing facility must have been in operation or under construction on November 19 , 1980 or have been in operation when the facility became subject to the RCRA requirements.

40 CFR 262.34 (subpart of 40 CFR 262 - IDAP'A 16.01.05.006), "Accumulation Time" and 40 CFR 268.50 (subpart of 40 CFR 268 - IDAPA 16.01.05.011), "Prohibitions on Storage of Restricted Wastes" contain requirements, conditions, and time limits for storage of hazardous wastes. Based on 40 CFR 262.34, a generator may accumulate hazardous waste on-site for 90 days or less without a permit or an interim status, provided that the generator meet the conditions specified in 40 CFR 262.34. A generator who accumulates hazardous waste for more than 90 days is an operator of a storage facility and is subject to the requirements of 40 CFR 264 and 40 . CFR 265, and the permit requirements of 40 CFR 270 unless the generator has been granted an extension to the 90-day period by the EPA. A 30-day extension may be granted at the discretion of the EPA on a case-by-case basis. A generator who accumulates hazardous waste greater than 100 kilograms but less than 1000 kilograms in a calendar month may accumulate hazardous .waste on-site for 180 days or less without a permit or without an interim status provided the generator meets the requirements stated in 40 CFR 262.34.

Under 40 CFR 268.50, the storage of hazardous wastes such as those present in the Tank Farm is prohibited, unless the following conditions are met:

(1) A generator stores such wastes in tanks, containers, or containment buildings on-site solely to facilitate proper recovery, treatment, or disposal and the generator complies with the requirements in 40 CFR 262.34, 40 CFR 264, and 40 CFR 265.

(2) An owner/operator of a hazardous waste treatment, storage, or disposal facility stores such wastes in tanks, containers, or containment buildings to facilitate proper recovery, treatment, or disposal, and an owner/operator must comply with the operating record requirements specified in 40 CFR 264.73 and 40 CFR 265.73.

An owner/operator of a treatment, storage, or disposal facility may store hazardous waste restricted from land disposal beyond one year provided that the owner/operator proves to the EPA that such storage was solely for the purpose of facilitating proper recovery, treatment, or disposal.

The prohibition in storage does not apply to hazardous wastes that meet the IDR treatment standards and treatment equivalency as defined in 268.42(b). However, if the waste is still listed, the generator must comply with the RCRA requirements for hazardous waste storage. 


\section{Permit Requirements}

Various permits are required prior to the construction and operations, and during the operations of the proposed facilities. These include RCRA permit, air emissions permit, wastewater discharges permit, etc. In addition, separate permits may be needed once the processes or activities are better known, as individual pilot plant operations or modifications to the existing facilities/systems may require separate permits. The applicable permit requirements are described below. A summary of the permit requirements and regulatory drivers is presented Section 5 .

\section{RCRAPermit}

All facilities that treat, store, or dispose of hazardous wastes are required to obtain a RCRA permit during the active life (including the closure period). $40 \mathrm{CFR} 270$ establishes the requirements for obtaining a Permit. A RCRA.Permit application consists of two parts, Part A and Part B. Part A of the permit application is a short standard form that collects general information about the treatment, storage, or disposal facility. Part B of the permit application includes a much more detailed technical description of the facility. The permit application covers all aspects of the design, construction, operation, monitoring, and maintenance of the facility. The requirements for Part A permit application are in 40 CFR 270.13, and for $P$ art $B$ Permit application are in 40 CFR 270.14 through 29.

Once the owner or operator $0 \hat{1}$ a facility has submitted a permit application, the regulator conducts an in-depth evaluation to determine if the application satisfies the RCRA requirements. For the new hazardous waste facilities, Parts A and Part B of the permit application must be submitted a least 180 days prior to physical construction is expected to commence. For the existing hazardous waste facilities, the requirements to submit an application is satisfied by submitting only Part A application to operate under interim status until the permitting agency sets a date for submitting Part $B$ of the application.

\section{Air Permit}

Air permits will be required from the State of Idaho and/or EPA Region X prior to construction and operations of each of the treatment, storage, and disposal facilities with radioactive and nonradioactive emissions sources. The existing facilities that will be used for the proposed waste processing activities may require permit modification if the existing permit does not satisfy the permit requirements for the proposed use.

The Clean Air Act (CAA) sets permit requirements and emission standard limits. The CAA requirements are implemented by the IDHW under Idaho codes (see Idaho Codes and regulations below) or by EPA Region X. The CAA implementing regulations are in 40 CFR $50,52,60,61$, $62,63,70,77$, and 124 . The air permit requirements are briefly described below. 
For nonrad emissions a Permit to Construct (PTC) will be required from the IDHW for each of the new emission points prior to the construction or modifications of a facility. Individual pilot plant systems may require separate PTCs once the processes are known. Hazardous Air Pollutants (HAPs) and the Toxic Air Pollutants (TAPs) will need to be quantified prior to permitting.

The National Emission Standards for Hazardous Air Pollutants (NESHAPs), Subpart $H$ sets the standards for the radioactive air emissions. The proposed facilities will qualify as radiological sources if they emit any radiological emissions. They are regulated by the EPA under the NESHAPS and the State of Idaho for radioactive air emissions. The state of Idaho treats the INEEL as one large facility. Currently, the maximum off-site limits for the total INEEL radiological emissions is $10 \mathrm{mrem} / \mathrm{yr}$.

The National Ambient Air Quality Pollutants Standards (NAAQS) has established requirements for particulate matter, sulfur dioxide, ozone, nitrogen dioxide, carbon monoxide, fluoride, and lead. If their emissions are significant as defined in IDAPA 16.01.01.88; they must comply with the requirements of the Prevention to Significant Deterioration (PSD). The Best Available Control Technology (BACT) must be used to control pollutants if compliance with the PSD is required.

The air emissions must be calculated for each of the new facilities to determine the permit -requirements and compliance with the regulatory standards, and to identify how they impact the sitewide total emissions at the INEEL because the State of Idaho treats the INEEL as one large facility. The determination of the expected air emissions is usually done during the Title II design.

Appendix $D$ to 40 CFR 61 should be used for estimating the radionuclide emissions from the new sources to determine if a NESHAPs approval to construct is needed. If the estimated dose is greater than $0.1 \mathrm{mrem} / \mathrm{year}$, the NESHAPs application will be required. In such case, a PSD evaluation will required by the State of Idaho.

Some of the process vents associated with the hazardous waste treatment units must meet the RCRA air emission standards in 40 CFR 264/265 Subparts AA. A process vent is any openended stack or pipe that is vented to the atmosphere. 40 CFR 264/265 Subparts BB and CC contain air emissions standards which are applicable only to certain types of processes such as equipment leaks, tanks, and containers.

The EPA proposed MACT Rule which enforces limits on air pollutants applies to hazardous waste incinerators or other comparable facilities which burn hazardous waste and/or are qualified as an incinerator by the EPA. This study assumes that the MACT Rule will be applicable to the vitrification and HIPing facilities. The MACT Rule sets emission limits for dioxin/furan, hydrocarbons, chlorine, carbon monoxide, lead, cadmium, mercury, antimony, arsenic, beryllium, chromium, and particulate matter. 


\section{Wastewater Effluent Discharges and Drinking Water Permit}

The EPA has established requirements for stormwater and nonstormwater discharges into the environment under the National Pollution Discharge Elimination System (NPDES). The NPDES contains the requirements that control the discharge of pollutants to waters of the U.S. (e.g., Big Lost River) as defined in the Clean Water Act (CWA) in 40 CFR 122. These sources can include sanitary, industrial processes, and storm water runoff from industrial and construction areas.

A permit under the NPDES is required for storm and nonstorm waters (e.g., service water, sewer discharges). The INEEL has a general NPDES permit. Therefore, the existing INEEL XPDES permit should be evaluated to determine if there is a need for modifications of the INEEL permit or addendum to the permit to satisfy the permit requirements for the proposed facilities.

Wastewater Land Application Permits are required for construction, modifications, and operation of facilities that dispose of municipal and industrial wastewater to the land surface. The requirements are defined in 40 CFR 122 (CWA).

Pursuant to the CWA, the facilities that engage in storing, transferring, and consuming oil and oil products which could reasonably be expected to discharge oil in the Big Lost River or other waters of the U.S. must have Spill Control Prevention and Countermeasures Plans. The Plans are required if the oil discharges are in harmful quantities that violate the applicable water quality standards and cause harm to the human health and environment.

\section{Idaho Laws and Regulations}

This section lists the Idaho codes and standards for air and water pollution control and for releases into the environment: The standards are based on the Federal requirements established by the CAA, the CWA, and the RCRA.

Idaho Code 39-44, "Hazardous Waste Management Act"

IDAPA 16.01.05, "Rules and Standards for Hazardous Waste" .

IDAPA 16.01.01, "Rules for the Control of Air Pollution in Idaho"

IDAPA.16.01.01.161, "Toxic Substances"

IDAPA 16.01.01.210, "Demonstration of Reconstruction Compliance with Toxic Standards"

IDAPA 16.01.01.575, "Air Quality Standards and Area Classification"

IDAPA 16.01.01.650, "Rules for Control of Fugitive Dust"

IDAPA 16.01.09, "Idaho Radiation Control Rules"

Idaho Code 39-36, "Water Quality Act"

IDAPA 16.01.02, "Water Quality Standards and Wastewater Treatment"

IDAPA 16.01.08.500, "Design Standards for Public Drinking water Systems".

IDAPA 16.01.08.551, "Construction Requirement for Public Water Systems". 
IDAPA 16.01.08, "Idaho Rules for Public Drinking Water Systems"

IDAPA 16.01.17, "Wastewater Land Application Permit Regulations"

\section{CFR 191, "Environmental Radiation Protection Standards for Management and} Disposal of Spent Nuclear Fuel, High-Level Waste and Transuranic Waste”

The EPA has set radiation protection requirements for management of radioactive waste in 40 CFR 19i. The radiation protection standards for management and storage of radioactive wastes apply to:

(a) Radiation doses received by members of the public as a result of the management (except for transportation) and storage of spent nuclear fuel or HLW or TRU waste at any facility regulated by'the NRC or by Agreement States, to the extent that such management and storage operations are not subject to the provisions of 40 CFR 190, "Environmental Radiation Protection Standards. for Nuclear Power Operations"; and

(b) Radiation doses received by members of the public as a result of the management and storage of spent nuclear fuel or HLW or TRU waste at any disposal facility that is operated by the DOE and that is not regulated by the NRC or by Agreement States.

\section{CFR 257, "Criteria for Classification of Solid Waste Disposal Facilities and Practices"}

The proposed LLW land disposal facility at the INEEL will be comparable to the LLW disposal facility of the Radioactive Waste Management Complex (RWMC). The Idaho:Department of Environmental Quality (DEQ) evaluated the applicability of federal and state regulations to the RWMC LLW disposal facility. The $\mathrm{DEQ}^{\mathrm{b}}$ has concluded that in addition to other requirements, disposal of waste at RWMC is subject to Subtitle $D$ landfill standards, 40 CFR 257. The environmental standards required by 40 CFR 257 are based on safety and health considerations which protect human health, wildlife, and the environment. The DOE or the NRC requirements for design and performance of a LLW disposal facility are much more stringent than those in 40 CFR 257 and supersede the subtitle D landfill standards.

40 CFR 257 requires that disposal facilities or practices in floodplains not restrict the flow of the base flood, reduce the temporary water storage capacity of the floodplain, or result in washout of solid wastes which pose a hazard to human life, wildlife, land or water resources. The disposal facilities shall not cause a discharge of pollutants into waters of the United States. Such a discharge would be a violation of the NPDES. Also, the facilities must not contaminate any underground drinking water source beyond solid waste facility boundary or beyond an alternative specified boundary.

'DEQ letter to Jay Mitchell, Manager of LMITCO NEPA/Permitting, July 23, 1996. 


\subsection{DEPARTMENT OF ENERGY}

The AEA of 1954 authorizes the DOE to establish standard criteria to ensure safe operations of its facilities, and to protect human health and to minimize dangers to life and property. The DOE has developed a series of Orders and Directives. They contain standards that require the planning, design, and construction of DOE facilities be performed in a manner that will satisfy all applicable Federal, State, and local environmental, safety and health laws and regulations, and the DOE criteria. The DOE and DOE-ID standards applicable to this study include the following:

\section{DOE Order 6430.1A, "General Design Criteria"}

Compliance with DOE Order $6430.1 \mathrm{~A}$ is mandatory under the current LMITCO contract. DOEID Notice 430.1A, "Life Cycle Asset Management- ID expectations," requires that for facilities under the purview of the Defense ${ }^{c}$ Nuclear Facilities Safety Board (DNFSB), the DOE Order 6430.1A remains effective until 10 CFR 830.340, "Maintenance Management", and DOE Order 420.1, "Facility Safety", are finalized and incorporated into the LMITCO contract.

DOE-ID Notice 430.1A establishes the DOE-ID expectations of the contractor in areas covered by DOE Order 430.1, 'Life Cycle Asset Management", in managing the INEEL. This order incorporates private industry standards safety design criteria, and requires additionai nuclear safety criteria for nuclear facilities.

DOE Order $6430.1 \mathrm{~A}$ provides general and specific design standards, guidance, and practices for use in the DOE facilities. The standards are to provide levels of design for occupant life safety, reduction in loss of government property, functioning essential operations and confinement of radioactive and hazardous material. Division 13, Section 1300, General Requirements, and Section 1324, Radioactive Solid Waste Facilities, address general and specific design criteria. Also, Section 0900-99.0, Nonreactor Nuclear Facilities, contains additional criteria relevant to

\footnotetext{
"The term "defense" nuclear facility has not been defined anywhere in the regulations or by the DOE yet. I have spoken with a number of people in the LMITCO Mechanical, Civil, and Industrial Engineering Department and the DOE-ID (David Crandall, Scott. Jensen, Lee Williams, and others) to learn what might constitute a "defense" nuclear facility and to find out the difference between a defense nuclear facility and a nuclear facility. They were not sure about the definition of a "defense" nuclear facility. However, they all believe that the facilities under consideration in this study could be considered "defense" nuclear facilities because they will be used for management of the DOE defense related wastes. According to Scott Jensen, the RWMC LLW disposal facility is considered a "defense" nuclear facility.

The definition of a nuclear facility is in MCP-2446. Based on this document, a "nuclear facility" is a facility with operations that involve radioactive and/or fissionable material in such form and quantity that a nuclear hazard potentially exits to the employees or the general public. A nuclear facility includes nonreactor and reactor facilities.
} 
facility design. All of these criteria provide minimally acceptable requirements for the facility design. It should be noted that the applicable local building codes and models always take precedence on the issues covered in the DOE order and provide additional design requirements not covered in the DOE order.

\section{DOE-ID, "Architectural Engineering Standards" (AE)}

The AE contains general design requirements such as those defined in DOE Order 6430.1A, and additional specific construction codes. The following is a list of some of the applicable standards and codes included in the AE documents:

ICBO UBC, "Uniform Building Code, latest edition"

ICBO UFC, "Uniform Fire Code, latest edition"

29 CFR 1910, "Occupational Safety and Health Standards"

29 CFR 1926, "Safety and Health Regulations for Construction"

ADAAG, "Americans with Disabilities Act (ADA) - Accessibility Guidelines"

ASCE-4-86, "Seismic Analysis of Safety-Related Nuclear Structures"

ASCE-7-93, "Minimum Design Loads for Buildings and Structures" .

DOE-STD-93, "Natural Phenomena Hazard Performance categorization"

DOE-STD-1020-94, "Natural Phenomena Hazard Design and Evaluation Criteria for

Department of Energy Facilities" :

DOE-STD-1021-93, "Natural Phenomena Hazards Performance Categorization Guidelines for Structures, Systems, and Components"

\section{DOE Order 5820.2A, "Radioactive Waste Management"}

DOE Order $5820.2 \AA$ established policies and criteria for management of HLW, TRU, and LLW. The Order requires that radioactive and mixed waste be managed in a manner that is in compliance with all applicable Federai, State, and local environmental, safety, and health regulations and laws and DOE criteria.

Design objectives for facilities shall assure protection of the public and operating personnel from hazards associated with normal HLW operations, accident conditions, and the effects of natural phenomena. Other objectives are compliance with the DOE policies regarding nuclear safety, quality assurance, contingency plans, training, fire protection, pollution control, and safeguards and security protection for waste and protection of essential operations from the effects of potential accidents.

The development of large scale waste treatment facilities shall be supported by the appropriate documentation such ás NEPA documentation, construction design report including projected 
waste throughputs, and treatment methods, construction and operating cost estimates, and Safety Analysis Report (SAR).

All new HLW handling, transfer, and storage facilities shall be doubly contained. Where required, ventilation and filtration systems shall be provided to maintain radionuclide releases' within the guidelines specified in DOE Order 5481.1B, "Safety Analysis and Review System", DOE Order 5480.23, "Nuclear Safety Analysis Report"and other applicable orders discussed in this EDF. Ventilation systems shall be provided where the possibility exists for generating flammable and explosive mixtures of gases.(e.g., hydrogen or organic).

Nuclear criticality safety considerations and controls shall be evaluated for normal operations and, before any significant operational changes are made, to protect against an uncontrolled nuclear criticality incident. Each facility shall utilize remote maintenance features and other appropriate techniques to minimize personnel radiation exposure in accordance with DOE . 5481.1B, "Environment, Safety, and Health Program for Department of Energy Operations," DOE Order 5480.23, and DOE Order 5480.24, "Nuclear Criticality Safety."

Monitoring; surveillance, and leak detection capability shall be incorporated in the engineering systems (e.g., liquid level sensing devices and alarms for high-level waste liquid systems) to provide rapid identification of failed containment, and measurement of abnormal temperatures. The following, at a minimum, shall be monitored: temperature; pressure; radioactivity in ventilation exhaust, and liquid' effluent streams associated with $\mathrm{HLW}$ facilities. Where the possibility exists for the generation of flammable and explosive mixtures of gases, monitoring shall be conducted.

Training and qualification standards shall be developed and an up-to-date record of training status shall be maintained. Worker safety training must comply with the requirements of DOE 5480.1B and applicable Orders. Quality Assurance consistent with DOE Order 5700.6C, "Quality Assurance", shall be conducted in accordance with applicable requirements of the American National Standards Institute and other applicable codes.

As in HLW facilities, the TRU and LLW treatment and storage facilities must be equipped with monitoring, surveillance, and leak detection capabilities. The DOE Order 5820.2A requires that the TRU temporary storage area at the generator site, prior to shipment to the WIPP, be designed, constructed, operated, and monitored to minimize the possibility of fire, explosion, or accidental release of waste to the environment. The activities to assure the self storage of TRU waste shall also be consistent with the RCRA requirements and 40 CFR 191. In this study, no interim storage area is planned for the TRU waste because it expected that the TRU waste will be roadready for shipment to the WIPP. A temporary staging or package transfer area may be required for the waste container handling prior to the TRU waste transfer to the WIPP.

LIW disposal performance must be in a manner that assures external exposure to the waste and concentrations of radioactive material which may be released into surface water, ground, water, soil, plants and animals results in and effective dose equivalent that does not exceed $25 \mathrm{mrem} / \mathrm{yr}$ 
to any member of the public. Releases to the atmosphere shall meet the requirements of $40 \mathrm{CFR}$ 61. Reasonable effort should be made to maintain releases of radioactivity in effluents to the general environment as low as is reasonably achievable.

\section{DOE Order 435.1 (Draft), "Radioactive Waste Management"}

Currently; a draft DOE Order 435.1 has been issued by the DOE for review. This order will replace the current DOE Order 5820.2A, Radioactive Waste Management. The cancellation of this order does not, by itself modify or otherwise affect contractual obligation with the order. Therefore, the provisions of the $5820.2 \mathrm{~A}$ will remain in effect until the LMITCO contract is modified to delete the reference to the requirements in the canceled order.

DOE Order 435.1 requires that facility siting and design be in compliance with all federal, state, and local laws and regulations, and be performed in accordance with the requirements in DOE Manual 435.1, Radioactive Waste Management Manual, and with other applicable DOE Orders.

DOE Manual 435.1 further describes and establishes the requirements ofDOE Order 435.1 for management of DOE HLW, TRU, and LLW. Based on the DOE Manual 435.1, waste storage, pre-treatment, and treatment facilities design and operation are required to comply with the following applicable Orders and regulations.

DOE 0 151.1, "Comprehensive Emergency Management System"

DOE O 420.1", "Facility Safety"

DOE 0 430.1, "Life-Cycle Asset Management"

DOE 0 440.1, "Worker Protection Management for DOE Federal and Contractor Employees"

DOE 0 460.1A, "Packaging and Transportation Safety"

DOE O 4330.4B, "Maintenance Management Program"

DOE O 5400.1, "General Environmental Protection Requirements."

DOE O 5400.5, "Radiation Protection of the Public and the Environment

DOE O 5480.19, "Cंonduct of Operations Requirements for DOE Facilities"

DOE O 5480.20A, "Personnel Selection, Qualification, añd Training Requirements for DOE Nuclear Facilities"

DOE O 5480.21, "Unreviewed Safety Questions".

DOE 05480.22 , "Technical Safety Requirements"

DOE O 5480.23, "Nuclear Safety Analysis Reports"

DOE 0 6430.1A, General Design Criteria"

10 CFR 820, "Procedural Rules for DOE Nuclear Activities"

10 CFR 830.120, "Quality Assurance Requirements 10 CFR 835, Occupational Radiation Protection"

10 CFR 1021, "National Environmental Policy Act Implementing Procedures"

29 CFR 1910, "Occupational Safety and Health Standards".

"DOE Order 420.1 will replace DOE Order 5480.24 , "Nuclear Criticality Safety." 
40 CFR 61, National Emission Standards for Hazardous Air Pollutants"

40 CFR 264, "Standards for Owners and Operators of Hazardous Waste Storage, Treatment, and Disposal Facilities"

40 CFR 265, "Interim Status Standards for Owners and Operators of Hazardous Waste Storage, Treatment, and Disposal Facilities"

49 CFR 106 - 110 Subchapter A, "Hazardous Materials Transportation"

49 CFR 171-180 Subchapter C, "Hazardous Materials Regulations"

\section{DOE Order 5400.5, "Radiation Protection of the Public and the Environment"}

DOE. Order 5400.5 establishes standards and requirements for operations of the DOE and contractors to protect the public and environment against undue risk from radiation

Chapter II, Requirements for Radiation Protection of the Public and the Environment, specifies that exposure of members of the public to radiation sources as a consequence of all routine DOE activities shall not cause, in a year, an effective dose equivalent greater than $100 \mathrm{mrem}$. The 100 mrem limit is the sum of the effective dose equivalent from exposure to radiation sources external to the body during the year plus the committed effective dose equivalent from radionuclides taken into the body (radioactive decay inside the body) during the year. Exposure of members of the public to radioactive materials released to the atmosphere as a consequence of routine DOE activities shall not cause members of the public to receive an effective dose equivalent greater than 10 mrem annually.

Chapter IV, Residual Radioactive Material, presents radiological protection requirements and guidelines for cleanup of residual radioactive material and management of the resulting residues and release of property. Basic dose limits, guidelines and authorized limits for allowable levels of residual radioactive material, and control of the radioactive wastes and residues are provided.

\section{DOE Order 5480.23, "Nuclear Safety Analysis Reports"}

This Order establishes requirements for DOE-owned nuclear facilities and operations, and for contractors responsible for the design, construction, operation, decontamination, or decommissioning of nuclear facilities to develop safety analyses that establish and evaluate the adequacy of the safety bases of the facilities. The SAR required by this Order documents the results of the safety analysis. 


\section{DOE Order 5480.28, "Natural Phenomena Hazard Mitigation"}

The requirements provided in this order shall be used in conjunction with the general design criteria in DOE $6430.1 \mathrm{~A}$ and other departmental design criteria as applicable. DOE Order 5480.28 requires that facilities structures, systems, and components (SSCs) be designed and constructed to. withstand the effects of natural phenomena hazards. An objective for all SSCs is to prevent loss of structural integrity endangering life safety. An additional objective for selected SSCs or site activities is to prevent loss of capability to perform functions consistent with: (1) importance to safety for workers and the public; (2) impact on the environment; (3) repair/replacement costs; or (4) programmatic mission.

\section{DOE Order 5700.6C, "Quality Assurance"}

The provisions of this Order apply to the work performed by all Departmental Elements and management and operating (M\&O) contractors as provided by law and/or contract and as implemented by the Department's Contracting Officer. If conflicts between this and other Departmental Orders exist, the quality assurance requirements of DOE 5700.6C take.precedent. Work licensed by the NRC or an NRC Agreement State and subject to the quality assurance requirements of that agency are excluded from this Order.

\subsection{NATIONAL ENVIRONMENTAL POLICY ACT}

Under 10 CFR 1021, the NEPA establishes national policy procedures promoting awareness of the environmental impacts of major federal activities during the planning and decisionmaking stages of a project. The NEPA requires all agencies of the federal government prepare a detailed EIS describing potential effects of the proposed major federal actions that may be significantly affect the quality of the human environment.

All federal facilities under the NEPA are encouraged, to the extent practicable, to incorporate Pollution Prevention/Waste Minimization (P2/WMin) criteria and recycling in the planning stages and in the design of the new facilities or modifications to the existing facilities. The P2/WMin and recycling activities will make facilities more efficient and compatible with future environmental regulations and increase energy efficiency and conservation.

\subsection{OTHER STANDARDS AND CODES}

In addition to the requirements discussed above, this section provides a list of other applicable standard codes pertinent to health and safety. 


\section{1) General design of structures, systems, and components}

Uniform Building Code, International Conference of Building Officials

American Institute of Steel Construction

American Welding. Society Standards

American Concrete Institute

DOE-ID Welding Procedure Specification Manual

DOE-STD-1027-92, "Hazard Categorization and Accident Analysis Techniques" for Compliance with DOE Order 5480.23; "Nuclear Safety Analysis Reports"

The hazard categorization is based on a simple approach which is intended to meet D,OE Order 5480.23 requirements for a preliminary assessment and hazard categorization. DOE Order 5480.23 , states that a hazard categorization of the DOE facilities is to be performed on processes, operations, or activities and not necessarily whole facilities.

DOE-STD-3007-93, "Guidelines for Preparing Criticality Safety Evaluations at Department of Energy Non-Reactor Nuclear Facilities" .

DOE-STD-3009-94, "Preparation Guide for U.S. DOE Nonreactor Nuclear Facility Safety Analysis Reports"

\section{2) Radiological control design features}

INEL Radiological. Control Manual

10 CFR 835e, "Occupational Radiation Protection"

\section{3) Fire design features}

Uniform Fire Code, Western Fire Chiefs Association and International conference of Building Officials

National Fire Protection Association (NFPA)

Uniform Building Code, Section 505 (e)

DOE Order 5480.7A, "Fire Protection"

\section{4) Seismic design feature, Flood design features, and Wind design features}

DOE-STD-1020-94(CH-1) Natural Phenomena Hazards Design and Evaluation Critéria for Department of Energy Facilities .

40 CFR 264.18

40 CFR 270.14

\footnotetext{
'DOE Order 5480.11 has been canceled and replaced by 10 CFR 835 .
} 


\section{SUMMARY OF REGULATORY DRTERS AND PERMTT REQUIREMENTS}

The following table presents a summary of the Federal and State regulatory requirements applicable to the construction and operations of the proposed facilities.

\begin{tabular}{|c|c|c|c|c|}
\hline $\begin{array}{l}\text { Media or type } \\
\text { of stream }\end{array}$ & $\begin{array}{c}\text { Requirements prior } \\
\text { to: }\end{array}$ & $\begin{array}{l}\text { Permit, approval, } \\
\text { or requirements }\end{array}$ & $\begin{array}{c}\text { Regulatory } \\
\text { Agency }\end{array}$ & Regulatory citation \\
\hline NEPA & $\begin{array}{l}\text { Title II Design and } \\
\text { procurement }\end{array}$ & NEPA documentation & DOE & 10 CFR 1021 \\
\hline $\begin{array}{l}\text { Nonrad air } \\
\text { emissions } \\
\text { : }\end{array}$ & Construction and operation & $\begin{array}{l}\text { PTC for new emission } \\
\text { sources }\end{array}$ & IDHW, EPA & $\begin{array}{l}40 \text { CFR } 50 \\
40 \text { CFR } 60 \\
40 \text { CFR } 62 \\
40 \text { CFR } 63 \\
40 \text { CFR } 70 \\
40 \text { CFR } 77 \\
40 \text { CFR } 124\end{array}$ \\
\hline $\begin{array}{l}\text { Nonrad air } \\
\text { emissions }\end{array}$ & Construction and operation & $\begin{array}{l}\text { NAAQS, PSD (if } \\
\text { significant emissions). }\end{array}$ & IDHW, EPA & 40 CFR 52 \\
\hline $\begin{array}{l}\text { Nonrad air } \\
\text { emissions }\end{array}$ & Construction and operation & HAPs and TAPs & $\begin{array}{r}\mathrm{DHW}, \mathrm{EPA} \\
.\end{array}$ & $\begin{array}{l}40 \text { CFR } 61 \\
40 \text { CFR } 63 \\
\text { IDAPA } 16.01 .01\end{array}$ \\
\hline $\begin{array}{l}\text { Hazardous waste air } \\
\text { emissions }\end{array}$ & Construction and operation & $\begin{array}{l}\text { Treatment, storage, and } \\
\text { disposal facilities }\end{array}$ & IDHW, EPA & $\begin{array}{l}40 \text { CFR } 264,40 \text { CFR } 265 \\
\text { (Subparts AA, BB, and CC), } \\
\text { IDAPA 16.01.01 } \\
\text { IDAPA } 16.01 .05\end{array}$ \\
\hline $\begin{array}{l}\text { Radioactive air } \\
\text { emissions }\end{array}$ & Construction and operation & NESHAPs & IDHW, EPA & $\begin{array}{l}40 \text { CFR 61, Subpart H } \\
\text { IDAPA } 16.01 .01\end{array}$ \\
\hline All air emissions & Operations & Air Operating Permit & IDHW, EPA. & $\begin{array}{l}40 \text { CFR } 70 \\
\text { IDAPA } 16.01 .01\end{array}$ \\
\hline Asbestos & Renovation and demolition & $\begin{array}{l}\text { Notification prior to } \\
\text { renovation or demolition }\end{array}$ & IDHW, EPA & 40 CFR 61, Subpart M \\
\hline $\begin{array}{l}\text { Ozone depleting } \\
\text { substances }\end{array}$ & $\begin{array}{l}\text { Operation, reporting, } \\
\text { training }\end{array}$ & $\begin{array}{l}\text { Release prevention, } \\
\text { recovery/recycle, } \\
\text { Certificate labeling }\end{array}$ & IDHW, EPA & 40 CFR 82 \\
\hline $\begin{array}{l}\text { Sanitary wastewater } \\
\text { discharges }\end{array}$ & Discharges & NPDES Permit & EPA, IDHW & $\begin{array}{l}40 \text { CFR } 122 \text {, and } 125 \\
\text { IDAPA } 16.01 .02\end{array}$ \\
\hline Land surface $:$ & Construction and & NPDES Permit & EPA, IDHW & 40 CFR 122 \\
\hline
\end{tabular}




\begin{tabular}{|c|c|c|c|c|}
\hline wastewater & Operations & & $\vdots$ & IDAPA 16.01 .02 \\
\hline $\begin{array}{l}\text { Storm wastewater } \\
\text { and nonstorm } \\
\text { wastewater } \\
\text { discharges }\end{array}$ & $\begin{array}{l}\text { Construction and } \\
\text { Operations }\end{array}$ & $\begin{array}{l}\text { NPDES Permit or } \\
\text { compliance with Idaho } \\
\text { Water Quality Standards. }\end{array}$ & EPA & 40 CFR 122 \\
\hline $\begin{array}{l}\text { Drinking water } \\
\text { supply }\end{array}$ & $\begin{array}{l}\text { Construction and } \\
\text { operations } \\
\text {. }\end{array}$ & $\begin{array}{l}\text { Approval of Engineering } \\
\text { Plans, Cross Connection } \\
\text { Control Plans, Report, } \\
\text { and Spec. }\end{array}$ & IDHW, EPA & $\begin{array}{l}40 \text { CFR 141, } \\
40 \text { CFR 143, } \\
\text { IDAPA 16.01.08. }\end{array}$ \\
\hline $\begin{array}{l}\text { Hazardous waste } \\
\text { treatment, storage, } \\
\text { Disposal }\end{array}$ & $\begin{array}{l}\text { Construction, operation, } \\
\text { and maintenance of new } \\
\text { facilities or modifications } \\
\text { of existing facilities }\end{array}$ & $\begin{array}{l}\text { Hazardous Waste Permit } \\
\text { (Part A and B) }\end{array}$ & IDHW, EPA & $\begin{array}{l}40 \text { CFR } 270 \\
40 \text { CFR } 264 \\
40 \text { CFR } 265\end{array}$ \\
\hline $\begin{array}{l}\text { Ünderground } \\
\text { storage Tanks } \\
\text { (UTSs) }\end{array}$ & $\begin{array}{l}\text { Construction and } \\
\text { operations } \\
\text {. }\end{array}$ & Technical standards & DHW, EPA & 40 CFR 280 \\
\hline $\begin{array}{l}\text { Land disposal of } \\
\text { waste }\end{array}$ & $\begin{array}{l}\text { Construction, operations, } \\
\text { disposal }\end{array}$ & LDRs & IDHW, EPA & $\begin{array}{l}40 \text { CFR } 268,40 \text { CFR } 257, \\
\text { IDAPA } 16.01 .05,10 \text { CFR } 61\end{array}$ \\
\hline
\end{tabular}

\section{SETTLEMENT AGREEMENT}

The State of Idaho and the DOE signed an agreement on October 16,1995. The Agreement contains several commitments for the treatment of the HLW and SBW and their transfer out of Idaho. Based on the Agreement, all remaining liquid HLW must be calcined by June 30, 1998, and calcination of all SBW must be completed by December 31, 2012. The Agreement requires that all HLW be treated and be road-ready to be moved out of Idaho for disposal by the year 2035. The calcination and the proposed treatment shall provide for completion of treatment of all calcine wastes by December 31, 2035.

It is stated in the Agreement that the DOE, as soon as practicable, commence the procurement of a treatment facility at INEEL for the treatment of mixed waste. The DOE shall execute a procurement contract for the Facility by June 1, 1997, complete construction of the Facility by December 31, 2002, and commence operation of the Facility by March 31, 2003. Commencement of construction is contingent upon Idaho approving necessary permits.

Based on the Agreement, the DOE shall accelerate efforts to evaluate alternatives for the treatment of calcined waste so as to put it into a form suitable for transport to a permanent repository or an interim storage facility outside Idaho. To support this effort, the DOE shall solicit proposals for feasibility studies by July 1, 1997, and shall commence negotiating a plan and schedule with the State of Idaho for calcine treatment by December 31, 1999. The plan and schedule shall provide for completion of the treatment of all calcined waste located at the INEEL 
by a date established by the Record of Decision (ROD) for the EIS that analyzes the alternatives for treatment of such waste. The State of Idaho expressly reserves its right to seek appropriate relief from the Court in the event that the date established in the ROD for the EIS that anaiyzes the alternatives for treatment of such waste is significantly later than the DOE's target date.

\section{NUCLEAR REGULATORY COMMISSION}

The purpose of this study is to provide an assessment of the current NRC regulations and their potential applicability to the proposed facilities if the facilities were to be licensed by the NRC in the future. The NRC requirement for regulating the $\mathrm{DOE}$ facilities or activities have not been defined yet. The requirements will need to be determined by the NRC and DOE Task Forces. Existing NRC regulations apply to commercial, non-DOE, facilities. The degree of applicability of the NRC requirements to the proposed facilities should be determined by the NRC and DOE, with input from the DOE contractor. If DOE facilities become regulated by NRC, the jurisdiction of other currently government applicable authorities will not automatically or necessarily cease. In particular, it is expected that local, State, Federal EPA, and some DOE regulațory tequirements would still apply.

Currently, the NRC-is not authorized by law to license DOE facilities for:

- HLW processing such as those for vitrification, solidification, Cs and Sr extraction,

- short term storage of HLW, for TRU waste storage and disposal from DOE activities, and

- DOE LLW processing; storage, and disposal.

However, based on the recent DOE proposal, the NRC could take responsibilities for regulating the DOE nuclear facilities. Existing NRC regulations are compiled in $10 \mathrm{CFR}$, titled "Energy". These regulations follow a similar philosophy as the DOE, the EPA, and other codes and standards previousiy discussed above. The Commission has also issued a number of regulatory guides (e.g., NUREG) and other guidance documents which provide acceptable methods for complying with the NRC regulations. They contain criteria for facility design, operations, and for safety and health.

Of the existing NRC regulations, it has been determined in this study that 10 CFR 61 will apply to the proposed LAW or the LLW disposal facility, and 10 CFR 72 will be applicable to the proposed interim storage facilities for the vitrified, HIPed, or grouted $\mathrm{HLW}$, and for the liquid HAW and vitrified HAW storage facilities. Independently, it was determined by Leroy and Morgan in "Nuclear Regulatory Commission (NRC) Licensing Assessment for the Idaho National Engineering and Environmental Laboratory (INEEL) High-Level Waste program," April 23, 1997, that 10 CFR 30 and 10 CFR 70 will apply to the following facilities: 
- 10 CFR 30 for the LAW collection and grouting facilities and for the collection and treatment of the LLW from the INEEL ongoing operations.

- 10.CFR 70 for the separations facility, for the interim storage of liquid HAW from the separation processes, and for the HAW vitrification facility.

- 10 CFR 70 for the HLW vitrification, HIPing, or grouting facilities.

- 10 CFR 70 for the calcine retrieval and dissolution facilities.

10 CFR 30, "Rules of General Applicability to Domestic Licensing of Byproduct Materialf", and 10 CFR 70, "Domestic Licensing of Special Nuclear Material", are not specifically or directly applicable to the facilities listed above. According to Steve LeRoy (personal communication, 12/03/97), they are the only ones which came close to being applicable to the proposed treatment, separations, and retrieval facilities. It is believed that certain elements of $10 \mathrm{CFR} 30$ and 10 CFR 70 could potentially be applicable to licensing of the proposed facilities. The fact remains that NRC will most likely have to promulgate new regulations specifically for the $D O E$ HLW, LLW, and calcine retrieval and treatment facilities or to revise the requirements of 10 CFR 30 and 10 CFR 70 if they were to apply them to the proposed facilities.

Additional NRC regulations that are applicable to all of the proposed facilities are in 10 CFR 2, 10 CFR 19,10 CFR 20, 10 CFR 21, 10 CFR 50, 10 CFR 51, 10 CFR 52, and 10 CFR 73. 10 CFR 71 and 49 CFR 173 (Department of Transportation) contain requirements for the packaging and transportation of radioactive wastes. These requirements would have impact on the design and operations of the storage facilities.

Appendix A provides a detailed source list of the regulations used by the NRE in commercial, non-reactor, nuclear facilities. The regulations are primarily based on the health and safety considerations. The list includes applicable parts and subparts of 10 CFR 20,21,30,50,51,61, 70 , and 72 as well as related guidance documents. The requirements and guidance documents are listed under the following categories: 1) radioactive waste management ,2) design of structures, components, equipment, and systems, 3 ) electric power, utility services, and fire protection, 4) radiation protection, 5) conduct of operation, 6) safety analysis report criteria, 7) quality assurance, and 8) decommissioning.

The existing facilities that will be modified to be used for storage of HLW are expected to be exempted by DOE from any further jurisdiction of NRC. Such a jurisdiction would be excessively difficult, costly, and complex to apply. All the work requirements for the modification of the existing facilities are expected to be performed in accordance with the DOE/RW/0333P, "Quality Assurance Requirements and Description." The requirements' in DOE/RW/0333P are endorsed by the Office of Civilian Radioactive Waste Management

\footnotetext{
'Byproduct material means any radioactive material (except special nuclear material) yielded in or made radioactive by exposure to the radiation incident to the process of producing or utilizing special nuclear material. Special nuclear material means (1) plutonium, uranium 233, uranium enriched in the isotope 233 or in the isotope 235, and any other material which the Commission determines to be special nuclear material, but does not include source -material; or (2) any material artificially enriched by any of the foregoing but does not include source material.
} 
(OCRWM) which carries out the DOE mission for safe design and operation of a HIW geologic repository and a HLW storage facility.

\section{Current Licensing Process}

Most of the discussion in this section is based on the information contained in the DOE-STD- . 101-92, "Compilation of Nuclear Safety Criteria Potential Application to the DOE Nonreactor Facilities" and in the report by Morgan and LeRoy, "Nuclear Regulatory Commission (NRC) Licensing Assessment for the Idaho National Engineering and Environmental Laboratory (INEEL) High-Level Waste program," April 23, 1997.

The applicable NRC regulations that define licensing processes are in 10 CFR 2, 10 CFR 30, 10 CFR 51, and 10 CFR 61 for LLW facilities and in 10 CFR 2, 10 CFR 50, 10 CFR 52,10 CFR 70 , and 10 CFR 72 for HLW or HAW facilities. The licensing of a nuclear facility requires preparation and submittal of an application and a number of supporting documents to the NRC such as SAR, environmental report (ER), quality assurance document, training plan, monitoring plan, and safeguards and security plan. The following is a generic description of the various documents that will be applicable to the proposed facilities.

The ER must meet the NRC requirements in 10 CFR 51. Appendix A, Section 7 provides regulatory sources containing quality assurance procedures for the facility design, construction, and operations. The quality assurance requirements in DOE/RW-0333P are expected to be used for the existing facilities that will be modified to be used for storage of HLW or HAW. The SAR documents the adequacy of safety analysis for a nuclear facility to ensure that the facility can be designed, constructed, operated, maintained, shut down, and decommissioned safely and in compliance with applicable laws and regulations. The SAR criteria must meet the regulations listed in Appendix A, Section 6. The training, monitoring, and safeguards and security plans used by the license applicant to protect health and minimize danger to life or property must be developed in accordance with the applicable regulations. The training program should include an analysis of the job, learning objectives and performance criteria, procedures for personnel monitoring, procedures to avoid accidents, etc. It is assumed that the DOE will retain the responsibility for safeguard and security for its facilities.

The NRC licensing process is divided into four stages: pre-application stage, application review stage, construction and operating license stage, and decontamination and site closure stage. The licensing duration from submittal of the application to receipt of the license is expected to take three to five years or longer.

Pre-application stage is prior to filing a license application with the NRC. It entails the development of the license application and the pre-submittal communications with the NRC. This includes the NRC and DOE interactions to clearly define the NRC acceptance criteria against which the ICPP proposed facilities license application will be reviewed. 
The application review stage describes the activities after submittal of the license application to the NRC. A notification will be published in the Federal Register for public hearing when the NRC receives the application. This application review stage begins with a review process referred to as a "Docketing Review" which is usually performed within 1 to 3 months. This review is to ensure that the application is complete and contains the necessary information. The Docketing Review process is followed by a detailed safety review of the application by the NRC staff. The NRC will ensure that the regulatory requirements are met as established in the . regulations. The NRC usually requests additional information during this review which can be extensive and delay the review. Submittal of high quality, complete, and detailed SAR will reduce the request for additional information, hence the review time.

The construction and operating license stage follows the receipt of the license. The NRC will have the regulatory oversight during construction and operations.

NRC issues a license for certain time period. Before a facility license expires, a decommissioning plan will be developed by the DOE for review by the NRC. It is expected that the EPA will regulate the decontamination and decommissioning activities. Before the final closure, the DOE must submit a closure plan to the NRC for review. The closure plan must describe how the owner/operator will conduct clean-up, what clean-up levels will be attained, and how clean-up will be verified. The plan also includes a post-closure, and long term monitoring and maintenance. Upon review and acceptance, the NRC will aithorize closure. Monitoring will be performed during the post-closure plan in accordance with the applicable requirements. When all the monitoring and control requirements are met, the license will be terminated.

\section{LLW Near-Surface Disposal}

10 CFR 61, Licensing Requirements for Land Disposal of Radioactive Waste

10 CFR 61 contains specific technical requirements and performance objectives applicable to near-surface disposal of radioactive wastes. It contains requirements for design, operation, closure and post-closure, and monitoring. Near-surface disposal involves disposal of waste in the uppermost portion of the earth, approximately 30 meters or 100 feet of natural grade. The NRC maintains that the use of shallow land disposal is adequate for protection of individuals and the public, when properly sited, designed, and operated, as required by 10 CFR 61 .

Design, operation, and closure of the land disposal facility must ensure protection of any individual inadvertently intruding into the disposal site and occupying the site or contacting the waste at any time after active institutional controls over the disposal site are removed. Operations at the land disposal facility must be conducted in compliance with the standards for radiation protection set in 10 CFR 20, except for releases of radioactivity in effluents from the land disposal facility, which shall be governed by 10 CFR 61.41. At the time a license application is submitted, the applicant shall have conducted a preoperational monitoring program to provide basic environmental data on the disposal site characteristics. The applicant shall 
obtain information about the ecology, meteorology, climate, hydrology, geology, geochemistry, and seismology of the disposal site. For those characteristics that are subject to seasonal variation, data must cover at least a twelve month period.

The regulations for near surface disposal of radioactive wastes include a waste classification system which divides the wastes into three classes: Class A, B, and C. The classification system is based on the overall disposal hazards of the wastes. Certain minimum requirements must be met for all waste Classes as provided in 10 CFR 61.56 (a). In addition; Class $B$ and $C$ wastes are required to have structural stability as discussed in 10 CFR $61.56(\mathrm{~b})$ : The detailed information regarding the NRC requirements for a LLW disposal facility can be found in EDF-FDO-008.

\section{HILW Storage}

10 CFR 72, "Licensing Requirements for the Independent Storage of Spent Nuclear Fuel and" High-Level Radioactive Waste"

10 CFR 72 contains regulations and procedures that are applicable to HLW or HAW interim. , storage facilities. The regulations in this part establish requirements for the issuance of licenses to the DOE to receive, transfer, package, and possess $H \mathrm{WW}$, spent fuel, and other radioactive " materials associated with spent fuel and HLW storage, in a monitored retrievable storage facility (MRS)g. This part also defines requirements for the safety design features of the facility structure and equipment. It requires that structures, systems, and components be designed, fabricated, erected, and tested to provide protection against environmental conditions and natural phenomena such as earthquakes, tornadoes, lighting, hurricanes, and floods. The facilities should also be designied to prevent massive collapse of building structures or the dropping of . heavy objects as a result of building structural failure on the spent fuel or high-level radioactive waste or on to structures, systems, and components important to safety. If the facilities are located over an aquifer which is a major water resource, measures must be taken to preclude the transport of radioactive materials to the environment through this potential pathway.

Structures, systems, and components against fires and explosions must be designed and located so that they can continue to perform their safety functions effectively under credible fire and explosion exposure conditions. Noncombustible and heat-resistant materials must be used . wherever practical, particularly in locations vital to the control of radioactive materials and to the maintenance of safety control functions. Explosion and fire detection, alarm, and suppression systems shall be designed and provided with sufficient capacity and capability to minimize the adverse effects of fires and explosions on structures, systems, and components important to safety.

\footnotetext{
${ }^{8}$ Pursuant to the Nuclear Waste Policy Act, a MRS is an option for providing safe and reliable long-term storage of HLW or spent nuclear fuel. However, disposal of HLW and spent fuel in a repository should proceed regardless of any construction of a MRS pursuant to the Act.
} 
Other features that are important to safety must be designed to permit inspection, maintenance, and testing. Emergency capability must be designed to provide for accessibility to the equipment of onsite and available offsite emergency facilities and services such as hospitals, fire and police departments, ambulance service, and other emergency agencies.

Ventilation systems and off-gas systems must be provided where necessary to ensure the confinement of airbome radioactive particulate materials during normal or off-normal conditions. Storage confinement systems must have the capability for continuous monitoring in a manner such that the licensee will be able to determine when corrective action needs to be taken to maintain safe storage conditions. Instrumentation and control systems must be provided to monitor systems that are important to safety over anticipated ranges for normal operation and off-normal operation. Those instruments and control systems that must remain operational under accident conditions must be identified in the SAR.

Control room or control area must be designed to permit occupancy and actions to be taken to monitor the facilities under normal conditions, and to provide safe control

of the facilities under off-normal or accident conditions. Utility or other services must be designed to meet emergency conditions.

It is required that $\mathrm{HLW}$ be packaged in a manner that allows handling and retrievability without the release of radioactive materials to the environment or radiation exposures in excess of 10 CFR 20, "Standards for Protection Against Radiation", limits. The package must be designed to confine the high-level radioactive waste for the duration of the license. During normal operations and anticipated occurrences, the annual dose equivalent to any real individual who is located beyond the controlled area must not exceed 25 mrem to the whole body, 75 mrem to the thyioid and 25 mrem to any other organ as a result of exposure to planned discharges of radioactive materials and decay products excepted, to the general environment, and direct radiation from operations. Operational restrictions must be established to meet as low as is reasonably achievable. (ALARA) objectives for radioactive materials in effluents and direct radiation levels associated with storage operations. Operational limits must be established for radioactive materials in effluents and direct radiation levels associated operations to meet the limits given above.

10 CFR 72.124, "Criteria for nuclear criticality safety"

The design of handling, packaging, transfer, and storage systems must include margins of safety for the nuclear criticality parameters that are commensurate. with the uncertainties in the data and methods used in calculations: It must demonstrate safety for the handling, packaging, transfer and storage conditions and in the nature of the immediate environment under accident conditions.

When practicable the design of an MRS must be based on favorable geometry, permanently fixed neutron absorbing materials (poisons), or both. Where solid neutron absorbing materials are used, the design shall provide for positive means to verify their continued efficacy. 
A criticality monitoring system shall be maintained in each area where special nuclear material is handled, used, or stored which will energize clearly audible alarm signals if accidental criticality occurs. Monitoring of dry storage areas where special nuclear material is packaged in its stored configuration under a license issued under this subpart is not required.

10 CFR 72.128, "Crriteria for spent fuel, high-level radioactive waste, and other radioactive waste storage and handling"

The regulations of this subpart require that HLW storage and other systems that might contain or handle radioactive materials be designed to ensure adequate safety under normal and accident conditions. These systems must be designed with: (1) a capability to test and monitor components important to safety, and suitable shielding for radioactive protection under normal and accident conditions, (2) confinement systems, (3) a heat-removal capability having testability and reliability consistent with its importance to safety, and (4) meanis to minimize the quantity of radioactive wastes generated. Provisions must be made for the packing of site-generated low: level wastes in a form suitable for storage onsite awaiting transfer to disposal sites. 


\section{APPENDIX A}

The following lists the current NRC requirements and guides applied to the areas of safety addressed in the SAR. The requirements are listed under the following categories: 1) radioactive waste management , 2) design of structures, components, equipment, and systems, 3) electric power, utility services, and fire protection, 4) radiation protection, 5) conduct of operation, 6 ). safety analysis report criteria, 7) quality assurance, and 8) decommissioning.

\section{1) Radioactive waste management}

This section identifies criteria for the control, collection, handling, processing, storage, and disposal of liquid, gaseous, and solid wastes that may contain radioactive materials, and the instrumentation used to monitor the release of radioactive materials. Also, as previously discussed, all RCRA hazardous and radioactive waste (mixed waste) management facilities are also subject to EPA RCRA regulations.

10 CFR 30, "Rules of General Applicability to Domestic Licensing of Byproduct Material" 10 CFR 61, "Licensing Requirements for Land Disposal of Radioactive Waste" 10 CFR 70̂.59,“"Effluent Monitoring Reporting Requirements".

10 CFR 72.104, "Criteria in Effluents and Direct Radiation in Effluents and Direct Radiation from an ISFSI or MRS"

D. 10 CFR 72.128, "Criteria for Spent Fuel, High-Level Radioactive Waste, and Other" . Radioactive Waste Storage and Handling"

Regulatory Guide 1.21, "Measuring, Evaluating, and Reporting Radioactivity in Solid Wastes and Releases of Radioactive Materials in Liquid and Gaseous Effluents from Light-WaterCooled Nuclear Power Plants."

Regulatory Guide 1.143, "Design Guidance for Radioactive Waste Management Systems, Structures, and Components Installed in Light-Water-Cooled Nuclear Power Plants."

Regulatory Guide 3.10, "Liquid Waste Treatment Design Guide for Plutonium Processing and Fuel Fabrication Plants."

Regulatory Guide 3.13, "Guide for Acceptable Waste Storage Methods at UF6 Production Plants." 
Regulatory Guide 3.20, "Process Off-gas Systems for Fuel Reprocessing Plants."

Regulatory Guide 3.49, "Design of an Independent Spent Fuel Storage Installation (Water-Basin Type)."

Regulatory Guide 3.60, "Design of an Independent Spent Fuel Storage Installation (Dry Storage)."

Reguilatory Guide 4.18, "Standard Format and Contents of Environmental Reports for Nearsurface Disposal of Radioactive Waste."

NUREG-1199, "Standard Format and Content of a License Application for a Low-level

Radioactive Waste Disposal Facility."

NUREG-1200, "Standard Review Plan for the Review of a License Application for a Radioactive Low-level Waste Disposal Facility."

NUREG-1300, "Standard Review Plan for the Review of a License Application for a Radioactive Low-level Waste Disposal Facility." .

NUREG-0800, Section 11.2, "Liquid Waste Management Systems."

NUREG-0800, Section 11.3, "Gaseous Waste Management Systems."

NUREG-0800, Section 11.4, "Solid Waste Management Systems:"

NUREG-0800, Section 11.5, "Process and Effluents radiological Monitoring."

NUREG-1567, "Offgas Treatment and Ventilation."

2) Design of structures, components, equipment and systems

10 CFR 21, "Reporting of Defects and Noncompliance"

10 CFR 50.34, "Contents of Applications: Technical Information"

10 CFR 50, Appendix F, "Policy Relating to the Siting of Fuel Reprocessing Plants and Related Waste Management Facilities"

10 CFR 61.51, "Disposal Site Design for Land Disposal"

10 CFR 61.52, "Land Disposal Facility Operation and Disposal Site Closure" 
10 CFR 61.54, "Alternative Requirements for Design and Operations"

10 CFR 70, "Domestic Licensing of Special Nuclear material"

10 CFR 72, "Licensing Requirements for the Independent Storage of Spent Nuclear Fuel and High-Level Radioactive Waste"

10 CFR 72.120, "General Considerations"

10 CFR 72.122, "Overall Requirements"

10 CFR 72.124, "Criteria for Nuclear Criticality Safety"

10 CFR 72.126, "Criteria for Radiological Protection"

10 CFR 72.128, "Criteria for Spent Fuel, High-Level Radioactive Waste, and Other Radioactive Waste Storage and Hand-ling"

10 CFR 72.130, "Criteria for Decommissioning"

Regulatory Guide, 3.10, "Liquid Waste Treatment System Design Guide for Plutonium Processing and Fuel Fabrication Plants."

Regulatory Guide 3.12, "General Design Guide for Ventilation Systems of Piuronium Processing and Fuel Fabrication Plants."

Regulatory Guide 3.20, "Process Off-gas Systems for Fuel Reprocessing Plants"

Regulatory Guide 3.32, "General Design Guide for Ventilation Systems for Fuel Reprocessing Plants."

Regulatory Guide 3.38, "General Fire Protection Guide for Fuel Reprocessing Plants."

Regulatory Guide 3.56, "General Guidance for Designing, Testing, Operating, and Maintaining Emission Control Devices at Uranium Mills."

Regulatory Guide 5.25, "Design Considerations for Minimizing Residual Holdups of Special Nuclear Material in Equipment for Wet process Operations:"

\section{Seismic systems criteria}

10 CFR 61.12, "Specific Technical Information" 
10 CFR 72.120, "General Considerations"

Regulatory Guide 1.29, "Seismic Design Classification."

Regulatory. Guide 3.14, "Seismic Design Classification for Plutonium Processing and Fuel Fabrication Plants.

\section{Wind and Tomado Loading criteria}

10 CFR 61.12, "Specific Technical Information"

10 CFR 72.40, "Issuance of License"

10 CFR 72.90, "General Considerations"

10 CFR 72.92, "Design Basis External Natural Events"

10 CFR 72.98, "Identifying Regions Around an ISFSI or MRS Site"

10 CFR 72.122, "Overall Requirements"

Regulatory Guide 1.76, "Design Basis Tornado for Nuclear Power Plants:"

Regulatory Guide 3.10, "Liquid Waste Treatment Design Guide for Plutonium Processing and Fuel Fabrication Plants."

Regulatory Guide 3.12, "General Design Guide for Ventilation Systems of Plutonium Processing and Fuel Fabrication Plants."

Regulatory Guide 3.16, "Géneral Fire Protection Guide for Plutonium Processing and Fuel Fabrication Plants."

Regulatory Guide 3.18, "Confinement Barriers and Systems for Fuel Reprocessing Plants.

Regulatory Guide 3.20, "Process Offgas Systems for Fuel Reprocessing Plants."

Regulatory Guide 3.31, "Emergency Water Supply Systems for Fuel Reprocessing Plants."

Regulatory Guide 3.32, "General Design Guide for Ventilation Systems for Fuel Reprocessing Plants."

Regulatory Guide 3.38, "General Fire Protection Guide for Fuel Reprocessing Plants." 
Regulatory Guide 3.49, "Design of an Independent Spent Fuel Storage Installation, (Water Basin Type)."

Regulatory Guide 3.53, "Applicability of Existing Regulatory Guides to the Design and Operation of an Independent Spent Fuel Storage Installation."

Regulatory Guide 3.60, "Design of an Independent Spent Fuel Storage Installation (Dry Storage)."

NUREG/CR-3874, "Near-Ground Tomado Wind Fields," McDonald, J.R., Texas Tech. University, July 1984.

NUREG/CR-3848, "Experimental Investigation of Unsteady Tomadic Wind Loads on Structures," Jischke, M.C., Oklahoma, Teaching Hospitals, June 1984.

NUREG/CR-3058, "A Methodology for Tornado Hazard Probability Assessment," McDonald, J.R, Texas Tech. University,.October 1983.

NUREG/CR-2944, “Tornado Damage Risk Assessment," Reinhold, T.A. and Ellingwood, B., National Bureau of Standards, February 1983.

NUREG/CR-2565, "Structural Performance of HEPA Filters Under Simulated Tornado Conditions," Horak, H.L. and Smith, P.R., Los Alamos National Laboratory, May 1982.

NUREG/CR-2014, "Kinematics of Translating Tornado Wind Fields," Peterson, R.E., Texas Tech. University, April 1981.

NUREG/CR-1585, "Modeling Tornado Dynamics," Aeronautical Research Association, September 1980.

\section{Water level (flood) design}

10 CFR 61.12, "Specific Technical Information".

10 CFR 61.50, "Disposal Site Suitability Requirements for Land Disposal"

10 CFR 72.40, "Issuance of License"

10 CFR 72.90, "General Considerations"

10 CFR 72.92, "Design Basis External Natural Ėvents"

10 CFR 72.94, "Design Basis External Man-Induced Events" 
10 CFR 72.98, "Identifying regions Around and ISFSI or MRS Site"

10 CFR 72.122, "Overall Requirements"

Regulatory Guide 3.10, "Liquid Waste Treatment Design Guide for Plutonium Processing and Fuel Fabrication Plants."

Regulatory Guide 3.11, "Design, Construction, and Inspection of Embankment Retention Systems for Uranium Mills."

Regulatory Guide 3.18, "Confinement Barriers and Systems for Fuel Reprocessing Plants.

Regulatory Guide 3.31, "Emergency Water Supply Systems for Fuel Reprocessing Plants."

Regulatory Guide 3.40, "Design Basis Floods for Fuel Reprocessing Plants and for Plutonium Processing and Fuel Fabrication Plants."

Regulatory. Guide 3.49, "Design of an Independent Spent Fuel Storage Installation, (Water Basin Type)."

Regulatory Guide 3.53, "Applicability of Existing Regulatory Guides to the Design and Operation of an Independent Spent Fuel Storage Installation:"

Reguilatory Guide 3:60, "D́esign of an Independent Spent Fuel Storage Installation (Dry Storage)."

NUREG/CR-2678, "Flood Risk Analysis Methodology Development Project - Final Report," Wagner, D.P. et al., Oak Ridge National Laboratory, July 1982.

\section{Missileprotection}

10 CFR 61.12, "Specific Technical Information"

10 CFR 72.40, "Issuance of License"

10 CFR 72.90, "General Considerations"

10 CFR 72.92, "Design Basis External Natural Events"

10 CFR 72.94, "Design Basis External Man-Induced Events"

10 CFR 72.98, "Identifying Regions Around an ISFSI or MRS Site" 
10 CFR 72.122, "Overall Requirements"

Regulatory Guide 3.10, "Liquid Waste Treatment Design Guide for Plutonium Processing and Fuel Fabrication Plants."

Regulatory Guide 3.12, "General Design Guide for Ventilation Systems of Plutonium Processing and Fuel Fabrication Plants."

Regulatory Guide 3.16, "General Fire Protection Guide for Plutonium Processing and Fuel Fabrication Plants."

Regulatory Guide 3.18, "Confinement Barriers and Systems for Fuel Reprocessing Plants."

Regulatory Guide 3.20, "Process Off-gas Systems for Fuel Reprocessing Plants."

Regulatory Guide 3.31, "Emergency Water Supply Systems for Fuel Reprocessing Plants."

Regulatory Guide 3.32, "General Design Guide for Ventilation Systems for Fuel Reprocessing Plants."

Regulatory Guide 3.38, "General Fire Protection Guide for.Fuel Reprocessing Plants."

Regulatory Guide 3.49, "Design of an Inüejendent Spent Fuel Storage Installation, (Water Basin Type)."

Regulatory Guide 3.53, "Applicability of Existing Regulatory Guides to the Design and Operation of an Independent Spent Fuel Storage Installation."

Regulatory Guide 3.60, "Design of an Independent Spent Fuel Storage Installation (Dry Storage)."

NUREG-0533, "Aircraft Impact risk Assessment, Data Base for Assessment of Air Carrier Impact Risk in the Vicinity of Airports," USNRC, July 1979.

NUREG/CR-2462, "Capacity of Nuclear Power Plant Structures to Resist Blast Loading," Kennedy, R.P. et al., Sandia National Laboratories, September 1983.

NUREG/CR-2859, "Evaluation of Aircraft Crash Hazards for Nuclear Power Plants," Kot, C.A. et al., Argonne National Laboratory, September 1982. 


\section{Seismic design}

10 CFR 61.12, "Specific Technical Information"

10 CFR 70.22, "Contents of Applications"

10 CFR 70.23, "Requirements for the Approval of Applications"

I0 CFR 72.40, "Issuance of License"

10 CFR 72.90, "General Considerations"

10 CFR 72.92, "Design Basis External Natural Events"

10 CFR 72.98, "Identifying Regions Around an ISFSI or MRS Site"

10 CFR 72.102 "Geological and Seismological Characteristics"

IO CFR 72.122, "Overall Requirements"

Regulatory Guide 3.10," "Liquid Waste Treatment Design Guide for Plutonium Processing and Fuel Fabrication Plants."

Regulatory Guide 3.12, "General Design Guide for Ventilation Systems of Plutonium-? Trocessing and Fuel Fabrication Plants."

Regulatory Guide 3.14, "Seismic Design Classification for Plutonium Processing and Fuel Fabrication Plants."

Regulatory Guide 3.16, "General Fire Protection Guide for Plutonium Processing and Fuel Fabrication Plants."

Regulatory Guide 3.17, "Earthquake Instrumentation for, Fuel Reprocessing Plants."

Regulatory Guide 3.18, "Confinement Barriers and Systems for Fuel Reprocessing Plants.

Regulatory Guide 3.20, "Process Off-gas Systems for Fuel Reprocessing Plants."

Regulatory Guide 3.31, "Emergency Water Supply Systems for Fuel Reprocessing Plants."

Regulatory Guide 3.32, "General Design Guide for Ventilation Systems for Fuel Reprocessing Plants."

Regulatory Guide 3.38, "General Fire Protection Guide for Fuel Reprocessing Plants." 
Regulatory Guide 3.49, "Design of an Independent Spent Fuel Storage Installation, (Water Basin Type)."

Regulatory Guide 3.53, "Applicability of Existing Regulatory Guides to the Design and Operation of an Independent Spent Fuel Storage Installation."

Regulatory Guide 3.60, "Design of an Independent Spent Fuel Storage Installation (Dry Storage)."

NUREG/CR-1069, "Effects of Earthquakes on Underground Facilities: Literature Review and Discussion," Carpenter, D.W. and Chung, D.C., Lawrence Livermore National Laboratory, June 1986.

\section{Yentilation and process off-gas systems}

10 CFR 72.132, "Overall Requirements"

Regulatory Guide 1.140, "Design, Testing, and Maintenance for Normal Ventilation Systems."

Regulatory Guide 1.52, "Design, Testing, and Maintenance aCriteria for Post-accident engineered Safety Feature Ventilation Systems."

Regulatory Guide 3.12, "General Design Guide for Ventilation Systems of Plutonium Processing and Fuel Fabrication Plants."

Regulatory Guide 3.20, "Process Off-gas Systems for Fuel Reprocessing Plants."

Regulatory Guide 3.32, "General Design Guide for Ventilation Systems for Fuel Reprocessing Plants."

Regulatory Guide 3.49, "Design of an Independent Spent Fuel Storage Installation (Water Basin Type)."

Regulatory Guide 3.60, "Design of an Independent Spent Fuel Storage Installation (Dry Storage)."

NUREG-1567, Section 11.4.1.3 (DRAFT), "Ventilation offgas System Design Feature." 
3) Electrical power, utility services, and fire protection

10 CFR 50.55(a), "Codes and Standards"

10 CFR 72.122, "Overall Requirements"

Regulatory Guide 1.108, "Periodic Testing of Diesel Generator Units Used as Onsite Electric Power Systems at Nuclear Power Plants."

Regulatory Guide 3.14, "Seismic Design Classification for Plutonium Processing and Fuel Fabrication Plants."

Regulatory Guide 3.16," "General Fire Protection Guide for Plutonium Processing and Fuel Fabrication Plants."

Regulatory Guide 3.38, "General Fire Protection Guide for Fuel Reprocessing Plants."

\section{4) Radiation protection}

The criteria identified in this chapter are for the radiation protection of operating, construction, and maintenance personnel during normal and anticipated operational occurrences. The compilation includes criteria for facility equipment design and programs to minimize and monitor radiation exposure to meet the standards for protection against radiation of 10 CFR 20 .

10 CFR 19.12, "Instructions to Workers"

10 CFR 20, "Standards for Protection Against Radiation"

10 CFR 61.41, "Protection of the General Population From Releases of Radioactivity"

10 CFR 61.43, "Protection of Individuals During Operations"

10 CFR 72.44, "License Conditions"

10 CFR 72.104, "Criteria for Radioactive Materials in Effluents and Direct Radiation from an ISFSI or MRS"

10 CFR 72.126, "Criteria for Radiological Protection"

Regulatory Guide 3.6, "Content of Technical Specifications for Fuel Reprocessing Plants."

Regulatory Guide 8.1, "Radiation Symbol." 
Regulatory Guide 8.2, "Administrative Practices in Radiation Monitoring."

Regulatory Guide 8.10, "Operating Philosophy for Maintaining Occupational Radiation Exposures as Low as is Reasonably Achievable."

Regulatory Gride 8.24, "Health Physics Surveys During Enriched Uranium Processing and Fuel Fabrication."

\section{5) Conduct of operations}

The criteria identified in this chapter address training, emergency planning, plant procedures, and the maintenance of records and reporting.

10 CFR 61, "Licensing Requirements for Land Disposal of Radioactive Waste"

10 CFRR 51, "Environmental Protection Regulations for Domestic Licensing and Related Regulatory Functions"

: 10 CFR 70, "Domestic Licensing of Special Nuclear Material"

10 CFR 72.190, "Operator Requirements"

- IU CFR 72.192, "Operator Training and Certification Program"

10 CFR 72.194, "Physical Requirements."

Regulatory Guide 3.28, "Welder Qualification for Welding in Areas of Limited Accessibility in Fuel Reprocessing Plants and in Plutonium Processing and Fuel Fabrication Plants.”

Regulatory Guide 3.42, "Emergency Planning for Fuel Cycle Facilities and Plants Licensed Under 10 CFR Parts 50 and 70.

\section{6) Safety analysis report criteria}

10 CFR 20, "Standards for Protection Against Radiation"

10 CFR 30, "Rules of General Applicability to Domestic Licensing of Byproduct Material"

10 CFR 50.33, "Contents of Application, General Information"

10 CFR 50.36(b), "Environmental Conditions" 
10 CFR 50.55, "Conditions of Construction Permits"

10 CFR 5 0.71, "Maintenance of Records, making Reports".

10 CFR 61, "Licensing Requirements for Land Disposal of Radioactive Waste"

10 CFR 61.10, "Contents of Application".

10 CFR 70, "Domestic Licensing of Special Nuclear Material"

10 CFR 70.22, "Contents of Application"

10 CFR 70.23, "Requirements for the Approval of Applications"

10 CFR 72, "Licensing Requirements for the Independent Storage of Spent Nuclear Fuel and High-Level Radioactive Waste”. .

10 CFR 72.22, "Contents of Application: General and Financial Information"

10 CFR 72.24, "Contents of Application: Technical Information"

10 CFR 72.30, "Decommissioning Planning, Including Financing and Reçord Keeping"

10 CFR 72.48, "Changes, Tests, and Experimonts"

- Regulatory Guide 3.15, "Standard Format and Content of License Application for Storage Only of Unirradiated Power Reactor Fuel and Associated Radioactive Material."

Regulatory Guide 3.25, "Standard Format and Content of Safety Analysis Reports for Uranium Enrichment Facilities."

Regulatory Guide 3.26, "Standard Format and Content of Safety Analysis Reports for Fuel Reprocessing Plants."

Regulatory Guide 3.39, "Standard Format and Content of License Applications for Plutonium Processing and Fuel Fabrication Plants."

Regulatory Guide 3.44, "Standard Format and Contènt for the Safety Analysis Report for an .Independent Spent Fuel Storage Installation (Water-Basin Type)."

Regulatory Guide 3.48, "Standard Format and Content for the Safety Analysis Report for an Independent Spent Fuel Storage Installation or Monitored Retrievable Storage Installation (Dry Storage)." 
Regulatory Guide 3.50, "Standard Format and Content for a License Application to Store Spent Fuel and High-Level Radioactive Waste."

Regulatory Guide 3.52, "Standard Format and Content for the Health and Safety Sections of License Renewal Applications for Uranium Processing and Fuel Fabrication.”

Regulatory Guide 3.55, "Standard Format and Content for the Health and Safety Sections of License Renewal Applications for Uranium Hexafluoride Production."

Regulatory Guide 3.61, "Standard Format and Content for a Topical Safety Analysis Report for a Spent Fuel Dry Storage Cask."

Regulatory Guide 3.62, "Standard Format and Content for the Safety Analysis Report for Onsite Storage of Spent Fuel Storage Casks."

\section{Accidentanalysis}

The criteria in this chapter are for initiating events that result in a criticality accident.

10 CFR 50.34, "Contents of Applications: Technical Information".

10 CFR 61.13, "Technical Analyses"

10 CFR 70.22, "Content of Applications"

10 CFR 70.23, "Requirements for the Approval of Applications"'

10 CFR 72.24, "Contents of Application: Technical Information"

10 CFR 100.11, "Determination of Exclusion Area, Low Population Zone, and Population Center Distance"

Regulatory Guide 1.25, "Assumptions Used for Evaluating the Potential Radiological Consequences of a Fuel-Handling Accident in the Fuel-Handling and Storage Facility for Boiling and Pressurized Water Reactors."

Regulatory Guide 1.91, "Evaluations of Explosions Postulated to Occur on Transportation Routes Near Nuclear Power Plants."

Regulatory Guide 1.113, "Estimating Aquatic Dispersion of Effluents from Accidental and Routine Releases." 
Regulatory Guide 1.145, "Atmospheric Dispersion Models for Potential Accident Consequence Assessments at Nuclear Power Plants."

NUREG-1320, "Nuclear Fuel Cycle Facility Accident Analysis Handbook," USNRC, May 1988.

NUREG-1179, "Rupture of Model 48Y UF6 Cylinder and Release of Uranium Hexafluoride," USNRC, February 1986.

NUREG-0772, "The Effects of Natural Phenomena on the Exxon Nuclear Company Mixed- : Oxide Fabrication Plant at Richland Washington," USNRC, September 1980.

NUREG/CR-4303, "High-Level Waste Preclosure Systems Safety Aralysis," GA Technologies, Inc., September 1985.

NUREG/CR-3682; 'Nuclear Fuel Cycle Risk Assessment-Review and Evaluation of Existing Methods," Pelto, P.J. et al., Battelle Pacific Northwest Laboratories, May 1984.

NUREG/CR-3210, "Low-Level Waste Risk Methodology Development" Cox, N.D. et al., EG\&G Inc., May 1983.

NUREG/CR-3139, "Scenarios and Analytical Methods for UF6 Releases at NRC-Licensed Fuel Cycle Facilities," Simantov, M. et al., Oak Ridge National Laboratory, July 1984.

7) Quality assurance during design, construction, and operation 10 CFR 50.4, "Written Communications"

10 CFR 50.55, "Conditions of Construction Permits"

10 CFR 50, Appendix B, "Quality Assurance Criteria for Nuclear Power Plants and Fuel Reprocessing Plants".

10 CFR 61.12, "Specific Technical Information"

10 CFR 72.40, "Issuance of License"

10 CFR 72 Subpart G, "Quality Assurance"

Regulatory Guide 1.30, "Quality Assurance Requirements for the Installation, Inspection, and Testing of Instrumentation and Electric Equipment."

Regulatory Guide 2.3, "Quality Verification for Plate-Type Uranium-Aluminum Fuel Elements for Use in Research Reactors." 
Regulatory Guide 3.3, "Quality Assurance Program Requirements for Fuel Reprocessing Plants and for Plutonium Processing and Fuel Fabrication Plants."

Regulatory Guide 3.10, "Liquid Waste Treatment System Design Guide for Plutonium Processing and Fuel Fabrication Plants.

Regulatory Guide 3.12, "General Design Guide for Ventilation Systems or Plutonium Processing and Fuel Fabrication Plants." .

\section{8) Decommissioning}

10 CFR 50, Appendix F, "Policy Relating to the Siting of Fuel Reprocessing Plants and Related Waste Management Facilities."

10 CFR 50.75, "Reporting and Record Keeping for Decommissioning Planning"

10 CFR 50.82, "Application for Termination of Licenses"

10 CFR 61.12, "Specific Technical Information"

10 CFR 61.14 , "Institutional Information"

10 CFR 61.23, "Standards for Issuance of a License"

10 CFR 61.24 , "Conditions of Licenses"

10 CFR 61.28, "Contents of Application for Closure"

10 CFR 61.29, "Post Closure Observation and Maintenance"

10 CFR 61.30, "Transfer of License"

10 CFR 61.31 , "Termination of License"

10 CFR 61.40, "General Requirement"

10 CFR 61.42, "Protection of Individuals From Inadvertent Intrusion"

10 CFR 61.44, "Stability of the Disposal Site After Closure"

10 CFR 61.52, "Land Disposal Facility Operation and Disposal Site Closure" 
10 CFR 61.53, "Environmental Monitoring"

10 CFR 61.62, "Funding for Disposal Site Closure and Stabilization"

10. CFR 70.25, "Financial Assurance and Record Keeping for Decommissioning"

10 CFR 70.38, "Expiration and Termination of Licenses"

10 CFR 72.30, "Decommissioning Planning Including Financing and Record Keeping"

10 CFR 72.40, "Issuance of License"

10 CFR 72.54, "Application for Termination of License"

10 CFR 72.130, "Criteria for Decommissioning"

Regulatory Guide 3.65, "Standard Format and Content of Decommissioning Plans for Licensees Under 10 CFR Parts 30, 40, and 70."

NUREG-0436, Rev. 1 and Supplements I and 2, "Plan for Reevaluation of NRC Policy on Decommissioning of Nuclear Facilities," USNRC, December 1978.

NUREG-0278, Vol. 1 \& Vol. 2, "Technology, Safety, and Costs of Decommissioning a Reference Nuclear Fuel Reprocessing Plant," Schneider, K.J. et al., Battelle Pacific Northwest" Laboratory, October 1977.

NUREG/CR-1754, Addendum 1, "Technology; Safety, and Costs of Decommissioning Reference Non-Fuel-Cycle Nuclear Facilities," Short, S.M., Pacific Northwest Laboratory, October 1989: 


\section{Project File Number $\quad$ 02BD7}

Project/Task Waste Treatment Project Feasibility Studies

Subtask WTF Design Requirements

Title:

Regulatory and Design Requirements for Waste Treatment Facilities

SUMMARY: The purpose of this document is to delineate the design requirements for the nonseparations and TRU only separations options for the Waste Treatment Facilities (WTF) feasibility studies. The facilities will be designed and constructed under one of three possible regulatory scenarios: 1) performance against US Department of Energy (DOE) Orders with maintenance of status-quo interfaces with other regulatory and oversight agencies such as the EPA (State of Idaho) and the Defēnse Nuclear Facilities Safety Boar̃d (DNFSB), 2) performance against DOE Or̃ders with all current regulatory/oversight relationships maintained and or Nuclear Regulatory Commission (NRC) oversight to achieve "NRC Equivalency" or, 3) NRC licensing though replacement of DOE Orders with NRC Regulations and replacement of DNFSB oversight with NRC licensing process. For the purposes of this study and at the direction of the high level waste alternatives feasibility studies project manager, the base case for this study is performance against DOE Orders (scenario 1, above). This is consistent with the approach taken by Fluor Daniel, Inc., at the direction of the HLW Program in the preparation of the planning alternative and will provide an apples-to-apples comparison of the alternatives.

\section{TABLE OF CONTENTS:}

Introduction

1.0 Facilities For Which This Document Applies.............................................2

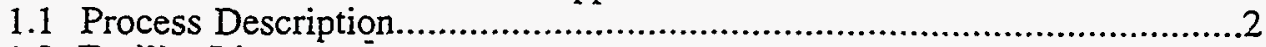

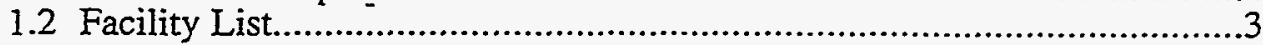

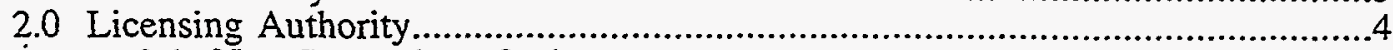

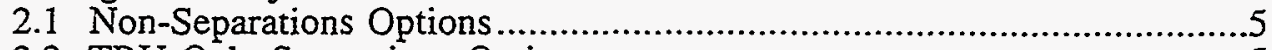

2.2 TRU-Only Separations Options.....................................................

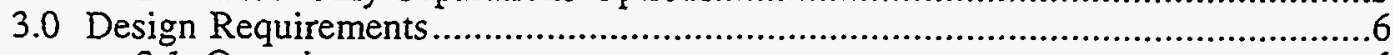

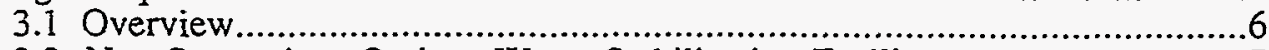

3.2 Non-Separations Options Waste Stabilization Facility ..............................

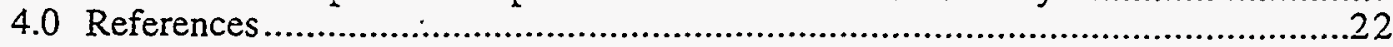

Distribution (complete package):

Distribution (summary package only):

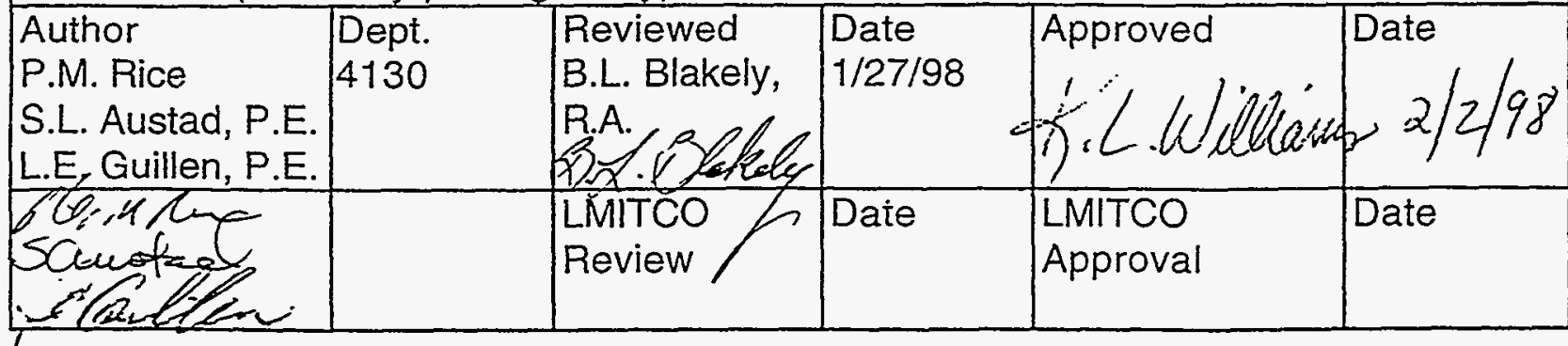




\section{Introduction}

The purpose of this document is to delineate the design requirements for the non-separations and TRU only separations options for the Waste Treatment Facilities (WTF) feasibility studies. The facilities will be designed and constructed under one of three possible regulatory scenarios: 1) performance against US Department of Energy (DOE) Orders with maintenance of status-quo interfaces with other regulatory and oversight agencies such as the EPA (State of Idaho) and the Defense Nuclear Facilities Safety Board (DNFSB), 2) performance against DOE Orders with all current regulatory/oversight relationships maintained and or Nuclear Regulatory Commission (NRC) oversight to achieve "NRC Equivalency" or, 3) NRC licensing though replacement of DOE Orders with NRC Regulations and replacement of DNFSB oversight with NRC licensing process. For the purposes of this study and at the direction of the high level waste alternatives feasibility studies project manager, the base case for this study is performance against DOE Orders (scenario 1, above). This is consistent with the approach taken by Fluor Daniel, Inc., at the direction of the HLW Program in the preparation of the planning alternative and will provide an apples-to-apples comparison of the alternatives.

With the ground rules clearly established above, the following is a discussion of how they will be selectively applied/ignored.

This EDF will identify the applicable DOE orders, regulations and guidance documents that would be used in the design of the facilities. There are currently no NRC regulations in place for the licensing of waste processing facilities such as those discussed herein. The only WTF activities for which the NRC has been routinely involved is the licensing of waste storage and disposal facilities. Never-the-less, NRC requirements are looming on the horizon. In addition, the waste products to be produced are in many cases destined for NRC licensed storage facilities. Therefore, there are some NRC requirements that just cannot be ignored. Thus, where appropriate, NRC regulations are explicitly specified in the design requirements. Where specific design criteria is provided under NRC regulations and guidance documents whether directly applicable or for similar facilities, it will be referenced. This will be useful in helping to determine the cost differential between DOE regulatory/oversite and NRC licensing requirements.

\subsection{Facilities For Which This Document Applies}

\subsection{Process Description}

The following is a very brief description of each of the processes that this document will consider:

Direct Vitrification Direct vitrification is a process for converting calcine into a glass waste. In the direct vitrification process, calcine is mixed with "frit" materials and fed to a melter, which would operate at a temperature of around $1100^{\circ} \mathrm{C}$. Numerous small-scale tests have been performed to determine frit formulations and glass properties, primarily leachability. No pilot data or design data is available for direct vitrification of calcine. 
Direct Grout Grouting is an ambient temperature process for solidifying or stabilizing a waste material. Grouting utilizes hydraulic cement that hardens by chemical interactions with water, and various additives which may aid dispersion, control hardening, control pumping characteristics or enhance retention of certain contaminants in the waste.

Cementitious Waste Process Darryl Siemer has proposed a direct grouting process ${ }^{33}$ with differences from that described above. Siemer suggests mixing existing calcine with existing SBW, and recalcining the resulting slurry in the existing calciner, using sugar as additive to permit calcination of high-sodium waste. This recalcined waste would then be mixed with a combination of cementitious agents and water, and transferred to a stainless steel can. After setting at ambient temperature, the can is transferred to an autoclave and cured with steam. If further processed by HIPing, the can would be vented and placed in a furnace to remove volatiles. Then the can would be transferred to the HIP chamber, an inert gas added to pressurize to $30-125 \mathrm{MPa}(4350-18,100$ psi), and the can heated at $850-1050^{\circ} \mathrm{C}$ for the required "soak" time.

Hot Isostatic Press (HIP) The HIP process uses high pressure and high temperature to convert calcine or other solid wastes to a glass-ceramic waste form. In a conceptual flowsheet proposed for processing calcine, calcine from storage is mixed with frit or other additives, fed to a HIP can, the can sealed and decontaminated, and then isostatically pressed in a furnace. Processing temperatures for the HIP process are similar to vitrification, typically $1050-1100^{\circ} \mathrm{C}$. The typical HIP operating pressure is 20,000 psi.

TRU Waste Alternative In this alternative, calcine is dissolved and actinides removed from the resulting solution by the TRUEX process. TRUEX wash effluents and raffinate along with other ICPP low level wastes, are evaporated, denitrated and grouted. The TRUEX strip effluent is evaporated, denitrated and then packaged for shipment to WIPP. An alternative to denitration would be to neutralize and evaporate the effluents from separation.

\subsection{Facility List}

Below is a list of the primary, main ancillary, and common support facilities that will be required for each of the options discussed herein:

1. Non-Separations Direct Vitrification Option:

- Vitrification Facility

2. Non-Separations Direct Grout Option:

- Grouting Facility

3. Non-Separations Calcine Hot Isostatic Press (HIP) Option:

- Calcine HIP Facility 
4. Non-Separations Cementitious Waste Option:

- Calcine Slurry and Grouting Facility

5. TRU-Only Separations:

- TRU Separations Facility

- TRU Product Handing, Packaging and Lag Storage Facility (TRU-only Separations Option):

- Class C Grouted Waste Interim Storage Facility:

In addition, a number of common support facilities/systems will be required to support the above facilities which include:

- Calcine Retrieval System (may vary depending on the process design for each option)

- Temporary Calcine (surge or staging) Storage

- Interim HLW Storage Facility

Note: For all of these alternatives, the study ends with interim storage of the waste product prior to shipment. Thus none of the studies includes facilities for receiving and internment of the final waste product at a repository.

\subsection{Licensing Authority}

As previously stated, for the purposes of this effort, the base case for this study is performance against DOE Orders. The following discussion is presented to defined under what NRC regulations each of the above referenced facilities would be licensed if NRC licensing were the preferred approach. This information is provided for reference only.

In early 1997 the INEEL Spent Nuclear Fuel, High-Level Waste and Related Programs prepared a licensing assessment report for proposed INEEL ICPP High Activity Waste Treatment Facilities (Idaho Chemical Processing Plant High Activity Waste Treatment Project Regulatory Assessment Report, prepared by R. G. Morgan and S. E. Leroy, Duke Engineering Services, Inc. S. E. Leroy letter to V. L. Jacobson, dated April 25, 1997) (1). The report provided an assessment of how the proposed ICPP Waste Treatment Facilities could be licensed under existing NRC regulations and processes. The report identifies the applicable NRC regulations and guidance documents that would be used in the licensing process. It also identifies those areas where additional NRC guidance documents, regulations, or rulemaking may be necessary.

The above referenced report specifically addresses the facilities defined in the 'preferred alternative', whereas this EDF is examining other methods of processing and disposing of the 
calcined wastes at ICPP. For the purpose of defining the licensing criteria for the options discussed in this EDF, the above referenced report will be used. Licensing criteria will be based on similarities between the preferred alternative facilities and the facilities described here.

\subsection{Non-Separations Options (Primary and Ancillary Facilities)}

The non-separations options include: 1) direct vitrification, 2) direct grouting, 3) calcine HIPing, and 4) the Cementitious Waste options. Each option will include a facility to perform the appropriate operations to produce the end product (e.g., vitrification facility, direct grouting facility, HIPing facility, and a calcine slurry and grouting facility for the Cementitious Waste option).

The non-separations options are similar in scope to the High Activity Waste Treatment (HAWT) Facilities described in reference 1. The HAWT facilities include a calcine retrieval, transport, and receiving system; a calcine dissolution process; a high activity waste vitrification process; and vitrified product storage. The non-separations options include a calcine retrieval, transport, and receiving system; a waste stabilization process (vitrification, grouting, HIPing); and product storage.

The facilities which will be licensed are the Waste Stabilization (WS) Facilities (e.g., the vitrification, grouting, HIPing facilities), the temporary calcine (surge or staging) storage tanks associated with the receipt of the feed stock, the calcine retrieval system, and the interim HLW storage facility.

Based on the existing NRC regulations and rulemaking activities, it is expected that the following licenses would be required to support the non-separations option plan:

- Waste Stabilization (WS) Facilities (e.g., the vitrification, grouting, HIPing facilities) would require a 10 CFR Part 70 license

- Temporary calcine (surge or staging) storage tanks associated with the receipt of the feed stock would require a 10 CFR Part 70 license.

- Calcine retrieval system would require a 10 CFR Part 70 license.

- Interim (temporary) HLW storage facility would require a 10 CFR Part 72 license.

Other NRC regulations that are applicable to the design, licensing, and operations of the facilities will be addressed later in this EDF.

\subsection{TRU-Only Separations Options (Primary and Ancillary Facilities)}

As with the non-separations options, the TRU-only separations options licensing requirements were derived by similarity to the preferred option HAWT facilities.

The facilities which will be licensed are the TRU Separations (TS) facility, TRU product handing, packaging and lag storage facility, class $C$ grouted waste interim storage facility, the 
temporary calcine (surge or staging) storage tanks associated with the receipt of the feed stock, and the calcine retrieval system.

Based on the existing NRC regulations and rulemaking activities, it is expected that the following licenses would be required to support the non-separations option plan:

- TRU Separations (TS) facilities, including product handling, packaging, and lag storage would require a 10 CFR Part 70 license

- Temporary calcine (surge or staging) storage tanks associated with the receipt of the feed stock would require a $10 \mathrm{CFR}$ Part 70 license.

- Calcine retrieval system would require a 10 CFR. Part 70 license.

Other NRC regulations that are applicable to the design, licensing, and operations of the facilities will be addressed later in this EDF.

\subsection{Design Requirements}

\subsection{Overview}

The criteria contained in this document are based only on the rudimentary descriptions of the processes presented in section 1.1. As the design is developed further, some of the criteria may become nonapplicable, and others will be identified. The purpose here is to provide a set of highlevel requirements to guide the development of the conceptual designs of the facilities and provide a reasonable basis for cost estimating purposes. In general this document will not attempt to cover criteria outside of the design and construction of the facilities. Process criteria such as the waste form acceptance criteria, treatment standards and so forth will be addressed by others.

\subsubsection{A Note on NRC Regulations}

NRC regulations are contained in Title 10, Energy of the Code of Federal Regulations. CFR's have the authority of legal mandates, and require compliance, under penalty of law, by all affected parties. NRC generates guidance documents as needed to provide clarification and elaboration of the regulations, describe information to be included in the reports, and give acceptance criteria. These publications truly are guidance documents which are not required to be followed but provide suggested methods for achieving successful licensing. Guidance documents include:

\section{NRC Regulatory Guides}

Regulatory Guides delineate acceptable methods of meeting NRC requirements. Different methods for meeting these requirements may be used if justified but the licensees usually attempt to use the Regulatory Guide methods because alternate approaches require extensive justification and additional NRC review. The use of the guides simplifies and shortens the licensing process. 
There are over 480 Regulatory Guides that have been issued to support the licensing of commercial nuclear facilities. While many of the guides apply to nuclear reactors, others, such as those describing waste storage may be viewed as applicable to the WTF. However, no definitive guidance is available for the type of facilities discussed below.

\section{$\underline{\text { NRC Reports }}$}

NRC Reports (NUREGs) and other NRC reports developed by contractors are published on a variety of technical and regulatory issues. They may pertain to specific proceedings such as Safety Evaluation reports or Environmental Impact statements.

\section{NRC Technical Positions}

Technical Position and Staff Position Papers are also prepared by the NRC as a means of providing guidance on requirements for specific facilities regulated by the agency.

\section{Generic Communications}

NRC Generic communications include NRC Information Notes, Generic Letters and NRC Bulletins. These documents provide the licensees with specific information on problems or matters of interest to the licensee.

\section{National Standards}

NRC regulations and documents often incorporate or refer to national codes such as the ASME boiler and pressure vessel codes. These codes then become a requirement and are used in developing design criteria. If the licensee wishes an exception, the exception must be identified and basis for the exception agreed to during the licensing process.

\subsection{Non-Separations Options Waste Stabilization Facility}

The non-separations options include: 1) direct vitrification, 2) direct grouting, 3) calcine HIPing, and 4) the Cementitious Waste options. Each option will include a facility to perform the appropriate operations to produce the end product (e.g., vitrification facility, direct grouting facility, HIPing facility, and a calcine slurry and grouting facility for the Cementitious Waste option). The requirements for supporting (ancillary) facilities will be discussed under separate headings.

\subsubsection{Civil Requirements}

\subsubsection{Site Development}

A suitable site shall be located for the Waste Stabilization Facility at the INEEL in the vicinity of the Idaho Chemical Processing Plant (ICPP) with the proximity to waste sources, utilities, other facilities, vehicular access, shipping and storage capability, and future growth. A study of the impact of this facility on site utilities and infrastructure shall be performed. Information regarding 
topography, soil conditions, subsurface rock formations, road and structure locations shall be included in the final site-decision process.

\subsubsection{Flood Design}

Flood design shall be in accordance with DOE-STD-1020. Additionally, if the facility is a RCRA facility, design shall be in accordance with 40 CFR 270.14. This standard requires the facility to be located above the $100-y r$ flood elevation or for engineered barriers against flooding of the site to be constructed. If the facility is a TSCA facility, 40 CFR 761.65 requires the facility to be located above the 100 -yr flood elevation with no allowance for engineered barriers against flooding.

\subsubsection{Surface Drainage}

Design for surface drainage from local precipitation shall be in accordance with DOE-STD1020 and should be consistent with the ICPP site drainage plan. The INEEL site specific local precipitation standard for a 25 -year, 6-hour storm is 1.4 inches total.

\subsubsection{Subsurface Investigation and Surveying}

Surveying and subsurface investigation for design shall be conducted to determine depth of rock, confirm soil characteristics and evaluate existing soil for chemical and radiological contamination. Locations of ground surface interferences and site characteristics shall be determined with a survey of the site.

\subsubsection{Soil Excavation and Shoring}

Specifications for excavation work shall require that excavations comply with OSHA Standards, 29 CFR 1926, Subpart P (and Subpart U if blasting is necessary), Subsection 1926.641. Where major complex temporary support systems such as shoring, cribbing, sheet piling, etc. are required, they shall be fully design by the $\mathrm{AE}$ as part of the design package.

\subsubsection{Paving and Surfacing}

Paving shall be provided around the building for parking areas and access roads. All paved areas adjacent to buildings and structures shall have a $1 \%$ minimum slope away from the buildings or structures.. Unpaved areas_shall.be sloped $2 \%$ minimum.

Design for paved roads shall conform to Idaho State Highway Standard Specifications and AASHTO HS-20 loading. Geometric design of all roads, streets, access drives and parking areas shall comply with AASHTO. Other loadings such as those imposed by transfer cask operations shall be incorporated into pavement design where applicable.

\subsubsection{Slabs, Sidewalks, and Stoops}


Sidewalks, door stoops, and approaches shall be provided at all building personnel exits or vehicle openings. Sidewalks shall be installed to provide a safe and efficient means for personnel to access doorways and walk to other nearby facilities. Concrete slabs, door stoops, truck ramps, etc., shall be sloped at least $2 \%$.

\subsubsection{Physical Protection}

The facility shall be located within the ICPP security system and fence. Construction of the new facility may take place outside of the existing main security fence if an equivalent level of security protection is established.

\subsubsection{Underground Utilities}

Existing underground ICPP utilities (sewer, potable and fire water systems) shall be extended as necessary to provide necessary services. Design of potable systems shall be in accordance with the State of Idaho Department of Health and Welfare, Idaho Regulations for Public Drinking Water Systems. Sanitary waste water shall be routed to the ICPP sewage treatment system. Water used for cleaning of the hot cells, if applicable, shall be removed by floor drains or sumps, filtered, contained in double containment tanks, monitored for hazardous materials, and if allowable, routed to the sanitary sewer system:

\subsubsection{Site Demolition}

Site demolition, as required, will be dependent of the final site location.

\subsubsection{Architectural Requirements}

\subsubsection{General .....}

Architectural designs shall be in accordance with the DOE-ID Architectural Engineering Standards, DOE $6430.1 \mathrm{~A}$, and the following design criteria. The facility shall have a minimum design life of 40 years. Interim Storage Facility design life shall be 50 years minimum. The facility shall be planned and laid out on the basis of repetitive or discrete processing steps. The need for safe normal and emergency access, egress and internal traffic flow shall be considered.

Energy conservation shall be given attention during planning and design in accordance with 10 CFR 435, Energy Conservation Voluntary Performance Standards for New Buildings, Mandatory for Federal Buildings.

\subsection{Hot Cell.}

Hot Cell design shall be based upon a Uniform Building Code (UBC) occupancy classification of Group $\mathrm{H}$, Division 7. Occupancy separations and construction types shall be designed in accordance with the UBC. 
Layout of the Hot Cell area shall include a buffer area for personnel entering and exiting the cell and shielded viewing windows for remote operations.

The Hot Cell shall include shielded, impervious and decontaminable walls, floors and ceilings as appropriate. The hot cell walls shall provide sufficient shielding to protect personnel from gamma and neutron radiation. The dose rate at the exterior of the Hot Cell wall in the operating gallery shall be below $0.1 \mathrm{mrem} / \mathrm{hr}$.

\subsection{Buffer Area.}

A Buffer Area shall be provided between the Hot Cell and other areas. Design shall be based upon a UBC occupancy classification of Group H, Division 7. Facilities for changing anti-c clothing and personnel monitoring (frisking) devices shall be provided adjacent to the Hot Cell. A shielding labyrinth leading from the Hot Cell to the Buffer Area and then to an Anti-C Change Room shall be provided. These areas. shall be separated from each other and the pressure barriers maintained.

The Buffer Area shall provide space for discarded protective clothing used in the Hot Cell and a step off pad for frisking of contamination by PCM machine. All surfaces in the Buffer Area shall be impervious and decontaminable as well as the floors and walls of the Anti-C room.

\subsection{Operating Galleries.}

Operating galleries shall be provided as required by view angles and retraction/repair of remote equipment. Space and utilities shall be provided to accommodate remote equipment operations. Operating galleries shall be separated from the Hot Cell by a concrete shielded wall.

\subsection{Utility Support Areas.}

Utility Support Areas design shall be based upon a UBC occupancy classification of $\mathrm{H}-7$ and shall be designed to accommodate remote and contact maintenance of equipment.

\subsection{Equipment Maintenance Areas.}

Crane maintenance areas shall be provided to support maintenance of in-cell equipment.

\subsection{Administrative Areas.}

The Administrative Area design, which includes office and support areas, shall be based upon a UBC occupancy classification of Group B.

The Administrative Area shall include a minimum of three offices for a shift supervisor and HP support personnel. A Ready Room shall be provided for conduct of meetings and work breaks. Men's and women's lavatories, showers, lockers, and change facilities shall be provided. Storage space and a janitor's room shall also be provided. 


\subsubsection{Building Features}

Materials selected for the walls shall address durability, low maintenance, shielding, insulation and decontamination. The walls shall meet the recommended $R$ value of the DOE-ID AVE Standards. The UBC Construction Type of II-N shall be used for the Facility.

The entire surface area of the contaminated work areas shall be decontaminable. Where wash down or decontamination activities are to be located, the floors shall be sloped to drains that lead to appropriate holding tanks.

Devices (such as door types or air lock arrangements) shall maintain pressure barriers for the hot cell and operating gallery areas.

\subsubsection{Structural-Requirements:-}

\subsubsection{General}

Structural design shall be in accordance with the DOE-ID Architectural Engineering Standards, DOE O 420.1, and DOE-STD-1020.

\subsubsection{Classification and Design Loads}

The performance categories for SSCs shall be established using DOE-STD-1021, Natural Phenomena Hazards Design and Evaluation Criteria for Department of Energy Facilities." Sitespecific studies and hazard assessments of the site, as needed, shall be developed in accordance with DOE-STD-1022, "Natural Phenomena Hazards Site Characterization Criteria" and DOE-STD1023, "Natural Phenomena Hazards Assessment Criteria."

All permanent and transient loads that could exist or be developed during normal operations of the facility shall be considered in the design of the facility. Loads to be considered shall include: dead, live, thermal, lateral soil, snow, natural phenomena, seismic, wind, flood, off-normal operating and accident loads, and load combinations.

Dead and live loads shall be determined in accordance with ANSI/ASCE 7, "Minimum Design Loads for Buildings and Other Structures." Loadings due to natural phenomena hazards (wind, seismic, flood, etc.) shall be determined in accordance with DOE-STD-1020, Natural Phenomena Hazards Design and Evaluation Criteria for Department of Energy Facilities."

In accordance with DOE-STD-1020, the Uniform Building Code shall be used as the basis for seismic design for Performance Category 1 and 2 SSCs. The seismic input control motion for the INEEL for Performance Category 3 SSCs is specified by appropriately scaling the USNRC R.G. 1.60 horizontal spectra $(0.18 \mathrm{~g})$. The input motion is assumed to occur in the free-field at the top of a real or hypothetical rock outcrop near the facility location. The vertical input spectra shall be taken as $2 / 3$ of the horizontal spectra. A detailed soil amplification analysis or the soil surface spectra shall be taken to equal the rock outcrop spectra multiplied by: 
(a) 1.2 for soil overburden up to $20 \mathrm{ft}$.

(b) 1.5 for soil overburden between $20 \mathrm{ft}$ and $50 \mathrm{ft}$.

Snow loads shall be determined in accordance with ANSI/ASCE 7, with a ground snow load of $35 \mathrm{psf}$ and a minimum roof snow load of $30 \mathrm{psf}$. Tornado loads are not anticipated and need not be included. Load combinations shall be determined in accordance with ANSI/ASCE 7.

3.2.4.2.1 NRC Specific-To meet NRC requirements, seismic loads shall be determined in accordance with 10 CFR 72, Subparts D and E, 10 CFR 100, and USNRC Reg. Guide 1.6. Tornado loads shall be determined in accordance with ANSI/ANS-2.3. Load combinations shall be designed using applicable load combinations and stress limits stipulated in ANSI/AISC N690 and ANSI/ACI349.

\subsubsection{Footings and Foundations}

Footings shall be designed to support the structure and keep differential settlement within allowable limits. Design frost depth shall be 5-ft below grade. The Hot Cell and shielded storage areas shall be provided with continuous reinforced grade beams or wall footings as required for shielding.

\subsubsection{Structural Features}

The Hot Cell walls and roof design shall be consistent with shielding and loading requirements. Other shielded area walls and roofs shall be designed consistent with shielding and loading requirements. The structural design must support crane systems.

\subsubsection{Handling Requirements}

\subsubsection{Cranes (Critical Lift Devices Only)}

All crane designs shall meet the ASME NOG-1 and where applicable, CMAA 70, Crane Manufacturers Association of America, Inc., Specification for Electric Overhead Traveling Cranes and CMAA 74-1987, Specifications for Top Running and Under Running Single Girder Electric Overhead Traveling Cranes Utilizing Under Running Trolley Hoist.

In addition, all cranes shall meet the requirements of NUREG-0612, Control of Heavy Loads at Nuclear Power Plants.

Cranes shall have true vertical lift on the hoist and all motions shall be the slowest that are commercially available to allow for more precise control when placing or picking objects.

\subsubsection{Work Platforms}


The facility shall be equipped with decontaminable work platforms that shall provide a safe and convenient elevated work area for personnel as required. Design of the platforms and their means of access shall be in accordance with OSHA 1910. Removable guard rails may be utilized as necessary to meet process and handling requirements.

\subsubsection{HVAC Requirements}

All HVAC systems shall be in accordance with Regulatory Guides RG 1.140 Design, testing, and maintenance for normal ventilation systems, RG 1 Design, testing, and maintenance criteria for post-accident engineered safety feature ventilation systems, NUREG-0800 Section 9.4.3 Auxiliary and radioactive waste area ventilation, NUREG-0800 Section 11.3 Gaseous waste management systems, NUREG 0800 Section 11.5 Process and effluent radiological monitoring, NUREG-1567 Offgas treatment and ventilation, NUREG-1567 (Draft) Section 11.4.1.3, Ventilation offgas system design features.

HVAC systems shall be in accordance with 29 CFR 1910, Occupational Safety and Health Standards for General Industry, Subpart G (Occupational Health and Environmental Control) and Subpart Z (Toxic and Hazardous Substances).

The HVAC systems shall meet the air quality requirements addressed in 40 CFR 50-53, and 58 .

The HVAC systems shall be in accordance with ANSI/ASME N509-1989, Nuclear Power Plant Air Cleaning Units and Components and ANSUASME N510-1989, Testing of Nuclear AirCleaning Systems.

The HVAC systems shall meet the requirements of Idaho Administrative Procedures Act (IDAPA) 16.01.01000-01999.

The HVAC systems shall be in accordance with MIL-F-51068C (Filter: Particulate High Efficiency, Fire Resistant) and MIL-F-51079A (Filter Medium: Fire Resistant, High Efficiency).

The Hot Cell atmospheric pressure shall be controlled during hot operations to $-0.6 \mathrm{~W}$. G. (or lower) below atmospheric pressure.

Heating loads shall be based on a minimum winter outdoor design temperature of $-14^{\circ} \mathrm{F}$. Cooling loads shall be based on temperatures of $93^{\circ} \mathrm{F}$ dry bulb and $61^{\circ} \mathrm{F}$ wet bulb. The HVAC system should maintain a minimum temperature of $65^{\circ} \mathrm{F}$ in the winter and approximately $76^{\circ} \mathrm{F}$ in the summer in the operations area (not including the vitrification cell). The HVAC system must maintain a minimum temperature of $65^{\circ} \mathrm{F}$ in the winter and approximately $72^{\circ} \mathrm{F}$ in the summer in the Administrative areas. HVAC design for indoor temperature conditioning shall be based on ASHRAE 90. 
Air shall flow from areas of least contamination potential to areas of highest contamination potential. The HVAC system shall collect exhaust air from contamination control areas and pass it through HEPA filters prior to discharge to the atmosphere.

\subsubsection{Mechanical Utilities Requirements}

Mechanical utilities systems shall meet the requirements of the ASME Code for Pressure Piping B31.

\subsubsection{Compressed Air}

Compressed air for plant and instrument air shall be provided for pneumatically operated HVAC system equipment and other pneumatic operations in the facility. The system design for compressed air shall be in accordance with 29 CFR 1910, Occupational Safety and Health Standards for General Industry, Subpart M (Compressed Gas and Compressed Air Equipment). Instrument air shall be ISO-141 Grade or better.

\subsubsection{Compressed Gas}

Argon compressed gas shall be supplied for welding processes

Helium compressed gas shall be supplied for pressure testing and inerting operations.

The system design for compressed gas shall be in accordance with 29 CFR 1910, Occupational Safety and Health Standards for General Industry, Subpart H (Hazardous Materials) and Subpantt M (Compressed Gas and Compressed Air Equipment).

\subsubsection{Potable Water}

Potäble water, including hot water where applicable, shall be provided to the facility to service water closets, urinal(s), sinks, showers, shower/eyewash facilities, evaporative coolant units, drinking fountains, and miscellaneous ports.

Cross-connection control shall be in accordance with the Idaho Code (IDAPA 16.01.08), "The Cross Connection Control Manual, Accepted Procedure and Practice" (Pacific Northwest Section of American Water Works Association), and the Foundation for Cross Connection Control and Hydraulic Research (University of Southern California.)

\subsubsection{Waste Systems}

3.2.9.4.1 Liquid Waste-Liquid waste system(s) shall be provided for in the Hot Cells and other process areas. The liquid waste systems shall be designed in accordance with NUREG-0800 Section 11.2 Liquid waste management systems. Condensate from HVAC equipment shall be disposed of using the liquid waste system. Liquid waste shall be collected and tested prior to being pumped into the waste line 
3.2.9.4.2 Sanitary Systems-The sanitation system design shall be in accordance with 29 CFR 1910, Occupational Safety and Health Standards for General Industry, Subpart J (General Environmental Controls). Sanitary sewer drains, cleanouts, and vents shall be provided as needed

\subsubsection{Fire Protection}

Fire water shall be provided in accordance with DOE 6430.1A, DOE Order 420.1, and the DOE-ID Architectural Engineering Standards. Fire protection systems shall ensure nuclear criticality and suppressant-HLW chemical reactions cannot occur. All underground fire water lines shall be cathodically protected and meet State of Idaho requirements for minimum distances from potable water piping.

\subsubsection{Steam}

Steam shall be provided and routed to the HVAC system as required. The steam lines shall be insulated.

\subsubsection{Electrical Requirements}

The criteria for the electrical design of the WTF is based on requirements from NFPA, ANSI, Factory Mutual (FM), DOE O 420.1 and 29 CFR 1910, Occupational Safety and Health Standards for General Industry, Subpart $S$ (Electrical).

Electrical design and installation shall incorporate the most efficient methods of penetration, shielding integrity retention, efficiency, and operational convenience.

The facility shall require an electrical room a communications room, and an Uninterruptable Power Supply (UPS) room.

\subsubsection{Power}

The electric power system shall be designed to provide standard power to the facility and emergency electrical supply to essential instrumentation, emergency lighting, emergency communications, and physical security systems. Standby power shall be supplied for the Hot Cells, process areas, and HVAC system exhaust fan.

An Uninterruptable Power Supply (UPS) shall provide emergency power. The UPS shall support the Fire Alarm, Voice Paging, HVAC, Radiation Monitoring and Alarm, and security systems. There shall sufficient battery capacity to carry the rated load for a minimum of 30 minutes.

\subsubsection{Grounding} standards.

Grounding shall be provided in accordance with the DOE-ID Architectural Engineering 


\subsubsection{Cathodic Protection}

Utility piping shall be protected through connection to the existing ICPP cathodic protection system. A testing/bonding station shall be included to periodically monitor the cathodic protection system.

\subsubsection{Lighting}

Interior and exterior lighting shall be designed and included in accordance with current Illuminating Engineering Society (IES) recommendations. Emergency and exit lighting shall be provided at each means of egress. Hot Cell lighting shall be provided by high-pressure sodium fixtures.

\subsubsection{Lightning Protection}

A lightning protection system shall be included and shall be designed in accordance with NFPA 780.

\subsubsection{HVAC Controls}

A HVAC control system shall be provided. It shall be a smart system that can automatically generate control signals to change HVAC equipment operating parameters based on signal received from various monitors. A computer monitor shall be provided in the Shift Supervisor's office for reviewing the operating status of the system and making adjustments to control setpoints.

Instrumentation shall be provided to detect and alarm both high and low differential pressure across filters in the HVAC system. Instrumentation shall be provided to initiate isolation of the HVAC system filters in the event of fire detection.

\subsubsection{Equipment Controls}

Facility control, process control, and data acquisition systems shall be provided.

Remotely controlled CCTV cameras shall be provided in the Hot Cells and process areas for general visual observation, operations, inspection, and documentation. Each Hot Cell window shall be equipped with a visual inspection station which shall include two high resolution cameras; a monitor; camera controls for pan, tilt, and zoom functions; and recording capability for archival purposes.

Instrumentation shall be provided to measure and record the facility structural response to an earthquake.

A system shall be provided for the collection of alarms from the HVAC system and other alarms. This shall be located in the Shift Supervisor's office. 


\subsubsection{Radiation Monitoring and Alarms}

Radiation detection instrumentation shall be provided to warn operating personnel of radiation and airborne radioactivity levels above set limits. The RAMs shall alarm locally and remotely in the RadCon office.

Stack monitoring shall be provided for the detection of radioactive particulates in the air exhaust stream. These instruments shall comply with ANSI-N42.17B-1989.

Provisions shall be made in the design for monitoring groundwater in the vicinity of the storage area for radioactive contamination.

Activity monitors shall be provided in the wash water collected from the Hot Cells and process areas.

\subsubsection{Communications and Alarms}

Voice and data telecommunications lines shall be provided throughout the occupied areas of the facility. The existing. Broadband Local Area Network (LAN) shall be made available in the facility. Access ports shall be provided in all normally occupied offices.

Fire alarm, emergency voice paging, and evacuation alarm systems shall be compatible with existing systems at ICPP.

\subsubsection{Data Acquisition and Recording}

A data entry station shall be provided to record and monitor all fuel movements. The stations shall be linked for data communications.

\subsubsection{Security Systems} Subpart $\mathrm{H}$.

Physical protection of the facility shall be in accordance with 10 CFR 73 and 10 CFR 72 ,

\subsubsection{Design Life Requirements}

Design life of the facility and equipment shall be 30 years and have maintainable or replaceable life of 60 years

\subsubsection{Safety Requirements}

\subsubsection{Safety Classification}

The facility is assumed to be a Hazard Category 2. 


\subsubsection{Construction}

The design of utility services and distribution systems that are important to safety shall include redundant systems to the extent necessary to maintain, with adequate capacity, the ability to perform safety functions assuming a single failure.'

The facility and its systems important to safety ${ }^{2}$ shall be designed to be evaluated by appropriate tests or by other means acceptable to the NRC to demonstrate that they will reasonably maintain confinement of radioactive material under normal, off-normal, and credible accident conditions.

Structures, systems, and components important to safety shall be designed and located so that they can continue to perform their safety functions effectively under credible fire and explosion exposure conditions.

The design of the facility shall include provisions to protect against nuclear criticality that might otherwise result from the operation or the failure of fire suppression or decontamination systems.

Material handling, packaging, transfer, and storage systems shall be designed to be maintained subcritical under the worst case moderated and reflected conditions, and to ensure that, before a nuclear criticality accident is possible, at least two unlikely, independent, and concurrent or sequential changes must occur in the conditions essential to nuclear criticality safety.

Each entrance or access point into a high radiation area shall have either a control device that energizes a conspicuous visible or audible alarm signal so that the individual entering the high radiation area and the supervisor of the activity are made aware of the entry; or entryways that are locked, except during periods when access to the areas is required, with positive control over each individual entry.

Process materials that are reactive with water or other chemicals shall be protected from exposure to those materials.

The facility shall be designed to prevent the dropping of critical loads under normal and off normal conditions including the design basis accidents (DBAs) that they shall withstand.

1. A single failure is an occurrence that results in the loss of capability of a component to perform its intended safety function(s). A multiple failure, i.e., loss of capability of several components, resulting from a single occurrence, is considered to be a single failure. Systems are considered to be designed against an assumed single failure if neither (1) a single failure of any active component (assuming passive components function properly) nor, (2) a single failure of any passive component (assuming active components function properly) results in loss of the system's capability to perform its safety function(s).

2. Structures, systems, and components important to safety mean those features of the Storage Facility whose function is: (1) To maintain the conditions required to store spent fuel safely, (2) To prevent damage to the spent fuel waste container during handling and storage, or (3) To provide reasonable assurance that spent fuel can be received, handled, packaged, stored, and retrieved without undue risk to the health and safety of the public. 
The facility shall be designed to be able to recover from accidents involving dropping of critical loads.

In-cell equipment shall be designed for recovery from all possible conditions to the extent that manned entry into the cell, for maintenance, can be accomplished.

Fire doors shall be provided as required by UBC, UFC, NFPA-80, and NFPA-101. In addition, all fire doors and frames shall meet all requirements of the Underwriters Laboratories and shall bear the UL or FM label. Fire doors and frames shall be constructed from metal. Structural members, such as steel channels embedded in wall openings, shall not substitute as door frames. All fire doors shall be provided with fitted frames which are anchored to, but separate from, the building structural members. The fire doors shall contain windows fitted with UL approved safety glass which is not removable from the outside of the door. Their installation shall meet all of the requirements of NFPA-80 and NFPA-101.

The facility design shall mitigate natural phenomena hazards. The design shall address common cause effects and interactions for: earthquakes, volcanic events, tornadoes, hurricanes, high winds, floods, excessive rains, excessive snow, ice cover, lightning, and fires. The secondary natural phenomena include drought, fog, frost, high temperatures, low temperatures, landslides, subsidence, surface collapse, uplift, storm surges, and waterspouts. Damage and failure will be considered for systems, structures, and components. In addition, the facility shall have instrumentation or other means to detect and record the occurrence and severity of seismic events.

\subsubsection{Operation}

Radiation protection for occupational workers shall be per $10 \mathrm{CFR} 835$ (Occupational Radiation Protection) and the INEEL Radiological Control Manual

Facility design features and physical controls shall ensure occupational exposure is maintained ALARA during normal and off-normal operations

Personnel radiation exposure levels throughout facility shall not exceed $0.1 \mathrm{mr} / \mathrm{hr}$ for continuously-occupied areas.

The following radiation zones (as described in the DOE-ID AE Standards) shall apply during operations: TBD

Safe access will be provided to all packages, vehicles, and installed components for purposes of testing, inspection, and maintenance.

\subsubsection{Environmental Requirements}

Facility emission limits shall be per requirements listed in EDF-WTS-003, Section 5. 
Administrative controls and Best Available Control Technology shall be used to minimize the impacts of air emissions

The facility processes and equipment shall be designed to limit solid waste generation of LLW and industrial (cold) waste

Solid radioactive waste produced by operations shall be packaged in standard RWMC $4 \times 4$ $\times 8$-ft plywood boxes for contact-handled (CH) LLW or INEL Mark III concrete containers for remote-handled (RH) LLW, and shipped to RWMC for disposal

Means for measuring the amount and concentrations of radionuclides in effluents during normal operations, and under accident conditions, shall be provided for effluent control systems

Warm liquid waste shall be controlled and verified to meet the criteria for existing ICPP handling systems, and shall be transferred to those systems

If all or part of the facility is located outside of existing ICPP fences, the use of new land shall not exceed 551 acres when combined with other storage systems included in DOE/EIS-0203-F.

\subsubsection{Safeguards and Security}

The materials are not attractive as defined in DOE Order 5633.3B.

A data management system shall be provided to keep records. The data management system shall meet the requirements of 10 CFR 72, Licensing Requirements for the Independent Storage of Spent Nuclear Fuel and High-Level Radioactive Waste, Subpart D (Records, Reports, Inspections, and Enforcement).

Dual records shall be maintained to ensure an off-normal event cannot result in the loss of the sole records. These records shall be retained for as long as the material is stored, and for a period of five years after the material is disposed of or transferred.

The following are NRC requirements that may or may not apply

Equipment shall be provided to conduct a physical inventory of all material in storage at intervals not to exceed 12 months unless otherwise directed by the Commission. A copy of the current inventory shall be retained as a record until the Commission terminates the license.

Physical protection of the facility and materials shall be in accordance with 10 CFR 72, Licensing Requirements for the Independent Storage of Spent Nuclear Fuel and High-Level Radioactive Waste, Subpart H (Physical Protection); 10 CFR 73, Physical Protection of Plants and Materials; and 10 CFR 1046, Physical Protection of Security Interests.

\subsubsection{Quality Assurance Requirements}


The applicable portions of DOE/RW-0333P, Quality Assurance Requirements and Description, shall be invoked as the baseline requirements document for developing and implementing quality assurance programs. These requirements apply to activities related to interim storage or disposal, including characterization for data collection, conditioning, or placing into a form for disposal. In addition, the EM-WAPS Rev. 01, Waste Acceptance Product Specifications for Vitrified High-Level Waste Forms, May 1995, also imposes a QA Program consistent with the QA requirements under the DOE/RW-0333P.

All purchased items will be restricted to those not suspect/counterfeit, misrepresented, used, or other than represented/advertised in accordance with INEL-95/227, "Guidelines for Identifying Suspect/Counterfeit Material."

Records, reports, and inspections shall be done in accordance with $10 \mathrm{CFR} 72$, Licensing Requirements for the Independent Storage of Spent Nuclear Fuel, Subpart D (Records, Reports, Inspections, and Enforcement).

Training of personnel shall be performed in accordance with 10 CFR 72, Licensing Requirements for the Independent Storage of Spent Nuclear Fuel, Subpart I (Training and Certification of Personnel). 


\subsection{References}

Nuclear Regulatory Commission (NRC) Licensing Assessment for the Idaho National Engineering and Environmental Laboratory (INEEL) High-Level Waste Program, prepared by R. G. Morgan and S. E. Leroy, Duke Engineering Services, Inc., April 23, 1997. Referenced in Idaho Chemical Processing Plant High Activity Waste Treatment Project Regulatory Assessment Report - SEL-11-97, prepared by S. E. Leroy, dated April 25, 1997. 
Project File Number $02 \mathrm{BEO}$

Project/Task Waste Treatment Project

Feasibility Studies

Subtask Direct Cementitious Waste Option Scoping Study

Title: Direct Cementitious Waste Option - Process Design

Summary: This EDF describes the proposed process for the Direct Cementitious Waste Option (DCWO) for treating high level radioactive waste calcine at the Idaho Chemical Processing Plant (ICPP) at the Idaho National Engineering and Environmental Laboratory (INEEL). The DCWO process is designed to stabilize previously calcined HLW and calcined SBW by combining this waste material with clay, blast furnace slag, and caustic soda to produce a hydroceramic form of feldspathoid/zeolite. The process includes mixing a thick paste of calcine and hydroceramic additives, casting the paste into a waste canister, curing the hydroceramic using elevated temperature and pressure, removing the free water from the hydroceramic by baking, and then sealing the canister The DCWO process facility will be designed to operate 10 hours per day and four days per week for casting grout. A process description, operational steps, processing rates, material balances, equipment lists, utility requirements and process flow sheets are provided.

Distribution (complete package): WTP EIS Studies Library, R. E. Dafoe M.S.3765, D. J. Harrell M.S. 3211 , B. R. Heim M. S. 3765 , K. L. Williams M. S. 3765

Distribution (summary package only):

\begin{tabular}{|c|c|c|c|c|c|}
\hline $\begin{array}{l}\text { Author } \\
\text { S.J. Losinski }\end{array}$ & $\begin{array}{l}\text { Dept. } \\
4170\end{array}$ & $\begin{array}{l}\text { Reviewed } \\
R \cdot \pi \cdot \text { Cemmets }\end{array}$ & $\begin{array}{l}\text { Date } \\
2 / 2 / 98\end{array}$ & Approved & Date \\
\hline $0+1 . h$ & $2 / 268$ & $\begin{array}{l}\text { LMITCO } \\
\text { Review }\end{array}$ & Date & $\begin{array}{l}\text { LN } \\
\text { Ap }\end{array}$ & \\
\hline
\end{tabular}




\section{DIRECT CEMENTITIOUS OPTION PROCESS DESIGN}

Page

$\begin{array}{ll}\text { I. REQUIREMENTS } & 3\end{array}$

II. PROCESS DESIGN BASIS AND ASSUMPTIONS 3

III. PROCESS DESCRIPTION 4

IV. ISSUES AND RECOMMENDATIONS 11

V. PROCESS FLOW DIAGRAMS

VI. MATERIAL BALANCES

$\begin{array}{ll}\text { VII. EQUIPMENT SIZING } & 48\end{array}$

$\begin{array}{ll}\text { VIII. UTILITIES } & 55\end{array}$ 


\section{REQUIREMENTS}

Process requirements are established by statutory laws, DOE orders, and the Batt agreement between DOE and the State of Idaho. These requirements are described in detail in Ref. 1.

\section{PROCESS DESIGN BASIS AND ASSUMPTIONS}

General assumptions used to develop this process design for the Direct Cementitous Option (DCWO) are included in this section. This design is based on hydroceramic stabilization technology developed by Dr. D. D. Seimer and others at the INEEL.

In this process, calcined High Level Waste (HLW) and calcined Sodium Bearing Waste (SBW) are combined with clay, blast furnace slag, and caustic soda such that analogs of naturally occurring feldspathoids/zeolites are generated.. The resulting stabilized waste forms are structurally sound, geologically stable and expected to not be considered RCRA hazardous, thus allowing permanent placement in a high level waste repository such as Yucca Mountain NV.

1. The DCWO alternative is based on processing existing calcine wastes and future calcine wastes generated through 2012. Future waste generation quantities are based on evaluations of the No Action Alternative and the assumption that calciner modifications will be made for higher temperature operation.( Refer to CMB-04-97)

2. It is assumed that the storage of SBW calcine is not included in this design. Furthermore, it is assumed that SBW calcine will be delivered to the DCWO facility through the common calcine retrieval system.

3. The DCWO alternative includes mixing a thick paste of calcine and hydroceramic additives, casting the paste into a $0.72 \mathrm{~m}^{3}$ waste canister, curing the hydroceramic under temperature and pressure, removing the free water from the hydroceramic by baking, and then sealing the canister so that it is "road ready" for shipment to a waste repository.

4. The total calcine quantity estimated to be stabilized in the DCWO facility is $5435 \mathrm{~m}^{3}$ and it is estimated that it will have a bulk average density of 1408 $\mathrm{Kg} / \mathrm{m}^{3}$.

5. The DCWO is based on the assumption that the final waste form will have a $35 \%$ weight loading of calcine and that it will have a density of $1700 \mathrm{Kg} / \mathrm{m}^{3}$.

6. The stabilized waste will meet the acceptance criteria for disposal at Yucca Mountain; and that "equivalency" will be demonstrated between the hydroceramic waste form generated in this process and vitrified waste which is the BDAT. 
7. Flow rates through the DCWO process are based on a 5 year processing cycle for the total calcine inventory with production starting in the year 2013 . A 50\% on-line factor is applied to processing equipment with batch operations occurring on a single $4 / 10$ days-only schedule

8. Material balances were calculated based on "average" calcine compositions. It is assumed that the DCWO process is capable of stabilizing all forms of calcine in inventory with minor variations of the hydroceramic ingredient recipe. The material balances provide the basis for sizing equipment and estimating utilities and quantities of stabilization chemicals required.

9. It is assumed that mercury contained in the calcine is not released during the autoclave cure cycle or high temperature drying process.

10. Heat transfer properties of the cast hydroceramic grout and cured grout strongly affect the process residence times of the autoclave step and the dewatering step. Because no empirical data on the heat transfer properties of the grout formulation have been collected, these properties were estimated. (See Engineering Design File on canister heat transfer. EDF-DCWO-003). Residence times, and hence the size of process equipment in the autoclave and dewatering steps, may change significantly when empirical data are collected.

11.It is assumed that one percent of all canisters processed through the DCWO will need to be recycled. Destructive analysis and the generation of off specification waste canisters are expected to be the major contributors.

\section{PROCESS DESCRIPTION}

The DCWO process is designed to stabilize previously calcined HLW and calcined SBW by combining this waste material with clay, blast furnace slag, and caustic soda to produce a hydroceramic form of feldspathoid/zeolite. The process includes mixing a thick paste of calcine and hydroceramic additives, casting the paste into a waste canister, curing the hydroceramic under temperature and pressure, removing the free water from the hydroceramic by baking, and then sealing the canister. Each of the various process systems are discussed below.

\section{A. Calcine Acceptance System}

A system for retrieval of calcine will be described in other documents. However, some equipment in the calcine retrieval system will be physically located at the DCWO facility. This equipment includes, two sets of cyclones and sintered filter assemblies, delivery piping, and ancillary equipment associated with recycle of entrainment air.

The calcine retrieval system will be capable of simultaneously delivering calcine from two bin sets. The calcine retrieval system will be operated in a batch mode 
such that it delivers one weeks worth of calcine inventory to the DCWO facility in approximately 11 hours. For the remainder of the week the calcine retrieval system will not operate.

The calcine will be air conveyed to the DCWO via two double walled and radiologically shielded process lines. Each line will feed a cyclone and filter system which will separate the calcine from the air. The calcine will be delivered to the gravity blender of the DCWO process, and the air will be recycled back to the calcine retrieval system. Each of the two feed lines is capable of delivering 2700 $\mathrm{Kg} / \mathrm{hr}$ of calcine for a total feed capacity of calcine of $5400 \mathrm{Kg} / \mathrm{hr}$.

Controls for the calcine retrieval system will be interlocked with controls of the DCWO process such that calcine can be delivered only when enabled by DCWO process operations.

\section{B. Calcine Blending}

Calcine blending at the DCWO facility is accomplished using two major pieces of process equipment. The first major piece of equipment is a static gravity blender and the second is a mechanically agitated dry process lot tank. The static gravity blender serves to moderate the variability of calcine composition as it is supplied by the calcine retrieval system. The mechanically agitated dry process lot tank serves to thoroughly blend one weeks worth of calcine, thus forming a production lot.

The calcine blending process consists of three separate steps.

In the first step calcine is delivered via the calcine retrieval system to the static gravity blender, which in turn fills the mechanically agitated dry process lot tank. When the dry process lot tank is filled the calcine retrieval system is turned off. The dry process lot tank is sized such that it has a working capacity of $42 \mathrm{~m}^{3}$ of calcine. (It should be noted that to operate properly the static gravity blender must remain full at all times. It should also be noted that the dry process lot tank will be full at the beginning of a weeks production and empty by the end of the week.)

The second step in the blending process is to operate the mechanically agitated dry process lot tank for a sufficient period of time to thoroughly blend the calcine. The calcine will then be chemically analyzed and assigned a process lot number. All calcine in the process lot will be stabilized using a stabilization recipe that is tailored to the chemical composition of the calcine process lot.

The third step in the blending process is to feed mixer-batch quantities of the blended calcine to the calcine batch bins of each grout mixer line. Each mixer batch of calcine is approximately $0.9 \mathrm{~m}^{3}$ 


\section{Grout Ingredient Delivery}

Grout ingredient delivery capability is provided for calcined clay, blast furnace slag, and caustic soda (aqueous 50\% sodium hydroxide). Each of these ingredients will be delivered in industry-standard semi truck quantities and processed through an off load station. Based on mass balance calculations the following delivery frequencies are anticipated; calcined clay 3 trucks/week, blast furnace slag 1 truck/2weeks, and caustic soda 1 truck/3weeks.

Calcined clay will be air lifted into storage silos using the standard air delivery system supplied by the clay vendor (standard equipment of the semi truck bulk delivery system). There are two $80 \mathrm{~m}^{3}$ storage silos which will provide storage capacity for approximately two weeks worth of production. Each storage silo is also provided with a dust abatement filter.

Blast furnace slag will be delivered in the same way as the calcined clay. There are two $32 \mathrm{~m}^{3}$ storage silos which will provide bulk storage capacity for approximately four weeks production. Each storage silo is sized such that it can accept the total volume of a semi truck delivery. Each silo is provided with a dust abatement filter.

Caustic soda will be delivered in industry-standard 48,000 pound $(22,000 \mathrm{Kg})$ shipments. The caustic soda will be pumped from the semi truck into a heat traced storage tank using the pump system provided on the semi truck. The heat traced storage tank is sized at $20 \mathrm{~m}^{3}$ to accept $150 \%$ of a standard delivery volume.

The grout ingredient delivery system also includes the equipment to feed the ingredients into the grout mixing line. Calcine clay and blast furnace slag are fed to the mixing line using an air ejector feed system. Caustic soda is fed to the mixing line using a positive displacement pump.

\section{Sodium Hydroxide Mixing}

The sodium hydroxide mixing system consists of two jacketed high shear mixers on load cells. The function of the mixing system is to combine caustic soda (concentrated sodium hydroxide) with water in the correct ratios to provide the liquid component for each mixer batch of grout. Each mixer volume of $1.5 \mathrm{~m}^{3}$ has adequate capacity to supply the liquid for one batch of grout. The cooling jacket on the mixer is provided to control the heat liberated during mixing. 
Each of the jacketed mixers is designed to provide mixed sodium hydroxide for two grout mixer lines. However, transfer piping is included so that each mixer can fill any one of the four grout mixers.

Caustic soda from the bulk storage tank will be metered into the high sheer mixer until the proper weight is added. While the mixer contents are being agitated, water from the mixer wash storage tank will be slowly metered into the mixer until the target batch weight is obtained. (Utilization of mixer wash water prevents the generation of a secondary waste stream.)

When the mixed sodium hydroxide has been cooled to the proper temperature it can then be transferred to the grout mixing process.

\section{E. Grout Mix and Place}

The grout mix and place operation is where the calcine, dry grout ingredients, and liquid grout ingredients are combined and mixed. After mixing, the paste-like grout is cast into a waste canister.

Calcined clay and blast furnace slag are fed into a $2.5 \mathrm{~m}^{3}$ ribbon blender which is on load cells. The proper weight of each dry ingredient for making one mixer batch of grout is added to the ribbon blender and then gently blended until homogeneous. The blended dry ingredients are then ready for introduction to the grout mixer.

Calcine from the dry process lot tank is metered into the $1.5 \mathrm{~m}^{3}$ calcine batch bin until the required weight for one mixer batch of grout is obtained. The calcine is then ready for introduction to the grout mixer.

Mixing of the grout is accomplished in a $3.6 \mathrm{~m}^{3}$ kneeder extruder mixer. First, the grout-recipe quantity of liquid ingredients from the high sheer mixer are added to the kneeder extruder. Then, under constant agitation in the kneeder extruder, the pre weighed quantity of calcine from the calcine batch bin is added. Upon completion of addition of the calcine, the calcined clay and blast furnace slag in the ribbon blender are added. All ingredients are mixed in the kneeder extruder for approximately 15 minutes. The grout is ready for casting into waste canisters.

After the grout is mixed in the kneeder extruder the grout is cast in the canisters using a robotically controlled injection head. In the casting process the canisters are placed under the injection head and the extruder portion of the kneeder extruder is actuated. The injection head controls flow of grout to the canister, ventilates the canister, obtains a test coupon sample, and then stops the flow of grout when the canister is full. Approximately three canisters are filled with the injection head per 
kneeder extruder mixer batch. Further detail on the function of the injection head system can be found in EDF-DCWO-005.

Canisters are placed on a mechanical conveyance system where they are transported to a surface decontamination/check station.

\section{F. Mixer Clean}

The mixer cleaning operation serves to wash residual grout from the kneeder extruder mixers at the end of each production day. Because the grout hardens slowly, washing of the mixers is required only at the end of each day.

An agitated $6 \mathrm{~m}^{3}$ mixer wash tank contains the wash water that will be used to wash the kneeder extruder mixer. High pressure pumps pump water from the mixer wash tank to a spray nozzle located in each kneeder extruder. The kneeder extruder mixing arms and extruder flights are rotated during the wash cycle to assure that all surfaces are cleaned. Wash water will be pumped through the robotic injection head at the end of each wash cycle to ensure that the injection head is cleaned as well.

To avoid the generation of a secondary waste stream the wash water is used as the water supply for the grout mix. Clean makeup water is added to the mixer wash tank to maintain an adequate supply of wash water.

\section{G. Surface Check and Decontamination}

The surface check and decontamination station is designed to detect and then remove any possible external contamination of the canister that may have occurred during the grout filling process. Although it is unlikely that any external contamination of the canister will occur, this station will provide assurance that contamination is not spread to other process equipment in the DCWO facility. Further detail on the design of the surface check and decontamination station can be found in EDF-DCWO-007.

\section{H. HEPA Filter Installation}

After the exterior surface of the canister is decontaminated a HEPA filter will be installed on the top of the canister. The function of the HEPA filter is to prevent radiologically contaminated particles from being released from inside the canister. Because the curing and drying steps are performed at elevated temperatures and pressures, the canister must be allowed to breathe so that a pressure differential is 
not generated across canister and so that water vapor can escape during the drying process. Further detail on the design of the HEPA filter can be found in EDFDCWO-006.

\section{Autoclave Cure}

The purpose of the autoclave cure cycle is to heat the cast grout canisters to ensure complete curing of the grout. For this design, cure of the grout is considered complete when the internal centerline temperature of the canister reaches $200^{\circ} \mathrm{C}$

In the autoclave cure process 18 grout canisters are loaded into one of four autoclaves and the autoclave is sealed. Saturated steam at $250^{\circ} \mathrm{C}$ is introduced to the autoclave so that condensing steam directly contacts the grout canister.

Heat transfer calculations indicate that 35 hours of residence time in the presence of saturated steam is required to drive the centerline temperature to $200^{\circ} \mathrm{C}$. After the centerline temperature of the grout canisters has reached $200^{\circ} \mathrm{C}$ the autoclave will be depressurized and purged with air. The autoclave will be opened and the grout canister will be moved to the dewatering station.

It is anticipated that one autoclave cure cycle, including loading and unloading of the canisters, can be accomplished in 48 hours.

It is assumed that the grout in the grout canisters will release approximately $30 \%$ of its moisture content during the depressurizarion of the autoclave. (Adiabatic decompression) This decompression step will also result in a lowering of the internal temperature of the grout to approximately $100^{\circ} \mathrm{C}$.

\section{J. Dewater Station}

The dewatering station serves to dry the cured grout in the canisters such that the residual moisture content of grout is less than $2 \%$ of the grout by weight.

Cured grout canisters from the autoclaves will be placed on a conveyor and moved into the dewatering station. The dewatering station is nothing more than a controlled environment room in which the temperature is maintained at $250^{\circ} \mathrm{C}$ and a relative humidity of less than $25 \%$. Under these conditions the remaining free water in the grout will slowly evaporate. Total residence time of the grout canister in the dewatering station is designed to be a minimum of 7 days. 


\section{K. Weld and Seal}

After the canister containing the grout is cured and dried a permanent cap will be welded onto the canister. The cap will be installed using a robotically controlled welder as described in EDF-DCWO-008.

\section{Final Contamination Check and Decontamination}

Although highly unlikely, it is possible that contamination of the exterior of the canister may occur in the curing, drying, or weld sealing steps of the DCWO process. A final contamination check and, if needed, decontamination of the canister will occur. The design of this station is detailed in EDF-DCWO-007.

\section{Sonic Tomography}

The sonic tomography process serves to provide quality assurance that the cured and dried grout meets minimum compressive strength and canister fill requirements.

In the sonic tomography process a grout canister is placed in a fixture, immersed in water and subjected to an ultrasonic signal. Via arrays of sensors detecting transmittance and back scatter of the ultrasonic signal, it is possible generate a three dimensional image of the waste in the canister. This image can be processed to interpret if there are any internal voids or uncured spots in the filled waste canister.

\section{N. Off-Specification Storage, Sizing, and Recycle}

While every effort will be made to avoid the generation of off-specification canisters, it is anticipated that the materials from some waste canisters will have to be recycled back into the DCWO process and regrouted. Destructive testing and upsets during the grout manufacturing process are anticipated as the major contributors of off-specification materials. It is assumed that one percent of all canisters made will have to be recycled.

This design includes the provision to store twenty canisters pending sizing and recycle. When it is determined that the canisters will be recycled they will be loaded into a concrete saw and sliced into sections. The sections will be crushed in a screw press and then further reduced in size in a jaw crusher. The resulting small particles will be stored in a collection hopper and reintroduced into the DCWO process. Further detail on this recycle system can be found in EDF-HWO-011. 


\section{O. Air Handling/Filter Systems}

Air handling and filtration systems in this process are not well defined. It is known that several different air handling and filtration systems will be required to support all the process needs of the DCWO facility. The larger systems are considered below.

An air handling and filtration system will be required to vent all radiologically contaminated tanks and process vessels operating at atmospheric pressure. This system is required to assure that the tanks and process vessels do not become pressurized or release radiological contaminants to the surrounding area. This system is expected to process a low volume of air.

An air handling and filtration system will be required to process the high temperature, high humidity, high volume, air flows associated with the autoclave purge cycles and grout drying process. These air streams are expected to be approximately $250^{\circ} \mathrm{C}$ and nearly saturated with water vapor when entering the filtration system. This system will present a significant process design challenge.

An air handling and filtration system will also be needed to address the HVAC requirements of the ambient temperature hot cells containing process equipment. Further detail on the design of this system can be found in EDF-DCWO-014.

\section{ISSUES AND RECOMMENDATIONS}

The hydroceramic stabilization technology utilized in this design has not been demonstrated or utilized at a scale or extent as large as that presented in this design. Physical and chemical properties associated with process reaction kinetics, mechanical properties, and heat transfer properties of the waste are not well understood and generate significant uncertainty in this design. It is recommended that research work be conducted to quantify the following:

1. Cure Reaction Kinetics

2. Cure Reaction Thermodynamics

3. Compressive Strength and Coefficient of Thermal Expansion

4. Specific Heat and Thermal Conductivity

It is suspected that the grout formulation used in this design is not fully optimized. It is recommended that additional research work be conducted to better understand the relationship between grout formulation and grout performance. Additionally, research should be conducted to demonstrate how variations in calcine properties affect grout performance. Specific research needs include: 
1. Chemical Constituent Variations

2. Trace Metals (Mercury in particular) Variations

3. Particle Size of Calcine

4. Function of Calcine Properties on Wasteform Porosity

Based on the experience of BNFL in completing a similar cement stabilization facility, (EP1, comp. 1990, Sellafield, United Kingdom) a phased approach to the research, concurrent with facility design can be accomplished. BNFL recommends the following product development phases:

1. Product Review

2. Matrix Definition

3. Detailed Product Evaluation and Long Term Studies

4. Establishing Product Envelope and Additional Product Evaluation Studies

BNFLs process design/process verification research for the Sellafield EP1 plant included the following phases:

1. Small Scale Non-Active

2. Small Scale Active

3. Full Scale Non-Active

4. Full Scale Active

This design was produced with limited knowledge of the quality assurance and waste acceptance criteria that will be imposed on the waste when it is shipped to the geological waste repository. Often these criteria significantly affect the process design because they dictate where, when, and how often process data, and sampling must occur: In a general sense, it is not known what "pedigree" of data must accompany each canister of waste. For the purposes of this design it was assumed that some form of statistical process control can be implemented such that infrequent sampling of the stabilized waste is required, but that critical process parameters and selected feed parameters are closely monitored and controlled. It was also assumed that non-destructive analysis techniques could be used to satisfy certain waste acceptance criteria. 


\section{PROCESS FLOW DIAGRAMS}

$\begin{array}{ll}\text { PFD Number } & \text { Title } \\ \text { DCWO-00 } & \text { Block Flow Diagram } \\ \text { DCWO-01 } & \text { Calcine Acceptance System } \\ \text { DCWO-02 } & \text { Calcine Blending } \\ \text { DCWO-03 } & \text { Grout Ingredient Delivery } \\ \text { DCWO-04 } & \text { Mixer - Line A } \\ \text { DCWO-05 } & \text { NaOH Mixing } \\ \text { DCWO-06 } & \text { Mixer Clean } \\ \text { DCWO-07 } & \text { Surface Contamination Check and Surface Decon - Line A } \\ \text { DCWO-08 } & \text { HEPA Filter Installation - Line A } \\ \text { DCWO-09 } & \text { Autoclave Cure Station - Line A } \\ \text { DCWO-10 } & \text { Dewater Station } \\ \text { DCWO-11 } & \text { Weld/Seal } \\ \text { DCWO-12 } & \text { Final Surface Contamination Check \& Surface Decon } \\ \text { DCWO-13 } & \text { Sonic Tomography } \\ \text { DCWO-14 } & \text { Off-Specification Storage, Sizing, and Recycle } \\ \text { DCWO-15 } & \text { Test Coupons and Chemical Analysis } \\ \text { DCWO-16 } & \text { Filter Systems }\end{array}$

Note: Four parallel process lines exist for the grout mixing through grout curing steps of the process. To simplify the PFDs, only process Line A is shown. Process lines $B, C$, and $D$ are identical to Line $A$. 


\section{MATERIAL BALANCES}

Material Balances for the DCWO are presented on the following thirteen pages. There are seven pages which quantify major chemical constituents for each flow number and there are six pages which quantify anticipated radionuclide concentrations. Quantities reported in the material balances are for the total DCWO production cycle. It should be noted that an air balance was not performed on this design so air stream mass quantities are reported as zero. A flow stream locator table is provided to correlate the process stream number with the relevant PFDs.

\section{Flow Stream Locator}

\begin{tabular}{|l|l|l|l|l|}
\hline $\begin{array}{l}\text { Stream } \\
\text { Number }\end{array}$ & Description & $\begin{array}{l}\text { Starting } \\
\text { Drawing }\end{array}$ & $\begin{array}{l}\text { Exists on } \\
\text { Drawings }\end{array}$ & $\begin{array}{l}\text { Ends on } \\
\text { Drawing }\end{array}$ \\
\hline 101 & Reserved for Calcine & & & \\
\hline 102 & Reserved for Calcine & & & \\
\hline 103 & Reserved for Calcine & & & \\
\hline 104 & Reserved for Calcine & & & \\
\hline 105 & Reserved for Calcine & & & \\
\hline 106 & Reserved for Calcine & & & \\
\hline 107 & Reserved for Calcine & & & \\
\hline 108 & Reserved for Calcine & & & \\
\hline 109 & Reserved for Calcine & & & \\
\hline 110 & Reserved for Calcine & & & \\
\hline 112 & Air Vent from Calcine Batch Bin & 04 & 04,16 & 16 \\
\hline 113 & Air Vent from Kneeder Extruder & 04 & 04,16 & 16 \\
\hline 114 & Calcine from Cyclone Separator & 01 & 01,02 & 02 \\
$\mathrm{~A}, \mathrm{~B}$ & & & & \\
\hline 115 & Calcine Retrieval System Purge & 01 & 01,16 & 16 \\
$\mathrm{~A}, \mathrm{~B}$ & Vent & & & \\
\hline 116 & Static Gravity Mixer Blend & 02 & 02, & 02 \\
\hline 1 (Calcine) & Mixed Calcine & 02 & 02,04 & 04 \\
\hline 118 & Calcined Clay & 03 & 03 & 03 \\
\hline 119 & Blast Furnace Slag & 03 & 03 & 03 \\
\hline 120 & $\begin{array}{l}\text { Caustic Soda, } \\
50 \% \text { Wt Sodium Hydroxide }\end{array}$ & & 03,05 & 05 \\
\hline 121 & Water to Grout Plant & 06 & 06 \\
\hline 122 & & & & \\
\hline
\end{tabular}




\begin{tabular}{|c|c|c|c|c|}
\hline $\begin{array}{l}\text { Stream } \\
\text { Number }\end{array}$ & Description & $\begin{array}{l}\text { Starting } \\
\text { Drawing }\end{array}$ & $\begin{array}{l}\text { Exists on } \\
\text { Drawings }\end{array}$ & $\begin{array}{l}\text { Ends on } \\
\text { Drawing }\end{array}$ \\
\hline 123 & Vent, Process Lot Tank & 02 & 02,16 & 16 \\
\hline 124 & Vent, Static Gravity Mixer & 02 & 02,16 & 16 \\
\hline $\begin{array}{l}125 \\
A, B, C, D\end{array}$ & Batch Bin Cleanout & 04 & 04,02 & 02 \\
\hline 126 & Ejector Air Clay & 03 & 03 & 03 \\
\hline 127 & Entrained Clay & 03 & 03,04 & 04 \\
\hline $\begin{array}{l}128 \\
A, B, C, D\end{array}$ & Ribbon Blender Air & 04 & 04,16 & 16 \\
\hline 129 & Ejector Air Slag & 03 & 03 & 03 \\
\hline 130 & Entrained Slag & 03 & 03,04 & 04 \\
\hline $\begin{array}{l}131 \\
A, B, C, D\end{array}$ & Blended Clay and Slag & 04 & 04 & 04 \\
\hline $\begin{array}{l}132 \\
A, B, C, D\end{array}$ & Mixed Caustic Soda/ Water & 05 & 05,04 & 04 \\
\hline $\begin{array}{l}133 \\
A, B, C, D\end{array}$ & Mixer Wash Water & 06 & 06,04 & 04 \\
\hline $\begin{array}{l}134 \\
A, B, C, D\end{array}$ & Injection Head Vents & 04 & 04,16 & 16 \\
\hline $\begin{array}{l}135 \\
A, B, C, D\end{array}$ & Test Coupons & 04 & 04,15 & 15 \\
\hline $\begin{array}{l}136 \\
A, B, C, D \\
\end{array}$ & Mixer Outlet Wash Water & 04 & 04,06 & 06 \\
\hline $\begin{array}{l}137 \\
A, B, C, D\end{array}$ & Cast Grout & 04 & $\begin{array}{l}04,07,08 \\
, 09,\end{array}$ & 09 \\
\hline 138 & Caustic Water Makeup & 06 & 06,05 & 05 \\
\hline 139 & Air to Dewater Station & 10 & 10 & 10 \\
\hline 140 & Air from Dewater Station & 10 & 10,16 & 16 \\
\hline 141 & Dewatered Grout & 10 & $\begin{array}{l}10,11,12 \\
, 13\end{array}$ & 13 \\
\hline 142 & Acceptable Grout & 13 & 13 & Product \\
\hline 143 & Unacceptable Grout & 13 & 13,14 & 14 \\
\hline 144 & Crushed Grout (Recycle) & 14 & 14,02 & 02 \\
\hline 145 & Test Coupon Waste & 15 & 15,04 & 04 \\
\hline 146 & Ribbon Blender Cleanout & 04 & 04,14 & 14 \\
\hline 147 & Vent, High Sheer Mixer & 05 & 05,16 & 16 \\
\hline 148 & Vent, Mixer Wash Tank & 06 & 06,16 & 16 \\
\hline
\end{tabular}




\begin{tabular}{|l|l|l|l|l|}
\hline $\begin{array}{l}\text { Stream } \\
\text { Number }\end{array}$ & Description & $\begin{array}{l}\text { Starting } \\
\text { Drawing }\end{array}$ & $\begin{array}{l}\text { Exists on } \\
\text { Drawings }\end{array}$ & $\begin{array}{l}\text { Ends on } \\
\text { Drawing }\end{array}$ \\
\hline $\begin{array}{l}149 \\
\mathrm{~A}, \mathrm{~B}, \mathrm{C}, \mathrm{D}\end{array}$ & Vent, Autoclave & 09 & 09,16 & 16 \\
\hline $\begin{array}{l}150 \\
\mathrm{~A}, \mathrm{~B}, \mathrm{C}, \mathrm{D}\end{array}$ & Autoclaved Grout & 09 & 09,10 & 10 \\
\hline $\begin{array}{l}151 \\
\mathrm{~A}, \mathrm{~B}, \mathrm{C}, \mathrm{D}\end{array}$ & Cure Steam & 17 & 17,09 & 09 \\
\hline $\begin{array}{l}152 \\
\mathrm{~A}, \mathrm{~B}, \mathrm{C}, \mathrm{D}\end{array}$ & Steam Condensate \\
\hline $\begin{array}{l}153 \\
\mathrm{~A}, \mathrm{~B}, \mathrm{C}, \mathrm{D}\end{array}$ & Calcine Batch & 09 & 09,17 & 17 \\
\hline $\begin{array}{l}154 \\
\mathrm{~A}, \mathrm{~B}, \mathrm{C}, \mathrm{D}\end{array}$ & Autoclave Purge Air & 04 & 04 & 04 \\
\hline
\end{tabular}




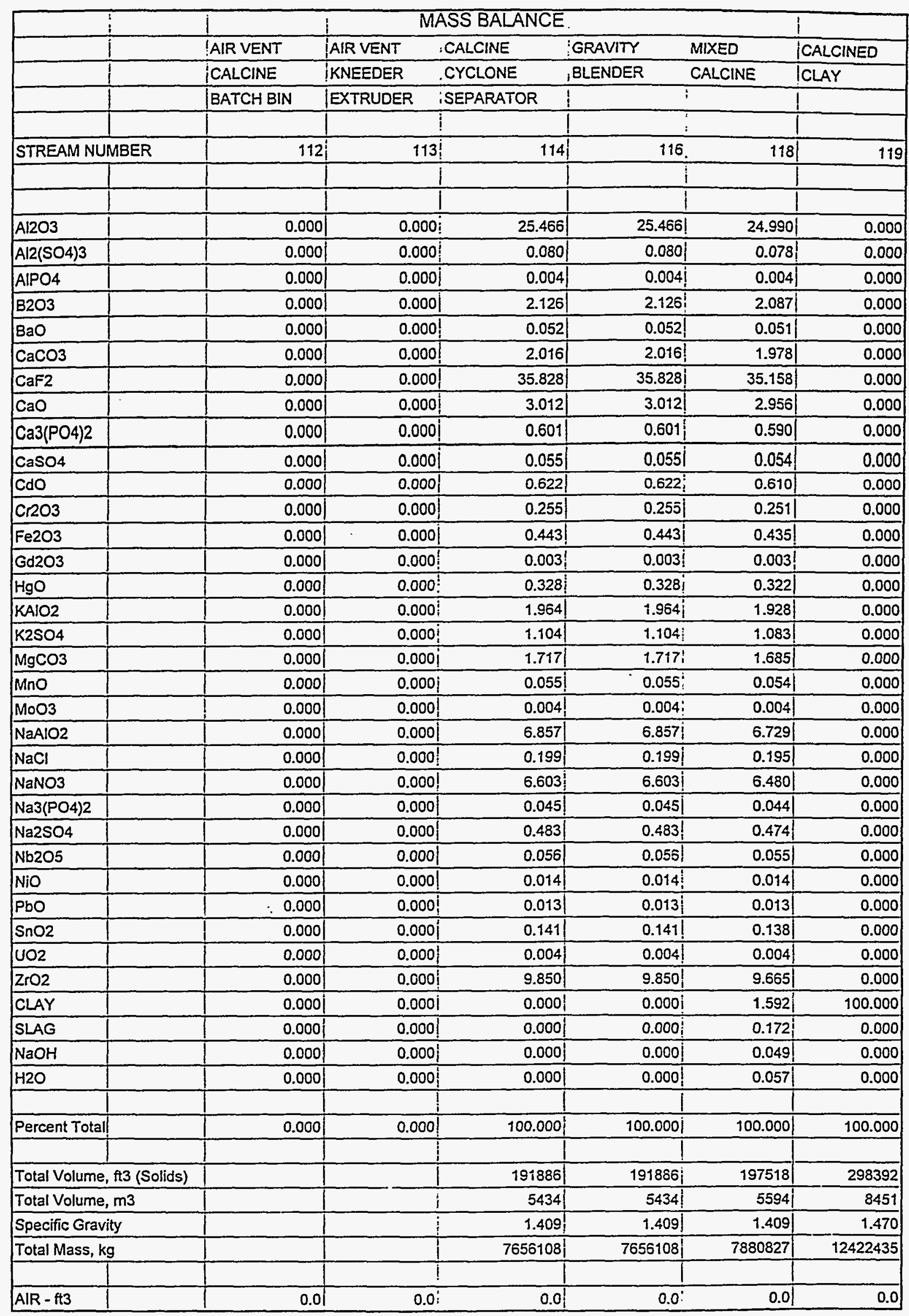




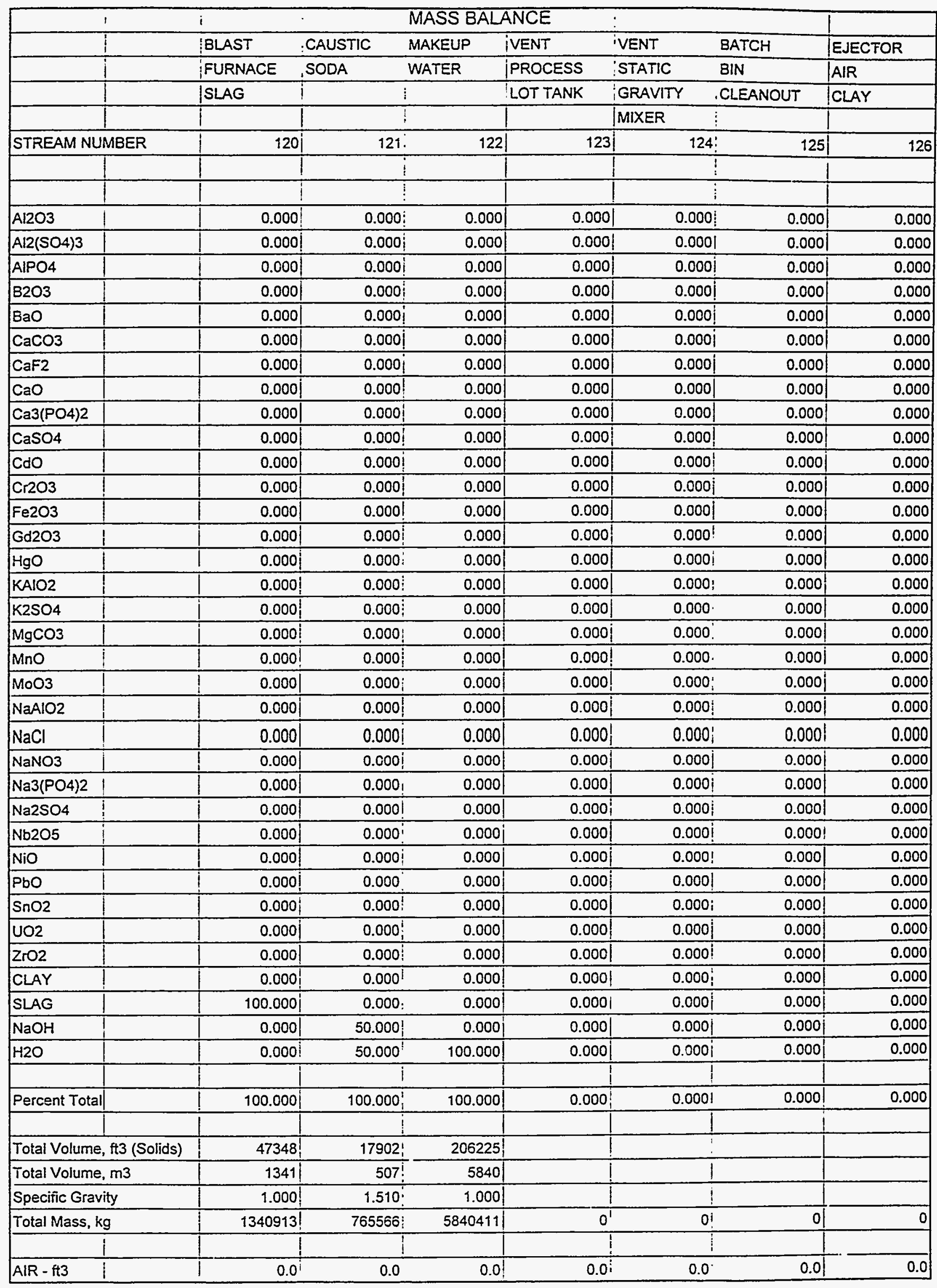




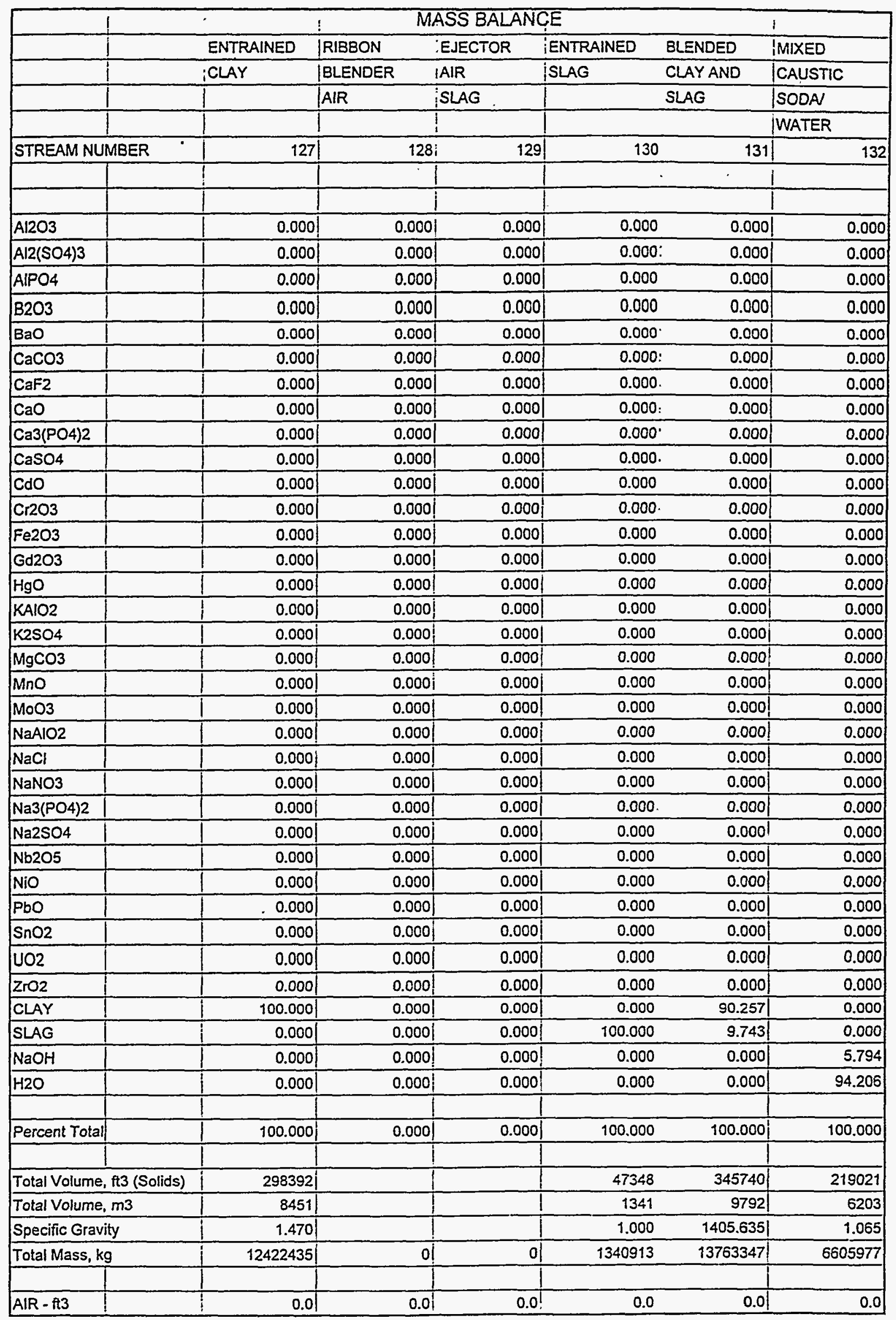




\begin{tabular}{|c|c|c|c|c|c|c|c|c|}
\hline & & & & & ASS BALANC & & & $!$ \\
\hline & & MIXER & & VENT & TEST & IMIXER & CAST & CAUSTIC \\
\hline & & WASH & & INJECTION & COUPONS & OUTLET & GROUT & IWATER \\
\hline & & WATER & & HEAD & $\vdots$ & WWASH & & MAKEUP \\
\hline & & & & 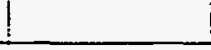 & ! & IWATER & $!$ & $!$ \\
\hline STREAM NU & MBER & & 133 & 134 & 135 & 136 & 137) & 138 \\
\hline & & & & 1 & $\vdots$ & & & \\
\hline & & & & & & & & \\
\hline $\mathrm{Al} 2 \mathrm{O}$ & & & 0.000 & 0.000 & 0.000 & 0.000 & $6.971 !$ & 0.000 \\
\hline $\mathrm{Al} 2(\mathrm{SO} 4) 3$ & & & 0.000 & 0.000 & 0.000 & 0.000 & $0.022 !$ & 0.000 \\
\hline AIPO4 & & & 0.000 & 0.000 & 0.000 & 0.000 & 0.001 & 0.000 \\
\hline $\mathrm{B2O3}$ & & & 0.000 & 0.000 & 0.000 & 0.000 & 0.582 & 0.000 \\
\hline $\mathrm{BaO}$ & & & 0.000 & 0.000 & 0.000 & 0.000 & 0.014 & 0.000 \\
\hline $\mathrm{CaCO} 3$ & i & & 0.000 & 0.000 & 0.000 & 0.000 & 0.552 & 0.000 \\
\hline CaF2 & & & 0.000 & 0.000 & 0.000 & 0.000 & 9.808 & 0.000 \\
\hline $\mathrm{CaO}$ & & & 0.000 & 0.000 & 0.000 & 0.000 & $0.825 !$ & 0.000 \\
\hline $\mathrm{Ca} 3(\mathrm{PO} 4) 2$ & & & 0.000 & 0.000 & 0.000 & $0.000:$ & 0.165 & 0.000 \\
\hline $\mathrm{CaSO} 4$ & & & 0.000 & 0.000 & 0.000 & 0.000 & 0.015 & 0.000 \\
\hline $\mathrm{CdO}$ & & & 0.000 & 0.000 & 0.000 & 0.000 & 0.170 & 0.000 \\
\hline $\mathrm{Cr} 2 \mathrm{O} 3$ & & & 0.000 & 0.000 & 0.000 & 0.000 & 0.070 & 0.000 \\
\hline $\mathrm{Fe} 2 \mathrm{O} 3$ & & & 0.000 & 0.060 & 0.000 & 0.000 & $0.121 \mid$ & 0.000 \\
\hline $\mathrm{Gd} 2 \mathrm{O} 3$ & & & 0.000 & 0.000 & $0.000^{1}$ & 0.000 & 0.001 & 0.000 \\
\hline $\mathrm{HgO}$ & & & 0.000 & 0.000 & 0.000 & 0.000 & 0.090 & 0.000 \\
\hline KAIO2 & & & 0.000 & 0.000 & 0.000 & 0.000 & 0.538 & 0.000 \\
\hline $\mathrm{K} 2 \mathrm{SO} 4$ & & & 0.000 & $0.000 !$ & 0.000 & 0.000 & 0.302 & 0.000 \\
\hline $\mathrm{MgCO} 3$ & & & 0.000 & 0.000. & 0.000 & 0.000 & $0.470 !$ & 0.000 \\
\hline $\mathrm{MnO}$ & & & 0.000 & 0.000. & 0.000 & 0.000 & 0.015 & 0.000 \\
\hline $\mathrm{MOO3}$ & & & 0.000 & 0.000 & 0.000 & 0.000 & $0.001]$ & 0.000 \\
\hline $\mathrm{NaAlO} 2$ & & & 0.000 & 0.000 & 0.000 & 0.000 & $1.877_{1}^{1}$ & 0.000 \\
\hline $\mathrm{NaCl}$ & & & 0.000 & 0.000 & 0.000 & 0.000 & 0.054 & 0.000 \\
\hline NaNO3 & & & 0.000 & 0.000 & 0.000 & 0.000 & 1.808 & 0.000 \\
\hline $\mathrm{Na3}(\mathrm{PO} 4) 2$ & & & 0.000 & 0.000 & 0.000 & 0.000 & 0.012 & 0.000 \\
\hline $\mathrm{Na} 2 \mathrm{SO}_{4}$ & & & 0.000 & 0.0001 & 0.000 & 0.000 & 0.132 & 0.000 \\
\hline $\mathrm{Nb2O5}$ & & & 0.000 & 0.000 & 0.0001 & 0.000 & 0.015 & 0.000 \\
\hline $\mathrm{NiO}$ & & & 0.000 & 0.000 & 0.000 & 0.000 & 0.004 & 0.000 \\
\hline $\mathrm{PbO}$ & & & 0.000 & 0.0001 & 0.000 & 0.000 & 0.004 & 0.000 \\
\hline $\mathrm{SnO} 2$ & & & 0.000 & 0.000 & 0.000 & 0.000 & 0.039 & 0.000 \\
\hline $\mathrm{UO2}$ & & & 0.000 & 0.000 & 0.000 & 0.000 & 0.001 & 0.000 \\
\hline $\mathrm{ZrO} 2$ & & & 0.000 & 0.000 & 0.000 & 0.000 & 2.696| & 0.000 \\
\hline CLAY & & & 0.000 & 0.000 & 0.000 & 0.000 & $44.417 \mid$ & 0.000 \\
\hline SLAG & & & 0.000 & 0.000 & 0.000 & 0.000 & $4.795 \mid$ & 0.000 \\
\hline $\mathrm{NaOH}$ & & & 0.000 & 0.000 & 0.000 & 0.000 & 1.369 & 0.000 \\
\hline $\mathrm{H} 2 \mathrm{O}$ & & & 0.000 & 0.000 & 0.000 & 0.000 & 22.045 & 100.000 \\
\hline Percent Total & & & 0.000 & 0.000 & $0.000 !$ & 0.000 & 100,000 & 100.000 \\
\hline & & & & 1 & 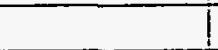 & & & \\
\hline Total Volume, & ft3 (Solids) & & & $T$ & 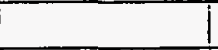 & & 466755 & 206225 \\
\hline Total Volume, & $\mathrm{m} 3$ & & & 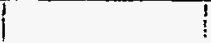 & & & 13219 & 5840 \\
\hline Specific Gravi & & & & 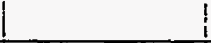 & & & 2.137 & 1.000 \\
\hline Total Mass, $\mathrm{k}$ & & & 0 & 0 & 이 & 0 & $28250: 51$ & 5840411 \\
\hline & & & & 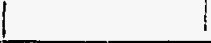 & 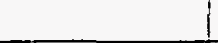 & & & \\
\hline AIR - $\mathrm{ft3}$ & & 1 & $0.0 !$ & 0.0 & 0.0 & 0.0 & $0.0 !$ & 0.0 \\
\hline
\end{tabular}




\begin{tabular}{|c|c|c|c|c|c|c|}
\hline \multirow{2}{*}{ 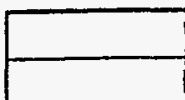 } & & i & \multicolumn{3}{|c|}{ MASS BALANCE } & \multirow[b]{2}{*}{ UNACCEPTABLE } \\
\hline & $i$ & AIR TO & AIR FROM & DEWATERED & 'ACCEPTABLE & \\
\hline & & DEWATER & DEWATER & GROUT & GROUT & GROUT \\
\hline & & STATION & ISTATION & 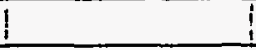 & & 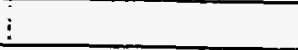 \\
\hline & & & 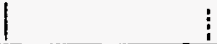 & 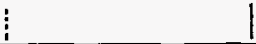 & & $:$ \\
\hline \multicolumn{2}{|c|}{ STREAM NUMBER } & 139 & $140^{\prime}$ & 141 & 142 & 143 \\
\hline & & & & & & $\vdots$ \\
\hline & & & & & & $!$ \\
\hline Al2O3 & & 0.000 & 0.000 & 8.764 & 8.764 & 8.764 \\
\hline $\mathrm{Al} 2(\mathrm{SO} 4) 3$ & & 0.000 & 0.000 & 0.027 & 0.027 & 0.027 \\
\hline AIPO4 & & 0.000 & 0.000 & 0.001 & 0.001 & 0.001 \\
\hline $\mathrm{B2O3}$ & & 0.000 & 0.000 & 0.732 & 0.732 & 0.732 \\
\hline $\mathrm{BaO}$ & & 0.000 & 0.000 & 0.018 & 0.018 & 0.018 \\
\hline $\mathrm{CaCO} 3$ & & 0.000 & 0.000 & 0.694 & 0.694 & 0.694 \\
\hline CaF2 & & 0.000 & 0.000 & 12.330 & 12.330 & 12.330 \\
\hline $\mathrm{CaO}$ & & 0.000 & 0.000 & 1.037 & 1.037 & 1.037 \\
\hline $\mathrm{Ca} 3(\mathrm{PO} 4) 2$ & & 0.000 & 0.000 & 0.207 & 0.207 & 0.207 \\
\hline $\mathrm{CaSO}_{4}$ & & 0.000 & 0.000 & 0.019 & 0.019 & 0.019 \\
\hline $\mathrm{CdO}$ & & 0.000 & 0.000 & 0.214 & 0.214 & 0.214 \\
\hline $\mathrm{Cr} 2 \mathrm{O}$ & & 0.000 & 0.000 & 0.088 & 0.088 & 0.088 \\
\hline $\mathrm{Fe} 2 \mathrm{O} 3$ & & 0.000 & 0.000 & 0.152 & 0.152 & 0.152 \\
\hline Gd2O3 & & 0.000 & 0.000 & 0.001 & 0.001 & 0.001 \\
\hline $\mathrm{HgO}$ & & 0.000 & 0.000 & 0.113 & 0.113 & 0.113 \\
\hline KAIO2 & & 0.000 & $0.000:$ & 0.678 & 0.676 & 0.676 \\
\hline $\mathrm{K} 2 \mathrm{SO} 4$ & & 0.000 & 0.000 & $0.380 !$ & 0.380 & 0.380 \\
\hline $\mathrm{MgCO} 3$ & & 0.000 & 0.000 & $0.591 \mid$ & 0.591 & 0.591 \\
\hline $\mathrm{MnO}$ & & 0.000 & 0.000 & 0.019 & 0.019 & 0.019 \\
\hline MoO3 & & 0.000 & 0.000 & 0.002 & $0.002:$ & 0.002 \\
\hline $\mathrm{NaAlO2}$ & & 0.000 & 0.000 & 2.360 & $2.360 !$ & 2.360 \\
\hline $\mathrm{NaCl}$ & & 0.000 & 0.000 & 0.068 & 0.068 & 0.068 \\
\hline $\mathrm{NaNO3}$ & & 0.000 & 0.000 & 2.272 & 2.272 & 2.272 \\
\hline $\mathrm{Na} 3(\mathrm{PO} 4) 2$ & & 0.000 & 0.000 & 0.016 & 0.016 & 0.016 \\
\hline $\mathrm{Na} 2 \mathrm{SO}_{4}$ & & 0.000 & 0.0001 & 0.166 & 0.166 & 0.166 \\
\hline $\mathrm{Nb2O5}$ & & 0.000 & 0.000 & 0.019 & 0.019 & 0.019 \\
\hline $\mathrm{NiO}$ & & 0.000 & $0.000 i$ & 0.005 & 0.005 & 0.005 \\
\hline $\mathrm{PbO}$ & & 0.000 & 0.0001 & 0.004 & 0.004 & 0.004 \\
\hline $\mathrm{SnO2}$ & & 0.000 & 0.000 & 0.048 & 0.048 & 0.048 \\
\hline $\mathrm{UO} 2$ & & 0.000 & 0.0001 & $0.001 \mid$ & 0.001 & 0.001 \\
\hline $\mathrm{ZrO2}$ & & 0.000 & 0.000 & 3.390 & 3.390 & 3.390 \\
\hline CLAY & & 0.000 & 0.000 & 55.838 & 55.838 & 55.838 \\
\hline SLAG & & 0.000 & 0.0001 & 6.027 & 6.027 & 6.027 \\
\hline $\mathrm{NaOH}$ & & 0.000 & 0.000 & 1.721 & 1.721 & 1.721 \\
\hline $\mathrm{H} 2 \mathrm{O}$ & & 0.000 & 100.000 & 2.000 & 2.000 & 2.000 \\
\hline Percent Total & & 0.000 & $100.000 !$ & 100.000 & 100.000 & 100.000 \\
\hline & & & $!$ & $!$ & & \\
\hline Total Volume & ft3 (Solids) & & $!$ & 466755 & 462087 & 4668 \\
\hline Total Volume & $\mathrm{m3}$ & & & 13219! & 13087 & 132 \\
\hline Specific Grav & & & & $1.700 !$ & 1.700 & 1.700 \\
\hline Total Mass, $k$ & & 0 & 3760479 & 22471900 & 22247181 & 224719 \\
\hline & & & 1 & I & & \\
\hline$A I R-f t 3$ & & 0.0 & 0.01 & 0.01 & 0.0 & 0.0 \\
\hline
\end{tabular}




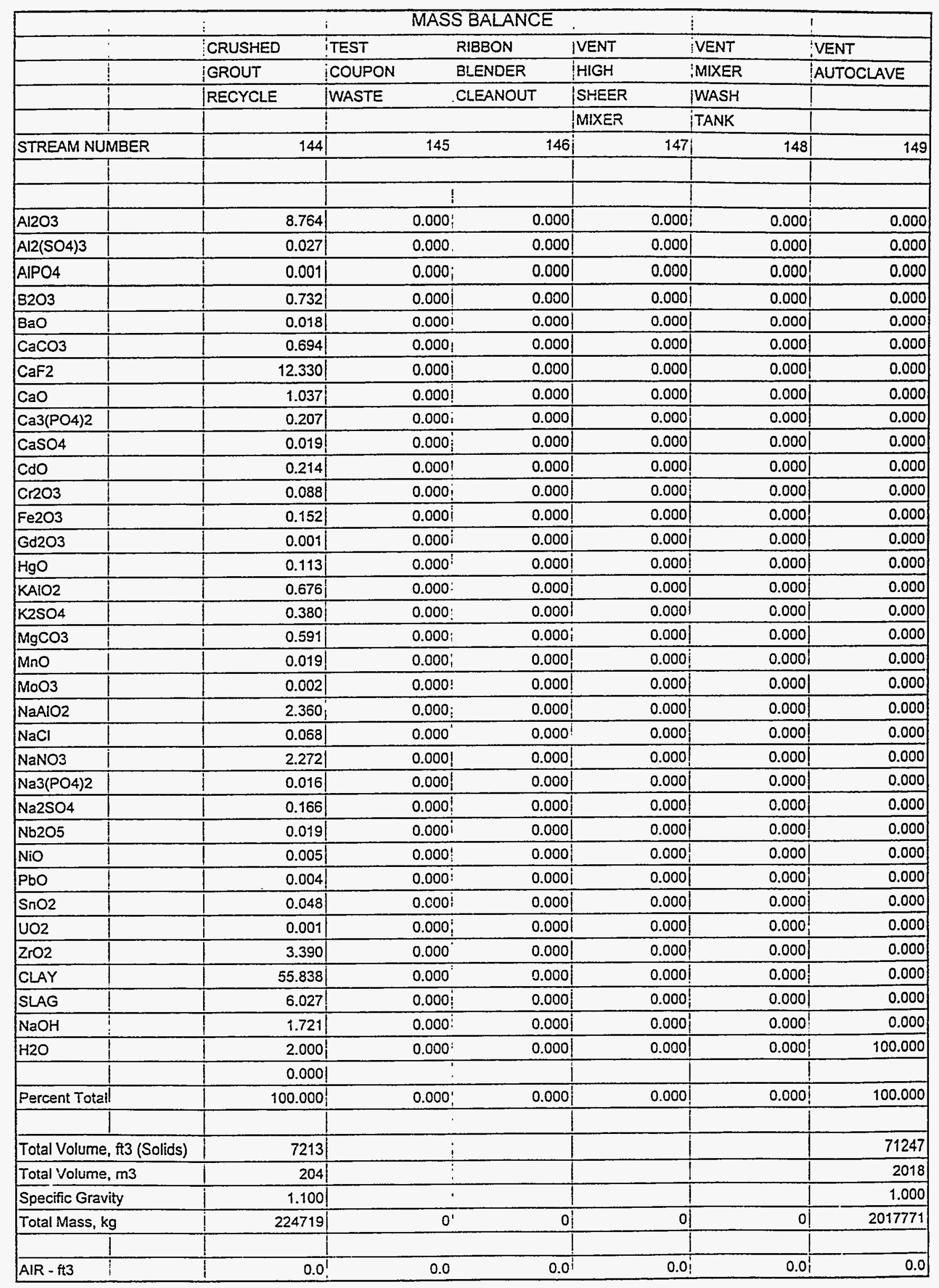




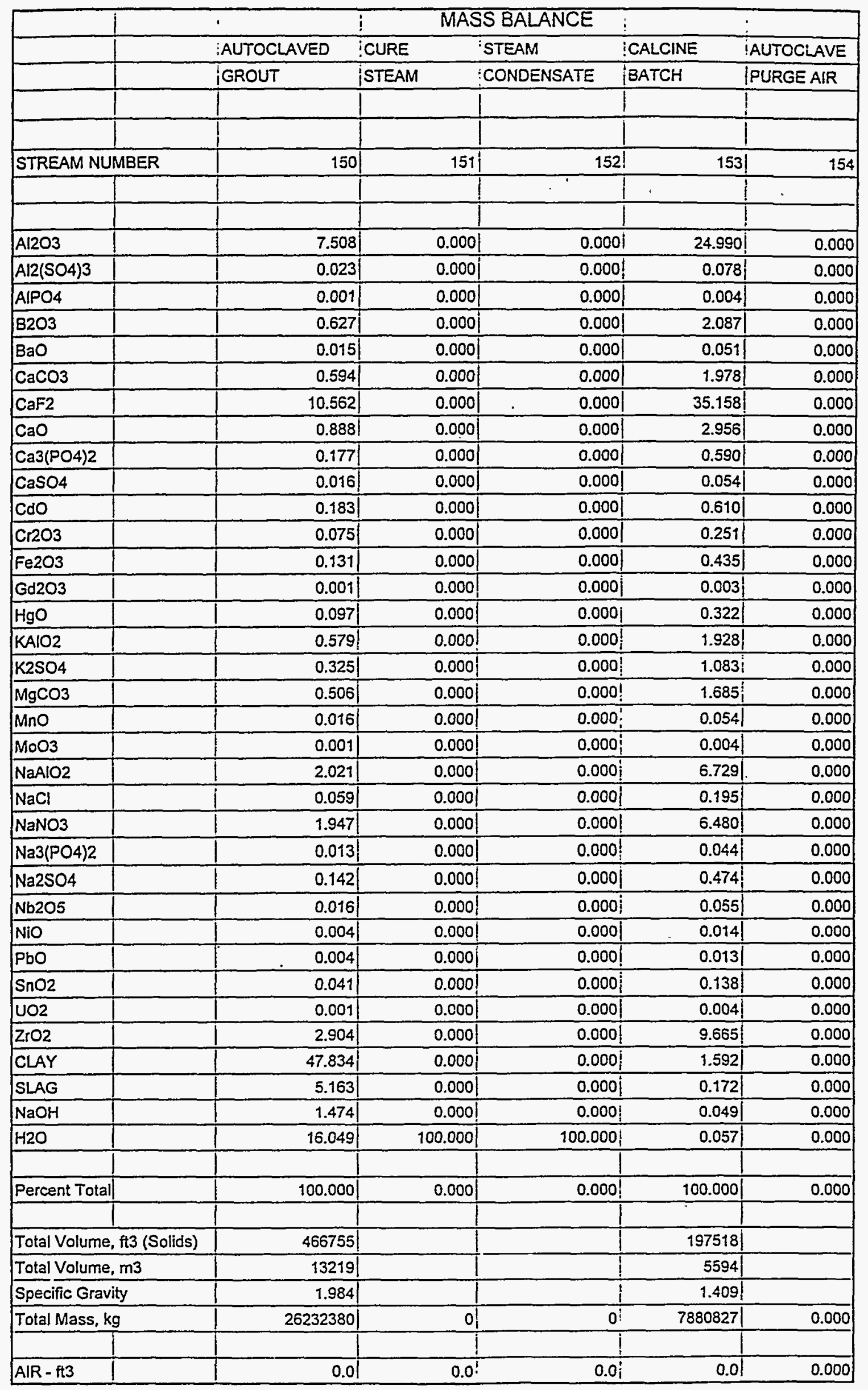


Rad Mass Balance

\begin{tabular}{|c|c|c|c|c|c|c|c|}
\hline & 1 & AIR VENT & AIR VENT & CALCINE & GRAVITY & MIXED & CALCINED \\
\hline & $!$ & CALCINE & IKNEEDER & CYCLONE & BLENDER & CALCINE & CLAY \\
\hline & $\vdots$ & BATCH BIN & EXTRUDER & SEPARATOR & & & $\bar{i}$ \\
\hline & STREAM NUMBER & 112 & 113 & $114^{\circ}$ & $116 !$ & 118 & 119 \\
\hline & STREAM WEIGHT kg & 이 & $0 !$ & $7656108^{\prime}$ & 7556108 & 7880827 & $\overline{12422435}$ \\
\hline $\mathrm{Ci} / \mathrm{g}$ & & & & 1 & & $!$ & \\
\hline Am-241 & i & 0 & $0 !$ & 1.99E-07: & $1.99 E-07$ & 1.99E-07 & 0 \\
\hline Am-243 & & ol & $0 !$ & $!$ & $0.00 E+00$ & $0.00 E+00$ & 0 \\
\hline $\mathrm{Cm}-242$ & & $0 !$ & 0 & I & $0.00 E \div 00$ & $0.00 \mathrm{E}+00$ & 0 \\
\hline $\mathrm{Cm}-244$ & & 0 & 0 & $i$ & $0.00 E+00$ & $0.00 \mathrm{E}+00$ & 0 \\
\hline $\mathrm{Np}-237$ & & 이 & $0 !$ & $8.73 E-09$ & $8.73 E-09$ & $8.73 E-09$ & 0 \\
\hline Pa-233 & & 0 & 0 & 1 & $0.00 E+00$ & $0.00 E+00$ & 0 \\
\hline$P u-238$ & & 0 & 0 & $2.64 E-06 i$ & $2.64 \mathrm{E}-06$ & $2.64 E-06$ & 0 \\
\hline Pu-239 & & 0 & 01 & $2.81 E-07$ & $2.81 E-07$ & 2.81E-07 & 0 \\
\hline Pu-240 & & 01 & 01 & $6.84 E-08$ & $6.84 E-08$ & $6.84 E-08$ & $\overrightarrow{0}$ \\
\hline Pu-241 & & 이 & 이 & 2.52E-06 & $2.52 E-06$ & $2.52 E-06$ & 0 \\
\hline Pu-242 & & 0 & of & $4.32 E-11$ & $4.32 E-11$ & $4.32 E-11$ & 0 \\
\hline U-233 & & $0 !$ & 0 & $6.03 E-10$ & $6.03 E-10$ & $6.03 E-10$ & 0 \\
\hline U-234 & & 이 & 이 & $3.89 \mathrm{E}-10 \mathrm{i}$ & $3.89 E-10$ & $3.89 E-10$ & 0 \\
\hline U-235 & & 0 & ol & $6.29 \mathrm{E}-11$ & $6.29 \mathrm{E}-11$ & $6.29 E-11$ & 0 \\
\hline U-236 & & 이 & o1 & $4.03 \mathrm{E}-12^{\prime}$ & $4.03 E-12$ & $4.03 E-12$ & 0 \\
\hline U-237 & & $0 !$ & o! & $\vdots$ & $0.00 E+00$ & $0.00 E+00$ & 0 \\
\hline$U-238$ & & 0 & 이 & $9.48 \mathrm{E}-11 \mathrm{i}$ & $9.48 E-111$ & $9.48 \mathrm{E}-11$ & 0 \\
\hline$T h-230$ & & 0 & 0 & 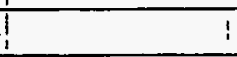 & $0.00 E+00$ & $0.00 E+00$ & 0 \\
\hline Th-231 & & $0 !$ & 0 & $;$ & $0.00 E+00 !$ & $0.00 E+00$ & 0 \\
\hline Ba-137m & & oj & 01 & 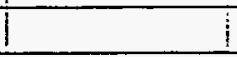 & $0.00 E \div 00$ & $0.00 E+00$ & 0 \\
\hline $\mathrm{Cd}-113 \mathrm{~m}$ & & 0 & o! & $:$ & $0.00 E=00$ & $0.00 E+00$ & 0 \\
\hline $\mathrm{Ce}-144$ & & 이 & o! & $2.13 E-05$ & 2.i3E-05| & 2.13E-05 & 0 \\
\hline Co-60 & & 0 & ol & 1.01E-06 & $1.01 E-06$ & 1.01E-06 & 0 \\
\hline Cs-134 & & 이 & of & $4.00 \mathrm{E}-06$ ! & $4.00 \mathrm{E}-06$ & $4.00 \mathrm{E}-06$ & 0 \\
\hline Cs-135 & & 01 & 이 & $1.93 E-09$ & $1.93 \mathrm{E}-09$ & $1.93 \mathrm{E}-09$ & 0 \\
\hline Cs-137 & & 이 & of & $1.45 E-04$ & $1.45 E-04$ & $1.45 \mathrm{E}-04$ & 0 \\
\hline Eu-152 & $!$ & 01 & 0 & 1 & $0.00 E+00$ & $0.00 \mathrm{E}+00$ & 0 \\
\hline Eu-154 & & 이 & 이 & $8.41 E-07$ & $8.41 E-07$ & $8.41 \mathrm{E}-07$ & 0 \\
\hline Eu-155 & . & 0 & of & 1.35E-06! & 1.35E-06 & $1.35 E-06$ & 0 \\
\hline $1-129$ & & $0 !$ & 0 & 6.64E-09: & 6.64E-09 & $6.64 \mathrm{E}-09$ & 0 \\
\hline $\mathrm{Nb}-93 \mathrm{~m}$ & & ol & of & 1 & $0.00 E+00$ & $0.00 E+00$ & 0 \\
\hline Pd-107 & & $0 j$ & 01 & $i$ & $0.005+00$ & $0.00 E+00$ & 0 \\
\hline $\mathrm{Pm}-147$ & $i$ & 01 & $0 !$ & 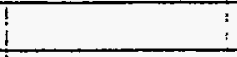 & $0.00 E+00$ & $0.00 \mathrm{E}+00$ & 0 \\
\hline$R u-106$ & & 01 & of & $8.65 E-06^{\circ}$ & $8.65 E-06$ & 8.65E-06 & 0 \\
\hline Sb-125 & & $0_{1}^{1}$ & o! & $1.70 \mathrm{E}-06$ & $1.70 \mathrm{E}-06$ & $1.70 E-06$ & 0 \\
\hline$S b-126$ & & ol & of & & $0.00 E+00$ & $0.00 E+00$ & 0 \\
\hline Sb-126m & & 이 & 0 & $\bar{\vdots}$ & $0.00 E+00$ & $0.00 E+00$ & 0 \\
\hline Se-79 & & 0 & 0 & 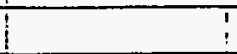 & $0.00 E \div 00$ & $0.00 E+00 j$ & 0 \\
\hline $\mathrm{Sm}-151$ & & 이 & 0 & $T$ & $0.00 E+00$ & $0.00 E+00$ & 0 \\
\hline$S n-121 m$ & & ol & 이 & 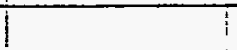 & $0.00 E+00$ & $0.00 E+00$ & 0 \\
\hline Sn-126 & & 01 & 0 & $!$ & $0.00 E+00$ & $0.00 E+00$ & 0 \\
\hline Sr-90 & & $0 j$ & $0 !$ & 1.37E-04: & $1.37 E-04$ & 1.37E-04 & 0 \\
\hline TC-99 & & 0 : & $0 !$ & 3.82E-08: & $3.82 E-08$ & $3.82 E-08$ & 0 \\
\hline$\overline{Y-90}$ & & 0 : & oi & $\bar{i}$ & $0.00 E+00$ & $0.00 E+00$ & 0 \\
\hline$Z r-93$ & & $0 !$ & $0 !$ & 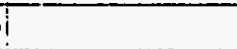 & $0.00 E+00$ & $0.00 E+00$ & 0 \\
\hline $\mathrm{Ni}-63$ & 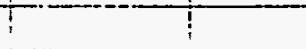 & 0 & oi & $1.70 E-07$ & $1.70 E-07^{\prime}$ & 1.70E-07! & 0 \\
\hline
\end{tabular}


Rad Mass Balance

\begin{tabular}{|c|c|c|c|c|c|c|c|c|}
\hline & BLAST & ¿CAUSTIC & MAKEUP & VENT & ¿VENT & BATCH & EJECTOR & ¿ENTRAINED \\
\hline & FURNACE & SODA & WATER & PROCESS & ISTATIC & BIN & AIR & CLAY \\
\hline & SLAG & & $\vdots$ & LOT TANK & GRAVITY & CLEANOUT & CLAY & \\
\hline & 120 & 121 & 122 : & 123 & $124 !$ & 125 & $126 i$ & 127 \\
\hline$E$ & $1340913 !$ & 765566 & $5840411^{\prime}$ & 01 & $0 !$ & $0:$ & 이 & 12422435 \\
\hline \multicolumn{9}{|l|}{$\mathrm{Ci} / \mathrm{g}$} \\
\hline Am-241 & 이 & 0 & $0 !$ & 이 & 이 & 1.99E-07. & 이 & 0 \\
\hline Am-243 & 01 & 0 & $0 !$ & 이 & 이 & $0.00 E+00$ & 이 & 0 \\
\hline $\mathrm{Cm}-242$ & 이 & 0 & $0 !$ & 이 & 이 & $0.00 E+00$ & 이 & 0 \\
\hline $\mathrm{Cm}-244$ & 이 & 0 & 0 & 0 & ol & $0.00 E+00$ & 0 & 0 \\
\hline $\mathrm{Np}-237$ & 이 & 이 & $0 !$ & ol & of & $8.73 E-09$ & 이 & 0 \\
\hline $\mathrm{Pa}-233$ & 이 & 이 & ㅇ! & 이 & ol & $0.00 E+00$ & 이 & 0 \\
\hline Pu-238 & o) & 이 & 01 & 0) & 이 & 2.64E-06! & 이 & 0 \\
\hline Pu-239 & 이 & 이 & 이 & 이 & ol & 2.81E-07| & 이 & 0 \\
\hline Pu-240 & 이 & 이 & 01 & 이 & 이 & $6.84 E-08$ & 01 & 0 \\
\hline Pu-241 & 0 & 이 & $0 !$ & 이 & $0_{1}^{1}$ & $2.52 \mathrm{E}-06$ & 이 & 0 \\
\hline Pu-242 & 0 & 이 & $0 !$ & 0) & 01 & $4.32 E-11$ & 이 & 0 \\
\hline$U-233$ & 0 & 이 & 이 & 이 & 이 & $6.03 E-10$ & 이 & 0 \\
\hline U-234 & 이 & 이 & 이 & 이 & 이 & $3.89 E-10 i$ & 이 & 0 \\
\hline U-235 & 이 & 01 & $0 !$ & 이 & 의 & $6.29 \mathrm{E}-11 !$ & of & 0 \\
\hline U-236 & 01 & $0 !$ & $0 !$ & 이 & 0 & 4.03E-12 & 이 & 0 \\
\hline$U-237$ & ol & 01 & $0 \vdots$ & o) & of & $0.00 E+00^{!}$ & 이 & 0 \\
\hline U-238 & 이 & 0 & $0 !$ & 이 & $0 i$ & $9.48 \mathrm{E}-11 !$ & 이 & 0 \\
\hline Th-230 & 0 & 0 & 0 & 이 & 0 & $0.00 E+00:$ & 0 & 0 \\
\hline Th-231 & 0 & $0 !$ & $0 ;$ & $0 !$ & 0 & $0.00 E+00$ & 0 & 0 \\
\hline$B a-137 m$ & 01 & 이 & 01 & 01 & $0 !$ & $0.00 E+00$ & 0 & 0 \\
\hline Cd-113m & 0 & 01 & 01 & 이 & o! & $0.00 E+00$ & 0) & 0 \\
\hline Ce-144 & 01 & $0 !$ & $0 i$ & 이 & 0 & $2.13 E-05$ & 01 & 0 \\
\hline Co-60 & 01 & 이 & $0 !$ & 이 & $0 !$ & 1.01E-06i & 이 & 0 \\
\hline Cs-134 & 01 & 이 & 이 & 이 & 0! & $4.00 \mathrm{E}-06$ & 이 & 0 \\
\hline Cs-135 & 이 & 이 & $0 !$ & 0 & o! & 1.93E-09! & 01 & 0 \\
\hline Cs-137 & 0 & 01 & $0 !$ & 이 & of & 1.45E-04 & 0 & 0 \\
\hline Eu-152 & 0 & 0) & $0 !$ & 0 & $0 !$ & $0.00 E+00$ & 0 & 0 \\
\hline Eu-154 & 0 & 이 & $0 !$ & 이 & 0 & 8.41E-07| & 01 & 0 \\
\hline Eu-155 & 이 & $\because \quad 0$ & $0 !$ & 이 & o! & 1.35E-06! & 0 & 0 \\
\hline $1-129$ & 이 & 이 & $0 !$ & 이 & ol & 6.64E-09! & 이 & 0 \\
\hline $\mathrm{Nb}-93 \mathrm{~m}$ & 이 & 0 & 0 & 0 & $0 !$ & $0.00 E \div 00$ & 이 & 0 \\
\hline Pd-107 & 이 & $0 !$ & 0 & 이 & 0 & $0.00 E+00$ & 이 & 0 \\
\hline$P m-147$ & 이 & $0 !$ & $0:$ & 0 & 0 & $0.00 E+00$ & 0 & 0 \\
\hline$R u-106$ & 이 & 0 & $0 !$ & 01 & 01 & $8.65 E-06$ & 이 & 0 \\
\hline$S b-125$ & 이 & 0! & 0 & 0 & of & $1.70 \mathrm{E}-06$ & 이 & 0 \\
\hline$S b-126$ & 0 & 이 & 01 & 0 & 이 & $0.00 E \div 00$ & 이 & 0 \\
\hline Sb-126m & ol & 이 & $0 i$ & 0 & $0 !$ & $0.00 \mathrm{E} \div 00$ & 이 & 0 \\
\hline Se-79 & 이 & 0 & $0 ;$ & $0 !$ & 이 & $0.00 \mathrm{E}+00$ & of & 0 \\
\hline$S m-151$ & ol & 이 & $0 !$ & 이 & 이 & $0.00 \mathrm{E}+00$ & 0 & 0 \\
\hline$S n-121 m$ & 01 & $0 !$ & $0 ;$ & 이 & 0 & $0.00 E+00$ & 이 & 우 \\
\hline Sn-126 & ol & 0 & $0:$ & 0 & $0 !$ & $0.00 E+00$ & 이 & 0 \\
\hline$S T-90$ & 의 & 0 & $0:$ & 0 & 0 & 1.37E-04! & of & - \\
\hline TC-99 & 0 & 0 & $0:$ & of & 0 & $3.82 \mathrm{E}-08_{\mathrm{i}}$ & 이 & 음 \\
\hline$Y-90$ & 0 & 0 & $0:$ & $0 \mid$ & $0_{i}^{\prime}$ & $0.00 \mathrm{E}+00$ & 01 & 의 \\
\hline$z r-93$ & $0 !$ & 0 & $0 ;$ & ol & $0 !$ & $0.00 E+00$ & 이 & - \\
\hline $\mathrm{Ni}-63$ & 0 & 0 & 0 & 01 & 0. & 1.70E-07: & $0 !$ & 0 \\
\hline
\end{tabular}


Rad Mass Balance

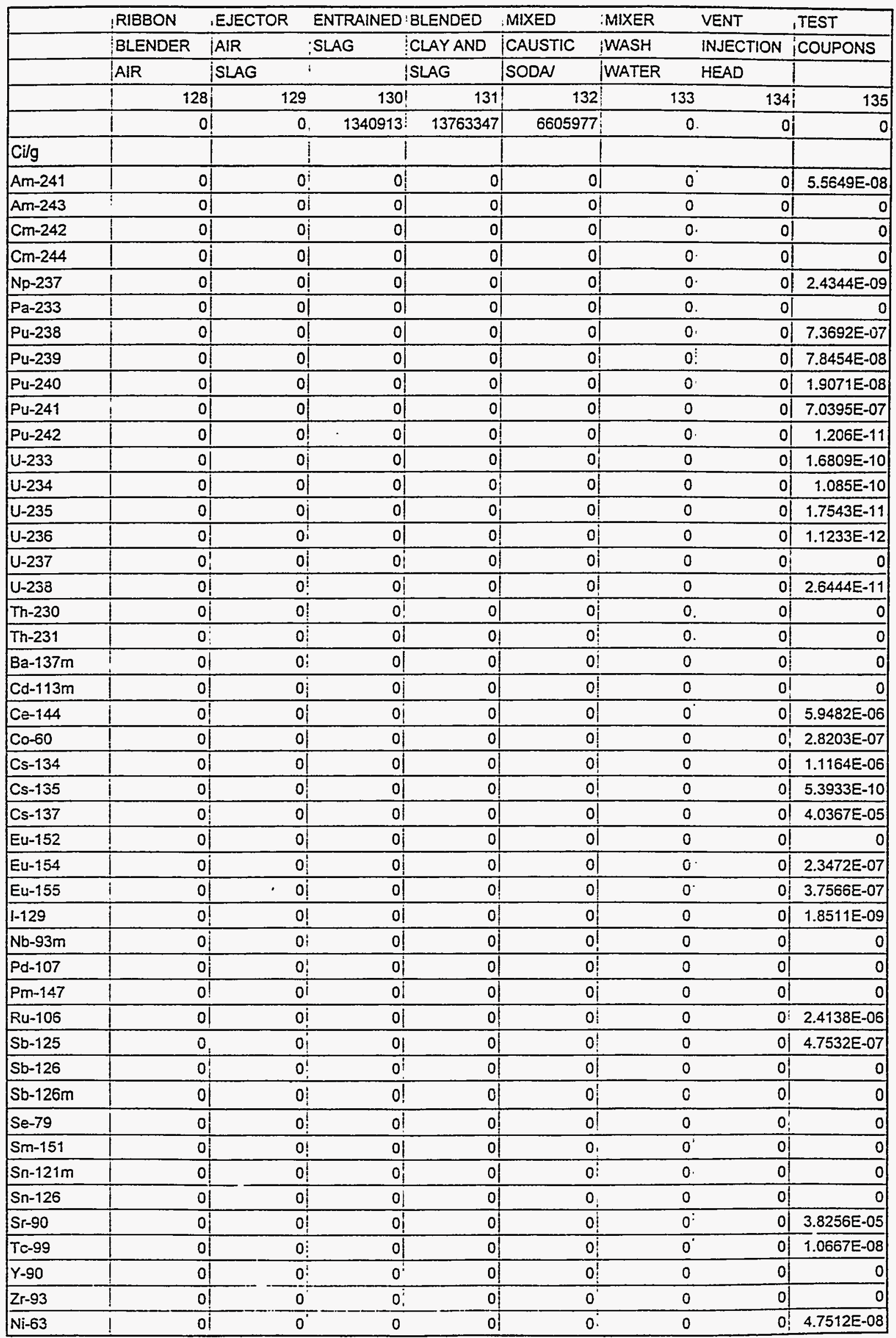




\begin{tabular}{|c|c|c|c|c|c|c|c|c|}
\hline & MIXER & CAST & CAUSTIC & :AIR TO & AIR FROM & DEWATERE & ACCEPTABL & IUNACCEPT \\
\hline & |OUTLET & GROUT & IWATER & IDEWATER । & IDEWATER & GROUT & !GROUT & GROUT \\
\hline & IWASH & & MAKEUP & STATION & ISTATION & & $!$ & \\
\hline & 13 & 137 & $138 !$ & $139 !$ & 140 & 141 & 142 & 143 \\
\hline & & 28250151 & 5840411 & 0 & 3760479 & $1 \quad 22471900$ & 22247181 & 224719 \\
\hline $\mathrm{Ci} / \mathrm{g}$ & & & & & & & 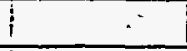 & \\
\hline$A m-241$ & & of $5.5649 \mathrm{E}-08$ & of & 이 & 0 & $6.9958 \mathrm{E}-08$ & $6.9958 E-08$ & $6.9958 \mathrm{E}-08$ \\
\hline Am-243 & & 0 & 이 & 0 & 0 & 0 & 0 & 0 \\
\hline $\mathrm{Cm}-242$ & & 0 & o! & $0 !$ & of & $0 !$ & 0 & 0 \\
\hline $\mathrm{Cm}-244$ & & 0 & 이 & of & 0 & 01 & 0 & 0 \\
\hline Np-237 & & \begin{tabular}{l|l|l|l|}
$0.4344 E-09$ \\
\end{tabular} & o) & $0 !$ & 0 & $3.0604 E-09$ & $3.0604 E-09$ & $3.0604 E-09$ \\
\hline $\mathrm{Pa}-233$ & & 0 & 이 & 0 & 이 & 0 & 0 & 0 \\
\hline Pu-238 & & $\begin{array}{lll}0.3692 \mathrm{E}-07 \\
\end{array}$ & ol & 이 & 이 & 9.2641E-07 & $9.2641 \mathrm{E}-07$ & $9.2641 \mathrm{E}-07$ \\
\hline Pu-239 & & \begin{tabular}{l|l|l|l|} 
& $7.8454 E-08$ \\
\end{tabular} & 이 & 이 & 0! & | S.8627E-08 & $9.8627 E-08$ & $9.8627 E-08$ \\
\hline Pu-240 & & $1.9071 \mathrm{E}-08$ & ol & 이 & 0 & 2.3975E-08 & $2.3975 E-08$ & 2.3975E-08 \\
\hline Pu-241 & & $7.0395 \mathrm{E}-07$ & of & 이 & 이 & $8.8496 E-07$ & $8.8496 \mathrm{E}-07$ & 8.8496E-07 \\
\hline PU-242 & & of $1.206 \mathrm{E}-11$ & 이 & 이 & 0 & $1.5161 E-11$ & $1.5161 \mathrm{E}-11$ & $1.5161 \mathrm{E}-11$ \\
\hline $\mathrm{U}-233$ & & of $1.6809 E-10$ & 이 & 이 & 0 & $2.1131 E-10$ & $2.1131 E-10$ & $2.1131 E-10$ \\
\hline U-234 & & $1.085 E-10$ & 이 & 의 & 0) & $1.3639 \mathrm{E}-10$ & $1.3639 \mathrm{E}-10$ & $1.3639 E-10$ \\
\hline U-235 & & o| $1.7543 \mathrm{E}-11$ & ol & $0 !$ & 0 & $2.2054 E-11$ & $2.2054 \mathrm{E}-11$ & $2.2054 \mathrm{E}-11$ \\
\hline$U-236$ & & 0) $1.1233 \mathrm{E}-12$ & of & 01 & $0 !$ & $1.4122 \mathrm{E}-12$ & $1.4122 \mathrm{E}-12$ & 1.4122E-12 \\
\hline$U-237$ & & 0 & $0 !$ & 01 & 0 & ol & 0 & 0 \\
\hline U-238 & & o| $2.6444 \mathrm{E}-11$ & 0 & 0 & 0 & $3.3243 E-11$ & 3.3243E-11 & $3.3243 E-11$ \\
\hline$T h-230$ & & 0 & o! & $0 !$ & $0 !$ & 0 & 이 & 0 \\
\hline Th-231 & & 0 & 0 & $0 !$ & 0 & $0:$ & 이 & 0 \\
\hline Ba-137m & & 이 & 0 & 이 & $0 i$ & 0 & 이 & 0 \\
\hline Cd-113m & & 0 & ol & of & 이 & 01 & 이 & 0 \\
\hline $\mathrm{Ce}-144$ & & | $5.9482 E-06$ & oi & of & 01 & $7.4777 \mathrm{E}-05$ & $7.4777 \mathrm{E}-06$ & 7.4777 E-06 \\
\hline Co-60 & & of 2.8203E-07 & $0 !$ & 이 & 이 & 3.5455E-07 & 3.5455E-07 & $3.5455 \mathrm{E}-07$ \\
\hline Cs-134 & & | $1.1164 \mathrm{E}-06$ & 이 & 01 & $0 !$ & $1.4035 E-05$ & $1.4035 E-06$ & $1.4035 E-06$ \\
\hline Cs-135 & & \begin{tabular}{l|l|}
$5.3933 E-10$ \\
\end{tabular} & of & 0 & 0 & $6.7801 E-10$ & $6.7801 E-10$ & $6.7801 E-10$ \\
\hline Cs-137 & & o| $4.0367 \mathrm{E}-05$ & $0 !$ & 0 & 01 & $5.0746 \mathrm{E}-05$ & $5.0746 \mathrm{E}-05$ & 5.0746E-05 \\
\hline Eu-152 & & 0 & o| & o! & 이 & 이 & 0 & 0 \\
\hline Eu-154 & & | $2.3472 \mathrm{E}-07$ & of & 01 & 이 & |2.9507E-07 & | 2.9507E-07 & 2.9507E-07 \\
\hline Eu-155 & & \begin{tabular}{|l|l|}
$0.7566 E-07$ \\
\end{tabular} & 이 & 이 & 이 & 4.7226E-07 & 4.7226E-07 & 4.7226E-07 \\
\hline $1-129$ & & O $1.8511 E-09$ & o) & 이 & 01 & $2.3271 E-09$ & $2.3271 E-09$ & 2.3271E-09 \\
\hline $\mathrm{Nb}-93 \mathrm{~m}$ & & of $\quad 0$ & o! & 이 & 0 & 이 & of & 0 \\
\hline Pd-107 & & 이 & 0 & 01 & 0 & o! & 이 & 0 \\
\hline$P m-147$ & & 0 & of & 0 & 이 & 이 & 0 & 0 \\
\hline RU-106 & & $\begin{array}{ll}0.4138 \mathrm{E}-06 \\
\end{array}$ & o) & of & $0 !$ & 3.0344E-06 & 3.0344E-06 & $3.0344 E-06$ \\
\hline$S b-125$ & & o| $4.7532 E-07$ & 01 & of & $0 !$ & $5.9754 \mathrm{E}-07$ & $5.9754 E-07$ & $5.9754 \mathrm{E}-07$ \\
\hline Sb-126 & & 0 & oi & 이 & 이 & 01 & 이 & 0 \\
\hline $\mathrm{Sb}-126 \mathrm{~m}$ & & 0 & ol & oi & 01 & 01 & 0 & 0 \\
\hline Se-79 & & 0 & 이 & 0 & 01 & 여 & 이 & 0 \\
\hline$S m-151$ & & 0 & 이 & of & 01 & 01 & 이 & -0 \\
\hline Sn-121m & & 0 & 이 & 0 & 01 & 01 & 이 & 0 \\
\hline $5 n-126$ & & 0 & of & $0 !$ & 0 & ol & 이 & 0 \\
\hline Sr-90 & & \begin{tabular}{l|l|}
$0.8256 \mathrm{E}-05$ \\
\end{tabular} & $0 !$ & 01 & $0 !$ & $4.8093 E-05$ & 4.8093E-05 & $4.8093 \mathrm{E}-05$ \\
\hline TC-99 & & \begin{tabular}{l|l}
0 & $1.0667 \mathrm{E}-08$ \\
\end{tabular} & $0 !$ & 이 & $0 i$ & $1.341 \mathrm{E}-08$ & $1.341 \mathrm{E}-08$ & $1.341 \mathrm{E}-08$ \\
\hline$Y-90$ & & a) & $0 !$ & $0 !$ & 0 & 0 & 0 & 0 \\
\hline$z r-93$ & & 0 & 0 & 0 & 0 & 0 & 0 & 0 \\
\hline Ni-63 & & o) $4.7512 E-08$ & $0^{i}$ & $0 !$ & 0 & $5.9729 \varepsilon-08$ & i $5.9729 \mathrm{E}-08$ & 15.9729E-08 \\
\hline
\end{tabular}




\begin{tabular}{|c|c|c|c|c|c|c|c|c|c|c|}
\hline & 'CRUSHED & ITEST & RIBBON & VENT & & VENT & & VENT & AUTOCLAV & |CURE \\
\hline & GROUT & COUPON & ¡BLENDER & $\mathrm{HIGH}$ & & MIXER & & AUTOCLAV & GROUT & STEAM \\
\hline & |RECYCLE | & IWASTE & CLEANOUT & |SHEER & & WASH & & & & \\
\hline & 144 & 145 & 146 & & $147 \mid$ & & $148 !$ & 149 & 150 & 15 \\
\hline & 224719 & 0 & 0 & & $0 !$ & & $0 !$ & 2017771 & 26232380 & c \\
\hline Civg & & & 1 & $i$ & & & $!$ & 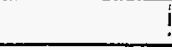 & 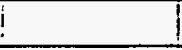 & \\
\hline$A m-241$ & $6.9958 \mathrm{E}-08$ & $3 \mid 6.9958 \mathrm{E}-08$ & 0 & & 0 & & 01 & 0 & : 5.9929E-08 & c \\
\hline Am-243 & 0 & 0 & 0 & & 01 & & ol & $0_{i}$ & 0 & $c$ \\
\hline $\mathrm{Cm}-242$ & 이 & 0 & 0 & & ol & & ol & $0:$ & 이 & c \\
\hline $\mathrm{Cm}-244$ & 이 & 0 & 0 & & o) & & o! & 01 & 이 & c \\
\hline $\mathrm{Np}-237$ & $3.0604 \mathrm{E}-09$ & \begin{tabular}{l|l|l|}
$9.0604 E-09$ \\
\end{tabular} & 0 & & 이 & & $0 !$ & $0 !$ & 2.6217E-09 & 0 \\
\hline Pa-233 & 이 & 0 & 0 & & o| & & $0 i$ & $0:$ & 이 & c \\
\hline Pu-238 & $9.2641 E-07$ & 9.2641E-07| & 0 & & 01 & & o! & 0 & $7.936 E-07$ & 0 \\
\hline Pu-239 & $9.8627 \mathrm{E}-08$ & $\mid 9.8627 E-08$ & 0 & & o! & & o! & $0 !$ & | 8.4489E-08 & c \\
\hline Pu-240 & $2.3975 \mathrm{E}-08$ & 2.3975E-08 & 0 & & 0 & & 이 & 0 & $2.0538 E-08$ & 0 \\
\hline Pu-241 & $8.8496 E-07$ & $\mid 8.8496 \mathrm{E}-07$ & 0 & & 이 & & ol & 0 & $7.581 \mathrm{E}-07$ & 0 \\
\hline Pu-242 & $1.5161 \mathrm{E}-11$ & $1.5161 \mathrm{E}-11$ & 0 & & o! & & $0:$ & $0 \mathrm{i}$ & $1.2987 \mathrm{E}-11$ & 0 \\
\hline U-233 & $2.1131 \mathrm{E}-10$ & $2.1131 E-10$ & 0 & & 이 & & $0 !$ & $0:$ & $1.8102 \mathrm{E}-10$ & 0 \\
\hline U-234 & $\mid 1.3639 \mathrm{E}-10$ & $|1.3639 \mathrm{E}-10|$ & 0 & & oj & & $0 !$ & 0 & $1.1684 \mathrm{E}-10$ & 0 \\
\hline$U-235$ & $2.2054 \mathrm{E}-11$ & $2.2054 \mathrm{E}-11$ & 0 & & 01 & & 01 & $0:$ & $1.8893 E-11$ & 0 \\
\hline U-236 & $1.4122 \mathrm{E}-12$ & $1.4122 \mathrm{E}-12$ & 0 & & 0 & & 0 & 0 & $\mid 1.2097 \mathrm{E}-12$ & 0 \\
\hline U-237 & ! ! & 0 & 0 & & 이 & & 0 & $0:$ & 이 & 0 \\
\hline$U-238$ & $3.3243 \mathrm{E}-11$ & $3.3243 \mathrm{E}-11$ & 0 & & 이 & & $0 !$ & $0:$ & $2.8478 \mathrm{E}-11$ & 0 \\
\hline Th-230 & 이 & 0 & 0 & & $0 !$ & & $0:$ & 0 & 0 & 0 \\
\hline Th-231 & 이 & 0 & 0 & & 이 & & 0 & 0 & 01 & 0 \\
\hline $8 a-137 m$ & 이 & 0 & 0 & & 0 & & $0 !$ & 0 & 이 & 0 \\
\hline $\mathrm{Cd}-113 \mathrm{~m}$ & ol & 0 & 0 & & $0 !$ & & $0:$ & 0 & 0 & 0 \\
\hline$C e-144$ & $7.4777 \mathrm{E}-06$ & |7.4777E-06 & 0 & & 이 & & 0 & $0:$ & $6.4058 \mathrm{E}-06$ & 0 \\
\hline $\mathrm{Co}-60$ & $3.5455 \mathrm{E}-07$ & $\mid 3.5455 \mathrm{E}-07$ & 0 & & 0 & & $0 !$ & 0 & $3.0372 \mathrm{E}-07$ & 0 \\
\hline Cs-134 & $1.4035 \mathrm{E}-06$ & $1.4035 \mathrm{E}-06$ & 0 & & 0 & & $0 !$ & $0:$ & $1.2023 \mathrm{E}-06$ & 0 \\
\hline Cs-135 & $6.7801 \mathrm{E}-10$ & $\mid 6.7801 \mathrm{E}-10$ & 0 & & 이 & & $0 !$ & $0:$ & $5.8082 \mathrm{E}-10$ & 0 \\
\hline Cs-137 & $5.0746 \mathrm{E}-05$ & $5.0746 \mathrm{E}-05$ & 0 & & 0 & & 0 & 0 & $4.3472 \mathrm{E}-05$ & 0 \\
\hline Eu-152 & 이 & 0 & 0 & & of & & 0 & 0 & 이 & 0 \\
\hline Eu-154 & $2.9507 E-07$ & $2.9507 \mathrm{E}-07$ & 0 & & 이 & & $0 !$ & $0 !$ & $2.5277 \mathrm{E}-07$ & 0 \\
\hline Eu-155 & $4.7226 \mathrm{E}-07$ & $\mid 4.7226 \mathrm{E}-07$ & 0 & & 0 & & $0 !$ & 0 & $4.0456 \mathrm{E}-07$ & 0 \\
\hline $1-129$ & $2.3271 E-09$ & |2.3271E-09 & 0 & & 01 & & $0 !$ & 0 & $1.9935 \mathrm{E}-09$ & 0 \\
\hline $\mathrm{Nb}-93 \mathrm{~m}$ & of & 01 & 0 & & 이 & & $0 !$ & 0 & 이 & 0 \\
\hline $\mathrm{Pd}-107$ & $0 !$ & 이 & 0 & & 이 & & 0 & 0 & 이 & 0 \\
\hline $\mathrm{Pm}-147$ & 이 & 0 & 0 & & $0 !$ & & 0 & $0^{2}$ & 이 & 0 \\
\hline Ru-106 & $3.0344 \mathrm{E}-06$ & $3.0344 \mathrm{E}-06$ & 0 & & $0 !$ & & 0 & 0 & 2.5994E-06 & 0 \\
\hline Sb-125 & $5.9754 \mathrm{E}-07$ & $5.9754 E-07 \mid$ & 0 & & 01 & & 0 & $0 !$ & $5.1188 \mathrm{E}-07$ & 0 \\
\hline Sb-126 & 이 & ol & 0 & & $0_{i}$ & & 0 & 0 & 이 & 0 \\
\hline Sb-126m & 이 & 이 & 0 & & of & & 0 & $0 !$ & 01 & 0 \\
\hline Se-79 & 이 & 0 & 0 & & 이 & & 01 & 01 & 이 & 0 \\
\hline Sm-151 & 이 & 0 & 0 & & 0 & & $0 !$ & $0 !$ & 이 & 0 \\
\hline $5 n-121 m$ & ol & 0 & 0 & & o! & & $0 !$ & $0:$ & 이 & 0 \\
\hline Sn-126 & 이 & of & 0 & & $0 !$ & & 0 & 0. & 0 & 0 \\
\hline Sr-90 & $4.8093 \mathrm{E}-05$ & $4.8093 E-05 !$ & 0 & & $0 i$ & & 0 & 0 & 4.1199E-05 & 0 \\
\hline TC-99 & $1.341 \mathrm{E}-08$ & $1.341 \mathrm{E}-08$ & 0 & & 0 & & 0 & 0 & $1.1488 \mathrm{E}-08$ & 0 \\
\hline$Y-90$ & 01 & 01 & 0 & & 이 & & 0 & 0 & 이 & 0 \\
\hline$Z r-93$ & 이 & 0 & 0 & & $0 !$ & & $0 i$ & 0 & oi & 0 \\
\hline$\sqrt{\mathrm{Ni}-63}$ & $5.9729 \mathrm{E}-08$ & | $5.9729 \mathrm{E}-08$ & $\overline{0}$ & & 0 & & 0 & 0 & $5.1166 \mathrm{E}-08$ & 0 \\
\hline
\end{tabular}




\begin{tabular}{|c|c|c|c|}
\hline & ISTEAM & ICALCINE & - AUTOCLAVE \\
\hline & |CONDENSATE| & BATCH & PURGE AIR \\
\hline & & & 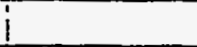 \\
\hline & 152 & 153 & 154 \\
\hline & 01 & 7880827 & 0.000 \\
\hline \multicolumn{4}{|l|}{$\mathrm{Ci} / \mathrm{g}$} \\
\hline$A m-241$ & ol & 1.99E-07) & 0 \\
\hline Am-243 & 이 & $0.00 E+00$ & 0 \\
\hline $\mathrm{Cm}-242$ & of & $0.00 E+00$ & 0 \\
\hline $\mathrm{Cm}-244$ & 이 & $0.00 E+001$ & 0 \\
\hline $\mathrm{Np}-237$ & 이 & $8.73 E-09$ & 0 \\
\hline $\mathrm{Pa}-233$ & o) & $0.00 E+00$ & $\overrightarrow{0}$ \\
\hline Pu-238 & 0 & 2.64E-06 & 0 \\
\hline Pu-239 & 0 & 2.81E-07 & 0 \\
\hline Pu-240 & 0 & $6.84 E-08$ & 0 \\
\hline Pu-241 & 이 & 2.52E-06 & 0 \\
\hline Pu-242 & 이 & $4.32 E-111$ & 0 \\
\hline U.233 & 이 & $6.03 E-10$ & 0 \\
\hline U-234 & 0) & $3.89 \mathrm{E}-10$ & 0 \\
\hline U-235 & 0 & $6.29 E-11$ & 0 \\
\hline U-236 & 0 & 4.03E-12 & 0 \\
\hline U-237 & 이 & $0.00 E+00$ & 0 \\
\hline U-238 & 이 & $9.48 \mathrm{E}-11$ & 0 \\
\hline Th-230 & 0 & $0.00 E+00$ & 0 \\
\hline Th-231 & $0 !$ & $0.00 E+00$ & 0 \\
\hline $8 a-137 m$ & 01 & $0.00 E+00$ & 0 \\
\hline$C d-113 m$ & $0 \mid$ & $0.00 E+00$ & 0 \\
\hline $\mathrm{Ce}-144$ & 0 & $2.13 E-05 \mid$ & 잉 \\
\hline Co-60 & 이 & $1.01 \mathrm{E}-06$ & 0 \\
\hline Cs-134 & 이 & $4.00 E-06$ & 0 \\
\hline Cs-135 & 이 & 1.93E-09 & 요 \\
\hline Cs-137 & 이 & $1.45 \mathrm{E}-04$ & 으 \\
\hline Eu-152 & 01 & $0.00 \mathrm{E}+00$ & 0 \\
\hline Eu-154 & 이 & 8.41E-07| & 0 \\
\hline Eu-155 & 이 & $1: 35 E-06$ & 이 \\
\hline $\mid-129$ & 이 & $6.64 E-09$ & 0 \\
\hline $\mathrm{Nb}-93 \mathrm{~m}$ & 이 & $0.00 E+00$ & 0 \\
\hline$P d-107$ & 이 & $0.00 E+00$ & 0 \\
\hline $\mathrm{Pm}-147$ & 0 & $0.00 E+00$ & 0 \\
\hline Ru-106 & 이 & $8.65 \mathrm{E}-06$ & 0 \\
\hline$S b-125$ & 이 & $1.70 \mathrm{E}-06$ & 은 \\
\hline$S b-126$ & 이 & $0.00 E+00$ & 0 \\
\hline Sb-126m & 이 & $0.00 \mathrm{E}+00$ & 0 \\
\hline Se-79 & 0 & $0.00 \mathrm{E}+00$ & 의 \\
\hline $\mathrm{Sm}-151$ & 01 & $0.00 E+00$ & 요 \\
\hline$S n-121 m$ & 이 & $0.00 E+00$ & 0 \\
\hline $5 n-126$ & 이 & $0.00 E+00$ & 0 \\
\hline Sr-90 & 01 & 1.37E-04 & 0 \\
\hline Tc.99 & 이 & $3.82 E-08$ & 0 \\
\hline$Y-90$ & 이 & $0.00 E+00$ & 0 \\
\hline Zr-93 & 이 & $0.00 E+00$ & 0 \\
\hline $\mathrm{Ni}-63$ & 이 & $1.70 \mathrm{E}-07$ & 0 \\
\hline
\end{tabular}




\section{EQUIPMENT SIZING}

Shown below is a table of major process equipment included in the DCWO design.

\begin{tabular}{|c|c|c|c|c|c|c|c|c|c|}
\hline $\begin{array}{l}\text { Equipment } \\
\text { ID }\end{array}$ & Name & $\begin{array}{l}\text { Design } \\
\text { Capacity }\end{array}$ & $\begin{array}{l}\text { Number } \\
\text { of Items }\end{array}$ & $\begin{array}{l}\text { Diameter } \\
\text { or Rating }\end{array}$ & Height & $\begin{array}{l}\text { Pressure } \\
\text { Rating } \\
\end{array}$ & Material & Power & PFD \\
\hline & & & & & & & & & \\
\hline$C Y-101$ & Cyclone Separator & & & & & & & & DCWO-01 \\
\hline CY -102 & Cyclone Separator & & 1 & & & & & & DCWO-01 \\
\hline$T-201$ & Static Gravity Mixer & $80 \mathrm{~m}^{3}$ & 1 & $4 m$ & $15 \mathrm{~m}$ & Atm & $304 \mathrm{~L}$ & & DCWO-02 \\
\hline$T-202$ & $\begin{array}{l}\text { Dry Process Tank with Ribbon } \\
\text { Blender Agitator }\end{array}$ & $50 \mathrm{~m}^{3}$ & 1 & $3 m \times 4 m$ & $4 m$ & Atm & $304 \mathrm{~L}$ & & DCWO-02 \\
\hline $\mathrm{P}-203$ & Mechanical Conveyor & $0.2 \mathrm{~m}^{3} / \mathrm{min}$ & 1 & & & Atm & $304 \mathrm{~L}$ & & DCWO-02 \\
\hline$F-204 A, B$ & Filter (Air) Auto Shake & $\begin{array}{l}5 \mathrm{micron} 0.2 \\
\mathrm{~m}^{3} / \mathrm{min}\end{array}$ & 2 & & & $\begin{array}{l}25 \mathrm{~cm} \\
\text { WC }\end{array}$ & $304 \mathrm{~L}$ & & DCWO-02 \\
\hline$T-301 \mathrm{~A}, \mathrm{~B}$ & Cone Bottom Storage Silo & $80 \mathrm{~m}^{3}$ & 2 & $4 m$ & $15 \mathrm{~m}$ & Atm & $\mathrm{CS}$ & & DCWO-03 \\
\hline $\begin{array}{l}F-302 \\
A, B, C, D\end{array}$ & Filter (Air) Auto Shake & $\begin{array}{l}5 \mathrm{micron} \\
0.2 \mathrm{~m}^{3} / \mathrm{min}\end{array}$ & 4 & & & $\begin{array}{l}25 \mathrm{~cm} \\
W C\end{array}$ & & & DCWO-03 \\
\hline $\begin{array}{l}E J-303 \\
A, B\end{array}$ & Air Ejector & & 2 & & & & & & DCWO-03 \\
\hline$T-304$ A,B & Cone Bottom Storage Silo & $32 \mathrm{~m}^{3}$ & 2 & $3 m$ & $9 m$ & Atm & CS & & DCWO-03 \\
\hline$P-305 A, B$ & $\begin{array}{l}\text { Positive Displacement Pump, } \\
\text { Heated Barrel }\end{array}$ & $20 \mathrm{l} / \mathrm{min}$ & 2 & & & $\begin{array}{l}20 \mathrm{~m}- \\
\text { head }\end{array}$ & CS & & DCWO-03 \\
\hline$T .306$ & Tank; Heat Traced @ 70 F & $20 \mathrm{~m}^{3}$ & 1 & $3 m$ & $3 m$ & Atm & CS & & DCWO-03 \\
\hline $\begin{array}{l}T-401 \\
A, B, C, D\end{array}$ & Cone Bottom Tank & $1.5 \mathrm{~m}^{3}$ & 4 & $1 \mathrm{~m}$ & $2 m$ & Atm & $304 \mathrm{~L}$ & & DCWO-04 \\
\hline $\begin{array}{l}M-402 \\
A, B, C, D\end{array}$ & Ribbon Blender & $2,5 \mathrm{~m}^{3}$ & 4 & $\begin{array}{l}1.5 \mathrm{~m} \\
\times 1.5 \mathrm{~m}\end{array}$ & $2 m$ & Atm & CS & & DCWO-04 \\
\hline $\begin{array}{l}L-403 \\
A, B, C, D\end{array}$ & Load Cell for Calcine Batch Bins & $\begin{array}{l}2000 \mathrm{Kg} \text { Full } \\
\text { Scale }\end{array}$ & 4 & $\begin{array}{l} \pm 0.5 \% \\
\text { FS }\end{array}$ & & & & & DCWO-04 \\
\hline $\begin{array}{l}L-404 \\
A, B, C, D\end{array}$ & Load Cell for Ribbon Blenders & $\begin{array}{l}3000 \mathrm{Kg} \text { Full } \\
\text { Scale }\end{array}$ & 4 & $\begin{array}{l} \pm 0.5 \% \\
\text { FS }\end{array}$ & & & & & DCWO-04 \\
\hline
\end{tabular}




\begin{tabular}{|c|c|c|c|c|c|c|c|c|c|}
\hline $\begin{array}{l}\text { Equipment } \\
\text { ID }\end{array}$ & Name & $\begin{array}{l}\text { Design } \\
\text { Capacity }\end{array}$ & $\begin{array}{l}\text { Number } \\
\text { of Items }\end{array}$ & $\begin{array}{l}\text { Diameter } \\
\text { or Rating }\end{array}$ & Height & $\begin{array}{l}\text { Pressure } \\
\text { Rating }\end{array}$ & Material & Power & PFD \\
\hline & & & & & & & & & \\
\hline $\begin{array}{l}F-405 \\
A, B, C, D\end{array}$ & Filter (Air) for Ribbon Blender & $\begin{array}{l}5 \mathrm{micron} \\
0.2 \mathrm{~m}^{3} / \mathrm{min}\end{array}$ & 4 & & & $\begin{array}{l}25 \mathrm{~cm} \\
W C\end{array}$ & & & DCWO-04 \\
\hline $\begin{array}{l}E J-406 \\
A, B, C, D\end{array}$ & Ejector for Ribbon Blenders & & 4 & & & & & & DCWO-04 \\
\hline $\begin{array}{l}E J-407 \\
A, B, C, D\end{array}$ & Ejector for Calcine Batch Bins & & 4 & & & & & & DCWO-04 \\
\hline $\begin{array}{l}1 J-408 \\
A, B, C, D\end{array}$ & Robotic Grout Injection Head & $\begin{array}{l}\text { Dennis } \\
\text { Keiser }\end{array}$ & 4 & & & & & & DCWO-04 \\
\hline $\begin{array}{l}\text { VP-409 } \\
A, B, C, D \\
\end{array}$ & Vibrator Platform & $\begin{array}{l}\text { Dennis } \\
\text { Keiser } \\
\end{array}$ & 4 & & & & & & DCWO-04 \\
\hline $\begin{array}{l}M-410 \\
A, B, C, D\end{array}$ & Mixer, Kneader/Extruder & $3.6 \mathrm{~m}^{3}$ & 4 & & & Atm & $304 \mathrm{~L}$ & & DCWO-04 \\
\hline $\begin{array}{l}F-411 \\
A, B, C, D\end{array}$ & Filter, (Air) Calcine Batch Bin & $\begin{array}{l}5 \mathrm{micron} 0.2 \\
\mathrm{~m}^{3} / \mathrm{min}\end{array}$ & 4 & & & $\begin{array}{l}25 \mathrm{~cm} \\
W C\end{array}$ & & & DCWO-04 \\
\hline$A-412$ & Filter, (Air) Kneeder/Extruder & $\begin{array}{l}5 \mathrm{micron} 0.2 \\
\mathrm{~m}^{3} / \mathrm{min}\end{array}$ & 4 & & & $\begin{array}{l}25 \mathrm{~cm} \\
W C\end{array}$ & & & DCWO-04 \\
\hline$M-501 A, B$ & $\begin{array}{l}\text { High Sheer Mixer with Cooling } \\
\text { Jacket }\end{array}$ & $1.5 \mathrm{~m}^{3}$ & 2 & $1 \mathrm{~m}$ & $2 m$ & Atm & $304 \mathrm{~L}$ & & DCWO-05 \\
\hline L-502 A,B & Load Cell for High Sheer Mixer & $\begin{array}{l}1500 \mathrm{Kg} \text { Full } \\
\text { Scale }\end{array}$ & 2 & $\begin{array}{l} \pm 0.25 \% \\
\text { FS }\end{array}$ & & & & & DCWO.05 \\
\hline P-503 A,B & Pump & $201 / \mathrm{min}$ & 2 & & & $\begin{array}{l}20 \mathrm{~m} \\
\text { head }\end{array}$ & $304 \mathrm{~L}$ & & DCWO-05 \\
\hline$M-601$ & Mixer, for Wash Tank & $6 \mathrm{~m}^{3}$ & 1 & $2 m$ & $2 m$ & Atm & $304 \mathrm{~L}$ & & DCWO-06 \\
\hline P-602 A,B & Pump, Wash Return & $31 / \mathrm{sec}$ & 2 & & & $\begin{array}{l}20 \mathrm{~m} \\
\text { head }\end{array}$ & $304 L$ & & DCWO-06 \\
\hline P-603 A,B & Pump, Mixer Spray/Wash & $31 / \mathrm{sec}$ & 2 & & & $\begin{array}{l}30 \mathrm{~m} \\
\text { head }\end{array}$ & $304 \mathrm{~L}$ & & DCWO-06 \\
\hline P-604 A,B & Pump, Caustic Makeup Water & $20 \mathrm{l} / \mathrm{min}$ & 2 & & & $\begin{array}{l}20 \mathrm{~m} \\
\text { head }\end{array}$ & $304 \mathrm{~L}$ & & DCWO-06 \\
\hline $\begin{array}{l}\text { CCS-701 } \\
A, B, C, D \\
\end{array}$ & $\begin{array}{l}\text { Robotic Contamination Check } \\
\text { Station } \\
\end{array}$ & $\begin{array}{l}\text { Dennis } \\
\text { Keiser }\end{array}$ & 4 & & & & & & DCWO-07 \\
\hline $\begin{array}{l}\mathrm{DS}-702 \\
\mathrm{~A}, \mathrm{~B}, \mathrm{C}, \mathrm{D}\end{array}$ & Decontamination Station & $\begin{array}{l}\text { Dennis } \\
\text { Keiser }\end{array}$ & 4 & & & & & & DCWO-07 \\
\hline
\end{tabular}




\begin{tabular}{|c|c|c|c|c|c|c|c|c|c|}
\hline $\begin{array}{l}\text { Equipment } \\
\text { ID }\end{array}$ & Name & $\begin{array}{l}\text { Design } \\
\text { Capacity }\end{array}$ & $\begin{array}{l}\text { Number } \\
\text { of ltems }\end{array}$ & $\begin{array}{l}\text { Diameter } \\
\text { or Rating }\end{array}$ & Height & $\begin{array}{l}\text { Pressure } \\
\text { Rating }\end{array}$ & Material & Power & PFD \\
\hline & & & & & & & & & \\
\hline $\begin{array}{l}R-801 \\
A, B, C, D\end{array}$ & Robot, HEPA Filter Placement & $\begin{array}{l}\text { Dennis } \\
\text { Keiser }\end{array}$ & 4 & & & & & & DCWO-08 \\
\hline $\begin{array}{c}A-901 \\
A, B, C, D \\
\end{array}$ & Autoclave & $\begin{array}{l}300 \mathrm{C} \\
1.1 \times 10^{4} \\
\mathrm{KPa}\end{array}$ & 4 & $4.9 \mathrm{~m}$ & $15.5 \mathrm{~m}$ & & & & DCWO-09 \\
\hline$R-1101$ & Robot, HEPA Filter Removal & $\begin{array}{l}\text { Dennis } \\
\text { Keiser }\end{array}$ & 1 & & & & & & DCWO-11 \\
\hline $\mathrm{R}-1102$ & Robot, Weld/Seal Check Cannister & $\begin{array}{l}\text { Dennis } \\
\text { Keiser }\end{array}$ & 1 & & & & & & DCWO-11 \\
\hline CCS-1201 & $\begin{array}{l}\text { Robotic Contamination Check } \\
\text { Station }\end{array}$ & $\begin{array}{l}\text { Dennis } \\
\text { Keiser }\end{array}$ & 1 & & & & & & DCWO-12 \\
\hline DS-1202 & Decontamination Station & $\begin{array}{l}\text { Dennis } \\
\text { Keiser }\end{array}$ & 1 & & & & & & DCWO-12 \\
\hline STS-1301 & Sonic Tomography & & 1 & & & & & & DCWO-13 \\
\hline & & & & & & & & & \\
\hline & & & & & & & & & \\
\hline & & & & & & & & & \\
\hline
\end{tabular}


In the following section a brief description of major process equipment and the parameters used to size the equipment are presented. Process equipment supporting calcine blending, grout mixing and canister casting is based on batch operations utilizing a 4-day work week with days-only operation. The autoclave cure process is batch by nature but because of the fact that it requires two days to complete a batch will be staffed as a continuous operation ( 24 hour shift coverage). The drying process which takes seven days to complete will also be staffed as a continuous process.

For both the days-only and continuous shifts it is assumed that the associated processes will achieve a $50 \%$ on line factor. To meet the requirement of stabilizing the entire inventory of calcine in 5 years the DCWO must average processing $29,450 \mathrm{Kg} /$ week of calcine. Therefore, because of the on-line factor, the DCWO calcine throughput design rate must be $58.900 \mathrm{Kg} /$ week of calcine/

The most feasible approach to obtaining the desired calcine stabilization rate is to have parallel grout processing lines. This approach was used because it allows for a reasonable time to fill a canister, and can use grout mixing equipment of reasonable capacity. For the DCWO facility four parallel grout processing lines are included.

\section{Cyclone Separator $(C Y-101,102)$}

The cyclone separators are used to separate the entrained solid calcine materials from the air stream which has delivered them. The entrained calcine is expected to be delivered from two different calcine bin sets with one cyclone for each bin set. The design and sizing of this equipment is being performed as a separate effort, however the separators and associated filtration equipment will be located in the DCWO facility. Each cyclone system will be designed to deliver approximately $2700 \mathrm{Kg} / \mathrm{hr}$ of calcine from the calcine bin sets.

The approximate dimensional characteristics for the cyclone are as shown in Perry's Handbook of Chemical Engineering (pg 20-82)

Shell Diameter $=$ Approximately $0.75 \mathrm{~m}$

Height $=$ Approximately $2.0 \mathrm{~m}$

\section{Static Gravity Mixer (T-201)}

This unit serves to moderate the variability of calcine composition with respect to time. This unit will remain full of calcine at all times. It is sized such that it will 
hold two weeks worth of calcine, based on full production capacity of the grout mixing lines. Experience indicates this will result in semi-uniform calcine blending. It should be noted that this type of device works only if it remains completely filled.

The conceptual operation is that calcine from the calcine retrieval system will be fed to the static gravity mixer once per week during the weekend when the grout mixing operation is off-line. The calcine retrieval system will feed the static gravity mixer at approximately $5400 \mathrm{~kg} / \mathrm{hr}$; and at the same rate calcine will automatically feed from the bottom of the static gravity mixer into the dry process tank. Feed of calcine from the calcine retrieval system will stop when the dry process lot tank is filled with one weeks worth of calcine.

Diameter $=$ Approximately $4 \mathrm{~m}$

Height $=$ Approximately $15 \mathrm{~m}$

\section{Dry Process Tank with Ribbon Blender Agitator T-202}

The dry process tank serves to provide a one weeks reservoir of calcine for use in grout mixing. Each tank quantity of calcine will be processed as a production lot. The dry process lot tank is sized such that it has a working capacity of $42 \mathrm{~m}^{3}$, with a total volumetric capacity of $50 \mathrm{~m}^{3}$. The ribbon blender agitator serves to mix the tank contents until they are homogenious.

Approximate physical dimensions are: $3 \mathrm{~m}(\mathrm{w}) \times 4 \mathrm{~m}$ (h) $\times 5 \mathrm{~m}$ (I)

\section{Bulk Hoppers Calcined Clay T-301 A, B}

Sizing of this bulk hopper system is based on the assumption that two weeks production of calcined clay must be in inventory at the DCWO. A two silo system is used so that calcined clay can be delivered to one silo while the other silo is supplying calcined clay to the process. Two $80 \mathrm{~m}^{3}$. silos are required

Diameter $=$ Approximately $4 \mathrm{~m}$

Height $=$ Approximately $15 \mathrm{~m}$

\section{Bulk Hoppers Blast Furnace Slag T-304 A, B}

Sizing of this bulk hopper system is based on the assumption that each individual silo must be able to receive $150 \%$ of a standard delivery volume of slag. A two 
silo system is used so that slag can be delivered to one silo while the other silo is supplying slag to the process. Two $32 \mathrm{~m}^{3}$. silos are required

Diameter $=$ Approximately $3 \mathrm{~m}$

Height $=$ Approximately $9 \mathrm{~m}$

\section{Bulk Hopper Sodium Hydroxide T-306}

Caustic soda (50\% wt sodium hydroxide) is delivered in standard shipments of $48,000 \mathrm{lb}(21,800 \mathrm{Kg})$, which is significantly more that the $5700 \mathrm{Kg} / \mathrm{wk}$ DCWO process utilization rate. Therefore the sodium hydroxide store tank size was based an the ability to accept a standard delivery volume. A single $20 \mathrm{~m}^{3}$ tank is required.

Because caustic soda freezes at approximately $60^{\circ} \mathrm{F}$ the tank must be heat traced.

Diameter $=$ Approximately $3 \mathrm{~m}$

Height $=$ Approximately $3 \mathrm{~m}$

\section{Cone Bottom Tank (Calcine Batch Bin) T-401 A,B,C,D}

The purpose of the calcine batch bin is to collect the calcine for one kneeder extruder batch of grout. Each batch of grout requires approximately $0.9 \mathrm{~m}^{3}$ of calcine and the tank is conservatively sized at $1.5 \mathrm{~m}^{3}$

Diameter $=$ Approximately $1 \mathrm{~m}$

Height $=$ Approximately $2 \mathrm{~m}$

\section{Ribbon Blender T-402 A,B.C.D}

The purpose of the ribbon blender is to collect and mix the calcine clay and blast furnace slag for one kneeder extruder batch of grout. After both the clay and slag are added to the mixer, they will be blended to assure that they are well mixed before introduction to the kneeder extruder. The combined volumes of clay and slag for a single batch is $1.52 \mathrm{~m}^{3}$ and the ribbon blender is conservatively sized at 2.5 $\mathrm{m}^{3}$. 
Approximate physical dimensions are: $1.5 \mathrm{~m}(\mathrm{w}) \times 2 \mathrm{~m}(\mathrm{~h}) \times 1.5 \mathrm{~m}$ (I)

\section{High Sheer Mixer with Cooling Jacket M-501 A,B}

The purpose of the high sheer mixers is to mix caustic soda with water to provide the liquid ingredients for one batch of grout. The DCWO design includes two high sheer mixers to service the four grout mixing lines. Each batch of grout requires $0.99 \mathrm{~m}^{3}$ of liquid ingredients and the mixer is conservatively sized at $1.5 \mathrm{~m}^{3}$. Mixing of water with caustic soda is exothermic and therefor requires a cooling jacket to reduce the liquid mixtures temperature before introduction to the kneeder extruder.

Diameter $=$ Approximately $1 \mathrm{~m}$

Height $=$ Approximately $2 \mathrm{~m}$

\section{Kneeder Extruder Mixer M-410 A,B,C,D}

The purpose of the kneeder extruder mixer is to combine the liquid and dry ingredients and generate the paste-like grout material which is then loaded into the canisters. First, the grout-recipe quantity of liquid ingredients from the high sheer mixer are added to the kneeder extruder. Then, under constant agitation in the kneeder extruder, the pre weighed quantity of calcine from the calcine batch bin is added. Upon completion of addition of the calcine, the calcined clay and blast furnace slag in the ribbon blender are added. All ingredients are mixed in the kneeder extruder for approximately 15 minutes. The grout is ready for casting into waste canisters. The actual volume of the grout ingredients is $2.38 \mathrm{~m}^{3}$ and the kneeder mixer is conservatively sized at $3.6 \mathrm{~m}^{3}$. Grout is cast in canisters by actuating the extruder portion of the mixer which will force mixed grout through the injection head and into the canister.

Approximate physical dimensions are: $2 \mathrm{~m}(\mathrm{w}) \times 2 \mathrm{~m}(\mathrm{~h}) \times 3 \mathrm{~m}(\mathrm{l})$ 


\section{UTILITIES}

\section{Electrical:}

There are no large electrical loads identified in the DCWO process. Process equipment electrical loads are expected to be minimal relative to the building electrical loads, therefore it is appropriate to use standard facility electric loads as an estimate. Please see EDF-DCWO-004

\section{Steam:}

Steam will be required in the autoclave cure process and the as the heat source in the drying process. Thermodynamic calculations indicate that the process load of steam for the autoclave cure process is $950 \mathrm{lb} / \mathrm{hr}$ and for the air drying process is $1200 \mathrm{lb} / \mathrm{hr}$ (see EDF-DCWO-003). These steam requirements are a small increment of the estimated HVAC heating load. 


\section{E-368}




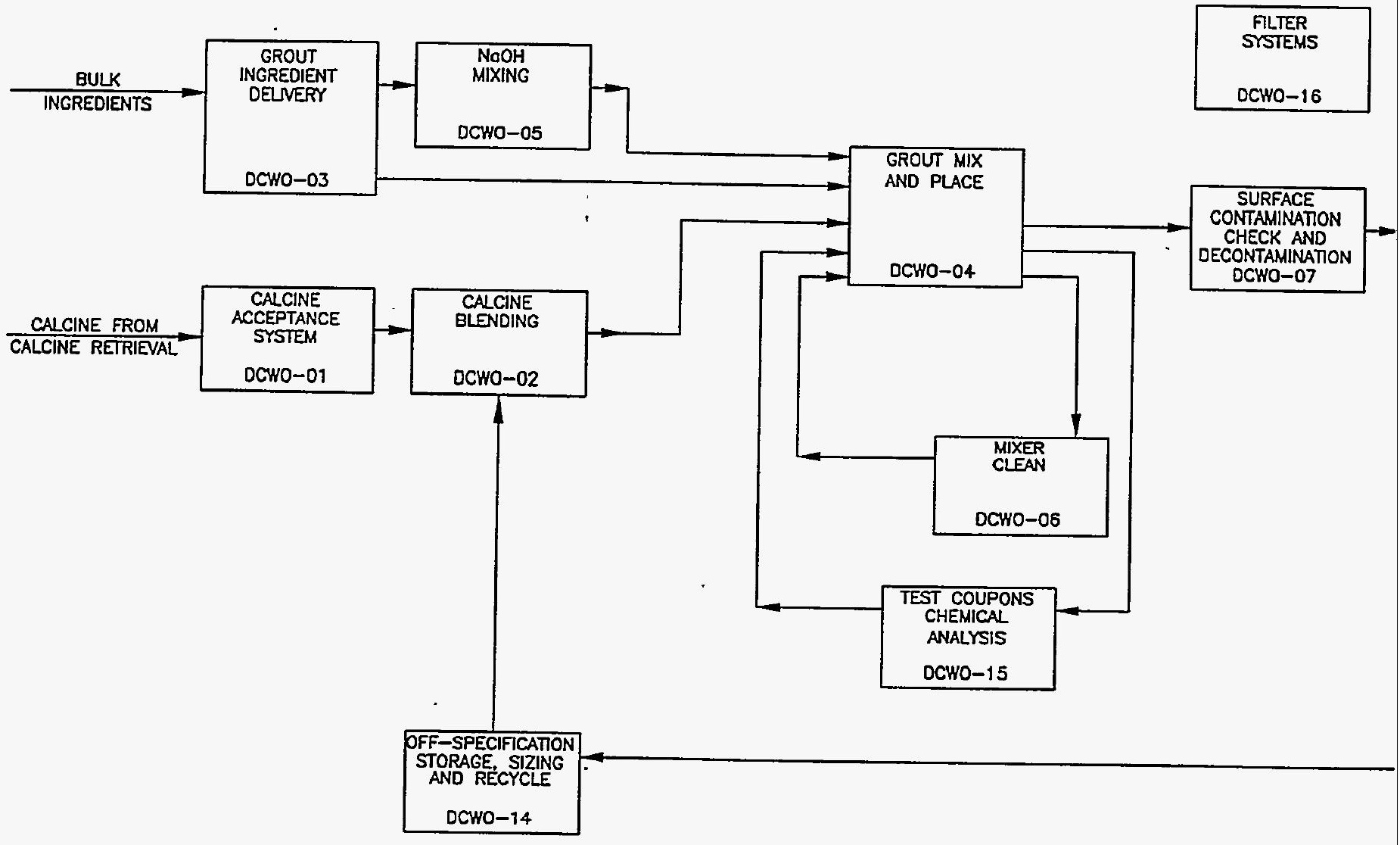

SALIENT PROCESS INFORMATION

PROCESS DURATION 5 YEARS 50\% O.L.F.

INITAL CALCINE VOLUME $5,435 \mathrm{~m} 3$

FINAL GROUT VOLUME $12,860 \mathrm{~m}^{3}$

NUMBER OF GROUT CANNISTERS 17,860

FINAL GROUT WASTEFORM-HYDROCERAMIC 


\begin{tabular}{|c|c|c|c|c|c|}
\hline $\begin{array}{l}\text { HEPA } \\
\text { FILTER } \\
\text { INSTALLATION }\end{array}$ & $\begin{array}{l}\text { AUTOCLAVE } \\
\text { CURE } \\
\text { STATION }\end{array}$ & $\begin{array}{l}\text { DEWATER } \\
\text { STATION }\end{array}$ & $\begin{array}{l}\text { HELD } \\
\text { SEAL }\end{array}$ & $\begin{array}{c}\text { FINAL } \\
\text { CONTANINATION } \\
\text { CHECK AND } \\
\text { DECON }\end{array}$ & $\begin{array}{c}\text { SONIC } \\
\text { TOMOGRAPHY }\end{array}$ \\
\hline DCWO-08 & DCWO-09 & DCWO-10 & DCWO-11 & DCWO-12 & DCWO-13 \\
\hline
\end{tabular}

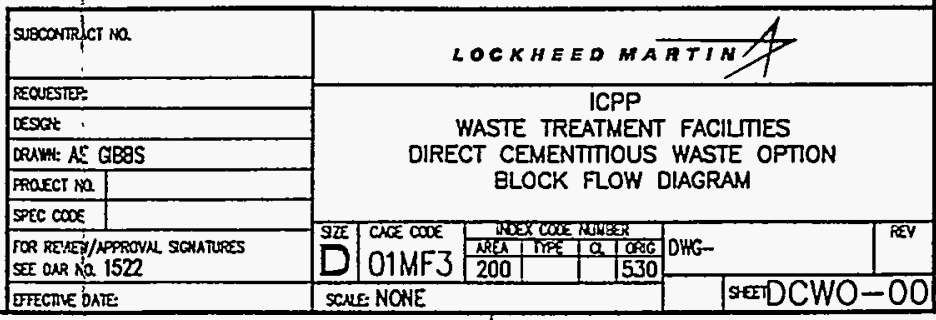




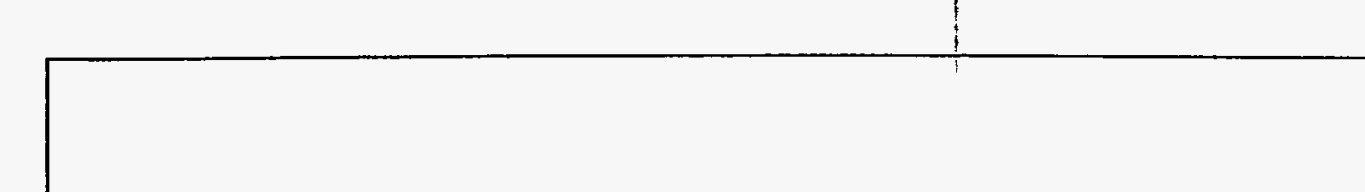

CALCINE BIN SETS
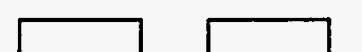

C

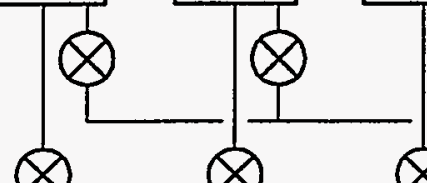

8
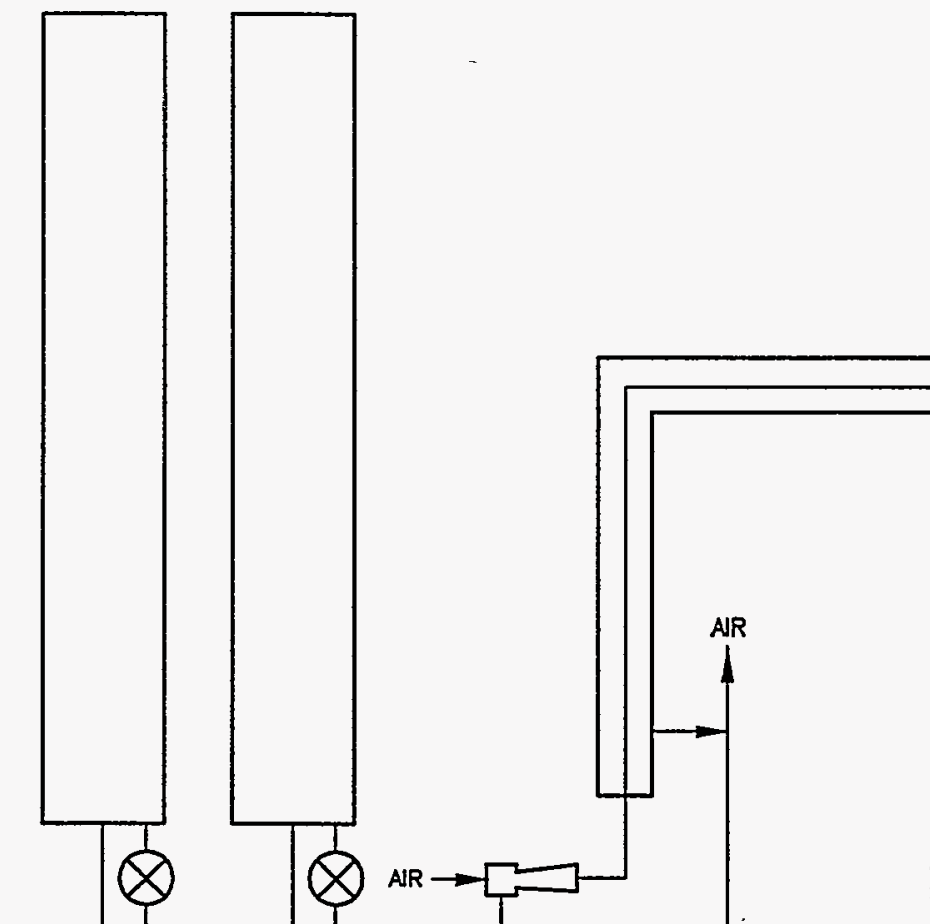

RETURN AIR TO CALCINE RETRIEVAL SYSTEM

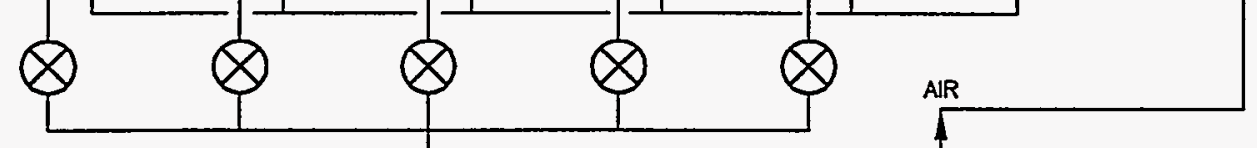

RETURN AIR TO

CALCINE RETRIEUA

STSTEM

CALCINE RETRIEVAL SYSTEM DESIGN

SECONDARY -

CONTAINMENT SYSTEM

BINS TO DCWO FACILTY

B
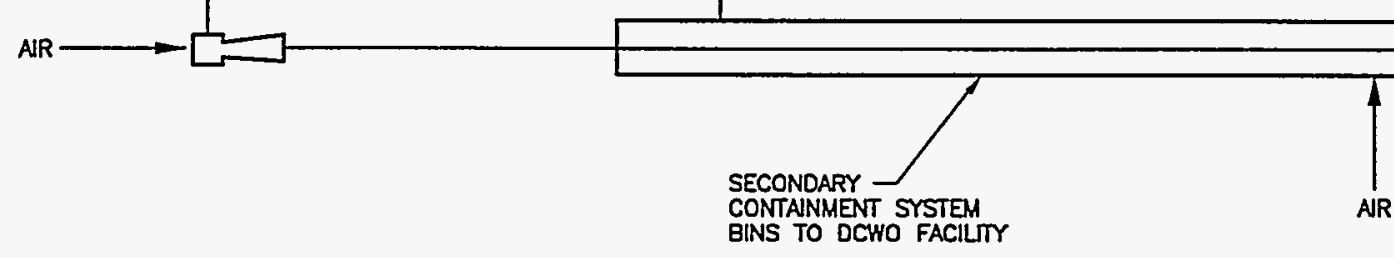


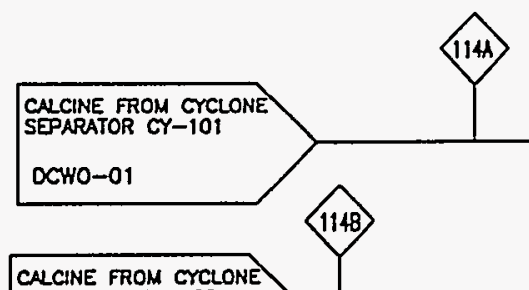

SEPARATOR CY-102

DCHO-01

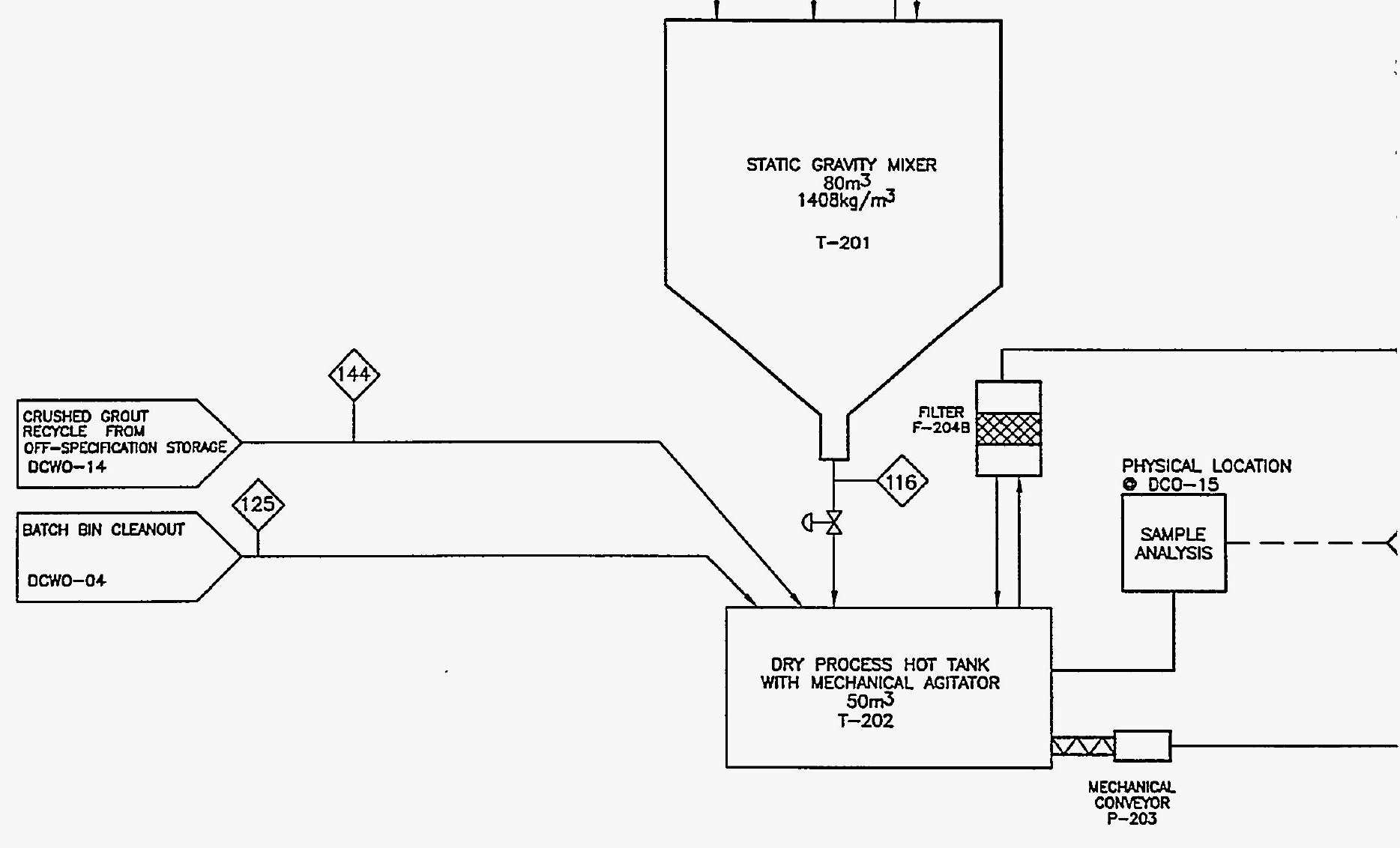

B

急

它范

A

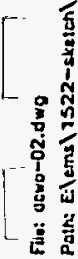


VENT TO AR HANOUNG FLTRATON SYSTEN

DCHO-16

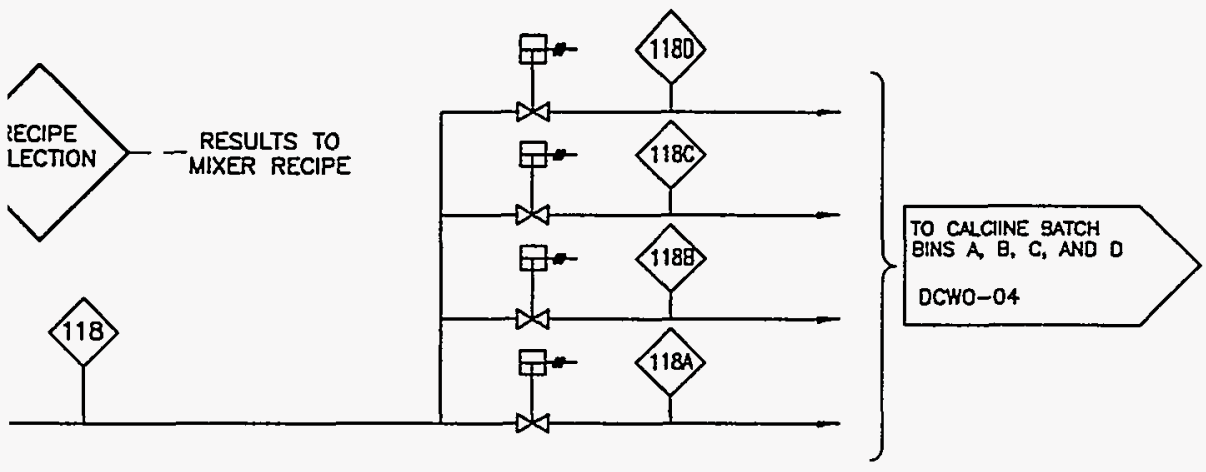

\begin{tabular}{|c|c|c|c|}
\hline 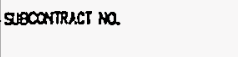 & \multicolumn{3}{|c|}{ LOCKHEEO MAATIN } \\
\hline REQESTER: & \multirow{5}{*}{\multicolumn{3}{|c|}{$\begin{array}{c}\text { ICPP } \\
\text { WASTE TREATMENT FACILTES } \\
\text { OIRECT CEMENTIMOUS WASTE OPTION } \\
\text { CALCINE BLENDING }\end{array}$}} \\
\hline cessetst & & & \\
\hline DRA Eर SNEl & & & \\
\hline FraECT RO & & & \\
\hline Prec $00 x$ & & & \\
\hline 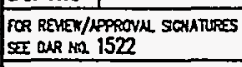 & 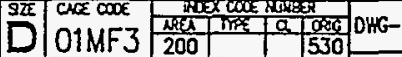 & & sov \\
\hline ETECARE OATE & SCNE NONE & senDCWC & .02 \\
\hline
\end{tabular}


CLAY - 3 TRUCKS/WK

SLAG - 1 TRUCK/2WKS

$\mathrm{NOOH}$ - 1 TRUCK/3WKS

D

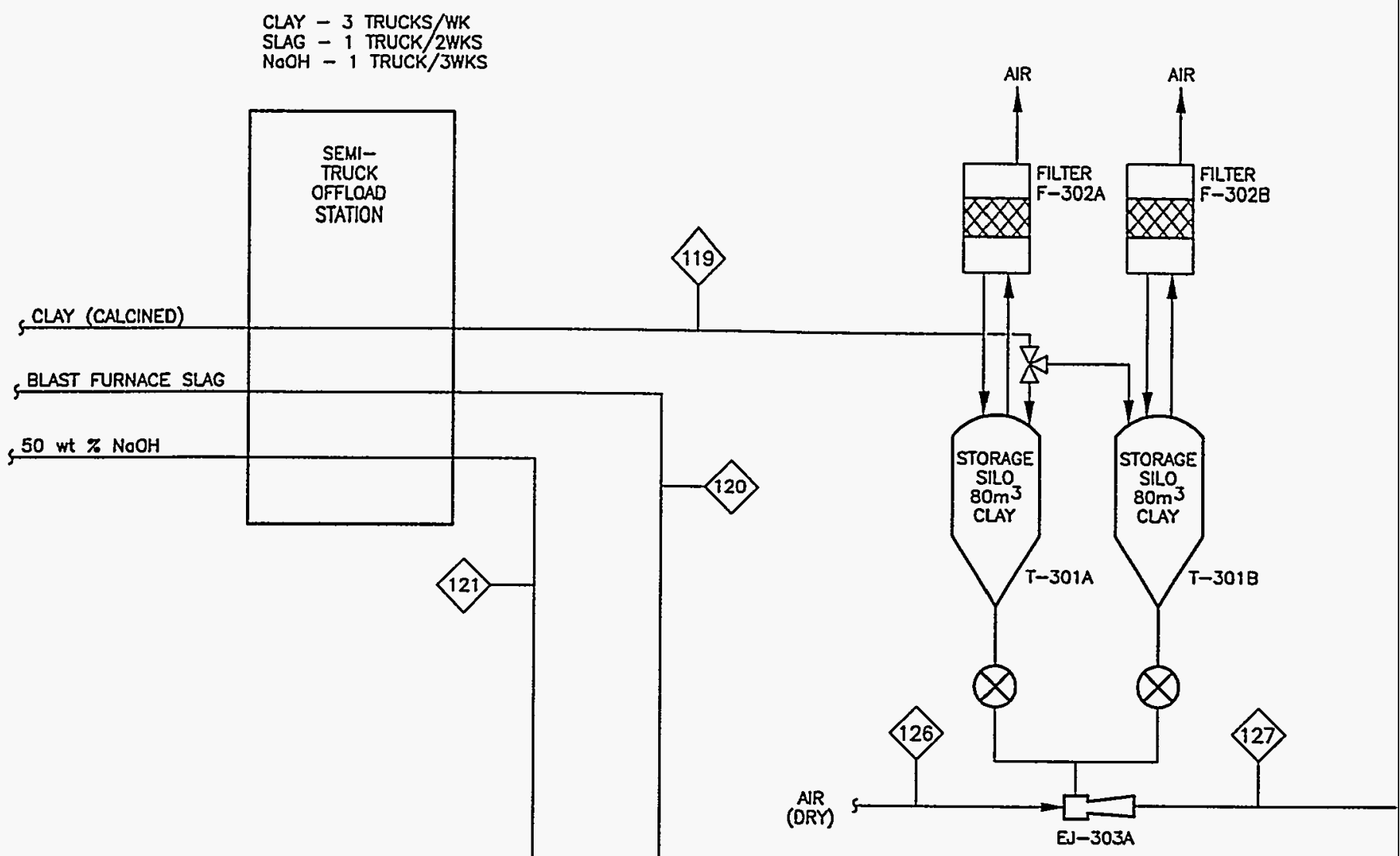

B

C
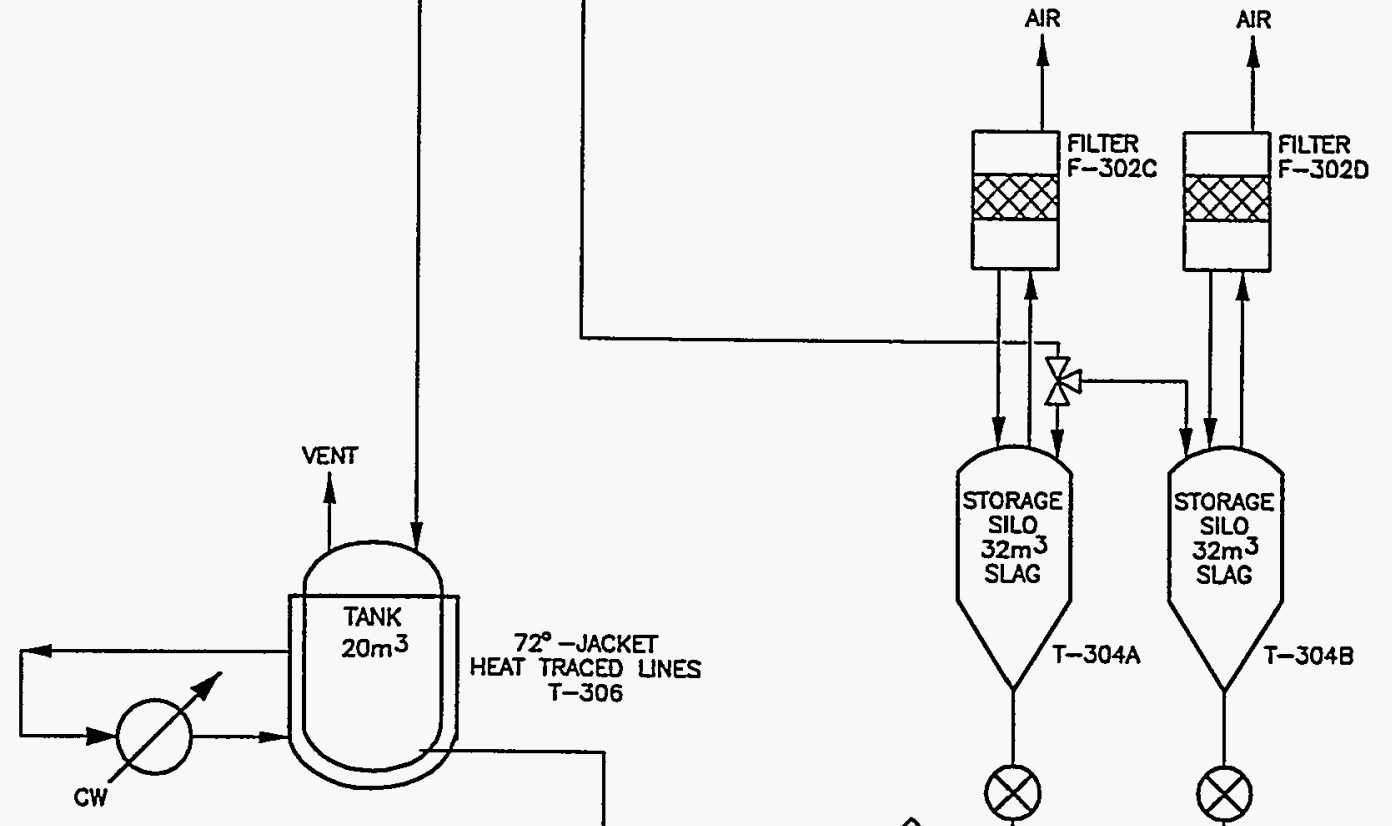

A
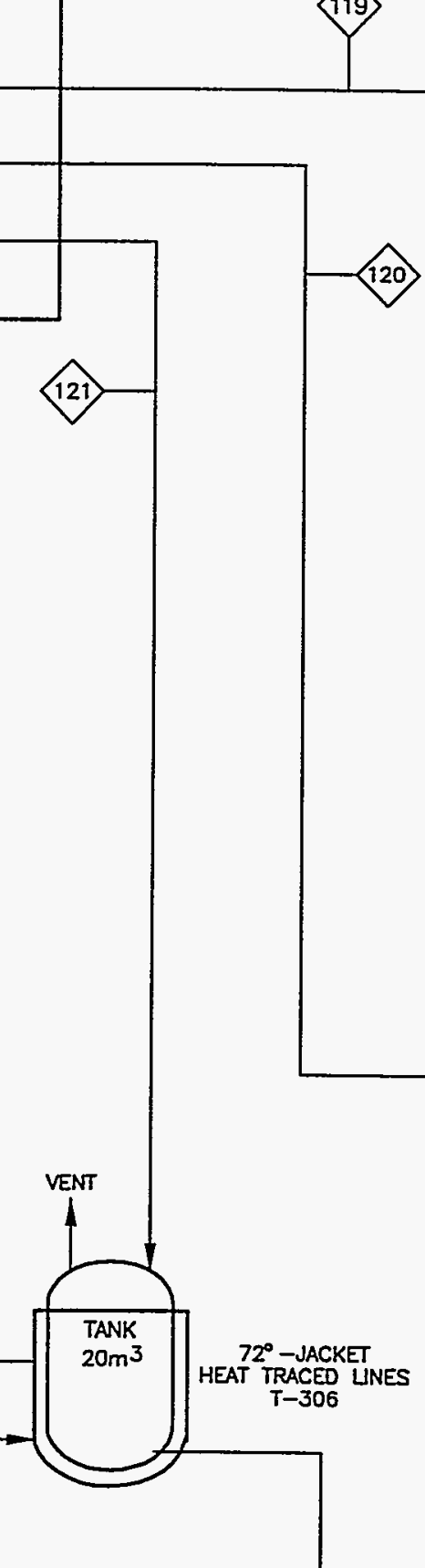


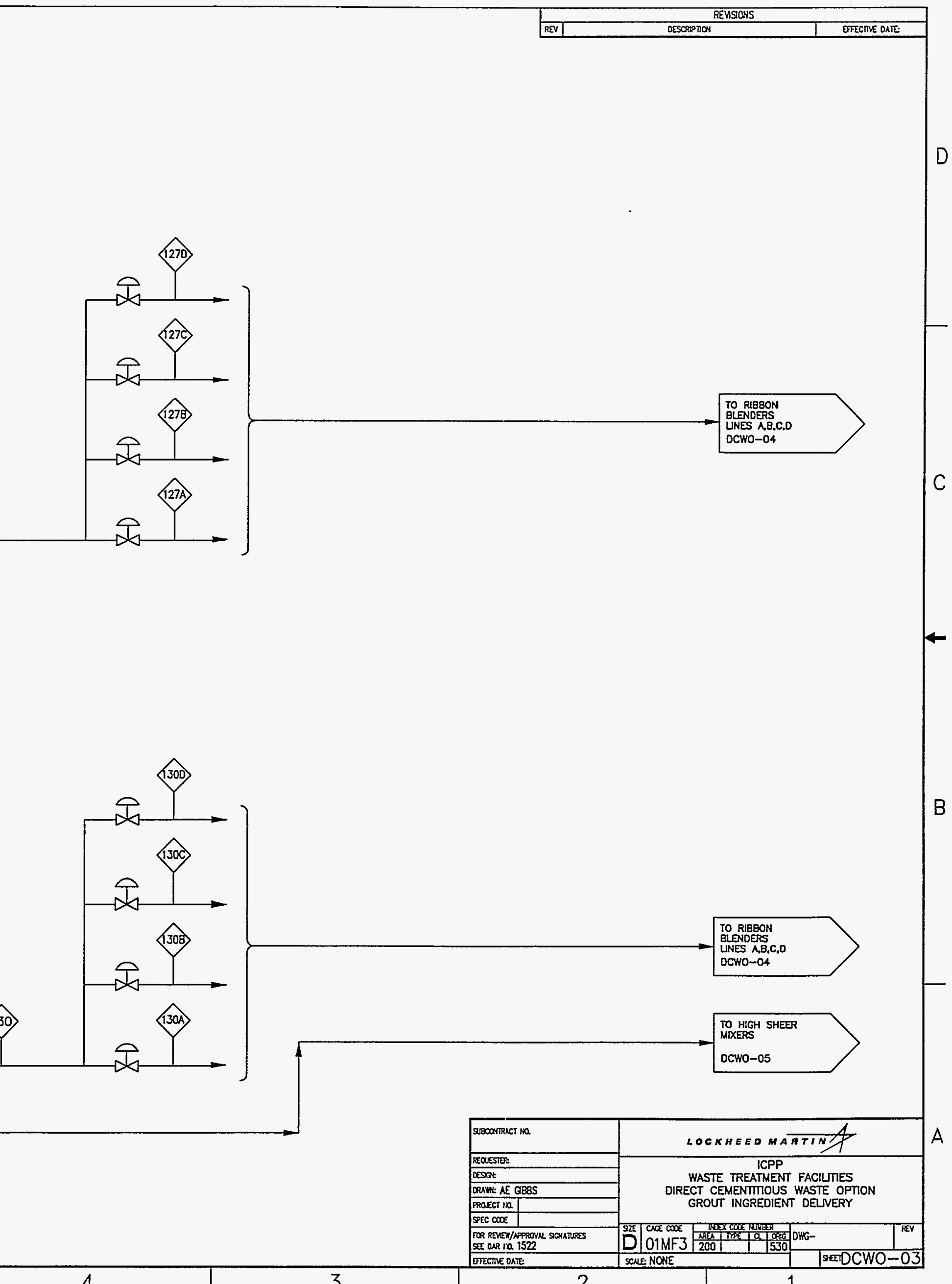




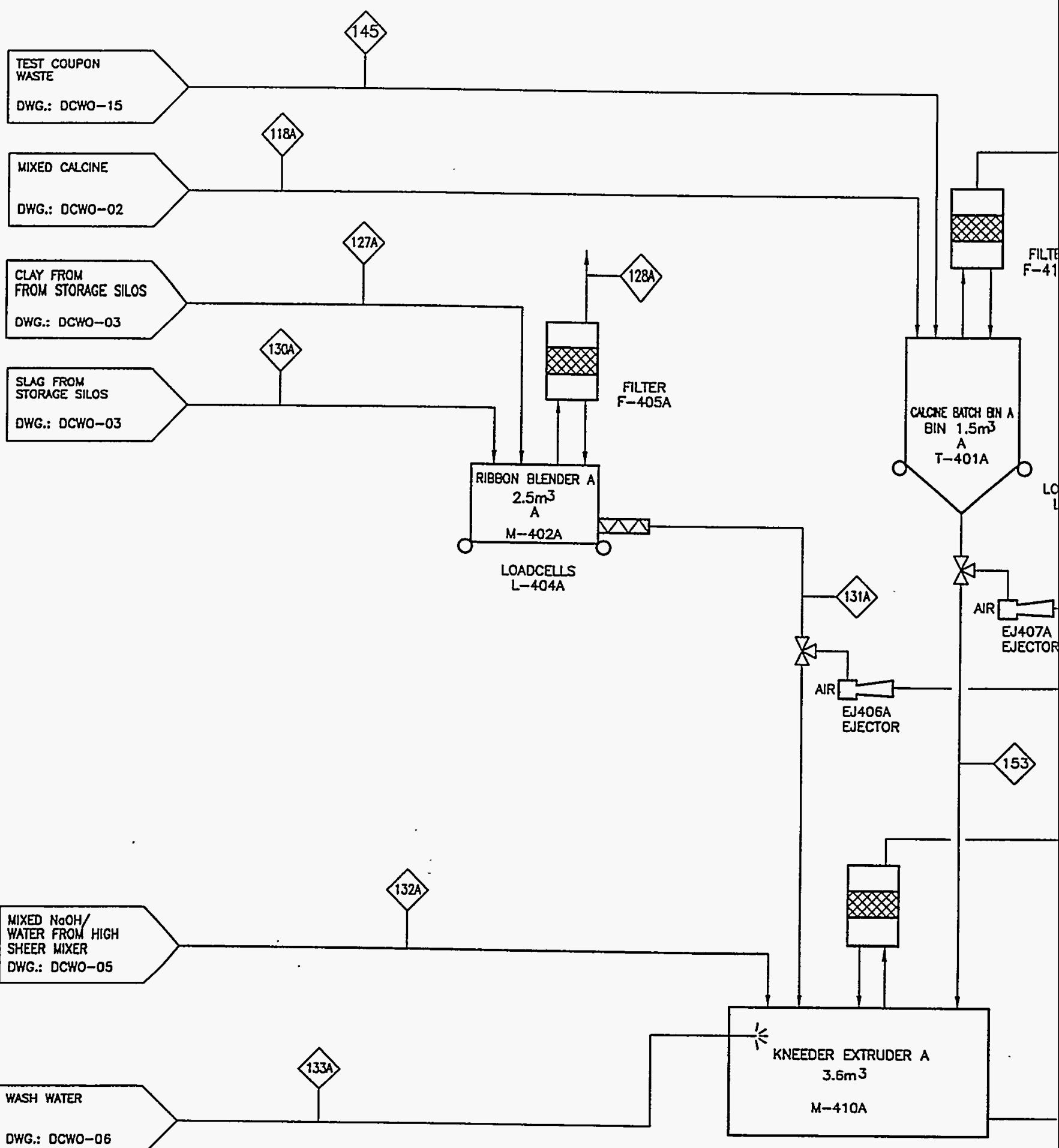

DWG.: DCWO-06 


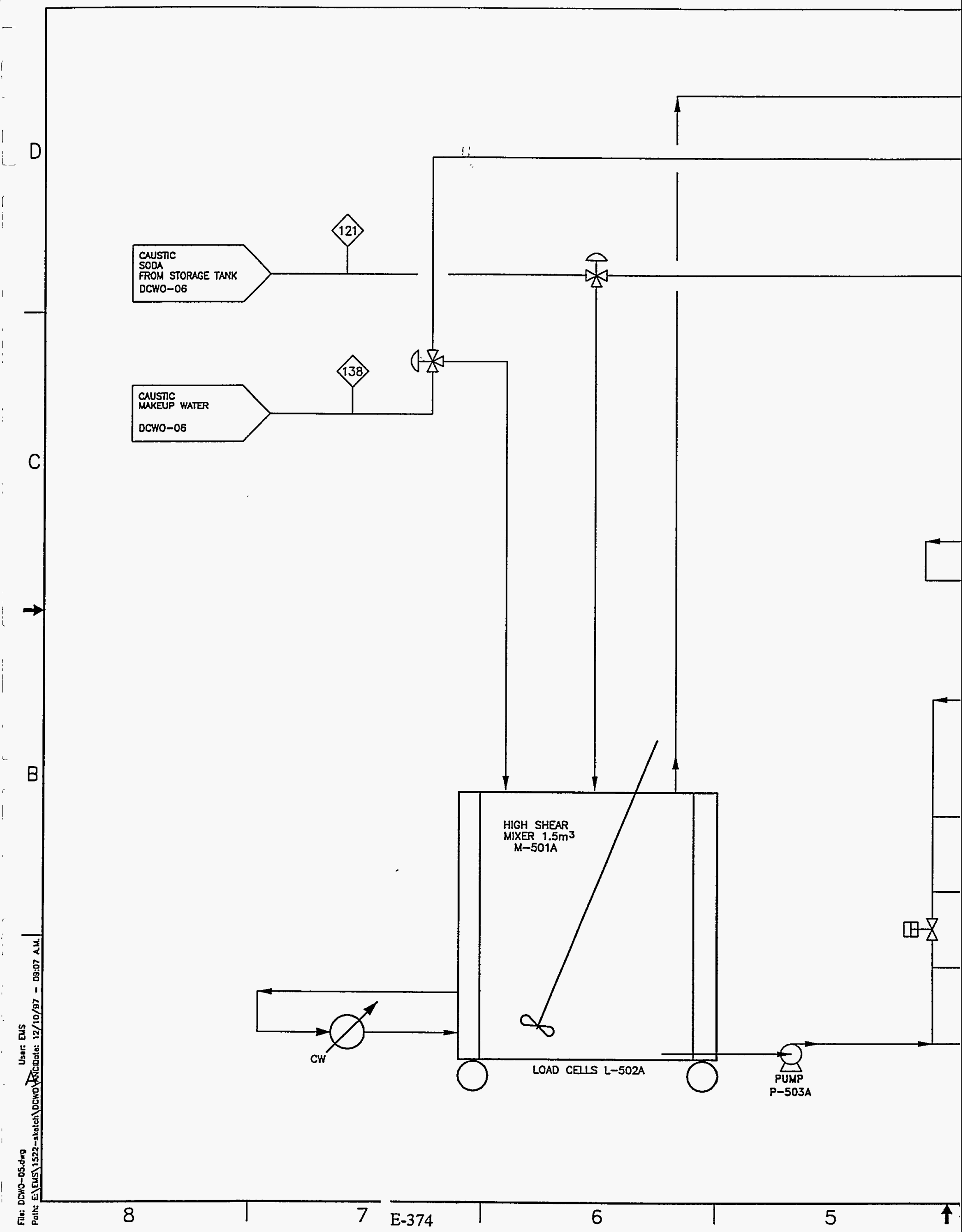




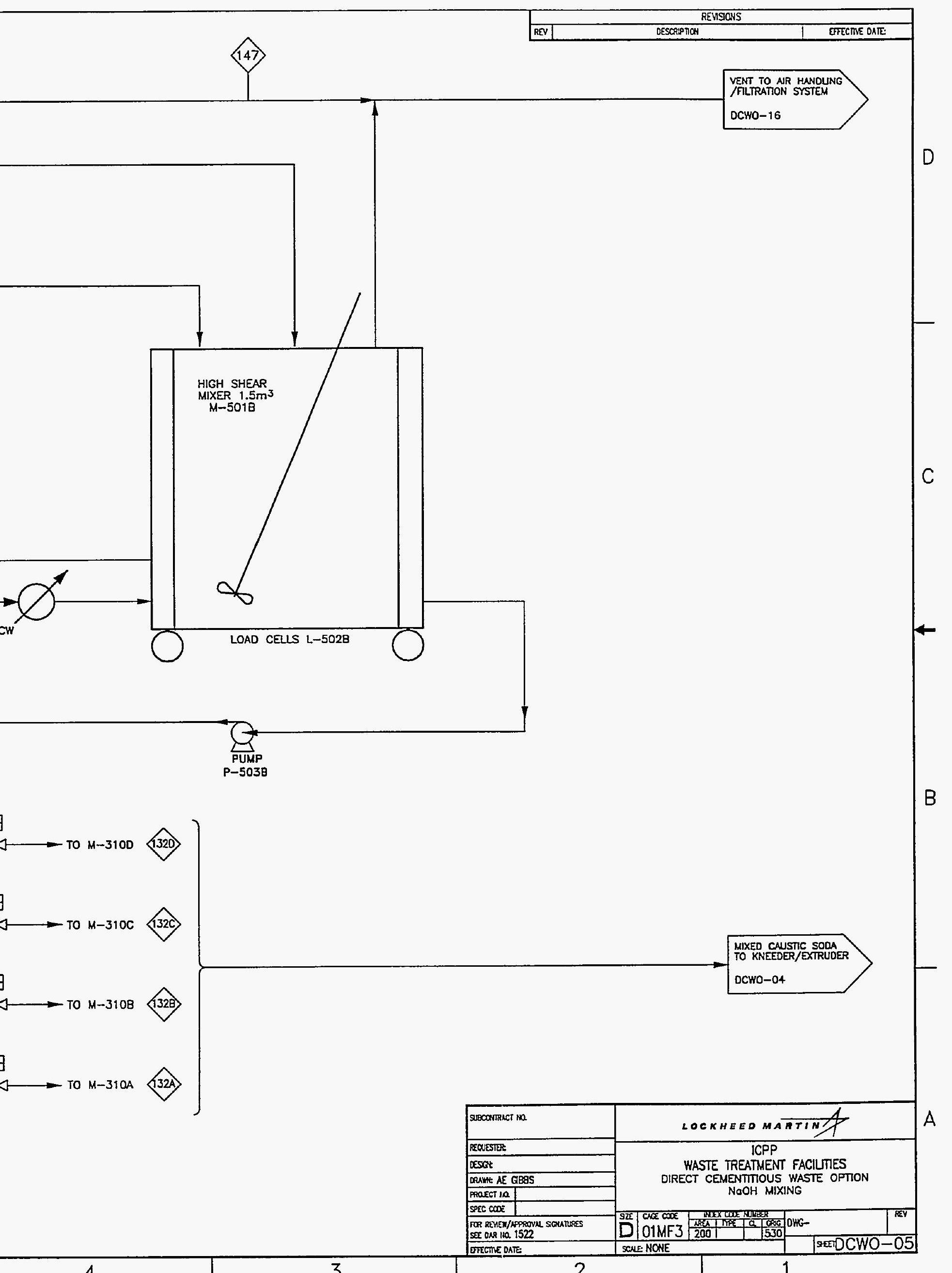




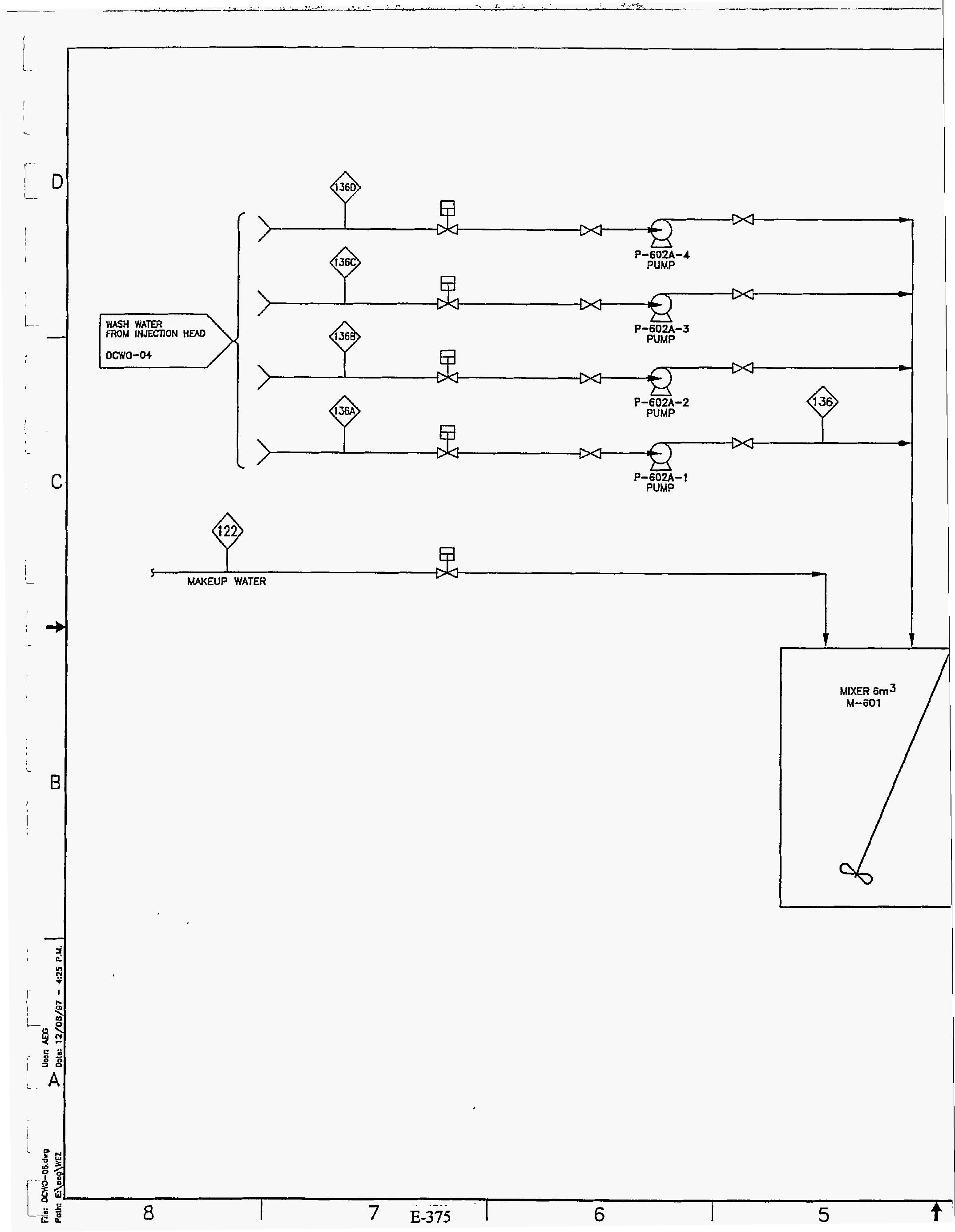




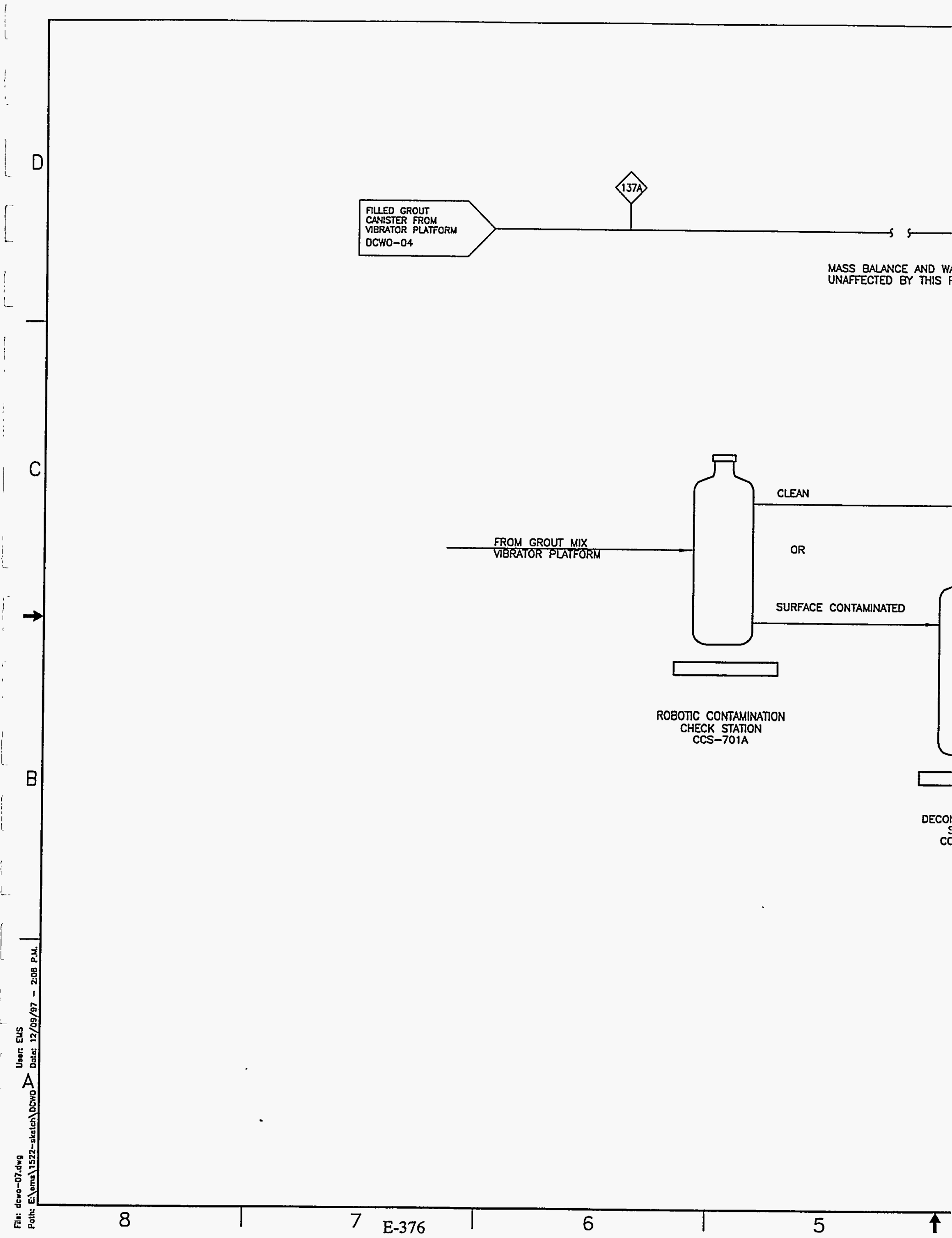


DECONTANINATED GROU CANISTER TO HEPA FLTER INSTALLATON

DCWO-08
COMPOSITON

ESS STEP

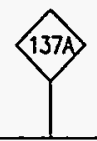

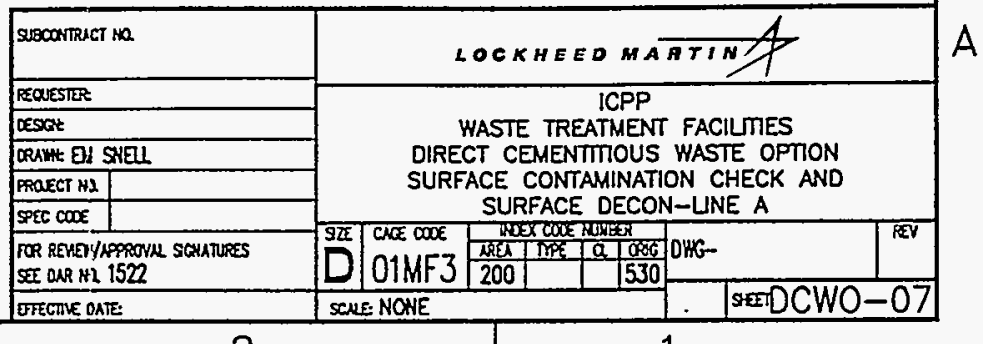




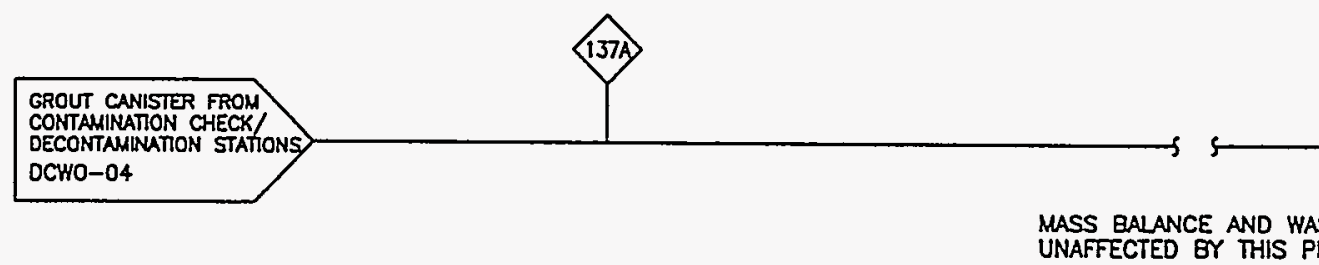

UNAFFECTED OY THIS PI $R-801 A$ 

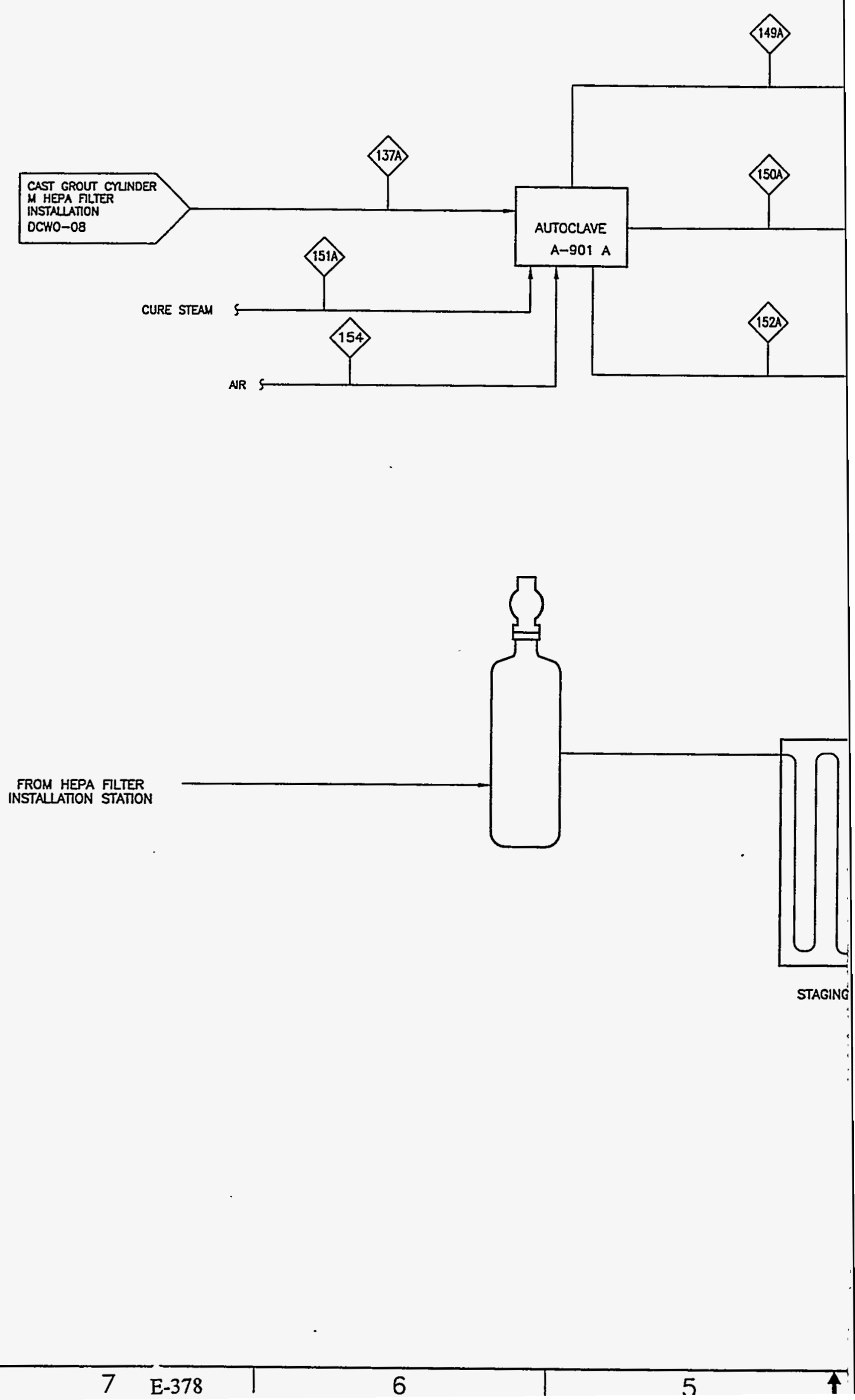


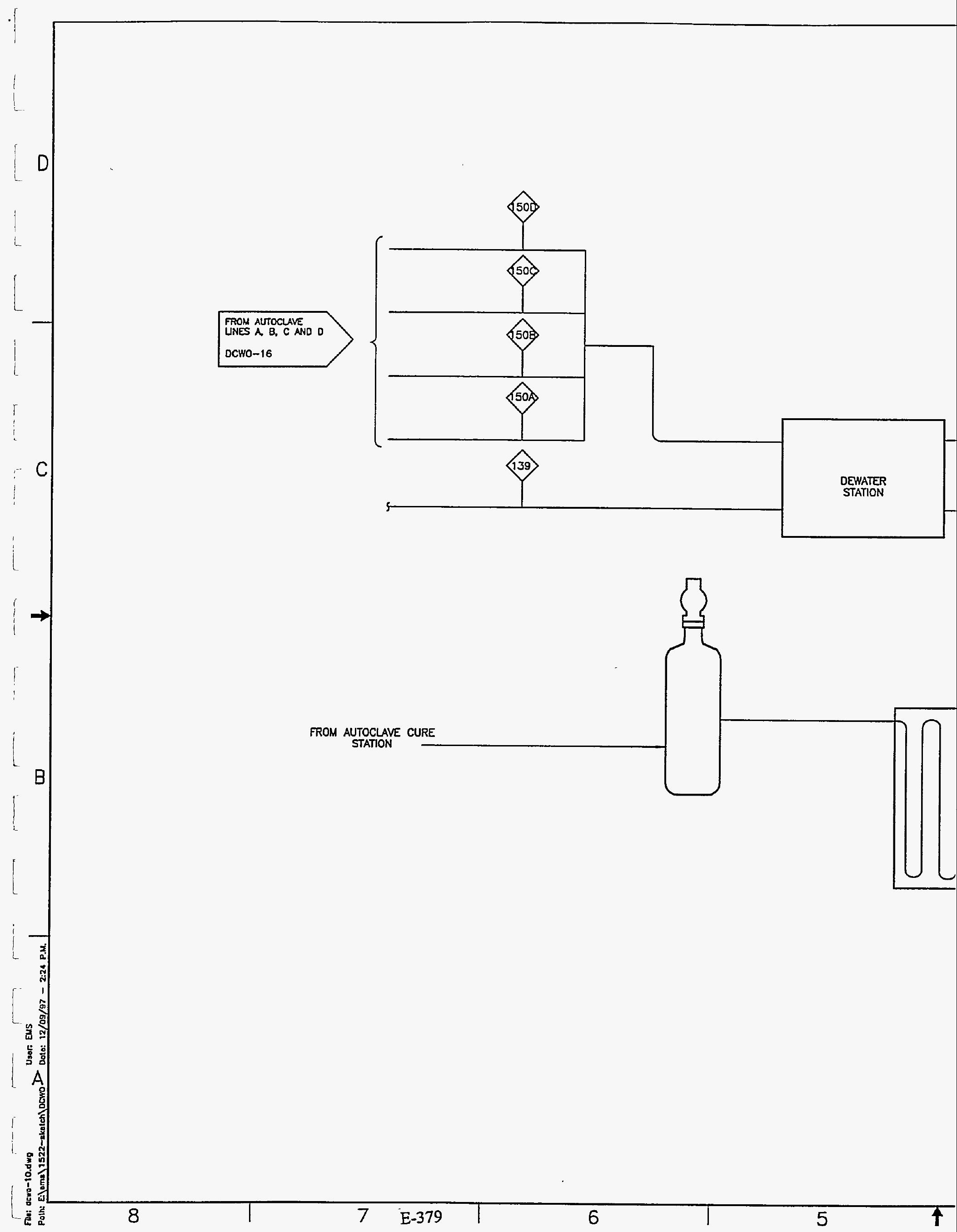




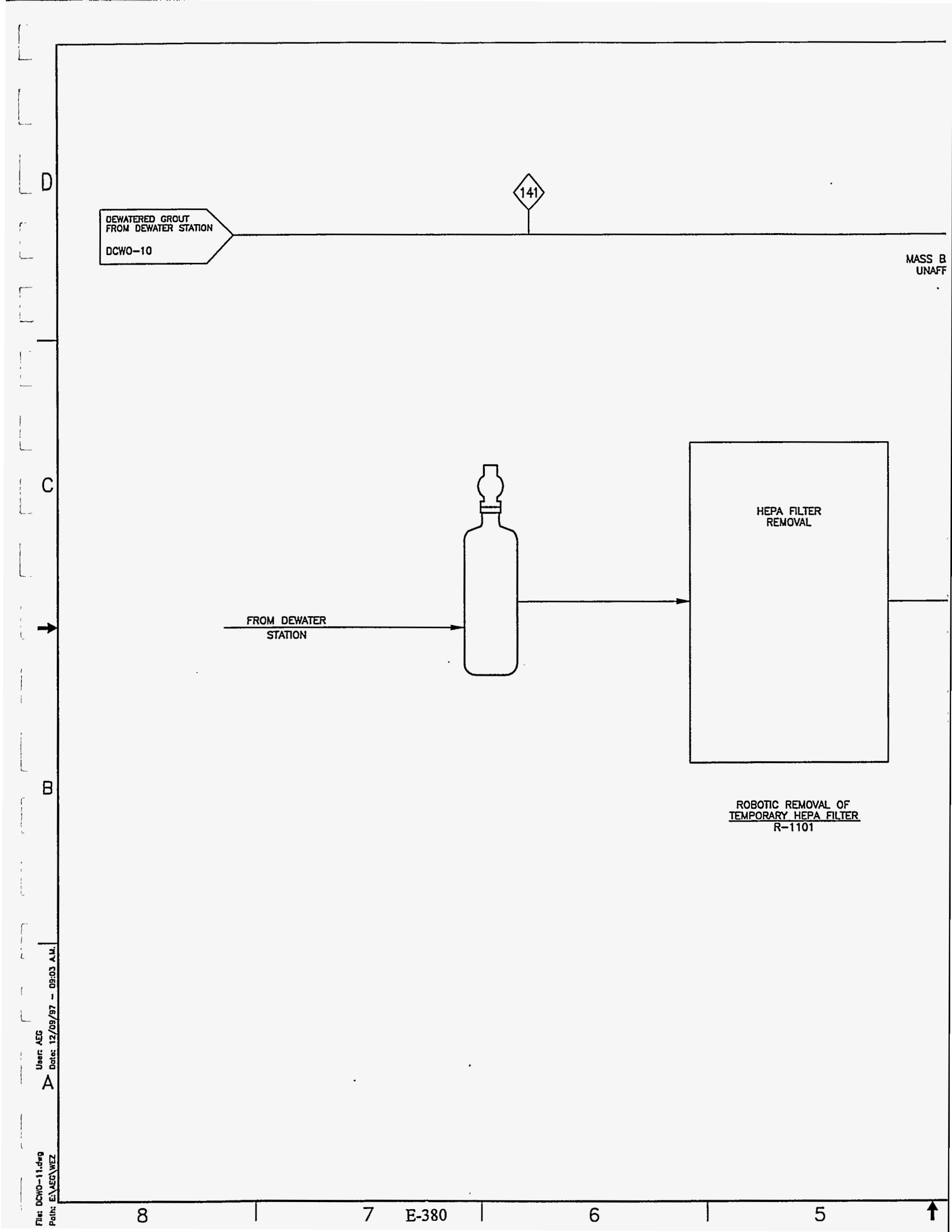


$L$

FROM WELO/SEAL

STATION

0.\%-11

UNAFFECTED BY THIS PROCE

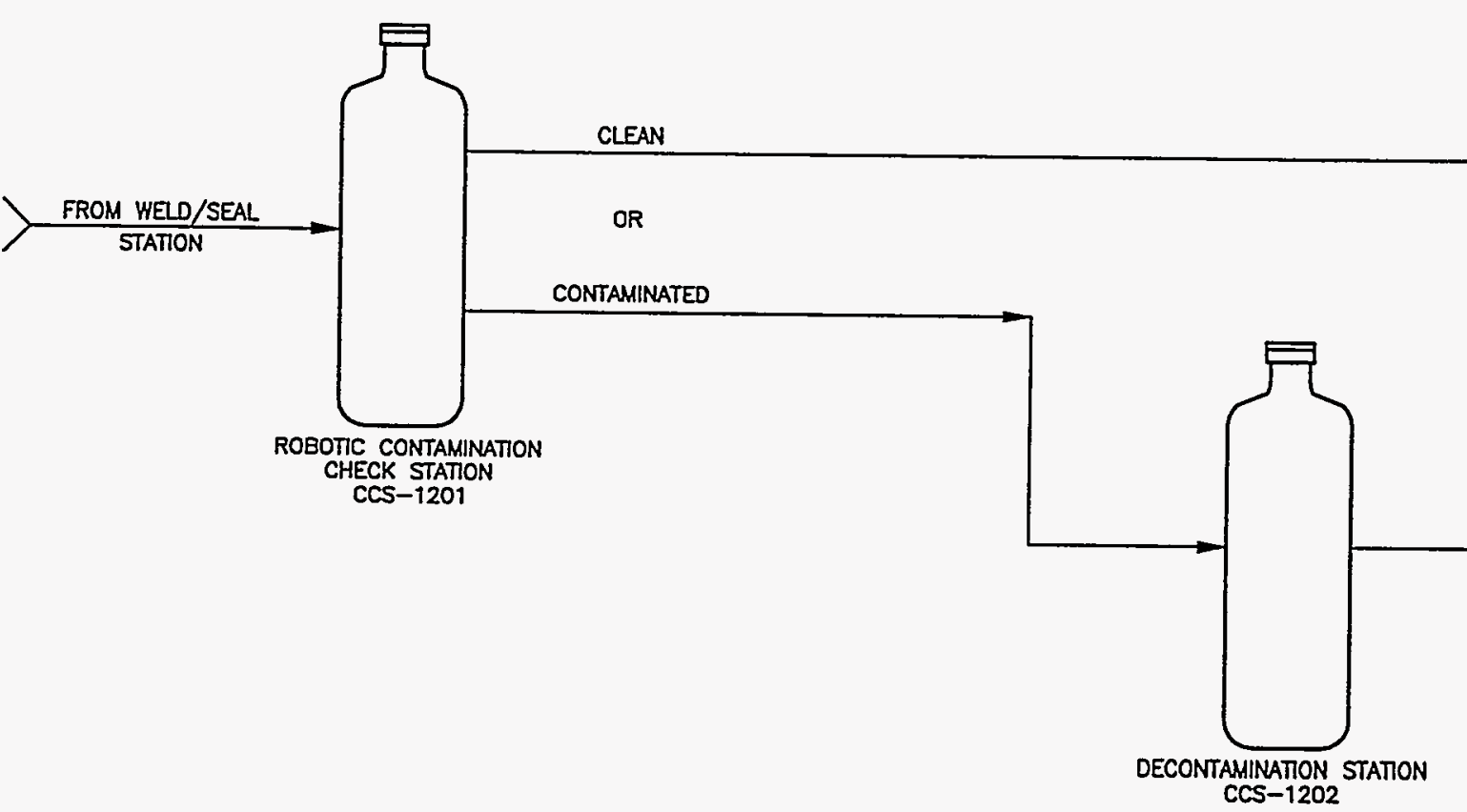

$B$

C

CCS -1202 
To sonic

TOMOGRAPHY STATON

ocwo-13

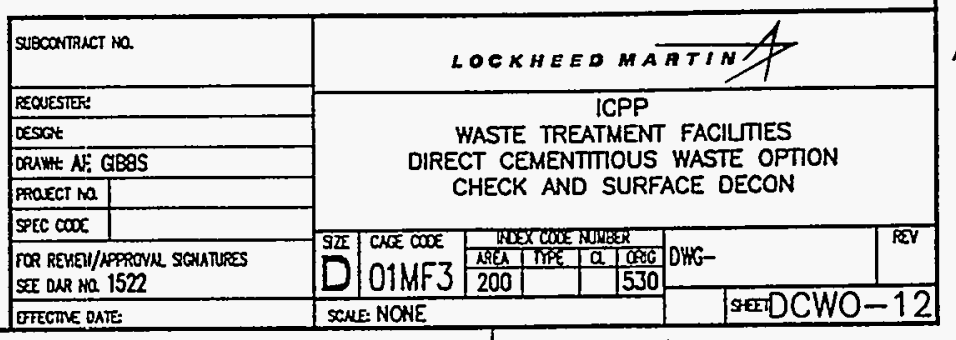




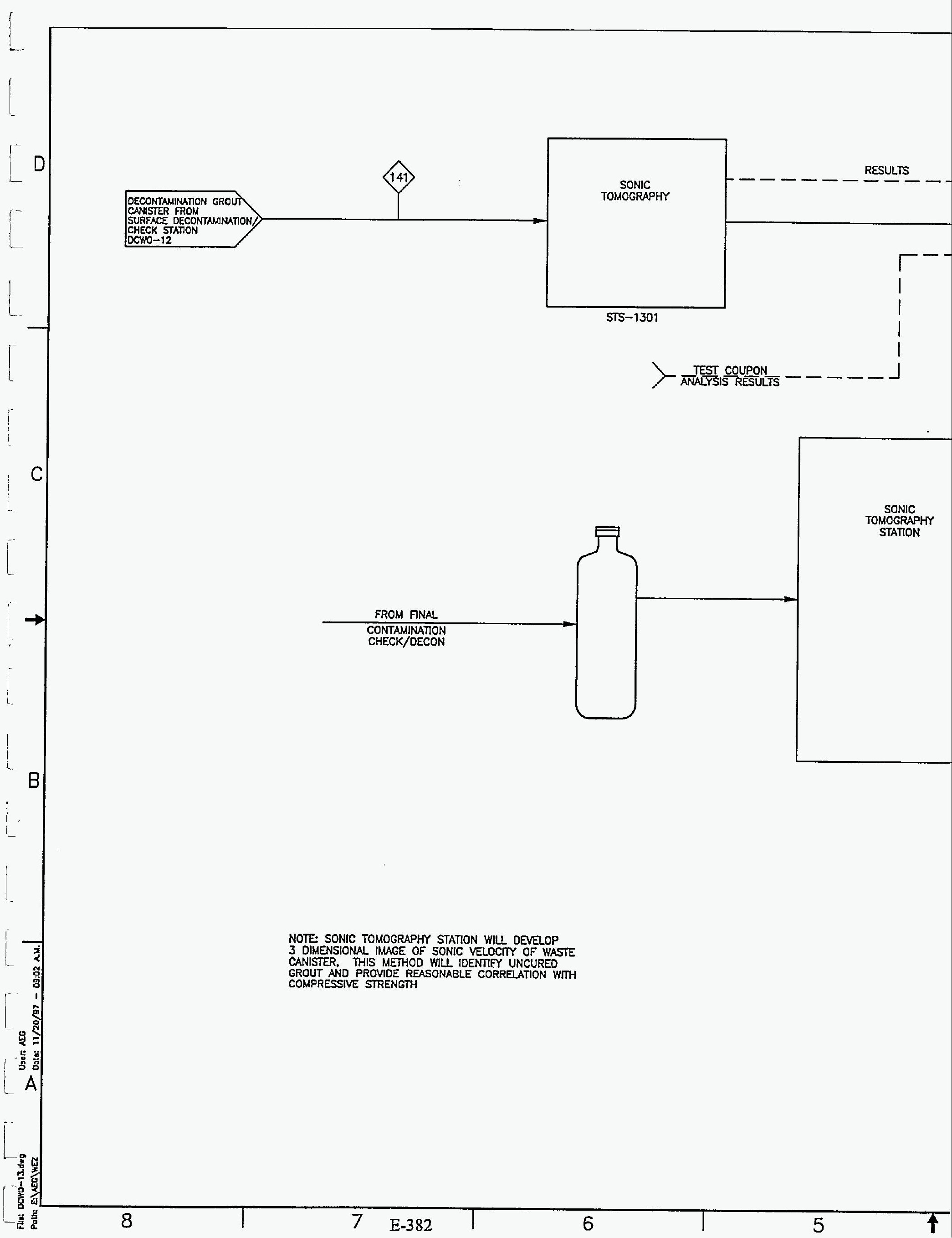




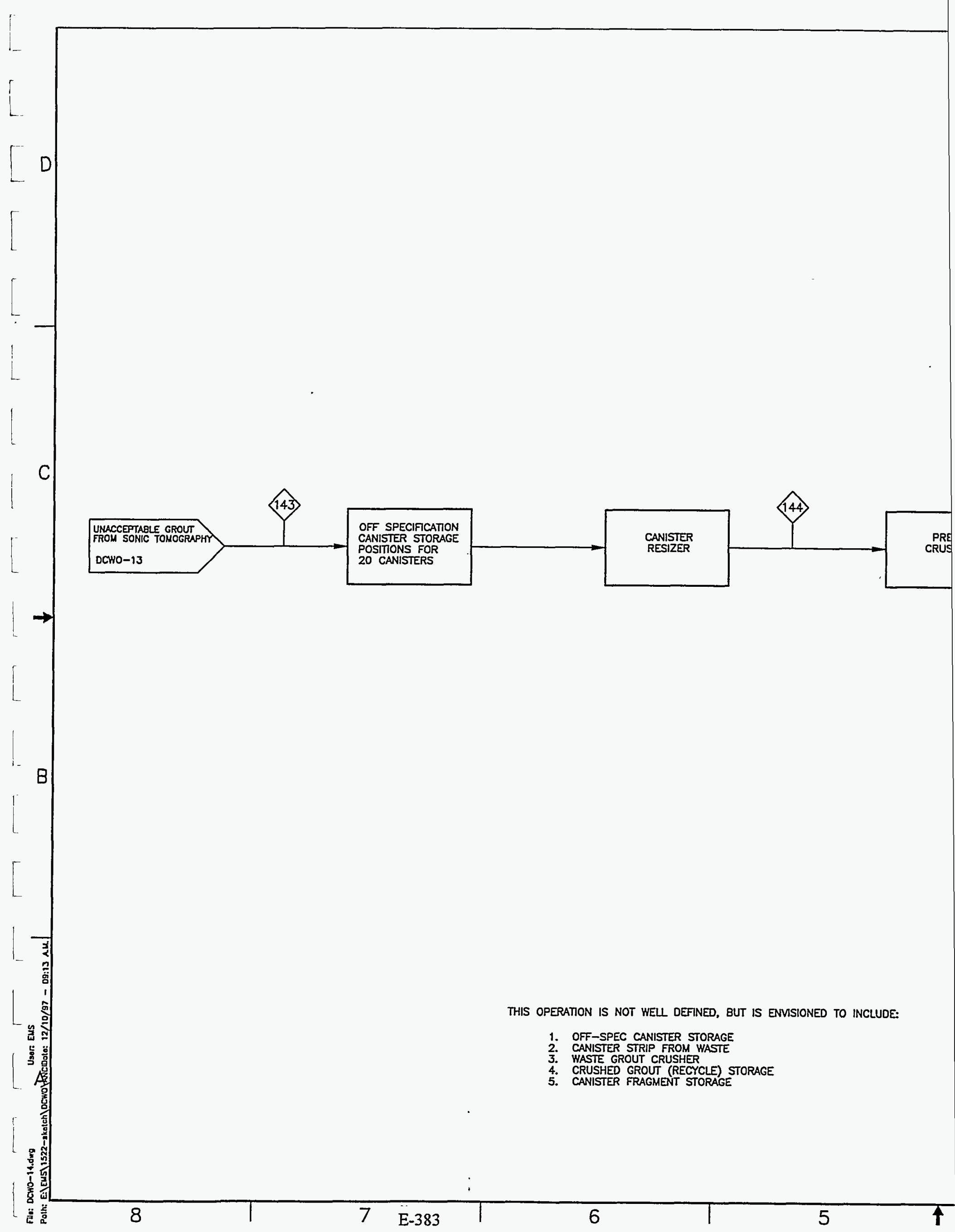




\begin{tabular}{|l|l|l|}
\hline REV & \multicolumn{2}{|c|}{ REVSSGNS } \\
\hline
\end{tabular}

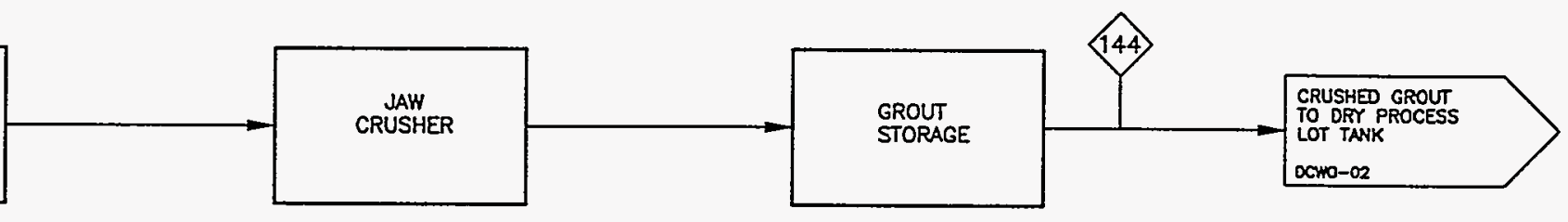

\begin{tabular}{|c|c|}
\hline SUBCartiRAst Na. & LOCKHEED MATTIN \\
\hline $\begin{array}{ll}\text { EEOUESTIE: } \\
\text { DESPAt }\end{array}$ & \multirow{5}{*}{$\begin{array}{l}\text { ICPP } \\
\text { WASTE TREATMENT FACIUTIES } \\
\text { DIRECT CEMENTTITOUS WASTE OPTION } \\
\text { SIZING AND RECYCLE }\end{array}$} \\
\hline DESATE & \\
\hline orAWt AE GBBS & \\
\hline PraECT Ho & \\
\hline SPEC COOEE & \\
\hline 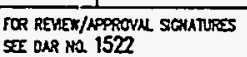 & 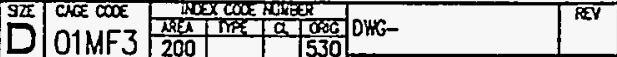 \\
\hline ETECDVE GATE: & SEEDCWO-14 \\
\hline
\end{tabular}


3. PHYSICAL PROPERTIES

FRON DEWATER STATION

4. SIZE REDUCE

DCWO-04

5. FEED to calCINe BATCH BIN

ᄃ

THIS SEGMENT IS NOT WEU DEFNED, BUT WILL BE VERY SMALL VOLUME.

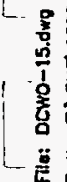





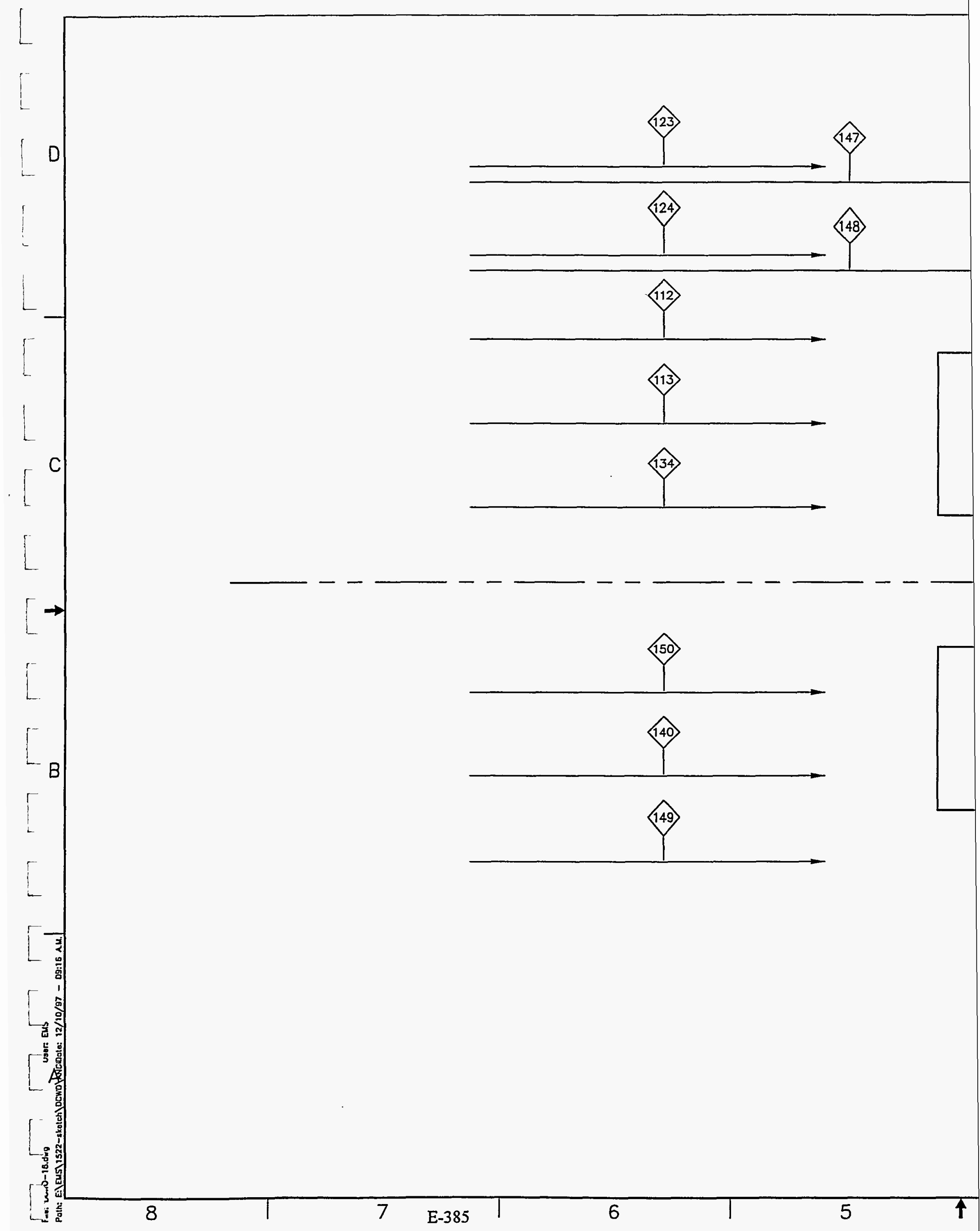




\begin{tabular}{|c|c|c|c|}
\hline SuBcontruct No. & \multicolumn{3}{|c|}{ LOCKHEED MATTIN/ } \\
\hline \begin{tabular}{|l} 
EEDESTIER \\
DESPEt
\end{tabular} & \multirow{4}{*}{\multicolumn{3}{|c|}{$\begin{array}{c}\text { ICPP } \\
\text { WASTE TREATMENT FACIUMES } \\
\text { DIRECT CEMENTIMOUS WASTE OPTION } \\
\text { AIR HANDUNG/FILTRATION SYSTEM }\end{array}$}} \\
\hline DRAWL AE QBBS & & & \\
\hline PAOECT HO & & & \\
\hline SEec coos & & & \\
\hline \multirow{2}{*}{ 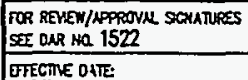 } & 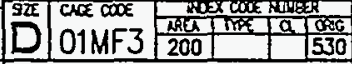 & DHG- & 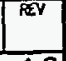 \\
\hline & SCNE: NONE & \multicolumn{2}{|c|}{ SEDCWO-16 } \\
\hline
\end{tabular}


Project File Number $02 \mathrm{BE} 0$

Project/Task Waste Treatment Project Feasibility Studies

Subtask Direct Cementitious Waste Option Scoping Study

Title: 20 Year Scaling

Summary:

The DCWO study used a model process and facility to obtain data for the DCWO and CWO grout facilities. The model used the larger of the two facilities (CWO) and was based on the CWO requirement to grout its calcine volume in 5 years. Due to study constraints, information for the DCWO 20 year grout process and facility was obtained by scaling the CWO 5 year grout process and facility. This EDF documents the steps taken in this scaling process.

References:

1. Plant Design and Economics for chemical Engineers, $4^{\text {th }}$ edition, Peters and Timmerhaus

2. EDF-DCWO-012, 20 Year Manloading and Physical Space Identification.

Conclusions:

Scaling used an industry guide for process reduction with resulting facility reduction of $31 \%$ over the 5 year facility based on estimated square footage reduction.

Distribution (complete package): WTP EIS Studies Library, R. E. Dafoe M.S. 3765, D. J. Harrell M. S. 3211, B. R. Heim M. S. 3765, S. J. Losinski M. S. 3625, T. E. Sivill M. S. 3655, K. L. Williams M. S. 3765

Distribution (summary package only):

\begin{tabular}{|c|c|c|c|c|c|}
\hline Author & $\begin{array}{l}\text { Dept. } \\
\text { if } 30\end{array}$ & Reviewed & $\begin{array}{l}\text { Date } \\
26 / 48\end{array}$ & Approved & $\begin{array}{l}\text { Date } \\
7 / 2 / 98\end{array}$ \\
\hline सि? & & $\begin{array}{l}\text { LMITCO } \\
\text { Review }\end{array}$ & Date & $\begin{array}{l}\text { LMITC } \\
\text { Approval }\end{array}$ & Date \\
\hline
\end{tabular}


The objective of the Direct Cementitious Waste Option (DCWO) was to provide a cost estimate for a grouting process and facility that would support the needs of both the DCWO and the Cementitious Waste Option (CWO). The operating schedule for the. CWO grout facility is 5 years and 20 years for the DCWO grout facility. Time alotted for this study did not lend itself to generating two complete process and facility scoping level designs. Because the actual grouting process does not change, a scaling approach was determined to be a legitimate method for obtaining the costs and related process and facility scoping level designs for one of the processes and facility. The model chosen was based on the CWO 5 year schedule requirement. Engineering judgement indicated that scaling down from a larger volume process and building was preferred to scaling up.

Scaling began with determining the quantity of process equipment required then estimating the amount of facility square footage reduction as a result. Personnel requirements were estimated also and documneted in reference 2 . This information was then used in determining a new cost estimate.

The ratio of 5 years to 20 years (1/4) was used to scale down equipment quantities or size. The CWO 5 year grouting process, where required, includes 4 process lines at the batch mixing, canister loading, grout curing (autoclaves), decontamination, and sonic tomography stations. For the DCWO the 4 lines were reduced to one. Single line components such as the static gravity mixer, dry process hot tank, and dewatering chamber were scaled down using percentages provided in reference 1. Some components such as the clay and slag bins were not changed because they were originally sized to accommodate standard delivery load quantities.

Building drawings (draft stage) included as part of this EDF, show where facility square footage was adjusted. Study constraints did not permit reconfiguring the building. The following is a comparison, by area type, between the CWO 5 year and DCWO 20 year estimated facility footage:

$$
\text { CWO } 5 \text { year DCWO } 20 \text { year }
$$

Admin

$103,000(9569)$

$92,050(8551)$

Non-Radiation

$57,100(5305)$

$43,625(4053)$

Radiation

$$
126,500(11,752)
$$$$
61,600(5723)
$$

$$
\text { in } \mathrm{ft}^{2}\left(\mathrm{~m}^{2}\right)
$$

Total

$$
286,600(26,626) \quad 197,275(18,327)
$$

From this evaluation, the DCWO 20 year facility square footage is approximately $69 \%$ of the CWO 5 year facility. This same percentage was applied to the grout facility support systems, such as power, I\&C, and HVAC. 
$20+2$

542

Hor len - 6,600sF HOT CFen - 126,500st

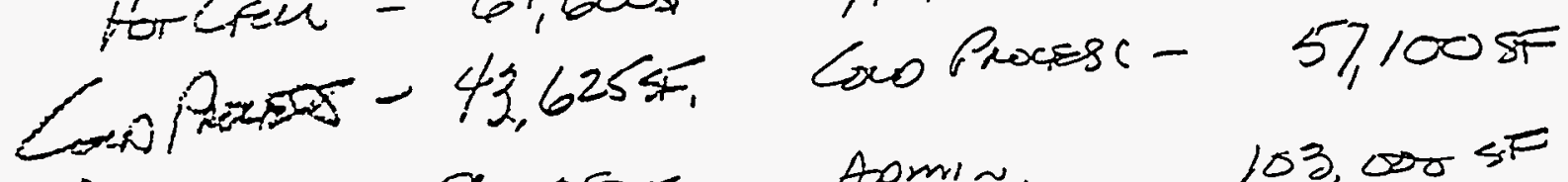

Anmis, T 92,050, Aomin. 103,000 sF

$197,27545 \cdot-320 .-286,5504$

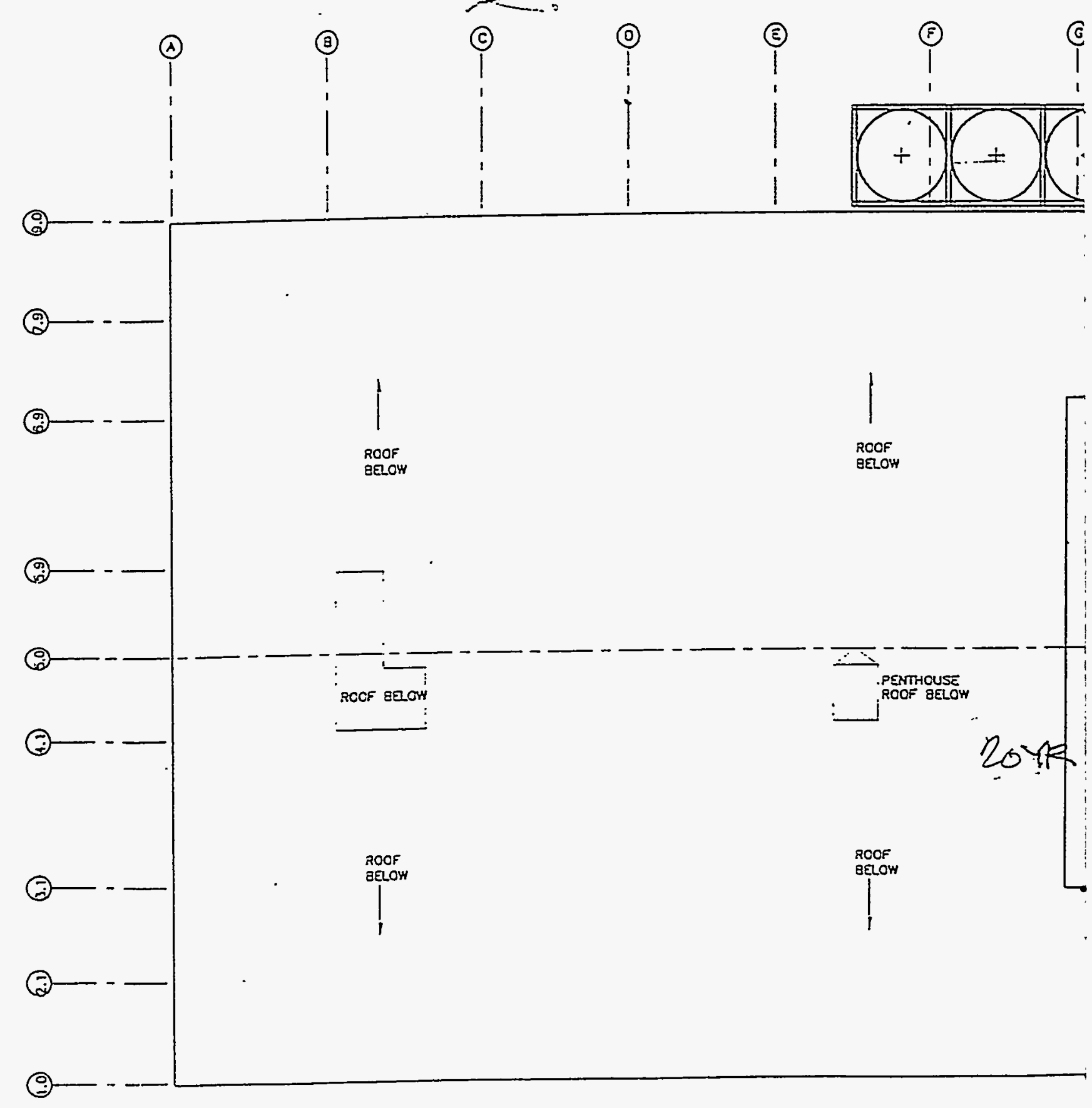

E-389

LEVEL +135'-0" (6) $=$ 
60:00

$\equiv_{0}, 0<\infty=$

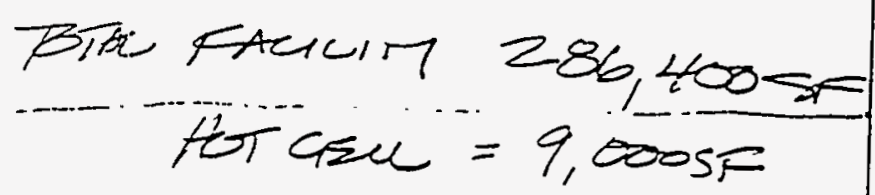

$20=3$

coco process

Aomin. $=3,6005 F$.

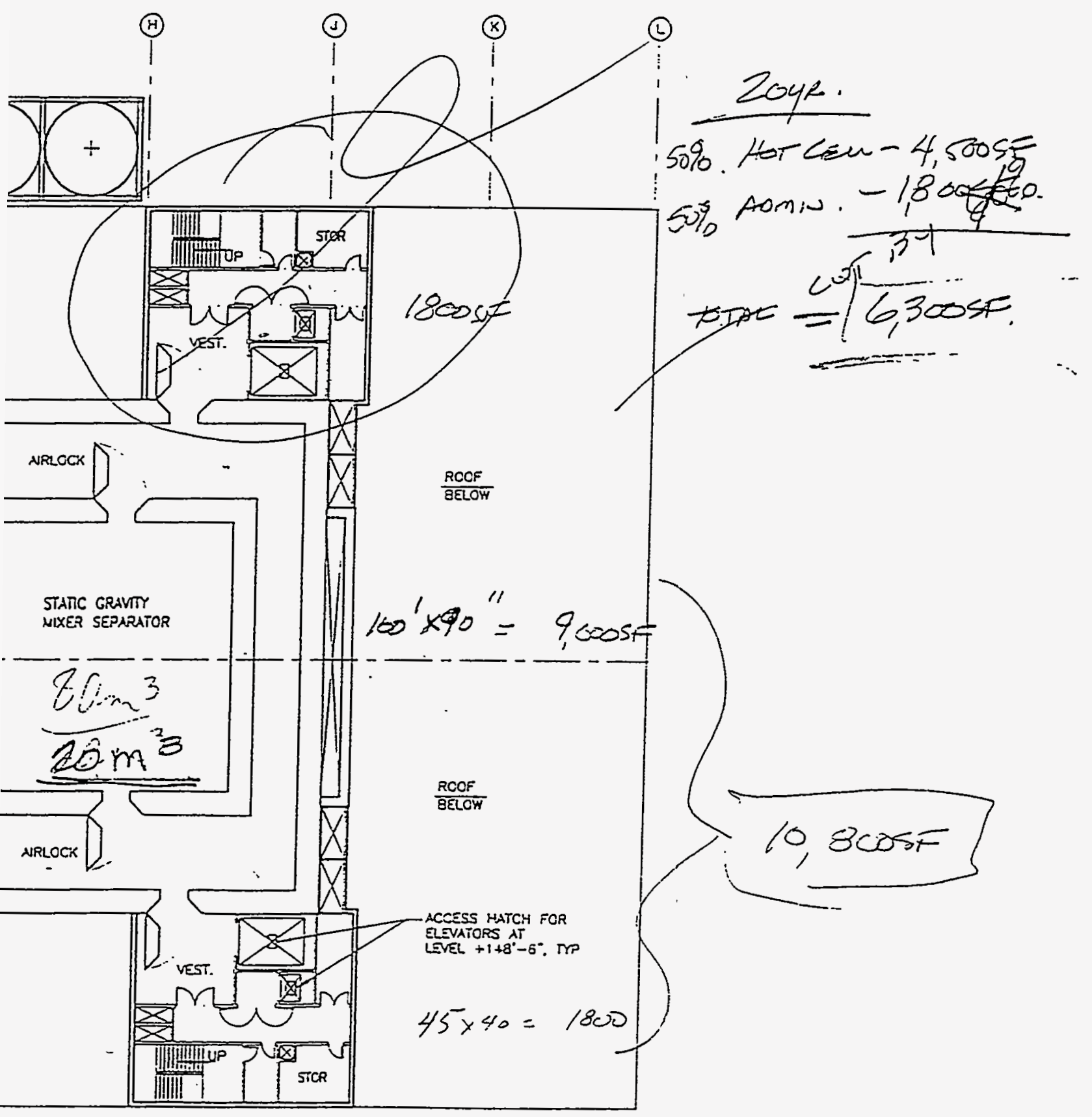




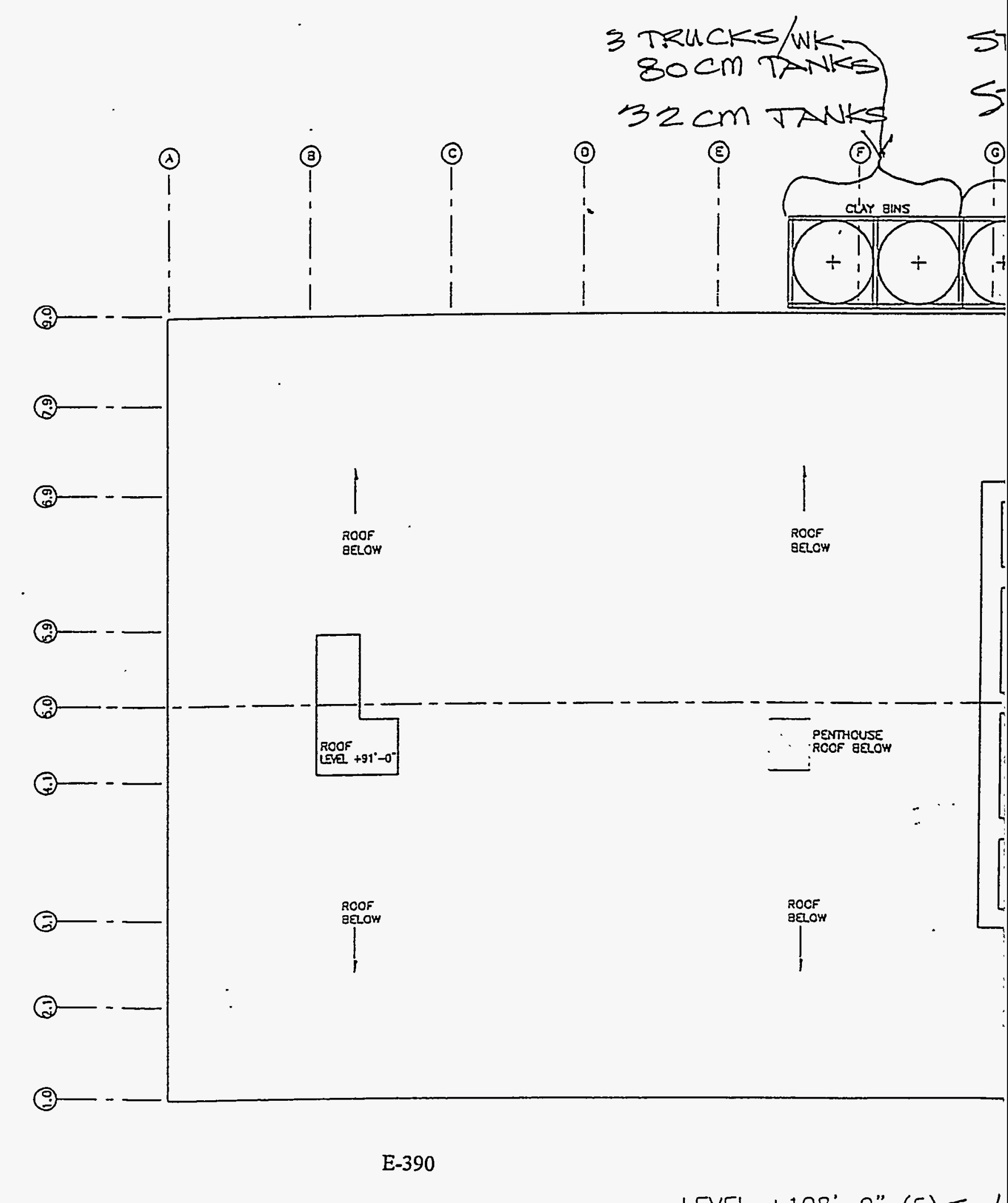




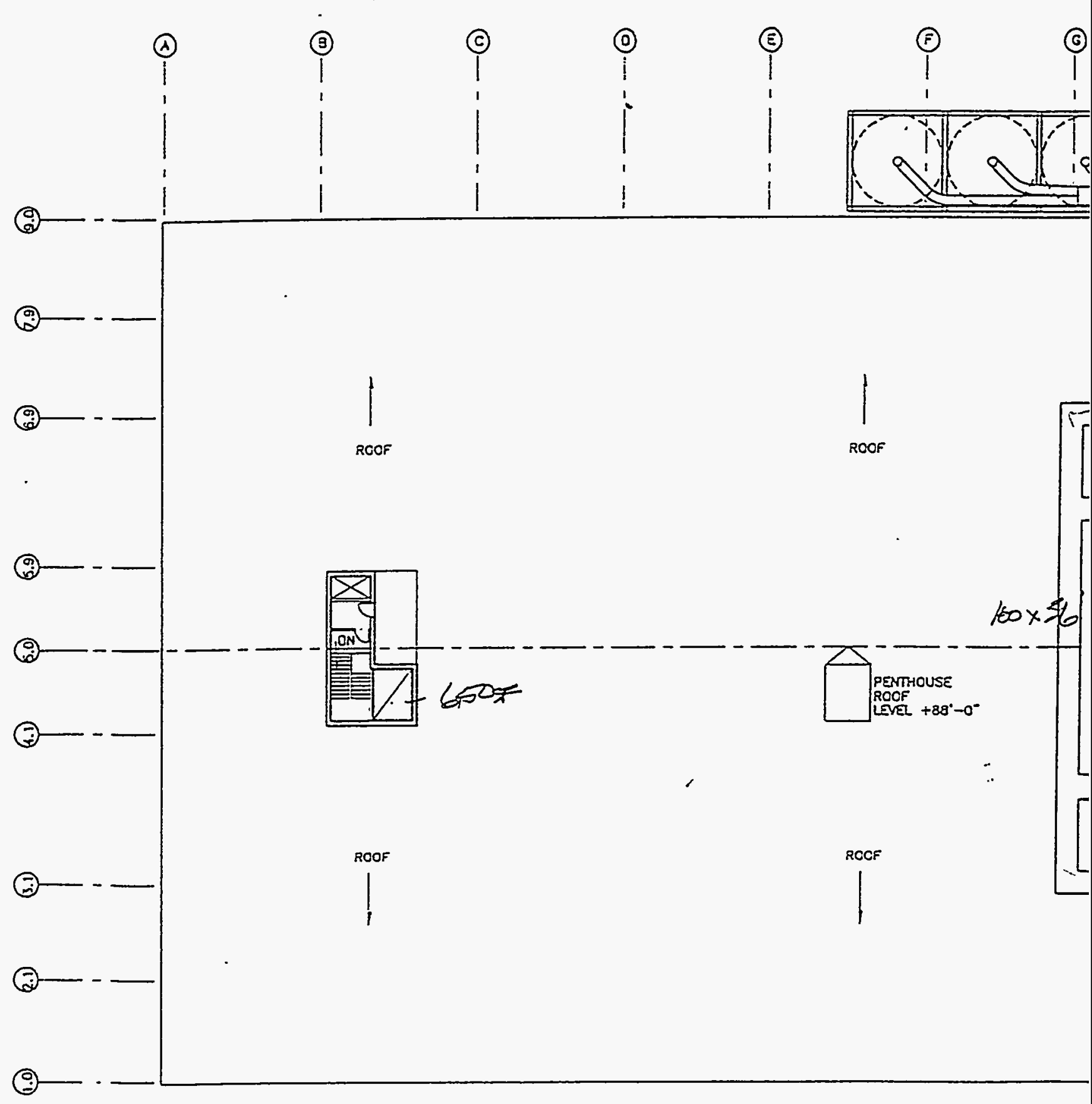

E-391 


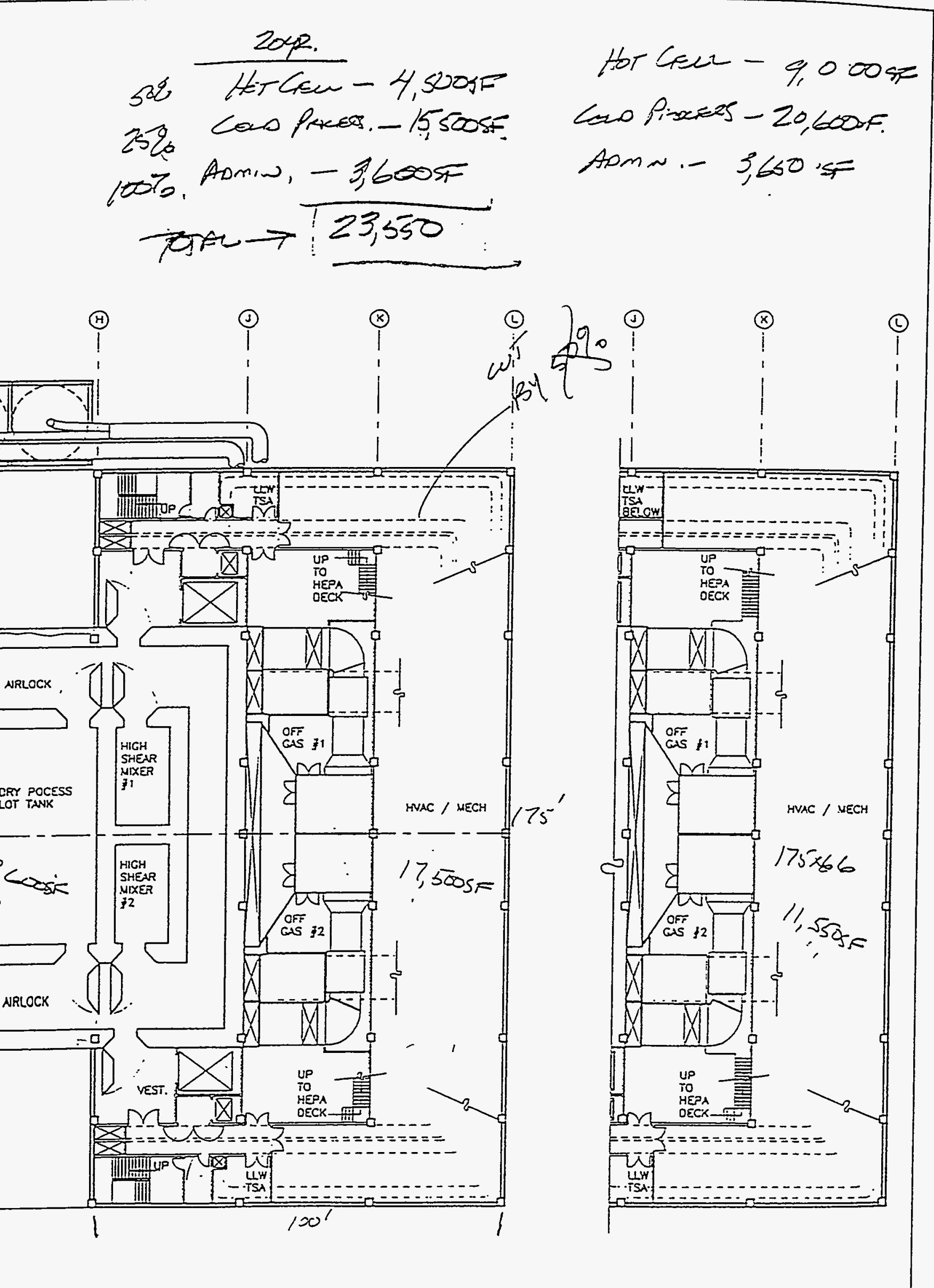




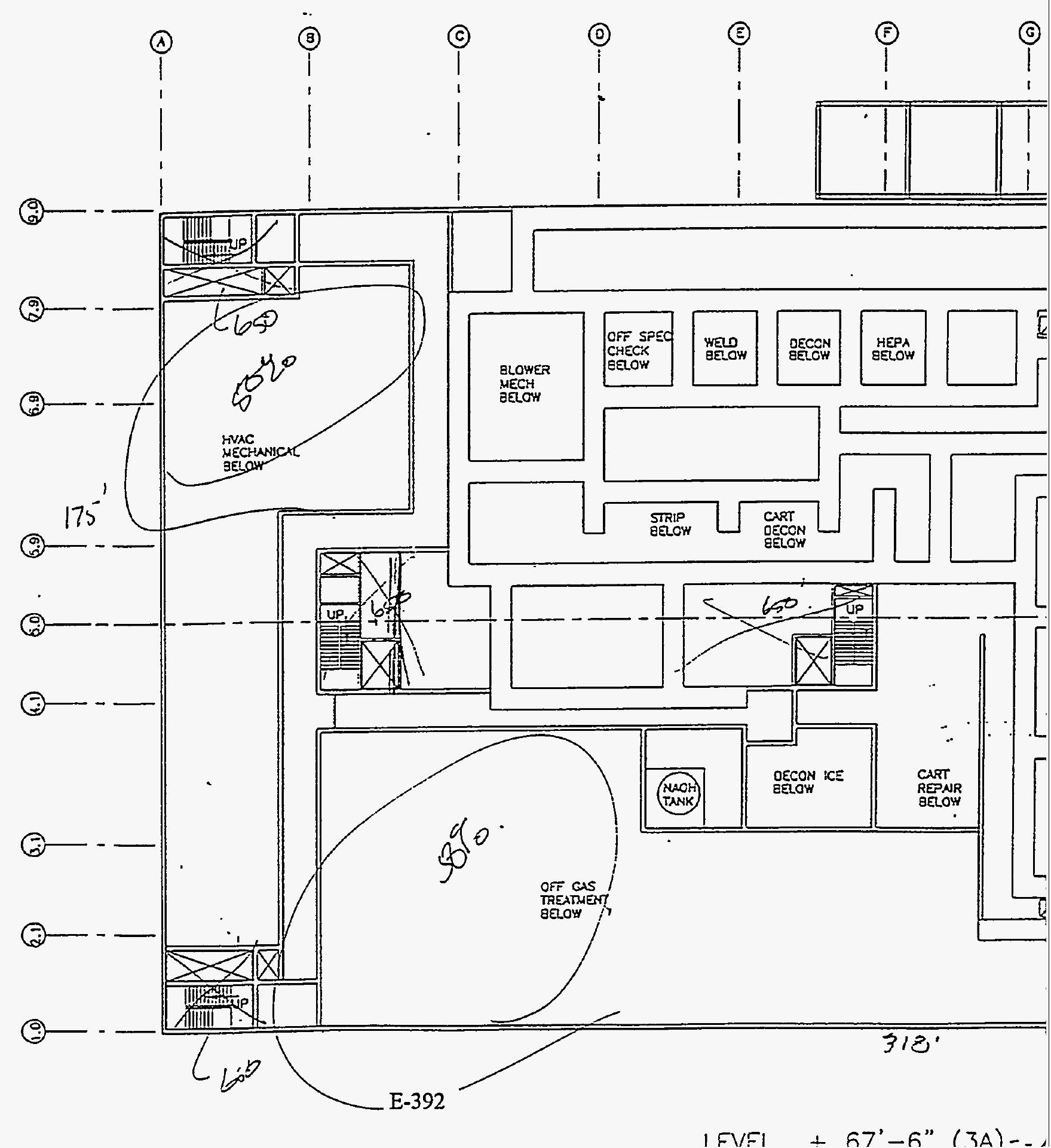




$$
\begin{aligned}
& \text { Horlize - } \phi \\
& \text { Cawpleases - } 9 \\
& \text { Aromin. - 18,000s }
\end{aligned}
$$

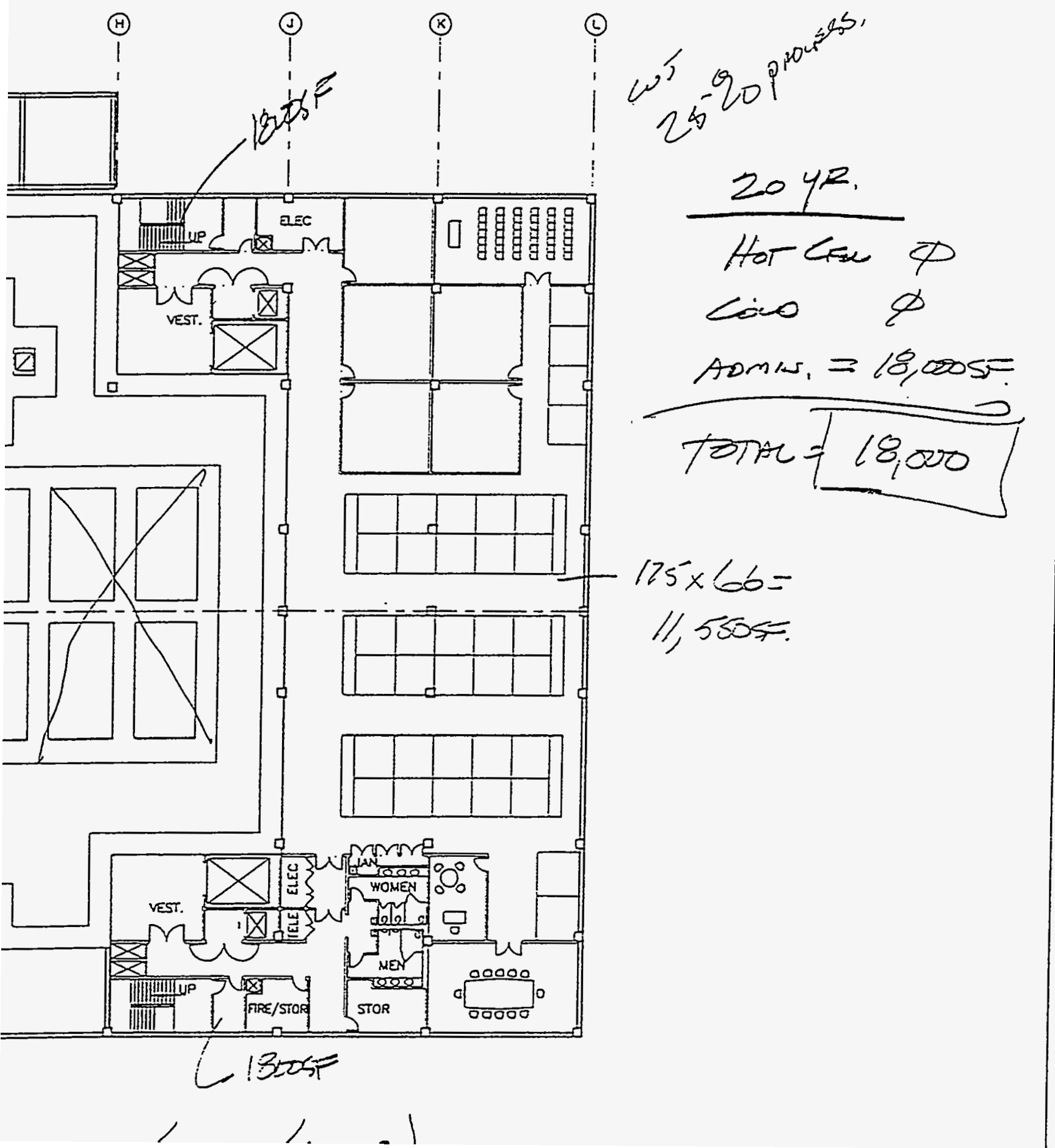




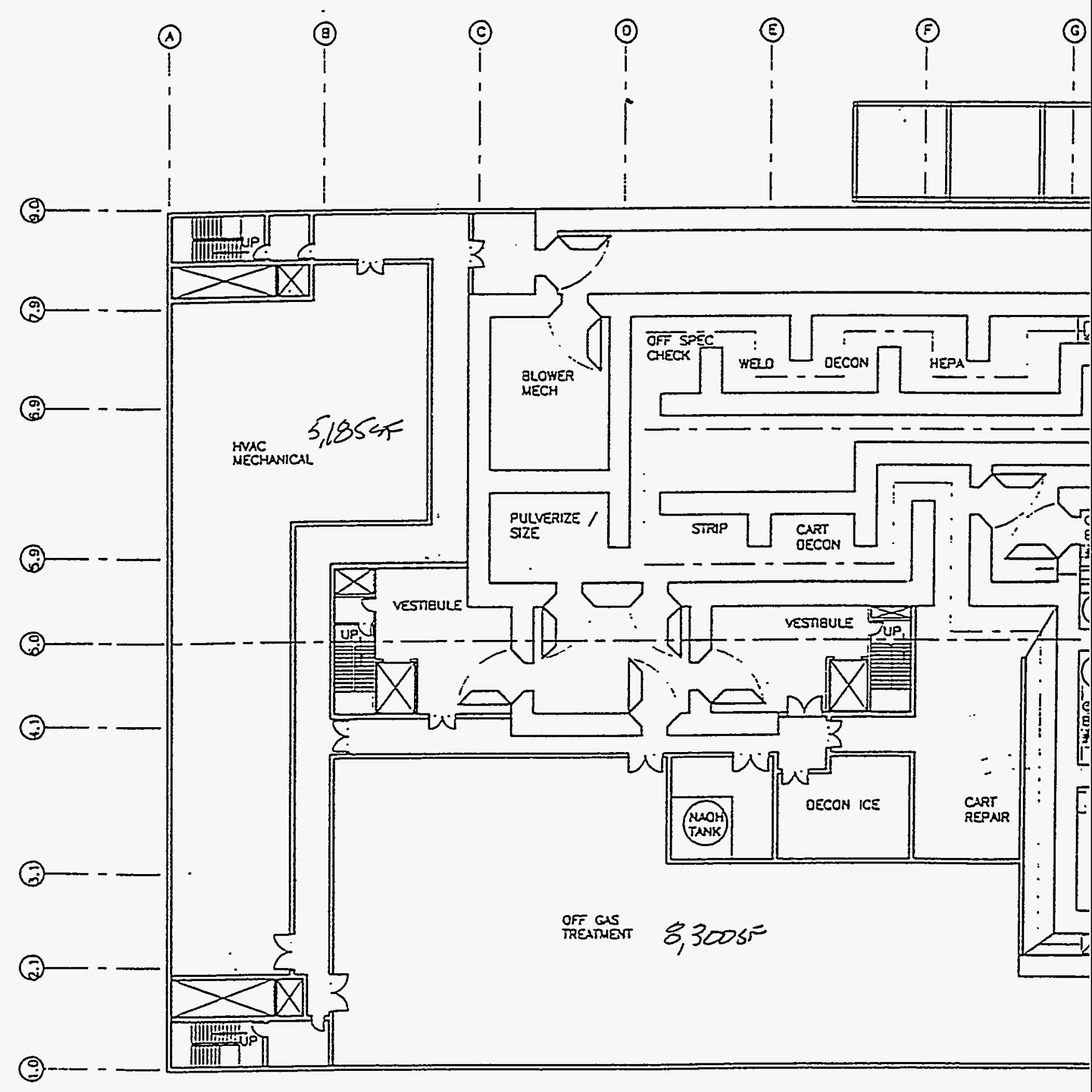

LEVEL $+54 ;-0 "$ (3) $=55$ 


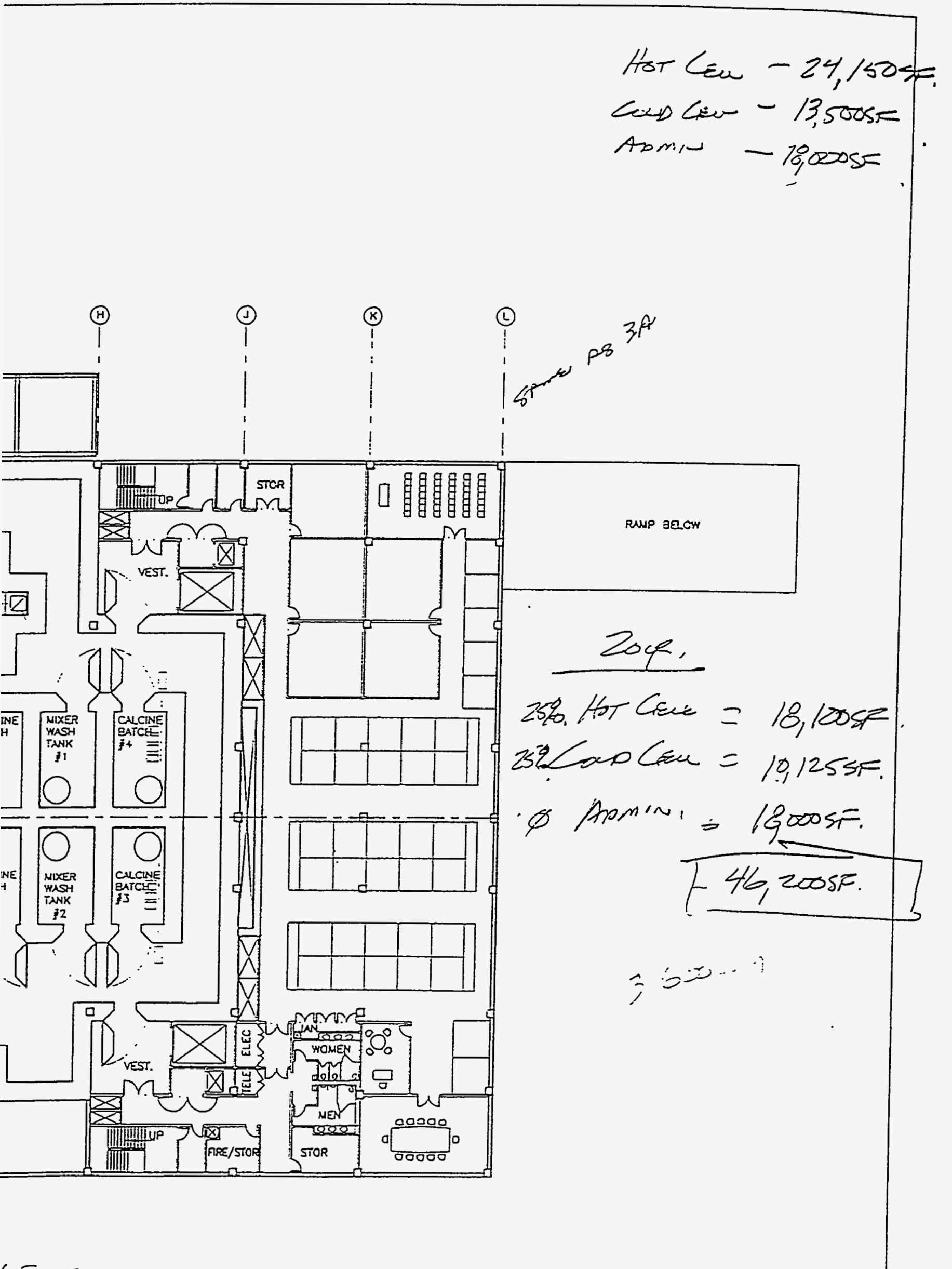




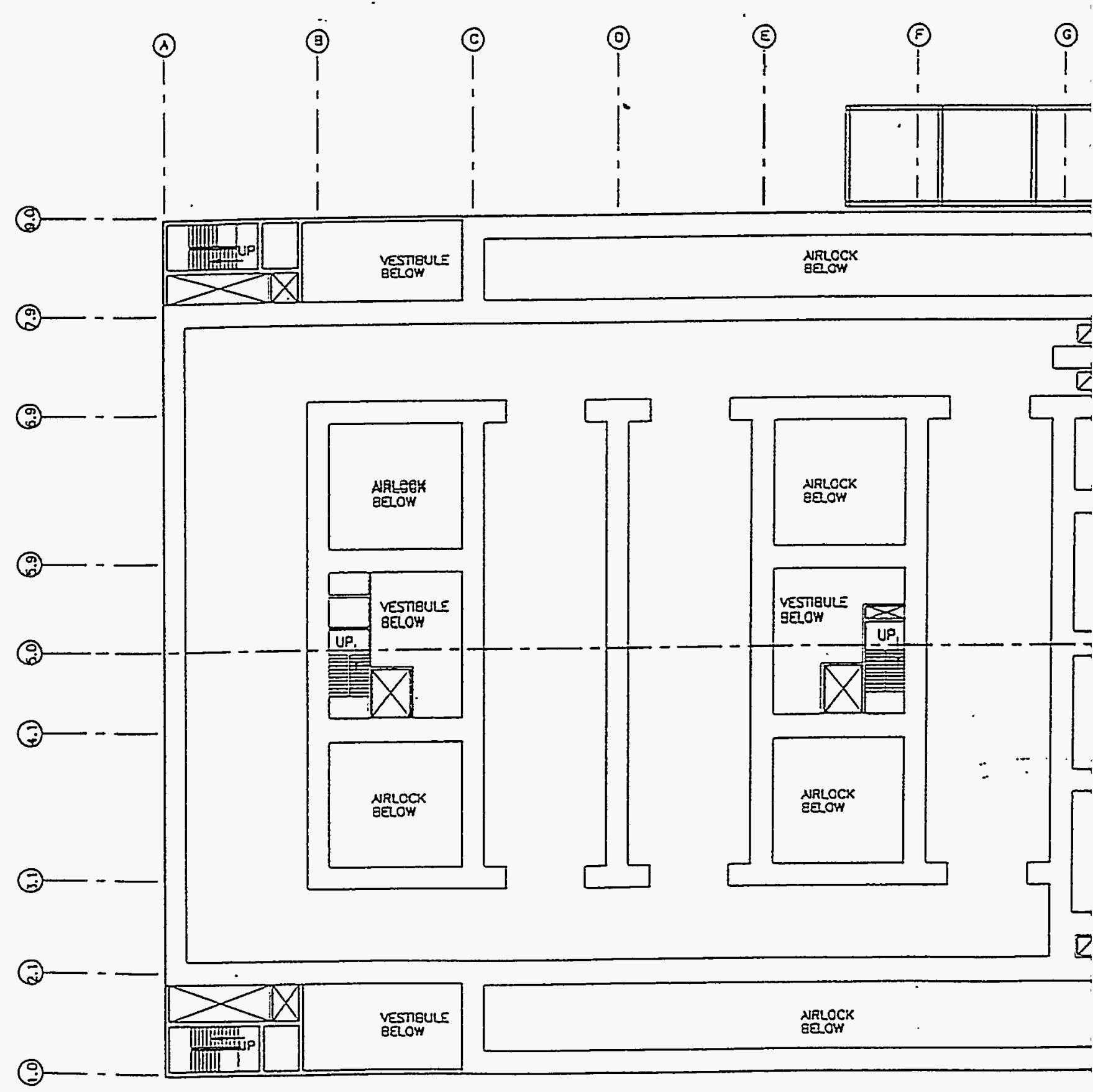

LEVEL $+40^{\prime}-6^{\prime \prime}(2 A)=18$ 


$$
\begin{aligned}
& \text { Hor Geen - } \phi \\
& \text { Canken - } \phi \\
& \text { Atmin - 18, coos: }=
\end{aligned}
$$

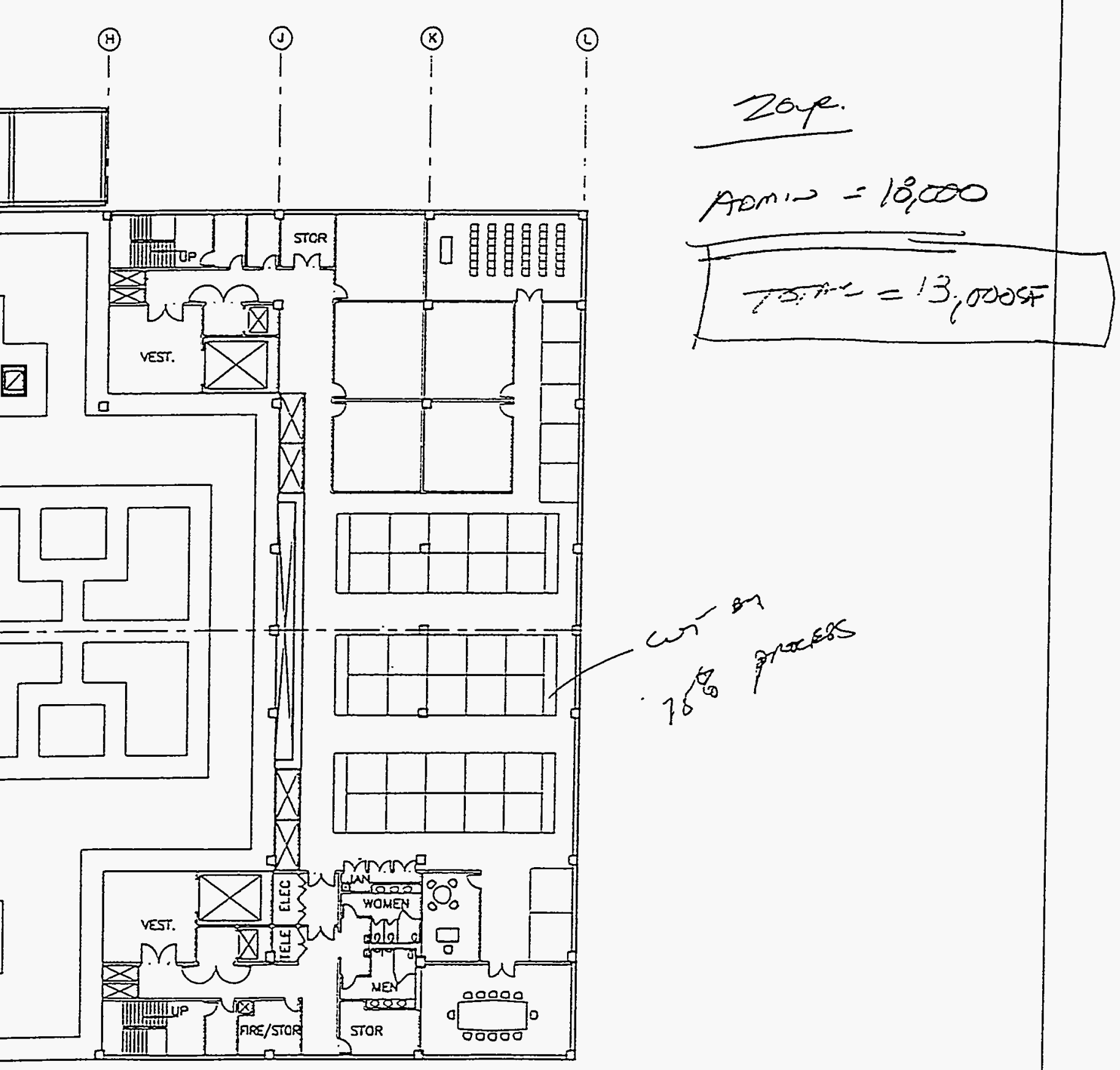




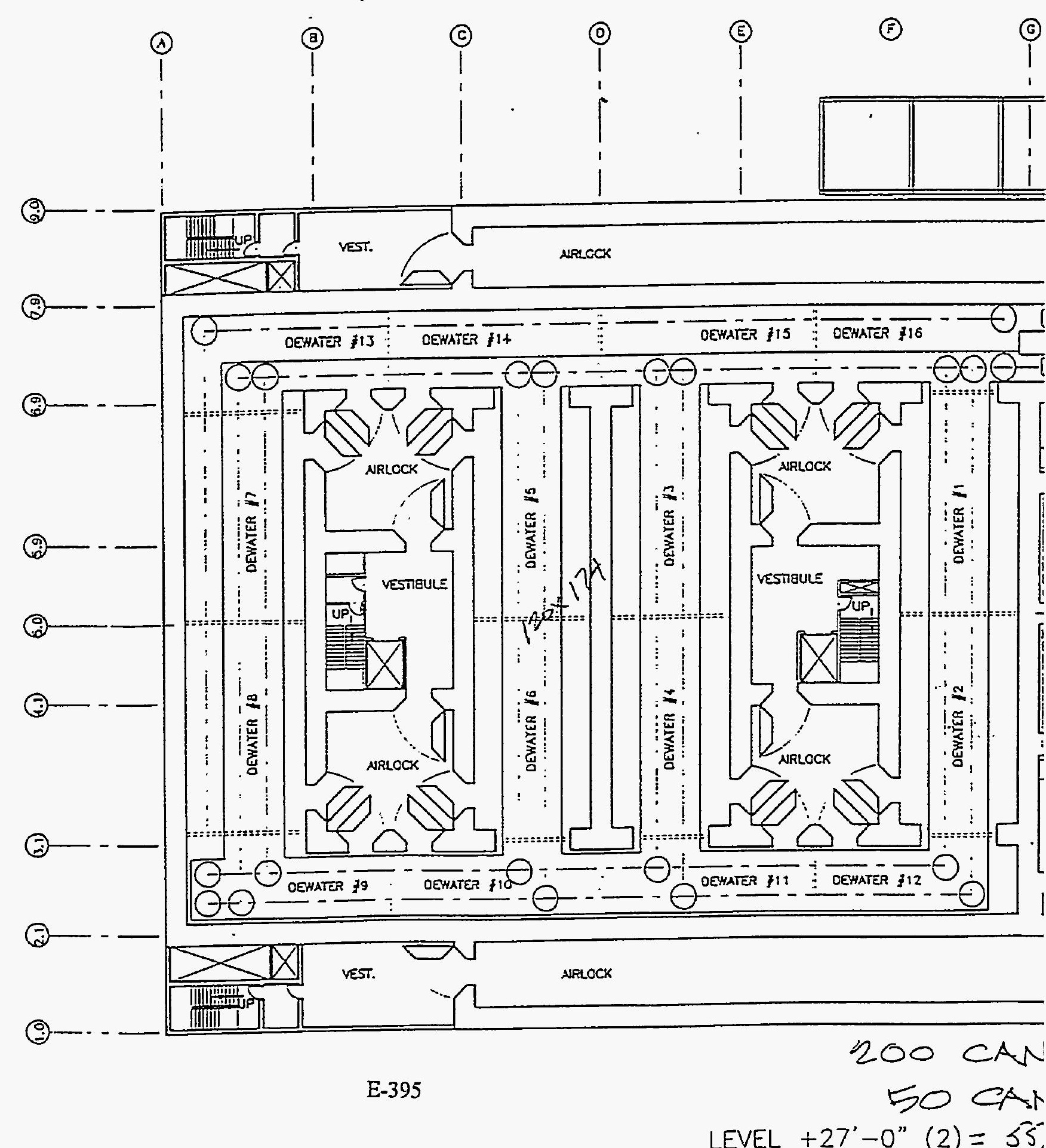




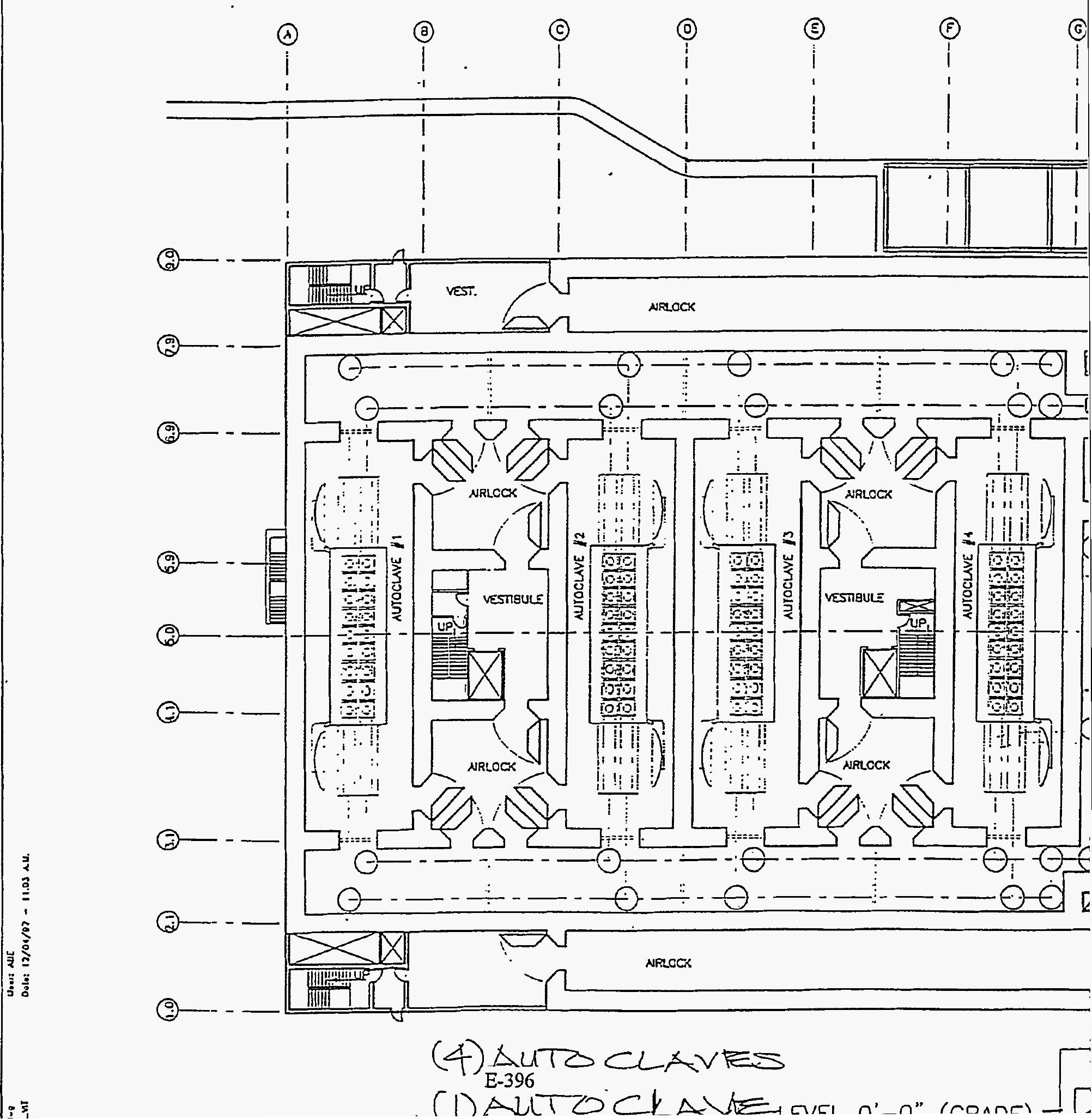




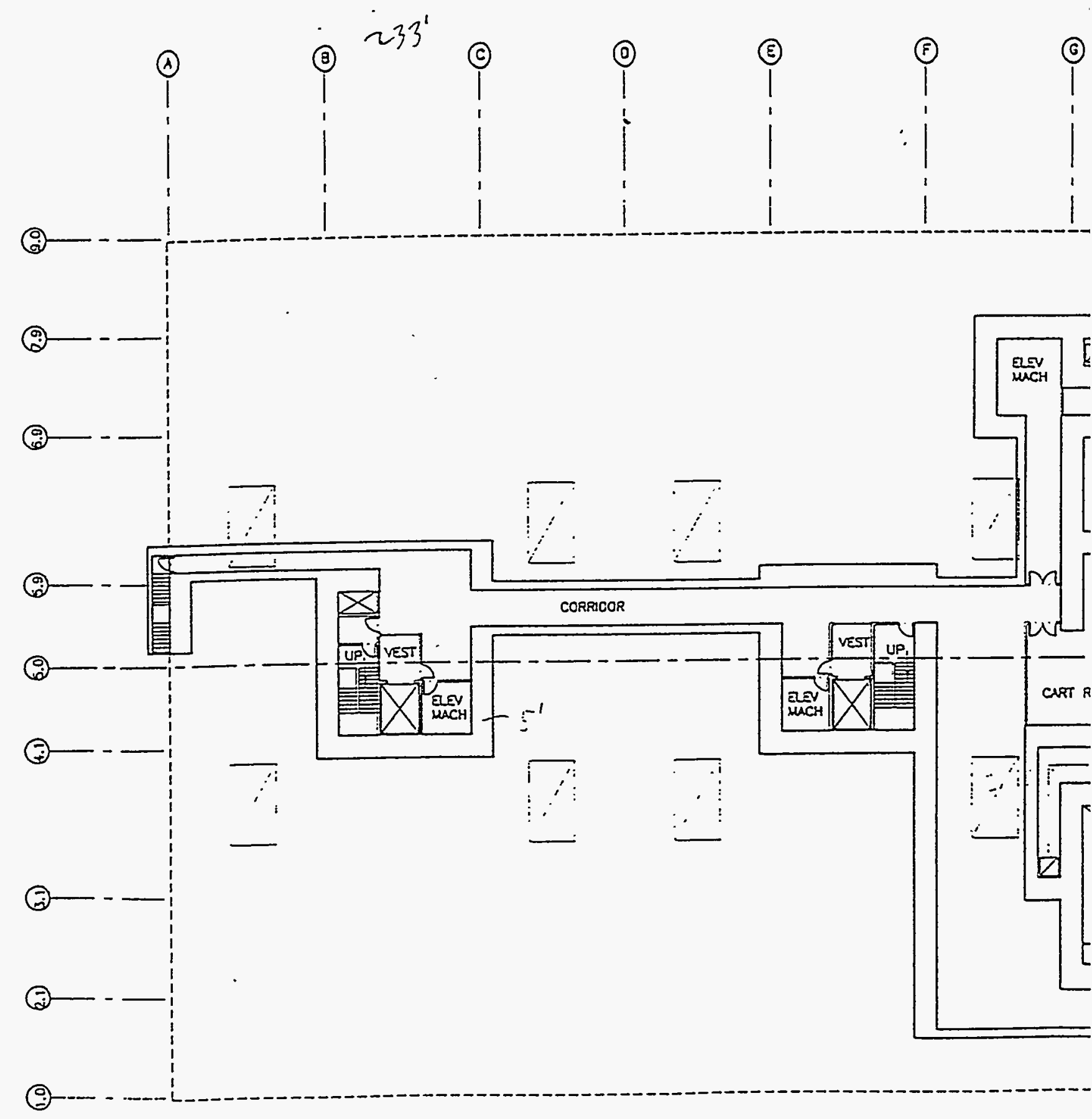




$$
\begin{aligned}
& \text { An, CSes - } \infty \\
& \text { Las prosess - 23.000s = } \\
& \text { fromis - 2,0005. }
\end{aligned}
$$

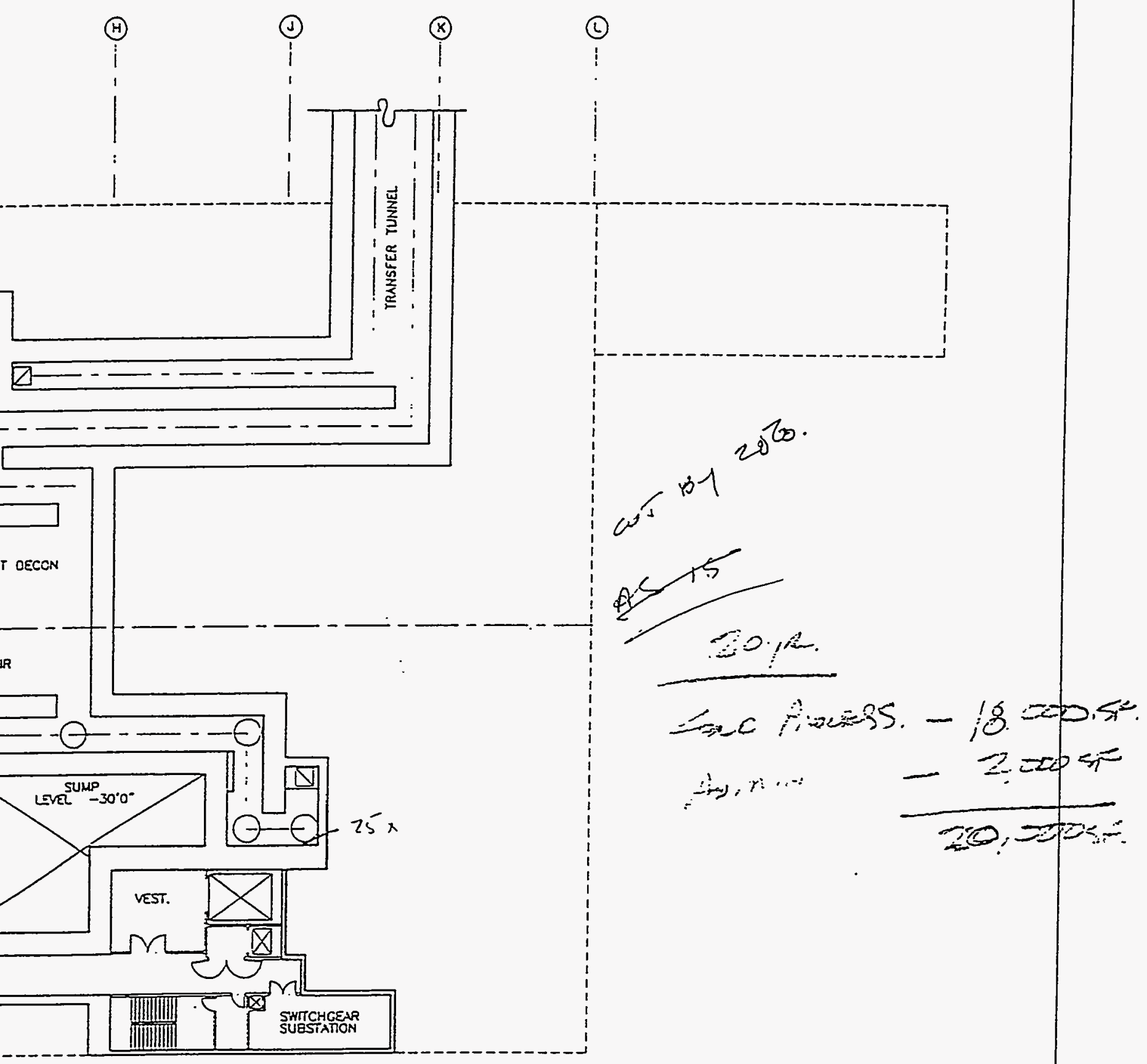




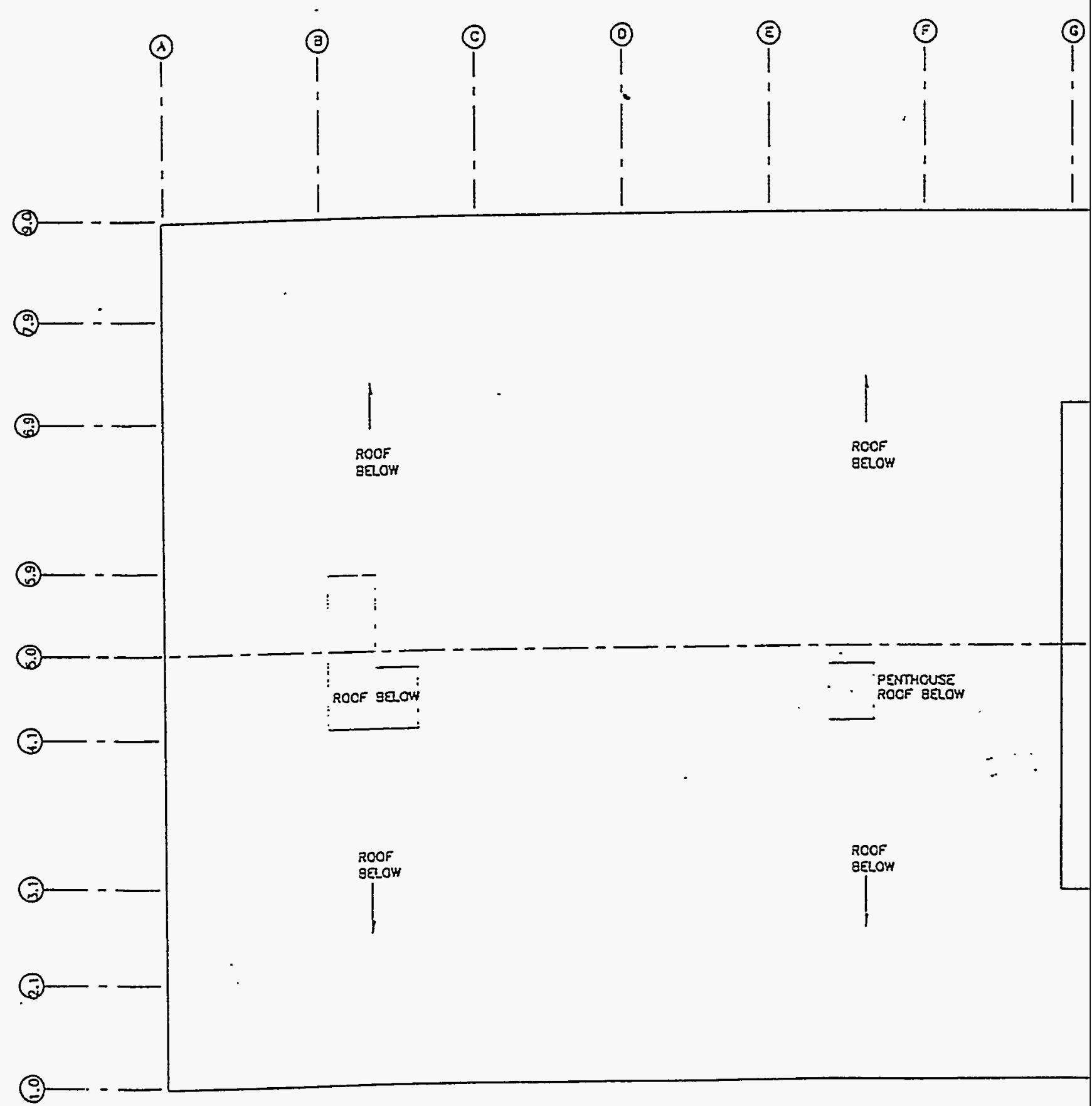




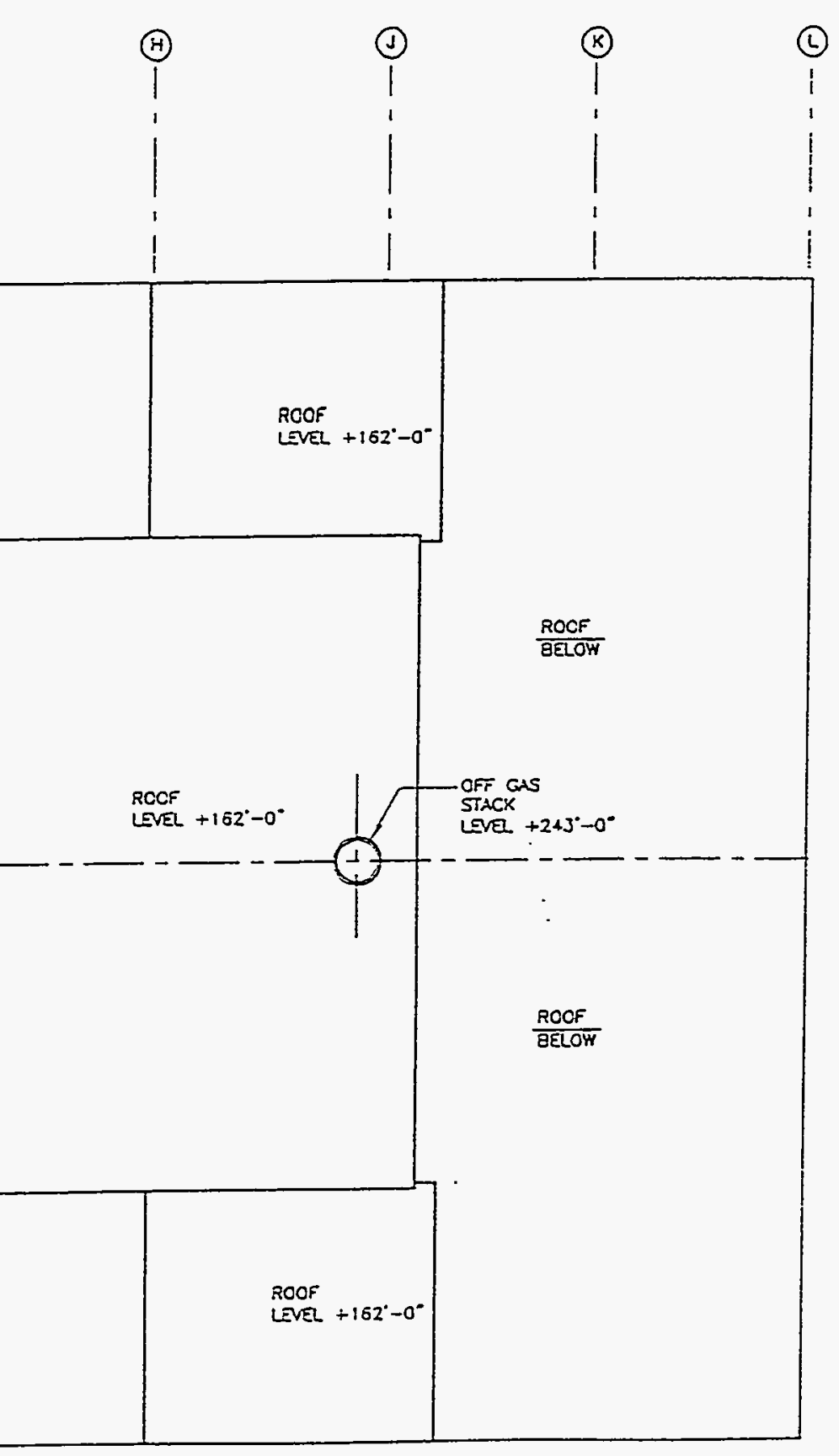




\section{Appendix F}

Risk Assessment Data Sheets 


\section{Risk Assessment - Data Sheet}

Risk Type $O$ Projec OTechnica OESH

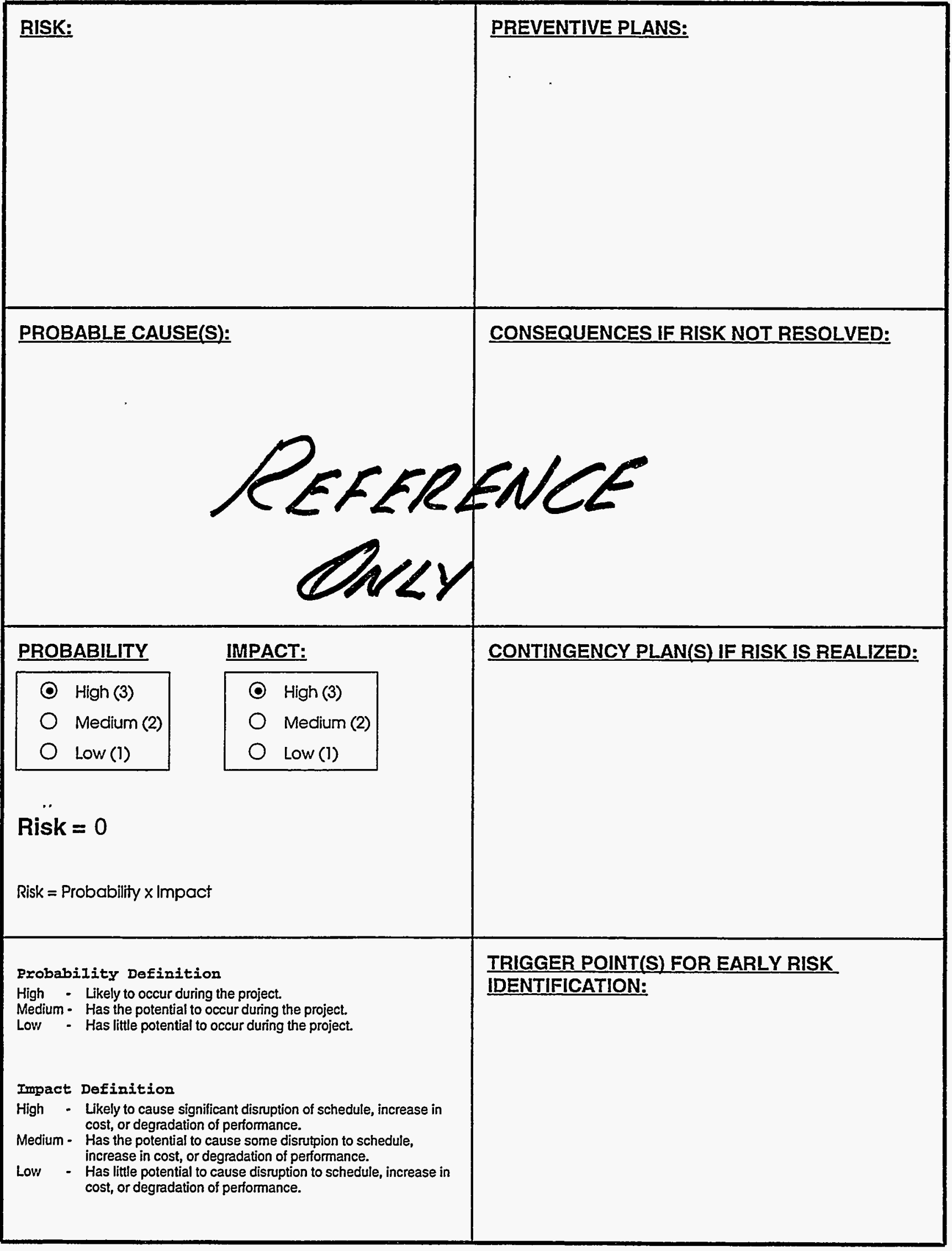




\section{Risk Assessment Data Sheet - Project}

\begin{tabular}{|c|c|}
\hline $\begin{array}{l}\text { RISK: P.1: Regulatory requirements change } \\
\text { Changing regulatory requirements may change CWO } \\
\text { design and delay startup. }\end{array}$ & $\begin{array}{l}\text { PREVENTTVE PLANS: } \\
\text { None }\end{array}$ \\
\hline $\begin{array}{l}\text { PROBABLE CAUSE: } \\
\text { EPA becoming more restrictive in regulation of thermal } \\
\text { treatment devices (incinerators). } \\
\text { Political pressure on permitting process for HLW } \\
\text { disposal sites. }\end{array}$ & $\begin{array}{l}\text { CONSEOUENCES IF RISK NOT RESOLVED: } \\
\text { Funding for title design would likely be delayed. } \\
\text { Design iteration to accommodate changing design requirements } \\
\text { would extend cost and schedule. }\end{array}$ \\
\hline $\begin{array}{l}\text { PROBABILITY: Medium (2) } \\
\text { IMPACT: Medium (2) } \\
\text { RISK: (probability x impact) } 4\end{array}$ & $\begin{array}{l}\text { CONTINGENCY PLAN(S) IF RISK IS } \\
\text { REALIZED: } \\
\text { none } \\
\text { TRIGGER POINT(S) FOR EARLY RISK } \\
\text { IDENTIFICATION: } \\
\begin{array}{l}\text { Input from regulatory oversight organizations (LMTCO, State of } \\
\text { Idaho, EPA Region X, NRC). }\end{array}\end{array}$ \\
\hline $\begin{array}{l}\text { RISK: P.2: Planned space for NWCF additions not } \\
\text { available } \\
\text { The area due east of CPP- } 659 \text { (NWCF) may not be } \\
\text { usable for the additional hot cell space required for } \\
\text { recalcination. Also, the area near the southwest corner of } \\
\text { CPP- } 659 \text { may not be usable for the MACT compliance } \\
\text { building. }\end{array}$ & $\begin{array}{l}\text { PREVENTIVE PLANS: } \\
\text { None }\end{array}$ \\
\hline $\begin{array}{l}\text { PROBABLE CAUSE: } \\
\text { Existing burried utilities in these areas may not be } \\
\text { movable at reasonable cost. } \\
\text { Continued access to other ICPP facilities may prohibit } \\
\text { placing the needed additions in the desired locations. }\end{array}$ & $\begin{array}{l}\text { CONSEQUENCES IF RISK NOT RESOLVED: } \\
\text { Space which is non-adjacent to NWCF would have to be used, } \\
\text { requiring shielded corridors (above or below ground) to link new } \\
\text { facilities with NWCF. Construction and capital costs would } \\
\text { increase. Safety \& environmental hazards would be incurred due } \\
\text { to piped transfer of radioactive waste and offgas between } \\
\text { buildings. }\end{array}$ \\
\hline $\begin{array}{l}\text { PROBABMITY: Low (1) } \\
\text { IMPACT: Medium (2) } \\
\text { RISK: (probability x impact) } 2\end{array}$ & $\begin{array}{l}\text { CONTINGENCY PLAN(S) IF RISK IS } \\
\text { REALIZED: } \\
\text { Identify where the facilities can be placed and redesign (as } \\
\text { necessary) to allow for piped transfer of materials between non- } \\
\text { adjacent processing facilities. }\end{array}$ \\
\hline & $\begin{array}{l}\text { TRIGGER POINT(S) FOR EARLY RISK } \\
\text { IDENTIFICATION: } \\
\text { Evaluation of planned locations for new buildings by ICPP } \\
\text { facilities personnel. }\end{array}$ \\
\hline
\end{tabular}




\section{Risk Assessment Data Sheet - Technical}

\begin{tabular}{l} 
RISK: T.1: Pipe failure from high erosion by \\
slurry \\
Pumping of slurried calcine, containing high \\
concentrations of undissolved solid, may result in rapid \\
erosion and failure of the piping, valves, and nozzles \\
required to transport the slurry from the blending tanks \\
to the NWCF calciner. \\
\hline PROBABLE CAUSE:
\end{tabular}

High concentrations of erosive solids in slurried waste. Inadequate testing/design of the slurry system prior to 'title design.

\section{PREVENTIVE PLANS:}

Early testing and development will be done to evaluate erosion rates, identify erosion-resistant alloys, and optimize the design of the piping system to accommodate the measured erosion rates.

\section{CONSEQUENCES IF RISK NOT RESOLVED:}

Frequent required maintenance/replacement of piping components, or reduced throughput of waste if slurry must be diluted. Either would result in adverse impact to processing schedule.

Alternatively, the slurry system may have to be redesigned and replaced, impacting the cost and schedule for construction \& modification to the NWCF.

\section{CONTINGENCY PLAN(S) IF RISK IS REALIZED:}

Identify alternative materials to replace the problematic piping components.

Increase the dilution of the slurry mixture with water.

Increase frequency of changeout of piping components which are subject to erosion.

\section{TRIGGER POINT(S) FOR EARLY RISK IDENTIFICATION:}

Slurry system erosion measurements during advanced conceptual design. Similar measurements during SO testing of installed system.

\section{PREVENTIVE PLANS:}

RISK: T.2: NWCF won't handle slurried wastes

The NWCF may not accommodate injection of solids in slurried wastes.

Determine whether cold slurried wastes can be processed in a pilot scale fluidized bed calciner, either at ICPP or at a subcontractor's facility.

\section{PROBABLE CAUSE:}

High solids throughput will reduce residence time of solids in calciner. This may limit the effectiveness of the calciner in destroying nitrates, resulting in too much nitrate in the recalcined solids, and/or bed agglomeration due to buildup of alkali nitrates inside the calciner.

Alternatively, the NWCF product takeoff system may not be able to accommodate the higher flow rate of solids from the calciner.

PROBABחLTY: Medium (2)

MMPACT: Medium (2)

RISK: (probability $\mathrm{x}$ impact) 4

\section{CONSEQUENCES IF RISK NOT RESOLVED:}

Frequent required shutdown and dissolution of calciner bed, resulting in adverse impacts to throughput and operational costs.

\section{CONTINGENCY PLAN(S) IF RISK IS} REALIZED:

Increase slurry dilution with water to reduce the solids concentration.

Increase calciner operating temperature to enhance thermal 


\begin{tabular}{|c|c|}
\hline & destruction of nitrates. \\
\hline & $\begin{array}{l}\text { TRIGGER POINT(S) FOR EARLY RISK } \\
\text { IDENTIFICATION: } \\
\text { Pilot scale testing of fluidized bed calcination of high-solids slurry } \\
\text { mixtures. }\end{array}$ \\
\hline $\begin{array}{l}\text { RISK: T.3: Low nitrate destruction in calciner } \\
\text { A high ( }>90 \% \text { ) destruction of nitrates in the slurried } \\
\text { wastes may not be achieved during recalcination. }\end{array}$ & $\begin{array}{l}\text { PREVENTIVE PLANS: } \\
\text { Evaluate effectiveness of sugar in reducing nitrates in } \\
\text { representative pilot scale tests. }\end{array}$ \\
\hline $\begin{array}{l}\text { PROBABLE CAUSE: } \\
\text { High solids throughput will reduce residence time of } \\
\text { solids in calciner. This may limit the effectiveness of the } \\
\text { calciner in destroying nitrates. } \\
\text { Alternatively, the high solids concentration may catalyze } \\
\text { preferential oxidation of sugar with oxygen from } \\
\text { fluidizing air, rather than from nitrates. }\end{array}$ & $\begin{array}{l}\text { CONSEQUENCES IF RISK NOT RESOLVED: } \\
\text { If nitrates accumulate and cause bed agglomeration, frequent } \\
\text { shutdown and dissolution of calciner bed, or increased slurry } \\
\text { dilution with water would be required, resulting in adverse } \\
\text { impacts to throughput and operational costs. } \\
\text { If high solids throughput inhibits bed agglomeration, but grout is } \\
\text { not tolerant of nitrates, an alternative denitration scheme (e.g., } \\
\text { elevated calciner temperature) would be required. } \\
\text { If high solids throughput inhibits bed agglomeration, and grout is } \\
\text { tolerant of nitrates, there would be little if any consequence. }\end{array}$ \\
\hline $\begin{array}{l}\text { PROBABMITY: Low (1) } \\
\text { IMPACT: Medium (2) } \\
\text { RISK: (probability x impact) } 2\end{array}$ & $\begin{array}{l}\text { CONTINGENCY PLAN(S) IF RISK IS } \\
\text { REALIZED: } \\
\text { Increase slurry dilution with water to reduce the solids } \\
\text { concentration. } \\
\text { Increase calciner operating temperature to enhance thermal } \\
\text { destruction of nitrates. }\end{array}$ \\
\hline & $\begin{array}{l}\text { TRIGGER POINT(S) FOR EARLY RISK } \\
\text { IDENTIFICATION: } \\
\text { Pilot scale testing of fluidized bed calcination of high-solids slurry } \\
\text { mixtures using sugar as a reducing agent. }\end{array}$ \\
\hline $\begin{array}{l}\text { RISK: T.4: Inadequate blending of calcines } \\
\text { Optimal calcine blends may not be achieved due to } \\
\text { calcine retrieval difficulties. }\end{array}$ & $\begin{array}{l}\text { PREVENTIVE PLANS: } \\
\text { Perform laboratory tests to develop acceptable grouting recipes for } \\
\text { all calcine types in the bins. Optimize these recipes for robustness } \\
\text { to variations in bulk calcine composition. }\end{array}$ \\
\hline $\begin{array}{l}\text { PROBABLE CAUSE: } \\
\text { Stratification/segregation of calcine types in bins. } \\
\text { Too many different types of calcine in bins, coupled with } \\
\text { too few extraction points. } \\
\text { Grouting method may not be sufficiently robust to } \\
\text { accommodate large variations in calcine composition due } \\
\text { to imperfect blending. }\end{array}$ & $\begin{array}{l}\text { CONSEQUENCES IF RISK NOT RESOLVED: } \\
\text { A larger number of grout recipes will have to be developed, and } \\
\text { each bin of accumulated recalcine solids will have to be } \\
\text { characterized for composition prior to grouting. The grout recipe } \\
\text { will then be tailored to each bin of recalcine. }\end{array}$ \\
\hline $\begin{array}{l}\text { PROBABILITY: High (3) } \\
\text { MMPACT: Low (1) } \\
\text { RISK: (probability x impact) } 3\end{array}$ & $\begin{array}{l}\text { CONTINGENCY PLAN(S) IF RISK IS } \\
\text { REALIZED: } \\
\text { Develop suitable grouting recipes for entire spectrum of calcines } \\
\text { and blends. In conjunction with this, develop real time (or near } \\
\text { real time) remote methods for characterizing calcine (e.g., X-ray }\end{array}$ \\
\hline
\end{tabular}




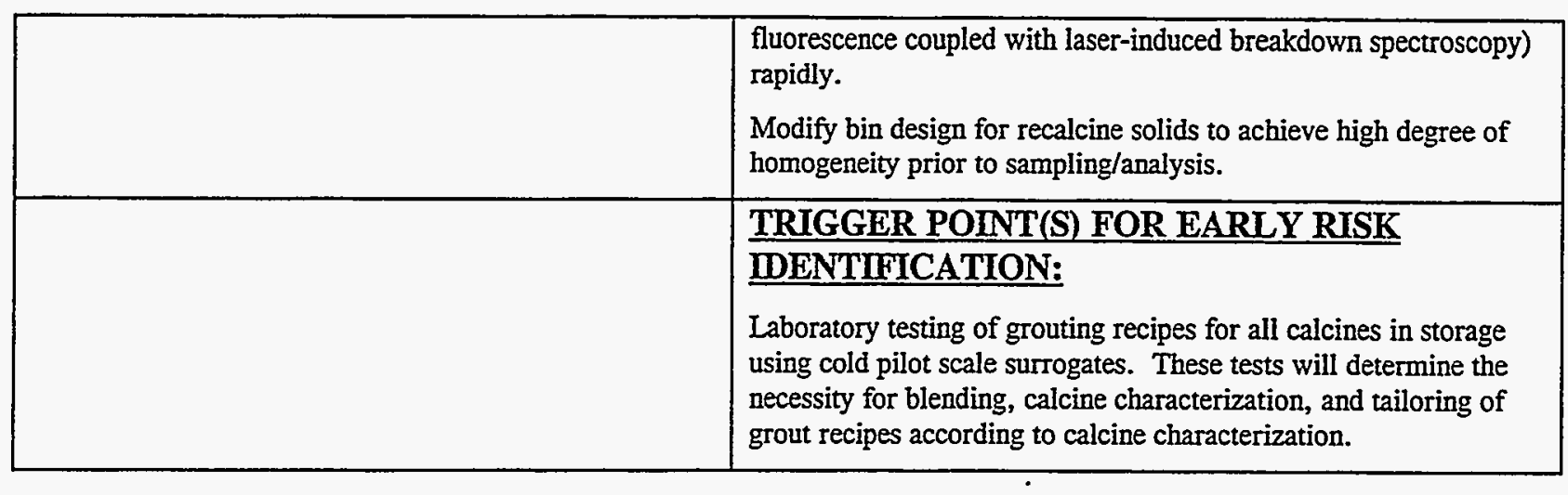




\section{Risk Assessment Data Sheet - ES\&H}

\section{RISK: ESH.1: Perceived safety hazard of sugar+nitrates \\ Safety experts may claim that the potential to form explosive nitrated organics from sugar and dissolved nitrates during recalcination cannot be disproven. \\ PROBABLE CAUSE: \\ Based on investigations to date, the risk is probably not real for the proposed process with ICPP waste. \\ However, nitrated organic explosions have occurred in other nuclear facilities. This being the case, absolute proof that nitration of sugars cannot occur may be very difficult to obtain due to the large number of tests, analyses, etc. that would likely be required by safety oversight organizations.}

\section{PREVENTIVE PLANS:}

Testing has already been done to show that calcines from sugar calcination are non-reactive. In addition, proposed NWCF modifications include a digestion tank to consume all residual hydrocarbons in scrub with nitric acid. Scrub would be sampled and analyzed to show that organics are not present prior to recycle to tank farm.

\section{CONSEOUENCES IF RISK NOT RESOLVED:}

A long and costly research program may be required to show conclusively that nitrated organics are not formed in any portion of the slurry recalcination process.

Barring conclusive proof that nitration of sugar cannot occur, sugar calcination would not be allowed in the NWCF calciner. An alternative (such as high temperature calcination) would have to be used to destroy nitrates. In this case the only consequences would be continued emission of $\mathrm{NO}_{2}$ from NWCF during recalcination, and some costs associated with retrofit of calciner piping to accommodate higher temperature operation.

\section{PROBABILITY: High (3)}

IMPACT: Low (1)

RISK: (probability $x$ impact) 3

\section{CONTINGENCY PLAN(S) IF RISK IS}

\section{REALIZED:}

Operate calciner at elevated temperature without using sugar as a reducing agent.

\section{TRIGGER POINT(S) FOR EARLY RISK IENTIFICATION:}

Early discussions with safety oversight personnel (e.g., Defense Nuclear Facilities Safety Board) will be initiated to determine early on whether sugar addition should be pursued.

\section{RISK: ESH.2: Leakage from recalcine transport} line

Because of the distance from the NWCF to the proposed grouting facility location, a pressurized pneumatic transport line is proposed. However, use of a pressurized line (as opposed to a vacuum system) involves risk of leakage, environmental contamination, and exposure to workers if a breach occurs.

\section{PROBABLE CAUSE:}

The risk is due to the fact that if a breach occurs in a pressurized pneumatic transport line, the air in the line would flow out, taking the radioactive solids with it. By comparison, if a breach occurs in a vacuum transport system, the air outside the line would flow in, confining the radioactive solids inside the line.

PROBABILITY: Medium (2)

IMPACT: Low (1)

RISK: (probability $\mathrm{x}$ impact) 2

\section{PREVENTIVE PLANS:}

Because of the higher air density at higher pressure, the required air velocity in the line will be lower than with a vacuum system. Thus, the potential for failure should be lower. In addition, most (if not all) past transport system line failures have occurred at cyclones or bends in the line. These "pressure points" can be designed to accommodate additional erosion without failing.

\section{CONSEQUENCES IF RISK NOT RESOLVED:}

If a breach occurs, the secondary containment line will be contaminated. To repair the breached transport line, a temporary containment structure would be required, and the secondary would have be thoroughly decontaminated. This would be more costly than repair of a breached vacuum line.

\section{CONTINGENCX PLAN(S) IF RISK IS REALIZED:}

Any transport line (vacuum or pressurized) will be contained within a secondary line. By making the secondary line a vacuum system, and monitoring the air in the line for radioactivity, any 


\begin{tabular}{|c|c|}
\hline & $\begin{array}{l}\text { breach in the primary line could be controlled and contained } \\
\text { without external contamination. The breach would be repaired } \\
\text { (possibly at considerable cost) as described above under } \\
\text { "Consequences". }\end{array}$ \\
\hline & $\begin{array}{l}\text { TRIGGER POINT(S) FOR EARLY RISK } \\
\text { DENTIFICATION: } \\
\text { Development testing of the proposed calcine transport system } \\
\text { during advanced conceptual design or title design will include } \\
\text { erosion testing. Data from these tests should indicate whether } \\
\text { suitable design allowances can be made to mitigate the risk. }\end{array}$ \\
\hline $\begin{array}{l}\text { RISK: ESH.3: High radiation exposure due to } \\
\text { frequent equipment failures } \\
\text { Because mechanical equipment is required to blend and } \\
\text { pump high-solids slurries, frequent maintenance and } \\
\text { changeout of equipment may be required, resulting in } \\
\text { high radiation exposure to workers. }\end{array}$ & $\begin{array}{l}\text { PREVENTIVE PLANS: } \\
\text { Components likely to require frequent changeout will be identified } \\
\text { during development testing and design measures will be taken to } \\
\text { place them in locations (in some cases in separate cells) which can } \\
\text { be decontaminated, shielded, and accessed. }\end{array}$ \\
\hline $\begin{array}{l}\text { PROBABLE CAUSE: } \\
\text { Pumping of high-solids slurries is potentially problematic } \\
\text { due to the erosiveness of such mixtures, and their } \\
\text { tendency to plug. }\end{array}$ & $\begin{array}{l}\text { CONSEQUENCES IF RISK NOT RESOLVED: } \\
\text { Higher radiation exposures of workers than is typical of NWCF } \\
\text { operation will likely be experienced. } \\
\text { Alternatively, if it is determined that higher rad exposures are not } \\
\text { acceptable, the NWCF addition will be redesigned for total remote } \\
\text { operation and maintenance. This would have a significant adverse } \\
\text { impact on cost, and possibly a minor impact on schedule. }\end{array}$ \\
\hline $\begin{array}{l}\text { PROBABILITY: Medium (2) } \\
\text { IMPACT: Medium (2) } \\
\text { RUSK: (probability } x \text { impact) } 4\end{array}$ & $\begin{array}{l}\text { CONTINGENCY PLAN(S) IF RISK IS } \\
\text { REALIZED: } \\
\text { The design of the hot cells housing the new systems includes } \\
\text { substantial shielding and provision for extensive decontamination. } \\
\text { Radiation exposure to workers will thus be minimized. } \\
\text { Alternatively, the new hot cells will be redesigned for total remote } \\
\text { operation and maintenance. }\end{array}$ \\
\hline 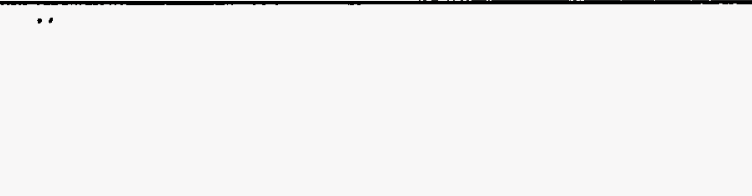 & $\begin{array}{l}\text { TRIGGER POINT(S) FOR EARLY RISK } \\
\text { DEENTIFICATION: } \\
\text { Development testing of the slurry pumping systems should indicate } \\
\text { whether major corrective action is needed. }\end{array}$ \\
\hline
\end{tabular}


Appendix G

Background Information for Project Data Sheets 


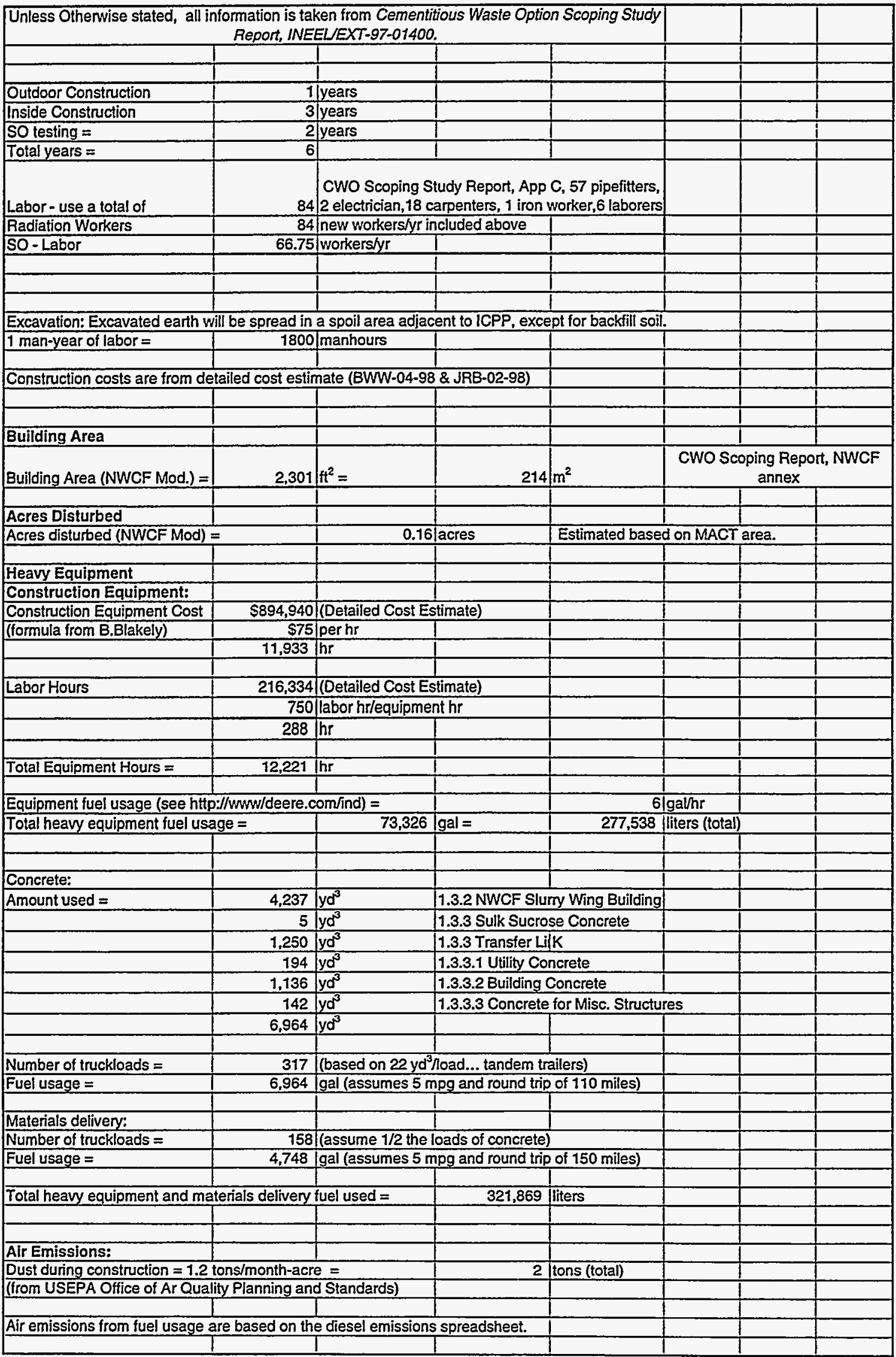




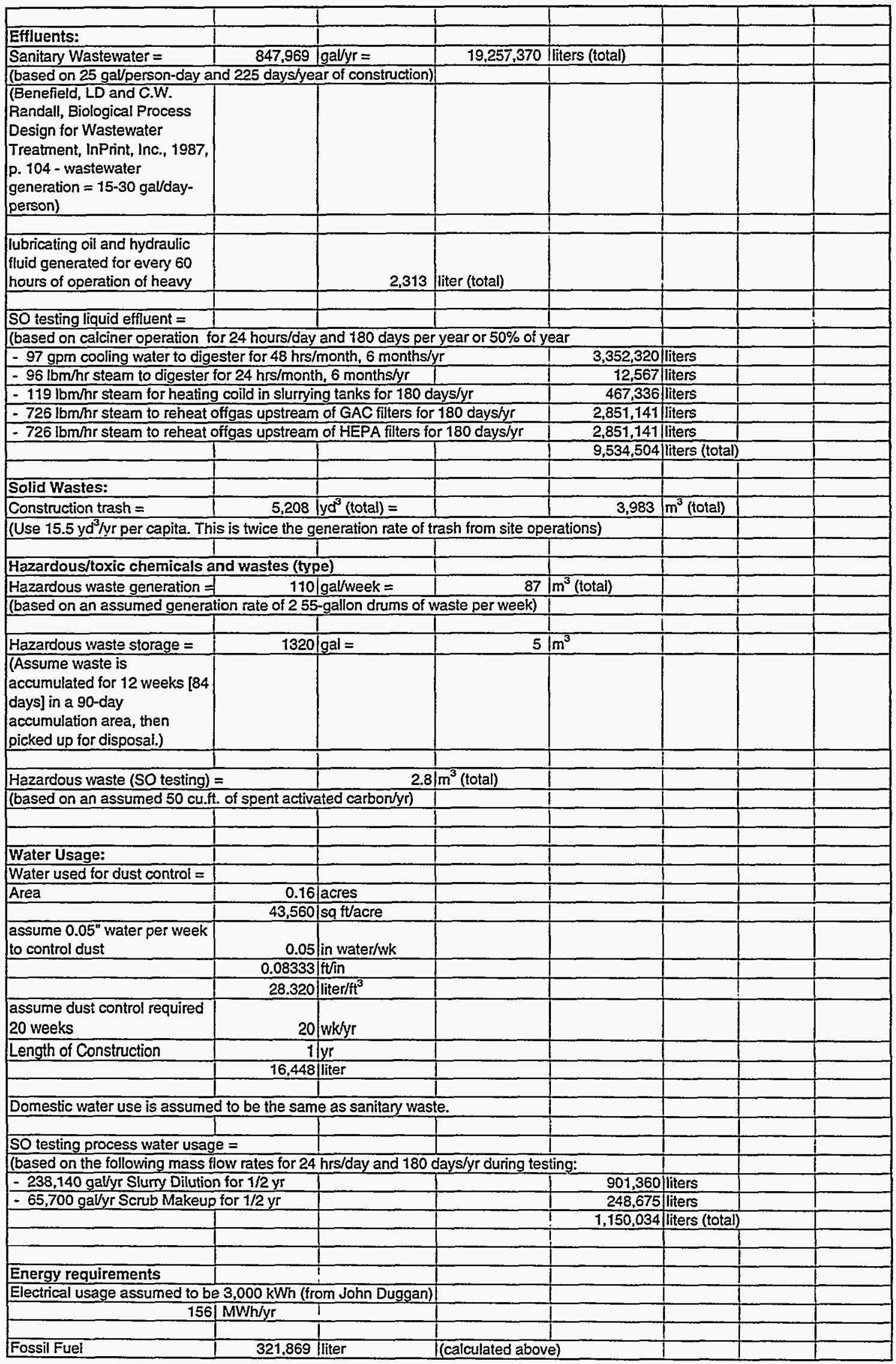




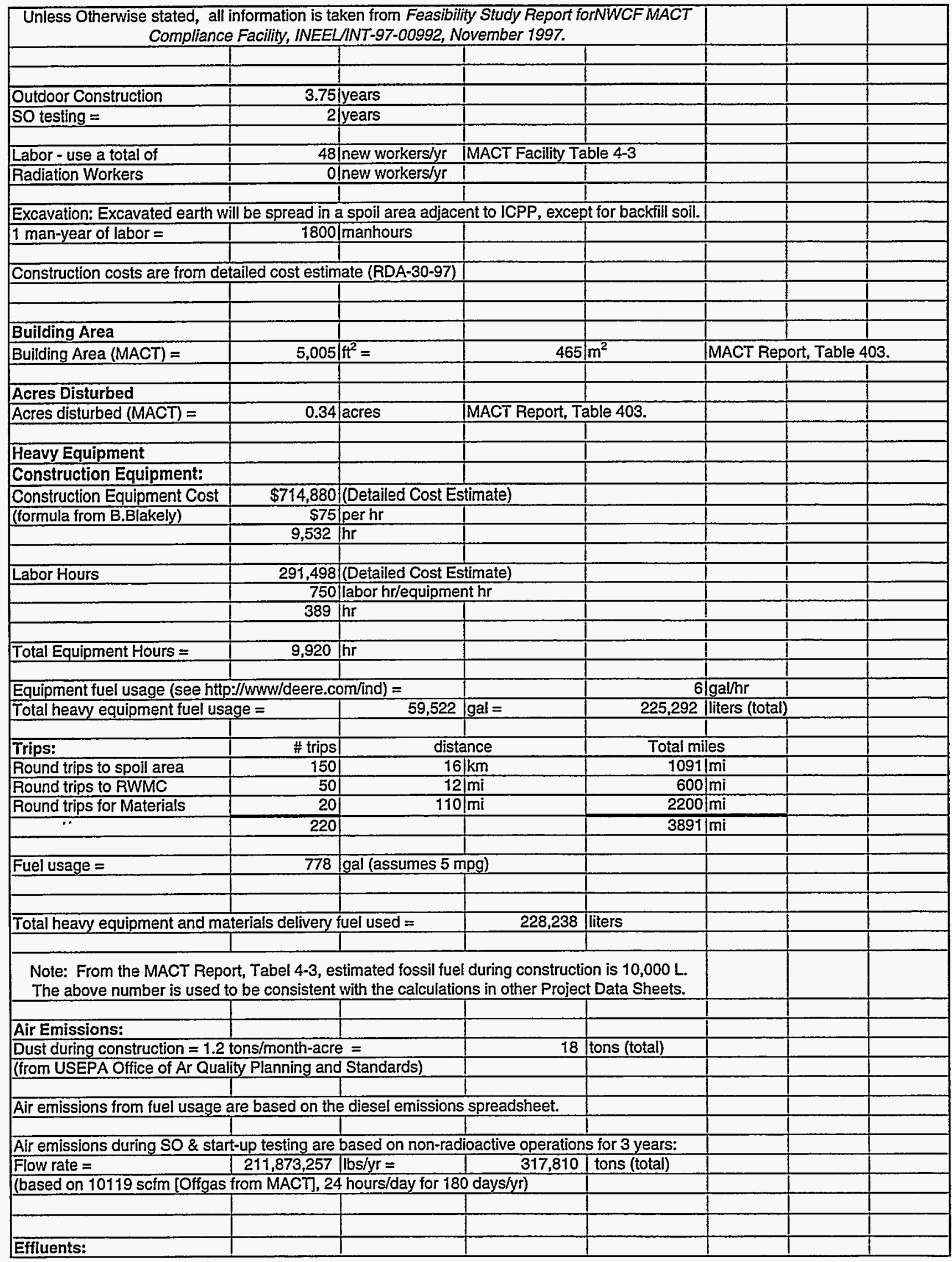




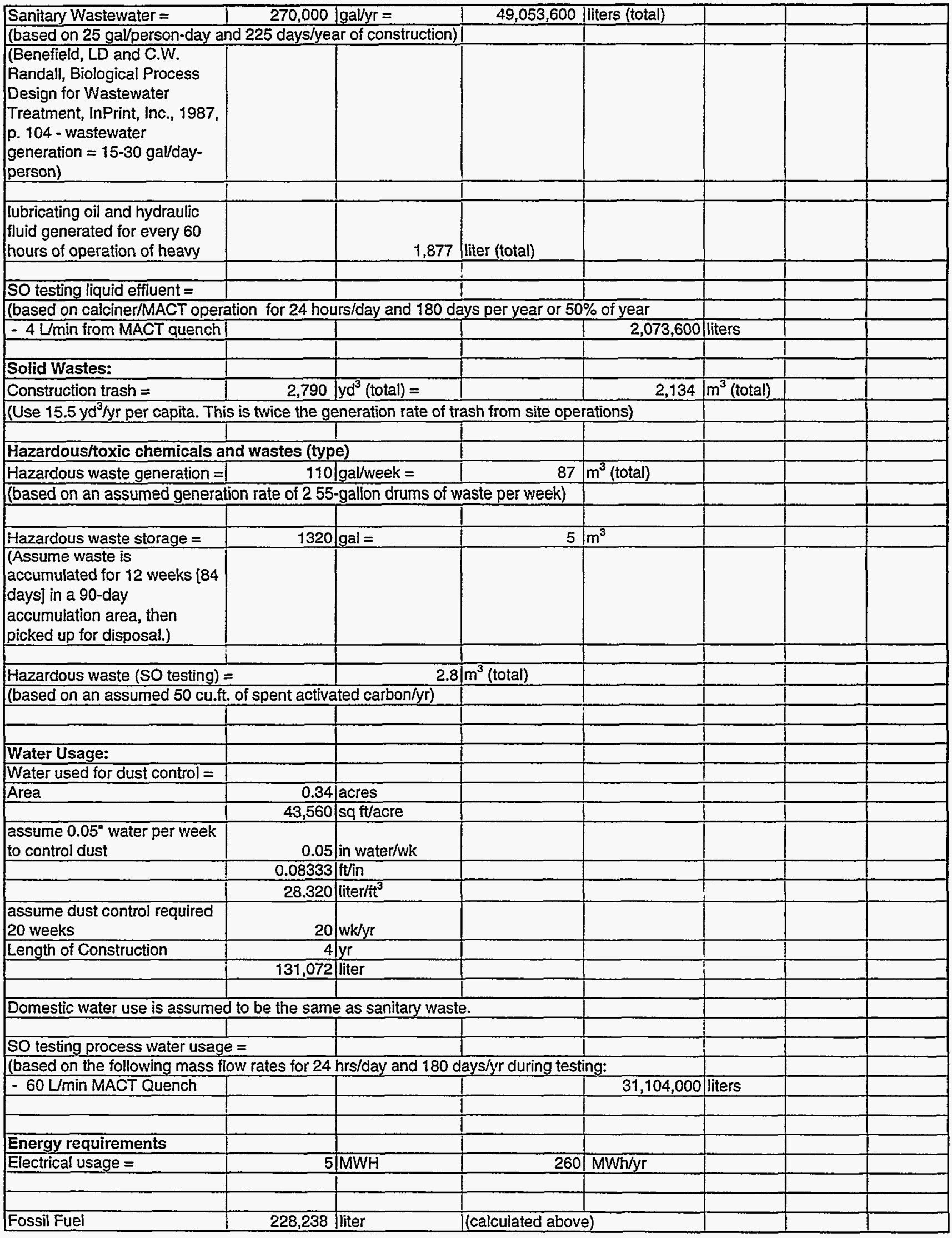




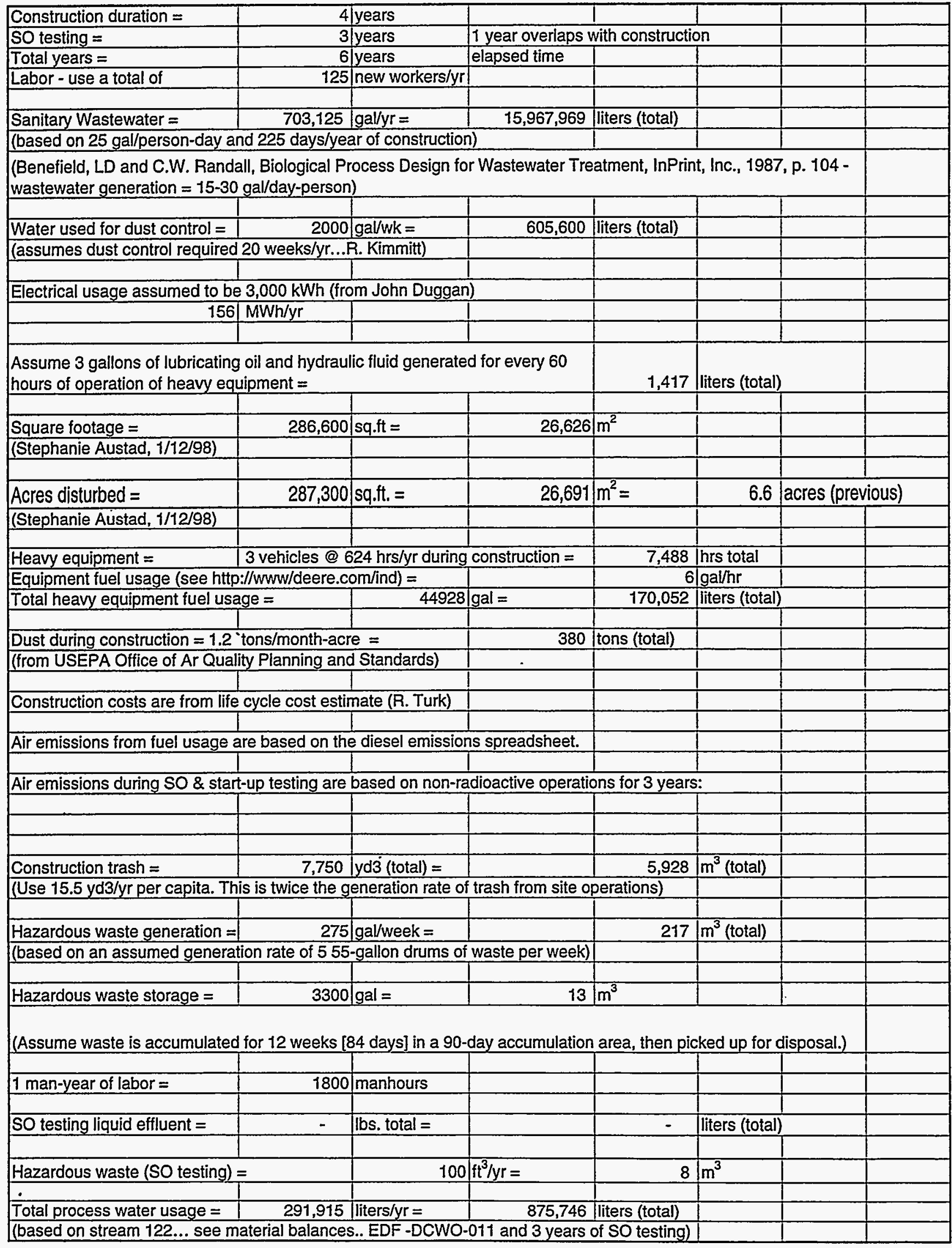


Construction Assumptions

(DCWO)

\begin{tabular}{|l|r|l|l|l|l|}
\hline & & & \\
\hline
\end{tabular}




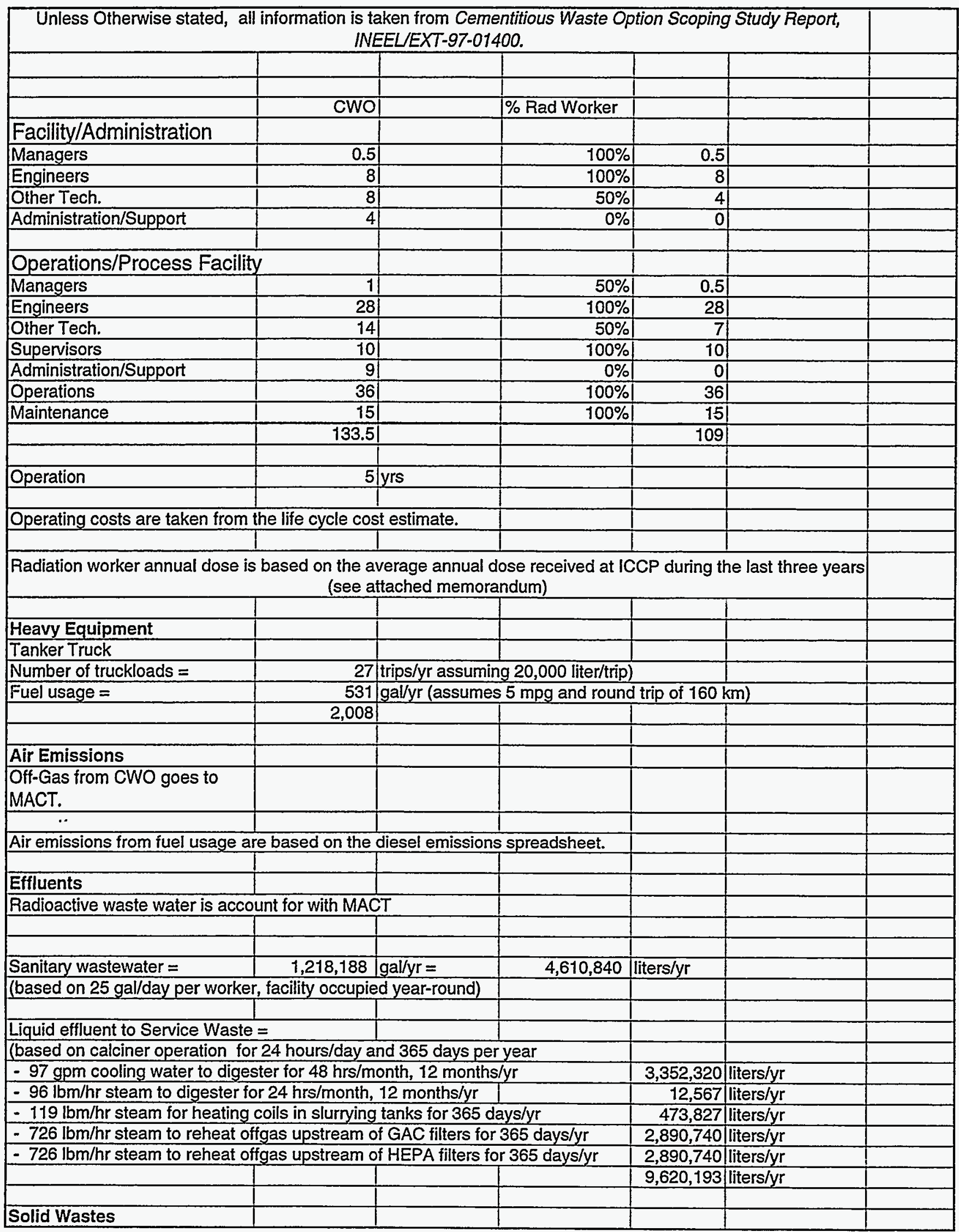




\begin{tabular}{|c|c|c|c|c|c|c|}
\hline Sanitary/Industrial trash $=$ & 968 & $y d^{3} / y r$ & 740 & $\mathrm{~m}^{3} / \mathrm{yr}$ & & \\
\hline \multicolumn{7}{|c|}{ (based on $7.25 \mathrm{yd}^{3} /$ person-year... Bob Skinner [cuber facility]) } \\
\hline & & & & & & \\
\hline \multicolumn{7}{|c|}{ Hazardous/toxic chemicals and wastes } \\
\hline Nitric Acid (HNO3), $13 \mathrm{M}=$ & 65,700 & gal/yr & & & & \\
\hline & & & & & & \\
\hline \multicolumn{7}{|l|}{ Water Usage } \\
\hline \multicolumn{7}{|l|}{ Process water usage $=$} \\
\hline \multicolumn{7}{|c|}{ (based on the following mass flow rates for $24 \mathrm{hrs} /$ day and 365 days/yr: } \\
\hline - $238,140 \mathrm{gal} / \mathrm{yr}$ Slurry Dilution & & & & 901,360 & liters & \\
\hline \multirow[t]{3}{*}{ - 65,700 gal/yr Scrub Makeup } & & & & 248,675 & liters & \\
\hline & & & & $1,150,034$ & liters (total) & \\
\hline & & & & & & \\
\hline \multicolumn{7}{|c|}{ Domestic water use is assumed to be the same as sanitary waste. } \\
\hline & & & & & & \\
\hline \multicolumn{7}{|l|}{ Energy Requirements: } \\
\hline Steam: & 1547 & $\mathrm{lbm} / \mathrm{hr}$ & \multicolumn{3}{|c|}{ CWO Scoping Study Report, Sect 3.4, p 33} & \\
\hline molecular wt & 18 & $\mathrm{lb} / \mathrm{lbm}$ & & & & \\
\hline \multirow[t]{3}{*}{ hrs/yr } & 8760 & hr/yr & & & & \\
\hline & 0.4536 & $\mathrm{~kg} / \mathrm{lb}$ & & & & \\
\hline & $110,647,083$ & $\mathrm{~kg} / \mathrm{yr}$ & & & & \\
\hline & & & & & & \\
\hline \multicolumn{7}{|l|}{ Kerosine: } \\
\hline \multirow[t]{4}{*}{ NWCF } & 16 & gph & \multicolumn{4}{|c|}{ CWO Scoping Study Report, Sect 3.2.3.1.1, p 13.} \\
\hline & 3.785 & Liter/gal & & & & \\
\hline & 8760 & $\mathrm{hr} / \mathrm{yr}$ & & & & \\
\hline & 530,506 & Lifyr & & & & \\
\hline & & & & & & \\
\hline \multicolumn{7}{|l|}{ Electrical: } \\
\hline \multirow[t]{4}{*}{ NWCF } & 85.2 & $\mathrm{~kW}$ & \multicolumn{3}{|c|}{$\begin{array}{l}\text { CWO Scoping Study Report, Sect 3.4, p } 33 . \\
(85.2 \mathrm{~kW})\end{array}$} & \\
\hline & 8760 & $\mathrm{hr} / \mathrm{yr}$ & & & & \\
\hline & 1.00E-031 & MWN & & & & \\
\hline & 7461 & MWH/yr & & & & \\
\hline
\end{tabular}




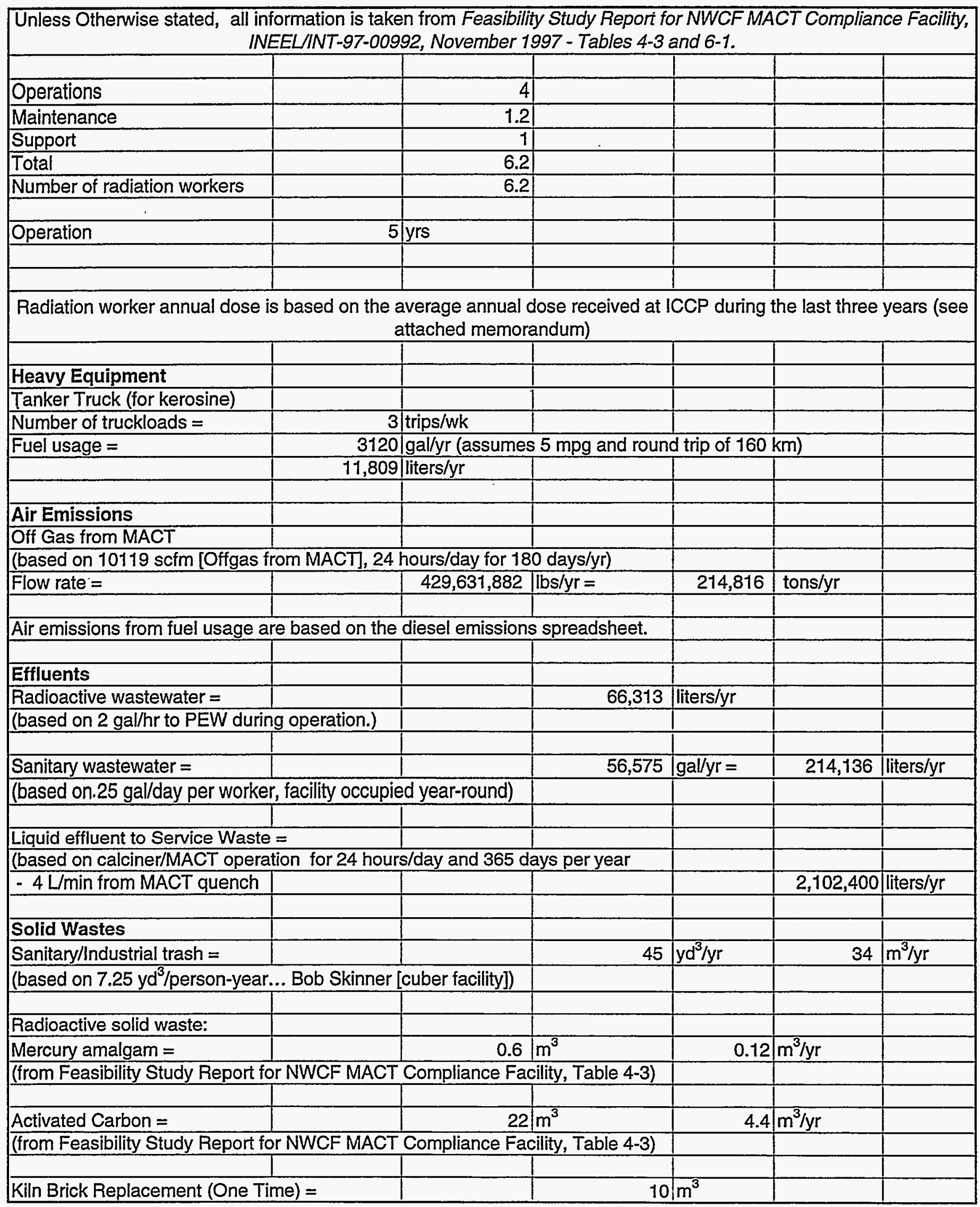


Operations Assumptions

(MACT)

\begin{tabular}{|c|c|c|c|c|c|c|}
\hline \multicolumn{7}{|c|}{ (from Feasibility Study Report for NWCF MACT Compliance Facility, Table 4-3) } \\
\hline & & & & & & \\
\hline \multicolumn{7}{|c|}{ Curie content of Refractory Brick } \\
\hline \multirow{2}{*}{\multicolumn{7}{|c|}{$\begin{array}{l}\text { To estimate the curie content of the refractory brick, analysis information for WERF refractory brick is used. Radiation } \\
\text { levels are taken from letter JAD-165-955 dated } 11 / 21 / 95 \text {. }\end{array}$}} \\
\hline & & & & & & \\
\hline & & & & & & \\
\hline \multicolumn{7}{|c|}{ Density of refractory brick used is 2.2 from Handbook of Chemistry \& Physics 55 th ED. } \\
\hline & & & & & & \\
\hline \multirow[t]{3}{*}{ Volume of refractory brick } & & 10 & $\mathrm{~m}^{3}$ & & & \\
\hline & density & 2.2 & $\mathrm{~g} / \mathrm{cm}^{3}$ & & & \\
\hline & conversion & $1,000,000$ & $\mathrm{~cm}^{3} / \mathrm{m}^{3}$ & & & \\
\hline & & & & & & \\
\hline & & & & & & \\
\hline \multirow[t]{2}{*}{ Total Weight of refractory brick } & & & $22.0 \mathrm{E}+6$ & grams & & \\
\hline & & & & & & \\
\hline Cs-137 & 8.2E-09 & $\mathrm{Ci} / \mathrm{g}$ & 180.40E-3 & $\mathrm{Ci}$ & & \\
\hline Co-60 & $1.41 E-09$ & $\mathrm{Ci} / \mathrm{g}$ & $31.02 \mathrm{E}-3$ & $\mathrm{Ci}$ & & \\
\hline Cs-134 & $6 E-11$ & $\mathrm{Ci} / \mathrm{g}$ & $1.32 \mathrm{E}-3$ & $\mathrm{Ci}$ & & \\
\hline Sb-125 & $7.5 E-111$ & $\mathrm{Ci} / \mathrm{g}$ & $1.65 \mathrm{E}-3$ & $\mathrm{Ci}$ & & \\
\hline $\mathrm{K}-40$ & $4.3 E-11$ & $\mathrm{Ci} / \mathrm{g}$ & $946.00 \mathrm{E}-6$ & $\mathrm{Ci}$ & & \\
\hline $\mathrm{Mn}-54$ & $1.9 E-12$ & $\mathrm{Ci} / \mathrm{g}$ & $41.80 \mathrm{E}-6$ & $\mathrm{Ci}$ & & \\
\hline \multirow{2}{*}{$\frac{E u-154}{E u-152}$} & $1.14 \mathrm{E}-11$ & $\mathrm{Ci} / \mathrm{g}$ & $250.80 E-6$ & $\mathrm{Ci}$ & & \\
\hline & 1.04E-11 & $\mathrm{Ci} / \mathrm{g}$ & $228.80 \mathrm{E}-6$ & $\mathrm{Ci}$ & & \\
\hline & & & & & & \\
\hline & & & $\overline{0.216}$ & $\mathrm{Ci}$ & & \\
\hline \multicolumn{7}{|l|}{ HEPA filters } \\
\hline HEPA filter volume $=$ & & 320 & & 30 & $m^{3}$ & \\
\hline \\
\hline \multicolumn{7}{|c|}{ (from Feasibility Study Report for NWCF MACT Compliance Facility, Table 4-3) } \\
\hline \multicolumn{7}{|l|}{ Curie Content } \\
\hline \multicolumn{7}{|l|}{ Assumptions } \\
\hline 1 & \multicolumn{5}{|c|}{$\begin{array}{c}\text { The HEPA filters in the MACT facility will be similar in function to the } \\
\text { HEPA filters in the APS. }\end{array}$} & \\
\hline 2 & \multicolumn{5}{|c|}{$\begin{array}{l}\text { The HEPA filters in the APS, Building CPP-649 are replaced when the } \\
\text { radiation levels reach } 5 \mathrm{R} / \mathrm{hr} \text { or the pressure drop across the filter } \\
\text { becomes to high. The worst case for Curie content is when the filter is at } \\
5 \mathrm{R} / \mathrm{hr} \text { contact. }\end{array}$} & \\
\hline 3 & \multicolumn{5}{|c|}{ Based on $320 \mathrm{ft}^{3}$ for 80 filters, a HEPA filter is $4 \mathrm{ft}^{3}$ in volume. } & \\
\hline 4 & \multicolumn{5}{|c|}{ Assume that the HEPA filter is the same dimension on each side. } & \\
\hline 5 & Assume that the & filter creates a & point source. & & & \\
\hline 6 & Use Curie - $N$ & $\begin{array}{l}\text { Meter - Rem rule } \\
\text { reading }\end{array}$ & $\begin{array}{l}\text { to estimate Curie } \\
5 \mathrm{R} / \mathrm{hr} \text { on contac }\end{array}$ & content of $\mathrm{H}$ & AEPA filter & \\
\hline & & & & & & \\
\hline Length per side assuming filter is & s cube $=$ & 1.59 & $\mathrm{ft}$ & & & \\
\hline & & & & & & \\
\hline $\begin{array}{l}\text { Assume distance from point } \\
\text { source is } 1 / 2 \text { the length of a }\end{array}$ & & 0.79 & ft & 0.24 & $\mathrm{~m}$ & \\
\hline & & & & & & \\
\hline Assuming a point source, the & strength of the & $\begin{array}{l}\text { rad field is prop } \\
\text { distance. }\end{array}$ & itional to the & rse & & \\
\hline
\end{tabular}


(MACT)

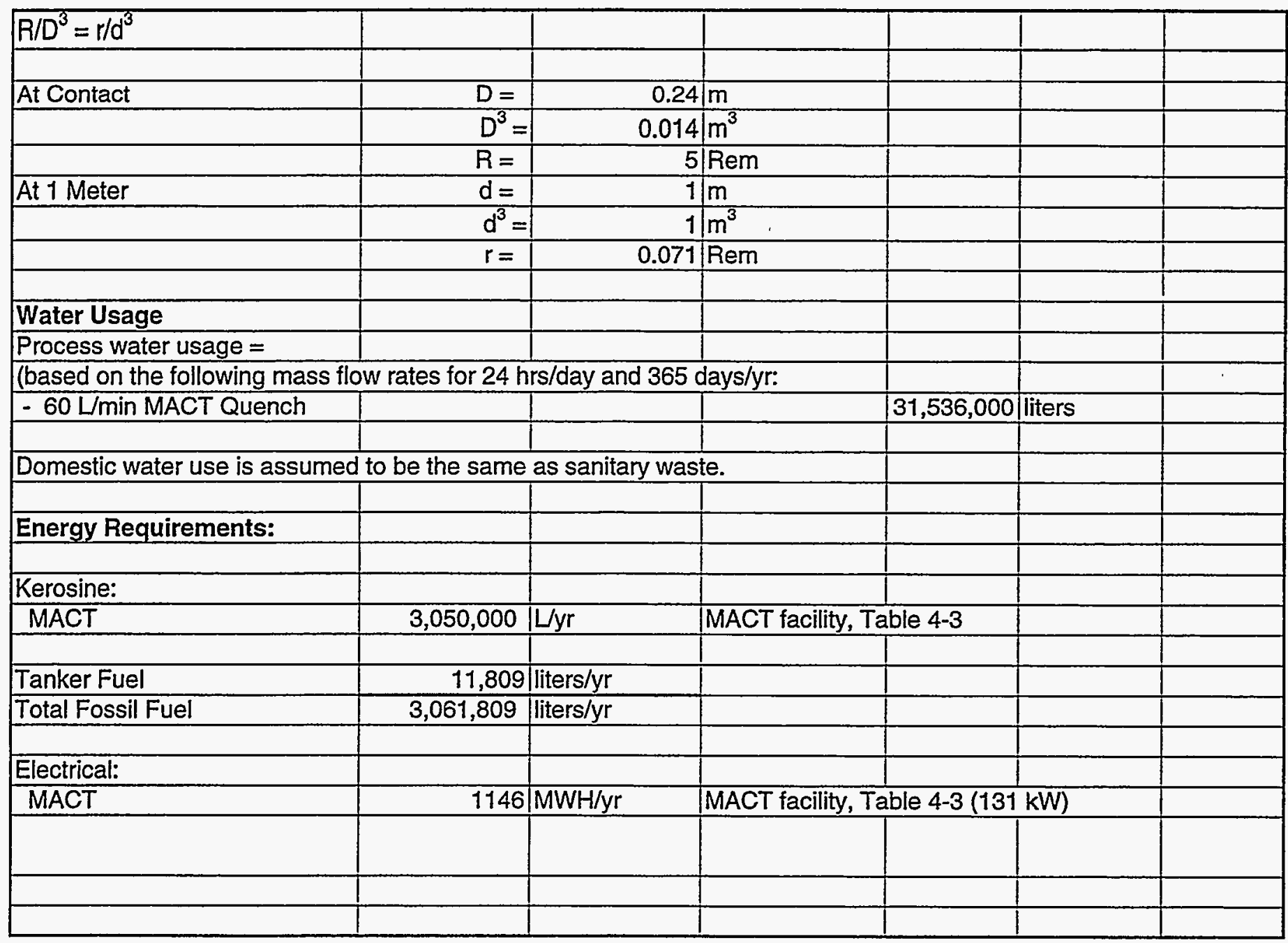




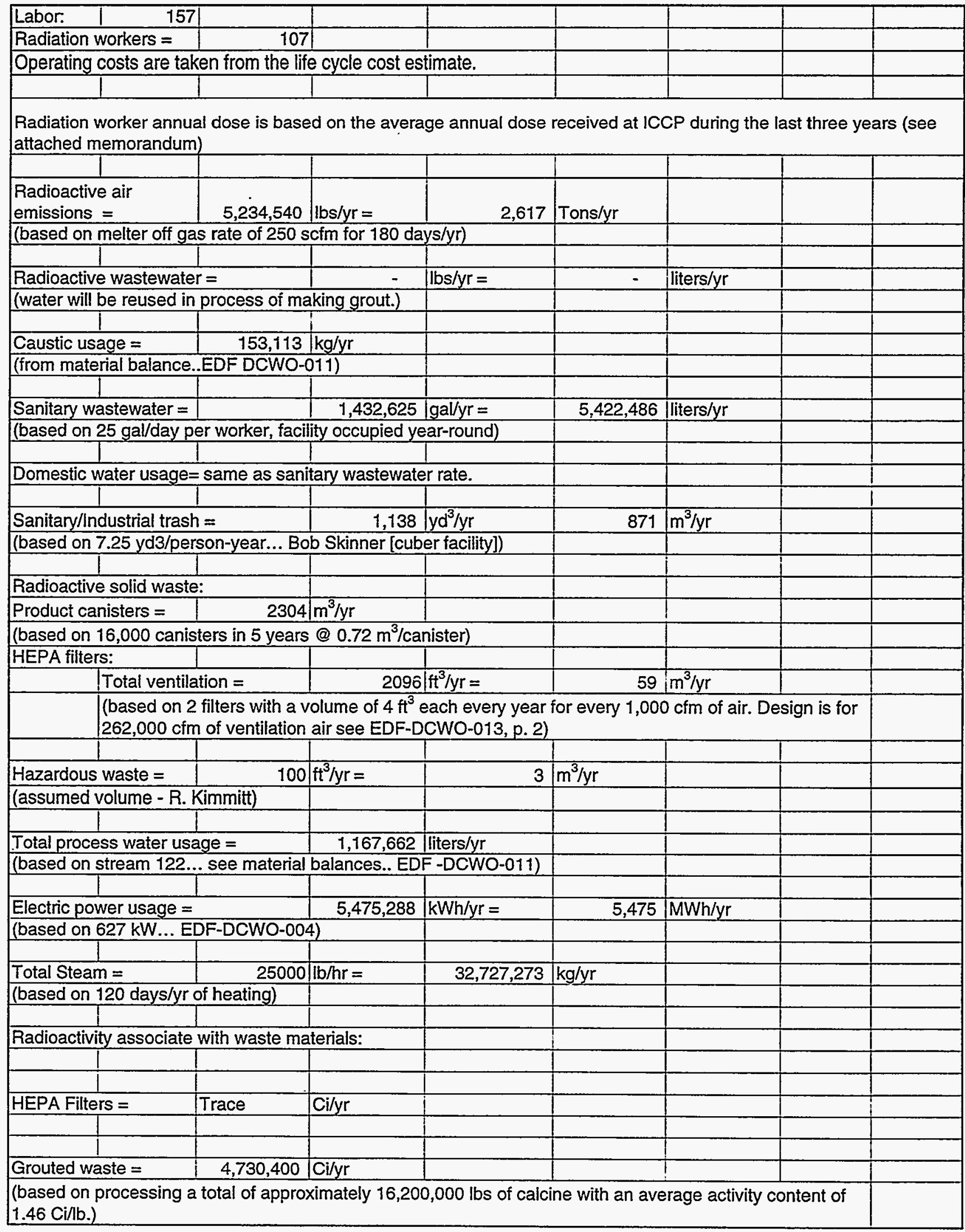




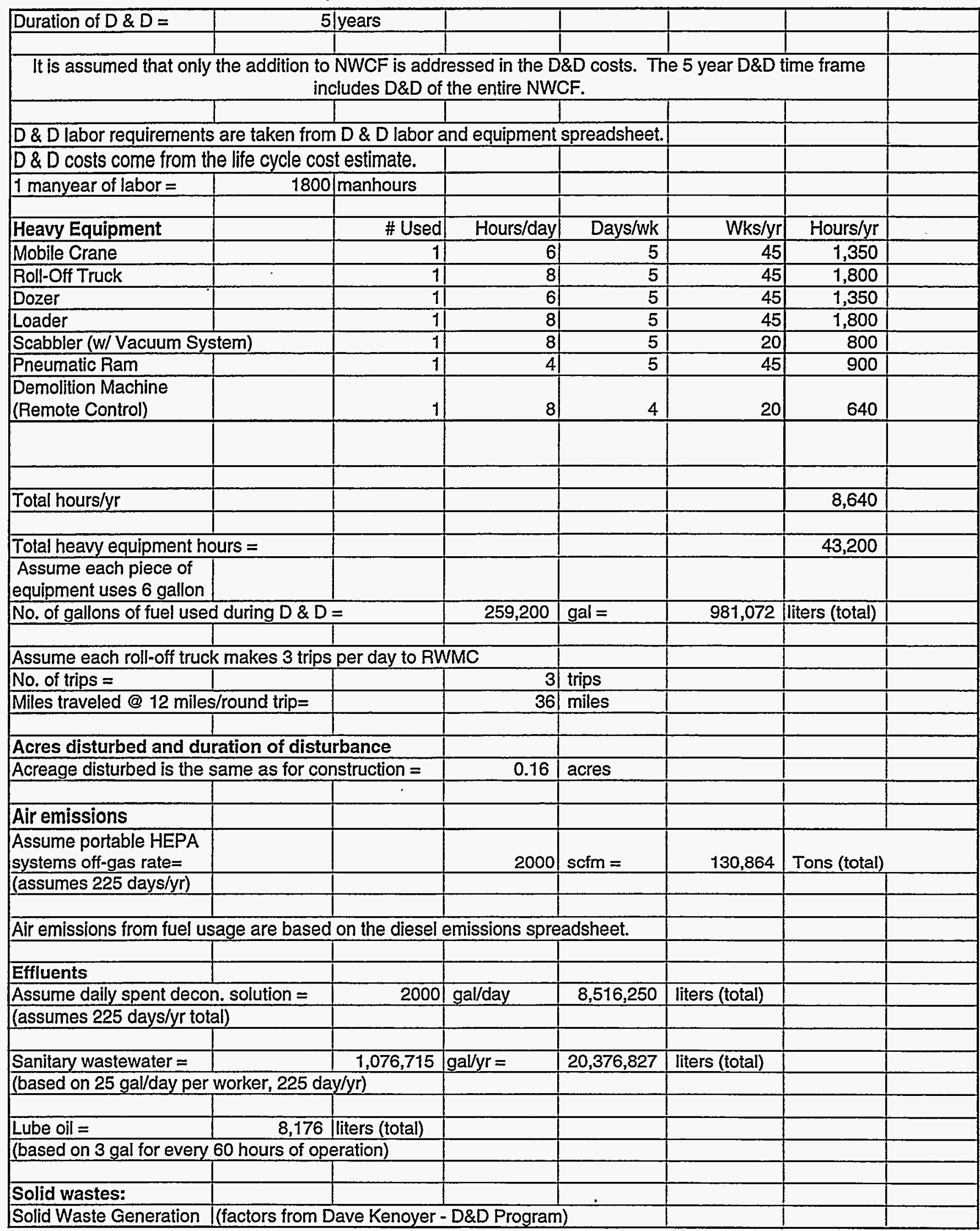


D D Assumptions

(CWO)

\begin{tabular}{|c|c|c|c|c|c|c|}
\hline Waste Type & & \begin{tabular}{|c|} 
Factor \\
(cu.ft./sq.ft.)
\end{tabular} & $\begin{array}{l}\text { Sq.Ft. in } \\
\text { Facility }\end{array}$ & $\begin{array}{l}\text { Cu.Ft. of } \\
\text { Waste }\end{array}$ & Cu. Meters & \\
\hline & & & & & & \\
\hline $\begin{array}{l}\text { WERF-LLW } \\
\text { Combustible PPEs }\end{array}$ & & 0.167 & 2,301 & 384 & 11 & \\
\hline $\begin{array}{l}\text { WERF-LLW } \\
\text { Combustible Building } \\
\text { Debris }\end{array}$ & & 0.128 & 2,301 & 295 & 8 & \\
\hline $\begin{array}{l}\text { WERF-LLW } \\
\text { Compactable Building } \\
\text { Debris }\end{array}$ & & 0.195 & 2,301 & 449 & 13 & \\
\hline $\begin{array}{l}\text { RWMC-LLW Non- } \\
\text { Compactable Equipment }\end{array}$ & & 0.513 & 2,301 & 1,180 & 34 & \\
\hline $\begin{array}{l}\text { RWMC-LLW Non-Compt } \\
\text { Building Debris }\end{array}$ & & 0.684 & 2,301 & 1,574 & 45 & \\
\hline $\begin{array}{l}\text { RWMC-LLW Non-Compt } \\
\text { Concrete Rubble * }\end{array}$ & & 3.44 & 2,301 & 7,915 & 226 & \\
\hline $\begin{array}{l}\text { RWMC-LLW Non-Compt } \\
\text { Scrap Metal }\end{array}$ & & 0.778 & 2,301 & 1,790 & 51 & \\
\hline $\begin{array}{l}\text { RWMC-LLW } \\
\text { Asbestos/ACM Covered }\end{array}$ & & 0 & 2,301 & - & & \\
\hline $\begin{array}{l}\text { CFA Landfill Non-Compt } \\
\text { Building Debris }\end{array}$ & & 1.99 & 2,301 & 4,579 & 131 & \\
\hline $\begin{array}{l}\text { CFA Landfill Non-Compt } \\
\text { Concrete Rubble * }\end{array}$ & & 2.454 & 2,301 & 5,647 & 161 & \\
\hline CFA Landfill Asbestos & & 0 & 2,301 & - & & \\
\hline $\begin{array}{l}\text { HWSF Hazardous Mtrls } \\
\text { (Hg/PCBs/etc) }\end{array}$ & & 0.002 & 2,301 & 5 & 0 & \\
\hline Metal Recycle & & 0.022 & 2,301 & 51 & 1 & \\
\hline$L L W=$ & & & & 13,587 & 388 & \\
\hline Non-Rad $=$ & & & & 10,226 & 292 & \\
\hline Hazardous $=$ & & & & 5 & 0 & \\
\hline Metal $=$ & & & & 51 & 1 & \\
\hline (Factor used is twice the & given by Dave & e Kenoyer due & to use of con & rete for shielc & ding) & \\
\hline & & & & & & \\
\hline Hazardous/toxic chemic & als and waste & S (type) & & & & \\
\hline Mixed waste $=$ & 44,075 & gal (total) $=$ & 1,515 & $\mathrm{m3}$ (total) & & \\
\hline (based on an assumed 5 & 5-gailon drum & is generated $p$ & r week... wor & only 225 da & $y /(y r)$ & \\
\hline Decontamination solution & stored $=$ & & 2,000 & gallons & 205 & $\mathrm{~m}^{3}$ \\
\hline & & & & & & \\
\hline Water Usage & & & & & & \\
\hline Daily process water usag & & 3000 & gal/day $=$ & $11,424,375$ & \begin{tabular}{|l} 
liters (total) \\
\end{tabular} & \\
\hline (washing, decon, etc.; ba & ed on 225 day & s/yr) & & & & \\
\hline & & & & & & \\
\hline Domestic water use is as & umed to be the & e same as san & tary waste. & & & \\
\hline & & & & & & \\
\hline Energy requirements: & & & & & & \\
\hline Electric power usage $=$ & 156,000 & $\mathrm{kWh} / \mathrm{yr}$ & 156 & MWh/yr & & \\
\hline (based on $3,000 \mathrm{kWh} / \mathrm{wk}$ & John Duggan & & & & & \\
\hline Fossil Fuels = & 981,072 & liters & & & & \\
\hline
\end{tabular}




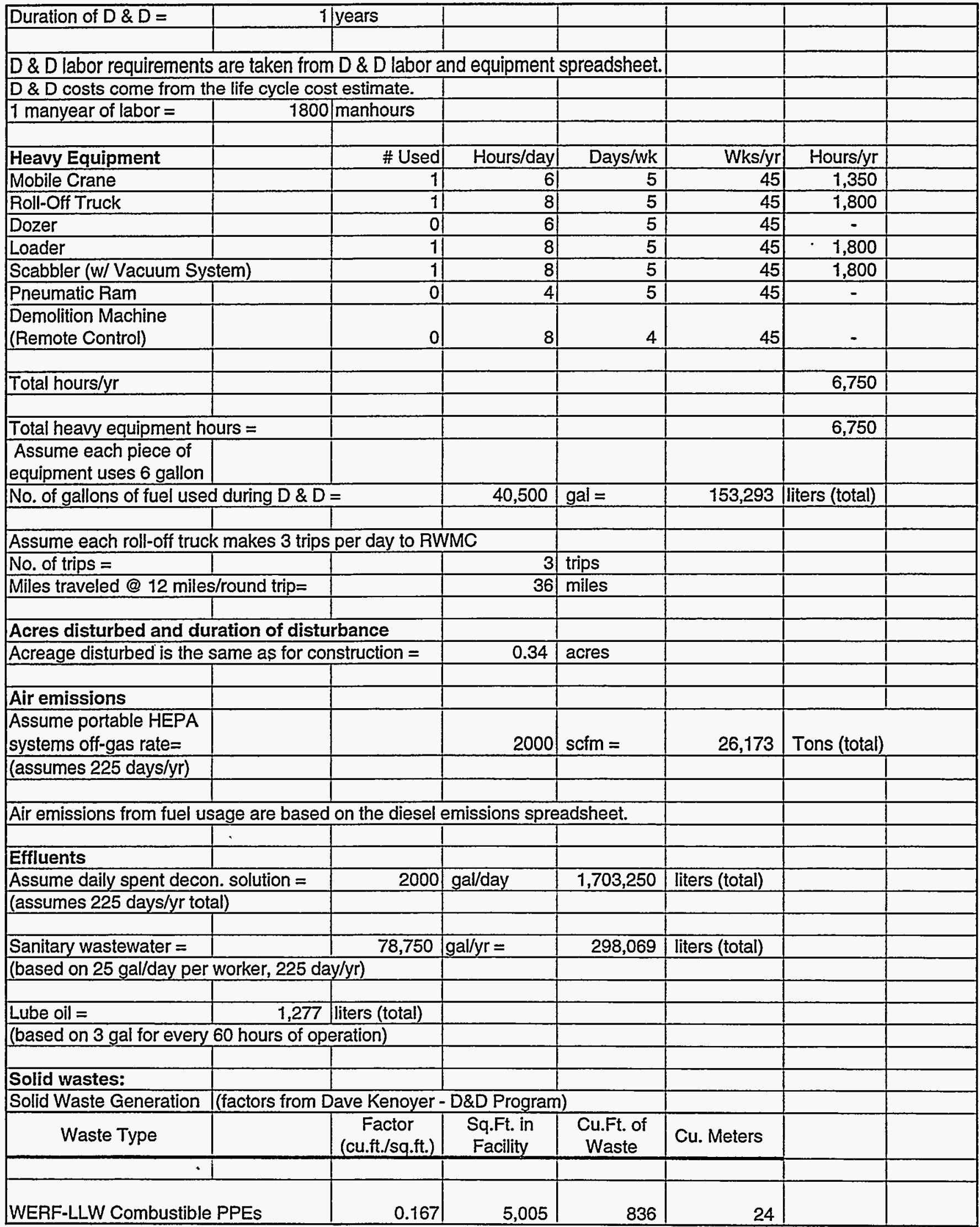


D D Assumptions

(MACT)

\begin{tabular}{|c|c|c|c|c|c|c|c|}
\hline $\begin{array}{l}\text { WERF-LLW Combustible } \\
\text { Debris * }\end{array}$ & Building & 0 & 5,005 & - & - & & \\
\hline $\begin{array}{l}\text { WERF-LLW Compactabl } \\
\text { Debris * }\end{array}$ & le Building & 0 & 5,005 & - & $=$ & & \\
\hline $\begin{array}{l}\text { RWMC-LLW Non-Compa } \\
\text { Equipment }\end{array}$ & actable & 0.513 & 5,005 & 2,568 & 73 & & \\
\hline $\begin{array}{l}\text { RWMC-LLW Non-Compt } \\
\text { Debris * }\end{array}$ & Building & 이 & 5,005 & - & - & & \\
\hline $\begin{array}{l}\text { RWMC-LLW Non-Compt } \\
\text { Rubble }\end{array}$ & Concrete & 1.72 & 5,005 & 8,609 & 246 & & \\
\hline RWMC-LLW Non-Compt & Scrap Metal & 0.778 & 5,005 & 3,894 & 111 & & \\
\hline $\begin{array}{l}\text { RWMC-LLW Asbestos/AC } \\
\text { Pipe * }\end{array}$ & CM Covered & 0 & 5,005 & - & & & \\
\hline $\begin{array}{l}\text { CFA Landfill Non-Compt } \\
\text { Debris * }\end{array}$ & Building & 의 & 5,005 & - & - & & \\
\hline $\begin{array}{l}\text { CFA Landfill Non-Compt } \\
\text { Rubble }\end{array}$ & Concrete & 1.227 & 5,005 & 6,141 & 175 & & \\
\hline CFA Landfill Asbestos & & 이 & 5,005 & - & & & \\
\hline HWSF Hazardous Mtrls ( & (Hg/PCBs/etc) & 0.002 & 5,005 & 10 & 0 & & \\
\hline Metal Recycle & & 0.022 & 5,005 & 110 & 3 & & \\
\hline $\begin{array}{l}\text { *These catagories are de } \\
\text { itself will not be demolish }\end{array}$ & $\begin{array}{l}\text { eleted since the } \\
\text { ed. }\end{array}$ & MACT will be & removed from & the building $\mathrm{bl}$ & ut the build & & \\
\hline & & & & & & & \\
\hline & & & & & . & & \\
\hline LLW $=$ & & & & 15,907 & 454 & & \\
\hline Non-Rad = & & & & 6,141 & 175 & & \\
\hline Hazardous $=$ & & & & 10 & 0 & & \\
\hline Metal = & & & & 110 & 3 & & \\
\hline & & & & & & & \\
\hline & & & & & & & \\
\hline Hazardous/toxic chemic & cals and waste & es (type) & & & & & \\
\hline Mixed waste $=$ & 3,526 & gal (total) $=$ & 121 & $\mathrm{~m} 3$ (total) & & & \\
\hline (based on an assumed & 55-gallon drums & is generated pe & er week... work & only 225 day & (s/yr) & & \\
\hline & & & & & & & \\
\hline Decontamination solution & stored at NWC & & & & & & \\
\hline & & & & & & & \\
\hline Water Usage & & & & & & & \\
\hline Daily process water usag & $\mathrm{e}=$ & 3000 & gal/day $=$ & $2,284,875$ & liters (total) & & \\
\hline (washing, decon, etc.; ba & sed on 225 day & s/yr) & & & & & \\
\hline & & & & & & & \\
\hline Domestic water use is as & sumed to be the & e same as san & itary waste. & & & & \\
\hline & & & & & & & \\
\hline Energy requirements: & & & & & & & \\
\hline Electric power usage $=$ & 156,000 & $\mathrm{kWh} / \mathrm{yr}$ & 156 & $M W h / y r$ & & & \\
\hline (based on $3,000 \mathrm{kWh} / \mathrm{wk}$ & - John Duggan & & & & & & \\
\hline Fossil Fuels = & 153,293 & liters & & & & & \\
\hline
\end{tabular}




\begin{tabular}{|c|c|c|c|c|c|c|c|}
\hline Duration of $D \& D=$ & & years & & & & & \\
\hline & & & & & & & \\
\hline Heavy Equipment & & \# Used & Hours/day & Days/wk & Wks/yr & Hours/yr & \\
\hline & Mobile Crane & 2 & $3 \mid$ & 4 & 45 & 1,080 & \\
\hline & Roll-Off Truck & 6 & 8 & 5 & 45 & 10,800 & \\
\hline & Dozer & 2 & 5 & 5 & 45 & 2,250 & \\
\hline & Loader & 6 & 8 & 5 & 45 & 10,800 & \\
\hline & $\begin{array}{l}\text { Scabbler (w/ } \\
\text { Vacuum } \\
\text { System) }\end{array}$ & 2 & 8 & 5 & 45 & 3,600 & \\
\hline & $\begin{array}{l}\text { Pneumatic } \\
\text { Ram }\end{array}$ & 2 & 4 & 4 & 45 & 1,440 & \\
\hline & $\begin{array}{l}\text { Demolition } \\
\text { Machine } \\
\text { (Remote } \\
\text { Control) } \\
\end{array}$ & 2 & 4 & 3 & 45 & 1,080 & \\
\hline Total hours/yr & & & & & & 31,050 & \\
\hline & & & & & & & \\
\hline Total heavy equipment ho & urs = & & & & & 93,150 & \\
\hline $\begin{array}{l}\text { Assume each piece of eq } \\
\text { Web Site (Construction E }\end{array}$ & $\begin{array}{l}\text { quipment uses } 6 \\
\text { quipment - http: }\end{array}$ & $\begin{array}{l}6 \text { gallon of dies } \\
: / / w w w . d e e r e . c\end{array}$ & $\begin{array}{l}\text { sel fuel per hou } \\
\text { com/ind/produc }\end{array}$ & $\begin{array}{l}\text { r. Consumpt } \\
\text { t/product.htn }\end{array}$ & $\begin{array}{l}\text { tion rate from Jol } \\
\text { nl) }\end{array}$ & hn Deere & \\
\hline No. of gallons of fuel used & during $D$ \& $D=$ & & 558,900 & gal $=$ & $2,115,437$ & liters (total) & \\
\hline . & & & & & & & \\
\hline Acreage disturbed is the $\mathrm{s}$ & same as for con & istruction $=$ & 6.6 & acres & & & \\
\hline & & & & 1 & & & \\
\hline $\mathrm{D} \& \mathrm{D}$ labor requirements & are taken from & D\& \& labor a & Ind equipment s & spreadsheet. & & & \\
\hline & & & & & & & \\
\hline D \& D costs come from th & e life cycle cost & t estimate. & & & & & \\
\hline & & & & & & & \\
\hline Assume each roll-off truck & makes 3 trips & per day to RW & IMC & & & & \\
\hline No. of trips $=$ & & & 18 & & & & \\
\hline Miles traveled @ 12 miles & /round trip = & & 216 & miles/day & & & \\
\hline & & & & & & & \\
\hline Decontamination solution & stored $=$ & & 2,000 & gallons & 205 & $m^{3}$ & \\
\hline & & & & & & & \\
\hline Daily process water usage & & & 3000 & gal/day $=$ & $6,854,625$ & liters (total) & \\
\hline (assumed for washing, de & con, etc.; based & d on 225 days & & & & & \\
\hline$\dot{-}$ & & & & & & & \\
\hline Domestic water usage = & & $56,445,812$ & liters (total) & & & & \\
\hline (based on $25 \mathrm{gal} /$ day for $\mathrm{e}$ & each worker) & & & & & & \\
\hline & & & & & & & \\
\hline Sanitary wastewater = sar & me as domestic & water usage & & & & & \\
\hline & & & & & & & \\
\hline Assume portable HEPA s & ystems off-gas r & rate $=$ & 2000 & scfm $=$ & 78,518 & Tons (total) & \\
\hline (assumes 225 days/yr) & & & & & & & \\
\hline & & & & & & Fitera (tatal) & \\
\hline Assume daily spent decon & n. solution $=$ & & 2000 & gal/day & $5,109,750$ & liters (total) & \\
\hline (assumes 225 days/yr tota & & & & & & $\therefore$ & \\
\hline & & & & & & & \\
\hline Solid Waste Generation & (factors from $\mathrm{D}$ & ave Kenoyer - & D\&D Program & & & & \\
\hline Waste Type & $\begin{array}{c}\text { Factor } \\
\text { (cu.ft./sq.ft.) }\end{array}$ & $\begin{array}{l}\text { Sq.Ft. in } \\
\text { Facility }\end{array}$ & $\begin{array}{l}\text { Cu.Ft. of } \\
\text { Waste }\end{array}$ & Cu. Meters & & & \\
\hline & & & & & & & \\
\hline $\begin{array}{l}\text { WERF-LLW } \\
\text { Combustible PPEs }\end{array}$ & 0.167 & 286,600 & 47,862 & 1,356 & & & \\
\hline $\begin{array}{l}\text { WERF-LLW } \\
\text { Combustible Building } \\
\text { Debris }\end{array}$ & 0.128 & 286,600 & 36,685 & 1,039 & & & \\
\hline
\end{tabular}




\begin{tabular}{|c|c|c|c|c|c|c|}
\hline \begin{tabular}{|l|} 
WERF-LLW \\
Compactable Building \\
Debris \\
\end{tabular} & 0.195 & 286,600 & 55,887 & 1,583 & & \\
\hline $\begin{array}{l}\text { RWMC-LLW Non- } \\
\text { Compactable Equipment }\end{array}$ & 0.513 & 286,600 & 147,026 & 4,165 & & \\
\hline $\begin{array}{l}\text { RWMC-LLW Non-Compt } \\
\text { Building Debris }\end{array}$ & 0.684 & 286,600 & 196,034 & 5,553 & & \\
\hline $\begin{array}{l}\text { RWMC-LLW Non-Compt } \\
\text { Concrete Rubble }\end{array}$ & 3.44 & 286,600 & 985,904 & 27,929 & $\begin{array}{l}\text { Factor is twice that used by } \\
\text { the D\&D program to } \\
\text { account for that large } \\
\text { amount of concrete used. }\end{array}$ & \\
\hline $\begin{array}{l}\text { RWMC-LLW Non-Compt } \\
\text { Scrap Metal }\end{array}$ & 0.778 & 286,600 & 222,975 & 6,317 & & \\
\hline $\begin{array}{l}\text { RWMC-LLW } \\
\text { Asbestos/ACM Covered } \\
\text { Pipe }\end{array}$ & 0.170 & 286,600 & $2<<, 910$ & 0,016 & & \\
\hline $\begin{array}{l}\text { CFA Landfill Non-Compt } \\
\text { Building Debris }\end{array}$ & 1.99 & 286,600 & 570,334 & 16,157 & & \\
\hline $\begin{array}{l}\text { CFA Landfill Non-Compt } \\
\text { Concrete Rubble }\end{array}$ & $\frac{2.45}{0^{1}}$ & 286,600 & 702,170 & 19,892 & $\begin{array}{l}\text { Factor is twice that used by } \\
\text { the D\&D program to } \\
\text { account for that large } \\
\text { amount of concrete used. }\end{array}$ & \\
\hline $\begin{array}{l}\text { HWSF Hazardous Mtrls } \\
\text { (Hg/PCBs/etc) }\end{array}$ & 0.002 & 286,600 & 573 & 16 & & \\
\hline Metal Recycle & 0.022 & 286,600 & 6,305 & 179 & & \\
\hline LLW $=$ & & & $1,692,373$ & 47,943 & & \\
\hline Non-Rad = & & & $1,272,504$ & 36,048 & & \\
\hline Hazardous $=$ & & & 573 & 16 & & \\
\hline Metal $=$ & & & 6,305 & 179 & & \\
\hline Electric power usage $=$ & 156.000 & kWh/vr & 156 & MWW/ur & & \\
\hline \multirow{2}{*}{\multicolumn{7}{|c|}{ (based on $3,000 \mathrm{kWh} / \mathrm{wk}$ - John Duggan) }} \\
\hline & & & & & & \\
\hline \multicolumn{7}{|c|}{ Air emissions from fuel are based on the diesel emissions spreadsheet. } \\
\hline 1 manyear of labor $=$ & 1800 & manhours & & & & \\
\hline & & & & & & \\
\hline Lube oil $=$ & 17,629 & liters (total) & & & & \\
\hline \multirow{2}{*}{\multicolumn{7}{|c|}{ (based on 3 gal for every 60 hours of operation) }} \\
\hline & & & & & & \\
\hline Mixed waste $=$ & 37,125 & gal (total) $=$ & 141 & m3 (total) & & \\
\hline \multirow{2}{*}{\multicolumn{7}{|c|}{ (based on an assumed 555 -gallon drums generated per week... work only 45 weeks/yr) }} \\
\hline \multicolumn{5}{|c|}{ Radioactivity associated with waste materials: } & & \\
\hline & & & & & & \\
\hline Spent decontamination sol & $\mathrm{on}=$ & 5,110 & $\overline{\mathrm{Ci}}$ & & & \\
\hline \multirow{2}{*}{\multicolumn{7}{|c|}{ (based on an assumed average activity concentration of $1 \mathrm{uCl} / \mathrm{ml}$ ) }} \\
\hline & & & & & & \\
\hline Radioactive solid waste $=$ & & 479 & $\overline{\mathrm{Ci}}$ & & & \\
\hline \multicolumn{7}{|c|}{ (based in an assumed activity concentration of $0.01 \mathrm{uCi} / \mathrm{cc}\left[0.01 \mathrm{Ci} / \mathrm{m}^{3}\right]$ ) } \\
\hline & & & & & & \\
\hline Mixed waste $=$ & & 1 & $\mathrm{Ci}$ & & & \\
\hline (based on an assumed a & concent & ion of $0.01 \mathrm{uC}$ & $\mathrm{i} / \mathrm{cc}[0.01 \mathrm{cl} / \mathrm{m}$ & & & \\
\hline
\end{tabular}




\begin{tabular}{|c|c|c|c|c|c|c|c|c|c|c|c|}
\hline \multicolumn{12}{|c|}{ D\&D Labor } \\
\hline \multirow{2}{*}{$\frac{\text { Crew \# }}{\text { D }}$} & \multirow{2}{*}{$\begin{array}{l}\text { Crew Function } \\
\text { Documentation }\end{array}$} & Total MH/day & Total \$/day & $\begin{array}{c}\text { Material } \\
\$ / \text { day }\end{array}$ & $\begin{array}{l}\text { Equipment } \\
\$ / \text { day }\end{array}$ & Total \$/day & \multicolumn{2}{|r|}{$\begin{array}{l}\text { D\&D Cost } \\
\text { Allocated }\end{array}$} & $\frac{\text { Total MH }}{89568}$ & Man-hours/yr & - \\
\hline & & 18 & $\$ 1,136$ & $\$ 114$ & $\$$ & $\$ 1,250$ & & $6,220,000$ & 89,568 & 17,914 & $\$$ \\
\hline 1 & Characterization & 44 & $\$ 2,302$ & $\$ 460$ & $\$ 691$ & $\$ 3,453$ & & $9,330,000$ & 118,888 & 23,778 & $\$ 1,867,081$ \\
\hline 2 & Rad Demolition-Systems & 77 & $\$ 4,091$ & $\$ 818$ & $\$ 1,023$ & $\$ 5,932$ & & $19,800,000$ & 257,013 & 51,403 & $\$ 3,414,599$ \\
\hline $2 A$ & Rad Demolition-Building & 99 & $\$ 5,319$ & $\$ 1,064$ & $\$ 1,596$ & $\$ 7,979$ & & $19,800,000$ & 245,670 & 49,134 & $\$ 3,960,496$ \\
\hline 3 & Demolition-Systems & 72 & $\$ 3,762$ & $\$ 752$ & $\$ 941$ & $\$ 5,455$ & & $7,500,000$ & 98,992 & 19,798 & $\$ 1,293,767$ \\
\hline $3 \bar{A}$ & Demolition-Building & 88 & $\$ 4,808$ & $\$ 962$ & $\$ 1,442$ & $\$ 7,212$ & & $7,500,000$ & 91,514 & 18,303 & $\$ 1,499,584$ \\
\hline 4 & Asbestos Abatement & 77 & $\$ 3,753$ & $\$ 375$ & $\$ 188$ & $\$ 4,316$ & & - & - & - & $\$$ \\
\hline 5 & Decontamination & 77 & $\$ 3,753$ & $\$ 751$ & $\$ 1,126$ & $\$ 5,630$ & & $\$ \quad 37,450,000$ & 512,194 & 102,439 & $\$ 7,490,000$ \\
\hline 6 & Prep/Fabrication & 61 & $\$ 3,217$ & $\$ 643$ & $\$ 965$ & $\$ 4,826$ & & $10,600,000$ & 133,983 & 26,797 & $\$ 2,119,561$ \\
\hline 7 & RADCON Surveys & 50 & $\$ 2,596$ & $\$ 519$ & $\$ 779$ & $\$ 3,894$ & & $\$ \quad 13,623,000$ & 174,923 & 34,985 & $\$ 2,725,300$ \\
\hline Total & & & & & & & & $\$ 131,823,000$ & $1,722,744$ & 344,549 & $\$ 24,370,388$ \\
\hline & & & & & & & & & & & \\
\hline Available & & & & & & & & $\$ 131,823,000$ & & & $10,168,446$ \\
\hline & & & & & & & & & & & \\
\hline Notes: & & & & & & & & & & & 8134.757041 \\
\hline 1 & Crew functions and daily & imates are from & the D\&D prog & Im (Dave Ha & (craft) & & & & & & \\
\hline 2 & Total costs are based on I & cycle estimate & py R. Turk & & & & & & & & \\
\hline 3 & Assume all workers in cre & $2,2 A, 5$, and 7 & are rad worke & & & & & & & & \\
\hline 4 & Assume a man-year is 18 & hours. & & & & & & & & & 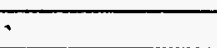 \\
\hline
\end{tabular}


D\&D Labor

(1)
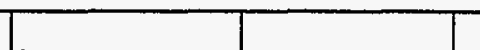

Labor for the MACT D\&D is calculated in a different manner than for the rest of the Process Data Sheets.

Labor costs for the MACT D\&D are given in the Feasibility Study Report for NWCF MACT Compliance Facility, INEEL/INT-97-00992, November 199 D\&D includes the gutting of the MACT building but does not include tearing down the building to bare earth.

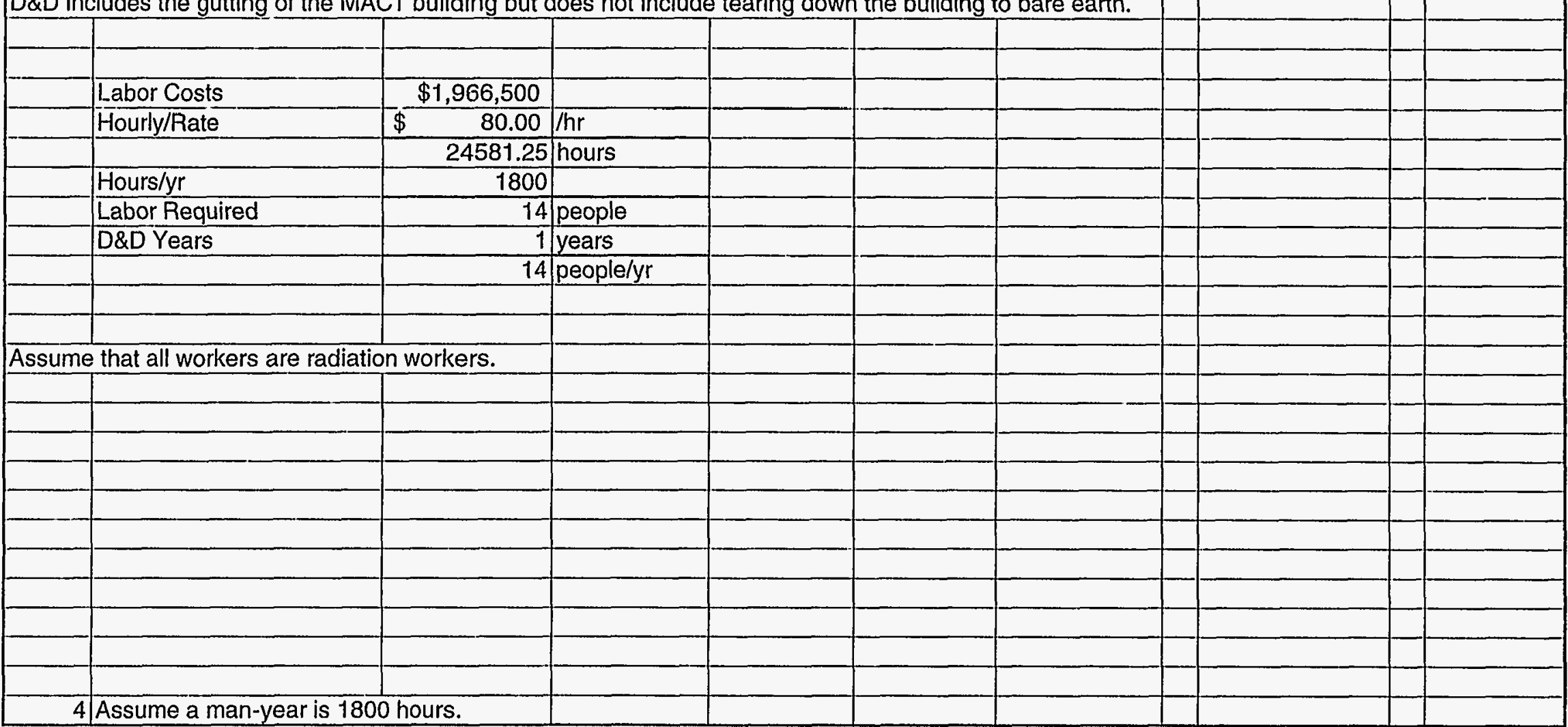


D\&D Labor-DCWO

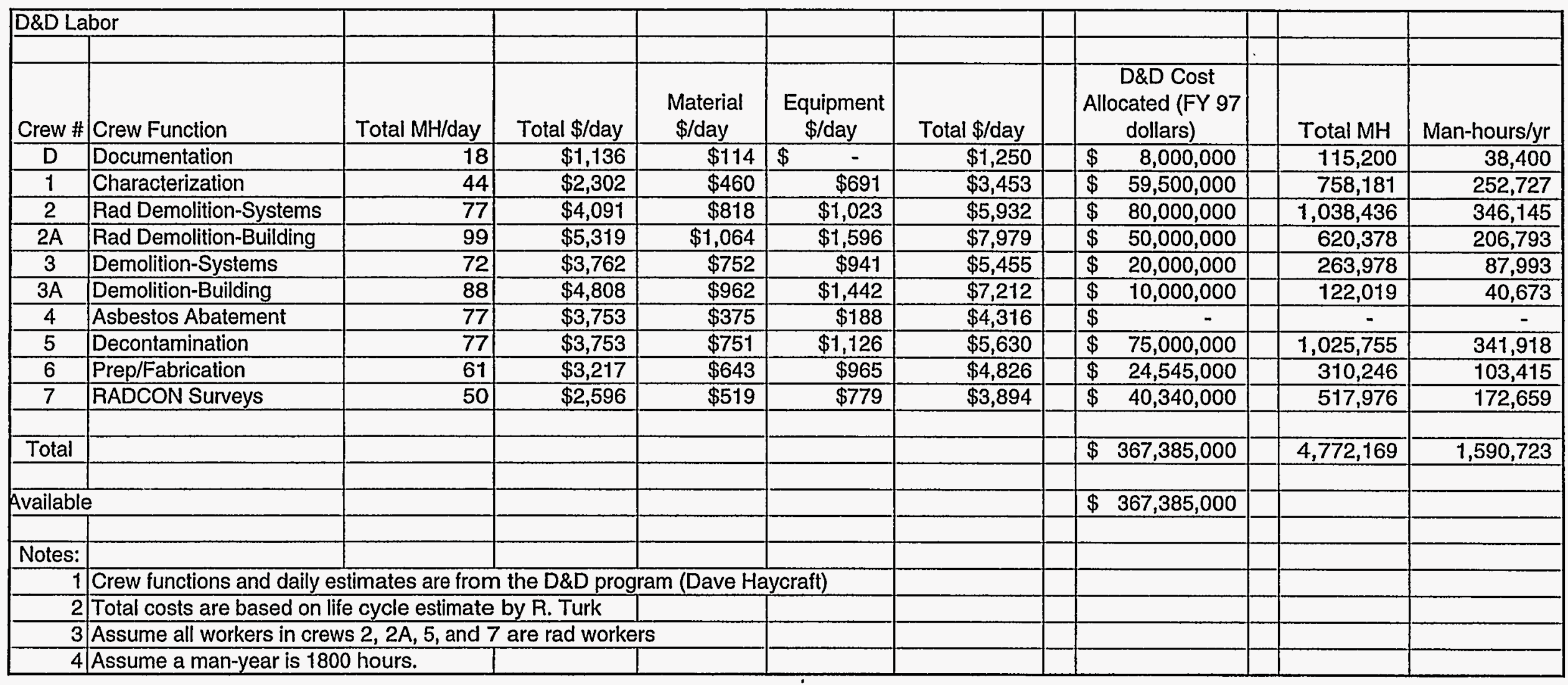




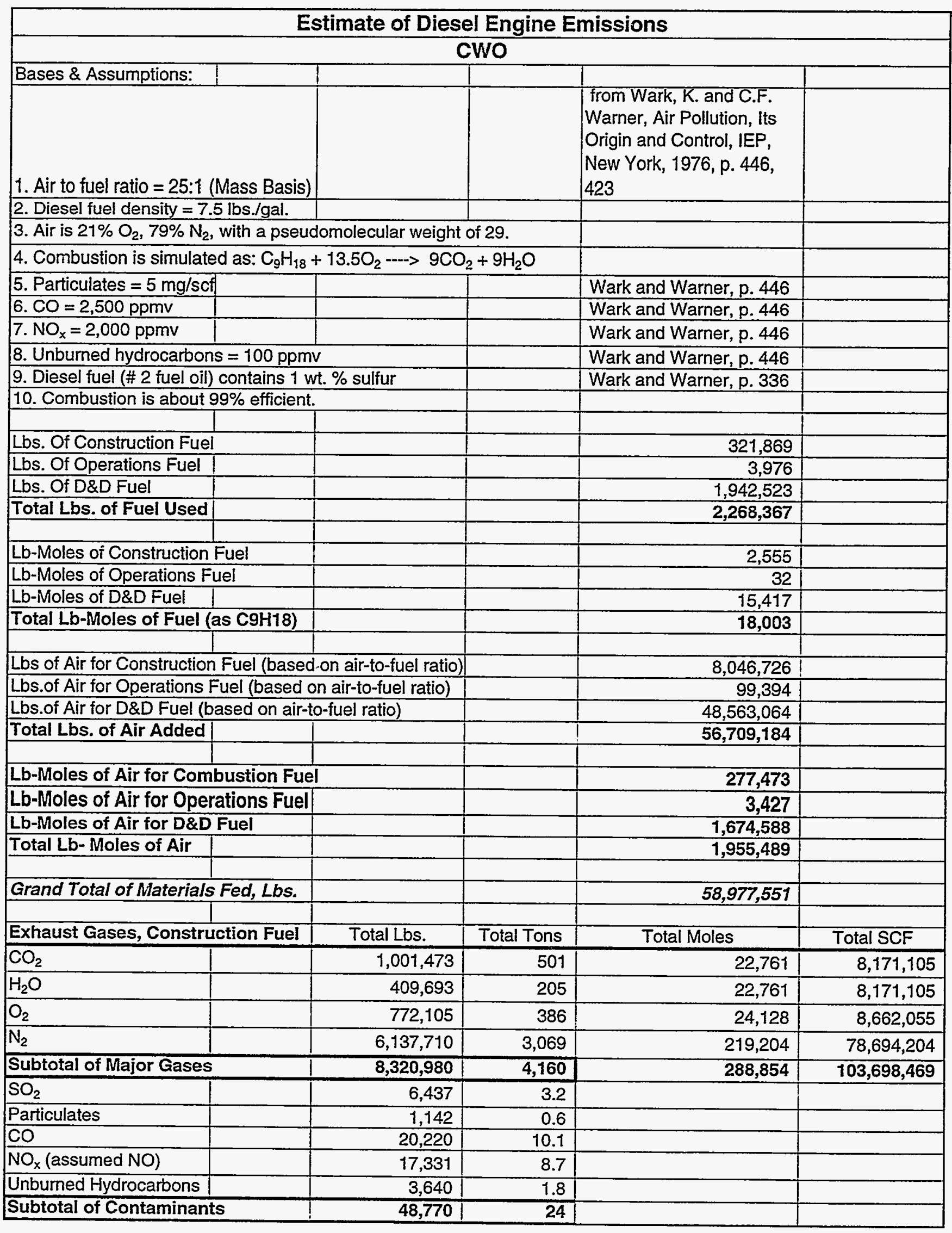




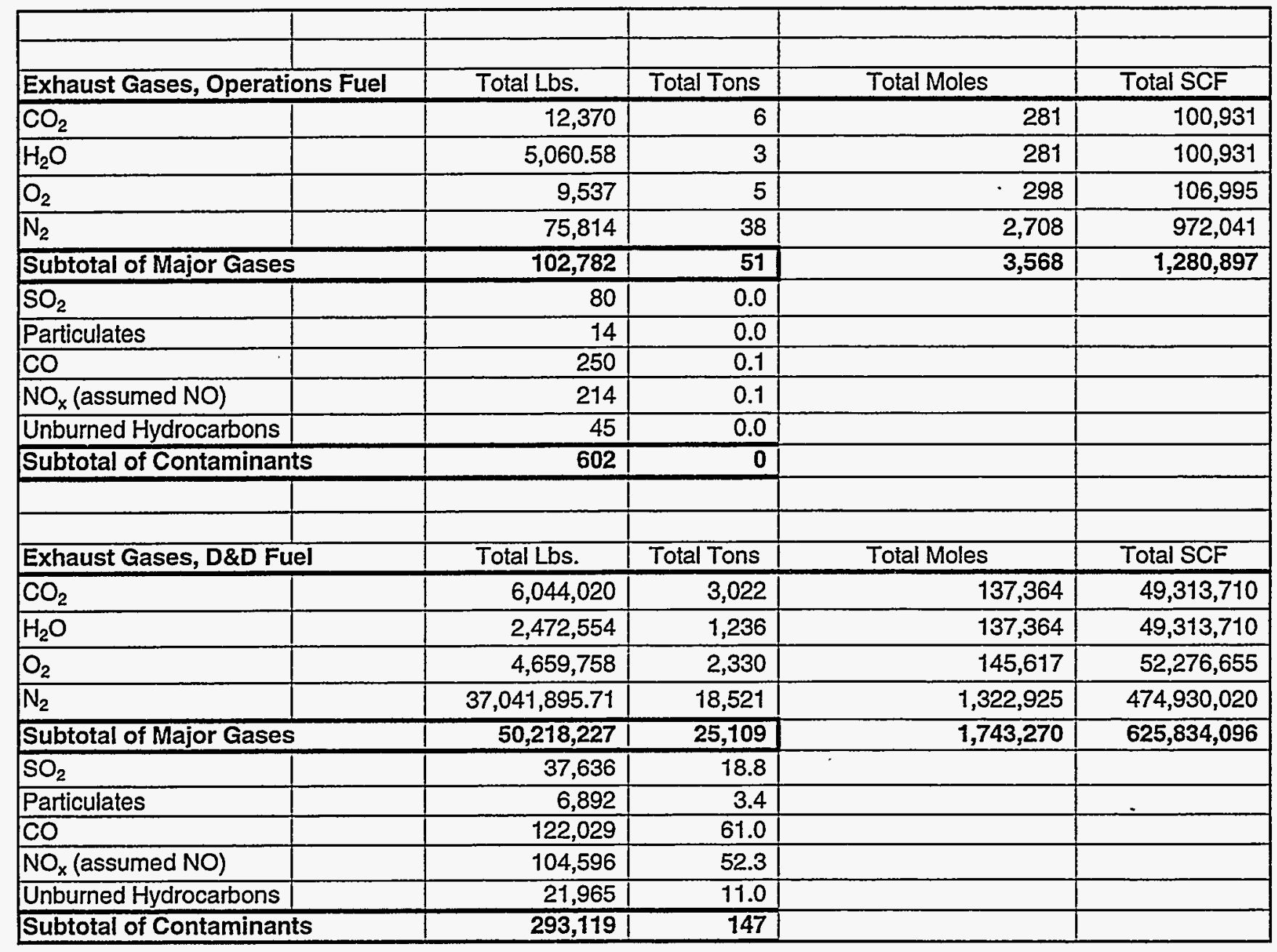




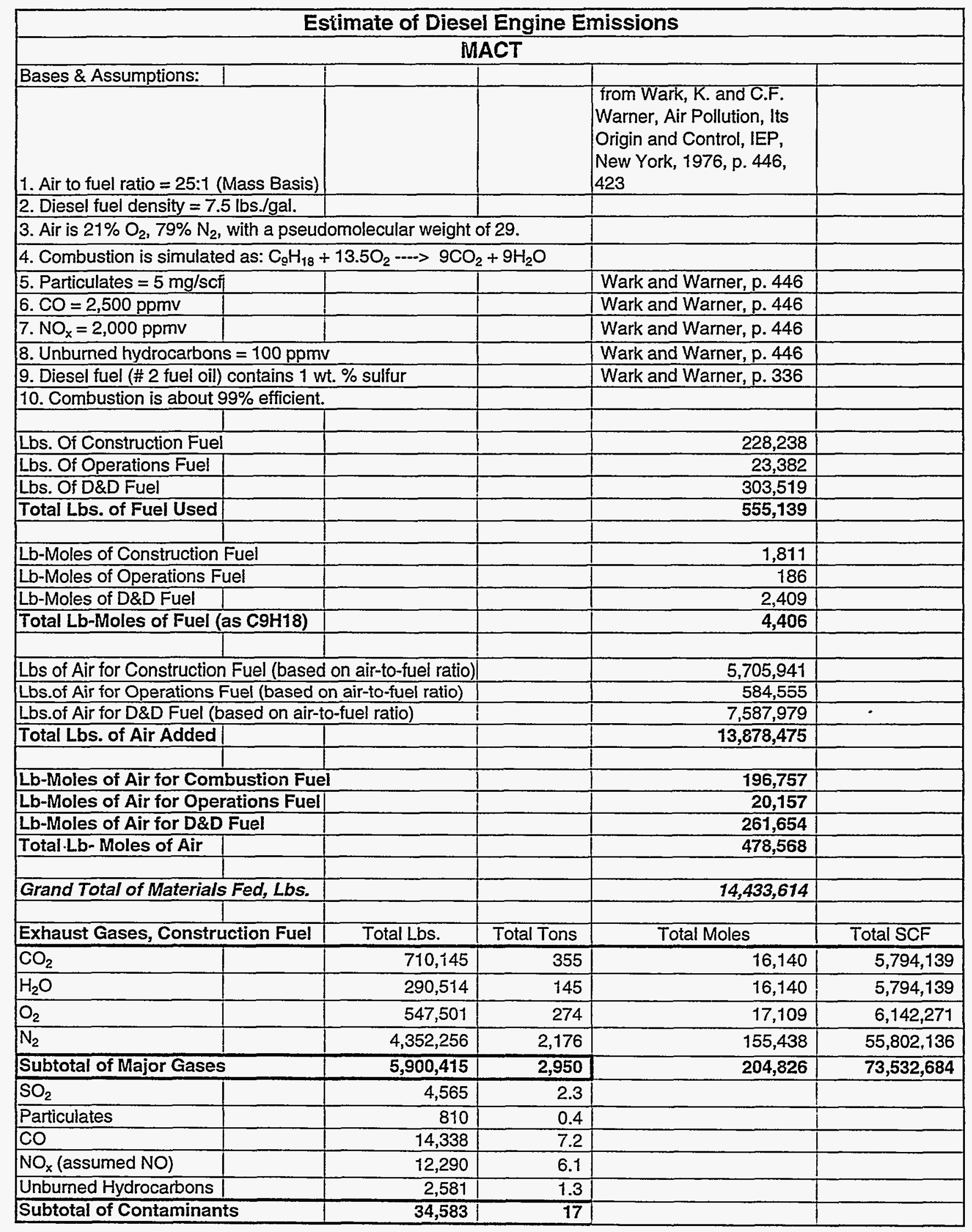




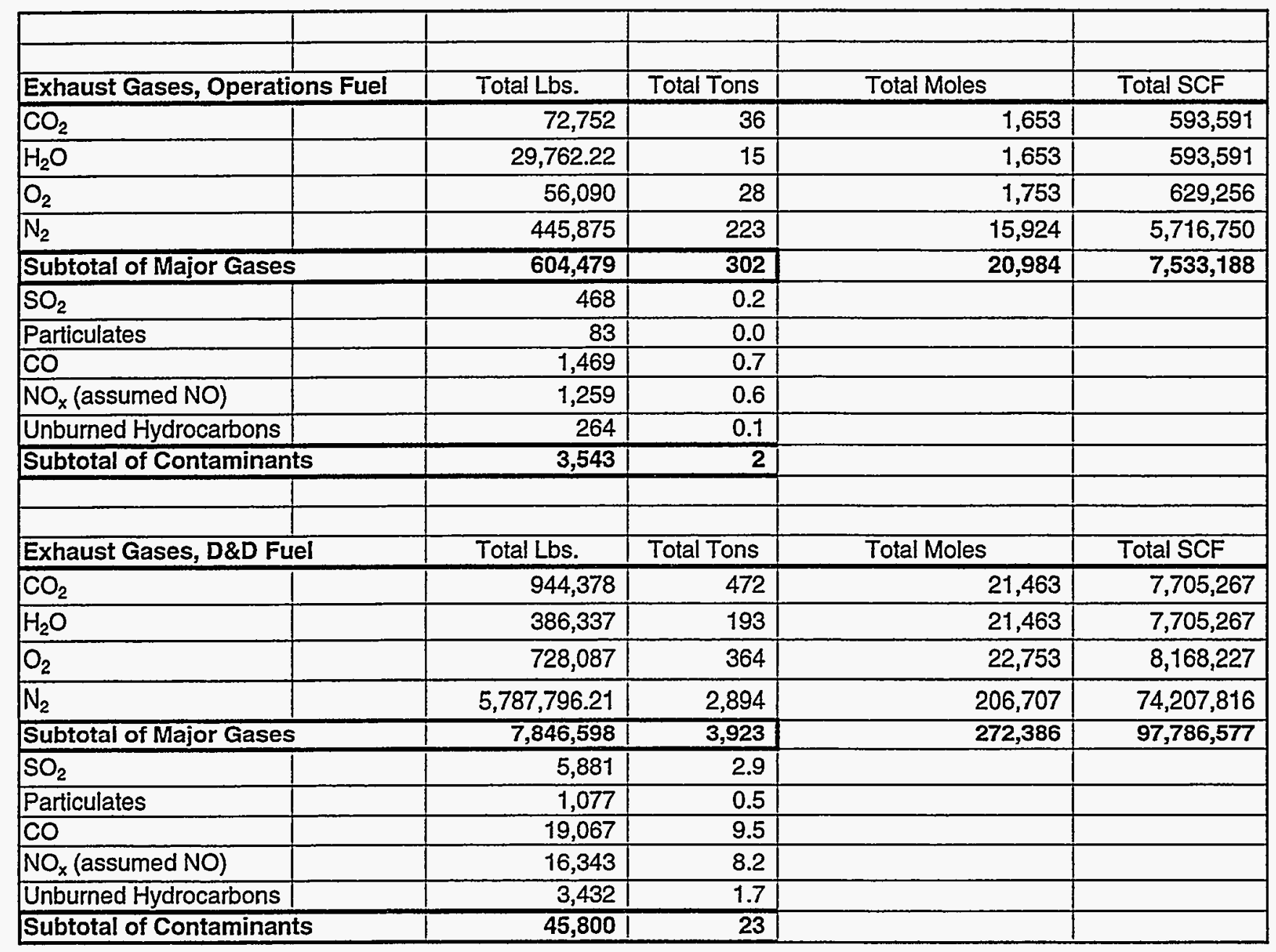




\begin{tabular}{|c|c|c|c|c|c|}
\hline \multicolumn{6}{|c|}{ Estimate of Diesel Engine Emissions } \\
\hline \multicolumn{6}{|c|}{ DCWO } \\
\hline \multicolumn{6}{|l|}{ Bases \& Assumptions: } \\
\hline \multirow{2}{*}{\multicolumn{2}{|c|}{$\begin{array}{l}\text { 1. Air to fuel ratio }=25: 1 \text { (Mass Basis) } \\
\text { 2. Diesel fuel density }=7.5 \mathrm{lbs} . / \text { gal. }\end{array}$}} & & & $\begin{array}{l}\text { from Wark, K. and C.F. } \\
\text { Warner, Air Pollution, Its } \\
\text { Origin and Control, IEP, } \\
\text { New York, 1976, p. 446, } \\
423\end{array}$ & \\
\hline & & & & & \\
\hline \multicolumn{6}{|c|}{ 3. Air is $21 \% \mathrm{O}_{2}, 79 \% \mathrm{~N}_{2}$, with a pseudomolecular weight of 29 . } \\
\hline \multicolumn{4}{|c|}{ 4. Combustion is simulated as: $\mathrm{C}_{9} \mathrm{H}_{18}+13.5 \mathrm{O}_{2} \rightarrow-9 \mathrm{CO}_{2}+9 \mathrm{H}_{2} \mathrm{O}$} & & \\
\hline \multicolumn{2}{|l|}{ 5. Particulates $=5 \mathrm{mg} / \mathrm{scf}$} & & & Wark and Warner, p. 446 & \\
\hline 6. $\mathrm{CO}=2,500 \mathrm{ppmv}$ & & & & Wark and Warner, p. 446 & \\
\hline 7. $\mathrm{NO}_{\mathrm{x}}=2,000 \mathrm{ppmv}$ & & & & Wark and Warner, p. 446 & \\
\hline \multicolumn{3}{|c|}{ 8. Unbumed hydrocarbons $=100 \mathrm{ppmv}$} & & Wark and Warner, p. 446 & \\
\hline \multicolumn{3}{|c|}{ 9. Diesel fuel (\#2 fuel oil) contains 1 wt. $\%$ sulfur } & & Wark and Warner, p. 336 & \\
\hline \multicolumn{6}{|c|}{ 10. Combustion is about $99 \%$ efficient. } \\
\hline & & & & & \\
\hline \multicolumn{2}{|l|}{ Lbs. Of Construction Fuel } & & & $1,380,802$ & \\
\hline Lbs. Of Operations Fuel & & & & - & \\
\hline Lbs. Of D\&D Fuel & & & & $4,188,564$ & \\
\hline \multirow[t]{2}{*}{ Total Lbs. of Fuel Used } & & & & $5,569,367$ & \\
\hline & & & & & \\
\hline \multicolumn{2}{|c|}{ Lb-Moles of Construction Fuel } & & & 10,959 & \\
\hline \multicolumn{2}{|l|}{ Lb-Moles of Operations Fuel } & & & - & \\
\hline Lb-Moles of D\&D Fuel & & & & 33,243 & \\
\hline \multicolumn{2}{|c|}{ Total Lb-Moles of Fuel (as C9H18) } & & & 44,201 & \\
\hline \multicolumn{3}{|c|}{ Lbs of Air for Construction Fuel (based on air-to-fuel ratio) } & & $34,520,057$ & \\
\hline \multicolumn{3}{|c|}{ Lbs. of Air for Operations Fuel (based on air-to-fuel ratio) } & & - & \\
\hline \multicolumn{3}{|c|}{ Lbs.of Air for D\&D Fuel (based on air-to-fuel ratio) } & & $104,714,107$ & \\
\hline Total Lbs. of Air Added & & & & $139,234,164$ & \\
\hline \multicolumn{3}{|c|}{\begin{tabular}{|l|} 
Lb-Moles of Air for Combustion Fuel \\
\end{tabular}} & & 1190.347 & \\
\hline \multicolumn{2}{|c|}{ Lb-Moles of Air for Operations Fuel } & & & - & \\
\hline \multicolumn{2}{|c|}{ Lb-Moles of Air for D\&D Fuel } & & & $3,610,831$ & \\
\hline Total Lb-Moles of Air & & & & $4,801,178$ & \\
\hline \multicolumn{2}{|c|}{ Grand Total of Materials Fed, Lbs. } & & & $144,803,531$ & \\
\hline \multicolumn{2}{|c|}{ Exhaust Gases, Construction Fuel } & Total Lbs. & Total Tons & Total Moles & Total SCF \\
\hline $\mathrm{CO}_{2}$ & & $4,296,268$ & 2,148 & 97,642 & $35,053,639$ \\
\hline $\mathrm{H}_{2} \mathrm{O}$ & & $1,757,564$ & 879 & 97,642 & $35,053,639$ \\
\hline $\mathrm{O}_{2}$ & & $3,312,293$ & 1,656 & 103,509 & $37,159,787$ \\
\hline $\mathrm{N}_{2}$ & & $26,330,471$ & 13,165 & 940,374 & $337,594,257$ \\
\hline Subtotal of Major Gases & & $35,696,596$ & 17,848 & $1,239,168$ & $444,861,322$ \\
\hline $\mathrm{SO}_{2}$ & & 27,616 & 13.8 & & \\
\hline Particulates & & 4,899 & 2.4 & & \\
\hline $\mathrm{CO}$ & & 86,742 & 43.4 & & \\
\hline $\mathrm{NO}_{\mathrm{x}}$ (assumed $\mathrm{NO}$ ) & & 74,350 & 37.2 & & \\
\hline Unbumed Hydrocarbons & & 15,614 & 7.8 & & \\
\hline Subtotal of Contaminan & & 209,221 & 105 & & \\
\hline
\end{tabular}




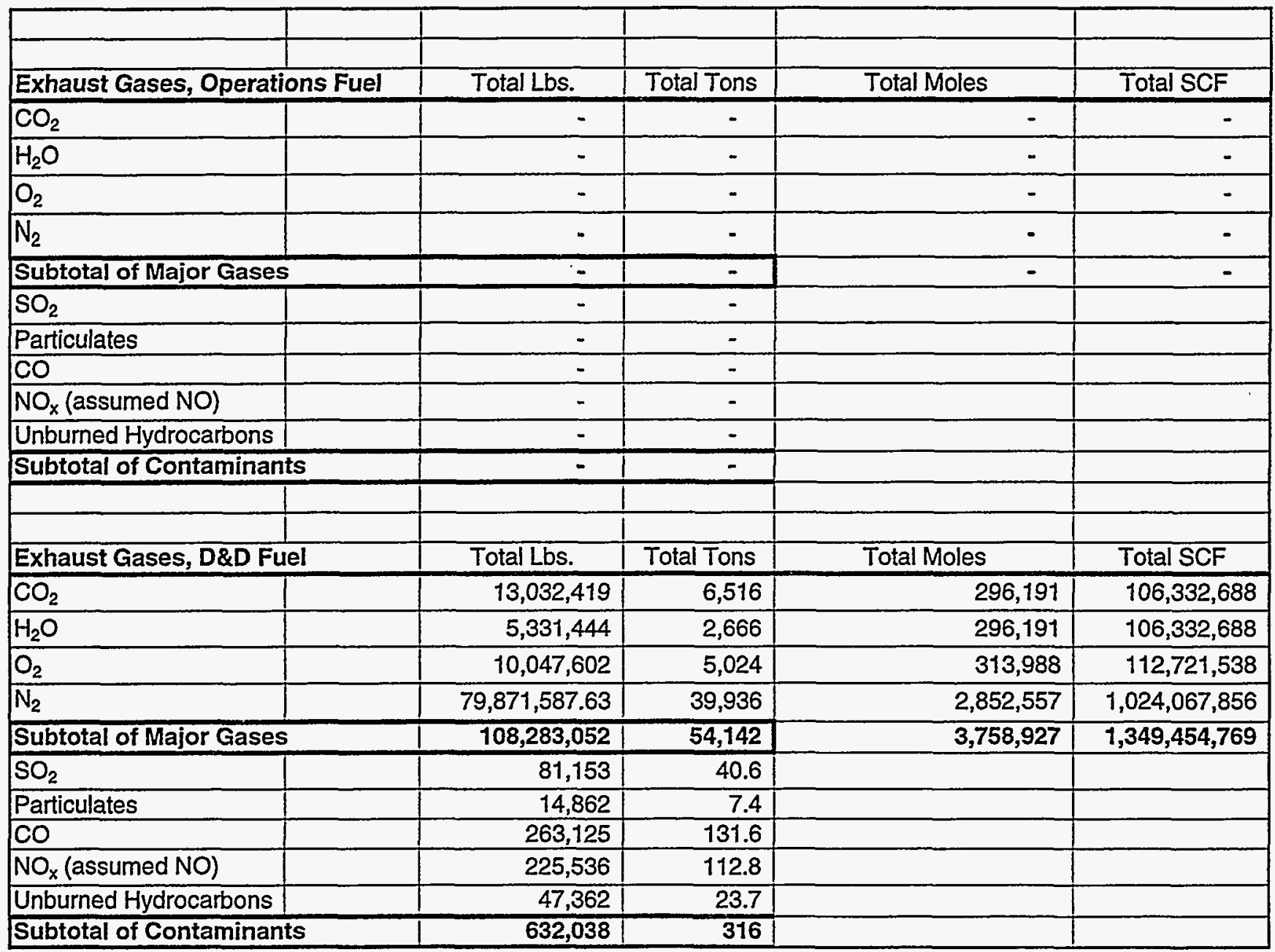

